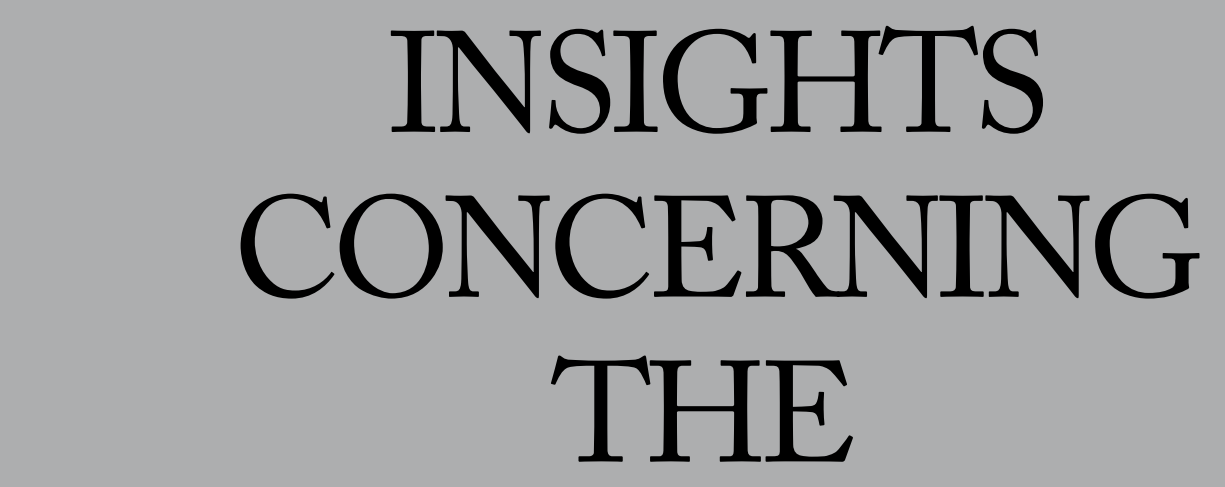

FUKUSHIMA DAIICHI

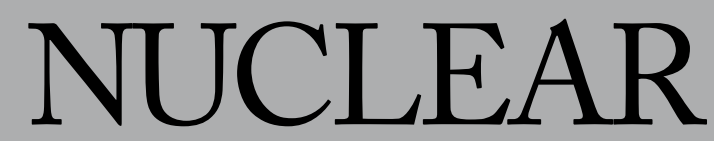

ACCIDENT

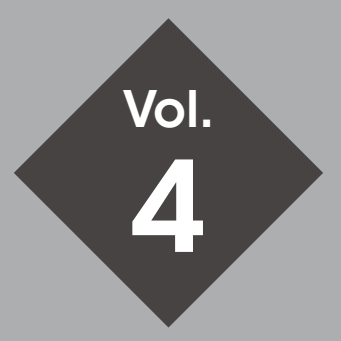

Endeavors by Scientists

Research and technical papers published in 2011-2016 in the Transactions of the Atomic Energy Society of Japan 


\section{Editor}

Editorial Committee, Atomic Energy Society of Japan

Editor-in-Chief: (Journal) Toyohiko Yano, (Bulletin) Tsutomu Sata

Associate Editor-in-Chief: (Journal) Toshihiko Ohnuki, Cheol Ho Pyeon

(Bulletin) Hidetoshi Okada, Masatoshi Kawashima

Special Working Group for this publication

Toshihiko Ohnuki, Toyohiko Yano, Naoki Yamano

PUBLISHED 2021

ISBN : 978-4-89047-182-9

DOI : 10.15669/fukushimainsights.Vol.4

Copyright @ Atomic Energy Society of Japan 2021. All right reserved.

Published by Atomic Energy Society of Japan

2-3-7, Shinbashi Minato-ku, Tokyo Japan

https://www.aesj.net

This book is not for sale.

This work is subject to copyright. All rights are reserved by the Atomic Energy Society of Japan whether the whole or part of the material is concerned, specifically the rights of translation reprinting reuse of illustrations, recitation, broad casting, reproduction on microfilms or in any other physical way, and transmission or information storage and retrieval, electronic adaptation computer software, or by similar or dissimilar methodology now known or hereafter developed. Exempted from this legal reservation are brief excerpt in connection with reviews or scholarly analysis or material supplied specifically for the purpose of being entered and executed on a computer system, for exclusive use by the purchaser of the work. Duplication of this publication or parts thereof is permitted only under the previsions of the Copyright Law of the Society's location, in its current version, and permission for use must always obtained from the Atomic Energy Society of Japan.

The use of general descriptive names, registered names, trademarks, service marks. etc. in this publication does not imply, even in the absence of a specific statement, that such names are exempt from the relevant protective laws and regulations and therefore free for general use.

While the advice and information in this book are believed to be true and accurate at the date of publication, neither the authors nor the editors can accept any legal responsibility for any errors or omissions that may be made.

Printed on acid-free paper

Printed by Sanbi Printing, Co. Ltd. 5-16-7, Nishi-Nippori, Arakawa-ku, Tokyo Japan https://www.sanbi.co.jp/company/corporate/ 


\section{Preface}

The March 11, 2011 off the Pacific coast of Tohoku Earthquake (also known as the Great East Japan Earthquake) and the severe nuclear accident that was initiated mainly by huge tsunami following the earthquake, inflicted enormous damage to a wide-spread area of Japan. The precious lives of nearly twenty thousand people were lost and a great number of residences and commercial buildings were destroyed by the 9.0 magnitude (Mw) earthquake, one of the greatest quakes in history, and its devastating tsunami. After almost a decade, a large number of people still continue to suffer because of this natural disaster and accident, and live in temporary housing as evacuees. Whereas reconstruction is proceeding in general, some areas have not been restored yet. The huge tsunami after the earthquake damaged equipment of the emergency core cooling systems such as diesel generators of the three nuclear reactors of Units 1 through 3 at the Tokyo Electric Power Company (TEPCO) Fukushima Daiichi Nuclear Power Plant. The subsequent failure of the reflooding resulted in multiple large-scale core meltdowns of Units 1 through 3 under station blackout. During these events, many kinds of radionuclides in large quantities including ${ }^{131} \mathrm{I}$ (estimated at about $150 \mathrm{PBq}$ ) and ${ }^{137} \mathrm{Cs}$ (13 PBq) were released into the atmosphere. This multiple large-scale core meltdown accident is one of the worst nuclear accidents in the world up to now.

After the accident, the Editorial Committee of the Atomic Energy Society of Japan published a large number of commentaries on the various aspects related to the accident through the Society's monthly Japanese language bulletin, Journal of the Atomic Energy Society of

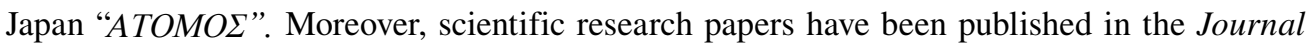
of Nuclear Science and Technology in English, and also in the Transactions of the Atomic Energy Society of Japan in Japanese; both are scientific journals of the Atomic Energy Society of Japan. Within these Fukushima Daiichi nuclear accident-related articles, those written in Japanese were crucial to understanding the situation at that time inside Japan, but they are not easily accessed and understood by persons worldwide. To remedy this situation, we as the Editorial Committee of the Atomic Energy Society of Japan decided to translate them into English and open them to researchers and the general public everywhere. We believe how the accident was tackled and the lessons learned from it are worth considering for preparing countermeasures against severe accidents and for pursuing safer and more secure nuclear power plants.

The collected commentaries and research articles were published in the five-year period beginning just after the accident. Each document has a "chronicle-like importance". What we thought, what we were anxious about, what we expected, what we believed, and what we should do to tackle the accident during that time line were recorded. Professionals in various academic fields were asked to provide these commentaries that represented the most up-todate information for the scientific community and the public. These records trace the recovery from this serious accident. Official reports on the nuclear accident at TEPCO's Fukushima Daiichi Nuclear Power Plant had been published by the Japanese Government, the IAEA, 
and the Atomic Energy Society of Japan; however it was not clear from these reports what we thought or what we were anxious about or what we wanted most to tackle at each time in Japan. Most of the commentaries were written not only for members of the Society but also for the public, including both those who were victims of the accident and those who were residents in Japan. The series of volumes that collected these articles has been entitled "Insights Concerning the Fukushima Daiichi Nuclear Accident, -Five Years' Comprehensive Archive Reports by Scientists and Engineers Published in Japanese from the Atomic Energy Society of Japan-". Each volume is entitled and includes selected articles as below.

\section{Volume 1: Fears and Concerns Just After the Accident, and Anxiety about Radiation}

Articles published in $A T O M O \Sigma$ within one year after the accident, and articles on radiation effects on human published in ATOMOS from April 2012 to 2016

Volume 2: Environmental Effects and Reconsideration of Nuclear Safety Articles on atmospheric dispersion, environmental remediation, decommissioning technology, nuclear safety and regulation published in ATOMO from April 2012 to FY2016

Volume 3: Impacts on the Public

Articles on social science published in ATOMOE from April 2012 to 2016

Volume 4: Endeavors by Scientists

Research and technical papers published in 2011-2016 in the Transactions of the Atomic Energy Society of Japan

Five years after the accident, many related articles still continued to be published. However, we thought that the situation during these first five years was more serious and, therefore it was more important to remember the articles appearing during this time. Many research papers published in the Journal of Nuclear Science and Technology (JNST) were written in English, and we have excluded them from these volumes. We ask interested readers to refer to these articles on the JNST journal web page, particularly in the special issues on the Fukushima Daiichi Nuclear Accident or papers identified as "Fukushima Daiichi NPP Accident related".

As we approach the end of the first quarter of the 21st century, populations are increasing rapidly in developing countries and improved living standards in those areas are sharply increasing energy consumption. At the same time, fears of great global environmental problems due to the consumption of fossil fuels are taking root, and there are also fears of food and safe water shortages. Among those issues, it is well accepted that an increase in atmospheric $\mathrm{CO}_{2}$ concentration is causing global warming and abnormal weather patterns that may cause serious damage to life as we know it on the Earth. To reduce emissions of greenhouse gases while securing the energy necessary for our life is a great challenge for all of us. Moreover, at present coronavirus COVID-19 is spreading worldwide rapidly, and more than 82 million people were suffered and 1.8 million people have passed away as of the end of 2020, and numbers still rapidly increasing. A higher quality of life supported by sufficient energy also can reduce susceptibility to unknown diseases. In these regards, there were great expectations 
for nuclear energy as well as renewable energies. The most dangerous aspect of nuclear energy was revealed to the public by the Fukushima Daiichi nuclear accident. We must humbly recognize the risks involved in the enormous energy release from the atomic nucleus, and then, reconstruct a safer nuclear system than ever before, in light of the important lessons learned from the 2011 accident. We believe the collected expertise in the volumes of this series forms an indispensable history for that purpose.

Finally, we would like to express our deep gratitude to Mrs. Kumiko Kishimoto of the Atomic Energy Society of Japan for her helpful assistance in all stages of this publication.

\author{
Toshihiko Ohnuki \\ Toyohiko Yano \\ Naoki Yamano
}

Note: The contents of Volumes 1 to 3 are only selected articles from the Journal of the Atomic Energy Society of Japan, ATOMOE. Original Japanese articles can be accessed free of charge through J-STAGE website with color figures. Besides articles included into these volumes, so many commentaries/opinions/technical reports related to the Fukushima Daiichi Nuclear Plant Accident were published in $A T O M O \Sigma$ from aftermath of the accident to date.

https://www.jstage.jst.go.jp/browse/jaesjb/63/2/_contents/-char/en

About the original Japanese papers in Volume 4, you can also access free of charge through web page of the Transactions of the Atomic Energy Society of Japan in J-STAGE.

https://www.jstage.jst.go.jp/browse/taesj/-char/en

Fukushima Daiichi Nuclear Plant Accident related papers already translated into English were excluded from this volume. 


\section{Administrative Map of East Part of Fukushima Prefecture}

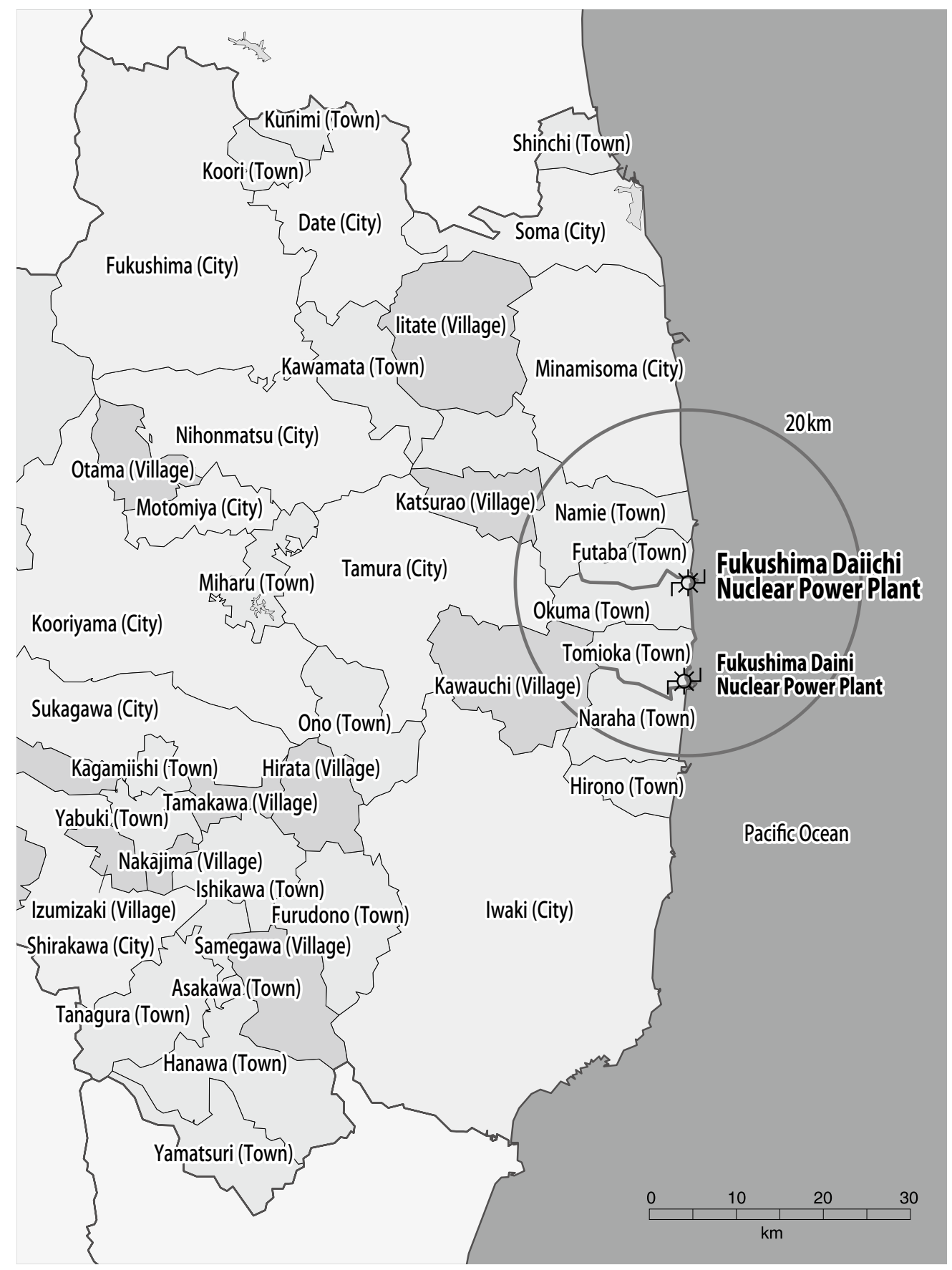




\section{CONTENTS OF VOLUME 4}

Survey of Living Environmental Land Contaminated with Radioactive Materials due to Fukushima Daiichi Nuclear Plant Accident

Sin-ya Hohara, Masayo Inagaki, Kiyoshi Kojima, Hirokuni Yamanishi, Genichiro Wakabayashi, Wataru Sugiyama and Tetsuo Itoh

$($ March, 2011)*

Evaluation of Averted Doses to Infants by Tap Water Restrictions after the Fukushima Daiichi Nuclear Power Plant Accident

Sakae Kinase, Masanori Kimura, Shogo Takahara and Toshimitsu Homma

(March, 2011)*

Time Variations in Dose Rate and $\gamma$ Spectrum Measured at Tsukuba City, Ibaraki, due to the Accident of Fukushima Daiichi Nuclear Power Station

Toshiya Sanami, Shinichi Sasaki, Kazuhiko Iijima, Yuji Kishimoto and Kiwamu Saito

(March, 2011)*

Removal of Radioactive Cesium from Surface Soils Solidified Using Polyion Complex -Rapid Communication for Decontamination Test at Iitate-mura in Fukushima Prefecture- Hirochika Naganawa, Noriyuki Kumazawa, Hiroshi Saitoh, Nobuyuki Yanase, Hisayoshi Mitamura, Tetsushi Nagano, Kaoru Kashima, Tatsuya Fukuda, Zenko Yoshida and Shun-ichi Tanaka (April, 2011)*

Measurement and Evaluation of Hydrogen Production from Mixtures of Seawater and Zeolite in Decontamination of Radioactive Water

-.-Yuta Kumagai, Ryuji Nagaishi, Atsushi Kimura, Mitsumasa Taguchi, Kenji Nishihara, Isao Yamagishi and Toru Ogawa

(April, 2011)*

\section{Analysis of BWR Station Blackout Accident}

-Thermal-Hydraulic Behavior up to Severe Core Damage in Fukushima Daiichi Power Plant Unit 2-

\section{Local Area Distribution of Fallout Radionuclides from Fukushima Daiichi Nuclear} Power Plant Determined by Autoradiography Analysis

-.-.- Fuminori Sakamoto, Toshihiko Ohnuki, Naofumi Kozai, Shosuke Igarashi, Shinya Yamasaki, Zenko Yoshida and Shunichi Tanaka (January, 2012)*

\section{Study on Public Awareness of Utilizing Nuclear Power in China}

-Changes in Public Awareness after the Accident of Fukushima Daiichi Nuclear Power Plants- Ting Xu and Toshio Wakabayashi (January, 2012)*

Effects of Ground Surface Decontamination on the Air Radiation Dose Rate -Results of a Decontamination Trial at a Playground Lot in a Fukushima Residential Area- 
Effect of Gamma Ray Irradiation on Deoxygenation by Hydrazine in Artificial Seawater

Practical Approach to Decontamination of Radioactive Cesium-Contaminated Matter in Agricultural Region by Improved Wet Classification and Use of Geomaterials Kenichi Ito, Hidetaka Miyahara, Toru Ujiie, Toshikatsu Takeshima, Shingo Yokoyama, Kotaro Nakata, Tetsushi Nagano, Tsutomu Sato, Tamao Hatta and Hirohisa Yamada (April, 2012)*

Testing of Multistep Soil Washing for Radiocesium-Contaminated Soil Containing Plant Matter

Long-Term Outlook for Global Natural Uranium and Uranium Enrichment Supply and Demand Situations after the Impact of Fukushima Daiichi Nuclear Power Plant Accident

Corrigendum: Survey of Living Environmental Land Contaminated with Radioactive Materials due to Fukushima Daiichi Nuclear Power Plant Accident Sin-ya Hohara, Masayo Inagaki, Kiyoshi Kojima, Hirokuni Yamanishi, Genichiro Wakabayashi, Wataru Sugiyama and Tetsuo Itoh (April, 2012)*

Design, Construction and Monitoring of Temporary Storage Facilities for Removed Contaminants

Hiromitsu Saegusa, Hironori Funaki, Hiroshi Kurikami, Yoshiaki Sakamoto and Takayuki Tokizawa (January, 2013)*

Changing Information Needs of Social Impact of Nuclear Power Plant Siting -Through a Comparison Before and After the Fukushima Daiichi Nuclear Power Plant Accident-- Takako Kashiwa and Yoshimi Kawamoto

Determination of Local-Area Distribution and Relocation of Radioactive Cesium in Trees from Fukushima Daiichi Nuclear Power Plant by Autoradiography Analysis -.. Fuminori Sakamoto, Toshihiko Ohnuki, Naofumi Kozai, Shinya Yamasaki, Zenko Yoshida and Kenji Nanba (April, 2013)*

Thermodynamic Evaluation of Sea Water to Degraded Nuclear Fuel in Severe Accident of LWR

Estimation of Radionuclide Concentration in Plume Using Pulse Height Distribution Measured by $\mathrm{LaBr}_{3}$ Scintillation Detector and Its Response to Radionuclides in Plume Calculated with egs5 
Leaching of Cs and Sr from Sewage Sludge Ash Buried in a Landfill Site

Periodical Public Opinion Survey on Nuclear Energy (Inhabitants Living in the Tokyo Metropolitan Area)

Transfer Behavior of Cesium Adsorbed on Clay Minerals in Aqueous Solution

Yoshihiro Okamoto, Takeshi Osugi, Hideaki Shiwaku and Mitsuo Akabori

Estimation of I-131 Concentration Using Time History of Pulse Height Distribution at Monitoring Post and Detector Response for Radionuclide in Plume

Hideo Hirayama, Masatsugu Kawasaki, Hiroshi Matsumura, Takehisa Ohkura, Yoshihito Namito, Toshiya Sanami, Mitsumasa Taki, Tetsuya Oishi and Michio Yoshizawa

(March, 2014)*

Treatment of Water Contaminated with Radiocesium Using Novel Complexes between Prussian-Blue and Bivalent Transition Metal Hydroxides

-.---.- Hisashi Ubara, Eiji Yuki, Kazuki Tsukiyama, Masato Kamizawa, Koichi Senoh, Kohei Ohta, Fumio Shimamoto and Nariyuki Ohta (April, 2014)*

Estimation of Time History of I-131 Concentration in Air Using NaI(TI) Detector Pulse Height Distribution at Monitoring Posts in Fukushima Prefecture Hideo Hirayama, Hiroshi Matsumura, Yoshihito Namito and Toshiya Sanami (January, 2015)*

Effect of $\mathrm{B}_{4} \mathrm{C}$ Absorber Material on Melt Progression and Chemical Forms of Iodine or Cesium under Severe Accident Conditions

Akihide Hidaka (January, 2015)*

Survey of Tsuruga Inhabitants Concerning Radiation and Its Risks

Yoshihiko Shinoda and Naoki Yamano (February, 2015)*

Rapid and Simple Measurement Method of ${ }^{90} \mathrm{Sr}$ Concentration in Water by Measuring $\beta$-rays from ${ }^{90} \mathbf{Y}$

- Hideo Hirayama, Kenjiro Kondo, Yasuhiro Unno, Hiroshi Matsumura, Hiroshi Iwase, Akira Yunoki and Shinichi Sasaki (March, 2015)*

Simple Measurement Method with High Detection Efficiency to Evaluate Sr-90 Concentration in Water by Measuring $\beta$ rays from Y-90 Using GM-Detector Kenjiro Kondo, Hideo Hirayama, Masafumi Taira, Hiroshi Matsumura, Hiroshi Iwase and Shinichi Sasaki (March, 2015)*

Multi-Physics Particle Method for the Simulation of Severe Accidents in Nuclear Power Plants 
Development of a Filtered Containment Venting System for Nuclear Power Plants

Shinichi Kawamura, Takeo Kimura, Shuichi Omori and Tadashi Narabayashi

(January, 2016)*

Measures to Improve Robustness of Primary Containment Vessel and Operation of Filtered Containment Venting System Based on the Lessons from the Containment Leak at Fukushima Dai-ichi Nuclear Power Station Unit 2

Shinichi Kawamura, Suguru Ooki and Tadashi Narabayash

(February, 2016)*

Improved Nuclear Emergency Management System Reflecting Lessons Learned from the Emergency Response at Fukushima Daini Nuclear Power Station after the Great East Japan Earthquake

Shinichi Kawamura and Tadashi Narabayash (February, 2016)*

Photon Spectra Measured above Operating Floor of Unit 3 Reactor at Fukushima Daiichi Nuclear Power Station

-.-.--.--..- Yoshihiko Tanimura, Hideo Hirayama, Kenjiro Kondo, Hiroshi Nagata, Kouhei Iwanaga, and Seishirou Suzuk

(March, 2016)*

Estimation of Radionuclide Intakes by Singular Value Decomposition

Development of an Organic Iodine Filter for Filtered Containment Venting Systems of Nuclear Power Plants

Shinichi Kawamura, Takeo Kimura, Fumitoshi Watanabe, Kazuki Hirao and Tadashi Narabayashi

* Original date of Publishing in ATOMO $\Sigma$ (in Japanese) 


\title{
Survey of Living Environmental Land Contaminated with Radioactive Materials due to Fukushima Daiichi Nuclear Plant Accident
}

\author{
Sin-ya HOHARA ${ }^{1, *}$, Masayo INAGAKI ${ }^{1}$, Kiyoshi KOJIMA ${ }^{1}$, \\ Hirokuni YAMANISHI ${ }^{1}$, Genichiro WAKABAYASHI ${ }^{1}$, \\ Wataru SUGIYAMA ${ }^{1}$ and Tetsuo ITOH $^{1}$ \\ ${ }^{1}$ Kinki University Atomic Energy Research Institute, 3-4-1 Kowakae, Higashi-osaka-shi, Osaka 577-8502, Japan
}

\begin{abstract}
Radioactive materials were released to the general environment due to the accident at the Fukushima Daiichi Nuclear Power Plant. The released radioactive materials fell and contaminated the land mainly in the Tohoku and Kanto areas of Japan. We surveyed the air dose rates in relation to the pave condition of the land, and investigated the contamination level in some nonpaved areas at the center of Fukushima City, Koriyama City, and Nasushiobara City. From the survey results, the dose rates of the nonpaved areas were found to be higher than those of the paved areas, and the dose rates of the paved areas depend on the paving materials of the area. The contamination level of the nonpaved area in Nasushiobara City was below the regulation level of specific activities in a radiation-controlled area in Japan. However, the contamination levels in the nonpaved areas in Fukushima City and Koriyama City were above the regulation level.
\end{abstract}

KEYWORDS: Fukushima Daiichi, contamination level, living environment, dose rate, pave condition

\section{Introduction}

As a result of the magnitude 9.0 earthquake off the Pacific Coast of Tohoku on March 11, 2011 and the subsequent tsunami, the residual heat removal system (RHRS) of the Fukushima Daiichi Nuclear Plant (hereinafter referred to as "1F") owned by Tokyo Electric Power Co., Inc. experienced functional failure ${ }^{1)}$. This failure resulted in the destruction of the building of Unit 1 of $1 \mathrm{~F}$ by an explosion at 15:36 on March 12th, 2011 ${ }^{2)}$ and the building of Unit 3 was also destroyed by another explosion at 11:01 on March 14th ${ }^{3)}$. The building of Unit 4 was also damaged by a fire ${ }^{4)}$, and Unit 2 experienced damage to its suppression chamber as well, due to the explosion ${ }^{5}$. The series of explosions and damage to the buildings released a significant amount of radioactive material into the general environment, and the soils of the Fukushima Prefecture and the Tohoku and Kanto areas became contaminated.

* Corresponding author, E-mail: hohara@kindai.ac.jp

DOI : 10.15669 /fukushimainsights.Vol.4.1

(C) 2021 Atomic Energy Society of Japan. All rights reserved.

Originally published in Transactions of the Atomic Energy Society of Japan (ISSN 1347-2879), Vol. 10, No. 3, p.145-148

(2011) in Japanese. (Japanese version accepted: May 2, 2011) 
An increased air dose rate caused by this radioactive contamination was immediately observed in various locations ${ }^{6}$, and since the incident, the air dose rate has been continuously monitored and opened for public. Furthermore, the density of the radioactive material contained in agricultural soils around Fukushima Prefecture was measured to monitor the potential influence on agricultural crops ${ }^{7,8)}$. The monitoring/measurements were conducted by local governments in various regions, and action directives for the residents were issued based on the observed radiation values.

However, the dynamic status of radioactive materials in the environment is not uniform because it depends significantly on peripheral factors. The environmental radiation dose rate investigation revealed the existence of places where air dose rates were higher than in the surrounding areas ${ }^{9-12}$. Therefore, a portable radiation position measurement system was used to follow the activity in locations with higher dose rates ${ }^{13)}$, and an exploration of high dose rate spots in the dynamic environment was conducted. Furthermore, in spots where a significantly higher dose rate was observed than in the surroundings, the soil was sampled, and radionuclide analyses were conducted. In this paper, the results of the air dose rate measurements and soil nuclide analyses are reported.

\section{Measurement Methodology and Locations}

A simplified radioactivity position measuring system consisting of a $\mathrm{NaI}(\mathrm{Tl})$ survey meter (Aloka: TCS-171) and a GPS receiver ${ }^{13)}$ was used. The air dose rate was measured once per second, and the results were displayed on a map for further analyses.

The survey meter probe was installed 50-60 $\mathrm{cm}$ above ground level, and the air dose rate was measured while moving at a walking speed of approximately $100 \mathrm{~m} / \mathrm{min}$. Measurements were made on April 16th and 17th, 2011 at three locations: Kuroiso Park, Nasushiobara City, Tochigi Prefecture $\left(100 \mathrm{~km}\right.$ southwest of $1 \mathrm{~F}$, at approx. $36.976^{\circ} \mathrm{N}$ and $\left.140.053^{\circ} \mathrm{E}\right)$, Hayama Park, Koriyama City, Fukushima Prefecture (60 km west of 1F, at approx. $37.395^{\circ} \mathrm{N}$ and $140.375^{\circ}$ E), and Arakawa Athletic Park, Fukushima City, Fukushima Prefecture (60 km northwest of $1 \mathrm{~F}$ at approx. $37.745^{\circ} \mathrm{N}$ and $140.461^{\circ} \mathrm{E}$ ). The approximate size of the surveyed areas was respectively $100 \mathrm{~m} \times 300 \mathrm{~m}, 130 \mathrm{~m} \times 300 \mathrm{~m}$ and $160 \mathrm{~m} \times 750 \mathrm{~m}$. The amount of precipitation per unit area for each location was considered to be equal because the survey areas were not large. Additionally, the weather during the measurements was clear at all locations.

Where the dose rate was significantly higher than in the surroundings, the top $1 \mathrm{~cm}$ of surface soil was sampled from an area of $30 \times 30 \mathrm{~cm}$ and sealed in a U-8 container at the site. The surface of the container was cleaned at the site and again at Kinki University and double-sealed with vinyl bags. The mass of the sampled soil was $160.9 \mathrm{~g}$ at Kuroiso Park, Nasushiobara, Tochigi Prefecture, 174.7 g at Hayama Park, Koriyama City, Fukushima Prefecture, and $149.5 \mathrm{~g}$ at Arakawa Athletic Park, Fukushima City, Fukushima Prefecture. The $\gamma$-ray spectra of the prepared soil samples were measured using a high-purity Ge detector at the Kinki University Atomic Energy Research Institute on April 18th, 2011, and a nuclide analysis was performed using the nuclide library of SEIKO EG\&G.

\section{Results and Discussion}

Measurements of air dose rates were classified by land surface conditions and observed for 
1 min (60 data points) with no significant fluctuation, and the maximum, minimum, and average air dose rates were obtained. The air dose rates are shown in Figures 1-3, where the plotted points are the average and the bars on the plotted points indicate the maximum and minimum of the 60 data points. Figure 1 shows the measurements taken at Kuroiso Park, Nasushiobara City, Tochigi Prefecture, Figure 2 shows the data from Hayama Park, Koriyama City, Fukushima Prefecture, and Figure 3 displays the data from Arakawa Athletic Park, Fukushima City, Fukushima Prefecture. The results show the trend of higher air dose rates above non-paved land surfaces and lower rates above paved areas.

Comparison of the air dose rates over soil and asphalt land surfaces shows that the soil locations exhibited approximately 1.4-fold higher values in the Kuroiso Park neighborhood,

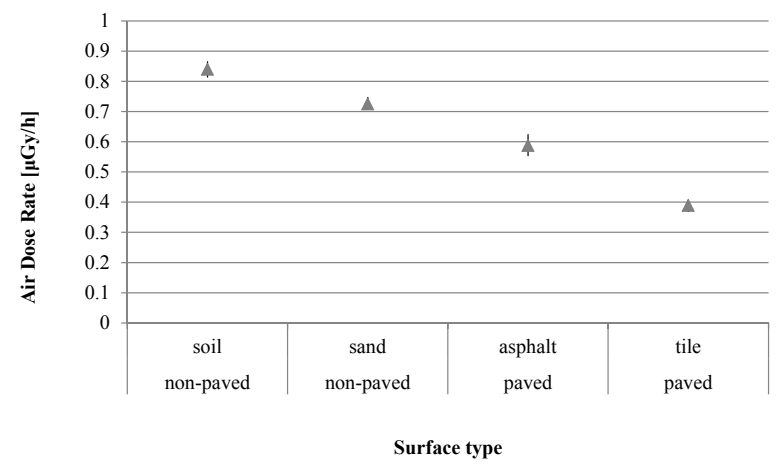

Figure 1 Air dose rate in the Kuroiso Park neighborhood, Nasushiobara City

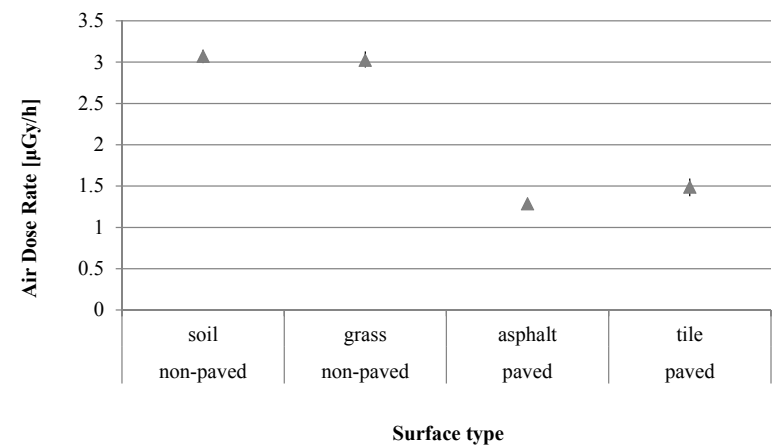

Figure 2 Air dose rate in the Hayama Park neighborhood, Koriyama City

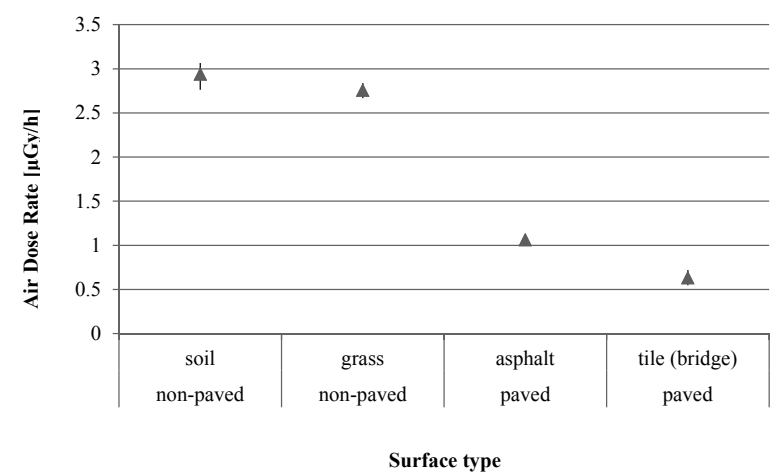

Figure 3 Air dose rate in the Arakawa Athletic Park neighborhood, Fukushima City 
Table 1 Specific activity of soil samples

\begin{tabular}{cccccc}
\hline Nuclide & $\begin{array}{c}\text { Half-life } \\
{[\text { day }]}\end{array}$ & $\begin{array}{c}\text { Nasushiobara city } \\
\text { Kuroiso park } \\
{[\mathrm{Bq} / \mathrm{kg}]}\end{array}$ & $\begin{array}{c}\text { Koriyama city } \\
\text { Hayama park } \\
{[\mathrm{Bq} / \mathrm{kg}]}\end{array}$ & $\begin{array}{c}\text { Fukushima city } \\
\text { Arakawa Athletic Park } \\
{[\mathrm{Bq} / \mathrm{kg}]}\end{array}$ & $\begin{array}{c}\text { Regulation level* } \\
{[\mathrm{Bq} / \mathrm{kg}]}\end{array}$ \\
\hline${ }^{95} \mathrm{Nb}$ & 35 & $2.87 \mathrm{E} 1 \pm 0.80 \mathrm{E} 1$ & $7.21 \mathrm{E} 1 \pm 0.02 \mathrm{E} 1$ & $1.12 \mathrm{E} 2 \pm 0.25 \mathrm{E} 2$ & $1.0 \mathrm{E} 4$ \\
${ }^{131} \mathrm{I}$ & 8 & $5.48 \mathrm{E} 2 \pm 0.16 \mathrm{E} 2$ & $3.51 \mathrm{E} 3 \pm 0.04 \mathrm{E} 3$ & $1.90 \mathrm{E} 4 \pm 0.01 \mathrm{E} 4$ & $1.0 \mathrm{E} 5$ \\
${ }^{129} \mathrm{Te}$ & 0.048 & $1.98 \mathrm{E} 3 \pm 0.18 \mathrm{E} 3$ & $5.74 \mathrm{E} 3 \pm 0.35 \mathrm{E} 3$ & $2.04 \mathrm{E} 4 \pm 0.06 \mathrm{E} 4$ & $1.0 \mathrm{E} 5$ \\
${ }^{129 \mathrm{~m} T e}$ & 34 & $2.68 \mathrm{E} 3 \pm 0.28 \mathrm{E} 3$ & $9.11 \mathrm{E} 3 \pm 0.56 \mathrm{E} 3$ & $3.26 \mathrm{E} 4 \pm 0.10 \mathrm{E} 4$ & $1.0 \mathrm{E} 5$ \\
${ }^{132} \mathrm{Te}$ & 3 & $2.44 \mathrm{E} 1 \pm 0.75 \mathrm{E} 1$ & $8.65 \mathrm{E} 1 \pm 1.54 \mathrm{E} 1$ & $2.29 \mathrm{E} 2 \pm 0.29 \mathrm{E} 2$ & $1.0 \mathrm{E} 4$ \\
${ }^{134} \mathrm{Cs}$ & 753 & $3.98 \mathrm{E} 3 \pm 0.04 \mathrm{E} 3$ & $1.71 \mathrm{E} 4 \pm 0.01 \mathrm{E} 4$ & $4.33 \mathrm{E} 4 \pm 0.01 \mathrm{E} 4$ & $1.0 \mathrm{E} 4$ \\
${ }^{136} \mathrm{Cs}$ & 13 & $1.54 \mathrm{E} 2 \pm 0.10 \mathrm{E} 2$ & $6.07 \mathrm{E} 2 \pm 0.19 \mathrm{E} 2$ & $1.61 \mathrm{E} 3 \pm 0.04 \mathrm{E} 3$ & $1.0 \mathrm{E} 4$ \\
${ }^{137} \mathrm{Cs}$ & 11020 & $4.46 \mathrm{E} 3 \pm 0.04 \mathrm{E} 3$ & $1.92 \mathrm{E} 4 \pm 0.01 \mathrm{E} 4$ & $4.89 \mathrm{E} 4 \pm 0.01 \mathrm{E} 4$ & \\
\hline
\end{tabular}

* minimum specific activity that defines a radiation area to be controlled under Japanese regulations ${ }^{14)}$

2.4-fold higher values at Hayama Park, and 2.8-fold higher values at Arakawa Athletic Park. This clearly shows a trend of higher levels of residual radioactive material at non-paved locations compared to the levels at paved locations.

Table 1 shows the nuclide analyses of the sampled soils. Radioactive materials, including ${ }^{137} \mathrm{Cs}$ and ${ }^{131} \mathrm{I}$ considered to have been released from $1 \mathrm{~F}$, were detected in all soil samples. Comparison of the detected radioactive material concentration with the specific isotope concentration (hereinafter referred to as the "lower limit concentration") ${ }^{14)}$ that releases the amount of radioactivity stipulated in Article 1 of the "Order for Enforcement of Act on Prevention of Radiation Disease Due to Radioactive Isotopes, etc". shows that the concentrations were below the lower limits for the detected nuclides at Kuroiso Park, Nasushiobara City, Tochigi Prefecture. However, the samples from Hayama Park, Koriyama City, Fukushima Prefecture and Arakawa Athletic Park, Fukushima City, Fukushima Prefecture showed concentrations above the lower limit for ${ }^{134} \mathrm{Cs}$ and ${ }^{137} \mathrm{Cs}$.

These measurements were conducted approximately 1 month after the release of a large amount of radioactive material from $1 \mathrm{~F}$, and the nonuniform pattern in the detected residual radioactive materials is considered to be the result of the precipitation amount of the radioactive materials, the dynamic status of the local conditions and soil properties, the deposition process of radioactive materials, and the wider distribution or outflow of radioactive materials due to rainfall, strong winds, and other environmental processes. The contribution of these processes to the dynamic status of the observed radioactive materials in the environment will be the subject of a future study.

\section{Conclusions}

A trend of higher air dose rates in non-paved areas compared to those observed in paved areas was observed approximately one month following contamination by radioactive materials. Furthermore, the nuclide analyses of soil specimens from the non-paved areas where contamination occurred showed that soil contamination exceeding the lower limit occurred in the non-paved locations in residential districts in Fukushima and Koriyama Cities as of April 17 th, 2011.

The places where we conducted the measurements were $60-100 \mathrm{~km}$ away from $1 \mathrm{~F}$, where soil contamination due to the accident was comparatively minor. However, a difference in air dose rate based on the land surface pavement status was observed. The data quantifying the difference in air dose rates associated with land surface pavement status will be helpful for future efforts to reduce air and exposure dose rates. 
Because this event is ongoing, we will continue to monitor the air dose rate, sample relevant soils, and perform nuclide analyses.

\section{References}

1) Nuclear and Industrial Safety Agency, Jishin Higai Jyoukyou, News Release, Dai 8 Hou, Ministry of Economy, Trade and industry, 12 Mar. 2011. [in Japanese]

2) Nuclear and Industrial Safety Agency, Jishin Higai Jyoukyou, News Release, Dai 16 Hou, Ministry of Economy, Trade and industry, 12 Mar. 2011. [in Japanese]

3) Nuclear and Industrial Safety Agency, Fukushima Daiichi Genshiryoku Hatsudensho 3 Gouki no Bakuhatsu Hassei nikakaru Houkoku nitsuite, News Release, Dai 1 Hou, Ministry of Economy, Trade and industry, 14Mar. 2011. [in Japanese]

4) Nuclear and Industrial Safety Agency, Jishin Higai Jyoukyou, News Release, Dai 24 Hou, Ministry of Economy, Trade and industry, 15 Mar. 2011. [in Japanese]

5) Nuclear and Industrial Safety Agency, Fukushima Daiichi Genshiryoku Hatsudensho 2 Gouki no Jyoukyou nitsuite, News Release, Ministry of Economy, Trade and industry, 15 Mar. 2011. [in Japanese]

6) Ministry of Education, Culture, Sports, Science and Technology, Kankyou Housyanou Suijyun Tyousa, Ministry of Education, Culture, Sports, Science and Technology, 15 Mar. 2011. [in Japanese]

7) Fukushima Prefecture, Nourin Suisan Bu, Fukushima kennai Kaku Shityouson no Dojyou niokeru Housyasei Busshitsu no Sokutei Kekka (Suiden/Tenkan Batake), Fukushima Prefecture, Nourin Suisan $\mathrm{Bu}, 6$ Apr. 2011. [in Japanese]

8) Fukushima Prefecture, Nourin Suisan Bu, Dai 2 Kai Fukushima kennai Kaku Shityouson no Nouyouchi Dojyou niokeru Housyasei Bussitsu no Sokutei Kekka, Fukushima Prefecture, Nourin Suisan Bu, 12 Apr. 2011. [in Japanese]

9) Fukushima Prefecture, Kankyou Housyasen Monitoring Mesh Tyousa Sokuhouchi, Fukushima Prefecture, 4 Gatsu 12 Nichi Tyousa Bun, 14 Apr. 2011. [in Japanese]

10) Fukushima Prefecture, Kankyou Housyasen Monitoring Mesh Tyousa Sokuhouchi, Fukushima Prefecture, 4 Gatsu 13 Nichi Tyousa Bun, 14 Apr. 2011. [in Japanese]

11) Fukushima Prefecture, Kankyou Housyasen Monitoring Mesh Tyousa Sokuhouchi, Fukushima Prefecture, 4 Gatsu 14 Nichi Tyousa Bun, 15 Apr. 2011. [in Japanese]

12) Fukushima Prefecture, Kankyou Housyasen Monitoring Mesh Tyousa Sokuhouchi, Fukushima Prefecture, 4 Gatsu 15/16 Nichi Tyousa Bun, 16 Apr. 2011. [in Japanese]

13) S. Hohara, S. Ito, "Development of a portable natural back-ground-radiation measurement system equipped with global positioning function and its application," Annu. Rep. Kindai Univ. At. Energy Res. Inst., 45, 1-10 (2008). [in Japanese]

14) Ministry of Education, Culture, Sports, Science and Technology, Housyasen wo Housyutsu suru Douigenso no Suuryou tou wo Sadameru Ken, Ministry of Education, Culture, Sports, Science and Technology, Monbu Kagaku Syou Kokuji Dai 154 Gou, 26 Dec. 2006. [in Japanese] 


\title{
Evaluation of Averted Doses to Infants by Tap Water Restrictions after the Fukushima Daiichi Nuclear Power Plant Accident
}

\author{
Sakae KINASE ${ }^{1, *}$, Masanori KIMURA ${ }^{1}$, Shogo TAKAHARA ${ }^{1}$ \\ and Toshimitsu HOMMA ${ }^{1}$ \\ ${ }^{1}$ Nuclear Safety Research Center, Japan Atomic Energy Agency, 2-4 Shirane, Shirakata, Tokai-mura, \\ Naka-gun, Ibaraki 319-1195, Japan
}

\begin{abstract}
There has been some concern in reviewing the effectiveness of making decisions on the implementation of protective measures in emergency exposure situations. After the Fukushima Daiichi Nuclear Power Plant accident, temporal changes in the concentration of iodine 131 in tap water were studied using published data from several authorities in Fukushima, Ibaraki, and Tokyo. Averted doses to infants (1-year-old children) due to the intake of iodine 131 through tap water intake restrictions were also evaluated. Consequently, it was found that the apparent half-life of iodine 131 in tap water was 2.8 days. The averted equivalent doses to the thyroids of 1-year-old children were found to have a maximum value of $8.3 \mathrm{mSv}$ in a local area of Fukushima. Hence, the tap water intake restrictions implemented by the authorities were considered to be effective in the early phase of the emergency exposure situation.
\end{abstract}

KEYWORDS: Fukushima, nuclear accident, iodine, tap water, infant, thyroid, dose

\section{Introduction}

In response to the Fukushima Daiichi Nuclear Power Plant Accident that occurred on March 11,2011 , tap water intake restrictions were implemented based on radioactive iodine 131 (hereinafter ${ }^{131} \mathrm{I}$ ) in Fukushima and in a wide range of areas including Ibaraki and Tokyo. However, there was no specific approach for canceling the tap water intake restrictions, which resulted in social confusion because the decision for the cancellation determination was left to the local governments that operated the water supply. Considering this situation, it is essential to evaluate the averted doses through the tap water intake restrictions, i.e., the effectiveness of preventive measures, and to conduct a survey on the characteristics of variations in ${ }^{131}$ I concentration in tap water. In this study, to contribute to the study on the establishment of standards for the cancellation of future tap water intake restrictions, the averted doses to infants (1-year-old children) through the tap water intake restrictions were evaluated. A survey on the variations in ${ }^{131}$ I concentration was also conducted in the tap water of Fukushima, Ibaraki and Tokyo, all of

\footnotetext{
${ }^{*}$ Corresponding author, E-mail: kinase.sakae@jaea.go.jp

DOI : 10.15669/fukushimainsights. Vol.4.6

(C) 2021 Atomic Energy Society of Japan. All rights reserved.

Originally published in Transactions of the Atomic Energy Society of Japan (ISSN 1347-2879), Vol. 10, No. 3, p.149-151

(2011) in Japanese. (Japanese version accepted: June 14, 2011)
} 
which were directly influenced by the accident at the Fukushima Daiichi Nuclear Power Plant.

\section{Actions Implemented by the Ministry of Health, Labour and Welfare (MHLW) on Tap Water Intake by Infants}

On March 19, 2011, the MHLW declared an index value (radioactive iodine concentration: $300 \mathrm{~Bq} / \mathrm{kg}$ ), which was determined by the Nuclear Safety Commission. Furthermore, on March 21,2011 , in order for the temporary restriction value to accord with the food sanitation law, it was recommended that the intake of the tap water, such as through the feeding of modified milk powder dissolved in tap water, should be avoided in infants when the radioactive iodine concentration exceeded $100 \mathrm{~Bq} / \mathrm{kg}$. On April 4, 2011, the time at which the tap water intake restrictions should be cancelled was defined as when the average of radioactive iodine concentration in the tap water, over a period of three days, falls below the prescribed standard and continues on a decreasing trend.

\section{Actions Implemented by the Local Governments on Tap Water Intake by Infants}

The local governments in Fukushima, Ibaraki and Tokyo implemented restrictions on tap water intake by infants in accordance with the standard established by the Nuclear Safety Commission or the MHLW, and then cancelled this preventative measure at their own discretion (Table 1). The restriction on tap water intake by infants in Tokyo was triggered by the $210 \mathrm{~Bq} / \mathrm{kg}$ detected in the sample taken at the Kanamachi Purification Plant on March 22, 2011.

Table 1 Tap water intake restrictions implemented by the authorities

\begin{tabular}{ccccc}
\hline Prefectures & Authority & The first day & The last day & Days \\
\hline Fukushima & Iitate & March 21st & May 10th & 50 \\
& $\begin{array}{c}\text { Koriyama, } \\
\text { Kawamata } \\
\text { Minamisoma }\end{array}$ & March 22nd & March 25th & 3 \\
& Iwaki & March 22nd & March 30th & 8 \\
& Tokai & March 23rd & March 31st & 8 \\
Ibaraki & The 23 wards and 5 cities & March 23rd & March 24th & 3 \\
Tokyo & &
\end{tabular}

\section{Methodology}

Using the data published by the MHLW, the Tokai Village Office and the Tokyo Metropolitan Office ${ }^{1)}$, a survey was conducted on the variations in ${ }^{131}$ I concentration in the tap water sampled from five local governments in Fukushima (Takishita Purification Plant, Iitate Village; Toyota-Machi-Jinai, Koriyama City; Aza Toinokuchi-Jinai, Kawamata Town; Minamisoma City government office complex; and Iwaki City government office complex); the Tojuku Purification Plant, Tokai Village, Ibaraki Prefecture; and Hyakunincho, Shinjuku, Tokyo. The time period for the survey was one month from March 16 to April 16, 2011 (till May 10, 2011 in Iitate Village). Furthermore, the amount of daily ${ }^{131} \mathrm{I}$ intake by 1 -year-old infants was estimated 
based on the ${ }^{131} I$ concentration in the tap water and the quantity of daily water intake. The equivalent infant thyroid dose rate and the effective dose rate were estimated by multiplying the ${ }^{131}$ I intake with the dose coefficient from the International Commission on Radiological Protection (ICRP) Publication 67 ${ }^{2}$. Referring to the values indicated in the Safety Series No. $81^{\text {3) }}$ of the International Atomic Energy Agency (IAEA), the volume of water intake of a 1-year-old infant was assumed to be $1.4 \times 10^{3} \mathrm{~mL}$ per day. Table 2 shows the dose coefficient of ${ }^{131} \mathrm{I}$. Note that for days on which sampling was not conducted, dose evaluation was conducted using the value obtained for a sample measured on the day before or the day after, whichever value was the higher. For days on which ${ }^{131} \mathrm{I}$ was not detected in the tap water, it was assumed that no significant exposure had occurred.

\begin{tabular}{|c|c|c|}
\hline Arc of intakes & Thyroid equivalent dose & Effective dose \\
\hline 1-year-old children & $3.6 \times 10^{-6}$ & $1.8 \times 10^{-7}$ \\
\hline Adult ${ }^{a)}$ & $4.3 \times 10^{-7}$ & $2.2 \times 10^{-8}$ \\
\hline
\end{tabular}

\section{Results and Discussions}

Figure 1 presents the variations in ${ }^{131} \mathrm{I}$ concentration in the tap water observed in the five local governments in Fukushima Prefecture (Iitate Village, Koriyama City, Kawamata Town, Minamisoma City, and Iwaki City), Tokai Village in Ibaraki and Shinjuku in Tokyo. As shown in the chart, ${ }^{131}$ I concentration in the tap water varied according to the sampling location, and on March 26, 2011, 37.2 Bq/kg was observed as the maximum for Shinjuku, which is 230 $\mathrm{km}$ from the Fukushima Daiichi Nuclear Power Plant. ${ }^{131}$ I concentration in the tap water from Tokyo was lower compared to that of a number of locations in Fukushima Prefecture and Tokai

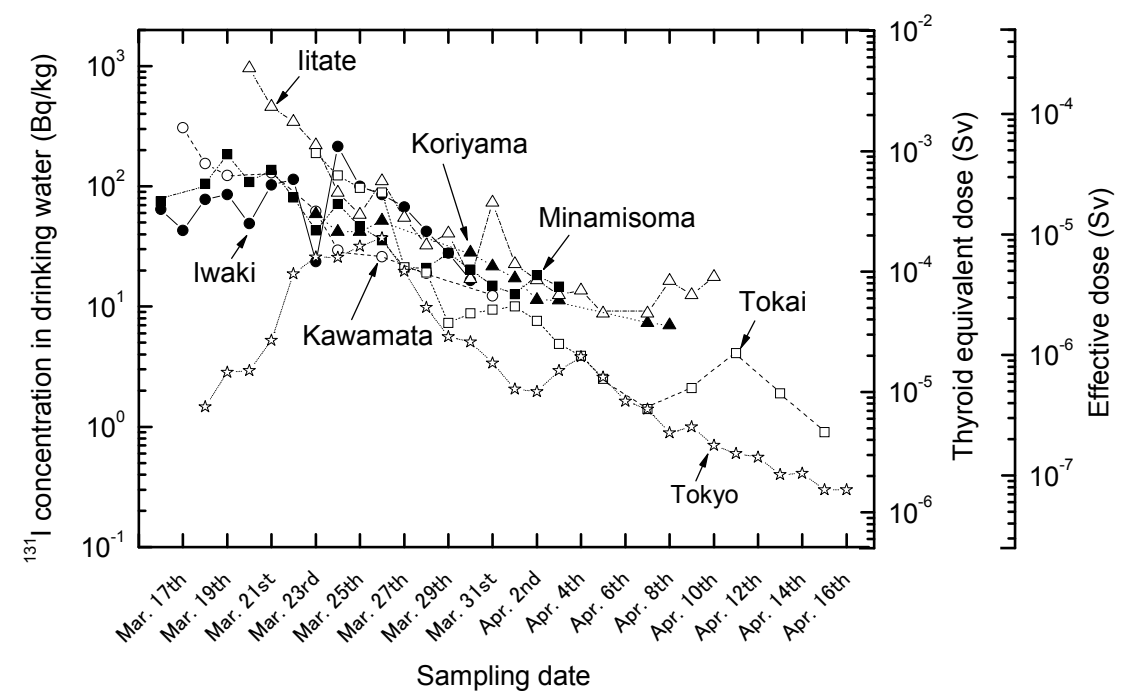

Figure 1 Concentration of ${ }^{131} \mathrm{I}$ in tap water, projected equivalent doses to thyroid, and effective doses of 1-year-old children, due to the intake of ${ }^{131} \mathrm{I}$ in tap water in local areas of Fukushima, Tokai and Tokyo 
Village in Ibaraki. Accordingly, it was confirmed that there had been no immediate influence traceable to the accident of Fukushima Daiichi Nuclear Power Plant. The general trend showed a decrease over time after the occurrence of the Fukushima Daiichi Accident in all locations. The reduction rate in the variations of ${ }^{131}$ I concentration in tap water displayed an apparent half-life period of approximately $2.8^{+/}-1.2$ days, which is shorter than the physical half-life of ${ }^{131} \mathrm{I}$ of 8.0 days. This trend was observed due to the reduced additional precipitation of ${ }^{131} \mathrm{I}$ in the relevant locations after March 16, 2011 when the survey began, and accordingly, the dilution effect in the environment was considered to have contributed significantly to these findings. After April 1, 2011, a low concentration of ${ }^{131}$ I was detected in tap water from Iitate Village, Koriyama City, and Minamasoma City in Fukushima, Tokai Village in Ibaraki, and Shinjuku in Tokyo. It is assumed that these results are due to the inflow of deposits and re-floating of matter with the rain water into the tap water source. During the survey period, the highest value was detected in Iitate Village $(965 \mathrm{~Bq} / \mathrm{kg})$.

Additionally, Figure 1 shows the variations in the thyroid equivalent dose and the effective dose rate for an infant. All dose rates demonstrated a decreasing trend over time along with the variations in ${ }^{131} \mathrm{I}$ concentration in the tap water. Note that in the case of long-term, continuous, and chronic intake of ${ }^{131} \mathrm{I}$ by an infant, it is assumed that ${ }^{131} \mathrm{I}$ in the thyroid or inside the body builds up and then reaches the equilibrium value (= (effective half-life (days) $) \times($ constant intake amount per day) / $\ln 2$ ) due to the balancing of intake and egestion. Consequently, the thyroid equivalent dose and the effective dose rate are expected to be lower than the total sum of the daily evaluated dose rate.

Table 3 lists the averted dose in infants resulting from the tap water intake restrictions implemented by local governments. During the continued emergency exposure conditions, the tap water intake restrictions were an effective measure for avoiding a thyroid equivalent dose of $8.3 \mathrm{mSv}$ and effective dose rate of $4.1 \times 10^{-1} \mathrm{mSv}$ and for reducing the residual dose rate, especially in Iitate Village, Fukushima Prefecture.

Given the risk of exposure under the circumstances that prevailed at the time, not only to ${ }^{131}$ I but also to radioactive cesium $\left({ }^{137} \mathrm{Cs}\right)$ and other radioactive nuclides, the tap water intake restrictions implemented by the MHLW can be justified as a protective measure for decreasing the thyroid equivalent dose and the effective dose rate in infants. Furthermore, in areas such as Tokai Village, the supply of tap water was delayed due to the power outage caused by the earthquake and the works undertaken to restore the tap water pipelines. This delay in tap water supply happened to reduce the exposure to ${ }^{131} \mathrm{I}$ in the tap water. In comparison, in Shinjuku, Tokyo, where the duration of the tap water intake restriction was shorter and the concentration of ${ }^{131} \mathrm{I}$ was low, the averted thyroid equivalent dose in infants was $1.3 \times 10^{-1} \mathrm{mSv}$.

Table 3 Averted doses in 1-year-old children due to the intake of ${ }^{131} \mathrm{I}$ by tap water restrictions (Sv)

\begin{tabular}{ccc}
\hline Authority & Thyroid equivalent dose & Effective dose \\
\hline Iitate, Fukushima & $8.3 \times 10^{-3}$ & $4.1 \times 10^{-4}$ \\
Koriyama, Fukushima & $5.1 \times 10^{-4}$ & $2.5 \times 10^{-5}$ \\
Kawamata, Fukushima & $1.1 \times 10^{-3}$ & $5.6 \times 10^{-5}$ \\
Minamisoma, Fukushima & $1.7 \times 10^{-3}$ & $8.7 \times 10^{-5}$ \\
Iwaki, Fukushima & $2.9 \times 10^{-3}$ & $1.5 \times 10^{-4}$ \\
Tokai, Ibaraki & $2.1 \times 10^{-3}$ & $1.0 \times 10^{-4}$ \\
Shinjuku, Tokyo & $1.3 \times 10^{-4}$ & $6.5 \times 10^{-6}$ \\
\hline
\end{tabular}




\section{Conclusions}

A survey was conducted on variations in ${ }^{131}$ I concentration in the tap water of Fukushima Prefecture, Ibaraki Prefecture and Tokyo after the Fukushima Daiichi Nuclear Power Plant Accident. In addition to evaluating the thyroid equivalent dose to infants and the effective dose rate of ${ }^{131} \mathrm{I}$ in the tap water, the averted dose rate resulting from the intake restrictions implemented by the local governments was calculated. As a result, it became clear that the ${ }^{131} \mathrm{I}$ concentration in tap water was influenced heavily by the sample locations, and it decreased with the rate of the apparent half-life of approximately 2.8 days. The tap water intake restriction related to the Fukushima Daiichi Nuclear Power Plant was effective in reducing the thyroid equivalent dose and the averted thyroid equivalent dose to infants was approximately $8.3 \mathrm{mSv}$ in Iitate Village, Fukushima Prefecture.

\section{References}

1) Ministry of Health, Labour and Welfare, Information about the Great East Japan Earthquake, Online, Available: http://www.mhlw.go.jp/english/topics/2011eq/index.html (2011, April 17).

2) ICRP, Age-dependent Doses to Members of the Public from Intake of Radionuclides: Part 2 Ingestion Dose Coefficients, ICRP Publication 67, Pergamon Press, Oxford (1993).

3) IAEA, Derived Intervention Levels for Application in Controlling Radiation Doses to the Public in the Event of a Nuclear Accident or Radiological Emergency, IAEA, Vienna, Safety Series No. 81, IAEA, Vienna (1986). 


\title{
Time Variations in Dose Rate and $\gamma$ Spectrum Measured at Tsukuba City, Ibaraki, due to the Accident of Fukushima Daiichi Nuclear Power Station
}

\author{
Toshiya SANAMI ${ }^{1, *}$, Shinichi SASAKI ${ }^{1}$, Kazuhiko IIJIMA ${ }^{1}$, \\ Yuji KISHIMOTO $^{1}$ and Kiwamu SAITO ${ }^{1}$ \\ ${ }^{1}$ Applied Physics Laboratory, High Energy Accelerator Research Organization, 1-1 Oho, Tsukuba-shi, Ibaraki 305-0801, Japan
}

\begin{abstract}
The time variations in the dose rate and $\gamma$ spectrum of radioactive nuclides originating from the Accident of Fukushima Daiichi Nuclear Power Station were measured at Tsukuba City, Ibaraki, during the period from 15th March to 9th April 2011. The radiation dose peaked three times during the period from 15 th to 16 th March $(1.27 \mu \mathrm{Sv} / \mathrm{h}$ at maximum). The contribution of Xe-133 to the dose rate was observed in the $\gamma$ spectrum obtained from the $5 \mathrm{~h}$ measurement during the peaks on 15th and 16th March, indicating that radioactive plume passed through Tsukuba City at that time. After the peaks, a dose rate increase with rainfall was observed on 21th March, dominating the integral dose rate measured at Tsukuba City. The dose after the rainfall comes from I-131, Cs-134, and Cs-137 that can be observed as peaks in the spectra.
\end{abstract}

KEYWORDS: variation in dose rate, NaI scintillator, Tsukuba City, Ibaraki, radioactive plume, Fukushima Daiichi Nuclear Power Station

\section{Introduction}

Due to an earthquake in the Pacific off the coast of Tohoku, which occurred at 14:56 on March 11, 2011 and the ensuing tsunami, Fukushima Daiichi Nuclear Power Station fell into a state of station blackout ${ }^{1}$. As a result, the nuclear reactors lost coolant and fuel rods were exposed, causing a hydrogen explosion. A significant amount of radioactive materials was released. The range of dispersion of these materials and the nuclide distribution were thought to be influenced by the conditions of release, wind direction, surrounding landscape, weather, etc. It was necessary to clarify the conditions under which measurements were taken and to compile the measured data regarding the radiation dose and radioactivity, such as ambient dose equivalent and fallout composition over a wide-ranging area and over different time spans.

The High Energy Accelerator Research Organization in Oho, Tsukuba-City, Ibaraki-Prefecture focused on the necessity of acquiring such data and started continuous measurement of dose rate and pulse-height spectrum using a $\mathrm{NaI}(\mathrm{Tl})$ scintillation detector (hereinafter $\mathrm{NaI}$

* Corresponding author, E-mail: toshiya.sanami@kek.jp

DOI : 10.15669 /fukushimainsights. Vol.4.11

(C) 2021 Atomic Energy Society of Japan. All rights reserved.

Originally published in Transactions of the Atomic Energy Society of Japan (ISSN 1347-2879), Vol. 10, No. 3, p.163-169

(2011) in Japanese. (Japanese version accepted: May 25, 2011) 
detector) starting at 14:13 on March 14 . The dose rate abruptly increased three times before dawn on March 15 and another increase in dose was observed on March 21 due to rainfall. The variation in the dose rate of $\gamma$-rays over time and the time-dependent change of the pulse-height spectrum measured using the NaI detector are summarized in this paper. Based on the data, the characteristics of the dose rate increase and residual radionuclides were discussed.

\section{Measurement Methodology}

\section{Measurement Location}

The measurement location was inside a prefabricated house (for radiation monitoring at the site boundary) at the High Energy Accelerator Research Organization in Oho, Tsukuba-City, Ibaraki-Prefecture (located $165 \mathrm{~km}$ from Fukushima Daiichi Nuclear Power Station by straightline distance, $36^{\circ} 08^{\prime} 50^{\prime \prime} \mathrm{N} 140^{\circ} 04^{\prime} 40^{\prime \prime} \mathrm{E}$ ). The monitoring house is shown in Figure 1 and the layout is depicted schematically in Figure 2. The house was placed in a wood near an asphalt paved road (20 m wide). The distance from the site boundary was $20 \mathrm{~m}$ and the distance from the asphalt paved road was $5 \mathrm{~m}$. The floor area of the house was $5.2 \times 2.3 \mathrm{~m}$ and the height was $2.1 \mathrm{~m}$. The floors, walls, and the roof were made of plywood and steel sheet. The house had two windows of $90 \times 170 \mathrm{~cm}$ each, and a ventilation fan. The ventilation fan operated continuously until a power outage on March 22. The installation location of the NaI detector is shown in the enlarged drawing in Figure 2. The NaI detector was installed at a height of $120 \mathrm{~cm}$ from the floor and $6 \mathrm{~cm}$ away from the window.

The dose rate was measured at a point inside the monitoring house and at a location outside the house using a $\mathrm{NaI}(\mathrm{Tl})$ scintillation survey meter at the time of installation and at the times when an increase in dose rate occurred. The doses inside were approximately the same as those outside; the dose rate level was confirmed for both conditions.

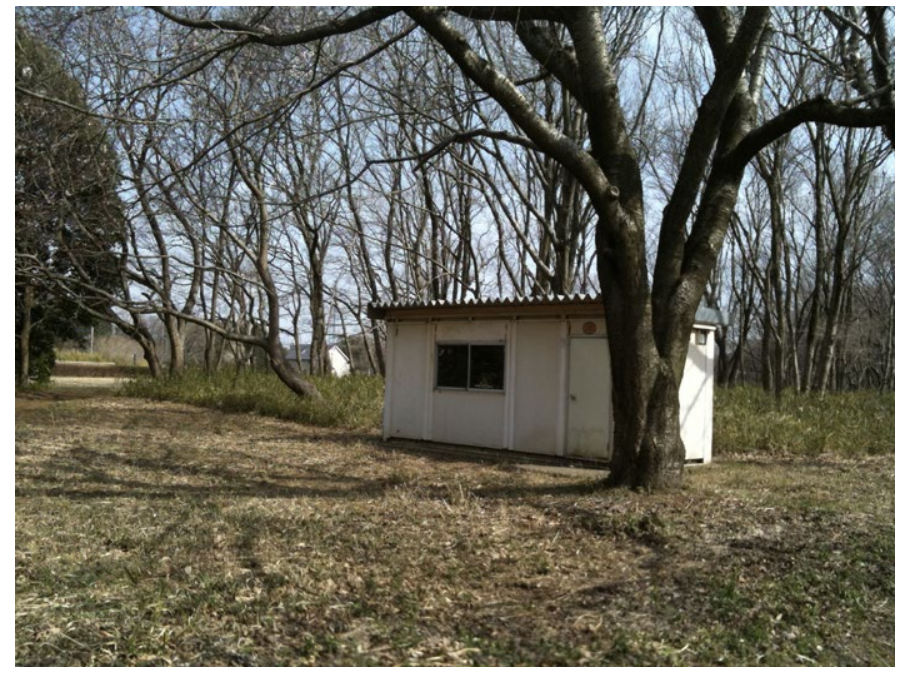

Figure 1 Picture of a monitoring house

The house was located at the High Energy Accelerator Research Organization at $36^{\circ} 08^{\prime} 50^{\prime \prime} \mathrm{N}$, $140^{\circ} 04^{\prime} 40^{\prime \prime} \mathrm{E}, 165 \mathrm{~km}$ south of the Fukushima-Daiichi Nuclear Power Station. A monitoring house containing a $\mathrm{NaI}$ scintillation detector was placed in a copse at the site. 


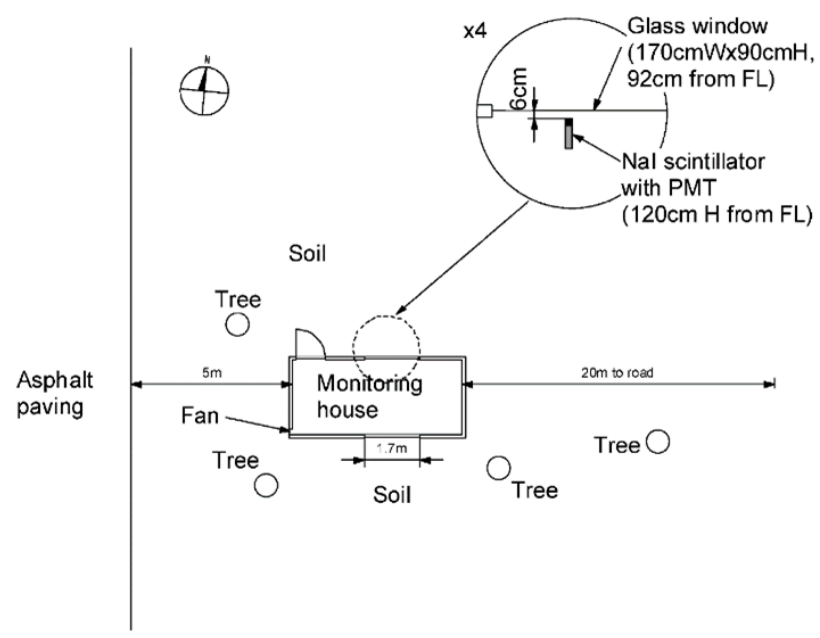

Figure 2 Plan view of the monitoring house

The house was placed on ground consisting of soil. The NaI scintillation detector was placed $120 \mathrm{~cm}$ above the floor, and $6 \mathrm{~cm}$ away from a north-facing window.

\section{NaI Detector and Data Recording System}

The $\mathrm{NaI}$ detector (Bicron, model 2M2/2) had a NaI(Tl) crystal ( 2 in. diameter $\times 2$ in. thick) mounted on the photomultiplier tube. The crystal was covered by a $0.02 \mathrm{in}$. thick aluminum casing coated with white reflective material. The output of the photomultiplier was amplified via a pre-amplifier and a main amplifier. The events having a pulse-height greater than a $\gamma$-ray energy of $118 \mathrm{keV}$ were selected using a single-channel analyzer. Counts of these pulse signals were recorded every 10 seconds and continuously written to a compact flash card. The output of the main amplifier, pulse height, was analyzed using a multi-channel pulse-height analyzer, and the pulse-height spectrum of $\gamma$-rays was acquired continuously, with measurement restart every 5 hours. Pulse-count data were recorded throughout the period from 14:57 on March 14 until 0:00 on April 9. The pulse-height spectrum data were acquired during that entire period excluding March 20 through March 22 and again from March 29 through March 31.

\section{Derivation of Dose Rate}

The time-dependent variation in dose rate was estimated based on the pulse-count data obtained by the $\mathrm{NaI}$ detector. The compensation of energy dependence in the detection efficiency based on the pulse-height signal should be applied to dose measurements using a NaI(Tl) scintillator, but this method was not available during this time because only part of the pulse-height distribution was acquired.

Furthermore, under these measuring conditions, it was likely that the $\gamma$-rays from the existing/adhered radionuclides in/on (1) the atmosphere, (2) the soil surface, and (3) roof, walls, windows, etc. of the prefabricated house were measured, so it was difficult to define the detection efficiency because the intensity and energy distribution fluctuated over time. Hence, the pulse-count rate was converted to a dose rate by standardizing to the measurement of a $1 \mathrm{~cm}$ dose equivalent on the $\mathrm{NaI}(\mathrm{Tl})$ scintillation survey meter implemented with an energy compensation circuit.

As a standard, a NaI(Tl) scintillation survey meter (Hitachi Aloka Medical Ltd., TCS-171, 
hereinafter $\mathrm{NaI}$ survey meter) was used. A NaI detector and a $\mathrm{NaI}$ survey meter were placed in the same position and the time constant was set to 30 seconds. The survey meter readings were recorded. The pulse-count rate of the $\mathrm{NaI}$ detector was normalized to this value. The normalization factor was $5.55 \times 10^{-4} \mu \mathrm{Sv} / \mathrm{h} / \mathrm{cps}$. It was assumed that this normalization factor could vary with fluctuations in the $\gamma$-ray energy distribution in the field. Hence, regarding the spectra that were acquired separately from the count rate and accumulated for 5 hours, a comparison was made on the $1 \mathrm{~cm}$ dose equivalent rate derived using the $\mathrm{G}$ function ${ }^{2)}$ of the 2 in. diameter $\times$ 2 in. thick NaI scintillation detector. The dose rate based on the above normalization factor was consistent with the value derived by the $\mathrm{NaI}$ survey meter within a margin of $10 \%$. Based on this, the dose rate during the measurement period was determined from the pulse-signal count rate, acquired over the entire period, using this normalization factor.

\section{Measured Results}

\section{Time-Variation of Dose Rate}

The time-valiation of dose rate is shown in Figure 3. The lines in the above figure show the dose rate converted from the count rate of the $\mathrm{NaI}$ detector and the dots indicate the measurement
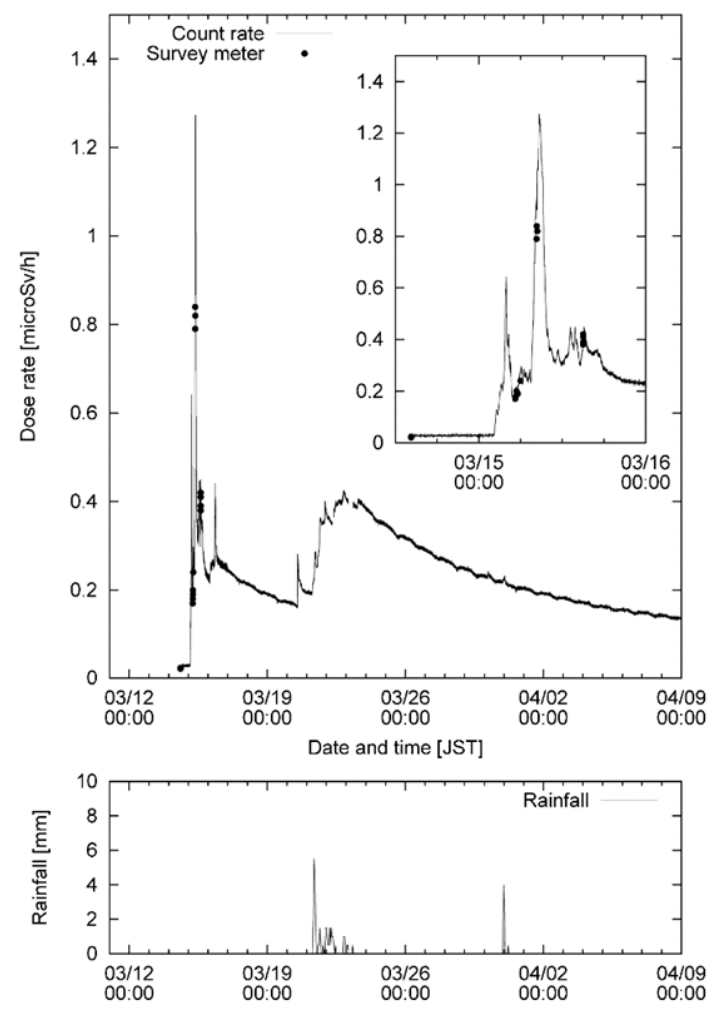

Figure 3 (Above) Dose rate for the entire period (from Mar. 12 to Apr. 9) and its increase from Mar. 14 to Mar. 16. The line shows the count rate above the threshold (118 keV equivalent) of the NaI scintillation detector. The points represent dose rate measured by a NaI scintillation survey meter with an energy compensation circuit. The count rate is normalized to the dose rate of the survey meter.

(Below) Rainfall data during the period near the site (Simotsuma area) 
by the NaI survey meter made at the same time. The two measurements are consistent with each other both in terms of the period of dose rate peaks and other periods having different pulseheight distributions. It was assumed that the variations in the pulse-height spectrum during this period did not seriously influence the conversion.

It is understood from these charts that the first arrival of the radioactive plume at Tsukuba-City, Ibaraki-Prefecture was at 2:13 on March 15. The first peak was $0.64 \mu \mathrm{Sv} / \mathrm{h}$ at 3:56 on March 15, the second was $1.27 \mu \mathrm{Sv} / \mathrm{h}$ at 8:42 on the same day. On the next day, March 16, a peak of $0.44 \mu \mathrm{Sv} / \mathrm{h}$ was observed at 8:38, and after that the dose monotonically decreased until the rate rise on March 20. In the lower chart of Figure 3, the precipitation data are shown at AMEDAS located at Nihongi, Shimotsuma-City, Ibaraki-Prefecture, which is the nearest one from the measuring point ${ }^{3}$. Rain was observed to fall from March 21 through 23 and on March 26, and a significant rise in the dose rate was seen on March 21. An increase in the dose rate, presumably due to rainfall, was observed after a small peak of $0.27 \mu \mathrm{Sv} / \mathrm{h}$ was observed at 13:36 on March 20, and the dose rate increased to $0.36 \mu \mathrm{Sv} / \mathrm{h}$ at 16:53 on March 21. The missing dose rate data during this period was caused by a power outage due to the electrical leakage that occurred at the monitoring house. The peak component on March 15 and that on March 21 were markedly different in terms of the time variation of the dose rate. While the peak component on March 15 showed a rapid increase and decrease in the dose rate, that on March 21 showed a slow increase and decrease in the dose rate. Accordingly, the increase in the dose rate was attributed to the radionuclides that were deposited near the monitoring house. Consequently, that increase in the dose rate dominated the dose rate changes after that and the cumulative dose at that point. Additionally, rainfall was observed at midnight on March 30, but no obvious increase in the dose rate was observed. Based on this observation, it was inferred that there was no significant increase in atmospheric radioactivity after the rain that fell on March 21.

It is important to understand the origin of the radioactivity on March 21 for radiological protection by elucidating the mechanism by which the dose rate increased due to rainfall.

\section{Time Variation of Pulse-height Spectrum}

Typical pulse-height spectra are shown together with the time-variation of the dose rate in Figures 4-10. The upper part in each chart shows the time-variation of the dose rate, and the marks on the graph indicate the start and stop time of the pulse-height spectrum shown in the lower part.

In the upper panel, the enlarged chart of the time-variation of the dose rate from March 15 through March 17 shows detail of the period. The measuring energy range of the pulse-height spectrum was $3 \mathrm{MeV}$, but to make the peaks easier to recognize, the range higher than $2 \mathrm{MeV}$ was omitted in Figures 4-9.

Figure 4 shows the pulse-height spectrum measured for approximately 4 hours. It shows the first increase of dose. Although there is a continuous spectrum due to various $\gamma$-ray peaks overlapping, the typical $\gamma$-ray energies for major nuclides are shown in this figure. The nuclides were chosen based on short half-life components that were deduced from the long half-life nuclides observed on the pulse-height spectrum after decay-out, the measured data on fallout substances in the Tsukuba area ${ }^{4)}$, and, the data measured by Fukushima-Prefecture ${ }^{5)}$. This is discussed later in the paper.

Figure 5 shows the pulse-height spectrum measured somewhat later than the spectrum shown in Figure 4. The time period was 5 hours from 15:15 until 20:19 on March 15. For comparison, the spectrum in Figure 4 is also shown. It can be seen from this chart that in this period, the first two increases in dose had already ended and the contribution of comparatively short half-life 

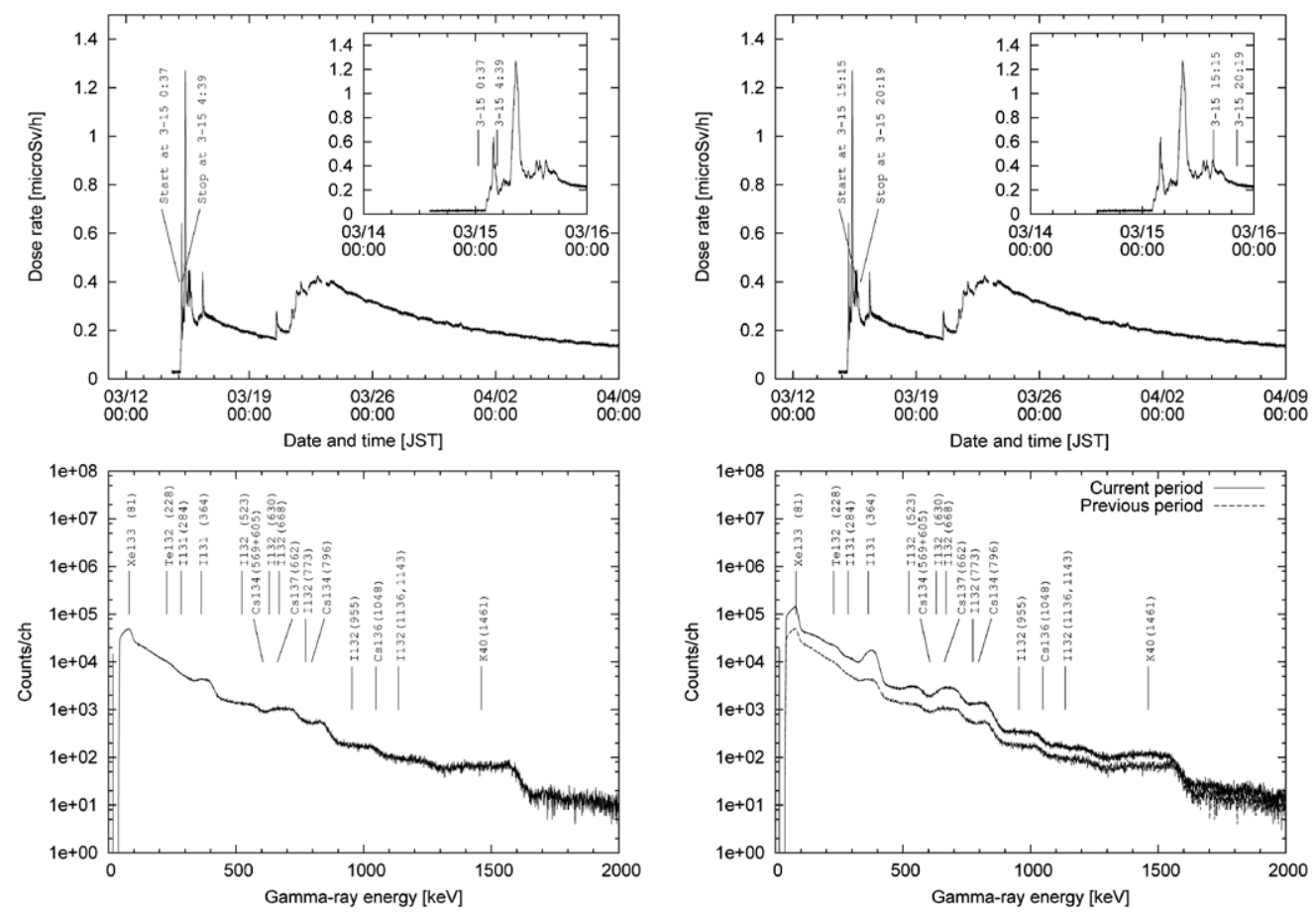

Figure 4 (Above) Dose rate for the entire period and its increase from Mar. 14 to Mar. 16. The period for the energy spectrum shown is indicated by the two lines with date and time.

(Below) Energy spectrum for the first period (from 3-15 0:37 to 3-15 4:39) The period contains the first dose rate peak due to the accident at the Fukushima Daiichi Nuclear Power Station.

The energies of major peaks from isotopes identified by other reports are indicated on the spectrum.

Figure 5 (Above) Dose rate for the entire period and its increase from Mar. 14 to Mar. 16. The period contains several small dose rate peaks after the largest peak.

(Below) Energy spectrum for the second period (from 3-15 15:15 to 3-15 20:19) in comparison with the first period Several broad peaks were observed because many $\gamma$ emissions of various energy levels contributed to the spectrum.

nuclides such as Xe-133 and Te-132 generally decreased. The main peak at $364 \mathrm{keV}$ of I-131 can be clearly seen for this reason.

Figure 6 shows the pulse-height spectrum measured from 20:19 on March 15 until 1:22 on March 16, immediately before the dose increase. The complete disappearance of Xe-133 can be seen. If the half-life of 5.243 days for Xe-133 is also considered, it becomes apparent that the variation in the dose rate was caused by the onward migration of Xe-133. An independent increase in Xe-133 is observed in Figure 7. The pulse-height spectrum more than 5 hours before the peak dose on March 16 indicates that it may be observed before the dose rise.

Figure 8 shows the pulse-height spectrum after the rise in dose on March 16. The comparison in the lower chart is the pulse-height spectrum in the period starting from 6:26 and ending at 11:29 on March 16, which includes the rise in dose on March 16. The rapid decrease of both Xe-133 and a $\gamma$-ray component from I-132, having various energy peaks, were clearly observed. Xe-133 was therefore considered a good marker for the instantaneous increase in dose. 

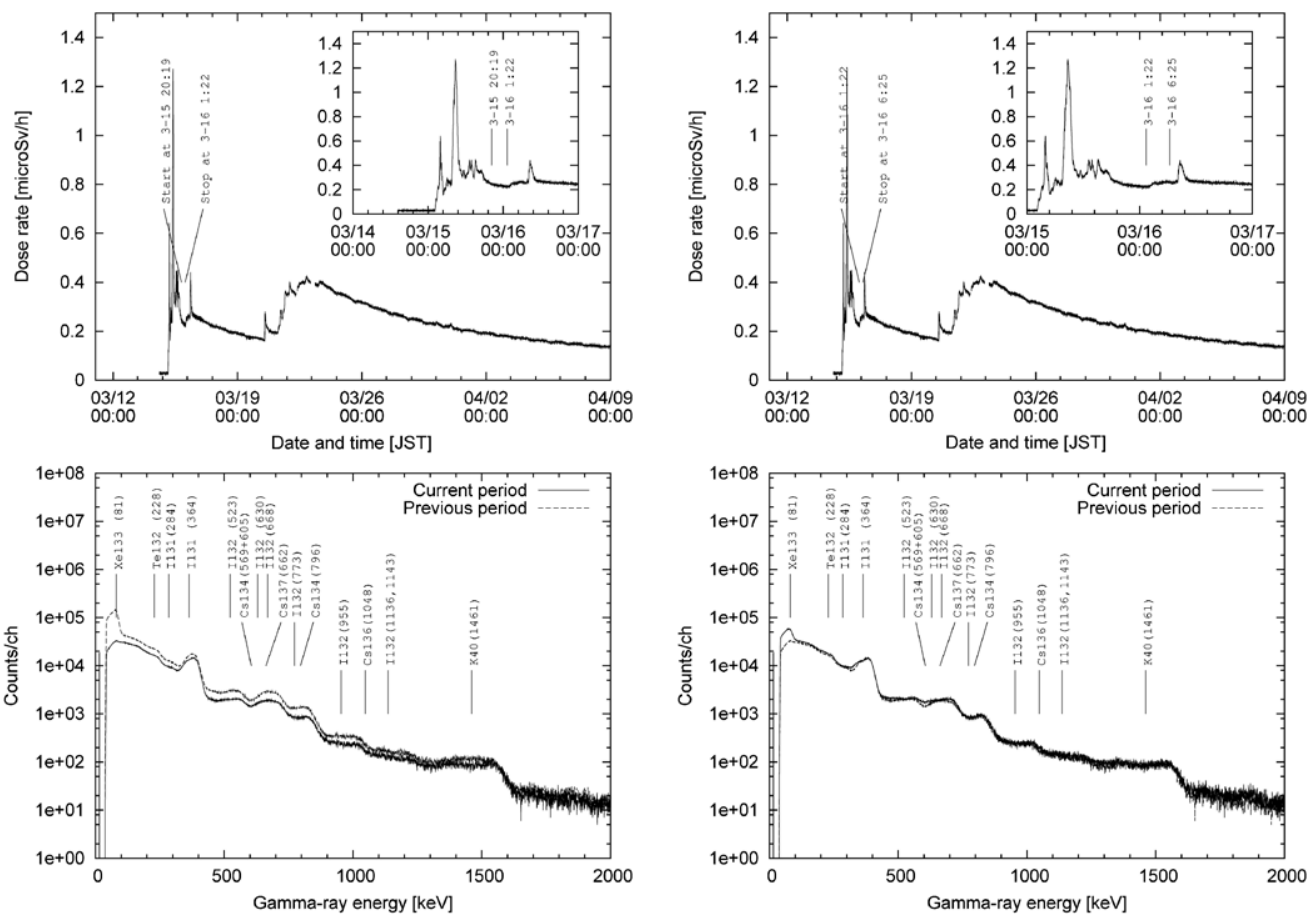

Figure 6 (Above) Dose rate over the entire period and its increase from Mar. 14 to Mar. 17. The period contains no dose rate peak. (Below) Energy spectrum for the third period (from 3-15 20:19 to 3-16 1:22) in comparison with the second period. The contribution from Xe-133 completely disappeared after the peaks.

Figure 7 (Above) Dose rate over the entire period and its increase from Mar. 15 to Mar. 17. The dose rate in the period increases slightly without any peak.

(Below) Energy spectrum for the fourth period (from 3-16 1:22 to 3-16 6:25) in comparison with the third period. The difference between the two spectra is entirely due to the contribution from $\mathrm{Xe}-133$, because Xe-133, an inert gas, can travel freely with the atmosphere.

Figure 9 shows the pulse-height spectrum (from 19:19 on March 23 until 0:21 on March 24) after the rain that fell on March 21. For the purpose of comparison, the pulse-height spectrum before the rain (from 11:04 on March 19 until 16:05 on March 19) is shown with the pulseheight spectrum after the rain. There was no obvious change in the shape of the spectrum before and after the rain. The rise in the dose rate after March 21 was clearly understandable as different from the rise in dose rate due to the radioactive plume accompanied by Xe-133. However, the rise in dose caused by the radioactive plume was only temporary as can be understood from the time-dependent fluctuations in the dose rate, but the duration of the rise in dose due to rain was long, and as a result, large cumulative values were acquired. The dose rate as of April 9 was inferred as being mainly due to the rain that fell on March 21.

Figure 10 shows the comparison of the pulse-height spectrum from 21:47 on March 31 until 2:49 on April 1 and the spectrum from 16:46 on March 31 through 21:47 on March 31, the measurement period immediately before it. Over this time period, there was no significant change in the pulse-height spectrum, instead the absolute value gradually decreased. The observed 

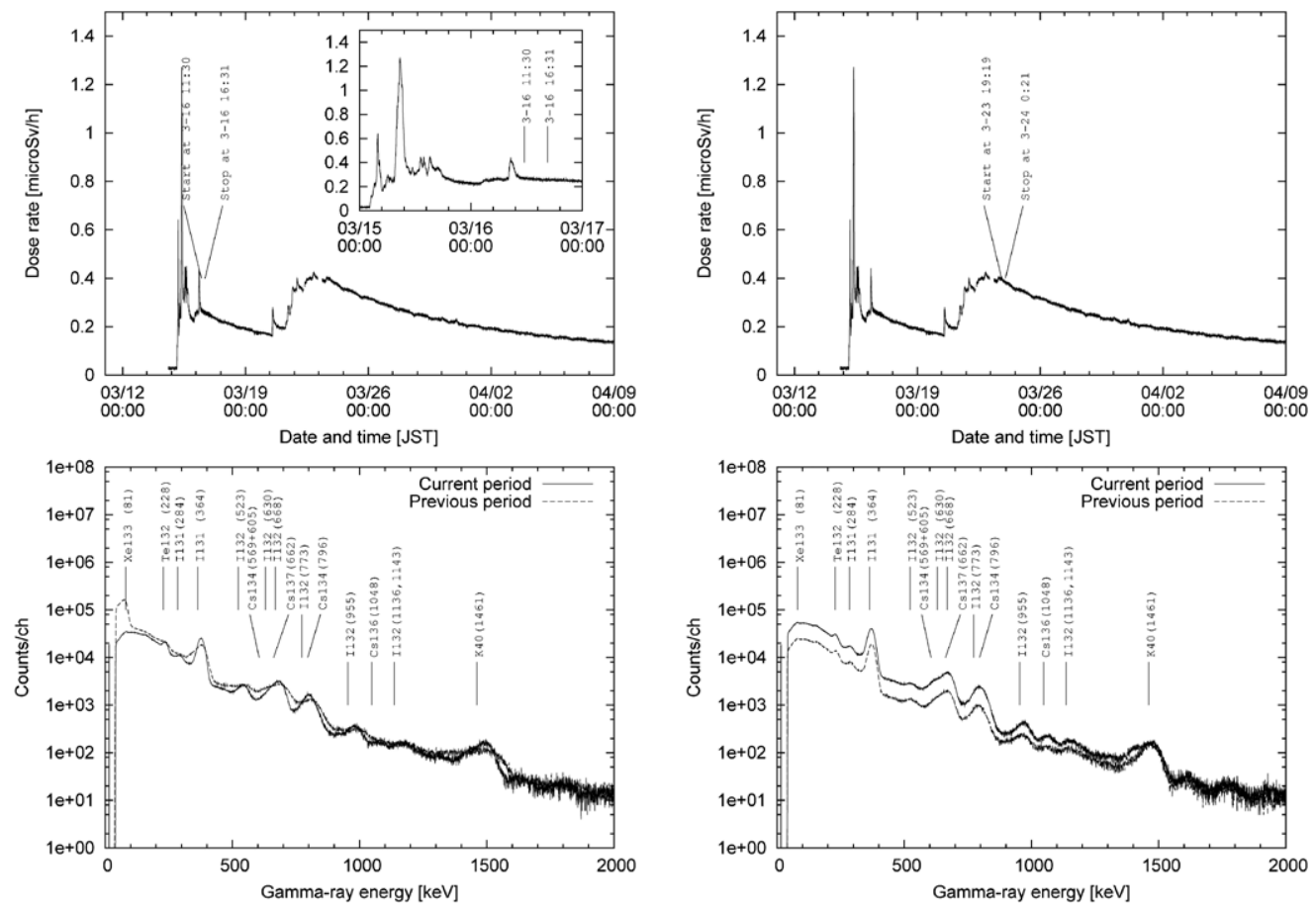

Figure 8 (Above) Dose rate over the entire period and its increase from Mar. 15 to Mar. 17.

This period corresponds to that after the dose rate peak on Mar. 16.

(Below) Energy spectrum for the sixth period (from 3-16 11:30 to 3-16 16:31) in comparison with fifth period (from 3-16 6:26 to $3-1611: 29)$

Contribution from Xe-133 has completely disappeared.

Figure 9 (Above) Dose rate over the entire period (Below) Energy spectrum for the 18th period (from 3-23 19:19 to 3-24 0:21) in comparison with 17 th period (from 3-19 11:04 to 3-19 16:05)

The 18th period corresponds to the time after rainfall and the 17 th refers to the time before rainfall. Both spectra have similar shapes, which indicates that the mechanism responsible for the increasing dose rate following rainfall was different from the mechanism that increased the dose rate during the plume.

nuclides over this time period were I-131, Cs-134, and Cs-137, and after this the dose rate declined according to the half-life of the nuclides.

\section{Time-Dependent Variation in Peak Components}

The time-dependent fluctuations in net yield with regard to each peak component of the pulse-height spectrum shown in Figures 4-10 are shown in Figure 11. The peak components were classified into four groups: (1) Xe-133 (energy $81 \mathrm{keV}$, branch ratio $38.4 \%$ (only values are listed in the following)), (2) I-131 (364 keV, 81.7\%), (3) I-132 (523 keV, 16.0\%, $630 \mathrm{keV}$, $13.3 \%, 668 \mathrm{keV}, 98.7 \%$ ), Cs-134 (605 keV, 97.6\%), Cs-137 (662 keV, 85.1\%), (4) I-132 (773 $\mathrm{keV}, 75.6 \%$ ), and Cs-134 (796 keV, 85.5\%), taking the measured nuclides, the energy and branch ratio of $\gamma$-rays, and the energy resolution of the $\mathrm{NaI}$ detector into consideration.

The chart of the peak yield of Xe-133, the second from the top in Figure 11, shows that Xe-133 has a high count rate during the dose rate peak period on March 15 and 16 and after that 

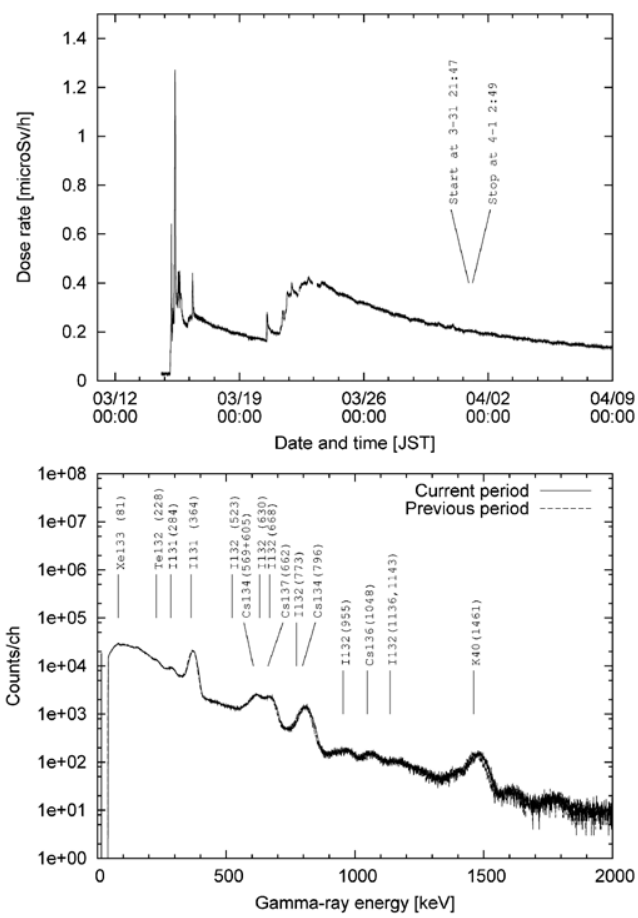

Figure 10 (Above) Dose rate over the entire period (Below) Energy spectrum for the $41 \mathrm{st}$ period (from 3-31 21:47 to 4-1 2:49) in comparison with that in the 40th period (from 3-31 16:46 to 3-31 21:47)

The 41 st spectrum is almost the same as that in the 40th period. Based on their energies, the peaks on the spectrum can be identified as $\gamma$-rays from I-131, Cs134, Cs-137, Cs-136 and K-40.
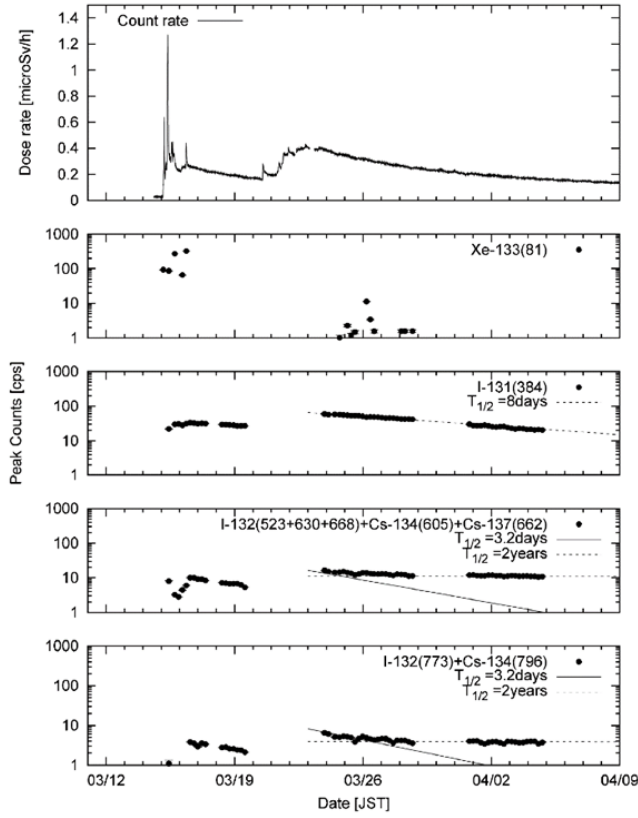

Figure 11 Relationship between dose rates over the entire period (top figure) and peak count rates for $\gamma$-rays of "Xe-133", "I-131", "I-132, Cs-134, and Cs-137", and "I-132 and Cs-134" (4 lower figures)

The line on the plot for I-131 shows the decay rate with the half-life of I-131. The solid and dotted lines on the plots of "I-132, Cs-134, and Cs-137" and "I-132 and Cs-134" correspond to the decay rates with the half-lives of Te-132 (3 days, parent of I-132) and Cs-134, respectively.

its count rate remains low. This low count rate was considered to be due to the shape of the pulseheight distributions near the peak of Xe-133 and the I-131 $\gamma$-rays. Thus, it did not indicate the real presence of Xe-133. Consequently, most the Xe-133 was measured on March 15 and 16. A small increase in the count rate on the order of several cps was measured on March 26 as well, which can be attributed to the contribution of Xe-133 based on a comparison between the pulse-height spectra during the period before and after, as shown in Figure 12.

The third chart from the top in Figure 11 shows the time-dependent fluctuation of the peak yield of I-131. Because it shows a higher count rate compared to other peak components after the increase in dose on March 21, it was assumed that it was responsible for a major portion of the dose rate. Because the count rate was a greater than $60 \mathrm{cps}$ on March 23, the contribution was expected to be less than that of $\mathrm{Cs}$, as shown below in late April after three half-life periods. The dotted line in the chart shows the decrease in count rate with a half-life of 8 days. The peak count rate was consistent with this dotted line.

The fourth and fifth charts from the top in Figure 11 show the peak count rates of Cs-134, 137, and I-132. Cs and I were not separated in either of the groups due to the energy resolution 


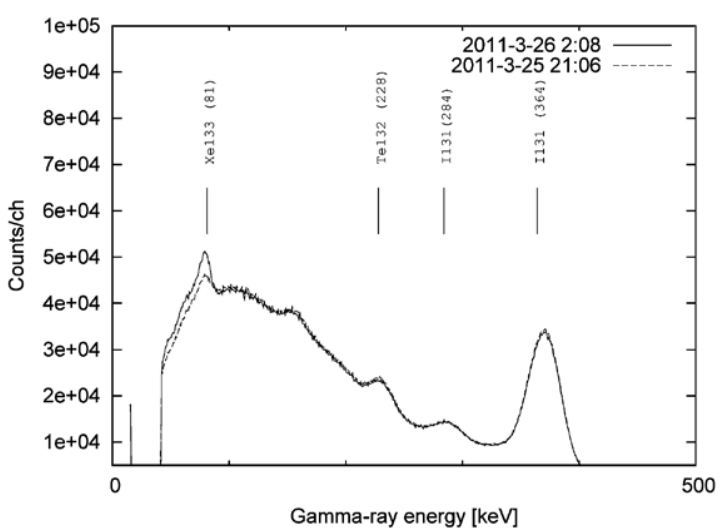

Figure $12 \mathrm{NaI}$ detector pulse-height spectrum for 5-hour periods starting at 2:08 on Mar. 26 and 21:06 on Mar. 25

The difference between the two spectra indicates the arrival of Xe-133, which suggests that additional, smaller amounts of gas were being released from the nuclear power station.

of the NaI detector. The solid line in the chart shows the decrease in count rate for a half-life of 3.2 days (equivalent to the half-life of the parent nuclide, Te-132 of I-132) and the dotted line shows that for a half-life of 2 years (equivalent to the half-life of Cs-134). The peak count rates in the chart are consistent with the half-life line of 3.2 days before March 28 and the half-life line of 2 years after March 28. Based on this, it was understood that I-132 was dominant during the period until March 28, while Cs was dominant after March 28. Furthermore, the radioactivity ratio was estimated based on the peak yields of Cs-137 and Cs-134 using the peak detection efficiency of the 2 in. diameter $\times 2$ in. thick $\mathrm{NaI}$ scintillator when a point source estimated by EGS5 ${ }^{6}$ ) was assumed. The ratio of Cs-137 to Cs-134 was 1:1.15 based on the data in the fourth and fifth charts of Figure 11 for the period after I-132 decayed. The uncertainty of this value was approximately $5 \%$, excluding the factors due to the distribution of radio active nuclides. In the future, a more precise ratio of Cs-137 to Cs-134 will be obtained by measuring the distribution for radionuclides adhering to the soil, structures, etc. around the measuring point.

\section{Conclusions}

The measurement of $\gamma$-rays derived from the Fukushima Daiichi Nuclear Power Station was conducted using a NaI scintillation detector in Tsukuba-City, Ibaraki-Prefecture, and the time-variation of dose rate and pulse-height spectra were measured. During the measurement period, the increase in dose rate due to the radioactive plume in the early stage and later as a result of rainfall were observed, and the different decay behavior of the two were clarified. Based on the pulse-height spectrum during the time when the radioactive plume passed over the city in the early stage, it was clarified that the plume contained a large quantity of Xe-133. Xe-133 was observed on March 26 as well. The pulse-height spectrum before and after the increase in dose due to rain that fell on March 21 did not show a significant difference, and it was found that I-131, Cs-134, and Cs-137 were the major components. The radioactivity ratio of Cs-134 to $\mathrm{Cs}-137$ was estimated to be $1: 1.15$.

We express our gratitude to Mrs. Takeshi Machida, Kenichi Yokota of JREC, who kindly cooperated in the calibration of detectors. 


\section{References}

1) Nuclear and Industrial Safety Agency, Conditions of Fukushima Dai-ichi Nuclear Power Station Unit 1-6 (As of 6:00 April 30th, 2011), News release, Ministry of Economy, Trade and Industry (2011). [in Japanese]

2) M. Tsutsumi, K. Saito, S. Moriguchi, Spectrum-dose Conversion Operators, G(E) Functions of NaI(Tl) Scintillators Adapted for Effective Dose Equivalent Quantities, JAERI-M 91-204. [in Japanese]

3) Japan Meteorological Agency, http://www.data.jma.go.jp/obd/stats/etrn/index.php. [in Japanese]

4) Environmental radiation at High energy accelerator research organization, http://www.kek.jp/quake/ radmonitor. [in Japanese]

5) H. Matsumura, K. Saito, J. Ishioka et al., "Behavior of radioactive materials from Fukushima Daiichi Nuclear Power Station obtained by radiation scan on the expressways," Trans. At. Energy Soc. Jpn., $10[3]$, to be published. [in Japanese]

6) H. Hirayama, Y. Namito, A. F. Bielajew et al., The EGS5 Code System, SLAC-R-730 (2005) and KEK Report 2005-8 (2005). 


\title{
Removal of Radioactive Cesium from Surface Soils Solidified Using Polyion Complex
}

\author{
-Rapid Communication for Decontamination Test \\ at Iitate-mura in Fukushima Prefecture-
}

\author{
Hirochika NAGANAWA ${ }^{1, *}$, Noriyuki KUMAZAWA ${ }^{2}$, Hiroshi SAITOH ${ }^{3}$, \\ Nobuyuki YANASE ${ }^{1}$, Hisayoshi MITAMURA ${ }^{1}$, Tetsushi NAGANO ${ }^{1}$, \\ Kaoru KASHIMA ${ }^{3}$, Tatsuya FUKUDA ${ }^{4,5}$, Zenko YOSHIDA ${ }^{1}$ \\ and Shun-ichi TANAKA ${ }^{5}$ \\ ${ }^{1}$ Nuclear Science and Engineering Directorate, Japan Atomic Energy Agency, 2-4 Shirane, Shirakata, Tokai-mura, Naka-gun, \\ Ibaraki 319-1195, Japan \\ ${ }^{2}$ Department of Biomolecular Functional Engineering, College of Engineering, Ibaraki University, 4-12-1 Nakanarusawa-cho, \\ Hitachi-shi, Ibaraki 316-8511, Japan \\ ${ }^{3}$ ATOX Co., Ltd., 161-2 Shirane, Muramatsu, Tokai-mura, Naka-gun, Ibaraki 319-1112, Japan \\ ${ }^{4}$ Chiyoda Technol Corporation, 1-7-12 Yushima, Bunkyo-ku, Tokyo 113-8681, Japan \\ ${ }^{5}$ NPO Radiation Safety Forum, Toranomonshiroyama Trust Tower (27F), Minato-ku, Tokyo 105-6027, Japan
}

\begin{abstract}
We tried the decontamination of surface soils for three types of agricultural land at Nagadoro district of Iitate-mura (village) in Fukushima Prefecture, which is highly contaminated by deposits of radionuclides from the plume released from the Fukushima Daiichi Nuclear Power Plant. The decontamination method consisted of the peeling of surface soils solidified using a polyion complex, which was formed from a salt solution of polycations and polyanions. Two types of polyion complex solution were applied to an upland field in a plastic greenhouse, a pasture, and a paddy field. The decontamination efficiency of the surface soils reached $90 \%$, and dust release was effectively suppressed during the removal of surface soils.
\end{abstract}

KEYWORDS: polyion complex, decontamination of surface soil, suppression of dust release, Iitate-mura, upland field in a plastic greenhouse, pasture, paddy field, radioactive cesium

\section{Introduction}

Because of the reactor meltdown and hydrogen explosion at the Fukushima Daiichi Nuclear Power Plant belonging to the Tokyo Electric Power Company Holdings, Inc. caused by the Great East Japan Earthquake that occurred on March 11, 2011, a large amount of radioactive material was released into the environment. In particular, a large amount of radioactive material was released from noon until $3 \mathrm{pm}$ on March 15, and this material was carried by the south-east

\footnotetext{
${ }^{*}$ Corresponding author, E-mail: naganawa.hirochika@jaea.go.jp

DOI : 10.15669 /fukushimainsights. Vol.4.22

(C) 2021 Atomic Energy Society of Japan. All rights reserved.

Originally published in Transactions of the Atomic Energy Society of Japan (ISSN 1347-2879), Vol. 10, No. 4, p.227-234

(2011) in Japanese. (Japanese version accepted: July 8, 2011)
} 
wind toward the north west of the nuclear power plant. Most of the radioactive materials are believed to have been precipitated in Namie-machi, Iitate-mura, and Kawamata-machi by rain and snow that fell from the evening until night on the same day ${ }^{1}$. Consequently, these highly contaminated areas were included in the planned evacuation areas because of concerns regarding accumulated doses exceeding $20 \mathrm{mSv}$ per year. For the residents to return to these evacuated areas and restart their lives, the radioactive material contaminating these areas must be removed.

In the present study, decontamination tests of sites including houses, farmlands, paddy fields, and pastures were selected as decontamination targets. We chose sites in the Nagadoro district, where the contamination was highest, particularly, Iitate-mura. The soil decontamination was tested via a series of decontamination tests using a method to remove surface soils solidified using a solid-state polyion complex (PIC) developed by Ibaraki University and Japan Atomic Energy Agency.

The decontamination method is based on the utilization of a PIC solid, which is uniformly dissolved in an aqueous solution by the addition of a salt, such as $\mathrm{KCl}$ or $\mathrm{NaCl}$. The salt can screen the electrostatic interactions between the polycations (positively charged polyions) and polyanions (negatively charged polyions), resulting in spontaneous precipitation of a PIC solid from the aqueous solution on the addition of water, which reduces the salt concentration. In the treatment process, an aqueous solution containing both polycations and polyanions is applied to the soil. The solution easily penetrates the soil to an appropriate depth. Then, a highly viscous PIC solid is formed by the runoff of the salt, solidifying the soil within the treated depth like an adhesive. The PIC solidified soil is stable for an extended period of time. In fact, PIC solids have a proven record: they were used after the Chernobyl Nuclear Power Plant Accident ${ }^{2)}$ to prevent the loss of radioactive materials as soil dust. Furthermore, experimental studies evaluating the applicability of PIC solids were carried out by Kumazawa et al. ${ }^{3,4}$. Methods using PIC solids have several advantages, for example, the ability to solidify the soil surface even with a comparatively small quantity of PIC (less than $2 \mathrm{wt} \%$ of soil), suppressing soil dust generation while solid removal work goes on, and easy post-processing of the solid material by gelation after the addition of water.

The features of this method and future subjects of investigation are described as rapid communication, together with the results of the decontamination experiments.

\section{Experimental}

\section{Reagents and Preparation of PIC Solutions}

(1) Reagents

(a) For a PIC solution of natural origin

Cationic cellulose ether chloride (hydroxyethylcellulose hydroxypropyltrimethylammonium chloride ether, HECHPTA, solid powder, $\mathrm{MW} \approx 50,000$, product name $\# \mathrm{QH} 200$ ) and anionic cellulose sodium salt (carboxymethyl cellulose sodium salt, CMC, solid powder, MW $\approx 750,000$, product number \#1350) were purchased from Daicel FineChem Ltd. and used without further purification.

(b) For a synthetic PIC solution

The cationic polyion poly (tetraalkyl ammonium) ion (polydiallyldimethyl ammonium chloride, PDADMAC, syrup-like state, $\mathrm{MW}=100,000-500,000$, product name \#Unisence FPA1001L) purchased from Senka Corporation and anionic polyacrylic acid (polyacrylic acid, PAA, solid powder, $\mathrm{MW} \approx 250,000$ ) purchased from Wako Pure 
Chemical Corporation were used without further purification.

(c) Salts

$\mathrm{KCl}$ and $\mathrm{NaOH}$ purchased from Wako Pure Chemical Corporation and $\mathrm{NaCl}$ purchased from The Salt Industry Center of Japan were used without further purification. Tap water was used without deionization and distillation.

\section{(2) Preparation of the PIC solutions}

(a) Preparation of the PIC solution of natural origin (200 L)

Approximately $100 \mathrm{~L}$ of water was placed in a $200 \mathrm{~L}$ tank. Then, $\mathrm{KCl}(4 \mathrm{~kg})$ or $\mathrm{NaCl}(4$ $\mathrm{kg}$ ) was added and dissolved in water by stirring using a power mixer (model PM-1011, Ryobi), yielding a salt solution. Meanwhile, HECHPTA (3 kg) and CMC (1 kg) were placed in another container and mixed well using a cooking spatula. Next, the solution was added to the above-mentioned tank containing the salt solution and mixed by stirring for $2 \mathrm{~h}$. Finally, the tank was filled with enough water to reach the full capacity of $200 \mathrm{~L}$ and stirred again, yielding the PIC solution.

(b) Preparation of synthetic PIC solution (200 L)

Approximately $100 \mathrm{~L}$ of water was placed in a $200 \mathrm{~L}$ tank. Then, $\mathrm{KCl}(10 \mathrm{~kg})$ and $\mathrm{NaOH}(0.48 \mathrm{~kg})$ were added and dissolved in water with stirring, yielding a salt solution. Then, PDADMAC (4.2 kg) was added to the tank containing the salt solution and stirred for about $1 \mathrm{~h}$ to facilitate complete dissolution. PAA $(0.87 \mathrm{~kg})$ was gradually added to the above-mentioned solution while stirring. PAA forms a rubber-like glob that is difficult to dissolve on mixing with water. Consequently, it required 2-3 days for the PAA to be dissolved completely. To speed up PAA dissolution, the undissolved glob was collected from the tank, transferred to a beaker, dissolved in the beaker, and returned to the tank. Finally, the tank was filled with water and stirred again to obtain $200 \mathrm{~L}$ of the synthetic PIC solution.

\section{Viscosity of the PIC Solution}

An SV-type viscosity meter (SV-10, A \& D Co., Ltd.) was used to measure the viscosity of the PIC solutions. The calibration was conducted using deionized water. The viscosity of the natural-origin PIC solution (approximately $150 \mathrm{mPa} \cdot \mathrm{s}$ ) is higher than that of the synthetic PIC solution (approximately $7 \mathrm{mPa} \cdot \mathrm{s}$ ), as shown in Table 1. Thus, the former seems to be suitable

Table 1 Viscosity of PIC solution applied and drying time for each zone

\begin{tabular}{|c|c|c|c|c|}
\hline Zone & & $\begin{array}{l}\text { PIC Solution } \\
\text { Sprayed }\end{array}$ & $\begin{array}{c}\text { Viscosity of } \\
\text { PIC Solution } \\
(\mathrm{mPa}-\mathrm{s})\end{array}$ & $\begin{array}{c}\text { Drying } \\
\text { Time }\end{array}$ \\
\hline \multirow{4}{*}{$\begin{array}{c}\text { Plastic } \\
\text { Greenhouse }\end{array}$} & Zone 1 & $\begin{array}{l}\text { Synthetic PIC } \\
\text { Solution }\end{array}$ & 6.72 & $4 \mathrm{~h}$ \\
\hline & Zone 2 & $\begin{array}{l}\text { Synthetic PIC } \\
\text { Solution }\end{array}$ & 6.72 & $4 \mathrm{~h}$ \\
\hline & Zone 3 & $\begin{array}{l}\text { Synthetic PIC } \\
\text { Solution }\end{array}$ & 6.72 & $6 \mathrm{~d}$ \\
\hline & Zone 4 & $\begin{array}{l}\text { Natural PIC }(\mathrm{KCl}) \\
\text { Solution }\end{array}$ & 152 & $6 \mathrm{~d}$ \\
\hline \multirow{2}{*}{ Pasture } & Zone 5 & $\begin{array}{l}\text { Natural PIC }(\mathrm{KCl}) \\
\text { Solution }\end{array}$ & 152 & $6 \mathrm{~d}$ \\
\hline & Zone 6 & $\begin{array}{l}\text { Natural PIC }(\mathrm{NaCl}) \\
\text { Solution }\end{array}$ & 149 & $6 \mathrm{~d}$ \\
\hline Paddy & Zone 7 & $\begin{array}{l}\text { Natural PIC (KCl) } \\
\text { Solution }\end{array}$ & 152 & $6 \mathrm{~d}$ \\
\hline
\end{tabular}


for the treatment of sandy soil and soft field soil, which aqueous solutions easily penetrate, and the latter seems to be suitable for well-trodden soil such as the front yards of private houses and the soil of dried paddy fields where the aqueous solution poorly penetrates because of its relatively high density.

\section{Target Soils for Decontamination}

\section{(1) Location of decontamination area and contaminating nuclides}

The Nagadoro district in Iitate-mura where the decontamination test was conducted is located at $37.6127^{\circ} \mathrm{N} 140.7493^{\circ} \mathrm{E}$. The major nuclides contaminating this area on May 19, 2011, just before the experiment was conducted, were ${ }^{137} \mathrm{Cs}$ (half-life 30 years) and ${ }^{134} \mathrm{Cs}$ (half-life 2 years) ${ }^{5)}$.

\section{(2) Types of PIC solutions for different land uses}

(a) Vegetable fields under plastic greenhouses

An area of $4 \mathrm{~m} \times 10 \mathrm{~m}$ under a plastic greenhouse $(5.3 \mathrm{~m} \times 44.5 \mathrm{~m})$ was adopted for a decontamination test (Figure 1).

The decontamination area was further divided into small zones. The synthetic PIC solution was sprayed onto zones $1-3$, and the natural-origin PIC $(\mathrm{KCl})$ solution was sprayed onto zone 4 (11 am to $0 \mathrm{pm}$, May 20) (see Figure 1 and Table 1). The volume of the PIC solution sprayed was about $5 \mathrm{~L} / \mathrm{m}^{2}$. To apply the synthetic PIC solution, which had a low viscosity, a commercially available gardening watering can with a shower

(1)

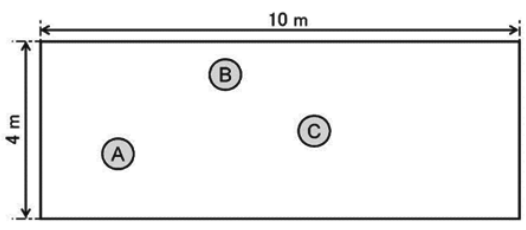

(2)

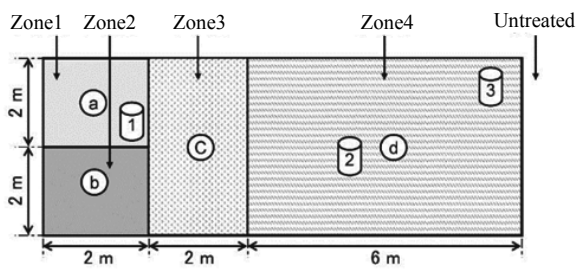

Figure 1 Layout of the decontamination area in a vinyl greenhouse before and after zoning

(1) Before zoning and (2) After zoning

Zone 1: Synthetic PIC solution sprayed and dried 4 hours and then $1-2 \mathrm{~cm}$ soil surface removed

Zone 2: Synthetic PIC solution sprayed and dried 4 hours and then $4-5 \mathrm{~cm}$ soil surface removed

Zone 3: Synthetic PIC solution sprayed and dried 6 days and then 3-4 cm soil surface removed

Zone4: Natural-origin PIC $(\mathrm{KCl})$ solution sprayed and dried 6 days and then 3-4 $\mathrm{cm}$ soil surface removed

A-C: Radioactivity measuring point (before spraying PIC solution)

a-d: $\quad$ Radioactivity measuring point (after spraying PIC solution and drying and then removing soil surface)

1-3: $\quad$ Sampling points for core samples 
head was used. To apply the natural-origin PIC solution, which had a high viscosity, the watering can was used with the shower head removed.

(b) Pasture

A decontamination area of $5 \mathrm{~m} \times 5 \mathrm{~m}$ of pasture was divided into small zones (Figure 2). A natural-origin PIC $(\mathrm{KCl})$ solution was sprayed onto zone 5, and the other natural-origin PIC $(\mathrm{NaCl}$ ) solution was sprayed in zone 6 (at 2 pm, May 20) (see Figure 2 and Table 1). The PIC solutions were sprayed with the same volume as that mentioned above and treated in a similar manner.

(c) Paddy fields

An area of $5 \mathrm{~m} \times 5 \mathrm{~m}$ of a paddy field was decontaminated (Figure 3). The natural-origin

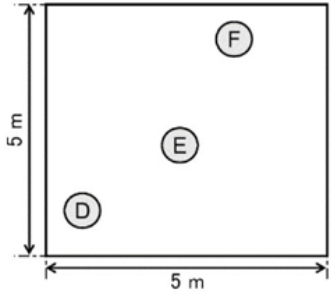

(1)

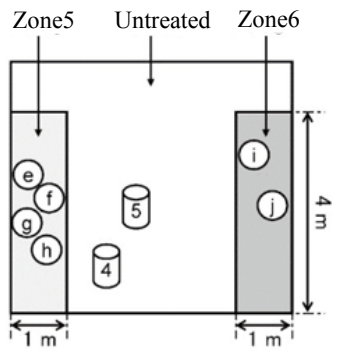

(2)

Figure 2 Layout of decontamination area in pasture

(1) Before zoning and (2) After zoning

Zone 5: Natural-origin PIC $(\mathrm{KCl})$ solution sprayed and dried 6 days and then 2-4 cm soil surface removed

Zone 6: Natural-origin PIC $(\mathrm{NaCl})$ solution sprayed and dried 6 days and then 2-4 cm soil surface removed

D-F: Radioactivity measuring point (before spraying PIC solution)

e-j: $\quad$ Radioactivity measuring point (after spraying PIC solution and drying and then removing soil surface)

4-5: $\quad$ Sampling points for core samples

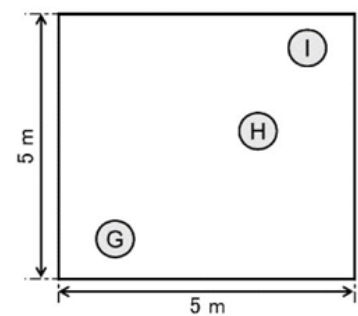

(1)

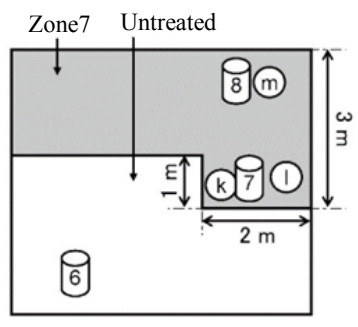

(2)

Figure 3 Layout of decontamination area in a paddy field

(1) Before zoning and (2) After zoning

Zone 7: Natural-origin PIC $(\mathrm{KCl})$ solution sprayed and dried 6 days and then 2-10 cm soil surface removed

G-I: $\quad$ Radioactivity measuring point (before spraying PIC solution)

$\mathrm{k}-\mathrm{m}$ : $\quad$ Radioactivity measuring point (after spraying PIC solution and drying and then removing soil surface)

6-8: $\quad$ Sampling points for core samples 
PIC ( $\mathrm{KCl}$ ) solution was sprayed in zone 7 (at $1 \mathrm{pm}$, May 20) (see Figure 3 and Table 1). The PIC solution was sprayed with the same volume as that mentioned above and treated in a similar manner.

\section{(3) Method for soil surface removal and drying time}

After applying the PIC solution to the surface of the soil for decontamination, the PIC was left for 4 or $6 \mathrm{~h}$ for solidification (see Table 1), and, subsequently, the soil surface layer of a specified depth was removed using a farm hoe.

The weather during the period from May 20 (the day when PIC solutions were applied) to May 26 (the day when the soil layer was removed) was as follows ("Past Weather Data" provided by Japan Meteorological Agency) ${ }^{6}$. 5/20: Fine, later cloudy, precipitation $0 \mathrm{~mm}$, and 6.5-29. ${ }^{\circ} \mathrm{C}$; $5 / 21$ : fine, $0 \mathrm{~mm}$, and $9.8-28.5^{\circ} \mathrm{C} ; 5 / 22$ : cloudy, later rainy, $2.5 \mathrm{~mm}, 7.5-22.1^{\circ} \mathrm{C}$; 5/23: cloudy, $0 \mathrm{~mm}, 4.2-17.1^{\circ} \mathrm{C} ; 5 / 24$ : cloudy, later fine, $0 \mathrm{~mm}, 5.7-18.2^{\circ} \mathrm{C} ; 5 / 25$ : fine, $0 \mathrm{~mm}$, 5.3-25. $3^{\circ} \mathrm{C}$; and $5 / 26$ : fine, later cloudy, $0 \mathrm{~mm}, 8.6-19.4^{\circ} \mathrm{C}$.

(a) Vegetable fields under plastic greenhouses

Concerning zones 1 and 2 in Figure 1, the removal of the soil surface layer was conducted $4 \mathrm{~h}$ after the application of the synthetic PIC solution. In zone 1, a soil surface layer of 1-2 cm was removed, and, in zone 2, a soil surface layer of 4-5 cm was removed. However, the aqueous PIC solutions had not sufficiently solidified. During the removal of the wet mixture of soil and PIC from the soil surface, the generation of soil dust was not observed.

On the other hand, in zone 3 in Figure 1 where the synthetic PIC solution had been sprayed and in zone 4 where the natural-origin PIC solution had been sprayed, soil surface layers of 3-4 cm were removed 6 days after the application of the PIC solutions (11 am, May 20 until 10 am, May 26).

(b) Pastures

The soil surface layers of specified depths were removed in zones 5 and 6 in Figure 2 after leaving these zones for 6 days after the application of the PIC solutions (at 2 pm, May 20 until 3pm, May 26).

(c) Paddy fields

The soil surface layer of a specified depth was removed in zone 7, as shown in Figure 3 , after leaving this zone for 6 days after the PIC solution had been applied ( $1 \mathrm{pm}$, May 20 until 2 pm, May 26).

\section{Measurement of Radioactive Cesium Contamination of the Soil Surface}

\section{(1) Measuring instrument}

A Geiger-Müller (GM) survey meter (TGS-133, Aloka) was used for measuring the $\beta$-ray count rate on site.

\section{(2) Measurement conditions}

The $\beta$-ray count rate of the soil surface was measured with a fixed time constant at $10 \mathrm{~s}$ using the GM survey meter TGS-133. Furthermore, to prevent the GM probe from becoming contaminated, the soil surface at the measurement points was covered with a polyethylene sheet $0.1 \mathrm{~mm}$ thick $\times 40 \mathrm{~cm}$ long $\times 20 \mathrm{~cm}$ wide and the GM probe was covered by a polyethylene sheet $0.05 \mathrm{~mm}$ thick and placed in close contact with the soil surface for the measurements. The $\beta$-ray count rate of the soil surface before decontamination was measured on May 20, and the soil surface measurement after decontamination was conducted on the day of soil removal. 


\section{(3) Measurement accuracy (uncertainty)}

The background was not corrected for the measurements of $\beta$-ray count rate on site because the whole area was contaminated in Nagadoro district and Iitate-mura. The standard deviation $(\sigma)$ of the $\beta$-ray count rate $(n)$ was obtained using the following equation considering time constant $\tau^{7)}$ :

$$
\sigma=n /(2 n \tau)^{1 / 2}
$$

Furthermore, when obtaining the average of the $\beta$-ray count rate in the same zone, the dispersion depending on the different measuring points was considered to be the error.

\section{(4) Definition of the decontamination rate}

The decontamination rate $(D)$ as a percentage $(\%)$ was obtained by using the $\beta$-ray count rate of the soil surface before decontamination $\left(n_{\mathrm{i}}\right)$ and that after decontamination $\left(n_{\mathrm{f}}\right)$ :

$$
D=\left\{\left(n_{\mathrm{i}}-n_{\mathrm{f}}\right) / n_{\mathrm{i}}\right\} \times 100
$$

\section{Measurement of $\gamma$-Ray Air Dose Rate}

To measure the $\gamma$-ray air dose rate at the site, a $\mathrm{NaI}(\mathrm{Tl})$ scintillation survey meter (TCS-161, Aloka, calibration constant 1.0) was used. The $\gamma$-ray air dose rate was measured at heights of 0 and $1 \mathrm{~m}$ while holding the scintillation probe horizontally and with a time constant of $10 \mathrm{~s}$. In addition, the scintillation probe was covered with a polyethylene sheet $0.05 \mathrm{~mm}$ thick to prevent the scintillation probe from becoming contaminated. Furthermore, for measurement at a height of $0 \mathrm{~m}$, the soil surface at the measuring points was covered by a polyethylene sheet $0.1 \mathrm{~mm}$ thick $\times 40 \mathrm{~cm}$ long $\times 20 \mathrm{~cm}$ wide, and the scintillation probe was placed on the sheet for measurement. The measurement was conducted on May 20 before decontamination, and the measurement after decontamination was conducted only at the points where soil removal was conducted on the same day.

\section{Measurement of the Depth Distribution of Radioactive Cesium}

\section{(1) Sampling and treatment of soil core samples}

Soil core samples $2.5 \mathrm{~cm}$ diameter were collected. A portion $0-5 \mathrm{~cm}$ depth and $1 \mathrm{~cm}$ thickness was cut on site and the deeper portion was placed whole into two polyethylene bags $0.04 \mathrm{~mm}$ thickness (with zippers) and taken to the laboratory for analysis.

(a) Vegetable fields under plastic greenhouses

Soil core samples 15-cm depth were collected at three points (1-3) shown in Figure 1.

(b) Pastures

Soil core samples were collected at two points (4 and 5) as shown in Figure 2. Because the pasture soil was too hard to obtain long soil core samples, samples $7 \mathrm{~cm}$ depth at point 4 and $4 \mathrm{~cm}$ depth at point 5 were collected.

(c) Paddy fields

Soil core samples were collected at three points (6-8) as shown in Figure 3. To avoid the influence of the crop stubble, where the cesium contamination is concentrated, samples were collected at points located between stubble. 


\section{(2) Measurement of radioactivity concentration of cesium}

The $\beta$-ray count rate of core samples was measured using a GM survey meter (model TGS133 and TG-136, Aloka) in the laboratory, and the depth distribution of the cesium radioactivity concentration in the soil was obtained from the above-mentioned measurements. The background values of the $\beta$-ray count rate were obtained $(50-60 \mathrm{cpm})$ in the laboratory, and these values were used to compensate for the background. A model TGS-133 meter was used with a conversion factor $4.3 \times 10^{-2} \mathrm{~Bq} / \mathrm{cpm}$ and a time constant of $30 \mathrm{~s}$ for measuring the core samples taken from the surface to a depth of $5 \mathrm{~cm}$. A model TGS-136 with a conversion factor $4.6 \times 10^{-2}$ $\mathrm{Bq} / \mathrm{cpm}$ operating in scaler mode was used for 1 min to measure the core samples taken from a depth greater than $5 \mathrm{~cm}$. In this $\beta$-ray count-rate measurement, we carefully sealed the soil samples in a polyethylene bag during measurement to maintain the wet condition. In addition, soil samples weighing more than $10 \mathrm{~g}$ obtained from a depth greater than $5 \mathrm{~cm}$ were cut into samples weighing less than $5 \mathrm{~g}$ and were measured in a similar manner using a double polyethylene bag $0.04 \mathrm{~mm}$ thickness (with a zipper). Furthermore, in the measurements, the samples were distributed as widely as possible within the range of the $5 \mathrm{~cm}$ diameter detection window of the GM probe, and the GM probe covered with a $0.05 \mathrm{~mm}$ thickness polyethylene sheet was pressed against the polyethylene bag. The weight of the soil samples was determined using an electronic scale. The radioactive concentration of soil samples was obtained by multiplying the $\beta$-ray count rate by each conversion factor mentioned above and then by dividing the product by the weight of the samples.

\section{Measurement of Floating Dust Concentration}

\section{(1) Dust collectors}

A portable air sampler (model L100, Munro Group Ltd.) was used for collecting floating dust.

Filter paper (\#HE-40TA, Advantec) having an outer diameter of $60 \mathrm{~mm}$ was used as the filter to collect floating dust. To increase the generation of floating dust, a household electric fan (MF-P30C, U-Ing) was used as a blower. The wind speed of the fan was measured in advance in the laboratory using a sensitive anemometer (ISA-80, Sibata Scientific Technology).

\section{(2) Measurement of radioactive cesium in floating dust}

The measurement of the floating dust concentration in air was conducted to determine the suppression of dust generation after the solidification of the soil surface layer with PIC in the vegetable fields under plastic greenhouses at the following three points (refer to Figure 1 for zone numbers): (1) Untreated points where no PIC solution had been sprayed, i.e., outside the decontamination area, (2) points in zone 4 where the natural-origin PIC solution had been sprayed, and (3) points in zone 3 where the synthetic PIC solution had been sprayed.

The equipment configuration used in the experiment is shown in Figure 4. The electric fan for blowing and the air sampler for collecting floating dust were $2 \mathrm{~m}$ from each other on the soil surface. The direction of the electric fan (having a height above ground level at its center of $33 \mathrm{~cm}$ ) was adjusted in such a way that the center of the air flow hit the filter of the floating dust collector (the height of its center was approximately $10 \mathrm{~cm}$ above ground level), and a high wind strength mode was selected to obtain a wind speed of $1.9 \mathrm{~m} / \mathrm{s}(10 \mathrm{~cm}$ above ground level) at the midpoint.

(a) Before removing the soil surface

The floating dust collector was operated immediately after blowing started, and the 


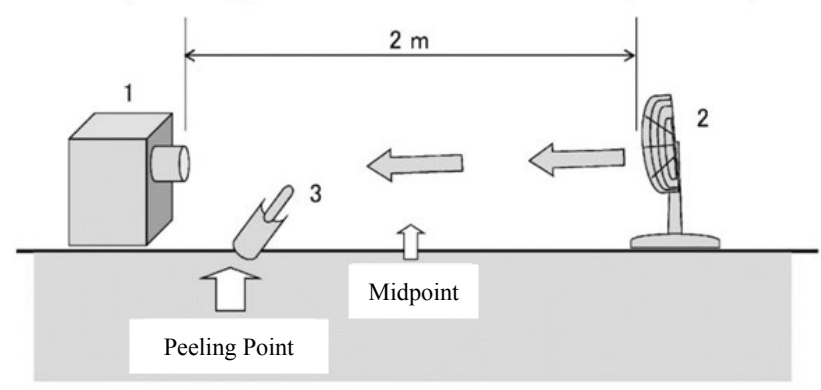

Figure 4 Equipment configuration used for collecting the floating dust 1: Floating dust collector, 2: electric fan (household), and 3: scoop for removing the soil surface

floating dust was collected in the filter for $5 \mathrm{~min}$ at a wind speed of $100 \mathrm{~L} / \mathrm{min}^{-1}$. The filter samples after dust collection were put into double polyethylene bags $0.04 \mathrm{~mm}$ thickness (with a zipper) and taken to the laboratory for analysis.

(b) In the course of soil surface removal

An electric fan was used to simulate wind flow over the soil surface during soil removal. The experiment was repeated 10 times (approximately 1 min was required for the removal of each sample) at the soil removal point shown in Figure 4 (in an area of $30 \mathrm{~cm} \times 30 \mathrm{~cm}$ approximately $10 \mathrm{~cm}$ away from the filter of the dust collector) using a gardening scoop. The floating dust generated during the operation was collected on the filter. Similarly, the filter samples after the dust collection operation were put into double polyethylene bags (each $0.04 \mathrm{~mm}$ thickness) with zippers and taken to the laboratory for analysis.

\section{(3) Measurement of filter radioactivity}

The $\beta$-ray count rate of the filter samples was measured using a GM survey meter (model TGS-136, Aloka) in the laboratory. The measurement was conducted using a TGS-136 (conversion factor $4.6 \times 10^{-2} \mathrm{~Bq} / \mathrm{cpm}$, operating in scaler mode to take measurements over $10 \mathrm{~min}$ ) with the GM probe covered by a polyethylene sheet $0.05 \mathrm{~mm}$ thickness. The probe was pressed against the polyethylene bag containing the filter sample. We took care to set the detection window of the GM probe, which had a diameter of $5 \mathrm{~cm}$, in such a way that the window covered the entire the floating dust collection area of the filter. The radioactive cesium concentration of the floating dust was obtained by multiplying the $\beta$-ray count rate by a conversion factor and dividing the product by the quantity of air collected.

\section{Results and Discussion}

\section{Soil Contamination of the Experimental Targets}

(1) Overview of land use (conditions of the soil surface, plants, etc.)

(a) Overview of vegetable fields under plastic greenhouses

By March 15, the vegetable fields under the plastic greenhouses had not been harvested for several months, and the vinyl sheets had been removed, leaving the fields open. For this reason, the contamination by rainfall on March 15 was almost the same as that in other fields used for agricultural purposes near the greenhouses. After March 15, no 
agricultural work was carried out. The land surface was dry on May 20 with neither agglomeration nor weeds; thus, the soil surface was easily scraped away with a scoop.

(b) Overview of pasture

The pastures targeted for decontamination were covered with grasses over the winter that had been mowed the previous autumn. By March 15, when the radioactive contamination occurred, some plants had begun to germinate. Based on visual observation on May 20, the land surface was covered with flat hay and cattle dung fertilizer, and pastures and weeds were sprouting through them. Based on the in situ measurement of the $\beta$-ray count rate using a GM survey meter, the radioactive cesium was concentrated in the roots of the dried hay.

(c) Overview of paddy fields

The paddy fields targeted for decontamination were contaminated on March 15; the rice had been harvested the previous autumn and the fields had then been left untouched. The soil surface was almost dry by May 20 and cracked in some spots. The withered stems and roots (stubble) after the crop of rice were observed remained intact. The in situ radioactivity measurements revealed that the radioactivity was concentrated in the stubble, and almost no radioactivity was observed on the bottom soil surface after pulling out the stubble. Consequently, it was found that removing this stubble was an effective way of decontaminating the paddy fields.

(2) $\gamma$-Ray air dose rate in each land-use classification and depth distribution of radioactive cesium

(a) Vegetable fields under plastic greenhouse

The $\gamma$-ray air dose rate at point $\mathrm{C}$ in Figure 1 was $19 \mu \mathrm{Sv} / \mathrm{h}$ at a height of $0 \mathrm{~m}$ and $12 \mu \mathrm{Sv} / \mathrm{h}$ at a height of $1 \mathrm{~m}$ (Table 2).

The depth distribution of radioactive cesium in the soil obtained from the measurement of core samples is shown in Figure 5. As a result of fitting with an exponential function, assuming a concentration of naturally derived $\beta$-nuclides in soil of $0.46 \mathrm{~Bq} / \mathrm{g}$ and because a net $\beta$-ray count rate of $10 \mathrm{cpm} / \mathrm{g}$ in soil deeper than $5 \mathrm{~cm}$ of the core sample was detected, we found that $99 \%$ of radioactive cesium was present to a depth of $5 \mathrm{~cm}$ from the surface, and the radioactivity did not penetrate deeper than $7 \mathrm{~cm}$.

(b) Pasture

The $\gamma$-ray air dose rates at points D, E, and F in Figure 2 were $20-21 \mu \mathrm{Sv} / \mathrm{h}$ at a height of $0 \mathrm{~m}$ and $12-13 \mu \mathrm{Sv} / \mathrm{h}$ at a height of $1 \mathrm{~m}$ (Table 2).

The depth distribution of radioactive cesium in soil obtained from the core sample measurement is shown in Figure 6. Compared to the results for vegetable fields under plastic greenhouses shown in Figure 5, the radioactive cesium in the pasture was found to be concentrated in a shallower region, and more than $99 \%$ of the radioactive cesium remained about $3 \mathrm{~cm}$ from the surface.

(c) Paddy fields

The $\gamma$-ray air dose rates at points G, H, and I in Figure 3 were $15 \mu \mathrm{Sv} / \mathrm{h}$ at a height of $0 \mathrm{~m}$ and $10 \mu \mathrm{Sv} / \mathrm{h}$ at a height of $1 \mathrm{~m}$ (Table 2).

The depth distribution of radioactive cesium in the soil obtained from the measurements of the core samples is shown in Figure 7. Using a similar fitting as described above, we found that more than $99 \%$ of the radioactive cesium remained as deep as $3 \mathrm{~cm}$ from the soil surface. It was also found that the depth distribution of radioactive cesium in paddy field soil was analogous to that of the pasture soil, and the radioactive cesium was distributed nearer to the surface compared to that of the soil for the vegetable fields 
Table 2 Measurements of radioactivity at the points targeted for decontamination.

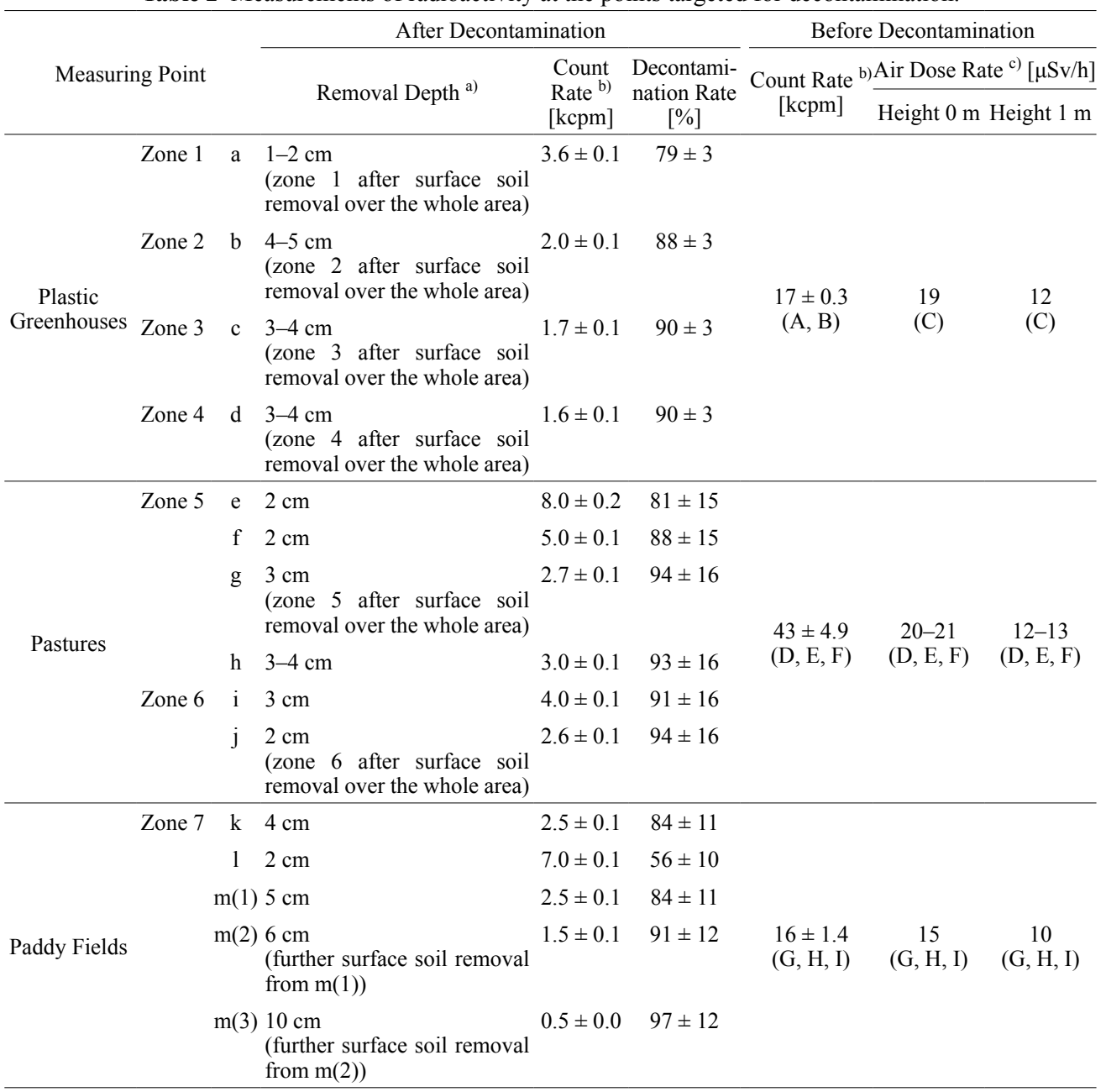

a) Surface soil removal after $4 \mathrm{~h}$ at points a and $\mathrm{b}$ and after $6 \mathrm{~d}$ at other points

b) Measurement of $\beta$-ray using a GM survey meter (including the background)

c) Measurement of $\gamma$-ray using a NaI scintillation survey meter

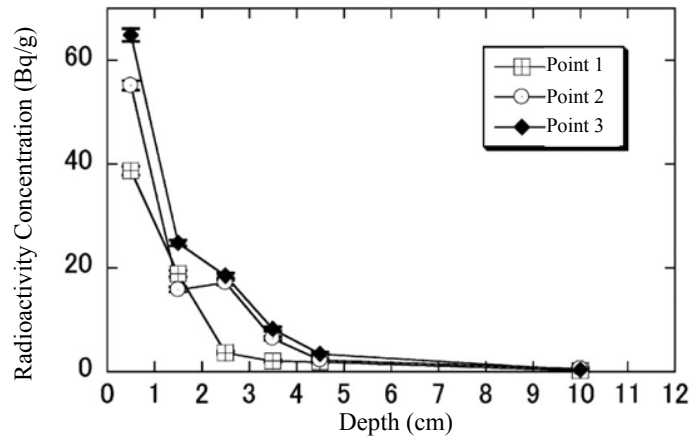

Figure 5 Depth distribution of radioactive cesium in the soil in vegetable fields under plastic greenhouses obtained by core sampling 


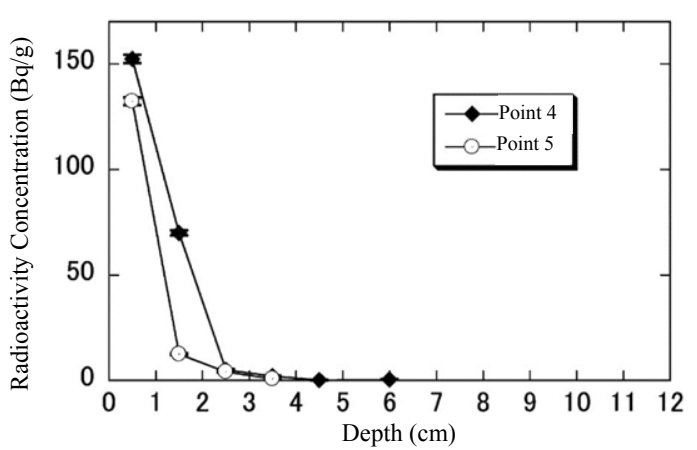

Figure 6 Depth distribution of radioactive cesium in the soil in pasture obtained by core sampling

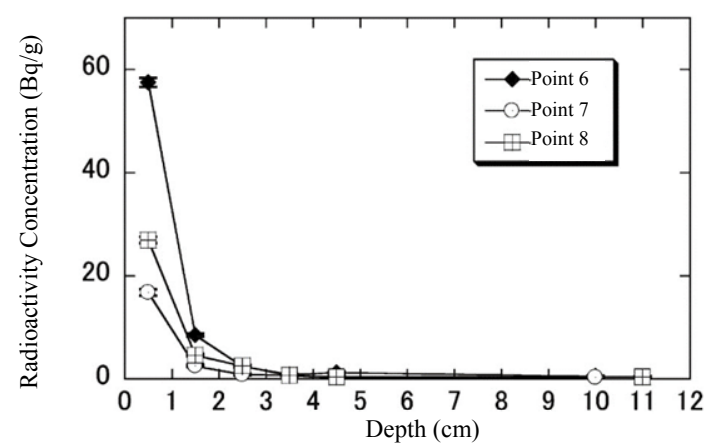

Figure 7 Depth distribution of radioactive cesium in the soil in paddy fields obtained by core sampling

under plastic greenhouses. The reason for this is assumed to be the lower penetration of rainfall in paddy fields and pastures than in the vegetable fields under the plastic greenhouses where the soil contained more voids.

\section{Decontamination Effect of the Removing the Soil Surface}

\section{(1) Vegetable fields in plastic greenhouses}

The $\beta$-ray count rates of the soil surface before decontamination at points A and B in Figure 1 are shown in Table 2 . The $\beta$-ray count rate on the soil surface before decontamination was almost uniform within the targeted decontamination range, and the average was $17 \mathrm{kcpm}$.

The $\beta$-ray count rates of the soil surface at points a-d shown in Figure 1 are summarized in Table 2. The removal of the soil surface $4 \mathrm{~h}$ after the PIC solution had been applied and penetrated the soil to a depth of 1-2 cm from the surface in zone 1 resulted in a decontamination rate of approximately $80 \%$ at point a. The removal of the soil surface in zone 2 after similar spraying and penetration to a depth of 4-5 $\mathrm{cm}$ from the soil surface resulted in a decontamination rate of about $90 \%$ at point $b$ (Table 2). The $\gamma$-ray air dose rates at point a and point $b$ after decontamination decreased respectively to 6.5 and $4.0 \mu \mathrm{Sv} / \mathrm{h}$ at a height of $0 \mathrm{~m}$ and 10.6 and $9.7 \mu \mathrm{Sv} / \mathrm{h}$ at a height of $1 \mathrm{~m}$.

The natural-origin PIC sprayed in zone 4 was fixed to a sufficient hardness after 6 days and could be removed as soil flakes having a thickness of $0.5-1 \mathrm{~cm}$ and a size ranging from that of a 
100 yen coin to the size of the palm of the hand by using a gardening scoop, as shown in Photo 1. By measuring the $\beta$-ray count rate of the soil surface after the removal of these soil flakes, a decontamination rate of $72-82 \%$ was obtained. In the actual decontamination operation, 3-4 $\mathrm{cm}$ of the soil surface layer was removed. As a result, a decontamination rate of $90 \%$ was obtained at points $\mathrm{c}$ and $\mathrm{d}$ by removing $3-4 \mathrm{~cm}$ of soil surface layer solidified by PIC for both zone 3 (solidified with synthetic PIC solution) and zone 4 (solidified with natural-origin PIC solution) (Table 2).

\section{(2) Pasture}

The results of the measurement of the $\beta$-ray count rate of the soil surface before decontamination at points D-F in Figure 2 are shown in Table 2. The average $\beta$-ray count rate of the pasture soil surface before decontamination was $43 \mathrm{kcpm}$, which is $2-3$ times higher than that on the soil surfaces of the vegetable fields under plastic greenhouses or the paddy fields targeted for the present decontamination test.

The natural-origin PIC sprayed on soil surface solidified to a sufficient hardness. The grasses growing in the pasture set their roots within a shallow range (range of depth about $2-3 \mathrm{~cm}$ ) from the soil surface; therefore, the soil and the solidified PIC were able to be removed uniformly together with grasses using a gardening scoop. A decontamination rate of $94 \%$ was obtained at both points $\mathrm{g}$ and $\mathrm{j}$ (removing 3 and $2 \mathrm{~cm}$ depth of soil, respectively) (Table 2).

\section{(3) Paddy fields}

The results of the measurements of the $\beta$-ray count rate of the soil surface before decontamination at points $\mathrm{G}-\mathrm{I}$ in Figure 3 are listed in Table 2 . The average $\beta$-ray count rate for paddy field soil surfaces before contamination was $16 \mathrm{kcpm}$.

The natural-origin PIC sprayed in paddy fields solidified to a sufficient hardness. The measured $\beta$-ray count rates of the soil surface after removing $2-10 \mathrm{~cm}$ of the soil surface layer at points $\mathrm{k}-\mathrm{m}$ shown in Figure 3 are summarized in Table 2. As a result, decontamination rates of $56 \%$ (removing a thickness of $2 \mathrm{~cm}$ ), 84\% (removing a thickness of $4 \mathrm{~cm}$ ), $84 \%$ (removing a thickness of $5 \mathrm{~cm}$ ), $91 \%$ (removing a thickness of $6 \mathrm{~cm}$ ), and 97\% (removing a thickness of 10 $\mathrm{cm}$ ) were obtained. These results would appear to indicate that the higher decontamination rate was obtained by removing a deeper soil level. However, because about $99 \%$ of the radioactive cesium is presumed to be removed by removing $3 \mathrm{~cm}$ of soil, based on the depth distribution of radioactive cesium obtained by measuring soil core samples, the decontamination rate listed

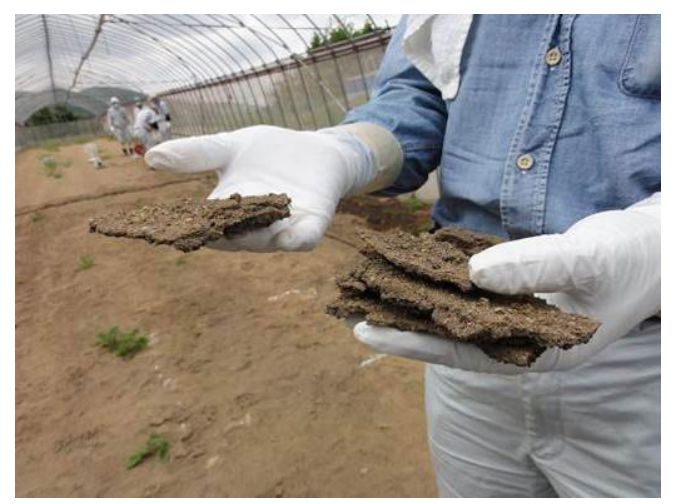

Photo 1 Soil flakes of the vegetable fields under plastic greenhouses solidified by natural-origin PIC 
in Table 2 would be even higher if in situ background compensation of measurements were possible.

\section{Suppression of Dust Generation}

\section{(1) Effect of PIC before surface soil removal}

The quantity of radioactive cesium in the captured floating dust was determined, and the results are shown in Figure 8 (1) as the radioactivity of the captured air. The quantity of airborne radioactive cesium produced from the soil surface captured by the solidified PIC solution is lower than that of the soil surface without solidification treatment, especially after solidification using the natural-origin PIC solution, that is, 0.36 times lower.

\section{(2) Effect of PIC during surface soil removal}

The quantity of radioactive cesium in the captured floating dust was obtained as above, and the results are shown in Figure 8 (2) as the radioactivity of the captured air. The quantity of airborne radioactive cesium produced during surface soil removal was reduced by the solidification treatment using the PIC solution, especially using natural-origin PIC solution. The radioactive cesium produced was 0.32 times smaller than the case without any soil surface treatment. For context, the radioactivity concentration in the captured air during surface soil removal operation was larger by one order of magnitude than that before operation.

\section{Application of PIC to Other Materials for Decontamination}

The decontamination of more complex environments, such as the surroundings of a house, is required; thus, the use of the PIC solution was tested for surface soil removal from the garden, the removal of solid material accumulated in the roof gutters, and the removal of withered Cryptomeria branches.

The soil of the gardens had been trodden down and compacted by vehicles, which made it difficult for the PIC solution to penetrate the soil. In particular, the natural-origin PIC solution did not penetrate the soil to a sufficient depth. For this reason, the soil must be treated (e.g., by pricking) after spraying in the case of using the natural-origin PIC solution.
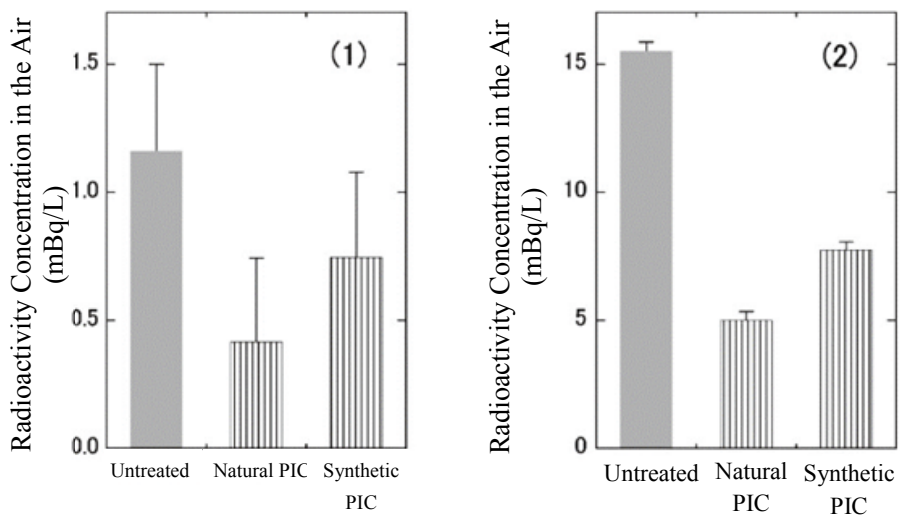

Figure 8 Suppression of floating dust generation using PIC solidification

(1) Quantity of airborne radioactive cesium before soil surface removal

(2) Quantity of airborne radioactive cesium during soil surface removal 
The amount of floating dust was reduced successfully after the advanced spraying of the PIC solution during various operations. The proper treatment of the waste collected during the decontamination operation is currently under investigation and further tests will be required. At that time, the use of the PIC solution for inhibiting dust generation from waste was considered to be effective.

\section{Summary}

We confirmed that the solidification of the soil surface layer using a polyion complex (PIC), followed by removal of the soil, is effective for the decontamination of soil contaminated by radioactive cesium. The decontamination rate of the soil surface layer of vegetable fields under plastic greenhouses, pastures, and paddy fields was higher than $90 \%$. Furthermore, the production of airborne radioactive cesium concentrated in the soil surface layer and scattered by the wind was effectively suppressed. From the experimental data, especially in the vegetable fields under plastic greenhouses, the method using high viscosity natural-origin PIC solution was particularly effective.

It is expected that the range of application of this method will be expanded in the future by the development of PICs having suitable properties for application to different types of soil.

\section{References}

1) M. Chino, H. Nakayama, H. Nagai et al., "Preliminary estimation of release amounts of ${ }^{131} \mathrm{I}$ and ${ }^{137} \mathrm{Cs}$ accidentally discharged from the Fukushima Daiichi nuclear power plant into the atmosphere," J. Nucl. Sci. Technol., 48(7), 1129-1134(2011).

2) A. B. Zezin, N. Kumazawa "Chemical Treatment of Chernobyl Nuclear Power Plant - How did the Russian Chemists do for Prevention of Expanded Radioactivity Contamination," Chem. Today, 339, 30-38(1999). [in Japanese]

3) N. Kumazawa, T. Ozawa, V. G. Sergeyev et al., "The Application of Chemical Treatment Used in Chernobyl Accident to Tokai-mura," Annual Report of the Institute of Regional Studies Ibaraki University Sup. Vol. The Tokai-mura Criticality Accident and Regional Society 69-75(2002). [in Japanese]

4) N. Kumazawa, T. Ozawa, V. G. Sergeyev et al., "Development of Soil Solidification Method using Natural Polyion - Aiming at Improvement of Treatment Method in Chernobyl Accident," Annual Report of the institute of Regional Studies Ibaraki University Sup. Vol. Tokai-mura Nuclear Anti-Disaster Measures and Regional Society 7-11(2005). [in Japanese]

5) MEXT, Dust Sampling around Fukushima Daiichi and Daini Nuclear Power Plants, Measurement Results of Environmental Specimens and Soil Monitoring: The measurements on soil monitoring (as of 10:00, Jun. 03, 2011), http://www.mext.go.jp (2011). [in Japanese]

6) JMA, Weather Statistical Information "Past Weather Data Search," (http://www.jma.go.jp/jma/index. html) (2011). [in Japanese]

7) G. F. Knoll (author), Itsuro Kimura, Eiji Sakai (translator), Radiation Measuring Handbook (Vol. 3), Nikkan Kogyo Shimbun, 708-711(2001). [in Japanese] 


\title{
Measurement and Evaluation of Hydrogen Production from Mixtures of Seawater and Zeolite in Decontamination of Radioactive Water
}

\author{
Yuta KUMAGAI ${ }^{1, *}$, Ryuji NAGAISHI ${ }^{1}$, Atsushi KIMURA ${ }^{2}$, \\ Mitsumasa TAGUCHI $^{2}$, Kenji NISHIHARA ${ }^{1}$, Isao YAMAGISHI ${ }^{1}$ \\ and Toru OGAWA ${ }^{1}$ \\ ${ }^{1}$ Nuclear Science and Engineering Directorate, Japan Atomic Energy Agency, 2-4 Shirane, Shirakata, Tokai-mura, \\ Naka-gun, Ibaraki 319-1195, Japan \\ ${ }^{2}$ Quantum Beam Science Directorate, Japan Atomic Energy Agency, 1233 Watanuki-machi, Takasaki-shi, Gunnma \\ 370-1292, Japan
}

\begin{abstract}
Zeolite is used for the decontamination of radioactive water, which contains salts from seawater, accumulated in the Fukushima Daiichi Nuclear Power Plant. The evaluation of hydrogen production by water radiolysis during the decontamination process is important for safe operation. Thus, hydrogen production from a mixture of zeolite and seawater was studied by a $\gamma$-radiolysis experiment, and the hydrogen production during the process was evaluated. The measured yield of hydrogen from seawater was comparable to the primary yield in the $\gamma$-radiolysis of water. This result indicates that the oxidation of hydrogen by the radical products of water radiolysis is not effective in seawater. The measured yield from the mixture decreased at a high weight fraction of zeolite. However, the measured yield was higher than that expected from the direct radiolysis of water in the mixture, which would decrease proportionally to the weight fraction of water. This result suggests that the radiation energy deposited on the zeolite is involved in hydrogen production. From the measured yields, the hydrogen production rate was evaluated to be $3.6 \mathrm{~mL} / \mathrm{h}$ per $\mathrm{t}$ of radioactive water before the process and $1.5 \mathrm{~L} / \mathrm{h}$ per $\mathrm{t}$ of waste adsorbent after the process.
\end{abstract}

KEYWORDS: hydrogen, radiolysis, zeolite, seawater, radioactive contaminated water, decontamination

\section{Introduction}

Inorganic adsorbents have been extensively studied in the removal of radioactive cesium and strontium from contaminated water and used in the treatment of the radioactive water from the Fukushima Daiichi Nuclear Power Plant (1F). Before practical application, safety during the adsorbing treatment and the temporary storage of radioactive waste after treatment must be evaluated. Under radiation environment, hydrogen is produced by the decomposition

\footnotetext{
${ }^{*}$ Corresponding author, E-mail: kumagai.yuta@jaea.go.jp

DOI : 10.15669 /fukushimainsights.Vol.4.37

(C) 2021 Atomic Energy Society of Japan. All rights reserved.

Originally published in Transactions of the Atomic Energy Society of Japan (ISSN 1347-2879), Vol. 10, No. 4, p.235-239

(2011) in Japanese. (Japanese version accepted: July 26, 2011)
} 
of water. Previous studies have pointed out that various inorganic solid substances affect the hydrogen production from the radiolysis of water ${ }^{1-4)}$. This radiolytic hydrogen production must be considered to ensure safety during and after treatment. Furthermore, the contaminated water in $1 \mathrm{~F}$ includes a considerable quantity of salts from seawater. The presence of salts makes the situation different from that experienced in the reactor water treatment ${ }^{5,6}$ after the accident at Three Mile Island Unit 2.

Hence, in this study, we focused on zeolites that are potential inorganic adsorbents mainly for radioactive cesium to conduct irradiation experiments and evaluate the quantity of hydrogen produced from the mixture of zeolites and seawater. Furthermore, we evaluated the radiolytic hydrogen production from the zeolite-seawater mixtures at elevated temperatures, because a temperature rise in the spent adsorbents is expected because of the decay heat of radioactive nuclide during the temporary storage.

\section{Experimental Methodology}

The mixtures of zeolite-type adsorbents and seawater were irradiated by $\gamma$-ray in a vial with a septum. After irradiation, the gas in the upper free space in the vial was analyzed by gas chromatography and hydrogen in the gas phase was determined.

The details of the experimental methodology are described below:

The zeolite-type adsorbents used in this study were Herschelite (bulk density $0.65 \mathrm{~g} / \mathrm{mL}$ ), engineered Herschelite (hereafter referred to as "E-Herschelite", $0.65 \mathrm{~g} / \mathrm{mL}$ ) provided by KURION, and natural mordenite mined in Ayashi by Shin Tohoku Chemical Industry ( $0.8 \mathrm{~g} /$ $\mathrm{mL}$ ). The seawater was sampled in Oarai Seaside, Ibaraki Prefecture, and filtrated through a filter paper (5C) before use.

In practical application, zeolite-type adsorbents may be used in batch processes or in column systems. Therefore, we prepared samples containing $1 \mathrm{wt} \%$ of adsorbents for simulating the batch processes and samples containing approximately $50 \mathrm{wt} \%$ of the adsorbents for simulating the column systems and the spent adsorbents during temporary storage. The adsorbents in the 1 $\mathrm{wt} \%$ samples settled on the bottom of the vials. The $50 \mathrm{wt} \%$ samples were prepared by gradually adding the seawater to the adsorbents until the seawater filled up the vacant space between the adsorbent grains. The adsorbents were weighed under a condition of approximately $40 \%$ relative humidity. Note that the adsorbents contained adsorbed water because they were not dehydrated before use. Each sample vial contained $3 \mathrm{~g}$ to $9 \mathrm{~g}$ of the mixture to produce sufficient hydrogen for measurement. The samples were sealed in glass vials with a septum. The vial used for the measurement at room temperature had a diameter of $23 \mathrm{~mm}$ and a volume of $8.7 \mathrm{~mL}$. For the measurements of temperature dependence, the sample vial was $12 \mathrm{~mm}$ in diameter and had a volume of $5.15 \mathrm{~mL}$. The septum was made of silicon and coated with Teflon on the liquid contacting surface. The atmosphere and pressure of the samples were not controlled and the upper free space was filled with air.

The cobalt irradiation facility of the Takasaki Advanced Radiation Research Institute was used for irradiating the $\gamma$-ray (mean energy $1.25 \mathrm{MeV}$ ) from the ${ }^{60} \mathrm{Co}$ radiation source on the specimen. During irradiation, the specimen was left in a static condition and was not stirred. The dose rate was adjusted in the range of $2 \mathrm{kGy} / \mathrm{h}$ to $5 \mathrm{kGy} / \mathrm{h}$ to obtain an irradiation time fewer than 2 hours with a range of absorbed dose rate from $1 \mathrm{kGy}$ to $10 \mathrm{kGy}$. A dichromate dosimeter was used for measuring the absorbed dose rate ${ }^{7)}$. For irradiating under a controlled temperature, an aluminum block isothermal bath (TAITEC) was used. 
After stirring the specimen after irradiation for $2 \mathrm{~min}$ at a speed of 3,000 rpm using a vortex mixer, the gas in the upper free space in the vial was sampled with a microsyringe and analyzed using the gas chromatograph (GC14A/Shimadzu) and the hydrogen concentration in the upper free space was measured. For the gas chromatograph analyses, a column of bulking agent molecular sieve $5 \mathrm{~A}$ with a $4 \mathrm{~m}$ long column was used for the measurement of hydrogen using a TCD detector with pure argon as a carrier gas. Furthermore, to determine the volume of the upper free space, pure water was added until the container was filled with the specimen after measurement and the weight increase was measured. Four rounds of measurements were conducted for specimens of identical composition, and an average of the volume of the upper free space was determined. The specific weight of pure water was assumed to be $1.00 \mathrm{~g} / \mathrm{mL}$. The errors were not more than $0.1 \mathrm{~mL}$ for a vial with a volume of $8.7 \mathrm{~mL}$, and not more than $0.06 \mathrm{~mL}$ for a vial with a volume of $5.15 \mathrm{~mL}$. These errors were not more than $3 \%$ of the average of the volume of upper free space. The quantity of hydrogen emitted from the specimen was measured using the hydrogen concentration and the volume of the vial's upper free space.

\section{Hydrogen Production Quantity due to the Radiolysis of Seawater}

First, the result of the measurement of hydrogen production due to the radiolysis of seawater is explained. The hydrogen production quantity of a specimen that consisted only of seawater was proportional to the absorbed dose and the measured yield $G\left(\mathrm{H}_{2}\right)$ was $(4.8 \pm 0.3) \times 10^{-8} \mathrm{~mol} / \mathrm{J}$. Hereafter, the measured hydrogen yield as a final product and the primary yield of hydrogen produced by the radiolysis of water are distinguished as $G\left(\mathrm{H}_{2}\right)$ and $\mathrm{g}\left(\mathrm{H}_{2}\right)$, respectively.

In the radiolysis of water, the production of hydrogen due to the reaction between the decomposition products progresses on a short timescale up to $0.1 \mu$ s from the energy transfer from the radiation to the water. The "primary yield $\mathrm{g}\left(\mathrm{H}_{2}\right)$ " is the yield of hydrogen at approximately $0.1 \mu \mathrm{s}$ after the energy transfer, and is $4.7 \times 10^{-8} \mathrm{~mol} / \mathrm{J}$ for $\gamma$-rays or electron beams that are classified as radiation with low linear energy transfer. Because the reaction of decomposition products and hydrogen still occurred $0.1 \mu$ s after the energy transfer, $G\left(\mathrm{H}_{2}\right)$ will be dependent on the reaction path of a decomposition product and ultimately takes a different value than $\mathrm{g}\left(\mathrm{H}_{2}\right)$. What is problematic in the treatment of contaminated water is the hydrogen released in the gas phase, and hence, $G\left(\mathrm{H}_{2}\right)$, not $\mathrm{g}\left(\mathrm{H}_{2}\right)$, becomes important in the evaluation of hydrogen production quantity.

A typical reaction that influences $G\left(\mathrm{H}_{2}\right)$ is the reaction with the hydroxylic radical $(\cdot \mathrm{OH})$ :

$$
\begin{aligned}
\mathrm{H}_{2}+\cdot \mathrm{OH} \longrightarrow \mathrm{H} \cdot+\mathrm{H}_{2} \mathrm{O} \\
\quad k_{(1)}=4.2 \times 10^{7} \mathrm{~L} \cdot \mathrm{mol}^{-1} \cdot \mathrm{s}^{-1}
\end{aligned}
$$

For example, it is known for deaerated pure water that the $G\left(\mathrm{H}_{2}\right)$ falls far below $4.7 \times$ $10^{-8} \mathrm{~mol} / \mathrm{J}$ due to this reaction ${ }^{8}$.

On the other hand, for potassium bromide solution, the oxidation of hydrogen via the reaction (1) is inhibited by the following reaction of the bromide ion and hydroxylic radical ${ }^{9,10)}$ :

$$
\begin{aligned}
\mathrm{Br}^{-}+\cdot \cdot \mathrm{OH} \rightleftarrows & \mathrm{BrOH}^{-} \\
& k_{(2) \mathrm{f}}=1.1 \times 10^{10} \mathrm{~L} \cdot \mathrm{mol}^{-1} \cdot \mathrm{s}^{-1} \\
& k_{(2) \mathrm{b}}=3.3 \times 10^{7} \mathrm{~s}^{-1}
\end{aligned}
$$




$$
\begin{aligned}
\mathrm{BrOH} & \cdot-+\mathrm{Br}^{-} \longrightarrow \mathrm{Br}_{2}^{--}+\mathrm{OH}^{-} \\
& k_{(3)}=1.9 \times 10^{8} \mathrm{~L} \cdot \mathrm{mol}^{-1} \cdot \mathrm{s}^{-1}
\end{aligned}
$$

In this experiment, it was confirmed that $G\left(\mathrm{H}_{2}\right)$ was $(4.6 \pm 0.2) \times 10^{-8} \mathrm{~mol} / \mathrm{J}$ because of the measurement of hydrogen production quantity in the deaerated $1 \mathrm{mmol} / \mathrm{L}$ potassium bromide solution.

Accordingly, the fact that the $G\left(\mathrm{H}_{2}\right)$ equivalent to the $\mathrm{g}\left(\mathrm{H}_{2}\right)$ measured in the specimen consisting only of seawater was similar to that of the potassium bromide solution, implies that the oxidation reaction of hydrogen was inhibited in the seawater. Regarding the mechanism, a more detailed study into the effect of dissolved species in seawater is needed. But, according to the analysis by Bjergbakke et al ${ }^{11}$., it is considered that the main reason for the effect is the reaction between the bromide ions, chloride ions, and the decomposition product of oxygen and water in the seawater.

\section{Hydrogen Production Quantity from Adsorbent-Seawater Mixture}

In this section, the influence of zeolite type adsorbent addition on the hydrogen production quantity is explained. The results of the measurements on hydrogen production quantity for the mixture of various zeolite types in the adsorbent and seawater are shown in Figure 1. The vertical axis in the figure is the hydrogen quantity released from the unit weight of the mixture. Because the absorbed dose was different due to the difference of the mass energy absorption coefficient between the adsorbent and water, even if the radiation fields were identical, a correction was applied to the absorbed dose on the horizontal axis in the figure. The absorbed dose measurement of the adsorbent was obtained using a dosimeter with a photon mass energy absorption coefficient of $1.25 \mathrm{MeV}$. The absorbed dose of the mixture was defined as the weighted sum with weight percent on the absorbed dose of adsorbent and water, i.e., the absorbed dose $D(\mathrm{kGy})$ of the mixture was acquired using the equation below:

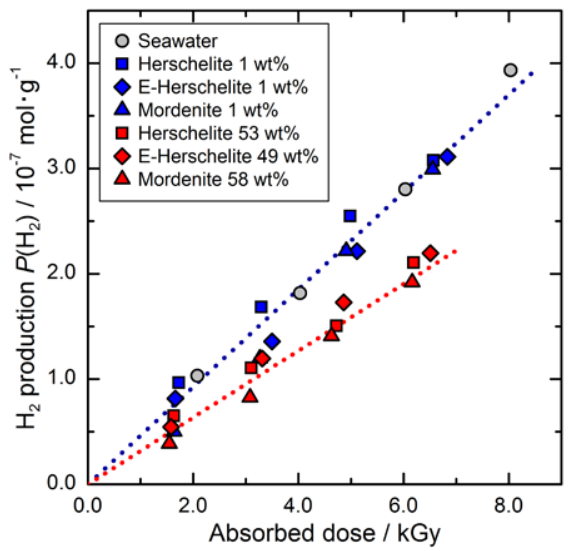

Figure 1 Hydrogen production by radiolysis of the zeolites and seawater mixtures Blue dotted line: linear fit for the samples containing $1 \mathrm{wt} \%$ zeolites. Red dotted line: linear fit for the samples containing approximately $50 \mathrm{wt} \%$ zeolites 


$$
D=D_{\text {solid }} \cdot w_{\text {solid }}+D_{\text {water }} \cdot w_{\text {water }}
$$

where $w$ indicates the weight percentage, the suffix "solid" and "water" indicate the adsorbent and seawater, respectively. The difference in the absorbed doses between seawater and the dichromate potassium solution used as a dosimeter was ignored. The absorption coefficient of water is $2.97 \times 10^{-2} \mathrm{~cm}^{2} / \mathrm{g}$ and the absorption coefficient of aluminosilicate such as zeolite shows a low dependence on the composition at approximately $2.7 \times 10^{-2} \mathrm{~cm}^{2} / \mathrm{g}^{12)}$. Accordingly, the absorption dose of adsorbent $D_{\text {solid }}$ is lower than that of water $D_{\text {water }}$ by approximately $10 \%$.

The hydrogen production quantity from the mixture of adsorbent and seawater was equivalent to the specimen containing only seawater, regardless of the adsorbent types for the specimen containing $1 \mathrm{wt} \%$ of adsorbent. While the specimen of approximately $50 \mathrm{wt} \%$ showed a lower hydrogen production quantity compared to the specimen containing only seawater, there was not a notable difference observed in the dependence on the adsorbent type.

Where the absorbed dose was $10 \mathrm{kGy}$ and under, the hydrogen production quantity and absorbed dose showed an approximately proportional relationship. Hence, the hydrogen production yield was obtained through proportional regression of the hydrogen production quantity to the absorbed dose. The hydrogen production yield $G\left(\mathrm{H}_{2}\right)(\mathrm{mol} / \mathrm{J})$ and hydrogen production quantity $P\left(\mathrm{H}_{2}\right)(\mathrm{mol} / \mathrm{g})$ has a relationship described by the following equation:

$$
P\left(\mathrm{H}_{2}\right)=G\left(\mathrm{H}_{2}\right) \times D
$$

The values of $G\left(\mathrm{H}_{2}\right)$ acquired for the various specimens are summarized in Table $\mathbf{1}$.

\begin{tabular}{|c|c|c|}
\hline \multicolumn{2}{|c|}{ Sample } & \multirow{2}{*}{$\frac{G\left(\mathrm{H}_{2}\right) / 10^{-8} \mathrm{~mol} \cdot \mathrm{J}^{-1}}{4.9 \pm 0.2}$} \\
\hline Herschelite & $1 \mathrm{Wt} \%$ & \\
\hline & $53 \mathrm{wt} \%$ & $3.4 \pm 0.1$ \\
\hline \multirow[t]{2}{*}{ E-Herschelite } & $1 \mathrm{wt} \%$ & $4.4 \pm 0.2$ \\
\hline & $49 \mathrm{wt} \%$ & $3.5 \pm 0.1$ \\
\hline \multirow[t]{2}{*}{ Mordenite } & $1 \mathrm{wt} \%$ & $4.4 \pm 0.3$ \\
\hline & $58 \mathrm{wt} \%$ & $3.0 \pm 0.1$ \\
\hline
\end{tabular}

Table $1 G\left(\mathrm{H}_{2}\right)$ measured in the mixtures of the zeolites and seawater

\section{Influence of Adsorbent on Hydrogen Production Yield}

In this experiment, no $G\left(\mathrm{H}_{2}\right)$ that exceeds $\mathrm{g}\left(\mathrm{H}_{2}\right)$ was observed for the mixture of adsorbent and seawater. However, the measured $G\left(\mathrm{H}_{2}\right)$ implied the influence of energy transfer from the radiations to the adsorbent on the radiolysis of water.

The dependence of $G\left(\mathrm{H}_{2}\right)$ to the weight ratio of adsorbent and seawater is shown in Figure 2. For comparison, a series of reports by Nakashima et al. regarding hydrous zeolite ${ }^{1-3)}$ are shown. As reported by Nakashima et al., $G\left(\mathrm{H}_{2}\right)$ was provided based on the weight ratio of zeolite dried at $350^{\circ} \mathrm{C}$ with moisture added. Nakashima et al. adopted the synthetic zeolite of type-A and type- $\mathrm{Y}$ with pure water added as specimens and investigated the hydrogen production quantity under a condition with less moisture content than that of this experiment. The blue and green solid lines in Figure 2 indicate the curves based on the evaluation formula for hydrogen production yield for type K-A and type Na-A zeolites by Nakashima et al., respectively. This evaluation formula assumes that hydrogen is produced with the yield of $4.7 \times 10^{-8} \mathrm{~mol} / \mathrm{J}$ from 


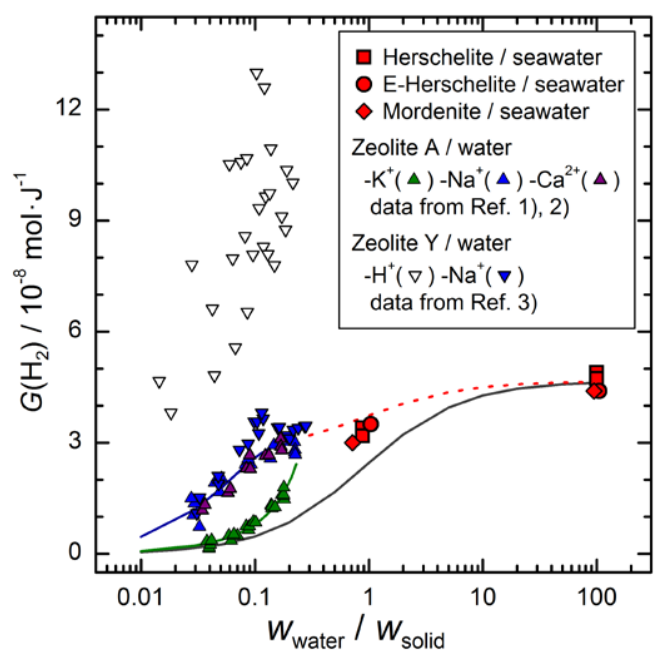

Figure 2 The yield of hydrogen as a final product, $G\left(\mathrm{H}_{2}\right)$, in the mixtures of the zeolites and water as a function of the weight ratio of water to zeolites Blue and green curves: formula by Nakashima and Aratono ${ }^{2)}$ Red dotted curve: extrapolation of the formula (Eq. 10) Gray curve: no $\mathrm{H}_{2}$ production from zeolites (Eq. 11)

the energy transferred to water and that the hydrogen production that occurs with the yield is dependent on the moisture content from the energy transferred to the zeolite ${ }^{1,2}$.

The results of the experiment by Nakashima et al. show the trend of convergence of the hydrogen production yield because of the energy transferred to the zeolite was $2.7 \times 10^{-8} \mathrm{~mol} / \mathrm{J}$ when the weight ratio of water to zeolite approached 0.25 for type A regardless of the contained cation ${ }^{2)}$. Hence, the evaluation formula by Nakashima et al. was extrapolated based on the assumption that the energy transferred to the zeolite contributed to hydrogen production with a certain yield of $2.7 \times 10^{-8} \mathrm{~mol} / \mathrm{J}$ in the range of higher moisture content and is indicated by the red dotted lines. The red dotted lines are the plots of $G\left(\mathrm{H}_{2}\right)$ obtained using the following equation:

$$
\begin{aligned}
P\left(\mathrm{H}_{2}\right) & =G\left(\mathrm{H}_{2}\right) \times \mathrm{D} \\
& =4.7 \times 10^{-8} \times D_{\text {water }} \cdot w_{\text {water }}+2.7 \times 10^{-8} \times D_{\text {solid }} \cdot w_{\text {solid }}
\end{aligned}
$$

Consequently, the red dotted line indicates the $G\left(\mathrm{H}_{2}\right)$ when it is assumed that the energy transferred to the zeolite produces hydrogen with approximately $60 \%$ efficiency compared to the energy transferred directly to the water.

The gray curve in Figure 2 is the plot of the following equation:

$$
\begin{aligned}
P\left(\mathrm{H}_{2}\right) & =G\left(\mathrm{H}_{2}\right) \times \mathrm{D} \\
& =4.7 \times 10^{-8} \times D_{\text {water }} \cdot w_{\text {water }}+0 \times D_{\text {solid }} \cdot w_{\text {solid }}
\end{aligned}
$$

More specifically, the gray curve in Figure 2 is equivalent to $G\left(\mathrm{H}_{2}\right)$ when only the energy transferred to the water in the mixture contributes to the hydrogen production with a yield of $4.7 \times 10^{-8} \mathrm{~mol} / \mathrm{J}$, and no hydrogen is produced from the energy transferred to the zeolite. However, based on the results of the measurements on specimens containing seawater only, the 
inhibition of hydrogen production by oxidation reaction was not considered.

Additionally, from the results of this experiment, it is evident that the value of the specimen with approximately $50 \mathrm{wt} \%$ of added adsorbent exceeds the anticipated $G\left(\mathrm{H}_{2}\right)$ from Eq. (11). For this reason, there is a possibility of underestimation when the hydrogen production quantity is evaluated using Eq. (11). Consequently, the evaluation that considers the hydrogen production from the energy transfer to the adsorbent, as shown in Eq. (10), is regarded as reasonable also for the mixture with seawater. However, because the weighed adsorbent contains the adsorbed water in this experiment, it was considered that the radiolysis of the adsorbed water also contributed to a higher observed value of $G\left(\mathrm{H}_{2}\right)$ than was predicted by Eq. (11).

The necessity for the consideration of hydrogen production caused by the energy transfer to the adsorbent was also indicated by the accident report of Three Mile Island Unit $2^{6}$. According to the report, due to the measurement of the hydrogen production quantity from the zeolite filled column used for reactor water treatment after the accident, the hydrogen production quantity was not regarded to be dependent on the moisture content in the range of water weight ratio to zeolite $0.2-0.7$.

The experiment results by Nakashima et al. report that, as shown in Figure 2, a higher $G\left(\mathrm{H}_{2}\right)$ was observed when the entrapped positive ion was the hydrogen ion. In a similar phenomenon, in the hydrogen production of a mixture of silica or alumina with water solution, a remarkably high hydrogen production quantity was reported under an acidic condition using sulfuric acid ${ }^{4}$. Accordingly, the hydrogen production quantity may increase under an acidic condition, but this was not presumed to be the case in the contaminated water treatment related to the Fukushima Daiichi Nuclear Power Station.

\section{Influence of the Specimen Temperature on Hydrogen Production Yield}

The temperature dependence of the hydrogen production quantity was measured for the mixture of Herschelite or mordenite and seawater. The temperatures for the experiments were the room temperature $\left(16 \pm 3^{\circ} \mathrm{C}, 40 \pm 1^{\circ} \mathrm{C}\right.$, and $\left.60 \pm 1^{\circ} \mathrm{C}\right)$. As a result, no significant temperature variation was observed on $G\left(\mathrm{H}_{2}\right)$ in the temperature range of the experiments, and the variations fell within the experimental error.

The primary yield of hydrogen due to the radiolysis of water $\mathrm{g}\left(\mathrm{H}_{2}\right)$ is known to vary little in the temperature range of these experiments ${ }^{13}$. Consequently, the fact that no significant temperature dependence was observed on the $G\left(\mathrm{H}_{2}\right)$ for the specimens containing only seawater indicates that the oxidation reaction of hydrogen in seawater was also ineffective at $60^{\circ} \mathrm{C}$. Furthermore, because no significant temperature dependence was observed on the $G\left(\mathrm{H}_{2}\right)$ for specimens with the adsorbent and seawater mixture, the hydrogen production due to energy transfer to absorbent was similarly regarded as not significantly temperature dependent.

\section{Evaluation of Hydrogen Production Ratio Assuming the Contaminated Water Treatment}

Assuming the treatment of contaminated water using zeolite type adsorbent, the production ratio of hydrogen emitted in the gas phase in each step of treatment was evaluated in the 
following manner.

First, based on the result of the nuclide analysis conducted on the contaminated water from the basement of the turbine building in Unit 2 of the Fukushima Daiichi Nuclear Power Plant ${ }^{14}$, the absorbed dose rate of the analyzed contaminated water was approximately $3.3 \mathrm{~Gy} / \mathrm{h}$ excluding ${ }^{131} \mathrm{I}$ with short half-life ${ }^{15)}$ —assuming that all $\beta$-rays and $\gamma$-rays from the radioactive nuclide were absorbed by the contaminated water. The oxidation of hydrogen in the contaminated water seems to be prohibited by the influence of the dissolved species in the seawater. For this reason, a similar level of yield as $\mathrm{g}\left(\mathrm{H}_{2}\right)$ should be expected assuming that all produced hydrogen is released in the gas phase. Here, the hydrogen production ratio before and during treatment with the batch method was estimated by regarding the hydrogen production yield as $4.9 \times 10^{-8} \mathrm{~mol} / \mathrm{J}$ based on the result of the specimen of seawater and $1 \mathrm{wt} \%$ adsorbent.

As a result, the value per $1 \mathrm{t}$ of contaminated water was determined to be approximately $1.6 \times 10^{-4} \mathrm{~mol} / \mathrm{h}$ in mol number, and $3.6 \times 10^{-3} \mathrm{~L} / \mathrm{h}$ in volume at a normal condition.

Next, the contaminated water was treated and the hydrogen production ratio was evaluated on the assumption that $0.1 \mathrm{wt} \%$ of cesium, a fission product, was adsorbed (total $4.4 \times 10^{-2} \mathrm{wt} \%$ of radioactive ${ }^{134} \mathrm{Cs}$ and ${ }^{137} \mathrm{Cs}$ ) on the adsorbent. Assuming that all of the $\beta$-ray and $\gamma$-ray from radioactive cesium are absorbed in the contaminated water and adsorbent mixture, the radiation energy was determined to be approximately $1.9 \times 10^{6} \mathrm{~J} / \mathrm{h}$ per $1 \mathrm{t}$ of adsorbent ${ }^{15}$. When considering a mixture, it is important to assume a higher hydrogen production yield than that expected from the moisture content in a mixture based on the recognition that hydrogen is produced by the energy transferred to absorbent in addition to the blocking of the oxidation reaction of hydrogen. The hydrogen production yield was assumed to be $3.5 \times 10^{-8} \mathrm{~mol} / \mathrm{J}$ based on the results acquired from a specimen containing approximately $50 \mathrm{wt} \%$ of adsorbent; additionally, the hydrogen production rate during temporary storage after treatment by the column method and treatment by the batch method and column method were estimated. As a result, the values were approximately $6.6 \times 10^{-2} \mathrm{~mol} / \mathrm{h}$ per $1 \mathrm{t}$ of adsorbent by mol number and approximately $1.5 \mathrm{~L} / \mathrm{h}$ in volume at a normal condition.

The experiments using mordenite in this study were conducted under the framework of the voluntary activities of the Atomic Energy Society of Japan, which conducted the evaluation tests on the adsorption properties of cesium and other compounds in various adsorbents useful for the treatment of the contaminated water in the Fukushima Daiichi Nuclear Power Station. KURION Inc. kindly offered zeolite through the Tokyo Electric Power Co., Inc.

\section{References}

1) M. Nakashima, E. Tachikawa, "Radiolytic gas production from tritiated water adsorbed on molecular sieve 5A," J. Nucl. Sci. Technol., 24(1), 41-46 (1987).

2) M. Nakashima, Y. Aratono, "Radiolytic hydrogen gas formation from water adsorbed on type A zeolites," Ratiat. Phys. Chem., 41, 461-465 (1993).

3) M. Nakashima, N. M. Masaki, "Radiolytic hydrogen gas formation from water adsorbed on type Y zeolites," Radiat. Phys. Chem., 47, 241-245 (1996).

4) R. Yamada, R. Nagaishi, Y. Hatano et al., "Hydrogen production in the $\gamma$-radiolysis of aqueous sulfuric acid solutions containing $\mathrm{Al}_{2} \mathrm{O}_{3}, \mathrm{SiO}_{2}, \mathrm{TiO}_{2}$ or $\mathrm{ZrO}_{2}$ fine particles," Int. J. Hydrogen Energy, 33, 929-936 (2008)

5) G. J. Quinn, J. O. Henrie, J. Greenborg, Submerged Demineralizer System Vessel Shipment Report, GEND-035, EG\&G Idaho, Inc. (1984).

6) J.O.Henrie, D. J. Flesher, G. J. Quinn et al., Hydrogen Control in the Handling, Shipping and Storage of Wet Radioactive Waste, GEND-052, EG\&G Idaho, Inc. (1986).

7) H. H. Mai, H. Tachibana, T. Kojima, "Effect of temperature during irradiation and spectrophotometry analysis on the dose response of aqueous dichromate dosimeters," Radiat. Phys. Chem., 53, 85-91 (1998). 
8) P. A. Yakabuskie, J. M. Joseph, J. C. Wren, "The effect of interfacial mass transfer on steady-state water radiolysis," Radiat. Phys. Chem., 79, 777-785 (2010).

9) D. Zehavi, J. Rabani, "The oxidation of aqueous bromide ions by hydroxyl radicals. A pulse radiolytic investigation,” J. Phys. Chem., 76, 312-319 (1972).

10) J. A. LaVerne, M. R. Ryan, T. Mu, "Hydrogen production in the radiolysis of bromide solutions," Radiat. Phys. Chem., 78, 1148-1152 (2009).

11) E. Bjergbakke, Z. D. Draganić, K. Sehested et al., "Radiolytic products in waters part II: computer simulation of some radiolytic processes in nature," Radiochim. Acta, 48, 73-77 (1989).

12) J. H. Hubbell, S. M. Seltzer, Tables of X-ray Mass Attenuation Coefficients and Mass Energy-absorption Coefficients from $1 \mathrm{keV}$ to $20 \mathrm{MeV}$ for Elements $Z=1$ to 92 and 48 Additional Substances of Dosimetric Interest, NISTIR 5632, National Institute of Standards and Technology (1995).

13) A. J. Elliot, Rate Constants and g-values for the Simulation of the Radiolysis of Light Water over the Range $0-300^{\circ} \mathrm{C}$, AECL Research/AECL-11073, COG-94-167, Atomic Energy of Canada Limited (1994).

14) The 2011 off the Pacific Coast of Tohoku Pacific Earthquake and the Seismic Damage to the NPPs, Nuclear and Industrial Safety Agency, Japan Nuclear Energy Safety Organization (2011).

15) J. Katakura, H. Yanagisawa, Photon and Decay Data Libraries for ORIGEN2 Code Based on JENDL FP Decay Data File 2000, JAERI-Data/Code 2002-021, Japan Atomic Energy Research Institute (1994). 


\title{
Analysis of BWR Station Blackout Accident -Thermal-Hydraulic Behavior up to Severe Core Damage in Fukushima Daiichi Power Plant Unit 2-
}

\author{
Tadashi WATANABE ${ }^{1, *}$, Masahiro ISHIGAKI ${ }^{1}$, Akira SATO $^{1}$ \\ and Hideo NAKAMURA ${ }^{1}$
}

${ }^{1}$ Safety Research Center, Japan Atomic Energy Agency, 2-4 Shirakata Shirane, Tokai-mura, Naka-gun, Ibaraki 319-1195, Japan

\begin{abstract}
The analysis of the long-term station blackout accident of BWR has been performed using the TRAC-BF1 code. The actuation of RCIC (reactor core isolation cooling) was assumed, and the results were compared with the data observed at the Fukushima Daiichi Power Plant Unit 2 reactor. BWR-5 of 1,100 MW was analyzed, while the unit 2 reactor was BWR-4 of $780 \mathrm{MW}$. The reactor pressure and the core liquid level were, however, in good agreement with the observed data. It was confirmed that the quasi-steady state continued for a long time with the RCIC actuation. The timing of recovery action, which was composed of depressurization and coolant injection, necessary for the maximum clad temperature being less than 1,500 $\mathrm{K}$ was studied and compared with that of the unit 2 reactor.
\end{abstract}

KEYWORDS: BWR, station blackout, RCIC, TRAC-BF1, Fukushima Daiichi

\section{Introduction}

During the Great East Japan Earthquake on March 11th 2011, a station blackout accident ensued at the Fukushima Daiichi Nuclear Power Plant due to the earthquake and tsunami that followed. Although the emergency safety equipment was partially operational, reactor core damage occurred in Units 1 through 3. The detailed development of events is unclear, but major issues were presumably the loss of external power, loss of emergency power, and loss of cooling function due to partial operation of emergency safety equipment after the reactor scram due to seismic acceleration. This rapid communication presents the analysis and study results of the thermal-hydraulic behavior of Unit-2, which was the last to experience core damage among the three units, using the safety analysis code TRAC-BF $1^{1)}$ up until the time the reactor core damage occurred. To allow prompt reporting, the input model of Unit-2 itself (BWR4, 780,000 KW) was not used, and the input data for analysis of the existing 1,100,000 KW class BWR-5 were prepared instead ${ }^{2}$. Because a comparatively steady reactor core cooling status continued as a result of the actuation of the reactor core isolation cooling (RCIC) system during the station blackout in Unit-2, actuation of RCIC was assumed in the analysis while

\footnotetext{
* Corresponding author, E-mail: watanabe.tadashi66@jaea.go.jp

DOI : 10.15669 /fukushimainsights. Vol.4.46

(C) 2021 Atomic Energy Society of Japan. All rights reserved.

Originally published in Transactions of the Atomic Energy Society of Japan (ISSN 1347-2879), Vol. 10, No. 4, p.240-244

(2011) in Japanese. (Japanese version accepted: August 3, 2011)
} 
investigating the pressure reducing operation after stoppage of RCIC and the variation in reactor conditions before injection of cooling water. The analysis was justified by comparison with the observed data for Unit-2, including nuclear reactor pressure, etc ${ }^{3)}$. A sensitivity analysis was conducted adopting the timing of RCIC stoppage and start of the reactor core cooling function recovery operation as parameters and evaluating the effectiveness of operation.

\section{Analysis of BWR Station Blackout Accident}

\section{Analysis Conditions}

The node assignment used for the analysis is shown in Figure 1. The object reactor is a $1,100,000 \mathrm{KW}$ class BWR-5 ${ }^{2)}$, modeled from the water supply line to the main steam pipe. In the analysis, input data were prepared by setting up the thermal-hydraulic values acquired by steady state simulation as the initial condition, and assuming reactor scram due to seismic acceleration at time "zero," turbine trip, stoppage of recirculation pump, closure of the main steam isolation valve (MSIV), turbine bypass valve close and main water supply stop due to station blackout ${ }^{4}$. Among the emergency safety equipment, only the actuation of RCIC was assumed.

The rated design flow rate and operational start/stop conditions based on the water level of the nuclear reactor were specified ${ }^{2)}$ but the water level of the reactor core was kept stable for an extended period in Unit-2. Therefore, in the analysis, the flow rate of cooling water injected

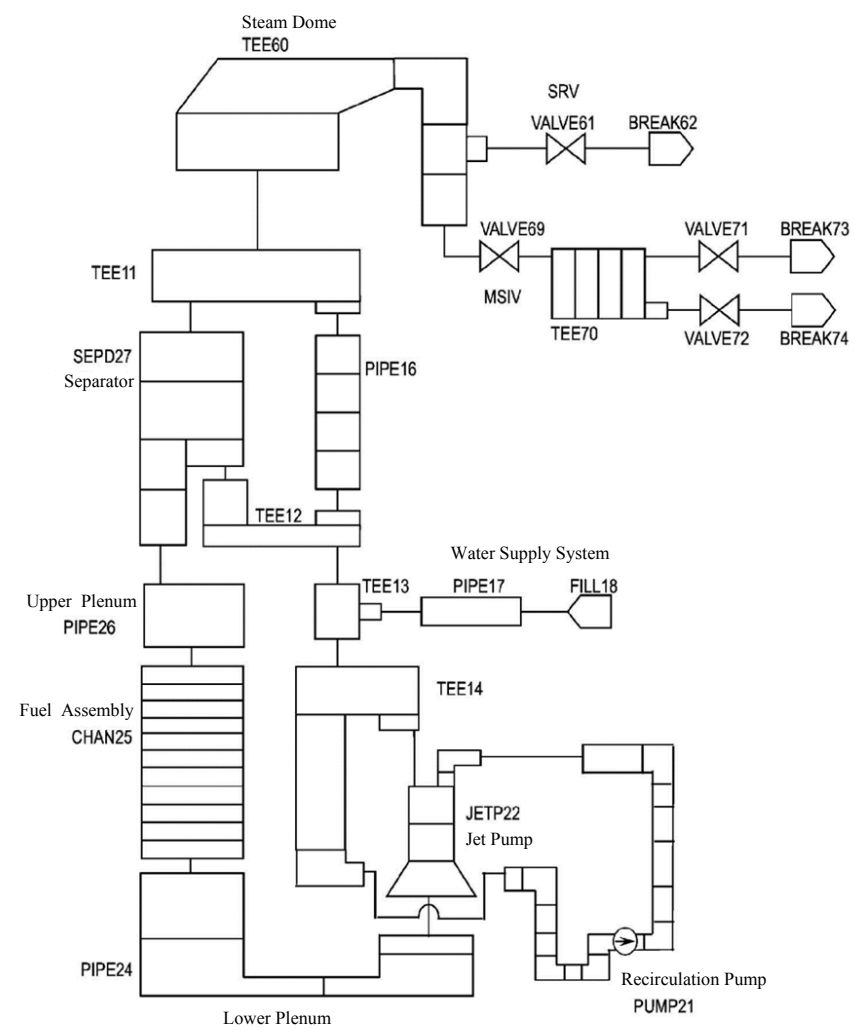

Figure 1 TRAC-BF1 nodding diagram for BWR analysis 
into the reactor core by the RCIC system and the extracted steam flow rate for driving the RCIC turbine were set up as boundary conditions to balance the nuclear reactor thermal output after the scram. The initial value was set to approximately $30 \mathrm{~kg} / \mathrm{s}$, and by gradual decrease the value fell to approximately $12 \mathrm{~kg} / \mathrm{s}$ after $50,000 \mathrm{~s}$, and approximately $10 \mathrm{~kg} / \mathrm{s}$ after 250,000 s. The line for VALVE72 was employed as the steam line for RCIC, and the steam flow rate was established by adjusting the opening of VALVE69 in the node assignment, as shown in Figure 1. The line for VALVE72 and VALVE69 were originally used to model the turbine bypass line and the MSIV, respectively, but because these were not used except for in the initial stage, they were used as substitutes for the steam lines for RCIC. The water supply line after stoppage of the main water line was directly employed for water injection. The water injection temperature was set to $300 \mathrm{~K}$ for the first $50,000 \mathrm{~s}$, then augmented to $373 \mathrm{~K}$. This is because the water source for water injection by the RCIC system was switched from the recovery tank at the initial stage to the suppression pool, but the temperature rise of the water source after switching was not taken into consideration.

Because the RCIC system is considered to have stopped at around 250,000 s for Unit-2, the operation up until then is assumed for the analysis as well. Afterwards, the injection quantity from the injection line and the extraction quantity from the steam line were both set to zero. The pressure reducing operation for the recovery of the cooling function in Unit-2 and the water injection by the fire engine was tried after 20,000 s. In the analysis, a water injection of $15 \mathrm{~kg} / \mathrm{s}$ was assumed at the time that pressure was reduced to approximately $0.6 \mathrm{MPa}$ by the pressure reducing operation after RCIC stop and these conditions were defined as the base case. The average water injection rate of Unit-2 was set up as the flow rate without considering differences in the reactor thermal output. The pressure reduction and water injection after RCIC stoppage was regarded as the reactor core cooling recovery operation. Furthermore, the time of operation start and the time RCIC stoppage were adopted as parameters for the sensitivity analysis, and the availability and effectiveness of the operation were studied.

\section{Comparison of Base Case and Unit-2}

The comparison of reactor pressure in the base case and observed data of Unit-2 are shown in Figure 2. The pressures from the analysis results are taken from the values for the steam

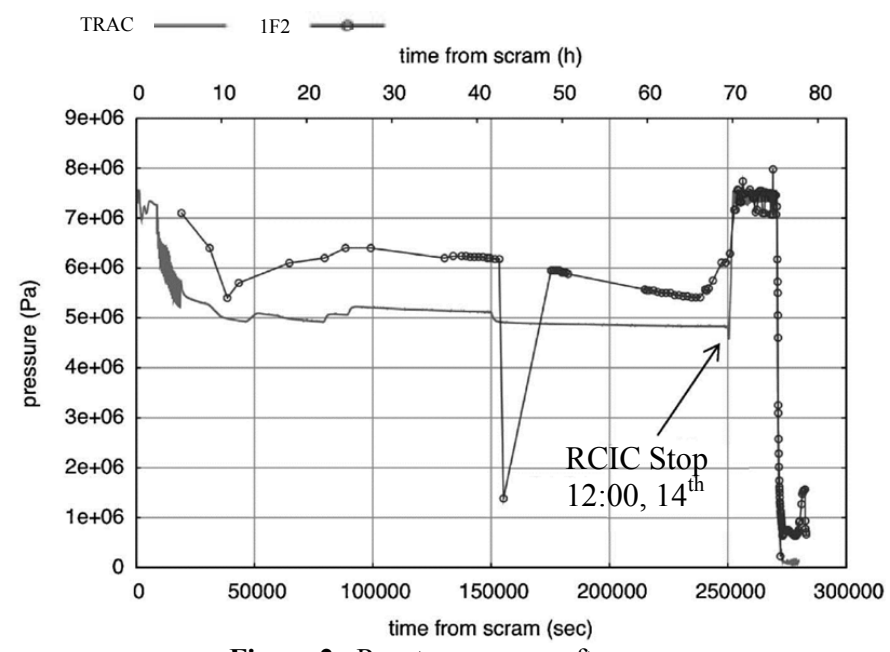

Figure 2 Reactor pressure after scram 
dome and depicted as solid lines. Furthermore, elapsed time after the scram is indicated in hours (h) in the upper part of the chart for comparison. The pressure increases slightly after the steady state of 7.0 MPa during the transition of events due to the blackout immediately after the scram, then gradually decreases accompanied by slight fluctuations, entering a stable condition at approximately $5 \mathrm{MPa}$ while wavering in a quasi-steady condition for approximately 200,000 s until the RCIC system ceases to function. The observed data indicated by circular symbols decrease from approximately $7 \mathrm{MPa}$ to $5.4 \mathrm{MPa}$ and show slight variations rising to approximately $6.4 \mathrm{MPa}$ and falling again to $5.4 \mathrm{MPa}$, exhibiting a quasi-steady condition during the period of $40,000 \mathrm{~s}$ to $240,000 \mathrm{~s}$. Accordingly, the analysis result portrays the nuclear reactor condition under the operation of RCIC rather well, even though the pressure is underestimated. In the analysis, RCIC stoppage was assumed to occur at 250,000 s, and the pressure rose due to the cutoff of the water supply and steam discharge to around the safety relief valve (SRV) setup pressure of 7.6 MPa. During this process, the steam generated in the reactor core was gradually discharged from the SRV. The pressure abruptly decreased at $270,000 \mathrm{~s}$ because the SRV was manually opened to reduce the pressure. The observed data exhibited a gradual increase of pressure from around 240,000 s, which was understood to indicate a gradual slowdown of RCIC and subsequent cessation, i.e., the speed of the turbine gradually slowed down and stopped within approximately $240,000-10,000 \mathrm{~s}$, causing the decrease in the steam flow rate. Then, an abrupt rise of pressure occurred as a result of the complete stop near $250,000 \mathrm{~s}$. Until the manual reduction of pressure, the pressure fluctuated during the SRV setup pressure, justifying the analysis. The pressure of Unit- 2 was reduced to the containment pressure of $0.6 \mathrm{MPa}$ at the time by manual reduction, while in the analysis the pressure reduced approximately to atmospheric pressure, as the increased pressure of containment was not taken into consideration.

A comparison of the reactor core water level of the study with the observed data (see Figure 3) shows them acting similarly to the pressure. For the purpose of comparison, the top of active fuel (TAF) was set to zero. In the analysis result, similarly to the pressure, an almost constant value of approximately $3.3 \mathrm{~m}$ was observed post-fluctuation after the scram and a quasi-steady state was realized until the RCIC stop, while exhibiting a gradual increase. The observed data showed a slightly higher value, but were stable until the RCIC stop, similarly to the analysis. This, likewise, demonstrates the justifiability of the analysis, i.e., in the analysis, the steam discharge and water supply quantities were specified in such a way that a balance with the

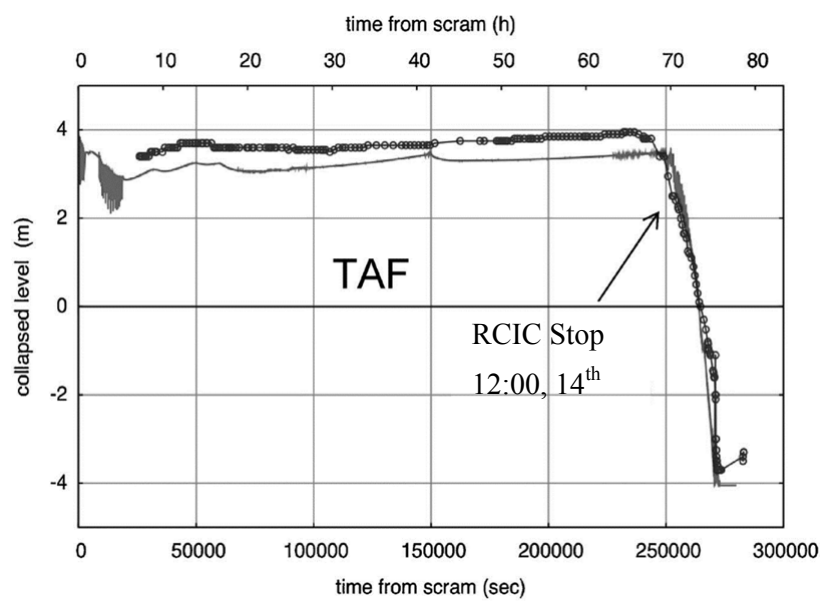

Figure 3 Core liquid level after scram 
reactor thermal output is maintained, and steady behavior without on/off of the RCIC seems to have taken place in Unit-2 as well. It is possible to adjust the analysis result to the observed data by finely calibrating the steam flow rate and water supply quantity. However, no further adjustment was made because it was considered that it would not greatly influence the development of events. In the analysis, a decrease in the water level was observed after the stoppage of RCIC at 250,000 s, while the observation data indicate a gradual decrease initiated before that. This can be explained by the water supply quantity starting to decrease due to the gradual slowdown of RCIC operation, with the decrease accelerating after a complete stop.

\section{Effect of Cooling Function Recovery Operation}

From the comparison of analysis and observed data, the development in the Unit-2 accident can be regarded as sufficiently replicated by this analysis, although the input data are different with respect to the reactor type and the power.

Furthermore, the assumption for the operation of RCIC can be regarded as being a reasonable one for achieving a stable reactor condition. For this reason, a sensitivity analysis was conducted with different times for RCIC stoppage and the reactor cooling recovery operation in the base case, and the resulting efficiency of operation was studied. The cooling recovery operation comprised two steps; pressure reduction by the SRV after a certain amount of time from the RCIC stop and the injection start when the pressure decreased to $0.6 \mathrm{MPa}$ by pressure reduction.

Mapping of the times of RCIC stop and the corresponding efficiency of operation is shown in Figure 4. The horizontal axis is the time of RCIC stop assuming that only the RCIC system was operational after the scram, and the vertical axis indicates the time from RCIC stop until the start of the cooling recovery operation. The symbol " $x$ " indicates the case where the peak fuel clad temperature in the reactor core is kept under $1,500 \mathrm{~K}$ and the symbol "+" indicates the case where the peak fuel clad temperature exceeds 1,500 K, i.e., the zone under the solid lines connecting these symbols can be regarded as the range in which the recovery operation is effectively functional after the RCIC system ceased to function. The solid lines indicate a gradual increase toward the right side with the lapse of time. This corresponds to a gradual increase in required time until operation start due to a decreased core output with respect to time.

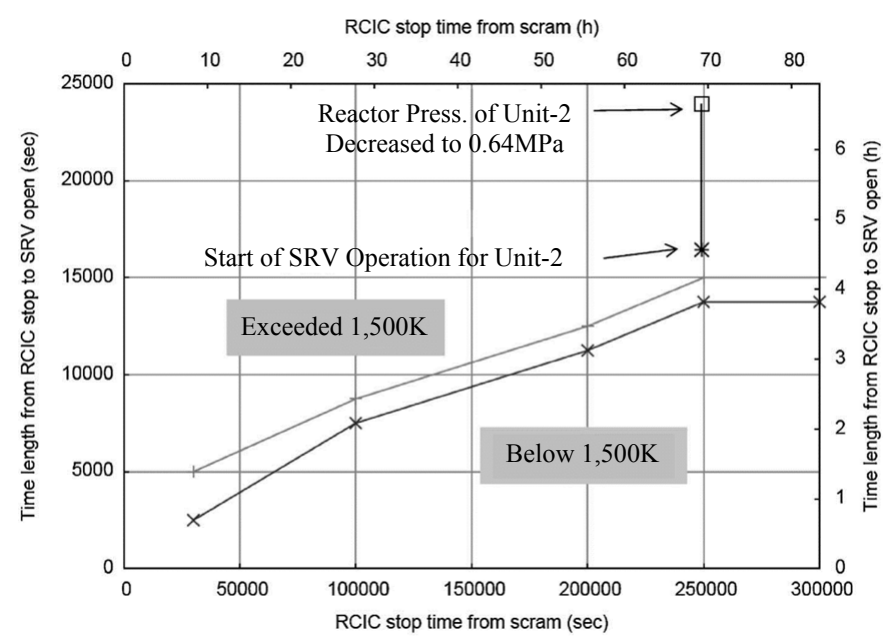

Figure 4 Timing map for cooling recovery operation 
Additionally, a band of time is observed on the boundary of the ranges where $1,500 \mathrm{~K}$ is exceeded and under it. This is caused by a change of several thousand seconds in the operation start time in the sensitivity analysis. Furthermore, 1,500 $\mathrm{K}$ was established as the value corresponding to the peak clad temperature of $1,200^{\circ} \mathrm{C}$ used in safety analyses. For completion at lower temperatures, an early operation start is required.

There is a possibility that the RCIC system stopped around 250,000 s in Unit-2, but for such a case, the chart implies that the recovery operation needs to start within $13,000 \mathrm{~s}$ after stoppage. The time when the pressure reduction operation actually started in Unit-2 and the time when the pressure decreased to $0.64 \mathrm{MPa}$ are shown for the purpose of comparison. The operation start time is in the slightly upper side of the range of analysis results, but if the assumption and errors in the analysis are considered, one can deduce that the timing is sensitive. However, some time before completion is required for actual pressure reduction after starting the operation, and thus the cladding tubes and fuels had already reached high temperatures by the time water injection began.

\section{Conclusions}

An analysis of the station blackout accident of a BWR was conducted over an extended time period using the TRAC-BF1 code. Only the RCIC operation was assumed. Comparison was made with observed data from Unit-2 of the Fukushima Daiichi Nuclear Power Plant, and the effectiveness of the cooling recovery operation after the RCC stop was evaluated. The analysis was conducted on a different 1,100,000 KW class BWR-5, but both the pressure of the nuclear reactor and the reactor water level showed good agreement with the data of the 780,000 KW BWR-4, in Unit-2 of the power plant. We confirmed that a quasi-steady thermal-hydraulic condition was preserved for an extended period of time. Furthermore, the necessary timing for starting the required operation in order to keep the peak clad temperature under $1,500 \mathrm{~K}$ was studied in the case of conducting pressure reduction and the subsequent injection of cooling water as steps in the cooling recovery operation after RCIC stop, and a comparison was made with the case of Unit-2.

We would like to express our gratitude to Dr. Masashi Hirano, the director of the Nuclear Safety Research Center, who gave us valuable suggestions and comments during the preparation of this rapid communication.

\section{References}

1) M. M. Giles, G. A. Jayne, S. Z. Rouhani et al., TRAC-BF1/MOD1: An Advanced Best-Estimate Computer Program for BWR Accident Analysis, NUREG/CR-4356 (1992).

2) J. Ishikawa, K. Muramatsu, T. Sakamoto, Systematic Source Term Analysis for Level 3 PSA of a BWR with Mark-II Type Containment with THALES-2 Code, JAERI-Research 2005-021, Japan Atomic Energy Research Institute (JAERI) (2005). [in Japanese]

3) http://www.tepco.co.jp, Tokyo Electric Power Co. (TEPCO) (2011).

4) D. D. Yue, W. A. Condon, Severe-Accident-Sequence Assessment of Hypothetical Complete Station Blackout at the Browns Ferry Nuclear Plant, CONF-810905-9 (1981). 


\title{
Local Area Distribution of Fallout Radionuclides from Fukushima Daiichi Nuclear Power Plant Determined by Autoradiography Analysis
}

\author{
Fuminori SAKAMOTO ${ }^{1}$, Toshihiko OHNUKI ${ }^{1, *}$, Naofumi KOZAI ${ }^{1}$, \\ Shosuke IGARASHI ${ }^{1,2}$, Shinya YAMASAKI ${ }^{1}$, Zenko YOSHIDA $^{1}$ \\ and Shunichi TANAKA ${ }^{3}$ \\ ${ }^{1}$ Japan Atomic Energy Agency, 2-4 Shirane, Shirakata, Tokai-mura, Naka-gun, Ibaraki 319-1195, Japan \\ ${ }^{2}$ Ibaraki University, 2-1-1 Bunkyo Mito-shi, Ibaraki 310-8512, Japan \\ ${ }^{3}$ NPO Radiation Safety Forum, 27F Shiroyama-Trust Tower, Toranomon, Minato-ku, Tokyo 105-6027, Japan
}

\begin{abstract}
The environmental behavior of radioactive Cs in the fallout from the accident of the Fukushima Daiichi Nuclear Power Plant has been studied by measuring its spatial distribution on/ in trees, plants, and surface soil beneath the plants using autoradiography analysis. The results of autoradiography analysis showed that radioactive Cs was distributed on the branches and leaves of the grown trees and that only a small fraction of radioactive Cs was transported to new branches and leaves merged after the accident. Radioactive Cs was present on the grass and rice stubble on the soils, but not in the soils beneath the grass and rice stubble, indicating that the radioactive Cs was deposited on the grass and the rice plant. In addition, a small fraction of the deposited radioactive Cs was released by weathering for two months after the accident. These results indicate that trees and other plants are the reservoir of the fallout Cs and function to retard the fallout Cs migration with rain water.
\end{abstract}

KEYWORDS: radioactive fallout, radioactive Cs, accumulation in plant, migration, autoradiography

\section{Introduction}

A large amount of various nuclear fission products (hereinafter referred to as "radioactive material") was released into the environment by the core meltdown and hydrogen explosion at the Tokyo Electric Power Fukushima Daiichi Nuclear Power Plant on March 11th, 2011. Judging from the observation that the radioactive materials released by the hydrogen explosion at Unit-2 on the morning of March 15th were transported to the northwest by southeast winds, followed by precipitation/snow from the evening into the night on that day, the radioactive materials probably fell on Namie-machi, Fukushima-Prefecture, Iitate-mura and Kawamata-machi of the same prefecture, and elsewhere ${ }^{1,2}$. The major portion of the radionuclides that fell in the area were ${ }^{131} \mathrm{I},{ }^{134} \mathrm{Cs}$, and ${ }^{137} \mathrm{Cs}$. Because of a short half-life of approximately 8 days for ${ }^{131} \mathrm{I}$,

\footnotetext{
* Corresponding author, E-mail: ohnuki.toshihiko@jaea.go.jp

DOI : 10.15669 /fukushimainsights. Vol.4.52

(C) 2021 Atomic Energy Society of Japan. All rights reserved.

Originally published in Transactions of the Atomic Energy Society of Japan (ISSN 1347-2879), Vol. 11, No. 1, p.1-7 (2012)

in Japanese. (Japanese version accepted: September 6, 2011)
} 
the radionuclides detected after May were mainly ${ }^{134} \mathrm{Cs}$ (half-life of approx. 2.1 years) and ${ }^{137} \mathrm{Cs}$ (half-life of approx. 30 years) ${ }^{3)}$.

The vertical distribution of the radioactive fallout was measured by Naganawa et al. using a $\mathrm{NaI}$ scintillation counter in such a manner that the soils were sampled at a spacing of approximately $10 \mathrm{~mm}$ for dry land soils, pastures, and paddy fields. It was clarified that nearly all of the radioactive fallout was distributed within the top $100 \mathrm{~mm}$ of the land surface ${ }^{4}$. However, it is believed that the radioactive fallout accumulated not only in soil but also in trees and chamaephytes, but its distribution and accumulation on soils, trees, chamaephytes, etc. has not yet been clarified.

To measure the distribution of radioactive materials, sampling the soil layer to a defined depth is effective, as previously explained. In such measurement, intervals in the vertical direction of the soil sample determine resolution in the depth direction. For this reason, $10 \mathrm{~mm}$ deep is considered the limit for an environmental specimen. Similarly, because reducing the cutting to obtain a higher resolution for the trees may result in measurement count decrease, $10 \mathrm{~mm}$ was considered the limit.

Using autoradiography, the distribution of radioactive materials is measured by leaving a specimen directly on an imaging plate sensitive to the radiation, for a certain period of time ${ }^{5}$. One disadvantage is that the individual identification of radioactive materials is not possible because all radiation is detected. Among the radioactive materials deposited in Fukushima-Prefecture, the radionuclides detected during May and after, as previously explained, were ${ }^{134} \mathrm{Cs}$ and ${ }^{137} \mathrm{Cs}$. Because of this, it was possible to measure the distribution of the radioactive fallout in a local area smaller than $10 \mathrm{~mm}$ using autoradiography, and to investigate its short-term mobility after the initial fallout.

In this report, the distribution of radionuclides was measured using autoradiography on trees, chamaephytes, and soils sampled on May 14th in Iitate-mura, Kitasoma-gun, and Fukushima-Prefecture, to study the local area distribution of fallout. Furthermore, as well as studying the distribution of radioactive Cs after deposition, decontamination was analyzed and discussed.

\section{Methodology}

\section{Specimen Sampling}

The sampled trees were Cryptomeria japonica, Torreya nucifera, and Prunus mume growing near the site of the decontamination experiment. Branches with leaves were sampled for Cryptomeria japonica and Torreya nucifera and used as specimens. Regarding the Torreya nucifera branches, those leaves with less green on the branch tips were considered to have grown following the spring and were selected. Regarding the Cryptomeria japonica, stems with a diameter of approximately $50 \mathrm{~mm}$ were sampled and used as specimens for measuring the distribution of radioactive materials in trees. The stems selected were those grown in a nearly horizontal direction. As for chamaephytes, Petasites japonicus and fern relatives were sampled. There were no plants such as trees that covered the Petasites japonicas. On the other hand, fern relatives were sampled in the grove. There were trees above the fern relatives, so it was assumed that direct accumulation of rainfall/snowfall did not occur.

Regarding the soil specimens, a location where pastures and Oryza sativa stubble remained was chosen and the soil layer was sampled to a depth of approximately $50 \mathrm{~mm}$, with care to avoid disturbing the soil layers. For information, stubbles was sampled with the roots and soil 
immediately under the stubble.

\section{Specimen Preparation}

No special preparation was conducted for sampling the branches and leaves of trees and chamaephytes to measure the distribution of radioactive materials using autoradiography. To measure radionuclide distribution in a Cryptomeria japonica stem section, a stem portion approximately $50 \mathrm{~mm}$ in diameter was cut into approximately 5 -mm-thick slices. This preparation was conducted at the stem sampling site. For soil sample preparation, vertical cuts were made in the pasture in the stubble zone, down into the soil, and the section was fixed by gluing and cutting to an approximately $5 \mathrm{~mm}$ thickness for autoradiograph measurement. The number of specimens used for measurement was one because the sampling was conducted in a planned evacuation area.

\section{Autoradiography Measurement}

The tool used for autoradiography measurement was a bio imaging analyzer BAS2500 (Fujifilm, Japan, BAS2500). The spatial resolution of the imaging plate was $0.05 \mathrm{~mm}$. After exposing the prepared samples on the imaging plate for 24 or $48 \mathrm{~h}$, the area exposed to radiation was developed using a reading and analysis unit of the imaging plate. In the resulting image, the higher the intensity of the radiation, which is directly related to the concentration of radionuclides, the darker the blackness of the image. Furthermore, it is possible to separately analyze two levels of intensity in a single image by varying the intensity gradient of the radiation. During measurement, the specimen was covered with a thin film of polyvinylidene chloride to prevent contamination of the imaging plate by the specimen. After measurement, an optical photo of the specimen was taken with the polyvinylidene chloride film in place. Before the autoradiography measurement, the intensity of the radiation from the specimen was measured with a NaI scintillation counter (Aloka, TCS161). Also, the contained radionuclides on some of the specimens were measured using a $\gamma$-ray spectrum analysis system. For the Torreya nucifera branches and leaves, the concentration of radionuclides was measured using a $\gamma$-ray spectrum analysis (Ge semiconductor detector) system after individually cutting off two portions showing the difference in blackness (see the Results and Discussion) after the completion of the autoradiography measurement.

\section{Results and Discussion}

\section{Distribution on Trees}

Cryptomeria japonica branches showed a slight blackening on the branches and leaves in the autoradiography analyses (Figure 1). Approximately 2-mm-wide black spots were observed on the branches. As shown in the optical photograph (Figure 2) of the sliced Cryptomeria japonica stems, distinct black spots are observed on the stem surface. The spots are not distributed all over the stem, but on the epidermis in the upper part of the stems. The images of the two sides are nearly symmetrical at the portion with an arrow in the autoradiograph image, while in the portion surrounded by a rectangle the spots were detected only on one of the surfaces. This was caused by the epidermis loss that occurred during the replacement of the specimen. Inside the 


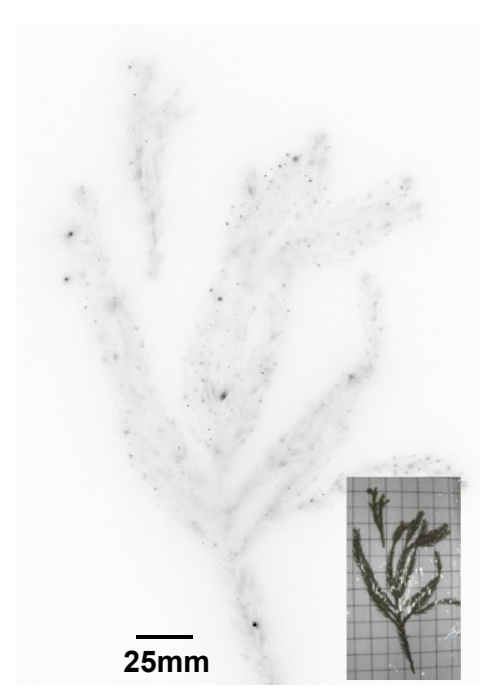

Figure 1 Autoradiograph image and optical photograph (inset) of branch and leaves of Cryptomeria japonica
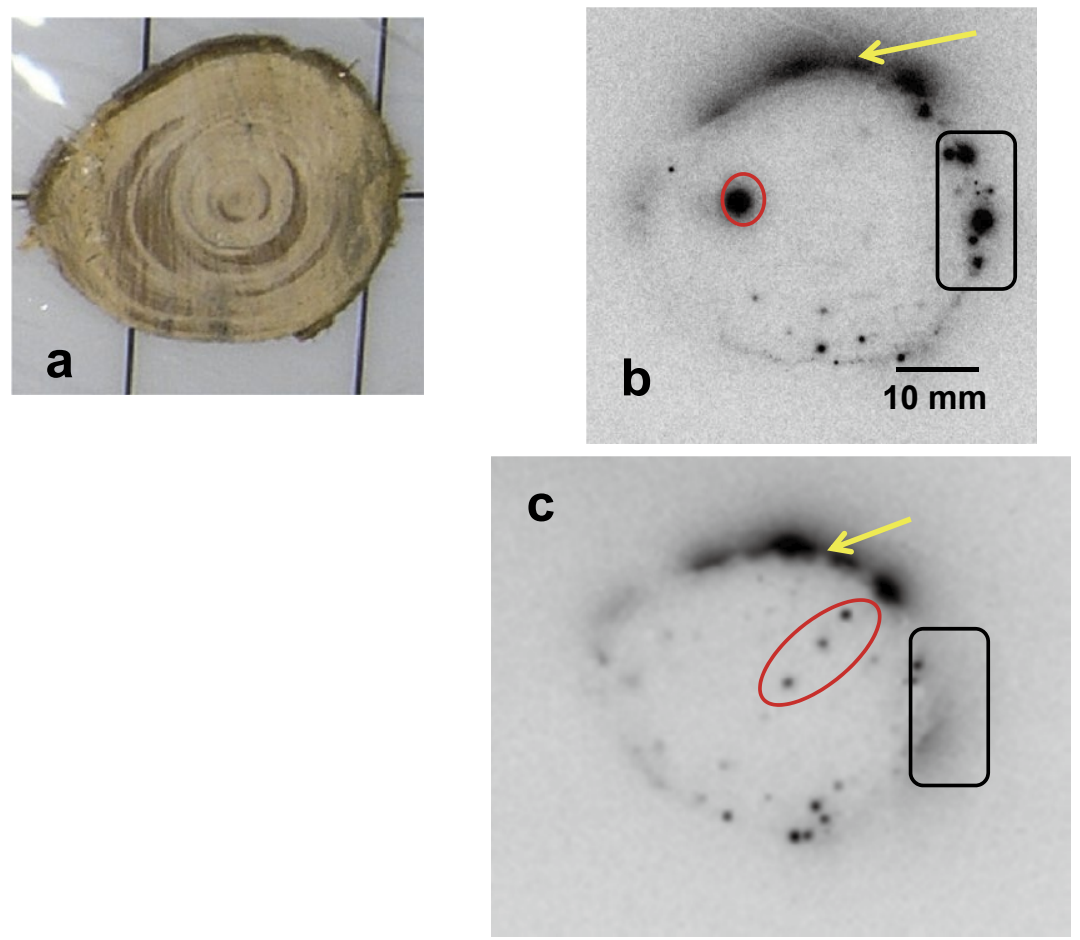

Figure 2 Cross sections of stem of Cryptomeria japonica

Optical photograph (a), autoradiograph images of both sides of the cross section (b), and (c). Note that image (c) is the result of a horizontal flip of the sample. 
stem, black spots were detected, but the spot positions differed between the two sides of the stem section. This indicated that the spots on the stem were not caused by radioactive materials that penetrated into the stem, but by contamination after the cutting of the stem. Additionally, because ${ }^{40} \mathrm{~K}$ is contained in the plants, there is a concern that the spots detected by autoradiography might have been caused by ${ }^{40} \mathrm{~K}$. The radioactivity measured on the stems, branches, and leaves using a scintillation counter showed several times higher intensity than that of the background radiation.

Based on the above discussion, most of the spots detected by autoradiography are considered the product of radioactive Cs. Thus, as the specimens' radioactivity as measured by the scintillation counter was more than twice that of the background, the influence of ${ }^{40} \mathrm{~K}$ will not be considered further.

Analysis of branches and leaves of Torreya nucifera using autoradiography (Figure 3) detected radioactive materials all over the branches, with numerous black spots on both branches and leaves. Furthermore, when the leaves of Torreya nucifera were separated according to their elevation on the tree, a series of scintillation counter measurements on the leaves showed 1.8 times higher radiation intensity than those collected at higher position. The trend of a higher intensity in the upper part was the same for three branches in different locations, confirming the greater accumulation of radioactive fallout on the higher leaves.

However, the blackness of the branch tips (surrounded by a red oval) is less intense than in other areas of the branch. So a $\gamma$-ray spectrum analysis was conducted on the low blackness (red oval 1) and high blackness (blue oval 2) regions. As a result, clear peaks of ${ }^{134} \mathrm{Cs}$ and ${ }^{137} \mathrm{Cs}$ were confirmed in the $\gamma$ spectrum in the red and blue areas and the Cs- 137 counts were 660 counts $/ g$ dry and 1,790 counts/g dry, respectively. Because the greenness of the leaves in the tip area of the branches was thinner than other areas in the optical photograph shown in Figure 3, the tip area is considered to be branches and leaves that grew after this spring. These results show lower radioactivity in the branches and the leaves that emerged this year than in those that grew in the previous year. Thus, it was concluded that the radioactive fallout did not deposit directly on the new branches and leaves, but was concentrated after deposition.

The autoradiography analysis of the Prunus mume branch and its sliced fruit (Figure 4) showed a clear black zone in the branch. On the other hand, the blackness of the fruits and

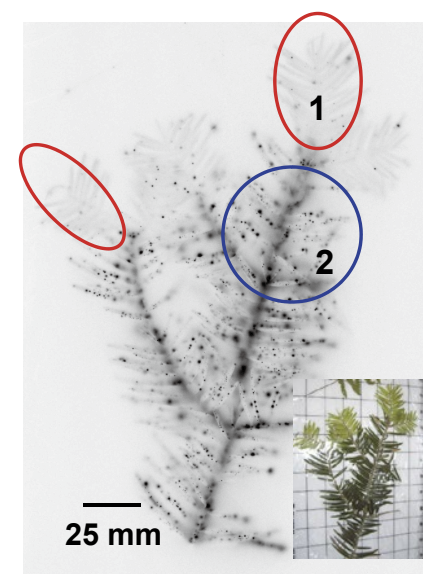

Figure 3 Autoradiograph image and optical photograph (inset) of branch and leaves of Torreya nucifera 

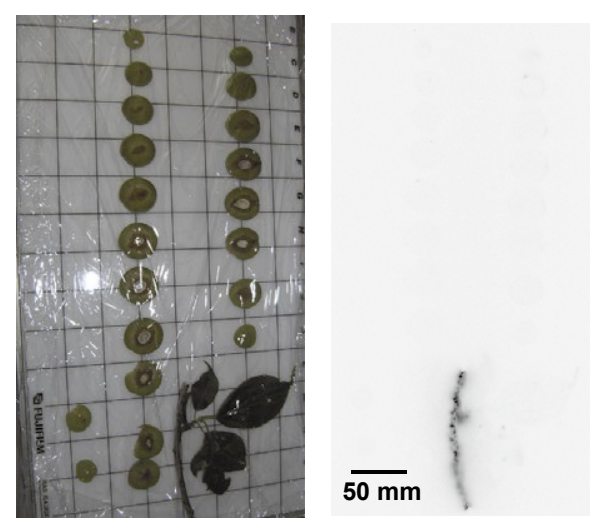

Figure 4 Autoradiograph image (right) and optical photograph (left) of branch and leaves, and cross sections of fruits of Prunus mume

leaves was extremely low compared to the branch area.

All the aforementioned results suggest that the radioactive materials concentrated in the trees were concentrated on the portion that had already grown during the previous year, i.e. before the accident at the Fukushima Nuclear Power Plant. Because the radioactive material concentration in the stem of the branches was extremely low, it is considered that the portion transported to the leaves from the roots through the stem was very low. Accordingly, direct adhesion of radioactive fallout to aged and therefore rougher-surfaced growth is a probable process. Furthermore, only some portion of the radioactive material was found in the new branches and leaves. Tsukada et al. conducted an experiment to affix ${ }^{137} \mathrm{Cs}$ to radish leaves and reported that part of the ${ }^{137} \mathrm{Cs}$ was absorbed from the leaf surface ${ }^{6}$. Our results indicate absorption and translocation from the leaves of the needle leaf tree, Torreya nucifera, as well.

\section{Distribution to Chamaephytes}

The autoradiography analysis of surface soil containing meadow grass sampled in pasture land is shown in Figure 5 together with optical photographs. A yellow dotted line in the optical photograph indicates the land surface. The meadow grass is present in the upper part of the land surface and roots are seen in the lower part. In the autoradiography image, a uniformly high blackness is seen in the upper part of the ground with some black spots and no black area lower than the land surface. The result indicates that nearly no penetration of radioactive fallout occurred below the land surface, but remained in the meadow grass. Naganawa et al. showed the possibility of distribution of most of the radioactive materials within $30 \mathrm{~mm}$ of the surface of pasture land ${ }^{4}$. Our results indicate that there was no penetration of radioactive materials into the soil immediately under the pasture where plants such as meadow grasses are growing.

The image acquired by autoradiography of a stubble section of Oryza sativa in the paddy fields (Figure 6) shows the black portion in the stubble. The blackness of the soil portion has the same level as that of the background indicating that the concentration of radioactive material is below the detection limit of autoradiography, showing that the radioactive fallout on stubble was concentrated in the stubble portion.

Analyses of Petasites japonicus are shown in Figure 7 together with an optical photograph. The area in the oval in the optical photograph indicates the root of Petasites japonicus. Black areas are uniformly seen in the stem and leaf area. In the stem, the shape of the black area nearly 


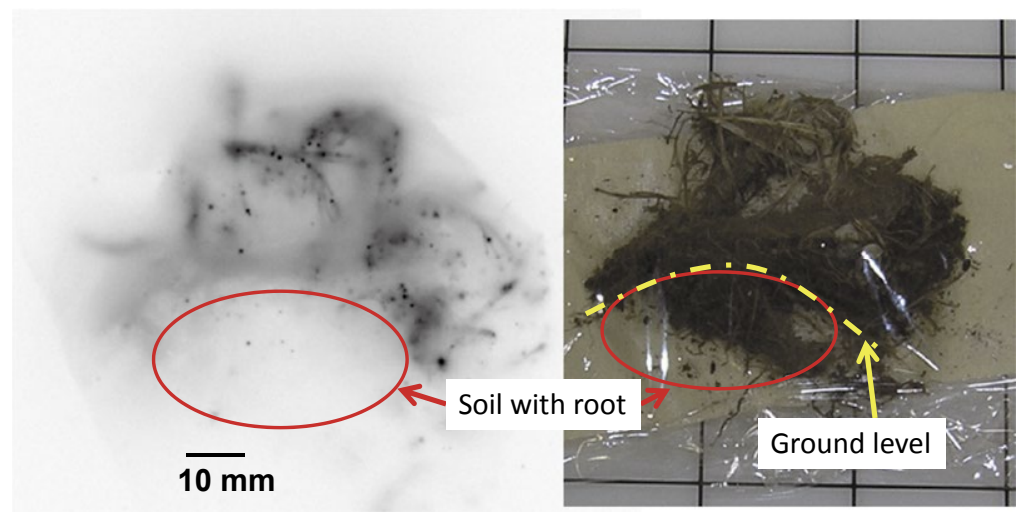

Figure 5 Autoradiograph image (left) and optical photograph (right) of cross section of meadow grass with soil beneath the grass

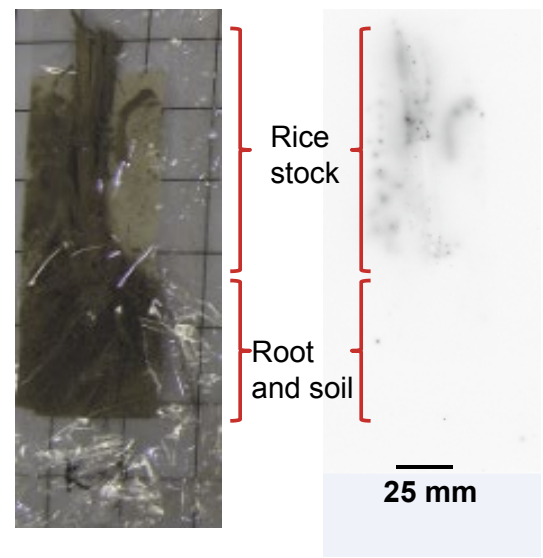

Figure 6 Autoradiograph image (right) and optical photograph (left) of stubble of Oryza sativa with paddy soil beneath the stubble

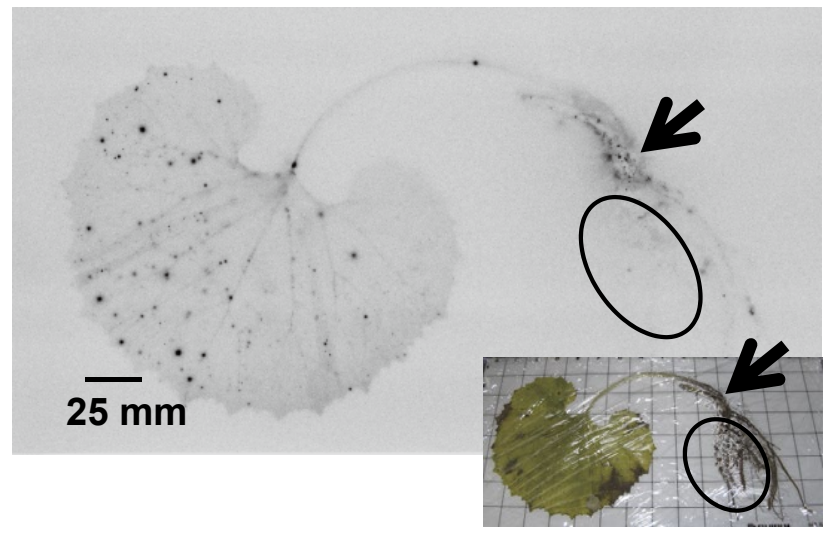

Figure 7 Autoradiograph image and optical photograph (lower right) of Petasites japonicus with soil 


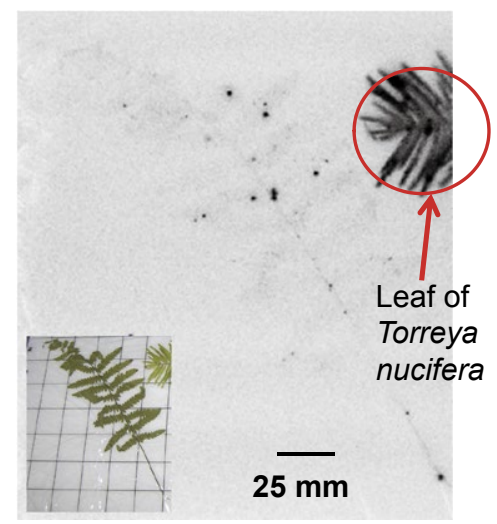

Figure 8 Autoradiograph image and optical photograph (inset) of fern relatives

coincides with that of the stem in the optical photograph. On the other hand, the blackness in the root is approximately the same as that of the background. The black spots, considered to indicate concentrations of radioactive materials, are seen in the leaf area of Petasites japonicus. This concentration is a result of the absorption/accumulation via the roots or secondary transmission of radioactive materials adhering to soil particles. Furthermore, radioactive materials are observed in the area of the ground surface (indicated with an arrow) and the blackness is darker than that of the stem and leaf areas. There was no tree (such as Cryptomeria japonica) over the sampling point of Petasites japonicus. It was assumed that Petasites japonicus had not grown when the deposition of radioactive fallout occurred. Because accumulation of radioactive materials is seen in the stem area, absorption from the roots is implied.

The autoradiography analysis of fern relatives sampled in the grove (Figure 8) shows that there was nearly no accumulation of radioactive materials. The radioactivity of the fern relatives measured with a scintillation counter was nearly the same as background, indicating that there was nearly no accumulation of radioactive Cs on the fern relatives. The upper parts of the sampled fern relatives were covered by trees such as Cryptomeria japonica. These results implied that most of the radioactive materials were intercepted by the trees above the fern relatives and did not fall on them.

\section{Local Area Translocation of Radioactive Materials from the Fukushima Nuclear Power Plant after Initial Deposition}

The radioactive materials released into the atmosphere from the Fukushima Nuclear Power Plant fell in Iitate-mura on March 15th. Based on the AMEDAS data of that day ${ }^{7}$, precipitation was $0.5-1.5 \mathrm{~mm} /$ hour after $17: 00$. Furthermore, the temperature was $2^{\circ} \mathrm{C}$ or lower, below $1^{\circ} \mathrm{C}$ after 20:00, and below $0^{\circ} \mathrm{C}$ after 24:00. Given these data, there was a high possibility of snow instead of rain, or freezing immediately after rainfall.

In the case of rainfall, water droplets that fall on the ground will flow along the gradient of the land surface or penetrate the soil vertically. Accordingly, the distribution of radioactive materials contained in the rain is considered not to be much influenced by the location of trees, chamaephytes, etc., but fairly uniform. However, in the case of rainfall/snow (hereinafter in this paper "rainfall/snow" refers to both snowfall and freezing after rainfall), stagnation occurs on the upper branches and leaves of trees. Furthermore, rainfall/snow will stagnate on the chamaephytes in pastures and soils and will have less of a chance to flow over the land surface 
than simple rainfall, whatever the gradient of the land. For this reason, any radioactive material contained in rainfall/snow remains on the precipitated location longer than rainfall during the early stage of deposition.

In our study, it was recognized that accumulation of fallout was seen in the upper part of the stem surface of the sliced Cryptomeria japonica, more accumulation on the leaves that were present in the upper part of Torreya nucifera leaves, and nearly none in the fern relatives that were sampled in the grove. This resulted from the non-uniform distribution of fallout under rainfall/snow conditions. Furthermore, the concentration of radioactive fallout in the upper part of the land surface, particularly on pastures, in the soils, and on rice stubble, can be explained as a peculiar behavior of radioactive materials in rainfall/snow.

The behavior of radioactive materials accumulating in snow piled up on trees and pastures post-melting has scarcely been studied until now. No rainfall was observed around Iitate-mura on March 16th, and the temperature was generally below $2^{\circ} \mathrm{C}$. This implies a slow melting rate for snow, i.e. a longer duration of the contact between the radioactive fallout contained in the snow or ice and the trees, pastures, and soils than during simple rainfall.

The radioactive Cs deposited on the surface of the chamaephytes is considered to have been transported to the inner plant and soil layers through leaf surface absorption or weathering, respectively ${ }^{8}$. According to Kawabata et al., more than $80 \%$ of the Cs deposited as solid aerosol on Raphanus sativus var. sativus was removed from the outer plant by weathering resulting from rainfall ${ }^{9)}$. The radioactive Cs transported to the soil was tightly adsorbed by a so-called 2:1 type clay mineral similar to mica ${ }^{10-12)}$. Based on this, during the translocation of radioactive Cs deposited on the plants via soil, a delaying role of the soil is expected. Our results imply that, in the actual conditions for 2 months after the fallout, the effect of weathering was very low because of the immobility of the radioactive Cs accumulated on the chamaephytes and trees. Accordingly, the pastures contributed greatly to a delay in translocation of the fallout as it penetrated the soil via rainfall water from plants and soil surfaces.

\section{Decontamination of Plants and Pasture Lands}

In regions in Fukushima-Prefecture, where the radioactive Cs concentration is high, radioactive Cs was detected on the stem epidermis, branches and leaves of trees. Detergent was considered as a decontamination tool ${ }^{6}$ to wash away Cs that had adhered to trees, etc. So, autoradiography measurement was conducted after dipping Torreya nucifera branches and leaves in a detergent solution. However, there was nearly no change after dipping in the detergent solution, perhaps because of strong binding of radioactive Cs to surfaces, or even actual accumulation into the trees. This suggested that trimming the trees might be more effective. Additionally, our results indicated higher contamination in the upper regions of the trees, which suggested reducing the dose of the whole tree by trimming just the upper part. Furthermore, because of the limited penetration of radioactive Cs in the stem, the possibility of preventing penetration from the surface to the inside by promptly trimming the branches and leaves with a high dose is recommended. Consequently, given the precondition of confirmed radioactivity, it is strongly indicated that trees such as Cryptomeria japonica be used as architectural material after removing their epidermis.

Nearly no penetration of radioactive Cs into the soil was observed in the decontamination of pasture lands. This implies the possibility of effective decontamination of pasture lands by collecting just their surface portion. However, because the rainfall total increases with time after the fallout, the possibility of re-translocation of concentrated radioactive Cs on the pastures cannot be denied. As well as the necessity of promoting studies on the chemical status of 
concentrated radioactive Cs, it would be effective to decontaminate the pasture by promptly collecting surface soils/plants.

These results are important knowledge for future decontamination operations. However, because our results are only from a single region, further data collection will be necessary for the decontamination of a wider range of area types. When conducting a survey of the distribution of radionuclides, visualized data from autoradiography as well as the concentration distribution will contribute to the preparation of a detailed decontamination plan and its understanding by residents.

\section{Summary}

The local area distribution of radioactive materials was measured using autoradiography, targeting trees, chamaephytes, and soils. The behavior of the radioactive Cs component of the fallout after precipitation was studied. As a result, the following conclusions can be made:

- The radioactive Cs that was deposited on the trees accumulated more on the stems and leaves that were present before the fallout. Furthermore, some of the radioactive Cs was distributed on the new branches and leaves that grew after the fallout.

- Most of the radioactive Cs that was deposited on pastures and rice stubble accumulated on the vegetation, and secondary movement to the soils immediately beneath was below the detection limit of this method.

- Rainfall/snow is considered to be one of the reasons for the non-uniform concentration of radioactive Cs on the plants.

Consequently, chamaephytes and trees have acted as accumulation media, greatly inhibiting the further translocation of radioactive Cs, either in the soil or in runoff.

\section{References}

1) M. Chino, H. Nakayama, H. Nagai et al., "Preliminary estimation of release amounts of ${ }^{131} \mathrm{I}$ and ${ }^{137} \mathrm{Cs}$ accidentally discharged from the Fukushima Daiichi Nuclear Power Plant into the atmosphere," J. Nucl. Sci. Technol., 48, 1129-1134 (2011).

2) A. Omoto, ICAPP2011 Special Japan Session: Presentation Nice, France, May 2-6, 2011. file is available athttp://www.aesj.or.jp/information/fnpp201103/icapp2011_Omoto_WEBrevision20110517.pdf

3) MEXT, Measurements on Dust Sampling, Environmental Specimens and Soil Monitoring around Tokyo Electric Fukushima Daiichi and Daini Nuclear Power Plants: Measurements of Soil Monitoring (as of 10:00, Jun.25, 2011) http://www.mext.go.jp

4) H. Naganawa, N. Kumazawa, H. Saitoh et al., "Removal of Radioactive Cesium from Surface Soils Solidified Using Polyion Complex - Rapid Communication on Decontamination Test at Iitate-mura in Fukushima Prefecture," Transactions of the Atomic Energy Society of Japan. (To be published).

5) F. Sakamoto, T. Ohnuki, T. Fujii, et al., "Response of Saccharomyces cerevisiae to heavy element stress: lead vs. uranium," Geomicrobiol. J. (To be published).

6) H. Tsukada, H. Hasegawa, H. Kawabata et al., "Leaf Surface Absorption of ${ }^{137} \mathrm{Cs}$ by Droplet Impregnation," 2009 Fall Meeting of Atomic Energy Society of Japan, Abstract A23 (2009).

7) JMA, AMEDAS Hp (http://www.jma.go.jp/jp/amedas/)

8) H. Amano, T. Takahashi, S. Uchida, et al., "Development of a code MOGRA for predicting the migration of ground additions and its application to various land utilization areas," J. Nucl. Sci. Technol., 40, 975-979 (2003).

9) H. Kawabata, H. Hasegawa, H. Tsukada et al. "Behavior on Leaf Surface of Crop Plants," Annual Report FY2009/Institute for Environmental Sciences/Institute for Environmental Science, 20-24 (2010).

10) T. Ohnuki, "Sorption characteristics of cesium on sandy soils and their components," Radiochimica Acta, 65, 75-80 (1994).

11) T. Ohnuki, N. Kozai, "Sorption characteristics of radioactive cesium and strontium on smectite," 
Radiochim. Acta, 66/67, 327-331 (1994).

12) D. J. Assinder, S. M. Mudge, G. S. Bourne, "Migration of ${ }^{137} \mathrm{Cs}$ and ${ }^{90} \mathrm{Sr}$ from Chernobyl fallout in Ukrainian, Belarussian and Russian soils," J. Environmental Radioactivity, 35, 1-19 (1997). 


\title{
Technical Material
}

\section{Study on Public Awareness of Utilizing Nuclear Power in China}

\author{
-Changes in Public Awareness after the Accident of \\ Fukushima Daiichi Nuclear Power Plants-
}

\author{
Ting XU ${ }^{1, *}$ and Toshio WAKABAYASHI ${ }^{1}$ \\ ${ }^{1}$ Department of Management Science and Technology, Nuclear Engineering, Graduate School of Engineering, Tohoku \\ University, 6-6-11 Aramaki-Aza-Aoba, Aoba-ku, Sendai-shi, Miyagi 980-8579, Japan
}

\begin{abstract}
The purpose of this study is to clarify public awareness of utilizing nuclear power in China and to determine the effects of the accident of Fukushima Daiichi Nuclear Power Plants. Web online surveys were carried out before and after the accident of Fukushima Daiichi Nuclear Power Plants. The online survey before the accident of Fukushima Daiichi Nuclear Power Plants had 4,255 adult respondents consisting of 1,851 males and 2,404 females. The online survey after the accident had 721 respondents consisting of 406 males and 315 females. The two online surveys about the attitude toward nuclear power plants consisted of 37 items, such as the necessity of nuclear power plants, the reliability of safety, and government confidence. As a result, respondents of the online surveys in China consider that nuclear energy is more important than the anxiety of accident. On the other hand, women have sensation of fear for the accident of Fukushima Daiichi Nuclear Power Plants and radiation.
\end{abstract}

KEYWORDS: Fukushima Daiichi Nuclear Power Plants, accident, public awareness, online survey, China

\section{Introduction}

At 2:46 pm on March 11, 2011, one of the largest earthquakes ever recorded hit the Pacific coast side of the Tohoku area. The epicenter was 130 kilometers below the surface of the sea east of Sendai City in Miyagi Prefecture and the magnitude was 9.0 (according to the Japan Meteorological Agency, 8.9 according to the U.S. Geological Survey). It was the fifth largest earthquake since 1900, when human beings started officially recording the intensity of earthquakes.

After the accident at the Fukushima Daiichi Nuclear Power Plant (hereinafter "the accident") caused by the tsunami following the earthquake, radioactive materials were released into the surrounding area, producing serious consequences. The Nuclear and Industrial Safety Agency of the Ministry of Economy, Trade and Industry (NISA) and the Nuclear Safety Commission (NSC) raised the INES (International Nuclear and Radiological Event Scale) level to Level

\footnotetext{
${ }^{*}$ Corresponding author, E-mail: xu.ting@most.tohoku.ac.jp

DOI : 10.15669 /fukushimainsights. Vol.4.63

(C) 2021 Atomic Energy Society of Japan. All rights reserved.

Originally published in Transactions of the Atomic Energy Society of Japan (ISSN 1347-2879), Vol. 11, No. 1, p.20-29 (2012)

in Japanese. (Japanese version accepted: October 31, 2011)
} 
7, a Major Accident, as the amount of radioactive materials released had been significant and had spread over an extensive area. It is comparable to the worst nuclear power plant accident, Chernobyl, in the former Soviet Union in 1986.

The accident is expected to have an impact on the energy strategies adopted and implemented in countries throughout the world. Specifically, one of the focuses is on how the accident may be reflected in the policies of China, which is planning to construct more nuclear power plants than any other country worldwide. The Chinese government declared on March 12 that they would not change their nuclear power promotion plan after the Great East Japan Earthquake. However, on the 16th of the month they suddenly changed the policy and clearly expressed their intention to call off the assessment and ratification (approval process) of nuclear power plant-related projects until they developed a safety plan for nuclear power.

Nevertheless, it is assumed that it is unavoidable for China to increase its dependency on nuclear power from the perspectives of resolving resource limits and promoting environmental conservation. China's dependency on thermal power is high at $90 \%$ and in 2009 they became a net importer of coal for fuel. While China depends on imports for petroleum, how it will decrease dependency on thermal power will be one of the most significant issues for the country in order to ensure stable economic growth.

At this stage, nuclear power generation accounts for only $2 \%$ of all the power generated in China. However, in order to achieve the grand goal of increasing the share of non-fossil fuels from the current $9 \%$ to $15 \%$ by 2020 , they have no choice but to pursue a nuclear promotion policy. They also have to pay attention to promoting sustainable energies such as wind and solar power. In China, 13 nuclear power plants are currently under operation and another 35 plants will be added over the medium term. It is the largest number in the world at the planning level and they will be distributed widely across the country, but mainly in coastal areas ${ }^{1)}$. Therefore, it is an important issue to ascertain public attitudes in China to nuclear power. While many public awareness surveys have been conducted in Japan ${ }^{2-7)}$, in China, only a few have been carried out ${ }^{8)}$.

\section{Study Objectives and Method}

China has a growing interest in the safety of nuclear power in response to the accident at the Fukushima Daiichi Nuclear Power Plant. Some cities in coastal areas in particular have growing concerns about radioactive dispersion. By performing a comparative analysis with survey data obtained before the accident, this study reveals the impact that the accident has had on public attitudes and awareness in China regarding nuclear power generation.

In this study, analysis was conducted based on an online questionnaire survey, which allows respondents who are interested in the survey or are conscious of the issue to be recruited. Respondents answered the questions online.

The first questionnaire survey was conducted between May 2 and June 30, 2010, which was before the accident, and the second survey was conducted between May 9 and May 16, 2011, which was after the accident. Based on the data, we captured what impact the accident had on the public in China.

In addition, the study revealed the changes in public awareness and attitudes of male and female respondents including the level of public knowledge on nuclear power, interest in nuclear power, concerns about nuclear reactor accidents, attitudes towards nuclear power generation (for or against), and the perceived reliability of operating bodies relevant to nuclear power. 


\section{Questionnaire Survey on Nuclear Power Use}

\section{Overview of the Questionnaire Survey}

We reviewed what the Chinese public feels about nuclear power and developed questions to ascertain their opinions on nuclear power use and to measure the awareness factors that could have an influence on their opinions. The questions are listed in the Appendix. Each question has four or more multiple-choice answers. The questions are about the utility and need for nuclear power, awareness and the level of knowledge on the use of nuclear power, attitudes (whether for or against) to nuclear energy policies, perceived reliability of nuclear energy operators, how information on nuclear power is obtained, and what the important points are when evaluating nuclear power. In addition, at the end of the questionnaire, the respondent's information, including his/her gender, age, place of birth and academic history, was collected.

The online questionnaire was conducted in Liaoning, Shandong, Jiangsu, Zhejiang, Fujian, and Guangdong. Currently, nuclear power plants in operation, under construction, and planned in China are concentrated in these coastal areas.

We chose these areas because we thought those who live in these areas have more knowledge about nuclear power, a higher interest in energy issues and nuclear power, and are more strongly impacted by the use of nuclear power. The respondents are aged 18 years or older, live in China and use the Internet. Internet users in China consist of people living in urban cities, people with superior education including business people working at foreign companies, and university students. Among them, 53.2\% are aged between 18 to 30 years. The Internet is also widely accepted by people in their 40's and 50's. Table 1 provides an overview of the public awareness survey conducted in China on nuclear power generation.

\section{Survey Results and Analysis before and after the Nuclear Accident}

\section{(1) Survey results before the accident}

Table 2 shows the age and gender composition of respondents who took the public awareness survey on the use of nuclear power conducted before the accident. According to Table $2,43.5 \%$ of the respondents were male and $56.5 \%$ were female. We were able to receive responses from a wide range of age groups. Their age groups were as follows; those in their 20 's represented highest proportion at $25 \%$, followed by 30 's at $22.8 \%, 40$ 's at $21 \%, 50$ 's at $13 \%$ and aged under 20 years and 60 years or older at $14 \%$. The following describes the features of the results of the main questions.

To find out the level of knowledge on nuclear power generation, the question asked was, "Do you have sufficient knowledge on nuclear power generation?"

The answer was scaled into four levels: "Yes, I have.", "Yes, I have a little.", "No, I don't

Table 1 Summary of the public awareness surveys on nuclear power generation

\begin{tabular}{|c|c|c|c|c|c|}
\hline Survey type & Survey content & Survey period & \multicolumn{2}{|c|}{ Survey regions } & $\begin{array}{l}\text { Number of valid } \\
\text { responses }\end{array}$ \\
\hline $\begin{array}{l}\text { Online questionnaire } \\
\text { survey }\end{array}$ & $\begin{array}{l}\text { Public awareness survey in China on } \\
\text { nuclear power }\end{array}$ & $\begin{array}{r}\text { May } 2 \text { to June } 30, \\
2010\end{array}$ & $\begin{array}{l}\text { Liaoning, } \\
\text { Jiangsu, } \\
\text { Guangdong }\end{array}$ & $\begin{array}{l}\text { Shandong, } \\
\text { Zhejiang, }\end{array}$ & 4,255 \\
\hline $\begin{array}{l}\text { Online questionnaire } \\
\text { survey }\end{array}$ & $\begin{array}{l}\text { Survey conducted } 2 \text { months after the } \\
\text { accident at the Fukushima Daiichi } \\
\text { Nuclear Power Plant }\end{array}$ & $\begin{array}{r}\text { May } 9 \text { to May } 16, \\
2011\end{array}$ & $\begin{array}{l}\text { Liaoning, } \\
\text { Jiangsu, } \\
\text { Guangdong }\end{array}$ & $\begin{array}{l}\text { Shandong, } \\
\text { Zhejiang, }\end{array}$ & 721 \\
\hline
\end{tabular}


have much." and "No, I don't have any." In this question, the level of knowledge is not measured in an objective manner but in a subjective, self-reported manner. According to the results on the level of knowledge on nuclear power measured by this question, you can see $11.4 \%$ of the respondents have knowledge regarding nuclear power.

As for interest in nuclear power in China, those who are interested ("Very interested" or "Somewhat interested") comprise 58.6\%, while those who are not interested ("Not very interested") account for $41.4 \%$. China actively promotes nuclear power generation and the survey found that people in China have high interest in it even after the accident.

To clarify attitudes (for or against) to nuclear energy policies, the following question was asked. "What do you think about China's future nuclear power generation?" The results showing the degree of support for nuclear energy policies as measured by this question are presented in Table 3. The question found that the majority of respondents (58.3\%) "Agree" or "Somewhat agree" and thus it seems that the respondents in this survey tend to support the government's nuclear energy policies.

Table 2 Age and gender composition of respondents who took part in the public awareness survey on nuclear power generation (before the accident)

\begin{tabular}{cccr}
\hline Age & Male (\%) & Female (\%) & \multicolumn{1}{c}{ Total (\%) } \\
\hline Under 20 & $214(11.6)$ & $196(8.2)$ & $410(9.6)$ \\
$20-29$ & $516(27.9)$ & $562(23.4)$ & $1,078(25.3)$ \\
$30-39$ & $427(23.1)$ & $545(22.7)$ & $972(22.8)$ \\
$40-49$ & $310(16.7)$ & $590(24.5)$ & $900(21.2)$ \\
$50-59$ & $236(12.7)$ & $330(13.7)$ & $566(13.3)$ \\
60 and older & $148(8.0)$ & $181(7.5)$ & $329(7.7)$ \\
\hline Total (\%) & $1,851(100.0)$ & $2,404(100.0)$ & $4,255(100.0)$ \\
\hline
\end{tabular}

Table 3 Results of the public awareness survey on nuclear power generation (before the accident)

\begin{tabular}{llccc}
\hline \multicolumn{1}{c}{ Question } & \multicolumn{1}{c}{ Response options } & Total & Male & Female \\
\hline $\begin{array}{l}\text { Level of } \\
\text { knowledge }\end{array}$ & Yes, I have. & $11.4 \%$ & $12.4 \%$ & $10.4 \%$ \\
on nuclear & Yes, I have a little. & $22.0 \%$ & $24.6 \%$ & $19.3 \%$ \\
power & No, I don't have much. & $32.3 \%$ & $28.1 \%$ & $36.5 \%$ \\
generation & No, I don't have any. & $34.4 \%$ & $35.0 \%$ & $33.8 \%$ \\
\hline & Very interested & $21.5 \%$ & $23.9 \%$ & $19.1 \%$ \\
$\begin{array}{l}\text { Interest in } \\
\text { nuclear }\end{array}$ & Somewhat interested & $37.1 \%$ & $39.0 \%$ & $35.2 \%$ \\
power & Not very interested & $32.5 \%$ & $29.7 \%$ & $35.3 \%$ \\
\hline $\begin{array}{l}\text { Attitude (for } \\
\text { or against) to }\end{array}$ & Not at all interested & $8.9 \%$ & $7.4 \%$ & $10.4 \%$ \\
nuclear & Somewhat agree & $21.8 \%$ & $25.1 \%$ & $18.4 \%$ \\
energy & Somewhat disagree & $36.5 \%$ & $38.9 \%$ & $34.1 \%$ \\
policy & Disagree & $27.2 \%$ & $22.2 \%$ & $32.1 \%$ \\
$\begin{array}{l}\text { Concerns } \\
\text { about }\end{array}$ & Very concerned & $14.6 \%$ & $13.8 \%$ & $15.4 \%$ \\
$\begin{array}{l}\text { nuclear } \\
\text { power } \\
\text { generation }\end{array}$ & Somewhat concerned & $33.5 \%$ & $35.4 \%$ & $31.5 \%$ \\
\hline $\begin{array}{l}\text { Reliability of } \\
\text { nuclear } \\
\text { energy }\end{array}$ & Not very concerned & $29.9 \%$ & $29.8 \%$ & $30.0 \%$ \\
operators & Not at all concerned & $16.8 \%$ & $16.6 \%$ & $17.0 \%$ \\
\hline & Nomewhat reliable & $44.3 \%$ & $47.1 \%$ & $41.5 \%$ \\
& Not at all reliable & $20.8 \%$ & $18.7 \%$ & $22.9 \%$ \\
& $12.5 \%$ & $10.8 \%$ & $14.1 \%$ \\
\hline
\end{tabular}


What people in China place most importance on when evaluating nuclear power energy was "Whether it contributes to solving environmental issues," which accounts for $47.4 \%$, while many other people (44.8\%) answered "Whether energy can be produced safely at low cost by nuclear power generation." Many people also answered, "Whether the country is capable of responding to an emergency issue when it happens," "Whether nuclear power generation technology is necessary for society," "Whether the energy-based society can manage nuclear power without causing problems," and "Whether the government has already anticipated what kind of influences may happen." This shows more people are interested in nuclear power technology and its social impact. Furthermore, some people place importance on "Whether nuclear power generation contributes to solving environmental issues," "Whether influences on unborn babies and children have already been investigated" and "Whether nuclear power generation technology is fully developed."

As for the issue regarding concerns about nuclear power generation, the question asked, "Do you have concerns that a major accident may happen at a nuclear power plant in China in the future?" The results on the degree of concern about nuclear energy measured by this question are shown in Table 3. It was found that $53.3 \%$ of the respondents are concerned about nuclear power generation (either "Very concerned" or "Somewhat concerned"). As for the reason for concern regarding nuclear energy, $40.6 \%$ of respondents answered, "China does not have sufficient response measures if a nuclear accident occurs." In addition, many people answered, "The electricity supplier does not disclose sufficient information or provide PR activities regarding nuclear power" and "Nuclear accidents have happened in the US, Russia and other countries."

\section{(2) Survey results after the accident}

In the same way as the survey before the accident, a public awareness survey on the use of nuclear power was performed two months after the accident. Table 4 shows the age and gender composition of respondents who took the public awareness survey on the use of nuclear power conducted after the accident.

This survey was performed to clarify how the accident changed public awareness of and attitudes to nuclear power generation. In the survey, a question was asked whether their thinking and attitudes regarding nuclear power generation in China had changed after the accident. Table 5 shows the results of this public awareness survey. In the survey, 79\% of the respondents answered that their awareness had "changed." The survey found that the accident had influenced many respondents' "attitude towards nuclear energy in China." The results of the survey after the accident found that respondents' interest in and sense of safety towards nuclear power generation had changed as had their confidence in the reliability of nuclear energy operators.

To be more specific, regarding the level of knowledge on nuclear power generation, the

Table 4 Age and gender composition of respondents who took part in the public awareness survey on nuclear power generation (after the accident)

\begin{tabular}{ccrrrrr}
\hline Age & \multicolumn{2}{c}{ Male } & \multicolumn{2}{c}{ Female } & \multicolumn{2}{c}{ Total (\%) } \\
\hline $20-29$ & 140 & $(34.5)$ & 122 & $(38.7)$ & 262 & $(36.3)$ \\
$30-39$ & 150 & $(36.9)$ & 107 & $(34.0)$ & 257 & $(35.6)$ \\
$40-49$ & 76 & $(18.7)$ & 57 & $(18.1)$ & 133 & $(18.4)$ \\
$50-59$ & 40 & $(9.9)$ & 29 & $(9.2)$ & 69 & $(9.6)$ \\
\hline Total (\%) & 406 & $(100.0)$ & 315 & $(100.0)$ & 721 & $(100.0)$ \\
\hline
\end{tabular}


Table 5 Results of the public awareness survey on nuclear power generation (after the accident)

\begin{tabular}{|c|c|c|c|c|}
\hline Question & Response options & Total & Male & Female \\
\hline \multirow{4}{*}{$\begin{array}{l}\text { Level of } \\
\text { knowledge on } \\
\text { nuclear power } \\
\text { generation }\end{array}$} & Yes, I have. & $39.0 \%$ & $39.9 \%$ & $38.1 \%$ \\
\hline & Yes, I have a little. & $35.2 \%$ & $37.4 \%$ & $33.0 \%$ \\
\hline & No, I don't have much. & $20.1 \%$ & $18.2 \%$ & $21.9 \%$ \\
\hline & No, I don't have any. & $5.7 \%$ & $4.4 \%$ & $7.0 \%$ \\
\hline \multirow{4}{*}{$\begin{array}{l}\text { Interest in } \\
\text { nuclear power }\end{array}$} & Very interested & $41.9 \%$ & $43.8 \%$ & $40.0 \%$ \\
\hline & Somewhat interested & $39.3 \%$ & $37.2 \%$ & $41.3 \%$ \\
\hline & Not very interested & $15.3 \%$ & $16.3 \%$ & $14.3 \%$ \\
\hline & Not at all interested & $3.6 \%$ & $2.7 \%$ & $4.4 \%$ \\
\hline \multirow{4}{*}{$\begin{array}{l}\text { Attitude (for } \\
\text { or against) to } \\
\text { nuclear energy } \\
\text { policy }\end{array}$} & Agree & $19.1 \%$ & $25.1 \%$ & $13.0 \%$ \\
\hline & Somewhat agree & $23.3 \%$ & $28.6 \%$ & $18.1 \%$ \\
\hline & Somewhat disagree & $29.4 \%$ & $25.4 \%$ & $33.3 \%$ \\
\hline & Disagree & $28.3 \%$ & $20.9 \%$ & $35.6 \%$ \\
\hline \multirow{4}{*}{$\begin{array}{l}\text { Concerns } \\
\text { about nuclear } \\
\text { power } \\
\text { generation }\end{array}$} & Very concerned & $24.8 \%$ & $25.1 \%$ & $24.4 \%$ \\
\hline & Somewhat concerned & $37.8 \%$ & $39.9 \%$ & $35.6 \%$ \\
\hline & Not very concerned & $26.1 \%$ & $22.9 \%$ & $29.2 \%$ \\
\hline & Not at all concerned & $11.5 \%$ & $12.1 \%$ & $10.8 \%$ \\
\hline \multirow{4}{*}{$\begin{array}{l}\text { Reliability of } \\
\text { nuclear energy } \\
\text { operators }\end{array}$} & Reliable & $20.0 \%$ & $21.6 \%$ & $18.3 \%$ \\
\hline & Somewhat reliable & $34.1 \%$ & $36.7 \%$ & $31.5 \%$ \\
\hline & Not very reliable & $26.3 \%$ & $23.4 \%$ & $29.2 \%$ \\
\hline & Not at all reliable & $19.7 \%$ & $18.3 \%$ & $21.0 \%$ \\
\hline
\end{tabular}

same question was asked; "Do you have sufficient knowledge on nuclear power generation?" The most common response was "Yes, I have." at 39.0\%, while those who answered "No, I don't have any." was 5.7\%.

The results show that the respondents learned more about nuclear power generation after being exposed to a lot of information following the accident. After the accident, the media in China broadcast information about nuclear power every day. The public has acquired knowledge and information about nuclear power through mass media including newspapers, magazines and TV, and via the Internet.

Regarding public interest in nuclear power in China, about $80 \%$ of the respondents answered they were interested ("Very interested" and "Interested"), while 3.6\% answered "Not at all interested" and $15.3 \%$ answered "Not very interested." China actively promotes nuclear power generation and this questionnaire found that people in China have become more interested in and aware of it.

Regarding attitudes (for or against) to nuclear energy policies in China, the responses to the question about the degree of support for nuclear energy policies revealed that $42.4 \%$ of the respondents supported (Agree or Somewhat agree) the country's nuclear energy policies, while $57.7 \%$ did not. In relation to technologies to be used as measures to solve energy issues, many respondents answered that "Solar energy should be developed" and "Cleaner fossil fuels should be developed and technologies should be developed and introduced to facilitate more efficient use of fossil fuels," accounting for 53.6\% and 50.1\% respectively. As measures to secure energy, many people answered that "Technologies should be developed to secure a stockpile of energy," "Energy-saving technology should be developed" and the "Development, implementation, and use of nuclear power should be promoted."

As for what people in China place importance on when they evaluate nuclear power use, many answered, "Whether the country is capable of responding to an emergency issue when 
it happens" and, "Whether energy can be produced safely at low cost by nuclear power generation," accounting for $58.3 \%$ and $55.2 \%$ respectively. Furthermore, $47.4 \%$ of respondents answered that "Whether the government has already anticipated what kind of influences may happen." Respondents also place importance on "Whether nuclear power generation technology is necessary for society" and "Whether influences on unborn babies and children have already been investigated."

As for concerns about nuclear power generation in China, $62.6 \%$ of the respondents answered that they were "Very concerned" or "Somewhat concerned," which shows many people became concerned about nuclear power after the accident. Among the respondents, more people answered that "Major nuclear accidents like the one in Japan can also happen in China" and "China does not have sufficient response measures if a nuclear accident occurs" compared to the previous survey. In addition, in this survey, $39.7 \%$ of the respondents answered that "Nuclear power technologies are immature in China compared to other technologically developed countries."

The survey results on the reliability of nuclear energy operators showed that $53.1 \%$ of the respondents thought that operators are reliable ("Reliable" or "Somewhat reliable"), while $46.9 \%$ thought that operators are not reliable ("Not very reliable" or "Not at all reliable"). Although even after the accident the Chinese government continued to assert, regarding nuclear energy operators in China, that "Our technology is advanced," "Nuclear problems cannot happen in China," "Nuclear power plants are not located where earthquakes happen from the start," and "There is nothing to worry about regarding nuclear power in China," it is assumed that many people in China wonder if these statements are really true.

\section{(3) Comparison of awareness related to nuclear power before and after the accident}

In this study, comparison and analysis were performed to ascertain public interest in nuclear power, their support for it and how reliable they think it is. Also, we wanted to find out how safe the public thought nuclear power is as well as the public image of nuclear power, both before and after the accident.

Figure 1 indicates the public interest in nuclear power generation before and after the accident. To the question as to whether they are interested in nuclear power generation in China, the number of respondents who answered "Very interested" increased by $20.4 \%$, while those who answered "Not at all interested" decreased by $5.3 \%$.

As for their opinions on the promotion of nuclear power in China, the percentage of those who responded it "Should be actively promoted" decreased by about $10 \%$ compared to the survey before the accident as indicated in Figure 2. On the other hand, the percentage of those who answered it "Should be carefully promoted" increased by $4.5 \%$ and reached $40.9 \%$.

Figure 3 indicates how reliable the operating bodies relevant to nuclear power are perceived to be before and after the accident. The reliability of nuclear energy operating bodies in China, which is assumed to have significant impact on the use of nuclear energy, has shifted in a negative direction. The percentage of those who answered "Reliable" decreased by $2.5 \%$ from $22.5 \%$ to $20.0 \%$.

On the other hand, the percentage of those who answered "Not reliable" has increased by $7.2 \%$, to $19.7 \%$. As for the reason, many people answered that "Nuclear accidents have happened in the US, Russia, and other countries," "Nuclear power technologies are immature in China," and "China does not have sufficient response measures if a nuclear accident occurs."

Regarding concerns about nuclear energy in China, those who are concerned about it ("Very concerned" and "Somewhat concerned") account for $62.6 \%$ of all respondents (see 
Figure 4). Compared to before the accident, those who answered, "Very concerned" and "Somewhat concerned" increased by $5 \%$ and $4.3 \%$ respectively.

Figure 5 shows a comparison of the sense of safety towards foods from the surrounding seas before and after the accident. For the question "Would you mind eating foods including vegetables and fish that come from the vicinity of a nuclear power plant?," 58.2\% of respondents answered "Yes" ("Yes, I would mind a little" or "Yes, I would mind"), while $41.8 \%$ of them answered "No" ("No, I would not mind at all" or "I would not mind much") after the accident. Compared to before the accident, the percentage of those who answered

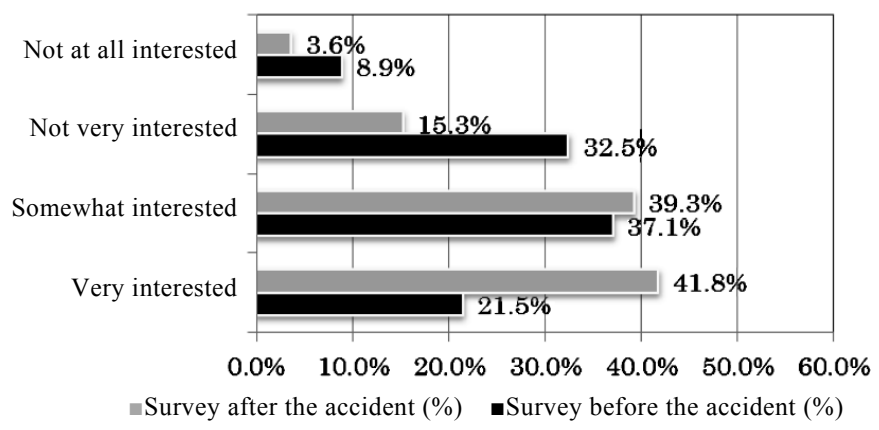

Figure 1 Interest in nuclear power generation before and after the accident

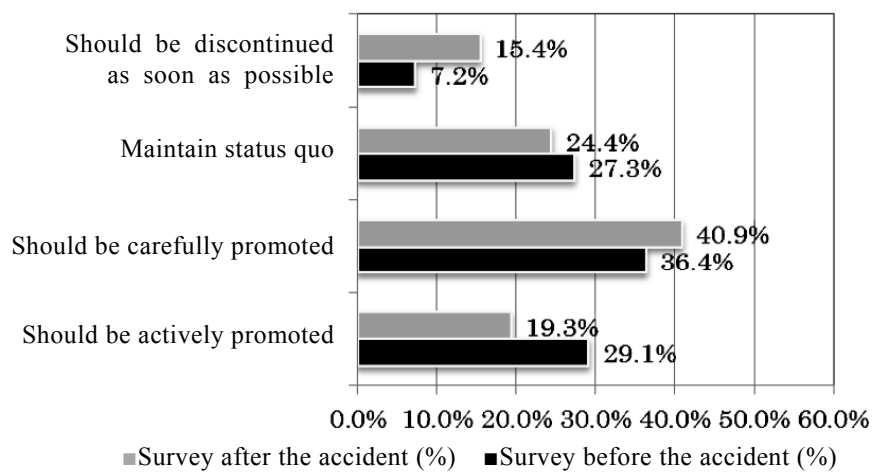

Figure 2 Opinions on the promotion of nuclear power before and after the accident

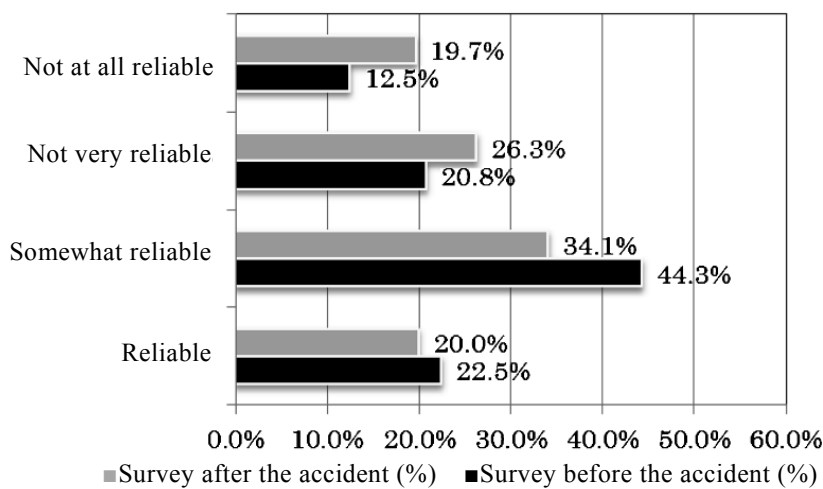

Figure 3 Reliability of operating bodies relevant to nuclear power before and after the accident 
"No" decreased by $18.9 \%$; especially after the accident, more people did not want to have food sourced from near a nuclear power plant, including seaweeds, due to concerns about radioactive contamination of fish from the surrounding seas.

Figure 6 shows a comparison of the image of nuclear power before and after the accident. The largest number of respondents gave the answer "Radioactive contamination" to the question "What's the first thing you think of when you hear the words 'nuclear power'?," which accounts for $33.8 \%$, an increase of $11.7 \%$ from the survey before the accident. Although the number of those who think "Nuclear power $=$ Nuclear reactor explosion"

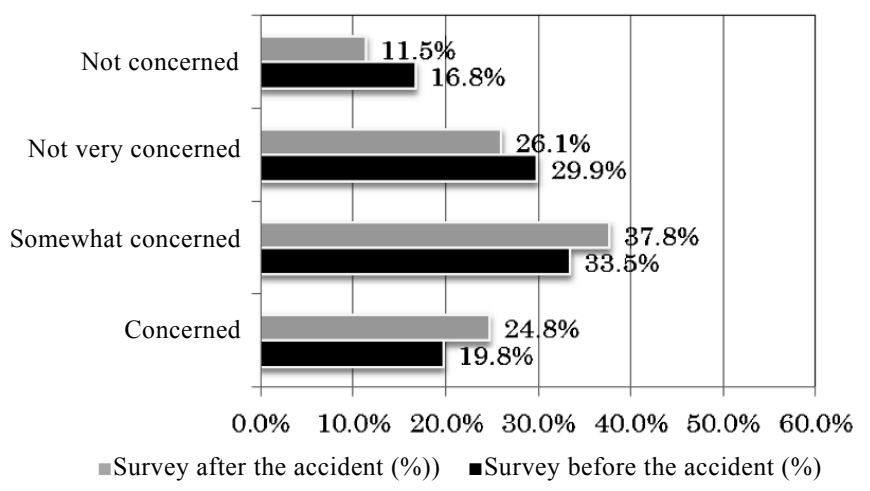

Figure 4 Concern about nuclear energy before and after the accident

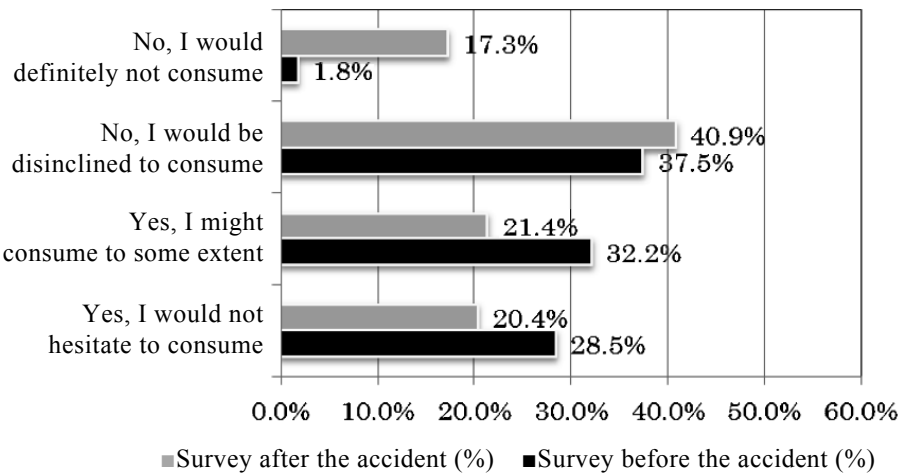

Figure 5 Sense of safety towards foods from the surrounding seas before and after the accident

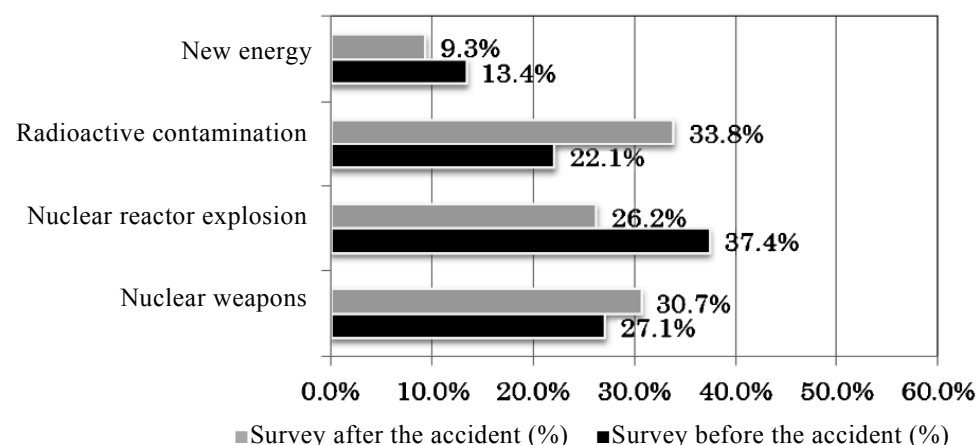

Figure 6 Image of nuclear power before and after the accident 
decreased slightly, the percentage of the public who think "Nuclear power = Radioactive contamination" increased.

\section{(4) Comparison of impact on males and females before and after the accident}

This study also analyzed if there were any differences between male and female respondents based on their answers to the questionnaire survey.

Figure 7 shows the comparison results of the level of knowledge on nuclear power generation between male and female respondents. Those who answered "Yes, I have." were $12.4 \%$ among males and $10.4 \%$ among females before the accident and $39.9 \%$ and $38.1 \%$, respectively, after the accident. It shows that males have more knowledge than females even after the accident.

Figure 8 shows the comparison results of interest in nuclear power between male and female respondents.

Those who answered "Very interested" were $23.9 \%$ among males and $19.1 \%$ among females before the accident and $43.8 \%$ and $40.0 \%$, respectively, after the accident. It shows that males have more interest in nuclear power than females.

Figure 9 shows the comparison results of attitudes (for or against) to nuclear energy policies between males and females. Before the accident $64 \%$ of males and $52.5 \%$ of females were supportive of nuclear energy policies and there was a $7.5 \%$ difference between males and females. However, after the accident, $53.7 \%$ of males and $31.1 \%$ of females were supportive of nuclear energy policies and the gap between them expanded significantly to

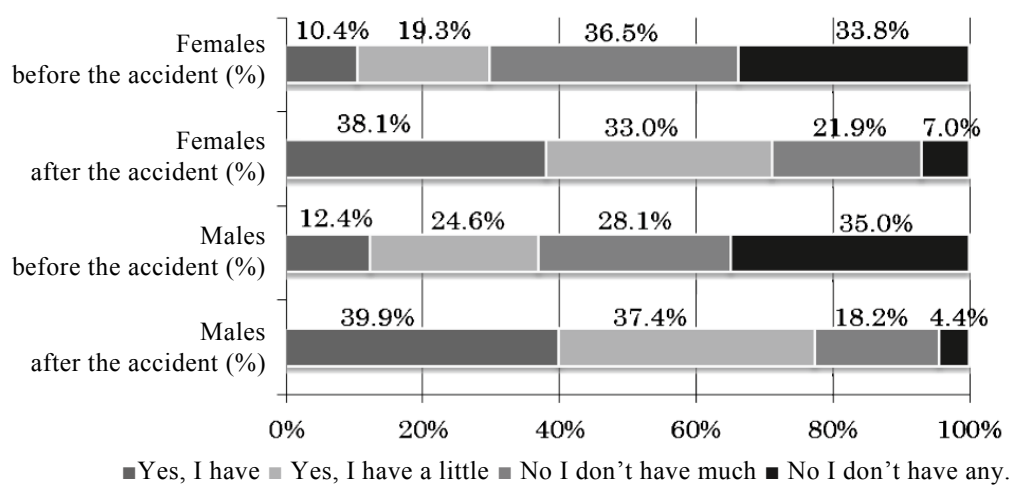

Figure 7 Comparison results of the level of knowledge on nuclear power generation between males and females

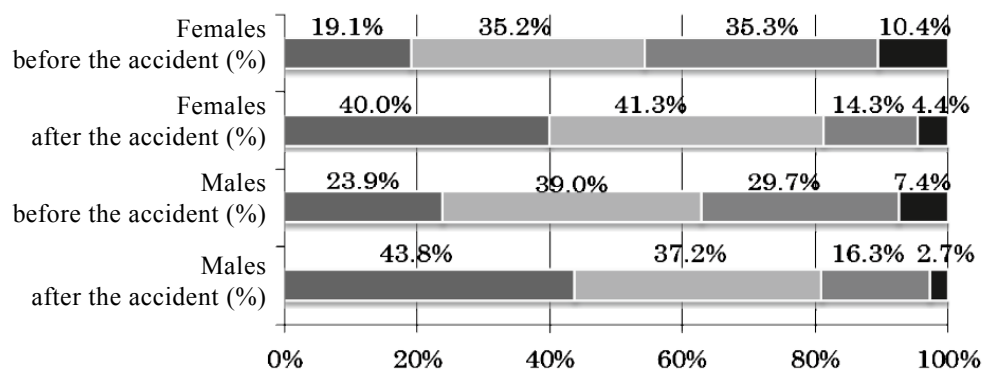

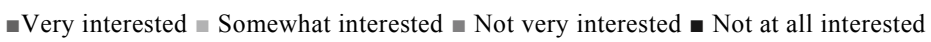

Figure 8 Comparison results of interest in nuclear power between males and females 


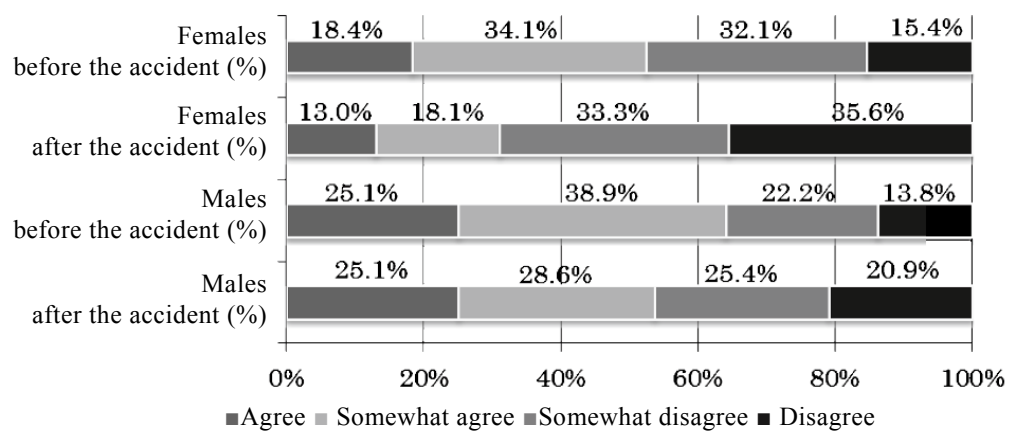

Figure 9 Comparison results of attitudes (for or against) to nuclear energy policies between males and females

$22.6 \%$. The survey found that while males tend to be positive about nuclear power generation as a new source of energy, females tend to fear accidents, rather than thinking of nuclear power as energy, and have a negative attitude to nuclear energy policies. Moreover, there are gaps between males and females in terms of how safe they think nuclear power is and how reliable nuclear energy operating bodies are.

\section{Summary}

For this study, a questionnaire survey was conducted before and after the accident at the Fukushima Daiichi Nuclear Power Plant and the survey results were compared and analyzed to ascertain the impact that the accident had on public awareness and attitudes in China towards nuclear power generation.

This study found that the accident lowered the public's sense of safety towards the use of nuclear energy, had a negative impact on support for nuclear energy policies, and nuclear energy-related businesses were seen as less reliable after the accident.

Furthermore, the analysis of the differences in awareness between males and females due to the impact of the accident revealed that the percentage in favor of nuclear power ("Agree" and "Somewhat agree") is higher among males than females both before and after the accident. We found that there is a correlation between interest in and level of knowledge on nuclear energy.

Although many people provided ambiguous answers such as "Somewhat" and "Not much" in the survey before the accident, their agreement or disagreement with propositions in the survey after the accident were more definite. This is probably because public interest in and the level of knowledge on nuclear power generation increased in China due to the accident and this meant respondents were more willing to make unambiguous statements.

We believe that these survey results will be a valuable resource when considering nuclear energy policies in not only Japan after the accident but also internationally and when responding to public awareness of and attitudes to the use of nuclear power.

\section{References}

1) Annual Report on China and Japanese Chamber of Commerce and Industry, and China Economy and Japanese Companies 2011, No. 2, 13 (2011).

2) Yasuko Nagai, Chikio Hayashi, "Public Attitudes toward Nuclear Power Generation - Focusing on 
Measurement of Attitude Intensity -," INSS J, 6, 24-54, Institute of Nuclear Safety System, Incorporated (1999).

3) Atsuko Kitada, Chikio Hayashi, "Effects of the Criticality Accident at Tokai-mura on the Public's Attitude to Nuclear Power Generation," INSS J, 7, 25-44, Institute of Nuclear Safety System, Incorporated (2000).

4) Naoko Maruyama, Ryozo Yanagihara, Juji Misumi, et al., "Public Attitudes toward Nuclear Power Generation - Preliminary Study Focusing on Characteristics of the Attitudes of Men and Women -," INSS J, 3, 5-45, Institute of Nuclear Safety System, Incorporated (1996).

5) Tetsuji Shibata, Hiroaki Tomokiyo, Public Opinion on Nuclear Power Plants - The Transitions of Consciousness about Nuclear Power Seen in Opinion Surveys, published by ERC (1999).

6) Yoshihiko Shinoda, "Consideration on the Interaction between Society and Nuclear Technology," Trans. At. Energy Soc. Jpn. 6(2), 97-112 (2007).

7) Tomoko Tsuchiya, Motoko Kosugi, Yasuko Senda, et al., "Study of Public Understanding and Evaluation of Messages Concerning the Policy of "Pu Thermal Utilization" based on Results of Interview Survey," Trans. At. Energy Soc. Jpn. 7(3), 280-296 (2008). [in Japanese]

8) Yasuhiro Sakai, Masashi Tajima, Chen Yuling, "An empirical study in risk perception and social culture: Japan versus China,” The Hikone Ronso, The Economic Society of Shiga University, 380, 49-65 (2009).

\section{-Appendix questions and results of the public awareness survey on future energy use in China-}

(For the percentage figures given below, the first (left) represents the value before the accident, and the second (right) is the value after the accident.)

Question 1: Did your opinion change towards nuclear power generation in China after the earthquake disaster and accident at the Fukushima Daiichi Nuclear Power Plant?

Choose the one that applies to you.

(1) Changed $42.2 \%$

(2) Slightly changed $36.8 \%$

(3) Not changed $16.5 \%$

(4) I don't know $5.5 \%$

(This question was for after the accident only.)

Question 2: Are you interested in topics on energy issues?

Choose the one that applies to you.

(1) Very interested $35.6 \% \quad 49.2 \%$

(2) Somewhat interested $32.0 \% \quad 39.9 \%$

(3) Not very interested $22.4 \% \quad 6.1 \%$

(4) Not at all interested $10.0 \% \quad 7.4 \%$

Question 3: The Earth's energy resources are limited. What do you think about energy issues in China?

Choose the one that applies to you.

(1) Very serious $40.6 \% 47.7 \%$

(2) Somewhat serious $42.1 \% 38.6 \%$

(3) Not very serious $14.4 \% 9.8 \%$

(4) Not at all serious $2.9 \% 3.9 \%$

Question 4: Do you have basic knowledge on nuclear power? 
Choose the one that applies to you.

(1) Yes, I have. $11.4 \% 39.1 \%$

(2) Yes, I have a little. $22.0 \% 35.2 \%$

(3) No, I don't have much. $32.3 \% 20.1 \%$

(4) No, I don't have. $34.2 \% 5.7 \%$

Question 5: Are you interested in nuclear power?

Choose the one that applies to you.

(1) Very interested $21.5 \% 41.9 \%$

(2) Somewhat interested $37.1 \% 39.3 \%$

(3) Not very interested $32.5 \% \quad 15.3 \%$

(4) Not interested $8.9 \% 3.6 \%$

Question 6: Do you think uranium, fuel for nuclear power, is found in broader areas in the world including politically stable countries and therefore the supply of uranium is more stable compared to petroleum?

Choose the one that applies to you.

(1) Yes, I definitely think so. $28.3 \% 30.8 \%$

(2) Yes, I somewhat think so. $34.1 \% 30.4 \%$

(3) No, I generally don't think so. $26.0 \% 23.4 \%$

(4) No, I definitely don't think so. $11.6 \% \quad 15.4 \%$

Question 7: When thinking about our energy usage in the future, do you think nuclear energy is necessary?

Choose the one that applies to you.

(1) Yes, I definitely think so. $27.1 \% 37.1 \%$

(2) Yes, I somewhat think so. $47.5 \% 35.4 \%$

(3) No, I generally don't think so. $17.0 \% 20.0 \%$

(4) No, I definitely don't think so. $8.4 \% \quad 7.5 \%$

Question 8: Do you think it would be better for China to increase the number of nuclear power plants?

Choose the one that applies to you.

(1) Yes, I definitely think so. $22.2 \% 29.0 \%$

(2) Yes, I somewhat think so. $38.5 \% 28.2 \%$

(3) No, I generally don't think so. $32.9 \% \quad 25.0 \%$

(4) No, I don't think so. $6.4 \% \quad 17.9 \%$

Question 9: What's the first thing you think of when you hear the words "nuclear power"? Choose the one that applies to you.

(1) Nuclear weapons $27.1 \% \quad 30.7 \%$

(2) Nuclear reactor explosion $37.4 \% 26.2 \%$

(3) Radioactive contamination $22.1 \% 33.8 \%$

(4) New energy $13.4 \% 9.3 \%$

Question 10: Would you mind eating foods including vegetables and fish that come from the vicinity of a nuclear power plant?

Choose the one that applies to you.

(1) No, I would not mind at all. $28.5 \% 20.4 \%$

(2) No, I would not mind much. $32.2 \% \quad 21.4 \%$ 
(3) Yes, I would mind a little. $37.5 \% 40.9 \%$

(4) Yes, I would mind. $1.8 \% \quad 17.3 \%$

Question 11: What would you think, if the city/ town/ village you live in or a city/ town/ village nearby planned to establish a nuclear power plant?

Choose the one that applies to you.

(1) I would agree. $19.8 \% \quad 19.1 \%$

(2) I would somewhat agree. $23.5 \% 23.3 \%$

(3) I would somewhat disagree. $29.9 \% 29.4 \%$

(4) I would disagree. $16.8 \% 28.3 \%$

Question 12: Are you anxious about the methods used to dispose of high-level radioactive waste?

Choose the one that applies to you.

(1) Yes, I am anxious. $16.5 \% 26.8 \%$

(2) Yes, I am somewhat anxious. $22.9 \% 25.5 \%$

(3) No, I am not particularly anxious. $48.5 \% 27 \%$

(4) No, I am not anxious. $12.1 \% \quad 20.7 \%$

Question 13: What do you think about China's future nuclear power generation?

Choose the one that applies to you.

(1) Should be actively promoted $29.1 \% \quad 19.3 \%$

(2) Should be carefully promoted $36.4 \% \quad 40.9 \%$

(3) Status quo should be maintained $27.3 \% 24.4 \%$

(4) Should be discontinued as soon as possible $7.2 \% \quad 15.4 \%$

Question 14: Do you think Chinese nuclear energy operators are reliable?

Choose the one that applies to you.
(1) Reliable $22.5 \% \quad 20.0 \%$
(2) Somewhat reliable $44.3 \% 34.1 \%$
(3) Not very reliable $20.8 \% 26.3 \%$
(4) Not reliable at all $12.5 \% \quad 19.7 \%$

Question 15: Are you concerned that a major accident may happen at a nuclear power plant in China in the future?

Choose one that applies to you.

(1) Very concerned $19.8 \% 24.8 \%$

(2) Somewhat concerned $33.5 \% 37.8 \%$

(3) Not very concerned $29.9 \% \quad 26.1 \%$

(4) Not at all concerned $16.8 \% \quad 11.5 \%$

Question 16: How do you obtain information on Chinese nuclear power generation?

(1) From mass media including newspapers, magazines and TV and on the Internet $68.2 \% 80.97 \%$

(2) From families, friends and coworkers $12.8 \% \quad 16.45 \%$

(3) From specialists and specialized books $13.2 \% 21.29 \%$

(4) From civil activities and environmental groups $9.6 \% \quad 14.52 \%$

(5) From publications issued by local government and public facilities $8.7 \% \quad 11.29 \%$

(6) From the Internet $58.9 \% \quad 63.55 \%$ 
Question 17: Why are you anxious about nuclear power in China.

Choose all that apply to you.

(1) Major nuclear accidents like the one in Japan can also happen in China. $25.8 \% \quad 45 \%$

(2) Nuclear accidents have happened in the US, Russia and other countries. $35.2 \% \quad 37 \%$

(3) Nuclear power technologies are immature in China compared to other technologically developed countries. $\quad 30.9 \% \quad 34.7 \%$

(4) China does not have sufficient response measures if a nuclear accident occurs. $40.6 \% 41 \%$

(5) I don't know what safety regulations are implemented in China. $22.3 \% 31 \%$

(6) A series of scandals implicating electrical power suppliers including false reports and data falsification have been exposed. $25.4 \% 21.5 \%$

(7) Sufficient information disclosure and PR activities are not provided by electrical power suppliers on nuclear power. $32.9 \% 23.8 \%$

Question 18: These days, countries are promoting measures to secure energy in order to maintain economic growth and support societies. What do you think we should do to address energy issues?

Choose all that apply to you.

(1) Energy-saving technology should be developed. $25.3 \% \quad 43.6 \%$

(2) Development, implementation and use of nuclear power should be promoted. $28.2 \% \quad 37 \%$

(3) Solar energy should be developed. $18.7 \% \quad 53.6 \%$

(4) Cleaner fossil fuels should be developed and technologies should be developed and introduced to facilitate more efficient use of fossil fuels. $13.4 \% 50.1 \%$

(5) Use of non-fossil energy and transfer to gas energy $10.4 \% 29.2 \%$

(6) Diversifying imported energy suppliers and strengthening relationships with major energy producing countries. $14.5 \% 39.0 \%$

(7) Technologies should be developed to secure a stockpile of energy. $23.7 \% 39.3 \%$

Question 19: What do you consider is the most important factor when evaluating nuclear power energy?

(1) Whether nuclear power generation technology is necessary for society. $32.9 \% \quad 44.8 \%$

(2) Whether researchers and engineers are reliable. 29\% 34.2\%

(3) Whether energy can be produced safely at low cost by nuclear power generation. $44.8 \% 55.2 \%$

(4) Whether the energy-based society can manage nuclear power without causing problems. $36.8 \% 43 \%$

(6) Whether the country is capable of responding to an emergency issue when it happens. $37 \% \quad 58.3 \%$

(7) Whether nuclear power generation contributes to solving environmental issues. 47.4\%. 33\%

(9) Whether the government has already anticipated what kind of influences may happen in the future. $33.8 \% 47.4 \%$

(10) Whether influences on unborn babies and children have already been investigated. $28.6 \% 35.2 \%$

(11) Whether nuclear power generation technology is fully developed. $29.35 \% 22.9 \%$

(12) Whether information issued by nuclear power plants is correct. $18.8 \% 25.2 \%$ 


\title{
Effects of Ground Surface Decontamination on the Air Radiation Dose Rate
}

\section{-Results of a Decontamination Trial at a Playground Lot in a Fukushima Residential Area-}

\author{
Akihiro TAGAWA ${ }^{1, *}$ \\ ${ }^{1}$ Headquarters of Fukushima Partnership Operations, Japan Atomic Energy Agency, 2-2-2 Uchisaiwai-cho, \\ Chiyoda-ku. Tokyo 100-8577, Japan
}

\begin{abstract}
The Japan Atomic Energy Agency decontaminated schools, playgrounds, swimming pools, and houses in nonevacuated, less-contaminated areas in Fukushima for environmental restoration. A small, $150 \mathrm{~m}^{2}$ playground lot in the residential area was chosen for decontamination demonstration, which used routinely available tools and commodities to carry out the work. The surfaces of playground lot equipment, such as swings, slides, and horizontal iron bars, were completely decontaminated by brushing with water and/or detergent. Side gutters around the playground lot were cleaned by removing the mud and then brushed and washed with a high-pressure water jet ( $7 \mathrm{MPa}$ ). The air dose rate at the playground lot was dominated by radiation from the ground surface and adjacent surroundings, such as apartments and rice fields. Two or three centimeters of the surface soil contaminated with cesium was removed manually with shovels, hoes, and other gardening tools. This significantly reduced the average air dose rate of the entire playground lot from $1.5 \mu \mathrm{Sv} / \mathrm{h}$ before decontamination to $0.6 \mu \mathrm{Sv} / \mathrm{h}$. These results showed that ground surface decontamination can contribute measurably to the reduction in air dose rate in relatively small areas in residential areas.
\end{abstract}

KEYWORDS: Fukushima, environmental restoration, playground lot, decontamination, side gutter, high-pressure water washing, air dose rate, ground surface, cesium, gardening tools

\section{Foreword}

As a result of the nuclear accident resulting from the Great East Japan Earthquake on March 112011 , it is necessary to decontaminate radioactive materials dispersed into the environment. The Japan Atomic Energy Agency is engaged in the decontamination and environmental restoration of schools, playgrounds, swimming pools, and houses in the areas in Fukushima where the order to evacuate was not issued. In the future, the general public is expected to participate in the decontamination work without prior training in environments other than the evacuation-directive areas. Therefore, it is important to show that the spaces in which children

\footnotetext{
${ }^{*}$ Corresponding author, E-mail: tagawa.akihiro@jaea.go.jp

DOI : 10.15669 /fukushimainsights. Vol.4.78

(C) 2021 Atomic Energy Society of Japan. All rights reserved.

Originally published in Transactions of the Atomic Energy Society of Japan (ISSN 1347-2879), Vol. 11, No. 2, p.111-117

(2012) in Japanese. (Japanese version accepted: January 6, 2012)
} 
stay for long periods of time, such as schools and parks, can be decontaminated using basic tools that can be easily purchased at DIY stores. This trial was intended to test and evaluate a reduction in the air dose rate through the decontamination of a playground and playground equipment within an area of approximately $150 \mathrm{~m}^{2}$ located in a residential area where the order to evacuate was not issued.

\section{Decontamination Test}

\section{Introduction}

This test was intended to demonstrate the effects of ground surface decontamination on the air dose rate by decontaminating a playground with contaminated soil. A relatively small playground (approximately $150 \mathrm{~m}^{2}$ ) was chosen in order to confirm the localized contamination effect in an environment that is susceptible to background conditions in the surrounding area.

\section{Decontamination Test}

\section{(1) Decontamination plan}

Prior to decontamination, decontamination goals and plans needed to be established. Figure 1 provides a full view of the playground targeted for decontamination. The triangle-shaped playground is surrounded by apartments, houses, parking lots, and rice fields.

In order to establish the decontamination goal, the air dose rate was measured at three heights above the surface of the asphalt outside the playground $(1 \mathrm{~cm}, 50 \mathrm{~cm}$, and $100 \mathrm{~cm})$, without using a lead collimator (hereinafter referred to as "collimator"). The average values calculated from four measurement points were: $1.5 \mu \mathrm{Sv} / \mathrm{h}$ on the asphalt surface; $1 \mu \mathrm{Sv} / \mathrm{h}$ at a height of $50 \mathrm{~cm}$; and $0.9 \mu \mathrm{Sv} / \mathrm{h}$ at a height of $100 \mathrm{~cm}$. Since cesium on asphalt is dissolved in rainwater and can easily run off, the dose rate was lower than that of soil. Furthermore, because this relatively small playground is affected significantly by gamma rays from the surrounding area, an air dose rate of $0.9 \mu \mathrm{Sv} / \mathrm{h}$ (recorded $100 \mathrm{~cm}$ above the ground) was set as the decontamination goal.

Next, the contamination of all of the playground equipment was checked. The playground

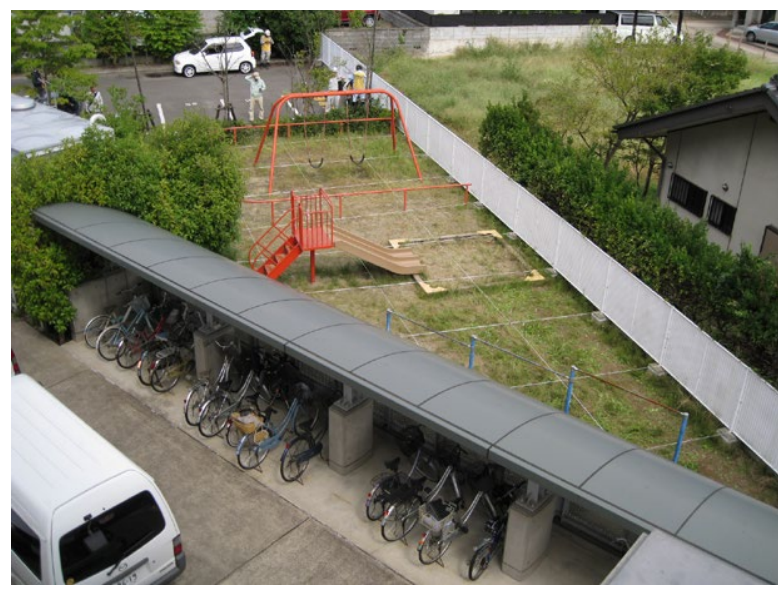

Figure 1 View of the playground before decontamination 
includes swings, a slide, a sandbox, equipment made of fiber reinforced plastic (FRP), and a horizontal bar, and plants are located at the stone-flagged entrance and around the gutters. Both concrete and iron grating covers are placed over the gutters, and the soil has been left unweeded.

At the time the test was carried out, Fukushima City made a recommendation to remove the top $5 \mathrm{~cm}$ of soil ${ }^{1)}$ and dig $1.5-\mathrm{m}$ deep burial holes (to be covered with $0.5 \mathrm{~m}$ of soil) ${ }^{1)}$. The demonstration aimed to remove a certain thickness of surface soil based on the measured radiation dose, but also to minimize the amount of material that had to be removed. Therefore, it was determined during the planning phase that the top $5 \mathrm{~cm}$ of soil would be removed from the entire playground area (approximately $150 \mathrm{~m}^{2}$ ) and that a burial hole would be dug that could contain the removed soil. The depth of the burial hole was designed so that $10.5 \mathrm{~m}^{3}$ of soil could be overlaid with a soil cover $0.5 \mathrm{~m}$ thick, based on the following formula: $150 \mathrm{~m}^{2} \times 5 \mathrm{~cm} \times$ loose soil volume factor. Since it was impossible to dig the hole by hand, heavy machinery was used to excavate a portion of planted area, where plants were pulled out. Concerning decontamination work in residential areas, it is important to demonstrate a method that the general public can implement. Therefore, except for the measurement instruments, we used tools that can be purchased from DIY stores, such as a high-pressure water sprayer and bamboo winnow. In addition, two working areas were created so that the work could proceed efficiently without the risk of re-contamination. Figure 2 shows the layout of the playground.

The decontamination was conducted in the order of playground equipment, planted areas, soil, and gutters, and ground leveling was performed last using Soilex ${ }^{2)}$, which generates less fugitive sand as additional soil. Considering the drainage function of the playground, surface compaction was performed during ground levelling so that the ground sloped toward the

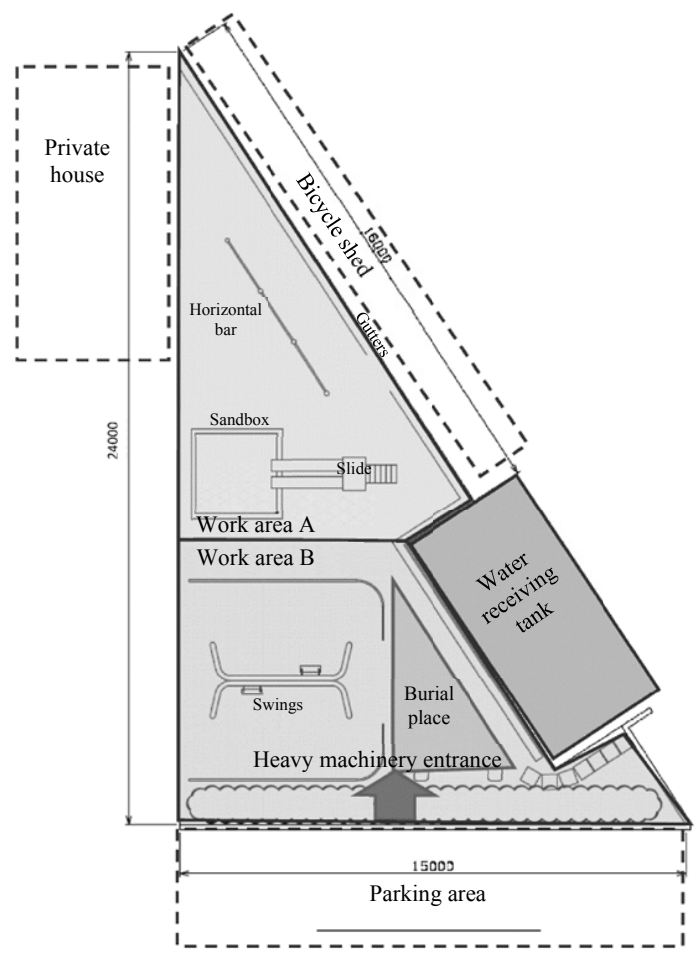

Figure 2 Layout of the playground lot 
gutters. Concerning the playground equipment, the elimination of loose contamination (which was not fixed firmly by paint, for example) was tested.

\section{(2) Radiation measurement method}

(a) Air dose rate

The air dose rate was measured for 30 seconds based on a time constant of $10 \mathrm{~s}$ using a NaI scintillation survey meter (Aloka, TCS-172) with a calibration constant of 1.0. The average of five measured values was recorded as the measurement value. The measurements were performed at the soil surface $(1 \mathrm{~cm})$, and at $50 \mathrm{~cm}$ and $100 \mathrm{~cm}$ above the ground.

When using the lead collimator for measurement on the surface, it was placed with the probe covered with a 7-mm-thick lead sheet (equivalent to a half-value layer of cesium (Cs) 137).

(b) Surface contamination

Surface contamination was measured at a distance of $1 \mathrm{~cm}$ from the measured object, using a GM counter tube (Aloka, TG133) with a counting efficiency of $43 \%$. When the background measurement was conducted, a 7-mm-thick lead sheet was placed between the measured object and the GM counter tube so that the detector would be hidden from the measured object.

When implementing the direct smear method, a filter paper used for the smears was placed inside a building with relatively low background radiation, enclosed with a lead wool mat $(760 \mathrm{~mm} \times 240 \mathrm{~mm}$, with a lead equivalent of $10 \mathrm{~mm})$, to create a background condition of around $100 \mathrm{cpm}$.

(c) Measurement before decontamination

We measured the air dose rate before decontamination. First, we divided the playground into a grid with each block measuring $2 \mathrm{~m} \times 2 \mathrm{~m}$. At the 43 intersection points, we measured the air dose rate at the three sample heights $(1 \mathrm{~cm}, 50 \mathrm{~cm}$, and $100 \mathrm{~cm}$ above the ground)

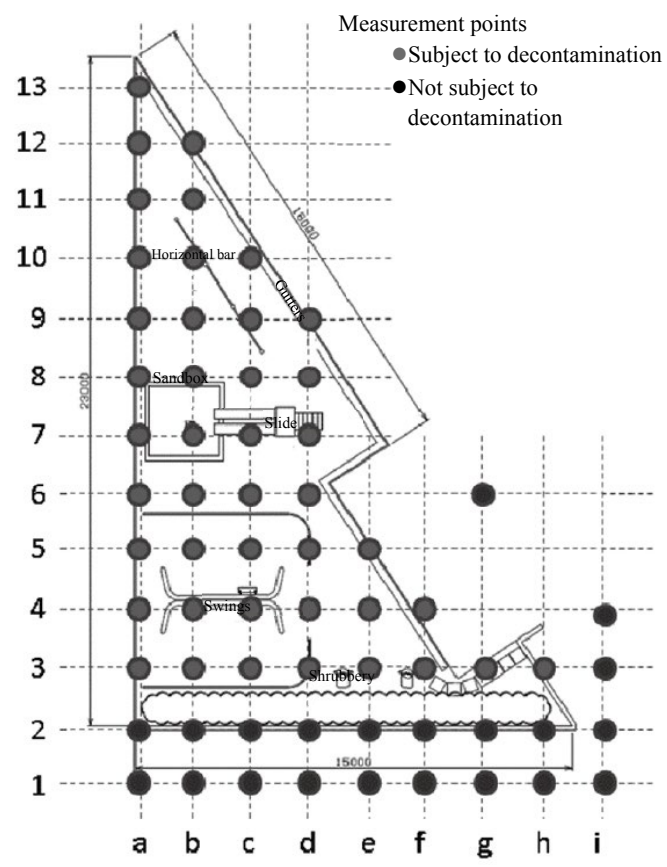

Figure 3 Air dose rate measurement points in the playground lot 
using the NaI scintillation survey meter. Figure 3 shows the positions of the measurement grid. Detailed measurement data are shown below in comparison with the values after decontamination; the average values were $2.4 \mu \mathrm{Sv} / \mathrm{h}$ at the soil surface, $1.8 \mu \mathrm{Sv} / \mathrm{h}$ at a height of $50 \mathrm{~cm}$, and $1.5 \mu \mathrm{Sv} / \mathrm{h}$ at a height of $100 \mathrm{~cm}$.

\section{(3) Decontamination method tested}

(a) Decontamination of horizontal bar

Before decontamination, measurements were conducted according to the smear method using smear filter papers. After this, the horizontal bar was washed using water, neutral detergent, orange oil-based detergent (orange oil acts as a surfactant), and a brush (car-washing brush with Pacquin and polypropylene bristles). Measurement was then repeated according to the smear method after decontamination. Rust was filed away with abrasive paper (\#80).

At the time of decontamination, the parts of the horizontal bar were washed in the order from higher to lower (while paying attention to the direction of water flow) and wiped with paper towels. However, we did not disassemble the fixing joints.

(b) Decontamination of the slide and swings

As described later, since washing with water and wiping produced a sufficient cleaning effect for the horizontal bar, the slide and swings were washed and brushed without detergent and eleven points were evaluated using the smear method. The same measurement method was used as described in Section II-2-(3)-(a) (Figure 4).

We decontaminated the fixing joints of the slide using a commercially available high-pressure water sprayer (water pressure $=7 \mathrm{MPa}$, straight nozzle) without disassembling.

(c) Decontamination of sandbox

As the area of the sandbox into which water flows from the slide was highly contaminated, and the frame of the sandbox was made of wood, decontamination of the sandbox was performed after dividing it into the following three areas: (a) an area with a high degree of

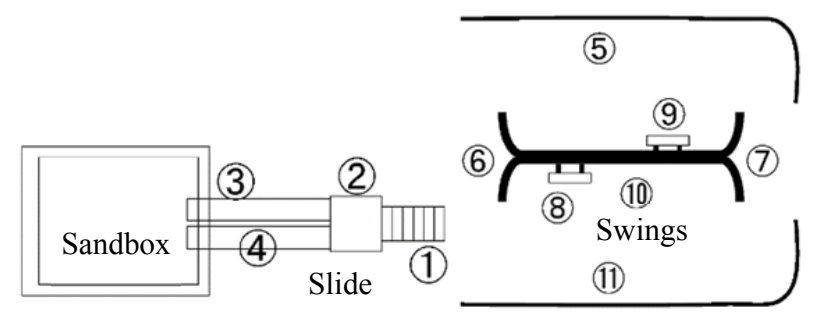

Figure 4 Air dose rate measurement points at and around the slide
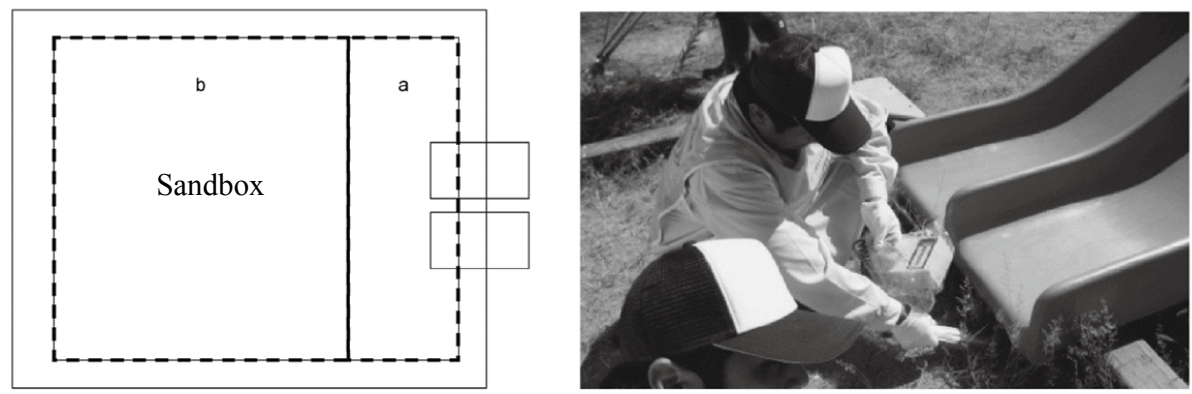

Figure 5 Air dose rate measurement points at the sandbox 
contamination, (b) an area with a low degree of contamination, and (c) the wooden frame (Figure 5). In each area, after eliminating garbage and weeds, and removing the top 1, 3, 5 , and $10 \mathrm{~cm}$ of the soil according to the topsoil removal method, we measured the air dose rate at the surface $(1 \mathrm{~cm})$ using a collimator and also measured the surface contamination density using a GM counter tube. For area (b), we performed the measurement at five points, positioned evenly, and evaluated the average value.

For the wooden frame, we implemented and evaluated four types of decontamination: water washing with a brush; wiping using a detergent containing orange oil; sanding with sandpaper; and sanding with an electric sander.

(d) Spot decontamination of soil

A circular area $100 \mathrm{~cm}$ in diameter was targeted for decontamination because it was less susceptible to the scattering effect of gamma radiation from the periphery of the site. After removing any garbage and weeds, the soil within the circle was then decontaminated according to the topsoil removal method. We removed the top $1 \mathrm{~cm}, 3 \mathrm{~cm}$, and $5 \mathrm{~cm}$ of soil and measured the air dose rate at heights of $1 \mathrm{~cm}, 50 \mathrm{~cm}$, and $100 \mathrm{~cm}$ above the surface using a GM counter tube.

(e) Decontamination of surface soil

As described later, since removing the soil up to $3 \mathrm{~cm}$ in depth was found to have a sufficient decontamination effect, decontamination was implemented by removing the top $3 \mathrm{~cm}$ of soil from the entire playground area. In this decontamination work, care was taken to remove the soil thinly and evenly using bamboo winnows and square shovels, which are used as farm tools. After decontamination, the air dose rate was measured at three heights $(1 \mathrm{~cm}, 50 \mathrm{~cm}$, and $100 \mathrm{~cm}$ ) at the same measurement points as shown in Figure 3. For measurements taken at the soil surface $(1 \mathrm{~cm})$, a collimator was also used as well as a GM counter tube.

(f) Decontamination of gutters

For the gutters (made of concrete and with a pit width of $18 \mathrm{~cm}$ and a pit depth of $17 \mathrm{~cm}$ ), garbage and sludge were first removed using tongs and shovels, respectively. The gutters were then washed with water and a brush (using scrubbing brushes with red fern bristles) before using a high-pressure washer (from a distance of $10 \mathrm{~cm}$ ). Since the gutters were approximately $30 \mathrm{~cm}$ deep, the air dose rate was measured at a height of $1 \mathrm{~cm}$ from the bottom.

(g) Decontamination of gutter covers

The gutters had both iron gratings and concrete covers $(25 \mathrm{~cm}$ wide $\times 60 \mathrm{~cm}$ long $\times$ $3.5 \mathrm{~cm}$ thick). Since the iron covers could be decontaminated by washing with water and brushes and the high-pressure washer, we conducted the test on the concrete covers. The decontamination effect was compared between water washing with brushes (scrubbing) and high-pressure washing.

(h) Shielding of buried soil

In order to check the shielding effect of the soil cover at the burial site, we measured the air dose rates $(1 \mathrm{~cm}, 50 \mathrm{~cm}$, and $100 \mathrm{~cm}$ above the surface $)$ with soil cover thicknesses of $10 \mathrm{~cm}, 20 \mathrm{~cm}, 30 \mathrm{~cm}$, and $35 \mathrm{~cm}$.

\section{(4) Test results}

(a) Decontamination of horizontal bar

Table 1 shows the results of decontamination of the horizontal bar. Each measured value (net) was calculated by deducting the background value from the raw (gross) measured value. As a result, we found that loose contamination (which was not fixed firmly by paint, for example) could be eliminated by water washing.

(b) Decontamination of slide and swings 
Table 1 Decontamination effects for the horizontal bar

\begin{tabular}{ccccc} 
& & & & (Unit: cpm) \\
\hline Measurement point & $\begin{array}{c}\text { Water } \\
\text { washing }\end{array}$ & $\begin{array}{c}\text { Mild } \\
\text { detergent }\end{array}$ & Sandpaper & $\begin{array}{c}\text { Detergent } \\
\text { containing } \\
\text { orange oil }\end{array}$ \\
\hline $\begin{array}{c}\text { Before } \\
\text { decontamination } \\
\text { value } \\
\text { (Gross) } \\
\text { Measured } \\
\text { value } \\
\text { (Net) }\end{array}$ & 100 & 80 & 130 & 270 \\
\hline $\begin{array}{c}\text { Measured } \\
\text { value } \\
\text { (Gross) } \\
\text { decontamination } \\
\text { Measured } \\
\text { value } \\
\text { (Net) }\end{array}$ & 100 & 100 & 100 & 100 \\
\hline Decline rate $(\%)$ & 0 & 0 & 0 & 0 \\
\hline
\end{tabular}

Table 2 Decontamination effects for the slide

\begin{tabular}{|c|c|c|c|c|c|}
\hline \multirow{2}{*}{\multicolumn{2}{|c|}{ Measurement point }} & & & \multicolumn{2}{|c|}{ (Unit:cpn } \\
\hline & & (1) & (2) & (3) & (4) \\
\hline \multirow{2}{*}{$\begin{array}{c}\text { Before } \\
\text { decontamination }\end{array}$} & $\begin{array}{l}\text { Measured } \\
\text { value } \\
\text { (Gross) }\end{array}$ & 180 & 200 & 190 & 200 \\
\hline & $\begin{array}{l}\text { Measured } \\
\text { value } \\
\text { (Net) }\end{array}$ & 80 & 100 & 90 & 70 \\
\hline \multirow{2}{*}{$\begin{array}{c}\text { After } \\
\text { decontamination }\end{array}$} & $\begin{array}{l}\text { Measured } \\
\text { value } \\
\text { (Gross) }\end{array}$ & 100 & 100 & 100 & 100 \\
\hline & $\begin{array}{l}\text { Measured } \\
\text { value } \\
\text { (Net) }\end{array}$ & 0 & 0 & 0 & 0 \\
\hline \multicolumn{2}{|c|}{ Decline rate $(\%)$} & 100 & 100 & 100 & 100 \\
\hline
\end{tabular}

Table 3 Decontamination effects for swings

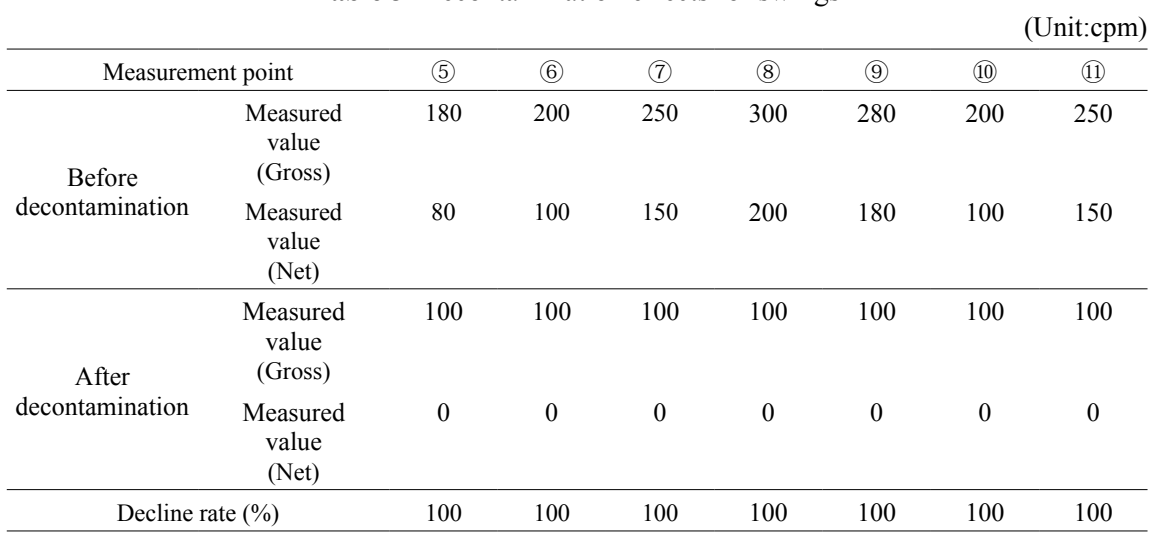

Table 2 and Table 3 show the results of decontamination of the slide and swings, respectively. Based on these data, it was found that loose contamination could be eliminated by water washing.

(c) Decontamination of sandbox

Table 4 shows the result of decontamination of the sandbox. By removing the top $5 \mathrm{~cm}$ of soil, areas a and b were decontaminated to $350 \mathrm{cpm}$ and $110 \mathrm{cpm}$, respectively. After 
removing the top $10 \mathrm{~cm}$ of soil, both areas were decontaminated to $30 \mathrm{cpm} ; 70-90 \%$ of the contamination was contained in the top $1 \mathrm{~cm}$ of soil. Table 5 shows the results of decontamination of the wooden frame. Sanding but not washing was required to achieve a sufficient decontamination effect.

(d) Effect of spot decontamination of soil

Table 6 shows the results of the measurements conducted at the center of a circle with a diameter of $1 \mathrm{~m}$. Although this showed $100 \%$ decontamination at the surface $(1 \mathrm{~cm}$ above the ground), the air dose rate at a height of $100 \mathrm{~cm}$ above the ground was reduced by only $11 \%$. This is because the greater the height, the more gamma rays from the periphery of the site reached the detector. This means that performing spot decontamination alone contributes little to the reduction of air dose rate in areas of widespread contamination.

(e) Effect of surface decontamination of soil

Table 7 shows the average, maximum, and minimum values for all measurement points. Not only was the soil surface decontaminated as in the case of spot decontamination, but the air dose rate also declined more than it did following spot decontamination.

(f) Decontamination of gutters

Table 8 shows the results of decontamination of the gutters. Although the elimination of

Table 4 Decontamination effects for the sandbox

\begin{tabular}{|c|c|c|c|c|c|c|c|c|}
\hline \multirow[b]{3}{*}{$\begin{array}{c}\text { Decontamination } \\
\text { item }\end{array}$} & \multicolumn{4}{|c|}{ Area a } & \multicolumn{4}{|c|}{ Area $b$ (average of 5 points) } \\
\hline & \multicolumn{2}{|c|}{ Air dose rate } & \multicolumn{2}{|c|}{ Surface contamination } & \multicolumn{2}{|c|}{ Air dose rate } & \multicolumn{2}{|c|}{ Surface contamination } \\
\hline & $\begin{array}{c}\text { Measured } \\
\text { value } \\
(\mu \mathrm{Sv} / \mathrm{h})\end{array}$ & $\begin{array}{l}\text { Decline } \\
\text { rate } \\
(\%)\end{array}$ & $\begin{array}{l}\text { Measured } \\
\text { value } \\
\text { Net }(\mathrm{cpm})\end{array}$ & $\begin{array}{l}\text { Decline } \\
\text { rate } \\
(\%)\end{array}$ & $\begin{array}{c}\text { Measured } \\
\text { value } \\
(\mu \mathrm{Sv} / \mathrm{h})\end{array}$ & $\begin{array}{l}\text { Decline } \\
\text { rate } \\
(\%)\end{array}$ & $\begin{array}{c}\text { Measured } \\
\text { value } \\
\text { Net }(\mathrm{cpm})\end{array}$ & $\begin{array}{c}\text { Decline rate } \\
(\%)\end{array}$ \\
\hline $\begin{array}{c}\text { Before } \\
\text { decontamination }\end{array}$ & 14 & - & 9,500 & - & $2.4 \pm 0.4$ & - & $842 \pm 207$ & - \\
\hline $\begin{array}{c}\text { Elimination of } \\
\text { garbage and weeds }\end{array}$ & 13 & 7 & - & - & $2.3 \pm 0.3$ & 5 & - & - \\
\hline $\begin{array}{l}\text { Removal of the top } \\
1 \mathrm{~cm} \text { of soil }\end{array}$ & 3.6 & 74 & 7,700 & 18.9 & $2.1 \pm 0.3$ & 11 & $900 \pm 89$ & -6.9 \\
\hline $\begin{array}{l}\text { Removal of the top } \\
3 \mathrm{~cm} \text { of soil }\end{array}$ & 2.1 & 85 & 1,000 & 89.5 & $1.3 \pm 0.3$ & 45 & $1,000 \pm 0$ & -18.8 \\
\hline $\begin{array}{l}\text { Removal of the top } \\
5 \mathrm{~cm} \text { of soil }\end{array}$ & 0.8 & 94 & 350 & 96.3 & $0.6 \pm 0.0$ & 74 & $110 \pm 34$ & 86.9 \\
\hline $\begin{array}{l}\text { Removal of the top } \\
10 \mathrm{~cm} \text { of soil }\end{array}$ & 0.5 & 96 & 30 & 99.7 & $0.4 \pm 0.1$ & 83 & $30 \pm 85$ & 96.4 \\
\hline
\end{tabular}

Table 5 Decontamination effects for sandbox wood frame

\begin{tabular}{|c|c|c|c|c|c|}
\hline & & & & & Unit:cpn \\
\hline \multicolumn{2}{|l|}{ Measurement point } & $\begin{array}{c}\text { Water } \\
\text { washing }\end{array}$ & $\begin{array}{l}\text { Detergent } \\
\text { containing } \\
\text { orange oil }\end{array}$ & $\begin{array}{l}\text { Sanding } \\
\text { with } \\
\text { sandpaper }\end{array}$ & $\begin{array}{l}\text { Sanding } \\
\text { with } \\
\text { electric } \\
\text { sander } \\
\end{array}$ \\
\hline \multirow{2}{*}{$\begin{array}{c}\text { Before } \\
\text { decontamination }\end{array}$} & $\begin{array}{l}\text { Measured } \\
\text { value } \\
\text { (Gross) }\end{array}$ & 4,500 & 4,500 & 4,500 & 4,500 \\
\hline & $\begin{array}{l}\text { Measured } \\
\text { value } \\
\text { (Net) }\end{array}$ & 4,200 & 4,200 & 4,200 & 4,200 \\
\hline \multirow{2}{*}{$\begin{array}{c}\text { After } \\
\text { decontamination }\end{array}$} & $\begin{array}{l}\text { Measured } \\
\text { value } \\
\text { (Gross) }\end{array}$ & 3,000 & 2,100 & 1,250 & 330 \\
\hline & $\begin{array}{l}\text { Measured } \\
\text { value } \\
\text { (Net) }\end{array}$ & 2,700 & 1,800 & 950 & 30 \\
\hline \multicolumn{2}{|c|}{ Decline rate $(\%)$} & 36 & 57 & 77 & 99 \\
\hline
\end{tabular}


sludge, water washing with brushes, and high-pressure washing had a high decontamination effect, the high-pressure washing showed the greatest effect.

(g) Decontamination of gutter cover

It was found that high-pressure washing produced a greater decontamination effect than water washing with brushes (Table 9).

(h) Results of shielding the buried soil

In order to check the shielding effect of soil cover at the burial site, air dose rates were

Table 6 Spot decontamination effects on soil

\begin{tabular}{|c|c|c|c|c|c|c|c|c|}
\hline \multirow[b]{3}{*}{ Decontamination item } & \multicolumn{4}{|c|}{ Surface $(1 \mathrm{~cm})$} & \multirow{2}{*}{\multicolumn{2}{|c|}{$\begin{array}{c}50 \mathrm{~cm} \\
\text { Air dose rate }\end{array}$}} & \multirow{2}{*}{\multicolumn{2}{|c|}{$\begin{array}{l}100 \mathrm{~cm} \\
\text { Surface contamination }\end{array}$}} \\
\hline & Air do & e rate & Surface col & amination & & & & \\
\hline & $\begin{array}{c}\text { Measured } \\
\text { value } \\
(\mu \mathrm{Sv} / \mathrm{h})\end{array}$ & $\begin{array}{c}\text { Decline } \\
\text { rate } \\
(\%)\end{array}$ & $\begin{array}{l}\text { Measured } \\
\text { value } \\
\text { Net }(\mathrm{cpm})\end{array}$ & $\begin{array}{c}\text { Decline } \\
\text { rate } \\
(\%)\end{array}$ & $\begin{array}{l}\text { Measured } \\
\text { value } \\
(\mu \mathrm{Sv} / \mathrm{h})\end{array}$ & $\begin{array}{c}\text { Decline } \\
\text { rate } \\
(\%)\end{array}$ & $\begin{array}{c}\text { Measured } \\
\text { value } \\
(\mu \mathrm{Sv} / \mathrm{h})\end{array}$ & $\begin{array}{c}\text { Decline } \\
\text { rate } \\
(\%)\end{array}$ \\
\hline Before decontamination & 2.6 & - & 1,400 & - & 2.1 & - & 1.8 & - \\
\hline $\begin{array}{c}\text { Elimination of garbage } \\
\text { and weeds }\end{array}$ & 2.5 & 4 & 1,400 & 0 & 2.1 & 0 & 1.7 & 6 \\
\hline $\begin{array}{c}\text { Removal of the top } 1 \mathrm{~cm} \\
\text { of soil }\end{array}$ & 1.3 & 50 & 400 & 71 & 1.9 & 10 & 1.7 & 6 \\
\hline $\begin{array}{c}\text { Removal of the top } 3 \mathrm{~cm} \\
\text { of soil }\end{array}$ & 0.9 & 65 & 150 & 89 & 1.7 & 19 & 1.6 & 11 \\
\hline $\begin{array}{l}\text { Removal of the top } 5 \mathrm{~cm} \\
\text { of soil }\end{array}$ & 0.8 & 69 & 0 & 100 & 1.7 & 19 & 1.6 & 11 \\
\hline
\end{tabular}

Table 7 Decontamination effects for ground surface soil

\begin{tabular}{|c|c|c|c|c|c|c|}
\hline \multirow{2}{*}{$\begin{array}{l}\text { Measurement } \\
\text { point }\end{array}$} & \multicolumn{3}{|c|}{ Before decontamination $(\mu \mathrm{Sv} / \mathrm{h})$} & \multicolumn{3}{|c|}{ After decontamination $(\mu \mathrm{Sv} / \mathrm{h})$} \\
\hline & Average $^{\text {a) }}$ & Max. & Min. & Average $^{\text {a) }}$ & Max. & Min. \\
\hline $100 \mathrm{~cm}$ & 1.5 & 1.9 & 1.0 & 0.6 & 1.1 & 0.4 \\
\hline $50 \mathrm{~cm}$ & 1.8 & 2.4 & 1.2 & 0.6 & 1.0 & 0.3 \\
\hline $1 \mathrm{~cm}$ & 2.4 & 5 & 1.6 & 0.4 & 1.1 & 0.3 \\
\hline (Collimate) & - & - & - & 0.2 & 0.4 & 0.1 \\
\hline $\begin{array}{c}\text { Surface } \\
\text { contamination } \\
(\mathrm{Net})(\mathrm{cpm})\end{array}$ & - & 一 & - & 18 & 550 & 0 \\
\hline
\end{tabular}

a) Measurements were taken at 43 intersection points generated by dividing the playground lot into a $2 \mathrm{~m} \times 2 \mathrm{~m}$ grid.

Table 8 Decontamination effects for gutters

\begin{tabular}{cccc}
\hline $\begin{array}{c}\text { Decontamination } \\
\text { method }\end{array}$ & $\begin{array}{c}\text { Before } \\
\text { decontamination } \\
(\mu \mathrm{Sv} / \mathrm{h})\end{array}$ & $\begin{array}{c}\text { After } \\
\text { decontamination } \\
(\mu \mathrm{Sv} / \mathrm{h})\end{array}$ & $\begin{array}{c}\text { Decline rate } \\
(\%)\end{array}$ \\
\hline Elimination of garbage & 2.28 & 3.32 & -46 \\
Elimination of sludge & 2.11 & 0.68 & 68 \\
Water washing with brushes & 1.4 & 0.43 & 69 \\
High-pressure washing & 2.75 & 0.74 & 73 \\
\hline
\end{tabular}

Table 9 Decontamination effects for gutter covers

\begin{tabular}{cccc}
\hline Decontamination method & $\begin{array}{c}\text { Before } \\
\text { decontamination } \\
(\mathrm{cpm})\end{array}$ & $\begin{array}{c}\text { After } \\
\text { decontamination } \\
(\mathrm{cpm})\end{array}$ & $\begin{array}{c}\text { Decline rate } \\
(\%)\end{array}$ \\
\hline $\begin{array}{c}\text { Water washing } \\
\text { with brushes } \\
\text { High-pressure } \\
\text { washing }\end{array}$ & 6,000 & 2,500 & 58 \\
\hline
\end{tabular}


Table 10 Shielding effect for removed topsoil

\begin{tabular}{cccccc}
\hline $\begin{array}{c}\text { Measurement } \\
\text { height }\end{array}$ & $0 \mathrm{~cm}$ & $10 \mathrm{~cm}$ & $20 \mathrm{~cm}$ & $30 \mathrm{~cm}$ & $35 \mathrm{~cm}$ \\
\hline $100 \mathrm{~cm}$ & 1.2 & 0.6 & 0.5 & 0.5 & 0.5 \\
$50 \mathrm{~cm}$ & 1.4 & 0.6 & 0.5 & 0.5 & 0.4 \\
$1 \mathrm{~cm}$ & 2.3 & 0.6 & 0.5 & 0.4 & 0.4 \\
$1 \mathrm{~cm}$ & - & - & - & - & 0.1 \\
\hline
\end{tabular}

measured at $50 \mathrm{~cm}$ and $100 \mathrm{~cm}$ above the ground at soil thicknesses of $10 \mathrm{~cm}, 20 \mathrm{~cm}, 30 \mathrm{~cm}$ and $35 \mathrm{~cm}$. The measurements were evaluated based on the average values measured at five points. Table 10 shows the measurement results.

\section{Evaluation of Decontamination}

In the case of loose contamination of the playground equipment, water washing produced a sufficient decontamination effect. However, the joint fixings of the playground equipment also need to be washed thoroughly and this involves disassembly and reassembly. Contamination adhering to the equipment also needs to be eliminated by removing the paint and replacing parts.

In the case of the sandbox, it was sufficiently decontaminated by removing the top $10 \mathrm{~cm}$ of sand. However, given that children dig and play in the sand, the landowner requested that the sand be replaced to a depth of $20 \mathrm{~cm}$.

Concerning the wooden frame of the sandbox, the decontamination using an electric sander resulted in the highest removal of radioactive materials. However, it is important to use dust collectors to prevent scattering of contaminated wood dust. Wearing a mask is also required to prevent inhalation of the contaminated wood dust.

Spot decontamination of the soil was found to be effective in removing contaminated soil, but to have very little effect in terms of decreasing the air dose rate. In addition, removing the top $3 \mathrm{~cm}$ of soil removed approximately $90 \%$ of the contamination. This accords with the fact that $95 \%$ of radioactive cesium was distributed in the soil up to a depth of $2.5 \mathrm{~cm}^{3)}$. In addition, there was a $2-3 \mathrm{~cm}$ layer generated by weed mulch on the top of the playground's soil. Therefore, removing this layer produced a sufficient decontamination effect.

The surface decontamination of the soil reduced the air dose rate to below $0.9 \mu \mathrm{Sv} / \mathrm{h}$, which had been set as the target value. Since a lower air dose rate was achieved when using a collimator, it would appear that the air dose rate was increased by radiation from the periphery of the site rather than from the playground itself. When a collimator was used, the average dose rate at a height of $1 \mathrm{~cm}$ was $0.2 \mu \mathrm{Sv} / \mathrm{h}$. On the other hand, the average air dose rate at a height of $100 \mathrm{~cm}$ was $0.6 \mu \mathrm{Sv} / \mathrm{h}$. This difference of $0.4 \mu \mathrm{Sv} / \mathrm{h}$ is considered to result from the influence of nearby buildings. On the other hand, the average air dose rate at a height of $100 \mathrm{~cm}$ was $1.5 \mu \mathrm{Sv} / \mathrm{h}$ before decontamination but declined by $0.9 \mu \mathrm{Sv} / \mathrm{h}$ after the topsoil was removed. From this, it was found that contamination from the soil surface contributed to more than half of the dose rate measured in the air at a height of $100 \mathrm{~cm}$. It was also found that air dose rates were effectively reduced by decontaminating the soil directly beneath the surface. The dose rate at which decontamination is required at present is $0.23 \mu \mathrm{Sv} / \mathrm{h}^{4)}$, which was calculated based on the annual additional radiation dose of $1 \mathrm{mSv}$. Since the average surface dose rate of soil is $0.2 \mu \mathrm{Sv} / \mathrm{h}$, the air dose rate is expected to be closer to $0.2 \mu \mathrm{Sv} / \mathrm{h}$ as the decontaminated area 
increases.

On the other hand, the dose rate of the gutters increased in spite of removing the garbage. This is considered to be the result of contaminated sludge being moved to the measurement site, although the measurements were taken in the same place before and after the garbage was removed. In addition, washing with brushes achieved the same level of decontamination as high-pressure washing.

The concrete covers were highly contaminated. Although high-pressure washing had a high decontamination effect, the contamination level could not be reduced to below $1,000 \mathrm{cpm}$. This means that radioactive cesium gained entry into the concrete joints. Therefore, a method that enables these joints to be washed is required in order to decontaminate the concrete completely. In this test, we used a 7-MPa high-pressure water sprayer equipped with a straight nozzle, and confirmed that high-pressure washing was effective to a certain degree. Although the effect of a device equipped with a rotary nozzle (which can produce higher pressure) needs to be tested for surface decontamination, such devices may be higher in cost than those easily purchased at DIY stores. Therefore, this is an issue for further study.

Concerning the shielding effect of the buried soil, it was found that a thickness of $30 \mathrm{~cm}$ could shield approximately $80 \%$ of the radiation dose rate. Since approximately $3 \mathrm{~cm}$ of topsoil was removed, there was no need to dig a $10-\mathrm{m}^{3}$ hole as originally planned; covering the soil to a thickness of approximately $0.3 \mathrm{~m}$ was sufficient to produce a superior shielding effect.

Finally, the following points were learned as a result of the decontamination tests, although more data need to be collected in the future:

First, a method for decontaminating the wooden frame as well as the rubber steps of the slide was also tested. For this, a mold-removing agent containing hypochlorous acid was used in order to remove cesium together with the dirt on the surface. This method produced decontamination effects but resulted in the bleaching of the wooden frame. In the case of the rubber steps, the dirt was removed by the foam that was generated when the detergent was sprayed on. There is a best moment to wipe off the detergent foam before the dirt adhered again, although it was not possible to conduct a test to verify this.

Second, combining an electrical sander with a dust collector expanded the scope of the decontamination. A field test of effective and efficient decontamination using general-purpose cleaning devices is expected before full-scale decontamination commences.

Figure 6 shows a view of the playground after decontamination. This shows how the

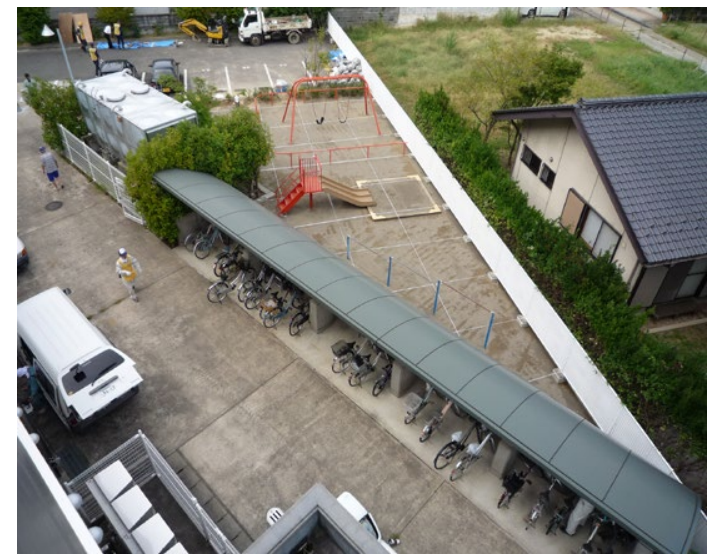

Figure 6 View of the playground after decontamination 
playground was cleaned in comparison to its condition before decontamination as shown in Figure 1

\section{Conclusions}

The loose contamination on the playground equipment was sufficiently eliminated by washing with water. Soil can be decontaminated by removing approximately $3 \mathrm{~cm}$ of topsoil. Although the gutters were decontaminated using water washing with brushes and high-pressure washing, there is an issue concerning the elimination of radioactive materials that have gained entry into concrete joints. On the whole, this demonstration has shown that implementing surface decontamination can reduce air dose rates at a height of $100 \mathrm{~cm}$, and surface decontamination was shown to be more effective at reducing air dose rates than spot decontamination.

\section{Afterword}

In this test, various decontamination methods were trialed for the purpose of checking the effect of surface decontamination on air dose rates for a playground with an area of $150 \mathrm{~m}^{2}$. Loose contamination on the playground equipment was shown to be removable by washing with water and brushes. For soil, implementing surface decontamination by removing approximately $3 \mathrm{~cm}$ of topsoil lowered the air dose rate at a height of $100 \mathrm{~cm}$ to $0.3 \mu \mathrm{Sv} / \mathrm{h}$ below the original target of $0.9 \mu \mathrm{Sv} / \mathrm{h}$. For the gutters and the concrete gutter covers, high-pressure washing at a pressure of $7 \mathrm{MPa}$ proved to be effective to a certain degree, but eliminating contamination in the concrete joints proved to be problematic. When placing a cover of soil on material removed during decontamination, a thickness of $30 \mathrm{~cm}$ was found to be effective in shielding approximately $80 \%$ of the radiation.

The data collected demonstrate that implementing surface decontamination can be more effective at reducing air dose rates than implementing spot decontamination in hot spots.

The results of this test, conducted in accordance with the decontamination instructions issued by the Fukushima Prefecture ${ }^{5}$, are summarized in a 17-minute video. The video is posted on the Japan Atomic Energy Agency website ${ }^{6}$ and is also utilized in decontamination seminars held by the Fukushima Prefecture.

We are very grateful to Fukushima Prefecture and Fukushima City for their supporting in helping us to implement this test and evaluation.

\section{References}

1) Fukushima City website: http://www.city.fukushima.fukushima.jp/. [in Japanese]

2) Highclay Corporation website: http: // www.highclay.co.jp /products/index.html. [in Japanese]

3) Agriculture, Forestry and Fisheries Research Council, Radioactive material removal technologies (decontamination technology) of agricultural soil are explained in [Appendix 4], http://www.s.affrc.go.jp/ docs/press/pdf/110914-10.pdf, 2011.9.14. [in Japanese]

4) The Ministry of the Environment, 1st Safety Assessment Committee / Environment Recovery Assessment Committee Appendix 2 Concept of Additional Exposure Dose of $1 \mathrm{mSv} /$ year, http://www.env. go.jp/jishin/rmp/conf/g01-mat4.pdf, 2011.10.10. [in Japanese]

5) Disaster Control Headquarters of Fukushima Prefecture, "Guidance on measures to reduce radiation dose in living areas," http://www.pref.fukushima.jp/j/tebiki0715.pdf, 2011.7.15. [in Japanese]

6) Japan Atomic Energy Agency, “About Decontamination Work,” http://www.jaea.go.jp/fukushima/josenvtr.html. [in Japanese] 


\title{
Rapid Communication
}

\section{Effect of Gamma Ray Irradiation on Deoxygenation by Hydrazine in Artificial Seawater}

\author{
Takafumi MOTOOKA $^{1, *}$, Tomonori SATO $^{1}$ and Masahiro YAMAMOTO ${ }^{1}$ \\ ${ }^{1}$ Nuclear Science and Engineering Directorate, Japan Atomic Energy Agency, 2-4 Shirane, Shirakata, Tokai-mura, Naka-gun, \\ Ibaraki 319-1195, Japan
}

\begin{abstract}
At the spent nuclear fuel pools in the Fukushima Daiichi Nuclear Power Plant, hydrazine has been added to reduce dissolved oxygen in the pool water containing salts. The reduction behavior of dissolved oxygen in seawater with hydrazine in the presence of radiation is unknown. The effect of gamma ray irradiation on deoxygenation by hydrazine in artificial seawater was investigated at room temperature. We placed the artificial seawater with a small amount of hydrazine under gamma ray irradiation at dose rates of $0.3-7.5 \mathrm{kGy} / \mathrm{h}$. The concentration of dissolved oxygen in the solutions was measured before and after the irradiation. The concentration of dissolved oxygen hardly decreased in the absence of gamma radiation in a few hours, whereas it markedly decreased in the presence of gamma radiation. The concentration of dissolved oxygen decreased with irradiation time. At this moment, hydrazine concentration decreased more than twice the dissolved oxygen concentration. This shows that some gamma radiolysis products of hydrazine act as deoxidizers. The concentration of dissolved oxygen in artificial seawater could be decreased by the addition of a small amount of hydrazine in the presence of gamma radiation at room temperature.
\end{abstract}

KEYWORDS: gamma ray, irradiation, solution, hydrazine, radiolysis, dissolved oxygen

\section{Introduction}

In the Great East Japan Earthquake, a station blackout occurred following the earthquake and subsequent tsunami at the Fukushima Daiichi Nuclear Power Plant. Consequently, seawater was injected into the spent fuel pool of Unit 2 through Unit 4 for the purpose of cooling the spent fuel, with freshwater injection switched to later. Currently, to inhibit the corrosion of materials in the spent fuel pool, water containing hydrazine $\left(\mathrm{N}_{2} \mathrm{H}_{4}\right)$ is injected into the spent fuel pool. This is done to reduce the oxidizer, the dissolved oxygen (DO), contained in the water in the pool.

As of September 2011, the values listed in Table 1 were disclosed as the water quality of the pool for Unit 4 of the Fukushima Daiichi Nuclear Power Plant ${ }^{1)}$. The concentration of chloride ions $\left(\mathrm{Cl}^{-}\right)$was $997 \mathrm{ppm}$. The $\mathrm{N}_{2} \mathrm{H}_{4}$ concentration was $59 \mathrm{ppm}$. The DO concentration

\footnotetext{
* Corresponding author, E-mail: takafumi.motoka@jaea.go.jp

DOI : 10.15669 /fukushimainsights. Vol.4.90

(C) 2021 Atomic Energy Society of Japan. All rights reserved.

Originally published in Transactions of the Atomic Energy Society of Japan (ISSN 1347-2879), Vol. 11, No. 4, p.249-254

(2012) in Japanese. (Japanese version accepted: July 18, 2012)
} 
Table 1 Concentration and activity of key aqueous solutes in the Unit 4 spent fuel pool in the Fukushima Daiichi Nuclear Power Plant

\begin{tabular}{lccccc}
\hline $\begin{array}{l}\text { Date } \\
\text { time }\end{array}$ & $\begin{array}{c}\mathrm{Cl}^{-} \\
\mathrm{ppm}\end{array}$ & $\begin{array}{c}\mathrm{N}_{2} \mathrm{H}_{4} \\
\mathrm{ppm}\end{array}$ & $\begin{array}{c}\mathrm{Cs}-137 \\
\mathrm{~Bq} / \mathrm{L}\end{array}$ & $\begin{array}{c}\mathrm{Cs}-134 \\
\mathrm{~Bq} / \mathrm{L}\end{array}$ & $\begin{array}{c}\mathrm{I}-131 \\
\mathrm{~Bq} / \mathrm{L}\end{array}$ \\
\hline $\begin{array}{l}2011.9 .2 \\
15: 00\end{array}$ & 997 & 59 & $3.1 \times 10^{4}$ & $2.2 \times 10^{4}$ & $\mathrm{ND}$ \\
\hline
\end{tabular}

ND: Not detected.

Table 2 Chemical composition of artificial seawater $\left(\mathrm{mol} / \mathrm{dm}^{3}\right)$

\begin{tabular}{ccccccccccc}
\hline $\mathrm{Na}^{+}$ & $\mathrm{Mg}^{2+}$ & $\mathrm{Ca}^{2+}$ & $\mathrm{K}^{+}$ & $\mathrm{Sr}^{2+}$ & $\mathrm{Cl}^{-}$ & $\mathrm{SO}_{4}{ }^{2-}$ & $\mathrm{HCO}_{3}{ }^{-}$ & $\mathrm{Br}^{-}$ & $\mathrm{BO}_{3}{ }^{3-}$ & $\mathrm{F}^{-}$ \\
0.478 & 0.0547 & 0.0104 & 0.0102 & 0.00016 & 0.560 & 0.0288 & 0.0024 & 0.00084 & 0.00045 & 0.00007 \\
\hline
\end{tabular}

is unknown because data are not provided. The spent fuel is stored in the spent fuel pool and the spent fuel radiates various radioactive products such as gamma rays. In the thermal power plant, and elsewhere, $\mathrm{N}_{2} \mathrm{H}_{4}$ is used as a deoxidizer for high temperature water, but the deoxidization effect is known to be limited in pure water at room temperature ${ }^{2)}$. When exposed to radiation, it is reported that $\mathrm{N}_{2} \mathrm{H}_{4}$ is degraded and the DO concentration is reduced by the reaction of its degradation products with $\mathrm{DO}^{3-6,8,9)}$. However, those are the results acquired with pure water, and the reaction behavior of $\mathrm{DO}$ and $\mathrm{N}_{2} \mathrm{H}_{4}$ is unclear in a system that contains seawater components, as in this case.

Hence, in this research, the influence of gamma rays on the reaction behavior of DO and $\mathrm{N}_{2} \mathrm{H}_{4}$ (deoxygenation) in artificial seawater was studied by irradiating pure water and artificial seawater containing an infinitesimal amount of $\mathrm{N}_{2} \mathrm{H}_{4}$ at room temperature with gamma rays, and then determining the quantity of $\mathrm{DO}$ and $\mathrm{N}_{2} \mathrm{H}_{4}$ in aqueous solution. In this paper, the comparison and study results are reported on the influences of gamma rays on the reaction behavior of $\mathrm{DO}$ and $\mathrm{N}_{2} \mathrm{H}_{4}$ in seawater and pure water.

\section{Experimental Methodology}

\section{Testing Solution}

Solutions of pure water and the artificial seawater Aquamarine (Yashima Pure Chemicals Co., Ltd.), with the addition of $10^{-3} \mathrm{~mol} / \mathrm{dm}^{3}$ of $\mathrm{N}_{2} \mathrm{H}_{4}$, were used for the test. The main components of artificial seawater are listed in Table 2. The chloride ion concentration was $0.560 \mathrm{~mol} / \mathrm{dm}^{3}$ (approximately 18,900 ppm). Special grade hydrazine 1 hydrate (purity $98.0 \%$ ) was used as $\mathrm{N}_{2} \mathrm{H}_{4}$. The $\mathrm{pH}$ of pure water and artificial seawater at $25^{\circ} \mathrm{C}$ with the addition of $10^{-3} \mathrm{~mol} / \mathrm{dm}^{3}$ of $\mathrm{N}_{2} \mathrm{H}_{4}$ was 9.3 .

\section{Gamma Ray Irradiation Test}

The gamma ray irradiation test was conducted in Irradiation Room 2 in Cobalt Building 1 of the Takasaki Advanced Radiation Research Institute of the Japan Atomic Energy Agency. The irradiation with gamma rays was started by pulling up the cobalt (Co-60) radiation source from the pool of the irradiation room and was stopped by pulling it down to the bottom of the pool. The average energy of emitted gamma rays from the cobalt radiation source was 1.25 $\mathrm{MeV}$. The absorption dose rate was controlled by varying the distance of the sample and the 


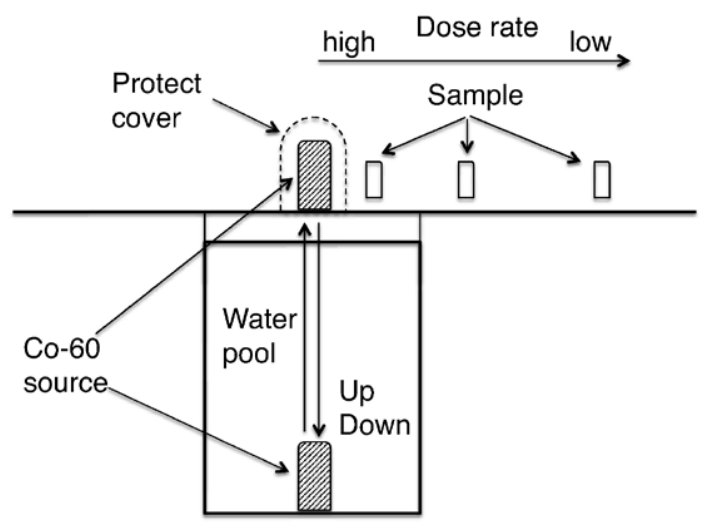

Figure 1 Schematic layout of Co-60 gamma-ray source and samples

Co- 60 radiation source, and the absorbed dose quantity by varying the duration of irradiation. Figure 1 shows a schematic of the positional relationship of the Co-60 radiation source and the samples. In this test, the gamma rays irradiated the samples at room temperature within the absorption dose rate range of $0.3-7.5 \mathrm{kGy} / \mathrm{h}$. The dose rates were within the dose rate distribution $\left(1-10^{6} \mathrm{~Gy} / \mathrm{h}\right)$ of the spent fuel pool of the Fukushima Daiichi Nuclear Power Plant as analyzed and evaluated by us ${ }^{10)}$.

The samples for irradiation were pure water and artificial seawater with added $\mathrm{N}_{2} \mathrm{H}_{4}$, contained in a $25 \mathrm{~mL}$ test tube with no air gap and tightly plugged. The absorption dose rate at the position of irradiation was evaluated using an alanine dose meter (Aminogray: Hitachi Cable, Ltd.) The measurement range of the Aminogray was 10-10 5 G.

\section{Measurement of DO and $\mathrm{N}_{2} \mathrm{H}_{4}$ Concentrations}

The concentrations of DO and $\mathrm{N}_{2} \mathrm{H}_{4}$ were measured before and after irradiation with gamma rays. The indigo carmine method was mainly used to determine the DO. The measurement range of this method was $3-34 \times 10^{-5} \mathrm{~mol} / \mathrm{dm}^{3}(1.0-11.0 \mathrm{ppm})$. When the determined value was one ppm and lower, a fluorescent DO concentration meter with a measurement range of $0-156 \times 10^{-5} \mathrm{~mol} / \mathrm{dm}^{3}(0-50.0 \mathrm{ppm})$ was used. For the determination of $\mathrm{N}_{2} \mathrm{H}_{4}$, the p-dimethylaminobenzaldehyde method was used. For adjusting the concentration within the measurement range when using this method, the sample was diluted with pure water. The measurement range was $2-234 \times 10^{-7} \mathrm{~mol} / \mathrm{dm}^{3}(0.005-0.750 \mathrm{ppm})$.

\section{Results and Discussion}

\section{Deoxygenation Behavior in Pure Water without Irradiation}

After tightly plugging a test tube containing $25 \mathrm{~mL}$ of pure water and $\mathrm{N}_{2} \mathrm{H}_{4}$, the temperature was maintained at a specified level for eight hours using an aluminum block constant-temperature tank (at $20,30,40,50$ and $65^{\circ} \mathrm{C}$ ). As a point of information, the initial DO concentration of the test fluid was in a supersaturation status higher than atmospheric concentration. The DO concentrations measured at specified times are shown in Figure 2. The DO concentration showed a slight decrease after eight hours at $20^{\circ} \mathrm{C}$. The decrease at $30^{\circ} \mathrm{C}$ was several ppm. At 


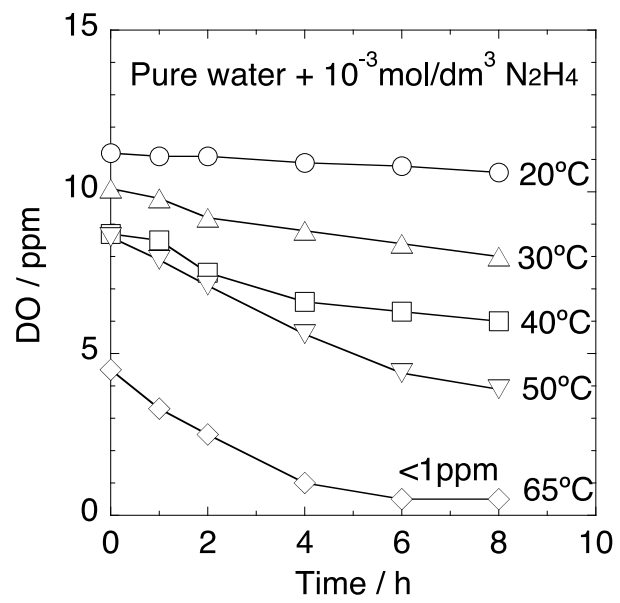

Figure 2 Decreases in dissolved oxygen in pure water with $10^{-3} \mathrm{~mol} / \mathrm{dm}^{3}$ hydrazine at different temperatures in the absence of gamma radiation

$65^{\circ} \mathrm{C}$, the $\mathrm{DO}$ concentration decreased to under one ppm in eight hours.

Thus, DO reduction by $\mathrm{N}_{2} \mathrm{H}_{4}$ was small at room temperatures of $20-30^{\circ} \mathrm{C}$, but increased as the temperature rose.

\section{Deoxygenation Behavior under Gamma Ray Irradiation}

Pure water and artificial seawater, with the addition of $\mathrm{N}_{2} \mathrm{H}_{4}$, were irradiated with gamma rays at $7.5 \mathrm{kGy} / \mathrm{h}$ for one hour. As before, the initial DO concentration of the test fluid was in supersaturation, higher than air saturation. The DO concentration before and after irradiation is shown in Figure 3. For comparison, the DO concentration of a test fluid sample left for one hour with no irradiation $(0 \mathrm{~Gy} / \mathrm{h})$ is indicated in the same chart. In the non-irradiated condition, the DO concentration after one hour was almost the same as before testing for both pure water and artificial seawater. After the gamma irradiation, both pure water and artificial seawater showed a DO concentration of one ppm or less. The reduction in DO concentration (deoxygenation) was clearly the result of the gamma irradiation. Incidentally, the DO concentration of artificial seawater before irradiation was lower than that in the pure water by approximately one ppm due to the salt content of the artificial seawater. The saturated DO concentration of a $3.5 \% \mathrm{NaCl}$ solution, with a chloride ion concentration equivalent to the artificial seawater at $20^{\circ} \mathrm{C}$, was $7.2 \mathrm{ppm}^{11)}$ and was lower than the saturated DO concentration of $9.1 \mathrm{ppm}$ of pure water at the same temperature.

In this test, the temperature of the test water before and after the gamma irradiation was $25-30^{\circ} \mathrm{C}$. Considering the temperature dependence of deoxygenation behavior in pure water shown in Figure 2, under the test conditions of this test, the influence of temperature on deoxygenation was small, while the influence of gamma irradiation was large.

\section{Relation of Absorption Dose Rate and Deoxygenation Behavior}

Pure water and artificial seawater, with the addition of $\mathrm{N}_{2} \mathrm{H}_{4}$, were irradiated with gamma rays at room temperature for one hour at different absorption dose rates within the range $0.3-7.5 \mathrm{kGy} / \mathrm{h}$. The DO concentration after irradiation is shown in Figure 4. For comparison, 


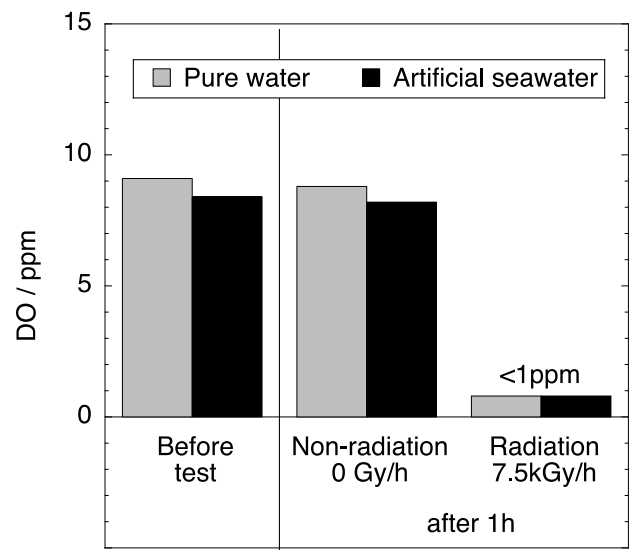

Figure 3 Concentrations of dissolved oxygen in pure water and artificial seawater containing $10^{-3} \mathrm{~mol} / \mathrm{dm}^{3}$ hydrazine at initial and after 1 hour without and with gamma ray irradiation at room temperature

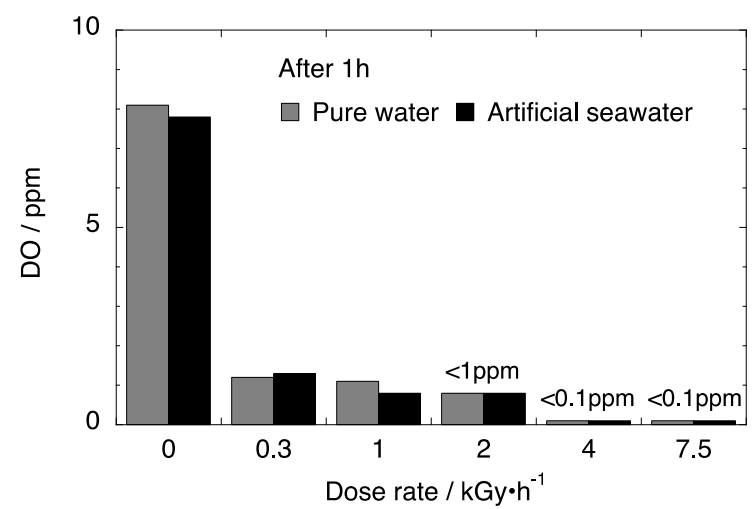

Figure 4 Comparison of concentration of dissolved oxygen with and without gamma ray irradiation at room temperature at different dose rates after 1 hour

the DO concentration of a test fluid sample left for one hour with no irradiation $(0 \mathrm{~Gy} / \mathrm{h})$ is indicated in the same chart. For both pure water and artificial seawater, the DO concentration was one ppm or less for absorption dose rates of $2 \mathrm{kGy} / \mathrm{h}$ and above. The deoxygenation took place within the absorption dose rates in this test.

\section{Time-Dependent Behavior of Dissolved Oxygen Concentration and Hydrazine Under Gamma Irradiation}

Pure water and artificial seawater, with the addition of $\mathrm{N}_{2} \mathrm{H}_{4}$, were irradiated with gamma rays at $1 \mathrm{kGy} / \mathrm{h}$ at room temperature. The time-dependent $\mathrm{DO}$ and $\mathrm{N}_{2} \mathrm{H}_{4}$ concentrations are shown in Figures 5 and 6, respectively. For both pure water and artificial seawater, the DO concentration was one ppm or less as the duration of irradiation increased. Only 10 minutes was required for pure water, and 30 minutes for artificial seawater, in order to decrease to one ppm or less. The concentration of $\mathrm{N}_{2} \mathrm{H}_{4}$ continuously decreased with increasing duration of irradiation. Both pure water and artificial seawater showed a decrease from the initial 
concentration of $10^{-3} \mathrm{~mol} / \mathrm{dm}^{3}$ (approximately $33 \mathrm{ppm}$ ) to approximately $0.25 \times 10^{-3} \mathrm{~mol} / \mathrm{dm}^{3}$ (approximately $8 \mathrm{ppm}$ ) from an irradiating duration of 60 minutes. Within the irradiating dose rate range $(0.3-7.5 \mathrm{kGy} / \mathrm{h})$ in this test, the $\mathrm{N}_{2} \mathrm{H}_{4}$ concentration showed a continuous decrease with increasing irradiating duration.

Usually, in the absence of irradiation, the chemical reaction of $\mathrm{N}_{2} \mathrm{H}_{4}$ and $\mathrm{O}_{2}$ is expressed in Eq. $(1)^{2)}$ :

$$
\mathrm{N}_{2} \mathrm{H}_{4}+\mathrm{O}_{2} \rightarrow \mathrm{N}_{2}+2 \mathrm{H}_{2} \mathrm{O}
$$

$\mathrm{N}_{2} \mathrm{H}_{4}$ and $\mathrm{O}_{2}$ react with equivalent weights, where the chemical reaction of Eq. (1) progresses with equal concentrations because the molecular masses are equal. However, with irradiation by gamma rays at room temperature, the concentration of $\mathrm{N}_{2} \mathrm{H}_{4}$ and $\mathrm{O}_{2}$ did not change with equivalent weights $(1: 1)$ as shown in Figures 5 and 6. With an irradiation duration of 60 minutes, the reduction in quantity of $\mathrm{N}_{2} \mathrm{H}_{4}$ was approximately $25 \mathrm{ppm}$ while the reduction in quantity of DO was approximately $10 \mathrm{ppm}$.

Based on this, it is considered that $\mathrm{N}_{2} \mathrm{H}_{4}$ transferred to another chemical species (radiolysis product) and reacted with DO. Ershov et al ${ }^{6}$. and Buxton et al ${ }^{8)}$. explain the deoxygenating reaction in pure water under the copresence of DO and $\mathrm{N}_{2} \mathrm{H}_{4}$ as follows, based on the measurement of pulse radiolysis:

$$
\cdot \mathrm{N}_{2} \mathrm{H}_{3}+\mathrm{O}_{2} \rightarrow \mathrm{O}_{2} \cdot{ }^{-}+\mathrm{N}_{2} \mathrm{H}_{2}+\mathrm{H}^{+}
$$

Here, $\cdot \mathrm{N}_{2} \mathrm{H}_{3}$ is a radiolysis of $\mathrm{N}_{2} \mathrm{H}_{4}$ and is formed by the reaction with $\mathrm{OH} \cdot$, a radiolysis of water ${ }^{6,8)}$.

$$
\mathrm{N}_{2} \mathrm{H}_{4}+\mathrm{OH} \cdot \rightarrow \cdot \mathrm{N}_{2} \mathrm{H}_{3}+\mathrm{H}_{2} \mathrm{O}
$$

On the other hand, for the radiolytic behavior of $\mathrm{N}_{2} \mathrm{H}_{4}$ in pure water with the absence of DO $(\mathrm{DO}=0 \mathrm{ppm})$, several researchers ${ }^{3,7}$ suggest the reaction of Eq. (4):

$$
\begin{aligned}
& 2 \mathrm{~N}_{2} \mathrm{H}_{4} \rightarrow 2 \mathrm{NH}_{3}+\mathrm{N}_{2}+\mathrm{H}_{2} \\
& \mathrm{NH}_{3}+\mathrm{H}_{2} \mathrm{O} \rightarrow \mathrm{NH}_{4}^{+}+\mathrm{OH}^{-}
\end{aligned}
$$

Using a test fluid from which DO is completely removed by irradiating with gamma rays for 60 minutes, the determination of ammonium ions $\mathrm{NH}_{4}{ }^{+}$was conducted using the indophenol blue method, and several ppm were detected. Because $\mathrm{NH}_{4}{ }^{+}$is considered to be formed by the dissolution of $\mathrm{NH}_{3}$ in water, it is further considered that deoxygenation progressed with the reaction of Eq. (2), and after deoxygenation, the reactions in Eq. (4) and Eq. (5) followed.

\section{G-Values of Dissolved Oxygen and Hydrazine Under Gamma Irradiation}

As shown in Figures 5 and 6, the concentration of DO and $\mathrm{N}_{2} \mathrm{H}_{4}$ decreased with increasing duration of gamma irradiation. Hence, the relation between gamma ray dose and the reduction in quantity of DO and $\mathrm{N}_{2} \mathrm{H}_{4}$ was quantified with regard to the G-value. From Figures 5 and 6 , the $G$-values $G\left(-\mathrm{O}_{2}\right)$ and $G\left(-\mathrm{N}_{2} \mathrm{H}_{4}\right)$, with regard to the reduction in DO and $\mathrm{N}_{2} \mathrm{H}_{4}$, were obtained at every measured time. $G\left(-\mathrm{O}_{2}\right)$ and $G\left(-\mathrm{N}_{2} \mathrm{H}_{4}\right)$ were summarized with regard to measured time and absorption dose in Table 3. The values were $G\left(-\mathrm{O}_{2}\right)=18.0 \times 10^{-7} \mathrm{~mol} \mathrm{~J}^{-1}$ and $G\left(-\mathrm{N}_{2} \mathrm{H}_{4}\right)=$ $7.6-21.5 \times 10^{-7} \mathrm{~mol} \mathrm{~J}^{-1}$ for pure water of $\mathrm{pH}=9.3$, and $G\left(-\mathrm{O}_{2}\right)=5.8-11.0 \times 10^{-7} \mathrm{~mol} \mathrm{~J}^{-1}$ and $G\left(-\mathrm{N}_{2} \mathrm{H}_{4}\right)=8.3-24.9 \times 10^{-7} \mathrm{~mol} \mathrm{~J}^{-1}$ for artificial seawater of $\mathrm{pH}=9.3 . G\left(-\mathrm{N}_{2} \mathrm{H}_{4}\right)$ of seawater 


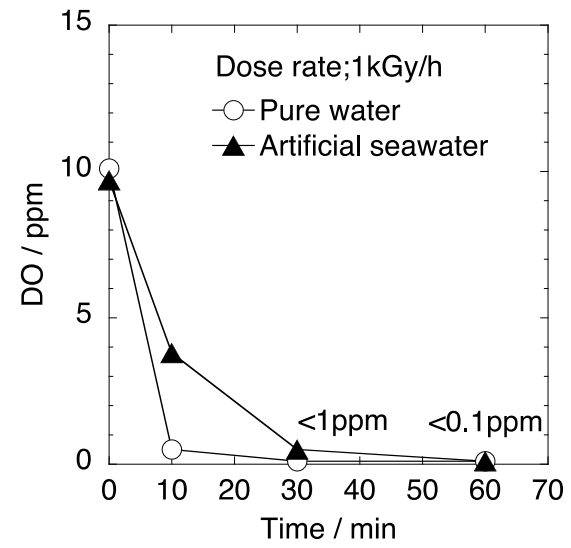

Figure 5 Changes of dissolved oxygen concentration for pure water and artificial seawater at room temperature at a dose rate of $1 \mathrm{kGy} / \mathrm{h}$

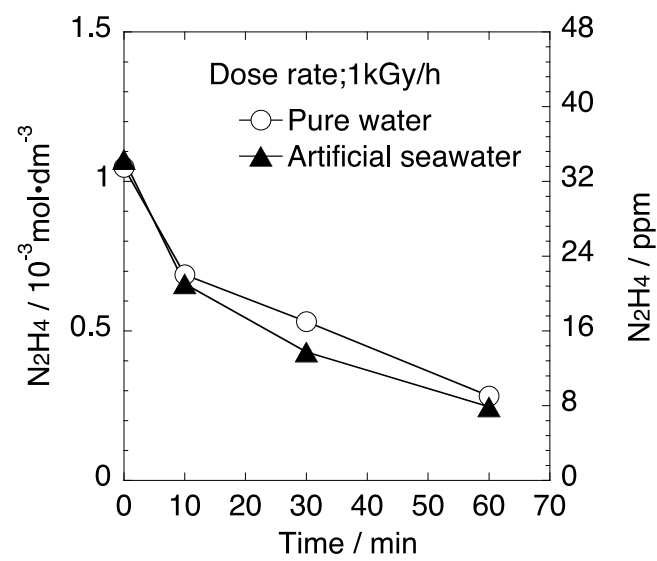

Figure 6 Change in hydrazine concentration in pure water and artificial seawater at room temperature at a dose rate of $1 \mathrm{kGy} / \mathrm{h}$

Table $3 G\left(-\mathrm{N}_{2} \mathrm{H}_{4}\right)$ and $G\left(-\mathrm{O}_{2}\right)$ values in pure water (a) and artificial seawater (b) with $10^{-3} \mathrm{~mol} / \mathrm{dm}^{3}$ hydrazine at a dose rate of $1 \mathrm{kGy} / \mathrm{h}$ (unit: $10^{-7} \mathrm{~mol} \mathrm{~J}{ }^{-1}$ )

\begin{tabular}{lccccc}
\hline \multirow{2}{*}{$\begin{array}{c}\text { Time } \\
\text { dose }\end{array}$} & \multicolumn{2}{c}{ (a) pure water } & & \multicolumn{2}{c}{ (b) artificial seawater } \\
\cline { 2 - 3 } \cline { 5 - 6 } & $G\left(-\mathrm{N}_{2} \mathrm{H}_{4}\right)$ & $G\left(-\mathrm{O}_{2}\right)$ & & $G\left(-\mathrm{N}_{2} \mathrm{H}_{4}\right)$ & $G\left(-\mathrm{O}_{2}\right)$ \\
\hline $0-10 \mathrm{~min}$ & 21.5 & 18.0 & & 24.9 & 11.0 \\
$167 \mathrm{~Gy}$ & 10.3 & & & & \\
$0-30 \mathrm{~min}$ & $13^{\mathrm{a}}$ & - & & 12.9 & 5.8 \\
$500 \mathrm{~Gy}$ & 7.6 & - & & 8.3 & - \\
$0-60 \mathrm{~min}$ & & & & \\
$1,000 \mathrm{~Gy}$ & & & & \\
\hline
\end{tabular}

a) from Ref. 3), 5).

was almost the same as that of pure water, and $G\left(-\mathrm{O}_{2}\right)$ was smaller.

The $G$-values obtained in this study were compared with those reported by other researchers. Lefort et al. ${ }^{3)}$ obtained $G\left(-\mathrm{N}_{2} \mathrm{H}_{4}\right)$ in pure water of $\left[\mathrm{N}_{2} \mathrm{H}_{4}\right]=10^{-3} \mathrm{~mol} \mathrm{dm}^{-3}$ and $\mathrm{pH}=9.5$ with an absorption dose rate of $0.293 \mathrm{~Gy} \mathrm{~s}^{-1}(1 \mathrm{kGy} / \mathrm{h})$, and obtained the result of $G\left(-\mathrm{N}_{2} \mathrm{H}_{4}\right)=$ 
$13 \times 10^{-7} \mathrm{~mol} \mathrm{~J}^{-1}$ with an absorption dose of $500 \mathrm{~Gy}$. Furthermore, Ershov et al ${ }^{5}$. obtained $G\left(-\mathrm{N}_{2} \mathrm{H}_{4}\right)$ in pure water of $\left[\mathrm{N}_{2} \mathrm{H}_{4}\right]=10^{-3} \mathrm{~mol} \mathrm{dm}^{-3}$ and $\mathrm{pH}=9.5$ with an absorption dose rate of $0.113 \mathrm{~Gy} \mathrm{~s}^{-1}$ (approximately $400 \mathrm{~Gy} / \mathrm{h}$ ), and obtained a result of $G\left(-\mathrm{N}_{2} \mathrm{H}_{4}\right)=13 \times 10^{-7} \mathrm{~mol} \mathrm{~J}^{-1}$ with an absorption dose of $500 \mathrm{~Gy}$. The $G$-value in this study in pure water of $500 \mathrm{~Gy}$ was $G\left(-\mathrm{N}_{2} \mathrm{H}_{4}\right)=10.3 \times 10^{-7} \mathrm{~mol} \mathrm{~J}{ }^{-1}$, not a large difference from the previous reports. Incidentally, Buxton et al ${ }^{9)}$. reported that a reason for large $G\left(-\mathrm{N}_{2} \mathrm{H}_{4}\right)$ values is that a chain reaction is involved in the reduction of $\mathrm{N}_{2} \mathrm{H}_{4}$.

Regarding the $G\left(-\mathrm{O}_{2}\right)$ value, nothing was reported by Lefort et al. ${ }^{3)}$ or Ershov et al. ${ }^{5}$.

\section{Influence of Seawater Components Under Gamma Irradiation}

The hydrated electron $\mathrm{e}^{-}$aq is known to react rapidly with $\mathrm{O}_{2}{ }^{12)}$. On the other hand, the reaction of $\mathrm{e}^{-}$aq and $\mathrm{N}_{2} \mathrm{H}_{4}$ is slow ${ }^{13)}$. The reaction and reaction rate constant $k$ are indicated in Eqs. (6) and (7) respectively:

$$
\begin{aligned}
& \mathrm{e}^{-}{ }_{\text {aq }}+\mathrm{O}_{2} \rightarrow \mathrm{O}_{2}^{-}+\mathrm{H}_{2} \mathrm{O} \\
& k_{(6)}=1.9 \times 10^{10} \mathrm{dm}^{3} \cdot \mathrm{mol}^{-1} \cdot \mathrm{s}^{-1} \\
& \mathrm{e}^{-}{ }_{\text {aq }}+\mathrm{N}_{2} \mathrm{H}_{4} \rightarrow \mathrm{H}^{+}+\cdot \mathrm{N}_{2} \mathrm{H}_{4}+\mathrm{OH}^{-} \\
& k_{(7)}=2.3 \times 10^{6} \mathrm{dm}^{3} \cdot \mathrm{mol}^{-1} \cdot \mathrm{s}^{-1}
\end{aligned}
$$

Based on these factors, there is a possibility of reduction in DO regardless of the presence of $\mathrm{N}_{2} \mathrm{H}_{4}$. Furthermore, because the reduction in DO was slower in artificial seawater than in pure water under gamma irradiation, it was hypothesized that a component of the artificial seawater was influencing the reduction of DO. This possibility was investigated.

First, an investigation was made to determine whether the DO is reduced regardless of the presence of $\mathrm{N}_{2} \mathrm{H}_{4}$ by irradiating pure water and artificial seawater with gamma rays without the addition of $\mathrm{N}_{2} \mathrm{H}_{4}$. The DO concentrations are shown in Figure 7 where the gamma irradiation is for one hour at a dosage rate of $7.5 \mathrm{kGy} / \mathrm{h}$. The data with the addition of $\mathrm{N}_{2} \mathrm{H}_{4}$ is also included in Figure 3 for the references.

The reduction in DO concentration was approximately one ppm for pure water. No reduction in DO concentration was observed in the artificial seawater. In contrast, under identical irradiation, the DO concentration was one ppm or less and the reduction exceeded seven ppm for both pure water and artificial seawater with the addition of $\mathrm{N}_{2} \mathrm{H}_{4}$. Based on these results, it has been shown that the DO concentration decreases with gamma irradiation without the addition of $\mathrm{N}_{2} \mathrm{H}_{4}$, but the effect of added $\mathrm{N}_{2} \mathrm{H}_{4}$ was stronger on the reduction of DO under irradiation with gamma rays. Furthermore, it was confirmed that the DO then decreased according to a mechanism different from that in pure water because a component of seawater is present in the artificial seawater.

Next, the influence of chloride ions, highly abundant in seawater, was studied. In pure water, the irradiation facilitated deoxygenation by $\mathrm{N}_{2} \mathrm{H}_{4}$ is considered to progress via the following

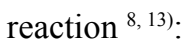

$$
\begin{aligned}
& \mathrm{N}_{2} \mathrm{H}_{4}+\mathrm{OH} \cdot \rightarrow \cdot \mathrm{N}_{2} \mathrm{H}_{3}+\mathrm{H}_{2} \mathrm{O} \\
& k_{(8)}=1.4 \times 10^{10} \mathrm{dm}^{3} \cdot \mathrm{mol}^{-1} \cdot \mathrm{s}^{-1} \\
& \cdot \mathrm{N}_{2} \mathrm{H}_{3}+\mathrm{O}_{2} \rightarrow \mathrm{O}_{2} \cdot{ }^{-}+\mathrm{N}_{2} \mathrm{H}_{2}+\mathrm{H}^{+} \\
& k_{(9)}=3.8 \times 10^{8} \mathrm{dm}^{3} \cdot \mathrm{mol}^{-1} \cdot \mathrm{s}^{-1}
\end{aligned}
$$


The chemical species that influences the reduction of DO is considered to be the hydroxyl radical $\mathrm{OH} \cdot$.

Because of the high chloride ion concentration in artificial seawater, it is presumed that the reaction in Eqs. (8) and (9) is blocked by the reaction of $\mathrm{OH} \cdot$ and $\mathrm{Cl}^{-}$according to the reaction of Eq. (10) ${ }^{14)}$.

$$
\begin{aligned}
& \mathrm{Cl}^{-}+\mathrm{OH} \cdot \rightarrow \mathrm{ClOH}^{-} \\
& k_{(10)}=4.3 \times 10^{9} \mathrm{dm}^{3} \cdot \mathrm{mol}^{-1} \cdot \mathrm{s}^{-1}
\end{aligned}
$$

In this experiment, gamma rays irradiated a $3.5 \% \mathrm{NaCl}$ solution containing almost the same amount of chloride ions as the artificial seawater with added $\mathrm{N}_{2} \mathrm{H}_{4}$ and it was found that the speed of DO reduction was slow, similar to that in artificial seawater. This fact supports the influence of chloride ions on the progress of deoxygenation under the irradiation of gamma rays.

To clarify the mechanisms for deoxygenation by $\mathrm{N}_{2} \mathrm{H}_{4}$ in water systems that contain seawater components in a radioactive environment, further research is necessary to build a system for evaluating the influence of individual seawater components and evaluating their interrelated influences.

\section{Conclusions}

Pure water and artificial seawater with the addition of an infinitesimal quantity of hydrazine, $\mathrm{N}_{2} \mathrm{H}_{4}$, at room temperature, were irradiated with gamma rays. In the absence of radiation, the DO concentration did not decrease in a short period of time for either pure water or artificial seawater. However, the DO concentration did decrease in a short period for both pure water and artificial seawater when irradiated with gamma rays. Because the $\mathrm{N}_{2} \mathrm{H}_{4}$ concentration decreased continuously after the removal of DO, it was further considered that the radiolysis products of $\mathrm{N}_{2} \mathrm{H}_{4}$ were involved in the reduction of DO (deoxygenation). Because the addition of $\mathrm{N}_{2} \mathrm{H}_{4}$ in the water containing seawater components removes the DO, a cause of corrosion, in a short period of time in a radioactive environment at room temperature, the addition of $\mathrm{N}_{2} \mathrm{H}_{4}$ to spent fuel pool water, under proper control of $\mathrm{N}_{2} \mathrm{H}_{4}$ concentration, is considered to be an effective countermeasure for inhibiting the corrosion of the metallic materials that make up the spent fuel pool.

\section{References}

1) http://www.tepco.co.jp/nu/fukushima-np/images/handouts_110903_04-j.pdf

2) S. Tubakizaki, M. Takada, H. Gotou, K. Hawatari et al., "Alternatives hydrazine in water treatment at thermal power plant," Mitsubishi Heavy Industries Tech. Rev., 46(2), 43 (2009).

3) M. Lefort, M. Hassinsky, J. Chim. Phys., 53, 527 (1956).

4) B. G. Ershov, T. L. Mikhailova, A. Yu. Emel'yanova, Izv. Akad. Nauk SSSR, Ser. Khim., 5, 1192 (1985). [in Russian]

5) B. G. Ershov, T. L. Mikhailova, A. Yu. Emel'yanova, Izv. Akad. Nauk SSSR, Ser. Khim., 11, 2450 (1988). [in Russian]

6) B. G. Ershov, T. L. Mikhailova, A. Yu. Emel'yanova, Izv. Akad. Nauk SSSR, Ser. Khim., 2, 341 (1991). [in Russian]

7) G. V. Buxton, C. R. Stuart, "Radiation chemistry of aqueous solutions of hydrazine at elevated temperatures Part 1.- Oxygen-free solutions," J. Chem. Soc., Faraday Trans., 92(9), 1519-1525 (1996).

8) G. V. Buxton, C. R. Stuart, "Radiation chemistry of aqueous solutions of hydrazine at elevated temperatures Part 2.- Solutions containing oxygen," J. Chem. Soc., Faraday Trans., 93(8), 1535-1538 (1997).

9) G. V. Buxton, D. A. Lynch, "Radiation chemistry of aqueous solutions of hydrazine at elevated 
temperatures Part 3. The chain reaction in oxygenated solutions irradiated with ${ }^{60}$ Co $\gamma$ - rays," Phys. Chem. Chem. Phys., 1, 3293-3296 (1999).

10) N. Sato, T. Motooka, C. Kato, M. Yamamoto, "Corrosion phenomena in spent nuclear fuel storage pool containing salinity -(1) determination of the corrosive environment by the water radiolysis calculation," Proc. 2012 Annual Meeting of AESJ, Mar. 27-29, 2012, Fukui, Japan, (2012). [in Japanese] [CD-ROM].

11) D.B.Radtke, A. F. White, J. V. Davis, F.D.Wilde, "National field manual for the collection of water-quality data-dissolved oxygen," Techniques of Water-Resources Investigations, book 9, U.S. Geological Survey, chap. A6 (1998).

12) A. J. Elliot, "A pulse radiolysis study of the temperature dependence of reactions involving $\mathrm{H}, \mathrm{OH}$ and $\mathrm{e}^{-}$aq in aqueous solutions," Radiat. Phys. Chem., 34, 753-758 (1989).

13) E. Hayon, M. Simic, "Intermediates produced from the oneelectron oxidation of hydrazine. Evidence for the formation and decay of tetrazane and triazene." J. Am. Chem. Soc., 94, 42-47 (1972).

14) G. G. Jayson, B. J. Parsons, A. J. Swallow, "Some simple, highly reactive, inorganic chlorine derivatives in aqueous solution. Their formation using pulses of radiation and their role in the mechanism of the Fricke dosimeter.” J. Chem. Soc., Faraday Trans. 1, 69, 1597-1607 (1973). 


\title{
Practical Approach to Decontamination of Radioactive Cesium-Contaminated Matter in Agricultural Region by Improved Wet Classification and Use of Geomaterials
}

\author{
Kenichi ITO $^{1, *}$, Hidetaka MIYAHARA ${ }^{2}$, Toru UJIIE ${ }^{3}$, \\ Toshikatsu TAKESHIMA ${ }^{4}$, Shingo YOKOYAMA ${ }^{5}$, Kotaro NAKATA ${ }^{5}$, \\ Tetsushi NAGANO ${ }^{6}$, Tsutomu SATO $^{7}$, Tamao HATTA ${ }^{8}$ and Hirohisa YAMADA ${ }^{2}$ \\ ${ }^{1}$ Department of Geo Environmental Preservation, Center for International Relations, University of Miyazaki, 1-1 Gakuenkibana- \\ dai-nishi, Miyazaki-shi, Miyazaki 889-2192, Japan \\ ${ }^{2}$ Environmental Remediation Materials Unit, Environment and Energy Materials Division, National Institute for Materials \\ Science, 1-1 Namiki, Tsukuba-shi, Ibaraki 305-0044, Japan \\ ${ }^{3}$ Japan Conservation Engineers \& Co., Ltd., 1-12-11 Kitaurawa, Urawa-ku, Saitama 330-0074, Japan \\ ${ }^{4}$ Astec Tokyo Co., Ltd., 5-21-3 Hirai, Edogawa-ku, Tokyo 132-0035, Japan \\ ${ }^{5}$ Nuclear Fuel Cycle Backend Research Center, Civil Engineering Research Laboratory, Central Research Institute of Electric \\ Power Industry, 1646 Abiko, Abiko-shi, Chiba 270-1194, Japan \\ ${ }^{6}$ Nuclear Science and Engineering Directorate, Japan Atomic Energy Agency, 2-4 Shirakatashirane, Tokai-mura, Naka-gun, \\ Ibaraki 319-1195, Japan \\ ${ }^{7}$ Division of Sustainable Resources Engineering, Department of Socio-Environmental Engineering, Faculty \\ and Graduate School of Engineering, Hokkaido University, Kita-13, Nishi-8, Kita-ku, Sapporo 060-8628, Japan \\ ${ }^{8}$ Environmental Resources Division, Japan International Research Center for Agricultural Sciences, \\ 1-1 Ohwashi, Tsukuba-shi, Ibaraki 305-8686, Japan
}

In the radiation dosimetry of radiocesium in Iitate, Fukushima, the level of radiocesium around the environment did not exceed the criteria in liquid phases such as puddle water, but was distributed in solid phases such as some soil types and organic matter. On the other hand, retting of the cut bamboo grass and hemlock fir in water allowed the release of radiocesium, about $230 \mathrm{~Bq} / \mathrm{kg}$ exceeding the criteria for a bathing area. The flow-thru test using zeolite showed the removal of radiocesium from the liquid phase. The wet classification test was performed for 3 types of radiocesium-contaminated soil. According to the results of wet classification, radiocesium was detected and its level exceeded the cropping restriction level in almost all classified particle fractions. The decontamination effect of wet classification on radiocesium contamination was smaller than that on heavy metal contamination. Specifically, the wet classification could not induce volume reduction. Accordingly, preprocessing and intermediate treatments such as dispersion or attrition by vibration or mixing in the wet classification process were devised and examined as improved processing techniques. As a result, the effectual volume reduction of the radiocesium-contaminated soil was confirmed by adding an intermediate process such as the surface attrition in the vibrator.

KEYWORDS: radiocesium, released radiocesium from organic matter, radiocesium-contaminated soil, wet classification, volume reduction

\footnotetext{
* Corresponding author, E-mail: itoken@cc.miyazaki-u.ac.jp

DOI : 10.15669/fukushimainsights. Vol.4.100

(C) 2021 Atomic Energy Society of Japan. All rights reserved.

Originally published in Transactions of the Atomic Energy Society of Japan (ISSN 1347-2879), Vol. 11, No. 4, p.255-271

(2012) in Japanese. (Japanese version accepted: July 23, 2012)
} 


\section{Introduction}

Due to the large quantity of radioactive material released during the Fukushima Daiichi Nuclear Power Plant Accident, caused by the Great East Japan Earthquake Disaster, the areas contaminated with comparatively high radiation are not only those surrounding the Fukushima Daiichi Nuclear Power Plant, but are distributed throughout various regions of South Tohoku and Kanto. The predominant radioactive materials in the contaminated areas, excluding the evacuation zone, are ${ }^{131} \mathrm{I},{ }^{134} \mathrm{Cs}$ and ${ }^{137} \mathrm{Cs}{ }^{1}$. Among these, almost no ${ }^{131} \mathrm{I}$, which has a short halflife of eight days, was detected by the surveys after May 2011. The radioactive cesium isotopes

${ }^{134} \mathrm{Cs}$ and ${ }^{137} \mathrm{Cs}$ were reported by environmental monitoring surveys to be residual materials that persist in the land surface due to forming cations with a low translocation tendency ${ }^{1)}$. Based on this, it is explained that reducing the influence of radiation on residential areas on the land surface and minimizing health risks is possible by actions such as inversion tillage, which digs or peels off the surface soil with high radioactivity (due to residual radioactive cesium) and replaces it with soil from a deeper zone ${ }^{2)}$. However, because of the wide area of contamination, the variety in geographical features such as ground water levels, land forms, and land use types, the necessity was felt for control over each piece of land for an extended period of time after any such action. Obstacles to land digging, land use, and land trading, and difficulty with gaining agreement and providing adequate comfort for nearby residents were anticipated. However, removing the surface soil layer by peeling, etc. and storing it in a controlled place (temporary storage yard, etc.) that satisfies certain requirements until the radioactivity doses go under a certain level, is regarded as a safety measure. Technologies such as safe peeling using polymers, etc. are already being studied as ways to prevent the surface layer from becoming airborne ${ }^{3,4)}$.

On the other hand, according to the Ministry of the Environment, if the areal decontamination of regions including forests emitting $5 \mathrm{mSv} / \mathrm{y}$ and over and of spots of $1 \mathrm{mSv} / \mathrm{y}$ and over is conducted, the area of regions requiring decontamination adds up to $2,419 \mathrm{~km}^{2,5}$. Furthermore, the quantity of generated debris is estimated at $28,785 \times 10^{3} \mathrm{~m}^{3}$, considering the $40 \%$ of land under buildings, peeling $100 \%$ of a $5 \mathrm{~cm}$ thick surface layer from agricultural lands, cutting $10 \%$ of branches and $10-100 \%$ recovery of fallen leaves, and mowing ${ }^{5)}$. For handling such an enormous amount of contaminated debris, an efficient removal method and volume reduction technology is expected to be easily possible at the relocation site. The extraction technology for radioactive cesium in the contaminated peripheral soils was studied after the Chernobyl Nuclear Power Plant Accident ${ }^{6,7}$. After the Great East Japan Earthquake, a method for extracting the radioactive cesium using acids was studied in Japan as well ${ }^{8}$. However, because the chemical processing will change the nature of the soil, its reuse after decontamination would be difficult. The use of this method in outdoor sites is problematic, and it may generate secondary wastes in the process of treating the soils with acid. Also, its operation at numerous and varied sites over a wide area is difficult. Consequently simpler and safer methods are expected.

In the field of soil contamination, Figure 1 shows how the soil purification technology termed "soil wet-classification" separates and classifies fine grains such as heavy metals and clays that are distributed throughout the soil by separation, classification, etc. and compresses and reduces the volume of contaminated soil that exceeds specified standards ${ }^{9,10)}$. The cesium in the contaminated soils is radioactive, but is also a non-organic harmful metal similar to heavy metals such as arsenic, lead and cadmium. Therefore, like heavy metals, cesium is also considered to be present in the finer grains in relatively large quantities. Consequently, volume reduction is expected by wet-classification of soils contaminated by radioactive cesium as well.

On the other hand, many agricultural lands and mountain forests are included in the contaminated regions, mainly in Fukushima Prefecture. According to a document of the Ministry of 


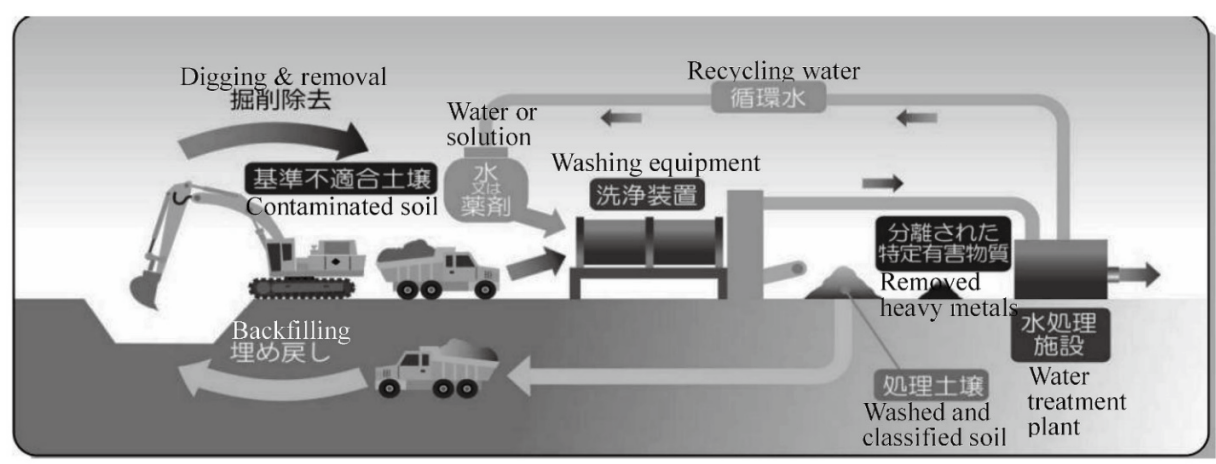

Figure 1 Conceptual scheme of wet-classification and washing for decontamination of heavy-metal contaminated soil ${ }^{9)}$

the Environment, of the total area of $2,373 \mathrm{~km}^{2}$ of lands emitting radiation exceeding $1 \mu \mathrm{Sv} / \mathrm{h}$, $26.5 \%$ is agricultural land and $62.6 \%$ forest ${ }^{5}$. However, in the Soil Contamination Countermeasures Act enforced in 2003, agricultural lands and mountain forests are outside of the scope of the law, whose major object is old factory sites that treated hazardous substances, or soil contamination in urban areas ${ }^{11)}$. Due to this, the available examples and data for soil wet-classification on agricultural lands, etc. are inadequate. Furthermore, because the soils of agricultural land, etc. contain finer grains such as clay, and soil enrichment agents such as fertilizer retainers, their physical and chemical properties presumably differ from urban area soils. For example, the ion exchange capacity (anion and cation exchange capacity, AEC and CEC) and water retaining capabilities are higher in agricultural soil.

Hence, a hot test was conducted in an agricultural village in a mountain area included in the planned evacuation area, Iitate-mura, Soma-gun, Fukushima-Prefecture It aimed at the validation of the volume reduction effect by soil wet-classification on the contaminated agricultural soils, etc. by tracking radioactive cesium.

An in-situ hot test was conducted twice, first from Aug. 23 to 25, and then from Oct. 16 to 21, 2011. All procedures, including the sampling of specimens, tests, measurements, etc. were conducted in Iitate-mura. Additionally, because no storage place had been selected, after the test the specimens were returned to the specimen sampling location, after confirmation from the village office of Iitate-mura.

\section{Measuring Method and Specimens}

\section{Workplace}

The specimens were sampled in Iitate-mura, mainly from the agricultural land around the Iitate-mura village office, and with the cooperation of the office, were contained in the indoor parking place on the premises of the office. The testing of the specimen was conducted in the building of Iitate-mura village office to avoid the radiation in the environment and to suppress the background during measurement.

\section{Measuring Methods}

For measuring the radioactivity of the radioactive cesium in the test specimens, an $\mathrm{NaI}$ (TI) 
scintillation type $\gamma$-ray spectrometer (EMF-211, $3 \times 3$ in./AMPTEK) was used. To suppress the background, measurements were conducted with equipment that was contained in a lead shielding system, covered with 5-cm-thick lead blocks together with the specimen being measured (Figure 2). For measuring, 100 to $300 \mathrm{~g}$ soil specimens and approximately $100 \mathrm{~g}$ fluid specimens were used. The measurements were converted to $\mathrm{Bq}$ (becquerel) equivalents of ${ }^{134} \mathrm{Cs}$ and ${ }^{137} \mathrm{Cs}$ and the sum was divided by the weight of the specimens used, to provide the radioactive cesium concentration as $\mathrm{Bq} / \mathrm{kg}$. The determined lower sensitivity limit was $33 \mathrm{~Bq} / \mathrm{kg}$, and the measurement error was approximately $\pm 10 \%$. For details please refer to the reports by Ujiie et al. (2012) and the documents from the device supplier ${ }^{12,13)}$. When sampling a specimen in the field, a $\beta / \gamma$-ray GM Survey meter was used (TGS-121/Hitachi Aloka Medical) to provide a rough standard for locating and identifying contaminated specimens. Also, some of the fluid specimens were measured by the Japan Atomic Energy Agency after the recovery of cesium using an Empore ${ }^{\mathrm{TM}}$ Rad Disk (Sumitomo 3M) ${ }^{14}$. The Empore ${ }^{\mathrm{TM}}$ Rad Disk is a disk-type solid phase extraction material that collects the specific elements, ions, etc. dissolved in the liquid phase. In this study, a cesium Rad Disk was used that incorporates a type of ferrocyanide, cobalt-modified hexacyanoferrate as the cesium capturing material that selectively adsorbs the dissolved cesium without being obstructively influenced by the major ions contained in the water present in the general environment, including potable water, river water, and ground water (Figure 3) ${ }^{14}$. The measuring methods are as follows: A $100 \mathrm{~mL}$ water specimen was vacuum-filtered, and the cesium in the liquid phase was collected in the Rad Disk. Next, the
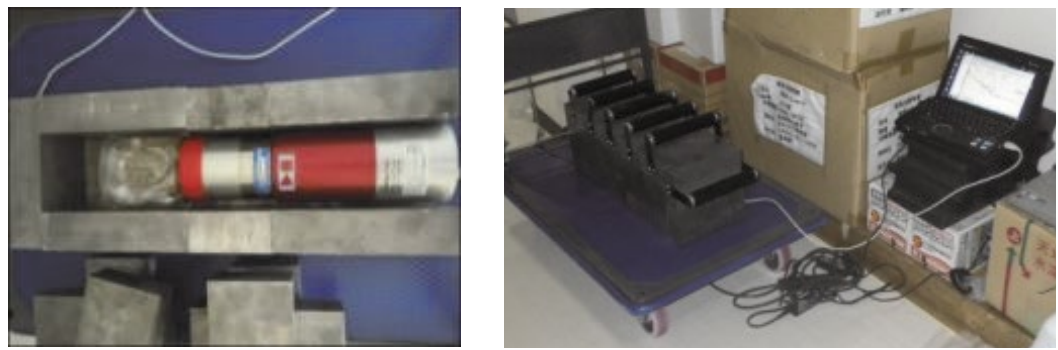

Figure 2 Gamma ray spectrometer detector enclosed by radioprotective lead blocks (left) and appearance of field assay (right)

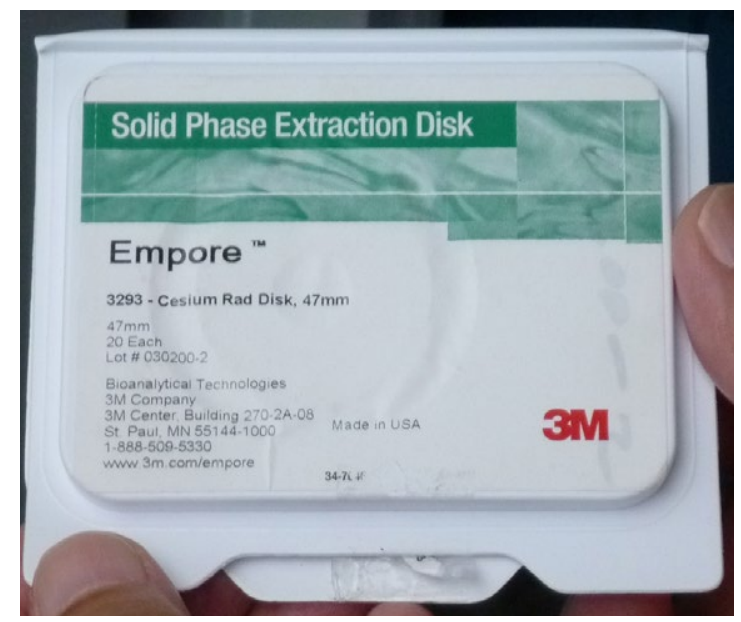

Figure 3 Empore ${ }^{\mathrm{TM}} \mathrm{RAD}$ disk for cesium 
Table 1 Two radiocesium measurement methods used on samples

\begin{tabular}{|c|c|c|}
\hline \multirow{4}{*}{ Instrument \& specification } & $\gamma$-ray spectrometer & GM survey meter with rad disk \\
\hline & $\begin{array}{c}\gamma \text {-ray spectrometer } \\
\text { EMF-211 (Amptek inc.) }\end{array}$ & $\begin{array}{c}\text { selective SPE disk } \\
\text { Empore }^{\mathrm{TM}} \text { RAD disk (3M) }\end{array}$ \\
\hline & \multirow{2}{*}{$\begin{array}{l}3 \times 3 \text { inch, } \\
\text { NaI(TI) scintillation counter }\end{array}$} & pore: $0.1 \sim 0.2 \mu \mathrm{m}$ \\
\hline & & $\begin{array}{c}\beta \text {-ray and } \gamma \text {-ray GM survey meter } \\
\text { TGS-136 (Hitachi Aloka Medical, } \\
\text { Ltd.) }\end{array}$ \\
\hline Measurement time & $10 \mathrm{~min}$. & $10 \mathrm{~min}$. \\
\hline Objective sample & Liquid and solid & Liquid \\
\hline Form in measuring & In vinyl bag. & RAD disk filtrated sample \\
\hline Sample amount in measuring & $\begin{array}{l}\text { Liquid: } 100 \mathrm{~g} \\
\text { Solid: } 300 \mathrm{~g}\end{array}$ & $100 \mathrm{~mL}$ \\
\hline Measurable form of radiocesium & All phase in sample & Soluble ion phase, fine particulate \\
\hline Unmeasurable form & - & Colloidal phase under $0.1 \sim 0.2 \mu \mathrm{m}$ \\
\hline
\end{tabular}

Rad Disk was assessed in the detection zone of a $\beta / \gamma$-ray GM Survey Meter (TGS-136/Hitachi Aloka Medical). The acquired $\beta$-ray dose count rate (cpm) of the Rad Disk was multiplied by a conservative conversion coefficient $(0.0644 \mathrm{~Bq} / \mathrm{cpm})$ for the radioactive cesium acquired, using a standard source. The value was then divided by the filtered water quantity and considered to be the water's radioactive cesium concentration $(\mathrm{Bq} / \mathrm{L})^{15,16)}$. The general detection limit of this method is $400 \mathrm{mBq} / \mathrm{L}$ and lower. This method is considered capable of collecting radioactive cesium with a high capture rate ${ }^{14}$.

Additionally, the physical hole diameter of a Rad Disk is $0.1-0.2 \mu \mathrm{m}^{17}$. Hereinafter this measuring method is referred to as the "Rad Disk method." The specific weights of liquid phase specimens such as water were regarded as $1 \mathrm{~kg} / \mathrm{L}$ and recorded as $\mathrm{Bq} / \mathrm{kg}$ in the measurement results. The above two types of measuring methods are shown in Table 1.

\section{Contamination Survey and Specimen Sampling of Agricultural Soils, etc.}

Specimens were sampled from substances such as the soils in the periphery of the Iitate-mura village office, agricultural land, branches and leaves, water, etc., and evaluated (Figure 4).

Because the fallout radioactive cesium or the fine grains that contain it might remain in the liquid phase in a closed system of water fields, water in a fireproof concrete water tank was sampled, since this water was presumably stored outdoors in the liquid phase for an extended period. For comparison purposes, water in a pool formed on a covering sheet near a forest was sampled as land surface water, reflecting the rainfall water after the accident. Also, in the paddy field in Iitate-mura, on Aug. 24, 2011 during this hot test period, sampling was conducted on the muddy water discharged during the 'soil pudding forced water falling' test, in which the paddy field soil with farming water was stirred and discharged together with surface soil. This sampling was conducted by the National Institute for Rural Engineering, and the National Agriculture and Food Research Organization as one of the potential physical methods for removing the radioactive material from the paddy field soil ${ }^{18)}$. This sample was a specimen containing the surface soil of a paddy field. Because the draining of water after a flood generally only involves the supernatant, two types of supernatant were prepared; the water after natural settling-out of soil pudding muddy water and the water treated by filtration through a sieve of $75 \mu \mathrm{m}$, stirring with $200 \mu \mathrm{L} / \mathrm{L}$ of added poly aluminum chloride (PAC), and concentrated sedimentation. These procedures were used on the liquid phase specimens representing flooding in the paddy field.

Solid specimens were the sampled plants and soils. Branches of bamboo and hemlock, and the grass around the office were cut for the purpose of decreasing the air doses, and these were sampled as plants on which the adhesion of fallout radioactive cesium was presumed. 

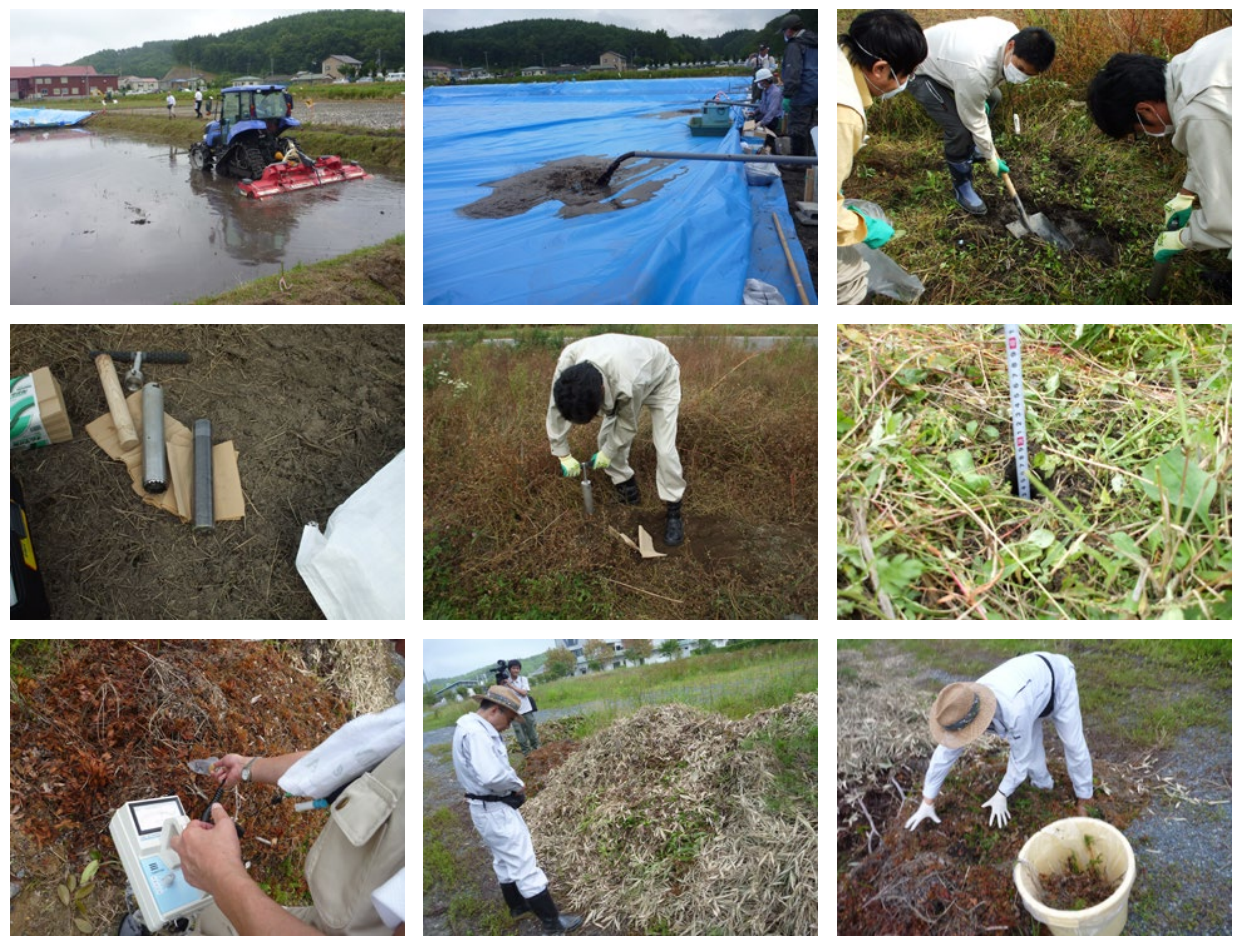

Figure 4 Field research and sampling in Iitate, Fukushima

(top left: rotary on paddy-field, top center: forced overfall, top right: topsoil sampling, middle left: handy sampler, middle center: soil sampling, middle right: borehole sampling, bottom left: dose measurement of pruned hemlock fir, bottom center: pruned bamboo grass, bottom right: pruned blanches being sampled for immersion test)

Because the plants in the grass, forest, etc., are exposed to rainfall outdoors, the cut branches and leaves of bamboo grass and hemlock were immersed in the ion-exchange water overnight (approximately 16 hours) and a solid/liquid separation was conducted through a $3 \mu \mathrm{m}$ porosity approximately $5 \mathrm{~mm}$ thick non-woven bag filter and the possibility of translocation of radioactive cesium to water was validated. The surface soils were sampled from the dry lands, ridge soils, grass lands, drained paddy lands, and sandy ground. Among these, in three places (paddy field, dry land and sandy ground), soil as deep as $30 \mathrm{~cm}$ was sampled, and the distribution of radioactive cesium was confirmed to the maximum sampling depth. The radioactive cesium concentration per dry weight of solid phase specimens was calculated after drying to a constant mass in a constant-temperature chamber at $110^{\circ} \mathrm{C}$ after measuring the as-is specimen and acquiring the water content ratio.

The specimens were selected from the survey results above, and a study was conducted mainly on the soil wet-classification and the processing of radioactive cesium by the minerals.

\section{Results and Discussion of Measurements}

\section{Land Surface Specimens}

The concentration of radioactive cesium in the samples and the various adjusted specimens 
are shown in Table 2. The concentration of radioactive cesium in the fireproof water tank was equal to or lower than the guideline of $50 \mathrm{~Bq} / \mathrm{L}$ of radioactive material in the bathing area, as specified by the Ministry of the Environment. When the specific weight of water was regarded as $1 \mathrm{~kg} / \mathrm{L}^{19)}$, the concentration was lower than the determination limit in a pool. The soil pudding muddy water showed $2,100 \mathrm{~Bq} / \mathrm{kg}$ and was not allowed to drain, but its supernatant water showed values lower than the determination limit for both natural settling and flocculent settling processing. This indicates that the radioactive fallout cesium in the hydrosphere from March was removed comparatively more quickly from the liquid phase after being distributed onto the soil grains and concrete surfaces. Additionally, the naturally generated water pool also showed values equal to or lower than the determination limit. This indicates that the radioactive cesium was distributed on the solid surfaces as explained above or was not contained in the rainwater.

All the soil samples showed a concentration of radioactive cesium, per dry weight, exceeding the standard restriction limit of $5,000 \mathrm{~Bq} / \mathrm{kg}$ for rice ${ }^{20)}$ but fell within the specified standard of 8,000-100,000 Bq/kg set by the Ordinance for Enforcement of the Act on Special Measures concerning the Handling of Contamination from Radioactive Materials (Special Measures Act), a standard for safe processing of wastes except for dry lands and grass field surface soils in August $^{21,22)}$. However, this exceeded the range of the standard specified by the Special Measures Act for grass-surfaced soils. Furthermore, the radioactive cesium concentration of cut branches and leaves showed high values of $53,000 \mathrm{~Bq} / \mathrm{kg}$ for bamboo grasses, and $65,000 \mathrm{~Bq} / \mathrm{kg}$ for hemlock in a wet condition.

Because the moisture content is presumed to be more than $50 \%$ in undried plants, the possibility of exceeding the upper limit specified in the Special Measures Act was pointed out for these as well. The water seeping from cut branches and leaves immersed in the water overnight showed a concentration of $230 \mathrm{~Bq} / \mathrm{kg}$, which exceeded the guideline of $50 \mathrm{~Bq} / \mathrm{L}$ for bathing areas, as set by the Ministry of the Environment (Table 2, Figure 5).

The results of the above survey implied that the objects that caused contamination were the soils and the vegetation, i.e. the solid phases in the muddy water. This further implied the possibility of translocation of radioactive cesium adhering to vegetation, or in water by eluviation due to rainfall. The radioactive cesium concentration on cut branches and leaves of bamboo

Table 2 Radioactive cesium concentrations of substances around Iitate village office

\begin{tabular}{|c|c|c|c|c|c|}
\hline \multirow{2}{*}{\multicolumn{2}{|c|}{ Time }} & \multirow[b]{2}{*}{ Sample } & \multirow[b]{2}{*}{ Aspect and sampling site } & \multicolumn{2}{|c|}{$\mathrm{Cs}(\mathrm{Bq} / \mathrm{kg})$} \\
\hline & & & & $\begin{array}{l}\text { Per wet } \\
\text { weight }\end{array}$ & $\begin{array}{l}\text { Per dry } \\
\text { weight }\end{array}$ \\
\hline \multirow{11}{*}{$\begin{array}{l}2012 . \\
\text { Aug. }\end{array}$} & \multirow{6}{*}{ Solid } & Soil: croft & Near Iitate village office & 3,640 & 5,620 \\
\hline & & Soil: line soil & Same as above & 24,800 & 35,900 \\
\hline & & Soil: grass topsoil & Same as above, twined soil round grasses & 93,900 & 208,700 \\
\hline & & Organic matter: bamboo grass & Near Iitate village office & 53,000 & - \\
\hline & & Organic matter: hemlock fir & Same as above & 65,000 & - \\
\hline & & Muddy water: Paddy-field & From rotary and forced overfall test & 2,100 & - \\
\hline & \multirow{5}{*}{ Liquid } & Rotary muddy water & Supernatent after spontaneous precipitation & $<33$ & - \\
\hline & & & Supernatent after sieve and precipitation with PAC & $<33$ & - \\
\hline & & Fire-fighting water tank & Near Iitate village office & 39 & - \\
\hline & & Puddle water & On the coating sheet near Iitate village office & $<33$ & - \\
\hline & & Seeping water & With organic matter & 230 & - \\
\hline \multirow{3}{*}{ Oct. } & \multirow{3}{*}{ Solid } & Soil: Paddy-field & Kusano area & 12,000 & 18,500 \\
\hline & & Soil: Croft & Akaishizawamaeda area & 13,000 & 18,255 \\
\hline & & Soil: Ground & In front of Iitate village office & 34,000 & 37,810 \\
\hline \multirow{3}{*}{\multicolumn{4}{|c|}{$\begin{array}{l}\text { Ministry of Agriculture, Forestry and Fisheries of } \\
\text { Environment Ministry: Criterion of designation } \\
\text { Environment Ministry: Criterion of bathing area }\end{array}$}} & \multicolumn{2}{|c|}{5,000} \\
\hline & & & & \multicolumn{2}{|c|}{$8,000 \sim 100,000$} \\
\hline & & & & \multicolumn{2}{|c|}{50} \\
\hline
\end{tabular}




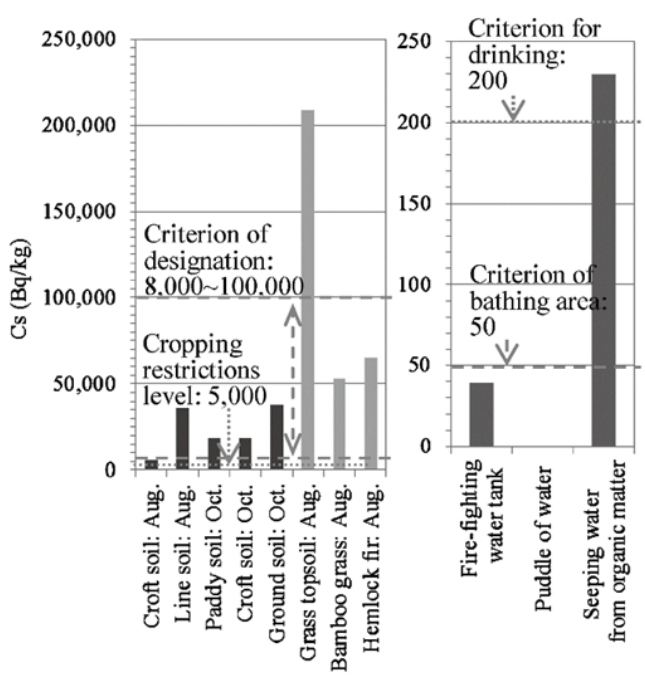

Figure 5 Radioactive cesium concentrations on substances around the Iitate village office

grass and hemlock used in the test showed $53,000 \mathrm{~Bq} / \mathrm{kg}$ for bamboo grass and $65,000 \mathrm{~Bq} / \mathrm{kg}$ for hemlock. The bulk volume of those was approximately $10 \mathrm{~L}$. Where the quantity ratio of tested bamboo grass and hemlock is assumed to be approximately the same and the bulk specific weight of branches and leaves of woody plants is $0.1 \mathrm{~kg} / \mathrm{L}$, the quantity of cut branches and leaves used in the test was estimated to be approximately $1 \mathrm{~kg}$ and the radioactive cesium concentration at $59,000 \mathrm{~Bq} / \mathrm{kg}$. Because the radioactive cesium concentration in seeping water acquired by immersing $1 \mathrm{~kg}$ of these cut branches and leaves in approximately $10 \mathrm{~L}$ of ion-exchange water was $230 \mathrm{~Bq} / \mathrm{kg}$, the quantity of radioactive cesium translocated from the immersed cut branches and leaves to the liquid phase was $2,300 \mathrm{~Bq}$ by rough calculation, and approximately $4 \%$ of the cesium contained in the branches and leaves leached into the liquid phase when immersed in the water.

\section{Depth Distribution of Radioactive Cesium}

Soils were sampled at three locations using a hand sampler (HS-25S/Fujiwara) in a paddy field, on dry land and in the ground as deep as $30 \mathrm{~cm}$. The radioactive cesium concentration was measured after sampling at $2 \mathrm{~cm}$ depth intervals.

The results are shown in Figure 6. The distribution of radioactive cesium was limited to less than the top $10 \mathrm{~cm}$ at all three locations, and was below detection limit in soils deeper than 10 $\mathrm{cm}$. Based on this, as seen in the surveillance research report by the Ministry of Agriculture, Forestry and Fisheries ${ }^{2)}$, it was confirmed that the radioactive cesium that fell out on the land surface did not penetrate deep into the soil but became fixed at a comparatively shallow depth. On the other hand, penetration as deep as approximately $20 \mathrm{~cm}$ was reported in the paddy fields, etc. in the surveillance research report of the Ministry of Agriculture, Forestry and Fisheries. The difference in the penetration of radioactive cesium with soil quality was presumably influenced by the soil's water permeability or the presence of clay minerals that easily adsorb and retain the radioactive cesium. Furthermore, in the agricultural lands, it is assumed that some regions were disturbed by plowing or cultivation during farming work after the accident. So although the penetration of radioactive cesium was $10 \mathrm{~cm}$ or less in this survey, but deeper 


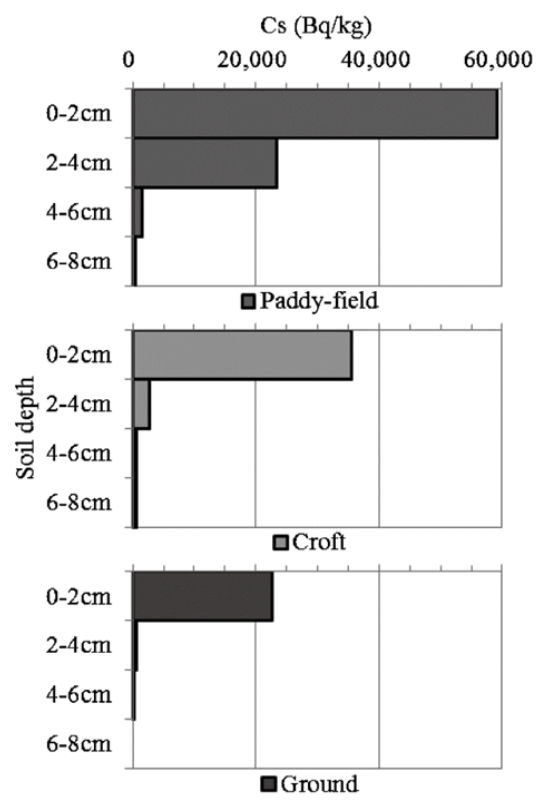

Figure 6 Radioactive cesium distribution in 3 types of soil

than that in other locations, it can be said that it is necessary to remove at least enough surface soil to provide a sufficient check of the local air dose and radioactive cesium concentration in soil during real decontamination work.

\section{Removal of Eluviated Cesium}

\section{Adsorption Test Method}

During the survey, the eluviation of radioactive cesium from plants to water was recognized. This implies the possibility of secondary contamination of the peripheral environment due to leaching of radioactive cesium by rainfall and runoff from the plants and forest plants, etc. cut/ stored to decrease the air dose. So the reduction of leaching water volume during storage and the volume reduction of stored plants were studied as possible methods to remove radioactive cesium by using a mineral to treat the water used for its active eluviation from plants. For this purpose, a contact adsorption test with a mineral was conducted by both batch and column methods on the leaching water (water in which cut branches and leaves were immersed) during the survey.

The batch adsorption test was as follows: For $1 \mathrm{~L}$ of plant leachate water, $100 \mathrm{mg}$ of powders of each mineral were added and stirred using a spoon, for dispersion. Then $200 \mu \mathrm{L}$ of PAC was immediately added for flocculent settling, and was stirred again. After leaving this for $30 \mathrm{~min}-$ utes, the liquid phase was collected using a $0.45 \mu \mathrm{m}$ membrane filter, as used for solid/liquid separation in the official test for soil contamination. Thus, the solid phase and liquid phases were separated and used as measurement specimens ${ }^{23)}$. Because the specimen was a liquid, the Rad Disk method was used to measure the radioactive cesium concentration. As a mineral powder, the Hokkaido Niki zeolite (clinoptilolite) of under 100 mesh, phlogopite, South African vermiculite powder and the mesoporous silica for cesium synthesized by the Natl. Inst. for Materials Science (NIMS) were used. PAC was not added to the mesoporous silica because a 
flocculant is not usually used.

Two types of Niki grain zeolite, Size-M (grain size 2-5 mm) and Size-L (grain size 5-10 mm) were used (Figure 7). Almost equal volumes of Size-M(27 g) and Size-L(25 g) were placed in a polypropylene resin column (ID $15 \mathrm{~mm} \times \mathrm{H} 175 \mathrm{~mm}$ ) and four test sections were set up with one each of Size-M and Size-L and two sets in which the same sizes were connected (Figure 8 and Figure 9). Through each of the columns, the plant leachate water was passed upward using a tubing pump. The water that came though was collected as two samples, during 0-30 min (first $30 \mathrm{~min}$ ) from the start and during 30-90 $\mathrm{min}$ (next $60 \mathrm{~min}$ ), and the as-found measurement of radioactive cesium concentration was conducted. The flow rate was SV6.6-7.6 $\mathrm{h}^{-1}$. SV (Space Velocity) is the space flow rate indicated by the water quantity per unit time divided by the volume of filtered material.

\section{Results and Discussion: Cesium Adsorption Test}

The results of batch adsorption tests are shown in Table 3. While the radioactive cesium concentration of plant leachate water measured by $\gamma$-ray spectrometer was $230 \pm 23 \mathrm{~Bq} / \mathrm{kg}$, the
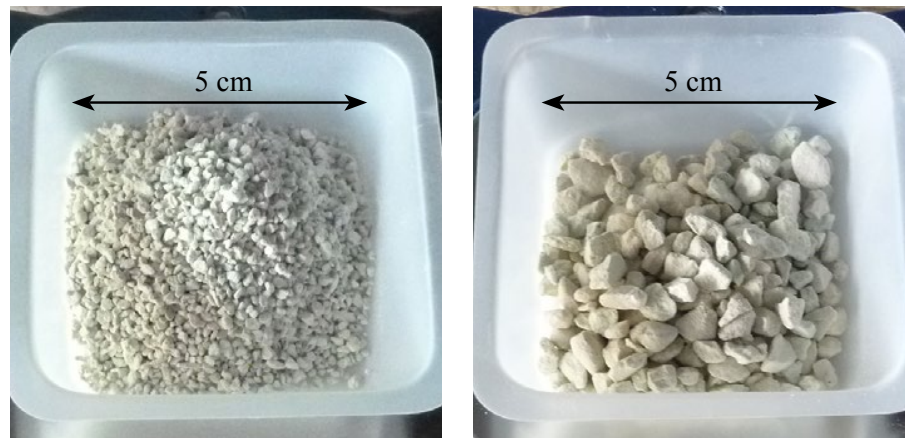

Figure 7 Granular Niki-zeolite

(left : M size, right : L size).

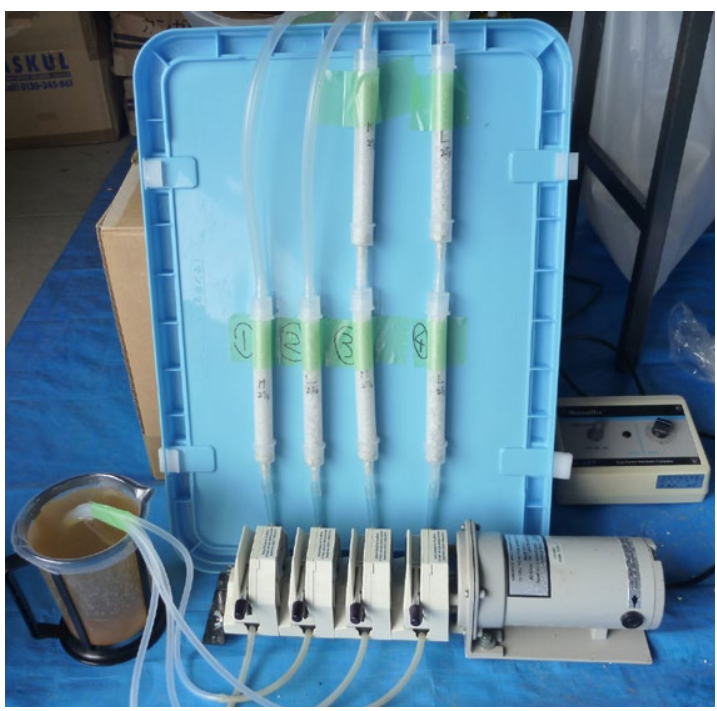

Figure 8 Appearance of the columns for flow-thru test 


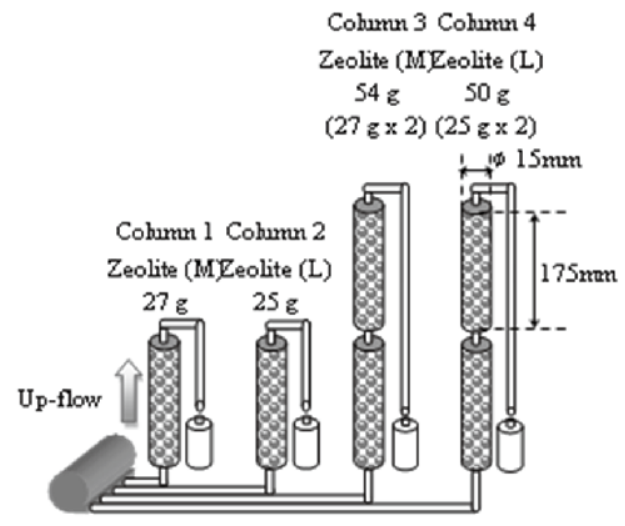

Figure 9 Scheme of the flow-thru test using columns

Table 3 Results of batch adsorption test for cesium eluted from organic matter using geomaterials

\begin{tabular}{|c|c|c|c|c|c|}
\hline Sample & $\begin{array}{c}\mathrm{Cs} \\
(\mathrm{Bq} / \mathrm{kg})\end{array}$ & $\begin{array}{c}\text { Removal efficiency } \\
(\%)\end{array}$ & $\underset{(\mathrm{Bq} / \mathrm{kg})}{\text { Adsorbed amount }}$ & $\begin{array}{l}\text { Partition coefficient } \\
(\mathrm{mL} / \mathrm{g})\end{array}$ & Comment \\
\hline \multirow{2}{*}{$\begin{array}{l}\text { Seeping water from } \\
\text { organic matter }\end{array}$} & $(230 \pm 23)$ & \multicolumn{2}{|c|}{$(\gamma$-ray spectrometer $)$} & & \\
\hline & $133.8 \pm 4.9$ & \multicolumn{2}{|c|}{ Measured using rad disk } & & \\
\hline Zeolite & $93.9 \pm 4.1$ & 29.8 & 399,000 & $4.2 \times 10^{3}$ & $\begin{array}{l}\text { At Niki, Hokkaido, } \\
<100 \text { mesh }\end{array}$ \\
\hline Phlogopite & $65.4 \pm 6.6$ & 51.1 & 684,000 & $1.0 \times 10^{4}$ & \\
\hline Vermiculite & $87.5 \pm 4.0$ & 34.6 & 463,000 & $5.3 \times 10^{3}$ & In South Africa \\
\hline Meso-porous silica & $110.9 \pm 8.0$ & 17.1 & 229,000 & $2.1 \times 10^{3}$ & Cs target type \\
\hline
\end{tabular}

radioactive cesium concentration of plant leachate water measured with the Rad Disk method was $133.8 \pm 4.9 \mathrm{~Bq} / \mathrm{kg}$. The difference of $68.3-124.1 \mathrm{~Bq} / \mathrm{kg}$ between the two measuring methods was recognized as being due to the error range of each measurement. The $\gamma$-ray spectrometer is a method to measure the $\gamma$-radiation emitted from a whole object in the lead shielded space, and the value reflects the concentration of total radioactive cesium contained in the object specimen.

The Rad Disk is a filter with an effective hole diameter of $0.1-0.2 \mu \mathrm{m}$ that selectively adsorbs the dissolved cesium ion with ferrocyanide ${ }^{14,17)}$. Consequently, the radioactive cesium measured by the Rad Disk method is considered to be the sum of the dissolved cesium ion adsorbed by the ferrocyanide and the radioactive cesium physically adhering to the fine grains larger than $0.1-0.2 \mu \mathrm{m}$ in the plant leachate water. Also, the difference between the two measuring methods $(68.3-124.1 \mathrm{~Bq} / \mathrm{kg})$ is assumed to be due to the radioactive cesium adsorbed by the colloid that passed through the holes of Rad Disk or that formed a complex with an organic acid, etc. Based on these test conditions and measurement techniques, the results of the batch test by the Rad Disk method are considered to reflect the removal effect of various minerals that target the radioactive cesium in dissolved form and in the adsorbed form on the fine grains exceeding $0.1-0.2 \mu \mathrm{m}$ in size.

Considerations have been made based on the results of the adsorption test of various minerals. The concentration of radioactive cesium after the batch adsorption test using minerals was $65.4-110.9 \mathrm{~Bq} / \mathrm{kg}$, the removal rate $17.1-51.1 \%$, and the distribution coefficient $2.1 \times 10^{3}$ $-1.0 \times 10^{4} \mathrm{~mL} / \mathrm{g}$ by the Rad Disk method; higher effects were observed, in the order of phlogopite, vermiculite, zeolite, and mesoporous silicate. For comparison of the cesium adsorption tests using identical minerals, the data for the cold state dissolved cesium adsorption test are shown in Table 4 and Figure 10. The test was conducted using a cesium solution on Niki zeolite and 
Table 4 Cesium removal efficiency of 2 geomaterials according to READS ${ }^{24)}$

\begin{tabular}{|c|c|c|c|c|c|}
\hline Sample & $\begin{array}{c}\text { Initial Cs } \\
(\mathrm{mg} / \mathrm{L})\end{array}$ & $\mathrm{pH}$ & $\begin{array}{c}\text { Removal efficiency } \\
(\%)\end{array}$ & $\begin{array}{l}\text { Partition coefficient } \\
(\mathrm{mL} / \mathrm{g})\end{array}$ & Condition \\
\hline \multirow{5}{*}{ Zeolite at Niki, Hokkaido } & 0.01 & 8.27 & 93.45 & $1.4 \times 10^{3}$ & \multirow{5}{*}{$\mathrm{L} / \mathrm{S}=100$ Shaking: $24 \mathrm{~h}$} \\
\hline & 0.1 & 8.11 & 96.60 & $2.8 \times 10^{3}$ & \\
\hline & 1 & 8.43 & 98.99 & $9.8 \times 10^{3}$ & \\
\hline & 2 & 8.25 & 99.26 & $1.3 \times 10^{4}$ & \\
\hline & 10 & 7.75 & 99.56 & $2.2 \times 10^{4}$ & \\
\hline \multirow{7}{*}{ Vermiculite in South Africa } & 0.04 & 6.0 & 84.0 & $5.2 \times 10^{2}$ & \multirow{7}{*}{$\mathrm{L} / \mathrm{S}=100$ Shaking: $24 \mathrm{~h}$} \\
\hline & 0.1 & 6.8 & 97.9 & $4.8 \times 10^{3}$ & \\
\hline & 2 & 6.1 & 98.9 & $8.6 \times 10^{3}$ & \\
\hline & 10 & 5.8 & 99.5 & $1.9 \times 10^{4}$ & \\
\hline & 20 & 5.9 & 99.4 & $1.7 \times 10^{4}$ & \\
\hline & 100 & 5.9 & 99.8 & $5.3 \times 10^{4}$ & \\
\hline & 200 & 5.6 & 99.9 & $7.1 \times 10^{4}$ & \\
\hline
\end{tabular}

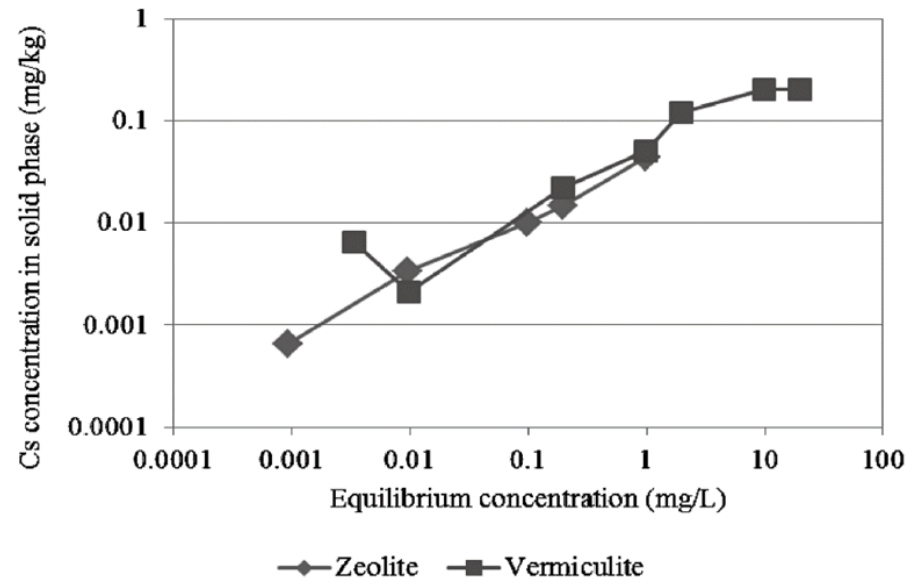

Figure 10 Relationship between the equilibrium concentration of cesium in the liquid phase and in the solid phase of 2 geomaterials according to READS ${ }^{24)}$

South African vermiculite from the same sampling batch as used for the radioactive material removal/collection technology by NIMS ${ }^{24)}$. The cesium removal rate of zeolite and vermiculite in Table 4 was $84.0-99.9 \%$ whereas the removal rates of this batch test in Table 3 were low.

However, because the test results from this batch test fell within the range of the distribution coefficients $5.2 \times 10^{2}-7.1 \times 10^{4} \mathrm{~mL} / \mathrm{g}$ of the cold test, the results of zeolite and vermiculite in this batch test are considered to have shown its effectiveness. This is because the data fell within the cesium removal performance of each mineral indicated by the cold tests although the removal behavior from the liquid phase is uncertain because the form of cesium in the plant leachate water was not identified. Furthermore, the minerals showed a trend of lower values on both removal rate and distribution coefficient with the lower initial cesium concentration in the cold test, while vermiculite showed a trend of slightly increased concentration in the solid phase at low concentration (Figure 10). Zeolite showed a slightly high value of $4.2 \times 10^{3} \mathrm{~mL} / \mathrm{g}$ in this batch test against the distribution coefficient $1.4 \times 10^{3} \mathrm{~mL} / \mathrm{g}$ when the cesium concentration was $0.01 \mathrm{mg} / \mathrm{L}$ in the cold test, and vermiculite showed a one digit higher value of $5.3 \times$ $10^{3} \mathrm{~mL} / \mathrm{g}$ in this batch test against the distribution coefficient $5.2 \times 10^{2} \mathrm{~mL} / \mathrm{g}$ when the cesium concentration was $0.04 \mathrm{mg} / \mathrm{L}$ in the cold test.

The concentration of radioactive cesium, $133.8 \pm 4.9 \mathrm{~Bq} / \mathrm{kg}$ in the plant leachate water in this batch test, shown in Table 3, is the sum of multiple cesium isotopes because it was calculated 
from the $\beta$-ray count of a Rad Disk, as measured by a $\beta / \gamma$-ray GM survey meter. However, if the contained radioactive cesium is assumed to be ${ }^{137} \mathrm{Cs}$, the concentration is approximately $4.16 \times$ $10^{-8} \mathrm{mg} / \mathrm{kg}$, with a decay constant of ${ }^{137} \mathrm{Cs}, \lambda=7.32 \times 10^{-10} \mathrm{~s}^{-1}$ and an atomic mass of approximately 137 , which is regarded as an extremely low concentration compared to the initial cesium in the test condition of READS in Table 4.

The removal of dissolved cesium ions by various minerals indicates that a lower initial cesium concentration resulted in a lower distribution coefficient. However, in this batch test with an extremely low cesium concentration compared with the cold test, the minerals showed a higher distribution coefficient than in the cold test with the lowest cesium concentration. Furthermore, as mentioned earlier, the radioactive cesium detected by the Rad Disk method is supposed to be from dissolution and adsorption on fine grains larger than $0.1-0.2 \mu \mathrm{m}$. Due to this, it is considered that the cesium removal effect of minerals from the plant leachate water shown by this batch test may not be solely the adsorption effect of the minerals on the dissolved cesium ion. As for the other actions, the influence of added PAC as a flocculant and the filtering through the membrane filter during solid/liquid separation needs to be considered. If all these factors are considered, then apart from the adsorption of cesium ions by the minerals, it is supposed that the fine grains and colloids that adsorbed the radioactive cesium may have caused a flocculent settling of minerals due to the addition of PAC and their physical removal by filtering through the $0.45 \mu \mathrm{m}$ membrane filter.

The water passing test results on the column filled with granular zeolite are shown in Table 5. The measurements were taken mainly using a $\gamma$-ray spectrometer and parts of columns 3 and 4 that could not be measured at the site were measured using the Rad Disk method. The plant leachate water that passed through showed lower values than the bathing area guideline of the Ministry of Environment of $50 \mathrm{~Bq} / \mathrm{L}$ for both the first $30 \mathrm{~min}(0-30 \mathrm{~min})$ and the next, after $60 \mathrm{~min}(60-90 \mathrm{~min})$. The specimens in columns 3 and 4 for $60-90 \mathrm{~min}$ measured with the Rad Disk method also showed a decreased radioactive cesium concentration that was lower than the lower determination limit of the $\gamma$-ray spectrometer. Furthermore, the Size-M columns 1 and 3 with smaller grain size showed a lower concentration of radioactive cesium after passing than

Table 5 Results of flow-thru test using columns filled with granular zeolite

\begin{tabular}{|c|c|c|c|c|c|c|c|}
\hline \multirow{2}{*}{\multicolumn{2}{|c|}{$\begin{array}{c}\text { Sample } \\
\& \\
\text { condition }\end{array}$}} & \multirow{2}{*}{$\begin{array}{l}\text { Seeping } \\
\text { water }\end{array}$} & \multirow{2}{*}{$\begin{array}{c}\text { Column } 1 \\
\text { M size } \\
\times 1 \text { stem }\end{array}$} & \multirow{2}{*}{$\begin{array}{c}\text { Column } 2 \\
\begin{array}{c}\text { L size } \\
\times 1 \text { stem }\end{array}\end{array}$} & \multirow{2}{*}{$\begin{array}{c}\text { Column } 3 \\
\begin{array}{c}\text { M size } \\
\times 2 \text { stems }\end{array}\end{array}$} & \multirow{2}{*}{$\begin{array}{c}\text { Column } 4 \\
\begin{array}{c}\mathrm{L} \text { size } \\
\times 2 \text { stems }\end{array}\end{array}$} & \multirow{2}{*}{$\begin{array}{l}\text { Criterion of } \\
\text { bathing area }\end{array}$} \\
\hline & & & & & & & \\
\hline \multicolumn{2}{|c|}{ Amount of mineral $(\mathrm{g})$} & & 27 & 25 & 54 & 50 & \\
\hline \multicolumn{2}{|c|}{ Column volume $(\mathrm{mL})$} & & 30 & 30 & 60 & 60 & \\
\hline \multirow{3}{*}{$\begin{array}{c}\text { 1st time: } \\
30 \mathrm{~min} \\
(0 \sim 30 \mathrm{~min})\end{array}$} & $\mathrm{Cs}(\mathrm{Bq} / \mathrm{L})$ & $230 \pm 4.6$ & $<33$ & $46 \pm 4.6$ & $<33$ & $32 \pm 3.2$ & 50 \\
\hline & $\begin{array}{l}\text { Treated water } \\
(\mathrm{mL})\end{array}$ & & - & 194.79 & 223.38 & 214.6 & \\
\hline & $\underset{(\mathrm{Bq})}{\operatorname{Removal}} \mathrm{Cs}$ & & 11.6 & 9.3 & 52.5 & 46.3 & \\
\hline \multirow{3}{*}{$\begin{array}{l}\text { 2nd time: } \\
60 \mathrm{~min} \\
(30 \sim 90 \mathrm{~min})\end{array}$} & $\mathrm{Cs}(\mathrm{Bq} / \mathrm{L})$ & $230 \pm 4.6$ & - & $<33$ & $8.3 \pm 2.7^{\mathrm{a})}$ & $27.2 \pm 2.9^{\mathrm{a})}$ & 50 \\
\hline & $\begin{array}{l}\text { Treated water } \\
(\mathrm{mL})\end{array}$ & & - & 101.11 & 456.09 & 468 & \\
\hline & $\underset{(\mathrm{Bq})}{\operatorname{Removal} \mathrm{Cs}}$ & & - & 23.3 & 101.1 & 94.9 & \\
\hline \multirow[t]{6}{*}{ Total: $90 \mathrm{~min}$} & $\begin{array}{l}\text { Treated water } \\
(\mathrm{mL})\end{array}$ & & - & 296 & 679 & 682.6 & \\
\hline & Ave. SV $\left(h^{-1}\right)$ & & - & 6.6 & 7.5 & 7.6 & \\
\hline & $\underset{(\mathrm{Bq})}{\operatorname{Removal} \mathrm{Cs}}$ & & 11.6 & 32.6 & 153.6 & 141.2 & \\
\hline & $\mathrm{L} / \mathrm{S}$ & & - & 11.8 & 12.6 & 13.7 & \\
\hline & $\begin{array}{l}\text { Ave. Reaction } \\
\text { time (min.) }\end{array}$ & & - & 9.1 & 7.9 & 7.9 & \\
\hline & $\begin{array}{l}\text { Partition } \\
\text { coefficient }\end{array}$ & & - & $7.8 \times 10^{1}$ & $5.1 \times 10^{2}$ & $9.6 \times 10^{1}$ & \\
\hline
\end{tabular}

a) Measured using rad disk. Others are measured by $\gamma$-ray spectrometer. The values are indicated as $\mathrm{Bq} / \mathrm{L}$ on the assumption that water density is 1 . 
the Size-L columns 2 and 4. It is considered that the processing efficiency was enhanced simply because the smaller grain size meant that a larger quantity of mineral, with a larger surface area, occupied the a same volume. The distribution coefficient in zeolite under the conditions in each column, measured in terms of the radioactive cesium removed and the quantity of water passed through was $7.8 \times 10^{1}-5.1 \times 10^{2} \mathrm{~mL} / \mathrm{g}$ and lower than the distribution coefficient in zeolite by the batch test, $4.2 \times 10^{3}$ as shown in Table 3 . This is because: (1) The adsorption efficiency per unit weight of mineral decreased because the total water quantity that passed through with regard to the mineral quantity in each column, i.e., the solid/liquid ratio, was 11.8 to 13.7 , which was extremely low compared to the solid/liquid ratio of 10,000 in the batch test, (2) The reaction time for a column test (7.9-9.1 $\mathrm{min}$ ) was less than $1 / 3$ of the $30 \mathrm{~min}$ reaction time of a batch test, but the ion-exchange reaction of zeolite seems to be relatively slow in spite of the long reaction time of SV of 6.6-7.6 $\mathrm{h}^{-1}$ compared to the SV 10-100, etc. when tap water is treated using the ion-exchange resin. As a result, the effect of zeolite was not fully used for the radioactive cesium in the plant leachate water within the reaction time available during water passage, (3) The physical removal effect on the adsorbed cesium by the fine grains and colloids due to the addition of PAC and filtering that was suggested for the batch testing was not part of the column water-through test. However, the concentration of radioactive cesium that passed through the column was lower than in the batch test, and lower than the guideline of treatment $50 \mathrm{~Bq} / \mathrm{L}$.

From all the above results, it can be concluded that the reduction in the distribution coefficient of minerals means the reduction of utilization efficiency of a material under those conditions, but it was shown that even if this value is lower, the concentration of radioactive cesium in the liquid phase could be sufficiently decreased by a treatment with a low liquid/solid ratio, such as having the water pass through a column.

\section{Wet-classification of Agricultural Soils}

\section{Soil Wet-classification Test Method}

A soil wet-classification test was conducted with the aim of decreasing the volume of surface layer soils containing radioactive cesium. For the test, surface soils such as ridge soil, paddy field, dry land and the ground were used, in which a high level of radioactive cesium was detected from bulk measurement. The survey showed that the concentration of radioactive cesium was the highest in the grass field surface soils, but these were excluded because there were local specimens of soil adhering to the grass roots, and a sufficient quantity of specimens for testing was not secured.

The soil wet-classification test was conducted as follows. The soils were separately sampled as-is in raw weights of $1.5 \mathrm{~kg}$ (equivalent to dry weight of approximately $1 \mathrm{~kg}$.) and wet-classification was conducted while water-washing using an electromagnetic experimental screening shaking apparatus (A-3/FRITSCH) (Figure 11). The tap water of the Iitate-mura village office, in which radioactive cesium was not detected, was used as the cleaning water and the quantity used was approximately 7 times as much as the raw weight of specimen soil.

Seven stages of sieve mesh were set up as grain size fractions: $<0.075 \mathrm{~mm}, 0.075-0.125 \mathrm{~mm}$, $0.125-0.25 \mathrm{~mm}, 0.25-0.5 \mathrm{~mm}, 0.5-2 \mathrm{~mm}, 2-4.75 \mathrm{~mm}$, and $>4.75 \mathrm{~mm}$. The $<0.075 \mathrm{~mm}$ specimen was collected as muddy water and processed with a $3 \mu \mathrm{m}$ bag filter similar to that used for filtering plant leachate water to remove the suspended component.

Since the radioactive cesium adsorbed due to ion-exchange in the clay minerals may be replaced by other cations, a study was also conducted on using an ammonium ion solution that 


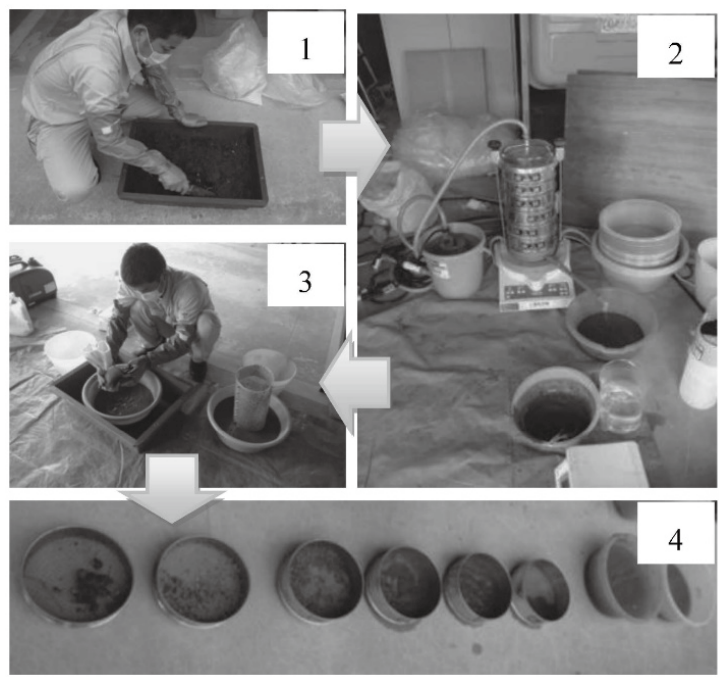

Figure 11 Soil wet-classification and washing procedure

(1: homogenization of soil samples, 2: electric test sieve shaker, 3: washing and re-sieving residue, and solid-liquid separation for muddy water, 4: classifying the particle fractions from soil samples)

is considered to have less influence on farming if added to the agricultural soil for the ridge soils in place of cleaning water. A $0.1 \mathrm{~mol} / \mathrm{L}$ ammonium nitrate solution was prepared and an ammonium nitrate solution of approximately $1.5 \mathrm{~L}$, an amount equal to the volume of the soil, was added to the specimen soil, which was left immersed for $1 \mathrm{~h}$. Then a soil wet-classification test was conducted.

\section{Soil Wet-classification Test Results and Discussion}

The results of the wet-classification of ridge soils conducted using water and ammonium nitrate are shown in Table 6. As all the desired equipment was not ready for in-situ measurement of soil moisture content after classification in August, when the ridge soils were tested, the concentration of radioactive cesium was measured using a $\gamma$-ray spectrometer on each $1 \mathrm{~kg}$ of agricultural field soil, from which water was removed by sieving. The original soil was dried in the wind and the dry weight was acquired.

The concentration of radioactive cesium was reduced as the grain size used became coarser compared to the original soil in both water cleaning and ammonium nitrate solution cleaning. However, the effect of the ammonium nitrate solution was not recognizable. Although not precise enough because the value was based on wet weight, it was presumed for the case of general wet soil of moisture content $30-50 \%$, that the radioactive cesium would be reduced to half of that in the original soil by wet-classification for under $6,000 \mathrm{~Bq} / \mathrm{kg}$ in wet weight and in the grain size larger greater than $0.5 \mathrm{~mm}$. However, because the concentration of radioactive cesium exceeded the acreage limit of 5,000 Bq/ $\mathrm{kg}$ for rice in the grain size fraction $0.075-4.75 \mathrm{~mm}$, the expected volume reduction effect on the processed soil by wet-classification was not achieved for the ridge soils.

The muddy water with grains under $0.075 \mathrm{~mm}$ was processed through a bag filter. However, the suspended substances in the muddy water managed to pass through the non-woven bag filter, which expanded due to the hydraulic pressure, so that the liquid still contained 
Table 6 Radioactive cesium in each particle fraction from each soil after wet-classification

\begin{tabular}{|c|c|c|}
\hline \multirow{3}{*}{ Line soil } & \multicolumn{2}{|r|}{$\mathrm{Cs}(\mathrm{Bq} / \mathrm{kg})$} \\
\hline & $25,794^{\text {a) }}$ & Per natural wet weight \\
\hline & 38,731 & Per dry weight \\
\hline Washing solvent & Water & $1 \mathrm{~mol} / \mathrm{L}$ ammonium nitrate \\
\hline$>4.75 \mathrm{~mm}$ & $2,200^{\mathrm{a})}$ & $5,500^{\text {a) }}$ \\
\hline $2 \sim 4.75 \mathrm{~mm}$ & $5,100^{\text {a) }}$ & $4,700^{\text {a) }}$ \\
\hline $0.5 \sim 2 \mathrm{~mm}$ & $5,300^{\text {a) }}$ & $5,600^{\text {a) }}$ \\
\hline $0.25 \sim 0.5 \mathrm{~mm}$ & $9,500^{\text {a) }}$ & $8,700^{\mathrm{a})}$ \\
\hline $0.125 \sim 0.25 \mathrm{~mm}$ & $10,000^{\mathrm{a})}$ & $9,800^{\text {a) }}$ \\
\hline $0.075 \sim 0.25 \mathrm{~mm}$ & $10,000^{\mathrm{a})}$ & $12,000^{\text {a) }}$ \\
\hline$<0.075 \mathrm{~mm}^{\mathrm{b})}$ & 320 & - \\
\hline$<0.075 \mathrm{~mm}^{\mathrm{c})}$ & $<33$ & - \\
\hline \multicolumn{3}{|c|}{ a) Per natural wet weight. } \\
\hline \multicolumn{3}{|l|}{ b) Muddy water. } \\
\hline \multicolumn{3}{|c|}{ c) Filtrated b water using $10 \mu \mathrm{m}$ membrane filter. } \\
\hline
\end{tabular}

suspended solids after filtration, and showed a radioactive cesium concentration of $320 \mathrm{~Bq} / \mathrm{kg}$. Because the forced filtration of muddy water is not possible using a non-woven bag filter, it is considered that filtration using a strong filtration cloth such as filter press is appropriate for in-situ dewatering of suspended solids and solid/liquid separation. Hence, a single sheet of $10 \mu \mathrm{m}$ membrane filter having an equivalent mesh opening to filtration cloth was used for filtering the water that passed through the bag filter. The fine soil grains, etc. were removed, the filtered liquid obtained was clear, and the radioactive cesium concentration was lower than the determination limit of $33 \mathrm{~Bq} / \mathrm{kg}$.

This implied that the radioactive cesium content may be reduced to half by removing soil particles under $0.5 \mathrm{~mm}$ with soil wet-classification on ridge soils. The presence of radioactive cesium in soil in the liquid phase was decreased by soil wet-classification.

Next, after the results of the soil classification test on ridge soils, tests were also conducted on three types of soils: from paddy fields, dry land, and the ground, to compare the effect of wet-classification on the agricultural soils under different conditions. The results are shown in Table 7 and Figure 12.

Of the radioactive cesium contained in these soils, $63-85 \%$ was present in grain sizes under $0.075 \mathrm{~mm}$. However, it was also present in the coarse grain size fractions, so the considered grain size fractions and weight percentage of soils under $5,000 \mathrm{~Bq} / \mathrm{kg}$ were $12.2 \%$ for paddy fields over $2 \mathrm{~mm}, 29.5 \%$ for dry lands over $0.25 \mathrm{~mm}$ and $0 \%$ for the ground. Furthermore, it may be considered that after removing the highly contaminated fine grains by wet-classification, an equivalent quantity of healthy soil can be mixed with the original ground soil and water to recover the physical properties of soil. When lower than $5,000 \mathrm{~Bq} / \mathrm{kg}$ is achieved by dilution, the paddy fields and dry lands of lower than $0.075 \mathrm{~mm}$ and the ground lower than $0.25 \mathrm{~mm}$ are removal object, and the collection rates are expected to be $39 \%, 44 \%$ and $75 \%$. To enhance the efficiency of volume reduction by the soil wet-classification method, it is necessary (1) to conduct further classification in the soils containing significant quantities of fine grains such as paddy fields and dry lands using a highly precise wet-classification apparatus such as cyclone for the soil grains smaller than $0.075 \mathrm{~mm}$ not separable by a simple mesh sieve, and (2) to conduct an additional treatment such as chemical treatment depending on the cost and effect for the sludges of high 
INSIGHTS CONCERNING THE FUKUSHIMA DAIICHI NUCLEAR ACCIDENT Vol. 4

Table 7 Particle size and radioactive cesium distribution in 3 types of soil in wet-classification

\begin{tabular}{|c|c|c|c|c|c|c|}
\hline Sample & $\begin{array}{c}\text { Water } \\
\text { content ratio } \\
(\%)\end{array}$ & \multicolumn{2}{|c|}{$\begin{array}{c}\text { Dry weight ratio of } \\
\text { particle fraction } \\
(\%)\end{array}$} & $\begin{array}{l}\text { Cs per dry weight } \\
(\mathrm{Bq} / \mathrm{kg})\end{array}$ & $\begin{array}{c}\text { Cs per } 1 \mathrm{~kg} \text { dry } \\
\text { weight sample } \\
(\mathrm{Bq})\end{array}$ & Cs distribution \\
\hline Paddy-field & \multicolumn{3}{|l|}{$35.1 \%$} & \multicolumn{2}{|l|}{18,500} & \\
\hline$>4.75 \mathrm{~mm}^{\mathrm{a})}$ & - & \multicolumn{2}{|l|}{-} & - & - & - \\
\hline $2 \sim 4.75 \mathrm{~mm}$ & 5.3 & 2.8 & & 4,011 & 111.3 & 0.8 \\
\hline $0.5 \sim 2 \mathrm{~mm}$ & 33.9 & 9.4 & & 3,782 & 356.5 & 2.5 \\
\hline $0.25 \sim 0.5 \mathrm{~mm}$ & 35.9 & 12.4 & & 8,733 & $1,084.4$ & 7.5 \\
\hline $0.125 \sim 0.25 \mathrm{~mm}$ & 43.0 & 7.4 & & 13,674 & $1,008.9$ & 7.0 \\
\hline $0.075 \sim 0.125 \mathrm{~mm}$ & 47.6 & \multirow{2}{*}{$\begin{array}{r}7.1 \\
60.9\end{array}$} & & 16,418 & $1,160.6$ & 8.0 \\
\hline$<0.075 \mathrm{~mm}$ & 77.9 & & & 17,618 & $10,736.1$ & 74.3 \\
\hline Croft & \multicolumn{3}{|l|}{28.8} & 18,255 & & \\
\hline$>4.75 \mathrm{~mm}$ & 23.9 & \multicolumn{2}{|c|}{3.1} & 1,445 & 45.1 & 0.3 \\
\hline $2 \sim 4.75 \mathrm{~mm}$ & 24.6 & 7.2 & \multirow{6}{*}{29.5} & 2,918 & 210.5 & 1.6 \\
\hline $0.5 \sim 2 \mathrm{~mm}$ & 39.6 & 11.0 & & 2,981 & 327.0 & 2.4 \\
\hline $0.25 \sim 0.5 \mathrm{~mm}$ & 39.6 & 8.2 & & 4,805 & 396.0 & 2.9 \\
\hline $0.125 \sim 0.25 \mathrm{~mm}$ & 45.2 & 8.5 & & 7,301 & 618.2 & 4.6 \\
\hline $0.075 \sim 0.125 \mathrm{~mm}$ & 41.6 & 6.2 & & 7,363 & 459.4 & 3.4 \\
\hline$<0.075 \mathrm{~mm}$ & 79.0 & 55.7 & & 20,525 & $11,440.9$ & 84.8 \\
\hline Ground & 10.1 & & & 37,810 & & \\
\hline$>4.75 \mathrm{~mm}$ & 22.5 & \multicolumn{2}{|l|}{1.1} & 30,968 & 325.9 & 1.1 \\
\hline $2 \sim 4.75 \mathrm{~mm}$ & 4.4 & \multicolumn{2}{|l|}{9.5} & 16,732 & $1,584.0$ & 5.3 \\
\hline $0.5 \sim 2 \mathrm{~mm}$ & 15.5 & \multicolumn{2}{|l|}{33.0} & 9,946 & $3,286.9$ & 11.1 \\
\hline $0.25 \sim 0.5 \mathrm{~mm}$ & 15.0 & \multicolumn{2}{|l|}{31.4} & 10,701 & $3,364.5$ & 11.3 \\
\hline $0.125 \sim 0.25 \mathrm{~mm}$ & 8.8 & \multicolumn{2}{|l|}{9.6} & 19,733 & $1,892.4$ & 6.4 \\
\hline $0.075 \sim 0.125 \mathrm{~mm}$ & 0.9 & \multicolumn{2}{|l|}{2.4} & 26,228 & 617.9 & 2.1 \\
\hline$<0.075 \mathrm{~mm}$ & 73.3 & \multicolumn{2}{|l|}{13.0} & 142,500 & $18,589.8$ & 62.7 \\
\hline \multicolumn{4}{|c|}{ Cropping restriction level about paddy-field } & $8,000 \sim 100,000$ & & \\
\hline \multicolumn{4}{|c|}{ Criterion of designation (storage direction) } & 5,000 & & \\
\hline
\end{tabular}

a) preclusion for less amount to measure.

※ The error range of all data in this table is $\pm 10 \%$.

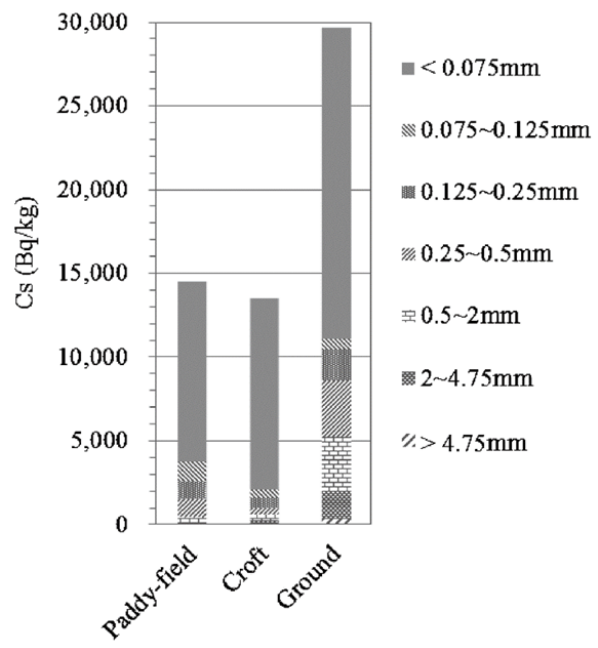

Figure 12 Radioactive cesium concentration and amount recovered from 3 types of soil

concentration that are finally discharged. Importantly, the concentration of radioactive cesium exceeded $8,000 \mathrm{~Bq} / \mathrm{kg}$ in all grain fractions including the coarse grain sizes in the sandy ground.

This indicates the possibility of residual radioactive cesium due to the adsorption by the coarse soil grain surfaces or due to the adhesion of clay minerals, etc. that adsorbed the 
radioactive cesium. The grain size fractions showing high and coarse values should be treated by the soil wet-classification process, not by an additional treatment from the viewpoint of volume reduction. For this case, it is necessary to reduce the radioactive cesium concentration in the coarse grain fractions by devising a sieving or pre-treatment process.

\section{Study of Pre-Treatment for Wet-classification}

\section{Study Method}

A pre-treatment was studied to improve the efficiency of wet-classification for the ground soils in which high radioactive cesium concentration was observed in the coarse grain fraction, also after wet-classification. The methods studied are listed in Table 8. Assuming in-situ operation, the methods applicable using general purpose instruments were used. Furthermore, either direct adsorption to coarse grains or the adhesion of fine grains such as clay that adsorb cesium are considered to be the causes of residual radioactive cesium, but the range of its presence is assumed to include at least the surface of coarse soil grains. So a study was conducted using four types of pre-treatment methods acting on the grain surfaces: an ultrasonic apparatus and a vibrator for fresh concrete whose functions are mainly dispersion and vibration, a pot mixer + weight and, a mortar + vibrator whose main function is grinding.

These methods, excluding mortar + vibrator, were used as a pre-treatment for wet-classification, and the mortar + vibrator was used for intermediate processing in wet-classification. However, the ultrasonic apparatus is not a general purpose in-situ instrument but is used to compare typical dispersion methods. The details of study methods are as follows (Figure 13):

(1) Pre-Treatment 1: Ultrasonic Device

A vinyl bag containing $1 \mathrm{~kg}$ of soil and $1 \mathrm{~kg}$ water was set in a laboratory compact ultrasonic shaker (As One) and a dispersion of $10 \mathrm{~min} \times 3$ times (total $30 \mathrm{~min}$ ) was conducted for wet-classification.

(2) Pre-Treatment 2: Vibrator for Fresh Concrete

Added $1 \mathrm{~kg}$ of soil and $1 \mathrm{~kg}$ of water to a $5 \mathrm{~L}$ stainless steel container and attached an iron vibrator (Iron type $50 \mathrm{~W}$ formwork vibrator, owned by AKTIO) and wet-classification was conducted after vibrating for 5 mins.

(3) Pre-Treatment 3: Pot Mixer + Weight

A $20.5 \mathrm{~cm}$ diameter basketball was filled with $8.5 \mathrm{~kg}$ of sand and $1.5 \mathrm{~kg}$ of soil, put in a $70 \mathrm{~L}$ pot mixer (owned by AKTIO) and rotated (at $24 \mathrm{rpm}$ ) for $3 \mathrm{~min}$ with a tilt of approximately 45 degrees. Then $1.5 \mathrm{~kg}$ of water was added and the pot rotated for another 2 mins because the soil

Table 8 Efficiency improvement methods for removal of radioactive cesium from coarse-grained particle fractions

\begin{tabular}{lc}
\hline \multicolumn{1}{c}{$\begin{array}{c}\text { Method } \\
\text { Target and equipment }\end{array}$} & Effect \\
\hline $\begin{array}{l}\text { Preprocessing 1: Ultrasonic vibration } \\
\text { Dispersing soil particles by about } 20 \mathrm{kHz} \text { Ultrasonic vibration. }\end{array}$ & Dispersion \\
Preprocessing 2: Vibration using degasser for fresh concrete & Dispersion, shaking \\
Dispersing and shaking soil particles using with about $120 \mathrm{~Hz}$ vibration using degasser for fresh concrete. & Weighted attrition \\
Preprocessing 3: Pot-mixing with rubber weight & Surface attrition \\
Mixing soil in rotary pot with rubber weight ball frictionally. & \\
Intermediate processing: Attrition by vibrator and mortar & \\
Rubbing soil with rubber coating 6,500 Hz vibrator in deep mortar. &
\end{tabular}



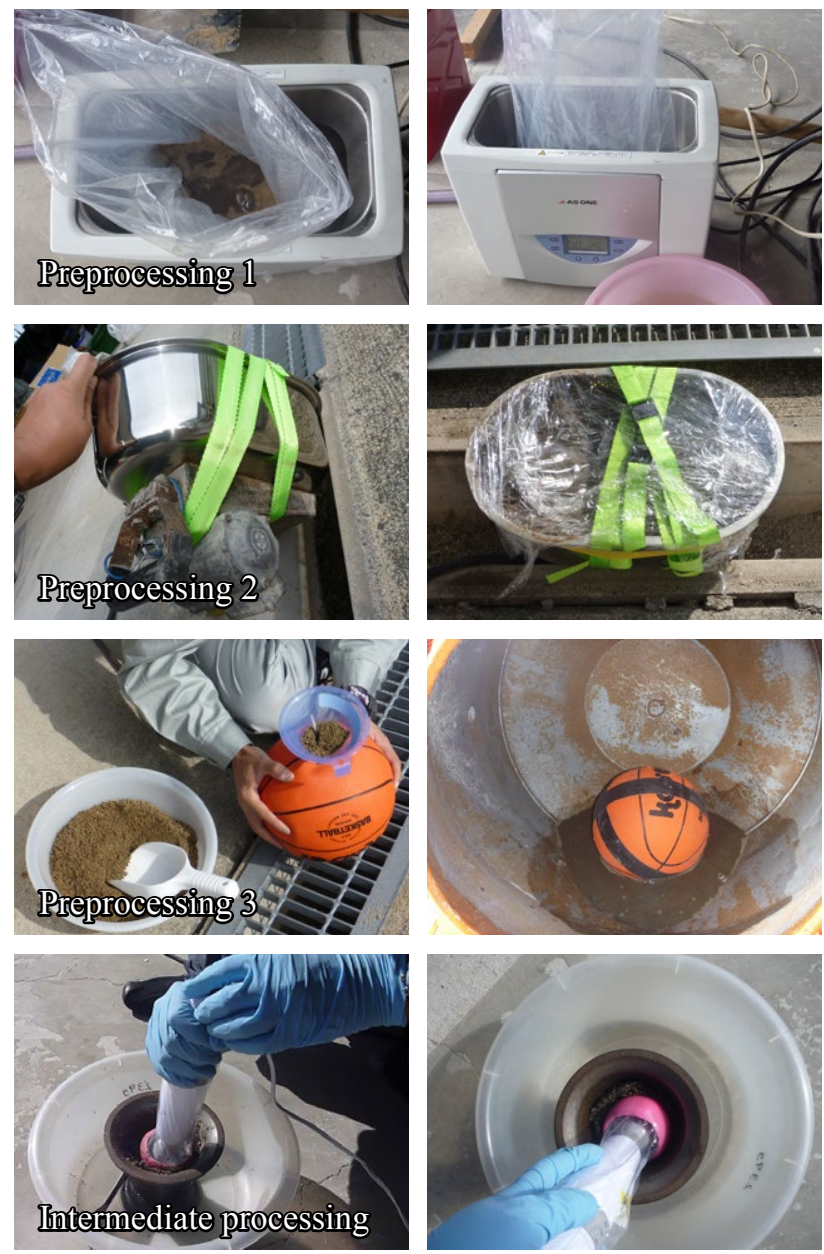

Figure 13 Efficiency improvement methods for the removal of residual radioactive cesium from coarse-grained particle fractions

(Preprocessing 1: Ultrasonic vibration, Preprocessing 2: Vibration using degasser for fresh concrete, Preprocessing 3: pot-mixing with rubber weight, Intermediate processing: Attrition by vibrator and mortar)

slipped in the mixer and no loss in weight occurred. Then the pot was tilted to approximately 10 degrees and was rotated for $30 \mathrm{~min}$, but again no loss in weight was observed as the abrasion was insignificant. After treatment, wet-classification was conducted.

(4) Intermediate Treatment: Mortar + Vibrator

This treatment aimed at the removal of residual radioactive cesium in the coarse soil grains after wet-classification. The grain fraction of $0.125-4.75 \mathrm{~mm}$ was separated as a coarse grain soil from the sample soil. Coarse grain soil $200 \mathrm{~g}$ and water $100 \mathrm{~g}$ were fed into the steel deep mortar. Treatment was applied using a bar-type vibrator with a rubber ball fitted on the contact zone, which was pressed lightly on the coarse grain soil with vibration (of 6,500 rpm) for 10 mins, sieving with a $0.125 \mathrm{~mm}$ filter. Concentration measurement was performed for both the remaining portion and the passed portion. Also, a study was conducted on the mixture of coarse soil $(100 \mathrm{~g})$ and water $(100 \mathrm{~g})$ for $20 \mathrm{~min}$ using the same procedure for increased water percentage and duration of treatment. 


\section{Results and Discussion on Three Types of Pre-Treatment}

The results of three pre-treatments for wet-classification are shown in Table 9. 1: Ultrasonic Device; 2: Vibrator pretreatment for Fresh Concrete; 3: Treatment in Pot Mixer + Weight. The study showed that the radioactive cesium concentration was slightly decreased by the dispersion treatment with an ultrasonic apparatus in Pre-Treatment 1 for grain size fractions $0.075-0.125 \mathrm{~mm}, 0.125-0.25 \mathrm{~mm}$ and $2-4.75 \mathrm{~mm}$, but on the other hand, an increase was observed in the grain size fractions $0.25-0.5 \mathrm{~mm}$ and $0.5-2 \mathrm{~mm}$. Pre-Treatment 2 and Pre-Treatment 3 reduced the concentration of radioactive cesium to half of the concentration left by Pre-Treatment 1 or less at $2-4.75 \mathrm{~mm}$, while the radioactive cesium concentration in other grain size fractions showed a similar trend as Pre-Treatment 1.

Soil wet-classification is a method aimed at separation by concentrating the harmful substances on finer grains. To assess the effect, the distribution of radioactive cesium onto each grain size fraction relative to the radioactive cesium concentration in whole soil was calculated using the radioactive cesium concentration of each grain size fraction and its weight percent. The radioactive cesium distribution onto each grain size fraction was compared to an untreated sample of all the grain size fractions of $0.25 \mathrm{~mm}$ and above. A significant reduction, especially by Pre-Treatment 3, was seen in $0.125-0.25 \mathrm{~mm}$ as well, and the effect was verified (Table. 9). The changes in weight percentage and the distribution ratio of radioactive cesium on the grain size fractions of $0.075 \mathrm{~mm}$ and below are shown in Figure 14. From Table 9 and Figure 13, after each Pre-Treatment (1, 2 and 3$)$ an increase was seen in the percentage of radioactive cesium distributed in grain size fractions $0.075 \mathrm{~mm}$ and smaller.

These results implied that part of the radioactive cesium present in the coarser grain size

Table 9 Results of wet-classification after 3 types of preprocessing

\begin{tabular}{|c|c|c|c|c|c|c|c|c|c|c|c|c|}
\hline \multirow{3}{*}{ Sample } & \multicolumn{4}{|c|}{$\mathrm{Cs}(\mathrm{Bq} / \mathrm{kg})$} & \multicolumn{4}{|c|}{ Particle size distribution (\%) } & \multicolumn{4}{|c|}{ Cs distribution (\%) } \\
\hline & \multirow{2}{*}{ Control } & \multicolumn{3}{|c|}{ Preprocessing } & \multirow{2}{*}{ Control } & \multicolumn{3}{|c|}{ Preprocessing } & \multirow{2}{*}{ Control } & \multicolumn{3}{|c|}{ Preprocessing } \\
\hline & & 1 & 2 & 3 & & 1 & 2 & 3 & & 1 & 2 & 3 \\
\hline$>4.75 \mathrm{~mm}$ & 30,968 & - & - & - & 1.1 & - & - & - & 1.1 & - & - & - \\
\hline $2 \sim 4.75 \mathrm{~mm}$ & 16,732 & 15,535 & 6,249 & 6,239 & 9.5 & 13.8 & 12.1 & 18.2 & 5.3 & 5.3 & 1.7 & 2.0 \\
\hline $0.5 \sim 2 \mathrm{~mm}$ & 9,946 & 10,252 & 10,449 & 9,555 & 33.0 & 16.7 & 26.3 & 19.5 & 11.1 & 4.2 & 6.3 & 3.3 \\
\hline $0.25 \sim 0.5 \mathrm{~mm}$ & 10,701 & 14,175 & 12,234 & 12,857 & 31.4 & 20.7 & 11.1 & 10.1 & 11.3 & 7.2 & 3.1 & 2.3 \\
\hline $0.125 \sim 0.25 \mathrm{~mm}$ & 19,733 & 15,206 & 12,879 & 12,762 & 9.6 & 17.0 & 19.6 & 12.0 & 6.4 & 6.4 & 5.8 & 2.7 \\
\hline $0.075 \sim 0.125 \mathrm{~mm}$ & 26,228 & 15,697 & 18,095 & 23,385 & 2.4 & 8.6 & 5.6 & 7.7 & 2.1 & 3.3 & 2.3 & 3.3 \\
\hline$<0.075 \mathrm{~mm}$ & 142,500 & 128,531 & 139,832 & 147,705 & 13.0 & 23.2 & 25.3 & 32.5 & 62.7 & 73.5 & 80.8 & 86.3 \\
\hline
\end{tabular}

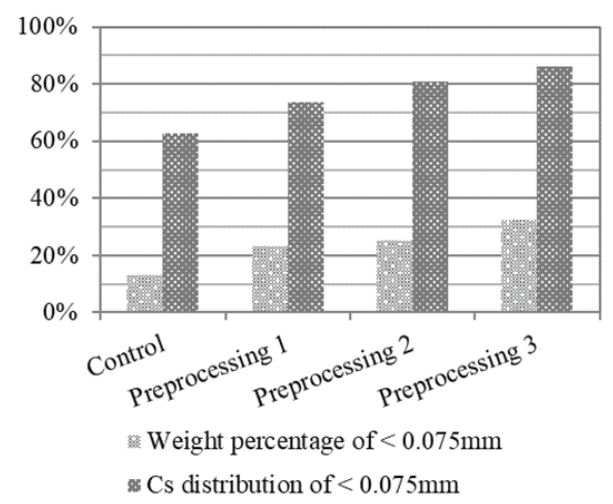

Figure 14 Results of wet-classification after 3 types of preprocessing 
fractions translocated to the grain size fractions of $0.075 \mathrm{~mm}$ and smaller, together with fine particles such as clay minerals that adsorb them in the pre-treatment.

Furthermore, the fine grain portion of $0.075 \mathrm{~mm}$ and lower increases from $13.0 \%$ to $32.5 \%$ by wet-classification following Pre-Treatment 3, that is Pot Mixer + Weight. As a result, it is considered that the removal rate of radioactive cesium when the above fine grain portion was separated from the contaminated soil can be enhanced from $62.7 \%$ to $86.3 \%$.

We also observed that the increase in the weight percentage and the distribution rate of radioactive cesium in the particle size fractions less than $0.075 \mathrm{~mm}$ were higher in order of the ultrasonic apparatus of Pre-Treatment 1, the vibrator for fresh concrete of Pre-Treatment 2 and the pot mixer + weight of Pre-Treatment 3 . This is thought to mean that a higher wet-classification effect may be achieved by methods that have the effect of grinding the soil grain surfaces, such as vibration and grinding as a pre-treatment of soil, rather than by applying a dispersion treatment, e.g. using a high frequency for the dispersion of powder in liquid.

\section{Results and Discussion on Intermediate Treatment}

When the soil is pre-treated in bulk, the fine grain portion of $0.075 \mathrm{~mm}$ and smaller that is separated by the wet-classification of original soil is obtained. This fine grain portion may inhibit the functioning, e.g., by damping the physical shocks by cushioning the coarse soil grains during the dispersion or grinding in the pre-treatment stage. Hence, a wet-classification was conducted on the original soil, only the grain size of $0.125-4.75 \mathrm{~mm}$ was collected, and water was added. The surface grinding was conducted using a rubber-coated vibrator, using a deep steel mortar as a capturing tray, and was sieved with a $0.125 \mathrm{~mm}$ sieve while washing with water. The grinding with soil:water $=2: 1$ for 10 mins was defined as Condition 1 and soil: water $=1: 1$, for $20 \mathrm{~min}$ as Condition 2 .

The results are shown in Table 10. As a result of grinding, a weight percentage of 6-10\% was classified as the fine grain portion of $0.125 \mathrm{~mm}$ and smaller. The radioactive cesium concentration did not go below $5,000 \mathrm{~Bq} / \mathrm{kg}$ but was at least less than the standard of $8,000 \mathrm{~Bq} / \mathrm{kg}$ subjected to storage, and the reduction of the Cs residual rate to as low as $53-55 \%$ was achieved.

From these results of the experiment, it was demonstrated that by applying grinding and wet-classification after removing the fine grains by classification in pretreatment greatly enhances the separation efficiency for radioactive cesium by wet-classification.

Table 10 Efficiency of intermediate processing on wet-classification

\begin{tabular}{cccc}
\hline Sample & $\begin{array}{c}\mathrm{Cs} \\
(\mathrm{Bq} / \mathrm{kg})\end{array}$ & $\begin{array}{c}\text { Soil residual } \\
\text { rate }(\%)\end{array}$ & $\begin{array}{c}\text { Cs residual } \\
\text { rate }(\%)\end{array}$ \\
\hline $\begin{array}{c}\text { Coarse-grained soil } \\
\text { 0.125 4.75 mm }\end{array}$ & 11,706 & 100.0 & 100.0 \\
Condition 1 & 6,490 & 93.6 & 55.4 \\
Condition 2 & 6,217 & 89.5 & 53.1 \\
\hline
\end{tabular}

※ The error range of all data in this table is $\pm 10 \%$.

\section{Summary of the Implemented Research}

Through the sampling of specimens and the survey conducted in Iitate-mura, it was confirmed that the radioactive cesium is distributed mainly on solids such as soils and plants on the 
land surface. Soil will hold it securely, while plants release it when exposed to water. There is thus a concern that the release of radioactive cesium distributed on plants by rainfall, etc., can cause secondary contamination in the peripheral environment.

On the other hand, it was confirmed that the radioactive cesium released from plants can be removed from the liquid phase by treatment with minerals such as zeolite, as was proved through the batch adsorption test and the column water passing test.

The possibility of reducing the concentration in the soil with grain sizes of $0.075-0.125 \mathrm{~mm}$ and above while in storage was confirmed by collecting the radioactive cesium in the fine grain portion of contaminated soil using the soil wet-classification test. The classification efficiency depended on the type of soil. Especially in sandy-ground soils, the radioactive cesium remained in the coarse-grain fractions when only the wet-classification of soils was conducted. It was confirmed that by using dispersion, vibration and grinding in the pre-treatment, and also using grinding in the intermediate treatment, and an additional wet-classification is conducted, that more effectively translocating the radioactive cesium to the fine grain portion was possible.

Some possible measures for the decontamination of agricultural soils, etc., are presented as follows. These are based on the results of work in Iitate-mura and studies of radioactive cesium removal methods in this research: First, when storing plants with a high concentration of radioactive cesium, use a waterproof sheet, foil, etc. as a cover to avoid exposure to rainfall, etc. and prevent any secondary damage due to leaching of the radioactive cesium from the plants. Also, as a countermeasure to the leaching of cesium during storage, it is considered effective to arrange natural minerals such as zeolite or vermiculite under or around the contaminated substances as adsorbents. Because the minerals can exert the desired effect not only in powder form but also in grains, it is worth noting that the grain form is easy to use in water-passing types of treatment, easy to handle, and does not disperse easily, making it better for use than powder. For contaminated soils, the wet-classification treatment of soil is suggested. The treatment flow chart is shown in Figure 15. First, collect the highly contaminated surface layer soil using a polymer, etc. and immerse it in water to avoid dispersion. In surface layer soils, organic substances such as the roots and leaves of plants and humus are present in relatively large amounts. Because organic substances such as plants may be highly contaminated, there is the possibility of effective volume reduction by drying or incineration separately from soils. Thus, they are separated from soil grains by floatation.

Separated organic substances such as plants, etc. are dehydrated, measured and stored. Storage procedures for the plants are explained above. Also, it is best to apply this volume reduction separately, through decomposition or by incineration, or to apply a treatment to prevent the release of radioactive cesium. For soils from which the organic substances have been removed, the soil mass is smashed and rough sorting of stones and gravel is conducted using a drum washer, a rougher or a sieve. The sieved soils are cleaned and separated using a trommel, sieving shaker, high-mesh separator, cyclone, etc. in the order of grain size from rough soil to fine, with the soil grains of $0.02-0.75 \mathrm{~mm}$ and above collected in stages. The fine grains smaller than that are sent as muddy water containing radioactive cesium to the solid/liquid separation process. Because the radioactive cesium tends to remain in the coarse grain size fractions, as seen in the classification result of ground soil in this study, the status of residual radioactive cesium is checked for the soil grains in each collected grain size fraction using a sieve shaker, etc. Those lower than the standard specified in the guidelines of government, etc. are regenerated as soil. For those higher than the standard, a surface grinding process is applied such as an attrition mixer, vibrator, or loaded grinding, and a wet-classification is conducted to remove the fine grains peeled from the coarse soil grains containing radioactive cesium. After the second wet-classification, each collected grain size fraction is re-measured to confirm its safety. The 


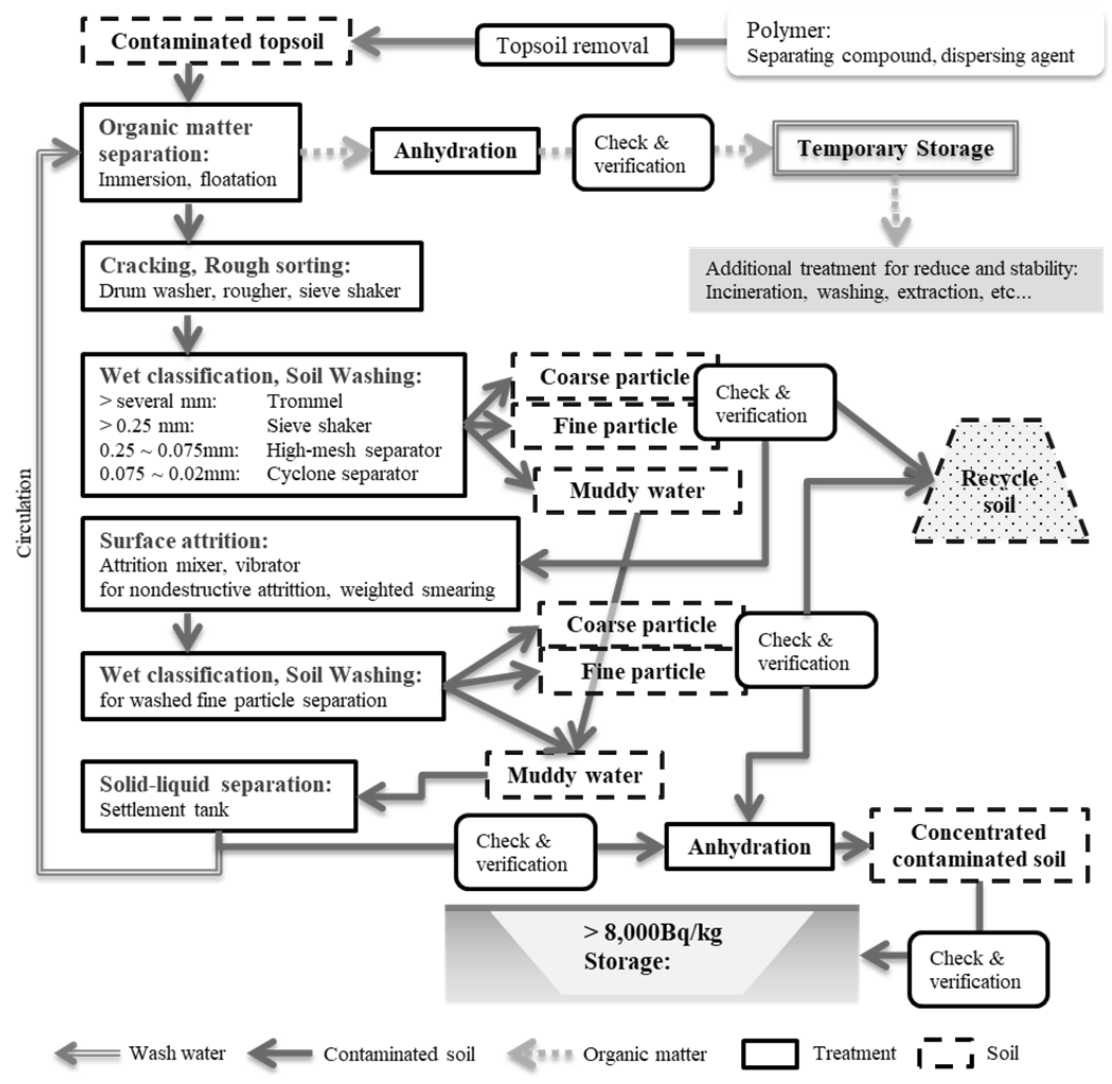

Figure 15 Conceptual schema for improved wet-classification decreasing the amount of radioactive cesium contaminated soil

muddy water is transferred to the solid/liquid separation process and separated into solid and liquid by flocculant settling. As shown in this study, almost no radioactive cesium in the soil is released into the water, the supernatant solution is used and recirculated as cleaning water. The fine grains in the muddy water collected in the solid/liquid separation process are dehydrated. The soil grains that exceeded the standard in the second wet-classification are stored as specified after evaluating their contamination level as a concentrated contaminated soil.

As explained above, it is expected that the efficient in-situ volume reduction treatment of agricultural land soils, etc. generated in the contaminated area becomes possible by implementing the storage procedures according to the results of this study. i.e. classification, grinding, etc.

\section{Future Subjects}

It was confirmed that the separation and volume reduction of contaminated soils was possible by the soil wet-classification method. However, in this study, the status or the presence of radioactive cesium in the soils sorted by the wet-classification could not be evaluated or discussed. However several different statuses of cesium have been reported, including its adsorption by ion-exchange between layers of micro lamellar clay minerals such as smectite, etc. having exchangeable cations ${ }^{25}$, and the adsorption/retention of illite on the frayed edge sites ${ }^{26,27)}$. 
Among the clay minerals universally present in the surface layer soils in Japan, mica and illite are the non-swellable lamellar silicate minerals in which the hydrated cations do not enter in between layers, and their cation exchange ability is regarded as inferior to that of swellable clays. However, the frayed gap on the edge of this mineral grain is called the "frayed edgesite" and it is known to be steadily restored if the potassium and cesium in between the layers are exchanged ${ }^{26,27)}$. However, because agricultural lands contain artificial materials such as those for soil enrichment, etc., and radioactive cesium is inevitably present in the coarse grain fractions that apparently contain pebbles with almost no clay mineral, the immediate cesium environment or chemical associations may not be limited to those suggested in the existing bibliography. Accordingly, for soils, the essential subjects for future decontamination and volume reduction treatment are to conduct the validation of mineral composition and surface observations with respect to grain size fraction after wet-classification of various soils and soil qualities. Also, on the fine grain portion containing the radioactive cesium removed from the coarse grain fraction by grinding, etc., clarification of the present form is required, especially in the coarse grain size fractions in the real soil contaminated with radioactive cesium. Further, the adsorption and desorption properties should be evaluated.

For conducting this test and summarizing the above report, we received a considerable amount of cooperation and advice from concerned persons. First, we express our gratitude to the people of the Iitate-mura village office, including Mr. Ryuji Honda/Industrial Promotion Section, and the residents in Iitate-mura who kindly permitted the sampling of specimens. When conducting the tests, Mr. Mitsuru Yamamura of JCE Co., Ltd. conducted in-situ measurement using a $\gamma$-ray spectrometer. During the in-situ testing, Mr. Takuya Echigo of NIMS, Dr. Hirochika Naganawa, Dr. Nobuyuki Yanase and Dr. Hisayoshi Mitamura of JAEA, Dr. Yujiro Watanabe of the Kanazawa Institute of Technology cooperated. Mr. Takashi Kobayashi of NIMS recorded images of the whole process. We are grateful to them, and to all those who supported and cooperated us.

Additionally, the contents of this research were acquired from the FY2011 Commissioned Project on Science and Technology Strategic Promotion "Development of Radioactive Materials Decontamination Technology for Agricultural Land Soils."

\section{References}

1) Survey and Research Report on Radioactive Material Concentration Distribution Mapping on Farmland Soils, MAFF, The Office of Agriculture, Forestry and Fisheries Research Council, Edition 3, p58 (2012). [in Japanese] http://radioactivity.mext.go.jp/ja/distribution_map_around_FukushimaNPP / 5600_201203131000_report3.pdf

2) On the Farmland Radioactive Material Removal Technologies (Decontamination Technology) Attachment 4, 4. Reversal Tillage, MAFF, Press Release on Sep.14, 2011, 11-12 (2011). [in Japanese] http:// www.s.affrc.go.jp/docs/press/110914.htm

3) H. Naganawa, N. Kumazawa, H. Saitoh et al., "Removal of radioactive cesium from surface soils solidified using polyion complex rapid communication for decontamination test at Iitate-mura in Fukushima prefecture," Trans. At. Energy Soc. Jpn., 10[4], 227-234 (2011). [in Japanese]

4) H. Naganawa, "Removal of radioactive cesium from contaminated soil by using poly-ion complex with clay," Nendo Kagaku, 50[2], 52-57 (2011). [in Japanese]

5) No.2 Environmental Restoration Commission, Distributed Material 7, No.2 Environmental Restoration Commission, MOE, Sep.27, 2011(2011). [in Japanese] http://www.env.go.jp/jishin/rmp/conf/02-mat4. pdf

6) S. V. Krouglov, A. D. Kurinov, R. M. Alexakhin, "Chemical fractionation of ${ }^{90} \mathrm{Sr},{ }^{106} \mathrm{Ru},{ }^{137} \mathrm{Cs}$, and ${ }^{144} \mathrm{Ce}$ in Chernobyl-contaminated soils: An evolution in the course of time," J. Environ. Radioact., 38, 59-76 (1998). 
7) K. Bunzl, W. Kracke, W. Schimmack, L. Zelles, "Forms of fallout ${ }^{137} \mathrm{Cs}$ and ${ }^{239+240} \mathrm{Pu}$ in successive horizons of a forest soil," J. Environ. Radioact., 39, 55-68 (1998).

8) Succeeded in Extracting Cesium from Soil Using Low-Level Acid, National Institute of Advanced Industrial Science and Technology, Press Release, Aug.31, 2011. [in Japanese] http://www.aist.go.jp/aist_j/ press_release/pr2011/pr20110831/pr20110831.html

9) Guidebook for Improved Measures in the District, MOE, Soil Environment Management Division, Water-Air Environment Management Bureau, 43-46 (2011). [in Japanese] http:/www.env.go.jp/water/ dojo/gb_me/

10) T. Futami, "Practical approach to remediating heavy metal-contaminated soil by grain sorting, gravity separation, and grain-surface attrition," Chigaku-zasshi (J. Geography), 116[6], 932-940 (2007). [in Japanese]

11) FY2010 The Statuses of Enforcement of Soil Contamination Countermeasures Act and the Survey Results on Soil Contamination Survey/Case Examples of Countermeasures, MOE, MOE Water-Air Environment Management Bureau, Mar.2012(2012). [in Japanese] http://www.env.go.jp/water/report/ h23-02/index.html

12) T. Ujiie, M. Yamamura, N. Narisawa, "Investigation of onsite analysis method for the concentrations of Radiocesium," Proc. 21st Symp. Geo-Environ. and Geo-Techn., Tsukuba, Japan, Jun. 24-25, 2012, 17-22 (2012). [in Japanese]

13) EMF211 $\gamma$-Ray Spectrometer, EMF Japan Co., Ltd., p5 (2012). [in Japanese] http://www.emf-japan. com/emf/img/PDF/EMF211.pdf

14) $3 M^{T M}$ Empore $^{T M}$ Rad Disk Cesium Catalog, Sumitomo 3M Ltd., p2 (2011). [in Japanese] http://www. mmm.co.jp/filter/empore/rad/pdf/sced11-014.pdf

15) T. Nagano, H. Mitamura, N. Yanase, H. Naganawa, K. Yasuda, Z. Yoshida, H. Yamaguchi, "On-site monitoring method of radioactive cesium in water sample using cesium adsorption disk and GM survey metereter," Nihon- Genshiryoku-Gakkai Shi (J. At. Energy Soc. Jpn.), in preparation (2012). [in Japanese]

16) D. M. Beals, W. G. Britt, J. P. Bibler, D. A. Brooks, "Radionuclide analysis using solid phase extraction disks," J. Radioanal. Nucl. Chem., 236[1-2], 187-191 (1998).

17) D. M. Beals, K. J. Hofstetter, V. G. Johnson, G. W. Patton, D. C. Seely, "Development of field portable sampling and analysis systems," J. Radioanal. Nucl. Chem., 248[2], 315-319 (2001).

18) On the Farmland Radioactive Material Removal Technologies (Decontamination Technology), 3. Stirring and Removal of Soil with Water, Press Release, MAFF, Sep.14, 2011, Attachment 4, 9-10 (2011). [in Japanese] http://www.s.affrc.go.jp/docs/press/110914.htm

19) Guidelines on Radioactive Substances in Bathing Areas, MOE, Water-Air Environment Management Bureau, EWAWR No. 110624001, Jun.24, 2011 (2011). [in Japanese] http://www.env.go.jp/jishin/attach/no110624001.pdf

20) Guidelines for Rice Acreage, Cabinet Office, Nuclear Emergency Headquarters, Apr.08, 2011 (2011). [in Japanese] http://www.maff.go.jp/j/kanbo/joho/saigai/ine_sakutuke.html

21) Policies on disposal methods for incineration ash exceeding $8,000 \mathrm{~Bq} / \mathrm{kg}$ and less than or equal to 100,000 Bq/kg, EDCR No.110831001, EDCR No.110831001, MOE, Minister's Secretariat/Waste Management and Recycling Department, Aug. 31, 2011. [in Japanese] http://www.env.go.jp/jishin/attach/ no110831001.pdf

22) On the difference of duplicated standards $100 \mathrm{~Bq} / \mathrm{kg}$ and $8,000 \mathrm{~Bq} / \mathrm{kg}, \mathrm{MOE}$, Waste Management and Recycling Department (2011). [in Japanese] http://www.env.go.jp/jishin/attach/waste_100-8000.pdf

23) On the environmental standards relevant to soil contamination, MOE Notification No.46, Aug.23, 1993(1993), Last Amendment: MOE Notification No. 37, Jun. 16, 2010). [in Japanese]

24) READS Database for radioactive material removal and collection technology, Natl. Inst. for Materials Science (2012). [in Japanese] http://reads.nims.go.jp/

25) H. Shirozu, Nendokobutsugaku Nendokagaku-no-kiso, Asakurashoten, Tokyo, 38-42, ISBN4-25416231-6 C 344 (1988). [in Japanese]

26) A. Cremers, A. Elsen, P. D. Preter, A. Maes, "Quantitative analysis of radiocaesium retention in soils," Nature, 335, 247-249 (1988).

27) N. Ishikawa, S. Uchida, K. Tagami, "Effects of clay minerals on radiocesium sorption behavior onto paddy field soils,” Radioisotopes, 56[9], 519-528 (2007). [in Japanese] 


\title{
Article
}

\section{Testing of Multistep Soil Washing for Radiocesium-Contaminated Soil Containing Plant Matter}

\author{
Masafumi FUNAKAWA ${ }^{1, *}$, Akihiro TAGAWA ${ }^{2}$ and Nobuyasu OKUDA ${ }^{3}$ \\ ${ }^{1}$ Environmental Engineering Department, Takenaka Corporation, 1-1-1 Shinsuna, Koto-ku, Tokyo 136-0075, Japan \\ ${ }^{2}$ Headquarters of Fukushima Partnership Operations, Japan Atomic Energy Agency, 2-2-2 Uchisaiwai-cho, Chiyoda-ku, Tokyo \\ 100-0011, Japan \\ ${ }^{3}$ Takenaka Research and Development Institute, Takenaka Corporation, 1-5-1 Otsuka, Inzai-shi, Chiba 270-1395, Japan
}

\begin{abstract}
Decontamination work following radiocesium exposure requires a vast reduction in the amount of contaminated soil generated. The current study subjected 4 types of contaminated soil with different properties to multistep soil washing under the same conditions. This study also determined the effectiveness of radiocesium decontamination and the extent to which the amount of contaminated soil was reduced. In addition, the effectiveness of plant matter separation, adsorbent addition, and grinding as part of multistep soil washing was determined using the same contaminated soil. Results of testing indicated that the rate of radiocesium decontamination ranged from 73.6 to $89.2 \%$ and the recovery rate ranged from 51.5 to $84.2 \%$ for twice-treated soil, regardless of the soil properties or cesium level. Plant matter in soil had a high radiocesium level. However, there was little plant matter in our soil sample. Therefore, plant matter separation had little effect on the improvement in the percentage of radiocesium decontamination of twice-treated soil. Soil surface grinding improved the radiocesium decontamination of twice-treated soil. However, radiocesium in soil tightly bound with minerals in the soil; thus, the addition of an adsorbent also failed to improve radiocesium decontamination.
\end{abstract}

KEYWORDS: decontamination, radiocesium-contaminated soil, multistep soil washing, plant matter, adsorbent, grinding

\section{Introduction}

The accident at the Tokyo Electric Power Company's Fukushima Daiichi Nuclear Power Plant, caused by the Great East Japan Earthquake on March 11th, 2011, released radioactive materials (mainly ${ }^{134} \mathrm{Cs}$, ${ }^{137} \mathrm{Cs}$ and ${ }^{131} \mathrm{I}$ ) over a wide area, making the reduction of the influence of environmental pollution on human health and the environment a challenging task. Investigation of the contamination and studies of decontamination technology were conducted for more than a year after the accident, and the removal of contamination by ${ }^{134} \mathrm{Cs}$ and ${ }^{137} \mathrm{Cs}$ (hereinafter referred to as "radioactive Cs") is now due to begin in earnest. According

\footnotetext{
* Corresponding author, E-mail: funakawa.masafumi@takenaka.co.jp

DOI : 10.15669/fukushimainsights.Vol.4.125

(C) 2021 Atomic Energy Society of Japan. All rights reserved.

Originally published in Transactions of the Atomic Energy Society of Japan (ISSN 1347-2879), Vol. 11, No. 4, p.272-280

(2012) in Japanese. (Japanese version accepted: August 22, 2012)
} 
to the decontamination-related guidelines ${ }^{1)}$, surface soil removal is the main recommended soil decontamination method. Plowing and removing the surface soil enables the soil to be decontaminated elsewhere. However, all of the decontaminated topsoil needs to be placed in interim storage facilities and, therefore, there is a possibility that the amount of radioactive Cs-contaminated soil will need to be reduced before this stage.

Soil washing can potentially reduce the amount of radioactive Cs-contaminated soil. Soil washing is a technology that has been applied in many cases to soils contaminated by heavy metals such as lead and arsenic, as well as oil ${ }^{2}$. In general, contaminants are present on the surface of soil particles, and the concentration of contaminants tends to be higher in clay and silt, which have large surface-to-weight and surface-to-volume ratios due to their small particle size, than in sand and gravel fractions, which have a larger particle size ${ }^{2)}$. The soil washing treatment recovers the sand with a larger particle size and gravel fractions as purified soil, and recovers the clay and silt fractions with a smaller particle size as a dehydrated cake which concentrates the contaminants. This is done by cleaning the contaminated soil mechanically with a wash solution, such as water. This is done by cleaning the contaminated soil mechanically with a wash solution, such as water. Since radioactive Cs tends to be adsorbed by the fine-particle fractions in the soil ${ }^{3}$, soil washing is expected to decontaminate radioactive Cs-contaminated soil.

The multistep soil washing described in this paper is a method to separate radioactive Cs-contaminated soil, containing plant matter, into processed twice-treated soil, a dehydrated cake in which the radioactive $\mathrm{Cs}$ is concentrated, and drainage water, by washing the contaminated soil according to the following process: (1) "Initial washing process" to slurry the contaminated soil, (2) "Plant matter separation process" to separate plant matter from the slurry and collect the once-treated soil, and (3) "Second washing process" to add water and an adsorbent into the once-treated soil and grind it. In particular, the plant matter separation process and the second washing process are expected to produce the following effects:

In the plant matter separation process, organic matter to which radioactive $\mathrm{Cs}$ is adsorbed is selectively separated and eliminated securely (with a high degree of accuracy) to improve decontamination and reduce the amount of soil to be processed.

In the second washing process, the surface of soil particles is ground to exfoliate the adsorbed radioactive $\mathrm{Cs}$ in order to enhance the decontamination effect. In addition, an adsorbent, the particle size of which is controlled, is added so that the radioactive Cs is adsorbed to the adsorbent rather than the soil particles. This allows the radioactive Cs to be collected as a dehydrated cake, even if water-soluble radioactive Cs is present in the soil.

As a result of dividing the soil washing treatment into three processes, the removal of radioactive Cs is improved and only the separated plant matter and the dehydrated cake need to be placed in interim storage facilities after soil washing. Therefore, the amount of radioactive Cs-contaminated soil is expected to be reduced.

In this paper, the multistep soil washing experimental results are reported. These experiments were conducted using four types of radioactive Cs-contaminated soil (hereinafter referred to as "contaminated soil"), to clarify the effectiveness of radioactive Cs removal, and the effect of the separation of fine-particle fractions through the separation of plant matter and physical grinding on the removal of radioactive $\mathrm{Cs}$, as well as to quantify the reduction of contaminated soil by the multistep soil washing process. 


\section{Experimental Methods}

\section{Radioactive Cs-contaminated Soils}

The contaminated soils used for the soil washing tests were collected from two locations: three samples from Fukushima Prefecture (Date, Fukushima and Minamisoma Cities) and one from Inzai City, Chiba Prefecture on December 14th-16th and 19th, 2012. At each sampling location, the contaminated soils were sampled by screening the soil using a stainless steel testing screen with a $9.5 \mathrm{~mm}$ mesh after removing the top $1.0-1.5 \mathrm{~cm}$ of soil using a long-shank sickle to eliminate pebbles (diameter $>9.5 \mathrm{~mm}$ ) and plant matter. The soils collected in Date and Fukushima cities are classified as lowland soils, and the ones collected in Minamisoma and Inzai cities are classified as andosols ${ }^{4)}$.

In this paper, the contaminated soils collected from the cities of Date, Fukushima, Minamisoma and Inzai are referred to as Soils A-D, respectively.

\section{Soil Washing Test}

Seven soil washing tests were conducted. Table 1 contains the test parameters. In Cases 1-4, the soil washing test was conducted on the four contaminated soils from A to D with different radioactive Cs concentrations, soil properties, and plant matter mixing conditions, using the same washing process (Protocol 1) to compare the decontamination effects achieved by washing soils with different properties. In addition, in Case 1 and Cases 5-7, the soil washing test was conducted on four samples of contaminated soil A, using different plant matter separation and second washing process conditions (Protocols 1-4) to compare the decontamination effects achieved by the plant matter separation and the second washing process. The conditions changed were: (1) Application of the plant matter separation process, (2) addition of an adsorbent, and (3) use of the grinding process. In this paper, the soil washing procedure in which plant matter separation, adsorbent addition, and grinding were all implemented is referred to as "Protocol 1"; "Protocol 2" is identical to Protocol 1 except for the omission of the plant matter separation process. In "Protocol 3", an adsorbent was not added during the test.

\section{Soil Washing Treatment Test Method}

For the initial washing, $10 \mathrm{~kg}$ of contaminated soil and $10 \mathrm{~kg}$ of water were placed in a drum washer (Kikosha Co., Ltd., KDW0602), and the washing process was conducted for one minute at $40 \mathrm{rpm}$. After the initial washing, the slurry was moved to a $20 \mathrm{~L}$ plastic bucket and agitated to separate the plant matter using a stainless steel testing screen with a $9.5 \mathrm{~mm}$ mesh. The remaining slurry was gravity-separated, at the classification point of $75 \mu \mathrm{m}$, to separate

Table 1 Test cases

\begin{tabular}{|c|c|c|c|c|c|c|c|c|}
\hline \multicolumn{2}{|c|}{ Case number } & Case 1 & Case 2 & Case 3 & Case 4 & Case 5 & Case 6 & Case 7 \\
\hline \multicolumn{2}{|c|}{ Soil } & Soil A & Soil B & Soil C & Soil D & & Soil A & \\
\hline \multicolumn{2}{|c|}{ Soil washing protgocol } & \multicolumn{4}{|c|}{ Protocol 1} & Protocol 2 & Protocol 3 & Protocol 4 \\
\hline${ }^{134} \mathrm{Cs}+{ }^{137} \mathrm{Cs}$ & {$[\mathrm{Bq} / \mathrm{kg}]$} & 12,600 & 9,200 & 12,500 & 5,400 & 12,100 & 12,500 & 12,000 \\
\hline \multicolumn{5}{|c|}{ Initioal soil washing } & Scrubbing & & & \\
\hline \multirow{3}{*}{$\begin{array}{l}\text { Plant matter separetion } \\
\text { and second soil washing }\end{array}$} & Plant matter separetion & 0 & 0 & $\mathrm{O}$ & O & - & 0 & 0 \\
\hline & Adsorbent addition & 0 & 0 & $\mathrm{O}$ & 0 & 0 & - & 0 \\
\hline & Grinding & $\bigcirc$ & 0 & 0 & $\bigcirc$ & $\bigcirc$ & $\bigcirc$ & - \\
\hline
\end{tabular}

* ' $\bigcirc$ ' in the table show the work carried out in the process of plant separation and second soil washing. 
the once-treated soil from the washed wastewater. The separated washed soil was put into the drum washer again after its weight was checked. Water, equivalent to half the weight of the once-treated soil, the adsorbent, equivalent to one percent of the weight of the once-treated soil (Katsuri Inc., with powdered hard zeolite of $75 \mu \mathrm{m}$ or lower), and an iron ball for grinding were put into the drum washer to perform a second washing for one minute at $40 \mathrm{rpm}$.

After the second washing, the slurry was moved to another $20 \mathrm{~L}$ plastic bucket. The slurry was gravity-separated, at the classification point of $75 \mu \mathrm{m}$, to separate the twice-treated soil from the second-washing drainage water. Some of the twice-treated soil was classified into size ranges using stainless steel testing screens and experimental shaking screens with $75 \mu \mathrm{m}$, $125 \mu \mathrm{m}, 250 \mu \mathrm{m}$ and $500 \mu \mathrm{m}$ meshes (Fritsch Japan Co., Ltd., 03-505).

The initial- and second-washed wastewaters were left to stand for over 30 minutes to separate the sediment from the muddy water. Flocculation agents (Taki Chemical Co., Ltd., PAC) (Nippon Poly-Glu Co., Ltd., PGa21Ca) were added to the two muddy water samples at concentrations of $200 \mathrm{mg} / \mathrm{L}$ and $50 \mathrm{mg} / \mathrm{L}$, respectively, to perform the coagulation and sedimentation processes. After the coagulation and sedimentation processes, the supernatant liquid was separated from the sediment. This sediment, mixed with the earlier sediment, was dehydrated using a filter press (Nippon Roka Co., Ltd., FS-076) to separate the filtrate water from the dehydrated cake. Figure 1 shows the sequence of the soil washing test.

\section{Confirmation Items Including Decontamination Effect}

\section{(1) Decontamination and weight reduction effects}

In order to check the effect of soil washing on decontamination and weight reduction, the

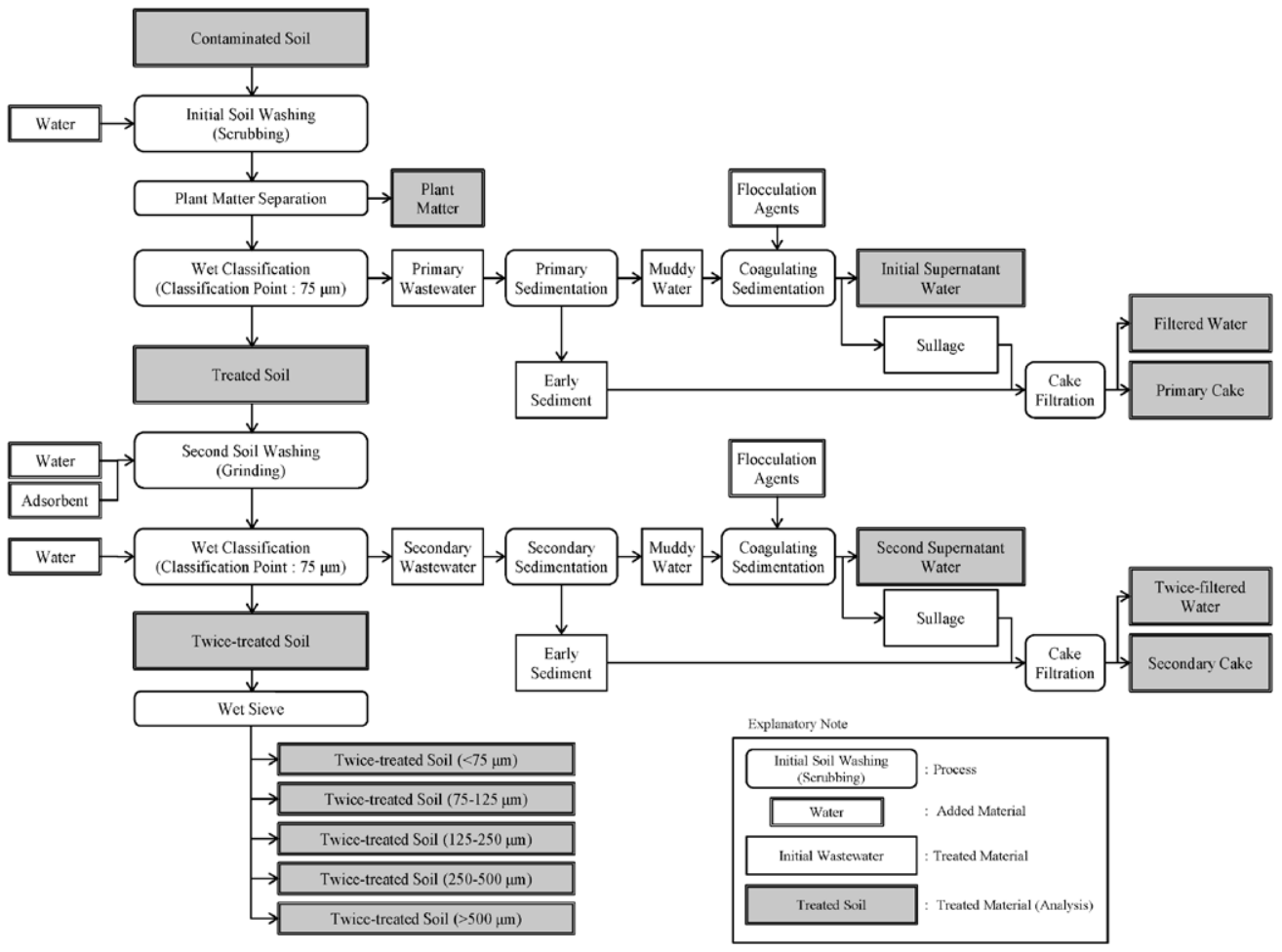

Figure 1 Schematic diagram of a soil washing test 
concentration, weight, and moisture content of the radioactive Cs in the soil and drainage water before and after the washing process were measured. In addition, the particle size distribution of the soil was measured before and after the washing process.

\section{(2) The influence of soil properties on soil washing}

In order to check the decontamination and weight reduction effects of soil washing, the organic carbon concentration and cation exchange capacity (CEC) of the contaminated soil were measured before washing. In addition, as the soil is washed using water, the heavy metals contained in the soil, such as lead and arsenic, may elute to cause yet another form of soil contamination. In order to determine the potential for the re-elution of heavy metals, the amount of heavy metals contained in the soil before the washing process, in the initial dehydrated cake, and the secondary dehydrated cake, was measured.

\section{Analysis Method}

\section{(1) Measurement of radioactive Cs concentration}

The concentration of radioactive Cs was measured in accordance with the method described in the manual on the measurement of the radioactivity of foods in the event of an emergency. A U-8 container and a 2-L Marinelli beaker were used for the soil sample and water sample, respectively. For the measurements, a gamma-ray spectrometer, based on a germanium semiconductor detector was used (manufactured by Canberra, 7500SL (P-type standard coaxial model) relative efficiency: $25 \%$, measurement time: 1,000 seconds).

\section{(2) Measurement of heavy metals etc.}

The heavy metals contained in the soil were analyzed according to the items that fall under the category of class II specified chemical substances stipulated in the Soil Contamination Countermeasures Act.

The soil elution amount and the soil content were analyzed in accordance with methods that comply with the Ministry of Environment Notification No. 18, and the Ministry of Environment Notification No. 19 , respectively.

\section{(3) Cation exchange capacity measurement}

The semimicro-Schollenberger method was used to measure the CEC of the soil.

\section{(4) Organic carbon measurement}

The organic carbon in the soil was measured using the CHN coder method. In addition, as pretreatment, the soil sample was air-dried and visible plant matter eliminated.

\section{(5) Particle size distribution measurement}

The particle size distribution in the soil was measured using a method compliant with JIS A 1204 (Test method for particle size distribution of soils). 
Table 2 Results of testing multistep soil washing (Cases 1-4)

\begin{tabular}{|c|c|c|c|c|c|c|c|}
\hline \multirow{2}{*}{\multicolumn{4}{|c|}{$\begin{array}{c}\text { Case number } \\
\text { Soil } \\
\text { Soil washing protocol }\end{array}$}} & $\begin{array}{l}\text { Case } 1 \\
\text { Soil A }\end{array}$ & $\begin{array}{l}\text { Case } 2 \\
\text { Soil B }\end{array}$ & $\begin{array}{l}\text { Case } 3 \\
\text { Soil C }\end{array}$ & $\begin{array}{l}\text { Case } 4 \\
\text { Soil D }\end{array}$ \\
\hline & & & & \multicolumn{4}{|c|}{ Protocol 1} \\
\hline \multirow{5}{*}{\multicolumn{2}{|c|}{ Contaminated soil }} & ${ }^{134} \mathrm{Cs}+{ }^{137} \mathrm{Cs}$ & {$[\mathrm{Bq} / \mathrm{kg}]$} & 12,000 & 9,200 & 12,500 & 5,400 \\
\hline & & Dry weight & {$[\mathrm{kg}]$} & 8.65 & 8.28 & 7.86 & 9.30 \\
\hline & & Gravel fraction & {$[\%]$} & 7.7 & 12.9 & 8.0 & 3.6 \\
\hline & & Sand fraction & {$[\%]$} & 62.1 & 53.3 & 46.7 & 82.4 \\
\hline & & Clay and silt fraction & {$[\%]$} & 30.2 & 33.8 & 45.3 & 14.0 \\
\hline \multirow{34}{*}{$\begin{array}{l}\text { Treated materials } \\
\text { from the soil } \\
\text { washing process }\end{array}$} & \multirow[t]{3}{*}{ Treated soil } & ${ }^{134} \mathrm{Cs}+{ }^{137} \mathrm{Cs}$ & {$[\mathrm{Bq} / \mathrm{kg}]$} & 2,800 & 2,110 & 4,500 & 1,880 \\
\hline & & Dry weight & {$[\mathrm{kg}]$} & 6.65 & 7.27 & 7.49 & 8.89 \\
\hline & & $\begin{array}{l}\text { Rate of recovery for } \\
\text { treated soil }\end{array}$ & {$[\%]$} & 76.9 & 87.8 & 95.3 & 95.6 \\
\hline & \multirow[t]{3}{*}{ Plant matter } & ${ }^{134} \mathrm{Cs}+{ }^{137} \mathrm{Cs}$ & {$[\mathrm{Bq} / \mathrm{kg}]$} & 30,000 & 7,700 & 6,100 & 24,000 \\
\hline & & Dry weight & {$[\mathrm{kg}]$} & 0.06 & 0.06 & 0.04 & 0.04 \\
\hline & & Enrichment ratio ${ }^{\text {a) }}$ & {$[-]$} & 2.5 & 0.84 & 0.49 & 4.4 \\
\hline & \multirow[t]{2}{*}{ Primary supernatant water } & ${ }^{134} \mathrm{Cs}+{ }^{137} \mathrm{Cs}$ & {$[\mathrm{Bq} / \mathrm{L}]$} & N.D. b) & N.D. & N.D. & N.D. \\
\hline & & Volume & {$[\mathrm{L}]$} & 3.09 & 2.85 & 2.56 & 4.80 \\
\hline & \multirow{2}{*}{ Filtered water } & ${ }^{134} \mathrm{Cs}+{ }^{137} \mathrm{Cs}$ & {$[\mathrm{Bq} / \mathrm{L}]$} & N.D. & N.D. & N.D. & N.D. \\
\hline & & Volume & {$[\mathrm{L}]$} & 3.79 & 1.91 & 2.87 & 1.92 \\
\hline & \multirow[t]{3}{*}{ Primary cake } & ${ }^{134} \mathrm{Cs}+{ }^{137} \mathrm{Cs}$ & {$[\mathrm{Bq} / \mathrm{kg}]$} & 21,100 & 13,300 & 18,700 & 22,700 \\
\hline & & Dry weight & {$[\mathrm{kg}]$} & 2.47 & 2.36 & 2.19 & 0.64 \\
\hline & & Enrichment ratio a) & {$[-]$} & 1.8 & 1.5 & 1.5 & 4.2 \\
\hline & \multirow[t]{2}{*}{ Secondary supernatant water } & ${ }^{134} \mathrm{Cs}+{ }^{137} \mathrm{Cs}$ & {$[\mathrm{Bq} / \mathrm{L}]$} & N.D. & N.D. & N.D. & N.D. \\
\hline & & Volume & {$[\mathrm{L}]$} & 6.88 & 9.34 & 8.07 & 9.91 \\
\hline & \multirow[t]{2}{*}{ Twice-filtered water } & ${ }^{134} \mathrm{Cs}+{ }^{137} \mathrm{Cs}$ & {$[\mathrm{Bq} / \mathrm{L}]$} & N.D. & N.D. & N.D. & N.D. \\
\hline & & Volume & {$[\mathrm{L}]$} & 2.13 & 3.34 & 3.70 & 1.51 \\
\hline & \multirow[t]{3}{*}{ Secondary cake } & ${ }^{134} \mathrm{Cs}+{ }^{137} \mathrm{Cs}$ & {$[\mathrm{Bq} / \mathrm{kg}]$} & 28,000 & 14,500 & 15,700 & 16,400 \\
\hline & & Dry weight & {$[\mathrm{kg}]$} & 0.43 & 0.79 & 1.14 & 0.38 \\
\hline & & Enrichment ratio a) & {$[-]$} & 2.3 & 1.6 & 1.3 & 3.0 \\
\hline & \multirow[t]{5}{*}{ Twice-treated soil } & ${ }^{134} \mathrm{Cs}+{ }^{137} \mathrm{Cs}$ & {$[\mathrm{Bq} / \mathrm{kg}]$} & 1,300 & 1,400 & 3,300 & 1,110 \\
\hline & & Dry weight & {$[\mathrm{kg}]$} & 5.37 & 4.34 & 4.05 & 7.83 \\
\hline & & Gravel fraction & {$[\%]$} & 12.9 & 19.5 & 11.2 & 4.7 \\
\hline & & Sand fraction & {$[\%]$} & 83.3 & 74.3 & 80.0 & 91.8 \\
\hline & & Clay and silt fraction & {$[\%]$} & 3.8 & 6.2 & 8.2 & 3.5 \\
\hline & \multicolumn{2}{|c|}{ Rate of recovery for twice-treated soil } & {$[\%]$} & 62.1 & 52.4 & 51.5 & 84.2 \\
\hline & \multicolumn{2}{|c|}{ Percentage of ${ }^{134} \mathrm{Cs}+{ }^{137} \mathrm{Cs}$ decontamination } & {$[\%]$} & 89.2 & 84.8 & 73.6 & 79.4 \\
\hline & \multirow[t]{5}{*}{ Thrice-treated soil } & ${ }^{134} \mathrm{Cs}+{ }^{137} \mathrm{Cs}$ & {$[\mathrm{Bq} / \mathrm{kg}]$} & 1,300 & 1,400 & 3,300 & 1,110 \\
\hline & & Dry weight & {$[\mathrm{kg}]$} & \multicolumn{4}{|c|}{ Not Analyzed } \\
\hline & & Gravel fraction & {$[\%]$} & \multicolumn{4}{|c|}{ Not Analyzed } \\
\hline & & Sand fraction & {$[\%]$} & \multicolumn{4}{|c|}{ Not Analyzed } \\
\hline & & Clay and silt fraction & {$[\%]$} & \multicolumn{4}{|c|}{ Not Analyzed } \\
\hline & \multicolumn{2}{|c|}{ Rate of recovery for thrice-treated soil } & {$[\%]$} & \multicolumn{4}{|c|}{ Not Analyzed } \\
\hline & \multicolumn{2}{|c|}{ Percentage of ${ }^{134} \mathrm{Cs}+{ }^{137} \mathrm{Cs}$ decontamination } & {$[\%]$} & 94.4 & 89.9 & 77.6 & 83.9 \\
\hline
\end{tabular}

* Comparison of test results at the time of processing 4 soils, using same soil washing protocol.

a) Enrichment ratio $=\left({ }^{134} \mathrm{Cs}+{ }^{137} \mathrm{Cs}\right.$ of treated materials $) /\left({ }^{134} \mathrm{Cs}+{ }^{137} \mathrm{Cs}\right.$ of contaminated soil $)$.

b) N.D.=Not Detected.

\section{Results and Discussion}

\section{The Radioactive Cs-Contaminated Soil Decontamination and Weight Reduction Effects of Multistep Soil Washing}

Table 2 shows the results of the multistep soil washing test for Cases 1-4.

The decontamination effect was evaluated based on the efficiency of radioactive Cs removal from the soil after multistep soil washing. Although the radioactive Cs concentrations in the soils were $12,000 \mathrm{~Bq} / \mathrm{kg}$ (Case 1), 9,200 Bq/kg (Case 2), 12,500 Bq/kg (Case 3) and 5,400 Bq/kg (Case 4) before soil washing, these values decreased to 2,800 Bq/kg (Case 1), 2,110 Bq/kg (Case 2), 4,500 Bq/kg (Case 3) and 1,880 Bq/kg (Case 4) after the initial washing. The initial 
washing and plant matter separation removed $60-80 \%$ of the radioactive Cs from the contaminated soil. The second washing, during which adsorbent addition and grinding were performed, decreased the radioactive Cs concentrations in the twice-treated soils to $1,300 \mathrm{~Bq} / \mathrm{kg}$ (Case 1), $1,400 \mathrm{~Bq} / \mathrm{kg}$ (Case 2), 3,300 Bq/kg (Case 3) and 1,110 Bq/kg (Case 4). The efficiency of radioactive Cs removal from the respective soils was, therefore, $89.2 \%$ (Case 1), $84.8 \%$ (Case 2), $73.6 \%$ (Case 3) and 79.4\% (Case 4), which were within the range of 70-90\%, regardless of the radioactive $\mathrm{Cs}$ concentrations and the particle size distribution of the contaminated soils. In all cases, the second washing increased the efficiency of radioactive Cs removal from the washed soil by approximately $10 \%$.

The weight reduction effect was evaluated based on the weight soil collection rate, after washing. The recovery rate for the once-treated soils was: $76.9 \%$ (Case 1), $87.8 \%$ (Case 2), 95.3\% (Case 3) and 95.6\% (Case 4), and for the twice-treated soils: $62.1 \%$ (Case 1), 52.4\% (Case 2), 51.5\% (Case 3) and 84.2\% (Case 4). The Cs removal from the once-treated soils after plant matter separation reduced the dry-weight percentage in the contaminated soils by 4.4-23.1\%. When the samples were washed a second time, the dry weight percentage was reduced to $15.8-48.5 \%$. In this test, the washed slurry was wet-classified at the classification point of $75 \mu \mathrm{m}$, and grains of $75 \mu \mathrm{m}$ or larger were collected as washed soils. Therefore, if the contaminated soils before washing contained high proportions of clay and silt fractions with a particle diameter of $75 \mu \mathrm{m}$ or smaller, the fraction of the soils larger than the sand fraction with a diameter of $75 \mu \mathrm{m}$ or larger was reduced, resulting in a decrease in the Cs removal rate from the twice-treated soils. As a result, when the proportion of clay and silt fractions in the contaminated soils was $30-40 \%$, the Cs removal rate from the twice-treated soils reached $50-60 \%$. When the proportion of clay and silt fractions in the contaminated soils was at the $10 \%$ level, the Cs removal rate from the twice-treated soils was around $80 \%$.

Compared to the radioactive $\mathrm{Cs}$ concentration in the contaminated soils, the radioactive Cs concentration in the separated plant matter was within the range of $0.5-4.5$ times higher/lower. From this, it was found that radioactive Cs was adsorbed to plant matter in the soils at concentrations similar to, or at higher levels than, the contaminated soils, although the concentration varied depending on the type of contaminated soil. In addition, the radioactive Cs concentration in the initial and secondary dehydrated cakes was 1.3-4.2 times higher than that in the contaminated soils, and it was found that radioactive Cs was concentrated in the dehydrated cakes.

On the other hand, in all cases, from 1 to 4, the concentration of radioactive Cs contained in the drainage waters (initial supernatant liquid, initial filtrate, secondary supernatant liquid, and secondary filtrate) was at, or below, the lower detection limit (approximately $10 \mathrm{~Bq} / \mathrm{L}$ ). It would appear that there is little possibility that the radioactive Cs contained in the soil would be eluted

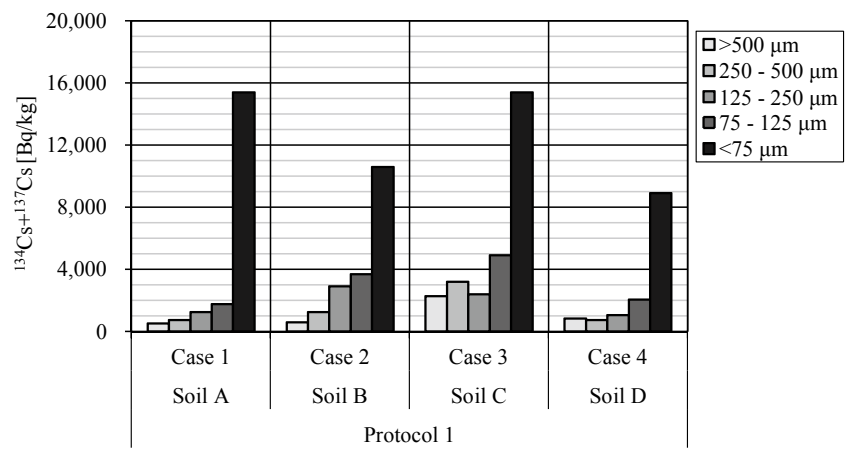

Figure $2{ }^{134} \mathrm{Cs}+{ }^{137} \mathrm{Cs}$ activity in twice-treated soil with different grain-size fractions 


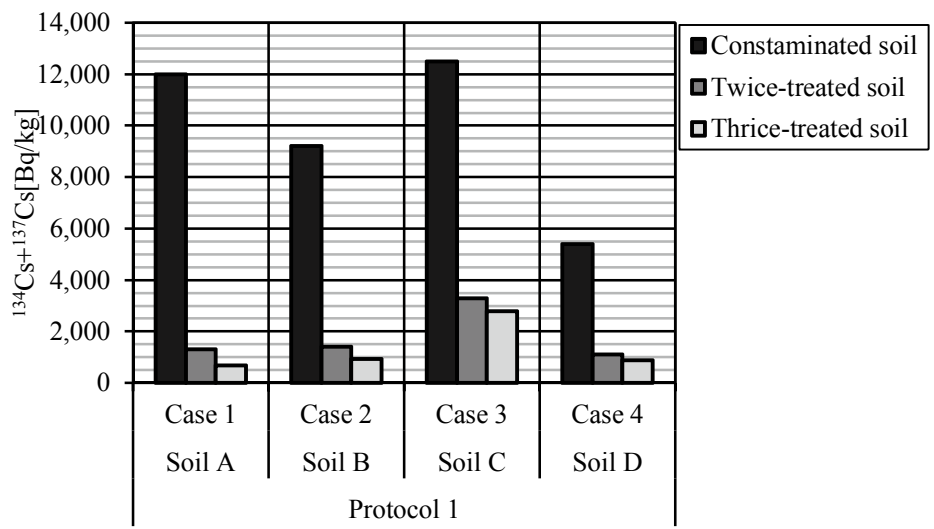

Figure $3{ }^{134} \mathrm{Cs}+{ }^{137} \mathrm{Cs}$ activity in contaminated soil, twice-treated soil, and thrice-treated soil

into water if this method were to be used.

Figure 2 shows the concentration of radioactive Cs in the twice-treated soils with different particle diameter ranges. In all cases, the radioactive Cs concentration was higher in soils with smaller particle diameters. In particular, the radioactive Cs concentration in clay and silt fractions with a particle diameter of $75 \mu \mathrm{m}$ or smaller was 2.8-7.9 times higher than that of the soils with a particle diameter of $75-125 \mu \mathrm{m}$ because radioactive Cs has a tendency to be adsorbed on fine particles. On the other hand, according to the particle size distribution in the twice-treated soils (Cases 1-4), 3-8\% of the silt fraction was present in the twice-treated soils. It is possible that the radioactive Cs concentration in the twice-treated soils was increased by the clay and silt fractions because of insufficient separation of soil particles with a diameter below $75 \mu \mathrm{m}$. Therefore, the twice-treated soils were diluted with an equal amount of water and agitated to re-slurry them. Then, the gravity separation operation was repeated three times at the classification point of $75 \mu \mathrm{m}$ in order to check the radioactive Cs concentration of the thrice-treated soils in which the clay and silt fraction was negligible. Table 2 and Figure 3 show the results. In all cases, the radioactive Cs concentration in the thrice-treated soil was lower than in the twice-treated soils. In order to improve rate of radioactive Cs removal, it is important to eliminate the clay and silt fractions using a mesh with a particle diameter of $75 \mu \mathrm{m}$ or less during multistep soil washing.

\section{Decontamination Effect Differences Due to Soil Washing Process Differences}

\section{(1) Effect of plant matter separation}

It was observed that plant matter in the contaminated soils contained high concentrations of radioactive Cs, so this was removed through gravity separation. Table 3 shows the concentration, weight and total amount of radioactive Cs in the contaminated soils, once-treated soils, eliminated plant matter, and initial dehydrated cakes of Case 5 (Soil A, Protocol 2), from which plant matter was eliminated, and Case 1 (Soil A, Protocol 1) where plant matter was not eliminated.

In Case 1 (Soil A, Protocol 1), the concentration of radioactive Cs in the eliminated plant matter was $30,000 \mathrm{~Bq} / \mathrm{kg}$, which was 2.5 times higher than that in the soil before soil washing, and it was found that the plant matter in the soils contained high concentrations of radioactive Cs. However, the removal rates in Case 1 (Soil A, Protocol 1), where plant matter was eliminated, and Case 5 (Soil A, Protocol 2), where plant matter was not eliminated, were $76.7 \%$ and 
Table 3 Results of testing multistep soil washing (Cases 1 and 5-7)

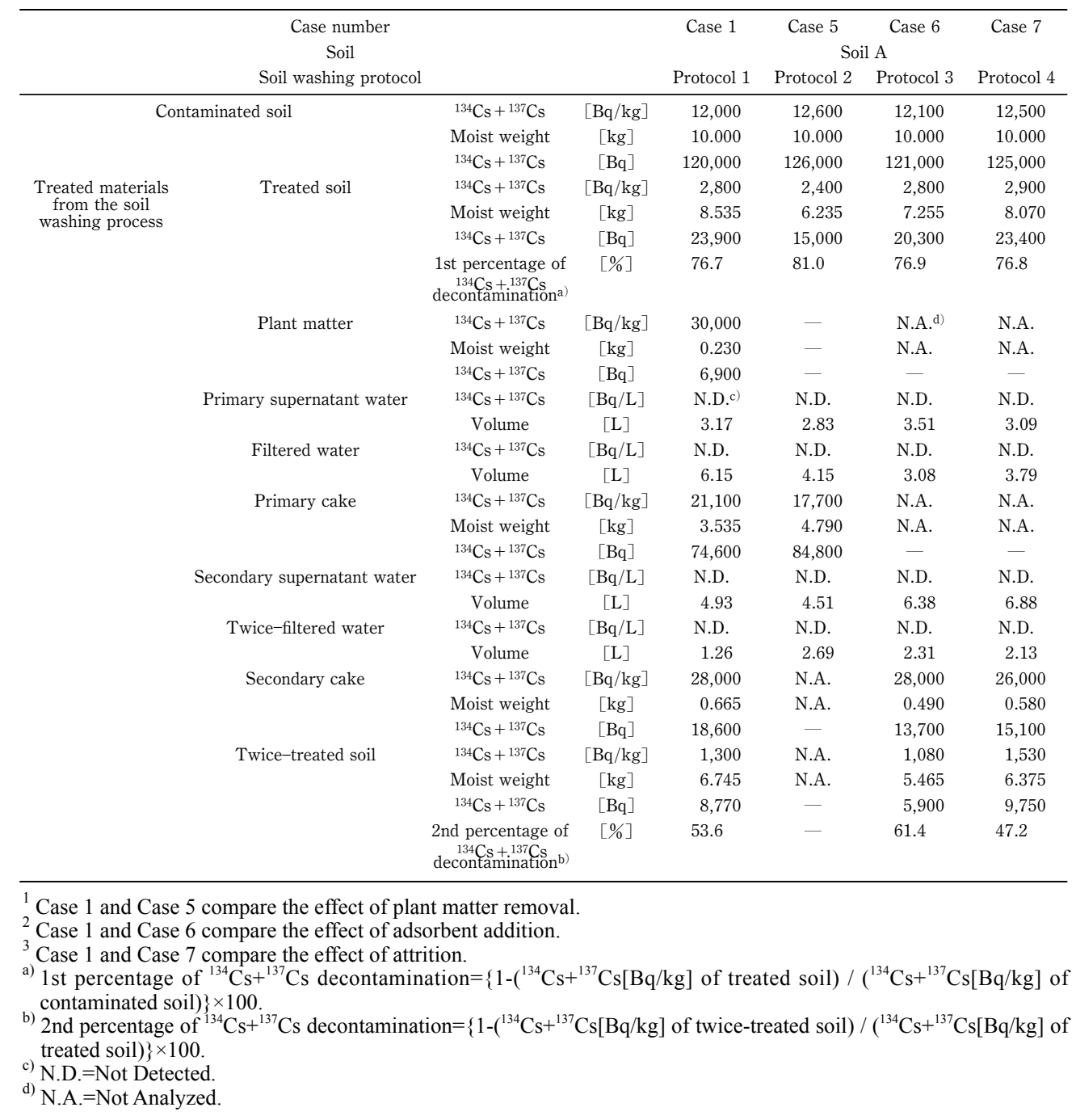

$81.0 \%$, respectively, and no significant difference was observed in the efficiency of radioactive Cs removal in the once-treated soils. The causes of this low removal efficiency are considered to be as follows: First, low amounts of plant matter were contained in the Soil A sample, which was used for the test. The wet weight of the plant matter eliminated in Case 1 (Soil A, Protocol 1) was $0.230 \mathrm{~kg}$, which was $2.3 \%$ of the wet weight of the soil before washing.

Therefore, since the concentration of radioactive Cs in the eliminated plant matter was high, but the amount of plant matter was small, the total amount of radioactive Cs contained in the plant matter was less than one-tenth of that in the once-treated soils and initial dehydrated cakes. Second, the wet classification, after the initial washing, was conducted based on wet separation. It would appear that the plant matter was removed from the contaminated soil regardless of whether the sample underwent the deliberate plant matter elimination process because the soil, with a high specific gravity, and the plant matter, with a low specific gravity, were separated in the initial washing drainage water during the gravity separation process. 


\section{(2) Effect of adsorbent addition}

Table 3 shows the concentration, weight and total amount of radioactive Cs in the once-treated soils, twice-treated soils and the secondary dehydrated cakes of Case 6 (Soil A, Protocol 3), where an adsorbent was not added, and Case 1 (Soil A, Protocol 1), when an adsorbent was added.

In the second washing process, no significant difference in the concentration of radioactive Cs was observed between the twice-treated soils and the secondary dehydrated cakes, regardless of the addition of an adsorbent. In addition, comparing the proportions of radioactive Cs present in the twice-treated soils after the second washing process, no significant difference was observed; these values were $30.1 \%$ in Case 6 (Soil A, Protocol 3) and 32.0\% in Case 1 (Soil A, Protocol 1). Initially, it was expected that the concentration of radioactive Cs in the twice-treated soils would be effectively decreased by preferentially adsorbing water-soluble radioactive Cs, which was present in the soils and eluted into water, to an adsorbent. In reality, however, adding an adsorbent contributed very little to the rate of Cs removal because radioactive Cs forms strong bonds with clay minerals in the soil and is not eluted into water as previously reported $^{3)}$.

\section{(3) Effect of grinding}

Grinding was effective in improving the rate of Cs removal in the twice-treated soils by removing the radioactive Cs adsorbed onto the soil particle surfaces. Table 3 shows the concentration, weight, and total amount of radioactive Cs in the once-treated soils, twice-treated soils, and secondary dehydrated cakes of Case 7 (Soil A, Protocol 4), where grinding was not performed during the second washing process, and Case 1 (Soil A, Protocol 1) where grinding was performed.

During the second washing process, the Cs removal rate was improved by $6.4 \%$ in Case 1 . In addition, comparing the amount of radioactive Cs present in the twice-treated soils, grinding reduced the level of radioactive Cs present by $7.3 \%$ (39.2\% in Case 7 to $32 \%$ in Case 1$)$.

It was confirmed that grinding the soil particle surfaces removed the radioactive Cs adsorbed onto the soil surface, together with the soil particle surfaces themselves, and concentrated them in the dehydrated cake.

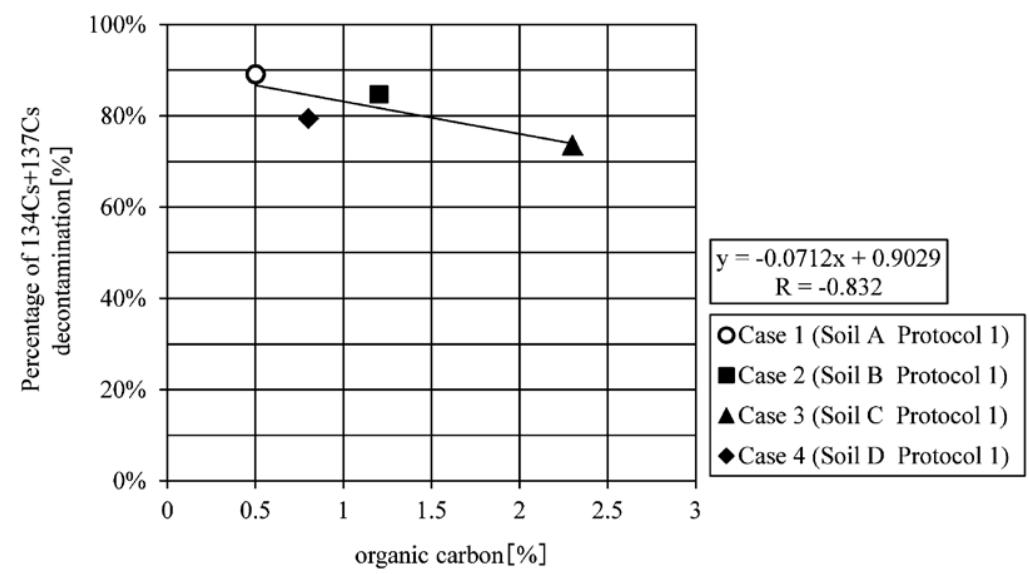

Figure 4 Relationship between organic carbon and the rate of ${ }^{134} \mathrm{Cs}+{ }^{137} \mathrm{Cs}$ decontamination 


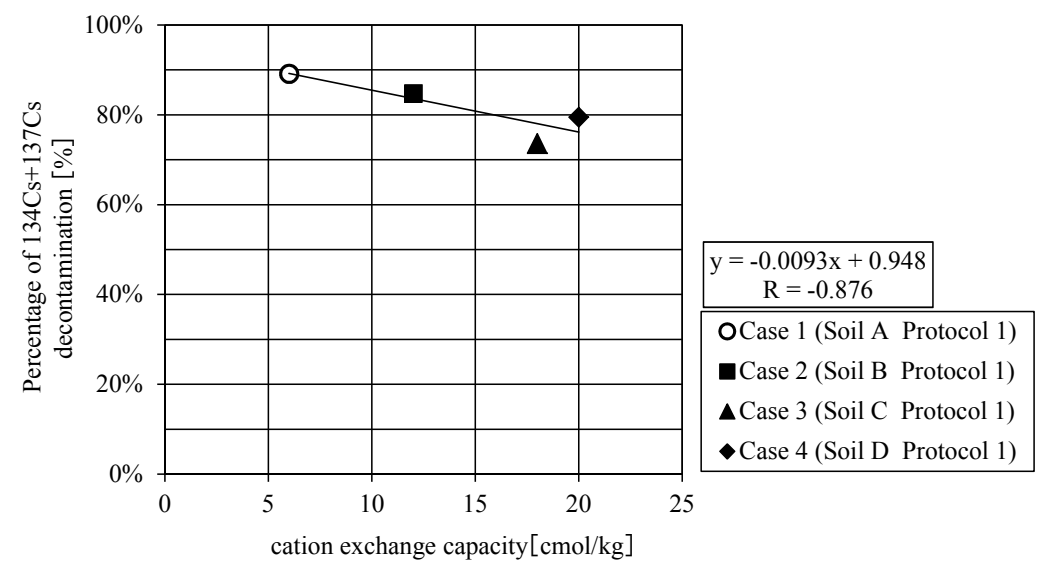

Figure 5 Relationship between cation exchange capacity and the rate of ${ }^{134} \mathrm{Cs}+{ }^{137} \mathrm{Cs}$ decontamination

Table 4 Resulting heavy metals in treated materials (Cases 1-4)

\begin{tabular}{|c|c|c|c|c|c|c|c|c|c|}
\hline \multirow{3}{*}{\multicolumn{2}{|c|}{ Case number and treated materials }} & \multicolumn{8}{|c|}{ Class 2 designated hazardous substances } \\
\hline & & \multicolumn{4}{|c|}{ Soil leachate ${ }^{\text {a) }}[\mathrm{mg} / \mathrm{L}]$} & \multicolumn{4}{|c|}{ Soil concentration $^{\text {b) }}[\mathrm{mg} / \mathrm{kg}]$} \\
\hline & & $\mathrm{Pb}$ & As & $\mathrm{F}$ & B & $\mathrm{Pb}$ & As & $\mathrm{F}$ & B \\
\hline \multirow{4}{*}{$\begin{array}{c}\text { Case 1 } \\
\text { (Soil A Protocol 1) }\end{array}$} & Contaminated soil & N.D.c) & N.D. & N.D. & N.D. & N.D. & N.D. & 69 & N.D. \\
\hline & Primary cake & N.D. & N.D. & N.D. & N.D. & N.D. & N.D. & 91 & N.D. \\
\hline & Secondary cake & N.D. & 0.001 & 0.42 & N.D. & 9 & N.D. & N.D. & N.D. \\
\hline & Twice-treated soil & N.D. & N.D. & N.D. & N.D. & N.D. & N.D. & N.D. & N.D. \\
\hline \multirow{4}{*}{$\begin{array}{c}\text { Case } 2 \\
\text { (Soil B Protocol 1) }\end{array}$} & Contaminated soil & 0.002 & N.D. & 0.13 & N.D. & 18 & N.D. & N.D. & N.D. \\
\hline & Primary cake & N.D. & N.D. & 0.21 & 0.01 & 26 & N.D. & N.D. & N.D. \\
\hline & Secondary cake & 0.001 & 0.002 & 0.34 & 0.01 & 33 & N.D. & N.D. & N.D. \\
\hline & Twice-treated soil & N.D. & N.D. & 0.15 & N.D. & 5 & N.D. & N.D. & N.D. \\
\hline \multirow{4}{*}{$\begin{array}{c}\text { Case } 3 \\
\text { (Soil C Protocol 1) }\end{array}$} & Contaminated soil & 0.002 & 0.001 & 0.49 & 0.01 & 18 & N.D. & 100 & N.D. \\
\hline & Primary cake & N.D. & N.D. & 0.23 & 0.01 & 33 & N.D. & 230 & N.D. \\
\hline & Secondary cake & 0.002 & 0.001 & 0.59 & 0.01 & 5 & N.D. & 220 & N.D. \\
\hline & Twice-treated soil & 0.002 & 0.001 & 0.59 & N.D. & N.D. & N.D. & N.D. & N.D. \\
\hline \multirow{4}{*}{$\begin{array}{c}\text { Case } 4 \\
\text { (Soil D Protocol 1) }\end{array}$} & Contaminated soil & N.D. & N.D. & 0.09 & 0.02 & N.D. & N.D. & N.D. & N.D. \\
\hline & Primary cake & N.D. & N.D. & N.D. & 0.02 & 19 & N.D. & 58 & N.D. \\
\hline & Secondary cake & 0.007 & 0.001 & 0.26 & 0.02 & 17 & N.D. & 76 & N.D. \\
\hline & Twice-treated soil & N.D. & 0.001 & 0.09 & N.D. & N.D. & N.D. & N.D. & N.D. \\
\hline \multicolumn{2}{|c|}{ Designation standard } & 0.01 & 0.01 & 0.8 & 1 & 150 & 150 & 4,000 & 4,000 \\
\hline \multicolumn{2}{|c|}{ Detamination limit } & 0.001 & 0.001 & 0.08 & 0.01 & 5 & 2 & 50 & 5 \\
\hline
\end{tabular}

a) Soil leachate: Quantity of the toxic substance eluted from soil to water.

b) Soil concentration: Quantity of the toxic substance taken into the body when contaminated soil is ingested.

c) N.D. $=$ Not Detected.

${ }^{*} \mathrm{Cd}, \mathrm{Cr}^{6+}, \mathrm{CN}, \mathrm{Hg}$, and Se were not detected in all test cases and all treated materials.

\section{Influence of Soil Properties on Soil Washing}

Figure 4 shows the relationship between organic carbon in the contaminated soils and the rate of radioactive Cs removal, while Figure 5 shows the relationship between the CEC and the rate of radioactive Cs removal. Both these figures are based on results from Cases 1-4.

According to Figure 4 and Figure 5, the reduction in radioactive Cs declined as the organic carbon and CEC increased. The correlation coefficients between the rate of radioactive Cs removal and organic carbon, and between the rate of radioactive Cs removal and CEC were -0.832 and -0.876 , respectively. The cause of the negative correlation is thought to be twofold: First, part of the radioactive Cs on the soil surface is adsorbed into organic substances in the 
soil, such as plant matter ${ }^{5)}$, and so the concentration of Cs is intrinsically linked with the amount of organic matter. In the experiments, the rate of radioactive Cs removal likely declined because organic substances were not eliminated by the soil washing process, causing a relative increase in the amount of organic substances, and therefore radioactive Cs, in the contaminated soil.

Second, the CEC, which shows the number of cations which can be retained in the soil, is also linked to the concentration of radioactive Cs. If the CEC is high, it means the number of cations, including radioactive Cs ions, per unit weight that can be adsorbed into the soil is high. Therefore, it is likely that the rate of radioactive Cs removal declined because soil, into which a larger amount of radioactive Cs was adsorbed, was included in the soil that underwent secondary soil washing.

Table 4 shows the concentration of heavy metals before and after multistep soil washing. In four kinds of contaminated soils, before undergoing multistep soil washing, contamination due to heavy metals was not observed. In addition, in the twice-treated soils, initial dehydrated cakes and secondary dehydrated cakes, which were generated as a result of multistep soil washing, the soil elution amount and the soil content of particular heavy metals did not exceed the criteria stipulated in the Soil Contamination Countermeasures Act. In this test, only water was used to clean the soils; the $\mathrm{pH}$ levels of the soil and water do not change significantly throughout the process. Therefore, the risk of heavy metal re-elution and subsequent recontamination is low, even if the radioactive Cs-contaminated soils are washed using only water.

\section{Conclusions}

Multistep soil washing experiments were conducted using radioactive Cs-contaminated soils collected from the Fukushima Prefecture and Inzai City, Chiba Prefecture.

As a result of initial and secondary washing, the radioactive Cs concentrations of the contaminated soils, which were initially between $9,200-12,500 \mathrm{~Bq} / \mathrm{kg}$, were reduced to 1,300 $3,300 \mathrm{~Bq} / \mathrm{kg}$. The rate of radioactive Cs removal was $73-90 \%$. In one case, where soil with a radioactive $\mathrm{Cs}$ concentration of $5,400 \mathrm{~Bq} / \mathrm{kg}$ was washed, the radioactive Cs concentration of the twice-treated soil was reduced to $1,110 \mathrm{~Bq} / \mathrm{kg}$, giving a rate of radioactive Cs removal of $79.4 \%$. Regardless of the radioactive Cs concentration and the soil properties, there was no significant difference in the removal rate of radioactive Cs removal. The radioactive Cs concentration in twice-treated soils tended to be higher in soils with smaller diameter particles. In particular, the radioactive Cs concentration in the clay and silt fractions, with a particle diameter of $75 \mu \mathrm{m}$, was 2.8-7.9 times higher than that in soils with a particle diameter of 75-125 $\mu \mathrm{m}$. Securely eliminating the clay and silt fractions was the most effective way of improving the rate of radioactive Cs removal from the twice-treated soils.

The efficiency of collection from the twice-treated soil is dependent on the proportion of the clay and silt fractions in the contaminated soil. When contaminated soils, in which the proportion of the clay and silt fractions was around $30 \%$, were washed, the recovery rate from the twice-treated soil was between $50-60 \%$. Collecting more than $50 \%$ of the contaminated soil as twice-treated soil, radioactive Cs-contaminated soil was reduced to the weight ratio of radioactive Cs-contaminated soil to $50 \%$ or lower. Since multistep soil washing reduces the weight ratio of contaminated soil by about half, it is expected to be utilized to reduce the weight of radioactive Cs-contaminated soils, enabling effective storage.

Multistep soil washing experiments were conducted with different plant matter elimination and secondary washing processes. The results confirmed that radioactive Cs was adsorbed to 
plant matter in the contaminated soils in high concentrations. In addition, grinding the soil surface improved the rate of radioactive Cs removal from the twice-treated soils. On the other hand, since the radioactive Cs contained in the soils formed strong bonds with the soil minerals and did not elute into water, adding an adsorbent during soil washing contributed very little to improving the rate of radioactive Cs removal. Furthermore, radioactive cesium did not elute into the treatment water, even though only water was used. In addition, no elution of heavy metals was observed from the samples used in this study. Therefore, there is little risk that the washing drainage water generated by this method would cause further soil contamination.

It was suggested that the rate of radioactive Cs removal had a negative correlation with the proportion of organic carbon in the contaminated soils as well as the CEC.

This test summarizes the results of the "Study On Multistage Soil Decontamination Treatment for the Radioactive Cesium-Contaminated Soil Mixed With Plants or Vegetables" conducted in 2011 as part of "Decontamination Pilot Projects for the Environmental Remediation of Evacuation Areas Contaminated With Radioactive Materials Discharged From The Fukushima Daiichi Nuclear Power Plant Accident" which was undertaken by the Japan Atomic Energy Agency, contracted by the Cabinet Office.

\section{References}

1) Ministry of the Environment, Josen Kankei Gaidorain, Ministry of the Environment, Tokyo, 2-63-2-65, (2011). [in Japanese]

2) Jiban Kogaku Kai, Zoku Dojo Chikasuiosen-notyosa Yosoku Taisaku, Jiban Kogaku Kai, Tokyo, 86-89, ISBN 978-4-88644-924-5 (2008). [in Japanese]

3) O. Nakamura, Y. Adachi, S. Iwata, Tutinokoroidogensyo Tutimizukankyonobutsurikagakutokogakutekikiso, Japan Scientific Societies Press, Tokyo, 23-41, ISBN 978-4762230103 (2003). [in Japanese]

4) National Institute for Agro-Environmental Sciences, Soil Database Browsing System, http://agrimesh. dc.affrc.go.jp/soil_db/index.phtml. [in Japanese]

5) H. Tsukada, A. Takeda, S. Hisamatsu, J. Inaba, "Concentration and specific activity of fallout ${ }^{137} \mathrm{Cs}$ in extracted and particle-size fractions of cultivated soils," J. Environ. Radioact., 99:6, 875-881 (2008). 


\title{
Long-Term Outlook for Global Natural Uranium and Uranium Enrichment Supply and Demand Situations after the Impact of Fukushima Daiichi Nuclear Power Plant Accident
}

\author{
Yuhji MATSUO ${ }^{1, *}$ and Tomoko MURAKAMI ${ }^{2}$ \\ 1,2 The Institute of Energy Economics, 1-13-1 Kachidoki, Chuo-ku, Tokyo 104-0054, Japan
}

\begin{abstract}
In this paper, the authors propose long-term projections of global nuclear power generation, uranium production, and uranium enrichment capacities by region, and estimate the trade flows of natural uranium and uranium enrichment activities in 2020 and 2035. In spite of the rapid nuclear power generation capacity growth expected especially in Asia, natural uranium and uranium enrichment trade will not be tightened by 2020 . This is due to the projected increase in both natural uranium production and uranium enrichment capacities, which may cause a drop in natural uranium and uranium enrichment prices. Therefore, there is a strong possibility that current capacity expansion projects will be considerably delayed. However, in the "high demand scenario", where nuclear expansion will be accelerated due to growing concerns about global warming and energy security issues, additional investments in uranium production and enrichment facilities will be required by 2035. In Asia, the self-sufficiency ratio for both natural uranium supply and uranium enrichment activities will remain relatively low until 2035. However, the Herfindahl-Hirschman $(\mathrm{HH})$ index of natural uranium and uranium enrichment activity trade to Asia will be lowered considerably up to 2035 , indicating that nuclear capacity expansion can contribute to enhancing energy security in Asia.
\end{abstract}

KEYWORDS: nuclear fuels, enrichment, natural uranium resources, uranium production, reprocessing, tail assay, outlook, trade balance, numerical analysis, self-sufficiency, energy security

\section{Introduction}

The Fukushima Daiichi Nuclear Power Plant Accident in the wake of the Great East Japan Earthquake caused the massive release of radioactive materials and the long-term evacuation of local residents. This accident had a large impact on nuclear energy policies both in Japan and overseas countries. According to the Strategic Energy Plan ${ }^{1)}$, the Japanese government had originally aimed to newly build nine nuclear power reactors and fourteen reactors by 2020 and 2030 , respectively. However, these plans are now under review because of the accident, and a significant revision of the nuclear energy policy is definitely required. Nevertheless, Japan is

* Corresponding author, E-mail: matsuo@edmc.ieej.or.jp

DOI : 10.15669/fukushimainsights. Vol.4.138

(C) 2021 Atomic Energy Society of Japan. All rights reserved.

Originally published in Transactions of the Atomic Energy Society of Japan (ISSN 1347-2879), Vol. 11, No. 4, p.281-303

(2012) in Japanese. (Japanese version accepted: August 31, 2012) 
required by international legislation to reduce greenhouse gas emissions, and it is unclear how the country now views the role of nuclear energy in this respect.

Immediately after the Fukushima Accident, Germany stopped the operation of seven old nuclear reactors and adopted a policy to successively decommission the remaining reactors by 2021. Furthermore, Italy had stopped operation of all nuclear power plants but had recently considered building new nuclear power plants; however, following a national referendum after the accident, over $90 \%$ of the nation were found to be opposed to resuming the program for building new nuclear power plants. Thus, the Fukushima Accident had a direct impact on certain European countries. In Asian countries (especially China and India), where a rapid future growth in power demand is expected, there has been no change in the situation, and a rapid expansion of the generating capacity of nuclear power plants is still expected. Similarly, in countries with advanced nuclear technologies (including the US, France, Russia, and Korea), no changes have been made to their nuclear policies, as these enable industrial development, the provision of a stable energy supply, and the potential to reduce greenhouse gas emissions.

Therefore, at least in the current situation, the expansion of nuclear energy use is expected to continue worldwide in the long term, because it provides the means to reduce fossil fuel consumption and greenhouse gas emissions.

When considering long-term perspectives of nuclear power generation, one of the most important issues is uranium resource availability. The demand for uranium will continue to expand in the long term, as long as we continue using the current light-water reactor (LWR) technologies. However, several factors could contribute to mitigating the demand expansion, such as policy changes after the Fukushima Accident, the introduction of Fast Breeder Reactors (FBRs), and a fuel transition by introducing the thorium cycle. Nevertheless, even when considering these factors, an increased demand is still expected. It is thus significantly important to evaluate whether a sufficient amount of uranium can be supplied, and if so, where and how much can be supplied, as this will enable the country to plan for the development of future nuclear power generation with respect to the growing demand for global energy and requirements to reduce greenhouse gas emission. In addition, for the use of existing LWRs, which use enriched uranium as fuel, it is necessary to consider the uranium enrichment (UE) supply capacity when considering the supply and demand of uranium, because demand for natural uranium (NU) and an amount of UE services have a trade-off relationship.

As nuclear power generation provides a significant proportion of energy supplies, various organizations (including the International Energy Agency (IEA) ${ }^{2,3)}$, the International Atomic Energy Agency (IAEA) ${ }^{4}$, the Organisation for Economic Co-operation and Development $(\mathrm{OECD})^{5)}$, United States Department of Energy (U.S. DOE) ${ }^{6}$ and the World Nuclear Association (WNA) ${ }^{7}$ have provided future nuclear outlooks. When future energy demands or power generation are forecast, it is common to use econometrics or cost-optimization models. However, as nuclear power development is largely dependent on national policies, nuclear power generation projections are made based on detailed research of current energy supplies, demand situations, and energy policies, rather than on these models. Such a method appears to be particular to the nuclear energy outlook.

The future uranium production outlooks include those provided by the $\mathrm{OECD}^{8)}$ and $\mathrm{WNA}^{9)}$; these examine uranium mines and their development projects and estimate the uranium production of each country, region, and the world, and associated future expansion based on the study results. However, these outlooks are more valuable as a reference material rather than providing a calculation method.

Few studies have used the supply and demand prospects to conduct a detailed analysis of future uranium demands. For example, Komiyama et al. ${ }^{10)}$ evaluated uranium supply and 
demand scenarios by 2030 and 2100 and derived policy implications by uniquely projecting the future global nuclear power generating capacity; their study referred to the OECD's uranium supply and demand forecast. However, the study separately simulated the global demand and production of uranium and evaluated surplus production and supply shortages by comparing simulation results, and it did not sufficiently consider elements specific to nuclear fuel, such as UE. Murakami ${ }^{11)}$ took this a step further and found that the possibility of tight supply-demand situations of NU in 2030 will largely change due to the changes in tail assay, considering demand of NU and UE service demand become trade-off relationship by changing tail assay in uranium enrichment. However, in this respect, only the relationship between the NU demand, the amount of UE services, and enriched uranium demand were globally recognized, and the study does not answer detailed questions such as what the supply and demand of uranium will be in Asia, which will be the core region where nuclear power generation will expand in the future. As most of the trade transactions and cost data are not disclosed, there are a lack of studies that forecast future trade flow by region.

Therefore, by referring to the world energy supply and demand outlook ${ }^{12)}$ created by the authors, this study provides a projection of nuclear power generation by global region, and evaluates the future demand for NU and the generating capacity of UE plants by referring to previous studies and using information obtained from websites of nuclear related companies. By considering the results and referring to current records as of 2009 , we then estimate the future trade flow based on the proposed evaluation model using the least-squares method.

The contribution of this study is twofold: first, the study includes observations about a possibility of long-run supply of uranium by estimating the world supply and demand amounts of NU and UE services until 2035 in consideration of their relationship with prices. Generally a shear of fuel cost on power generation cost of nuclear power is low, and then, nuclear power plant construction plans are affected by national policies as first, and construction cost and money supply circumstances as second. Once a nuclear power plant is established, it generates power as much as possible as a basic electricity supplier, from the view points of economy. Therefore, fuel prices have a low impact on the amount of electricity generated by nuclear power and the demand for uranium fuel. On the other hand, the relative relationship between prices of NU and UE affects cost optimum tails, and changes the demands of NU and UE through tails. This study evaluates their supply and demand balance in 2020 and 2035 based on this relationship.

Second, this study provides a regional fuel supply and demand balance by estimating future trade flow, especially the supply and demand trends in Asia, where a rapid growth in future energy demand is expected. It is anticipated that the domestic production of fossil fuels in Asia will not be able to meet the demand expansion, and Asian countries will need to depend more on other regions, such as the Middle East ${ }^{12}$. Therefore, increasing the amount of nuclear power generation would mean diversification of energy supply; however, it is unclear how much dependence on foreign energy sources, through production and UE service, can be reduced.

From these perspectives, providing an estimate of future trade flows has not yet been attempted in previous studies.

\section{Methodology and Assumptions}

\section{Outlook for Nuclear Generating Capacity and Demand for Uranium}

\section{(1) Nuclear generating capacity}

Immediately after the Fukushima Accident, Germany halted operation of its seven domestic 
nuclear reactors that had been in operation prior to 1980 . This halt was said to have been expedited in lieu of the facilities being confirmed safe; however, on June 6, 2011, the government adopted a policy to immediately decommission the seven reactors, and to decommission the remaining nuclear reactors from 2013 to 2021. A national referendum was held in Italy to obtain consensus as to whether operation of the nuclear reactors should be resumed, and over $90 \%$ of the nation opposed the motion; therefore, Italy will not resume its nuclear program for the foreseeable future. However, while the Fukushima Accident had a direct impact on some countries, especially those in Europe, and forced them to stop depending on nuclear power, certain countries retained their nuclear promotion policies: countries such as the US, France, Russia, and Korea, which have actively promoted nuclear energy generation for many years, and emerging countries such as China and India.

In light of such circumstances, this study uses current policies, power development plans, and energy supply and demand trends to provide a forecast until the year 2035 of the nuclear generating capacities of all countries that currently use and will possibly use nuclear power. Based on this forecast, we then estimate nuclear generating capacities by region for North America, Latin America, Europe, Africa, the former Soviet Union (FSU), the Middle East, and Asia. The estimation is based on two scenarios: a high demand scenario and a low demand scenario. In the former scenario, it is assumed that countries will actively continue expanding their nuclear generating capacities; and in the latter, it is assumed the Fukushima Accident had a maximum impact on nuclear policies or that other issues, such as funding, are hampering nuclear development. Overviews of these scenarios per region are as follows:

(a) North America

The US is the largest nuclear power player with 103 nuclear power reactors in operation. However, as previously mentioned, recent nuclear power plant construction initiatives have not been very successful despite it previously being referred to as the Nuclear Renaissance. This is because of the rapid growth in unconventional natural gas production and also that the use of nuclear energy is not the largest mean of reducing greenhouse gas emissions for the US ${ }^{13)}$. However, a combined construction and operation license (COL) was issued in 2012 for the first time, their policy to retain and expand their nuclear generating capacity is still firmly maintained: a 60-year operational life extension has been approved for over half of existing nuclear reactors and another 20-year operational life extension is also being considered. In the low demand scenario, it is assumed that the generation capacity will increase by approximately only $10 \mathrm{GW}$ by 2035 , which is in line with the projection by the U.S. Department of Energy ${ }^{14)}$; and in the high demand scenario, it is assumed power plant capacity will increase up to $130 \mathrm{GW}$ by 2035 , with respect to construction of nuclear reactors that are currently in the planning stage.

Canada is a country with a long nuclear power development history that mainly uses the country's unique heavy water reactor (HWR) technology. Ontario province, which has the largest energy demand and is home to 16 of the 18 nuclear reactors in the country, plans to expand its nuclear generating capacity ${ }^{15)}$.

Therefore, it is assumed that the nuclear generating capacity of North America will gradually increase from $119 \mathrm{GW}$ in 2010 up to $129 \mathrm{GW}$ in 2035 in the low demand scenario, and up to $147 \mathrm{GW}$ in the high demand scenario.

(b) Latin America

The Latin American countries currently using nuclear energy are Mexico, Brazil, and Argentina. In Mexico, it is assumed that the capacity of nuclear power plants will increase slightly due to power uprates. In Brazil, two power plants are currently in operation at $2.0 \mathrm{GW}$. The construction of Angra III has been suspended, but this is expected to be resumed, and operation is scheduled to start in 2013. Brazil's nuclear power generation capacity may increase 
significantly with a future growth in energy demands. In Argentina, two nuclear reactors are in operation at $1 \mathrm{GW}$, one of which is likely to be decommissioned before 2030. In this country, construction of a heavy-water reactor (Atucha II) was underway as the third nuclear reactor, but this was suspended in 1994 due to financial difficulties and other reasons. Although construction of this plant is expected to be resumed in the future, in the low demand scenario it was considered that the construction would not be resumed, mainly because of financial issues. However, a few new constructions were assumed in the high demand scenario.

Thus, it is assumed that the nuclear generating capacity of Latin America will increase from $4 \mathrm{GW}$ in 2010 to $6 \mathrm{GW}$ in 2035 in the low demand scenario, and to $17 \mathrm{GW}$ in the high demand scenario.

(c) Europe

In Europe, many nuclear reactors were constructed between 1970s and 1980s and the generation capacity has rapidly expanded. However, owing to the lack of relative competitiveness of nuclear power associated with low crude oil prices, there was a reduction in the construction of new nuclear power plants. Moreover, in some countries, policies included decommissioning of nuclear reactors stepwise have been taken.

In France, where utilization of nuclear power is widespread most, François Hollande expressed a commitment to reducing the share of nuclear power in national electricity generation down to $50 \%$ by 2025 , when he won the presidential election in 2012 . However, it is highly likely that the country will not to make any changes for a while, given that a schedule for closing nuclear reactors has not yet been provided, and it was announced in April 2012 that the nuclear agreement previously signed with the Green Party would be terminated.

In this study, we assume that France will retain its current high nuclear share until 2035. However, we assume that countries including Germany, Italy, Switzerland, and Belgium, that identified their clear direction toward a nuclear phase-out after the Fukushima Accident, will completely eliminate their nuclear power plants by 2030 in the low demand scenario. Meanwhile, as in the example of Sweden, that has not yet achieved nuclear power phase-out policies that were previously determined, we assumed that these countries will also retain parts of their nuclear generating capacities in 2035 in the high demand scenario.

In the future, although new nuclear power plants will be constructed at a certain level in Europe, it is supposed that the overall nuclear generating capacity will remain at the same level, or it will slightly decline because many existing aging reactors will be decommissioned. In consideration of the above situations, it is assumed that the nuclear generating capacity of $141 \mathrm{GW}$ in 2010 will decline to $107 \mathrm{GW}$ in 2035 in the low demand scenario and increase to $161 \mathrm{GW}$ in the high demand scenario.

(d) Former Soviet Union

The countries in the former Soviet Union with large nuclear generating capacities are Russia and the Ukraine. Russia is one of the world's largest oil and gas producers, and it regards its energy resources as key tools in achieving diplomatic relations. No major changes have been seen in Russia's nuclear policies, as previously mentioned. In addition, the Ukraine is supposed to continue building new nuclear reactors and has been planning to construct 14 new units by 2030 .

This region (and Russia in particular) is expected to largely enhance its nuclear generating capacity by 2035 . In this study, the capacity is assumed to increase from $37 \mathrm{GW}$ in 2010 to $59 \mathrm{GW}$ in 2035 in the low demand scenario, and to $85 \mathrm{GW}$ in the high demand scenario in 2035. In the high demand scenario, new nuclear power plant construction in Kazakhstan is also assumed.

(e) Middle East, Africa, and Oceania 
The Middle East is a large oil and gas producing area; however, many countries in the area have recently begun to consider implementing nuclear energy with the aim of diversifying energy resources and desalinating seawater. To be more specific, not only Iran, which has imported an LWR from Russia and has been uniquely developing nuclear energy, but also the UAE, Saudi Arabia, Kuwait, and Jordan are considering constructing new nuclear power plants. The UAE has already decided to construct a new nuclear plant and placed an order to a Korean plant manufacturer. In this study, we assumed that only the UAE would construct new nuclear reactors by 2035 in the low demand scenario, and in the high demand scenario other countries also proceed with power plant construction.

In the African continent, French LWRs have been in operation since 1984 in South Africa. The country planned to introduce their uniquely developed gas reactor, called the Pebble Bed Modular Reactor (PBMR), in the future, but this has been delayed due to the country's economic deterioration. We assume that South Africa's nuclear generating capacity will not increase from $1.9 \mathrm{GW}$ in 2010 in the low demand scenario, but that it will increase up to $6 \mathrm{GW}$ in 2035 in the high demand scenario.

(f) Asia

Even in the 1990s, when new nuclear power plant construction was stagnant in Europe and the US, Asian countries (including Japan and Korea) continued to construct new nuclear power plants. Today, emerging Asian countries (such as China, India, and those in Southeast Asia) have begun to show a rapid increasing interest in nuclear power generation, and it is expected that most new global nuclear power plant construction will be conducted in Asia.

Prior to the Fukushima Accident, China had 13 nuclear reactors in operation in early 2011, with an output of $10.85 \mathrm{GW}$ and another 30 power plants with an output of $33 \mathrm{GW}$ (which is about three times more than its current capacity) under construction at that time. However, after the accident, China suspended approvals for new construction plans, which forced them to slightly delay their new plans to construct plants providing a capacity of 70 to $80 \mathrm{GW}$ by 2020. Nevertheless, they moved steadily forward with the construction of the new plants that had been approved before the accident, and are expected to resume construction of new power plants once their safety has been confirmed. Rapid progress is expected in China towards such new power plant construction. India is also planning the large scale construction of LWRs with foreign technology, along with development employing the thorium cycle using their domestic resources, which they have been engaged with for a long period of time. No change was made to India's nuclear policy following the Fukushima Accident, as seen by the launch of the new Rajasthan VII and VIII constructions in July 2011.

In the high demand scenario, it is assumed that a great number of power plants will be constructed in line with national targets, whereas in the low demand scenario, we assumed lower nuclear capacities due to minor changes in nuclear policies made after the Fukushima Accident. In Southeast Asian countries, nuclear policies are being reviewed in the wake of the accident. In the low demand scenario, no new construction is expected until 2035, except in Vietnam where a concrete construction plan is already underway. The high demand scenario assumes that about four nuclear power plants per country will be constructed.

Therefore, in Asia, it is assumed that the nuclear power generation capacity will increase from $85 \mathrm{GW}$ in 2010 to $190 \mathrm{GW}$ in 2035 in the low demand scenario, but this will rapidly increase to $347 \mathrm{GW}$ in the high demand scenario.

These results indicate that the global generating capacity of nuclear power plants will increase from $390 \mathrm{GW}$ in 2010 to $795 \mathrm{GW}$ in 2035 in the high demand scenario and to $500 \mathrm{GW}$ in 2035 in the low demand scenario. The assumptions by country are shown in Table 1.

However, even in the high demand scenario, if we assume a future power demand growth, 
Table 1 Nuclear generating capacity outlook

Unit: GW

\begin{tabular}{|c|c|c|c|c|c|}
\hline & \multirow[t]{2}{*}{2010} & \multicolumn{2}{|c|}{$\begin{array}{l}\text { Low demand } \\
\text { scenario }\end{array}$} & \multicolumn{2}{|c|}{$\begin{array}{l}\text { High demand } \\
\text { scenario }\end{array}$} \\
\hline & & 2020 & 2035 & 2020 & 2035 \\
\hline North America & 118.6 & 129.0 & 129.0 & 129.1 & 146.7 \\
\hline USA & 105.3 & 115.8 & 115.8 & 114.7 & 130.4 \\
\hline Canada & 13.3 & 13.2 & 13.2 & 14.4 & 16.3 \\
\hline Latin America & 4.4 & 6.0 & 5.6 & 9.2 & 16.6 \\
\hline Mexico & 1.4 & 1.6 & 1.6 & 1.6 & 1.6 \\
\hline Brazil & 2.0 & 3.4 & 3.4 & 5.8 & 12.2 \\
\hline Argentina & 1.0 & 1.0 & 0.6 & 1.8 & 2.7 \\
\hline Europe & 141.3 & 126.5 & 107.3 & 146.0 & 161.3 \\
\hline UK & 12.0 & 7.8 & 6.5 & 10.4 & 11.7 \\
\hline Germany & 21.5 & 8.6 & 0.0 & 12.7 & 12.7 \\
\hline France & 66.0 & 67.5 & 67.5 & 67.5 & 69.1 \\
\hline Italy & 0.0 & 0.0 & 0.0 & 3.2 & 6.4 \\
\hline Turkey & 0.0 & 0.0 & 0.0 & 2.6 & 5.2 \\
\hline Sweden & 9.4 & 10.0 & 8.9 & 10.0 & 11.3 \\
\hline Spain & 7.7 & 7.3 & 7.3 & 7.3 & 8.6 \\
\hline Belgium & 6.2 & 4.3 & 0.0 & 5.5 & 5.5 \\
\hline Switzerland & 3.4 & 1.2 & 0.0 & 3.4 & 1.9 \\
\hline Finland & 2.8 & 5.8 & 5.8 & 7.4 & 7.4 \\
\hline Netherlands & 0.5 & 0.0 & 0.0 & 0.5 & 3.1 \\
\hline Slovakia & 1.9 & 1.9 & 1.9 & 2.6 & 2.6 \\
\hline Hungary & 1.9 & 2.0 & 0.0 & 2.0 & 2.0 \\
\hline Czech & 3.9 & 4.0 & 4.0 & 4.0 & 5.1 \\
\hline Burgaria & 2.0 & 4.2 & 4.2 & 4.2 & 4.2 \\
\hline Slovenia & 0.7 & 0.7 & 0.0 & 0.7 & 1.8 \\
\hline Romania & 1.4 & 1.4 & 1.4 & 2.1 & 2.8 \\
\hline Africa & 1.9 & 1.9 & 1.9 & 1.9 & 5.8 \\
\hline S. Africa & 1.9 & 1.9 & 1.9 & 1.9 & 5.8 \\
\hline $\mathrm{FSU}^{\mathrm{a})}$ & 37.4 & 43.7 & 59.3 & 61.5 & 85.5 \\
\hline Russia & 23.2 & 26.2 & 39.5 & 42.5 & 57.7 \\
\hline Ukraine & 13.8 & 16.0 & 18.2 & 16.0 & 20.4 \\
\hline Armenia & 0.4 & 0.0 & 0.0 & 0.4 & 0.4 \\
\hline Kazakhstan & 0.0 & 0.0 & 0.0 & 1.1 & 4.4 \\
\hline Lithuania & 0.0 & 1.5 & 1.5 & 1.5 & 1.5 \\
\hline Belarus & 0.0 & 0.0 & 0.0 & 0.0 & 1.1 \\
\hline Middle East & 1.0 & 1.0 & 6.6 & 6.2 & 31.8 \\
\hline Iran & 1.0 & 1.0 & 1.0 & 2.3 & 4.9 \\
\hline UAE & 0.0 & 0.0 & 5.6 & 2.8 & 8.4 \\
\hline Saudi Arabia & 0.0 & 0.0 & 0.0 & 0.0 & 7.8 \\
\hline Jordan & 0.0 & 0.0 & 0.0 & 1.1 & 1.1 \\
\hline Kuwait & 0.0 & 0.0 & 0.0 & 0.0 & 4.4 \\
\hline Qatar & 0.0 & 0.0 & 0.0 & 0.0 & 2.6 \\
\hline Oman & 0.0 & 0.0 & 0.0 & 0.0 & 2.6 \\
\hline Asia & 85.4 & 139.0 & 190.1 & 179.2 & 347.2 \\
\hline China & 9.1 & 60.0 & 103.6 & 69.8 & 158.1 \\
\hline Japan & 48.8 & 31.3 & 10.3 & 42.4 & 31.5 \\
\hline Taiwan & 5.1 & 5.2 & 3.9 & 7.7 & 7.7 \\
\hline S. Korea & 17.7 & 24.0 & 34.0 & 32.0 & 48.0 \\
\hline Indonesia & 0.0 & 0.0 & 0.0 & 0.0 & 5.2 \\
\hline Malaysia & 0.0 & 0.0 & 0.0 & 0.0 & 5.2 \\
\hline Philippines & 0.0 & 0.0 & 0.0 & 0.0 & 5.2 \\
\hline Thailand & 0.0 & 0.0 & 0.0 & 0.0 & 5.2 \\
\hline India & 4.1 & 17.8 & 34.9 & 25.8 & 72.5 \\
\hline Vietnam & 0.0 & 0.0 & 2.6 & 0.0 & 5.2 \\
\hline Pakistan & 0.5 & 0.8 & 0.8 & 1.4 & 3.4 \\
\hline World & 390.1 & 447.1 & 499.8 & 533.0 & 794.8 \\
\hline
\end{tabular}

a) FSU: Former Soviet Union. 
as reported in literature ${ }^{12}$, the nuclear share in global electricity generation will stay at around $18 \%$ by 2035 . Therefore, even in this scenario, there is still room for a further increase in nuclear capacities after 2035.

Figure 1 shows a comparison between the low and high demand scenarios presented here and scenarios presented by leading international organizations.

The IAEA presents two scenarios: high and low estimates ${ }^{4)}$. In the 2010 version, they estimated that the current net capacity of $372 \mathrm{GW}$ would increase to $546 \mathrm{GW}$ by 2030 in the low estimate and $843 \mathrm{GW}$ in the high estimate. Immediately following the Fukushima Accident in March 2011, the IAEA published a slight downward revision of its latest estimates, where the low estimate would have a capacity of $501 \mathrm{GW}$ and the high estimate of $746 \mathrm{GW}$ by 2030 . As can be seen from Figure 1, the IAEA estimates have similar trends to the low and high demand scenarios presented in this study.

Three scenarios were defined in the IEA's estimates ${ }^{2)}$ : the Current Policies Scenario (CPS), the New Policies Scenario (NPS) and the 450 Scenario. The nuclear capacities are the lowest in the CPS and the highest in the 450 Scenario. As seen in Figure 1, the growth in the generating capacity of the 450 Scenario is similar to that in the high demand scenario presented in this study, and that of the CPS is slightly higher than that in the low demand scenario presented here. It is clear that nuclear generating capacities estimated in the present study based on policies of each country fairly matched with those published by the representative international agencies, and then there should be no discomfort.

\section{(2) Nuclear power output}

The nuclear power output can be estimated by multiplying the generating capacity by assumed capacity factors. For countries that have already been using nuclear energy, the average of the capacity factors over the past five years was used, whereas for newcomer countries, a capacity factor of $80 \%$ was assumed.

From the nuclear power generation estimates calculated above, it is then possible to estimate the demand for NU and UE services in Separative Work Unit (SWU). It should be noted that each demand differs depending on enrichment tails assays: the NU demand is higher and UE services demand is lower with higher tails assays.

There is thus a trade-off relationship between NU and the amount of UE services with respect to tails assays. Tables $\mathbf{2}$ and $\mathbf{3}$ show the NU and UE services demands with high $(0.3 \%)$

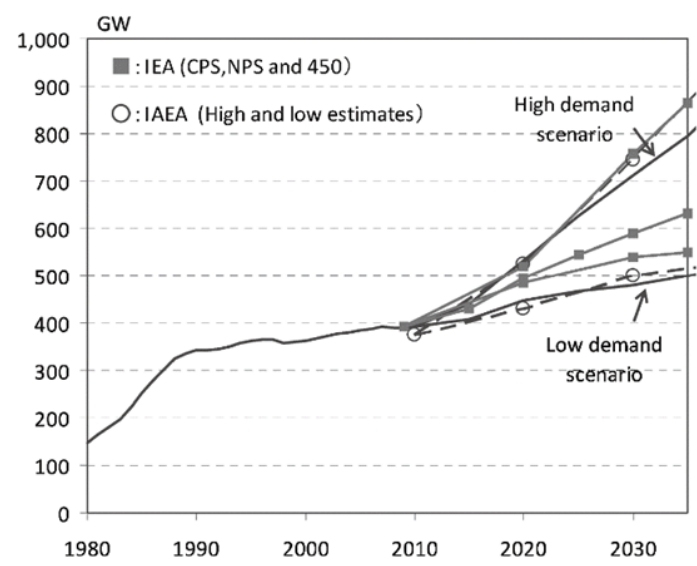

Figure 1 Comparison with nuclear capacity projections by IEA and IAEA 
Table 2 Uranium and uranium enrichment demand (Low demand scenario)

\begin{tabular}{|c|c|c|c|c|c|c|c|c|c|c|c|c|c|c|}
\hline & \multirow{2}{*}{\multicolumn{2}{|c|}{$\begin{array}{c}\text { Electricity } \\
\text { generation } \\
\text { (TWh) }\end{array}$}} & \multirow{2}{*}{\multicolumn{2}{|c|}{$\begin{array}{l}\text { Nuclear power } \\
\text { generation } \\
\text { (TWh) }\end{array}$}} & \multirow{2}{*}{\multicolumn{2}{|c|}{$\begin{array}{l}\text { Nuclear } \\
\text { share }\end{array}$}} & \multicolumn{4}{|c|}{$\underset{(\mathrm{tU})}{\operatorname{Uranium} \text { demand }}$} & \multicolumn{4}{|c|}{$\underset{(\mathrm{tSWU})}{\text { Uranium }} \underset{\mathrm{t} \text { enichment demand }}{ }$} \\
\hline & & & & & & & \multicolumn{2}{|c|}{$0.1 \%$} & \multicolumn{2}{|c|}{$0.3 \%$} & \multicolumn{2}{|c|}{$0.1 \%$} & \multicolumn{2}{|c|}{$0.3 \%$} \\
\hline & 2020 & 2035 & 2020 & 2035 & 2020 & 2035 & 2020 & 2035 & 2020 & 2035 & 2020 & 2035 & 2020 & 2035 \\
\hline USA & 4,673 & 5,261 & 867 & 912 & $19 \%$ & $17 \%$ & 15,109 & 15,886 & 21,326 & 22,423 & 21,127 & 22,214 & 12,421 & 13,060 \\
\hline Canada & 658 & 684 & 81 & 81 & $12 \%$ & $12 \%$ & 1,554 & 1,554 & 1,554 & 1,554 & 0 & 0 & 0 & 0 \\
\hline L. America & 1,812 & 2,749 & 40 & 38 & $2 \%$ & $1 \%$ & 703 & 657 & 992 & 927 & 982 & 918 & 578 & 540 \\
\hline Europe & 4,270 & 4,689 & 887 & 747 & $21 \%$ & $16 \%$ & 15,453 & 13,004 & 21,813 & 18,356 & 21,609 & 18,185 & 12,705 & 10,692 \\
\hline FSU & 1,675 & 1,863 & 276 & 375 & $17 \%$ & $20 \%$ & 4,816 & 6,533 & 6,798 & 9,221 & 6,735 & 9,135 & 3,960 & 5,371 \\
\hline Middle East & 1,140 & 1,589 & 7 & 46 & $1 \%$ & $3 \%$ & 122 & 806 & 172 & 1,137 & 171 & 1,127 & 100 & 662 \\
\hline Africa & 1,023 & 1,930 & 13 & 13 & $1 \%$ & $1 \%$ & 221 & 221 & 312 & 312 & 309 & 309 & 182 & 182 \\
\hline Asia & 10,096 & 13,836 & 1,174 & 1,627 & $12 \%$ & $12 \%$ & 20,447 & 28,341 & 28,861 & 40,004 & 28,592 & 39,631 & 16,810 & 23,300 \\
\hline Oceania & 392 & 514 & 0 & 0 & $0 \%$ & $0 \%$ & 0 & 0 & 0 & 0 & 0 & 0 & 0 & 0 \\
\hline World & 25,737 & 33,115 & 3,346 & 3,838 & $13 \%$ & $12 \%$ & 58,424 & 67,000 & 81,828 & 93,934 & 79,525 & 91,518 & 46,756 & 53,807 \\
\hline
\end{tabular}

Table 3 Uranium and uranium enrichment demand (High demand scenario)

\begin{tabular}{|c|c|c|c|c|c|c|c|c|c|c|c|c|c|c|}
\hline & \multirow{2}{*}{\multicolumn{2}{|c|}{$\begin{array}{l}\text { Electricity } \\
\text { generation } \\
\text { (TWh) }\end{array}$}} & \multirow{2}{*}{\multicolumn{2}{|c|}{$\begin{array}{l}\text { Nuclear power } \\
\text { generation } \\
\text { (TWh) }\end{array}$}} & \multirow{2}{*}{\multicolumn{2}{|c|}{$\begin{array}{l}\text { Nuclear } \\
\text { share }\end{array}$}} & \multicolumn{4}{|c|}{$\underset{(\mathrm{tU})}{\text { Uranium demand }}$} & \multicolumn{4}{|c|}{ Uranium enrichment demand } \\
\hline & & & & & & & \multicolumn{2}{|c|}{$0.1 \%$} & \multicolumn{2}{|c|}{$0.3 \%$} & \multicolumn{2}{|c|}{$0.1 \%$} & \multicolumn{2}{|c|}{$0.3 \%$} \\
\hline & 2020 & 2035 & 2020 & 2035 & 2020 & 2035 & 2020 & 2035 & 2020 & 2035 & 2020 & 2035 & 2020 & 2035 \\
\hline USA & 4,673 & 5,261 & 903 & 1,027 & $19 \%$ & $20 \%$ & 15,736 & 17,895 & 22,212 & 25,259 & 22,005 & 25,024 & 12,937 & 14,712 \\
\hline Canada & 658 & 684 & 88 & 100 & $13 \%$ & $15 \%$ & 1,687 & 1,910 & 1,687 & 1,910 & 0 & 0 & 0 & 0 \\
\hline L. America & 1,812 & 2,749 & 61 & 107 & $3 \%$ & $4 \%$ & 1,064 & 1,868 & 1,501 & 2,636 & 1,487 & 2,611 & 874 & 1,535 \\
\hline Europe & 4,270 & 4,689 & 1,028 & 1,139 & $24 \%$ & $24 \%$ & 17,903 & 19,835 & 25,271 & 27,997 & 25,035 & 27,736 & 14,719 & 16,307 \\
\hline FSU & 1,675 & 1,863 & 389 & 541 & $23 \%$ & $29 \%$ & 6,779 & 9,426 & 9,569 & 13,305 & 9,479 & 13,181 & 5,573 & 7,749 \\
\hline Middle East & 1,140 & 1,589 & 43 & 223 & $4 \%$ & $14 \%$ & 757 & 3,882 & 1,068 & 5,480 & 1,058 & 5,428 & 622 & 3,192 \\
\hline Africa & 1,023 & 1,930 & 13 & 39 & $1 \%$ & $2 \%$ & 221 & 677 & 312 & 955 & 309 & 946 & 182 & 556 \\
\hline Asia & 10,096 & 13,836 & 1,562 & 2,846 & $15 \%$ & $21 \%$ & 27,211 & 49,568 & 38,408 & 69,966 & 38,050 & 69,313 & 22,371 & 40,752 \\
\hline Oceania & 392 & 514 & 0 & 0 & $0 \%$ & $0 \%$ & 0 & 0 & 0 & 0 & 0 & 0 & 0 & 0 \\
\hline World & 25,737 & 33,115 & 4,088 & 6,021 & $16 \%$ & $18 \%$ & 71,357 & 105,059 & 100,029 & 147,508 & 97,424 & 144,240 & 57,279 & 84,804 \\
\hline
\end{tabular}

and low $(0.1 \%)$ tails assays in the high and low demand scenarios, respectively. Here, for simplicity, all nuclear power plants are assumed to be LWRs, with the exception of HWRs in Canada, and the UE services demand is calculated for LWRs with an assumption of UE, the fuel burnup, and a thermal efficiency of $4 \%, 45 \mathrm{GWd} / \mathrm{t}$, and $34.5 \%$, respectively. The tables also show the electricity demand and nuclear share in the Advanced Technologies Scenario presented in literature ${ }^{12}$. As the high and low demand scenarios in this study refer only to nuclear energy demand, rather than to the electricity demand, the nuclear share is higher in the high demand scenario than in the low demand scenario.

\section{Uranium Production Forecast}

With recent increases in the price of uranium, investments in uranium resource development are rapidly expanding. Accordingly, uranium production capacities are expected to drastically improve by 2020. For the future expansion of uranium production capacities, we refer to the evaluation by $\mathrm{OECD}^{8)}$ (Table 4).

A-II in Table 4 shows the forecast based on existing and committed projects, while B-II shows the forecast based on existing, committed, planned, and prospective projects. It is assumed that the committed projects will be launched by 2020 and existing uranium mines will diminish; therefore, the production capacity in 2035 is assumed to be less than that in 2025 . Note that in this forecast, Kazakhstan's production capacity is expected to increase from the current 18,000 tU to 24,000 tU by 2020 , but will decrease to 5,000 to $6,000 \mathrm{tU}$ by 2035 . With 
Table 4 Uranium production forecast $\left(\mathrm{OECD}^{8)}\right)$

\begin{tabular}{|c|c|c|c|c|c|}
\hline & \multirow[b]{3}{*}{2010} & & & \multicolumn{2}{|c|}{ Unit: tU/yr } \\
\hline & & \multicolumn{2}{|c|}{2020} & \multicolumn{2}{|c|}{2035} \\
\hline & & $\begin{array}{c}\text { A-II } \\
\text { (Production } \\
\text { case A) }\end{array}$ & $\begin{array}{c}\text { B-II } \\
\text { (Production } \\
\text { case B) }\end{array}$ & A-II & B-II \\
\hline Argentina & 120 & 500 & 500 & 500 & 500 \\
\hline Australia & 9,700 & 10,100 & 24,200 & 9,800 & 27,600 \\
\hline Brazil & 340 & 2,000 & 2,000 & 2,000 & 2,000 \\
\hline Canada & 16,430 & 17,730 & 19,000 & 17,730 & 19,000 \\
\hline China & 940 & 1,200 & 1,200 & 1,200 & 1,200 \\
\hline Czech & 500 & 50 & 50 & 20 & 20 \\
\hline India & 295 & 980 & 1,200 & 1,000 & 2,000 \\
\hline Iran & 20 & 100 & 100 & 100 & 100 \\
\hline Jordan & 0 & 2,000 & 2,000 & 2,000 & 2,000 \\
\hline Kazakhstan & 18,000 & 24,000 & 24,000 & 5,000 & 6,000 \\
\hline Malawi & 0 & 1,425 & 1,100 & 0 & 0 \\
\hline Mongolia & 0 & 150 & 1,000 & 150 & 1,000 \\
\hline Namibia & 5,000 & 8,000 & 19,000 & 5,000 & 7,500 \\
\hline Niger & 4,000 & 9,500 & 10,500 & 5,000 & 5,000 \\
\hline Pakistan & 65 & 140 & 155 & 140 & 650 \\
\hline Romania & 230 & 350 & 475 & 350 & 630 \\
\hline Russia & 3,520 & 7,600 & 11,990 & 6,800 & 13,400 \\
\hline S. Africa & 4,860 & 4,860 & 6,320 & 4,860 & 6,320 \\
\hline Ukraine & 960 & 810 & 5,500 & 170 & 5,500 \\
\hline USA & 2,900 & 3,800 & 6,600 & 3,100 & 5,600 \\
\hline Uzbekistan & 2,300 & 3,000 & 3,750 & 3,500 & 3,500 \\
\hline World & 70,180 & 98,295 & 140,640 & 68,420 & 109,520 \\
\hline
\end{tabular}

the future growth in global nuclear power generation, irrespective of the scenario, it seems unlikely that uranium production will significantly decrease once it has increased. As diminished existing uranium mines are likely to be compensated for by new uranium resource development after 2020, it is therefore considered that global uranium production will remain stable or increase in the future.

Here, we established two cases, where the uranium production increases until 2020 according to forecast A-II or B-II, and then remains constant until 2035: these are respectively named Uranium Production Cases A and B. In addition, for the high demand scenario in 2035, we also established another case named Production Case $\mathrm{C}$ for when the highest demand occurs and production increases more than in B-II in relation to uranium resource development after 2020. The regional differences between Cases B and C are assumed to be proportional to the differences between Cases A and B.

\section{Outlook for UE Capacities}

Global uranium enrichment facilities currently in operation and in the planning stage are shown in Table $5^{16-19)}$. It is apparent that these facilities are dominated by a few countries, and four companies (including USEC (US), Areva NC (France), Urenco (UK, Germany, and the Netherlands), and Atomenergoprom (Russia)) dominate more than $95 \%$ of the global enrichment capacity.

The Paducah enrichment plant in the US (run by USEC), and the George Besse enrichment plant in France (run by EURODIF, which is a subsidiary of Areva) have played leading role in UE in their respective countries since they were established in 1952 and 1979, respectively. However, as these are inefficient gaseous diffusion plants, they are no longer operating 
Table 5 World's enrichment facilities

\begin{tabular}{|c|c|c|c|c|}
\hline Country & Company & Location & Type & $\begin{array}{l}\text { Capacity, } \\
\text { tSWU/yr }\end{array}$ \\
\hline \multirow[t]{5}{*}{ USA } & \multirow[t]{2}{*}{ USEC } & Paducah & Gaseous diffusion & 11,300 \\
\hline & & Piketon & Centrifugation & 3,800 \\
\hline & Urenco & Eunice & Centrifugation & 3,800 \\
\hline & Areva $\mathrm{NC}$ & Idaho falls & Centrifugation & 6,600 \\
\hline & GE Hitachi Nuclear Energy & Wilmington & Laser & 6,000 \\
\hline Europe & Eurodif (Areva NC etc.) & Georges Besse (Tricastin) & Gaseous diffusion & 10,800 \\
\hline France & Areva NC & Georges Besse II (Tricastin) & Centrifugation & 7,500 \\
\hline \multirow{3}{*}{$\begin{array}{l}\text { UK, Germany and } \\
\text { Netherlands }\end{array}$} & \multirow[t]{3}{*}{ Urenco } & Capenhurst (UK) & Centrifugation & 5,000 \\
\hline & & Almelo (Netherlands) & Centrifugation & 5,000 \\
\hline & & Gronau (Germany) & Centrifugation & 4,200 \\
\hline Japan & JNFL & Rokkasho & Centrifugation & 1,050 \\
\hline \multirow[t]{4}{*}{ Russia } & Atomenergoprom & Angarsk & Centrifugation & 2,500 \\
\hline & Atomenergoprom & Novouralsk & Centrifugation & 13,200 \\
\hline & Atomenergoprom & Zelenogorsk & Centrifugation & 7,800 \\
\hline & Atomenergoprom & Seversk & Centrifugation & 3,700 \\
\hline \multirow[t]{2}{*}{ China } & \multirow[t]{2}{*}{$\mathrm{CNNC}$} & Lanzhou & Centrifugation & 500 \\
\hline & & Hanzhong & Centrifugation & 500 \\
\hline India & Department of Atoic Energy & Ratnahalli & Centrifugation & 25 \\
\hline \multirow[t]{3}{*}{ Iran } & & Natanz & Centrifugation & $\mathrm{n} / \mathrm{a}$ \\
\hline & & Natanz & Centrifugation & \\
\hline & & Qom & Centrifugation & \\
\hline Brazil & INB & Fábrica de Combustivel Nuclear & Centrifugation & 200 \\
\hline Pakistan & & Kahuta & Centrifugation & 5 \\
\hline North Korea & & Nyeongbyeon & Centrifugation & $\mathrm{n} / \mathrm{a}$ \\
\hline
\end{tabular}

at full capacity for economic reasons, and they only operate at around 5,000 tSWU/yr and $3,000 \mathrm{tSWU} / \mathrm{yr}$ out of a capacity of $11,300 \mathrm{tSWU} / \mathrm{yr}$ and 10,800 tSWU/yr, respectively ${ }^{20}$.

Therefore, these companies are currently establishing, or planning to establish, new centrifuge plants to replace the old plants, which is further expected to increase the growing enrichment demand. In France, the George Besse II enrichment plant, which is adjacent to the existing plant, began operation in 2010, and its production capacity is expected to rise to 7,500 tSWU/yr at full operation. In the US, USEC is planning to establish a new centrifuge plant in Piketon in Ohio called the American Centrifuge Plant, and Urenco constructed an enrichment plant in Eunice, New Mexico, that began operating in 2010. Furthermore, Areva NC is planning the construction of a new plant in Eagle Rock, Idaho, and a construction and operation license was acquired in October 2011 from the Nuclear Regulatory Commission (NRC). Moreover, GE-Hitachi is currently constructing an advanced enrichment plant equipped with laser enrichment processes that launched test operations in 2010 .

In Europe, Urenco, which is jointly owned by the UK, Germany, and the Netherlands, has enrichment plants in each of these countries and a total capacity of 14,200 tSWU/yr as of 2011. In addition to the above-mentioned plant in the US, the company plans to expand its capacity up to $18,000 \mathrm{tSWU} / \mathrm{yr}$ by $2015^{21)}$.

Russia has the largest enrichment capacity in the world and owns enrichment plants in Angarsk, Novouralsk, Zelenogorsk, and Seversk that have a total capacity of 27,200 tSWU/yr. In 2007, they established the International Uranium Enrichment Center (IUEC) in Angarsk, as advocated by President Vladimir Putin. Russia aims to prevent UE technologies from being promulgated and also prevent their nuclear fuel supplies from being disrupted by stockpiling enriched uranium under the supervision of the IAEA.

In cooperation with Russia, China has constructed and is operating UE plants in Lanzhou and Hanzhong, each with a capacity of 500 tSWU/yr. In addition, Russian President Dmitry Medvedev and Chinese Premier Wen Jiabao have signed an agreement and contract to build a 
new enrichment plant in 2008 (the 4th plant including those which have already been closed), and if this is completed, China's enrichment capacity will be 1,500 tSWU/yr.

The world's enrichment capacity as of 2011 is estimated at approximately 42,200 tSWU/yr, excluding declines due to economic and other reasons ${ }^{21)}$. In addition, enriched uranium from dismantled nuclear weapons was exported from Russia to the US between 1994 and 2013 under the "Megatons to Megawatts" program, and it is estimated that this amounted to 5,500 tSWU annually. In addition, these covered the UE service demand. In this study, we assume that the uranium supply from dismantled weapons will come to an end by 2020, and that gaseous diffusion plants will be closed in a phased manner, mainly due to economic reasons. Under such circumstances and if the above stated new plants are completed, the world's enrichment capacity will reach 72,300 tSWU by 2020 . This UE capacity is sufficient or not is depends on the amount of UE demand.

If the global enrichment capacity is insufficient in the future and needs to be expanded further, which corresponds to the high demand case and the Enrichment Case C in 2035, the associated regional capacities are assumed to be as follows. In this respect, we firstly restrict the additional capacity expansion to regions (countries) that currently have enrichment facilities and have clear intentions of further expansion. If we exclude Japan, these regions (countries) are the US, Europe, former Soviet Union, and China. Of these, Europe and the US are already expected to construct new plants, as mentioned above, but the nuclear power generation growth will not be as great as that in China and Russia, and may even decline. Consequently, in Europe, where the supply of UE will exceed demand when the new plants that are currently under construction or planning are in operated, it is assumed that enrichment plants will not be further established. Furthermore, in the US, where the demand will slightly exceed the supply in the high demand scenario (as mentioned later), it is assumed only one enrichment plant with a capacity of 6,000 tSWU/yr, which is equivalent to the capacity of the latest laser enrichment plant, will be established. To cover the rest of the increased demand, we assume that enrichment plants will be constructed in Russia and China, where the establishment of no new large facilities have been assumed in Enrichment Case B, in spite of the expected significant increased future demand. Considering the differences between the maturity of domestic technologies, we assume that $70 \%$ of the additional capacity will be constructed in Russia and $30 \%$ in China.

Table 6 shows the assumed capacities of UE plants. Considering all current construction plans, the global enrichment capacity is expected to grow to $72,300 \mathrm{tSWU} / \mathrm{yr}$, as shown in Enrichment Case B. Meanwhile, considering that existing Russian plants currently generate only $18,000 \mathrm{tSWU} / \mathrm{yr}^{20)}$, in the case where the UE services demand is eased, we assume that plants would not be operated in Russia to generate more than the current level, and the global capacity would be 63,100 tSWU/yr (Enrichment Case A). Meanwhile, in the high demand scenario (in 2035), when enrichment facilities become insufficient in relation to the current established plans, we established Enrichment Case C, where Russia and China will expand

Table 6 Enrichment capacity projection

\begin{tabular}{lrrrr} 
& & \multicolumn{2}{c}{ Unit: tSWU/yr } \\
\hline Americas & 5,400 & 20,400 & 20,400 & 26,400 \\
Europe & 18,200 & 21,700 & 21,700 & 21,700 \\
FSU & 18,000 & 18,000 & 27,200 & 35,472 \\
Asia & 2,050 & 3,000 & 3,000 & 6,545 \\
Total & 43,650 & 63,100 & 72,300 & 90,117 \\
\hline
\end{tabular}


their enrichment facilities.

\section{Prices of NU and UE Services}

Although large parts of NU and UE services are procured under long-term contracts, some are purchased at spot markets where prices are disclosed ${ }^{22}$. However, even with long-term contracts, NU and UE services are often traded based on spot prices. Therefore, changes in spot prices are supposed to reflect the trends of overall NU and UE service prices. Past trends in spot prices are shown in Figure 2.

Prior to 2003, the price of NU was very low at around $10 \mathrm{USD} / \mathrm{lb}-\mathrm{U}_{3} \mathrm{O}_{8}$, which reflected the fact that uranium production, including that for military purposes, exceeded commercial uranium demand until the early-90s, and that enriched uranium supply was abundant after the mid-90s because of secondary sources. Thereafter however, nuclear energy was politically promoted in each country due to global environmental concerns, which upwardly revised estimates of nuclear energy generation. Consequently, the price of NU dramatically increased after 2004 and reached nearly $140 \mathrm{USD} / \mathrm{lb}-\mathrm{U}_{3} \mathrm{O}_{8}$ in 2007 , due to the increased societal awareness of the finite nature of natural resources, an understanding that the uranium supply chain depends on a few important facilities, the increased price of primary energies (including crude oil), and intervention by speculators. Although the dramatically increased price entered an adjustment phase, the price stabilized at a higher than previous level and reached approximately $51 \mathrm{USD} / \mathrm{lb}-\mathrm{U}_{3} \mathrm{O}_{8}$ in June 2012.

While the UE service price does not fluctuate as much as that of the price of NU (in the past it fluctuated between 80 USD and 110 USD per kgSWU), it continued to rise after 2005 and temporarily reached 160 USD per kgSWU. It then slowly declined and reached 130 USD per $\mathrm{kgSWU}$ as of June 2012.

As seen in Figure 2, in this study we set two future price scenarios for NU and UE services. First, in the low price case for NU we assumed that although it will not decline to the previous record of $10 \mathrm{USD} / \mathrm{lb}-\mathrm{U}_{3} \mathrm{O}_{8}$, it will remain stable at the lowest price after price adjustment of $40 \mathrm{USD} / \mathrm{lb}-\mathrm{U}_{3} \mathrm{O}_{8}$, because secondary uranium supplies will be terminated. Second, in the high price case for NU, it is estimated to rise again to $140 \mathrm{USD} / \mathrm{lb}-\mathrm{U}_{3} \mathrm{O}_{8}$ (which is the price reached when prices soared in the past).

For the UE service price, in the low price case we estimated that it would drop to 100 USD per $\mathrm{kgSWU}$, which is the price reached prior to the sudden rise, but in the high price case we estimated it would rise to $160 \mathrm{USD}$ per $\mathrm{kgSWU}$, which is the price reached when prices soared.
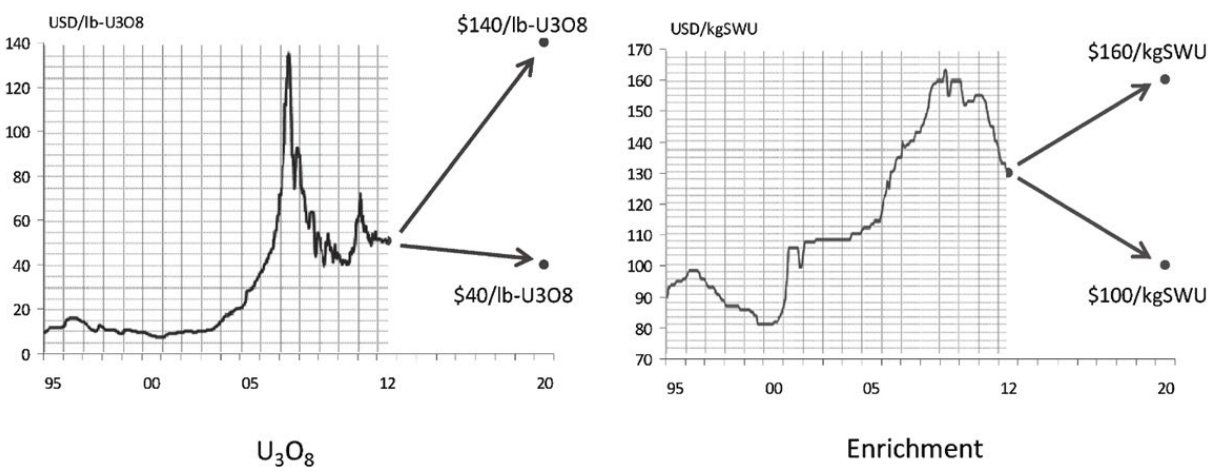

Figure $2 \mathrm{U}_{3} \mathrm{O}_{8}$ and uranium enrichment spot prices ${ }^{22)}$ 


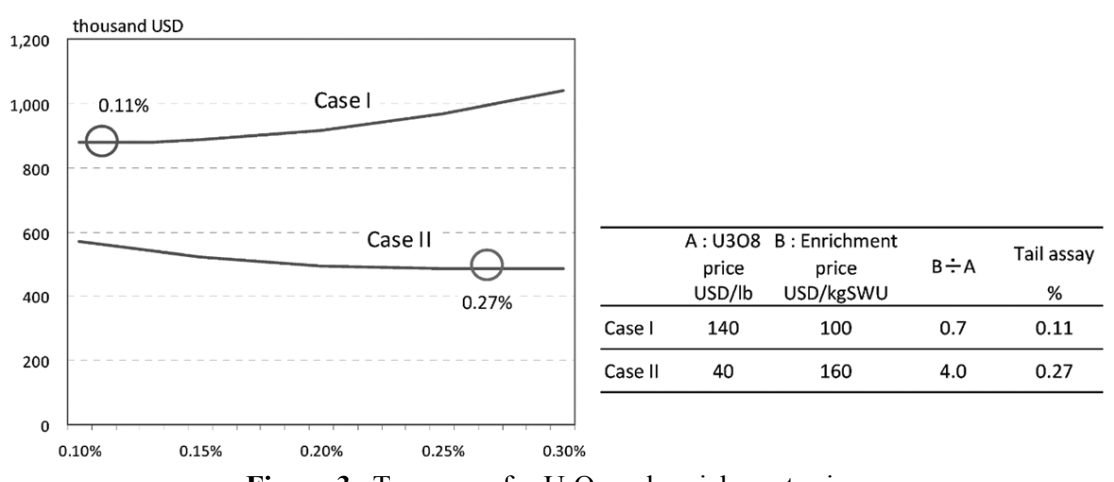

Figure 3 Two cases for $\mathrm{U}_{3} \mathrm{O}_{8}$ and enrichment prices

The demand for NU and UE services have a trade-off relationship in relation to tails assays. Therefore, when the NU unit price and UE service unit price are determined, the total price (the NU purchase price and the UE service price) is calculated based on their demand amounts according to tails assays, and the optimum tails assay is calculated using the condition that provides the lowest total cost. Therefore, to estimate the prices of NU and UE services in 2020 and 2035, we established two representative price cases: Price Case I and Price Case II. In Price Case 1, NU is estimated to be as high at $140 \mathrm{USD} / \mathrm{lb}-\mathrm{U}_{3} \mathrm{O}_{8}$ and UE services as 100 USD per $\mathrm{kgSWU}$, whereas in Price Case 2, NU is estimated to be as low as $40 \mathrm{USD} / \mathrm{lb}-\mathrm{U}_{3} \mathrm{O}_{8}$ and UE services as 160 USD per $\mathrm{kgSWU}$. These cases provide an estimate that optimum tails assays are $0.11 \%$ and $0.27 \%$, respectively (Figure 3). In this study, these two price cases were set as the upper and lower tails assays limits, and the future demand amounts of NU and UE services were then calculated. The optimum tails assay is determined by the ratio of the UE service price (USD/kg-SWU) to the NU price (USD/lb- $\mathrm{U}_{3} \mathrm{O}_{8}$ ), which is 0.7 and 4.0 for Price Case I and II, respectively.

\section{Method used to Estimate Trade Flows}

Based on the above assumptions, we made an estimate for NU and EU trade flows in 2020 and 2035.

Global trade flow estimations of oil products or natural gas are usually conducted using cost optimization methods ${ }^{23,24}$. For example, using linear programming as an optimization method, costs of production, shipping, transportation, and refining of petroleum and natural gas are calculated for every country in the world and the optimal supply-demand balance where the total cost takes the minimal value is obtained to make trade flow.

In the above estimations, selection of the LNG supplier is determined by the balance between the local production costs and those related to transportation to the destination. In the case of nuclear power, however, unlike oil and natural gas, it is practically impossible to obtain accurate data on costs relating to shipment, transportation, conversion, enrichment, reconversion, and other processes, as most of them are undisclosed. At the same time, as often pointed out, because the energy density of uranium fuel is far higher than that of fossil fuels, costs related to its transportation are proportionally low. For example, one reference ${ }^{25}$ estimated the transportation cost of uranium hexafluoride from Europe to an enrichment plant in Russia as 1.5 USD per $\mathrm{kg}$, which is almost equivalent to $1 / 100$ of LNG's transportation cost from Indonesia to Japan $(0.5 \mathrm{USD} / \mathrm{MMBtu})^{26)}$, if costs per unit electricity generation are compared. However, the cost per unit mass for intercontinental transportation of spent nuclear fuels is much higher at 200 
$\mathrm{USD} / \mathrm{kg}^{27)}$, which suggests that nuclear-related transportation costs are largely related to safety measures and regulations rather than distance.

The actual procurement of uranium fuel by power utilities is mainly based on long-term contracts. When selecting a supplier, the purchasers usually consider the price for the fuel itself, past contracts, various rights and interests, and the political partnership between the two countries, and the transportation costs are not the most important factor considered. Therefore, unlike conducting analyzes of petroleum product trade flows, calculating trade balances based on differences in transportation costs is not appropriate when analyzing nuclear fuels.

Therefore, this study uses actual recent trade flow data (i.e., the share of NU and UE services supplied to a country from various countries), and estimates future trade flows by revising the data according to future conditions. In other words, we assumed that past trade flows contain various information about political and geopolitical influences, existing contracts, rights and interests, and other important factors, and that these situations will not change greatly in the future from those in 2009 because of the rigidity associated with long-term contracts. To be more specific, we calculated the demand for NU and UE services in 2020 and 2035 in each region with the assumption of tails assays for each case (as described later). On this basis, and supposing that global supply meets global demand, we assumed that the supply will decrease with a uniform decline in operation rates among regions, when it has a capacity lower than Production and Enrichment Case A. When the supply lies between that of Cases A and B, we set capacities assuming that the proportion between the two cases was uniform for each region. Furthermore, when it exceeds Case B, we calculated the supply for each region by using Case $\mathrm{C}$, as previously mentioned.

In the past, global uranium production has never exceeded $90 \%$ of its production capacity, and production has been between $75 \%$ to $84 \%$ since $2003^{8)}$. We assumed here that the actual uranium production cap was $80 \%$ of the uranium production capacity, as shown in Table 4 .

Using these assumptions for the demand by region for NU and UE services as boundary conditions, we evaluated a flow that minimizes the following objective function $x$,

$$
\chi=\sum_{i, j}\left(x_{i, j}-x_{i, j}^{0}\right)^{2}
$$

where $x_{i, j}^{0}$ and $x_{i, j}$ indicate the share of import from region $\mathrm{j}$ to region $\mathrm{i}$ in the entire import to $\mathrm{i}$ in 2009 (actual value), and its future estimates for the years 2020 or 2035, respectively.

Actual trades in 2009 were calculated by modifying data ${ }^{28)}$, where the supply of NU, uranium conversion and UE services, and fuel fabrication services for PWRs and BWRs is estimated by region and by company. Tables $\mathbf{7}$ and $\mathbf{8}$ show aggregated inter-regional trade of NU and UE services for 2009.

It is of note that, in line with actual trade, the trade flow of NU refers to the flow from the uranium production country to the country where the final fuel is consumed, rather than to the country where uranium is enriched.

The actual data from 2009 include the enrichment service flow from Europe to the US, and this shows that uranium enriched in Areva's plant in Europe is consumed in the US. However, as can be seen from Table 5, Areva is currently constructing an enrichment plant in the US for use in the US market. Therefore, in this study, we assumed the future operation and launch of this plant, and thus the UE service supply from Europe to the US is not included in the future estimation. It was not possible to obtain the historical input share of the Middle East for use in equation (1) because there has been no actual nuclear power generation or production/supply of NU. Therefore, we assumed that if the Middle East exports NU, the destination will be Asia or 
Table 7 Uranium trade (2009)

\begin{tabular}{|c|c|c|c|c|c|c|c|c|c|c|c|}
\hline From: $\quad$ To: & USA & Canada & L. America & Europe & FSU & Middle East & Africa & Asia & Oceania & Unknown & World \\
\hline USA & 1,921 & & & & & & & & & & 1,921 \\
\hline Canada & 3,867 & 1,600 & 21 & 3,664 & 19 & & & 1,819 & & 1,913 & 12,904 \\
\hline L. America & & & 330 & & & & & & & & 330 \\
\hline Europe & & & & 1,174 & & & & & & & 1,174 \\
\hline FSU & 3,789 & & 62 & 8,480 & 8,856 & & 131 & 12,013 & & 5,466 & 38,796 \\
\hline Middle East & & & & & & & & & & & 0 \\
\hline Africa & 1,425 & & 52 & 2,188 & 62 & & & 2,904 & & 3,315 & 9,946 \\
\hline Asia & & & & & & & & 1,274 & & & 1,274 \\
\hline Oceania & 522 & & 47 & 2,048 & 61 & & & 3,924 & & 2,900 & 9,503 \\
\hline Unknown & 51 & & 10 & 883 & 13 & & & 2,496 & & 101 & 3,554 \\
\hline World & 11,576 & 1,600 & 522 & 18,438 & 9,011 & & 131 & 24,431 & & 13,695 & 79,402 \\
\hline
\end{tabular}

Table 8 Enrichment trade (2009)

\begin{tabular}{|c|c|c|c|c|c|c|c|c|c|c|c|}
\hline \multicolumn{12}{|c|}{ Unit: tSWU } \\
\hline From: & USA & Canada & L. America & Europe & FSU & Middle East & Africa & Asia & Oceania & Unknown & World \\
\hline USA & 7,063 & & & 909 & & & & 4,536 & & 1,219 & 13,727 \\
\hline Canada & & & & & & & & & & & 0 \\
\hline L. America & & & 236 & & & & & & & & 236 \\
\hline Europe & 5,133 & & 160 & 16,875 & & & & 2,958 & & 1,146 & 26,272 \\
\hline FSU & 5,500 & & 2 & 4,213 & 5,540 & & 94 & 1,078 & & 4,899 & 21,326 \\
\hline Middle East & & & & & & & & & & & 0 \\
\hline Africa & & & & & & & & & & & 0 \\
\hline Asia & & & & & & & & 955 & & & 955 \\
\hline Oceania & & & & & & & & & & & 0 \\
\hline World & 17,696 & 0 & 398 & 21,997 & 5,540 & 0 & 94 & 9,527 & 0 & 7,264 & 62,516 \\
\hline
\end{tabular}

Europe, where the demand is high. For $x_{i, j}^{0}$ we used the production in the Middle East divided by the demand in each region, and for the case where the Middle East imports NU, we assumed that the source regions would be the former Soviet Union, Africa, and Oceania, where resources are abundant. If the Middle East imported UE services, we assumed that the source regions would be the former Soviet Union and Europe, which have greater export capacities. We used values of $1 / 3$ for NU and $1 / 2$ for UE services as $x_{i, j}^{0}$ in equation (1), respectively.

In these estimates, the cost of transportation between regions was not considered to be an influential factor when calculating future trade flow. This is because, as stated above, the impact of transportation costs is considered to be relatively low when determining the uranium fuel flow. To evaluate the uncertainty of the estimates, we also estimated a case (as a reference) where trade flow is determined based on transportation cost between regions. In this case, we firstly set the supply and demand by region, as described above, and then calculated the flows of trade across the world where transportation costs are the lowest, assuming (for simplicity) that transportation costs would proportionally increase with the distance between regions. We set the distance between regions by referring to Ref. 29, which is widely used for oil tankers. To simplify this estimation, we used the distance between representative ports, such as St. Petersburg and Hamburg, for each region. It is of note that ports used for the actual shipping and receiving uranium are sometimes other neighboring ports. As we considered that the former Soviet Union requires land transportation to ports in addition to marine transportation, we added the distance from Kazakhstan to St. Petersburg for NU and that from Angarsk to St. Petersburg for UE. In the case of oil, for example, land transportation is more expensive than marine transportation, but in the case of uranium, as the transportation lot size is small, 
marine transportation is relatively costly. In this study, we assumed that both land and marine transportation costs for uranium would work out the same.

We also assumed (as discussed later) that the US and Europe would use at least $50 \%$ of the output from newly established UE plants in US and EU to cover the demand of their own regions.

\section{Scenario Setting}

Table 9 shows the scenario setting. We estimated the supply and demand balance in 2020 and 2035 and calculated trade flows for the low and high demand scenarios.

As described in detail in the following section, in both scenarios for 2020 and in the low demand scenario for 2035, the demand for enriched uranium can be met with existing uranium resource development projects and enrichment plant construction plans.

In the low demand scenario in 2020, in particular, both the supply and demand balance of NU and UE services will be significantly eased. In this case, we assumed the minimum cases, Production Case A and Enrichment Case A, for the NU production capacity and the UE capacity, respectively. However, even in these cases, the supply and demand balance is eased and facilities will not be required to work at full operation. In contrast, for the low demand scenario (2035) and high demand scenario (2020), we assumed the planned expansion of Case B's estimation of capacities. In the high demand scenario in 2035, the demand for NU and UE services cannot be satisfied by the existing plan only; therefore, in these cases, we established Case $\mathrm{C}$, where both the uranium production capacity and enrichment capacity are additionally expanded to a greater extent than previously planned. We then defined the tails assay for each case based on the supply and demand status of NU and UE services. The details of the assumptions are described in each subsection in Chapter III. For the high demand scenario in 2035, we made a separate estimation for the reference case (where supply and demand regions are matched based on transportation costs) for comparison purposes.

\section{Usability of Reprocessed Uranium}

Reprocessed uranium is that generated when spent fuel is reprocessed, and it usually contains around $1 \% \mathrm{U} 235$, which is higher than $0.72 \%$ for NU. By enriching and using reprocessed uranium, it is possible to reduce the demand for NU.

According to Ref. 30, an accumulated amount of 6,350 MOX fuel assemblies, which was generated by reprocessing spent fuel, had been loaded into 58 reactors in France, Germany, Switzerland, Belgium, the US, and other countries by the end of 2008. When we calculate the power output with MOX fuel using actual values of the loading rate estimated in Ref. 30, and supposing a facility capacity factor of $80 \%$, it provides 83 TWh per year, which is about $3 \%$ of the global nuclear power generation in 2009.

Because producing fuel using reprocessed uranium costs more than that by enriching NU, as more reprocessed uranium is used, costs are higher in most cases. However, if the price of NU

Table 9 Scenario setting

\begin{tabular}{ccccc}
\hline Scenario & & $\begin{array}{c}\text { Uranium } \\
\text { production } \\
\text { capacity }\end{array}$ & $\begin{array}{c}\text { Enrichment } \\
\text { capacity }\end{array}$ & $\begin{array}{c}\text { Tail assay } \\
\%\end{array}$ \\
\hline Low demand & 2020 & Case A & Case A & 0.22 \\
& 2035 & Case B & Case B & 0.27 \\
High demand & 2020 & Case B & Case B & 0.27 \\
& 2035 & Case C & Case C & 0.27 \\
\hline
\end{tabular}


costs more than $150 \mathrm{USD} / \mathrm{lb}-\mathrm{U}_{3} \mathrm{O}_{8}$, expanding the use of reprocessed uranium would cost the same as using NU ${ }^{11}$. As of 2010, the reprocessing facilities at Sellafield (UK), la Hague (France), and Chelyabinsk (Russia) have capacities of 4,600 tHM/yr. New construction is planned for a RT-2 reprocessing facility (1,000 to $1,500 \mathrm{tU} / \mathrm{yr})$ in Russia, but it is currently suspended due to economic reasons ${ }^{17)}$. If these facilities other than RT-2 run in a stable manner at an operation rate of $80 \%$, for example, about $3,700 \mathrm{tU}$ of reprocessed uranium will be available at the most. This is equivalent to approximately $3 \%$ to $6 \%$ of the NU demand indicated in Tables 2 and 3 (in 2020 and 2035) and the required production amount will thus decrease by this amount. In addition, as the $\mathrm{U} 235$ assay of reprocessed uranium is around $1 \%$ and that of $\mathrm{NU}$ is $0.72 \%$, the demand for UE services would decrease due to the difference. However, the decline would be small compared to the decline in the demand for NU. In this study, we did not explicitly consider the use of reprocessed uranium, and if this is taken into consideration, the demand for NU may slightly decrease. Although the impact is not significant with reference to the current reprocessing facilities only, it would be more significant if a new large-scale construction of reprocessing facility was planned responding to a trend of the reprocessed and natural uranium costs.

\section{Results and Discussion}

\section{Trade flows for the Low Demand Scenario}

Figure 4 presents estimates of global NU and UE services using the low demand scenario.

\section{(1) Year 2020}

The demands for NU in 2020 are 59,232 tU and 76,978 tU, and those for UE services are 76,372 tSWU and 49,685 tSWU in Price Cases I and II (tails assays are $0.11 \%$ and $0.27 \%$ ), respectively.

The production capacity of NU (multiplying operating rate of 0.8 ) is between 78,636 and 112,512 tU. In Case A, where no new project proceeds, if any price cases (tails assays) are applied, the demand for NU can be satisfied by the production of 78,636 tU. However, although in Price Case I the demand for UE services slightly exceeds the capacity (Enrichment Case B), which includes the capacity of enrichment facilities under construction and in the planning
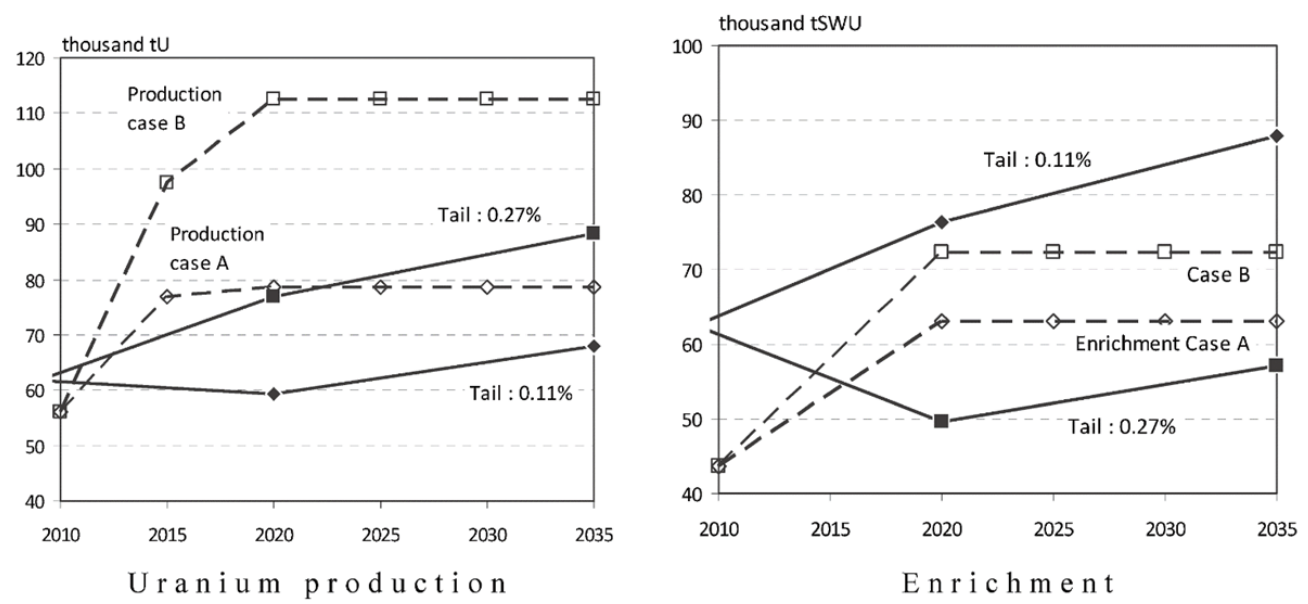

Figure 4 Uranium production and enrichment projections (Low demand scenario) 
phase, it will significantly fall below the capacity in Price Case II.

Considering these, it is highly likely in the low demand scenario that the supply and demand balance of NU, in particular, will be significantly eased, and the price will fall far below 40 $\mathrm{USD} / \mathrm{lb}-\mathrm{U}_{3} \mathrm{O}_{8}$, which is the assumption for Case II. In addition, as the supply capacity of NU becomes excessive, it will be enriched at high tails assays. As a result, the price of UE services is also likely to decline, due to easing of the supply and demand balance. Under such circumstances, it is also likely that NU resource development projects will stagnate, and that enrichment facilities that are currently in the planning phase on a large scale (particularly in the US) will be delayed or cancelled.

In this case with the lowest tails assays, UE services will surpass the estimated facility capacity, and the demand for NU will fall significantly below supply. As such a price could not be accepted by the market, it is supposed that tails assays will remain at a high level. Here we obtained a tails assay value for a case where the NU demand and the demand for UE services have the same ratio in Production Case A and Enrichment Case A (0.88 times: their supply amounts are less than the lowest supply capacities because their supply and demand balances are eased), which provides a tails assay of $0.22 \%$. This occurs when the UE service price in $\mathrm{USD} / \mathrm{kg}-\mathrm{SWU}$ is about 2.5 times that of $\mathrm{NU}$ in $\mathrm{USD} / \mathrm{lb}-\mathrm{U}_{3} \mathrm{O}_{8}$, and the UE service price is relatively high. If we suppose that the NU price falls below $40 \mathrm{USD} / \mathrm{lb}-\mathrm{U}_{3} \mathrm{O}_{8}$, as stated above, the UE service price will drop below $100 \mathrm{USD} / \mathrm{kg}$-SWU. Therefore, this case would also cause the UE price to significantly drop from its current price of $130 \mathrm{USD} / \mathrm{kg}-\mathrm{SWU}$.

The projected trade flows are shown in Tables 10 and 11. Asia, where the demands significantly increase, will be supplied NU from the former Soviet Union (Russia, Kazakhstan, and

Table 10 Uranium trade (Low demand scenario: 2020)

\begin{tabular}{|c|c|c|c|c|c|c|c|c|c|c|}
\hline & & & & & & & & & & Unit: $t U$ \\
\hline From: $\quad$ To: & USA & Canada & L. America & Europe & FSU & Middle East & Africa & Asia & Oceania & World \\
\hline USA & 2,690 & & & & & & & & & 2,690 \\
\hline Canada & 5,855 & 1,554 & & 3,752 & & & & 1,389 & & 12,549 \\
\hline L. America & 542 & & 840 & & & & & 387 & & 1,769 \\
\hline Europe & & & & 283 & & & & & & 283 \\
\hline FSU & 3,596 & & & 6,580 & 5,760 & & 264 & 8,863 & & 25,063 \\
\hline Middle East & & & & 702 & & 146 & & 638 & & 1,486 \\
\hline Africa & 4,590 & & & 4,916 & & & & 7,328 & & 16,835 \\
\hline Asia & & & & & & & & 1,748 & & 1,748 \\
\hline Oceania & 798 & & & 2,249 & & & & 4,102 & & 7,149 \\
\hline World & 18,070 & 1,554 & 840 & 18,483 & 5,760 & 146 & 264 & 24,455 & 0 & 69,573 \\
\hline
\end{tabular}

Table 11 Enrichment trade (Low demand scenario: 2020)

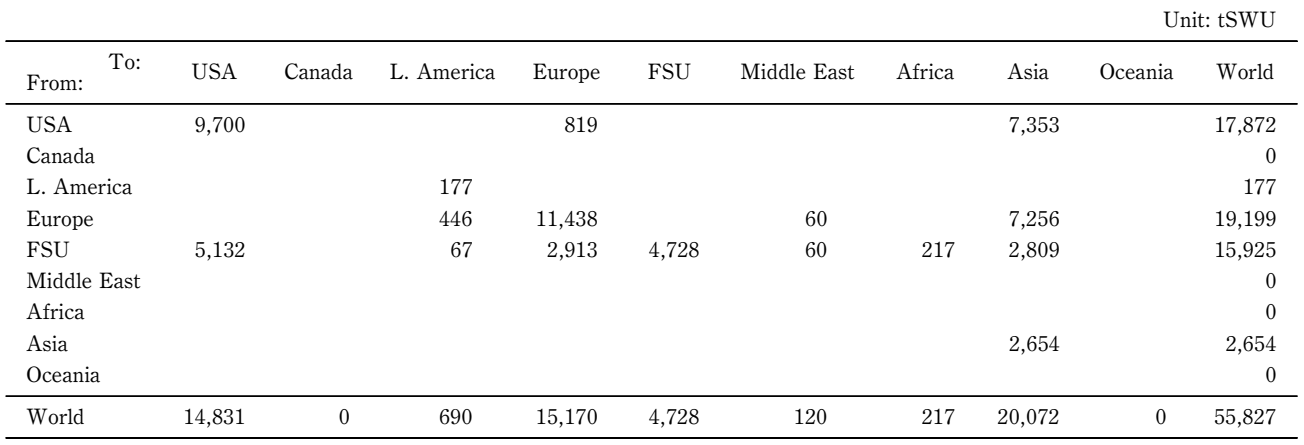


other countries), Africa, and Australia. Europe will be supplied from the former Soviet Union and Africa, and the US will be supplied from Canada. The balance of supply and demand of NU will be eased by 2020, and particularly in the low demand scenario, no large-scale project will be implemented other than those that are already fixed. In such a case, the production amount will not increase from the current level in Australia, and there will be an increase in the export from the former Soviet Union and Africa to other regions.

In addition, if an amount of 2,000 tU is produced in Jordan as planned, it will far exceed production in the Middle East, and they will need to export uranium to other regions (Europe, Asia, and other regions).

Even in this low demand scenario, the UE service demand is supposed to increase, especially in Asia. Consequently, the import of UE services from Europe, Russia, and the US to Asia will increase towards 2020. However, if this region (especially China) tries to substantially expand the enrichment capacity to satisfy the UE service demand in the region, there is a concern that the global supply of UE services may be excessive. It is thus difficult for Asian countries to substantially lower their dependence on imported enrichment services.

\section{(2) Year 2035}

The demands for NU in 2035 are 67,931 tU and 88,352 $\mathrm{tU}$, and those for UE services are $87,890 \mathrm{tSWU}$ and 57,178 tSWU in Price Cases I and II, respectively (with tails assays of $0.11 \%$ and $0.27 \%$ ).

For the NU production capacity in 2035, the production capacity of A-II and B-II in 2020 is assumed to be retained in Production Cases A and B, as stated above, which is 78,636 tU and $112,512 \mathrm{tU}$, respectively. If there is no increased demand, there will be a decline in the production capacity. The production capacity multiplied by an availability of $80 \%$ will then drop to $54,736 \mathrm{tU}$ of A-II in 2035 at the lowest, and this would occur in a case where the NU price declines and remains substantially low due to a reason such as secondary supply.

In the low demand scenario, the NU demand of 67,000 tU in 2035 will not exceed the lower limit $(78,636 \mathrm{tU})$ of the production capacity in Price Case I. In addition, for Price Case II, the NU demand of 93,934 tU will be slightly above the lower limit. This means that whatever the tails assay amount, the supply of NU will remain excessive. Whereas, although the demand for UE services will fall far below Enrichment Case A in Price Case II, it will be far above that of Enrichment Case B in Price Case I. This shows that tails assays will not significantly decline in the low demand scenario, even in 2035, and that it will remain at high level. If a same ratio is assumed with respect to the supply capacity to the cases where all the projects currently in the planning stage are implemented (Production Case B and Enrichment Case B), both demands will remain at $79 \%$ of the supply capacities, and tails assays will be around $0.27 \%$, which is equivalent to that in Price Case II.

If enrichment plants that are currently in the planning stage begin operation by 2035 , the supply and demand relation between NU and UE projects will not become tight even in 2035, and instead the production amount will decline with respect to the easing of the supply and demand balance. In such a case, it will be difficult to construct any further enrichment plants by 2035 . Furthermore, it is supposed that the prices of NU and enrichment service will fall to easing of the supply and demand balance. The $0.27 \%$ tails assay indicates that the optimal ratio of the UE service price in USD/kg-SWU to the NU price in USD $/ \mathrm{lb}-\mathrm{U}_{3} \mathrm{O}_{8}$ is about 4:1. In this case, even if the UE price remains at $130 \mathrm{USD} / \mathrm{kg}-\mathrm{SWU}$, which is the same level as the current price, there will be a significant drop in the NU price to about $30 \mathrm{USD} / \mathrm{lb}-\mathrm{U}_{3} \mathrm{O}_{8}$. This means that in the low demand scenario, even if only the existing uranium resource development plans and enrichment facility constructions that are currently in the planning stage are implemented, 
the supply will remain excessive until 2035. In addition, it is supposed that prices will drop due to easing of the supply and demand balance. Therefore, it is highly likely in this case that both projects currently in the planning stage will not only be delayed but will be called off by 2035 .

The estimated results for a tails assay of $0.27 \%$ are shown in Tables 12 and $\mathbf{1 3}$ and in Figures 5 and 6.

In this case, $70 \%$ of the increased demand from 2020 to 2035 for global NU will come from Asia. This increased demand will mainly be covered by supplies from Africa and Australia. The demand in Europe will decrease from 2020, which will slightly reduce the amount traded from the former Soviet Union and Africa.

In this scenario, as the tails assay is supposed to increase from that in 2020 , there will be a relatively small increase in the UE service demand. Although the demand in Asia will increase, that in the US and Europe will decrease. In particular, there will be a significant decline in the demand in Europe, and the export of UE services from Europe and Russia to Asia will increase.

\section{High Demand Scenario}

Estimates of global NU and UE services in the high demand scenario are shown in Figure 7.

\section{(1) Year 2020}

The demands for NU in 2020 in the high demand scenario are 72,348 $\mathrm{tU}$ and 94,086 tU, and those for UE services are 93,561 tSWU and 60,868 tSWU in Price Cases I and II (tails assays are $0.11 \%$ and $0.27 \%$ ), respectively.

Table 12 Uranium trade (Low demand scenario: 2035)

\begin{tabular}{|c|c|c|c|c|c|c|c|c|c|c|}
\hline & & & & & & & & & & Unit: $\mathrm{tU}$ \\
\hline From: $\quad$ To: & USA & Canada & L. America & Europe & FSU & Middle East & Africa & Asia & Oceania & World \\
\hline USA & 3,703 & & & & & & & & & 3,703 \\
\hline Canada & 6,808 & 1,554 & & 3,583 & & & & 2,540 & & 14,485 \\
\hline L. America & 465 & & 874 & & & & & 661 & & 2,000 \\
\hline Europe & & & & 350 & & & & & & 350 \\
\hline FSU & 3,890 & & & 6,473 & 8,695 & 0 & 294 & 11,303 & & 30,655 \\
\hline Middle East & & & & 606 & & 1,072 & & 2 & & 1,680 \\
\hline Africa & 5,074 & & & 3,946 & & 0 & & 13,117 & & 22,138 \\
\hline Asia & & & & & & & & 2,233 & & 2,233 \\
\hline Oceania & 1,202 & & & 2,351 & & 0 & & 7,866 & & 11,419 \\
\hline World & 21,144 & 1,554 & 874 & 17,309 & 8,695 & 1,072 & 294 & 37,721 & 0 & 88,662 \\
\hline
\end{tabular}

Table 13 Enrichment trade (High demand scenario: 2035)

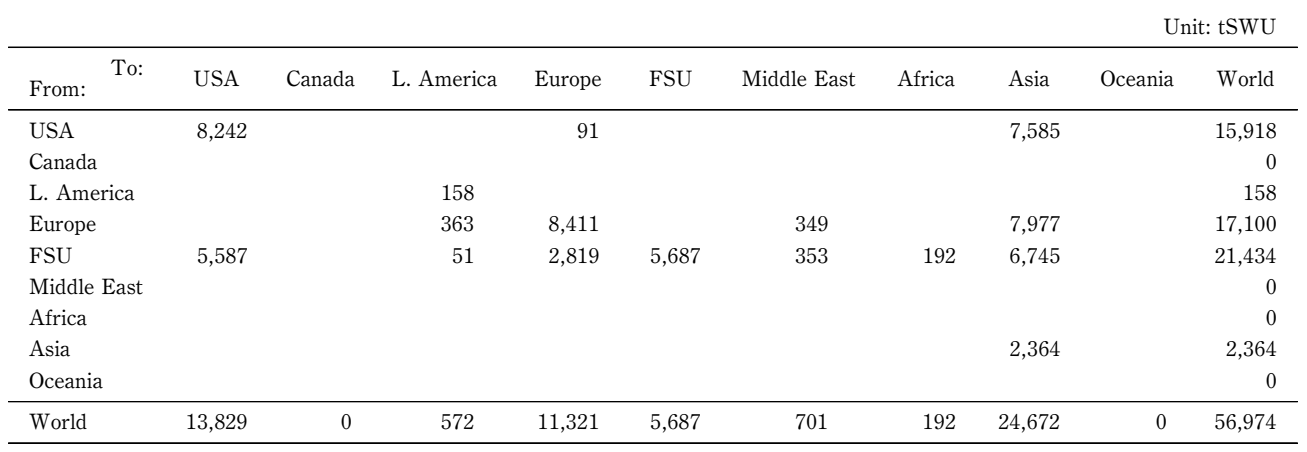




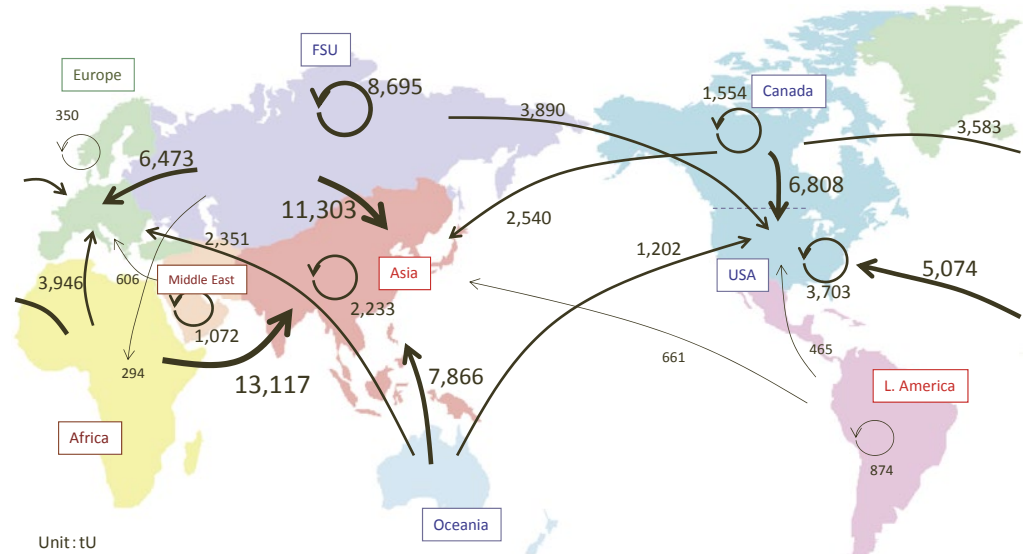

Figure 5 Uranium trade (Low demand scenario: 2035)

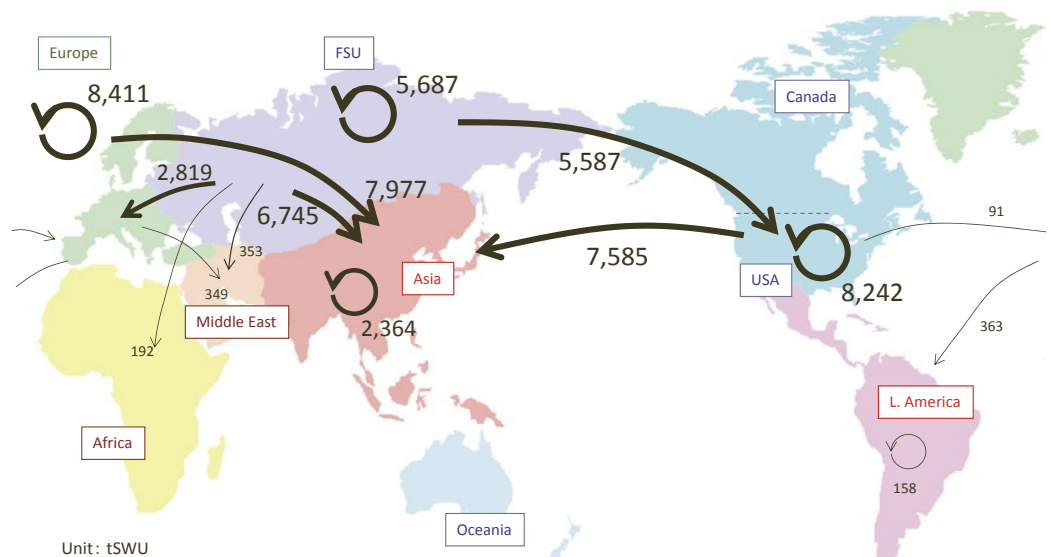

Figure 6 Enrichment trade (Low demand scenario: 2035)
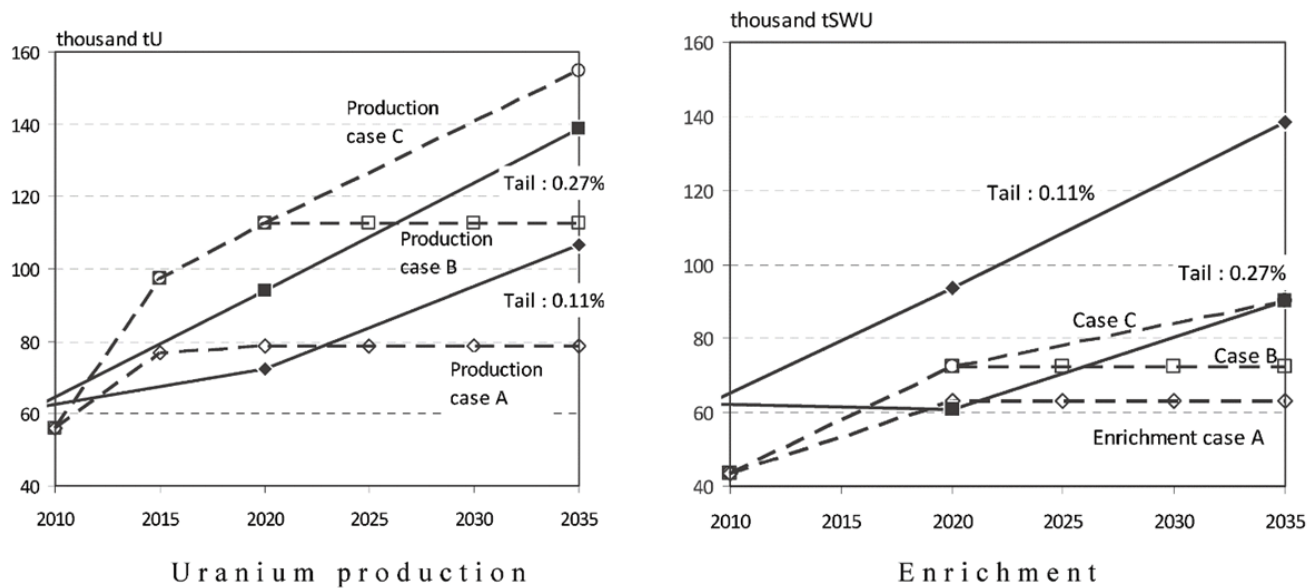

Figure 7 Uranium production and enrichment projections (High demand scenario) 
The demand for NU will fall below that in Production Case B, even with Price Case II where the tails assay is high. This means that if uranium resource development projects that are currently in the planning stage are implemented, the supply will exceed the demand, even in the high demand scenario. The demand for UE services will be at around the same level as in Enrichment Case A with Price Case II where the tails assay is high, and it will far exceed that of Enrichment Case B with Price Case I, where the tails assay is low. This means that the supply and demand for NU will be relatively eased compared to that of UE services, and in this case, therefore, it is supposed that the tails assay will remain at a high level.

If we suppose that the ratio of both NU production and UE services will be the same with respect to the supply capacities for Production Case B and Enrichment Case B, where all projects currently in the planning stages are implemented, demands will be $84 \%(94,400 \mathrm{tU}$ and 60,662 tSWU) of the capacity, and tails assay will again be around $0.27 \%$, which is at a similar level to that in Price Case II.

If we suppose that all new projects currently in the planning stages have been planned to provide reasonable profits at the current price level, the demand growth to attain levels of Production Case B and Enrichment Case B, where implementation of the new projects are taken into account, will be that sustains the current price level. If demand falls lower than this, it is supposed that only low-cost projects will be implemented and uranium prices will decline. As in the case presented in the previous section, the $0.27 \%$ tails assay in this case indicates that the optimal ratio between the UE service price in USD/kg-SWU to the NU price in USD/lb- $\mathrm{U}_{3} \mathrm{O}_{8}$ is about $4: 1$. Even if the UE price remains at the current level of $130 \mathrm{USD} / \mathrm{kg}-\mathrm{SWU}$, the NU price will drop significantly to about $30 \mathrm{USD} / \mathrm{lb}-\mathrm{U}_{3} \mathrm{O}_{8}$. This means that even in the high demand scenario, if we consider the operation launch of the currently planned UE plants, it is highly likely that the supply and demand balance of NU will be eased, and resource development investment will decline.

The estimated results of trade flows are presented in Tables 14 and 15.

In response to the increased demand, the trade in NU will increase more than in the low demand scenario. In particular, exports from the former Soviet Union, Africa, and Australia to Asia will increase. With an increase in the global uranium demand, it is expected that Australia will become increasingly important as a uranium producing country, because of its large uranium resources. However, the NU production capability of Australia in 2020 is not so large.

The UE service demand will also increase, particularly in Asia. In this respect, imports from North America, Russia, and Europe will increase. Compared to the current situation, import expansion will be significant, particularly from North America where many new enrichment plants are being constructed, and from Russia, which has a significant capacity margin. However, as stated above, even in this case, no further enrichment facilities will be required, other

Table 14 Uranium trade (High demand scenario: 2020)

\begin{tabular}{|c|c|c|c|c|c|c|c|c|c|c|}
\hline From: $\quad$ To: & USA & Canada & L. America & Europe & FSU & Middle East & Africa & Asia & Oceania & World \\
\hline USA & 4,082 & & & & & & & & & 4,082 \\
\hline Canada & 6,450 & 1,687 & & 4,670 & & & & 1,850 & & 14,657 \\
\hline L. America & 293 & & 1,415 & & & & & 291 & & 2,000 \\
\hline Europe & & & & 367 & & & & & & 367 \\
\hline FSU & 3,769 & & & 7,886 & 9,021 & 0 & 294 & 11,017 & & 31,988 \\
\hline Middle East & & & & 667 & & 1,007 & & 6 & & 1,680 \\
\hline Africa & 4,984 & & & 6,480 & & 0 & & 12,454 & & 23,918 \\
\hline Asia & & & & & & & & 2,380 & & 2,380 \\
\hline Oceania & 1,362 & & & 3,755 & & 0 & & 8,212 & & 13,329 \\
\hline World & 20,941 & 1,687 & 1,415 & 23,824 & 9,021 & 1,007 & 294 & 36,210 & 0 & 94,400 \\
\hline
\end{tabular}


Table 15 Enrichment trade (High demand scenario: 2020)

\begin{tabular}{|c|c|c|c|c|c|c|c|c|c|c|}
\hline & & & & & & & & & \multicolumn{2}{|c|}{ Unit: tSWL } \\
\hline From: $\quad$ To: & USA & Canada & L. America & Europe & FSU & Middle East & Africa & Asia & Oceania & World \\
\hline USA & 8,276 & & & 468 & & & & 8,205 & & 16,948 \\
\hline Canada & & & & & & & & & & c \\
\hline L. America & & & 168 & & & & & & & 168 \\
\hline Europe & & & 629 & 10,818 & & 327 & & 6,433 & & 18,207 \\
\hline FSU & 5,426 & & 129 & 4,302 & 5,902 & 332 & 192 & 6,537 & & 22,821 \\
\hline Middle East & & & & & & & & & & c \\
\hline Africa & & & & & & & & & & c \\
\hline Asia & & & & & & & & 2,517 & & 2,517 \\
\hline Oceania & & & & & & & & & & c \\
\hline World & 13,701 & 0 & 926 & 15,588 & 5,902 & 659 & 192 & 23,692 & 0 & 60,662 \\
\hline
\end{tabular}

than those that are currently in the planning stages, and it is more likely that the expansion plans of enrichment plants will be delayed.

\section{(2) Year 2035}

The demands for NU in 2035 in the high demand scenario will be 106,525 tU and 138,710 $\mathrm{tU}$, and those for UE services will be 138,521 tSWU and 90,117 tSWU in Price Cases I and II, respectively (with tails assays of $0.11 \%$ and $0.27 \%$ ).

In such a case, the demand for NU in Price Case II will exceed 112,512 tU in Production Case B, and even in Price Case I, the demand will be close to the level in Production Case B. The demand for UE services will significantly exceed the supply capacity in Enrichment Case $\mathrm{B}$, even in the Price Case II. This means it is highly likely that the supply-demand balance of NU will become tight in the high demand scenario, unless the NU production capacity and EU facilities steadily expand.

However, as in this case, if a steady growth in uranium production is expected, even after 2020 , it would be natural to assume that greater resources development investment will occur accordingly, and uranium production will further increase. Assuming Production Case C, where uranium production will grow at a rate that is half of that occurring between 2010 and 2020, production in 2035 will be 154,788 $\mathrm{tU}$ and will exceed demand with the tails assay of $0.27 \%$. Therefore, as long as steady development investment in uranium resources continue, a sufficient supply is expected, even if demand rises and the tails assay is high.

With respect to UE facilities, the required facility growth between 2020 and 2035 in Price Cases II and I is 17,817 tSWU and 66,221 tSWU, respectively. In particular, growth in Price Case $I$ is at a level that exceeds the current total capacity of global enrichment facilities, and to construct new facilities to cover the level, a significantly tight balance of supply and demand and a price rise in UE service costs would be required. However, as mentioned above, the supply and demand of NU itself would be satisfied, even if such rapid resource development was not be made. Therefore, in this case, it is also expected that tails assays will remain high and that construction of UE facilities will be minimal. The facility growth in Price Case II is $17,817 \mathrm{tSWU}$, which is equivalent to the capacity of three to four large-scale enrichment plants. It is not unrealistic to expect this level of facility construction between 2020 and 2035 . In this study, we established Case $\mathrm{C}$ which assumes that an enrichment plant with a capacity of $6,000 \mathrm{tSWU} / \mathrm{yr}$ would be constructed in the US, and that the remaining required facility growth would be covered by new construction in the former Soviet Union and China. The estimated results are presented in Tables 16 and 17 and Figures 8 and 9.

With respect to the supply and demand of NU, the demand in Asia will be significant, and 
INSIGHTS CONCERNING THE FUKUSHIMA DAIICHI NUCLEAR ACCIDENT Vol. 4

Table 16 Uranium trade (High demand scenario: 2035)

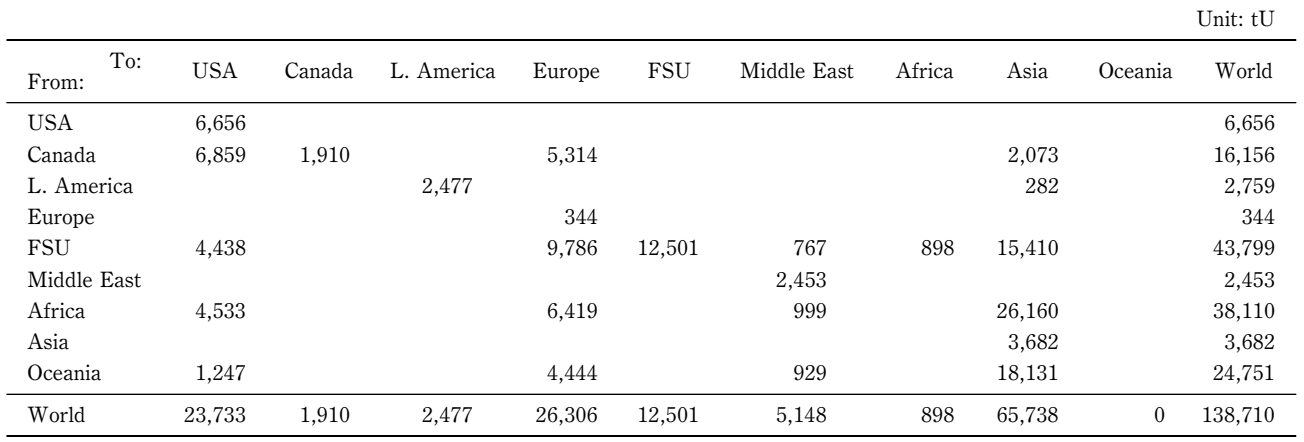

Table 17 Enrichment trade (High demand scenario: 2035)

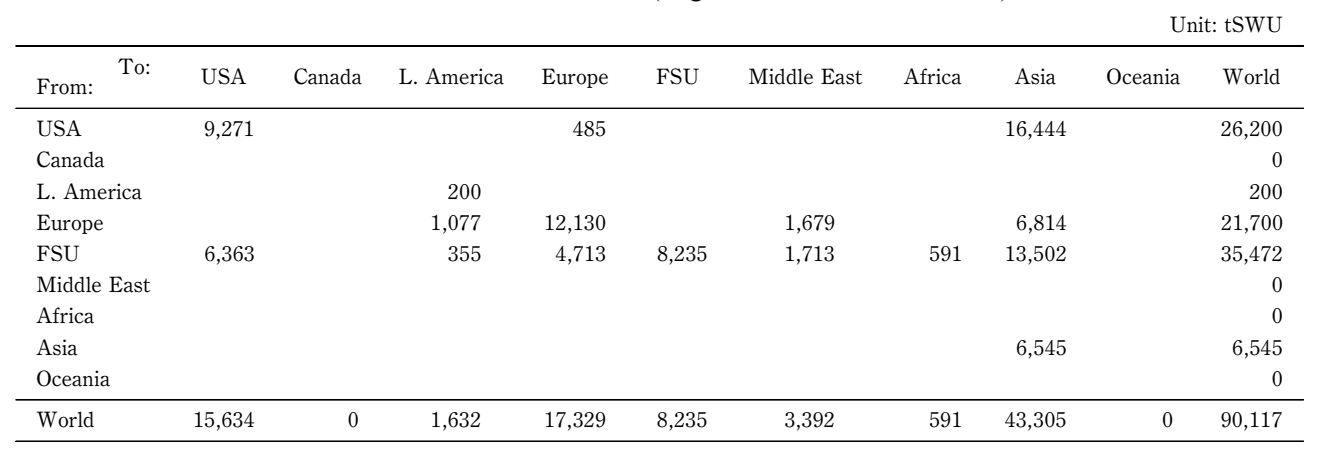

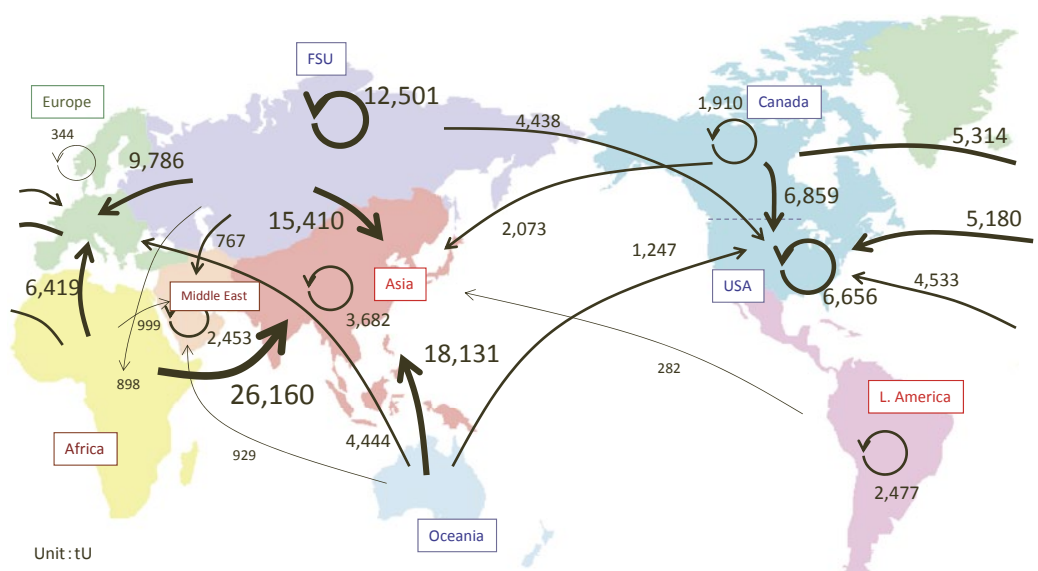

Figure 8 Uranium trade (High demand scenario: 2035)

$67 \%$ of global growth after 2020 will occur in this area. The demand for this growth will be covered by imports from the former Soviet Union, Africa, and Australia. However, as the demand for uranium will also increase in the former Soviet Union, the amount exported will be limited. In contrast, imports from Africa and Australia will largely expand.

Despite the new large-scale enrichment facilities assumed in China, the supply for UE 


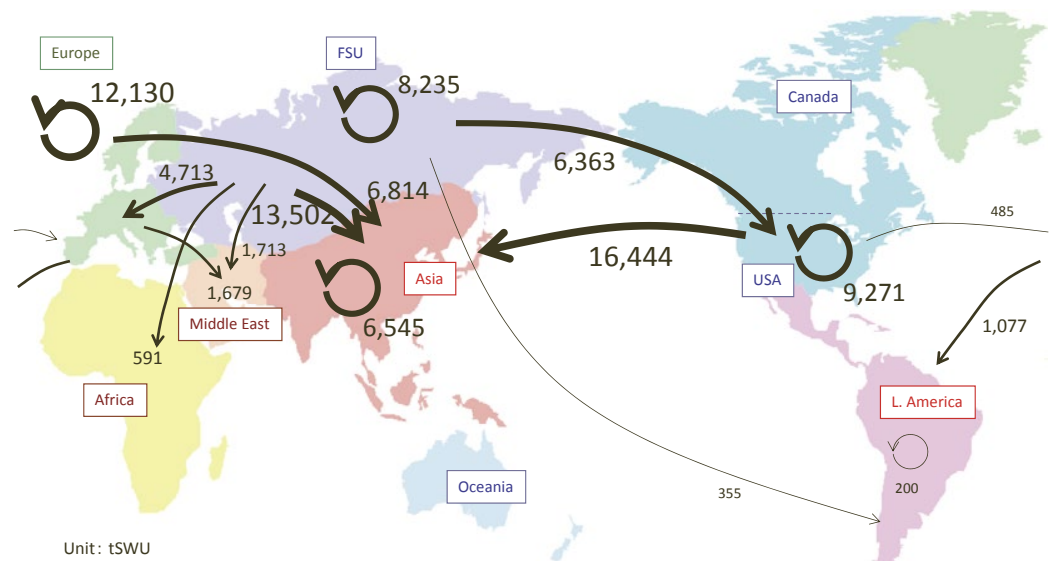

Figure 9Enrichment trade (High demand scenario: 2035)

Table 18 Uranium transport to enrichment facilities (High demand scenario: 2035, optimized with freight)

\begin{tabular}{|c|c|c|c|c|c|c|c|c|c|c|}
\hline & & & & & & & & & & Unit: $\mathrm{tU}$ \\
\hline From: $\quad$ To: & USA & Canada & L. America & Europe & FSU & Middle East & Africa & Asia & Oceania & World \\
\hline USA & 6,656 & & & & & & & & & 6,656 \\
\hline Canada & 11,655 & & & 4,501 & & & & & & 16,156 \\
\hline L. America & 2,450 & & 308 & & & & & & & 2,759 \\
\hline Europe & & & & 344 & & & & & & 344 \\
\hline FSU & 4,043 & & & 1,536 & 38,220 & & & & & 43,799 \\
\hline Middle East & & & & 2,453 & & & & & & 2,453 \\
\hline Africa & 13,914 & & & 7,816 & 16,380 & & & & & 38,110 \\
\hline Asia & & & & & & & & 3,682 & & 3,682 \\
\hline Oceania & 1,609 & & & 16,751 & & & & 6,391 & & 24,751 \\
\hline World & 40,328 & 0 & 308 & 33,401 & 54,599 & 0 & 0 & 10,074 & 0 & 138,710 \\
\hline
\end{tabular}

Table 19 Enrichment trade (High demand scenario: 2035, optimized with freight)

\begin{tabular}{|c|c|c|c|c|c|c|c|c|c|c|}
\hline \multicolumn{11}{|c|}{ Unit: tSWU } \\
\hline From: $\quad$ To: & USA & Canada & L. America & Europe & FSU & Middle East & Africa & Asia & Oceania & World \\
\hline USA & 13,100 & & & 17 & & & & 13,083 & & 26,200 \\
\hline Canada & & & & & & & & & & 0 \\
\hline L. America & & & 200 & & & & & & & 200 \\
\hline Europe & & & 0 & 12,927 & & 0 & & 8,773 & & 21,700 \\
\hline FSU & 2,534 & & 1,432 & 4,384 & 8,235 & 3,392 & 591 & 14,904 & & 35,472 \\
\hline Middle East & & & & & & & & & & 0 \\
\hline Africa & & & & & & & & & & 0 \\
\hline Asia & & & & & & & & 6,545 & & 6,545 \\
\hline Oceania & & & & & & & & & & 0 \\
\hline World & 15,634 & 0 & 1,632 & 17,329 & 8,235 & 3,392 & 591 & 43,305 & 0 & 90,117 \\
\hline
\end{tabular}

services in Asia will not be satisfied and will be supplied from Russia and the US.

In the high demand scenario for 2035, estimated results for the reference case where trade flows are optimized depending on the cost of transportation between regions is shown in Tables 18 and 19. Table 18 shows flows between NU production areas and UE facilities areas, unlike Table 16, which shows flows from uranium production to fuel demand areas. The values in 
Table 19 are largely similar to the estimated results shown in Table 17. In this case, as mentioned above, $50 \%$ of the amount provided by enrichment facilities in the US is assumed to be supplied for US demand. If estimations that eliminate this constraint are made, all enriched uranium in the US would be exported to Asia, and the supply from Russia would be sent to the US (because the total distance from Russia to the US and then the US to Asia is shorter than that from Russia to Asia via the Cape of Good Hope).

However, the supply of fuel between the US and Russia is determined based on sensitive political conditions and these have a large impact on the global supply and demand of fuel.

\section{Supply of NU and UE Services to Asia}

As mentioned above, Asia will be the center of nuclear power plants expansion. The demand share of NU and UE services in the region is assumed to increase from the current level of around $30 \%$ to around $40-50 \%$ in 2035 , depending on the case. It is thus crucial to determine how the demand growth in Asia can be met with regard to the future issue of uranium supply and demand.

Figures 10 and 11 show the supply of NU and UE services from other regions to Asia in the above cases. It is currently estimated that a considerable amount of NU will be supplied to Asia from the former Soviet Union (Russia, Kazakhstan, and other countries). However, in future, as seen from the estimated growth of nuclear power plants in this region, the former Soviet Union

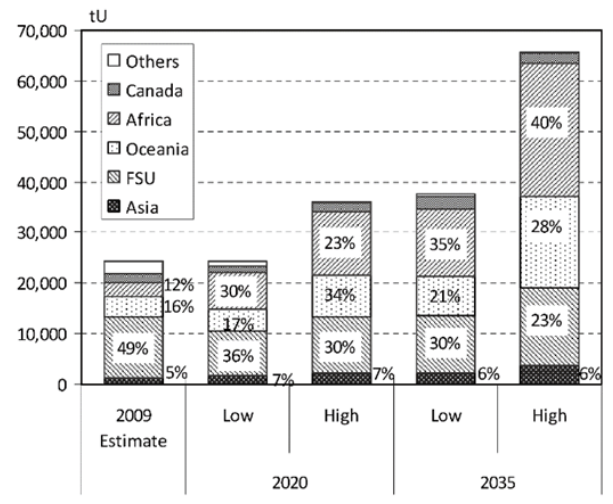

Figure 10 Uranium supply to Asia

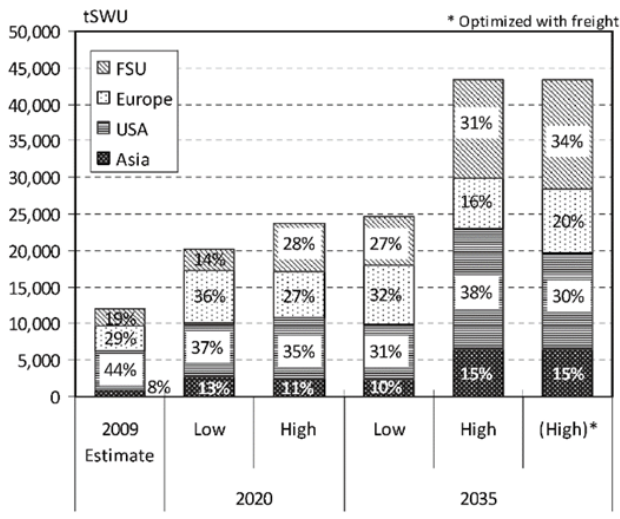

Figure 11 Enrichment supply to Asia 
(FSU) is not the region that will cover the growth demand. Instead, Africa will increase their supply in the medium and short term. As mentioned above, in the low demand scenario, where the growth of the global nuclear capacity is slower, it is supposed that the supply and demand balance of NU will be largely eased and that resource development will stagnate. In this case, there will be no significant increase in imports from any region other than Africa.

In the high demand scenario, where there is a maximum expansion of nuclear power capacities, imports from Australia and Africa will cover the rapid growth in demand, and imports from these two areas will cover $70 \%$ of the Asian demand in 2035 . However, production within Asia will only cover about $6 \%$ of the demand, and it is supposed their resource dependency on foreign countries will continue in the long run.

With respect to UE services, imports from the US, where the large-scale construction of new enrichment plants is planned, will increase in the medium term. However, the former Soviet Union is the area where exports will grow in accordance with long-term demand increases, and their importance will increase. In contrast, the supply share from Europe to Asia will decline, and there will be no significant increase in the supply share from Asia. As with the case of NU, it is supposed that Asia will depend on imports from other regions for long-term UE services. Figure 11 also shows results of the reference case, where the trade flow is optimized based on the cost of transportation between regions; results are largely similar to those for the high demand scenario in 2035 .

Figures 12 and 13 show changes in the self-sufficiency rate and the Herfindahl-Hirshman

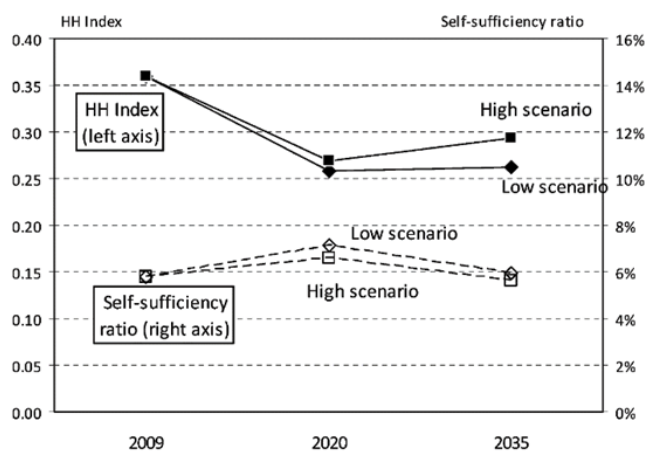

Figure $12 \mathrm{HH}$ index and self-sufficiency ratio (Uranium)

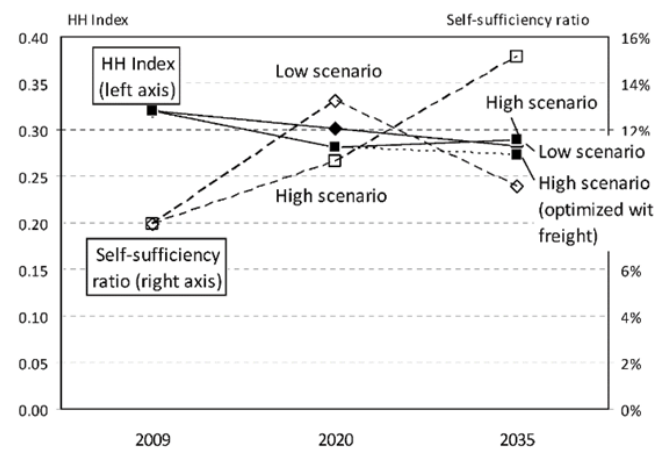

Figure $13 \mathrm{HH}$ index and self-sufficiency ratio (Enrichment) 
(HH) index of NU and UE services in Asia. As mentioned above, it is expected that NU self-sufficiency will remain at a very low level of about $6 \%$ until 2035, and UE services will increase up to about $15 \%$ in the high demand scenario in 2035 , due to the operation and launch of new enrichment facilitates. However, as also mentioned above, it is supposed that improving the self-sufficiency rate will be more difficult than this level from the view points of price and supply-demand balance.

The HH index indicates the diversity of NU and UE service suppliers to Asia, which can be expressed by Eq. (2). A lower value shows that the suppliers are more diverse, which is desirable for an importer from the perspectives of energy security,

$$
H H=\sum_{i}\left(\frac{a_{i}}{X}\right)^{2}
$$

where, $a_{i}$ indicates the supply amount from region i to Asia, and $\mathrm{X}$ indicates the total supply to Asia. As of 2009, the estimated HH index for NU is 0.36 and that of UE services is 0.32 . This index for NU is a little lower than the oil supply value (0.37) in Asia estimated based on the BP statistics ${ }^{31)}$. This is because, according to the actual record in 2009 , the supply ratio from the former Soviet Union is high. However, as seen in Figures 12 and 13, both indexes for NU and UE services will fall below 0.3, due to the increased supplies from Africa, Australia, and other regions for NU, and the well-balanced supply from three main regions (including Russia, the US, and Europe) for UE services. With respect to the reference case, the self-sufficiency rate in Figure 13 is the same value in the high demand scenario, and the HH index in Figure 13 is also nearly the same as the value in the high demand scenario.

\section{Conclusions}

This study estimates the trade flows of NU and UE services in the long term by considering nuclear power generation growth and increases in the NU production and UE capacities. It is expected that although nuclear capacity will continue to expand mainly in Asia in the future, the balance between the supply and demand of NU and UE services will be eased until 2020, due to NU resource development and the enhancement of UE facilities that are currently in the planning stage.

As a result, resource development and enrichment facility enhancement projects are likely to be significantly delayed in the mid-term. In the case where the growth of nuclear power generating capacities is slow, this trend will probably continue until 2035, and uranium production might decline from 2020. However, in the case where countries throughout the world promote nuclear power generation at a maximum level to maintain a stable supply of energy, and to provide measures to prevent global warming, more projects plans will need to be implemented (other than those that currently exist). Even if the implementation speed is relatively slow compared to those currently in the planning stage, it is projected that this will be sufficient to satisfy demand. These projections suggest that it is unlikely that the supply and demand balance will be tight for the next 20 to 30 years, as long as the development plans currently underway are implemented by around 2020 and new projects are launched in an appropriate manner that considers the future growth demand. From the relation between supply and demand of NU and UE services, it is projected that the tails assay will remain at a high level in the long term, which means that UE service prices will remain at a relatively high level compared to the price of NU.

Asia will be at the center of future demand growth, and its dependence on foreign resources 
for both NU and UE services will stay at a high level in the long term. With respect to NU, the demand will be covered by imports from Africa and Australia; and for UE services, it is estimated that Asia will rely on imports to a greater degree from the US and then from Russia. Nuclear energy is often considered a quasi-domestic energy from the perspective of its long fuel storage capability, in addition to domestic implementation of the nuclear fuel cycle and the reusability of spent fuel. As suggested by this study, it is supposed that a rise in the self-sufficiency rate of the production of NU and UE services will be difficult in Asia in the medium-term. However, the diversification of supply regions ( $\mathrm{HH}$ index) is projected to drastically improve compared to that of the oil supply, owing to progress made in global development plans. We can thus estimate that progress made in providing nuclear energy would contribute to energy security in Asia at a certain level.

If nuclear power plants expand even after 2035, which is the period studied in this paper, the supply and demand balance of NU resources and UE will become gradually tighter, depending on development trends in new uranium resources and UE facilities. However, with respect to the time span, it will be necessary to consider introducing various technologies that help mitigate the growth in demand for NU, including those relating to the use of FBRs and thorium resources, uranium extraction from seawater, and nuclear fusion. This also means that, as this estimation suggests, as long as the supply and demand for uranium is considered only until 2035, such innovative new technologies are not necessarily indispensable. Future studies will need to be conducted to determine in the longer term how we regard the use of such technologies.

Part of this research was conducted with the support of the Environment Research and Technology Development Fund of the Ministry of the Environment (S-6-1:General Research for Development, Estimation and Evaluation Methods for Meddle to Long Term Political Options and their Promotion for Low Carbon Society in Asia; Scenario Development for Establishment of Low Carbon Society for Asian Countries). We would like to express our deepest gratitude to the Ministry.

\section{References}

1) Ministry of Economy, Trade and Industry, The Strategic Energy Plan of Japan (2010).

2) International Energy Agency, World Energy Outlook 2011, IEA Publications, ISBN 9789264124134 (2011).

3) International Energy Agency, Energy Technology Perspectives 2010, IEA Publications, ISBN 9789264085978 (2011).

4) International Atomic Energy Agency, Energy, Electricity and Nuclear Power Estimates for the Period up to 2050, IAEA Vienna, ISBN 9789201194107 (2011).

5) OECD, Nuclear Energy Agency, Nuclear Energy Outlook 2008, OECD Publishing, ISBN 9789264054103 (2008).

6) U.S. Energy Information Administration, International Energy Outlook 2011, DOE/EIA-0484 (2011).

7) World Nuclear Association, The WNA Nuclear Century Outlook: Averting the Danger of Catastrophic Climate Change: Is the Nuclear Renaissance Essential?, WNA (2011).

8) OECD, International Energy Agency, Uranium 2009 Resources, Production and Demand, OECD Publishing, ISBN 9789264047907 (2009).

9) World Nuclear Association, The Global Nuclear Fuel Market: Supply and Demand 2011-2030, WNA (2011).

10) R. Komiyama, T. Kakinoki, "Long-term outlook for nuclear power generation and uranium demand and supply situations up to 2030 and 2100," Energy Keizai, 33[3], 1-24 (2007). [in Japanese]

11) T. Murakami, "Nuclear fuel demand and supply outlook up to 2030," Energy Keizai, 33[5], 22-34 (2007). [in Japanese]

12) Y. Matsuo, M. Aoshima, M. Kako et al., "Asia/world energy outlook 2011 - Growing uncertainty over international energy trends and the future of Asia-," IEEJ Energy J., 7[1], 17-55 (2012). 
13) Y. Matsuo, “U. S. nuclear energy policy and Japan's nuclear industry expansion trends," IEEJ Energy J., 6[2], 27-42 (2011).

14) U. S. Energy Information Administration, Annu. Energy Outlook 2011, DOE/EIA-0383 (2011).

15) National Energy Board, Canada's Energy Future: Energy Supply and Demand Projections to 2035, ISBN 9781100194646 (2011).

16) Japan Atomic Industrial Forum, World Nuclear Power Plants 2011, JAIF (2011).

17) Denki Shimbun, Nuclear Pocket Book, The Japan Electric Association, ISBN 9784905217053 C0550 (2011). [in Japanese]

18) World Nuclear Association Website, http://www.worldnuclear.org/

19) Japan Atomic Energy Agency, Oubei ni Okeru Kakunenryou Saikuru ni Kansuru Chousa (A Report on Nuclear Fuel Cycle in Europe and North America FY 2007), (2008). [in Japanese]

20) Areva, 2011 Reference Document (2011).

21) URENCO, Annual Report and Accounts 2011 (2011).

22) Ux Consulting Website, http://www.uxc.com/

23) H. Hirai, Y. Matsuo, Y. Nagatomi, "Supply and demand analysis on petroleum products and crude oil for Asia and the world," IEEJ Energy J., 3[3], 29-60 (2008).

24) Y. Kobayashi, "Natural gas situation and LNG supply/ demand trends in Asia-Pacific and Atlantic markets," IEEJ Energy J., 5[1], 65-96 (2010).

25) P. Diehl, Re-enrichment of West European Depleted Uranium Tails in Russia, WISE Uranium Project, http://www.wiseuranium.org, (2004).

26) Poten and Partners, Estimated Voyage Costs — Round Trip Economics (2011).

27) U. S. Committee on the Internationalization of the Civilian Nuclear Fuel Cycle, Committee on International Security and Arms Control, National Academy of Sciences and National Research Council, Internationalization of the Nuclear Fuel Cycle: Goals, Strategiesand Challenges, The National Academies Press, ISBN 9780309126601 (2009).

28) NAC International, Selected Aspects of the Fuel Cycle Front End (2011).

29) World Scale Association, New Worldwide Tanker Nominal Freight Scale (2010).

30) PESCO Co., Ltd, Kakunenryou Saikuru Shisetsu, Gijutsu tou ni Kakaru Doukou Chousa (A Report on Nuclear Fuel Cycle Facilities and Technologies) (2009). [in Japanese]

31) BP, Statistical Review of World Energy June 2012 (2012). 


\section{Corrigendum}

\section{Survey of Living Environmental Land Contaminated with Radioactive Materials due to Fukushima Daiichi Nuclear Power Plant Accident}

\section{Sin-ya HOHARA*, Masayo INAGAKI, Kiyoshi KOJIMA, Hirokuni YAMANISHI, Genichiro WAKABAYASHI, Wataru SUGIYAMA and Tetsuo ITOH}

Correction of Table 1 in page 4 has been made as shown below by author declaration.

Table 1 Specific activity of soil samples

\begin{tabular}{cccccc}
\hline Nuclide & $\begin{array}{c}\text { Half-life } \\
{[\text { day }]}\end{array}$ & $\begin{array}{c}\text { Nasushiobara City } \\
\text { Kuroiso Park } \\
{[\mathrm{Bq} / \mathrm{kg}]}\end{array}$ & $\begin{array}{c}\text { Koriyama City } \\
\text { Hayama Park } \\
{[\mathrm{Bq} / \mathrm{kg}]}\end{array}$ & $\begin{array}{c}\text { Fukushima City } \\
\text { Arakawa Athletic } \\
\text { Park }[\mathrm{Bq} / \mathrm{kg}]\end{array}$ & $\begin{array}{c}\text { Regulation } \\
\text { level* }[\mathrm{Bq} / \mathrm{kg}]\end{array}$ \\
\hline${ }^{95} \mathrm{Nb}$ & 35 & $3.26 \mathrm{E} 1 \pm 0.91 \mathrm{E} 1$ & $8.33 \mathrm{E} 1 \pm 1.79 \mathrm{E} 1$ & $1.26 \mathrm{E} 2 \pm 0.28 \mathrm{E} 2$ & $1.0 \mathrm{E} 4$ \\
${ }^{131} \mathrm{I}$ & 8 & $6.49 \mathrm{E} 2 \pm 0.19 \mathrm{E} 2$ & $4.25 \mathrm{E} 3 \pm 0.04 \mathrm{E} 3$ & $2.21 \mathrm{E} 4 \pm 0.01 \mathrm{E} 4$ & $1.0 \mathrm{E} 5$ \\
${ }^{129} \mathrm{Te}$ & 0.048 & $2.31 \mathrm{E} 3 \pm 0.21 \mathrm{E} 3$ & $6.84 \mathrm{E} 3 \pm 0.41 \mathrm{E} 3$ & $2.34 \mathrm{E} 4 \pm 0.07 \mathrm{E} 4$ & $1.0 \mathrm{E} 5$ \\
${ }^{129 \mathrm{~m}} \mathrm{Te}$ & 34 & $3.05 \mathrm{E} 3 \pm 0.32 \mathrm{E} 3$ & $1.06 \mathrm{E} 4 \pm 0.07 \mathrm{E} 4$ & $3.67 \mathrm{E} 4 \pm 0.11 \mathrm{E} 4$ & $1.0 \mathrm{E} 6$ \\
${ }^{132} \mathrm{Te}$ & 3 & $2.98 \mathrm{E} 1 \pm 0.92 \mathrm{E} 1$ & $1.09 \mathrm{E} 2 \pm 0.19 \mathrm{E} 2$ & $2.76 \mathrm{E} 2 \pm 0.34 \mathrm{E} 2$ & $1.0 \mathrm{E} 5$ \\
${ }^{134} \mathrm{Cs}$ & 753 & $4.83 \mathrm{E} 3 \pm 0.04 \mathrm{E} 3$ & $2.11 \mathrm{E} 4 \pm 0.01 \mathrm{E} 4$ & $5.18 \mathrm{E} 4 \pm 0.01 \mathrm{E} 4$ & $1.0 \mathrm{E} 4$ \\
${ }^{136} \mathrm{Cs}$ & 13 & $1.74 \mathrm{E} 2 \pm 0.11 \mathrm{E} 2$ & $6.99 \mathrm{E} 2 \pm 0.22 \mathrm{E} 2$ & $1.80 \mathrm{E} 3 \pm 0.04 \mathrm{E} 3$ & $1.0 \mathrm{E} 4$ \\
${ }^{137} \mathrm{Cs}$ & 11020 & $5.10 \mathrm{E} 3 \pm 0.05 \mathrm{E} 3$ & $2.24 \mathrm{E} 4 \pm 0.01 \mathrm{E} 4$ & $5.50 \mathrm{E} 4 \pm 0.02 \mathrm{E} 4$ & $1.0 \mathrm{E} 4$ \\
\hline
\end{tabular}

* minimum specific activity stipulated for handling in a radiation controlled area under Japanese regulation ${ }^{14)}$.

\footnotetext{
* Corresponding author, E-mail: hohara@kindai.ac.jp DOI : 10.15669/fukushimainsights. Vol.4.169

(C) 2021 Atomic Energy Society of Japan. All rights reserved.

Originally published in Transactions of the Atomic Energy Society of Japan (ISSN 1347-2879), Vol. 11, No. 4, p.340 (2012)

in Japanese.
} 


\title{
Technical Data
}

\section{Design, Construction and Monitoring of Temporary Storage Facilities for Removed Contaminants}

\author{
Hiromitsu SAEGUSA ${ }^{1}$, Hironori FUNAKI ${ }^{1, *}$, Hiroshi KURIKAMI ${ }^{1}$, \\ Yoshiaki SAKAMOTO ${ }^{1}$ and Takayuki TOKIZAWA ${ }^{1}$ \\ ${ }^{1}$ Fukushima Environmental Safety Center, Japan Atomic Energy Agency, 1-29 Okitama-cho, Fukushima-shi, \\ Fukushima 960-8034, Japan
}

\begin{abstract}
Since the Fukushima Daiichi Nuclear Power Plant Accident caused by the Tohoku Region Pacific Coast Earthquake on March 11, 2011, decontamination work has been conducted in the surrounding environment within the Fukushima Prefecture. Removed contaminants including soil, grass and trees are to be stored safely at temporary storage facilities for several years, after which they will be transferred to a planned interim storage facility. The decontamination pilot project was carried out in both the restricted and planned evacuation areas in order to assess decontamination methods and demonstrate measures for radiation protection of workers. Fourteen temporary storage facilities of different technical specifications were designed and constructed under various topographic conditions and land use. In order to support the design, construction and monitoring of temporary storage facilities for removed contaminants during the full-scale decontamination within the prefecture of Fukushima, technical know-how obtained during the decontamination pilot project has been identified and summarized in this paper.
\end{abstract}

KEYWORDS: Fukushima Daiichi Nuclear Power Plant Accident, removed contaminants, temporary storage facility, facility requirements, management-related requirements, decontamination pilot project, technical know-how

\section{Introduction}

A large amount of radioactive material has been released into the environment because of the accident at the Tokyo Electric Power Co. Inc. Fukushima Daiichi Nuclear Power Plant, as a result of the Tohoku Region Pacific Coast Earthquake that occurred on March 11, 2011. The decontamination effort to reduce risks to human health and the living environment is still ongoing in various areas. To carry out the decontamination, the soil, fallen leaves, and vegetation (hereinafter referred to as "removed contaminants") must be stored safely in a temporary storage yard until being transferred to interim storage facilities ${ }^{1)}$.

The Japan Atomic Energy Agency (hereinafter referred to as "JAEA") has been making use of the RD findings regarding the disposal of radioactive waste, and formulating organizational

* Corresponding author, E-mail: funaki.hironori@jaea.go.jp

DOI : 10.15669/fukushimainsights. Vol.4.170

(C) 2021 Atomic Energy Society of Japan. All rights reserved.

Originally published in Transactions of the Atomic Energy Society of Japan (ISSN 1347-2879), Vol. 12, No. 1, p.1-12 (2013)

in Japanese. (Japanese version accepted: November 21, 2012) 
policies to secure the safety of temporary storage yards, construction methods and policies for monitoring after construction, and has been providing technical support regarding site selection, design, etc. of temporary storage for the local governments and residents in the region. Furthermore, our work forms part of the Government-commissioned project "Decontamination Demonstration Projects on Evacuation Areas, etc., for the Fukushima Daiichi Nuclear Power Plant Accident," and the "Decontamination Demonstrations Projects in the Restricted Area, Planned Evacuation Area, etc." (hereinafter referred to as "Decontamination Pilot Project") conducted in eleven municipal government zones designated as restricted zones, etc. This decontamination pilot project mainly involved the regions where a high dose rate was recorded, and where the annual additional exposure dose exceeded $20 \mathrm{mSv}$, and was aimed at establishing efficient and effective measures for decontamination, etc., and for securing radioactivity protection for workers.

One or two construction locations per municipal government, for a total of fourteen temporary storage yards and storage sites (both hereinafter referred to as "temporary storage yards") were selected in accordance with the agreed adjustments made between the local governments, the Fukushima decontamination promotion team, and the cabinet support team for the livelihood of disaster victims. As defined, a "site storage place" is a facility for temporarily storing removed contaminants for a certain period until the temporary storage yards are ready. During the selection of construction sites for temporary storage yards, the authors provided advice for site selection under the Fukushima decontamination promotion team, and was involved in the communications, etc. designed to promote understanding among the residents. The selected sites for temporary storage yards represented a wide variety of land use types, such as flat land, national forests in mountainous areas, cultivated land, pastures, and steep valleys.

The authors executed the design, construction, maintenance, and management of the temporary storage yards according to these various conditions.

In this paper, as well as describing the policies for securing the safety of the temporary storage yards for the purpose of contributing to future maintenance, such as full-scale decontamination in accordance with the decontamination guidelines ${ }^{2)}$ "Edition 4: The Guidelines for Storage of Removed Soils," we extracted and reported technical know-how (e.g., important points, site selection of types, etc., relevant to each operation) through actual experience in relation to the design, construction, maintenance, and management of temporary storage sites.

\section{Fundamental Policies to Secure the Safety of Temporary Storage Yards}

The decontamination pilot project was started in November 2011. At the beginning of this project, the design of temporary storage yards was conducted based on fundamental policies for safety, considering case examples of the final disposal site design for municipal solid wastes and industrial wastes ${ }^{3)}$, case examples of studies regarding fire prevention of combustible disaster wastes ${ }^{4)}$, case examples of design for safe demonstration testing for the disposal of very low-level concrete waste conducted by JAEA ${ }^{5)}$, etc. This was necessary because policies for the maintenance/preservation of temporary storage yards have not been formulated by Government. After that, the guidelines on decontamination ${ }^{2)}$ (hereinafter, this stands for "Edition 4: The Guidelines for Storage of Removed Soils") were drawn up, and specific measures for the maintenance/restoration of temporary storage yards were presented. Comparison of these showed a general match, but the measures for earthquake resistance proposed in the guidelines 
on decontamination were not included in the safety policies that we had formulated previously. Therefore, policies for earthquake resistance were added to the fundamental policies for securing safety. An overview of the fundamental policies for securing the safety of temporary storage yards based on the contents of the guidelines on decontamination ${ }^{2)}$ is presented below and shown in Figure 1.

(a) Radiation shielding and isolation

These are required to prevent the public from being exposed to additional $\gamma$-radiation from the removed/concentrated contaminants, to be achieved by covering the facility with soil, etc., to isolate it from the human environment.

(b) Prevention of removed contaminants scattering

To prevent the dispersion of radioactive materials when bringing the removed/concentrated contaminants into the facility, a closeable container will be used, covered during transport.

(c) Prevention of infiltration of rainwater, etc.

Preventing the infiltration of rainwater is essential to avoid the translocation and outflow of soils, vegetation, etc. that may contain contaminants such as radioactive cesium.

(d) Prevention of outflow of removed contaminants and radioactive materials

It is necessary to prevent the contamination of public water and groundwater by soil outflow carrying adhered radioactive cesium, or by the migration of radioactive cesium dissolved in water. For this purpose, countermeasures were implemented to prevent the outflow of removed contaminants and radioactive cesium in the base of the temporary storage yard.

(e) Prevention of influence by components other than radioactive materials

In a separate section where organic substances such as grass and fallen leaves are stored, which may spontaneously combust, a heat accumulation prevention measure was implemented to avoid fires that could ignite from accumulated heat if internal heat generation exceeded balancing heat radiation.

(f) Earthquake resistance, etc.

Measures must be taken for the prevention of damage to shielding, and the prevention of

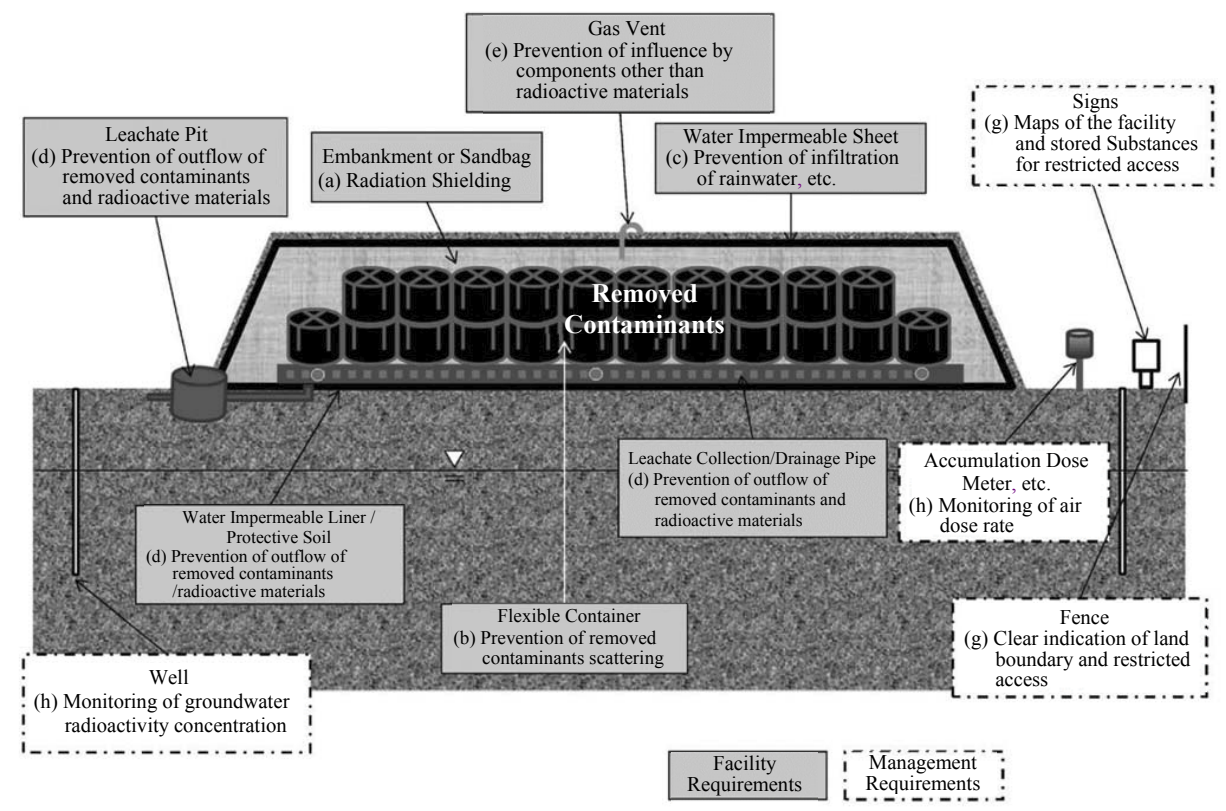

Figure 1 Facility requirements and management-related requirements of a temporary storage facility 
outflow of removed contaminants and radioactive materials, such as the collapse of removed contaminant containers and shielded soils during an earthquake.

(g) Restricted access

In addition to implementing other measures to prevent unauthorized entry into the facility by the public, signs must be visibly posted indicating a storage facility for removed radioactive contaminants, and enabling emergency communications.

(h) Radiation monitoring and recovery, etc.

To confirm the safe storage of removed contaminants during and after the transport of removed contaminants, and to check the outflow of radioactive material from the facility, if an abnormality is found attributable to the temporary storage yard, necessary actions will be taken.

(i) Preservation of records

Records will be kept to track the transportation and storage of removed contaminants in temporary storage yards and interim storage facilities, as well as collection sites, which specify the contents and their location in the temporary storage yard, and other relevant factors.

Furthermore, removed contaminants are generally classified into combustible and incombustible materials. Soils, stones, etc. are classified as incombustible materials, while asphalt, vinyl sheets, filters, rubber gloves, vegetation, Tyvec suits, waste cloths, etc., are classified as combustible materials.

\section{Case Examples of Design and Construction of Temporary Storage Yards}

\section{Temporary Storage Yard Type}

For sites suggested by local governments for the construction of temporary storage yards, it was decided to investigate the possibility of using a design that satisfies the requirements in Section II, based partly on the type of temporary storage yard, whether above ground, underground, or semi-underground (Figure 2). The most important factors are mainly the usable area of the premises with regard to the estimated generated quantity of removed contaminants, the

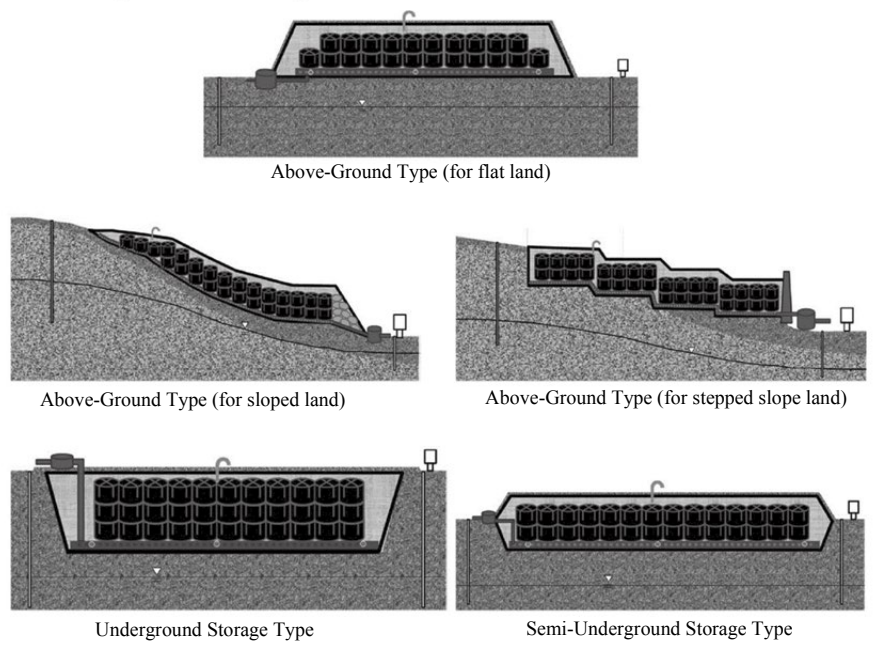

Figure 2 Various types of temporary storage facilities 
topographical conditions, land use status, and the ground characteristics.

\section{Specifications for Facility Requirements}

Reasonable specifications were set up for facility requirements considering the wait time until transportation to the interim storage facilities, and the cost effectiveness of the process. Practical specifications according to facility requirements are summarized as follows:

\section{(1) Shielding and isolation}

It is possible to shield $98 \%$ of $\gamma$-rays generated by the removed contaminants with a 30 $\mathrm{cm}$ layer of soil, or a $30 \mathrm{~cm}$ layer of concrete (such as a wall block) ${ }^{6}$. In the decontamination pilot project, if shielding is required, uncontaminated soil, which is less costly than a concrete retaining wall, was selected as the shielding material. To install the shielding material in either case, uncontaminated soil thicker than $30 \mathrm{~cm}$ was used for covering and surrounding the removed contaminants, plus the impermeable sheet mentioned later (Figure 3(1)). Alternatively, the removed contaminants were covered with an impermeable sheet, and weatherproof flexible containers were filled with uncontaminated soil (about $1 \mathrm{~m}$ wide) (Figure 3(2)). In the first case, a weatherproof sheet or a light-resistant sheet was placed over the impermeable sheet as an anti-ultraviolet ray measure. The second case focused on protecting the weatherproof sheet from ultraviolet rays and from damage by birds and animals.
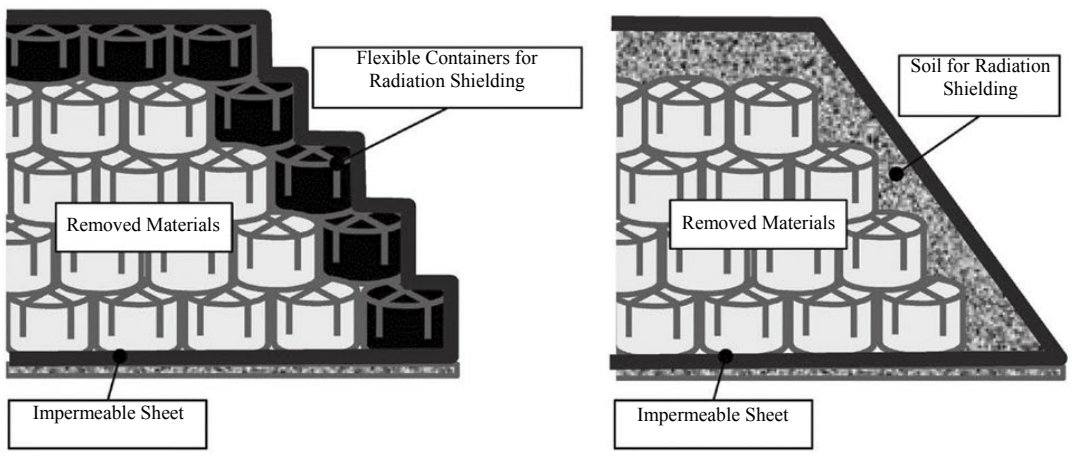

(1) Cover the removed materials with soil, then cover all with aimpermeable sheet

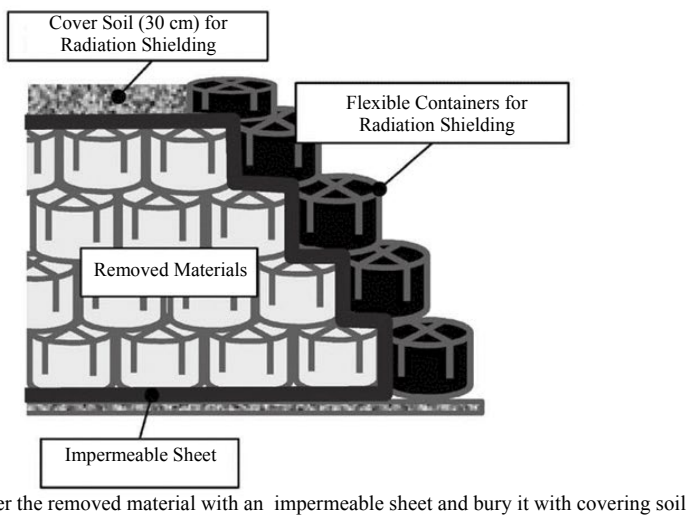

(2) Cover the removed material with an impermeable sheet and bury it with covering soil

Figure 3 Conceptual designs of shielding methods 


\section{(2) Prevention of removed contaminant dispersion}

To prevent the dispersion of removed contaminants when bringing them into a temporary storage yard, the contaminants were carried in flexible containers during transportation. Furthermore, to prevent dispersion when the removed contaminants were taken out at the end of the storage period in the temporary storage yard, flexible containers with a durable lifetime of a several years were used. After transporting the removed contaminants, impermeable sheets and covering soil were used as dispersion prevention measures.

Furthermore, special weatherproof flexible containers were not used because the measures stated in III-2-(3) and item (4) were applied.

(3) Prevention of infiltration of rainwater, etc.

The surface and slope side were covered with an impermeable sheet to prevent the infiltration of rainwater, etc.

(4) Prevention of outflow of removed contaminants and radioactive materials

To prevent the outflow of radioactive cesium in the leachate, an impermeable sheet was laid under the temporary storage yard. To prevent sheet damage due to the roughness of uneven ground, an asphalt-type impermeable sheet was sprayed directly on the ground to allow for plastic deformation after drying. For other locations, an impermeable sheet made of synthetic rubber or synthetic resin materials, or a bentonite impermeable sheet was laid to allow elastic deformation.

When bringing in the removed contaminants, to avoid damage to the impermeable sheet, as well as to lay a protective mat on the impermeable sheet, protective soil was laid between the removed contaminants and the impermeable sheet to adsorb radioactive cesium and inhibit translocation. Furthermore, when heavy machinery was used on the impermeable sheet, protection mat, and protective soil, a covering such as a steel sheet was used to prevent damage. To collect leachate from the removed contaminants, a collection pipe with holes and pits was installed. Moreover, crushed stone, etc., was laid around the pipes with holes to prevent clogging by sand and mud as much as possible.

\section{(5) Prevention of influence except from radioactive materials}

For enhanced heat dissipation, and to exhaust heat generated by the decomposition of organic materials, a gas vent pipe was mounted at the top part of the covered contaminants. Furthermore, a separate section in which organic materials could be stored was designed in an area less than $200 \mathrm{~m}^{2}$ with a height of 2-5 $\mathrm{m}$ based on case examples regarding the aforementioned fire prevention of combustible disaster wastes ${ }^{4)}$. Furthermore, if separation into several sections was required, and to implement a spacing between sections of at least $2.0 \mathrm{~m}$, sand was prepared for easier fire extinguishing in the event of fire.

\section{Construction of Temporary Storage Yards and Placement of Removed Contaminants}

\section{(1) Construction of temporary storage yard}

The construction of the temporary storage yards began with tree trimming and ground leveling, excavation as necessary, the setting up of a section for storing removed contaminants (building a dam), laying impermeable sheets and leachate drainage pipes, laying protective soil, positioning the removed contaminants, and then laying down the upper impermeable sheets and covering them with soil, regardless of topographical status, land use status, or type of 
temporary storage yard.

Additionally, equipment such as the collection pit and gas vent pipe, etc., were installed. Furthermore, light-shielding protection mats and fire extinguishing sand were provided in addition to the top impermeable sheets when necessary.

The condition of removed contaminants in above-ground temporary storage yards, (for flat land, slopes, and steep valleys), underground storage types, semi-underground storage types, and the external appearance after completion are shown in Figure 4. As seen in these case examples, the design and construction were done in a flexible way in accordance with topographical status and land use status, etc., of each installation site.

Moreover, in spite of the site-to-site differences in scale, such as placement height, and the difference in the percentage of combustible and incombustible materials, there is a rough correlation between the placement area for removed contaminants $\left(\mathrm{m}^{2}\right)$ and the quantity of removed contaminants (the number of flexible containers) as shown in Figure 5. Furthermore, a fixed percentage (0.5-2\%) of the area was dedicated to decontamination (Figure 6).

\section{(2) Placement of removed contaminants}

There were two methods for controlling combustible and incombustible materials in different

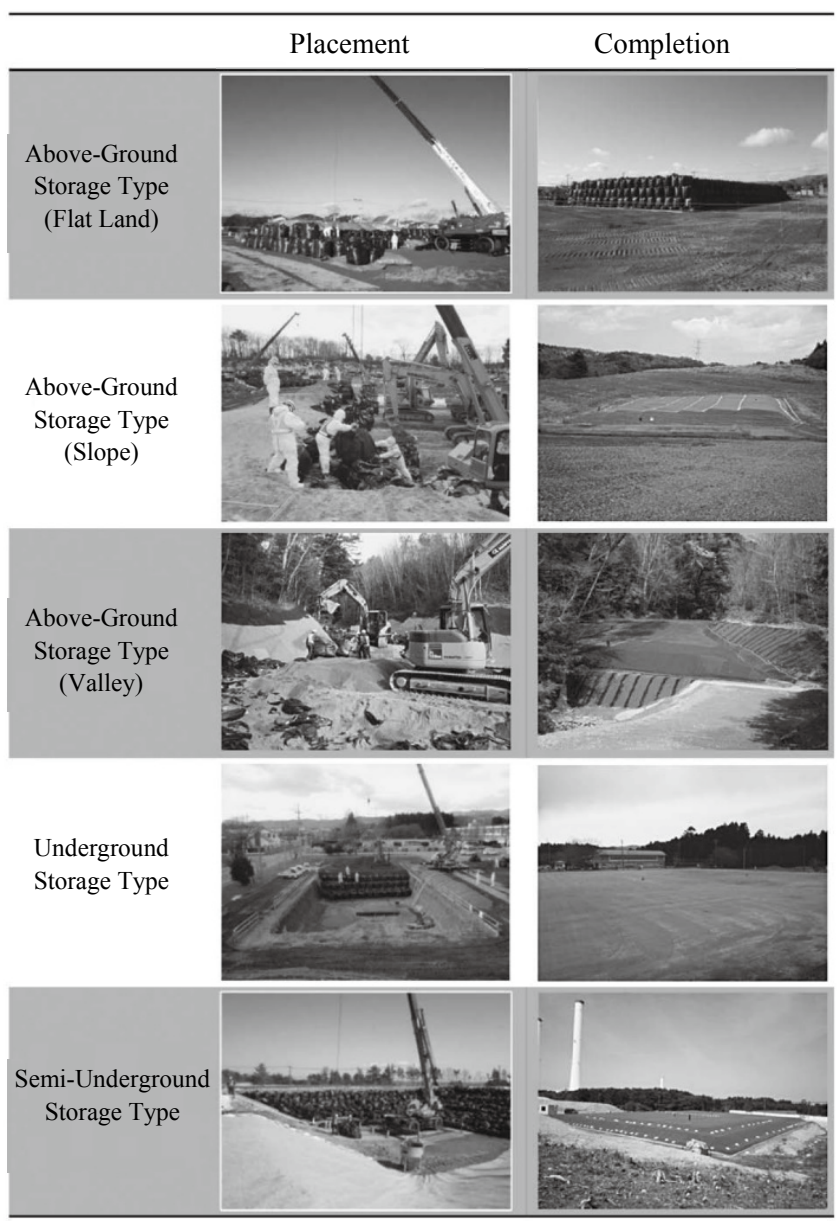

Figure 4 Waste-storage scenarios, and completed temporary storage facilities 


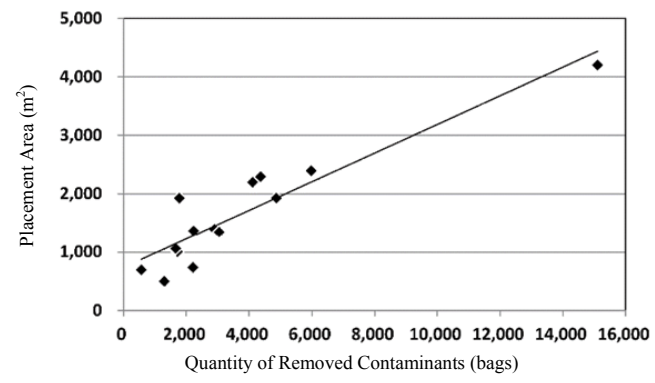

Figure 5 Amount of removed contaminants, and size of temporary storage site

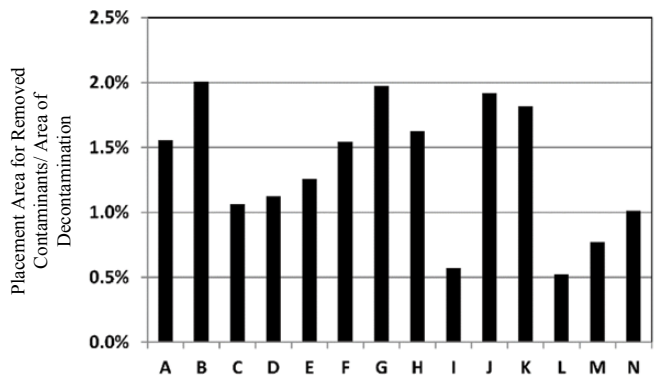

Figure 6 Ratio of area of temporary storage site to area of decontamination target

Table 1 Measures to subsidence of flammable removed contaminants

\begin{tabular}{|c|c|c|}
\hline $\begin{array}{l}\text { Storage of Combustible } \\
\text { Materials }\end{array}$ & Type & Actions \\
\hline \multirow{2}{*}{$\begin{array}{l}\text { Stored separately from } \\
\text { incombustible materials }\end{array}$} & \multirow[t]{2}{*}{ Above-Ground Storage } & -Advance volume reduction using a chipper, etc. \\
\hline & & $\begin{array}{l}\text {-Limit the number of stacking stages to as few as possible to make } \\
\text { the load from upper removed contaminants as low as possible. }\end{array}$ \\
\hline \multirow[t]{5}{*}{$\begin{array}{l}\text { Stored in the same section as } \\
\text { combustible materials }\end{array}$} & \multirow[t]{3}{*}{ Above-Ground Storage } & $\begin{array}{l}\text {-Sandwich of combustible and incombustible materials by making } \\
\text { use of the weight of incombustible materials for compression, and } \\
\text { by making the residual subsidence as small as possible. }\end{array}$ \\
\hline & & $\begin{array}{l}\text {-To limit the deformation of whole earth filling, only incombustible } \\
\text { materials are stacked in the core portion. }\end{array}$ \\
\hline & & $\begin{array}{l}\text {-Place combustible materials on top of the stack of incombustible } \\
\text { materials. }\end{array}$ \\
\hline & \multirow[t]{2}{*}{ Underground Storage } & -Limit the number of stacking stages to as few as possible. \\
\hline & & $\begin{array}{l}\text {-Finish the surface by banking up the soil with a slope of several } \\
\text { degrees in consideration of later subsidence. }\end{array}$ \\
\hline
\end{tabular}

sections and in the same section due to the limited area of the temporary storage yards. When storing combustible materials such as grass and fallen leaves, deformation by subsidence due to the reduced volume and the compression of combustible materials can be expected. Countermeasures for this were implemented as listed in Table $\mathbf{1 .}$

The surface dose rate and weight of flexible containers brought into a temporary storage yard were measured in terms of selecting placement positions for the removed contaminants, and controlling the exposure of workers during placement. Specifically, because radiation from the removed contaminants in the inner part of the temporary storage yard is shielded by contaminants located in the outer part, removed contaminants with a higher surface dose rate were placed as close to the center as possible. 
Furthermore, traceability during transport to the interim storage facilities, etc., was secured by attaching a tag to each flexible container indicating the surface dose rate of the flexible container, and its weight, collection site, specification of its contents, and its storage location in the temporary storage yard.

\section{Monitoring and Maintenance of Temporary Storage Yard}

To verify that the soundness of the shielding and radioactivity containment functions in the temporary storage yard is maintained until the removed contaminants are taken out of the interim storage facilities, monitoring was conducted for the following items:

- Air dose rate

- Water levels and radioactivity concentration in leachate and groundwater

- Carbon monoxide concentration and temperature of generated gases

- Deterioration or damage status of equipment in the temporary storage yard

(a) Air dose rate

The air dose rate was measured at several predetermined locations, such as at the site boundary or in temporary storage sections. For underground storage, measurement was conducted above the storage facility, i.e., on the surface of the ground. As a result, it was verified that the air dose rate of the temporary storage yards when completed was less than before bringing the removed contaminants into the storage facility (Figure 7). Continuous monitoring was conducted approximately once every week after that. No increase in air dose was observed after approximately three months had passed; the shielding function was maintained.

(b) Water levels and radioactivity concentration in leachate and groundwater

The monitoring interval for leachate was defined based on the relation between water storage capacity and leachate quantity. As a result, the generation of a large amount of water linked to the rise of atmospheric temperature was observed in some of the temporary storage yards. It is thought that the water originated from the frozen peeled surface soil and snow that had fallen into the temporary storage yard during placement. In the temporary storage yard, water quantity and radioactivity concentration were measured once every one or two days, and a dose lower than the concentration limit for water based on the "Notification for Dose Equivalent Limits on the Basis of the Ministerial Ordinance for Installation and Operation of Commercial Power Reactors Mar. 21, FY2001, Ministry of Economy, Trade and Industry (METI) Notification No. 187)" (hereinafter referred to as "concentration limit") was confirmed in the area other than the peripheral monitoring area. Thus the water could be safely discharged. Conversely, the radioactivity level of leachate in some of the facilities exceeded the concentration limit. This

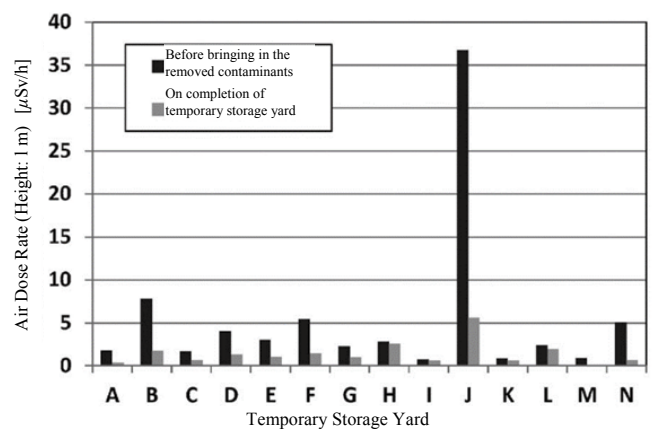

Figure 7 Air dose rates before and after construction of temporary storage facilities 
was observed only in sections where combustible materials were stored without a protective soil layer to function as an adsorbent for the dissolved radioactive cesium in the leachate. Furthermore, the flocculent settling process was used on the leachate and discharged after verifying that the concentration level was lower than the specified limit.

The groundwater monitoring interval was set to once every month in accordance with the decontamination guidelines. All the measurements for radioactivity concentration in groundwater were lower than the concentration limit.

(c) Carbon monoxide concentration and temperature of generated gases

The carbon monoxide concentration, and the temperature of generated gases were measured at an interval of approximately once a week, similar to the measurement of the air dose rate, to determine the fluctuation trend. A gas vent pipe was used to measure the carbon monoxide concentration, and the temperature was measured at a depth of approximately $1 \mathrm{~m}$. Furthermore, temperature monitoring with thermal sensors and an underground wireless compact transmitter was tried in some of the temporary storage yards.

As a result, some temporary storage yards showed an increase in temperature up to approximately $55^{\circ} \mathrm{C}$ in sections where combustible materials were stored, due to the fermentation decomposition of vegetation (Figure 8). As the rise in temperature continued, it was necessary to increase the number of gas vent pipes to accelerate the heat dispersion, but this countermeasure was not implemented because the temperature stopped rising and then began to fall. In addition, the generation of carbon monoxide exceeding a concentration of $100 \mathrm{ppm}^{4}$, in such environment a fire might happen, did not occur.

(d) Deterioration and damage status of equipment in temporary storage yards

Visual inspection was conducted for the purpose of verifying deterioration and damage in shielding materials, collection pits, gas vent pipes, etc., in the temporary yards. In the extremely

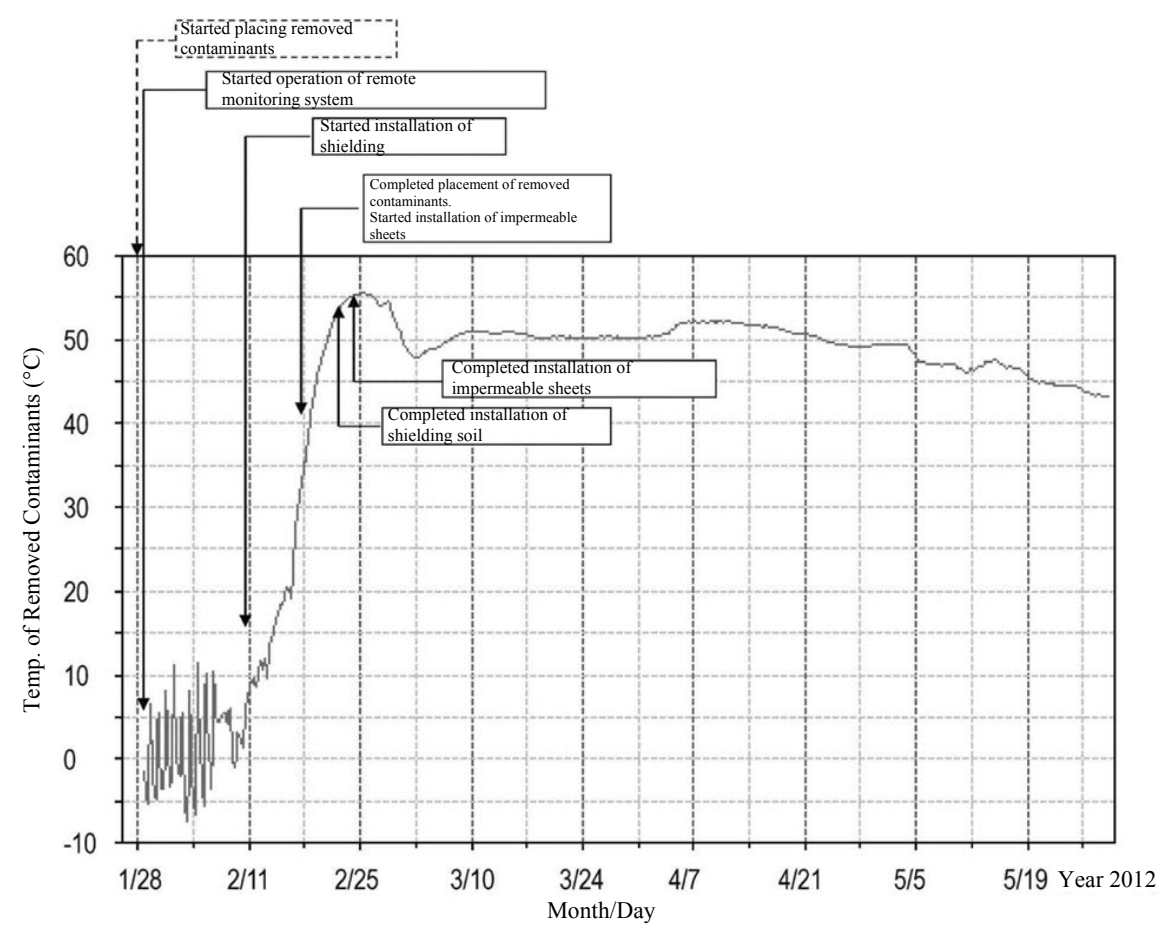

Figure 8 Monitored temperature inside the yard for flammable removed contaminants 
cold area of the Abukuma highlands, the removed contaminants and shielding soils were frozen when the flexible containers were filled. Because of this, as the temperature rose, the flexible containers became deformed due to the melting of the shielding soil, and the flexible containers that were installed outside of the impermeable sheets on the sides shifted or partially collapsed, as shown in Figure 9(1). Furthermore, in this temporary storage yard, the air dose rate did not increase because the sandbags for shielding were placed on the inner side of the flexible containers as well. In some of the temporary storage yards, subsidence occurred in the flexible containers

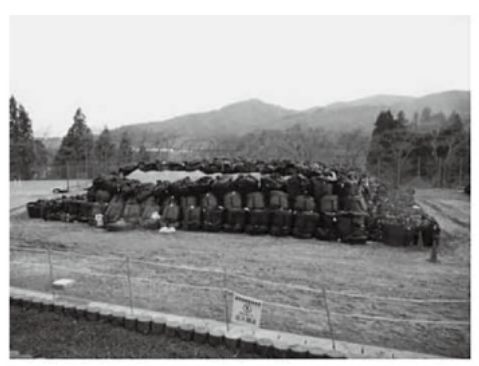

(1) Shifted and collapsed shielding around flexible containers

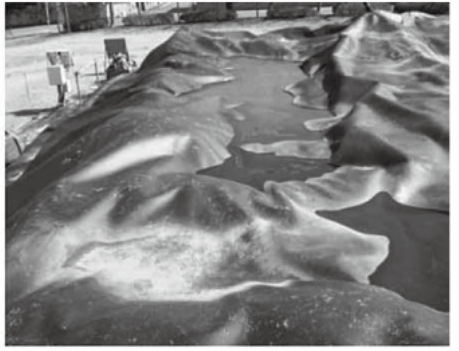

(2) Water pooling due to subsidence

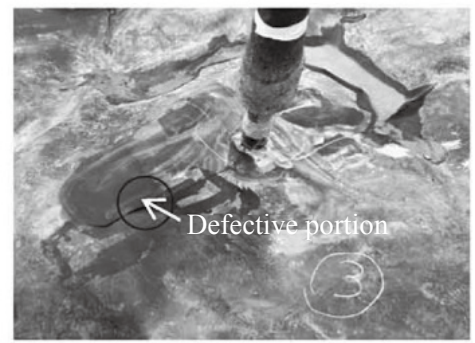

(3) Defective portion around gas vent pipe (after the peeling of cover soil)

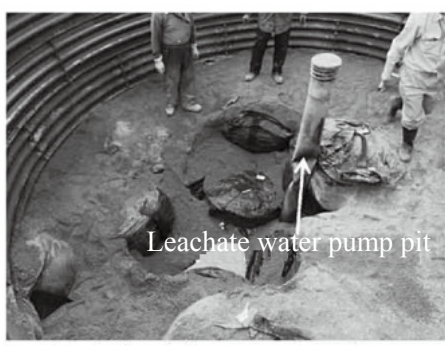

Deformation of leachate bilge pump pit due to the weight of flexible containers

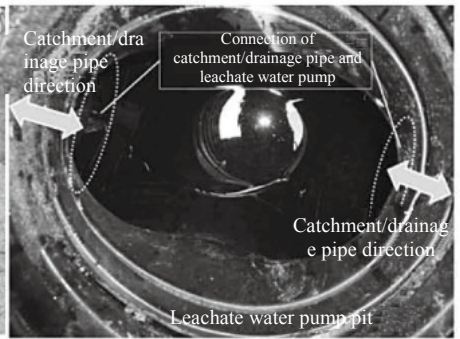

Clogged connection with

collection/drainage pipe due to the deformation of leachate bilge pump pit chate Water Pump Pit

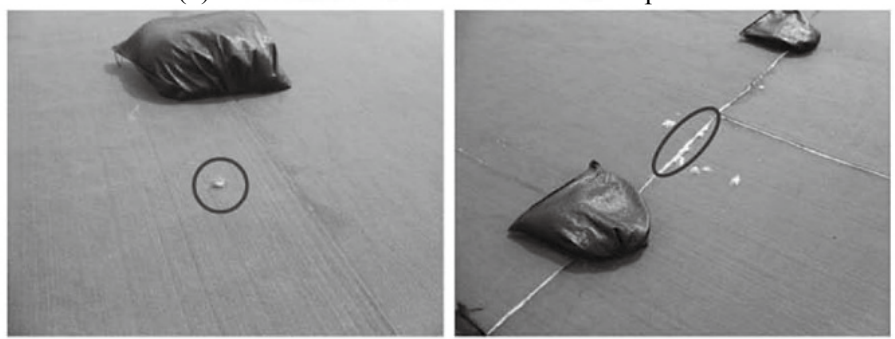

(5) Damage to Protection Mat by Wild Birds (circled)

Figure 9 Problems that occurred at temporary storage facilities 
due to the humification of combustible materials and the melting of frozen soils (Figure 9(2)).

In some of the temporary storage yards in which uneven subsidence occurred, there was defective welding between the gas vent pipe and the impermeable sheet (Figure 9(3)), and infiltration of rainwater through the defective area occurred during heavy rainfall. Defective welding was found on part of the upper impermeable sheet, and there was evidence of the infiltration of rainwater in some yards.

The low workability may have promoted the occurrence of defects because the impermeable sheet had to be welded on an uneven surface above a group of flexible containers, instead of on a flat one.

In addition, there was a problem with water drainage due to the deformation of the leachate bilge pump pit to drain the leachate stored in the dam (Figure 9(4)). The cause was considered to be that the leachate bilge pump pit was located near a slope, so horizontal stress was generated by the weight of the removed contaminants, and at the same time, the flexible container and the leachate bilge pump pit prevented the dispersion of the weight of the removed contaminants in the leachate bilge pump pit.

Partial damage to the protective mat in the upper part of contaminants occurred as well, which was attributed to a wild bird (Figure 9(5)). Furthermore, some signs of intrusion by wild pigs and feral cows on the premises of some of the temporary storage yards were observed, but until now, no specific problem has been observed.

\section{Technical Know-How}

The technical know-how that is considered applicable to future temporary storage yards is identified and summarized.

\section{Design and Construction of Temporary Storage Yards}

\section{(1) Types of temporary storage yards}

The advantages and disadvantages of temporary storage yard types are summarized in Table 2. Considering their advantages and disadvantages, the types of temporary storage yards were selected according to the procedure indicated in Figure 10. In Table 2, "Easy construction according to topographical conditions" is an advantage for above-ground storage, but the following points must be considered if the location is a slope or a valley:

- For sloped land, earth retaining walls and dams must be installed in lower areas according to the gradient. Furthermore, land preparation with cutting or filling may be necessary.

- Where the slope is utilized as a direct loading area for the removed contaminants, such as in a valley, radiation shielding measures in the slope zone are not required. However, providing a means to securely guide the slope surface water downstream are needed, as are measures to lower the groundwater level because the water pressure from the back end tends to put pressure on the impermeable sheets.

\section{(2) Specifications for facility requirements}

The advantages and disadvantages of various specifications are listed in Tables 3-5, such as the shielding and placement methods for the removed contaminants, which differ depending on the temporary storage yard. It is necessary to optimize the specifications while considering these advantages and disadvantages when maintaining temporary storage yards during 
Table 2 Advantages and disadvantages of temporary storage yard types

\begin{tabular}{|c|c|c|}
\hline Type & Advantage & Disadvantage \\
\hline $\begin{array}{l}\text { Above- } \\
\text { Ground } \\
\text { Storage }\end{array}$ & $\begin{array}{l}\text { - Easy removal for transport to interim storage } \\
\text { facilities, etc. } \\
\text { - Easy to transport the removed contaminants after } \\
\text { placement (ease of inspection/repair). } \\
\text { - Easy installation according to topographical } \\
\text { conditions. }\end{array}$ & $\begin{array}{l}\text { - The shielding soil must be secured from other regions. } \\
\text { - Construction on a soft footing requires improvement } \\
\text { of soil. }\end{array}$ \\
\hline $\begin{array}{l}\text { Underground } \\
\text { Storage }\end{array}$ & $\begin{array}{l}\text { - Shielding soil is secured at the site (low activity } \\
\text { concentration in dug out soil, excluding surface soil). } \\
\text { - Construction is possible without improvement to soil } \\
\text { footing, even in locations with soft footing. } \\
\text { - Easy repair/inspection of covering soil. } \\
\text { - Maintained landscapes. }\end{array}$ & $\begin{array}{l}\text { - Requires time for digging and building the underground } \\
\text { zone. } \\
\text { - When the removed contaminants are placed lower than } \\
\text { groundwater level, groundwater infiltration prevention } \\
\text { and groundwater drawdown measures are required. } \\
\text { - Digging operations are required for transporting } \\
\text { removed contaminants. }\end{array}$ \\
\hline $\begin{array}{l}\text { Semi- } \\
\text { Underground } \\
\text { Storage }\end{array}$ & $\begin{array}{l}\text { - It is possible to increase the placement quantity in a } \\
\text { small area with a comparatively greater number of } \\
\text { stages compared to stacking in above-ground and } \\
\text { underground zones. } \\
\text { - Easier shielding by placing the higher-concentration } \\
\text { removed contaminants in an underground zone, and } \\
\text { placing removed contaminants with comparatively } \\
\text { lower concentration in the above-ground zone. } \\
\text { - Shielding soil is secured at the site (low activity } \\
\text { concentration in dug-out soil, excluding surface soil). }\end{array}$ & $\begin{array}{l}\text { - Requires time for digging and building the underground } \\
\text { zone. } \\
\text { - Rainwater infiltration prevention measure is required } \\
\text { at the boundary of the above-ground and underground } \\
\text { zones. } \\
\text { - When the removed contaminants are to be placed lower } \\
\text { than the groundwater level, groundwater infiltration } \\
\text { prevention and groundwater suppression measures are } \\
\text { required. }\end{array}$ \\
\hline
\end{tabular}

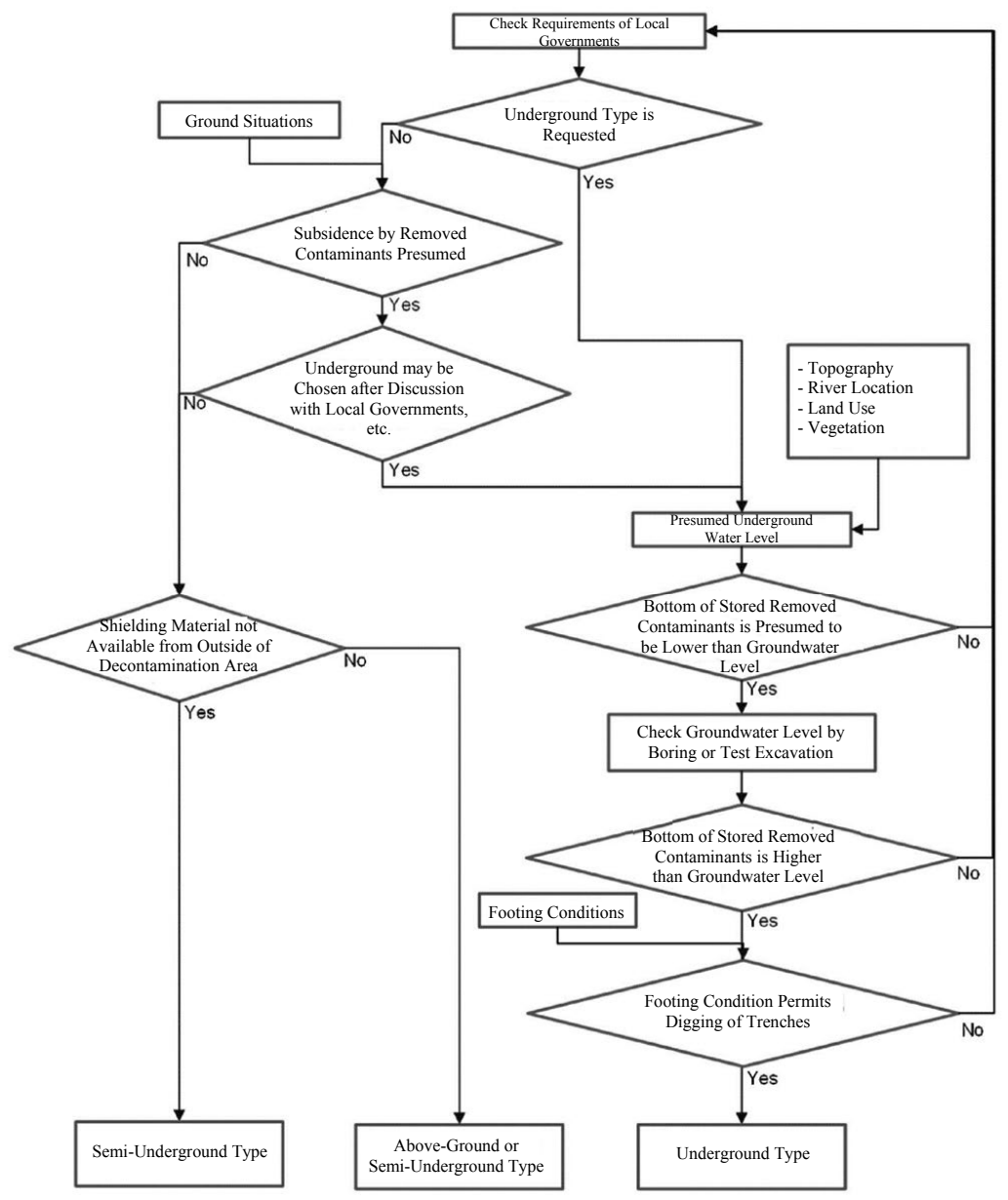

Figure 10 Flowchart for type selection of temporary storage facilities 
Table 3 Advantages and disadvantages of shielding methods for upper faces

\begin{tabular}{|c|c|c|}
\hline $\begin{array}{c}\text { Position and Method of Placement } \\
\text { of Shielding Materials }\end{array}$ & Advantage & Disadvantage \\
\hline \multicolumn{3}{|l|}{ Inside of Impermeable Sheet } \\
\hline Earth Fill & $\begin{array}{l}\text { - The necessary quantity of } \\
\text { soil can be used to provide a } \\
\text { shielding function } \\
\text { - Easily adapts to variations in } \\
\text { volume }\end{array}$ & $\begin{array}{l}\text { - Damage to sheets by ultraviolet rays, birds, and animals } \\
\text { must be anticipated and avoided (Damage is prevented } \\
\text { by covering with a protective light-shielding mat). } \\
\text { - Large-scale installation work } \\
\text { - Checking for earth fill contamination is required when } \\
\text { contaminants are taken out, and if contaminated, the } \\
\text { interim storage quantity increases. }\end{array}$ \\
\hline Flexible Container & $\begin{array}{l}\text { - Easy installation and removal } \\
\checkmark \text { Easy installation on a slope } \\
\checkmark \text { Easy to locate defects in the } \\
\text { impermeable sheet, and easy } \\
\text { to repair. }\end{array}$ & $\begin{array}{l}\text { - Damage to sheets by ultraviolet rays, birds, and animals } \\
\text { must be anticipated and avoided (Damage is prevented } \\
\text { by covering with a protective light-shielding mat). } \\
\text { - Excessive quantity may be used for shield. } \\
\text { - Checking of shielding soil contamination is required } \\
\text { during removal because the flexible containers come } \\
\text { into contact with removed contaminants, and if } \\
\text { contaminated, the interim storage quantity increases. }\end{array}$ \\
\hline \multicolumn{3}{|l|}{ Outside of Impermeable Sheet } \\
\hline Earth Fill & $\begin{array}{l}\text { - The necessary quantity of soil } \\
\text { can be used as a shield } \\
\text { - Easy installation work }\end{array}$ & $\begin{array}{l}\text { - The impermeable sheets become stressed when } \\
\text { subsidence occurs due to the reduction in volume of } \\
\text { the removed contaminants. } \\
\text { - For any repair, careful operation is required to remove } \\
\text { the earth fill without damaging the impermeable } \\
\text { sheets. }\end{array}$ \\
\hline Flexible Container & $\begin{array}{l}\text { - Damage to sheets by } \\
\text { ultraviolet rays, birds, and } \\
\text { animals is prevented. }\end{array}$ & $\begin{array}{l}\text { - The impermeable sheets are stressed when subsidence } \\
\text { occurs due to the volume reduction of removed } \\
\text { contaminants. }\end{array}$ \\
\hline
\end{tabular}

Table 4 Advantages and disadvantages of shielding methods for lateral faces

\begin{tabular}{|c|c|c|}
\hline $\begin{array}{c}\text { Position and Method of Placement } \\
\text { of Shielding Materials }\end{array}$ & Advantage & Disadvantage \\
\hline \multicolumn{3}{|l|}{ Inside of Impermeable Sheet } \\
\hline Earth Fill & $\begin{array}{l}\text { - The necessary quantity of soil } \\
\text { can be used as a shield } \\
\text { - Easily adapts to variations in } \\
\text { volume }\end{array}$ & $\begin{array}{l}\text { - Damage to sheets by ultraviolet rays, birds, and animals } \\
\text { must be anticipated and avoided. (Damage is prevented } \\
\text { by covering with a protective light-shielding mat.) } \\
\text { - Large-scale installation work } \\
\text { - Check of earth fill contamination is required during } \\
\text { removal, and if contaminated, the interim storage } \\
\text { quantity increases. } \\
\text { - For any kind of repair, construction work will be } \\
\text { difficult. } \\
\text { - Temporary storage yard area is increased due to the } \\
\text { requirements of certain degrees of slope to secure } \\
\text { stability. }\end{array}$ \\
\hline Flexible Container & $\begin{array}{l}\text { - Easy installation and removal } \\
\checkmark \text { Easy installation } \\
\checkmark \text { Easy to find a defect in the } \\
\text { impermeable sheet, easy to } \\
\text { repair. } \\
\text { - A steeper slope than for earth } \\
\text { filling provides rationalization } \\
\text { for the temporary storage yard } \\
\text { area. }\end{array}$ & $\begin{array}{l}\text { - Damage to sheets by ultraviolet rays, birds, and animals } \\
\text { must be anticipated and avoided. (Damage is prevented } \\
\text { by covering with a protective light-shielding mat.) } \\
\text { - Excess may be used for shielding. } \\
\text { - Checking of shielding soil contamination is required } \\
\text { during removal because the flexible containers come } \\
\text { into contact with the removed contaminants, and if } \\
\text { contaminated, the interim storage quantity increases. }\end{array}$ \\
\hline \multicolumn{3}{|l|}{ Outside of Impermeable Sheet } \\
\hline Earth Fill & - & - \\
\hline Flexible Container & $\begin{array}{l}\text { - Damage to sheets by } \\
\text { ultraviolet rays, birds, and } \\
\text { animals is prevented. }\end{array}$ & $\begin{array}{l}\text { - Possibility of shifting due to deformation caused by } \\
\text { the melting of frozen shielding soil in extremely cold } \\
\text { areas. }\end{array}$ \\
\hline
\end{tabular}


Table 5 Advantages and disadvantages of each technical specification (except shielding method)

\begin{tabular}{|c|c|c|c|}
\hline Specification & & Advantage & Disadvantage \\
\hline \multirow[b]{2}{*}{$\begin{array}{l}\text { Placement of } \\
\text { combustible } \\
\text { incombustible } \\
\text { material }\end{array}$} & $\begin{array}{l}\text { Separated } \\
\text { into Sections }\end{array}$ & $\begin{array}{l}\text { - Easy sorting during removal } \\
\text { for transport to interim storage } \\
\text { facilities. }\end{array}$ & $\begin{array}{l}\text { - A large temporary storage yard area is required due to } \\
\text { the limitation in area/height of temporary storage yards } \\
\text { for prevention of combustible material fires }\end{array}$ \\
\hline & Same Area & $\begin{array}{l}\text { - Minimized roughness on } \\
\text { the sheet surfaces due to } \\
\text { the subsidence of flexible } \\
\text { containers for combustible } \\
\text { material by devising the } \\
\text { layout of combustible and } \\
\text { incombustible materials } \\
\text { - Low risk of fire }\end{array}$ & $\begin{array}{l}\text { - Possibility of breakage due to loading on the } \\
\text { welded portion of impermeable sheets because of } \\
\text { unequal subsidence rates between combustible and } \\
\text { incombustible materials }\end{array}$ \\
\hline \multirow[t]{2}{*}{$\begin{array}{l}\text { Active use of } \\
\text { slopes as direct } \\
\text { loading zones }\end{array}$} & Yes & $\begin{array}{l}\text { - Easy adaptation to narrow lots. } \\
\text { - Shielding not required. }\end{array}$ & $\begin{array}{l}\text { - Water pressure tends to put pressure on the impermeable } \\
\text { sheets when the backend groundwater level is high (a } \\
\text { countermeasure for groundwater, such as the application } \\
\text { of waterproof treatment on slopes, etc., depending on } \\
\text { the situation). }\end{array}$ \\
\hline & No & $\begin{array}{l}\text { - No influence due to the pressure } \\
\text { of groundwater from the slopes. }\end{array}$ & - A larger cleared area is required in mountainous areas. \\
\hline \multirow[t]{2}{*}{$\begin{array}{l}\text { Storage of Leachate } \\
\text { Water }\end{array}$} & $\begin{array}{l}\text { Catchment } \\
\text { Pit }\end{array}$ & $\begin{array}{l}\text { - Easy monitoring of leached } \\
\text { quantity from the temporary } \\
\text { storage yard, and the outflow } \\
\text { of cesium. }\end{array}$ & - Determining catchment pit volume is difficult \\
\hline & Dam & $\begin{array}{l}\text { - Storage of large volumes of } \\
\text { leachate water. }\end{array}$ & - Larger installation area \\
\hline \multirow[b]{2}{*}{$\begin{array}{l}\text { Presence of } \\
\text { Protective Soil }\end{array}$} & Yes & $\begin{array}{l}\text { - Function as a cesium-adsorbing } \\
\text { layer is expected when the } \\
\text { leakage of cesium occurs } \\
\text { accidentally. } \\
\text { - Possible to protect of } \\
\text { impermeable sheet at bottom }\end{array}$ & $\begin{array}{l}\text { - Increased volume of temporary storage yard } \\
\text { - Possibility of protective soil contamination by cesium }\end{array}$ \\
\hline & No & $\begin{array}{l}\text { - The volume of the temporary } \\
\text { storage yard does not increase. } \\
\text { - Securing non-contaminated soil } \\
\text { is not necessary. } \\
\text { - No possibility of increased } \\
\text { interim storage quantity due to } \\
\text { the contamination of protected } \\
\text { soil. }\end{array}$ & $\begin{array}{l}\text { - Separate action is required if the accidental leakage of } \\
\text { cesium occurs. } \\
\text { - There is a risk of damage to the water impermeable } \\
\text { sheets from protruding objects such as branches and } \\
\text { leaves. } \\
\text { - It is not possible for heavy machinery to enter the } \\
\text { section. }\end{array}$ \\
\hline \multirow{2}{*}{$\begin{array}{l}\text { Subsidence } \\
\text { Prevention Measure }\end{array}$} & Yes & $\begin{array}{l}\text { - No stagnation etc. of rainwater } \\
\text { on top because there is no } \\
\text { subsidence. }\end{array}$ & $\begin{array}{l}\text { - A pre-treatment facility or place is required. } \\
\text { - There may be barriers to the decontamination operation } \\
\text { because the contaminated materials are not promptly } \\
\text { brought into the temporary storage yard. }\end{array}$ \\
\hline & No & $\begin{array}{l}\text { - Easy installation of temporary } \\
\text { storage yards. } \\
\text { - Pre-treatment is not required. }\end{array}$ & $\begin{array}{l}\text { - Rainwater, etc., may stagnate due to subsidence, and } \\
\text { the stagnated water may flow into the temporary storage } \\
\text { yard. }\end{array}$ \\
\hline
\end{tabular}

full-scale decontamination, etc., to be conducted in the future.

\section{(3) Construction of temporary storage yards}

The estimation of the quantity of removed contaminants and the real data may be significantly different due to the various decontamination methods, etc. used during the decontamination operation. Because of this concern, it is safer to carry out step-wise construction in accordance with the status of the generation of removed contaminants during the decontamination operation by setting up multiple sections, instead of constructing all the storage areas for the removed contaminants at once. As preventive measures for volume reduction or compression 
of combustible materials, and subsidence due to the melting of frozen soils, it is possible to fill the gaps between flexible containers with filling sand, etc.

\section{(4) Maintenance and management of temporary storage yards}

(a) Air dose rate

- The air dose rate is an indicator for locating defects, and for the early detection of defects in shielding by measuring between sections as well when there are multiple temporary storage yards in addition to land boundaries.

- It is desirable to monitor the upper part of covering soils because verification of shielding performance on the upper surface is important for underground temporary storage yards.

(b) Radioactivity concentration in groundwater

- The installation of observation wells for groundwater on upstream and downstream sides of the temporary storage yard facilitate the verification of radioactive material leaching from the temporary storage yard when radioactive material is detected in the groundwater.

- The direction of overall groundwater flow is estimated from the topography and location of rivers, etc.

- When an abnormality is recognized in radioactivity concentration in groundwater, action is required to prevent the spread of groundwater contamination after identifying the cause. Specific actions include the inspection and repair of impermeable sheets, pumping contaminated groundwater, etc. Furthermore, where there are domestic wells in the vicinity, it is necessary to conduct a prompt analysis of the relevant wells and to implement water restriction.

(c) Radioactivity concentration in leachate

- It is expected that radioactive cesium may be adsorbed by the protective soil before leachate containing dissolved radioactive cesium flows into the collection pit.

- When the radioactivity concentration of the leachate in the collection pit is higher than the concentration limit, a treatment to remove the radioactive cesium must be implemented. To do this, materials capable of cesium adsorption, such as zeolite, etc., are used for ionized cesium. Conversely, filtering or flocculent settling is considered appropriate for muddy water that contains soil with adhered radioactive cesium.

- Increased radioactivity concentration is possible in the collection pit due to the mixture of water and removed contaminants from water infiltration caused by degradation of the impermeability of the upper impermeable sheets. Considering the increased leachate water quantity in this case, the monitoring of leachate water flow is summarized in Figure 11.

(d) Deterioration and damage status of temporary storage yards

- Normally, visual inspection is conducted for deterioration and damage to shielding materials and impermeable sheets, collection pit, and gas vent pipes in the temporary storage yard, but the following phenomena enable inference due to deterioration or damage of impermeable sheets for which full visual inspection is difficult:

$\checkmark$ When the upper impermeable sheets are damaged, an increase in the water quantity due to precipitation in the collection pit is observed.

$\checkmark$ The water quantity flowing into the collection pit decreases due to the degraded shielding performance of the impermeable sheets at the bottom.

- To identify and repair the damaged parts of impermeable sheets in the upper part, visual inspection is mainly conducted when the impermeable sheets are exposed, and when they are covered with flexible containers, these are moved as necessary before inspection.

- If damage to the impermeable sheets in the bottom part is in question, the damaged part must be located by moving the removed contaminants.

(e) Automatic monitoring system 


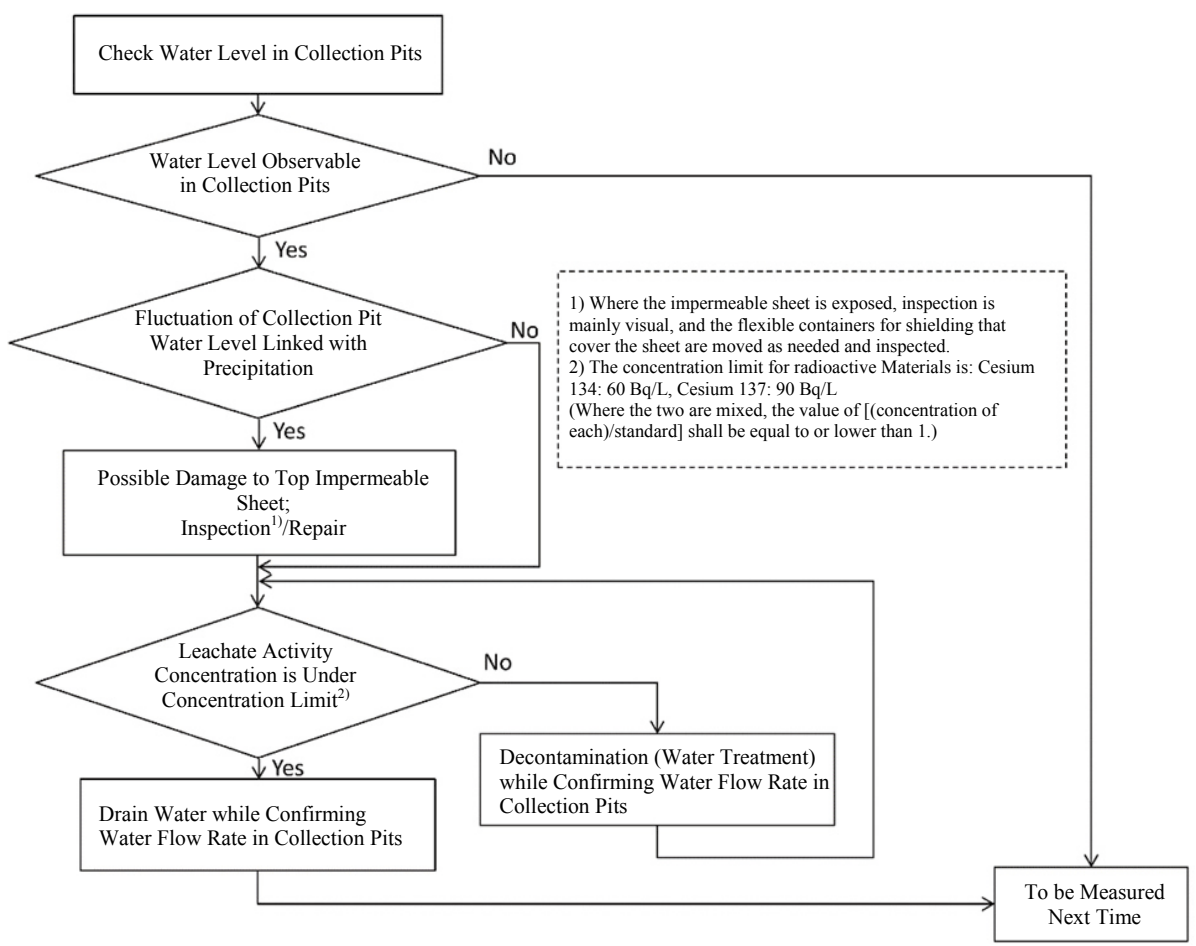

Figure 11 Flowchart of monitoring of seepage water

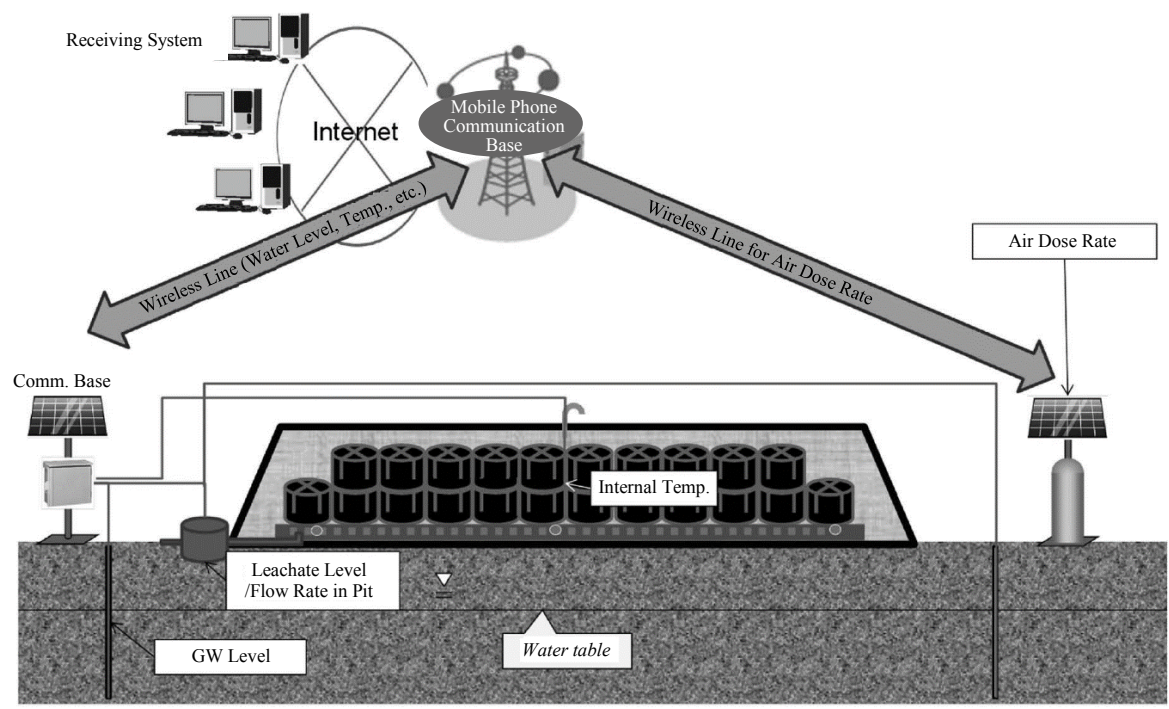

Figure 12 Concept of automatic monitoring system

An automatic monitoring system was implemented to facilitate the remote monitoring of the air dose rate and the temperature in some temporary storage yards during the decontamination pilot projects. Remote monitoring of the fluctuation in air dose, leachate quantity, groundwater level, temperature, etc. via an automatic monitoring system using wireless communications, such as mobile phones, as shown in Figure 12, is considered advantageous in terms of the 
following points:

- Live monitoring of the soundness of radioactive shielding, and containment of radioactive materials.

- Prompt action in the event of an abnormality

- Reduced labor for monitoring personnel and reduced time in high dose areas, such as a caution zone, and in mountainous areas.

- Timely publication of monitored results.

\section{Summary}

With this paper, we aim to contribute to the maintenance of temporary storage yards constructed in the future. Technical know-how was extracted and organized based on actual experience.

Based on actual experience with the design construction, maintenance, and management of multiple temporary storage yards having different specifications, technical know-how regarding the site selection, type, and specifications of facility requirements were extracted and organized. For the maintenance of temporary storage yards during full-scale decontamination in the future, it is considered beneficial to actively apply this technical know-how, and focus on the justification for specifications according to the conditions at the installation site.

In the future, it will be necessary to continue monitoring to verify the soundness of radioactivity shielding and the containment of radioactive materials in the temporary storage yards. Whenever an abnormality is detected, the appropriate action will need to be taken. It is considered beneficial to extract and organize the technical know-how acquired by those responsible, as this paper does, and to share this among the many temporary storage yards to be constructed in the future to maintain highly safe, stable, and evidence-based storage facilities, and to promote the understanding of local residents.

We thank Taisei JV, Kajima JV, Obayashi JV, and the Fukushima Environmental Safety Center, Fukushima Technology Headquarters, JAEA for their valuable cooperation and advice for this research.

This research adds to the achievements already made by the commissioned Government project "Decontamination Demonstration Projects on Evacuation Areas, etc., for the Fukushima Daiichi Nuclear Power Plant Accident."

\section{References}

1) Ministry of Environment. The Fundamental Concept of Interim Storage Facilities Required for the Management of Environmental Contamination by Radioactive Materials Released in the TEPCO Fukushima Daiichi Nuclear Power Plant Accident; 2011. [in Japanese]

2) Ministry of Environment. Decontamination Guidelines,Edition 1, Dec. 2011, 2011. [in Japanese]

3) Japan Industrial Waste Information Center. Lecture Class Textbooks Regarding Permission and Application for Industrial Waste or the Specially-Controlled Waste Treatment Industry, 2011. [in Japanese]

4) Earthquake Disaster Network (field of wastes, human waste, etc.). Fire Prevention of Combustible Waste in Temporary Storage Facilities (Edition 2); 2011, 20110919. [in Japanese]

5) M. Abe, M. Okoshi, M. Yoshimori. "Safety Demonstration Test for the Disposal of Very Low-Level; Concrete Waste," Journal of RANDEC; 1996, 15:50-58. [in Japanese]

6) Nuclear Emergency Response Headquarters. Guidelines for Decontamination Implemented by Municipal Governments (Aug.26, 2011), 2011. [in Japanese] 


\title{
Changing Information Needs of Social Impact of Nuclear Power Plant Siting
}

\author{
-Through a Comparison Before and After the Fukushima \\ Daiichi Nuclear Power Plant Accident-
}

\author{
Takako KASHIWA $^{1, *}$ and Yoshimi KAWAMOTO ${ }^{1}$ \\ ${ }^{1}$ Nuclear Power and Energy Safety Engineering, University of Fukui, 3-9-1 Bunkyo, Fukui-shi, Fukui 910-8507, Japan
}

\begin{abstract}
In the light of the Fukushima Daiichi Nuclear Power Plant Accident, we need to consider a symbiosis method based on the diminution of the nuclear power industry. To find a region that does not excessively depend on the nuclear power industry, it is necessary to examine and discuss the social impact of nuclear-related industries. In this study, we compared people's changing information needs of social impact before and after the Fukushima Daiichi Nuclear Power Plant Accident. It was found that the need for information increased after the accident. In particular, there were three research areas where the need for information increased: the consideration of building nuclear power plants, the influence of harmful rumors on the region, and influence on the nuclear power industry. Next, attempts were made to understand whether there is a difference between information needs of social impact by attributes, such as age, sex and knowledge of nuclear power. The information needs of the following categories of people increased after the accident: people aged between 10 and 50 years, women, people who do not have a clear opinion about the use of a nuclear power plant, and people who do not have any knowledge of nuclear power.
\end{abstract}

KEYWORDS: nuclear power plant siting, social impact, information needs, opinion survey

\section{Introduction}

Potential risks and economic impacts of nuclear power are related not only to the municipality where the nuclear power plant is located but also the entire society. Therefore, the relation between a nuclear power plant and the municipality where the power plant is sited has increasingly been reported on by the media after the Fukushima Daiichi Nuclear Power Plant Accident (hereinafter referred to as "the accident"), and we are now required to consider how to live with nuclear power as one of our future directions, while assuming that the size of the nuclear industry will diminish. As one of the options, in order for local communities not to overly depend on the nuclear industry, unlike the current situation where the nuclear industry is the main industry of a region, we will have to take a multi-phased approach to recognize and become interested

\footnotetext{
* Corresponding author, E-mail: pabrofukenspring@yahoo.co.jp

DOI : 10.15669/fukushimainsights. Vol.4.188

(C) 2021 Atomic Energy Society of Japan. All rights reserved.

Originally published in Transactions of the Atomic Energy Society of Japan (ISSN 1347-2879), Vol. 12, No. 2, p. 128-137

(2013) in Japanese. (Japanese version accepted: January 9, 2013)
} 
in the social impacts of the location of a nuclear power plant on everyday life and industries, be aware of questions, develop our own opinions, and understand a diverse range of values ${ }^{\mathrm{a}}$.

Looking at the information on nuclear power plants that has been provided by the government and power companies, their focus was on safety measures and operations. Also, previous studies ${ }^{2-4)}$ focusing on nuclear power information were based on the idea of understanding what information citizens need, from a list of items including the nuclear power generation mechanisms and safety measures, technical and engineering information on radiation, and providing support for risk communication. However, these previous studies do not examine whether people are aware of and interested in information that presents the social impacts of nuclear power plant siting. Therefore, to explore these, we need to understand what information on social impacts people feel they need.

According to a paper by Tsujikawa ${ }^{5)}$ investigating how people's perceptions of the need for, and apprehension towards, nuclear power influence their attitudes to nuclear power, the more people are aware of the need, the more they are likely to be motivated to collect and examine information on nuclear power.

Tsujikawa also mentioned that the fact that people recognize the importance of nuclear power. For example, the fact that they understand that nuclear energy supports our power supply and has become a public benefit indicates that people recognize that nuclear energy is closely related to the smooth functioning of modern society, and that those who recognize its importance have, at least to a certain degree, a detailed knowledge of nuclear power. This leads us to assume that those who are relatively favorably disposed towards and knowledgeable about nuclear energy are actively trying to understand the social impacts that nuclear power has. For example, what role nuclear power plays in modern society. On the other hand, those who do not take a definite stance on and are not interested in nuclear energy may be less interested in any information about the social impacts that nuclear power has, or any overall information on nuclear energy.

In this study, we attempt to understand the changes in people's perceived information needs regarding the social impact that nuclear power plant siting has by examining people's awareness and attitudes both before and after the accident. Then, to find out whether people have different information needs concerning the social influences that nuclear power has, depending on their general interest in nuclear power, we endeavor to understand the changes in information needs before and after the accident by focusing on "people's attitudes toward nuclear power," "those who are less interested in nuclear power," and "those who have relatively less knowledge on nuclear power." Lastly, we will perform a detailed analysis to find out what specific information people are interested in through a qualitative evaluation using open-ended questions.

\section{Survey Overview}

After the accident, it became clear that the impacts of a nuclear accident do not affect only the immediate area where the power plant is located but also involve wider areas. Moreover, economic benefits, including the subsidy on "Electric Power Development based on the Three Laws" also affect not only the immediate area but also the entire prefecture where the plant is located.

\footnotetext{
a According to "Model of knowledge cultivation activity" proposed by Kimura et al. ${ }^{1)}$, there are five stages, namely, "Recognition," "Showing interest," "Having doubts," "Forming one's own opinion," "Knowing various values" and these form the knowledge base for making individual judgments.
} 
In order to explore new ways to live with nuclear power, we think it necessary to examine the local community from a wide perspective. In this study, setting the study target as Fukui Prefecture, where the power plant is located, we would like to study Fukui City, which is the center of the economy and government of Fukui Prefecture.

The authors conducted interview surveys between 2009 and 2010 of local residents of the area where the nuclear power plant is located to investigate the impact of the plant siting on their living environment ${ }^{\mathrm{b}}$. We categorized the social impacts of the nuclear power plant's siting into 10 items by referring to the opinions acquired through the survey, and formulated survey items. Then, we created a leaflet including this content in questionnaire form. In the leaflet, we provided simple examples of the social impacts of the nuclear power plant and asked whether the respondents had wanted to hear about the information contained in the questionnaire before the accident, or only now want to hear about this information as a result of the accident. Tables 1 and $\mathbf{2}$ show the content of the leaflet and the survey overview, respectively.

As the response collection rate was low, we would like to provide the general features of collected data. According to the national census in 2010, the total population of Fukui City was 266,796. The gender composition was: Male 48\%; Female 52\%. Age composition: teens $11 \%$, 20 's $12 \%$, 30's $16 \%$, 40's $15 \%$, 50's $17 \%$, 60's $14 \%$, 70's and older $15 \%$. In this study, the respondents consisted of $52 \%$ males and $45 \%$ females, which shows a little bias towards males. Also, participants included $1 \%$ teens, 6\% 20's, 10\% 30's, 17\% 40's, 19\% 50's, $28 \% 60$ 's, and $15 \% 70$ 's and older, which shows a lack of response from the younger generation between teens and 30's and is biased toward the middle-aged and old-age groups. Also, for the question that asked whether the respondents are interested in nuclear power, $64 \%$ of them, more than half, answered they were "Interested" or "A little interested" since before the Fukushima Daiichi Nuclear Power Plant Accident, showing that many respondents were interested in nuclear power plants before the accident. It is assumed that this shows those who are not interested in the study theme did not answer the questions in the first place. When we have a bias in the sample, in general, it needs to be corrected. However, if it is corrected, the number of samples will decline, and we won't be able to reflect many opinions. Also, some responses questioned the format, such as "how was it before the accident," which ask how their awareness or attitudes changed after the accident. Therefore, such results are not fully valid comparisons before and after the accident.

\section{Trends of Information Needs}

\section{Overall Trends}

We made comparisons before and after the accident to understand the changes in information needs pertaining to the social impact of nuclear power siting. In the survey, respondents were asked to select their answer from four choices: "Would like to hear," "Would somewhat like to hear," "Would not particularly like to hear" and "Would not like to hear" — to 10 inquiry items concerning the social impact (hereinafter referred to as "items") of nuclear power plant siting before and after the accident. Example questions are provided in Figure 1, and answers

\footnotetext{
${ }^{\mathrm{b}}$ For the Hearing Survey in 2010, we selected 18 areas of concern (Nature, Sewage, Communication and Exchange, Children, Roads, Facilities, Fishery, Medicine, Tradition, Shopping, Welfare, Disasters, Emergency, Employment, Tourism, Transfer, Traffic, Agriculture) that are considered relevant to daily life in the area where a nuclear power plant is located. For the Hearing Survey in 2009, we selected 8 items (Human Resources, Culture, Communication and Exchange, Education, Nature, Primary Industry, Secondary Industry, Tertiary Industry) that are considered to be regional resources.
} 
Table 1 Leaflet contents

Item

(1) Awareness of and attitudes to nuclear power

(2) Influence of harmful rumors

(3) Relationship with the power company

(4) Impacts on activities

(5) Changes in scenery

(6) Changes in infrastructure development

(7) Opinions about energy education

\section{(8) Impacts on
industries}

\section{(9) Opinions about} PR activities about the region cultural and sports

mar

\author{
Examples of residents' opinions
}

I am not concerned about nuclear power as long as the power plant is safely operated/I am not concerned about nuclear power as long as we understand the risks and management structure of it/We have less reason to remonstrate against the plant due to the expansion of employment and economic benefits/We still have some concern about the power plant.

The number of tourists will decrease when an accident happens/The media were annoying when the accident happened/The media were apparently trying to pick up only negative comments and ignore positive opinions/Local people do not recognize dangerous impacts as much as people from outside do/There are harmful rumors that agricultural products and fish are contaminated by radiation.

We have relationships with nuclear-related workers through events and the obon dance festival in the Nuclear PR Center/We have no contact with management company and power company in everyday life/By actively associating with nuclear-related workers living in the neighborhood, we can develop relationships with each other and build trust in the company through them/Although we used to actively associate with the power company and residents, now we have fewer opportunities.

More people participate in the festival, and the size has become larger/The size of the festival has become larger, but we have less autonomy/As people can exhibit their artworks at the nuclear power-related facility (PR Center), their financial burden has been reduced and are more motivated, which promotes cultural activities/We have organized an official art organization through association with various people from outside/As some nuclear-related male employees have become coaches of sport teams such as junior sports teams, sports activities have become more active.

The power plant will not damage the natural environment/The scenery is beautiful around here, and I think the natural environment is preserved/I don't feel that nature is considered important in daily life. If a new plant is constructed, some aspects of nature will be damaged and roads will be redeveloped. This will markedly change the scenery.

It is good that the sewage system and roads have been well developed. Roads used to be narrow, and when it snowed, we had to go to school and work by walking along the beach. New roads have made our lives very easy/Although sewage system and roads have been improved in the early stage, I think they would have been improved one day anyway even if the power plant was not constructed/Community centers have been renovated to a better standard, and more public facilities are provided/There are few facilities really needed by the citizens because they may not have been built based on actual community requirements/I don't think the power company provides us something special because they have built their power plant in our area.

I think it is good to provide energy education at an early age/I want energy education that contributes to human resources development/We are not familiar with the research center as part of our daily life, and we have no idea what is held there/I think it will be great if local human resource development measures are provided and research is performed in collaboration with local companies in the research center.

Employment by the power plant-related companies has increased/Not only tourists but also temporary regular inspection workers use Minshuku guest houses/Fewer people inherit their family fishing businesses because more people go to work outside/Construction businesses receive more orders for construction works/More restaurants and supermarkets have been constructed and made our life more convenient/The industry of the area has basically become dependent on the nuclear power plant.

I know about the nuclear-related PR magazine, but I have never read it carefully/Information on nuclearrelated accidents is provided by media such as TV sooner than in the PR magazine/I would like not only the people in the area where the power plant is located but also energy consumption areas to gain an understanding of nuclear power. Also, I want the power company to not only try to tell us about the safety of nuclear power but also allow us to have positive discussions.

(10) Technology Research on jellyfish guiding techniques and processing technologies/Made Heshiko production more collaboration and efficient by controlling temperature and pressure/Development of work clothes with well-balanced water development resistance and air permeability.

*Examples of residents' opinions on the leaflet are a representation of some responses to the interview survey, and only 3 to 5 examples (100 to 200 characters) are provided for each item.

\section{are provided in Figure 2.}

Of the 10 items, the one that the largest number of people answered "Would like to hear" was "Impact on industries" with $14 \%$ and the smallest number was "Impacts on cultural and sport activities" with $5 \%$ before the accident. When we look at the overall trend, only about $10 \%$ of respondents answered, "Would like to hear," and there was no item where more than half of respondents answered either "Would like to hear" or "Would somewhat like to hear."

As for the results after the accident, more respondents answered they "Would like to hear" 
Table 2 Survey overview

\begin{tabular}{ll}
\hline Target audience & Fukui citizens \\
Survey period & May to June 2011 \\
& Questionnaire survey \\
Area sampling \\
(Randomly selected from the index of the central city of Fukui City in Zenrin Residential \\
Map of Fukui City, Fukui Prefecture \\
Survey method \\
Distribution: Posting/Collection: Postal mail \\
4istribution/ \\
4,000 \\
(Two forms were distributed to 2,000 households) \\
3umber of distributions & 1. Attributes (Age/Gender/Occupation) \\
2. Interest in social impact of nuclear power plant siting (the following 10 items) & (1) Awareness of and attitudes to nuclear power \\
(2) Influence of harmful rumors about the region & (3) Relationship with the power company \\
(4) Impacts on cultural and sports activities & (5) Changes in scenery \\
(6) Changes in infrastructure development & (7) Opinions about energy education \\
(8) Impact on industries \\
(9) Opinions about PR activities \\
(10) Technology collaboration and development \\
3. Questions for residents who live in the area where the nuclear power plant is located \\
4. Interest in the nuclear power plant, and energy and environmental issues \\
5. Knowledge of nuclear power \\
6. How to acquire information on nuclear power and energy issues \\
7. Image of nuclear power \\
8. Attitudes to nuclear power
\end{tabular}

*In Questions 2, 4, 5, and 8, comparisons were made before and after the Fukushima Daiichi Nuclear Power Plants Accident in the same questionnaire form.

Questions (Sample) Influence of harmful rumors about the region

Question 1-1. Present

\begin{tabular}{|l|l|l|l|l|}
\hline $\begin{array}{l}\text { Would like to } \\
\text { hear }\end{array}$ & $\begin{array}{l}\text { Would } \\
\text { somewhat like } \\
\text { to hear }\end{array}$ & $\begin{array}{l}\text { Would } \\
\text { particularly } \\
\text { want to hear }\end{array}$ & $\begin{array}{l}\text { Would not want } \\
\text { to hear }\end{array}$ \\
\hline
\end{tabular}

What did you think about it before the accident?

\begin{tabular}{|l|l|l|l|}
\hline Wanted to hear & $\begin{array}{l}\text { Somewhat } \\
\text { wanted to hear }\end{array}$ & $\begin{array}{l}\text { Didn't } \\
\text { particularly } \\
\text { want to hear }\end{array}$ & $\begin{array}{l}\text { Didn't want to } \\
\text { hear }\end{array}$ \\
\hline
\end{tabular}

Question 1-2. This question is for those who answered, "Would like to hear" (Wanted to hear) and "Would somewhat like to hear" (Somewhat wanted to hear) now and before the accident. Why do/did you want to hear about it?
(1) It is interesting because we have hardly had the opportunity to hear about it.
(2) It is easy to understand the feelings and lives of the residents who live in the area where a power plant is located.
(3) It is helpful to reduce harmful rumors about the region.
(4) Others ( )

Question 1-3. Provide your questions on "Influence of harmful rumors about the region," if you have any.

Figure 1 Sample questions 

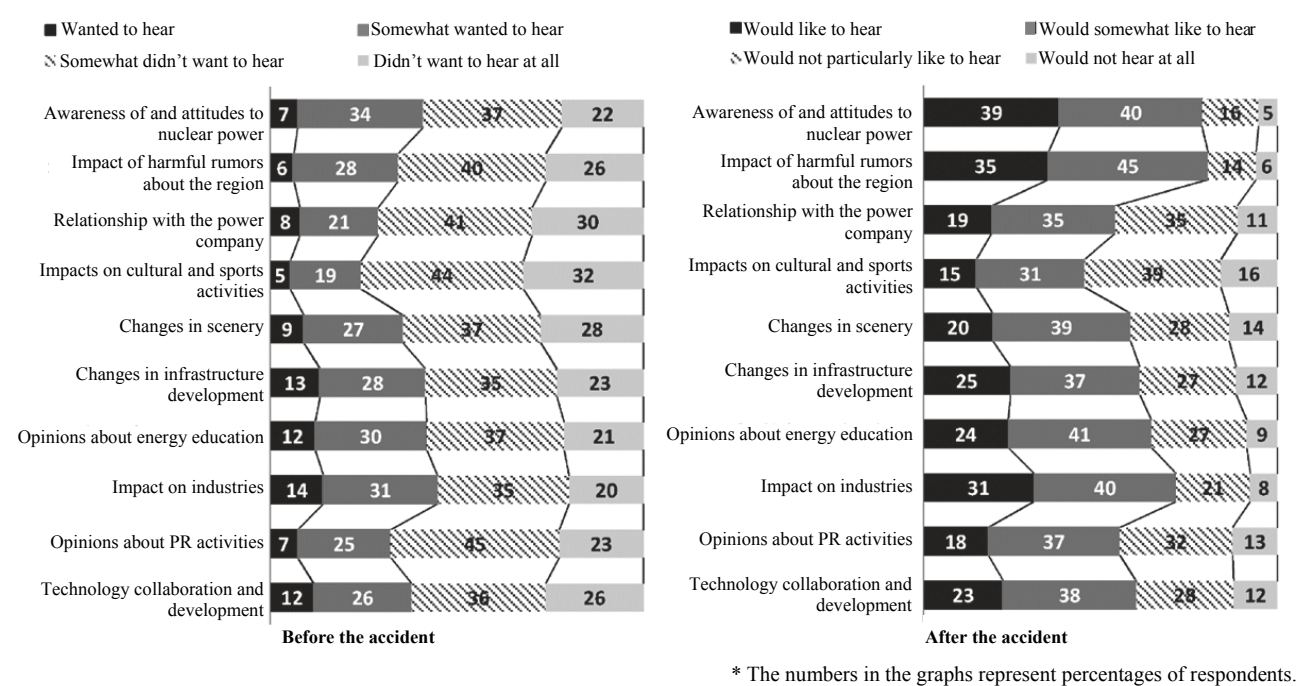

Figure 2 Proportions of interest by item before and after the Fukushima Daiichi Nuclear Power Plant Accident

for the question item on "Awareness of and attitudes to nuclear power," "Influence of harmful rumors about the region," and "Impact on industries," which shows that more respondents are willing to hear about social impact than before.

Also, although there was no item where more than half of respondents answered "Would like to hear" or "Would somewhat like to hear" before the accident, over half of the respondents answered "Would like to hear" or "Would somewhat like to hear" for 9 items out of 10 after the accident. This shows that the need for information on the social impact of nuclear power plant siting has increased after the accident. Also, before the accident, the information needs for "Impact on industries" were the highest.

However, more respondents answered "Would like to hear" about "Awareness of nuclear power," "Influence of harmful rumors about the region," "Impact on industries" and more than $70 \%$ of respondents answered either "Would like to hear" or "Would somewhat like to hear" after the accident. This means that more people showed their interest in more items after the accident.

Next, we scored respondents' answers to quantify the need for each item. Four points were given to "Would like to hear," 3 points to "Would somewhat like to hear," 2 points to "Would not particularly like to hear," and 1 point to "Would not like to hear." Note that the score does not indicate an absolute evaluation but rather a relative evaluation. The results are shown in Table 3.

The items where average scores became highest after the accident were three: "Awareness of and attitudes to nuclear power," "Influence of harmful rumors about the region," and "Impact on industries." Among them, the average score gap of "Influence of harmful rumors about the region" before and after the accident was 1 , which is the highest, and we can say that this is an item where the need has become higher after the accident. Also, although "Impact on industries" was the item with the highest need at the average score of 2.40 before the accident, the item with the highest average score was "Awareness of and attitudes to nuclear power" after the accident, so the item that draws people's interest has changed after the accident. Because of the increasing general interest in nuclear power after the accident, the need for information on "Awareness of and attitudes to nuclear power" also increased. Furthermore, after the accident, 
Table 3 Average scores before and after the accident

\begin{tabular}{|c|c|c|c|c|c|}
\hline Item & Before & After & $\begin{array}{c}\text { Difference } \\
\text { between } \\
\text { average scores }\end{array}$ & $\begin{array}{l}\text { Standard } \\
\text { deviation } \\
\text { (Before) }\end{array}$ & $\begin{array}{c}\text { Standard } \\
\text { deviation } \\
\text { (After) }\end{array}$ \\
\hline $\begin{array}{l}\text { Awareness of and attitudes to } \\
\text { nuclear power }\end{array}$ & 2.30 & 3.10 & 0.80 & 0.88 & 0.86 \\
\hline $\begin{array}{l}\text { Influence of harmful rumors about } \\
\text { the region }\end{array}$ & 2.10 & 3.10 & 1.00 & 0.88 & 0.85 \\
\hline $\begin{array}{l}\text { Relationship with the power } \\
\text { company }\end{array}$ & 2.10 & 2.60 & 0.50 & 0.91 & 0.92 \\
\hline $\begin{array}{l}\text { Impacts on cultural and sports } \\
\text { activities }\end{array}$ & 2.00 & 2.40 & 0.40 & 0.85 & 0.93 \\
\hline Changes in scenery & 2.20 & 2.60 & 0.40 & 0.93 & 0.95 \\
\hline $\begin{array}{l}\text { Changes in infrastructure } \\
\text { development }\end{array}$ & 2.30 & 2.70 & 0.40 & 0.97 & 0.96 \\
\hline Opinions about energy education & 2.30 & 2.80 & 0.50 & 0.94 & 0.90 \\
\hline Impact on industries & 2.40 & 3.00 & 0.60 & 0.96 & 0.91 \\
\hline Opinions about PR activities & 2.20 & 2.60 & 0.40 & 0.86 & 0.93 \\
\hline $\begin{array}{l}\text { Technology collaboration and } \\
\text { development }\end{array}$ & 2.20 & 2.70 & 0.50 & 0.97 & 0.95 \\
\hline Total average & 2.21 & 2.77 & 0.57 & & \\
\hline
\end{tabular}

the impact of restrictions and harmful rumors related to food products on tourism, scheduled power outages, and other direct impacts on the local people's lives probably increased the need for information in an attempt to gain insight into economic consequences, such as the impact of the harmful rumors on the local area or those on the industry of the area.

\section{Trends by Attribute}

People may have different needs for information on social impact depending on differences in their attitude to and knowledge of nuclear power. According to the survey examples ${ }^{5,6)}$ to find out the differences in interest in nuclear power by attribute, we can see that people have different interests in nuclear power depending on their gender, attitude to, and knowledge of, nuclear power. Also, as it has been about 40 years since the nuclear power plant was established in Fukui, the age groups of 10's to 40's, who were born after the plant establishment, and those in their 50's and older, who were born before the establishment, have different interests in nuclear power.

Therefore, we would like to understand the changes in people's needs for information on social impact depending on their age, gender, and attitude to and knowledge of nuclear power.

\section{(1) Age}

When we compare the average scores of the age groups from 10's to 40's and those of 50's and older (Table 4) before the accident, the average scores of the former age group are lower for 8 items except for "Awareness of and attitudes to nuclear power" and "Relationship with 
Table 4 Average score by age

\begin{tabular}{|c|c|c|c|c|c|c|}
\hline \multirow{2}{*}{ Gender } & \multicolumn{3}{|c|}{$10 \sim 40$ 's $(102)^{\mathrm{b})}$} & \multicolumn{3}{|c|}{50 's and older $(198)^{\mathrm{b})}$} \\
\hline & Before & After & $\begin{array}{c}\text { Difference } \\
\text { between } \\
\text { average } \\
\text { scores }\end{array}$ & Before & After & $\begin{array}{c}\text { Difference } \\
\text { between } \\
\text { average } \\
\text { scores }\end{array}$ \\
\hline $\begin{array}{l}\text { Awareness and attitudes } \\
\text { to nuclear power }\end{array}$ & 2.38 & 3.20 & $0.83^{\text {a) }}$ & 2.20 & 3.08 & $0.87^{\mathrm{a})}$ \\
\hline $\begin{array}{l}\text { Influence of harmful } \\
\text { rumors about the region }\end{array}$ & 2.07 & 3.13 & $1.06^{\mathrm{a})}$ & 2.18 & 3.08 & 0.90 \\
\hline $\begin{array}{l}\text { Relationship with the } \\
\text { power company }\end{array}$ & 2.07 & 2.77 & $0.70^{\mathrm{a})}$ & 2.07 & 2.55 & 0.47 \\
\hline $\begin{array}{l}\text { Impacts on cultural and } \\
\text { sports activities }\end{array}$ & 1.90 & 2.53 & $0.63^{\mathrm{a})}$ & 2.01 & 2.40 & 0.39 \\
\hline Changes in scenery & 2.13 & 2.82 & $0.69^{\mathrm{a})}$ & 2.19 & 2.56 & 0.36 \\
\hline $\begin{array}{l}\text { Changes in } \\
\text { infrastructure } \\
\text { development }\end{array}$ & 2.24 & 2.81 & $0.56^{\mathrm{a})}$ & 2.35 & 2.71 & 0.35 \\
\hline $\begin{array}{l}\text { Opinions about energy } \\
\text { education }\end{array}$ & 2.17 & 2.81 & $0.64^{\mathrm{a})}$ & 2.41 & 2.79 & 0.38 \\
\hline Impact on industries & 2.30 & 3.01 & $0.71^{\text {a) }}$ & 2.44 & 2.92 & 0.48 \\
\hline $\begin{array}{l}\text { Opinions about PR } \\
\text { activities }\end{array}$ & 2.00 & 2.54 & $0.54^{\text {a) }}$ & 2.26 & 2.65 & 0.39 \\
\hline $\begin{array}{l}\text { Technology } \\
\text { collaboration and } \\
\text { development }\end{array}$ & 2.16 & 2.73 & $0.57^{\mathrm{a})}$ & 2.27 & 2.69 & 0.42 \\
\hline
\end{tabular}

the power company" than the latter age group. However, after the accident, their average scores were higher for 9 items, except for "Opinions about PR activities", than the 50's and older age group. Also, for all items, the difference between average scores of the age group 10's to 40's is larger than those of all respondents collectively. In addition, when we performed a $t$-test to see whether there are differences in average scores before and after the accident, as for those aged in their 10 's to 40 's, there were significant differences at the $1 \%$ level for all items. It is assumed that although the information needs of those aged in their 10's to 40's, who were born after the plant establishment, were lower than those aged 50's and older, who were born before the establishment and before the accident, their needs increased to a greater extent than those aged 50's and older after the accident.

\section{(2) Gender}

When we compare the average scores of males and females (Table 5), the average score of females before the accident is lower than males for 9 items, but not for "Influence of harmful rumors about the region." Also, for all items, the difference between the average scores of females is larger than those of all respondents. In addition, when we performed $t$-tests to see whether there were differences in average scores before and after the accident, for females, there were significant differences at the $1 \%$ level for all items.

Females originally had low needs for information. However, it is assumed that their needs grew due to food intake restrictions after the accident, which is directly related to their lives.

\section{(3) Attitude to nuclear power}

According to the study of Shinoda ${ }^{7)}$, who found the factors that determine whether citizens 
Table 5 Average score by gender

\begin{tabular}{|c|c|c|c|c|c|c|}
\hline \multirow{2}{*}{ Gender } & \multicolumn{3}{|c|}{ Male $(158)^{\mathrm{b})}$} & \multicolumn{3}{|c|}{ Female $(135)^{\mathrm{b})}$} \\
\hline & Before & After & $\begin{array}{c}\text { Difference } \\
\text { between } \\
\text { average } \\
\text { scores }\end{array}$ & Before & After & $\begin{array}{c}\text { Difference } \\
\text { between } \\
\text { average } \\
\text { scores }\end{array}$ \\
\hline $\begin{array}{l}\text { Awareness of and } \\
\text { attitudes to nuclear } \\
\text { power }\end{array}$ & 2.32 & 3.09 & 0.77 & 2.20 & 3.18 & $0.98^{\mathrm{a})}$ \\
\hline $\begin{array}{l}\text { Influence of harmful } \\
\text { rumors about the region }\end{array}$ & 2.12 & 3.03 & 0.91 & 2.16 & 3.19 & $1.03^{\mathrm{a})}$ \\
\hline $\begin{array}{l}\text { Relationship with the } \\
\text { power company }\end{array}$ & 2.16 & 2.66 & 0.50 & 1.96 & 2.58 & $0.62^{\mathrm{a})}$ \\
\hline $\begin{array}{l}\text { Impacts on cultural and } \\
\text { sports activities }\end{array}$ & 1.97 & 2.39 & $0.41^{\mathrm{a})}$ & 1.95 & 2.50 & $0.56^{\mathrm{a})}$ \\
\hline Changes in scenery & 2.22 & 2.61 & 0.40 & 2.12 & 2.67 & $0.56^{\mathrm{a})}$ \\
\hline $\begin{array}{l}\text { Changes in } \\
\text { infrastructure } \\
\text { development }\end{array}$ & 2.41 & 2.78 & 0.38 & 2.21 & 2.68 & $0.47^{\mathrm{a})}$ \\
\hline $\begin{array}{l}\text { Opinions about energy } \\
\text { education }\end{array}$ & 2.42 & 2.80 & 0.38 & 2.19 & 2.78 & $0.59^{\mathrm{a})}$ \\
\hline Impact on industries & 2.48 & 2.93 & 0.45 & 2.28 & 2.97 & $0.69^{\mathrm{a})}$ \\
\hline $\begin{array}{l}\text { Opinions about PR } \\
\text { activities }\end{array}$ & 2.25 & 2.66 & $0.41^{\mathrm{a})}$ & 2.05 & 2.54 & $0.49^{\mathrm{a})}$ \\
\hline $\begin{array}{l}\text { Technology } \\
\text { collaboration and } \\
\text { development }\end{array}$ & 2.35 & 2.75 & 0.40 & 2.09 & 2.64 & $0.56^{\mathrm{a})}$ \\
\hline
\end{tabular}

are for or against the use of nuclear power, we can well understand the situation where nuclear power is placed by dividing a group of people who negatively agree with the use of nuclear power into two groups: people who are rather for and who are rather against nuclear power use. Thus, for the attitudes to nuclear power, we cannot divide people into just two groups, with one for and the other against. Therefore, we provided five multiple-choice options (1. should definitely develop and use, 2. should develop and use until alternative energy is fully available, 3 . should maintain the status quo, 4 . should discontinue use in a phased manner, and 5. Should discontinue immediately) for the question concerning attitude to nuclear power use, and allowing for multiple answers. Then, we divided the respondents into three groups: people who accept nuclear power at this stage, people who oppose nuclear power at this stage, and people who suspend judgment because they may change their attitude depending on the future energy situation. The first option clearly shows the acceptance of nuclear energy at this stage. Therefore, we categorized those who solely chose the first option, and those who chose the first plus other options, as the Acceptance Group. Then, the fifth option clearly shows opposition to nuclear power at this stage, so we categorized those who solely chose the fifth option, and those who chose the fifth and other options as the Opposition Group. Although option 4 also shows opposition, those who chose this option can be categorized into the Acceptance Group and Suspended Attitude Group depending on what other options they chose. Therefore, we categorized those who solely chose option 4 as the Opposition Group and categorized those who chose option 4 plus other options into a group that also reflected the other options they chose. Option 3 is an option that clearly shows respondents' suspended attitude. Therefore, we categorized the respondents who solely chose the third option and chose the third and other 
Table 6 Categories of attitude to nuclear power

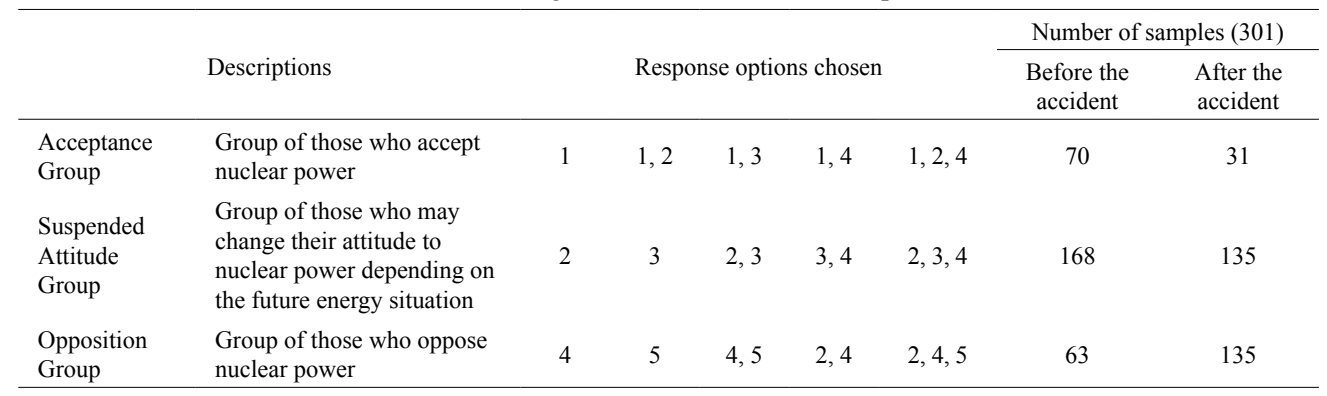

1: Should definitely develop and use; 2 : Should develop and use until alternative energy is fully available; 3 : Should maintain the status quo; 4: Should discontinue in a phased manner; 5: Should discontinue immediately

options as the Suspended Attitude Group. Those who chose option 2 can be categorized into both the Acceptance Group and the Opposition Group. We determined their group depending on what other options they chose. Table 6 shows the categorizing method.

To verify the validity of the classifications, we performed an analysis of variance for the average scores of each of the three groups on each item and examined whether there were differences between groups. We found significant differences at the 5\% level for the following four items: "Awareness of and attitudes to nuclear power," "Impacts on cultural and sports activities," "Opinions about energy education," and "Technology collaboration and development"; there were significant differences at the $1 \%$ level in the following three items: "Influence of harmful rumors about the region," "Relationship with the power company," and "Opinions about PR activities." No difference was observed in the following three items: "Changes in scenery," "Changes in infrastructure development," and "Impact on industries." Then, we compared the average scores between three groups for the 7 items, where significant differences were observed at $1 \%$ and $5 \%$ levels.

The average score results are shown in Table 7. When we compare each group, the average score of the first group (acceptance) was high for all seven items before the accident. However, after the accident, the average scores of "Awareness of and attitudes to nuclear power" and "Influence of harmful rumors about the region" are high for all groups (Acceptance/Suspended Attitude/Opposition). Differences between the average scores of the Suspended Attitude Group are higher than those of all the other respondents for all seven items. Also, a $t$-test on the differences of the average scores before and after the accident showed that the Suspended Attitude Group displayed significant differences at the $1 \%$ level for all items. The information needs of the Acceptance Group have been high since before the accident and the needs of the Suspended Attitude Group increased after the accident.

\section{(4) Knowledge of nuclear power}

The respondents were asked to respond to the questions on their knowledge of nuclear power from four multiple-choice answers: "I know a lot," "I know a little," "I don't know very much," and "I don't know anything." We considered those who answered either "I know a lot" or "I know a little" to be a group with relatively high knowledge and those who answered either "I don't know very much" and "I don't know anything" to be a group with relatively less knowledge.

When we compared the respondents' knowledge of nuclear power before and after the accident (Figure 3), we found that before the accident, the group with relatively high knowledge was less than half of all respondents at $44 \%$ but was more than half at $57 \%$ after the accident. 
Table 7 Average scores by attitude toward nuclear power

\begin{tabular}{|c|c|c|c|c|c|c|c|c|c|}
\hline \multirow[b]{2}{*}{ Item } & \multicolumn{3}{|c|}{ Acceptance } & \multicolumn{3}{|c|}{ Suspended } & \multicolumn{3}{|c|}{ Opposition } \\
\hline & $\begin{array}{l}\text { Before } \\
(70)^{\mathrm{c})}\end{array}$ & $\begin{array}{l}\text { After } \\
(31)^{c)}\end{array}$ & $\begin{array}{c}\text { Difference } \\
\text { between } \\
\text { average } \\
\text { scores }\end{array}$ & $\begin{array}{l}\text { Before } \\
(168)^{c)}\end{array}$ & $\begin{array}{l}\text { After } \\
(135)^{\mathrm{c})}\end{array}$ & $\begin{array}{c}\text { Difference } \\
\text { between } \\
\text { average } \\
\text { scores }\end{array}$ & $\begin{array}{l}\text { Before } \\
(63)^{c)}\end{array}$ & $\begin{array}{l}\text { After } \\
(135)^{\mathrm{c})}\end{array}$ & $\begin{array}{c}\text { Difference } \\
\text { between } \\
\text { average } \\
\text { scores }\end{array}$ \\
\hline $\begin{array}{l}\text { Awareness of and } \\
\text { attitudes to nuclear } \\
\text { power }\end{array}$ & 2.47 & 3.06 & 0.59 & 2.14 & 3.15 & $1.01^{\mathrm{a})}$ & 2.35 & 3.10 & 0.75 \\
\hline $\begin{array}{l}\text { Influence of harmful } \\
\text { rumors about the } \\
\text { region }\end{array}$ & 2.40 & 3.06 & 0.66 & 1.96 & 3.07 & $1.10^{\mathrm{a})}$ & 2.33 & 3.13 & 0.80 \\
\hline $\begin{array}{l}\text { Relationship with the } \\
\text { power company }\end{array}$ & 2.39 & 2.90 & $0.52^{\mathrm{b})}$ & 1.91 & 2.65 & $0.74^{\text {a) }}$ & 2.14 & 2.53 & 0.38 \\
\hline $\begin{array}{l}\text { Impacts on cultural } \\
\text { and sports activities }\end{array}$ & 2.19 & 2.68 & $0.49^{\mathrm{b})}$ & 1.89 & 2.52 & $0.63^{\mathrm{a})}$ & 1.97 & 2.32 & 0.35 \\
\hline Changes in scenery & 2.36 & 2.81 & $0.45^{\mathrm{b})}$ & 2.12 & 2.65 & $0.53^{\text {a) }}$ & 2.10 & 2.60 & $0.50^{\mathrm{a})}$ \\
\hline $\begin{array}{l}\text { Changes in } \\
\text { infrastructure } \\
\text { development }\end{array}$ & 2.51 & 2.77 & 0.26 & 2.28 & 2.88 & $0.60^{\mathrm{a})}$ & 2.19 & 2.59 & $0.40^{\mathrm{a})}$ \\
\hline $\begin{array}{l}\text { Opinions about } \\
\text { energy education }\end{array}$ & 2.59 & 3.00 & 0.41 & 2.25 & 2.83 & $0.58^{\text {a) }}$ & 2.24 & 2.72 & 0.48 \\
\hline Impacts on industries & 2.63 & 3.06 & 0.44 & 2.33 & 3.01 & $0.68^{a)}$ & 2.29 & 2.86 & 0.57 \\
\hline $\begin{array}{l}\text { Opinions about PR } \\
\text { activities }\end{array}$ & 2.44 & 2.97 & $0.52^{\mathrm{a})}$ & 2.03 & 2.67 & $0.64^{\text {a) }}$ & 2.24 & 2.47 & 0.24 \\
\hline $\begin{array}{l}\text { Technology } \\
\text { collaboration and } \\
\text { development }\end{array}$ & 2.49 & 2.97 & 0.48 & 2.14 & 2.84 & $0.69^{a)}$ & 2.19 & 2.51 & 0.32 \\
\hline
\end{tabular}

a) The numbers indicate scores larger than the difference of average scores and are significant at the $1 \%$ level using a $t$-test.

b) The numbers indicate scores larger than the difference of average scores and are significant at the $5 \%$ level using a $t$-test.

c) The numbers in brackets indicate the number of samples.

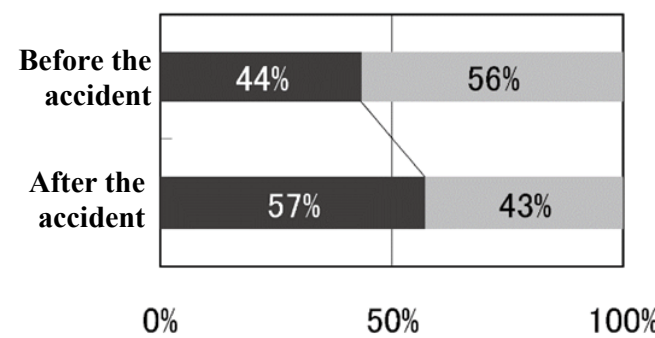

Group with relatively high knowledge

Group with relatively less knowledge

Figure 3 Comparison of knowledge of nuclear power before and after the accident

Respondents' knowledge of nuclear power increased after the accident.

Next, when we compared the average scores of the group with relatively high knowledge and the group with relatively less knowledge (Table 8), the average scores of the group with high knowledge were higher before and after the accident and their information needs had been high since before the accident. However, after the accident, the average scores of the group with relatively less knowledge increased more than the average score of all the respondents for all items. In addition, when we performed a $t$-test to see whether there were differences in average scores before and after the accident, for the group with relatively less knowledge, differences 
Table 8 Average scores by group (from the perspective of knowledge of nuclear power) on nuclear power

\begin{tabular}{|c|c|c|c|c|c|c|}
\hline \multirow[b]{2}{*}{ Item } & \multicolumn{3}{|c|}{ Group that has more knowledge } & \multicolumn{3}{|c|}{ Group that has less knowledge } \\
\hline & $\begin{array}{l}\text { Before } \\
(131)^{\text {b) }}\end{array}$ & $\begin{array}{l}\text { After } \\
(172)^{\text {b) }}\end{array}$ & $\begin{array}{c}\text { Difference } \\
\text { between } \\
\text { average } \\
\text { scores }\end{array}$ & $\begin{array}{l}\text { Before } \\
(170)^{\text {b) }}\end{array}$ & $\begin{array}{l}\text { After } \\
(129)^{\text {b) }}\end{array}$ & $\begin{array}{c}\text { Difference } \\
\text { between } \\
\text { average } \\
\text { scores }\end{array}$ \\
\hline $\begin{array}{l}\text { Awareness of and } \\
\text { attitudes to nuclear } \\
\text { power }\end{array}$ & 2.46 & 3.09 & 0.63 & 2.11 & 3.16 & $1.05^{\mathrm{a})}$ \\
\hline $\begin{array}{l}\text { Influence of harmful } \\
\text { rumors about the region }\end{array}$ & 2.34 & 3.05 & 0.71 & 1.99 & 3.16 & $1.17^{\mathrm{a})}$ \\
\hline $\begin{array}{l}\text { Relationship with the } \\
\text { power company }\end{array}$ & 2.31 & 2.61 & 0.30 & 1.88 & 2.64 & $0.75^{\mathrm{a})}$ \\
\hline $\begin{array}{l}\text { Impacts on cultural and } \\
\text { sports activities }\end{array}$ & 2.15 & 2.44 & 0.30 & 1.84 & 2.45 & $0.61^{\mathrm{a})}$ \\
\hline Changes in scenery & 2.37 & 2.76 & 0.38 & 2.01 & 2.50 & $0.48^{\text {a) }}$ \\
\hline $\begin{array}{l}\text { Changes in } \\
\text { infrastructure } \\
\text { development }\end{array}$ & 2.50 & 2.77 & 0.28 & 2.18 & 2.70 & $0.52^{\mathrm{a})}$ \\
\hline $\begin{array}{l}\text { Opinions about energy } \\
\text { education }\end{array}$ & 2.59 & 2.85 & 0.26 & 2.12 & 2.73 & $0.61^{\text {a) }}$ \\
\hline Impact on industries & 2.65 & 2.99 & 0.34 & 2.19 & 2.90 & $0.71^{\mathrm{a})}$ \\
\hline $\begin{array}{l}\text { Opinions about PR } \\
\text { activities }\end{array}$ & 2.38 & 2.65 & 0.26 & 2.01 & 2.57 & $0.56^{\mathrm{a})}$ \\
\hline $\begin{array}{l}\text { Technology } \\
\text { collaboration and } \\
\text { development }\end{array}$ & 2.48 & 2.74 & 0.26 & 2.04 & 2.66 & $0.62^{\mathrm{a})}$ \\
\hline
\end{tabular}

a) The numbers indicate scores larger than the difference of the average scores of all the respondents and are significant at the $1 \%$ level using a $t$-test.

b) The numbers in brackets indicate the number of samples.

were significant at the $1 \%$ level for all items.

As the information needs of the group with less knowledge increased after the accident, and there were no significant differences between their average scores and those of the group with relatively high knowledge, we can assume that the information needs increased regardless how much knowledge people had originally.

\section{Detailed Analysis of Items with High Information Needs}

Based on the analysis so far, as the average scores for "Awareness of and attitudes to nuclear power," "Influence of harmful rumors about the region," and "Impacts on industries" were high after the accident, they are assumed to have high information needs (Table 3). However, from only the names of the items, it is unknown what particular information people are interested in. Therefore, in this section, we would like to clarify the three items in greater detail.

In this survey, we provided free-answer fields to gain information on what they wanted to hear regarding each item. Then, using the free answers, we categorized and conducted analysis on information they wanted regarding "Awareness of and attitudes to nuclear power," "Influence of harmful rumors about the region," and "Impact on industries." Their free answers are provided in Table 9. Answers containing several questions were categorized across several items. Also, respondents' provided opinions that were not questions they would like to ask the 
Table 9 Free answers

\begin{tabular}{|c|c|c|c|}
\hline Item & Contents of free answers & $\begin{array}{l}\text { \# of respondents } \\
\text { who provided } \\
\text { free answers }\end{array}$ & $\begin{array}{l}\text { \# of free } \\
\text { answers }\end{array}$ \\
\hline $\begin{array}{l}\text { Awareness of and } \\
\text { attitudes to nuclear } \\
\text { power }\end{array}$ & $\begin{array}{l}\text { Awareness of potential hazards and safety/For or against the } \\
\text { acceptance of nuclear power/Changes in awareness/attitudes after the } \\
\text { accident/Local growth strategies and employment/Safety measures/ } \\
\text { Accident and evacuation coverage/Anxiety/Balance between risks } \\
\text { and benefits/Radiation effects/Feeling when accepting/Real feeling/ } \\
\text { Emotional distress/Demand/Others/Opinion }\end{array}$ & 88 & 106 \\
\hline $\begin{array}{l}\text { Influence of harmful } \\
\text { rumors about the } \\
\text { region }\end{array}$ & $\begin{array}{l}\text { Impacts on lives/Measures/Residents' opinion/Actual harm of } \\
\text { radiation/Balance between risks and benefits/Impacts on agriculture } \\
\text { and fishery/Influence of harmful rumors in mass media/Termination } \\
\text { period/Influence on areas where no accident occurred/Impact on } \\
\text { tourism industry/For or against the acceptance of nuclear power/ } \\
\text { Others/Opinion }\end{array}$ & 59 & 63 \\
\hline $\begin{array}{l}\text { Relationship with } \\
\text { the power company }\end{array}$ & $\begin{array}{l}\text { PR Methods/Positions of those who interact with/Details of } \\
\text { interactions/Opinions of nuclear-related businesses/Opportunity to } \\
\text { interact (frequency and period)/Contribution to the region/Others/ } \\
\text { Opinion }\end{array}$ & 47 & 47 \\
\hline $\begin{array}{l}\text { Impacts on cultural } \\
\text { and sports activities }\end{array}$ & $\begin{array}{l}\text { Effects and contributions/Activity details/Funds/Continuity/Others/ } \\
\text { Opinion }\end{array}$ & 27 & 32 \\
\hline Changes in scenery & $\begin{array}{l}\text { Scenic attraction/Protection and destruction of nature/Impact on } \\
\text { tourism industry/Others/Opinion }\end{array}$ & 36 & 38 \\
\hline $\begin{array}{l}\text { Changes in } \\
\text { infrastructure } \\
\text { development }\end{array}$ & $\begin{array}{l}\text { Financial perspective/Balance between risks and benefits/ } \\
\text { Evacuation road/Demand for the maintenance of roads and facilities/ } \\
\text { Convenience/Others/Opinion }\end{array}$ & 47 & 50 \\
\hline $\begin{array}{l}\text { Opinions about } \\
\text { energy education }\end{array}$ & $\begin{array}{l}\text { Education details/Human resource development/Current status of } \\
\text { energy supply/Research details/Others/Opinion }\end{array}$ & 54 & 54 \\
\hline Impact on industries & $\begin{array}{l}\text { Positive and negative perspectives/Employment/R\&D and technical } \\
\text { collaborations/Ties with industries/Balance between risks and } \\
\text { benefits/Human resource development/Tax revenue/Others/Opinion }\end{array}$ & 50 & 53 \\
\hline $\begin{array}{l}\text { Opinions about PR } \\
\text { activities }\end{array}$ & $\begin{array}{l}\text { Bias of PR content/Safety measures/Reliability of PR contents/ } \\
\text { Necessary information/Others/Opinion }\end{array}$ & 47 & 49 \\
\hline $\begin{array}{l}\text { Technology } \\
\text { collaboration and } \\
\text { development }\end{array}$ & $\begin{array}{l}\text { Benefits/Balance between risks and benefits/Human resource } \\
\text { development/Safety measures/Anxiety/Others/Opinion }\end{array}$ & 37 & 40 \\
\hline
\end{tabular}

local residents, were categorized as "Opinion." c The content of free answers, their description examples, and the number of free answers of "Awareness and attitudes to nuclear power," "Influence of harmful rumors about the region," and "Impact on industries" are provided in Tables 10 to 12.

When we look at respondents' free answers for "Awareness of and attitudes to nuclear power," we found questions raised about "Awareness of potential hazards and safety," "For or against the acceptance of nuclear power," and "Changes in awareness after the accident," and they focus on how the local residents perceived risks and safety measures in regard to nuclear power and how these perceptions changed after the accident.

As for "Influence of harmful rumors about the region," questions were raised about the course of improvement such as "Measures," "Termination period," "Balance between risks and benefits," and "Impact on lives," and they tend to show high interest in measures to counter harmful rumors about the region and the actual current status.

When we look at free answers for "Impact on industries," questions such as "Positive and negative perspectives," "Employment," and "Balance between risks and benefits" are raised

\footnotetext{
${ }^{\mathrm{c}}$ Research associates and students who major in nuclear power (2 students in secondary doctoral course and 2 students in primary doctoral course) categorized respondents into the set categories, which was regarded as the final decision.
} 
Table 10 Free answers by attribute for awareness/attitudes to nuclear power

\begin{tabular}{|c|c|c|}
\hline Content of free answers & Answer examples & $\begin{array}{l}\# \text { of free } \\
\text { answers }\end{array}$ \\
\hline $\begin{array}{l}\text { Awareness of and attitudes } \\
\text { to potential hazards and } \\
\text { safety }\end{array}$ & $\begin{array}{l}\text { How much do local residents hear about safety and what is the level of their } \\
\text { understanding? How much do local residents want to know about the risks } \\
\text { associated with nuclear power? }\end{array}$ & 13 \\
\hline $\begin{array}{l}\text { For or against the } \\
\text { acceptance of nuclear } \\
\text { power }\end{array}$ & Do we need nuclear power or not? Are we able to live with nuclear power? & 12 \\
\hline $\begin{array}{l}\text { Changes in awareness after } \\
\text { the accident }\end{array}$ & $\begin{array}{l}\text { Feeling of local residents regarding the collapse of safety myth due to the } \\
\text { accident/I would like to know what the local residents think about disasters } \\
\text { such as the Great Eastern Japan Earthquake }\end{array}$ & 12 \\
\hline $\begin{array}{l}\text { Local growth strategies and } \\
\text { employment }\end{array}$ & $\begin{array}{l}\text { Does a nuclear power plant provide job opportunities? Aren't there any (work- } \\
\text { related) safety issues? }\end{array}$ & 11 \\
\hline Safety measures & $\begin{array}{l}\text { Are measures established to deal with nuclear power-related problems when } \\
\text { they occur? }\end{array}$ & 6 \\
\hline $\begin{array}{l}\text { Accident and evacuation } \\
\text { coverage }\end{array}$ & What coverage do residents wish when an evacuation order is issued? & 6 \\
\hline Anxiety & $\begin{array}{l}\text { What are residents' feelings and concerns about the safety and security of } \\
\text { living in the region? }\end{array}$ & 6 \\
\hline $\begin{array}{l}\text { Balance between risks and } \\
\text { benefits }\end{array}$ & $\begin{array}{l}\text { What are the residents' views on (a) benefits including improvement of } \\
\text { local economy in the area where nuclear power plant is located and (b) } \\
\text { improvement of infrastructure and disadvantages including harmful effects if } \\
\text { a nuclear accident occurred? }\end{array}$ & 4 \\
\hline Radiation effects & Are there more or less some sort of health-related changes? & 4 \\
\hline Feeling when accepting & How did residents start accepting nuclear power? & 3 \\
\hline Real feeling & I would like to know the true opinions of local residents. & 3 \\
\hline Emotional distress & $\begin{array}{l}\text { Are there any people who oppose the establishment of a nuclear power plant? } \\
\text { If you are opposed, will it be hard to live in the area? }\end{array}$ & 2 \\
\hline Demand & $\begin{array}{l}\text { Demands to the government and nuclear power plant from safety and security } \\
\text { perspectives }\end{array}$ & 2 \\
\hline Others & $\begin{array}{l}\text { Differences between reported information and actual information, changes in } \\
\text { the future visions due to nuclear power plant siting }\end{array}$ & 5 \\
\hline Opinion & $\begin{array}{l}\text { Anxiety about safety, lack of residents' knowledge of nuclear power, and } \\
\text { reluctance of residents to express their opinions }\end{array}$ & 17 \\
\hline Total & & 106 \\
\hline
\end{tabular}

* Actual free answers are provided except those in the categories of "Others" and "Opinion." (Some changes are applied to the extent that such changes do not change the meaning.)

and respondents are interested in the economic benefits and disadvantages of plant siting.

Also, for all of the following items, "Awareness of and attitudes to nuclear power," "Influence of harmful rumors about the region," and "Impact on industries," respondents provide not questions but opinions and thoughts relevant to the examples of the social impact items. This shows that respondents want specific information on social impacts rather than the current situations in the power plant siting area.

\section{Conclusions}

Comparing the need for information on social impacts due to nuclear power plant siting before and after the accident, information needs have increased for three items: "Awareness of and attitudes to nuclear power," "Influence of harmful rumors about the region," and "Impact on industries" after the accident. The need for information on social impacts increased after the accident among those aged in their 10 's to 40 's, who were born after the power plant 
Table 11 Free answers by attribute for the influence of harmful rumors about the region

\begin{tabular}{|c|c|c|}
\hline Contents of free answers & Examples & $\begin{array}{l}\text { \# of free } \\
\text { answers }\end{array}$ \\
\hline Impacts on lives & $\begin{array}{l}\text { I would like to know how harmful rumors can change residents' lives/How } \\
\text { they actually change their life. }\end{array}$ & 7 \\
\hline Measures & $\begin{array}{l}\text { What measures did the government and mass media take for harmful rumors? } \\
\text { What measures do residents want for harmful rumors? }\end{array}$ & 6 \\
\hline Residents' opinion & $\begin{array}{l}\text { I would like to know the current feeling of neighboring residents/I would like } \\
\text { to know what they think about harmful rumors. }\end{array}$ & 6 \\
\hline Actual harm of radiation & $\begin{array}{l}\text { Effect on body/It's been } 40 \text { years since the power plant was built, and I wonder } \\
\text { if there has been any leakage of radiation or any problems with the ocean } \\
\text { environment. }\end{array}$ & 4 \\
\hline $\begin{array}{l}\text { Balance between risks and } \\
\text { benefits }\end{array}$ & $\begin{array}{l}\text { Do the residents live there because they believe they have benefits even } \\
\text { though harmful rumors can have some impacts? }\end{array}$ & 3 \\
\hline $\begin{array}{l}\text { Impacts on agriculture and } \\
\text { fishery }\end{array}$ & $\begin{array}{l}\text { We didn't think having a nuclear power plant was dangerous before the } \\
\text { accident, but we are worried about contamination in agriculture and fishery } \\
\text { ecosystems. }\end{array}$ & 3 \\
\hline Impact on tourism industry & Impacts on tourists/Impacts on Minshuku and accommodation businesses & 2 \\
\hline $\begin{array}{l}\text { Influence of harmful } \\
\text { rumors in mass media }\end{array}$ & $\begin{array}{l}\text { Specific details of harmful rumors in mass media and the extent of effects on } \\
\text { the region from harmful rumors }\end{array}$ & 2 \\
\hline Termination period & How long did it take before harmful rumors disappeared? & 2 \\
\hline $\begin{array}{l}\text { Influence on areas where } \\
\text { no accident occurs }\end{array}$ & $\begin{array}{l}\text { Are there harmful rumors even in areas where nuclear power plants are located } \\
\text { but no nuclear accidents have occurred? }\end{array}$ & 2 \\
\hline $\begin{array}{l}\text { For or against the } \\
\text { acceptance of nuclear } \\
\text { power }\end{array}$ & $\begin{array}{l}\text { Do residents think they can live with nuclear power even after such a } \\
\text { disastrous accident? }\end{array}$ & 3 \\
\hline Others & Influence on terrorism, information reliability, government's opinions, etc. & 5 \\
\hline Opinion & $\begin{array}{l}\text { Inevitability of harmful rumors, insufficient information disclosure, anxiety, } \\
\text { etc. }\end{array}$ & 18 \\
\hline Total & & 63 \\
\hline
\end{tabular}

* Actual free answers are provided except those in the categories of "Others" and "Opinion." (Some changes are applied to the extent that such changes do not change the meaning.)

establishment, in females, who seem to be less interested in nuclear power, in the Suspended Attitude Group, which consists of people who do not clearly show their attitude to nuclear power, and in the group with relatively less knowledge about nuclear power.

At the end of the survey, we categorized and analyzed questions given to local residents about "Awareness of and attitudes to nuclear power," "Influence of harmful rumors about the region," and "Impact on industries" using free answer questions. The results showed that respondents are interested in economic impacts such as regional development projects and employment due to the nuclear power plant siting, they also revealed residents' subjective opinions about risks of nuclear power, and the need for information on the economic and mental benefits and disadvantages related to the power plant siting.

We assumed that those who had never clearly revealed their attitudes to nuclear power and those with no knowledge of or interest in nuclear power may not be particularly interested in information on social impacts as well as in information on nuclear power in general. However, now that we have confirmed the information needs of these groups, we believe it is useful to distribute this information on social impacts to the group whose interest has increased after the accident so that they will recognize the social impact and be motivated to become interested in nuclear power. However, since respondents did not register an opinion in the free answer field in many cases, there is a gap between what residents want to hear and the information provided in this leaflet; we still have a little more work to do on the information that needs to be provided. Also, we found that residents are interested in specific information on social impacts 
Table 12 Free answers by attribute for impact on industries

\begin{tabular}{|c|c|c|}
\hline Free answers & Examples & $\begin{array}{l}\text { \# of free } \\
\text { answers }\end{array}$ \\
\hline $\begin{array}{l}\text { Positive and negative } \\
\text { perspectives }\end{array}$ & $\begin{array}{l}\text { The region has accrued benefits in various ways, but I wonder if everyone has } \\
\text { been the beneficiary of such benefits. I wonder if the residents are forced to } \\
\text { put up with many things in return/I wonder how much benefit the region has } \\
\text { received and whether residents can actually feel any benefit. }\end{array}$ & 10 \\
\hline Employment & $\begin{array}{l}\text { I wonder to what extent nuclear-related employment opportunities have been } \\
\text { created for neighboring residents. }\end{array}$ & 5 \\
\hline $\begin{array}{l}\text { R\&D and technical } \\
\text { collaborations }\end{array}$ & $\begin{array}{l}\text { I wonder whether we can expect development in other industries such as } \\
\text { medical and bio technologies. }\end{array}$ & 3 \\
\hline Ties with industries & $\begin{array}{l}\text { I would like to know what percentage of businesses in Fukui Prefecture are } \\
\text { related to the nuclear plant. }\end{array}$ & 3 \\
\hline $\begin{array}{l}\text { Balance between risks and } \\
\text { benefits }\end{array}$ & $\begin{array}{l}\text { I wonder if the government thinks they should retain the employment } \\
\text { opportunities and economic effects in exchange for accepting the risks } \\
\text { associated with nuclear power. }\end{array}$ & 3 \\
\hline $\begin{array}{l}\text { Human resource } \\
\text { development }\end{array}$ & Current state of human resource development & 2 \\
\hline Tax revenue & Impact on the local government in terms of tax-related matters & 2 \\
\hline Others & $\begin{array}{l}\text { Impacts on agriculture and fishery, impact of accident/How to develop } \\
\text { industries independent of nuclear power. }\end{array}$ & 13 \\
\hline Opinion & $\begin{array}{l}\text { Economic revitalization utilizing nuclear industry, regional economic decline } \\
\text { due to the power plant siting }\end{array}$ & 12 \\
\hline Total & & 53 \\
\hline
\end{tabular}

* Actual free answers are provided except those in the categories of "Others" and "Opinion." (Some changes are applied to the extent that such changes do not change the meaning.)

rather than the current status of the area where the power plant is located. To utilize information on social impacts, we need to provide not only the information people need to affirm or revise their opinions, and history of nuclear power plant siting, but also to study just what information to deliver, and how that information should be delivered to offer future prospect, and how to enable people to think about the region in a comprehensive manner and carry out plans to provide information appropriately.

\section{References}

1) H. Kimura, C. Katsuki, H. Madarame, T. Miyazawa, Study on "Facilitation Forum" for Nuclear Energy with Social and Technological Knowledge Circulation, NV Laboratory, Feb. 2008. [in Japanese]

2) Y. Tada, C. Hayashi, "A survey study about what an exhibition hall of nuclear power station should be," INSS J., 8, 15-26 (2004). [in Japanese]

3) Y. Shinoda, H. Torii, N. Yamano, Heisei 19 Nendo Oarai Fieldwork Cyosahokokusho, Tokyo Institute of Technology, Feb. 2 (2008). [in Japanese]

4) Heisei 22 Nendo, Genshiryokuriyo no Chishiki Fukyu Keihatsu ni Kansuru Yoronchosa, Japan Atomic Energy Relations Organization, Feb. (2010). [in Japanese]

5) N. Tsujikawa, S. Tsuchida, T. Shiotani, "Effects of perceptions of the necessities and anxieties associated with nuclear power on motivation for considering nuclear power generation," Shakai-gijutu Kenkyu Ronbunshu, 8, 74-81 (2011). [in Japanese]

6) Heisei 19, Nendo Enerugi ni Kansuru Koshu no Ishikichosa Hokokushu, The Institute of Applied Energy, Mar. (2008). [in Japanese]

7) Y. Shinoda, "Consideration on the interaction between society and nuclear technology," Trans. At. Energy Soc. Jpn., 6 [2], 97-112 (2007). [in Japanese] 


\title{
Determination of Local-Area Distribution and Relocation of Radioactive Cesium in Trees from Fukushima Daiichi Nuclear Power Plant by Autoradiography Analysis
}

\author{
Fuminori SAKAMOTO $^{1}$, Toshihiko OHNUKI ${ }^{1, *}$, Naofumi KOZAI ${ }^{1}$, \\ Shinya YAMASAKI ${ }^{1}$, Zenko YOSHIDA ${ }^{2}$ and Kenji NANBA ${ }^{3}$ \\ ${ }^{1}$ Japan Atomic Energy Agency, 2-4 Shirane, Shirakata, Tokai-mura, Naka-gun, Ibaraki 319-1195, Japan \\ ${ }^{2}$ ATOX Co. Ltd., 161-2 Muramatsu, Tokai-mura, Naka-gun, Ibaraki 319-1112, Japan \\ ${ }^{3}$ Fukushima University, 1 Kanayagawa, Fukushima-shi, Fukushima 960-1296, Japan
}

\begin{abstract}
The local area distribution and relocation of radioactive cesium deposited in trees after the 2011 tsunami-related accident at the Fukushima Daiichi Nuclear Power Plant (FDNPP) have been studied by measuring the spatial distribution of cesium on/in trees by autoradiography analysis. Samples of trees were collected from places located between 4 and $55 \mathrm{~km}$ from FDNPP approximately 2, 8, 20, and 22 months after the accident. The autoradiography analyses of Cryptomeria japonica, Torreya nucifera, and Thujopsis dolabrata var. hondae samples collected approximately 2 and 8 months after the accident showed that radioactive Cs was mainly distributed as spots on the branches and leaves of the trees emerged before the accident, and was detected in negligible amounts in new branch and leaves that emerged after the accident. On the contrary, radioactive Cs was detected at the outermost tip of the branches in the trees collected 20 months after the accident. Morus alba samples collected 22 months after the accident contained radioactive Cs inside and outside their stems, even though no radioactive Cs was detected in their roots, strongly suggesting that a certain amount of radioactive Cs was translocated from the outside to the inside of stems. These results indicate that the distribution of radioactive Cs deposited on/in the trees gradually changes with time (scale: year).
\end{abstract}

KEYWORDS: radioactive fallout, radioactive Cs, translocation, migration, autoradiography

\section{Introduction}

A large amount of nuclides produced by nuclear fission (hereinafter referred to as "radionuclides") was released into the environment due to the reactor melt down and hydrogen explosion at the Tokyo Electric Power Company (TEPCO) Fukushima Daiichi Nuclear Power Plant (hereinafter referred to as the " $1 \mathrm{~F}$ accident") that occurred on March 11, 2011. The total amount of radioactive $\mathrm{Cs}$ and radioactive $\mathrm{I}$ released in the atmosphere was $10^{16}-10^{17} \mathrm{~Bq}$, according to the report by Chino et al ${ }^{1)}$. and Brumfiel ${ }^{2)}$. Of the radioactive I, ${ }^{131} \mathrm{I}$ decayed out within

\footnotetext{
* Corresponding author, E-mail: ohnuki.toshihiko@jaea.go.jp

DOI : 10.15669/fukushimainsights. Vol.4.204

(C) 2021 Atomic Energy Society of Japan. All rights reserved.

Originally published in Transactions of the Atomic Energy Society of Japan (ISSN 1347-2879), Vol. 12, No. 4, p.257-266

(2013) in Japanese. (Japanese version accepted: May 24, 2013)
} 
about two months of the $1 \mathrm{~F}$ accident due to its short half-life of approximately eight (8) days; therefore, the residual radioactive nuclides in the environment are mainly ${ }^{134} \mathrm{Cs}$ (half-life of approximately 2.1 years) and ${ }^{137} \mathrm{Cs}$ (half-life of approximately 30 years) ${ }^{3)}$.

Decontamination activities were conducted at a good pace for the purpose of removing radioactive Cs from contaminated areas ${ }^{3)}$. Through such decontamination activities, a large amount of waste materials were packed in flexible container bags (hereinafter referred to as "flecons") and stored in temporary storage places. Given this situation, the environmental influence of radioactive Cs contained in the decontamination waste should be evaluated. To evaluate any leakage of radioactive Cs from decontamination waste requires recognition of the conditions surrounding radioactive $\mathrm{Cs}$ in the flecons. As trees and grasses were discarded from home gardens, back hills, and fruit farms and then packed with other decontamination wastes in flecons, there is concern of relocation of radioactive $\mathrm{Cs}$ in the environment through solubilization due to the decomposition of trees and grasses.

Sakamoto et al. conducted an autoradiograph analysis of the local distribution of the deposited radionuclides in the trees and grasses, targeting samples taken two months after the $1 \mathrm{~F}$ accident ${ }^{4}$. Their study demonstrated that the radioactive Cs that deposited on trees showed higher concentrations on the stem and leaves that had emerged before the $1 \mathrm{~F}$ accident, while almost no relocation took place to the branches and leaves that emerged after the deposition, but the major part of the radioactive Cs that deposited on pasture grass and rice stubs accumulated there in situ. Similar results were found in research by Tanaka et al. that analyzed C. japonica ${ }^{5}$.

The long-term relocation of radioactive $\mathrm{Cs}$ is being studied in Chernobyl. Reports show that radioactive $\mathrm{Cs}$ is accumulated in biological bodies such as trees and mushrooms ${ }^{6}$. On the other hand, based on study of the distribution of radioactive Cs by atomic bomb tests, it is reported that $80 \%$ of radioactive Cs has been distributed in the soil layer near the ground surface as deeply as $5 \mathrm{~cm}$ in pine forest, with the remainder distributed deeper in the soil or in the plants ${ }^{7}$. The radioactive Cs deposited on tree crowns in Fukushima was considered to be leached by rain or snow and thereby joined in the environmental cycle. However, it is considered that the relocation of radioactive $\mathrm{Cs}$ accumulated in trees has not been completely clarified.

To measure the distribution of radioactive Cs in tree samples, the trees should be divided into several parts to obtain adequate spatial resolution. Higher spatial resolution is achieved by dividing into small pieces, which leads to decreased measurement count. Unfortunately, as a result, spatial resolution may be limited to $10 \mathrm{~mm}$. In contrast, autoradiography analyzes the distribution of radionuclides by placing the samples directly on imaging plates sensitive to radiation ${ }^{8}$. Autoradiography's shortcoming is its inability to identify the radionuclides because it cannot distinguish between the types and energies of radiation detected. Among the radionuclides in fallout in Fukushima-Prefecture, the radionuclides that were detected after May are, as mentioned above, ${ }^{134} \mathrm{Cs}$ and ${ }^{137} \mathrm{Cs}$. Thus it is possible to study the behavior of radioactive Cs accumulated in the trees after the $1 \mathrm{~F}$ accident by measuring the local distribution to an accuracy of well under $10 \mathrm{~mm}$.

This report details the autoradiography study of the samples of trees collected (1) in May and November 2011, and December 2012 in Iitate-Village Kita-Soma-County Fukushima-Prefecture, (2) in May 2012 and February 2013 in Nihonmatsu-City Fukushima-Prefecture, (3) in October 2011 in Date-City Fukushima-Prefecture, and (4) in December 2012 in Okuma-Machi Futaba-County Fukushima-Prefecture Also, the relocation of radioactive Cs within a tree following precipitation was investigated, and the leaching of radioactive $\mathrm{Cs}$ from decontamination wastes including grasses was studied. 


\section{Experimental Methodology}

\section{Sampling and Preparation of Samples}

The collected trees were Japanese cedar (C. japonica), Japanese torreya (T. nucifera), var. hondae makino (T. dolabrata var. hondae), peach (Prunus percica) and mulberry (M. alba). $C$. japonica, Torreya and var. makino are evergreen needle-leaved trees. For the purpose of observing any variation with aging, evergreen trees that would not experience defoliation were selected. Peach and M. alba are defoliating trees, but were selected because there was concern about the effects on fruit trees. For C. japonica, sampling of developed male and female flowers was conducted. The samples of Torreya branches were selected from those with branches and leaves that were considered to have grown before the 1F accident in 2011, in the spring of 2011 and in the spring of 2012. The samples of var. hondae makino were taken from the branches and leaves. Peach stems (diameter approximately $25 \mathrm{~mm}$ ) and $M$. alba stems (diameter approximately $50 \mathrm{~mm}$ ) were cut into round slices and the cross sections were used as samples (peach stem cross section and $M$. alba stem cross section, respectively). The underground root portion (diameter approximately $50 \mathrm{~mm}$ ) of $M$. alba was also cut into round slices and used as a sample (mulberry root cross section sample). For peach trees, the peeled stem bark sample (peach bark sample) and vertically torn twig sample (twig sample) were used. Furthermore, the Parmeliaceae (Parmotrema tinctorum) that lived on the torreya stem in Nagadoro Iitate-Village was collected. For madake (Phyllostachys bambusoides), samples (bamboo sample) of stem cut into round slices and tender shoots (bamboo shoot sample) were used. The samples and the location and time of sampling are summarized in Table 1. The number of samples was one (1) for each because the sampling was conducted in areas that were difficult to revisit.

\section{Autoradiography Measurement}

The system used for autoradiography measurement (hereinafter abbreviated as "AR") was the Bioimaging Analyzer BAS2500 (Fuji Film/Japan). The space resolution of the imaging plate (IP) was $0.05 \mathrm{~mm}$. After exposing the collected/prepared sample for 24-72 hours on the IP, the area on which the radioactive sample had lain was imaged using an IP reading and analyzing device.

In the images acquired, the higher the radiation dose, i.e., the higher the concentration of radioactive nuclides, the more intense the blackness. Furthermore, a unique analysis of two images with different intensities is possible by changing the intensity gradient of the radioactivity dose. During a measurement, to avoid contamination of the IP by the sample, the sample was covered with a thin film of polyvinylidene chloride. After completion of the measurement, an optical photo of the sample, which was covered with the polyvinylidene chloride film, was taken. Additionally, the radioactivity of the sample was measured using a $\mathrm{NaI}$ scintillation counter (Aloka TCS161) before conducting the AR measurement. Furthermore, the radioactive nuclides contained in some of the samples were measured using the Ge semiconductor $\gamma$ ray spectrum analysis system (ORTEC). The M. alba stem samples and root samples were crushed at the same position as AR measurements were made, and were encapsulated in a $1 \mathrm{~L}$ Marinelli container, and then the contained radioactive nuclides were measured with $\gamma$ ray spectrum analysis (NaI scintillation spectrometer ATOMEXAT1320A). The radioactive doses measured using the scintillation counter for all samples were twice as high as background, so it was understood that there was almost no influence from ${ }^{40} \mathrm{~K}$. The grid in the AR and photos is $2.54 \mathrm{~cm}$ $\times 2.54 \mathrm{~cm}$ on the IP. 
Table 1 Tree samples and their parts analyzed by autoradiography, with places of origin and collection dates

\begin{tabular}{|c|c|c|c|c|}
\hline Sample & Sample Portion & Location of Sampling & Time of Sampling & $\begin{array}{c}\text { Distance from } 1 \mathrm{~F} \\
(\mathrm{~km})\end{array}$ \\
\hline C. japonica & Branches and leaves & May, 2011 & $\begin{array}{l}\text { Nagadoro, Iitate- } \\
\text { Village }\end{array}$ & 33 \\
\hline C. japonica & Branches and leaves & Nov., 2011 & $\begin{array}{l}\text { Nagadoro, Iitate- } \\
\text { Village }\end{array}$ & 33 \\
\hline C. japonica & Branches and leaves & Nov., 2012 & $\begin{array}{l}\text { Nagadoro, Iitate- } \\
\text { Village }\end{array}$ & 33 \\
\hline C. japonica & Branches and leaves & Dec., 2012 & $\begin{array}{c}\text { Kodate, Okuma- } \\
\text { Machi }\end{array}$ & 4 \\
\hline $\begin{array}{l}\text { T. dolabrata var. } \\
\text { hondae }\end{array}$ & Branches and leaves & Oct., 2011 & Ryozen, Date-City & 50 \\
\hline $\begin{array}{l}\text { T. dolabrata var. } \\
\text { hondae }\end{array}$ & Branches and leaves & Nov., 2012 & $\begin{array}{l}\text { Nagadoro, Iitate- } \\
\text { Village }\end{array}$ & 33 \\
\hline T. nucifera & Branches and leaves & Dec., 2012 & $\begin{array}{c}\text { Kodate, Okuma- } \\
\text { Machi }\end{array}$ & 4 \\
\hline P. persica & Branches & Oct., 2011 & Oguni, Date-City & 55 \\
\hline P. persica & Stem & Oct., 2011 & Oguni, Date-City & 55 \\
\hline P. persica & Epidermis & Oct., 2011 & Oguni, Date-City & 55 \\
\hline M. alba & Stem & Feb., 2013 & $\begin{array}{c}\text { Tosawa, Nihonmatsu- } \\
\text { City }\end{array}$ & 43 \\
\hline M. alba & Roots & Feb., 2013 & $\begin{array}{c}\text { Tosawa, Nihonmatsu- } \\
\text { City }\end{array}$ & 43 \\
\hline M. alba & Branches and leaves & May., 2012 & $\begin{array}{c}\text { Takatsuki, } \\
\text { Nihonmatsu-City }\end{array}$ & 47 \\
\hline Parmeliaceae & & May., 2011 & $\begin{array}{l}\text { Nagadoro, Iitate- } \\
\text { Village }\end{array}$ & 33 \\
\hline P. bambusoides & Stem & May., 2012 & $\begin{array}{l}\text { Kohata, Ninonmatsu- } \\
\text { City }\end{array}$ & 47 \\
\hline Bamboo Shoot & Tender Shoot & May., 2012 & $\begin{array}{l}\text { Kohata, Ninonmatsu- } \\
\text { City }\end{array}$ & 47 \\
\hline
\end{tabular}

\section{Results}

Autoradiography of branches and leaves of $C$. japonica collected in Iitate-Village in May 2011 (Figure 1) yielded a slight blackening from the branches and leaves, and dark spots all over the range of $C$. japonica. The growth of tender shoots at the tip of branches is seen in the photograph of branches and leaves. On the other hand, in the AR images, no specific accumulation of radioactive Cs on the male flower was observed. Similarly, in the AR images of branch and leaf samples collected in Iitate-Village in November 2011 (Figure 2), an increase in blackness and density of black spots was observed all over as in Figure 1. In the optical photographs, the growth of a male flower is observed in the area surrounded by a light blue circle, but no obvious concentration of radioactive $\mathrm{Cs}$ on any male flower is observed in the AR images. In the AR images, large and dense black spots are visible in the area surrounded by a blue circle. Although it is not clearly visible in the optical photos, the brown female flowers were identified, i.e. the female flowers that grew before the $1 \mathrm{~F}$ accident. This suggests that radioactive Cs was deposited directly on the male flower during precipitation. Although the results are not shown, the black spots were observed all over on the C. japonica sample collected in Date-City on October 2011 similarly to Figure 2.

The presence of scattered black spots was observed in the analysis of $C$. japonica branches and leaves collected in Iitate-Village in November 2012 (Figure 3). Furthermore, it was 

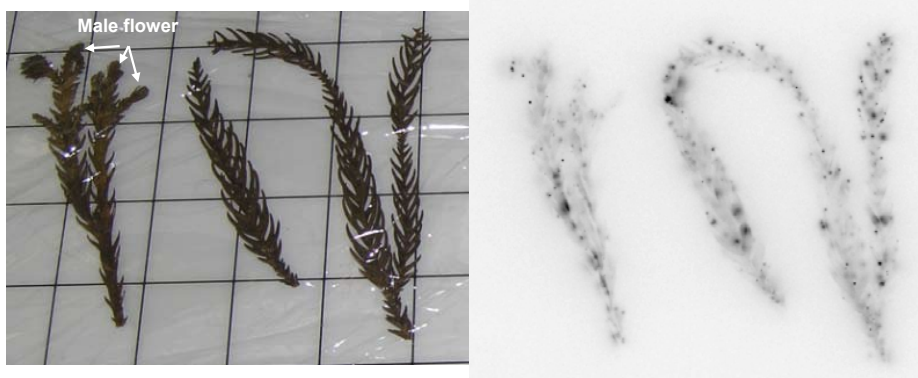

Figure 1 Autoradiograph image (left) and optical photograph (right) of branch and leaves of C. japonica collected in Iitate 2 months after the FDNPP accident

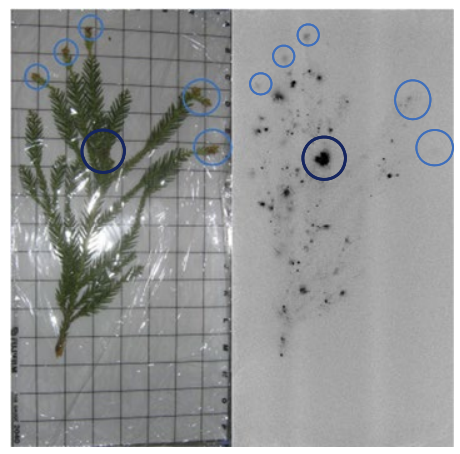

Figure 2 Autoradiograph image (left) and optical photograph (right) of branch and leaves of C. japonica collected in Iitate 8 months after the FDNPP accident. Light blue circles show a male flower that emerged before the FDNPP accident, and the dark blue shows one female flower that emerged before the FDNPP accident.

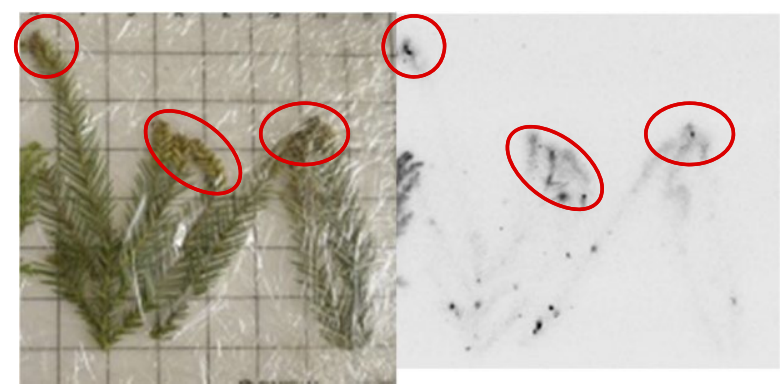

Figure 3 Autoradiograph image (left) and optical photograph (right) of branch and leaves of $C$. japonica collected in Iitate 20 months after the FDNPP accident. Red circles show a male flower that emerged after the FDNPP accident.

observed that the blackness at the tip of twigs surrounded by a red line is darker than that in other areas. In the optical photos, a male flower was found in the area, showing that the concentration of radioactive Cs on the male flower is higher than in the branches and leaves at the tip portion, although the blackness on the male flower is less than that of the black spots. In the AR analysis conducted on the C. japonica branches and leaves collected at Kodate, Okuma-Machi in December 2012 (Figure 4), the AR image shows the dense black spots in 
the female flower area surrounded by a blue line in the optical photo. In this area, black spots are smaller than those of the female flower. As the female flower areas are brown and assumed to have grown before the $1 \mathrm{~F}$ accident, the radioactive $\mathrm{Cs}$ is considered to have been directly deposited and to have remained in the area of deposition. The blackness density is lower in the upper part than in the female flower area, i.e., the branches and leaves in the tip area, and again a dense black area is recognized in the tip area of branches and leaves. As male flowers are found in the area surrounded by a red line, the density of radioactive Cs is comparatively higher in the male flower. Furthermore, in the areas where a male flower is not found, surrounded by a yellow-green line in the optical photo, a similar level of blackness is seen in the AR image as in the male flower area.

The AR analysis results on T. dolabrata var. hondae collected in Date-City in October 2011 are shown in Figure 5. The black spots appear in all areas of branches and leaves in the AR image of T. dolabrata var. hondae. However, in the T. dolabrata var. hondae collected in Iitate-Village in November 2012, as shown in the AR image (Figure 6), almost no black spots were found, but dense black areas were found in the tip area of twigs.
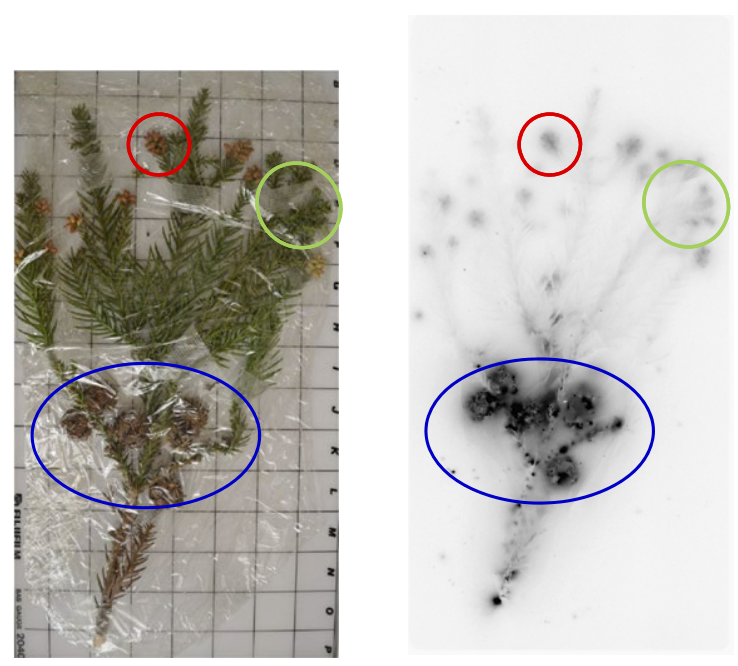

Figure 4 Autoradiograph image (left) and optical photograph (right) of branch and leaves of C. japonica collected in Ookuma 21 months after the FDNPP accident. The blue circle shows a female flower that emerged before the FDNPP accident, and the red circle shows one male flower that emerged before the FDNPP accident. The green circle indicates the outermost tip of the branch.
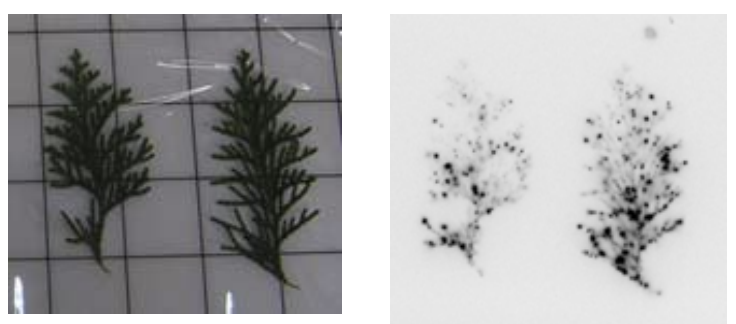

Figure 5 Autoradiograph image (right) and optical photograph (left) of branch and leaves of T. dolabrata var. hondae collected in Date 7 months after the FDNPP accident. 
In the AR results (Figure 7) from T. nucifera collected in Okuma-Machi in December 2012, the branches and leaves that had grown before the 1F accident (area surrounded with a blue line in the photo), those grown in 2011 after the $1 \mathrm{~F}$ accident (area surrounded with a blue dot-anddash line) and those grown in 2012 (area surrounded with a blue dotted line) were observed. In the AR images, many black dots were found in the area that had grown before the $1 \mathrm{~F}$ accident. The more recent the year of growth, the fewer black dots were found. Almost no such dots were found in the area that grew in 2012. This indicates with high probability that most of the radioactive $\mathrm{Cs}$ that directly deposited from the $1 \mathrm{~F}$ accident remained on the branches and leaves where deposited. The blackness on branches and leaves that had grown after the $1 \mathrm{~F}$ accident does not show noteworthy concentration in any area, but rather a uniform distribution.

In the AR analysis of a twig sample of P. persica collected in Date-City in October 2011 (Figure 8), black spots are visible on the bark of twigs that had grown before the $1 \mathrm{~F}$ accident. In contrast, on the twigs that grew after the $1 \mathrm{~F}$ accident, although black spots are visible on the branches near the stem surrounded by a black line, they are not visible at all in other areas. Measurement of the ${ }^{137} \mathrm{Cs}$ concentration using a Ge semiconductor detector on crushed twig samples separated into those that had been grown before and after the $1 \mathrm{~F}$ accident showed that the branches and leaves grown before the $1 \mathrm{~F}$ accident yielded $30,000 \mathrm{~Bq} / \mathrm{kg}$ and those grown after the $1 \mathrm{~F}$ accident just $500 \mathrm{~Bq} / \mathrm{kg}$. The AR analysis of a $P$. persica stem section and bark
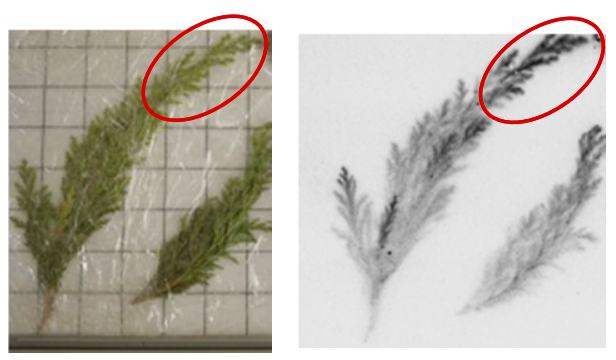

Figure 6 Autoradiograph image (right) and optical photograph (left) of branch and leaves of T. dolabrata var. hondae collected in Iitate 20 months after the FDNPP accident.
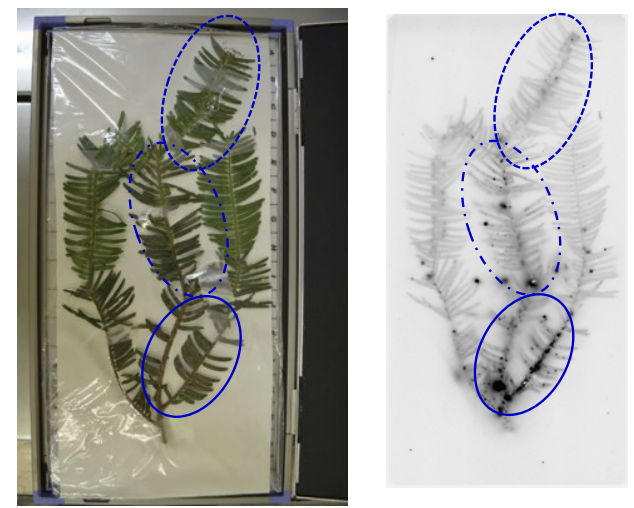

Figure 7 Autoradiograph image (right) and optical photograph (left) of branch and leaves of T. nucifera collected in Ookuma 22 months after the FDNPP accident. The blue solid oval shows the branch portion that emerged before the FDNPP accident, the blue dash-dot oval the branch portion that emerged after the FDNPP accident, and the blue dashed oval the branch that emerged 1 year after the FDNPP accident. 
sample (Figure 9) showed black spots on the stem bark, but almost no spots inside of the stem (Figure $9(a, b)$ ). In the AR image of the P. persica bark sample (Figure $9(\mathrm{~d})$ ), there were black spots on the bark as well as a dark area that spread over the surface with a similar blackness, as indicated with an arrow.

These results indicate that there is radioactive Cs on the stem bark that is deposited like spots and that is also seen as a dark area.

The AR analysis results for a $M$. alba stem section sample and $M$. alba root section sample collected in Nihonmatsu-City in February 2013 are shown in Figure 10. The AR image of the stem sample shows high grayness in the area without the sample. In the area without the sample, background $\alpha$ or $\beta$ radiation was detected, while the area with the sample

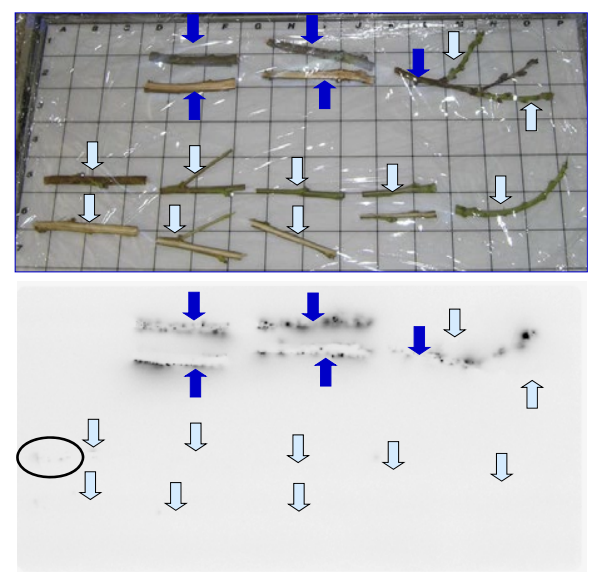

Figure 8 Autoradiograph image (lower) and optical photograph (upper) of branches of $P$. percica collected in Date 20 months after the FDNPP accident. Blue arrows show branches that emerged before the FDNPP accident, and light blue arrows branches that emerged after the FDNPP accident; the black circle shows spotted deposits of radioactive Cs on a branch that emerged after the FDNPP accident.

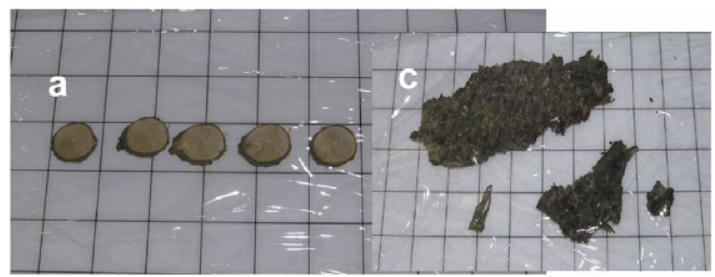

b

d

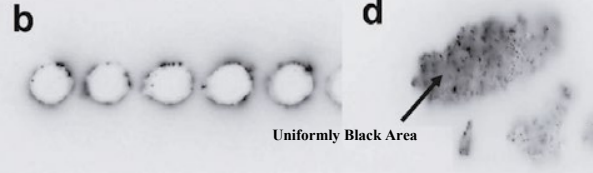

Figure 9 Autoradiograph images (b, d) and optical photographs (a, c) of cross sections of branches (a, b) and bark (c, d), respectively, of $P$. percica collected in Date 20 months after the FDNPP accident. 
did not show grayness, presumably due to the shielding effect of the samples. Black spots were observed on the stem bark area in the AR image (Figure 10 (b)) of the M. alba stem section sample (Figure 10 (a)). However, no black spots were seen in the AR image (Figure 10 (d)) of the cross section of the $M$. alba sample (Figure 10 (c)). Comparing the blackness of the inside of stem and root in the AR images shows a slightly higher grayness of the inside of the stem section sample than the inside of the root section sample.

As the AR images of stem and root were acquired with identical exposure times, they indicate the transport of radioactive Cs into the inside of the stem. Measurements indicate that order of ${ }^{137} \mathrm{Cs}$ concentration is: stem bark $(500 \mathrm{~Bq} / \mathrm{kg})>$ stem inside $(150 \mathrm{~Bq} / \mathrm{kg})>\operatorname{root}(18 \mathrm{~Bq} / \mathrm{kg})$. These results agreed with AR analyses, and supported the near absence of radioactive Cs in the root area, and the accumulation of radioactive $\mathrm{Cs}$ in the stem bark and inside. From the AR analysis result (Figure 11) on the branches and leaves of $M$. alba collected in Nihonmatsu-City in May 2012, a uniform distribution of radioactive Cs in the twig area was confirmed by the uniformly high blackness in the twig area. In the leaf area as well, the uniform distribution of radioactive Cs was indicated by the uniform black areas recognized similarly on twigs.

AR analysis of foliaceous lichen (P. tinctorum) collected in Iitate-Village in May 2011 (Figure 12) shows many black spots on the lichen that forms the yellow-green area in the optical photos, and black surface areas appear. On the other hand, with regard to the blackness of the bark areas appearing as brown in the optical photos, the blackness is thinner compared to
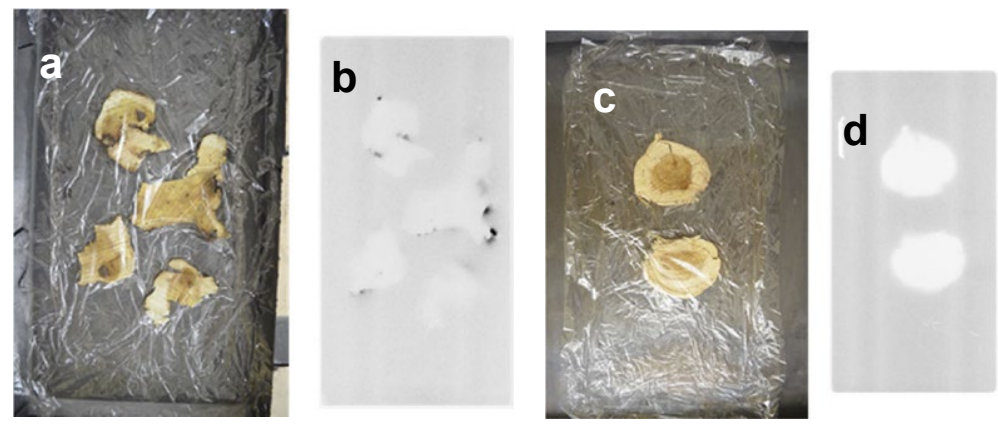

Figure 10 Autoradiograph images (b, d) and optical photographs (a, c) of cross sections of branches (a, b) and root (c, d), respectively, of M. alba collected in Nihonmatsu 23 months after the FDNPP accident
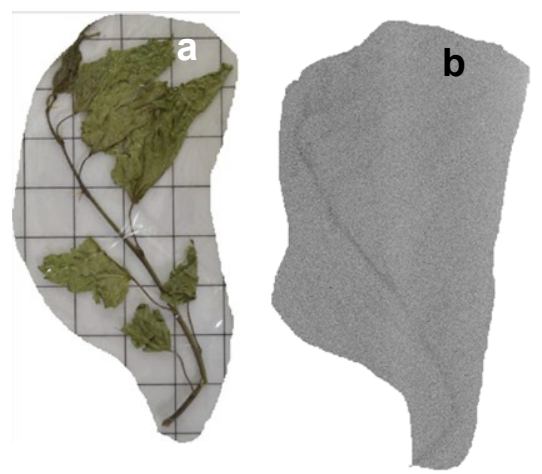

Figure 11 Autoradiograph image (b) and optical photograph (a) of branch and leaves of $M$. alba collected in Nihon-matsu 15 months after the FDNPP accident 
that in the foliaceous lichen areas. This indicates a higher concentration of radioactive Cs on foliaceous lichen than on the bark area.

$\mathrm{AR}$ analyses were conducted on the bamboo samples and bamboo shoot samples collected in Nihonmatsu-City in May 2012 using the round slice bamboo section samples (a, b), bamboo shoot section samples (c, d), the bamboo shoot vertical section samples (e, d), and bamboo shoot bark samples $(\mathrm{g}, \mathrm{h})$. The AR analysis showed (Figure 13) that the inside of the stem of bamboo section samples was uniformly black (Figure 13 (b)). The uniform blackness was also observed on the bamboo shoot section samples and the bamboo shoot vertical section samples, although the blackness was thinner than that of the bamboo section samples.

The AR images of bamboo shoot vertical section samples showed a uniform blackness of the plate and the internode of the bamboo shoot. This indicated that radioactive Cs had accumulated in the bamboo stems. As the AR image of the bamboo shoot bark sample shows, the bark area is blackened as well.

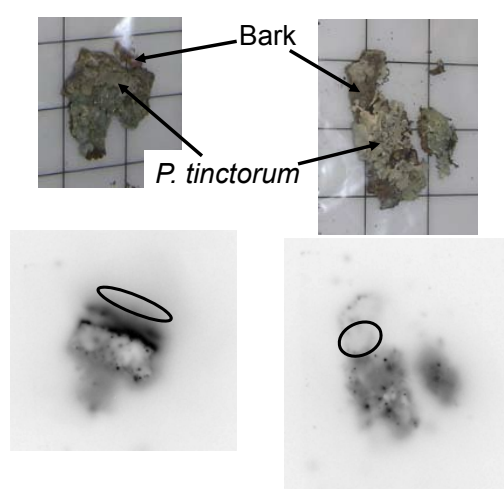

Figure 12 Autoradiograph image (lower) and optical photograph (upper) of lichen P. tinctorum collected in Iitate 2 months after the FDNPP accident

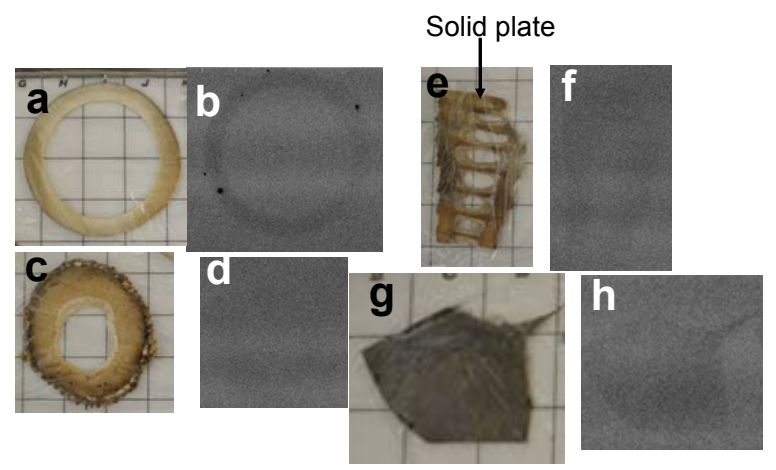

Figure 13 Autoradiograph images (b, d, f, h) and optical photographs (a, c, e, g) of cross section of internode $(\mathrm{a}, \mathrm{b})$ of $P$. bambusoides, vertical cross section of the sheath of a bamboo shoot $(\mathrm{e}, \mathrm{f})$, cross section of the internode of a sheath of a bamboo shoot, and the peel of bamboo shoot $(\mathrm{g}, \mathrm{h})$ 


\section{Discussion}

\section{Relocation of Radioactive Cs in a Tree}

The AR analyses conducted on the tree samples collected two months after the $1 \mathrm{~F}$ accident in March 2011 showed that the radioactive Cs deposited on trees was more concentrated on the stems and leaves that were present before the $1 \mathrm{~F}$ accident ${ }^{4}$. As explained above, in the $C$. japonica samples collected two months after the $1 \mathrm{~F}$ accident, no accumulation on the male flower was observed and a heterogeneous distribution of black spots of radioactive Cs was confirmed. The area of the spots in an AR image will vary not only according to the size of the radionuclide deposit, but also to its radioactivity and the AR exposure time. Hence, radioactive Cs was directly deposited on the trees like an aerosol, and deposited on the trees as spots.

In the AR analyses of the trees of $C$. japonica and T. dolabrata var. hondae collected six months after the $1 \mathrm{~F}$ accident, most of the radioactive Cs had a spotty distribution on trees (Figure 2). Furthermore, in the C. japonica sample collected in Okuma-Machi in December 2012, radioactive Cs was present on the female flower and branches and leaves that had grown before the $1 \mathrm{~F}$ accident (Figure 4). The samples in which the black spots were also found are C. japonica, P. persica, and T. dolabrata var. hondae sampled in Date-City in October 2011 (Figures 5, 8, and 9 respectively). Because the distance of the sampling locations from $1 \mathrm{~F}$ was $\sim 4 \mathrm{~km}$ (Okuma-Machi) and $\sim 55 \mathrm{~km}$ (Date-City), it was elucidated that radioactive Cs adhered as spots on the trees grown before the $1 \mathrm{~F}$ accident, not depending on the type of tree nor the distance from $1 \mathrm{~F}$. Because the same spotty adhesion of radioactive Cs was found in the $C$. japonica sampled six months after the $1 \mathrm{~F}$ accident, the distribution is considered to be a specific distribution of radioactive $\mathrm{Cs}$ that deposited directly on $C$. japonica, soon after the $1 \mathrm{~F}$ accident.

The distribution of radioactive $\mathrm{Cs}$ in the sample of $C$. japonica that was collected more than one year after the $1 \mathrm{~F}$ accident was completely different from that collected after six months. As shown in the AR analysis of $C$. japonica, the ratio of spots of radioactive Cs decreases in the branches and leaves that grew after the $1 \mathrm{~F}$ accident, and in the specific local distribution in the tips of branches and leaves or on male flowers (Figures 3 and 4). This distribution is different from the spotty distribution observed on the tree surface. Thus, it is considered that the distribution of radioactive Cs was not due to direct adherence on the tree surfaces. One of the possible pathways, such as by the adhesion of soil contaminated with radioactive $\mathrm{Cs}$, has been considered, but in this case the expected distribution is spots on the tips of branches and leaves. This hypothesis agrees with the scenario of a uniform aerial distribution like that observed in AR images of $C$. japonica. It is reasonable to conclude that the distribution of radioactive Cs was not on the stem surface but inside of $C$. japonica. Consequently, it is conceivable that the radioactive Cs deposited on the stem surface was absorbed into the inside of stem, and relocated to the tips of branches and leaves. This trend is seen not only in C. japonica, but also in $T$. dolabrata var. hondae (Figure 6).

On the other hand, the distribution in the branches and leaves of T. nucifera is different from that of C. japonica and T. dolabrata var. hondae. On the branches and leaves of T. nucifera, the radioactive Cs shows a spotty deposition on the branches and leaves that had grown before the $1 \mathrm{~F}$ accident, and a uniform distribution on the branches and leaves that had grown after the $1 \mathrm{~F}$ accident. Similarly, the uniform distribution in the branches and leaves that had grown after the $1 \mathrm{~F}$ accident is seen on M. alba. These results indicate two distribution patterns for the radioactive $\mathrm{Cs}$ in the branches and leaves that had grown after the $1 \mathrm{~F}$ accident, i.e., its concentration in the tip area in C. japonica, and its uniform distribution in T. nucifera and M. alba.

In the AR analyses of $M$. alba, the spotty adhesion of radioactive Cs on the bark as well as 
the distribution in the stem was observed on the M. alba stem that was collected approximately two years after the $1 \mathrm{~F}$ accident (Figure 10), while radioactive Cs was not present in the root area. These results indicate a low likelihood of absorption of radioactive Cs present in the stem via the roots. Kozai et al. studied the chemical species of radioactive Cs in the soil of Iitate-Village using the selective extraction method and clarified that more than $70 \%$ of radioactive Cs in the soil is in a chemical form that does not exchange cations ${ }^{9}$. This result points to problems for the idea of direct uptake of radioactive Cs from soil into the root shown in the AR analysis of a root of $M$. alba. Hasegawa et al. conducted the experiment of attaching ${ }^{137} \mathrm{Cs}$ to a leaf of radish and reported that a portion of ${ }^{137} \mathrm{Cs}$ is absorbed via the phylloplane ${ }^{10)}$. Tagami et al. measured the radioactive $\mathrm{Cs}$ on the leaves of evergreen trees and deciduous trees in Chiba-City and reported that several tens to several hundreds of $\mathrm{Bq} / \mathrm{kg}$ were detected ${ }^{11)}$. The results of our research indicated the relocation of radioactive $\mathrm{Cs}$ in $M$. alba from epidermis to the inside by translocation.

P. persica showed that radioactive Cs was distributed both evenly and in a spotty manner on the bark of trees that had grown before the $1 \mathrm{~F}$ accident (Figure 9). This result indicates that the radioactive Cs distributed as spots relocated into the bark surface during the six months after the $1 \mathrm{~F}$ accident. As almost no radioactive Cs was detected inside the stem of $P$. persica, it is considered that the radioactive Cs deposited on the bark remained in position. This implies that most of the deposited radioactive Cs stayed in the epidermis of $P$. persica trees. In contrast, as shown in Figure 8, almost no radioactive Cs was detected in the tree stems of those that had grown before or after the $1 \mathrm{~F}$ accident. This indicates that the radioactive Cs stayed in the bark of $P$. persica for at least six months after the $1 \mathrm{~F}$ accident.

The radioactive Cs was also distributed on the surface of foliaceous lichen (Figure 12). The blackness in the AR image of the foliaceous lichen is denser than that of the bark in Figure 12, indicating the accumulation of radioactive $\mathrm{Cs}$ on the inside of the lichen. Similarly to the above mentioned C. japonica, the even distribution also indicates the relocation of radioactive Cs to the inside of the lichen. Such accumulation of radioactive Cs in lichen in Austria was reported at a level 120 times higher than that before the accident of Chernobyl ${ }^{12)}$. The high accumulation of radioactive Cs in foliaceous lichen is not only due to the spot adhesion, but also the accumulation on the inside.

As the C. japonica sample shows accumulation of radioactive $\mathrm{Cs}$ in the male flower area, radioactive $\mathrm{Cs}$ is considered to have been accumulated in the region of active growth in a tree. Based on analyses conducted on pine trees during the twelve years after the accident at Chernobyl, it was pointed out that the radioactive Cs was concentrated in the tender shoot area with predominant growth, together with stable $\mathrm{Cs}^{13)}$. The results of our research on $C$. japonica and $T$. dolabrata var. hondae indicate that the radioactive $C s$ was accumulated in the active growth areas on the tips of twigs by translocation from the bark to the inside of the stem in the second year after the $1 \mathrm{~F}$ accident. It is considered that the concentration of radioactive Cs in the area of bamboo shoot nodes and sheathes in the AR images of the bamboo shoot vertical section sample was caused by the selective concentration of radioactive Cs in the areas of predominant growth. On the other hand, in T. nucifera and M. alba, the radioactive Cs showed spatial distribution. Furthermore, some $P$. persica trees showed stagnated radioactive $\mathrm{Cs}$ in the bark even a half year after the $1 \mathrm{~F}$ accident. Mori et al. refers to the accumulation on the inside of trees by translocation based on the significant increase in the radioactive Cs concentration in the tree sap of Rhus vernicifera ${ }^{14)}$.

Thus, the redistribution of the radioactive Cs deposited on the bark is not the same among different species of plants. In the future, it will be necessary to clarify the differences in the redistribution behavior in different plant species after the $1 \mathrm{~F}$ accident. 


\section{Chemical forms of Radioactive Cs in Trees Containing Decontamination Waste}

To evaluate the influence of radioactive Cs on the environment when the decontamination waste is stored or disposed of, leakage and migration of radioactive Cs from the decontamination waste must be predicted. As the migration behavior of radioactive Cs from decontamination waste depends on its chemical form, we should identify the chemical forms of radioactive Cs in decontamination waste.

Decontamination waste is considered to contain soil and vegetation. Some waste includes material whose decay and its prevention must be considered ${ }^{3)}$. In the soils contained in the decontamination waste, multiple chemical forms of radioactive $\mathrm{Cs}$ were found to be present ${ }^{9,15)}$. However, almost no analysis has been conducted on plants. Our results indicate a time-dependent change of the chemical form of radioactive Cs spread by the $1 \mathrm{~F}$ accident. That is, radioactive Cs exists in spots on the surface of branches and leaves that grew before the $1 \mathrm{~F}$ accident, but radioactive Cs was found to be present inside of the stem of branches and leaves that grew during the year following the $1 \mathrm{~F}$ accident.

To study the solubility of the deposited radioactive Cs on the bark surface, a desorption experiment was conducted using $1 \mathrm{M} \mathrm{CH}_{3} \mathrm{COOH}$ solution to accelerate its eluviation by rainwater on the branches and leaves that grew before the $1 \mathrm{~F}$ accident. The results of the desorption experiment on two T. nucifera samples are shown in Table 2. The radioactive Cs content of Samples 1 and 2 differ by 1 digit, but almost no radioactive $\mathrm{Cs}$ was desorbed with the $1 \mathrm{M} \mathrm{CH}_{3} \mathrm{COOH}$ solution. This result indicates that the leach-out due to the desorption of radioactive Cs from the tree by the infiltration of rainwater is extremely low. The AR analysis of the T. nucifera sample processed with the $1 \mathrm{M} \mathrm{CH}_{3} \mathrm{COOH}$ solution showed unchanged spotty distribution of radioactive Cs. Relocation of radioactive Cs deposited on the plant surface to the inside of the plant and to the soil layer by absorption in foliage or weathering has been reported ${ }^{10)}$. Kawabata et al. reported that more than $80 \%$ of solid aerosol deposition of Cs on the Raphanus sativus var. sativus was relocated to the outside of the plant ${ }^{16}$. These results, corroborated by our results, imply that the eluviation of radioactive Cs by weathering is extremely low for any trees contained in the decontamination wastes.

On the other hand, because the radioactive Cs contained in the branches and leaves that grew after the $1 \mathrm{~F}$ accident is present inside the tree, it is considered more difficult to desorb than the radioactive Cs that exists in the bark. As it is implied that the Co captured inside of a microbial cell is more difficult to be removed than Co adsorbed on cell surface ${ }^{17)}$, the desorption of radioactive $\mathrm{Cs}$ accumulated inside the tree is considered to be less than the radioactive Cs on the bark surface. Other living organisms such as algae or lichens inhabit plants as well, and lichen is known to condense radioactive $\mathrm{Cs}^{18)}$. AR analysis of lichens indicated the presence of radioactive $\mathrm{Cs}$ in the form of spots on the surface, and also accumulated within the plant. Radioactive Cs in such chemical forms may exhibit different desorption behavior from the radioactive Cs distributed in the bark or inside the tree. Furthermore, as there are various microorganisms living in decontamination waste, the radioactive Cs may be desorbed through cracking by the microorganisms in the tree. It is considered that the time required for cracking is different for

Table 2 Radioactive Cs desorbed from an old branch of $T$. nucifera by a $1 \mathrm{M} \mathrm{CH}_{3} \mathrm{COOH}$ solution

\begin{tabular}{cccc}
\hline \multirow{2}{*}{ T. nucifera Sample } & \multicolumn{3}{c}{ Radioactivity $\left(\mathrm{Bq} \cdot \mathrm{g}^{-1}\right)$} \\
\cline { 2 - 4 } & Before Treatment & After Treatment & In $1 \mathrm{M} \mathrm{CH}_{3} \mathrm{COOH}$ solution \\
\hline 1 & $6.6 \times 10^{2}$ & $6.6 \times 10^{2}$ & $1.0 \times 10^{-2}$ \\
\hline 2 & $1.8 \times 10^{3}$ & $1.8 \times 10^{3}$ & $2.2 \times 10^{-1}$ \\
\hline
\end{tabular}


the bark and inside the tree. Furthermore, it is reported that the microorganisms themselves preserve the radioactive $\mathrm{Cs}^{19,20)}$. Consequently, for the evaluation of the leaching behavior of radioactive $\mathrm{Cs}$ from any tree, it will be necessary to identify the type of tree, time of sampling, and presence of organisms, such as lichen, on the tree surface.

\section{Summary}

Using autoradiography, the local area distribution of radioactive Cs was measured in trees sampled in different locations and at different time periods since the $1 \mathrm{~F}$ accident, and the behavior of radioactive Cs contained in the radioactive fallout in the tree was studied. As a result, the following has been clarified:

- The distribution in the tree was mainly in the form of spots on the branches and leaves that grew before the $1 \mathrm{~F}$ accident, and this form persisted for approximately a year after the $1 \mathrm{~F}$ accident.

- The spotty distribution of radioactive Cs has been clarified as a typical form of distribution of radioactive Cs fallout, and is not dependent on the type of tree nor the distance from $1 \mathrm{~F}$.

- One year after the $1 \mathrm{~F}$ accident, the presence of radioactive Cs contained inside the trees was observed, instead of just the spotty radioactive Cs on the branches and leaves. Three types of distribution were observed in contaminated trees: (1) accumulation in the tip area of male flowers, branches, and leaves such as C. japonica or T. dolabrata var. hondae, (2) uniform distribution in the tree such as T. nucifera or M. alba, and (3) preservation in the bark of $P$. persica.

These results indicate that the radioactive Cs that fell out on the trees varied its chemical forms with time. Consequently, it is necessary to study the contaminated types in the trees, time of sampling, and the presence of living organisms, such as lichens on the tree surface.

We express our gratitude to Dr. Hirofumi Tsukada of the Institute for Environmental Sciences for the valuable advice we received from him while we worked on completing this research paper. Part of this research was conducted with the aid of Grants-in-Aid for Scientific Research by MEXT, subject No. 25289355.

\section{References}

1) M. Chino, H. Nakayama, H. Nagai, H. Terada, G. Katata, H. Yamazawa, "Preliminary estimation of release amounts of 131I and 137Cs accidentally discharged from the Fukushima Daiichi Nuclear Power Plant into the atmosphere," J. Nucl. Sci. Technol., 48, 1129-1134 (2011).

2) A. Omoto, ICAPP2011 Special Japan Session: Presentation (2-6 May, 2011 Nice, France). file is available at, http://www.aesj.or.jp/information/fnpp201103/icapp2011_Omoto_WEBrevision20110517.pdf

3) Ministry of the Environment, Off-site Decontamination Measures, available at, http://josen.env.go.jp/en/.

4) F. Sakamoto, T. Ohnuki, N. Kozai, S. Igarashi, S. Yamasaki, Z. Yoshida, S. Tanaka, "Local area distribution of fallout radioanuclides from Fukushima Daiichi Nuclear Power Plant determined by autoradiography analysis," Trans. At. Energy Soc. Jpn., 11, 1-7 (2012). [In Japanese]

5) K. Tanaka, H. Iwatani, A. Sakaguchi, Y. Takahashi, Y. Onda, "Local distribution of radioactivity in tree leaves contaminated by fallout of the radionuclides emitted from the Fukushima Daiichi Nuclear Power Plant," J. Radioanal. Nucl. Chem., 295, 2007-2014 (2013).

6) S. Yoshida, Y. Muramatsu, A. M. Dvornik, T. A. Zhuchenko, I. Linkv, "Equilibrium of radiocesium with stable cesium within the biological cycle of contaminated forest ecosystems," J. Environ. Radioact., 75 301-313 (2004).

7) N. Yamagata, S. Matsuda, M. Chiba, "Radioecology of cesium-137 and strontium-90 in a forest," $J$. Radiat. Res., 10, 107-112 (1969). 
8) F. Sakamoto, T. Ohnuki, T. Fujii, H. Iefuji, "Response of Saccharomyces cerevisiae to heavy element stress: lead vs. uranium," Geomicrobiol. J., 27, 240-244 (2010).

9) N. Kozai, T. Ohnuki, M. Arisaka, M. Watanabe, F. Sakamoto, S. Yamasaki, M. Jiang, "Chemical states of fallout radioactive Cs in the soils deposited at Fukushima Daiichi Nuclear Power Plant accident," $J$. Nucl. Sci. Technol., 49, 473-478 (2012), (Doi: 10.1080/00223131.2012.677131).

10) H. Hasegawa, H. Tsukada, H. Kawabata, Y. Chikuchi, Y. Takaku, S. Hisamatsu, "Effect of the counter anion of cesium on foliar uptake and translocation," J. Enviorn. Radioact., 100, 54-57 (2009).

11) K. Tagami, S. Uchida, N. Ishii, S. Kagiya, "Translocation of radiocesium from stems and leaves of plants and the effect on radiocesium concentrations in newly emerged plant tissues," J. Enviorn. Radioact., 111, 65-69 (2012).

12) G. Heinrich, K. Oswald, H. J. Muller, "Lichens as monitors of radiocesium and radiostrontium in Australia," J. Enviorn. Radioact., 45, 13-27 (1999).

13) S. Yoshida, M. Watanabe, A. Suzuki, "Distribution of radiocesium and stable elements within a pine tree," Radiat. Prot. Dosim., 146, 1-3, 326-329 (2011).

14) S. Mori, A. Hirato, K. Tanoi, K. Takeda, T. Yamakawa, H. Nakanishi, "Radioactive cesium flow in Rhus vernicifera," Soil Sci. Plant Nutri., 58, 611-617 (2012).

15) K. Tanaka, Y. Takahashi, A. Sakaguchi et al., "Vertical Pro-files of Iodine-131 and Cesium-137 in Soils in Fukushima Prefecture related to the Fukushima Daiichi Nuclear Power Station Accident," Geochem. $J ., 46,73-76$ (2012).

16) K. Kawabata, H. Hasegawa, H. Tsukada, Y. Takaku, S. Hisamatsu, "Behavior of Trace Elements on Leaf Surfaces of Crop Plants," Annu. Rep. Inst. Environ. Sci., 20-24 (2010). [in Japanese]

17) N. Kozai, T. Ohnuki, F. Sakamoto, Y. Suzuki, K. Tanaka, H. Iefuji, T. Sakai, "Accumulation of Co in yeast cells under metabolically active condition - Implication for role of yeast in migration of radioactive Co-," J. Nucl. Sci. Technol., 48, 1206-1213 (2011).

18) G. Heinrich, K. Oswald, H. J. Muller, "Lichens as monitors of radiocesium and radiostrontium in Australia," J. Enviorn. Radioact., 45, 13-27 (1999).

19) R. A. Olsen, E. Joner, L. R. Bakken, Soil Fungi and the Fate of Radiocaesium in the Soil Ecosystemda Discussion of Possible Mechanisms Involved in the Radiocaesium Accumulation in Fungi and the Role of Fungi as a Cs-sink in the Soil. In: Desmet, G., Nassimbeni, P., Belli, M. (Eds.), Transfer of Radionuclides in Natural and Semi-natural Environments. Elsevier Applied Science, London, New York, pp. 657-663 (1990).

20) B. H. Fawaris, K. J. Johanson, "Fractionation of ${ }^{137} \mathrm{Cs}$ in coniferous forest soil in central Sweden," Sci. Total Environ., 170, 221-228 (1995). 


\title{
Article
}

\section{Thermodynamic Evaluation of Sea Water to Degraded Nuclear Fuel in Severe Accident of LWR}

\author{
Masaki KURATA $^{1, *}$, Noriko SHIRASU ${ }^{1}$ and Toru OGAWA ${ }^{2}$ \\ ${ }^{1}$ Japan Atomic Energy Agency, Nuclear Science and Engineering Directorate, 2-4 Shirane, Shirakata, Tokai-mura, Naka-gun, \\ Ibaraki 319-1195, Japan \\ ${ }^{2}$ Nagaoka University of Technology, 1603-1 Kamitomioka-cho, Nagaoka-shi, Niigata 940-2188, Japan
}

\begin{abstract}
In the Fukushima Daiichi Nuclear Power Plant Accident, a large amount of seawater was introduced into reactor pressure vessels. Sodium and chloride ions, as well as several minor elements contained in seawater, such as $\mathrm{K}, \mathrm{Ca}, \mathrm{Mg}$, and $\mathrm{S}$, possibly reacted with degraded fuel debris or molten corium. Considering possible chemical reactions, various concerns such as volatilization of fission products, characterization of fuel debris, and formation of corrosive gases are discussed. Thermodynamic evaluation can give useful information on the general tendency of these seawater effects. The volatility of Cs, Sr, and Te potentially increases because of the change in stable chemical species. Corrosive gases, such as $\mathrm{HCl}$ and $\mathrm{H}_{2} \mathrm{~S}$, may be generated by the heating of seawater to high temperatures. These phenomena are predicted to vary according to the oxygen potential.
\end{abstract}

KEYWORDS: thermodynamic evaluation, severe nuclear accident, fission product, volatilization, source term, seawater, fuel degradation, corium, fuel debris

\section{Introduction}

In the severe accident at the Fukushima Daiichi Nuclear Power Plant (FDNPP), seawater was used as the water injection source after the loss of cooling ability. Furthermore, the Nuclear and Industrial Safety Agency (NISA) selected seawater as the final water source when a severe accident occurs. However, the influence of seawater injected into the damaged reactor core in a severe accident has rarely been studied. In addition to the main solutes in seawater, sodium and chloride ions, a considerable quantity of other elements are present, including potassium, calcium, magnesium, and sulfur (sulfate group). These elements readily form compounds with various other elements, which are concentrated when the moisture evaporates at high temperatures and may cause various chemical reactions with damaged fuel. Takano et al. investigated high temperature reaction tests on seawater condensates and uranium dioxide. The results indicated that calcium and magnesium are likely to react with fuel debris, although no obvious chemical reaction takes place between sodium chloride and uranium dioxide, which

* Corresponding author, E-mail: kurata.masaki@jaea.go.jp

DOI : 10.15669 /fukushimainsights.Vol.4.219

(C) 2021 Atomic Energy Society of Japan. All rights reserved.

Originally published in Transactions of the Atomic Energy Society of Japan (ISSN 1347-2879), Vol. 12, No. 4, p.286-294

(2013) in Japanese. (Japanese version accepted: July 25, 2013) 
was a point of concern in the beginning ${ }^{1)}$. On the other hand, the evaporation of fission products (FP) changes because of the variation in stability of chemical species due to their interaction with seawater condensates. Furthermore, various gases may be generated from the exposure of seawater to high temperatures, and some of them may be transformed to corrosive gases.

These chemical reactions are expected to drastically change according to the oxygen potential of the system. In a severe accident, it is considered that one of the major factors that control the oxygen potential of degraded fuel is the oxidation reaction of zircalloy ${ }^{2}$. In the early stages of an accident, the oxygen potential in the degraded fuel, excluding the surface that lies in direct contact with cooling water or steam, is presumably maintained at a low value because a large quantity of metallic zirconium remains. As the accident progresses, the oxygen potential is considered to rapidly increase when almost all the zirconium is oxidized. This implies that the influence of seawater on FP evaporation, the chemical status of fuel debris, and the generation of corrosive gases will vary depending on which stage in the severe accident the seawater is supplied. Furthermore, it also implies that the influence of seawater depends on the components of the structural substances in the reactor, which differ according to reactor type (PWR or BWR). For a BWR that contains a large quantity of metallic zirconium in the reactor, a status of low oxygen potential is likely to be maintained for an extended period, compared to a PWR. Furthermore, for a BWR, the high percentage of structural materials, such as stainless steel and inconel alloy, has to be carefully considered in advance.

Because boric acid is contained in the coolant of a PWR, the focus is on $\mathrm{CsBO}_{2}$, as a form of cesium that may be significant in a severe accident ${ }^{3}$. A large amount of $\mathrm{B}_{4} \mathrm{C}$ is used in a BWR as control blade material, and the boric acid or carbon may cause complicated chemical reactions with FP. Regarding this point, Shirasu et al. conducted an analysis using a similar method as in this report ${ }^{4}$.

It requires great effort to deal with these phenomena of concern that are likely occur with exposure to introduced seawater. Acquiring experimental data with repeatability is expected to be difficult because the events in an accident form a complex system with many components. In such a case, a chemical equilibrium computation based on a thermodynamic approach is actively applied as an evaluation method that approximates the chemical events. In this study, the evaluation of various concerns that are presumed to be brought about by the introduction of seawater was attempted.

\section{Analysis Method}

\section{Thermodynamic Database and Equilibrium Computation Tool}

For this study, the European SGTE (Scientific Group Thermodynamics Europe) database SSUB4 was adopted ${ }^{5)}$. As an equilibrium computation tool, chemical equilibrium computation software Thermocalc ver.S by European Thermo-Calc Software ${ }^{6}$ was used.

For proper understanding of the information acquired from the chemical equilibrium computations, the following issues are important. First, a preliminary survey of the contents of the database needs to be conducted in detail. In a severe accident, it is known that organic iodine compounds may be generated ${ }^{7}$. However, because there is almost no existing thermodynamic database, including SSUB4, that integrates the data of organic iodine compounds, the evaluation of the behavior of iodine is incomplete. The database provides only information about the thermal decomposition of cesium iodide (CsI), which has a high probability of formation. Furthermore, where mutual solubility data between compounds is necessary, for instance, in the 
calculation of the chemical state of the matrix phase of fuel debris, existing databases are not suitable for use because they rarely include any data regarding the mutual solubility of nuclear fuel or zircalloy. In this area, evaluations by Gueneau et al ${ }^{8)}$. and by Shirasu et al ${ }^{9}$. are in progress. Second, it is essential to conduct a parallel computation under various assumptions in addition to the simulated computation of actual reactor conditions. The chemical status varies from hour to hour in a severe accident. Based on various assumptions, it is possible to survey the trend of the chemical reactions by performing a so-called "mapping" of thermodynamic states. In this research, a model computation is conducted hypothetically, assuming such conditions as the progress of oxidation not only on zircalloy but also on the steel structural materials, and the reactions between the fuel rods and seawater.

\section{Analysis Conditions}

The quantity of different materials used in the calculation was determined in the following manner. The estimated values for the fuel, cladding, and structural materials for Unit 2 were taken from the FDNPS ${ }^{10}$. Regarding seawater, the values determined by Nozaki were adopt$\mathrm{ed}^{11)}$. The concentrations of the FP were calculated using the ORIGEN-II code ${ }^{12)}$, by referring to the averaged burn-up of Unit 2, summarized in the first step, and then considering the amount of volatile FP, such as cesium (Cs), strontium ( $\mathrm{Sr}$ ), iodine (I), tellurium (Te), molybdenum $(\mathrm{Mo})$, ruthenium $(\mathrm{Ru})$, and niobium $(\mathrm{Nb})$, in the second step. These elements are presumed to interact significantly with seawater. Values were standardized with respect to 1 mole of $\mathrm{UO}_{2}$ and were used as a standard condition in the present study. These are summarized in Table 1 and Table 2. Here, the quantity of actinide elements in the fuel was represented by $\mathrm{UO}_{2}$, the quantity of claddings and channel boxes was represented by $\mathrm{Zr}$, and the quantity of structural materials was represented by Fe. The oxygen potential in the fuel before the severe accident was assumed to be represented by a chemical condition in which some of the Mo is oxidized. Furthermore, the pressure was assumed to be $1 \mathrm{~atm}$. Current thermodynamic data stored in the database do not have proper description regarding influence of pressure. Present evaluation was performed by assuming $1 \mathrm{~atm}$.

\section{Analysis Method}

To evaluate the chemical status of various elements in the degraded fuels, it is necessary to evaluate the oxygen potential of the system in an analysis. In this study, the evaluation was

Table 1 Initial conditions for degraded fuel

\begin{tabular}{cc}
\hline Component & $\begin{array}{c}\text { Mole amount } \\
\text { (normalized with respect to } 1 \mathrm{~mol}-\mathrm{UO}_{2} \text { ) }\end{array}$ \\
\hline Fuel $\left(\mathrm{UO}_{2}\right)$ & $1.00 \mathrm{E}+00$ \\
\hline Zry $(\mathrm{Zr})$ & $1.28 \mathrm{E}+00$ \\
S.S. $(\mathrm{Fe})$ & $8.27 \mathrm{E}-01$ \\
\hline $\mathrm{Sr}$ & $1.82 \mathrm{E}-03$ \\
$\mathrm{Nb}$ & $4.05 \mathrm{E}-05$ \\
$\mathrm{Mo}$ & $5.89 \mathrm{E}-03$ \\
$\mathrm{Ru}$ & $3.67 \mathrm{E}-03$ \\
$\mathrm{Te}$ & $6.13 \mathrm{E}-04$ \\
$\mathrm{I}$ & $2.82 \mathrm{E}-04$ \\
$\mathrm{Cs}$ & $3.63 \mathrm{E}-03$ \\
\hline
\end{tabular}


Table 2 Chemical composition of sea-water

\begin{tabular}{cc}
\hline Component & Concentration $(\mathrm{mg} / \mathrm{L})$ \\
\hline $\mathrm{Na}$ & $1.08 \mathrm{E}+04$ \\
$\mathrm{Mg}$ & $1.28 \mathrm{E}+03$ \\
$\mathrm{~S}$ & $8.98 \mathrm{E}+02$ \\
$\mathrm{~K}$ & $3.99 \mathrm{E}+02$ \\
$\mathrm{Ca}$ & $4.12 \mathrm{E}+02$ \\
$\mathrm{Cl}$ & $1.94 \mathrm{E}+04$ \\
\hline
\end{tabular}

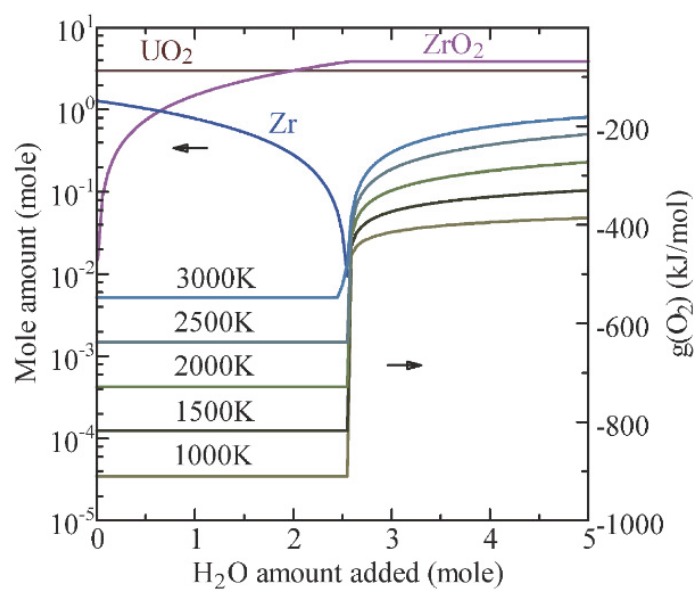

Figure 1 Variation in oxygen potential, $\mathrm{g}\left(\mathrm{O}_{2}\right)$, and progress in $\mathrm{Zr}$ oxidation with respect to added water amount

implemented as follows.

First, the amount of $\mathrm{H}_{2} \mathrm{O}$ added to 1 mole of $\mathrm{UO}_{2}$ and 1.282 mole of $\mathrm{Zr}$ was varied, and the changes in the chemical forms and oxygen potential $\left(\mathrm{g}\left(\mathrm{O}_{2}\right)\right)$ were evaluated.

Figure 1 shows the variation in $\mathrm{g}\left(\mathrm{O}_{2}\right)$ at several temperatures, and the degree of oxidation of $\mathrm{Zr}$ as a function of the amount of added $\mathrm{H}_{2} \mathrm{O}$. The conditions in the leftmost of the figure correspond to no oxidation of $\mathrm{Zr}$ (i.e., the condition before operation of nuclear reactor). In the early phases of a severe accident, the fuel temperature increased due to the decay heat. When the temperature reached approximately $1,473 \mathrm{~K}$, the rapid oxidation of $\mathrm{Zr}$ began, leading to the collapse of fuel ${ }^{2)}$. In a thermodynamic evaluation, this phenomenon is shown as a variation in the molar ratio between $\mathrm{Zr}$ and $\mathrm{ZrO}_{2}$, when the molar quantity of steam that effectively reacts with $\mathrm{Zr}$ is indicated on the horizontal axis (as given in the left half of the figure). In this zone, $\mathrm{g}\left(\mathrm{O}_{2}\right)$ is maintained at a constant value for each temperature because the oxidation of $\mathrm{Zr}$ specifies the $\mathrm{g}\left(\mathrm{O}_{2}\right)$ of the system. The value of $\mathrm{g}\left(\mathrm{O}_{2}\right)$ increases with temperature. In this study, case-I (added $\mathrm{H}_{2} \mathrm{O}$ amount: 1.5 mole) was selected as a typical condition in the early phases of a severe accident. This is equivalent to the chemical condition in which approximately $50 \%$ of $\mathrm{Zr}$ is oxidized. Here, the gram atomic weight of the chemical compound is treated as the sum of the atom weight that compose it. For example, 1 mole of $\mathrm{ZrO}_{2}$ is plotted as 3 moles, the sum of 1 atom of $\mathrm{Zr}$ and 2 atoms of $\mathrm{O}$, in the figure. In an actual severe accident, a higher value of $\mathrm{g}\left(\mathrm{O}_{2}\right)$ on the periphery of claddings is expected due to the interaction with coolant (steam), but this effect is not considered in the present analysis. Furthermore, in real fuel rods, the change in $\mathrm{O} / \mathrm{M}$ of $\mathrm{UO}_{2}$ with respect to the oxygen partial pressure is not negligible when evaluating the chemical properties of degraded fuel. However, in this study, the latter was not considered for conducting a thermodynamic evaluation of the chemical status of FP or the seawater 


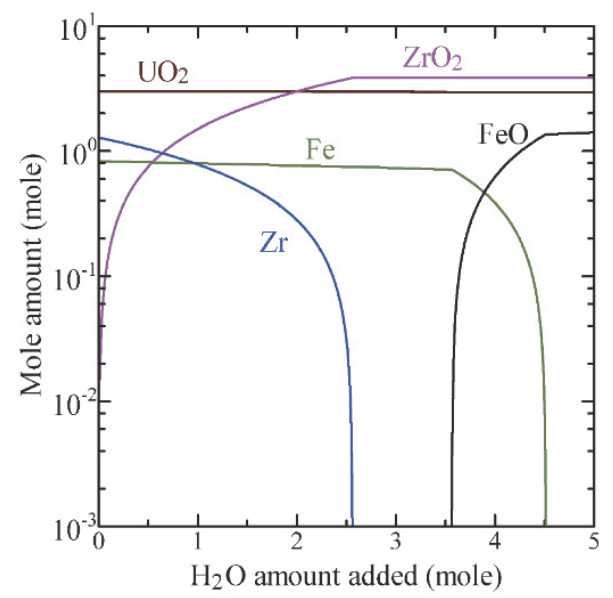

Figure 2 Variation in mole-amount of $\mathrm{U}, \mathrm{Zr}$, and Fe species with respect to added water amount (at 2,500 K)

components because the Gibbs free energy for the dissolution of the elements in $\mathrm{UO}_{2}$ is far smaller than that for the formation of compounds between FP and the seawater components. The change in $\mathrm{O} / \mathrm{M}$ scarcely influences these changes in chemical conditions, as $\mathrm{UO}_{2}$ was treated with a fixed $\mathrm{O} / \mathrm{M}=2.0$.

In the late phases of a severe accident, $\mathrm{g}\left(\mathrm{O}_{2}\right)$ rapidly increases due to the oxidation of almost all the $\mathrm{Zr}$ and is regulated by the partial pressure of $\mathrm{H}_{2} / \mathrm{H}_{2} \mathrm{O}$ (as shown in the right half of Figure 1). In this zone, the oxidation of $\mathrm{Fe}$ that was originally part of the structure begins when $\mathrm{g}\left(\mathrm{O}_{2}\right)$ exceeds a certain value. The relation of the added amount of $\mathrm{H}_{2} \mathrm{O}(0.827$ mole of $\mathrm{Fe}, 1$ mole of $\mathrm{UO}_{2}$, and 1.282 mole of $\mathrm{Zr}$ ), and the oxidation status of $\mathrm{U}, \mathrm{Zr}$, and $\mathrm{Fe}$ is shown in Figure 2 (at a temperature of 2,500 K). In an oxidative atmosphere, the oxidation of Fe occurs by the addition of a certain amount of $\mathrm{H}_{2} \mathrm{O}$. Based on these observations, case-II (added $\mathrm{H}_{2} \mathrm{O}$ amount: 3.7 mole) was selected as a typical condition for the partial oxidation of structural materials in the late phases of a severe accident. In an actual severe accident, a portion of the structural materials might be locally oxidized in the early phases as well, but that is not considered here. Furthermore, when an absorber material such as $\mathrm{B}_{4} \mathrm{C}$ contributes to the chemical reactions, the change in oxygen potential will further differ, but the analysis was conducted elsewhere ${ }^{9)}$.

In the present analyses, assuming that the fuel containing FP and zircalloy reacted with pure water, as in case-I and case-II, respectively, the variation in composition of the stable solid phases and those in the gaseous phase as functions of temperature and oxygen potential were evaluated in the first step, and the reaction with seawater was discussed in the second step. Then, the difference in the trend was discussed. Furthermore, the contribution of the structural materials was discussed as well as the contribution of the oxidative atmosphere.

\section{Results}

\section{Chemical Reaction of $\mathrm{UO}_{2} / \mathrm{FP} / \mathrm{Zr}$ Mixture and Pure Water}

Figure 3 shows the relationship between the added water amount and the gram atomweight quantity of solid phases, and the relation between the added water amount and the mole fraction of gaseous phases, evaluated at 1,500 K and 2,500 K, respectively. A significant variation in the chemical species between oxidative and reductive atmospheres is seen in both the gaseous 

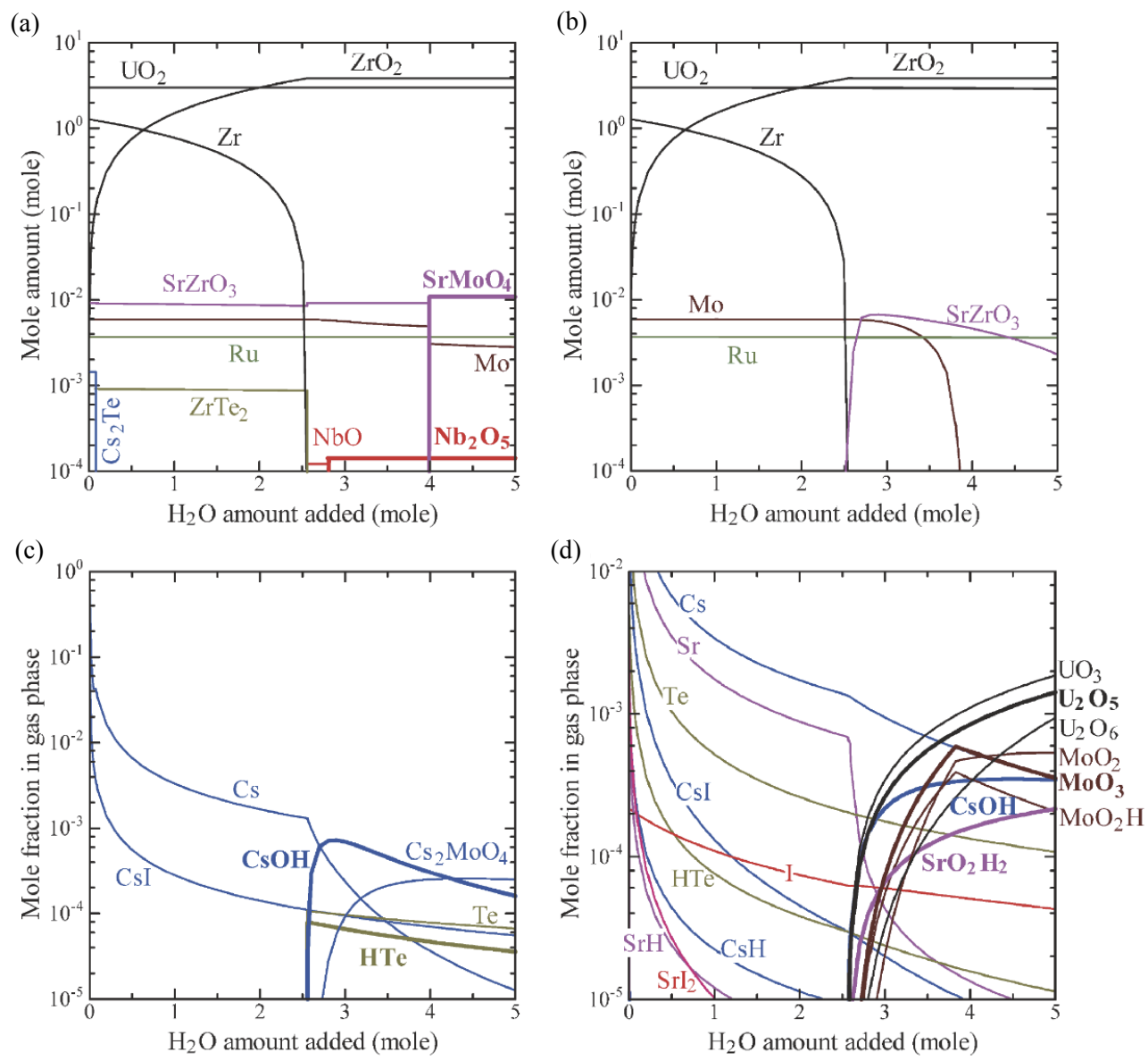

Figure 3 Variation in fission product species with respect to water amount added

(a) Mole amount of solid species (1,500 K), (b) Mole amount of solid species (2,500 K), (c) Mole fraction of gas species $(1,500 \mathrm{~K})$, and (d) Mole-fraction of gas species $(2,500 \mathrm{~K})$

and solid phases. In the solid phase, $\mathrm{ZrTe}_{2}$ and $\mathrm{Cs}_{2} \mathrm{Te}$ are stable in a reductive atmosphere at $1,500 \mathrm{~K}$. In an oxidative atmosphere, $\mathrm{Nb}$ oxides are stable. $\mathrm{SrZrO}_{3}$ is stable as well and is evaluated as transforming to $\mathrm{SrMoO}_{4}$ as $\mathrm{g}\left(\mathrm{O}_{2}\right)$ increases. Mo is generally stable as metallic Mo, but when a certain $\mathrm{g}\left(\mathrm{O}_{2}\right)$ is exceeded, it will form complex oxides with Sr. The metal solid phase of $\mathrm{Ru}$ is stable in this $\mathrm{g}\left(\mathrm{O}_{2}\right)$ range, and volatile high-order oxides are rarely formed. When the temperature rises to $2,500 \mathrm{~K}$, no $\mathrm{Sr}$ oxides are formed in a reductive atmosphere.

Furthermore, metallic Mo disappears in an oxidative atmosphere. This is attributable to the evaporation of $\mathrm{Sr}$ in a reductive atmosphere and Mo oxides in an oxidative atmosphere.

Regarding the gaseous phase, the elements that show significant evaporation in a reductive atmosphere at $1,500 \mathrm{~K}$ are $\mathrm{Cs}$ and $\mathrm{I}$, and it is shown that all the I evaporates as CsI and a part of Cs evaporates as single atoms of Cs. In an oxidative atmosphere, the evaporation of Mo and Te is observed in addition to $\mathrm{Cs}$ and $\mathrm{I}$, and it is shown that $\mathrm{CsOH}$ and $\mathrm{Cs}_{2} \mathrm{MoO}_{4}$ are added to the evaporating chemical forms of Cs. Te evaporates as single Te and in HTe. Because there are many evaporating species at 2,500 K, the scale factor of the y-axis is changed (Figure 3 (d)). In a reductive atmosphere, $\mathrm{Sr}$ and $\mathrm{Te}$ evaporate in addition to $\mathrm{Cs}$ and $\mathrm{I}$. As for $\mathrm{Sr}$, evaporation of single $\mathrm{Sr}$ is dominant. Te evaporates as single Te or as HTe. At this temperature, it is evaluated 
(a)

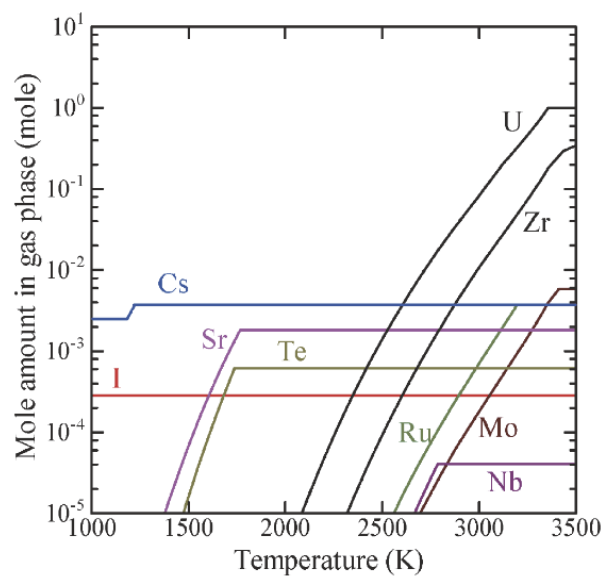

(b)

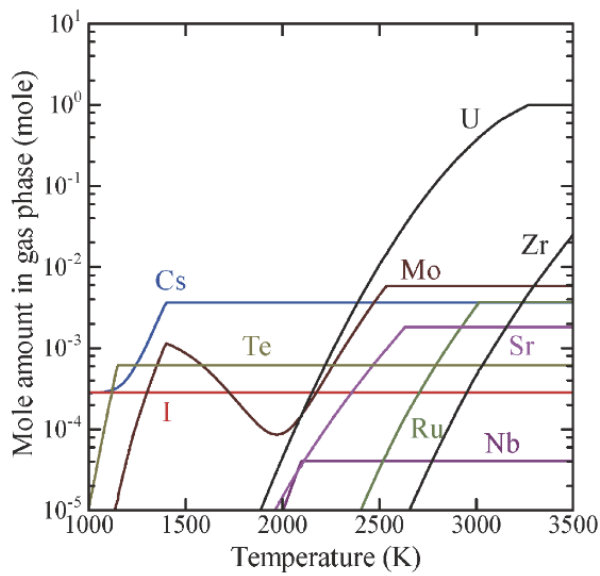

Figure 4 Mole amount in gas phase as a function of temperature

(a) reductive atmosphere (case-I) and (b) oxidative atmosphere (case-II)

that a portion of the gaseous phase of CsI decomposes, and single I and $\mathrm{SrI}_{2}$ evaporate as well. Meanwhile, in an oxidative atmosphere, high-order oxides of elements such as U and Mo evaporate. The percentage of single $\mathrm{Cs}$ decreases, and $\mathrm{CsOH}$ becomes the major component of $\mathrm{Cs}$, and as high-order oxides of Mo become stable, the complex oxides of the Cs-Mo system are not formed. $\mathrm{Sr}(\mathrm{OH})_{2}$ becomes the major form of $\mathrm{Sr}$.

The evaluation results of the molar quantity of gaseous phases for various elements in case-I and case-II are shown in Figure 4. By observation, including Figure 3, the general trend of evaporation is understood for both reductive and oxidative atmospheres for various elements:

$\mathrm{U}$ : Slightly evaporates in an oxidative atmosphere because of the formation of vapor species of high-order oxides.

$\mathrm{Zr}$ : Slightly evaporates in an oxidative atmosphere because of the slightly higher vapor pressure of metals compared with that of oxides.

$\mathrm{Sr}$ : Readily evaporates in a reductive atmosphere because of the easily decomposing nature of $\mathrm{SrZrO}_{3}$ in a reductive atmosphere.

$\mathrm{Nb}$ : Readily evaporates in an oxidative atmosphere because of the formation of vapor species of high-order oxides.

Mo: Readily evaporates in an oxidative atmosphere because of the formation of vaporous high-order oxides.

$\mathrm{Ru}$ : Slightly evaporates in an oxidative atmosphere because of the slightly higher vapor pressure of oxides compared with that of metals.

Te : Readily evaporates in an oxidative atmosphere because of the formation of the comparatively stable solid phase $\mathrm{ZrTe}_{2}$ on the reductive side.

I : Readily evaporates under this condition.

Cs : Readily evaporates but slightly inhibited because of the formation of a $\mathrm{Cs}_{2} \mathrm{MoO}_{4}$ type compound in a lower temperature zone of an oxidative atmosphere.

\section{Chemical Reaction of $\mathrm{UO}_{2} / \mathrm{FP} / \mathrm{Zr}$ Mix and Seawater}

It is highly unlikely that seawater reacts directly with the damaged fuel as it contains the original concentrations. Hence, a condition is assumed in which the $\mathrm{H}_{2} \mathrm{O}$ molar quantity is 1.0 , 
and an analysis was conducted by varying the $\mathrm{H}_{2} \mathrm{O}$ quantity by a factor of 5 , so the $\mathrm{H}_{2} \mathrm{O}$ molar quantity is 5.0. Figure 5 shows the relation between added seawater amount and the molar quantity of solid formed at 1,500 K and 2,500 K. By comparison with Figure 3, the expected influence of the reaction with seawater, when it occurs, is evaluated.

(Temperature: $1,500 \mathrm{~K}$ )

$\mathrm{Sr}$ : The chlorides and sulfates are formed in a reductive atmosphere. No change occurs in an oxidative atmosphere.

$\mathrm{Nb}$ : The oxide species are changed to higher-order species in an oxidative atmosphere.

Mo : Complex oxides of $\mathrm{Na}$ and $\mathrm{Ca}$ are formed in an oxidative atmosphere. No change occurs in a reductive atmosphere.

$\mathrm{Ru}$ : No change.

Te : CaTe is formed in a reductive atmosphere.

I : Solid phase is not formed.

$\mathrm{Cs}: \mathrm{Cs}_{2} \mathrm{Te}$ is decomposed in a reductive atmosphere.

(Temperature: $2,500 \mathrm{~K}$ )

$\mathrm{Sr}$ : Decomposition of $\mathrm{SrZrO}_{3}$ is slightly accelerated in an oxidative atmosphere.
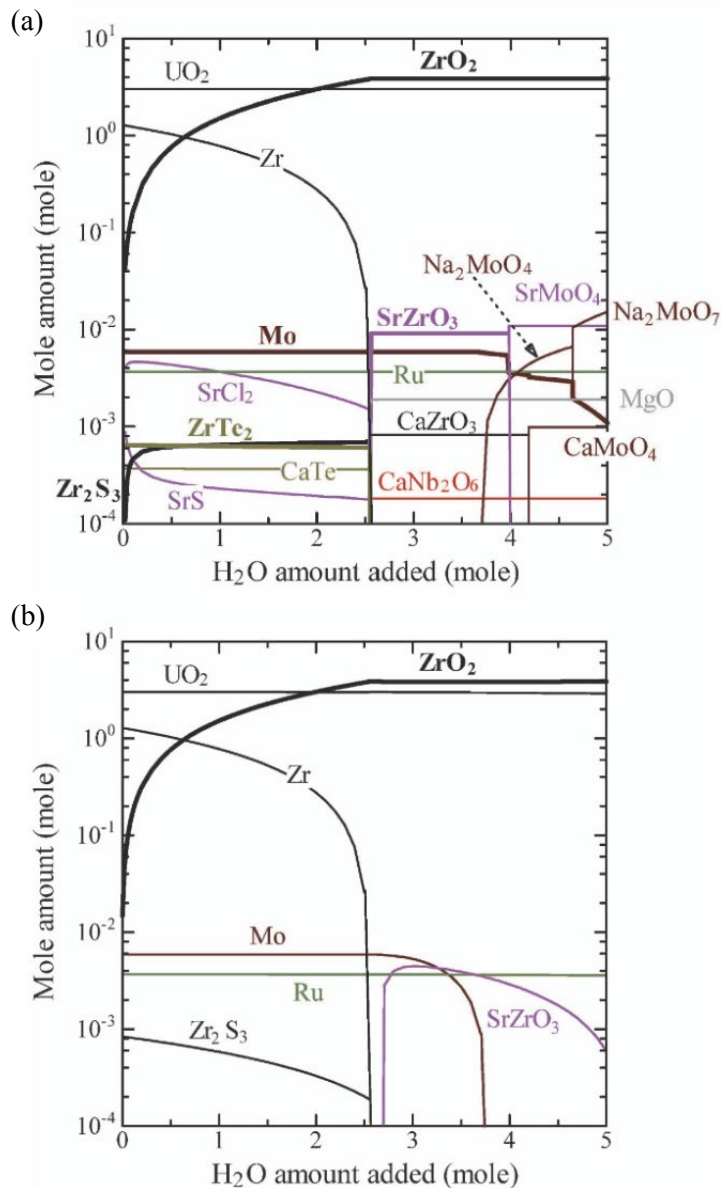

Figure 5 Mole amount of solid species as a function of water amount added in the case of seawater (a) $1,500 \mathrm{~K}$ and (b) $2,500 \mathrm{~K}$ 
Mo : Evaporation of Mo is slightly accelerated in an oxidative atmosphere.

$\mathrm{Ru}$ : No change.

Other FPs: Solid phase does not exist.

In a similar manner, the relation between added seawater amount and the molar fraction of the gaseous phase is shown in Figure 6. Here, the components originating mainly from FP and other components are separately shown for $2,500 \mathrm{~K}$ due to space limitations.

(Temperature: $1,500 \mathrm{~K}$ )

$\mathrm{Sr}$ : Chloride vapor species are formed in a reductive atmosphere, and evaporation is accelerated.

Mo: Vapor species of composite oxides with Cs are not formed in a reductive atmosphere, and evaporation is suppressed.

I : Formation of $\mathrm{NaI}$ becomes dominant in an oxidative atmosphere. Even in a reductive atmosphere, $\mathrm{NaI}$ exists in addition to CsI.

$\mathrm{Cs}: \mathrm{CsCl}$ is the major component of vapor species in both reductive and oxidative atmospheres, and evaporation is accelerated. The vapor species of complex compounds with Mo are not formed in an oxidative atmosphere.

Others: Significant generation of $\mathrm{HCl}$ in an oxidative atmosphere.

(Temperature: $2,500 \mathrm{~K}$ )

$\mathrm{U}$ : Evaporation is slightly accelerated in a reductive atmosphere because of the formation of vapor species of chlorides.

$\mathrm{Sr}$ : Chloride vapor species are formed in a reductive atmosphere, and evaporation is accelerated. Vapor species are transformed in an oxidative atmosphere.

Mo: Vapor species are transformed in an oxidative atmosphere.

I : $\mathrm{NaI}$ is formed in a reductive atmosphere.

$\mathrm{Cs}: \mathrm{CsCl}$ and $\mathrm{Cs}$ become the major components of vapor species both in reductive and oxidative atmospheres.

The temperature dependence of the evaporation rate for various elements in a reductive atmosphere (case-I) and an oxidative atmosphere (case-II) is shown in Figure 7. Trends for each element are evaluated by comparison with Figure 4.

(Reductive atmosphere: case-I)

$\mathrm{U}, \mathrm{Zr}, \mathrm{Nb}, \mathrm{Mo}, \mathrm{Ru}, \mathrm{Te}$, and I: No significant influence

$\mathrm{Sr}$ and $\mathrm{Cs}$ : Acceleration of evaporation

(Oxidative atmosphere: case-II)

$\mathrm{U}, \mathrm{Zr}, \mathrm{Ru}, \mathrm{Te}$, and I: No significant influence

$\mathrm{Sr}$ and $\mathrm{Cs}$ : Acceleration of evaporation

$\mathrm{Mo}$ and $\mathrm{Nb}$ : Inhibition of evaporation

\section{Generation of Corrosive Gas from Seawater}

Figure 8 extracts the evaluation results on corrosive gas components from the temperature dependence in the molar quantity of vapor species for case-I and case-II. The trends for each element are shown in Figure 7, and the trends are indicated for each vapor species in Figure 8. It is confirmed that the dominant vapor species for evaporation of each element changes depending on the temperature, and the trends vary significantly between reductive and oxidative atmospheres. The generation of the major corrosive gases is shown in the same figure. In a reductive atmosphere, corrosive gases such as $\mathrm{HCl}, \mathrm{HS}, \mathrm{H}_{2} \mathrm{~S}$, and $\mathrm{S}$, are generated. In an oxidative atmosphere, $\mathrm{SO}$ and $\mathrm{SO}_{2}$ are generated in addition to the gases observed in a reductive atmosphere. These corrosive gases likely start to form in the comparatively low temperature 

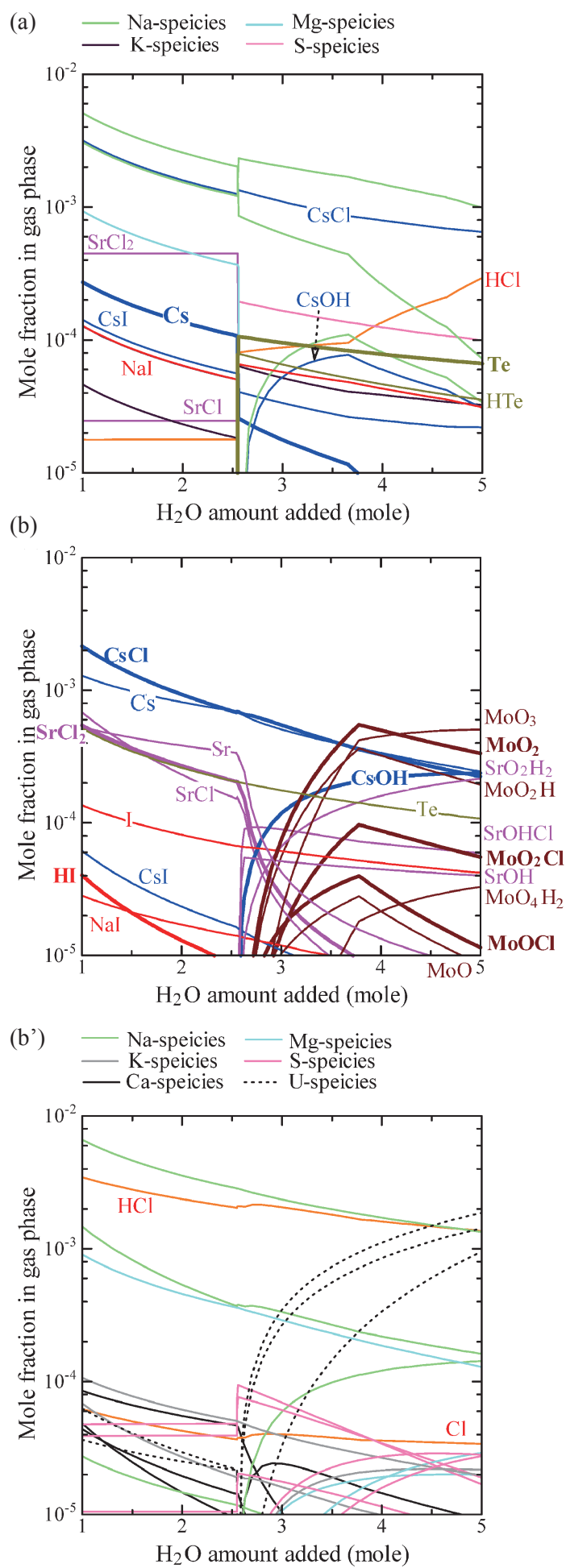

Figure 6 Mole fraction of gas species as a function of water amount added in the case of seawater (a) 1,500 K, (b) 2,500 K(FP), and (b') 2,500 K(others) 
(a)

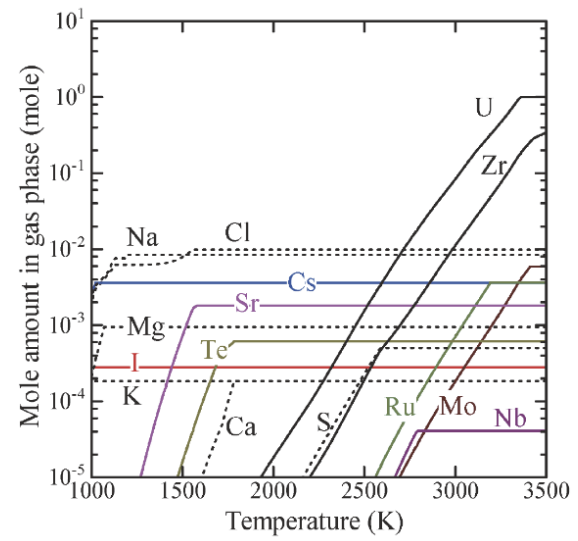

(b)

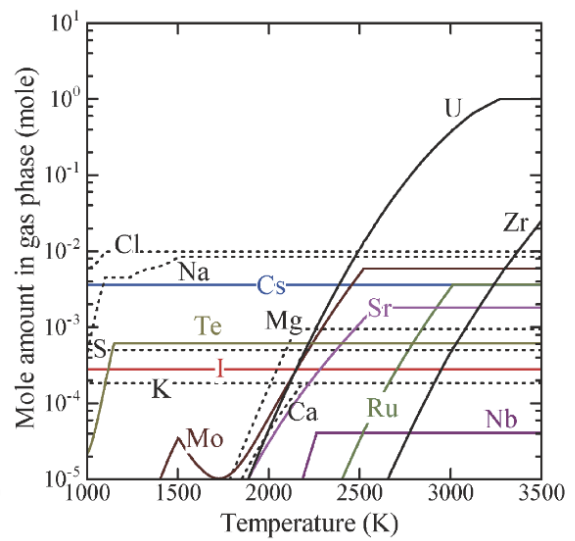

Figure 7 Mole amount in gas phase as a function of temperature in the case of seawater (a) reductive atmosphere (case-I) and (b) oxidative atmosphere (case-II)

(a)

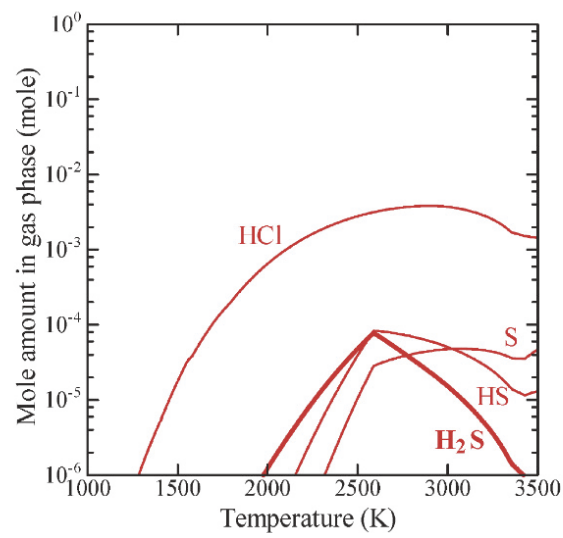

(b)

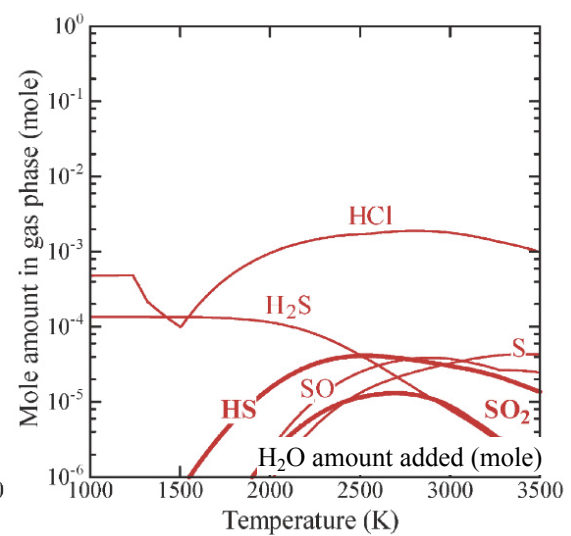

Figure 8 Mole amount in gas phase as a function of temperature in the case of seawater (a) reductive atmosphere (case-I) and (b) oxidative atmosphere (case-II)

regions in an oxidative atmosphere. This implies the possibility of a significant change in the trend of corrosive gas generation, depending on the time when seawater is supplied to the damaged reactor core. The later the seawater is supplied after the increase in $g\left(\mathrm{O}_{2}\right)$ in the system in the late stages of an accident, the more corrosive gases are likely to be generated.

\section{Chemical Reaction of $\mathrm{UO}_{2} / \mathrm{FP} / \mathrm{Zr} / \mathrm{Fe}$ Mix and Seawater}

For similar conditions as above, an evaluation was performed for the Fe from structural materials. Figure 9 shows the temperature dependence of the evaporated molar quantity for each element in case-I and case-II. Because $\mathrm{g}\left(\mathrm{O}_{2}\right)$ changes in an oxidative atmosphere through the coexistence of $\mathrm{Fe}$ oxide, a slight difference in the evaporation properties of Mo and $\mathrm{Te}$ is observed. It is shown that for both elements, an acceleration of evaporation takes place at low temperatures. A slight acceleration in the evaporation of seawater components such as $\mathrm{Na}$ and $\mathrm{Cl}$ likely takes place at low temperatures in an oxidative atmosphere. 
(a)

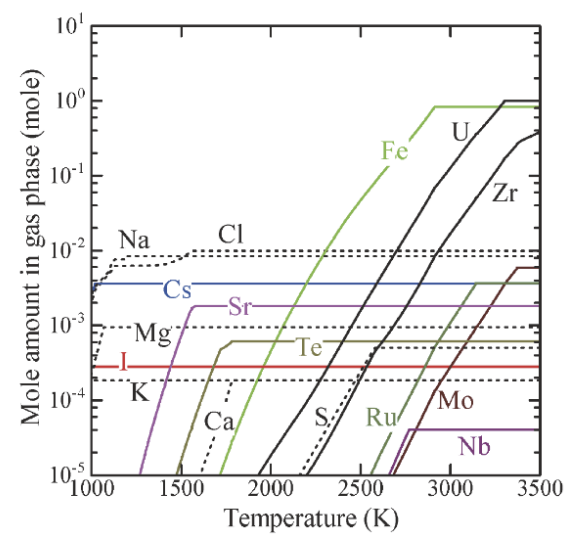

(b)

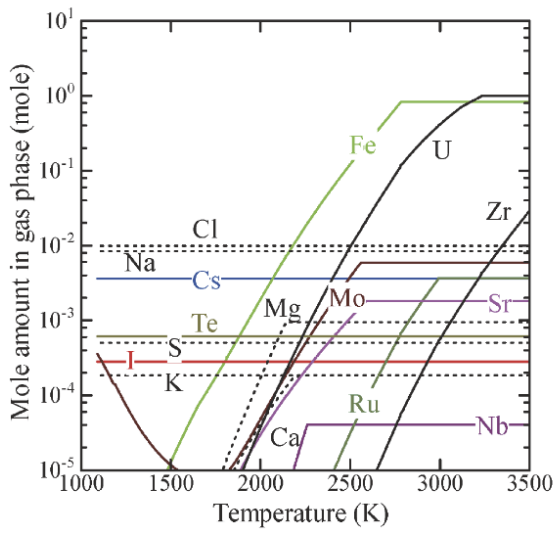

Figure 9 Mole amount in gas phase as a function of temperature in the case of the coexistence of seawater and steel (a) reductive atmosphere (case-I) and (b) oxidative atmosphere (case-II)

However, the possibility of complicated chemical reactions due to the coexistence of seawater and $\mathrm{Fe}$ is considered to be low. The details of the reaction of damaged fuel and structural materials is explained elsewhere ${ }^{4)}$.

\section{Discussion}

Based on the present evaluation results, concerns requiring further discussion are presented regarding the degradation of fuel.

\section{Heterogeneity of Damaged Reactor Vessel Interior}

As shown in Figure 5, when the reaction between damaged fuel and seawater takes place in a reductive atmosphere, $\mathrm{Zr}_{2} \mathrm{~S}_{3}$ may be formed. On the other hand, as shown in Figure 8, $\mathrm{SO}_{\mathrm{x}}$ gas is generated if seawater is heated in an oxidative atmosphere. The temperature and $g\left(\mathrm{O}_{2}\right)$ differ markedly at each location in a damaged reactor vessel in an actual severe accident and is further expected to vary minute by minute. Consequently, fuels are considered to collapse in a heterogeneous manner. Concerns caused by such heterogeneity are revealed by the current thermodynamic approach. In an example of sulfide formation, $\mathrm{g}\left(\mathrm{O}_{2}\right)$ increases in the molten corium when the damage of fuel progresses. The $\mathrm{SO}_{\mathrm{x}}$ gas, which is considered to be generated when the molten corium and seawater react, is transferred to the comparatively low temperature regions, where zircalloy metal remains, because the damage is not as thorough and extensive, and may sulfurize zircalloy and accelerate the damage. It is necessary to continue the analyses of these heterogeneous processes in future study.

\section{Extension of Database}

As explained in section II, the organization of a thermodynamic database is essential to the improved accuracy of thermodynamic evaluation. Because almost no data regarding oxy-chlorides of $\mathrm{U}$ and $\mathrm{Zr}$ (e.g., $\mathrm{UOCl}, \mathrm{ZrOCl}$ ) are available in the SSUB4 database, the influence of 
the evaporation of $\mathrm{U}$ and $\mathrm{Zr}$ in the case of reaction with seawater was not predicted. However, oxy-chlorides may accelerate the evaporation of $U$ and $\mathrm{Zr}$ because they have higher vapor pressures than the oxides of those elements. This implies the possibility of the adhesion of $U$ in an unanticipated location in the reactor core during an accident. Furthermore, it is necessary to consider the effect of various compounds present as solid or liquid solutions as related to the evaporation behavior or formation of chemical compounds. Because there are no thermodynamic data on $\mathrm{CsBO}_{2}$, relevant evaluation was not possible in this study. It is essential to systematically upgrade that data by referring to current evaluation results and various experimental data currently being collected. Here, a certain prioritization is necessary for extracting important data. For this purpose, sensitivity analysis assuming hypothetical data is likely to be considered. Research and development should be conducted both experimentally and analytically.

\section{Evaluation of Hydrogen Concentration and Prediction of Oxygen Potential $\left(\mathrm{g}\left(\mathbf{O}_{2}\right)\right)$}

In the late phases of a severe accident, the hydrogen partial pressure in steam is considered to be extremely low because the oxidation of zirconium almost ceases. In the current evaluation, all the analyses were conducted based on the assumption of a closed system. Therefore, they were always treated under the condition of a coexistence of a certain amount of hydrogen. To simulate the late phases of a severe accident with better accuracy, it is necessary to switch to an evaluation condition in which no residual hydrogen is assumed in the system at any stage of the accident. This analysis will be conducted along with simulation tests in the future. The properties of debris sampled from the reactors will also be referred to. A reverse estimation of $\mathrm{g}\left(\mathrm{O}_{2}\right)$ and temperature during real accident progression might be performed based on this knowledge of chemical behavior. This will contribute to the presumption of evaporation for the main FPs. It can be pointed out that the location of Mo and $\mathrm{Nb}$, potentially detected in the future in the damaged reactors, might be a very useful hint in evaluating accident progression.

\section{Summary}

A thermodynamic evaluation was conducted on the predicted chemical reactions when seawater is injected and contacts the degraded fuel under severe accident conditions, yielding the following results:

(1) Influence on volatile FPs: In an environment where a large amount of $\mathrm{Zr}$ remains in the early phases of a severe accident, due to the reaction between $\mathrm{FP}$ and $\mathrm{Na}, \mathrm{Cl}$, or other sub-components, such as $\mathrm{K}, \mathrm{Ca}, \mathrm{Mg}$, and $\mathrm{S}$ in seawater, the evaporation of $\mathrm{Sr}$ and $\mathrm{Cs}$ is likely to be accelerated compared to the situation in pure water or steam. Furthermore, in an environment with progressing oxidation of $\mathrm{Zr}$ in the late phases of a severe accident, the evaporation of $\mathrm{Sr}$ and $\mathrm{Cs}$ is likely to be accelerated, while that of $\mathrm{Mo}$ and $\mathrm{Nb}$ is suppressed.

(2) Generation of corrosive gases: Where the seawater is exposed to the high temperature environment in the reactor under the conditions of the early phases of a severe accident, corrosive gases, such as $\mathrm{HCl}, \mathrm{HS}, \mathrm{H}_{2} \mathrm{~S}$, and $\mathrm{S}$, are likely to be generated. In the late phases of a severe accident, those gases are even more likely to be generated, and $\mathrm{SO}$ and $\mathrm{SO}_{2}$ might be generated as well.

(3) Influence of structural materials: The presence of structural materials is likely to influence the variation in the oxygen potential in the reactor under severe accident conditions, but 
collateral chemical reactions due to the coexistence of the structural materials and seawater is unlikely to be common.

(4) Others: One of the key questions in the evaluation of the degraded core status is, "in which location in the degraded reactor core are $\mathrm{Mo}$ and $\mathrm{Nb}$, whose evaporation properties are presumably greatly influenced by chemical conditions (e.g., oxygen potential), detected?"

In the next step of our study, the analyses of heterogeneous open systems will be continued in parallel with the progress in various other experiments.

The authors are grateful to Drs. Kazuo Minato, Yasuo Arai, Fumihisa Nagase and Masahide Takano of the Japan Atomic Energy Agency for their valuable comments. The authors are also grateful to Dr. Kenji Nishihara of the same agency, who kindly provided the evaluation values of FP using the ORIGEN-II code.

\section{References}

1) M. Takano, T. Nishi, M. Kurata et al., "High temperature reaction between $\mathrm{UO}_{2}$ and sea salt deposit," Proc. of 5th European Review Meeting on Severe Accident Research, Mar. 21-23, 2012, Cologne, Germany.

2) P. Hofmann, S. Hagen, G. Schanz, A. Skokan, "Reactor core materials interactions at very high temperatures," Nucl. Technol., 87, 146-186 (1989).

3) A. C. Gregoire, T. Haste, "Material release from degraded bundle during the Phebus FP tests," presented at Final seminar of the Phebus FP Programme, Jun. 13-15, 2012, Aix-en-Provence, France, [CD-ROM].

4) Noriko Shirasu, Masaki Kurata, Toru Ogawa "A Thermodynamic Evaluation on the Behavior of $\mathrm{B}_{4} \mathrm{C}$ in an LWR Severe Accident," Journal of Nuclear Science and Technology (in Japanese). (to be published)

5) A. T. Dinsdale, "SGTE data for pure elements," CALPHAD, 15, 317 (1991).

6) B. Sundman, B. Jansson, J.-O. Andersson "The Thermo-Calc databank system," CALPHAD, 9, 153 (1985).

7) L. Cantrel, F. Cousin, L. Bosland, "Advances in mechanistic understanding and modeling of iodine behavior," presented at Final seminar of the Phebus FP Programme, Jun. 13-15, 2012, Aix-en-Provence, France, [CD-ROM].

8) C. Gueneau, N. Dupin, B. Sundman et al., "Thermodynamic modelling of advanced oxide and carbide nuclear fuels: Description of the U-Pu-O-C systems," J. Nucl. Mater., 419, 145 (2011).

9) N. Shirasu, H. Otobe, M. Kurata, "Thermodynamic assessment of the ZrO2-PuO1.5-PuO2 system," $J$. Nucl. Mater., (to be published).

10) F. Tanabe, “Analysis of core melt accident in Fukushima Daiichi-Unit 1 Nuclear Reactor,” J. Nucl. Sci. Technol., 48, 1135 (2011).

11) Y. Nozaki, “A fresh look at element distribution in the North Pacific Ocean,” Eos, 78, 221 (1997).

12) A. G. Croff, A user's Manual for the ORIGEN2 Computer Code, ORNL/TM-7175 (1980). 


\title{
Article
}

\section{Estimation of Radionuclide Concentration in Plume Using Pulse Height Distribution Measured by $\mathrm{LaBr}_{3}$ Scintillation Detector and Its Response to Radionuclides in Plume Calculated with egs5}

\author{
Hideo HIRAYAMA ${ }^{1, *}$, Hiroshi MATSUMURA ${ }^{1}$, \\ Yoshihito NAMITO ${ }^{1}$ and Toshiya SANAMI ${ }^{1}$ \\ ${ }^{1}$ High Energy Accelerator Research Organization, 1-1 Oho, Tsukuba-shi, Ibaraki 305-0801, Japan
}

\begin{abstract}
A method was presented to estimate radionuclide concentration in plume using the pulse height distribution measured by a $\mathrm{LaBr}_{3}$ scintillation detector and its calculated response to radionuclides in plume with egs5. Radionuclide concentration was estimated from the ratio between the peak count rates corresponding to each radionuclide in the measured pulse height distribution on an expressway on March 15 and in the calculated one from each radionuclide in plume using the egs5 Monte Carlo code. The pulse height distribution reconstructed based on the estimated concentrations agrees well with the measured one at the time that the contribution from radionuclides deposited on a ground surface is negligible.
\end{abstract}

KEYWORDS: radionuclide concentration, iodine 131, plume, Monte Carlo, egs5, $\mathrm{LaBr}_{3}$ scintillator

\section{Introduction}

The radionuclide concentrations in plumes dispersed over a wide area due to the accident that occurred at the Fukushima Daiichi Nuclear Power Plant (hereinafter referred to as "plume") are important in evaluating the internal radiation dose from inhalation. Although the concentration of I-131 is particularly important from the perspective of radiation-induced thyroid cancer risk assessment, the direct measurement of radionuclide concentrations in plumes was conducted only in limited places far from the Fukushima Daiichi Nuclear Power Plant. Therefore, the most direct method is estimation from the density of I-131 deposited on the soil surface. However, since the half-life of I-131 is as short as about eight days, the measurement data are extremely limited compared to that of Cs-134 and Cs-137. As a result, estimating from an atmospheric dispersion model and air dose rate, as well as estimating from the analysis of I- 139 with a very long half-life, have been attempted. Although it is essential to perform evaluation based on an atmospheric dispersion model to estimate the concentration distribution over a wide area, to increase accuracy, it would be desirable to obtain the data on the concentration of radionuclides,

* Corresponding author, E-mail: hideo.hirayama@kek.jp

DOI : 10.15669 /fukushimainsights.Vol.4.233

(C) 2021 Atomic Energy Society of Japan. All rights reserved.

Originally published in Transactions of the Atomic Energy Society of Japan (ISSN 1347-2879), Vol. 12, No. 4, p.304-310

(2013) in Japanese. (Japanese version accepted: October 1, 2013) 
such as I-131, contained in the plumes, but not the air dose rate and the amount of deposition on the soil surface. In this paper, we attempted to estimate the concentrations of radionuclides contained in the plumes that passed overhead using the pulse height distribution measured by Matsumura, et al. at expressway service areas (SA), parking areas (PA), some interchanges (IC), and junctions (JCT) with a $\phi 1.5^{\prime \prime} \times 1.5^{\prime \prime} \mathrm{LaBr}_{3}$ scintillation spectrometer (manufactured by Canberra, IN1KL-1, hereinafter referred to as " $\mathrm{LaBr}_{3}$ detector") on March 15, 2011 ${ }^{1)}$. We calculated the detector response of $\mathrm{LaBr}_{3}$ to the radionuclides in plumes by applying a method ${ }^{2)}$ whereby the photon flux from a plane isotropic source is converted into a point isotropic source, and a plane detector to an egs $5^{3)}$ electromagnetic cascade Monte Carlo code to estimate the concentrations of radionuclides in the plumes from the ratio between the peak count rates per $\mathrm{Bq} / \mathrm{cm}^{3}$, corresponding to each radionuclide calculated and the corresponding peak count rate measurements.

\section{Calculation of the Response of $\mathrm{LaBr}_{3}$ Detector with egs5}

\section{Photon Spectra of Radionuclides Contained in a Plume $1.3 \mathrm{~m}$ above the Ground Surface}

The distribution of radionuclides when a plume arrived was assumed to correspond to the case where there was a plane isotropic source spreading infinitely and horizontally from the ground surface to the plume height $\left(h_{0}\right)$ with a uniform density.

We calculated the photon spectra $1.3 \mathrm{~m}$ above the soil surface, assuming that the vertical distribution was uniform from the soil surface to $h_{0}$, by applying the method ${ }^{2)}$ of converting the photon flux from a plane isotropic source into a point isotropic source, and a plane detector to egs $5^{3)}$. To validate this method, Figure 1 shows the comparison between the result of the analytical method described in EPA-402-R-93-081 ${ }^{4}$ and the result of calculation with egs5 for the scattered photon spectrum in an infinite air system in which one $100-\mathrm{keV}$ photon is emitted per cubic meter every second. In the calculation with egs 5 , an air density of $0.001205 \mathrm{~g} / \mathrm{cm}^{3}$ was used under $1 \mathrm{~atm}$ (atmospheric pressure) at $20^{\circ} \mathrm{C}$ (hereinafter referred to as "NTP state"). As the simulation of an infinite system, $h_{0}$ was set to ten times of the mean free path of a 100 $\mathrm{keV}$ photon in the air. Both the shape and size of the spectra were found to be identical. Figure 1 also shows the result of calculating the photon spectrum at a height of $1.3 \mathrm{~m}$ taking the soil into

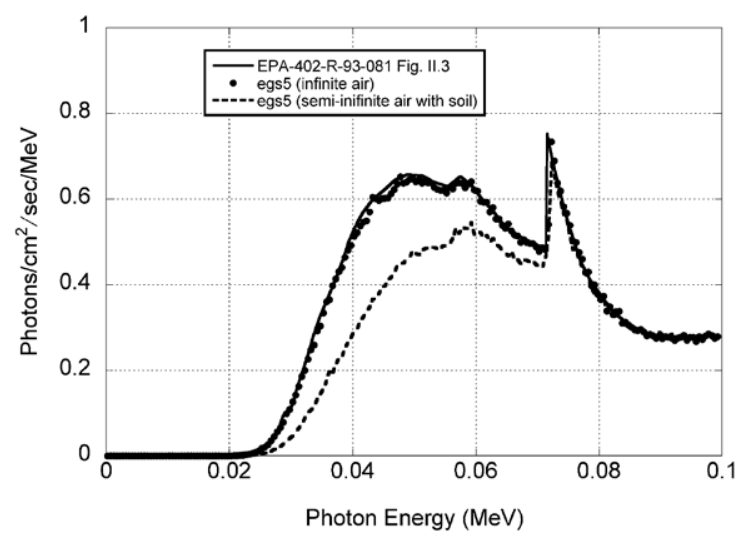

Figure 1 Comparison of energy spectrum of scattered photons for submersion in a $1 \mathrm{~Bq} / \mathrm{m}^{3} 100 \mathrm{keV}$ contaminated air source 
consideration. When the soil was taken into consideration, the calculation result was doubled so that the total of the radiation source would be the same. Although there was little change in the area close to the energy of the radiation source, it was found that there was less backscattering in a remote area when the soil was taken into account. The number of unscattered photons was $5.52 \times 10^{-3} \mathrm{photons} / \mathrm{cm}^{2} / \mathrm{sec}$ in the case of EPA-402-R-93-081. The result of egs 5 where the soil was not considered was $5.50 \times 10^{-3}$ photons $/ \mathrm{cm}^{2} / \mathrm{sec}$, and the result of egs 5 at a height of $1.3 \mathrm{~m}$ considering the ground was $6.03 \times 10^{-3}$ photons $/ \mathrm{cm}^{2} / \mathrm{sec}$. We can see that they are identical.

To see the impact of plume height $h_{0}$ from the plume containing I-132, which emits high energy gamma rays, Figure 2 shows the comparison of spectra at a height of $1.3 \mathrm{~m}$ from the soil surface between the case where the height is $500 \mathrm{~m}$ and the case where the height is $2,219 \mathrm{~m}$, which is ten times of the mean free path for the gamma ray with the highest energy in air, and is regarded as infinite in thickness. It was found that there was little difference between either case. Although the plume height is said to be between $100 \mathrm{~m}$ and $1,000 \mathrm{~m}$, based on the result shown in Figure 2, the plumes up to $500 \mathrm{~m}$ in height are to be studied.

Figure 3 shows the calculated photon spectra at a height of $1.3 \mathrm{~m}$ above the soil surface from plumes 100, 200, and $500 \mathrm{~m}$ high containing I-131 at a concentration of $1 \mathrm{~Bq} / \mathrm{cm}^{3}$ in the air in NTP state. The figure shows that the scattering portion increases as the plume height

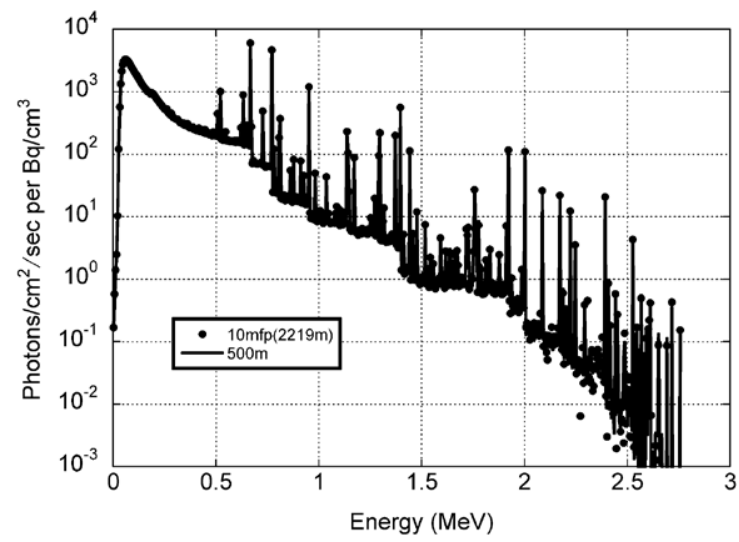

Figure 2 Comparison of photon spectrum at $1.3 \mathrm{~m}$ above soil surface from I-132 contained in plumes $500 \mathrm{~m}$ and 2,219 $\mathrm{m}$ (10 mfp) high

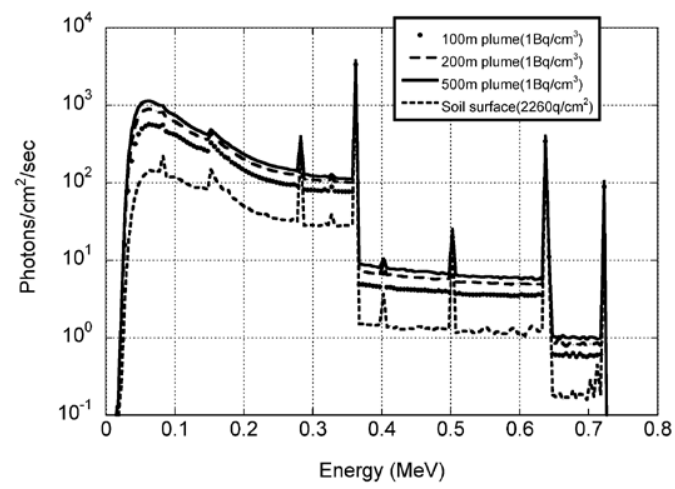

Figure 3 Comparison of photon spectrum at $1.3 \mathrm{~m}$ above soil surface from I-131 contained in plume or deposited on soil surface 
$h_{0}$ increases, but there is little difference between $200 \mathrm{~m}$ and $500 \mathrm{~m}$. Figure 3 also shows the calculated photon spectrum at a height of $1.3 \mathrm{~m}$ from the soil surface when I-131 is distributed uniformly over a wide area on the soil surface at a concentration of $2,260 \mathrm{~Bq} / \mathrm{cm}^{2}$, where the peak gamma ray count rate of $0.364 \mathrm{MeV}$ becomes almost identical to the result for a plume $500 \mathrm{~m}$ high. In the case of ground surface distribution, it was found that the ratio of scattered photons to unscattered photons that were emitted from a radionuclide and then were not subjected to scattering was small compared with that from the plume.

The density of air varies depending on the weather conditions. Figure 4 shows the air density dependency of the spectra for a plume $200 \mathrm{~m}$ high containing I-131. If the density of the air is increased by half without changing the composition, the spectrum changes a little in shape but varies almost inversely with the density, as it is $1 / 1.44$ at the peak of $0.365 \mathrm{MeV}$. This is because the attenuation in the air increases due to the increase in the density.

\section{Response of $\mathrm{LaBr}_{3}$ Detector}

We calculated the absorption energy in the detector based on the assumption that photons that have the spectrum calculated in Section II-1 in the air in the NTP state enter a $\phi 1.5^{\prime \prime} \times 1.5^{\prime \prime}$ $\mathrm{LaBr}_{3}$ detector uniformly, and also corrected the resolution using the detector performance achieving a FWHM of $3.5 \%$ relative to $0.662 \mathrm{MeV}$ photons. Based on these calculations, we determined the detector response in the case where various nuclides were contained at a concentration of $1 \mathrm{~Bq} / \mathrm{cm}^{3}$. Figure 5 shows the detector response according to I-131, I-132, Cs-134, Te-132, and Xe-133 when the plume height is 100,200 , and $500 \mathrm{~m}$. In the case of nuclides other than Xe-133, the detector response is also shown for a height of $1.3 \mathrm{~m}$ from the soil surface, where respective nuclides are distributed uniformly over a wide area on the soil surface at a density which makes major peak count rates almost identical. The lower the energy of the gamma rays emitted from various types of radionuclides, the smaller the contribution made by distance becomes, showing that the plume height has a smaller impact. The detector response to Te-132 shows peaks around 0.04 and $0.03 \mathrm{MeV}$ only in the case of soil surface distribution. These peaks are due to the gamma rays emitted by the decay of Te-132 and the K X-ray of I. Since the contribution from a region near the detector is relatively large in the case of soil surface distribution, the contribution of the low-energy gamma rays and X-rays is reflected in the pulse height distribution.

Since $\mathrm{La}$, which is a major constituent of the detector, contains $0.09 \%$ of La-138 with a

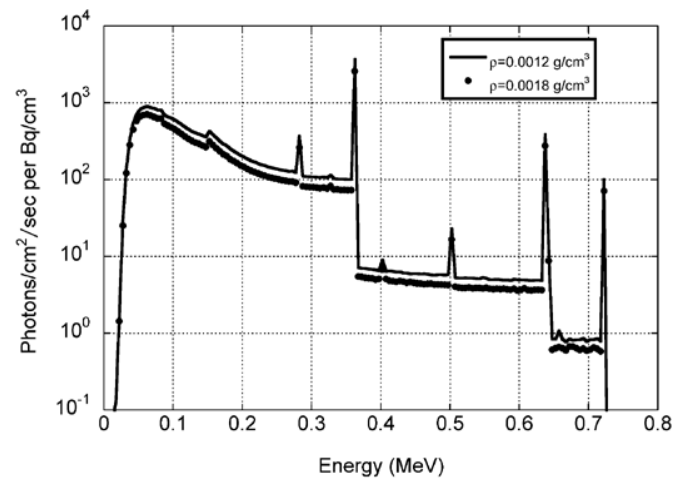

Figure 4 Air density dependence of photon spectrum at $1.3 \mathrm{~m}$ above soil surface from $200 \mathrm{~m}$ plume containing I-131 


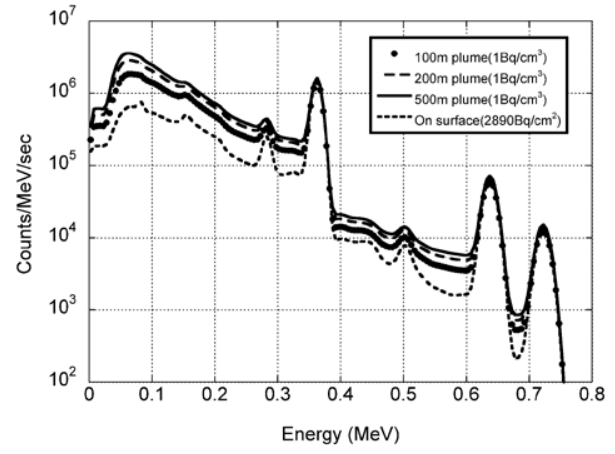

(a) I-131

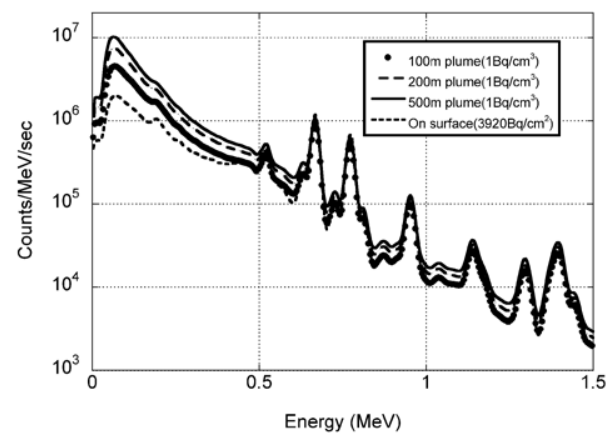

(b) I-132

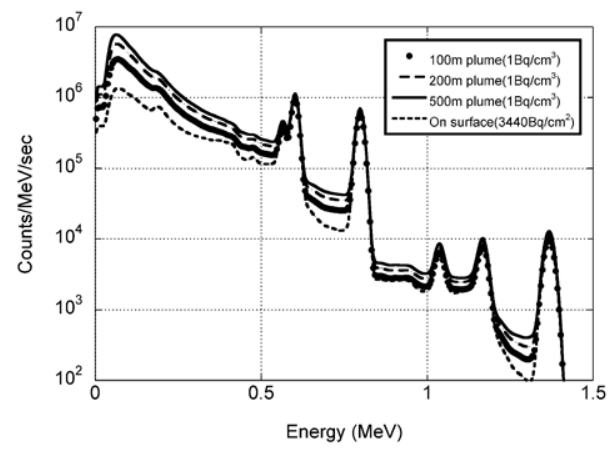

(c) $\mathrm{Cs}-134$

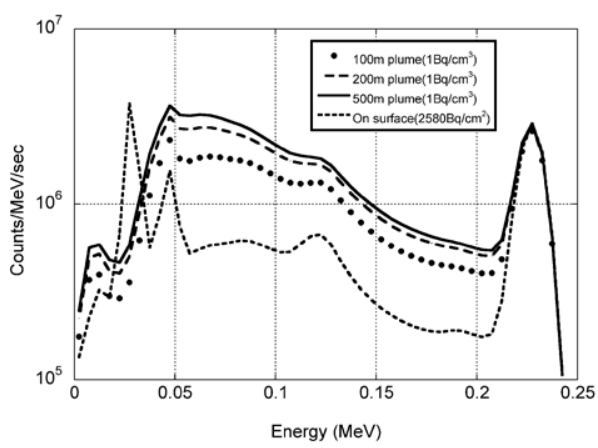

(d) $\mathrm{Te}-132$

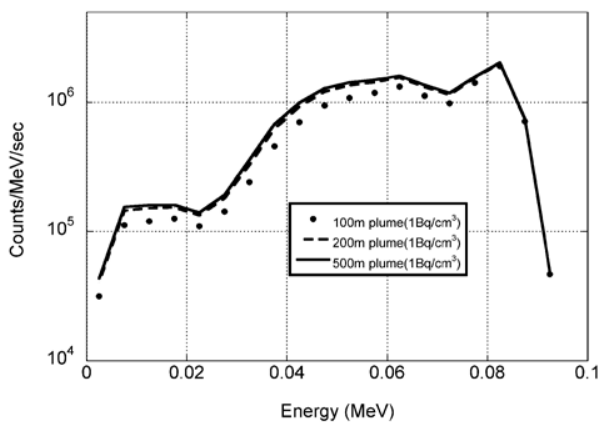

(e) $\mathrm{Xe}-133$

Figure 5 Comparison of $\mathrm{LaBr}_{3}$ response $1.3 \mathrm{~m}$ above soil surface from I-131(a), I-132(b), Cs-134(c), Te132(d) and Xe-133(e) contained in plume or deposited on soil surface

half-life of $1.06 \times 10^{11}$ years, the $\mathrm{LaBr}_{3}$ detector has a unique background consisting of the gamma and beta rays emitted as a result of the decay of La-138. Figure 6 shows the background pulse height distribution of the detector, which was calculated with egs5 based on the decay mode of La-138, assuming that La-138 was distributed uniformly in the $\mathrm{LaBr}_{3}$ detector. The detector response calculated had very similar amplitude and shape to the measurement value obtained by placing the $\mathrm{LaBr}_{3}$ detector in a lead shield, and it can be said that the result of calculation with egs 5 reproduced the background due to decay of La-138. In the comparison of pulse height distributions performed in Chapter III, the pulse height distributions calculated with egs 5 include this background unique for the $\mathrm{LaBr}_{3}$ detector. 


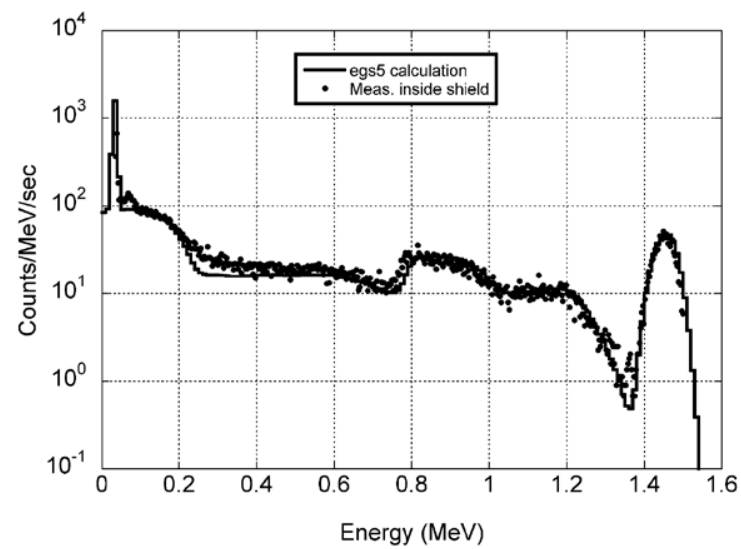

Figure 6 Comparison of $\mathrm{LaBr}_{3}$ detector response due to La-138 between egs 5 calculation and measurement inside $\mathrm{Pb}$ shield

\section{Concentration of Radionuclides in Plume}

\section{Method of Estimating Radionuclide Concentrations}

From the measurements of the pulse height distributions, Te-132, I-132, I-131, Xe-133, Cs-134, and Cs-136 were detected. To estimate the concentrations of respective radionuclides, we used the peaks corresponding to the energies shown in Table 1, which were considered to contain fewer gamma rays emitted from other nuclides.

The concentrations of respective nuclides were estimated from the pulse height distribution of the $\mathrm{LaBr}_{3}$ detector according to the following procedures:

(1) To determine the peak count rate from which the tail of the nuclide identified from the measurement value is deducted (A).

(2) To determine the corresponding peak count rate from which the tail is deducted from the detector responses calculated for respective nuclides, as is the case with the measurement value (B). This value is the peak count rate per $\mathrm{Bq} / \mathrm{cm}^{3}$.

(3) The radionuclide concentration $\left(\mathrm{Bq} / \mathrm{cm}^{3}\right)$ is obtained from $\mathrm{B} / \mathrm{A}$.

\section{Comparison of the Pulse Height Distributions of $\mathrm{LaBr}_{3}$}

Figures 7 and $\mathbf{8}$ show the comparison between the measured pulse height distributions and the reconstructed distributions using the detector responses in the case where the plume height was $200 \mathrm{~m}$, and where radionuclides deposited uniformly on the ground surface over a wide area at Koriyama-Higashi IC of the Ban-Etsu Expressway (March 15, 15:25-15:26) and at Abukuma-Kogen SA of the Ban-Etsu Expressway (March 15, 15:51-15:52). The reconstruction was based on the assumption that Cs-137, with the same concentration as Cs-134, was contained in the plume. Since Xe-133 does not deposit on soil surfaces, it was considered that Xe-133 existed in the plumes even in the case of calculation of soil surface contamination. In the comparison at Koriyama-Higashi IC, the calculated values had a tendency to be overestimated in the range from $0.081 \mathrm{MeV}$ to $0.228 \mathrm{MeV}$, but it can be said that the measured pulse height distributions were very similar to the reconstructed distributions. On the other hand, in the comparison at Abukuma-Kogen SA, the situation is different from Koriyama-Higashi IC in that the scattering portion of the pulse height distribution reconstructed at low energy was 
Table 1 Gamma-ray energies used for analysis

\begin{tabular}{cc}
\hline Radionuclide & Gamma-ray energy \\
\hline Xe-133 & $81 \mathrm{keV}$ \\
Te-132 & $228 \mathrm{keV}$ \\
$1-131$ & $365 \mathrm{keV}$ \\
Cs-134 & $605 \mathrm{keV}$ \\
$1-132$ & $955 \mathrm{keV}$ \\
Cs-136 & $1,048 \mathrm{keV}$ \\
\hline
\end{tabular}

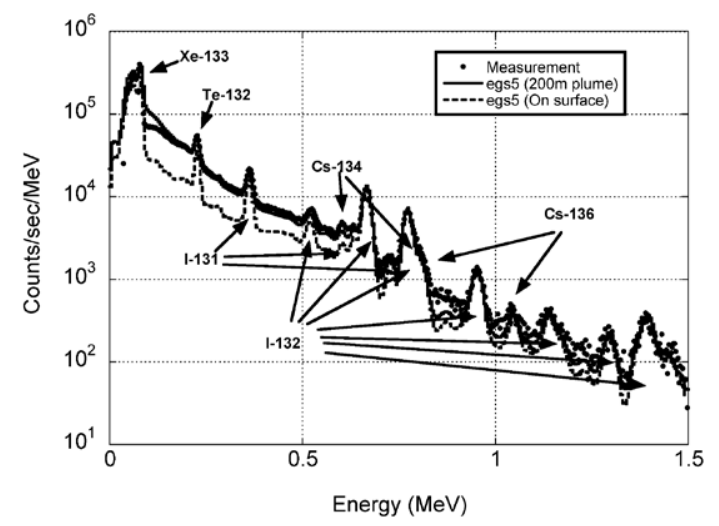

Figure 7 Comparison of $\mathrm{LaBr}_{3}$ pulse-height distribution between measurement at Koriyama-higashi IC at 15:25 March 15, 2011 and egs5 calculation

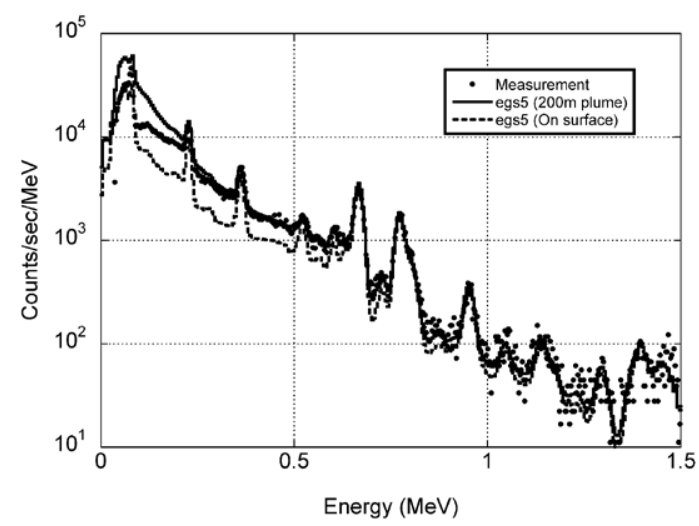

Figure 8 Comparison of $\mathrm{LaBr}_{3}$ pulse-height distribution between measurement at Abukuma-kogen SA at 15:51 March 15, 2011 and egs5 calculation

significantly larger than the measured one.

Based on the results of the analysis of radionuclides mention in the next section 3, it was clear that the concentrations varied markedly with time in both areas, which were located west of the Fukushima Daiichi Nuclear Power Plant. The measurement at Abukuma-Kogen SA was performed after a certain period of time had elapsed following the passage of the plume containing high concentrations of radionuclides. Therefore, it was surmised that this difference could be attributed to the contribution of radionuclides deposited on ground surfaces, etc. when 
the passing plume containing high concentrations of radionuclides was relatively large.

\section{Estimation of the Concentrations of Radionuclides in Plumes}

From the measurement values obtained on March 15, we estimated the concentrations of radionuclides from the pulse height distributions measured at six places, shown in Table 2, which were located 38.3-57.4 km west of the Fukushima Daiichi Nuclear Power Plant. Table 3 shows the concentrations of radionuclides, and measured peak count rates corresponding to respective radionuclides contained in the plumes. Although the radionuclide concentration becomes higher as the effective height of the plume is lower, the concentration is as low as $30 \%$ in the case of I-132, which means the largest difference occurs at $100 \mathrm{~m}$ and $500 \mathrm{~m}$. The lower

Table 2 Place and time used for the estimation of radionuclide concentration in plume

\begin{tabular}{|c|c|c|c|}
\hline Place of measurement & Time & Measurement time & $\begin{array}{l}\text { Direction and distance from Fukushima } \\
\text { No. } 1 \text { NPP }\end{array}$ \\
\hline Adatara SA & $15: 03$ & $60 \mathrm{sec}$. & West $56.0 \mathrm{~km}$ \\
\hline Motomiya IC & $15: 11$ & $60 \mathrm{sec}$ & West $57.4 \mathrm{~km}$ \\
\hline Koriyama-higashi IC & $15: 22$ & $60 \mathrm{sec}$. & West $52.4 \mathrm{~km}$ \\
\hline Miharu PA & $15: 33$ & $60 \mathrm{sec}$. & West $47.5 \mathrm{~km}$ \\
\hline Funehiki-Miharu IC & $15: 42$ & $60 \mathrm{sec}$. & West $41.7 \mathrm{~km}$ \\
\hline Abukuma-kogen SA & $15: 51$ & $60 \mathrm{sec}$. & West $38.3 \mathrm{~km}$ \\
\hline
\end{tabular}

Table 3 Estimated upper limit of radionuclide concentration inside plume

\begin{tabular}{|c|c|c|c|c|c|c|}
\hline \multirow{3}{*}{ Nuclide } & \multicolumn{6}{|c|}{ Concentration of radionuclide $\mathrm{Bq} / \mathrm{cm}^{3}$} \\
\hline & \multicolumn{3}{|c|}{ Adatara SA (15:03) } & \multicolumn{3}{|c|}{ Motomiya IC (15:11) } \\
\hline & $100 \mathrm{~m}$ plume & $200 \mathrm{~m}$ plume & $500 \mathrm{~m}$ plume & $100 \mathrm{~m}$ plume & $200 \mathrm{~m}$ plume & $500 \mathrm{~m}$ plume \\
\hline $\mathrm{Te}-132$ & 0.00224 & 0.00209 & 0.00206 & 0.00732 & 0.00683 & 0.00675 \\
\hline $1-131$ & 0.00190 & 0.00173 & 0.00169 & 0.00564 & 0.00514 & 0.00503 \\
\hline $1-132$ & 0.00290 & 0.00245 & 0.00226 & 0.00966 & 0.00814 & 0.00749 \\
\hline Xe-133 & 0 & 0 & 0 & 0 & 0 & 0 \\
\hline Cs-136 & 0.000102 & 0.0000867 & 0.0000810 & 0.000418 & 0.000355 & 0.000331 \\
\hline \multirow[t]{2}{*}{ Cs-134 } & 0.000268 & 0.000237 & 0.000227 & 0.00105 & 0.000924 & 0.000888 \\
\hline & \multicolumn{6}{|c|}{ Concentration of radionuclide $\mathrm{Bq} / \mathrm{cm}^{3}$} \\
\hline \multirow[t]{2}{*}{ Nuclide } & \multicolumn{3}{|c|}{ Koriyama-Higashi IC (15:25) } & \multicolumn{3}{|c|}{ Miharu PA (15:33) } \\
\hline & $100 \mathrm{~m}$ plume & $200 \mathrm{~m}$ plume & $500 \mathrm{~m}$ plume & $100 \mathrm{~m}$ plume & $200 \mathrm{~m}$ plume & $500 \mathrm{~m}$ plume \\
\hline Te-132 & 0.0103 & 0.00962 & 0.00952 & 0.00425 & 0.00396 & 0.00392 \\
\hline $1-131$ & 0.00847 & 0.00772 & 0.00755 & 0.00374 & 0.00340 & 0.00333 \\
\hline $1-132$ & 0.0138 & 0.0116 & 0.0107 & 0.00559 & 0.00471 & 0.00434 \\
\hline Xe-133 & 0.120 & 0.118 & 0.118 & 0.03306 & 0.03238 & 0.03238 \\
\hline Cs-136 & 0.000418 & 0.000355 & 0.000331 & 0.000158 & 0.000134 & 0.000125 \\
\hline \multirow[t]{2}{*}{ Cs-134 } & 0.00170 & 0.00150 & 0.00145 & 0.000945 & 0.000835 & 0.000802 \\
\hline & \multicolumn{6}{|c|}{ Concentration of radionuclide $\mathrm{Bq} / \mathrm{cm}^{3}$} \\
\hline \multirow[t]{2}{*}{ Nuclide } & \multicolumn{3}{|c|}{ Funehiki-Miharu IC (15:42) } & \multicolumn{3}{|c|}{ Abukuma-kogen SA (15:51) } \\
\hline & $100 \mathrm{~m}$ plume & $200 \mathrm{~m}$ plume & $500 \mathrm{~m}$ plume & $100 \mathrm{~m}$ plume & $200 \mathrm{~m}$ plume & $500 \mathrm{~m}$ plume \\
\hline Te-132 & 0.00353 & 0.00329 & 0.00325 & 0.00285 & 0.00266 & 0.00263 \\
\hline $1-131$ & 0.00329 & 0.00300 & 0.00293 & 0.00212 & 0.00193 & 0.00189 \\
\hline $1-132$ & 0.00478 & 0.00402 & 0.00370 & 0.00345 & 0.00291 & 0.00268 \\
\hline Xe-133 & 0.0317 & 0.0311 & 0.0311 & 0.0124 & 0.0122 & 0.0122 \\
\hline Cs-136 & 0.000195 & 0.000166 & 0.000155 & 0.000102 & 0.0000867 & 0.0000810 \\
\hline Cs-134 & 0.000733 & 0.000647 & 0.000622 & 0.000697 & 0.000616 & 0.000592 \\
\hline
\end{tabular}


the energy of the gamma rays emitted from radionuclides, the smaller the difference is. Figure 9 shows the variation in concentration with time, provided the effective plume height is $200 \mathrm{~m}$. Although it seems that Xe-133, which does not deposit on the soil surface, etc. best reflects the plume condition, the energy of the gamma rays emitted from Xe-133 is as low as $81 \mathrm{keV}$. As a result, the detection limit of the concentration is high due to the scattered photons from other nuclides, and it may possibly be zero at a place where the concentration of Xe-133 is low. From this figure, it is estimated that the plume that traveled westwards arrived at a place located approximately $50 \mathrm{~km}$ from Fukushima Daiichi Nuclear Power Plant at around 3:00 p.m., and then reached its highest concentration around 3:25 p.m. Furthermore, the concentration of I-131 dropped to about a quarter of the maximum concentration after 3:50 p.m.

Since it was drizzling during the measurement, it is considered that some radionuclides contained in the plume deposited on the ground surface as the plume passed. Therefore, the concentrations shown in Table 3 are the upper limits of the concentrations of radionuclides contained in the plumes. Since the measurement described in reference 1 used for estimation is not a continuous measurement at a fixed point, it is difficult to estimate the contribution of deposition directly from the measured pulse height distributions. In addition, although it is considered possible to estimate the contribution of the deposition on the soil surface from the difference in the ratio between unscattered and scattered photons (Figure 7), if the measurement is performed over a wide area consisting of a single material, such as soil, it is too difficult to apply this method because the measurement sites located on an expressway are quite different from such conditions. At Adatara SA on the Tohoku Expressway, which is one of the six estimation sites, the measurement was also performed on March 16 (twice), March 17 and April 8. Although the measurements were performed at the same service area, they were not performed continuously at a fixed point. Therefore, we attempted to estimate the influence of deposition from the change in the point of measurement under the assumption that measurements were not conducted at the same exact point. Table 4 shows the measured peak count rates of the respective nuclides, and Figure 10 shows the change over time in the peak count rates of Te-132, I-131, Xe-133, and Cs-134. Since the peak count rates of these nuclides increased on the evening of March 16, it would appear that another plume arrived during this period. It was also found that gaseous I-131 as well as particulate Te-132 and Cs-134 showed different change trends over time. In the case of I-131 and Cs-134, the values for which the half-life was corrected from the values measured on April 8 based on the assumption of $100 \%$ deposition on the soil surface were larger than the values measured on March 17. It is possible

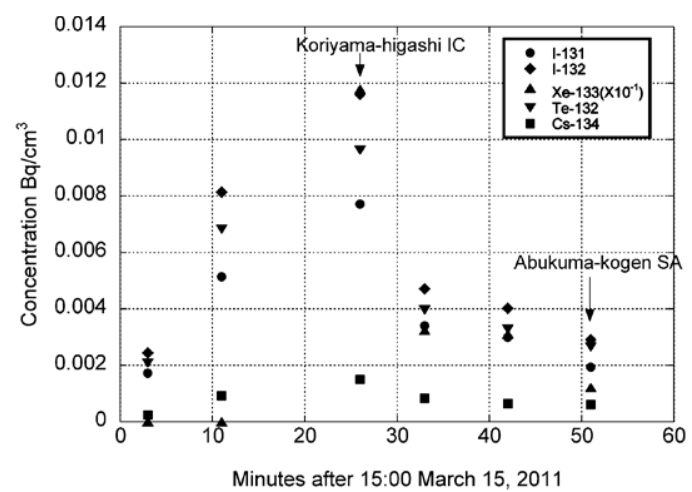

Figure 9 Estimated time variation of concentration 38.3-57.4 km west of the Fukushima Daiichi Nuclear Power Plant between 15:03 and 15:51 in March 15, 2011 
Table 4 Measured peak counts rate at Adatara SA

\begin{tabular}{cccccc}
\hline \multirow{2}{*}{ Nuclide } & \multicolumn{5}{c}{ Measured peak count rates (cps) } \\
\cline { 2 - 5 } & March 15 15:03 & March 16 12:18 & March 16 17:30 & March 17 11:28 & April 8 13:13 \\
\hline Te-132 & $80.1 \pm 3.3$ & $400 \pm 6$ & $660 \pm 7$ & $381 \pm 6$ & $0 \pm 0$ \\
$1-131$ & $47.2 \pm 2.6$ & $16 \pm 4.5$ & $80.9 \pm 5.2$ & $12.2 \pm 4.2$ & $2.2 \pm 0.4$ \\
$1-132$ & $5.45 \pm 0.8$ & $19.1 \pm 1.4$ & $31 \pm 1.7$ & $17.3 \pm 1.4$ & $0 \pm 0$ \\
Xe-133 & $0 \pm 0$ & $0 \pm 0$ & $33.1 \pm 6.4$ & $0 \pm 0$ & $0 \pm 0$ \\
Cs-136 & $1.1 \pm 0.5$ & $2.77 \pm 0.92$ & $6.7 \pm 1$ & $3.97 \pm 0.63$ & $1.18 \pm 0.13$ \\
Cs-134 & $5.3 \pm 0.3$ & $30.3 \pm 0.8$ & $41.2 \pm 0.9$ & $23.4 \pm 0.7$ & $33.1 \pm 0.5$ \\
\hline
\end{tabular}

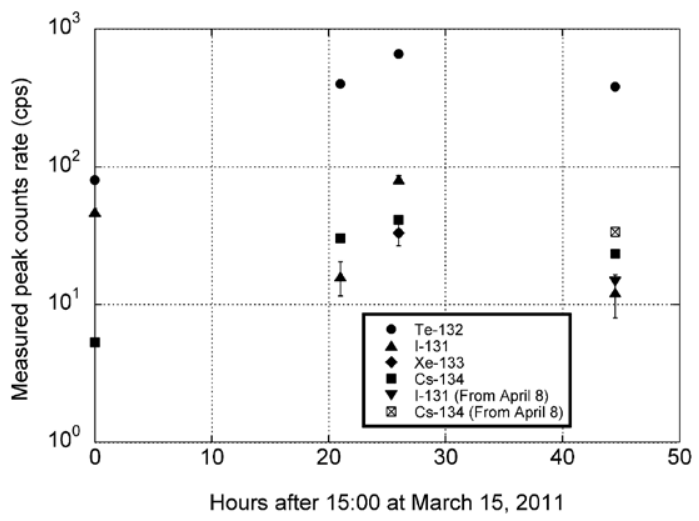

Figure 10 Time variation of measured peak counts rate for Te-132, I-131, Xe-133 and Cs-134 at Adatara SA

that the deposition due to the arrival of the plume on or after March 17 had an influence on this result. Assuming that the result of the measurement performed on March 17 was due to I-131 (which exhibited $100 \%$ deposition), the count rate of I-131 due to the deposition at the time of measurement on March 15 was $13.8 \mathrm{cps}$, which was equivalent to the peak count rate of $29 \%$ measured on March 15.

In the case of Miharu PA and Abukuma-Kogen SA on the Ban-Etsu Expressway, only the measurement result obtained on April 8 was available on and after March 15. The peak count rates of I-131 obtained on March 15 after correcting the half-life were $26.3 \mathrm{cps}$ and $16.0 \mathrm{cps}$, which were $28 \%$ and $30 \%$ of the peak count rates measured on March 15, respectively. As shown in the case of Adatara SA, it is possible that the contribution of the deposition estimated according to this method contained the deposition due to the plume that arrived on and after March 15. Therefore, it would appear that these values are the maximum values caused by the influence of deposition.

Since the measurements at Abukuma-Kogen SA, which are compared in Figure 8, were conducted starting at 3:51 p.m., it would appear that measurements were conducted more than 25 min after the plume with the maximum concentration had passed. Therefore, it can be estimated that the contribution of the radionuclides deposited on the soil surface reached $30 \%$, as shown above. It is thought that this is the cause of the low energy scattering component of the pulse height distributions reconstructed only by the radionuclide in the plume being larger than the measured ones. 


\section{Conclusions}

We estimated the concentrations of radionuclides contained in the plumes when they passed over, from the peak count rates corresponding to the radioisotopes of the $\mathrm{LaBr}_{3}$ detector measured on the expressways inside and outside Fukushima Prefecture on March 15, 2011, and the corresponding peak count rates in the plumes containing the radioisotopes calculated with egs5 at a concentration of $1 \mathrm{~Bq} / \mathrm{cm}^{3}$. The pulse height distributions of the $\mathrm{LaBr}_{3}$ detector reconstructed using the estimated radionuclide concentrations reproduced the measured values almost perfectly at places where the contribution of the deposition was considered to be small, thus the present method is adequate for estimation. From the radionuclide concentrations estimated at six places located 41.7-57.4 km west of Fukushima Daiichi Nuclear Power Plant, we estimated the change over time as the plume passed over. Although it is difficult to estimate the influence of deposition because it is affected by the plumes that arrived later as well as the conditions around the places of measurement, we could estimate that the contribution of the deposition of I- 131 was up to $30 \%$, as a result of considering the values obtained at three locations where measurement was performed at the same place on and after March 15.

Although in this paper we used $\mathrm{LaBr}_{3}$, whose pulse height distribution data were available, it is also possible to apply the same method to a $\mathrm{NaI}(\mathrm{Tl})$ scintillation detector. If the pulse height distribution data obtained at a monitoring post using a $\mathrm{NaI}(\mathrm{Tl})$ scintillation detector were to be available, it would be possible to evaluate the influence of deposition accurately from the change over time in the peak count rates corresponding to respective nuclides. It is expected to estimate the concentration of I-131 in the plume immediately after the accident, as well as the information on the change in its concentration over time, by the pulse height distribution data at a monitoring post together with an atmospheric dispersion model and to use obtained results for estimation of internal exposure by I- 131 .

\section{References}

1) H. Matumura, K. Saito, J. Ishioka, Y. Uwamino, "Diffusion of radioactive materials from Fukushima Daiichi Nuclear Power Station obtained by Gamma-ray measurements on expressways," Trans. At. Energy Soc. Jpn, 10, 152-162 (2011). [in Japanese]

2) Y. Namito, H. Nakamura, A, Toyoda, K. Iijima, H. Iwase, S. Ban, H. Hirayama, "Transformation of a system consisting of plane isotropic source and unit sphere detector into a system consisting of point isotropic source and plane detector in Monte Carlo calculation," J. Nucl. Sci. Technol., 49, 167-172 (2012).

3) H. Hirayama, Y. Namito, A. F. Bielajew, S. J. Wilderman, W. R. Nelson, The EGS5 Code System, SLAC-R-730 (2005) and KEK Report 2005-8 (2005).

4) K. F. Eckerman, J. C. Ryman, External Exposure to Radionuclides in Air, Water, and Soil, Federal Guidance Report No. 12, EPA-402-R-93-081 (Sep. 1993). 


\title{
Article
}

\section{Leaching of Cs and Sr from Sewage Sludge Ash Buried in a Landfill Site}

\author{
Nao ISHIKAWA ${ }^{1, *}$, Ayumi ITO ${ }^{2}$ and Teruyuki UMITA ${ }^{1}$ \\ ${ }^{1}$ Department of Civil and Environmental Engineering, Iwate University, 4-3-5 Ueda, Morioka-shi, Iwate 020-8551, Japan \\ ${ }^{2}$ Department of Frontier Materials and Function Engineering, Graduate School of Engineering, Iwate University, \\ 4-3-5 Ueda, Morioka-shi, Iwate 020-8551, Japan
}

\begin{abstract}
Radionuclide contamination from the nuclear accident at the Fukushima Daiichi Nuclear Power Plant has been found in sewage sludge ash produced in eastern Japan. When such contaminated waste contains less than $8,000 \mathrm{~Bq} / \mathrm{kg}$ radiocesium, it is being disposed in controlled landfill sites. In order to assess the possible spread of the radionuclides by their leaching from the landfill sites, it is important to know the leaching behavior of the radionuclides from the sewage sludge ash and factors influencing the leaching behavior. In this study, leaching experiments using stable $\mathrm{Cs}$ and $\mathrm{Sr}$ were conducted for sewage sludge ash under several conditions to investigate effects of chemical composition of leachate, $\mathrm{pH}$, and solid/liquid ratio on $\mathrm{Cs}$ and $\mathrm{Sr}$ leaching behaviors. In the $\mathrm{pH}$ range from 6 to 12, the leaching ratio of Cs or Sr was less than 5.2 or $0.21 \%$, respectively. Additionally, the leaching ratio of $\mathrm{Sr}$ decreased with increasing $\mathrm{pH}$ of the leachate. In contrast, the higher the $\mathrm{pH}$ in the leachate was, the higher the leaching ratio of Cs was. Finally, possible radionuclide leaching from contaminated sewage sludge ash and then radionuclide concentrations in an actual landfill leachate were assessed. It could be suggested that ${ }^{90} \mathrm{Sr}$ leaching from the landfill site had the least effect on the environment, whereas ${ }^{134+137} \mathrm{Cs}$ leaching needed to be taken into account for spreading radioactive materials from the landfill site to the environment.
\end{abstract}

KEYWORDS: cesium, strontium, sewage sludge ash, leaching, landfill site, $p H$

\section{Introduction}

Radioactive materials were released into the environment during the Fukushima Daiichi Nuclear Power Plant Accident in 2011, and they migrated via various pathways such as water bodies, soil, and living organisms ${ }^{1-4)}$. Therefore, many places are currently suffering contamination problems. It has been reported that radioactive materials flowed into sewers and migrated into sewage sludge which was then converted to sewage sludge ash and condensed ${ }^{5}$, and periodic measurements of radioactive cesium $\left({ }^{134} \mathrm{Cs}\right.$ and $\left.{ }^{137} \mathrm{Cs}\right)$ concentrations in sewage sludge ${ }^{6}$ confirm this occurrence. However, concentrations differ depending on the area and the sewage system involved, and maximum concentrations have been reported as $446,000 \mathrm{~Bq} / \mathrm{kg}$ in Fukushima Prefecture, $1,430 \mathrm{~Bq} / \mathrm{kg}$ in Miyagi Prefecture, and

\footnotetext{
* Corresponding author, E-mail: naoki@iwate-u.ac.jp

DOI : 10.15669 /fukushimainsights. Vol.4.244

(C) 2021 Atomic Energy Society of Japan. All rights reserved.

Originally published in Transactions of the Atomic Energy Society of Japan (ISSN 1347-2879), Vol. 13, No. 3, p.87-93

(2014) in Japanese. (Japanese version accepted: April 14, 2014)
} 
$2,510 \mathrm{~Bq} / \mathrm{kg}$ in Iwate Prefecture ${ }^{7)}$. The government permits the disposal of sewage sludge and sewage sludge ash (after appropriate treatment) with radioactive Cs concentrations under $8,000 \mathrm{~Bq} / \mathrm{kg}$ in existing controlled landfill sites ${ }^{8}$, and landfill disposal of sewage sludge ash containing under $8,000 \mathrm{~Bq} / \mathrm{kg}$ is occurring in accordance with this stipulation.

Leachate water is generated from controlled final disposal sites after rainwater infiltrates and passes through the waste (sludge ash, glasses, ceramics and concrete rubbish) and landfill deposited therein. Radioactivity has been reported in rainwater leachate from waste sites ${ }^{9}$. It is known that the leachate from sewage sludge ash contains much less radioactive Cs than that from general refuse incineration ash ${ }^{10,11)}$. However, the leachate characteristics depend on the type of waste disposed of in the landfill site, and are also related to $\mathrm{pH}$ conditions, which generally range from neutral to alkaline, with high concentrations of salts with cations such as $\mathrm{Ca}^{12-14)}$. To date, there have been no reports on the leachate properties of radioactive materials from sewage sludge ash with respect to alkaline or high salt concentration solutions. This study therefore aims to clarify the $\mathrm{pH}$ dependence (in a range of neutral to alkaline) of the solute content of leachate from sewage sludge ash generated in Iwate Prefecture and to determine the influence of the leachate salt type and solid/liquid ratio during leaching.

The radioactive materials of concern are radioactive $\mathrm{Cs}\left({ }^{134} \mathrm{Cs}\right.$ and $\left.{ }^{137} \mathrm{Cs}\right)$ and radioactive $\mathrm{Sr}$

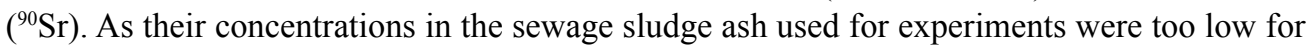
use in conducting direct measurements, their leaching properties were estimated by determining the leaching properties of stable isotopes ${ }^{133} \mathrm{Cs}$ and ${ }^{88} \mathrm{Sr}$.

\section{Experimental Methodology}

\section{Sampling Collection and Method of Analysis}

This study used samples of sewage sludge ash from the Tonan Purification Center in Morioka Iwate Prefecture. The concentrations of Fe, P, and Si in the sewage sludge ash were measured using a sequential X-ray fluorescence spectrometer (Shimadzu, XRF-1800). For other elements, the concentrations of $\mathrm{Cs}$ and $\mathrm{Rb}$ were measured using inductively coupled plasma mass spectrometry (ICP-MS, Thermo, iCAP Qs), and the concentrations of $\mathrm{Al}, \mathrm{Ca}, \mathrm{K}, \mathrm{Mg}, \mathrm{Mn}$, and $\mathrm{Sr}$ were measured using inductively coupled plasma atomic emission spectrometry (ICPAES, Shimadzu, ICPE9000) after decomposing each sample in accordance with the boiling and elution method and using nitric acid and hydrochloric acid in the sewage testing method ${ }^{15}$. The concentrations of elements within the sewage sludge ash are shown in Table 1, where it is evident that the content of $\mathrm{Al}, \mathrm{Ca}, \mathrm{P}$, and $\mathrm{Si}$ was high.

\section{Leaching Experiment}

(1) Experiment to determine leaching equilibrium time

The batch leaching experiment involved conducting the following experiments to determine the time until equilibration was reached in the leaching of Cs and $\mathrm{Sr}$ from sewage sludge ash.

A mixture of $3 \mathrm{~g}$ of sewage sludge ash and $30 \mathrm{~mL}$ of leachate was prepared in a $50-\mathrm{mL}$ polyethylene bottle, then shaken at 25 degrees $\mathrm{C}$ and $120 \mathrm{rpm}$. Ultrapure water, $10^{-4} \mathrm{~mol} / \mathrm{L} \mathrm{NaOH}$ solution, and $10^{-2} \mathrm{~mol} / \mathrm{L} \mathrm{Ca}(\mathrm{OH})_{2}$ solution were used as leaching liquids. After shaking, the samples were collected at various intervals ranging from 30 minutes to 96 hours. The samples were then filtered through a $0.45 \mu \mathrm{m}$ membrane filter, element concentrations in the filtrate 
Table 1 Element concentrations leaching from ash

\begin{tabular}{|c|c|c|c|}
\hline & {$[\mathrm{mg} / \mathrm{kg}$} & & {$[\mathrm{g} / \mathrm{kg}]$} \\
\hline Cs & 0.63 & $\mathrm{~A} 1$ & 39.7 \\
\hline \multirow[t]{8}{*}{$\mathrm{Rb}$} & 8.27 & $\mathrm{Ca}$ & 96.4 \\
\hline & & $\mathrm{Fe}$ & 33.3 \\
\hline & & K & 6.37 \\
\hline & & $\mathrm{Mg}$ & 28.4 \\
\hline & & $\mathrm{Mn}$ & 1.34 \\
\hline & & $\mathrm{P}$ & 89.5 \\
\hline & & $\mathrm{Si}$ & 74.0 \\
\hline & & $\mathrm{Sr}$ & 0.44 \\
\hline
\end{tabular}

were measured using ICP-MS and ICP-AES, and the leaching ratio was calculated using the following equation,

Leaching Ratio [\%]

$$
=\frac{\text { Amount of Element Leaching from Ash }[\mathrm{mg} / \mathrm{kg}]}{\text { Element Content in Ash used for Leaching Experiment }[\mathrm{mg} / \mathrm{kg}]} \times 100
$$

Also, the $\mathrm{pH}$ of the filtrate was measured using a $\mathrm{pH}$ meter (TOADKK, HM-25R).

\section{(2) Leaching experiment}

To clarify the leaching of $\mathrm{Cs}$ and $\mathrm{Sr}$ from sewage sludge ash, a batch leaching experiment was conducted. Three solution compositions, containing either $\mathrm{NaOH}, \mathrm{KOH}$, or $\mathrm{Ca}(\mathrm{OH})_{2}$ were used as the leaching liquids. The several initial molar concentrations (and $\mathrm{pH}$ ) of each were as follows. $\mathrm{NaOH}: 10^{-4}(6.7), 10^{-3}(9.0), 10^{-2}(11.5), 5 \times 10^{-2}(12.3)$ and $10^{-1}(12.6)$; $\mathrm{KOH}: 10^{-4}(6.6)$, $10^{-3}(8.0), 10^{-2}(11.4), 0.5 \times 10^{-1}(12.3)$, and $10^{-1}(12.6)$; and $\mathrm{Ca}(\mathrm{OH})_{2}: 10^{-5}(7.2), 10^{-4}(10.5)$, $10^{-3}(11.9)$, and $10^{-2}(12.9)$. An experiment using ultrapure water as the leaching liquid was also conducted.

A mixture of sewage sludge ash and leaching liquid (in a solid/liquid ratio of 0.1 ( $3 \mathrm{~g}$ : $30 \mathrm{~mL}$ )) was prepared in a $50 \mathrm{~mL}$ polyethylene bottle and then shaken by a shaker at 120 rpm and 25 degrees C. As leaching equilibration was reached within three days after shaking started (in accordance with information presented in Section III-1), the shaking duration was set at three days. After shaking, suction filtration of the sample was conducted using a $0.45-\mu \mathrm{m}$ membrane filter, the element concentration in the filtrate was measured using ICP-MS and ICP-AES, and the leaching ratio was acquired.

Also, the $\mathrm{pH}$ of the filtrate was measured using a $\mathrm{pH}$ meter (TOADKK, HM-25R).

Observations of the influence of the solid/liquid ratio on the leaching ratio were conducted using the $\mathrm{NaOH}$ solution and $\mathrm{KOH}$ solution at $10^{-2} \mathrm{~mol} / \mathrm{L}$ and five varied solid/liquid ratios of sewage sludge ash and leaching liquids in the range of 0.01 to $0.5(0.3 \mathrm{~g}: 30 \mathrm{~mL}, 1.5 \mathrm{~g}: 30 \mathrm{~mL}$, $3 \mathrm{~g}: 30 \mathrm{~mL}, 9 \mathrm{~g}: 30 \mathrm{~mL}, 15 \mathrm{~g}: 30 \mathrm{~mL}$ ). A similar experiment was conducted as described above and the leaching ratio was acquired. 


\section{Results and Discussions}

\section{Determination of Leaching Equilibration Time}

The leaching ratios of $\mathrm{Cs}$ and $\mathrm{Sr}$ in the sample with respect to the shaking duration are shown in Figure 1, where it is evident that a constant leaching ratio was reached in approximately three days. The $\mathrm{pH}$ fluctuations in the samples were determined as follows: for the $\mathrm{NaOH}$ solution, the initial $\mathrm{pH} 7.1$ stabilized at around 6.9 on the third day, and for the $\mathrm{Ca}(\mathrm{OH})_{2}$ solution, the initial pH 11.7 stabilized at around 8.6 within three days. From the above, it was considered that equilibration was reached after a shaking duration of three days.

The decrease in $\mathrm{pH}$ could be attributed to the dissolution of $\mathrm{Al}$ that existed in the sewage sludge ash, which caused the reaction $\mathrm{Al}^{3+}+4 \mathrm{OH}^{-} \rightarrow\left[\mathrm{Al}(\mathrm{OH})_{4}\right]^{-16)}$, or related to $\mathrm{OH}^{-}$consumption by the leaching of $\mathrm{AlPO}_{4}$ existing in the ash by the following reaction ${ }^{17}$,

$$
\mathrm{AlPO}_{4}+4 \mathrm{NaOH} \longrightarrow \mathrm{NaAlO}_{2}+\mathrm{Na}_{3} \mathrm{PO}_{4}+2 \mathrm{H}_{2} \mathrm{O}
$$

\section{Leaching Properties of Cs and Sr}

The leaching ratios of $\mathrm{Cs}$ at the $\mathrm{pH}$ of each leachate are shown in Figure 2, where it is evident that the leaching ratio is generally lower than $6 \%$ between $\mathrm{pH} 6$ and 12, showing that leaching is restricted. Leaching ratios were approximately $0.4 \%$ for all leachates in the neutral $\mathrm{pH}$ range, but $\mathrm{Cs}$ showed a higher leaching ratio tendency with increasing $\mathrm{pH}$. Furthermore,

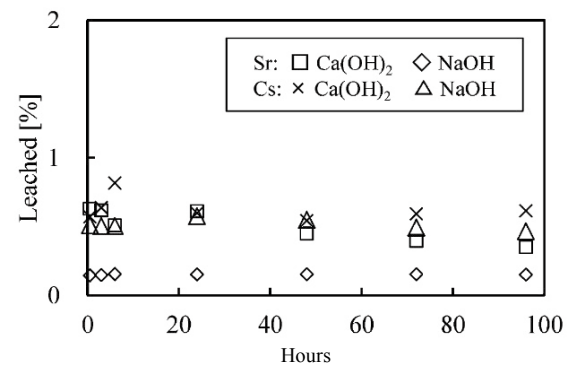

Figure 1 Effect of contact time on the leaching of $\mathrm{Cs}$ and $\mathrm{Sr}$

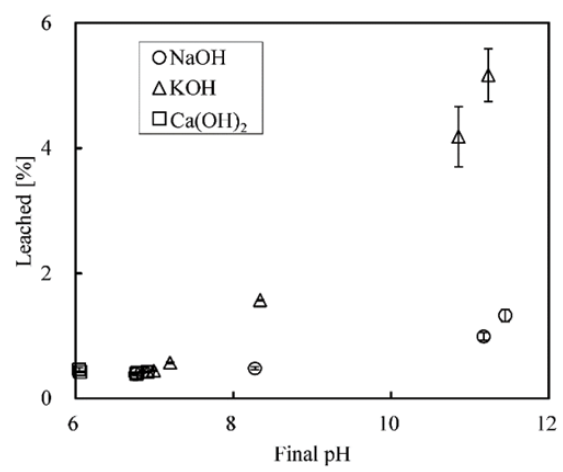

Figure $2 \mathrm{pH}$ dependence of leaching of $\mathrm{Cs}($ solid/liquid ratio $=0.1)$ 
a higher leaching ratio was seen when leached with $\mathrm{KOH}$ solution than with $\mathrm{NaOH}$ solution, even at the same $\mathrm{pH}$. This is because $\mathrm{K}$ has a behavior similar to $\mathrm{Cs}$, and the exchange reaction is occurred between Cs existing in the ash in a comparatively mobile form and $\mathrm{K}$ in the leachate, so that the leaching rate of $\mathrm{Cs}$ may be high if the $\mathrm{K}$ concentration is high. To determine the influence of $\mathrm{K}$ concentration on Cs leaching, the leaching ratio between $\mathrm{K}$ and $\mathrm{Cs}$ with $\mathrm{NaOH}$ was determined by subtraction from that with $\mathrm{KOH}$ at almost equal final $\mathrm{pHs}(\mathrm{pH} \mathrm{6.9,} \mathrm{8.3,}$ and 11.2). The value of $L_{\mathrm{KOH}-\mathrm{NaOH}}$ represents increments of the leaching ratio due to increases in the $\mathrm{K}$ concentration when the influence of $\mathrm{pH}$ is eliminated. Figure 3 shows fluctuations of the $L_{\mathrm{KOH}-\mathrm{NaOH}}$ value related to concentrations of $\mathrm{K}$. Linear regression shows that the rate of increase in the Cs leaching ratio relative to the increase in the $\mathrm{K}$ concentration can be estimated as $0.82 \%$ per $\mathrm{K}$ concentration increment of $0.01 \mathrm{~mol} / \mathrm{L}$ (in the range of $\mathrm{K}$ concentrations lower than $0.05 \mathrm{~mol} / \mathrm{L}$ ).

Figure 4 shows the leaching ratio of $\mathrm{Sr}$ in leachate with varying $\mathrm{pH}$. The leaching ratio of $\mathrm{Sr}$ was lower than $0.3 \%$ between $\mathrm{pH} 6-12$, which is one order of magnitude lower than that of Cs. The $\mathrm{pH}$ dependence in the leaching of $\mathrm{Sr}$ shows a leaching ratio that decreases with increasing $\mathrm{pH}$, unlike the behavior of Cs. The leaching ratio was less than $0.004 \%$ (and almost no leaching was observed) for all types of leachate at $\mathrm{pH} 8$ and above. Also differing from $\mathrm{Cs}$, there were no recognizable differences in the leaching ratios between different salt types of leaching liquids.

Figure 5 shows the leaching ratios of $\mathrm{Cs}$ and $\mathrm{Sr}$ congeners when $\mathrm{NaOH}$ is used for leaching. There are increases in the leaching ratios with $\mathrm{Cs}$ congeners $\mathrm{K}$ and $\mathrm{Rb}$ and increases in $\mathrm{pH}$, but decreases in leaching ratios with congeners $\mathrm{Mg}$ and $\mathrm{Ca}$ and increases in $\mathrm{pH}$, which is similar

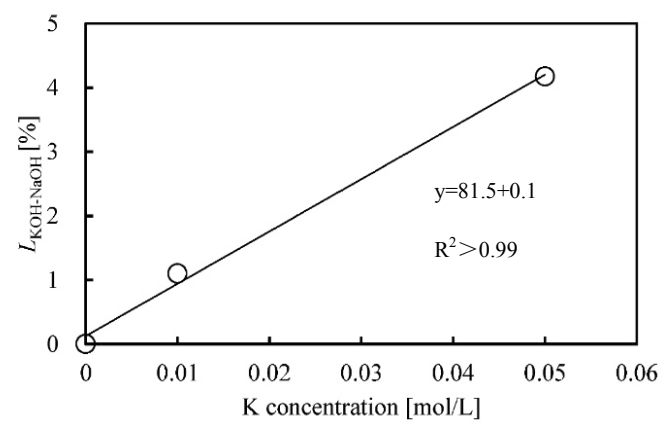

Figure 3 Effect of $\mathrm{K}$ concentration on $L_{\mathrm{KOH}-\mathrm{NaOH}}$ value

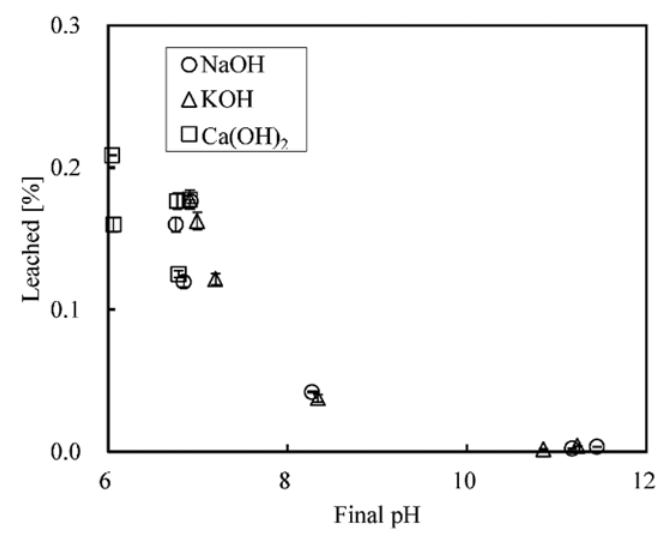

Figure $4 \mathrm{pH}$ dependence of leaching of $\mathrm{Sr}$ ( solid/liquid ratio $=0.1$ ) 
to the behavior or $\mathrm{Sr}$.

$\mathrm{Ca}$ is reported to exist in sewage sludge ash as calcium phosphate $\left(\mathrm{Ca}_{3}\left(\mathrm{PO}_{4}\right)_{2}\right)$ or hydroxyapatite $\left(\mathrm{Ca}_{10}\left(\mathrm{PO}_{4}\right)_{6}(\mathrm{OH})_{2}\right)^{17,18)}$. The solubility products indicate low solubilities of $10^{-26}$ for $\mathrm{Ca}_{3}\left(\mathrm{PO}_{4}\right)_{2}$ and $10^{-108}-10^{-128}$ for $\mathrm{Ca}_{10}\left(\mathrm{PO}_{4}\right)_{6}(\mathrm{OH})_{2}$ and show higher values of still difficult solubilities with increasing $\mathrm{pH}$, with a peak at around $\mathrm{pH} 9{ }^{19}$. $\mathrm{Sr}$ and $\mathrm{Ca}$ show similar behavior, and therefore, $\mathrm{Sr}$ is considered to exist in ash as strontium phosphate $\left(\mathrm{Sr}_{3}\left(\mathrm{PO}_{4}\right)_{2}\right)$, solubility product $10^{-27.4}$. and insoluble under alkaline conditions.

The possibility of Cs existing in sewage sludge ash as an alminosilicate compound (CsAl$\mathrm{Si}_{2} \mathrm{O}_{6}$, etc.) has also been reported ${ }^{11)}$. The leaching ratio of $\mathrm{Al}$ was $0.01 \%$ at $\mathrm{pH} 8.3$, but increased to $14.7 \%$ at $\mathrm{pH} 11.4$ when leached by an $\mathrm{NaOH}$ solution. These results indicate that some of the $\mathrm{Al}$ or $\mathrm{Si}$ within aluminosilicate compounds in the sewage sludge ash was dissolved due to the increased $\mathrm{pH}$, and $\mathrm{Cs}$ was then accordingly leached.

\section{Change in Leaching Ratio and pH in Relation to Solid/Liquid Ratio}

A higher solid/liquid ratio is expected at final disposal sites where precipitation levels are high, as a larger amount of liquid will contact the solid phase over time. To confirm whether the leaching ratio varies with rainfall, we studied the influence of the solid/liquid ratio on the leaching ratio.

The leaching ratios of $\mathrm{Cs}$ and $\mathrm{Sr}$ with respect to the solid/liquid ratio are shown in Figure 6. It is evident that the leaching ratio of Cs decreased with an increase in the solid/liquid ratio, and

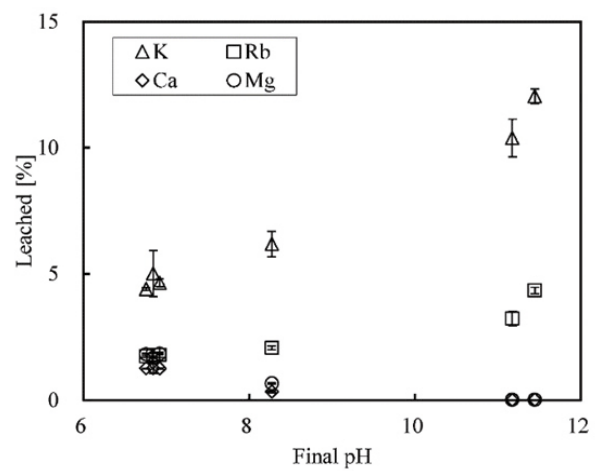

Figure $5 \mathrm{pH}$ dependence of leaching of $\mathrm{K}, \mathrm{Rb}, \mathrm{Ca}$, and $\mathrm{Mg}$ in $\mathrm{NaOH}$ solution

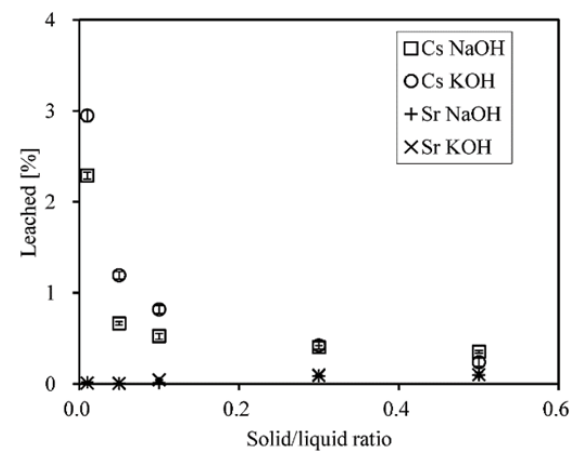

Figure 6 Solid/liquid ratio dependence of leaching of $\mathrm{Cs}$ and $\mathrm{Sr}$ 
the leaching ratio was higher when the solid/liquid ratio was low and when the $\mathrm{KOH}$ solution was used compared to when leaching with the $\mathrm{NaOH}$ solution. However, no differences were seen between the leaching ratios of the two solutions when the solid/liquid ratio was 0.3 and over. The leaching ratio of $\mathrm{Sr}$ differed from that of $\mathrm{Cs}$; although it showed a low leaching rate itself, this amount increased 10 -fold within a solid/liquid ratio range of 0.01 to 0.5 . Furthermore, no difference was observed in leaching ratios when leaching with either $\mathrm{NaOH}$ or $\mathrm{KOH}$ solution.

The $\mathrm{pH}$ decreased with an increase in the solid/liquid ratio; this is considered to be due to the higher $\mathrm{OH}^{-}$consumption with a higher solid phase percentage via the reaction of $\mathrm{Al}^{3+}+$ $4 \mathrm{OH}^{-} \rightarrow\left[\mathrm{Al}(\mathrm{OH})_{4}\right]^{-}$, which occurs when the ash and solution make contact. Additionally, the variation in leaching ratio with various solid/liquid ratios showed a match with the influence of $\mathrm{pH}$ on the leaching ratio for a solid/liquid ratio of 0.1, as shown in Figures 2 and 4, for Cs and $\mathrm{Sr}$ (Figures $\mathbf{7}$ and $\mathbf{8}$ ). Therefore, a study investigating leaching from ash was conducted to separately observe the influences of the solid/liquid ratio and the $\mathrm{pH}$, where the leaching quantity per $1 \mathrm{~L}$ of liquid $\left(L_{1}[\mu \mathrm{g} / \mathrm{L}]\right)$ was first calculated using the Cs leaching ratio $(L[\%])$ when leached at various $\mathrm{NaOH}$ concentrations, and then with a constant solid/liquid ratio of 0.1 . Figures 2 and 4 show the relationship between $\mathrm{pH}$ and the leaching ratio $(L[\%])$ for $\mathrm{Cs}$ and $\mathrm{Sr}$ when leached with the $\mathrm{NaOH}$ solution, and this can be expressed by the following equation as

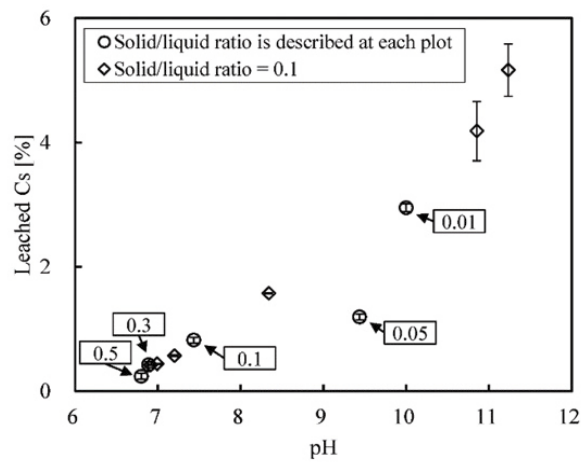

Figure 7 Comparison of leaching of $\mathrm{Cs}$ with $\mathrm{pH}$ at different experimental conditions using $\mathrm{KOH}$ as leaching solution

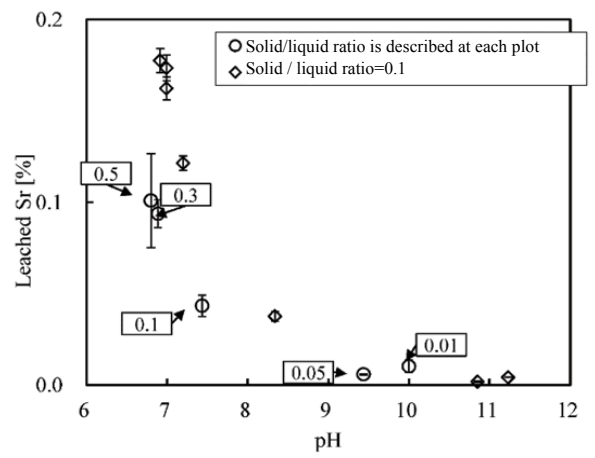

Figure 8 Comparison of leaching of $\mathrm{Sr}$ with $\mathrm{pH}$ at different experimental conditions using $\mathrm{KOH}$ as leaching solution 

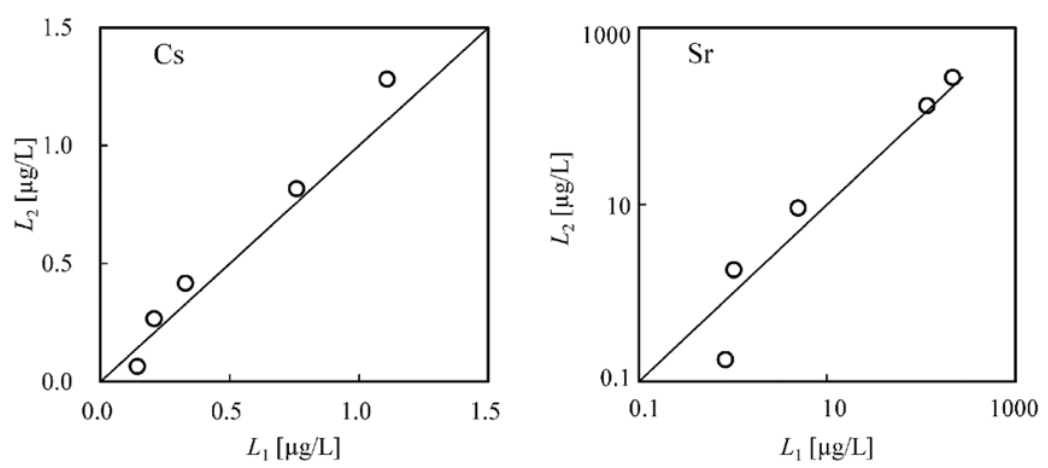

Figure 9 Relationships between $L_{1}$ and $L_{2}$ values

$$
\begin{aligned}
& \text { Cs }: \mathrm{L}=0.076 \mathrm{e}^{0.24 \mathrm{pH}} \quad\left(R^{2}=0.96\right) \\
& \mathrm{Sr}: \mathrm{L}=62.47 \mathrm{e}^{-0.88 \mathrm{pH}} \quad\left(R^{2}=0.98\right)
\end{aligned}
$$

Using the leaching ratio acquired by substituting the equations above with the final $\mathrm{pH}$ at various solid/liquid ratios, the leached quantity $\left(L_{2}[\mu \mathrm{g} / \mathrm{L}]\right)$ per liquid quantity was calculated. The relationship of $L_{1}$ and $L_{2}$ is shown in Figure 9. As the ratios of $L_{1}$ and $L_{2}$ were approximately 1 for both $\mathrm{Cs}$ and $\mathrm{Sr}$, the behavior of leaching from sewage sludge ash can only be explained with respect to the influence of $\mathrm{pH}$. It is thus indicated that $\mathrm{pH}$, rather than the solid/liquid ratio, is the dominant factor in the leaching of $\mathrm{Cs}$ and $\mathrm{Sr}$ from sewage sludge ash.

\section{Quantity Leached from Sewage Sludge Ash in Landfill Site}

Based on this study, the amounts of radioactive $\mathrm{Cs}$ and $\mathrm{Sr}$ leached in leachate, and their concentrations in leachate, were evaluated at a landfill site where sewage sludge ash was deposited. First, the leaching ratio was determined, and the leaching experiment indicated that the leaching ratio of $\mathrm{Cs}$ and $\mathrm{Sr}$ from sewage sludge ash is dependent on $\mathrm{pH}$. In addition to the actual $\mathrm{pH}$ of leachate at landfill sites, which differs greatly between sites, from near $\mathrm{pH} 7^{20)}$ to approximately $\mathrm{pH} 6-8^{14)}$ or $\mathrm{pH} 11.5-11.8^{21)}$, inter-annual variability may have an influence. Accordingly, as an evaluation on the safe side, the highest Cs leaching ratio of $5.2 \%$ (solid/liquid ratio $0.1, \mathrm{KOH} 10^{-1} \mathrm{~mol} / \mathrm{L}$ ) and $\mathrm{Sr}$ leaching ratio of $0.18 \%$ (solid/liquid ratio 0.1 , ultrapure water) were adopted for this calculation.

The upper limit for radioactive Cs in the waste permitted in landfill at existing controlled final disposal sites is $8,000 \mathrm{~Bq} / \mathrm{kg}$. For sewage sludge ash containing radioactive Cs producing $8,000 \mathrm{~Bq} / \mathrm{kg}$, the highest leaching rate $\left(L_{\max }\right)$ in this experiment was $5.2 \%$; therefore, the maximum quantity of leaching radioactive $\mathrm{Cs}$ was $416 \mathrm{~Bq} / \mathrm{kg}$. One study determined that the optimum moisture content $(\mathrm{w}=($ moisture weight $[\mathrm{g}] /$ soil weight $[\mathrm{g}]) \times 100)$ for compacting sewage sludge ash is $58 \%{ }^{22)}$; therefore, the maximum radioactive $C$ s concentration $\left(C_{\max }\right)$ in the leachate water was estimated as $717 \mathrm{~Bq} / \mathrm{L}$ using the equation below

$$
C_{\max }=\frac{8000 \times L_{\max }}{w / 100}
$$

The standard values for radioactive material concentration in landfill effluent are $60 \mathrm{~Bq} / \mathrm{L}$ for ${ }^{134} \mathrm{Cs}$ and $90 \mathrm{~Bq} / \mathrm{L}$ for ${ }^{137} \mathrm{Cs}^{7}$; therefore, our estimated concentration exceeds the effluent standard. Consequently, the possibility of leachate containing a high radioactive Cs concentration 
that exceeds the effluent standard value is indicated if it contains a high concentration of radioactive $\mathrm{Cs}$, even in sewage sludge ash with a low Cs leaching ratio.

Sewage sludge ash is disposed of as landfill as a three-meter-thick swathe that is covered by a soil layer measuring $50 \mathrm{~cm}$ to retain radioactive $\mathrm{Cs}$, or by proper treatment of radioactive Cs and leachate treatment. Using the experimental results and data from existing research, the concentration of radioactive $\mathrm{Cs}$ in the leachate generated from a final disposal site where ash is disposed of as landfill was estimated and studied. Various waste in addition to sewage sludge ash is disposed of at final disposal sites, and therefore leaching from these wastes also needs to be considered. However, data regarding the leaching of ${ }^{133} \mathrm{Cs}$ from other waste, such as cement-type material, has not been reported and is thus difficult to consider. Ishikawa et al. ${ }^{23)}$ made several measurements of ${ }^{133} \mathrm{Cs}$ in leachate at a controlled final disposal site where sewage sludge ash was disposed of as landfill and reported an average of $2.78 \mu \mathrm{g} / \mathrm{L}$, while the concentration of ${ }^{133} \mathrm{Cs}$ leached from sewage sludge ash measured in this research was $3.4 \mu \mathrm{g} / \mathrm{L}$. Furthermore, the concentration of $\mathrm{K}$ (a congener of $\mathrm{Cs}$ that has an adsorbing behavior similar to soil) has been reported as $423 \pm 172 \mu \mathrm{g} / \mathrm{L}^{20)}$ for leachate, while the maximum leaching concentration of $\mathrm{K}$ was $806 \mathrm{mg} / \mathrm{L}$ in the sewage sludge ash acquired in this research. It is considered that the rate of reduction in the Cs or K concentration in leachate occurs when the salt concentration in the leachate is high; the adsorption of Cs or K to soil is low when the leachate infiltrates the soil layer because of competition with other elements present in leachate. The result shows the same order for the leaching concentration, and the concentration in the leachate implies a certain level of reasonability. From the evaluation conducted in this research, ${ }^{133} \mathrm{Cs}$ in leachate is assumed to be derived from sewage sludge ash.

The highest reported ${ }^{133} \mathrm{Cs}$ concentration in sewage sludge ash was approximately $0.85 \mathrm{mg} / \mathrm{kg}^{24)}$, and based on this result, the ratio of ${ }^{133} \mathrm{Cs} /$ radioactive $\mathrm{Cs}$ can be determined to be $0.106 \mathrm{mg} / \mathrm{Bq}$ when sewage sludge ash of $8,000 \mathrm{~Bq} / \mathrm{kg}$ is disposed of as landfill. The maximum concentration of radioactive $\mathrm{Cs}\left(C_{\max }{ }^{\prime}\right)$ in leachate can then be calculated using the formula below,

$$
C_{\max }{ }^{\prime}=\frac{2.78}{0.106}=26.2
$$

$C_{\text {max }}{ }^{\prime}$ was estimated as $26.2 \mathrm{~Bq} / \mathrm{L}$, which does not exceed the effluent standard, and this result indicates that the current prevailing method for retaining radioactive Cs using a soil layer is working.

An increase in the $\mathrm{pH}$ of leachate has been observed in the dry season when there is less rainfall at final disposal sites ${ }^{25}$. The $\mathrm{pH}$ of leachate lowers during heavy rain, and the leaching ratio from ash is thus accordingly lower; therefore, it is not possible for the concentration in the leachate to exceed the level evaluated above.

The effluent standard for radioactive $\mathrm{Sr}$ is not stipulated, but the concentration limit for ${ }^{90} \mathrm{Sr}$ in effluent has been defined as $30 \mathrm{~Bq} / \mathrm{L}$ in an announcement that specifies the dose limits, etc. in accordance with stipulations relating to the installation or operation of nuclear reactors used for experiments and research. With respect to the Fukushima Daiichi Nuclear Power Plant Accident, the ${ }^{90} \mathrm{Sr} /{ }^{137} \mathrm{Cs}$ ratio in contaminated material is estimated as $3 \times 10^{-3}{ }^{26}$ ), and if it is assumed that all of the $8,000 \mathrm{~Bq} / \mathrm{kg}$ of radioactive Cs consists of ${ }^{137} \mathrm{Cs}$, the concentration of ${ }^{90} \mathrm{Sr}$ is thus assumed to be $24 \mathrm{~Bq} / \mathrm{kg}$. The highest leaching ratio of $\mathrm{Sr}$ in this experiment was $0.18 \%$, therefore the leaching rate of radioactive $\mathrm{Sr}$ estimated by this research is $43.2 \mathrm{mBq} / \mathrm{kg}$, and the concentration in the leachate is $74 \mathrm{mBq} / \mathrm{L}$ if the optimum moisture content above is considered. This value is thus lower than the limit concentration in effluent, as explained above. 
This shows that the contribution of ${ }^{90} \mathrm{Sr}$ to the leaching of radioactive materials from the landfill site to the leachate is extremely small (for sewage sludge ash containing radioactive materials), and results indicate that it is only necessary to consider the effects of ${ }^{137} \mathrm{Cs}$.

\section{Summary}

In this research, a series of batch leaching experiments was conducted to determine the leaching properties of $\mathrm{Cs}$ and $\mathrm{Sr}$ from sewage sludge ash. The leaching ratio of $\mathrm{Sr}$ was found to be $0.21-0.004 \%$ with $\mathrm{pH} 6-12$. As with $\mathrm{Ca}$, a higher $\mathrm{pH}$ inhibited sewage sludge ash leaching, and almost no leaching occurred with a $\mathrm{pH}$ of 8 and above. In contrast to $\mathrm{Sr}$, Cs showed an increased leaching ratio with higher $\mathrm{pH}$ : the maximum leaching ratio was $5.16 \%$ with $\mathrm{pH} 11.2$. These results show that it is mainly necessary to consider the leaching of radioactive Cs to prevent excessive radioactive material from escaping in the leachate when sewage sludge ash is disposed of as landfill.

This research was conducted under a JSPS Sciences Research Grant 12014030.

\section{References}

1) Ministry of the Environment, Radionuclide Monitoring Report in Water Environment 2011, available at, http://www.env.go.jp/jishin/monitoring/results_r-pw-h23/1-1.pdf

2) K. Ito, H. Miyahara, T. Ujiie et al., "Practical approach to decontamination of radioactive cesium-contaminated matter in agricultural region by improved wet classification and use of geomaterials," Trans. At. Energy Soc. Jpn. 11, 255-271 (2012). [in Japanese]

3) Ministry of Agriculture, Forestry and Fisheries, Monitoring Results of Strontium in Fisheries Products Conducted by Fisheries Research Agency, available at, http://www.jfa.maff.go.jp/e/inspection/ pdf/131126_strontium.pdf

4) F. Sakamoto, T. Ohnuki, N. Kozai et al., "Determination of local-area distribution and relocation of radioactive cesium in trees from Fukushima daiichi nuclear power plant by autoradiography analysis," Trans. At. Energy Soc. Jpn. 12, 257-266 (2013). [in Japanese]

5) N. K. Ishikawa, A. Ito, T. Umita, "Fate of radiocesium in sewage treatment process released by the nuclear accident at, Fukushima," Chemosphere, 93, 689-694 (2013).

6) Ministry of Economy, Trade and Industry, Immediate Handling of Byproducts from Water and Sewage Treatment etc. that have been Detected as Radioactive Materials, Ministry of Economy, Trade and Industry (2011). Available at http://www.meti.go.jp / press / 2011 / 06 / 20110616006/20110616006-2.pdf. [in Japanese]

7) Ministry of Land, Infrastructure, Transport and Tourism, Report on Radionuclide Concentrations in Sewage Sludge etc., Ministry of Land, Infrastructure, Transport and Tourism (2014). available at, http:// www.mlit.go.jp/mizukokudo/sewerage/crd_sewerage_tk_000168.html. [in Japanese]

8) Ministry of the Environment, Management of Off-site Waste Contaminated with Radioactive Materials Due to the Accident at Fukushima Nuclear Power Stations, Ministry of the Environment (2012). available at, http://www.env.go.jp/en/focus/docs/files/20121128-58.pdf

9) T. Takano, Y. Tamura, Y. Nishizaki et al., "Separation and removal of radiocesium from MSW final disposal site leachate using zeolite," Toshi Seisou, 65, 218-222 (2012). [in Japanese]

10) National Institute for Environmental Studies, Appropriate Waste Disposal Taking into Account Behavior of Radionuclides, National Institute for Environmental Studies (2011). Available at, http://www.env. go.jp/jishin/attach/haikihyouka_kentokai/10-mat_3.pdf. [in Japanese]

11) I. Thushima, M. Ogoshi, "Long term leaching study in sewage sludge contaminated with radioactive materials," J. Jpn. Soc. Water Environ., 50, 108-112 (2013). [in Japanese]

12) S. Urabe, "Treatment of leaching water from landfill disposal of solid wastes," Environ. Conservation Eng., 11, 275-282 (1982). [in Japanese]

13) H. Fukui, T. Yokoyama, "Long-term alteration of water quality in leachate from waste landfills," $J$. Environ. Lab. Assoc., 29, 162-166 (2004). 
14) R.K. Rowe, R. M. Quigley, R. W. I. Brachman, J. R. Booker, Barrier Systems for Waste Disposal Facilities, Second Edition, E\&FN Spon Press, London, 50-62, ISBN 0419226303 (2004).

15) Japan Sewage Works Association, Wastewater Examination Method, vol. 2, Japan Sewage Works Association, Tokyo, 215-216 (1997). [in Japanese]

16) K. Kaikake, T. Sekito, M. Tsunomori, Y. Dote, "Lead stabilization mechanisms of $\mathrm{AlPO}_{4}$ prepared from waste acid etchant in municipal solid waste incineration fly ash," J. Jpn. Soc. Mater. Cycles Waste Manage., 24, 53-62 (2013). [in Japanese]

17) Y. Yoshida, T. Noike, "Influence of temperature on alkaline elution of incinerator ash of sewage sludge," J. Environ. Syst. Eng., 762, 83-90 (2004). [in Japanese]

18) M. Sasaki, Y. Adachi, M. Ika, H. Nishikawa, "Studies on effective use of phosphorus resource obtained from sewage sludge," Rep. Gifu Pref. Res. Inst. Health Environ. Sci., 18, 13-17 (2010). [in Japanese]

19) M. Akiyama, S. Kawasaki, "Fundamental study on new grouting material using calcium phosphate compounds-Crystal precipitation test and unconfined compression test of sand test pieces-," Jpn. Geotech. J., 6, 341-350 (2011). [in Japanese]

20) N. Ishikawa, A. Ito, T. Umita, "Fundamental study about radionuclides removal from landfill leachate," Proc. 50th Environmental engineering research forum, Sapporo, Japan, Nov. 19-21, 2013 (2013).

21) T. Sasajima, K. Kawasaki, Y. Tuchihara, T. Mori, Stabilization of Controlled Landfill of Industrial Waste (X), Bulletin of Toyama Prefectural Environmental Science Research Center, No. 32-2, 32-37 (2004). [in Japanese]

22) M. Kamon, T. Katsumi, T. Inui et al., "Evaluation of geo-environmental impact due to the leachate from solid wastes," Ann. Disas. Prev. Res. Inst., Kyoto Univ., 42 B-2, 459-469 (1999).

23) N. K. Ishikawa, A. Ito, T. Umita, Evaluating Removal of Radionuclides from Landfill Leachate Using Generally Practiced Wastewater Treatment Processes, Radiation Monitoring and Dose Estimation of the Fukushima Nuclear Accident, Springer, Tokyo, 127-134, ISBN 9784431545828 (2014).

24) N. K. Ishikawa, A. Ito, K. Tagami, T. Umita, "Fate of radiocesium in sewage treatment process released by the nuclear accident at Fukushima," Chemosphere, 93, 689-694 (2013).

25) H. Fukui, M. Takahashi, K. Saitoh, H. Sakamoto, Research on the stabilization to judge the abolition of the landfill site, Bulletin of Kanagawa Environmental Research Center, 29, 80-89 (2006). [in Japanese]

26) Ministry of Health, Labour and Welfare, The Philosophy of Contributing Ratio of Radiocesium to Internal Radiation Dose Due by Food Ingestion, Ministry of Health, Labour and Welfare, available at, http:// www.mhlw.go.jp/stf/shingi/2r9852000001w5ek-att/2r9852000001w5je.pdf. [in Japanese] 


\title{
Periodical Public Opinion Survey on Nuclear Energy (Inhabitants Living in the Tokyo Metropolitan Area)
}

\author{
Yoshihiko SHINODA ${ }^{1,2,3, *}$, Shouji TSUCHIDA ${ }^{4}$ and Hiroshi KIMURA ${ }^{5}$ \\ ${ }^{1}$ Wakasa Wan Energy Research Center, 64-52-1 Nagatani, Tsuruga-shi, Fukui 914-0192, Japan \\ ${ }_{2}^{2}$ Nuclear Power and Energy Safety Engineering, University of Fukui, 3-9-1 Bunkyo, Fukui-shi, Fukui 910-8507, Japan \\ ${ }^{3}$ Japan Atomic Energy Agency, Tsuruga Head Office, 65-20 Kizaki, Tsuruga-shi, Fukui 914-8585, Japan \\ ${ }^{4}$ Graduate School and Faculty of Safety Science, Kansai University, 7-1 Hakubai-cho Takatsuki-shi, Osaka 569-1098, Japan \\ ${ }^{5}$ Public Outreach, NPO, 1-1-11 Nedsu, Bunkyou-ku, Tokyo 113-0031, Japan
}

\begin{abstract}
Fukushima Daiichi Nuclear Power Plant Accident (Fukushima Accident) has brought about a great change in many people's perceptions about nuclear power plant safety. When discussing future energy options for Japan, it is important to have a full grasp of the attitude of a large number of people towards nuclear energy. The Atomic Energy Society of Japan has conducted annual questionnaire survey of 500 adults who live within 30 kilometers of Tokyo Station. The aim of this survey is to assess trends in public attitude towards nuclear energy. The authors that designed the questionnaire entries of this survey have been managing questionnaire data as members of the Data Management Working Group under the Social and Environmental Division of the Atomic Energy Society of Japan. We confirmed the change in public attitude towards nuclear energy through this periodical survey after the Fukushima Accident. In particular, public concerns about the use of nuclear energy increased after the Fukushima Accident, and many people have raised doubts over the use of nuclear energy in the future.
\end{abstract}

KEYWORDS: nuclear energy, survey of public opinion, trends in public attitude, Fukushima Daiichi Nuclear Power Plant Accident

\section{Introduction}

An awareness survey called the "Survey on Energy and Nuclear Power" (hereinafter referred to as the "Survey") was conducted each year between 2007 and 2012 to examine and analyze trends in the public's attitudes to energy and nuclear power ${ }^{1,2)}$. The survey was conducted by a special committee (Atomic Energy Society of Japan) that examined the "Development and expansion of a database on mass media reports and public opinion about nuclear power" up until 2011 and has been led by another special committee (Atomic Energy Society of Japan) that has conducted an "Awareness survey and analysis of citizens and professionals" since 2012.

In March 2011, the Great East Japan Earthquake occurred, followed by the accident at the

* Corresponding author, E-mail: shinoda.yoshihiko@plum.plala.or.jp

DOI : 10.15669/fukushimainsights. Vol.4.255

(C) 2021 Atomic Energy Society of Japan. All rights reserved.

Originally published in Transactions of the Atomic Energy Society of Japan (ISSN 1347-2879), Vol. 13, No. 3, p.94-112

(2014) in Japanese. (Japanese version accepted: April 30, 2014) 
Fukushima Daiichi Nuclear Power Plants (hereinafter referred to as the "Fukushima Accident"). The Fukushima Accident caused extensive damage and was thought to have significantly changed people's attitudes to nuclear power, which created the need to continuously explore how nuclear power should be used in the future. Knowing that attitudes and awareness changed before, during and after the accident is useful for discussion within society to explore how nuclear power should be used in the future. It is desirable that a study that seeks to understand these changes in public awareness is based on information obtained by conducting consistent surveys, in line with the method commonly employed in social surveys, over a period of several years both before after the Fukushima Accident.

Regular awareness surveys on whether nuclear power should be used have been performed by various organizations, including the Cabinet Office ${ }^{3)}$, Japan Productivity Center for Socio-Economic Development ${ }^{4)}$ (its name was changed to the Japan Productivity Center in 2009), the Research Council for Energy and Information Technology ${ }^{5)}$ (dissolved in October 2011), the Institute of Applied Energy 6), Institute of Nuclear Safety System Incorporat$\mathrm{ed}^{7}$, and the Japan Atomic Energy Relations Organization ${ }^{8}$. Among the studies that analyzed and discussed changes in attitudes and awareness most probably caused by the impact of the Fukushima Accident are Kitada's ${ }^{9)}$, from the Institute of Nuclear Safety System Incorporated, and Yokote's ${ }^{10)}$, from Japan Atomic Energy Relations Organization. Their findings are based on the results of surveys conducted around the time of the Fukushima Accident.

Kitada discusses the impact of the Fukushima Accident based on the results of fourteen surveys conducted between 1993 and December 2011. She conducted two surveys after the accident targeting residents in areas where the Kansai Electric Power Company (KEPCO) supplies electricity and evaluated the changes in attitudes four and seven months after the accident.

Yokote reported changes in awareness and attitudes based on the results of six nationwide surveys conducted between January 2007 and November 2012. After the accident, surveys were conducted twice, once in November 2011 and again in November 2012. In these surveys, there was no question that directly asked whether nuclear power should continue to be used. Instead, the question was directed at ascertaining if the respondents thought that nuclear energy was necessary and whether they were for or against the use of nuclear power. No survey was published after the accident by the Cabinet Office, the Japan Productivity Center for Socio-Economic Development, the Research Council for Energy and Information Technology, or the Institute of Applied Energy.

However, due to the increased public interest in the trends and status of the national energy policy after the Fukushima Accident, surveys are being actively conducted, mainly by the media. For example, the NHK Broadcasting Culture Research Institute performed telephone surveys in June 2011, October 2011, and March 2012, referred to as the Awareness Survey on Nuclear and Energy ${ }^{11}$, and published the results after the Fukushima Accident. However, since there is no information about public attitudes and awareness before the accident, this limits the extent to which the impact of the accident can be discussed.

In this study, all surveys use the same sampling method and respondents use the same selection method with the focus on understanding changes in attitudes and awareness over time. Five surveys were performed between May 2007 and January 2011 (before the accident) and two surveys were performed after the accident, in January 2012 and January 2013. They were conducted on a regular basis, at almost equal intervals. The surveys include questions that directly ask whether respondents think nuclear power should continue to be used as well as questions designed to ascertain the respondents' attitudes and awareness regarding nuclear power. The responses will be useful in a structural analysis of respondents' attitudes, to identify whether they are for or against the use of nuclear power. From these perspectives, the results of the 
survey and analysis will be useful and very distinctive basic information.

These surveys facilitate discussions on the trends and status of public opinion about longterm nuclear use over a period of several years around the time of the accident. It is one of the few studies that attempts to delve into the respondents' subconscious mind based on a diverse range of questions. The study aims to examine factors that have an impact on the development of a social consensus on nuclear power use from a macro viewpoint by analyzing changes in awareness and attitudes in society, over time, based on the surveys.

\section{Survey Results}

\section{Survey Overview}

The survey participants were selected from the general public, were aged 20 years or older, and lived within a 30-kilometer radius of Tokyo Station. Quota sampling was used in line with the demographic composition of the survey audience and 500 sample results were acquired using the leaving method. The area covered 23 Wards from Tokyo to Tachikawa City, Yokohama City in Kanagawa Prefecture, Saitama City in Saitama Prefecture, and Narashino City in Chiba Prefecture. This is one of the patterns that defines the Tokyo area as a large metropolitan conurbation. In this study, the survey area is referred to as the "Tokyo metropolitan area within a 30-kilometer radius". The survey has been conducted every year and the overview, including the survey periods, is provided in Table 1.

The impact of The Fukushima Accident can be evaluated and analyzed based on the survey results. Moreover, following the sixth survey conducted in January 2013, about two years after the Fukushima Accident, we can obtain information on how public awareness and attitudes have evolved since any initial changes immediately following the accident. Furthermore, in July 2007, between the first survey in May 2007 and second survey in December 2008, the Niigata Chuetsu-oki Earthquake occurred, and we can compare the impact of this earthquake with that of the Fukushima Accident.

The survey conducted as part of this study included questions used in all the other six surveys (hereinafter referred to as repeated questions) and additional or revised questions (hereinafter referred to as topic questions). Some questions do not appear in all the surveys and the form questions take is not necessarily identical; for example, the question sequence of repeated questions may change due to the relation to topic questions. Taking this into account, the study discusses the results of the repeated questions and elucidates the impact of the Fukushima Accident and changes in public attitudes and awareness changes over time of those living within a 30-kilometer radius in the Tokyo metropolitan area.

Table 1 Survey overview of the residents living in the Tokyo metropolitan area

\begin{tabular}{cccc}
\hline \# of times & Survey period & \#of samples & Methods \\
\hline First & May 2007 & 500 & Leaving \\
\hline Second & Niigata Chuetsu-oki Earthquake (July 2007) & Leaving \\
\hline Third & December 2008 & 500 & Leaving \\
\hline Fourth & January 2010 & 500 & Leaving \\
\hline & January 2011 & 500 & Leaving \\
\hline Fifth & Great East Japan Earthquake and the Fukushima Accident & Larch 2011) & \\
\hline Sixth & January 2012 & 500 & 500 \\
\hline
\end{tabular}




\section{Overview and Results of Repeated Questions}

\section{(1) Questions on interest in, promotion, usefulness, and safety of nuclear power}

The common questions in this survey consisted of four items; "level of interest", "need to promote nuclear power", "usefulness/necessity", and "safety/anxiety". They are hereinafter referred to as interest, promotion, usefulness, and safety. Appendix A shows some of the actual questions excerpted from the survey forms. In the question form, we tried to minimize distortion caused by one-sided questions; for example, by using a neutral question text, "Are you interested in nuclear power or not?" we tried to ascertain if the respondent had any interest or not. Then, we provided multiple choice options "(A) I am interested”, "(B) I am somewhat interested”, “(C) Neither”, “(D) I am not particularly interested” and "(E) I am not interested”.

All the questions have five response options provided in the order from positive to negative, including a neutral response.

The letters (A) through (E), prefixed with the answer options, have been added in this study report only to distinguish them and do not appear on the actual survey forms. Tables 2 through 5 show changes over time in the survey results for the four question items. In some questions, the total percentage of the five answer results does not total $100 \%$, due to some invalid answers and rounding errors.

The answer options for these questions are based on an ordinal scale, not on an interval scale, in a strict sense. Or, even if they are on an interval scale, the intervals (difference) between options are not perfectly even. They are bipolar (ensuring the symmetry of response options) 5-point scale options with the neutral response "Neither" as option (C). In this survey, we analyzed the scores assigned to the 5 options, from -2 to 2 , at one-point intervals. In this case, in order to evaluate and analyze the responses as numerical information, a negative score represents a positive direction, a positive score represents a negative direction, and 0 was considered neutral (mid-point). This approach to scoring is referred to as the semantic differential (SD) method and is often used in psychology experiments; accordingly, it can be regarded as a reasonable method in this context also.

Table 2 Responses to the question on respondent's level of interest in nuclear power

\begin{tabular}{|c|c|c|c|c|c|c|}
\hline \multirow{2}{*}{$\begin{array}{l}\text { Level of interest } \\
\text { Options and response rate }\end{array}$} & \multicolumn{4}{|c|}{ Before the Fukushima Accident } & \multicolumn{2}{|c|}{ After the Accident } \\
\hline & First & Second & Third & Fourth & Fifth & Sixth \\
\hline (A) I am interested & $12.6 \%$ & $11.4 \%$ & $10.4 \%$ & $10.4 \%$ & $29.2 \%$ & $27.8 \%$ \\
\hline (B) I am somewhat interested & $29.8 \%$ & $26.4 \%$ & $28.2 \%$ & $27.6 \%$ & $35.4 \%$ & $44.2 \%$ \\
\hline (C) Neither & $35.0 \%$ & $34.4 \%$ & $30.8 \%$ & $29.6 \%$ & $25.8 \%$ & $21.0 \%$ \\
\hline (D) I am not particularly interested & $16.4 \%$ & $20.2 \%$ & $21.2 \%$ & $23.0 \%$ & $7.6 \%$ & $6.2 \%$ \\
\hline (E) I am not interested & $6.0 \%$ & $7.4 \%$ & $9.4 \%$ & $9.4 \%$ & $1.8 \%$ & $0.6 \%$ \\
\hline Invalid answers & $0.2 \%$ & $0.2 \%$ & - & - & $0.2 \%$ & $0.2 \%$ \\
\hline
\end{tabular}

Table 3 Responses to the question regarding the need to promote the use of nuclear power

\begin{tabular}{|c|c|c|c|c|c|c|}
\hline Need to promote nuclear power & \multicolumn{4}{|c|}{ Before the Fukushima Accident } & \multicolumn{2}{|c|}{ After the Accident } \\
\hline Options and Response rate & First & Second & Third & Fourth & Fifth & Sixth \\
\hline (A) Should use & $14.4 \%$ & $13.2 \%$ & $11.2 \%$ & $14.2 \%$ & $5.8 \%$ & $5.6 \%$ \\
\hline (B) May consider using & $28.8 \%$ & $28.6 \%$ & $28.6 \%$ & $29.6 \%$ & $14.8 \%$ & $17.6 \%$ \\
\hline (C) Neither & $39.8 \%$ & $42.0 \%$ & $43.0 \%$ & $42.4 \%$ & $30.4 \%$ & $26.6 \%$ \\
\hline (D) May consider stop using & $13.2 \%$ & $12.6 \%$ & $12.2 \%$ & $11.2 \%$ & $27.0 \%$ & $31.2 \%$ \\
\hline (E) Should stop using & $3.8 \%$ & $3.6 \%$ & $5.0 \%$ & $2.6 \%$ & $21.8 \%$ & $19.0 \%$ \\
\hline Invalid answers & - & - & - & - & $0.2 \%$ & - \\
\hline
\end{tabular}


Table 4 Responses to the question regarding the usefulness/necessity of nuclear power

\begin{tabular}{|c|c|c|c|c|c|c|}
\hline Usefulness/Necessity & \multicolumn{4}{|c|}{ Before the Fukushima Accident } & \multicolumn{2}{|c|}{ After the Accident } \\
\hline Options and Response rate & First & Second & Third & Fourth & Fifth & Sixth \\
\hline (A) I think it is useful & $21.8 \%$ & $17.0 \%$ & $17.2 \%$ & $21.2 \%$ & $11.2 \%$ & $11.2 \%$ \\
\hline (B) I think it is somewhat useful & $37.2 \%$ & $38.4 \%$ & $37.0 \%$ & $40.6 \%$ & $29.6 \%$ & $30.4 \%$ \\
\hline (C) Neither & $34.2 \%$ & $37.8 \%$ & $37.2 \%$ & $32.8 \%$ & $34.8 \%$ & $32.8 \%$ \\
\hline (D) I think it is somewhat unnecessary. & $5.6 \%$ & $4.8 \%$ & $5.6 \%$ & $4.4 \%$ & $13.2 \%$ & $14.2 \%$ \\
\hline (E) I think it is unnecessary & $1.2 \%$ & $2.0 \%$ & $3.0 \%$ & $1.0 \%$ & $11.0 \%$ & $11.4 \%$ \\
\hline Invalid answers & - & - & - & - & $0.2 \%$ & - \\
\hline
\end{tabular}

Table 5 Responses to the question regarding safety/anxiety about nuclear power

\begin{tabular}{|c|c|c|c|c|c|c|}
\hline Safety/anxiety & \multicolumn{4}{|c|}{ Before the Fukushima Accident } & \multicolumn{2}{|c|}{ After the Accident } \\
\hline Options and Response rate & First & Second & Third & Fourth & Fifth & Sixth \\
\hline (A) I feel it is safe & $3.4 \%$ & $2.6 \%$ & $0.8 \%$ & $1.6 \%$ & $0.6 \%$ & $0.4 \%$ \\
\hline (B) I feel it is somewhat safe & $8.6 \%$ & $14.2 \%$ & $14.2 \%$ & $17.0 \%$ & $4.6 \%$ & $5.6 \%$ \\
\hline (C) Neither & $35.0 \%$ & $33.8 \%$ & $34.8 \%$ & $31.6 \%$ & $24.0 \%$ & $20.2 \%$ \\
\hline (D) I feel somewhat anxious & $37.4 \%$ & $36.2 \%$ & $39.0 \%$ & $39.0 \%$ & $35.4 \%$ & $40.0 \%$ \\
\hline (E) I feel anxious & $15.4 \%$ & $13.2 \%$ & $11.2 \%$ & $10.8 \%$ & $35.4 \%$ & $33.8 \%$ \\
\hline Invalid answers & $0.2 \%$ & - & - & - & - & - \\
\hline
\end{tabular}

(2) Questions to ascertain the respondents' level of agreement with statements on the use of nuclear power

Next, in this survey, we provided general statements on the use of nuclear power as answers to questions designed to ascertain the respondents' levels of agreement or disagreement. Some of the actual questions are listed in the Appendix B. In these questions, we provided six answer options to show their level of agreement given in the order from agreement to disagreement and another option to show their lack of opinion/neutrality regarding the statement in question: "I don't know". In the surveys, these questions immediately follow the questions on the respondents' interest in and perception of the safety of nuclear power mentioned in the previous section II-2-(1).

In this study, we discuss the questions based on the responses that "I have confidence in the security consciousness and efforts of those who are engaged in the generation of nuclear power" (hereinafter referred to as "trust"), and "It is dangerous to have nuclear power plants in a country like Japan, which experiences frequent earthquakes" (hereinafter referred to as "risk of earthquake"). It is often reported ${ }^{12)}$ that the sense of trust has a strong direct or indirect impact on attitudes about nuclear power. The above-stated four questions do not include the perspective of trust in nuclear power. Therefore, through the questions on trust in those who are engaged in nuclear power, an attempt was made to evaluate the impact of public trust in nuclear power. Here, we regard public trust in those who are engaged in ensuring the safety (so-called "risk manager") of nuclear power as representing confidence in nuclear power. Moreover, this study focuses on discussing the impact of the Fukushima Accident. We consider that direct questions about the risk of earthquakes are important, and therefore, intend to discuss them in this paper.

Figure 1 shows the changes over time in the response rates for "trust" and "risk of earthquake". In the graph, the answer options (A), (B), (C), (D), (E) and (F) correspond to "I agree", "I somewhat agree", "Neither", "I somewhat agree", "I disagree", and "I don't know", respectively.

The lines that connect each symbol indicating time of measurement $(\boldsymbol{\bullet}, \square$, $\diamond$, etc.) distinguish between respective options. 


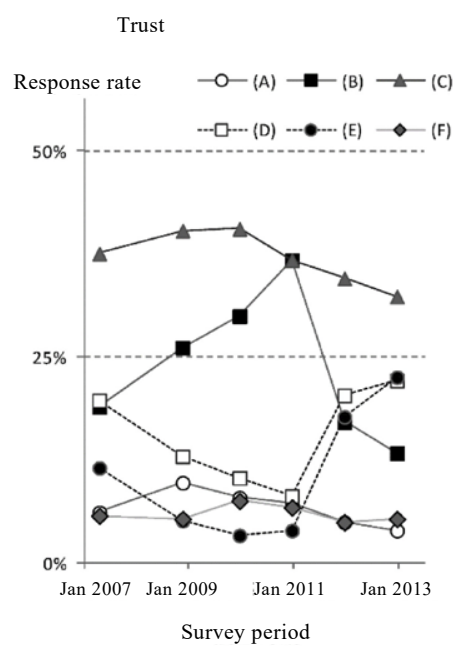

(1) Trust

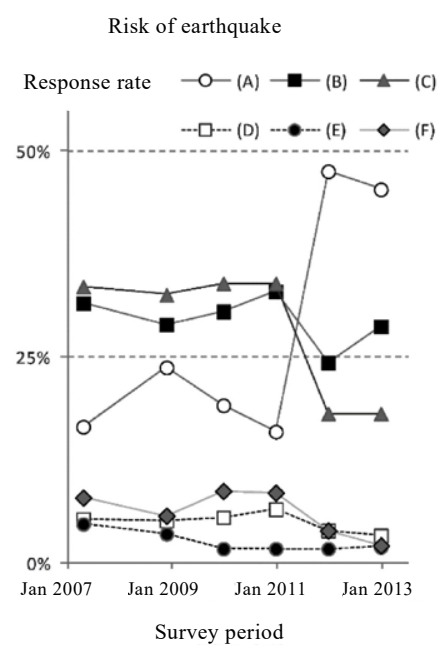

(2) Risk of earthquake

Figure 1 Examples of changes over time in the level of agreement on the use of nuclear power

(3) Questions on matters that respondents are interested in and/or are concerned about in everyday life

Examples of the questions that ask about respondents' interests and concerns about matters in society are provided in Appendix C. These questions included answer options such as Imported food, Sickness, Nuclear power, Nuclear-related accidents, Natural disasters, and Traffic accidents and the respondents had to choose all options that were applicable to them, with no restriction on the number of options they could select. Note that some of the answer options were changed in the course of the study and all surveys did not include exactly the same questions.

However, there is no change in the major topics of the questions, and we handle matters that have not changed as continuously evaluable questions. Figure 2 shows the changes over time in the responses to questions on interest in and concerns about Imported food, Sickness, Nuclear power, and Nuclear-related accidents as representative and characteristic matters of evaluable questions. These questions are provided at the beginning of all the surveys.

\section{Analysis and Evaluation of Survey Results}

\section{Analysis and Evaluation of Repeated Questions}

(1) Analysis and evaluation of awareness and attitudes in relation to interest in, promotion, usefulness, and safety of nuclear power

(a) Analysis and evaluation of survey results

a) Analysis and evaluation of changes in responses

Table 6 shows statistically tested results of changes in the responses between the surveys shown in Tables 2 to 5 . In Table $6,++(--)$ represents the increase (decrease) in the response rate, with $99 \%$ confidence, and $+(-)$ represents the increase (decrease) in response rate, with $95 \%$ confidence.

Each question item showed significant changes around the time of the Fukushima Accident. 


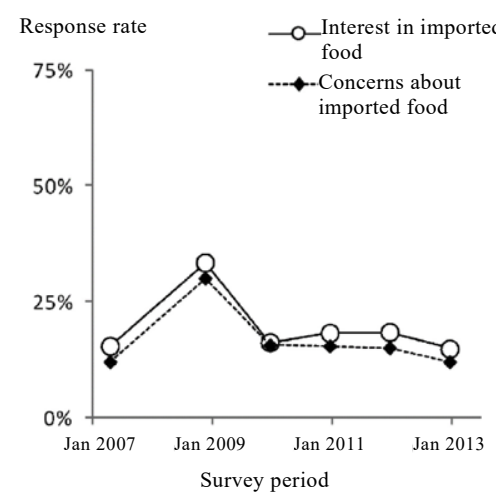

(1) Interest in/Concerns about imported food

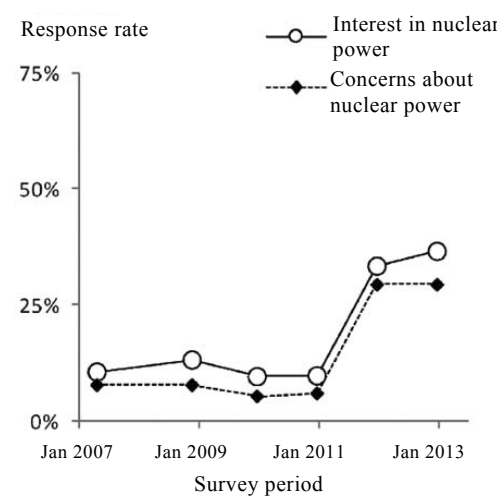

(3) Interest in/Concerns about nuclear power

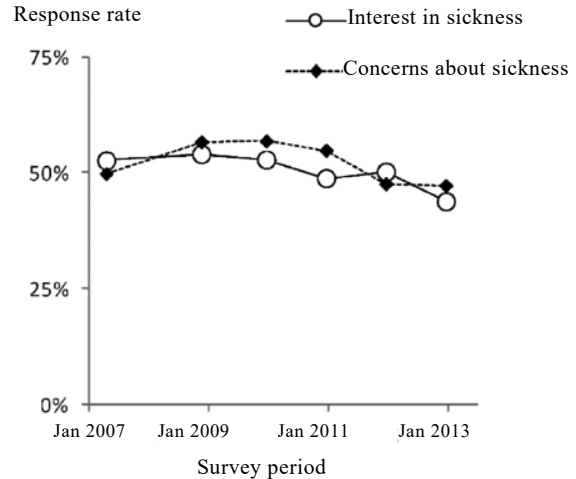

(2) Interest in/Concerns about sickness

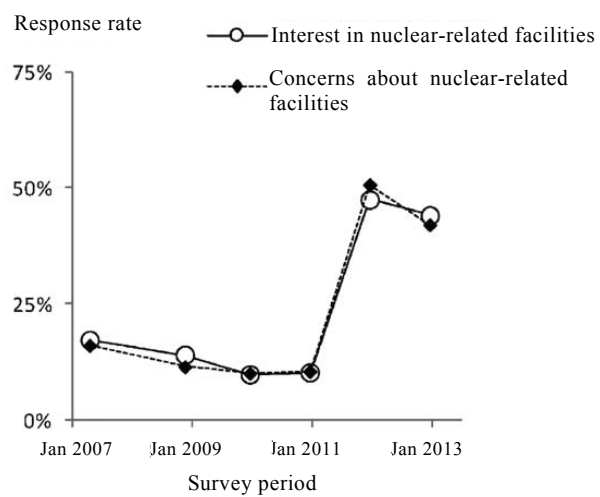

(4) Interests in/Concerns about nuclear-related facilities

Figure 2 Changes over time in response rates on matters that respondents have interest in and concerns about in everyday life

Table 6 Changes in response rates

\begin{tabular}{|c|c|c|c|c|c|c|c|c|c|c|c|}
\hline \multicolumn{6}{|c|}{ (1) Level of interest } & \multicolumn{6}{|c|}{ (2) Need to promote nuclear power } \\
\hline $\begin{array}{l}\text { Test of } \\
\text { significant } \\
\text { differences in } \\
\text { response rate } \\
\text { changes }\end{array}$ & $\begin{array}{c}\text { First } \\
\downarrow \\
\text { Second }\end{array}$ & $\begin{array}{c}\text { Second } \\
\downarrow \\
\text { Third }\end{array}$ & $\begin{array}{c}\text { Third } \\
\downarrow \\
\text { Fourth }\end{array}$ & $\begin{array}{c}\text { Fourth } \\
\downarrow \text { Accident } \\
\text { Fifth }\end{array}$ & $\begin{array}{c}\text { Fifth } \\
\quad \downarrow \\
\text { Sixth }\end{array}$ & $\begin{array}{c}\text { Test of } \\
\text { significant } \\
\text { differences in } \\
\text { response rate } \\
\text { changes }\end{array}$ & $\begin{array}{c}\text { First } \\
\downarrow \\
\text { Second }\end{array}$ & $\begin{array}{c}\text { Second } \\
\downarrow \\
\text { Third }\end{array}$ & $\begin{array}{c}\text { Third } \\
\downarrow \\
\text { Fourth }\end{array}$ & $\begin{array}{c}\text { Fourth } \\
\downarrow \text { Accident } \\
\text { Fifth }\end{array}$ & $\begin{array}{c}\text { Fifth } \\
\quad \downarrow \\
\text { Sixth }\end{array}$ \\
\hline (A) & & & & ++ & & (A) & & & & -- & \\
\hline (B) & & & & ++ & ++ & (B) & & & & -- & \\
\hline (C) & & & & & & (C) & & & & -- & \\
\hline (D) & & & & -- & & (D) & & & & ++ & \\
\hline (E) & & & & -- & & (E) & & & - & ++ & \\
\hline \multicolumn{6}{|c|}{ (3) Usefulness/necessity } & \multicolumn{6}{|c|}{ (4) Safety/anxiety } \\
\hline $\begin{array}{c}\text { Test of } \\
\text { significant } \\
\text { differences in } \\
\text { response rate } \\
\text { changes }\end{array}$ & $\begin{array}{c}\text { First } \\
\downarrow \\
\text { Second }\end{array}$ & $\begin{array}{c}\text { Second } \\
\downarrow \\
\text { Third }\end{array}$ & $\begin{array}{c}\text { Third } \\
\downarrow \\
\text { Fourth }\end{array}$ & $\begin{array}{c}\text { Fourth } \\
\downarrow \text { Accident } \\
\text { Fifth }\end{array}$ & $\begin{array}{c}\text { Fifth } \\
\quad \downarrow \\
\text { Sixth }\end{array}$ & $\begin{array}{c}\text { Test of } \\
\text { significant } \\
\text { differences in } \\
\text { response rate } \\
\text { changes }\end{array}$ & $\begin{array}{c}\text { First } \\
\downarrow \\
\text { Second }\end{array}$ & $\begin{array}{c}\text { Second } \\
\downarrow \\
\text { Third }\end{array}$ & $\begin{array}{c}\text { Third } \\
\downarrow \\
\text { Fourth }\end{array}$ & $\begin{array}{c}\text { Fourth } \\
\downarrow \text { Accident } \\
\text { Fifth }\end{array}$ & $\begin{array}{c}\text { Fifth } \\
\downarrow \\
\text { Sixth }\end{array}$ \\
\hline (A) & & & & -- & & (A) & & - & & & \\
\hline (B) & & & & -- & & (B) & ++ & & & -- & \\
\hline (C) & & & & & & (C) & & & & -- & \\
\hline (D) & & & & ++ & & (D) & & & & & \\
\hline (E) & & & - & ++ & & (E) & & & & ++ & \\
\hline
\end{tabular}

$++(--)$ : Increase (decrease) with $99 \%$ confidence, $+(-)$ : Increase (decrease) with $95 \%$ confidence 
Such changes are probabilistically unlikely to be a coincidence. However, there were only a few significant changes before the accident. Before the Fukushima Accident, option (E) decreased between the third and fourth surveys for both Promotion and Usefulness, option (B) significantly increased in the first and second surveys for Safety, and option (A) decreased between the second and third surveys.

As unique features for changes around the time of the Fukushima Accident, option (C) showed no changes for Interest and Usefulness but there were changes (decreased) for Promotion and Safety, and option (D) (weak negative answer = "I am somewhat concerned") showed no changes for Safety. If we assume that the pattern of changes for option (C) was caused by hesitation, we can say that people no longer hesitated when deciding how to respond to questions regarding Promotion and Safety.

b) Analysis and evaluation by assigning scores to options

Scores were assigned to options, the total scores of each respondents' answers were calculated and the (arithmetic) means and unbiased variances are listed in Table 7. The means represent the positive/negative trends of all respondents; the values and positive/negative directions made the trends in attitudes/awareness easy to understand. Unbiased variances were the measure of degree of dispersion among responses.

For example, although Promotion was assigned negative values up until the fourth survey before the Fukushima Accident, values turned positive in the fifth and sixth surveys. This shows that many people were positive about promoting nuclear power before the Fukushima Accident, but overall attitudes about the promotion of nuclear power shifted in a negative direction

Table 7 Overall survey trends based on scores assigned to answer options (Means and unbiased variances)

(1) Level of interest

\begin{tabular}{|c|c|c|c|c|c|}
\hline & \multicolumn{4}{|c|}{ Before the Fukushima Accident } & \multirow{2}{*}{$\begin{array}{c}\text { After the Accident } \\
\text { Fifth Sixth }\end{array}$} \\
\hline & First & Second & Third & Fourth & \\
\hline Mean & -0.27 & -0.14 & -0.09 & -0.07 & $-0.83-0.92$ \\
\hline $\begin{array}{l}\text { Unbiased } \\
\text { variance }\end{array}$ & 1.14 & 1.20 & 1.28 & 1.30 & $0.99 \quad 0.79$ \\
\hline
\end{tabular}

(2) Need to promote nuclear power

\begin{tabular}{|c|c|c|c|c|c|c|}
\hline & \multicolumn{4}{|c|}{ Before the Fukushima Accident } & \multicolumn{2}{|c|}{ After the Accident } \\
\hline & First & Second & Third & Fourth & Fifth & Sixth \\
\hline Mean & -0.37 & -0.35 & -0.29 & -0.42 & 0.44 & 0.40 \\
\hline $\begin{array}{l}\text { Unbiased } \\
\text { variance }\end{array}$ & 1.01 & 0.96 & 0.98 & 0.91 & 1.33 & 1.31 \\
\hline
\end{tabular}

(3) Usefulness/necessity

\begin{tabular}{|c|c|c|c|c|c|c|}
\hline & \multicolumn{4}{|c|}{ Before the Fukushima Accident } & \multicolumn{2}{|c|}{ After the Accident } \\
\hline & First & Second & Third & Fourth & Fifth & Sixth \\
\hline Mean & -0.73 & -0.64 & -0.60 & -0.77 & -0.17 & -0.16 \\
\hline $\begin{array}{l}\text { Unbiased } \\
\text { variance }\end{array}$ & 0.82 & 0.79 & 0.88 & 0.75 & 1.29 & 1.33 \\
\hline
\end{tabular}

(4) Safety/anxiety

\begin{tabular}{|c|c|c|c|c|c|c|}
\hline & \multicolumn{4}{|c|}{ Before the Fukushima Accident } & \multicolumn{2}{|c|}{ After the Accident } \\
\hline & First & Second & Third & Fourth & Fifth & Sixth \\
\hline Mean & 0.53 & 0.43 & 0.46 & 0.40 & 1.00 & 1.01 \\
\hline $\begin{array}{l}\text { Unbiased } \\
\text { variance }\end{array}$ & 0.94 & 0.95 & 0.81 & 0.89 & 0.83 & 0.80 \\
\hline
\end{tabular}


after the accident. Significant changes from positive to negative were also seen in relation to Promotion. Also, the changes in unbiased variances showed that, although the variation in attitudes was becoming less (meaning neutral attitudes increased and strong negative/positive attitudes decreased) before the Fukushima Accident, this variation became more pronounced after the accident (meaning neutral attitudes increased and negative attitudes decreased). The mean of Safety, which was a positive value reflecting a tendency towards anxiety, increased because of the Fukushima Accident and demonstrated that there was a tendency towards higher levels of anxiety. There was only a negligible change in unbiased variances before and after the accident, indicating that there was no significant change in variance in attitudes.

Table 8 shows the results of statistically tested changes (expressed as the mean value). Before the Fukushima Accident, the means of Promotion and Usefulness declined significantly between the third and fourth surveys. It shows that prior to the accident, people were positive about using nuclear power and strongly agreed that nuclear power was useful. All question items changed significantly before and after the accident.

There were no significant changes between the fifth and sixth surveys and the changes that did occur seem to be the result of the Fukushima Accident.

c) Summary of analysis and evaluation of the survey results around the time of the Fukushima Accident

A description of the changes between the fourth survey conducted immediately before the Fukushima Accident and the fifth survey conducted after the accident follows. The survey analysis ${ }^{13)}$ performed by Shinoda and others revealed that the evaluations of the strength of positive and negative answers and neutral answers are important and, in this study, each answer option was examined carefully.

(1) Interest: Respondents' interest increased. Option (A) increased by approximately $20 \%$ and option (D) decreased by approximately $15 \%$. The variation in interest decreased.

(2) Promotion: The mean shifted from positive to negative. Option (E) increased by $20 \%$ and option (C) decreased. The variation in this attitude increased.

(3) Usefulness: Fewer respondents came to feel that nuclear power was useful, but no respondents thought it had no utility at all. Both options (A) and (B) decreased by approximately $10 \%$ and options (D) and (E) increased by approximately $10 \%$. No change was observed in option (C). The variation in this attitude increased.

(4) Safety: The tendency to be anxious was further accentuated. The mean of option (A) had been low since before the accident and hardly changed. The means of options (B) and (C) decreased. That of option (D) did not change and that of option (E) increased by approximately $25 \%$. The changes in variation in the attitude to safety were small.

The changes in unbiased variances were due to the distribution leaning towards positive

Table 8 Changes in means based on scores assigned to answer options

\begin{tabular}{cccccc}
\hline & $\begin{array}{c}\text { First } \\
\downarrow \\
\text { Second }\end{array}$ & $\begin{array}{c}\text { Second } \\
\downarrow \\
\text { Third }\end{array}$ & $\begin{array}{c}\text { Third } \\
\downarrow \\
\text { Fourth }\end{array}$ & $\begin{array}{c}\text { Fourth } \\
\downarrow \text { Accident } \\
\text { Fifth }\end{array}$ & $\begin{array}{c}\text { Fifth } \\
\downarrow \\
\text { Sixth }\end{array}$ \\
\hline Level of interest & & & -- & \\
\hline Need to promote nuclear power & & & - & ++ \\
\hline Usefulness/necessity & & -- & ++ \\
\hline Safety/ anxiety & & & ++ \\
\hline
\end{tabular}

Welch's t-test for changes in means

$++(--)$ : Increase (decrease) with $99 \%$ confidence

$+(-)$ : Increase (decrease) with $95 \%$ confidence 
responses, options (A) and (B) (=I am interested) for Interest, wide distribution with decreased weak positive responses, option (B) for Promotion and Usefulness, and the whole distribution shift in a negative direction for Safety. It can be assumed that more respondents who initially had a neutral response to the statement about safety, started responding more negatively.

Only the number of those who chose option (B) for Interest increased significantly following the Fukushima Accident, which indicates that the changes due to the accident are likely to increase even further. No significant change was seen in other options and means, which suggests the changes that appear to be due to the Fukushima Accident have not yet returned to their previous status. This trend does not coincide with the evaluation ${ }^{10)}$ conducted and summarized by the Japan Atomic Energy Relations Organization, which concluded that the objection to the use of nuclear power would decrease and shift towards a neutral attitude. On the other hand, it conformed to the evaluation conducted by Hirose ${ }^{14)}$, who found a continuing negative trend after the Fukushima Accident.

In July 2007, between the first and second surveys, the Niigata Chuetsu-oki Earthquake (hereinafter referred to as the Chuetsu-oki Earthquake) occurred, followed by a fire in a transformer at the Kashiwazaki Kariwa Nuclear Power Plant Unit 3, all of which were widely reported in the media including the publication of aerial photos. Although the impact of the earthquake is not prominently reflected in the analysis results for Interest and Promotion, the responses rate for Safety slightly increased. We can assume that changes in attitudes/awareness occurred due to the size of the earthquake and post-earthquake status.

(b) Analysis and evaluation of relations

a) Analysis and evaluation of correlation coefficients

First, to understand the relation between the four question items (Interest, Promotion, Usefulness, and Safety), we performed correlation analysis based on the numerical information obtained by assigning scores to answer options. Figure 3 shows the changes in correlation coefficients between each question item over time. The error bars in the graphs indicate the $99 \%$ confidence interval of the correlation coefficients. Table 9 shows the statistically tested results of the differences (changes) in correlation coefficients. The changes between the fourth and fifth surveys are assumed to be due to the impact of the Fukushima Accident. Changes before the
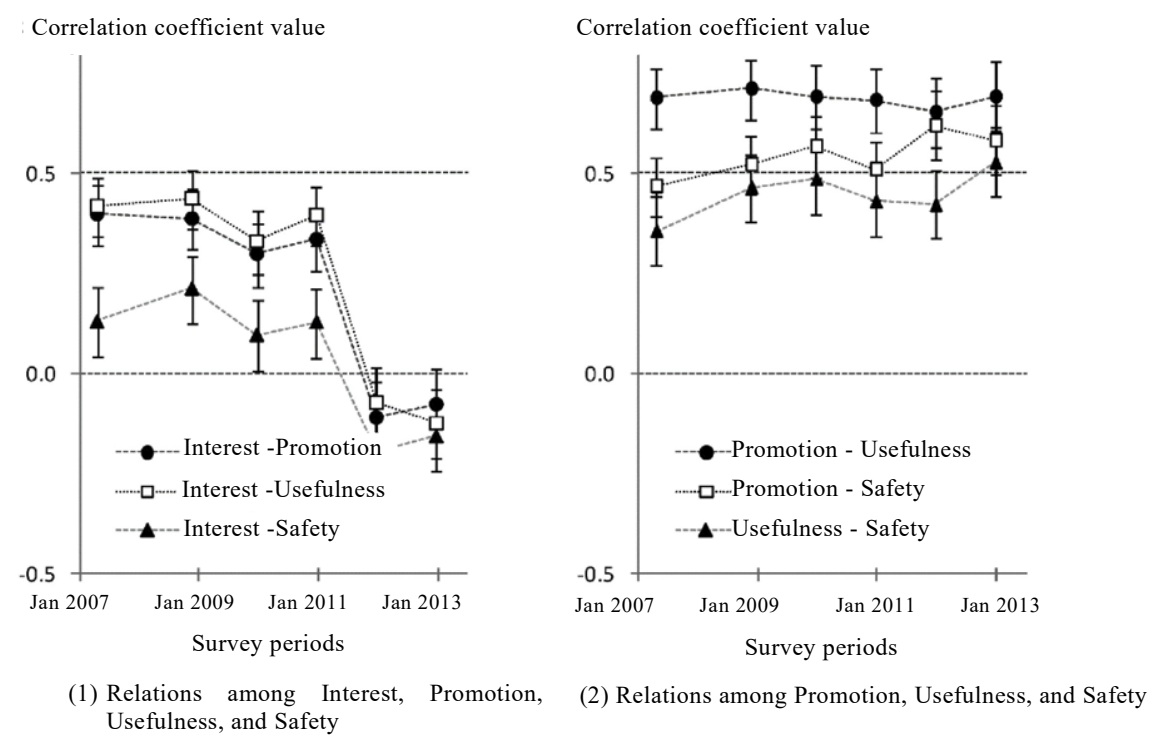

Figure 3 Changes in correlation coefficients between question items over time 
Table 9 Differences in correlation coefficients between question items

\begin{tabular}{cccccc}
\hline & $\begin{array}{c}\text { First } \\
\downarrow \\
\text { Second }\end{array}$ & $\begin{array}{c}\text { Second } \\
\downarrow \\
\text { Third }\end{array}$ & $\begin{array}{c}\text { Third } \\
\downarrow \\
\text { Fourth }\end{array}$ & $\begin{array}{c}\text { Fourth } \\
\downarrow \text { Accident } \\
\text { Fifth }\end{array}$ & $\begin{array}{c}\text { Fifth } \\
\downarrow \\
\text { Sixth }\end{array}$ \\
\hline Interest-Promotion & & & $* *$ & \\
\hline Interest-Usefulness & & $*$ & $* *$ \\
\hline Interest-Safety & & & $*$ \\
\hline Promotion-Usefulness & & & $*$ \\
\hline Promotion-Safety & $*$ & & $*$ \\
\hline Usefulness-Safety & & & \\
\hline
\end{tabular}

Test for differences in correlation coefficients

$* *(*)$ : Significant with $99 \%(95 \%)$ confidence

accident are small. Changes between the fifth and sixth surveys, which were conducted one and two years after the accident, are not significant except for the categories Usefulness and Safety.

In Figure 3 (1), which shows changes in relation to Interest, over time, there were changes from positive to negative correlations around the time of the Fukushima Accident in relation to Promotion, Usefulness and Safety. After the Fukushima Accident, the correlation coefficients between Interest and Usefulness $(\square)$ in the fifth survey and those between Interest and Promotion (๑) in the sixth survey are not statistically significant and their relationship is lost. Other relationships have statistically significant correlation coefficients and are related to each other.

In Figure 3 (2), which shows changes in the relations among Promotion, Usefulness, and Safety, correlation coefficients are significant throughout all surveys and all are related. The relation between Promotion and Usefulness $(\bullet)$ is especially strong and maintains a constant level throughout all surveys. Only the correlation coefficient between Promotion and Safety $(\square)$ around the time of the Fukushima Accident and those between Usefulness and Safety ( $(\mathbf{\Delta})$ after the accident show a significant increase.

b) Analysis and evaluation of partial correlation coefficients

It is hard to evaluate relations simply by means of correlation coefficients when the relations between question items are complex. As for relations between multiple question items that are related to each other, relations between only question items can be found by obtaining partial correlation coefficients. Figure 4 describes the changes in partial correlation coefficients over time.

The partial correlation coefficients between Interest and Safety (A) shown in Figure 4 (1) were negative across all the surveys and the level of interest and strength of concerns were related. The values were close to zero in the first, second, and fourth surveys; no statistical relations were observed and there was no significant relation before the Fukushima Accident. However, significant relations were evident in the fifth and sixth surveys after the accident. The changes in the relation between Interest and Usefulness $(\square)$ were significant after the Fukushima Accident and it continues to increase afterward. Although the level of interest in nuclear power and the degree to which the public saw nuclear power as useful tended to be related before the Fukushima Accident, the relation was lost and started to reverse after the accident. The relation between Interest and Promotion ( ) was lost after the Fukushima Accident, which shows that there was a change in the level of public interest.

The partial correlation coefficient values between Promotion, Usefulness, and Safety, shown in Figure 4 (2), are mostly significant and relations are observed between the question items. However, the partial correlation coefficient values between Usefulness and Safety $(\boldsymbol{\Delta})$ in the first and fifth surveys are not significant.

Another prominent feature is that the relation between Promotion and Usefulness 


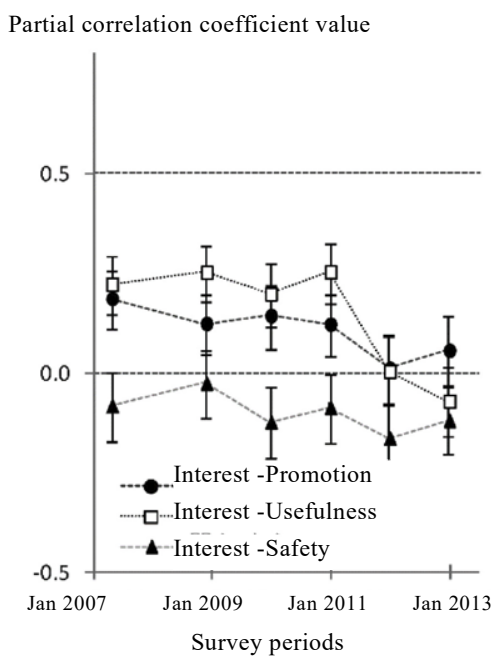

(1) Relations among Interest, Promotion, Usefulness, and Safety

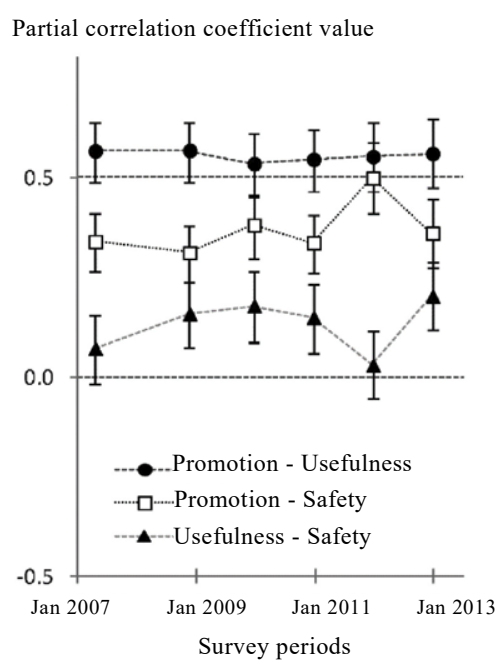

(2) Relations among Promotion, Usefulness, and Safety

Figure 4 Changes in partial correlation coefficients between question items over time

maintains a certain level throughout all the surveys and Need to promote nuclear power and Usefulness/necessity are strongly related. Around the time of the Fukushima Accident, the relations between Promotion and Safety $(\square)$ and Usefulness and Safety increased and then decreased. However, as for the changes over time in the fifth and six surveys after the accident, the changes in relations due to the Fukushima Accident returned to the statuses before the accident. The feeling of safety increased slightly around the time of the Chuetsu-oki Earthquake and the correlation between Usefulness and Safety became strong, which indicates a change in awareness and attitudes caused by the earthquake.

(c) Analysis and evaluation by principal component analysis (PCA)

PCA is a statistical method used to extract common components from multidimensional observed variables (actually measured variables; here they refer to survey results) and newly obtained non-correlated composite variables. The extracted components are called principal components. They are ranked in the order of how well they account for (contribution rate) all the information included in question items. The loads of principal components are between -1 and 1 and indicate the impact (weight) of observed variables against the principal components. Because the sum of the observed variables multiplied by the loads of principal components is principal components (composite variables), it allows us to establish a new standard where information is condensed and explore the structure of observed variable groups. This analysis allows us to deepen the above stated correlation analysis further. PCA is formulated as follows and principal components are represented by observed variables.

jth principal component

$=($ Load of the jth principal component of observed variable 1$)$

$\times($ Observed variable 1$)$

+ (Load of the jth principal component of observed variable 2$)$

$\times$ (observed variable 2$)$

$+($ Load of the jth principal component of observed variable $\mathrm{N})$

$\times($ Observed variable $\mathrm{N})$ 
$\mathrm{jth}$ Principal component ( $\mathrm{j}=1 \sim \mathrm{L}: \mathrm{L}$ matches the number $\mathrm{N}$ of observed variables at most)

a) Promotion, usefulness and safety of nuclear power

First, for three question items (Promotion, Usefulness and Safety) we performed analysis using the numerical information obtained by assigning scores to answer options. The number of observed variables becomes $\mathrm{N}=3$. Figure 5 shows the changes over time in the loads and contribution rates of the first to third principal components.

For the first principal component for which the contribution rate is the largest at approximately $70 \%$ throughout all the surveys, the three question items line up in the same direction. It means the three question items move in the same positive/negative directions and this connects the attitude of being "positive about promotion/usefulness/safe" and the attitude of being "negative about promotion/unnecessary/anxious". These variables indicate the public's attitude (for or against) to nuclear power by representing their feelings either for or against its use. The responses to questions about Promotion, Usefulness, and Safety account for approximately $70 \%$ of the public's attitude (for/against) to nuclear power; the load of the principal component for Safety decreased after the Fukushima Accident.

The second principal component shows an approximately $20 \%$ contribution rate, where Usefulness and Safety move in opposite directions in all the surveys. The direction of Promotion is reversed and moves in the same direction as Safety around the time of the Fukushima Accident. As for the second principal component, the attitude to the promotion of nuclear power does not directly reflect the feeling of being either for or against it. The second principal component represents a dissociation between the attitude to promotion and the feeling of being for or against nuclear power per se. We can see that the second principal component is a variable that represents the conflict between usefulness and anxiety and indicates ambiguity (in people's stances). From this perspective, although people were anxious before the Fukushima Accident, their attitude regarding the usefulness of nuclear power was linked to their attitude regarding its promotion.

However, after the accident, people's anxiety resulted in an increasingly negative attitude

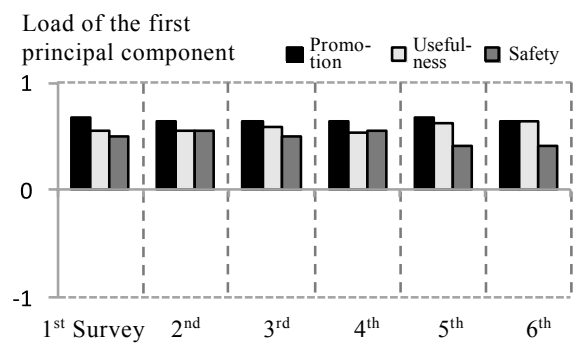

(1) Load of the first principal component

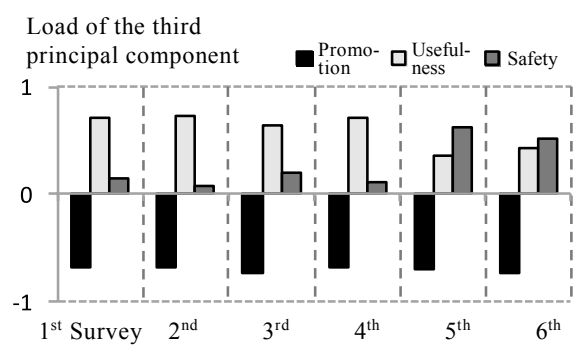

(3) Load of the first principal component

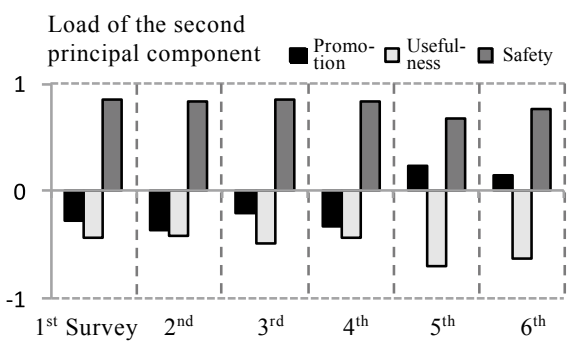

(2) Load of the first principal component

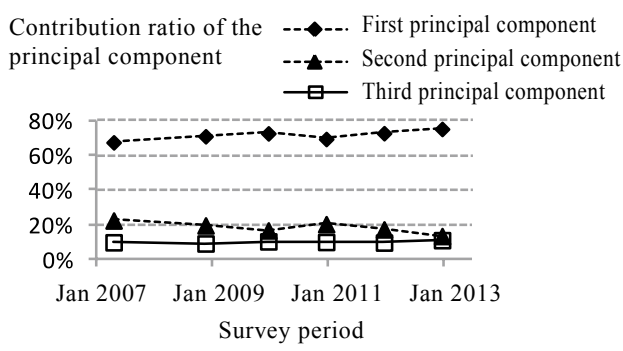

(4) Changes in contribution ratio over time

Figure 5 Principal component analysis results between three question items (based on load of principal component and contribution rate) 
toward the promotion of nuclear power. This is a reversal of the attitude that they would like to "promote nuclear power because it is useful although we have concerns" to they would like to "phase out nuclear power because it is not safe, even if it is useful". The contribution rate decreased after the accident and accompanied by a declining trend is seen in people's recognition of ambiguity. The decline in ambiguity is in response to a strengthening of the relations among "perception of usefulness/uselessness", "feeling of safety/anxiety towards nuclear power", and "need to promote nuclear power".

The third principal component has a less than $10 \%$ contribution rate, where Promotion and Usefulness and Safety move in opposite directions. Although Usefulness was assigned a high value and Safety a low value before the Fukushima Accident, they have both increased in the same direction following the accident. The first principal component shows that the awareness structure is directly linked to the attitude of being for or against the use of nuclear power; "As I think nuclear power is useful and safe and I would like the government to use it, therefore I am for = positive about nuclear power" or "I think the use of nuclear power is dangerous and I feel anxious and I would like the government to stop using it, therefore I am against = negative about nuclear power". It also shows that the perception of usefulness (necessity) and feelings of safety (anxiety) have become the major determining factors of the attitude (for or against) to the use of nuclear power. On the other hand, the third principal component shows an awareness structure that reflects the following attitude to nuclear power; "Although I think nuclear power is useful and safe, I am opposed to its use" or "Although I think nuclear power is dangerous and I feel anxious, I support its use", which are clearly contradictory and they cannot be understood in a logical manner. We can assume that it also suggests that other determining factors have had an impact.

b) Interest in and promotion, usefulness, and safety of nuclear energy

Next, we performed a similar analysis adding Interest into the question items; the results are shown in Figure 6. The impact of interest was evident in the first principal component, which

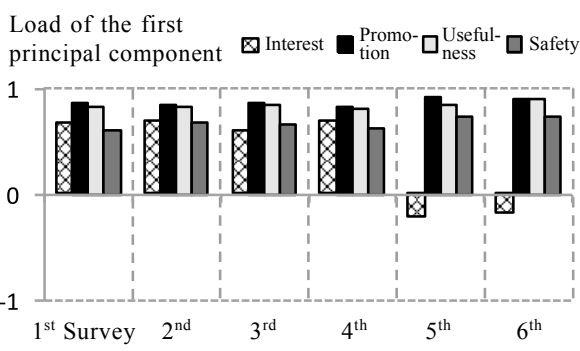

(1) Load of the first principal component

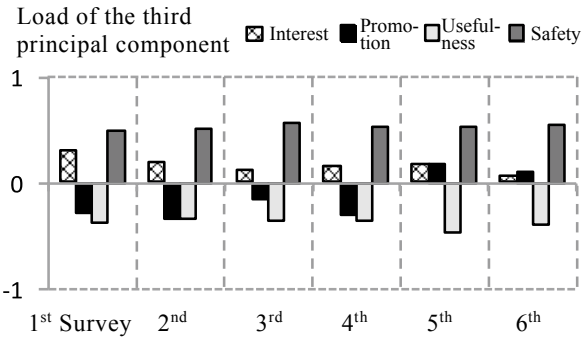

(3) Load of the third principal component

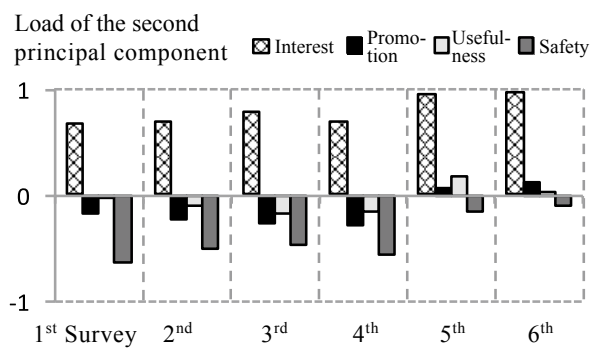

(2) Load of the second principal component

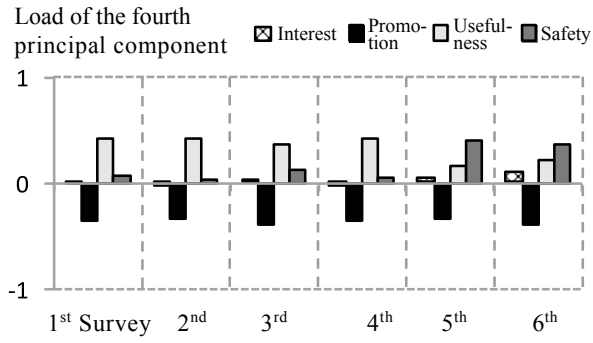

(4) Load of the fourth principal component

Figure 6 Analysis results of principal components between four question items (load of principal component) 
reflects the attitude of being either for or against the use of nuclear power. The four question items were oriented in the same direction before the Fukushima Accident, but the direction of Interest was reversed with small values after the accident. This reversal indicates that the relation between the attitude of being for or against nuclear power and the level of interest in nuclear power observed before the Fukushima Accident became weak after the accident. The growing interest after the accident indicates that people's interest is no longer related to whether they are for or against nuclear power and it supplements the analysis results by partial correlation coefficients.

In the second principal component, Interest received high scores in all the surveys. The scores are values that indicate the strength of public interest in nuclear power without factoring in the impact of being either for or against it as indicated by the first principal component. Before the Fukushima Accident only Interest was oriented in a direction opposite to that of the other question items. The values for Interest and Safety are higher than those of the others; thus, it can be concluded that anxiety leads to interest. Scores other than those for Interest decreased and only Safety was oriented in the opposite direction and registered low scores after the Fukushima Accident. This indicates that interest led by anxiety, regardless of respondent's attitude towards nuclear power, became low after the accident.

The pattern of the third principal component was similar to that of the second principal component based on an analysis of the three question items. Both Interest and Safety were oriented in the same direction and registered low scores. The pattern of the fourth principal component was similar to that of the third principal component based on an analysis of the three question items.

(d) Analysis and evaluation by quantification method III

Quantification method III enabled analysis even though it was not an interval scale and captured the relations between answer options and respondents. It calculated several axes that categorized options' features and that were not related to each other based on the relations of response, and provided scores that represented the features of each axis.

In the previous reports released by the Institute of Nuclear Safety System, Incorporated ${ }^{7)}$ and Shinoda ${ }^{13)}$, quantification method III was used to analyze attitudes regarding the use of nuclear power and interesting findings were obtained. As with principal component analysis, the axes were ranked in the order of how well they accounted for (contribution rate) all the information included in the question items.

Quantification method III is different from principal component analysis in that it analyzes without assigning scores. Compared to the PCA, in quantification method III, the contribution rates of calculated variables were relatively low, and interpretation of results is difficult. However, it provides information for multiple answer options. Exploring the relations between answer options allows the study of the respondents'.

First, three question items were analyzed (Promotion, Usefulness, and Safety). If options scores are plotted on a plane having two axes with high contribution rates, the trends (statistically similar tendency of trends) in the responses to questions can be captured. The contribution rates of the first and second axes were approximately $20 \%$ and $15 \%$, respectively.

Figure 7 shows the results of the third survey before the Fukushima Accident and fourth survey shows the results after the accident, which represent the features of the results around the time of the Fukushima Accident. The letters $\mathrm{P}, \mathrm{U}$, and $\mathrm{S}$ respectively represent the question items Promotion, Usefulness, and Safety and A to E represent the answer options (A) to (E) in Tables 3 to 5. The first axis represents public attitudes (for or against) to nuclear power and all three question items are ranked in the order of the strength of the attitude for or against nuclear power. $\mathrm{A}$ and $\mathrm{E}$, in the second axis, which show strongly positive and negative responses, 


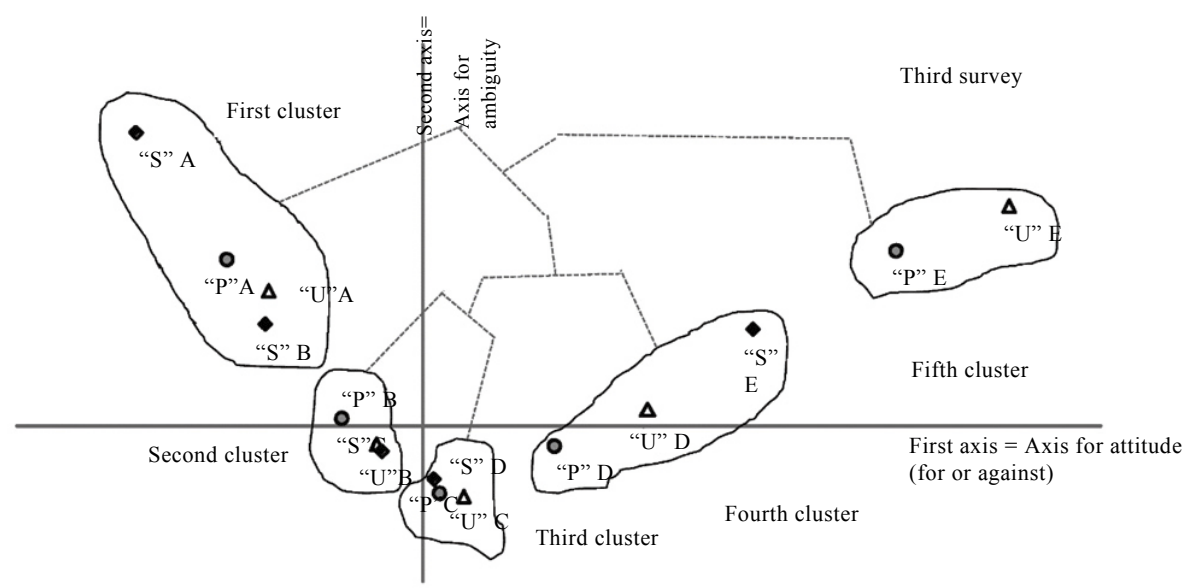

(1) Third survey (Before the Fukushima Accident)

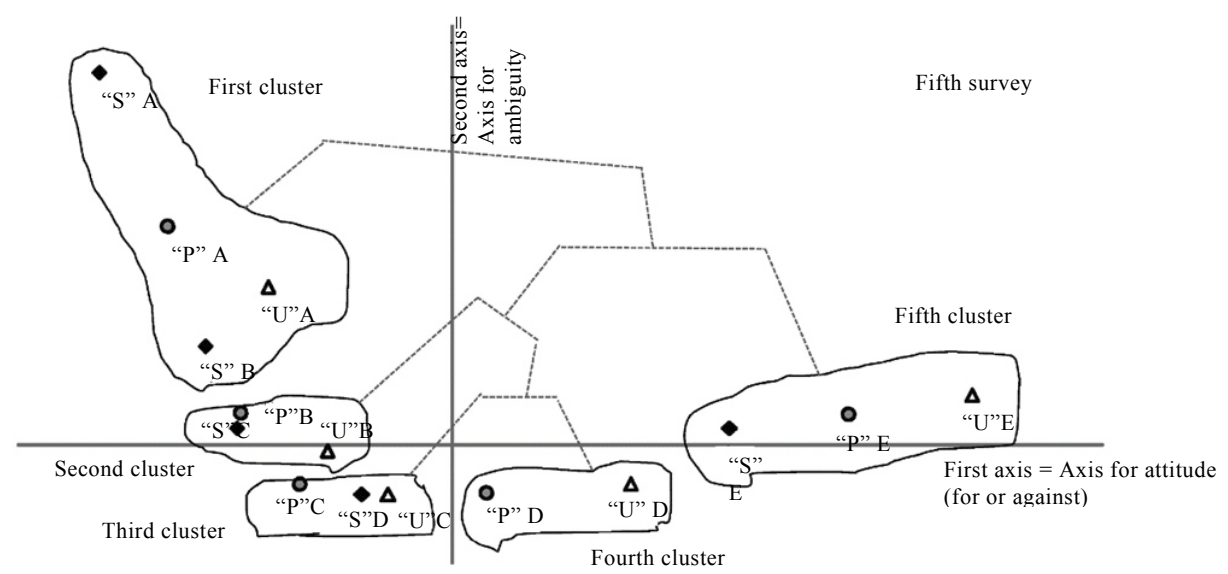

(2) Fifth survey (After Fukushi ma Accident)

Figure 7 Results of the analysis between question items by quantification method III (First and Second axes plane)

indicate the strength of opinions and number of opinions along it and the axis can be regarded as representing awareness of ambiguity. The first axis indicates respondents' attitudes to the use of nuclear power (for or against) and the second axis indicates a sense of ambiguity and these reflect the same trend that was seen in PCA.

If cluster analysis is performed on the relations between answer options plotted on the graph, the differences before and after the Fukushima Accident become clear. In cluster analysis, the target numerical value group is divided into "subsets = cluster", (which achieve internal connections and external separation). Using the option score distribution obtained by quantification method III, the option groups that are likely to be chosen by a respondent can be identified simultaneously.

We used Ward's method (Euclidean distance) for phased clustering and determined the division number and cluster characteristics by dendrogram, which represent the process through which individual data is aggregated.

In Figure 7, solid curved lines indicate clusters of several answer options placed based on 
the analysis results and dotted lines connect these clusters. Before and after the Fukushima Accident, we can observe differences in the connections between options (B), (C) and (D), which indicate weak positive, negative and neutral opinions respectively. Before the accident, the fifth cluster, which aggregates options reflecting strong negative opinions, was connected to another last. However, after the accident, the first cluster, which aggregates options reflecting strong positive opinions, is connected to another last. Getting connected to another cluster last indicates that the options in the cluster are most isolated, which means the attitudes of the respondents who chose the options in the cluster are most isolated from those of other survey audiences.

Shinoda ${ }^{12)}$ analyzed the backgrounds of those who tend to choose neutral answers, options (B), (C), and (D), using quantification method III and demonstrated that the public's stance or disposition can be understood by finding out whether the neutral group agrees with/feels empathy towards a group with strong positive opinions or a group with strong negative opinions. Such a neutral group accounts for the majority of respondents. The analysis results shown in Figure 7 indicate that the neutral group gradually came to adopt a favorable stance by creating the group with positive opinions before the Fukushima Accident, but this stance was reversed (into opposition) after the accident.

The orientation of each option did not significantly differ before and after the Fukushima Accident, but the orientation of the cluster of options indicating neutral opinions (third cluster) changed. We can find the features of the options from this orientation. Figure 8 shows the changes over time in distinctive values of the first axis, which shows the public attitude (for or against) to nuclear power. The attitudes of those who chose option (C) for Promotion and Usefulness in Figure 8 (1) included negative opinions before the Fukushima Accident, but which shifted to positive after the accident.

In Figure $8(2)$ the values $(\diamond)$ of option $(C)$ are in the positive direction throughout all the surveys for Safety and they further inclined in the positive direction after the Fukushima Accident. The values $(\boldsymbol{\Delta})$ of option (D) shifted directions (for or against) after the accident. People tend to avoid clearly stating their opinion by choosing options such as "Neither" because they sense the prevailing public sentiment at the time and only weakly oppose it. Respondents' choice of option (C), in other words avoiding making a clear indication of their opinion, depends on the degree to which they sense the social sentiment at the time. Respondents' choice of option (D), which is weak opposition, regarding Safety after the Fukushima Accident means "they do not utterly reject concerns about safety" and that they feel somewhat positive about the promotion of nuclear power.

When an evaluation is made together with the changes in survey responses around the time of the Fukushima Accident as indicated in Table 6, it is observed that people's shift from option (C) for Promotion from negative to positive around the time of the Fukushima Accident represents an alteration in their perception of social sentiment. We can see that the rejection of the promotion nuclear power became the prevailing social atmosphere after the accident. Also, we can assume that the unchanged response rate for option $(\mathrm{C})$ for Usefulness indicates that it is harder for people to decide whether nuclear energy is useful or unnecessary compared to deciding if nuclear power should be promoted or not.

When we analyze the three question items as well as Interest using quantification method III, we can see that before the Fukushima Accident, "Level of Interest (degree)" and "attitude (for or against) to nuclear power" faced in the same direction along the horseshoe-shaped distribution shown in Figure 7 (1), which means interest and attitude (for or against) were related in the same direction, along with an ambiguous stance as indicated by the second axis. On the other hand, after the accident the distribution of the options selected for Interest changed; the value 


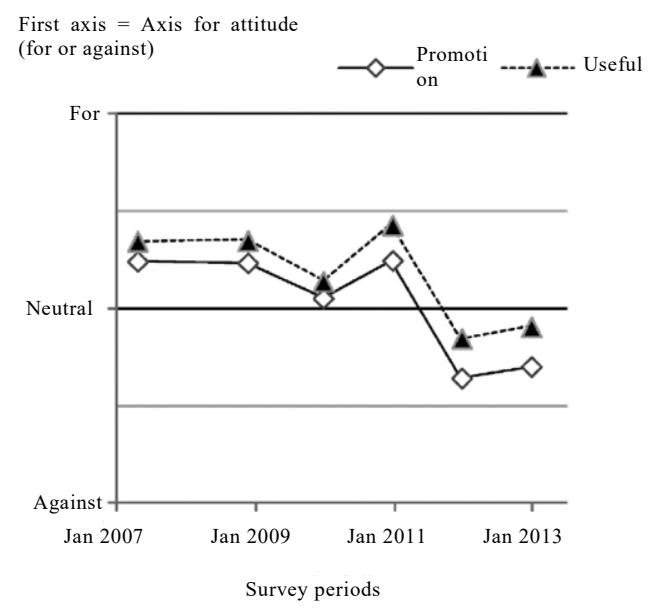

(1) Changes over time in the response rate for option (C) "Neither" question items Promotion and Useful on the first axis (Axis representing attitude (for or against))

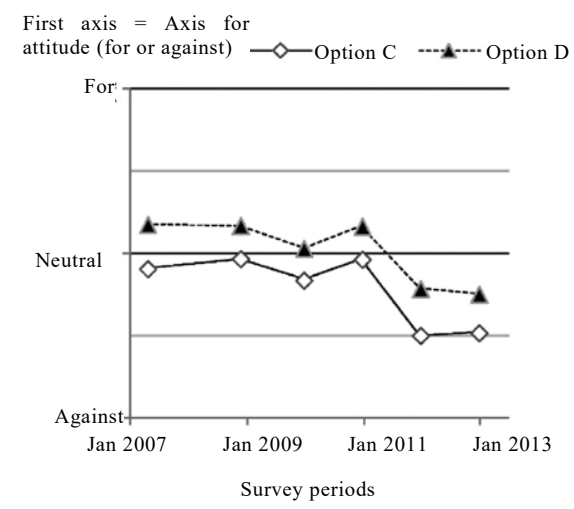

(2) Changes over time in the response rate for option (C) "Neither" option (D) "I feel somewhat anxious" for question item Safety ol the first axis (Axis representing attitude (for or against))

Figure 8 Changes over time in analysis results between question items using quantification method III (First axis $=($ Axis representing attitude (for or against $))$

for the first axis, which indicates the attitude (for or against) to nuclear power, became nearly zero and only option (A) for Interest had a high value on the second axis.

Regardless of the attitude (for or against) to nuclear power, we can see from this analysis that a high level of awareness of nuclear power among the public and the strength of their interest are related.

\section{(2) Analysis and evaluation of respondents' levels of agreement based on their opinions on the use of nuclear power}

In this question, as the response rate for option (F) "I don't know" was high, an evaluation was conducted by assigning scores to options. One of the factors that has a large impact on the attitude (for or against) to nuclear power is trust. We can gauge this impact through the question in this section concerning trust in those who are engaged in the generation of nuclear power. As we focus on changes before and after Fukushima Accident, the responses to Risk of earthquake 
are also covered in this section.

Table 10 shows the results of a statistical test on the changes over time in the response rates for Trust and Risk of earthquake. Regarding the latter, the rate for option (A), which represents an affirmative response to the question regarding the risk, increased around the time of the two earthquakes. Before and after the Chuetsu-oki Earthquake, the changes in response rates for options other than option (A) were not significant. The response rate for option (A) changed after the Chuetsu-oki Earthquake and gradually shifted back later. Around the time of the Fukushima Accident, there was a significant decrease in the selection of options (B), (C), and (F), and we can see that people chose option (A) due to an increased awareness of the risk posed by earthquakes. Although we cannot ascertain attitudes and awareness before 2007, as far as the present study is concerned, significant changes occurred after the Fukushima Accident. To be more specific, vague awareness of issues related to nuclear power changed into firm convictions; for example, people became increasingly convinced that a nuclear power plant accident caused by an earthquake would lead to serious damage. The most significant change in this attitude occurred after the Fukushima Accident. The response rates for options (D) and (E), which represent a rejection of the notion that earthquakes are a risk, were low and remained the same before and after the earthquake.

From the Trust perspective, the change trends vary between the two earthquakes. It significantly increased around the time of the Chuetsu-oki Earthquake and then gradually increased until before the Fukushima Accident. Considering this together with the changes in responses to Safety, it is assumed that the impact of the Chuetsu-oki Earthquake and the actions taken in response to it were viewed favorably. From the changes over time in the response rates as shown in Figure 1, it can be seen that the response rate for option (B), which represents trust, increased and those for options (D) and (E), which represent a negative trend, decreased between the Chuetsu-oki Earthquake and the Fukushima Accident. In the fourth survey, the "trusting" group that chose options (A) and (B) increased to its highest level at $44.2 \%$ and the "distrusting" group that chose options (D) and (E) decreased to its lowest level at 12.2\%. These results suggest that there was no outstanding change in status during this period and the smooth operation of nuclear power plants increased the level of public trust.

However, trust decreased after the Fukushima Accident. The change was not particularly significant, and the level of trust increased up until before the accident and then declined back to a level close to that in 2007 , when this study was initiated. It did not change markedly after the accident compared to "risk of earthquake". The trust built up in many people who never imagined that a nuclear power plant accident would occur before the Fukushima Accident was lost due to the impact of the accident. However, when we evaluate impact on trust, it is

Table 10 Test results of changes in response rates

(1) "Trust"

\begin{tabular}{cccccc}
\hline $\begin{array}{c}\text { Test of } \\
\text { significant } \\
\text { differences in } \\
\text { response rate } \\
\text { changes }\end{array}$ & $\begin{array}{c}\text { First } \\
\text { Second }\end{array}$ & $\begin{array}{c}\text { Second } \\
\downarrow\end{array}$ & $\begin{array}{c}\text { Third } \\
\downarrow\end{array}$ & $\begin{array}{c}\text { Fourth } \\
\text { Thecident } \\
\text { Fourth }\end{array}$ & $\begin{array}{c}\text { Fifth } \\
\text { Fifth } \\
\text { Sixth }\end{array}$ \\
\hline (A) & + & & & \\
\hline (B) & ++ & + & -- \\
\hline (C) & & & \\
\hline (D) & -- & & ++ \\
\hline (E) & -- & & ++ \\
\hline
\end{tabular}

(F)
(2) "Risk of earthquake"

\begin{tabular}{cccccc}
\hline $\begin{array}{c}\text { Test of } \\
\text { significant } \\
\text { differences in } \\
\text { response rate } \\
\text { changes }\end{array}$ & $\begin{array}{c}\text { First } \\
\downarrow \\
\text { Second }\end{array}$ & $\begin{array}{c}\text { Second } \\
\downarrow \\
\text { Third }\end{array}$ & $\begin{array}{c}\text { Third } \\
\downarrow \\
\text { Fourth }\end{array}$ & $\begin{array}{c}\text { Fourth } \\
\text { Accident } \\
\text { Fifth }\end{array}$ & $\begin{array}{c}\text { Fifth } \\
\downarrow \\
\text { Sixth }\end{array}$ \\
\hline (A) & ++ & & & ++ & \\
\hline (B) & & & -- & \\
\hline (C) & & & -- & \\
\hline (D) & & & & \\
\hline (E) & & & \\
\hline (F) & & & -- \\
\hline
\end{tabular}

$++(--)$ : Increase (decrease) with $99 \%$ confidence, $+(-)$ : Increase (decrease) with $95 \%$ confidence 
important to take into account the subject upon which trust was evaluated. In this study, the subject was "safety ensured by those who are engaged in the generation of nuclear power". Further study is required regarding trust.

(3) Analysis and evaluation of matters respondents are interested in and/or are concerned about in daily life

In this question, about 20 matters were presented as answer options. Most of the matters in which respondents have an interest in and/or anxiety about tend to be similar. Some of the matters in this question sensitively reflect the social situations at that time. For example, people's interest in and concerns about imported food, shown in Figure 2, significantly increased between the first and second surveys. We can say that this was due to the impact of the Chinese toxic dumplings incident in January 2008. A year later, the values returned to nearly the same levels as those before the incident. Each matter has their own specific features; for example, the number of selections was highest for Sickness throughout all the surveys and shows a stable trend.

The matters presented include Nuclear power and Nuclear-related accident. The former evokes a vaguer image of nuclear power in general, while the latter refers to a more specific topic with the addition of the word "accident".

The numbers of selections for "interest in" and "concerns about" for both matters significantly increased around the time of the Fukushima Accident. The increment for "Nuclear-related accident" is higher than for "Nuclear power" after the Fukushima Accident, and we can assume that this can be attributed to the impact of the accident. The concerns about "Nuclear-related accident" significantly decreased in the fifth and sixth surveys after the accident. However, this decrease needs to be examined in light of the next survey (scheduled to be conducted in January 2014).

In this study, interest in and concerns about nuclear power have already been covered by the question II-2-(1) in the section "Interest and concerns" for which five answer options were provided. It could be assumed that the results for the two questions would be different; one is a question that asks respondents to select an option corresponding to their interest in and concerns about nuclear power in relation to several specific topics including Sickness and Natural disaster and another is a direct question that asks about their "interests and concerns" by specifying these in the question. Providing these two types of questions in a survey allows us to see the influence of being conscious of interest and concerns by explicit representation.

Figure 9 (1) shows the changes over time in the response rates of those who chose both Nuclear power and Nuclear-related accident $(\boldsymbol{\Delta})$, Nuclear power only $(\bullet)$, Nuclear-related accident only $(\boldsymbol{\square})$ and at least one of them in a question regarding respondents' interest in Nuclear power and Nuclear-related accident. Figure 9 (2) shows the response rates of the same respondents in a question about concern. The response rate of those who chose at least one of those matters $(\bigcirc)$ is the same rate as those who expressed whether they are interested in/concerned about nuclear power in response to the questions about interest and concerns.

Figure 10 shows the changes over time in interest $(\bigcirc)$ and concerns $(\diamond)$ depicted in Figure 9 by adding "matters selected". In this figure we added the sum of the response rates for options (A) and (B) as (-), which represents the rate of respondents who expressed interest, for the question item Interest in the question mentioned in Section II-2-(1) and the sum of those for option (D) and (E) as $(\bullet)$, which represents the rate of respondents with concerns, for the question item Safety, as an "explicit question". We categorized respondents based on whether they are interested in and/or concerned about nuclear power, but disregarded the strength of that interest and concerns. 


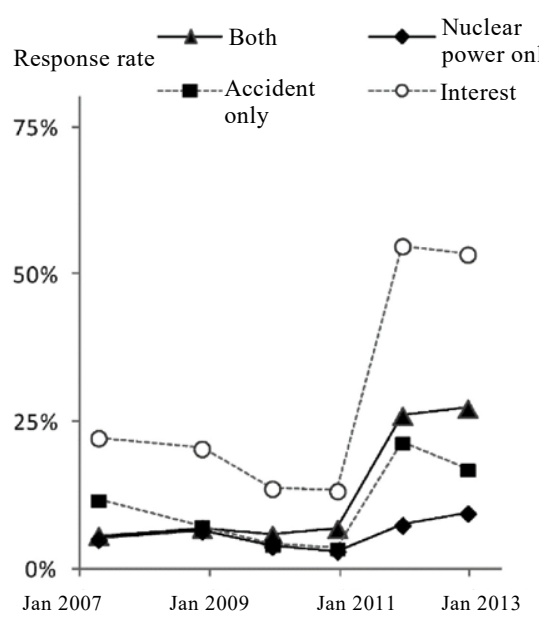

Survey periods

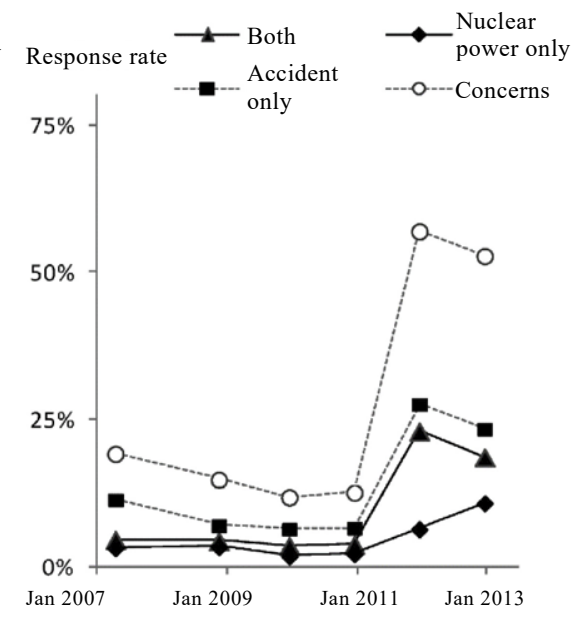

Survey periods

(2)Concerns

(1)Interest

$\boldsymbol{\Delta}$ : Both— Rate of respondents who chose both "Nuclear power" and "Nuclear-related facility accident"

•: Nuclear only— Rate of respondents who chose "Nuclear power" only

a: Accident only—Rate of respondents who chose "Nuclear facility accident" only

○: At least one of them— Rate of respondents who chose both or one of either "Nuclear power" or "Nuclear facility accident"

Figure 9 Changes over time in the response rates for interest in and concerns about nuclear power

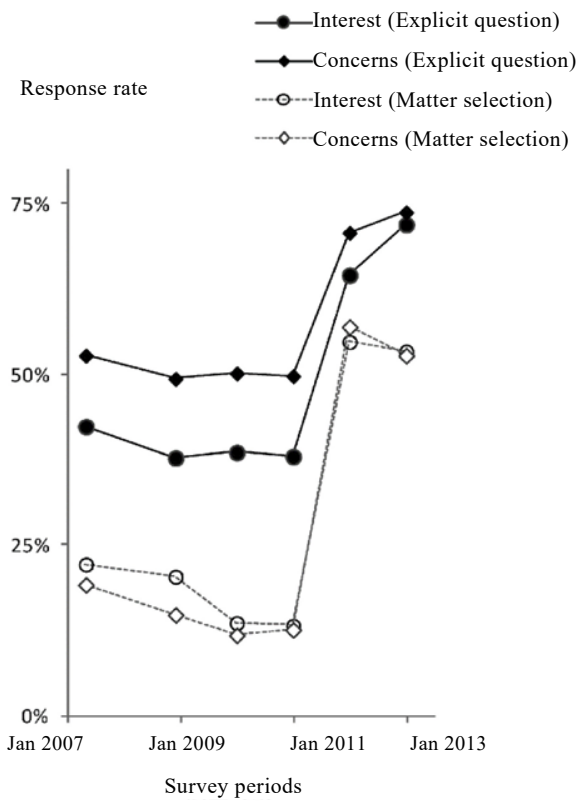

Figure 10 Differences between the results of two types of questions regarding interest in and concerns about nuclear power 
Before the Fukushima Accident, there were differences in the results between two different types of questions ("explicit questions" and "matter selection") regarding respondents' interest in and concerns about nuclear. After the accident, although the rate for interest and concerns increased, and the gap between the results for the two questions was narrowed. We can say that it partially captures the feature of interest and concerns led by an increase in awareness of nuclear-related issues. These results suggest that for the survey respondents, who were living in the Tokyo metropolitan area, awareness or concerns about nuclear power-related issues was low before the Fukushima Accident. It is highly likely that some trigger (e.g. the survey in this case) induced them to respond that they had interest in and concerns about nuclear power even if they were not aware of it in everyday life.

It is assumed that they started to become aware of nuclear power in everyday life after the Fukushima Accident. We can say that it narrowed the gap between the results of two types of questions.

\section{Summary of Analysis Results and Discussion}

By using various statistical tools including testing for changes, analysis of relations, principal component analysis, applying quantification method III, and cluster analysis with the changes over time in simple survey results, we attempted to explore the underlying factors of people's awareness of and attitudes to nuclear power, which are difficult to discover based on simple survey results only. For example, about $20 \%$ of respondents chose the same answer options for the question items Promotion, Usefulness, and Safety throughout all the surveys. Although in the early surveys about 10 percent of respondents chose option (C) for all the questions, this percentage gradually decreased at a constant rate in the two years prior to the Fukushima Accident and had decreased to about $4 \%$ in the sixth survey accelerated by the Fukushima Accident. It should not be assumed that the selection of option (C) Neither indicates that the respondent has no opinion, but such respondents started to show their opinions more clearly. At the same time, their hesitation and ambivalence decreased. Many respondents chose different options for each question item. This influence is shown by the statistical analysis used in this study.

By using principal component analysis, we were able to reaffirm that people's attitude (for or against) to nuclear power is determined primarily by opinions regarding whether or not they think nuclear power should be promoted, nuclear energy is either useful or not, and feel safe/ anxious about nuclear power. Also, it showed that their feelings of safety and anxiety have less impact on their attitude (for or against) to nuclear use than the other two question items. The main purpose of the use of principal component analysis is to condense information and conventional analysis rarely refers to principal components with a low contribution rate. In this study, we tried exploring the structures of the question item groups by also taking into account principal components with a low contribution rate and examined the psychologically complex way respondents thought about these matters, such as their perception of usefulness/necessity and feelings of safety and anxiety towards nuclear power. We found that such thinking changed in response to the Fukushima Accident.

We also revealed changes in awareness and attitudes among people in the middle layer (the middle layer in this study refers to the group of people who have neutral opinions that may vary depending on the survey) before and after the accident using quantification method III. To gain a deeper understanding of these changes among people in the middle layer resulting from the Fukushima Accident, we must evaluate respondents from the middle layer whose attitudes changed; for example, those who left the layer and those who moved into the layer. This study is not a follow-up study and we therefore cannot examine the changes over time in the 
responses of individual respondents. We tried exploring the factors underlying the awareness and attitudes of people with neutral opinions although these could be regarded as a limitation of the sample survey.

The study evaluated the impact of the Fukushima Accident based on the changes in people's awareness and attitudes over several years before and after the accident, taking advantage of previous surveys that were conducted at regular intervals. The shift to negative attitudes towards nuclear power generation, which seems to be an impact of the Fukushima Accident, has generally been maintained even though two years have passed since the accident occurred. After the accident, public interest in nuclear power generation has increased and it has become something that the public are aware of in everyday life. People's attitude towards the use of nuclear energy has shifted from positive to negative; they have become less positive about its usefulness, their anxiety has increased, and they have less confidence in nuclear power. There are no strong signs that such attitudinal changes are returning to the status that existed before the Fukushima Accident. This is due to the strong influence of the changes in awareness and attitudes in respondents occupying the middle layer arising from the situation where "nuclear power has become a common component of our daily lives".

In this survey, we confirmed some changes in awareness and attitudes had returned to their previous status after one year or so depending on which factor had been instrumental in changing it (e.g. Chuetsu-oki Earthquake and Chinese toxic dumplings incident). Also, one survey ${ }^{15}$ ) reported that public sentiment having changed due to the accident at the Mihama Unit 3 reverted back to its previous status after about one year. Shinohara ${ }^{12)}$ also reported that based on analysis of the results of a nuclear study conducted by various organizations, no other impact of nuclear accidents and incidents have lasted for a long period with the exception of the impact of the Chernobyl Accident. From the survey results, we can see that the changes in attitudes and awareness have not returned to their previous status and it is clear that the Fukushima Accident had a significant impact on people.

As for changes that seem to have originated from the impact of the Fukushima Accident, how people become interested in nuclear power needs to be noted. This means it is important to explore further how people's interests have increased and how that affects the future use of nuclear power. Our analysis revealed that, before the Fukushima Accident, there was a tendency for an interest in nuclear power to be linked positively to the use of nuclear power and so, too, was lack of interest in nuclear power linked to a negative perception of nuclear power. If we interpret the data to mean that it shows people's hesitation to show a positive attitude to nuclear power even though they are not interested in it or a negative attitude even though they are interested in it, we can say that, based on this psychological tendency, people's interest and attitude (for or against) to nuclear power were linked before the accident.

Such relationships are likely to change depending on external factors. After the accident, those having a high interest in nuclear power tend to show either a very positive attitude or very negative attitude towards it, or we can say that the accident simultaneously evoked a strong negative attitude to and very high interest in nuclear power. This was also demonstrated by the analysis using quantification method III. Public interest in nuclear power appears to be unprecedented. When policies on how nuclear power should be used are formulated, it is desirable to consider how and why people are interested in it.

People have also become less convinced of its usefulness as a result of the accident. Their feelings about its usefulness/necessity contributed to their attitude (for or against) to the use of nuclear energy rather than anxiety and their feelings regarding its safety. This study could not determine if the accident was the trigger that changed people's values and awareness in everyday life and consequently their belief that nuclear-generated power was unnecessary, or if their 
heightened concerns about nuclear power was the trigger for an attitudinal change to stop using nuclear power and consequently their belief that nuclear-generated power was unnecessary in an effort to be consistent with their own attitude. This must be studied in the future.

\section{Suggestions based on the Analysis of the Study Results}

The study examined the features of changes in awareness and attitudes around the time the Fukushima Accident occurred as well as before and after it. It is obvious that the accident has changed people's attitudes towards the use of nuclear power especially in regard to the safety of nuclear power plants. The study showed that a significant number of people became very interested in the use of nuclear energy in the future but became suspicious of it at the same time. Even two years after the accident, the news related to this event (about such as decontamination, leakage of contaminated water, restart of nuclear reactors and the nuclear power free movement) is still continuously reported and it is assumed that interest will not dissipate rapidly. We think it is natural that whenever energy and nuclear-related news is reported in the future, the Fukushima Accident will definitely be discussed. Thus, the public's focus on and interest in nuclear power have increased given the fact that nuclear power has become an inseparable part of daily life. This situation has drastically changed people's interest in and their attitude (for or against) to nuclear use.

The higher their interest becomes, the less dependent the public will be on the government and experts. Having experienced the Fukushima Accident, new initiatives (determination process) will be needed when determining policy related to the trends and status of nuclear power generation. In such a case, it is not reasonable to expect the public to shed their negative attitude by waiting for them to lose interest in this issue and forget that nuclear power is an intrinsic part of daily life; in other words, by waiting for things to "cool down" and "to be forgotten". There were several prominent incidents that strongly raised awareness of nuclear power generation a few years before the Fukushima Accident. The situation where "no incident happened and nothing was reported" lowered our awareness of nuclear energy in daily life and increased our trust in it with our interest remaining low. It would be reasonable to think such a situation will not happen considering how public awareness and attitudes changed after the Fukushima Accident. It is also clearly demonstrated by the responses to the questions in the survey asking about matters of interest and the concerns of respondents and the fact that nuclear power is becoming an integral part of daily life.

However, regarding trust, it must also be borne in mind that trust in nuclear power has not necessarily unprecedentedly declined since the Fukushima Accident. It is not true that trust was completely lost due to the accident; in fact, nuclear power plants were being operated in the past even though the level of trust was the same as that after the accident. However, if we hypothesize that the increase in trust before the accident represents an increase in a positive view of nuclear power, the trust that was subsequently lost is nothing more than the loss of this positive view. Also, when trust in nuclear power is assessed, how nuclear power has become a common part of daily life needs to be taken into account as this represents the degree to which the public are interested in nuclear power.

In such a situation, first, a positive attitude to nuclear power should be rebuilt; trust must be reestablished for this to happen. To achieve that, restoring trust to a level equivalent to that before the Fukushima Accident is one of the prerequisites for recommissioning nuclear power plants. There is no silver bullet that will restore public trust. Under the circumstances where 
trust cannot be established without information, those who are engaged in the nuclear industry must adopt a fair and transparent approach to nuclear-related matters so that the public can clearly see their commitment. Such efforts would be an effective way to rebuild a positive perception of the nuclear industry.

Deliberative polling on energy and environmental choices based on thorough deliberation conducted under the auspices of the Democratic Party of Japan is an exciting initiative. The increased interest in nuclear issues due to the Fukushima Accident was a great opportunity for constructive discussion on the use of nuclear power. Allowing a numerous people who were not proactive in such deliberations before to get involved in such an initiative has had an influence on the awareness and attitudes of people in the middle layer and may lead to a change in the social atmosphere. Citizens' participation in this form of deliberation that ensures representativeness will enhance the image of nuclear energy in the eyes of the public and convert non-participating citizens into active participants in such deliberations. The situation today where nuclear power has become an intrinsic part of our lives can be a tremendous opportunity for the country to explore the future direction of the use of nuclear power together with the participation of forward-thinking citizens. Also, placing importance on discussions with citizens in the decision-making process when formulating nuclear strategies is an essential element to ensure fairness and transparency in the promotion of nuclear power.

Promoting continuous implementation of deliberative polling on energy and environmental choices by pro-nuclear advocates will have a significant and major effect on the awareness and attitudes of the general public. When developing trust through the process of deliberation what is important is not the approval of decisions per se but an approach that emphasizes procedural justice in the decision-making process. Such an approach emphasizes fairness and transparency and consequently reestablishes a national feeling of trust in nuclear power ${ }^{17-19)}$.

The relationship between the attitude (for or against) to nuclear use and the attitude towards the usefulness/necessity of nuclear power is a representative awareness with little change. Also, this study showed that the attitude regarding the usefulness/necessity of nuclear power and the sense of safety/anxiety towards nuclear are not so directly related. Making people more aware of the usefulness of nuclear power is an effective way to promote the use of nuclear energy. Making people more aware of the usefulness of nuclear power could be the key to the recommissioning of light water reactors. However, if persuasion about usefulness involves raising public fears about an electricity shortage, this may lead to questions regarding fairness and transparency. For the public to develop a favorable view of nuclear power generation, continuous activities that ensure pro-nuclear advocates act fairly and transparently is an important measure. In order to recommission nuclear power plants, those who are engaged in promoting nuclear power must conduct themselves honestly and honorably based on a strong sense of social responsibility.

\section{Conclusions}

This study analyzed changes in public awareness and attitudes among residents living in the Tokyo metropolitan area over six years prior to and after the Fukushima Accident. The changes caused by the Fukushima Accident must not be considered as transient but as something that transformed people's awareness and attitudes. In particular, there have been marked changes in interest in the use of nuclear power. In this study, the heightened level of interest is seen as a good opportunity. Although we can discern some signs of a return to sense of safety and an 
acceptance of the usefulness of nuclear power, the evidence is not sufficient to make a definitive statement. We need to re-evaluate these matters based on the results of the survey scheduled to be conducted in January 2014.

This study uses some of the results of studies conducted under the Initiatives for Atomic Energy Basic and Generic Strategic Research (2012) and "Efforts in the communication field to go beyond the boundaries of the nuclear power village (2013)."

\section{Notes and Acknowledgements}

The authors were involved in the overall design of the survey including preparation of the survey form, aggregation and analysis of survey results and data management and publication of the survey results. The survey results were managed by a data management working group established under the Social and Environmental Division of the Atomic Energy Society of Japan. Third parties can use individual data and aggregated results provided they comply with the terms of use stipulated by the working group and use them for academic and educational purposes only. The authors participate in the working group as committee members.

Detailed information on the data management working group is provided on the following website. (As of February 2014) http://www.ponpo.jp/DMWG/index.html

The survey sheets of all the surveys and results of simple tabulation including cross tabulation are available on the website. Also, through the website, interested parties can submit an application for the use of survey data.

We have received useful advice and cooperation from Associate Professor Yoshimi Kawamoto of the Department of Nuclear Power and Energy Safety Engineering in the Graduate School of Engineering at the University of Fukui and Specially Appointed Professor Naoki Yamano of the Research Institute of Nuclear Engineering of the University of Fukui. We would like to express our gratitude to them.

Also, we could not have succeeded in conducting the study without the support of the Japan Nuclear Energy Safety Organization and Initiatives for Nuclear System Development and Atomic Energy Basic and Generic Strategic Research. Their allowing us to perform academic survey and disclose data helped dramatically raise the value of this study. We would like to once again express our gratitude.

\section{References}

1) Hiroshi Kimura, "The Gap of Perception towards Nuclear Energy between the Inhabitants in the Tokyo Metropolitan Area and the Members of the Atomic Energy Society of Japan," Journal of the Atomic Energy Society of Japan, 51 [9], 683-685 (2009).

2) Hiroshi Kimura, "What Happened to the Perception Gap between the Inhabitants in the Tokyo Metropolitan Area and the Members of the Atomic Energy Society of Japan Concerning Nuclear Energy since the Fukushima Nuclear Accident," Journal of the Atomic Energy Society of Japan, 54 [9], 606-610 (2012).

3) Cabinet Office, Outline of the "Special Public Opinion Survey on Nuclear Power," http://www8.cao. go.jp/survey/tokubetu/h21/h21-genshi.pdf, 2009 (final confirmation in February 2014).

4) Japan Productivity Center for Socio-Economic Development, 16th (FY 2002) Report on Public Opinion Poll on Energy, 2003.

5) Research Council for Energy and Information Technology, Report on Public Opinion Poll on Energy/ Nuclear Power and International Comparison Thereof, 2004.

6) Institute of Applied Energy, Report on Public Consciousness Survey on Energy in 2009, http://www.iae. or.jp/research/project/gen_2009_r2.pdf, 2009 (final confirmation in February 2014).

7) Institute of Social System (editing), Institute of Nuclear Safety System, Public Opinion of Nuclear 
Power by Data -10-year Continuation Survey-. President Inc., ISBN 4-8334-9098-6, 2004.

8) Japan Atomic Energy Relations Organization, Public Opinion Poll on Knowledge Dissemination and Enlightenment of Nuclear Energy Utilization in 2012. http://www.jaero.or.jp/data/01jigyou/tyousakenkyu.html. 2012 (final confirmation in February 2014).

9) Junko Kitada, "Public Opinion on Nuclear Power Revealed by the Continuation Survey, the Past 30 Years and the Change after the Fukushima Daiichi Nuclear Accident," Transactions of the Atomic Energy Society of Japan, 12[3], 177-196 (2013).

10) Mitsuhiro Yokote, "Result of Public Opinion Poll on Nuclear Power in 2012," Journal of the Atomic Energy Society of Japan, 55 [6], 43-45 (2013).

11) NHK Broadcasting Culture Research Institute, Consciousness Survey on Nuclear Plant and Energy, http://www.nhk.or.jp/bunken/summary/yoron/social/pdf/130523.pdf, 2013 (final confirmation in April 2013.

12) Yoshihiko Shinoda, "Study on the Interaction between Nuclear Power and Society," Transactions of the Atomic Energy Society of Japan, 6[2], 97-112 (2007).

13) Yoshihiko Shinoda, Naoki Yamano, Hiroyuki Torii, "Social Survey on the Relationship between Society and Nuclear Power," Transactions of the Atomic Energy Society of Japan, 7[4], 350-369 (2008).

14) Hirotada Hirose, Change in Public Opinion over Nuclear Power Generation, Document No. 2 of the 27th Nuclear Energy Council, 2013.

15) Tadzuko Kita, "Continuation Opinion Survey on Nuclear Power Generation-Result after One Year after the Mihama Unit 3 Accident-," INSS J, 13, 303 (2006).

16) Discussion Type Opinion Poll on Energy/Environmental Choices. http://www.cas.go.jp/jp/seisaku/npu/ kokumingiron/dp/index.htm. Psychology of Fairness and Procedures, translated by Ikuo Sugawara and Ken`ichi Ofuchi. Plain Publishing (1995).

17) E. Allan Lind et al., Social Psychology of Fairness and Procedures, translated by Ikuo Sugawara and Ken'ichi Ofuchi. Plain Publishing (1995).

18) Satoshi Fujii, Conditions for Creating Trust in Administration, Research on Experimental Social Psychology, 45[1], 27-41 (2005).

19) Susumu Onuma, Yukio Hirose, Kan Nonami, Junkichi Sugiura, Effect of Fairness on Policy Acceptance Case Study on Deliberative Social Decisions in the EU, Collection of Papers of the 48th Annual Conference of Japanese Society of Social Psychology, 2007. 


\section{-Appendix A) Questions concerning public interest in, attitudes to, and awareness of the usefulness/necessity of nuclear power-}

\begin{tabular}{|c|c|c|c|c|c|}
\hline \multirow[t]{3}{*}{$\begin{array}{l}\text { Are you interested in nuclear power or not? Please circle the number of the } \\
\text { response that best represents your opinion from the options on the right. }\end{array}$} & 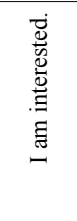 & 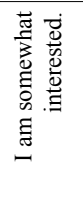 & 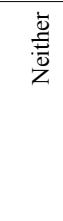 & 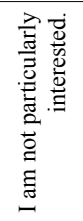 & 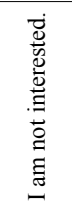 \\
\hline & $\downarrow$ & $\downarrow$ & $\downarrow$ & $\downarrow$ & $\downarrow$ \\
\hline & 1 & 2 & 3 & 4 & 5 \\
\hline \multirow[t]{3}{*}{$\begin{array}{l}\text { Do you think we should continue using nuclear power or stop using it? Please } \\
\text { circle the number of the response that best represents your opinion from the } \\
\text { options on the right. }\end{array}$} & 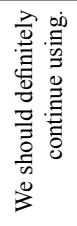 & 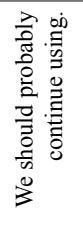 & 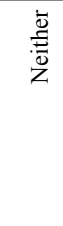 & 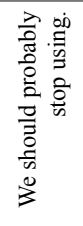 & 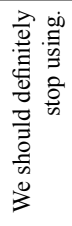 \\
\hline & $\downarrow$ & $\downarrow$ & $\downarrow$ & $\downarrow$ & $\downarrow$ \\
\hline & 1 & 2 & 3 & 4 & 5 \\
\hline \multirow[t]{3}{*}{$\begin{array}{l}\text { Do you think nuclear power is useful or unnecessary for today's society and } \\
\text { our livelihoods? Please circle the number of the response that best represents } \\
\text { your opinion from the options on the right. }\end{array}$} & 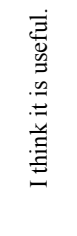 & 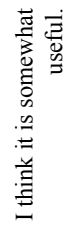 & 离 & 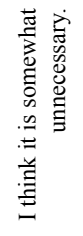 & 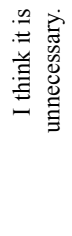 \\
\hline & $\downarrow$ & $\downarrow$ & $\downarrow$ & $\downarrow$ & $\downarrow$ \\
\hline & 1 & 2 & 3 & 4 & 5 \\
\hline \multirow[t]{3}{*}{$\begin{array}{l}\text { Do you feel safe or anxious about the use of nuclear power? Please circle the } \\
\text { number of the response that best represents your opinion from the options on } \\
\text { the right. }\end{array}$} & 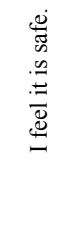 & 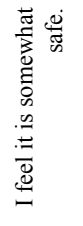 & 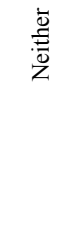 & 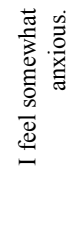 & 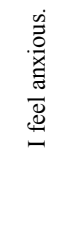 \\
\hline & $\downarrow$ & $\downarrow$ & $\downarrow$ & $\downarrow$ & $\downarrow$ \\
\hline & 1 & 2 & 3 & 4 & 5 \\
\hline
\end{tabular}




\section{-Appendix B) Questions concerning agreement/disagreement about statements on nuclear power use-}

\begin{tabular}{llll}
\hline As for opinions concerning nuclear power use, statements such as & \\
the ones listed below are heard. For each statement, indicate whether \\
you agree or not. Please circle the number of the response that best \\
represents your opinion from the options on the right.
\end{tabular}

The following questions/statements were not used in this study but have been asked in all six surveys. (equivalent to questions)

-We should not extract plutonium, which can be used for nuclear weapons, by reprocessing spent fuel.

-We can provide sufficient electricity without resorting to nuclear power.

- Since nuclear power does not emit $\mathrm{CO}_{2}$ when generating power, it contributes to the prevention of global warming.

- Considering the current electricity generating capacity in Japan, there is no alternative way of generating power in the near future.

-We have to place importance on new energy and human resource developments rather than technology development for nuclear power generation.

- Since there are more nuclear power plants with a long operating life, the level of safety is declining.

-We can use uranium resources semi-permanently for power generation by reprocessing spent fuel.

-We must determine the final repository for high-level radioactive waste as soon as possible.

-We cannot determine the final repository for high-level radioactive waste at the present time. 


\section{- Appendix C) Questions concerning interests and concerns in everyday life-}

Question Which following matters are you usually interested in? Please circle the numbers of the matters (Interests) that you are specifically interested in. You may choose as many answers as you like.

Question What are you usually concerned about? Please circle the numbers of the matters that you are (Concerns) specifically concerned about. You may choose as many answers as you like.

\begin{tabular}{|c|c|}
\hline \multicolumn{2}{|c|}{ Options (matters) are common in "Interests" and "Concerns". } \\
\hline From the first to fourth surveys & After the fifth survey \\
\hline 1. Politics & 1. Politics and economy \\
\hline 2. Price of commodity & 2. Low birthrate and longevity \\
\hline 3. Low birthrate and longevity & 3. Science and technology \\
\hline 4. Science and technology & 4. Energy and resources \\
\hline 5. Energy and resources & 5. Nuclear \\
\hline 6. Nuclear & 6. Environmental issues including global warming \\
\hline 7. Environment & 7. Imported food \\
\hline 8. Global warming & 8. GM food \\
\hline 9. Imported food & 9. Traffic accident \\
\hline 10. GM food & 10. Nuclear-related accident \\
\hline 11. Traffic accidents & 11. Natural disaster \\
\hline 12. Nuclear-related accidents & 12. Crime \\
\hline 13. Natural disasters including earthquake and typhoon & 13. Diplomacy \\
\hline 14. After retirement & 14. Nuclear nonproliferation issue \\
\hline 15. Crime & 15. War and terrorism \\
\hline 16. War & 16. Education \\
\hline 17. Diplomacy & 17. Waste disposal problems \\
\hline 18. Nuclear nonproliferation issues & 18. Radioactive waste disposal problems \\
\hline 19. Terrorism & 19. Sickness \\
\hline 20. Employment & 20. Hobbies and entertainment \\
\hline 21. Education & 21. Volunteer and community activities \\
\hline 22. Waste disposal problems & 22. Others: \\
\hline 23. Radioactive waste disposal problems & \\
\hline 24. Sickness & \\
\hline 25. Hobbies and entertainment & \\
\hline 26. Volunteering and community activities & \\
\hline 27. Others: & \\
\hline
\end{tabular}

Note) After the fifth survey ("Politics" and "Price of commodity"), ("Global warming" and "Environment"), and ("War" and "Terrorism") were integrated into "Politics and economy", "Environmental issues including global warming" and "War and terrorism" respectively. "After retirement" and "Employment" were removed from the list after the fifth survey. 


\title{
Article
}

\section{Transfer Behavior of Cesium Adsorbed on Clay Minerals in Aqueous Solution}

\author{
Yoshihiro OKAMOTO ${ }^{1, *}$, Takeshi OSUGI ${ }^{2}$, Hideaki SHIWAKU ${ }^{1}$ \\ and Mitsuo AKABORI ${ }^{3}$ \\ ${ }^{1}$ Quantum Beam Science Directorate, Japan Atomic Energy Agency (JAEA), 1-1-1 Kouto, Sayo-cho, Sayo-gun, \\ Hyogo 679-5148, Japan \\ ${ }^{2}$ Nuclear Science Research Institute, Japan Atomic Energy Agency (JAEA), \\ 2-4 Shirane, Shirakata, Tokai-mura, Naka-gun, Ibaraki 319-1195, Japan \\ ${ }^{3}$ Nuclear Science and Engineering Directorate, Japan Atomic Energy Agency (JAEA), \\ 2-4 Shirane, Shirakata. Tokai-mura, Naka-gun, Ibaraki 319-1195, Japan
}

\begin{abstract}
The transfer behavior of cesium adsorbed on some clay minerals in aqueous solution was investigated by X-ray absorption fine structure (XAFS) analysis of the Cs K-edge. The sample was prepared by mixing Cs-adsorbed mineral with a different pure clay mineral in water. The XAFS results of the dried mixture powder were compared with those obtained before mixing. It was recognized from the XAFS analysis for three kinds of clay minerals illite, kaolinite and vermiculite, that cesium was transferred from kaolinite to illite and vermiculite, and from illite to vermiculite. It can be concluded that cesium is transferred to and accumulated in vermiculite.
\end{abstract}

KEYWORDS: XAFS, cesium, transfer behavior, adsorption, X-ray, synchrotron radiation, clay mineral, migration, mixture

\section{Introduction}

Amid the extended decontamination conducted on radioactive cesium released during the accident at the Fukushima Daiichi Nuclear Power Plant, the understanding and elucidation of cesium behavior in the environment are important areas of investigation. From an early stage after the accident, efforts to elucidate the adsorption mechanism for each type of clay mineral have been made and tests on desorption have been carried out ${ }^{1-4)}$. Relevant to this, an understanding of the status of bound cesium and its changes during the decontamination process is required, and several projects have recently been initiated and are making progress. According to the large pool of knowledge accumulated to date, it has been determined that the desorption of cesium adsorbed by soil is not easy to achieve ${ }^{5)}$. If so, an important part of the question is the degree to which cesium is transferred among the mineral components in the soil, which is a mixture of various clay mineral components. One of the major natural phenomena that occurs when cesium is released into the environment is its adsorption by soil during an

* Corresponding author, E-mail: okamoto.yoshihiro@jaea.go.jp

DOI : 10.15669 /fukushimainsights.Vol.4.285

(C) 2021 Atomic Energy Society of Japan. All rights reserved.

Originally published in Transactions of the Atomic Energy Society of Japan (ISSN 1347-2879), Vol. 13, No. 3, p.113-118

(2014) in Japanese. (Japanese version accepted: June 16, 2014) 
extended period of repeated cycles of rainfall and drying. Puddles left on the ground following rainfall, and most of the cesium remaining in the shallow portion of the ground surface are then temporarily infiltrated in the water phase along with the clay minerals. The infiltration and drying are then repeated until the rainwater finally disappears. In this study, a test simulating an environment in which rainfall and its disappearance are repeated was conducted using synchrotron X-ray absorption fine structure (XAFS) analysis.

The main advantage of XAFS analysis is that it allows local environmental information regarding the specific element[s] in a multi-elemental composition system to be analyzed. Furthermore, it is an appropriate analysis method for determining the chemical status and coordination geometry of Cs adsorbed by clay minerals because it can precisely detect low concentrations of the targeted element. In this research, by taking advantage of the way that the chemical status of Cs in clay minerals is reflected in the XAFS spectrum, we tracked the variation of the chemical status in the minerals by soaking them in water.

\section{Experiments}

Three types of minerals were adopted for the test, namely, illite, vermiculite, and kaolinite (all of them purchased from Nichika, Inc., Japan). These minerals were soaked in a $0.02 \mathrm{M}$ $\mathrm{CsOH}$ solution for 1 week to allow Cs adsorption to occur. Next, the non-adsorbed Cs was removed through a cleaning process and dried. The results of a fluorescent X-ray analysis showed that the amount of $\mathrm{Cs}$ in the specimen (adsorbed amount) was approximately $0.4 \mathrm{wt} \%$ based on the equivalent oxide concentration. Furthermore, the amount of adsorption was similarly checked through the XAFS measurement of K absorption edge of Cs.

In spite of the possibility of influencing the skeleton of clay minerals by soaking them in an alkaline solution, there was no variation regarding the acquired EXAFS function, i.e., in the coordination geometry around Cs in spite of the different adsorption amounts of Cs between the tests with different soaking times of 3 h, 1 day, 1 week, and 2 weeks. Furthermore, it is considered that the influence was negligible because an almost identical EXAFS function was acquired through the tests when soaking in $0.02 \mathrm{M} \mathrm{CsCl}$ and $0.02 \mathrm{M} \mathrm{Cs}_{2} \mathrm{CO}_{3}$ including various clay minerals. After confirming the above, three prepared sets were adopted as the specimens for the Cs adsorption side. Next, two groups of large and small sized specimens were classified with a boundary of $75 \mu \mathrm{m}$ for three types of the same clay minerals in which Cs is not adsorbed. Three non-Cs adsorbing specimens no larger than $75 \mu \mathrm{m}$ were adopted. A total of $0.5 \mathrm{~g}$ of each specimen was mixed for the Cs adsorption and non-adsorption groups, placed in $10 \mathrm{~mL}$ of pure water, shaken, and left to stand at $25^{\circ} \mathrm{C}$. After the mineral components were deposited, the supernatant was removed and dried for measurement. The specimens used for testing are listed in Table 1. Three types of minerals with Cs adsorption (illite-Cs, vermiculite-Cs, and kaolinite-Cs), and six types of specimens after mixing (mix-1a through mix-6a) were subjected to measurement. To confirm repeatability, supplemental tests (mix-1b through mix-6b) were conducted using the same preparation and XAFS measurement methods. Furthermore, the cleaning agent was also measured (liq-1b through liq- $6 \mathrm{~b}$ ) because the possibility of migration of $\mathrm{Cs}$ to the cleaning agent side of the supernatant solution removed after mixing during the aqueous phase could not be ignored.

Synchrotron XAFS measurement was conducted using the BL11XU beamline of SPring- 8 . In this beamline, a monochromatic X-ray as high as $70 \mathrm{keV}$ is available using a liquid-nitrogen cooled $\mathrm{Si}(311)$ spectrometer ${ }^{6,7)}$. Using a monochromatic X-ray stabilized through a 
Table 1 Samples used in the XAFS measurement

\begin{tabular}{|c|c|c|c|c|}
\hline & $\begin{array}{l}\text { Cs-adsorbed } \\
\text { clay mineral }\end{array}$ & Pure clay mineral & Mixture & $\begin{array}{l}\text { Rinse } \\
\text { water }\end{array}$ \\
\hline \multirow{2}{*}{$\frac{1}{2}$} & \multirow{2}{*}{ Illite-Cs } & Pure Kaolinite & Mix-1a, mix-1b & Liq-1b \\
\hline & & Pure Vermiculite & Mix-2a, mix-2b & Liq-2b \\
\hline \multirow{2}{*}{$\frac{3}{4}$} & \multirow{2}{*}{ Vermiculite-Cs } & Pure Illite & Mix-3a, mix-3b & Liq-3b \\
\hline & & Pure Kaolinite & $\operatorname{Mix}-4 a, \operatorname{mix}-4 b$ & Liq $-4 b$ \\
\hline \multirow{2}{*}{$\frac{5}{6}$} & & Pure Illite & Mix $-5 a, \operatorname{mix}-5 b$ & Liq-5b \\
\hline & domte- & Pure Vermiculite & $\operatorname{Mix}-6 a, \operatorname{mix}-6 b$ & Liq-6b \\
\hline
\end{tabular}

monochromator stabilizer (MOSTAB) that stabilizes the dispersive crystal according to fluctuation in the incident X-rays, measurement was conducted within the range of $35.65-37.30 \mathrm{keV}$ on the targeted $\mathrm{K}$ absorption edge $\left(\mathrm{E}_{0}=35.985 \mathrm{keV}\right)$ of the Cs. For the XAFS measurement, a quick-scan XAFS (QAFS) measurement sweeping this energy range at high speed was conducted. The time required for an energy scan, i.e., the time needed to acquire the XAFS spectrum, was approximately $5 \mathrm{~min}$. The acquired data were compared after the background removal and normalization using WinXAS version 3.2 ${ }^{8}$. Furthermore, an EXAFS function $\mathrm{k}_{x}^{3}(\mathrm{k})$ was derived and the radial structural function $\left|\mathrm{FT}\left(\mathrm{k}_{x}^{3}(\mathrm{k})\right)\right|$ was acquired using a Fourier transformation.

\section{Results and Discussions}

\section{Cs in Three Types of Minerals}

The EXAFS function $\mathrm{k}_{x}^{3}(\mathrm{k})$ and the radial structural function of $\mathrm{Cs}$ adsorbed by illite, vermiculite, and kaolinite are shown in Figure 1. Illite and kaolinite showed quite similar functions, whereas vermiculite showed an EXAFS function with large amplitude and a radial structural function having a large second peak.

Illite has a fundamental 2:1-type layer structure consisting of two tetrahedral layers and an octahedral layer in between. Whereas vermiculite has the same 2:1-type structure, kaolinite has a 1:1-type form. The first peak of the radial structural function has a nearest-neighbor Cs-O correlation. Although the second peak is believed to have a Cs-Si correlation, a considerably large difference was observed between illite/kaolinite and vermiculite. The difference in the peak size of the radial structural function is largely attributable to the size of the coordination number and Debye-Waller factor, although the large difference in the second peak appears to be too big to be attributable to only one of them. Furthermore, vermiculite also shows higher peaks in a farther area than the others, which indicates a larger coordination number and the preservation of a good structural order over a long distance of the coordination environment of Cs captured in vermiculite. These differences [support][show a match with] the deduction that, while the Cs captured by illite and kaolinite is adsorbed mainly around the ends of the clay mineral layers, in vermiculite it is captured inside of the layer by swelling.

A characteristic difference is also observed between the Cs adsorbed by illite and kaolinite, which showed similar XAFS spectra. The EXAFS functions look almost identical, although the curve of illite shows a shoulder near $\mathrm{k}=4 \AA^{-1}$. This small difference was commonly recognizable in all XAFS spectra of the other Cs adsorbed by illite and kaolinite. Furthermore, while the 


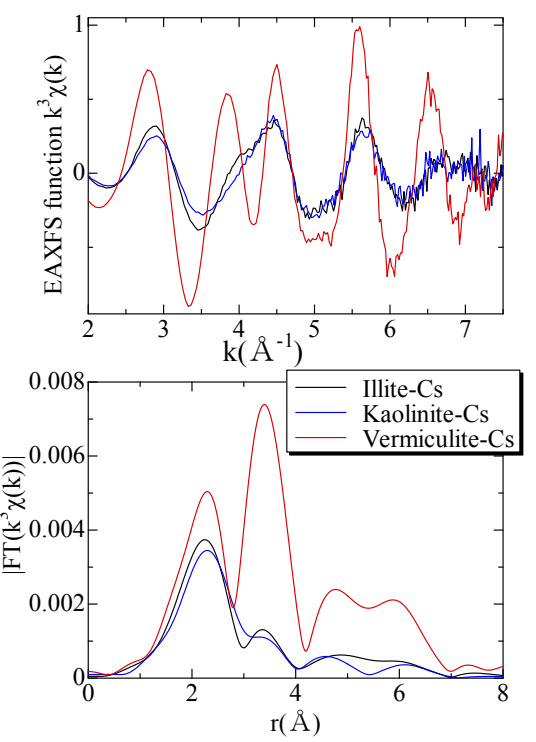

Figure 1 EXAFS functions $\mathrm{k}_{x}^{3}(\mathrm{k})$ and radial structural functions $\left|\mathrm{FT}\left(\mathrm{k}_{x}^{3}(\mathrm{k})\right)\right|$ of Cs-adsorbed illite, vermiculite and kaolinite

second peak of Cs adsorbed by illite shows a comparatively clear separation from the first peak in the radial structural function, kaolinite shows some ambiguity. The third and fourth peaks also show a difference. The existence of correlations such as Cs-Cs are presumed at around this distance, but are too obscure to be adopted for the purposes of comparison in this research owing to the fact that the effect of multiple scattering and other factors cannot be ignored.

The features of the clay minerals above were commonly observed in the Cs solution during the test at various soaking times. The attributions of the third peak and after the radial structural function are unclear, although it is thought that the determination of the difference in the coordination geometry around Cs captured in the three minerals through the XAFS analysis is possible with reasonable probability.

\section{Migration from Illite in Aqueous Phase}

The XAFS spectra of specimens prepared with a combination of illite on the Cs adsorption side and vermiculite and kaolinite on the non-adsorption side are shown in Figure 2. If the Cs adsorbed by illite does not move from the current position, no changes in spectra are expected. However, if Cs adsorbed by illite is desorbed and remains in an aqueous phase, the XAFS of Cs itself will not be observed through this measurement, or it will become difficult to observe owing to a small jump at the absorption edge proportional to the amount of Cs present. The results were clearly classified in two types of spectra. No changes were observed in the mixture with kaolinite from the spectrum of illite in which Cs is adsorbed. However, the mixture with vermiculite appears to show a match with the spectrum of Cs adsorbed by vermiculite. These results indicate that $\mathrm{Cs}$ adsorbed by illite migrated toward vermiculite whereas no migration toward kaolinite occurred.

The EXAFS and radial structural functions derived from various spectra are shown in Figure 3. Supporting the results derived through the comparison in Figure 2, the acquired results can be classified into two types of functions. 


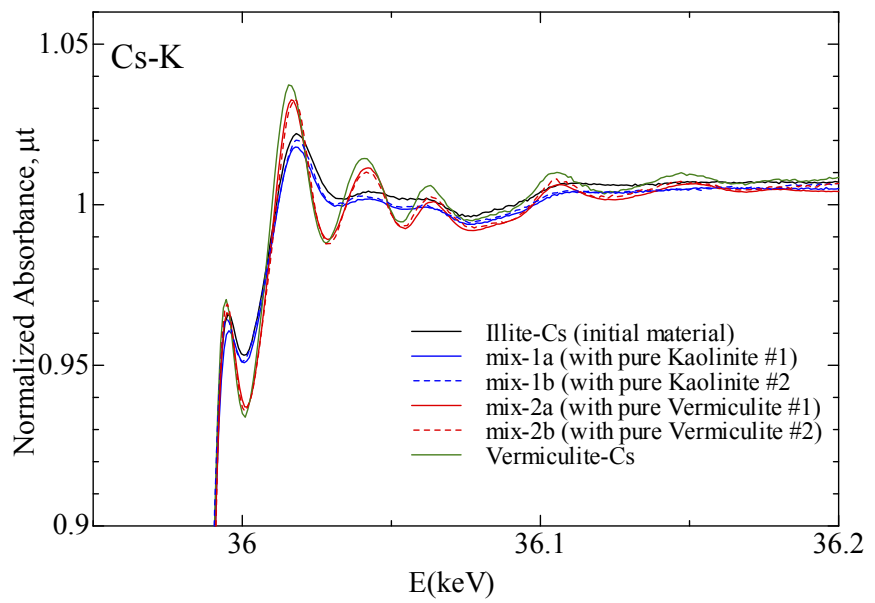

Figure 2 Normalized XAFS spectra of mixtures of Cs-adsorbed illite with pure kaolinite and pure vermiculite
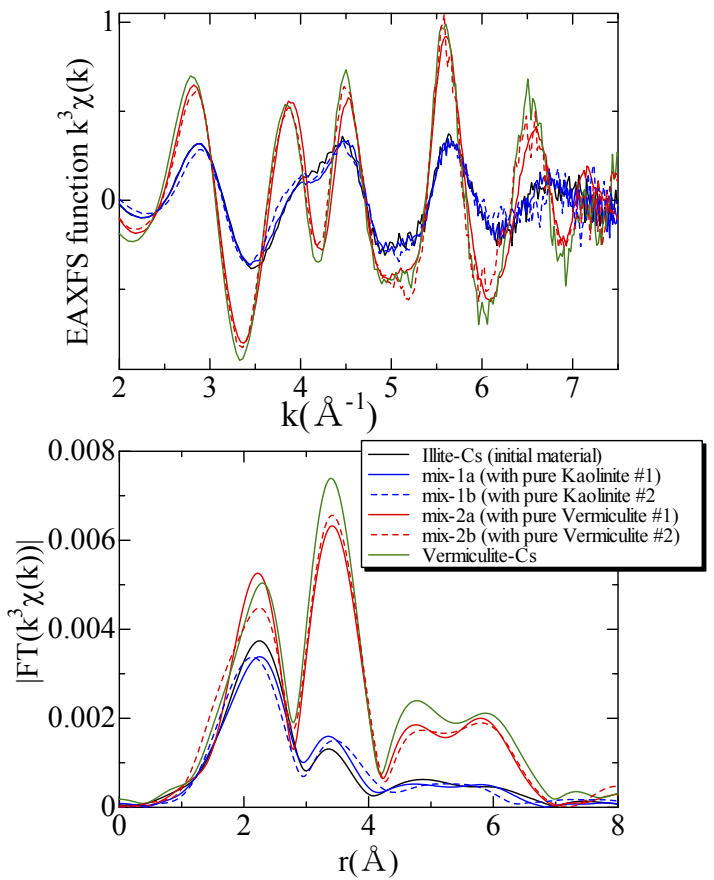

Figure 3 EXAFS functions $\mathrm{k}^{3}{ }_{x}(\mathrm{k})$ and radial structural functions $\left|\mathrm{FT}\left(\mathrm{k}^{3}{ }_{x}(\mathrm{k})\right)\right|$ of mixtures of Cs-adsorbed illite with pure kaolinite and pure vermiculite

The illite adsorbing Cs shows no change in the shoulder, which indicates that the characteristics of illite near $\mathrm{k}=4 \AA^{-1}$ of the EXAFS function and the second peak of the radial structural function remain clear. Consequently, it can be concluded that the Cs captured in the illite did not migrate toward the kaolinite. 


\section{Migration from Vermiculite in Aqueous Phase}

The XAFS spectra of the combination of vermiculite on the Cs adsorption side, and illite and kaolinite on the non-adsorption side, are shown in Figure 4. As can be clearly seen in the chart, all XAFS spectra show an identical feature, i.e., no change from the spectrum of Cs adsorbed in the vermiculite. This indicates that the Cs captured in vermiculite does not migrate even if mixed with illite and kaolinite in an aqueous phase.

Figure 5 shows the EXAFS and radial structural functions derived from each spectrum. All functions show a good match as if acquired from the same specimen. This indicates that both the EXAFS and radial structural functions have identical coordination geometries. Accordingly, this leads to the conclusion that the Cs adsorbed by vermiculite does not migrate even if mixed with illite or kaolinite in an aqueous phase, and remains in the vermiculite.

\section{Migration from Kaolinite in Aqueous Phase}

The XAFS spectra of the combination of kaolinite on the Cs adsorption side, and illite and vermiculite on the non-adsorption side, are shown in Figure 6. The spectra of specimens mixed with illite do not show a recognizable change, but the spectra of Cs adsorbed on illite and kaolinite exhibit an indistinguishably strong resemblance. However, when mixed with vermiculite, the features of kaolinite apparently disappear and the spectrum takes the form of Cs adsorbed by vermiculite.

Similarly, as in the case of Cs-adsorbed illite, the migration of Cs appears in an aqueous phase from kaolinite to vermiculite.

The EXAFS function derived from various spectra and the radial structural function are shown in Figure 7. For the specimens mixed with illite, a shoulder appears at approximately $\mathrm{k}=4 \AA^{-1}$ of the EXAFS function, and the second peak in the radial structural function is slightly clearer, indicating the features of $\mathrm{Cs}$ adsorbed by illite. This comparison implies the migration of Cs in kaolinite toward the illite side in the aqueous phase. In contrast, the specimen mixed with vermiculite shows that $\mathrm{Cs}$ is adsorbed by vermiculite, as confirmed through the comparison of the XAFS spectra.

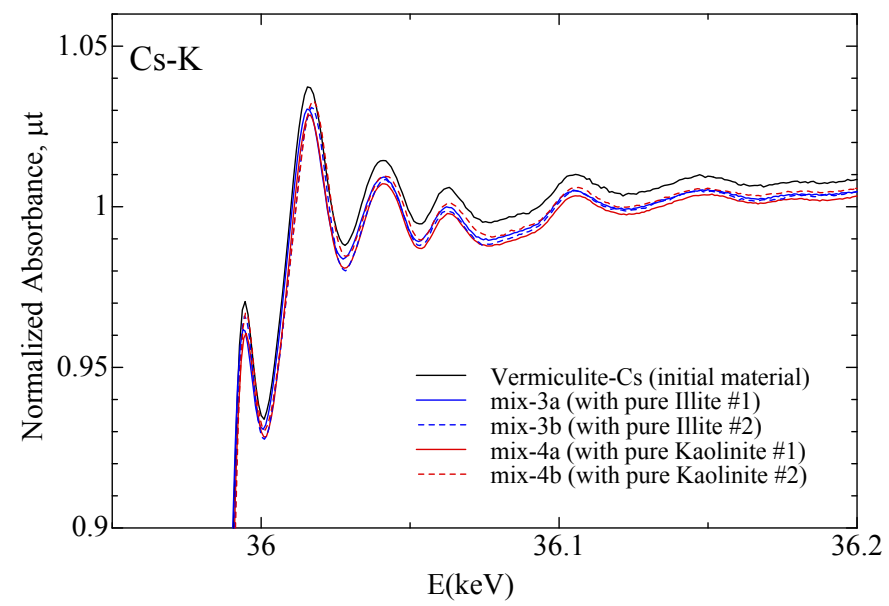

Figure 4 Normalized XAFS spectra of mixtures of Cs-adsorbed vermiculite with pure illite and pure kaolinite 

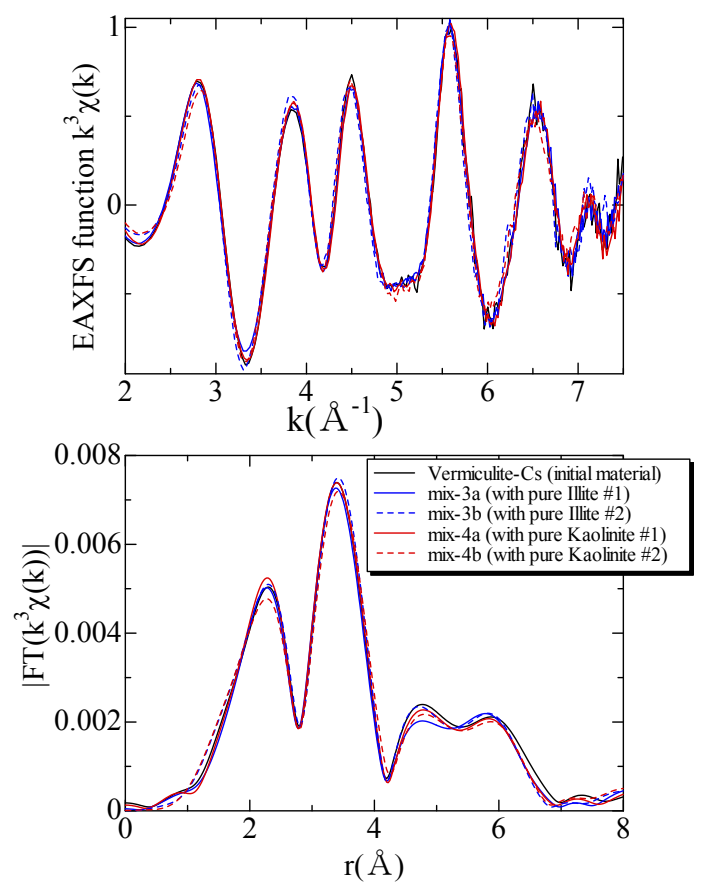

Figure 5 EXAFS functions $\mathrm{k}_{x}^{3}(\mathrm{k})$ and radial structural functions $\left|\mathrm{FT}\left(\mathrm{k}_{x}^{3}(\mathrm{k})\right)\right|$ of mixtures of Cs-adsorbed vermiculite with pure illite and pure kaolinite

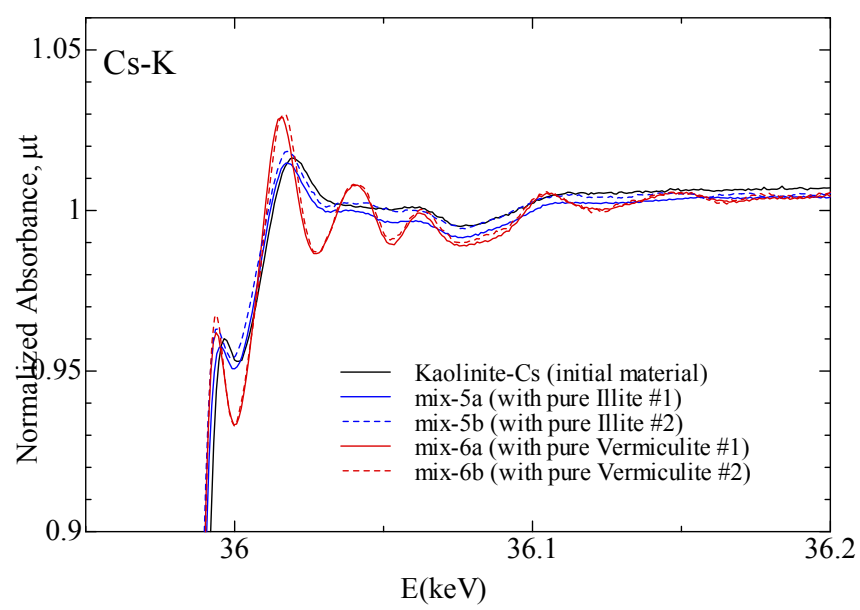

Figure 6 Normalized XAFS spectra of mixtures of Cs-adsorbed kaolinite with pure illite and pure vermiculite

The results above indicate that the Cs adsorbed by kaolinite migrates both toward illite and vermiculite in the aqueous phase.

\section{Analysis of Cleaning Agent}

The XAFS spectra of the cleaning agent sampled in the second experiment are shown in Figure 8. In two of the results, Cs-adsorbed illite (liq-1b) and Cs-adsorbed kaolinite (liq-5b) 

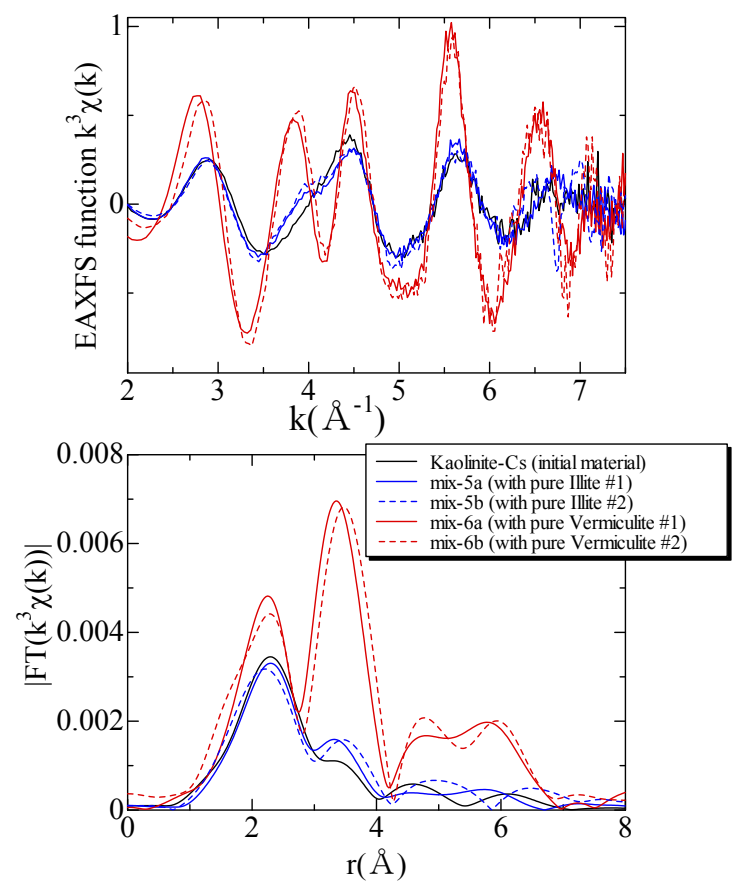

Figure 7 EXAFS functions $\mathrm{k}_{x}^{3}(\mathrm{k})$ and radial structural functions $\left|\mathrm{FT}\left(\mathrm{k}_{x}^{3}(\mathrm{k})\right)\right|$ of mixtures of Cs-adsorbed kaolinite with pure illite and pure vermiculite

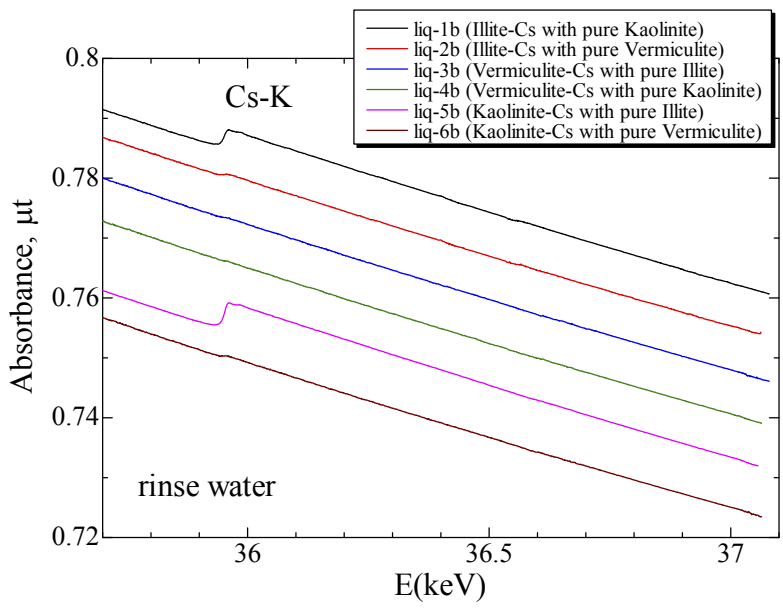

Figure 8 XAFS spectra of rinse water obtained in each mixture

clearly show the presence of Cs, which is confirmed through the presence of the absorption edge jump and the size, whereas such presence was not confirmed at all in the vermiculite. This matches the result showing that $\mathrm{Cs}$ does not migrate from vermiculite, i.e., it is possible to conclude that in the aqueous phase, Cs is not desorbed from vermiculite in such an amount that it can be confirmed using radio ray XAFS. The illite and kaolinite in which the presence of Cs is confirmed indicate the desorption of Cs in the aqueous phase. However, the level of the absorption edge jump is on the borderline for visual detection when the admixture is vermiculite. 
Table 2 Transfer behavior results of cesium adsorbed on clay minerals in aqueous solution

\begin{tabular}{lllll}
\hline $\begin{array}{c}\text { Cs-adsorbed } \\
\text { (initial metarial })\end{array}$ & $\begin{array}{c}\text { Pure clay } \\
\text { minerals }\end{array}$ & Transfer of Cs & $\begin{array}{c}\text { Rinse } \\
\text { water }\end{array}$ \\
\hline 1 & Illite & Kaolinite & No transfer & Detected \\
\hline 2 & Illite & Vermiculite & $\begin{array}{l}\text { Move to } \\
\text { Vermiculite }\end{array}$ & $\begin{array}{l}\text { Slightly } \\
\text { detected }\end{array}$ \\
\hline 3 & Vermiculite & Illite & No transfer & N.D. \\
\hline 4 & Vermiculite & Kaolinite & No transfer & N.D. \\
\hline 5 & Kaolinite & Illite & Move to Illite & Detected \\
\hline 6 & Kaolinite & Vermiculite & $\begin{array}{l}\text { Move to } \\
\text { Vermiculite }\end{array}$ & $\begin{array}{l}\text { Slightly } \\
\text { detected }\end{array}$ \\
\hline
\end{tabular}

These results indicate the re-adsorption of almost all desorbed Cs by vermiculite. They also indicate that a re-adsorption of Cs desorbed from illite into kaolinite, or Cs desorbed from kaolinite into illite, does not occur. However, as the results of an analysis on the main specimen side show, no migration from illite to kaolinite was seen, whereas there was migration from kaolinite to illite. Based on these results, it is easy to see that the amount of desorption of Cs from illite was small, whereas a large amount of Cs desorbed from kaolinite and migrated toward illite.

\section{Migration Phenomenon of Cs}

The integration of the above results and the considerations are shown in Table 2. There is a high possibility of migration of Cs released into the environment and adsorbed in the soil from kaolinite to illite and vermiculite, and from illite to vermiculite, during repeated rainfall and drying. The possibility that different results could be acquired is undeniable if the actual mineral composition in the soil is different because the tests conducted in this research were primitive, namely, the adoption of a mixture of two equal weights of minerals that adsorbed and did not adsorb Cs during an aqueous phase. There is another possibility of a different trend depending on the minerals or substances used.

The three clay minerals adopted for this research are contained in the soils of Fukushima Prefecture and its peripheral areas in considerably large quantities; it is quite significant that a clear migration of adsorbed Cs was confirmed between the materials during an aqueous phase. In particular, the migration toward vermiculite was clear, and the results of this research imply the possibility of a variation such as environmental condensation when Cs adsorbed in soil migrates toward vermiculite and accumulates during repeated rainfall.

\section{Conclusions}

The migration of Cs among three clay minerals contained in the soil of Fukushima Prefecture and its peripheral areas, namely, illite, kaolinite, and vermiculite, was investigated by mixing the adsorbed and non-adsorbed Cs during an aqueous phase using the synchrotron XAFS analysis method. As a result of the analysis, the migration of adsorbed Cs from kaolinite to illite or vermiculite, and from illite to vermiculite, was confirmed. It was also confirmed that there was no migration toward kaolinite, and almost no desorption of Cs took place from 
vermiculite. Among the three minerals, the migration toward vermiculite was noticeable, which indicates the possibility of the progress of migration and accumulation in vermiculite owing to the natural occurrence of repeated rainfall and drying.

The synchrotron radiation experiment conducted in this research applied research subjects 2012A3504 and 2012B3504 of SPring-8, a large synchrotron radiation facility.

\section{References}

1) S. Nakayama, "The Results of the decontamination pilot project and their application to the regional decontamination," Sangyo \& Kankyo, 41, 19-24 (2012). [in Japanese]

2) H. Sakurai, T. Kawamoto, K. Saito, M. Aritomi, H. Yamada et al., Kagaku, Kagaku-Dojin, 67, 16-46 (2012). [in Japanese]

3) H. Itoh, N. Seko, R. Kuroki, T. Yaita, H. Naganawa, S. Nakayama, "R\&D of removal technology of radioactive contaminants from the environment by using polymer absorbents," Housyasen-Kagaku, 93, 31-36 (2012). [in Japanese]

4) H. Naganawa, "Removal of radioactive cesium from contaminated soil by using poly-ion complex with clay,” J. Clay Sci. Soc. Jpn., 50, $52-57$ (2012). [in Japanese]

5) Department of Partnership Operations for Environmental Remediation, Headquarters of Fukushima Partnership Operations et al., "Investigation of radiocesium volatilization from soil by in-situ ground heating," JAEA-Res., 2011-026 (2011).

6) H. Shiwaku, T. Mitsui, K. Tozawa, K. Kiriyama, T. Harami, T. Mochizuki, AIP Conf. Proc., Eighth Int. Conf. on Synchrotron Radiation Instrumentation, 705, 659-662 (2004).

7) H. Shiwaku, T. Mitsui, K. Tozawa, "Introduction of liquid nitrogen cryogenic cooling system and development of multicrystal switching system on BL11XU at SPring-8," JAEA-Res., 2009-009 (2009). [in Japanese]

8) T. Ressler, "WinXAS: a program for X-ray absorption spectroscopy data analysis under MS-Windows," J. Synch. Rad., 5, 118-122 (1998). 


\title{
Estimation of I-131 Concentration Using Time History of Pulse Height Distribution at Monitoring Post and Detector Response for Radionuclide in Plume
}

\author{
Hideo HIRAYAMA ${ }^{1, *}$, Masatsugu KAWASAKI ${ }^{2}$, Hiroshi MATSUMURA ${ }^{1}$, \\ Takehisa OHKURA ${ }^{2}$, Yoshihito NAMITO ${ }^{1}$, Toshiya SANAMI ${ }^{1}$, \\ Mitsumasa TAKI $^{2}$, Tetsuya OISHI $^{2}$ and Michio YOSHIZAWA ${ }^{2}$ \\ ${ }^{1}$ High Energy Accelerator Research Organization, 1-1 Oho, Tsukuba-shi, Ibaraki 305-0801, Japan \\ ${ }^{2}$ Nuclear Science Research Institute, Japan Atomic Energy Agency, 2-4 Shirane, Shirakata, Tokai-mura, Naka-gun, \\ Ibaraki 319-1195, Japan
}

\begin{abstract}
A method of deducing the $\mathrm{I}-131$ concentration in a radioactive plume from the time history of peak count rates determined from pulse height spectra obtained from an $\mathrm{NaI}(\mathrm{Tl})$ scintillation detector employed as a detector of a monitoring post was presented. The contribution to the count rates from I-131 accumulated around the monitoring post was subtracted in accordance with the time history, taking into consideration the cumulative attachment and its decay. The concentrations of I-131 in the plumes were estimated from the count rates using the calculated response of the $\mathrm{NaI}(\mathrm{Tl})$ detector with egs 5 for a model of a plume uniformly containing I-131. This method was applied to the data from the monitoring posts at Nuclear Science Research Institutes of Japan Atomic Energy Agency (JAEA). The estimated time history variation of I-131 concentrations in plumes was in fair agreement with those measured directly by an air sampling method. The difference was less than a factor of 4 for plumes that arrived on March 15 and March 21, indicating relatively high I-131 concentrations among the plumes studied in this work.
\end{abstract}

KEYWORDS: iodine 131, plume, time history, Monte Carlo, egs5, Nal scintillator

\section{Introduction}

The radionuclide concentrations in plumes dispersed over a wide area resulting from the accident that occurred at the Fukushima Daiichi Nuclear Power Plant (hereinafter referred to as the "plume") are important in evaluating the internal exposure dose via inhalation. In particular, the concentration of I-131 is important from the perspective of radiation-induced thyroid cancer risk assessment. Hirayama et al. attempted to estimate the concentrations of radionuclides contained in the plumes that arrived using the pulse height distribution measured on expressways with a $\phi 1.5^{\prime \prime} \times 1.5^{\prime \prime} \mathrm{LaBr}_{3}$ scintillation spectrometer ${ }^{1)}$. Because the measurements on

\footnotetext{
* Corresponding author, E-mail: hideo.hirayama@kek.jp

DOI : 10.15669/fukushimainsights. Vol.4.295

(C) 2021 Atomic Energy Society of Japan. All rights reserved.

Originally published in Transactions of the Atomic Energy Society of Japan (ISSN 1347-2879), Vol. 13, No. 3, p.119-126

(2014) in Japanese. (Japanese version accepted: July 2, 2014)
} 
expressways were not continuously performed at the same location, it was difficult to deduce the contribution of I-131 deposited on the ground surface. In this study, we propose a method to estimate the contribution of radionuclides deposited on the soil surface ${ }^{\text {a }}$ resulting from the arrival of the plume, separately from the contribution of radionuclides contained in the plume, from the time histories of peak count rates obtained from the pulse height distribution at monitoring posts.

To validate this method, it was applied to monitoring posts placed in the area around the perimeter monitoring zone set up by the Nuclear Science Research Institute (NSRI) of the Japan Atomic Energy Agency (JAEA) as well as in Tokai Village (hereinafter referred to as "the village"). We estimate the time history of I-131 in the air using the pulse height distribution data measured at the monitoring posts to compare it to the time history of the concentration measured by sampling the air in the NSRI premises ${ }^{2)}$.

The peak count rates for I-131 in the plume were converted to I-131 concentrations using the peak detection efficiency per $\mathrm{Bq} / \mathrm{cm}^{3}$ of $365 \mathrm{keV} \gamma$ ray of $\mathrm{I}-131$, which was obtained by applying the method to replace a photon flux from a plane isotropic source with a point isotropic source and plane detector ${ }^{3)}$ to the egs $5^{4)}$ electromagnetic cascade Monte Carlo code, as described in reference 1 .

\section{Values Measured at Monitoring Posts}

\section{Layout of Monitoring Posts}

NSRI has established monitoring posts at 14 locations in the perimeter monitoring zone and the village as shown in Firure 1 to perform continuous measurement of the air absorbed dose rate. As a detector, a $\phi 2^{\prime \prime} \times 2^{\prime \prime} \mathrm{NaI}(\mathrm{Tl})$ scintillator, which was partially surrounded by a 7-mmthick lead shield to improve the photon energy characteristics to the air absorbed dose rate, was placed at a height of $2.7 \mathrm{~m}$ above the ground surface. Among the monitoring posts, the measurement results obtained at MP-11, MP-21, MP-22, MP-24, and MP-25 were used for analysis.

The conditions of the areas surrounding the respective monitoring posts were as follows. MP11 is on the western boundary near the north gate of the NSRI. There are roads on the premises and pine trees along its southern and northern sides. MP-21 is on the premises of the elementary school of the village (at the time of placement; it is currently used as a schoolchildren's club), and is surrounded by several types of trees. At the south side is a dry field. MP-22 is on a street in an industrial complex in the village and faces a road and a slope on its northeast and southwest sides, respectively. MP-24 is in a residential district and faces a road and houses on its north and south sides, respectively. MP-25 is in a dry field, and at its southeast side is a descending slope.

\section{Time History of Air Absorbed Dose Rate and I-131 Peak Count Rate}

For each monitoring post, we determined the net count rate corresponding to the total absorption peak of $365 \mathrm{keV}$ of I-131 using the pulse height distribution data of the $\mathrm{NaI}(\mathrm{Tl})$ detector measured every $10 \mathrm{~min}$ and adopted this value as the peak count rate. Figures $\mathbf{2}$ and $\mathbf{3}$ show the time history of the air absorbed dose rate (10-min average) from March 15 to March 24 and the time history of the peak count rate, respectively. These figures show that the time history of

\footnotetext{
a Here, "radionuclides deposited on the soil surface" represent the radionuclides which exist not only on the soil surface but also at monitoring posts and surrounding areas, but excluding the ones contained in the plume.
} 


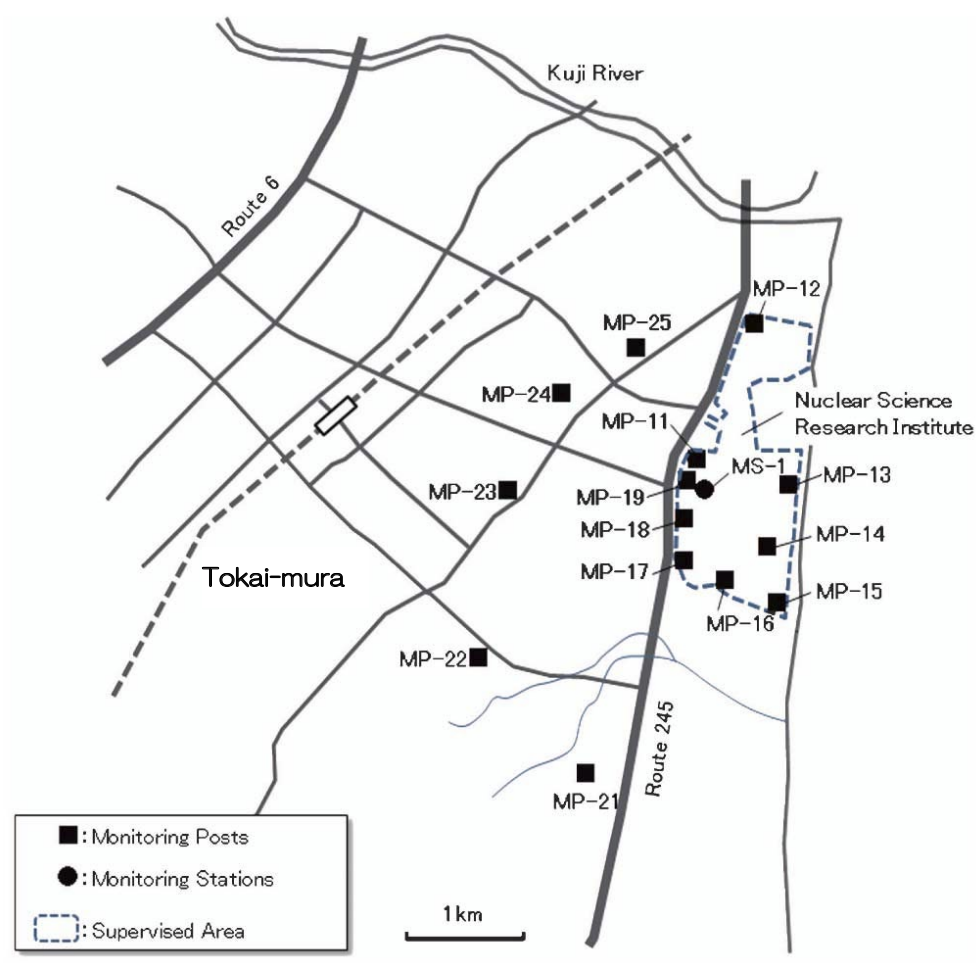

Figure 1 Map of monitoring posts at Nuclear Science Research Institutes of JAEA

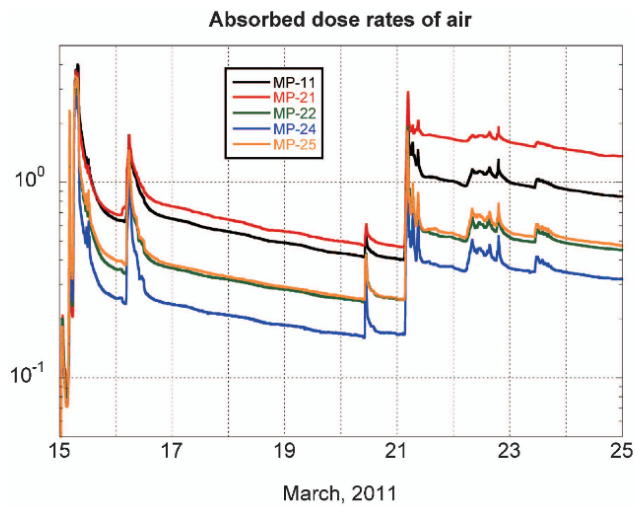

Figure 2 Time variation in absorbed dose rates of air from March 15 to 24 in 2011

the I-131 peak count rate is different from the time history of its air absorbed dose rate. At all of the monitoring posts, while the I-131 peak count rate showed the most noticeable increase on March 21, the air absorbed dose rate was highest on March 15. This means the compositions of the radionuclides contained in the plumes that arrived differ depending on the plume. In addition, the attenuation trend of the air absorbed dose rate resulting from the radionuclides which were deposited after the plume left is different from the I-131 attenuation trend. Therefore, it was found that the I-131 concentration in the plume cannot be estimated only from the air absorbed dose rate and the ambient dose equivalent rate. 


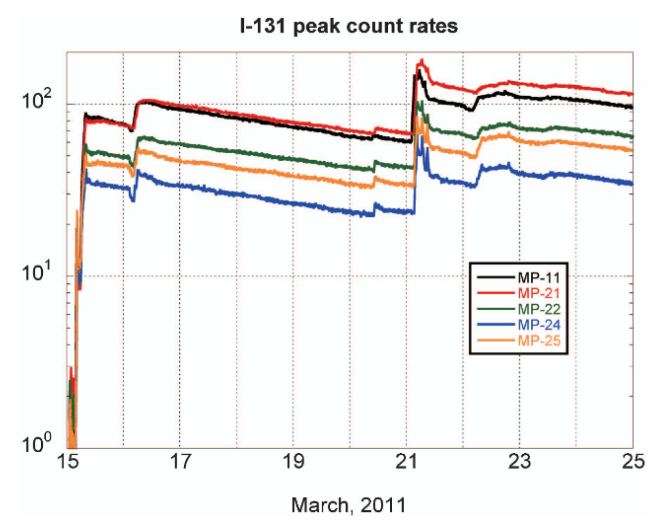

Figure 3 Time variation in I-131 peak count rates from March 15 to 24 in 2011

\section{Estimation of Plume Arrival Time}

Although the plume arrival time and passage completion time can be determined from the time history of the I-131 concentration in the sampled air, we used the method described in the following to determine the arrival time and passage completion time of the locations where the only data available were the data obtained at a monitoring post.

In the time history of the peak count rate corresponding to $365 \mathrm{keV}$ of each monitoring post used for estimating the concentration, the time of measurement when the count rate began to increase was determined as the plume arrival time and the time of measurement when the count rate completed its increase and began to attenuate with a half-life of I-131 was determined as the plume completion time. When it was difficult to determine based on the time history of the I-131 peak count rate, we estimated the time when the plume passed based on the time history of the air absorbed dose rate which changed more significantly. Table 1 shows the plume arrival time and completion time obtained using this method. Because the monitoring posts which were considered this time were located in a small area, the arrival time did not appear to vary significantly among the monitoring posts. The reason why the estimated time, particularly the completion time, varied significantly is because the change in the count rate after the plume passes is relatively small as the deposition increases and uncertainty remains over the determination of the time when the plume has passed. The start and stop times shown in Table 1 are a result of the uncertainty of the process used to determine the arrival time based on this method.

\section{Estimation of the Contribution of the Deposited Radionuclides Based on the Time History of the Peak Count Rates of Pulse Height Distribution}

At the monitoring posts, measurements were repeatedly performed for a predetermined measurement time (measurement section). The average count rate (cps) calculated from the peak counts corresponding to the radionuclides considered within the measurement time was used for the analysis.

For the plumes that arrived, the variables are defined as follows:

$D_{0}$ : The count rate in the measurement section immediately before a plume arrives, in cps

(the count rate corresponding to the contribution of the radionuclides deposited before 
Table 1 Start and stop time used for analysis at each plume and monitoring post

\begin{tabular}{|c|c|c|c|c|c|}
\hline & MP-11 & MP-21 & MP-22 & MP-24 & MP-25 \\
\hline & \multicolumn{5}{|c|}{ March 15} \\
\hline Plume start & $0: 54$ & $0: 47$ & $0: 48$ & $0: 41$ & $0: 41$ \\
\hline Plume stop & $3: 34$ & $3: 27$ & $3: 38$ & $2: 21$ & $2: 21$ \\
\hline Plume start & $3: 34$ & $3: 27$ & $3: 38$ & $3: 41$ & $2: 21$ \\
\hline Plume stop & $5: 34$ & $4: 57$ & $5: 28$ & $5: 41$ & $3: 21$ \\
\hline Plume start & $5: 44$ & $5: 37$ & $5: 58$ & $5: 51$ & $3: 31$ \\
\hline \multirow[t]{2}{*}{ Plume stop } & $11: 14$ & $9: 57$ & $10: 38$ & $8: 51$ & $9: 21$ \\
\hline & \multicolumn{5}{|c|}{ March 16} \\
\hline Plume start & $4: 16$ & 4:07 & $4: 28$ & $4: 21$ & $3: 51$ \\
\hline \multirow[t]{2}{*}{ Plume stop } & $10: 06$ & $8: 37$ & $11: 28$ & $12: 51$ & $9: 31$ \\
\hline & \multicolumn{5}{|c|}{ March 20} \\
\hline Plume start & $9: 46$ & $9: 37$ & 10:08 & $9: 21$ & $10: 11$ \\
\hline \multirow[t]{2}{*}{ Plume stop } & $13: 26$ & $13: 37$ & $13: 48$ & $12: 51$ & $13: 51$ \\
\hline & \multicolumn{5}{|c|}{ March 21} \\
\hline Plume start & $2: 46$ & 3:07 & $3: 18$ & $3: 11$ & $2: 51$ \\
\hline \multirow[t]{2}{*}{ Plume stop } & $10: 56$ & $10: 27$ & $14: 25$ & $10: 51$ & $11: 11$ \\
\hline & \multicolumn{5}{|c|}{ March 22} \\
\hline Plume start & $5: 16$ & $5: 28$ & 5:06 & $6: 21$ & $5: 11$ \\
\hline \multirow[t]{2}{*}{ Plume stop } & $23: 36$ & $23: 38$ & 21:26 & $23: 11$ & $21: 41$ \\
\hline & \multicolumn{5}{|c|}{ March 23} \\
\hline Plume start & $10: 26$ & $11: 08$ & 10:46 & $10: 31$ & $9: 51$ \\
\hline Plume stop & $14: 56$ & $20: 58$ & $16: 36$ & $14: 51$ & $17: 41$ \\
\hline
\end{tabular}

a plume arrives)

$D_{1}$ : The count rate after a plume passes, in cps (the count rate including the contribution of the radionuclides deposited when the plume passes)

$\lambda$ : The decay constant of the radionuclide $(1 / \mathrm{min})$

$t_{c}:$ Measurement time (min)

$i$ : The measurement number in the plume

$m$ : The number of measurements conducted while the plume is arriving

$n_{i}:$ The count rate in the case of the measurement number $i$, in cps

$p_{i}$ : The count rate based on the radionuclide in the plume in the case of the measurement number $i$, in cps

$d_{i}$ : The increment of the count rate based on the radionuclide deposited in the case of the measurement number $i$, in cps

$n_{i}^{\prime}:$ The count rate in the case of the measurement number $i$ from which the count rate of the contribution of the radionuclides deposited before the plume arrives taking into account the attenuation from the time of plume arrival, in cps

$$
\begin{aligned}
& n_{i}^{\prime}=n_{i}-D_{0} \times \exp \left(-(i-1) \times t_{c} \times \lambda\right) \\
& n_{i}^{\prime}=0.0
\end{aligned}
$$

$n_{m}{ }^{\prime}$ : The count rate based on the radionuclides deposited which increased as a result of the passage of the relevant plume, in cps

$$
n_{m}{ }^{\prime}=D_{1}-D_{0} \times \exp \left(-(m-1) \times t_{c} \times \lambda\right)
$$

We assume that a new deposition proportional to the count rate obtained by deducting the contribution of the radionuclides deposited before the relevant measurement section from $n_{i}{ }^{\prime}$ 
(The count rate based on the radionuclides in the plume in the measurement section) was generated as a result of the passage of the plume in each measurement section, and that the count rate obtained as a result of the deposition in the next measurement section accordingly increased. For the contribution of the radionuclides deposited, the attenuation after deposition was considered. The proportionality coefficient of the depositional rate was assumed to be constant while a plume was arriving, and the initial value was set to $f_{0}=0.1^{\mathrm{b}}$.

While applying the aforementioned assumption to the actual measurement values, the calculation was repeated until the relative error between the count rate based on the radionuclides deposited immediately after the plume passed and $n_{m}{ }^{\prime}$ became $1 \%$ or less with the proportionality coefficient which uses the ratio of both values as a correction. The count rate based on the radionuclides contained in the plume in each measurement section was obtained using $d_{i}$ in cases where the relative error was $1 \%$ or less.

During the first phase, the increment $d_{i}$ of the count rate based on the depositional amount increased in each measurement section because of the passage of the plume, using $f_{0}$.

$d_{i}$ does not become negative. Therefore, when $d_{i}$ becomes negative, it should be regarded as zero.

$$
\begin{aligned}
& d_{1}=0.0 \\
& d_{2}=n_{1}{ }^{\prime} \times f_{0}=0.0 \\
& d_{3}=n_{2}{ }^{\prime} \times f_{0} \\
& d_{4}=\left(n_{3}{ }^{\prime}-d_{3}\right) \times f_{0} \\
& d_{5}=\left[n_{4}{ }^{\prime}-\left(d_{3} \times \exp \left(-t_{c} \times \lambda\right)+d_{4}\right)\right] \times f_{0} \\
& \cdots \cdots \\
& d_{m}=\left[n_{m-1}{ }^{\prime}-\sum_{k=3}^{m-1}\left(d_{k} \times \exp \left(-(m-1-k) \times t_{c} \times \lambda\right)\right)\right] \times f_{0} \quad(m \geq 3) \\
& d_{\text {total }, 1}=\sum_{k=3}^{m}\left(d_{k} \times \exp \left(-(m-k) \times t_{c} \times \lambda\right)\right)(m \geq 3)
\end{aligned}
$$

Compare the aforementioned to $n_{m}{ }^{\prime}$, and when

$$
\frac{\left|d_{\text {total }, 1}-n_{m}{ }^{\prime}\right|}{n_{m}{ }^{\prime}}>0.01
$$

$f_{1}=f_{0} \times n_{m}{ }^{\prime} / d_{\text {total }, 1}$ as the new proportionality coefficient and repeat the same operation.

If $d_{\text {total, }, j}$ which satisfies the condition of $\left|d_{\text {total }, j}-n_{m}{ }^{\prime}\right| / n_{m}{ }^{\prime}<0.01$ is obtained, the value $d_{i}$ should be regarded as an increment of the count rate based on the deposition in the case of the measurement number $i$.

Next, the count in each measurement section based on the radionuclides contained in the relevant plume was determined using $d_{i}$. The value $f_{a}$, which is the ratio of the sum of the counts obtained $T_{p}$ to the count rate based on the depositional increase as a result of the passage of the relevant plume $n_{m}{ }^{\prime}$, is a parameter that shows the difference in depositional conditions because of the environment around the monitoring posts and the weather conditions at the time of plume arrival.

\footnotetext{
${ }^{\mathrm{b}}$ If the proportionality coefficient is assumed to be 1 , which is the largest value, the repeat count slightly increases but it has limited influence on the result. Because the results of analyzing several examples converged into a value of approximately 0.1 in many cases, 0.1 was adopted as the initial value.
} 


$$
\begin{aligned}
& T_{p}=n_{1}{ }^{\prime}(=0.0)+\left(n_{2}{ }^{\prime}-d_{1}\right)+\left(n_{3}{ }^{\prime}-\left(d_{1} \times \exp \left(-t_{c} \times \lambda\right)\right)+d_{2}\right)+\cdots+ \\
& \quad\left(n_{m-1}{ }^{\prime}-\sum_{i=1}^{m-2}\left(d_{i} \times \exp \left(-(m-2-i) \times t_{c} \times \lambda\right)\right)\right)=\sum_{i=1}^{m-1} p_{i} \\
& f_{a}=\frac{n_{m}{ }^{\prime}\left(=D_{1}-D_{0}\right)}{T_{p}}
\end{aligned}
$$

Figure 4 shows the schematic relation between the following equation and $p_{i}$ when the convergence conditions are met in the case of $m=8$.

$$
n_{i}^{\prime}, d_{i}, \sum_{k=3}^{i}\left(d_{k} \times \exp \left(-(i-k) \times t_{c} \times \lambda\right)\right)
$$

$d_{i}$ changes in proportion to $p_{i-1}$, and $p_{i}$ and $d_{i}$ becomes zero when $i=8$, showing $n_{i}^{\prime}$ is equivalent to the following equation:

$$
n_{i}^{\prime}=\sum_{k=3}^{i}\left(d_{k} \times \exp \left(-(i-k) \times t_{c} \times \lambda\right)\right)
$$

\section{Calculation of I-131 Detection Efficiency of a Lead- Shielded NaI(TI) Detector with egs5}

As shown in reference 1, the unscattered photons which enter the detector increase slightly as the plume height increases, but the difference is small between $200 \mathrm{~m}$ and $500 \mathrm{~m}$. The plume height was set to $500 \mathrm{~m}$, considering the distance from the Fukushima Daiichi Nuclear Power Plant to Tokai Village, and I-131 was assumed to be spreading infinitely at a uniform density in a horizontal direction from the ground surface to the plume height. By applying the method to replace the gamma-ray flux from a plane isotropic source with a point isotropic source and plane detector ${ }^{3)}$ to egs $5^{4)}$ as described in reference 1 , the photon spectrum of each angle section enters the detector at a height of $2.7 \mathrm{~m}$ from the ground surface in the air of the NTP state $\left(20^{\circ} \mathrm{C}, 1 \mathrm{~atm}\right)$, assuming that the vertical direction distribution is uniform from the ground surface to $500 \mathrm{~m}$.

The $\mathrm{NaI}(\mathrm{Tl})$ detector is structured to project into the upper part of a monitoring post. The

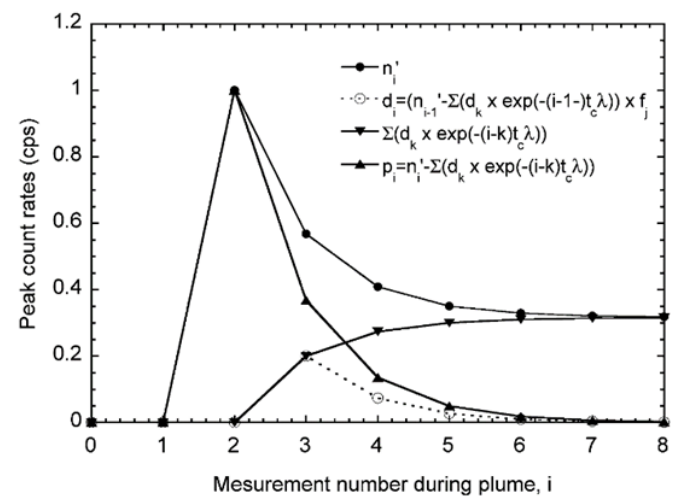

Figure 4 Schematic relation between $n_{i}{ }^{\prime}, d_{i}, \sum_{k=3}^{i}\left(d_{k} \times \exp \left(-(i-k) \times t_{c} \times \lambda\right)\right)$ and $p_{1}$ 
lower structure of the detector and monitoring post contribute to scattered photons but have a limited influence on the non-scattered photons, which may possibly generate a total absorption peak. In the calculation of detection efficiency, considering only the aluminum cover of the $\mathrm{NaI}(\mathrm{Tl})$ detector and lead-shielded $\mathrm{NaI}(\mathrm{Tl})$ detector, the photons having the photon spectra of the respective angle sections previously obtained were assumed to enter the detector at corresponding angles, as the peak detection efficiency corresponding to $365 \mathrm{keV} \gamma$ radiation of I-131, $2.38 \times 10^{4} \mathrm{cps}$ per $\mathrm{Bq} / \mathrm{cm}^{3}$, was obtained. Using this value as a conversion factor, the concentration in the plume was determined from the I-131 peak count rate in the plume obtained according to the method described in Chapter III.

\section{Results and Discussion}

\section{Time History of the Peak Count Rate Caused by the I-131 Contained in the Plumes that Arrived as well as the Deposited I-131}

Figure 5 shows the time history of the peak count rate at MP-21 and MP-24 caused by I-131 contained in the plumes as well as the deposited I-131 during the entire period obtained according to the method described in Chapter III. The peak count rate because of the deposited I-131 was the highest at MP-21 and the lowest at MP-24. Although the difference in the peak count rate resulting from the I-131 contained in the plume was not significant, a difference

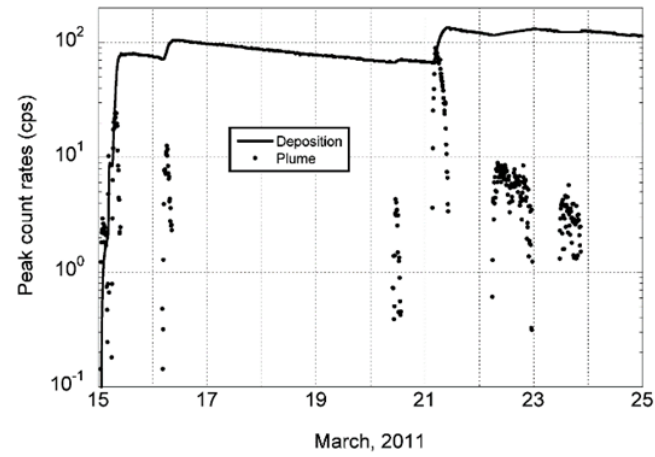

(a) MP-21

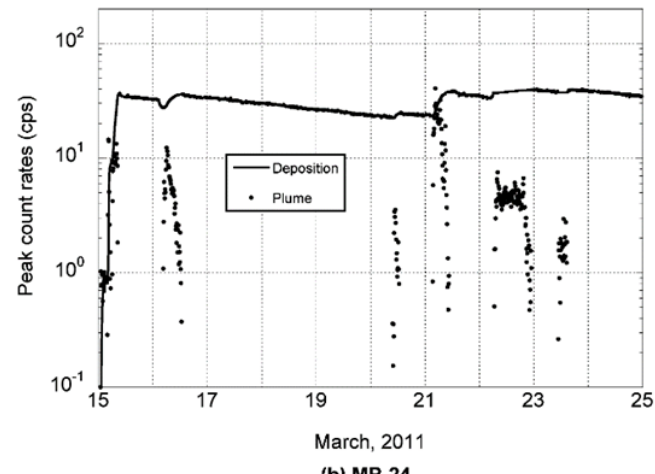

(b) MP-24

Figure 5 Time variation in peak count rates due to I-131 contained in the plume and deposited around (a) MP-21 and (b) MP-24 from March 15 to 24 in 2011 
of nearly 2 or 3 to 1 in the peak count rate resulting from the deposited I-131 was observed between these monitoring posts. This shows that the difference in the environment around the monitoring post makes a difference of from 2 or 3 to 1 in the depositional amount of I-131 and that it is extremely difficult to measure the I-131 concentration contained in the plume from the I-131 depositional density in the way that it has been measured. In both cases, it was found that the plumes that arrived on March 15 and March 21 had a significant influence. A significant decrease in the I-131 peak count rate was observed at MP-24 before the plume arrived on March 16, and the air absorbed dose rate also showed a similar tendency for unknown reasons.

\section{Comparison between the Estimated I-131 Concentration and that Measured using Air Sampling}

Figures 6-8 show the results of comparing the concentrations on March 15, 21, and 22 estimated using the measured pulse height distributions obtained at MP-21 and MP-24 to the measurements obtained by air sampling ${ }^{2}$. The monitoring of the radioactive concentration in the air by air sampling was performed from 01:25 on March 15 to 09:00 on June 6. During the sampling conducted until 7:05 on March 21, a monitoring vehicle was parked in front of MP11 to sample the air using a dust sampler mounted on the vehicle. Subsequent sampling was conducted using a sampler installed at the monitoring station (MS-1).

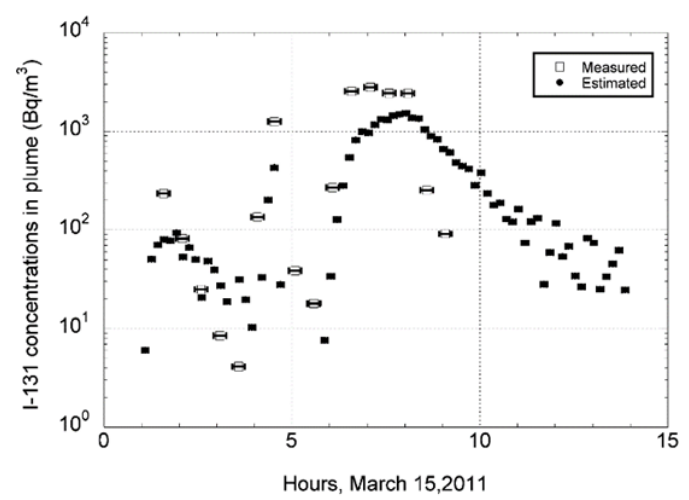

(a) MP-21

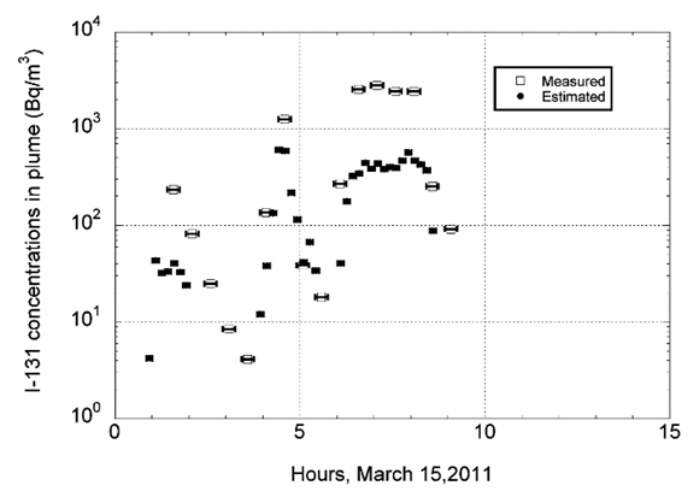

(b) MP-24

Figure 6 Comparison of estimated I-131 concentrations in the plume at (a) MP-21 and (b) MP-24 with those measured by air sampling at March 15, 2011 


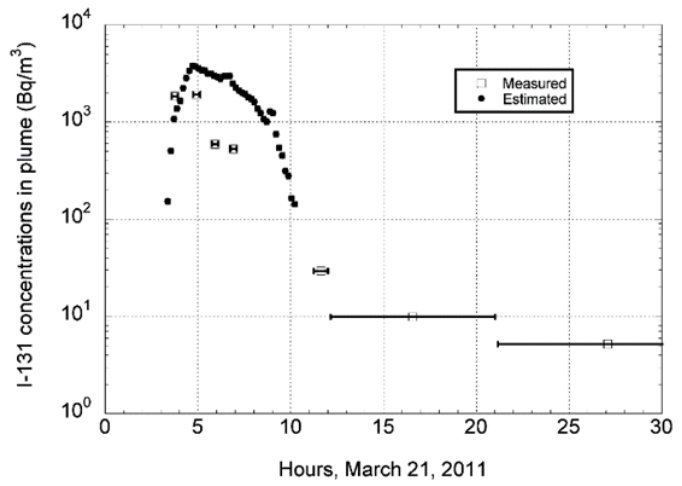

(a) MP-21

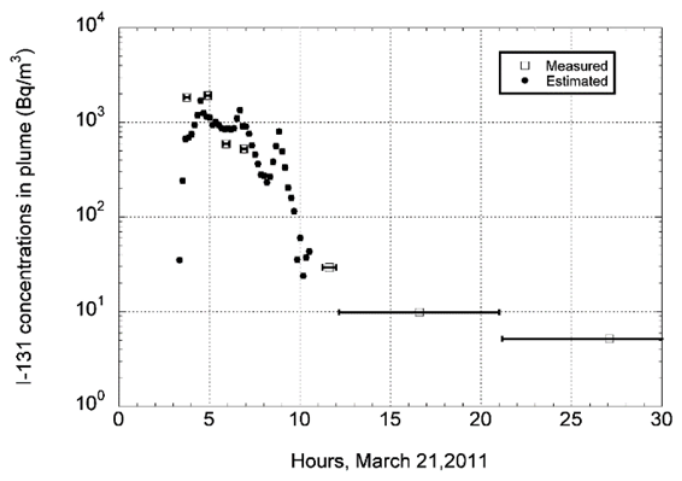

(b) MP-24

Figure 7 Comparison of estimated I-131 concentrations in the plume at (a) MP-21 and (b) MP-24 with those measured by air sampling at March 21, 2011

On March 15, the arrival of a plume was observed for the first time in Tokai Village and the I-131 peak count rate changed very significantly. Concerning the arrival time of the plume, both monitors showed very similar results to those of the air sampling. A significant difference of the time when the plume passed between the results of air sampling and estimated using the measured pulse height distributions was observed at MP-21, reflecting the difficulty in clearly determining the time at which the plume passed. Focusing on the plume at approximately 7:00 when the concentration was highest, the estimated I-131 concentrations at five monitoring posts were underestimated, and were $0.23-0.63$ those of the air sampling measurements.

The results on March 21 correspond to the plumes with the highest I-131 peak count rate. Concerning the time history of the arrival of the plume, both monitors showed results corresponding to the air sampling results. At off-peak times, the estimated concentrations were higher than the measurements at MP-21. At approximately 4:00 when the peak in the measurements was observed, the estimated I-131 concentrations at five monitoring posts were $0.58-1.4$ those of the air sampling measurements.

The results on March 22 were influenced markedly by deposition, and it was difficult to determine the time period of the plume from the time history of the I-131 peak count rate. Although a detailed comparison such as in the case of other plumes could not be performed because the prolonged air sampling time, in terms of the time history of the arrival of the plume there was a good correspondence between the monitoring and air sampling results. However, the estimated I-131 concentrations were consistently higher. The estimated I-131 


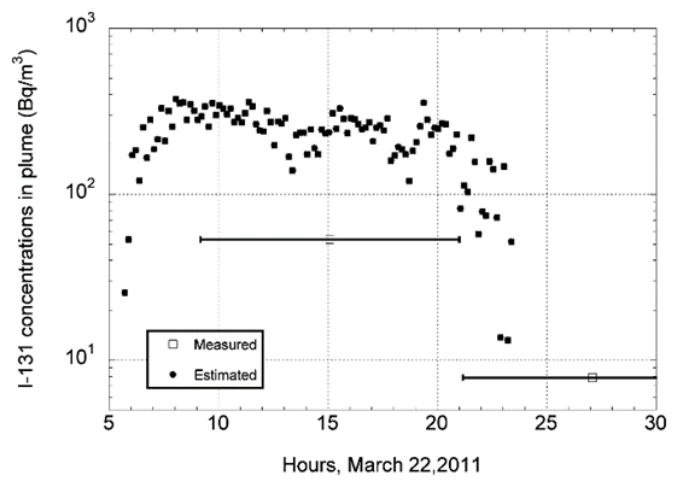

(a) MP-21

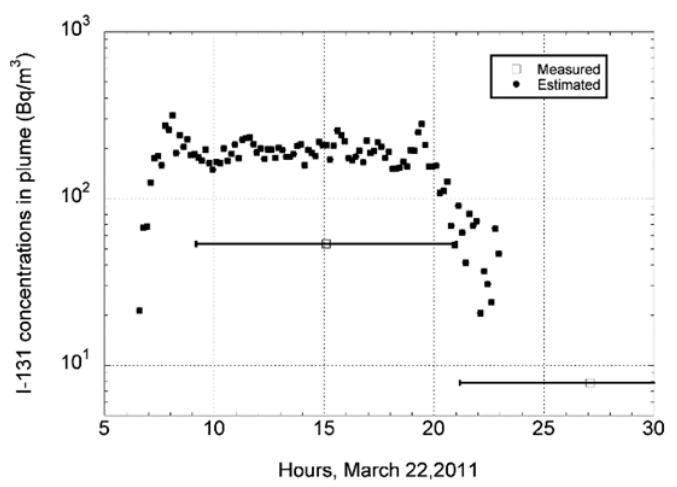

(b) MP-24

Figure 8 Comparison of estimated I-131 concentrations in the plume at (a) MP-21 and (b) MP-24 with those measured by air sampling on March 22, 2011

concentrations at the five monitoring posts were overestimated, and were 3.8-3.9 those of the air sampling measurements.

From the aforementioned results, it can be stated that using this method enables estimation of the time history of the I-131 concentrations in the plume that are near the actual values, although there may be uncertainty in the plume arrival completion time. The extent of the difference from the concentrations obtained through air sampling is up to a factor of 4 in the case of the plume that initially arrived on March 15 as well as the plume containing I-131 at a high concentration such as that which arrived on March 21, corresponding to a factor of 9 in the case where the concentration was low and the plume arrival completion time was difficult to determine. The comparison of the absolute value of the concentrations of sampled air was intended to verify the accuracy of this method in the case where it is used for estimation at a location where no information on the I-131 concentration in the air is available. It was shown that this method can provide much more important information compared to that of the former method in which the time history of the concentration in the air was estimated from the result of calculating an atmospheric dispersion model based on integrated information, the air absorbed dose rate and ambient dose equivalent rate of the radionuclides, which were deposited on the ground surface by the arrival of several plumes, although differing by up to a factor of 9 , which is not insignificant. 


\section{Change in $f_{a}$ Depending on the Time of Plume Arrival}

From the analysis of the change in the peak count rate of I-131 as described in Chapter III, the ratio $f_{a}$ of the total count rate based on I-131 which arrived as a plume to the count rate based on I-131 which was deposited by the plume upon arrival can be determined for each plume.

Regarding the same monitoring post, $f_{a}$ was considered to be a parameter that shows the difference in the depositional conditions caused by weather and the I-131 conditions. In addition, the difference among the monitoring posts in the case of the same plume was regarded as a parameter which depended on deposition condition of each monitoring post due to the difference in surrounding environment. Figure 9 represents $f_{a}$ values of each monitoring post during the observed period. It shows the March 21 to 23 period had smaller $f_{a}$ values than those which arrived on March 15 and 16. While conditions for "dry deposition" occurred on March 15 and 16 because no rainfall was recorded, conditions for "wet deposition" occurred from March 21 to 23 because there was some precipitation. Therefore, this tendency seemed to be in contradiction to "conventional thinking" that the deposition rate was higher in the case of wet deposition. Because the deposition subjected to this analysis included I-131 that adhered to monitoring posts and the areas around them, it is possible that the I-131 which adhered to the surface of the monitoring post near the detector was washed away by the rain and thereby there was a change in the distribution of the I-131 deposited and this had an influence on the readings. In addition, the I-131 conditions such as chemical form varied depending on the time of arrival, which may have had a significant influence on the rate of deposition. To investigate the various factors that affected the relation between the concentration of the radionuclides in a plume and depositional amount, it is necessary to study the change in $f_{a}$ for other nuclides and I-131 observed in other areas.

\section{Conclusions}

We proposed a method for estimating the contribution of radionuclides deposited on the ground surface resulting from plume arrival, separately from the contribution of radionuclides contained in the plume, from the time history of the peak count rates of the radionuclides obtained from the data of the pulse height distributions. We applied this method to five monitoring posts placed in an area around the perimeter monitoring zone of the NSRI to estimate the time history of the I-131 concentrations contained in the plumes that arrived from March

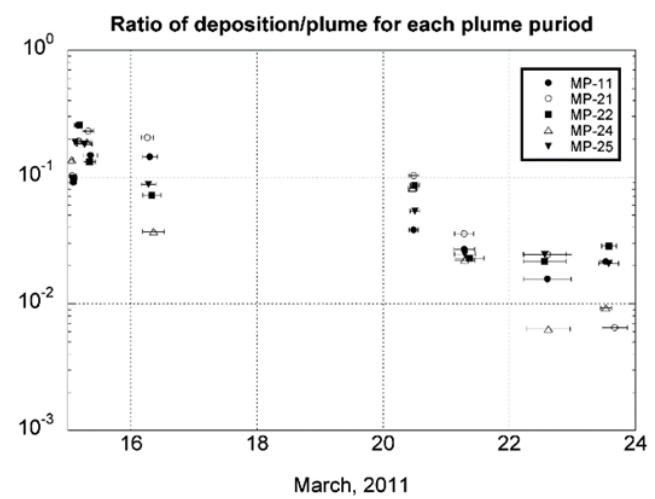

Figure 9 Variation in $f_{a}$ between the plumes and the monitoring posts 
15 to 24,2011 . The time history of the I-131 concentrations obtained showed generally good correspondence to the time history measured by air sampling. Concerning the absolute values of the concentrations, although they varied depending on the ratio of the peak count rate based on the deposited I-131 to the peak count rate based on the I-131 contained in the plume, it was found to be estimated within a range of up to a factor of 4 in the case of the plumes that arrived initially or those containing I-131 at a high concentration as well as within the range of up to a factor of 9 if the concentration was low and the plume completion time was difficult to determine. At the same time, it was also found that the rate of I-131 deposition in the surrounding area varied depending on the conditions around the monitoring posts and the arrival time.

The method we proposed is new and useful in that the time history of the I-131 concentration in the plume, which could not be previously measured without using the air sampling method, can be estimated from the pulse height distribution of the monitoring posts although the accuracy is limited. In the case of the accident that occurred at the Fukushima Daiichi Nuclear Power Plant, the time history of the I-131 concentration in the plumes was not measured in Fukushima Prefecture immediately after the accident. Therefore, the time history of the I-131 concentration in the air was estimated from an atmospheric dispersion model based on dose rate information such as the air absorbed dose rate and ambient dose equivalent rate, data on the density of I-131 deposited on the ground, and data on the density of I-131 estimated from the densities of Cs-134 and Cs-17 deposited on the ground or I-129 with a long half-life period.

However, as shown in Section II-2, it cannot be maintained that the dose rate information consistently corresponded to the time history of the I-131 concentration in the plume. In addition, the deposition on the ground surface is an integral value of the deposition caused by the plumes that arrived, and the deposition conditions varied significantly depending on the surrounding environment. Therefore, there are limitations to the estimation of the time history based on such data. By applying this method to the data of the monitoring posts in various locations where a $\mathrm{NaI}(\mathrm{Tl})$ scintillation detector was used, including the monitoring posts in Fukushima Prefecture for which the measurement data have already been disclosed, there is a possibility that the time history of the I-131 concentration in the plumes in various areas during the early stages of the accident that occurred at Fukushima Daiichi Nuclear Power Plant can be obtained, although the areas are limited to those near the monitoring posts. It is considered that the time history of the I-131 concentration in the plumes at the monitoring posts is useful in improving the accuracy of estimation from atmospheric dispersion models and also enables a more accurate evaluation of the equivalent dose to which the thyroid gland is exposed through linking this information with behavioral data.

\section{References}

1) H. Hirayama, H. Matumura, Y. Namito, T. Sanami, "Estimation of radionuclide concentration in plume using pulse height distribution measured by $\mathrm{LaBr}_{3}$ scintillation detector and its response to radionuclides in plume calculated with egs5," Trans. At. Energy Soc. Jpn., 12, 304-310 (2012). [in Japanese]

2) T. Ohkura, T. Oishi, M. Taki, Y. Shibanuma, M. Kikuchi, H. Akino, Y. Kikuta, M, Kawasaki, J. Saegusa, M. Tsutsumi, H. Ogose, S. Tamura, T. Sawahata, Emergency Monitoring of Environment Radiation and Atmospheric Radionuclides at Nuclear Science Research Institutes, JAEA Following the Accident of Fukushima Daiichi Nuclear Power Plant, JAEA-DATA/Code 2012-010 (2012).

3) Y. Namito, H. Nakamura, A. Toyoda, K. Iijima, H. Iwase, S. Ban, H. Hirayama, "Transformation of a system consisting of plane isotropic source and unit sphere detector into a system consisting of point isotropic source and plane detector in Monte Carlo calculation." J. Nucl. Sci. Technol., 49. 167-172 (2012).

4) H. Hirayama,Y. Namito, A. F. Bielajew, S. J. Wilderman, W. R. Nelson. The EGS5 Code System. SLAC-R-730 (2005) and KEK Report 2005-8 (2005). 


\title{
Treatment of Water Contaminated with Radiocesium Using Novel Complexes between Prussian-Blue and Bivalent Transition Metal Hydroxides
}

\author{
Hisashi UBARA ${ }^{1, *}$, Eiji YUKI ${ }^{1}$, Kazuki TSUKIYAMA ${ }^{1}$, Masato KAMIZAWA ${ }^{1}$, \\ Koichi SENOH ${ }^{1}$, Kohei OHTA ${ }^{1}$, Fumio SHIMAMOTO ${ }^{1}$ and Nariyuki OHTA ${ }^{1}$ \\ ${ }^{1}$ Mie Plant, Mie Chuo Kaihatsu Co., Ltd., 4713 Hachiya, Yono, Iga-shi, Mie 518-1152, Japan
}

\begin{abstract}
A variety of technologies are applied to the decontamination of radiocesium from water using inorganic adsorbents such as zeolites, Prussian blue (PB) and its analogues. However, these adsorbents are difficult to apply. Although zeolites work as good adsorbents for cesium (Cs) in freshwater, their adsorption ability is extremely low in seawater and fly ash extracts with a high salt concentration. In contrast, PB and its analogues maintain their selective adsorption ability for Cs even in water containing salts, but a high level of cyan remains in the treated water. In this study, we introduce a new technology that utilizes complexes between PB and hydroxides of transition metals (PB-X) for the decontamination of Cs from water and report results of demonstration tests on simulated seawater and fly ash extract. Furthermore, the excellent results of the PB-X method applied to the extracts from fly ash contaminated with radiocesium (more than $8,000 \mathrm{~Bq} / \mathrm{kg}$ ) are also shown. It has been proved that radiocesium activities are not only below the detection limit $(<10 \mathrm{~Bq} / \mathrm{kg})$ and the content of cyan can be controlled under the regulation value of tap water in the water treated with PB-X.
\end{abstract}

KEYWORDS: Prussian blue, PB, PB-X, radiocesium, Cs, cyan, decontamination, zeolite, transition meal seawater, fly ash extract

\section{Introduction}

Various treatment technologies have been developed and applied that use non-organic adsorbents such as zeolites or Prussian blue (PB) ${ }^{1-3)}$ and its analogs to decontaminate water containing radiocesium (Cs). Unfortunately, these adsorbents present several troublesome problems. For example, zeolite exhibits excellent Cs-adsorbing performance in contaminated water with low concentrations of sodium or potassium salts. However, in contaminated water with a high salt content, such as seawater, its adsorption performance is significantly degraded ${ }^{6)}$.

Burnable radioactive wastes, such as vegetation, are disposed of by incineration to reduce the volume of waste. After this process, a large quantity of fly ash is generated that exceeds

\footnotetext{
* Corresponding author, E-mail: ubara8379@dinsgr.co.jp

DOI : 10.15669 /fukushimainsights. Vol.4.308

(C) 2021 Atomic Energy Society of Japan. All rights reserved.

Originally published in Transactions of the Atomic Energy Society of Japan (ISSN 1347-2879), Vol. 13, No. 4, p.127-135

(2014) in Japanese. (Japanese version accepted: August 30, 2014)
} 
the radioactive limit designated for waste material $(8,000 \mathrm{~Bq} / \mathrm{kg})$ because the radioactive Cs migrates to the fly ash and condenses ${ }^{7}$. Thus, a study of the decontamination of fly ash by cleaning is required. However, the decontamination of fly ash contaminated with radioactive Cs by water extraction and treatment using zeolite is not efficient because of the high concentrations of $\mathrm{Na}^{+}, \mathrm{K}^{+}$, and $\mathrm{Ca}^{2+}$.

On the other hand, PB maintains its excellent selectivity for Cs adsorption from contaminated water containing salts; however, because of its ultrafine particle size (100 nm and below), the discharge limit for the total cyanide concentration (T-CN) $(<0.5 \mathrm{mg} / \mathrm{L})$ stipulated by Fukushima Prefecture code may be exceeded because of the elution of soluble $\mathrm{PB}^{8)}$. Furthermore, when PB is used as a particulate or powder, a long time is required before adsorption equilibrium is reached (as for zeolite) because of the slow diffusion in the crystal lattice, which limits the rate of Cs adsorption.

In this study, we report a new processing technology for Cs-contaminated water that insolubilizes the soluble PB by quickly adsorbing $\mathrm{Cs}$ at the molecular level during the synthesis of $\mathrm{PB}$ in contaminated water. Subsequently, the synthesis of metal $(\mathrm{Me})$ hydroxide $\left(\mathrm{Me}(\mathrm{II})(\mathrm{OH})_{2}\right)$ with divalent transition metals (Me(II): $\mathrm{Mn}(\mathrm{II}), \mathrm{Fe}(\mathrm{II}), \mathrm{Co}(\mathrm{II}), \mathrm{Ni}(\mathrm{II}), \mathrm{Cu}(\mathrm{II})$, and $\mathrm{Zn}(\mathrm{II})$ ) occurs, forming a new complex, PB-X. Using this method, the direct discharge of treated water is possible. In summary, the process shows (1) quick and highly efficient Cs adsorption and (2) a total cyanide concentration in the treated water below the discharge limit stipulated by the Fukushima Prefecture code.

Possible insolubilization mechanisms for soluble PB by the PB-X material are insolubilization by the interaction of the d-orbitals of the $\mathrm{Me}(\mathrm{II})$ ion of $\mathrm{Me}(\mathrm{II})(\mathrm{OH})_{2}$ or the $\mathrm{d}-\pi$ interactions of the cyano ligand of $\mathrm{PB}$. Alternatively, insolubilization by the intercalation of soluble $\mathrm{PB}$ in the lamellar structure of $\mathrm{Me}(\mathrm{II})(\mathrm{OH})_{2}{ }^{12}{ }^{13)}$ is possible because $\mathrm{Me}(\mathrm{II})(\mathrm{OH})_{2}$ has a hexagonal crystal form with a lamellar structure ${ }^{9-11)}$. A survey of time-dependent Cs removal by $\mathrm{PB}-\mathrm{Me}(\mathrm{II})(\mathrm{OH})_{2}$ treatment was conducted with simulated contaminated seawater and fly ash extract water containing stable Cs. The relationship between the $\mathrm{Me}(\mathrm{II})(\mathrm{OH})_{2} / \mathrm{PB}$ molar ratio and the T-CN concentration in the treated water and the influence of $\mathrm{K}^{+}$on the Cs removal were investigated. The radioactive Cs concentration in the treated water was lower than the stipulated limit $(<10 \mathrm{~Bq} / \mathrm{L})$. Furthermore, after the purification of fly ash extract water containing a high concentration of $\mathrm{K}^{+}(2 \%)$ and a radioactive Cs concentration exceeding $8,000 \mathrm{~Bq} / \mathrm{kg}$, the $\mathrm{T}-\mathrm{CN}$ concentration was in compliance with the limit stipulated by Fukushima Prefecture. In this paper, " $X$ " in PB-X represents $\mathrm{Me}(\mathrm{II})(\mathrm{OH})_{2}$.

\section{Experimental}

In this study, commercial special grade chemicals were used unless noted.

\section{Cs removal from Contaminated Simulated Seawater using Stable ${ }^{133} \mathrm{Cs}$}

(1) Preparation of simulated seawater contaminated with ${ }^{133} \mathrm{Cs}$

Cs-contaminated simulated seawater was prepared by adding cesium chloride ${ }^{133} \mathrm{CsCl}$, $12.7 \mathrm{mg}$ ) to simulated seawater ( $1 \mathrm{~L})$ prepared from distilled water containing sodium chloride ( $\mathrm{NaCl}, 3.0 \%)$, magnesium chloride $\left(\mathrm{MgCl}_{2}, 0.5 \%\right)$, and potassium chloride $(\mathrm{KCl}, 0.05 \%)$. This yielded a concentration of stable ${ }^{133} \mathrm{Cs}$ of $10 \mathrm{mg} / \mathrm{L}(0.075 \mathrm{mmol} / \mathrm{L})$. 


\section{(2) Treatment of ${ }^{133} \mathrm{Cs}$-contaminated simulated seawater using PB-X}

Sodium ferrocyanide decahydrate $\left(0.85 \mathrm{mmol}, \mathrm{Na}_{4} \mathrm{Fe}(\mathrm{II})(\mathrm{CN})_{6} \cdot 10 \mathrm{H}_{2} \mathrm{O}\right)$ obtained from Alfa Aesar was added to $500 \mathrm{~mL}$ of simulated seawater and stirred. Then, ferric chloride hexahydrate $\left(0.9 \mathrm{mmol}, \mathrm{Fe}(\mathrm{III}) \mathrm{Cl}_{3} \cdot 6 \mathrm{H}_{2} \mathrm{O}\right)$ was added, resulting in the formation of $\mathrm{PB}$. The $\mathrm{pH}$ of the treatment liquid was adjusted with an aqueous solution of sodium hydroxide $(5 \mathrm{~mol} / \mathrm{L}, \mathrm{NaOH})$ to $6-8$, and the solution was stirred for $10 \mathrm{~min}$.

Next, a Me(II) salt $(1.7 \mathrm{mmol})$, which is twice the molar concentration of $\mathrm{Na}_{4} \mathrm{Fe}(\mathrm{II})(\mathrm{CN})_{6} \cdot 10 \mathrm{H}_{2} \mathrm{O}$, was added. Thus, $\mathrm{Me}(\mathrm{II})(\mathrm{OH})_{2}$ was prepared by hydrolytic degradation induced by adjusting the $\mathrm{pH}$ to $7-9$ with $5 \mathrm{~mol} / \mathrm{L}$ aqueous $\mathrm{NaOH}$. The solution was stirred for a specified period, and the time-dependent $\mathrm{Cs}$ removal was recorded. Subsequently, the $\mathrm{pH}$ was adjusted to $7-8$, and $1 \mathrm{~mL}$ of a $0.1 \%$ aqueous anionic polymer flocculant (A-115/MT AquaPolymer Inc.) was added; this resulted in the deposition of a coarse floc while gently stirring the mixture. After leaving the solution under static conditions, the treated liquid was filtered through a $5 \mathrm{C}$ filter paper with a $1-\mathrm{mm}$ mesh, and the filtrate and filtered residue were obtained. The time from the synthesis of PB until the formation of the coarse floc on the addition of the polymer flocculant was defined as the processing time. The Cs concentration and T-CN of the filtered liquid were measured.

The $\mathrm{Me}(\mathrm{II})$ salts for insolubilizing the PB used were chlorides or hydrosulfates of $\mathrm{Mn}$ (II), $\mathrm{Fe}(\mathrm{II}), \mathrm{Co}(\mathrm{II}), \mathrm{Ni}(\mathrm{II}), \mathrm{Cu}(\mathrm{II})$, and $\mathrm{Zn}(\mathrm{II})$. In the cases of $\mathrm{Fe}(\mathrm{II})$ and $\mathrm{Mn}(\mathrm{II})$, the dissolved oxygen was removed using sodium sulfite $\left(\mathrm{Na}_{2} \mathrm{SO}_{3}\right)$ to prevent oxidation to $\mathrm{Fe}(\mathrm{III}), \mathrm{Mn}(\mathrm{III})$, or $\mathrm{Mn}(\mathrm{IV})$, and the treatment was conducted in the presence of bubbling nitrogen gas. For comparison with $\mathrm{Fe}(\mathrm{II})$, the insolubilization of $\mathrm{PB}$ was conducted using $\mathrm{Fe}(\mathrm{III}) \mathrm{Cl}_{3}$.

In addition, a commercially available PB pigment ( $0.8 \mathrm{~g}, 2.8 \mathrm{mmol}$, MILORI BLUE, Dainichiseika Color \& Chemicals Mfg., $\mathrm{NH}_{4} \mathrm{Fe}(\mathrm{III}) \mathrm{Fe}(\mathrm{II})(\mathrm{CN})_{6}$ ) was added to $500 \mathrm{~mL}$ of simulated seawater, and the time-dependent variation in the Cs concentration in the treated water was recorded.

\section{(3) Influence of molar ratio of $\mathrm{Me}(\mathrm{II})(\mathrm{OH})_{2} / \mathrm{Na}_{4} \mathrm{Fe}(\mathrm{II})(\mathrm{CN})_{6} \cdot 10 \mathrm{H}_{2} \mathrm{O}$}

The treatments were conducted under similar conditions as II-1-(2) with a fixed amount of added $\mathrm{Na}_{4} \mathrm{Fe}(\mathrm{II})(\mathrm{CN})_{6} \cdot 10 \mathrm{H}_{2} \mathrm{O}(0.85 \mathrm{mmol})$ but varying amounts of added $\mathrm{Me}(\mathrm{II})$ salt, and the relationship between the molar ratio $\mathrm{Me}(\mathrm{II})(\mathrm{OH})_{2} / \mathrm{Na}_{4} \mathrm{Fe}(\mathrm{II})(\mathrm{CN})_{6} \cdot 10 \mathrm{H}_{2} \mathrm{O}$ and the concentration of $\mathrm{Cs}$ and $\mathrm{T}-\mathrm{CN}$ were investigated.

\section{Test of Cs Removal from Simulated FLy Ash Extract Water Containing Stable ${ }^{133} \mathrm{Cs}$}

The following experiments were conducted on the simulated fly ash extract water having different $\mathrm{K}^{+}$concentrations, to investigate the inhibiting effect of $\mathrm{K}^{+}$on Cs removal.

(1) Preparation of ${ }^{133} \mathrm{Cs}$-contaminated simulated fly ash extract water

Four simulated fly ash extract samples were prepared by adding $\mathrm{NaCl}\left(1.2 \%, \mathrm{Na}^{+}: 0.47 \%\right)$, $\mathrm{MgCl}_{2}\left(0.2 \%, \mathrm{Mg}^{2+}: 0.05 \%\right), \mathrm{CaCl}_{2}\left(0.83 \%, \mathrm{Ca}^{2+}: 0.3 \%\right)$, and $\mathrm{KCl}$ to distilled water to yield four different $\mathrm{K}^{+}$concentrations of $0.05 \%, 0.1 \%, 0.5 \%$, and $1.0 \%$ respectively. Cs-contaminated simulated fly ash extract water was prepared by adding $12.7 \mathrm{mg}$ of ${ }^{133} \mathrm{CsCl}$ to $1 \mathrm{~L}$ of each simulated fly ash extract sample to yield a stable ${ }^{133} \mathrm{Cs}$ concentration of $10 \mathrm{mg} / \mathrm{L}(0.075 \mathrm{mmol} / \mathrm{L})$.

(2) Treatment of ${ }^{133} \mathrm{Cs}$-contaminated simulated fly ash extract

$\mathrm{Na}_{4} \mathrm{Fe}(\mathrm{II})(\mathrm{CN})_{6} \cdot 10 \mathrm{H}_{2} \mathrm{O}(1.0 \mathrm{mmol})$ was added to the four simulated fly ash extract 
samples with different $\mathrm{K}^{+}$concentrations $(500 \mathrm{~mL})$ and stirred. Then, ferric chloride anhydride $\left(1.2 \mathrm{mmol}, \mathrm{Fe}(\mathrm{III}) \mathrm{Cl}_{3}\right)$ was added to yield $\mathrm{PB}$. The $\mathrm{pH}$ of the treatment liquid was adjusted to 6-8 with a $5 \mathrm{~mol} / \mathrm{L}$ aqueous $\mathrm{NaOH}$ solution and stirred for $20 \mathrm{~min}$. Next, manganese sulfate hydrate $\left(2.1 \mathrm{mmol}, \mathrm{Mn}(\mathrm{II}) \mathrm{SO}_{4} \mathrm{H}_{2} \mathrm{O}\right)$ or copper sulfate anhydride $\left(2.1 \mathrm{mmol}, \mathrm{Cu}(\mathrm{II}) \mathrm{SO}_{4}\right)$ was added, and the $\mathrm{pH}$ was adjusted to $7-9$ with $5 \mathrm{~mol} / \mathrm{L}$ aqueous $\mathrm{NaOH}$ solution and stirred for $20 \mathrm{~min}$. Subsequently, the $\mathrm{pH}$ was adjusted to $7-8$, and $1 \mathrm{~mL}$ of $0.1 \%$ aqueous A-115 polymer flocculant solution was added, resulting in the deposition of a coarse floc on gentle stirring. After leaving in a static condition, the treated liquid was filtered through a $5 \mathrm{C}$ filter paper with a 1-mm mesh and the filtrate and filtered residues were acquired. The Cs and T-CN concentrations of the filtered liquid were measured.

(3) Elution test of filtered residue according to Notification No. 13 from the Ministry of the Environment ${ }^{14)}$

The filtered substance was weighed wet, soaked in 10 times as much distilled water, and elution was conducted for $6 \mathrm{~h}$ in a shaker. Then, the eluted liquid was filtered through a $5 \mathrm{C}$ filter paper with $1-\mu \mathrm{m}$ screen mesh, and the filtered liquid was acquired. The Cs and T-CN concentrations of the filtered liquid were measured.

\section{Radioactive Cs-Contaminated Fly Ash Extract}

The purification treatment was conducted on fly ash extract with a radioactive concentration of over $8,000 \mathrm{~Bq} / \mathrm{kg}$ provided by Tokyo Power Technology Ltd. under the conditions described in Cases 1-3.

\section{(1) Case 1: Radioactive concentration of $8,900 \mathrm{~Bq} / \mathrm{kg}$}

After the $\mathrm{pH}$ of the fly ash cleaning water $(500 \mathrm{~mL}, \mathrm{pH} 10.7)$ had been adjusted to $\mathrm{pH} 6.9$ with $2 \mathrm{~mol} / \mathrm{L}$ sulfuric acid, $1.75 \mathrm{mmol}$ of $\mathrm{Na}_{4} \mathrm{Fe}(\mathrm{II})(\mathrm{CN})_{6} .10 \mathrm{H}_{2} \mathrm{O}$ was added while stirring. Next, $1.75 \mathrm{mmol}$ of $\mathrm{Fe}(\mathrm{III}) \mathrm{Cl}_{3}$ was added and stirred for $20 \mathrm{~min}$ while adjusting the $\mathrm{pH}$ of the treated liquid to $6.8-7.5$ with $5 \mathrm{~mol} / \mathrm{L}$ aqueous $\mathrm{NaOH}$ solution.

Next, $3.5 \mathrm{mmol}$ of $\mathrm{Cu}$ (II) $\mathrm{SO}_{4}$ was added, and the hydrolytic degradation of $\mathrm{Cu}(\mathrm{II}) \mathrm{SO}_{4}$ was conducted for $20 \mathrm{~min}$ while adjusting the $\mathrm{pH}$ to $7.0-8.0$ with $5 \mathrm{~mol} / \mathrm{L}$ aqueous $\mathrm{NaOH}$ solution. Subsequently, $1 \mathrm{~mL}$ of $0.1 \%$ A- 115 polymer flocculant was added, and a coarse floc deposit was formed on gentle stirring. The treated liquid was filtered through the $5 \mathrm{C}$ filter paper, and the filtrate and filtered residues were acquired.

\section{(2) Case 2: Radioactive concentration of $9,200 \mathrm{~Bq} / \mathrm{kg}$}

After the $\mathrm{pH}$ of the fly ash extract water $(500 \mathrm{~mL}, \mathrm{pH} 11.1)$ had been adjusted to $\mathrm{pH} 4.1$ with $2 \mathrm{~mol} / \mathrm{L} \mathrm{H}_{2} \mathrm{SO}_{4}, 1.75 \mathrm{mmol}$ of $\mathrm{Na}_{4} \mathrm{Fe}(\mathrm{II})(\mathrm{CN})_{6} \cdot 10 \mathrm{H}_{2} \mathrm{O}$ was added while stirring. Next, $1.75 \mathrm{mmol}$ of $\mathrm{Fe}(\mathrm{III}) \mathrm{Cl}_{3}$ was added and stirred for $20 \mathrm{~min}$ while adjusting the $\mathrm{pH}$ of the treated liquid to $7.0-8.0$ with a $5 \mathrm{~mol} / \mathrm{L}$ aqueous $\mathrm{NaOH}$ solution.

Next, $4.0 \mathrm{mmol}$ of $\mathrm{Cu}(\mathrm{II}) \mathrm{SO}_{4}$ was added and stirred for 20 min while adjusting the $\mathrm{pH}$ to 7.0-8.0 with $5 \mathrm{~mol} / \mathrm{L}$ aqueous $\mathrm{NaOH}$ solution. Then, $0.2 \mathrm{~g}$ of $\mathrm{PG} \alpha 21 \mathrm{Ca}$ polymer flocculant provided by Nippon Poly-Glu was added, and a coarse floc deposit was formed on gentle stirring. The treated liquid was filtered through the $5 \mathrm{C}$ filter paper, and the filtrate and filtered residues were acquired.

(3) Case 3: Radioactive concentration of $13,000 \mathrm{~Bq} / \mathrm{kg}$

First, $2.0 \mathrm{mmol}$ of $\mathrm{Na}_{4} \mathrm{Fe}(\mathrm{II})(\mathrm{CN})_{6} \cdot 10 \mathrm{H}_{2} \mathrm{O}$ and $2.0 \mathrm{mmol}$ of $\mathrm{Fe}(\mathrm{III}) \mathrm{Cl}_{3}$ were added in series 
to $500 \mathrm{~mL}$ (pH 5.4 ) of fly ash water and stirred for $20 \mathrm{~min}$ while adjusting the $\mathrm{pH}$ to $7.0-8.6$ with $5 \mathrm{~mol} / \mathrm{L}$ aqueous $\mathrm{NaOH}$ solution. Next, $4.0 \mathrm{mmol}$ of $\mathrm{Cu}(\mathrm{II}) \mathrm{SO}_{4}$ was added. The solution was stirred for $20 \mathrm{~min}$ while adjusting the $\mathrm{pH}$ to $7.0-8.0$ with $5 \mathrm{~mol} / \mathrm{L}$ aqueous $\mathrm{NaOH}$ solution. After that, $1 \mathrm{~mL}$ of $0.1 \%$ A-115 polymer flocculant was added, and a coarse floc deposit was formed on gentle stirring. The treated liquid was filtered through the $5 \mathrm{C}$ filter paper, and the filtrate and filtered residues were acquired.

(4) Elution test according to Notification No. 13 from the Ministry of the Environment

An elution test was conducted on the filtered residue obtained using Case 1 according to Notification No. 13 from the Ministry of the Environment ${ }^{14}$. Here, $90 \%$ of the weight of the filtered residues was moisture, $1 \mathrm{~g}$ in $3.8 \mathrm{~g}$ of solid content was the active material, and the residues were insoluble components such as supernatant that coprecipitated with the active material and $\mathrm{CaSO}_{4}$. Because a quantity of specimen was required for the measurement of T-CN and radioactivity, the filtered residues were used for elution tests with the contained moisture.

\section{Analysis Methodology}

(1) Quantitative analysis of ${ }^{133} \mathrm{Cs}$ in simulated test water

The ${ }^{133} \mathrm{Cs}^{+}$-ion concentrations of the eluted liquid obtained from elution tests of the filtered water and the filtrates acquired after $\mathrm{PB}-\mathrm{X}$ treatment of the ${ }^{133} \mathrm{Cs}$-contaminated simulated seawater and simulated fly ash extract were measured using inductively coupled plasma mass spectrometry (ICP-MS, Agilent 7000x). The lower limit for determination was set to $0.001 \mathrm{mg} / \mathrm{L}$.

(2) Ion analysis of radioactive Cs-contaminated fly ash extract

After diluting the fly ash extract water by 1,000 , a quantitative ion analysis was conducted. The positive ions were measured by ICP-MS (Agilent 7000x) and negative ions were measured by ion chromatography (ICS2000, Dionex).

\section{(3) Total cyanide analysis}

The measurement of the T-CN of the treated water was conducted in accordance with methods 38.1.2 and 38.2 of JIS K0102. The lower limit for determination was set to $0.05 \mathrm{mg} / \mathrm{L}$.

\section{(4) Measurement of radioactive Cs concentration}

The measurement of the concentrations of radioactive $\mathrm{Cs}\left({ }^{134} \mathrm{Cs}\right.$ and $\left.{ }^{137} \mathrm{Cs}\right)$ were conducted on the radioactive-Cs-contaminated fly ash cleaning water, its $\mathrm{PB}-\mathrm{X}$ treated filtrate, and the eluted liquid of elution test for filtered residues under the following conditions.

(a) Measuring device: germanium semiconductor detector (GR3019-7935-7F-RDC-42002C(S/N6516)/Canberra, Japan).

(b) Measuring methodology: $\gamma$-ray spectrometry using germanium semiconductor detector (Ministry of Education, Culture, Sports, Science and Technology, Science and Technology Policy Bureau, Revised in 1992). 


\section{Results and Discussion}

\section{PB-X treatment of ${ }^{133}$ Cs-contaminated Simulated Seawater and Simulated Fly Ash Cleaning Water}

(1) Cs-contaminated simulated seawater

(a) Time dependent change in Cs concentration in the simulated seawater during PB-X treatment

Figure 1 shows the time-dependent fluctuation in Cs concentration of simulated seawater in the treated water using $\mathrm{PB}-\mathrm{Fe}(\mathrm{III})(\mathrm{OH})_{3}$ and $\mathrm{PB}$ pigment for comparison with that treated with $\mathrm{PB}-\mathrm{Me}(\mathrm{II})(\mathrm{OH})_{2}$.

If the 20-min PB synthesis process during PB-X treatment is considered, the adsorption removal rate for $\mathrm{Cs}$ in the simulated seawater should reach the highest value within $1 \mathrm{~h}$ for both processes. It is thought that the Cs adsorption by PB proceeds in molecular level because the PB is synthesized in aqueous solution. On the other hand, for the PB pigment, $24 \mathrm{~h}$ were required to reach equilibrium because of the slow adsorption speed of the $\mathrm{PB}$ pigment. One possible reason for this is that the $\mathrm{Cs}^{+}$-ion adsorption speed is controlled by the diffusion of $\mathrm{Cs}^{+}$ ions in the PB crystal lattice in the case of PB pigment particles. Table 1 Concentration of Cs in the finished water and $\mathrm{Cs}$ removal ratio after $1 \mathrm{~h}$ treatment of the simulated seawater using $\mathrm{PB}-\mathrm{Me}(\mathrm{II})(\mathrm{OH})_{2}, \mathrm{~PB}-\mathrm{Fe}(\mathrm{III})(\mathrm{OH})_{3}$, and $\mathrm{PB}$ dye.

The Cs concentration and Cs removal rate from treated water with treatment time $(1 \mathrm{~h})$ are shown in Table 1. The order of Cs removal rates is $\mathrm{Co}(\mathrm{II})-\mathrm{Ni}(\mathrm{II})>\mathrm{Cu}(\mathrm{II})-\mathrm{Mn}$ (II) $>\mathrm{Fe}(\mathrm{II})>$ $\mathrm{Zn}(\mathrm{II}) \gg \mathrm{Fe}(\mathrm{III})>\mathrm{PB}$ pigment.

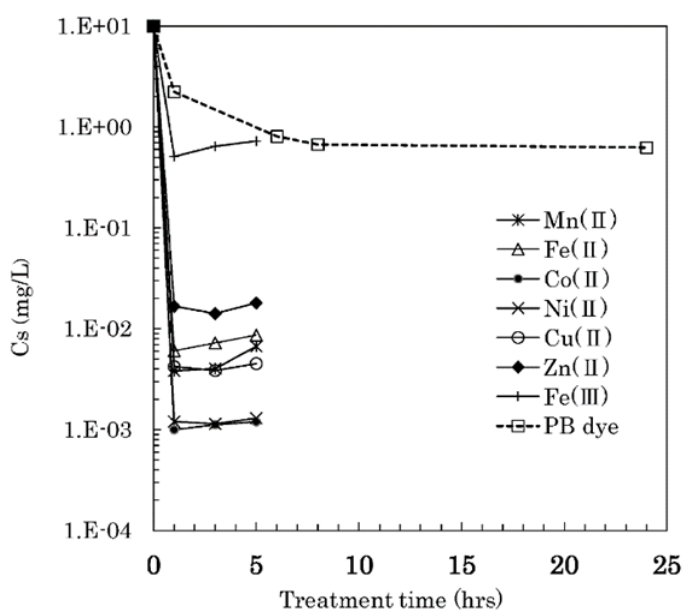

Figure 1 Change in Cs concentration in the filtrates of the simulated seawater treated with $\mathrm{PB}-\mathrm{Me}(\mathrm{II})(\mathrm{OH})_{2}$ and $\mathrm{PB}$ dye with respect to treatment time

Table 1 Concentration of $\mathrm{Cs}$ in the finished water and $\mathrm{Cs}$ removal ratio after $1 \mathrm{~h}$ of treatment of the simulated seawater using $\mathrm{PB}-\mathrm{Me}(\mathrm{II})(\mathrm{OH})_{2}$, $\mathrm{PB}-\mathrm{Fe}(\mathrm{III})(\mathrm{OH})_{3}$, and $\mathrm{PB}$ dye

\begin{tabular}{lcccccccc}
\hline $\mathrm{Me}(\mathrm{II})$ & $\mathrm{Mn}(\mathrm{II})$ & $\mathrm{Fe}(\mathrm{II})$ & $\mathrm{Co}(\mathrm{II})$ & $\mathrm{Ni}(\mathrm{II})$ & $\mathrm{Cu}(\mathrm{II})$ & $\mathrm{Zn}(\mathrm{II})$ & $\mathrm{Fe}(\mathrm{III})$ & $\mathrm{PB}$ dye \\
\hline $\mathrm{Cs}(\mathrm{mg} / \mathrm{L})$ & 0.004 & 0.006 & 0.001 & 0.001 & 0.004 & 0.017 & 0.51 & 2.2 \\
\hline Removal ratio (\%) & 99.96 & 99.94 & 99.99 & 99.99 & 99.96 & 99.83 & 94.91 & 77.69 \\
\hline
\end{tabular}


The distribution coefficient $\left(\mathrm{K}_{\mathrm{d}}\right)$, an index of the adsorption performance of Cs, is expressed by the ratio of $\mathrm{Cs}$ concentration in solid phase to that in the liquid phase. Here, the volume of Cs-contaminated water is $\mathrm{V}(\mathrm{mL})$, the Cs concentration before and after treatment are $\mathrm{C}_{0}$ and $\mathrm{C}_{1}$ $(\mathrm{mg} / \mathrm{L}$ or $\mathrm{Bq} / \mathrm{L})$, respectively, and the quantity of adsorbent is defined as $\mathrm{w}(\mathrm{g}) . \mathrm{K}_{\mathrm{d}}$ is expressed by equation (1):

$$
\mathrm{K}_{\mathrm{d}}=\left\{\left(\mathrm{C}_{0}-\mathrm{C}_{1}\right) / \mathrm{w}\right\} /\left(\mathrm{C}_{1} / \mathrm{V}\right)(\mathrm{mL} / \mathrm{g})
$$

In the PB-X treatment, two moles of Me(II) salt were added per mole of PB. Some $\mathrm{Me}(\mathrm{II})(\mathrm{OH})_{2}$ generated as a result of hydrolytic degradation of the Me(II) salt is dissolved, depending on the solubility product; here, using equation (1) the molecular formula of the active material that insolubilizes the soluble PB was determined to be $\mathrm{NaFe}(\mathrm{III}) \mathrm{Fe}(\mathrm{II})(\mathrm{CN})_{6} \cdot 2 \mathrm{Me}(\mathrm{II})(\mathrm{OH})_{2}$. Table 2 shows the $\mathrm{K}_{d}$ values of the active materials after $1 \mathrm{~h}$ of PB-X treatment. The $\mathrm{K}_{\mathrm{d}}$ values are high, $10^{6}$ to $10^{7} \mathrm{~mL} / \mathrm{g}$, except for that of $\mathrm{Zn}(\mathrm{II})$. On the other hand, the $\mathrm{K}_{\mathrm{d}}$ values of PB-Fe(III)(OH) and PB-dye are low, 22,000 and 2,200 $\mathrm{mL} / \mathrm{g}$.

(b) Concentration of T-CN and transition metal (Me) in treated water

In the PB-X treatment, T-CN was not detected $(<0.005 \mathrm{mg} / \mathrm{L})$ in any of water samples treated for $1 \mathrm{~h}$. On the other hand, the T-CN concentrations of water treated with $\mathrm{PB}-\mathrm{Fe}(\mathrm{III})(\mathrm{OH})_{3}$ and PB pigment were 110 and $1.8 \mathrm{mg} / \mathrm{L}$, respectively, thus exceeding the discharge limit $(0.5 \mathrm{mg} / \mathrm{L})$ stipulated by the Fukushima Prefecture code. The inferior performance of $\mathrm{Fe}(\mathrm{III})(\mathrm{OH})_{3}$ with respect to Cs adsorption and T-CN concentration compared to those of $\mathrm{Fe}(\mathrm{II})(\mathrm{OH})_{2}$ can be explained by the difference in affinity of $\mathrm{Fe}(\mathrm{II})$ and $\mathrm{Fe}(\mathrm{III})$ for the $\mathrm{PB}$ ion $\left[\mathrm{Fe}(\mathrm{III}) \mathrm{Fe}(\mathrm{II})(\mathrm{CN})_{6}\right]^{-}$.

According to the HSAB rules, Fe(III) is classified as a harder acid than Fe(II). Because the PB ion is a soft base, a hard acid like Fe(III) cannot form a sufficiently strong interaction with the $\mathrm{PB}$ ion for insolubilization. That is, part of $\mathrm{Fe}(\mathrm{III})(\mathrm{OH})_{3}$ may be eluted by disassociation even if the PB ion is insolubilized by attractive forces. On the other hand, for $\mathrm{Fe}(\mathrm{II})(\mathrm{OH})_{2}$, the interactions between the d-orbital of the soft acid (Fe(II)) and the $\pi$-electrons of the soft base $\left(\mathrm{CN}^{-}\right)$contribute to the insolubilization of soluble PB. In addition, the difference in the physical structures of $\mathrm{Fe}(\mathrm{II})(\mathrm{OH})_{2}$ and $\mathrm{Fe}(\mathrm{III})(\mathrm{OH})_{2}$ should be mentioned ${ }^{19)}$. A possible mechanism for the insolubilization is via the intercalation of soluble $\mathrm{PB}$ in $\mathrm{Fe}(\mathrm{II})(\mathrm{OH})_{2}$, which has a hexagonal crystal structure with a lamellar structure. For example, according to a report by Takahashi et al ${ }^{13)}$., when the $\mathrm{Zn}(\mathrm{II})$ salt is hydrolyzed under the presence of anions, the coprecipitation of zinc hydroxide $\mathrm{Zn}(\mathrm{II})(\mathrm{OH})_{2}$ occurs with the intercalation of the anions between layers. The distance between layers varies according to the molecular length of the coprecipitated anion. For example, the layer distance is $12 \AA$ in coprecipitation with succinic acid, which has an ion length of $7.6 \AA$, while coprecipitation with dodecanedioic acid ion increases the layer distance to $18 \AA$. Consequently, the layer distance will also be increased to more than $5 \AA$, the ion length of the co-present $\mathrm{PB}$ ion, in the case of $\mathrm{Fe}(\mathrm{II})(\mathrm{OH})_{2}$, which has a lamellar structure; thus, the intercalation of $\mathrm{PB}$ ions seems possible.

Table 2 Distribution coefficient $\left(\mathrm{K}_{\mathrm{d}}\right)$ for $\mathrm{Cs}$ of the active materials, $\mathrm{PB} \cdot 2 \mathrm{Me}(\mathrm{II})(\mathrm{OH})_{2}$

\begin{tabular}{lr}
\hline $\mathrm{Me}(\mathrm{II})$ & $\mathrm{Kd}(\mathrm{mL} / \mathrm{g})$ \\
\hline $\mathrm{Mn}(\mathrm{II})$ & $3,100,000$ \\
$\mathrm{Fe}(\mathrm{II})$ & $1,600,000$ \\
$\mathrm{Co}(\mathrm{II})$ & $12,000,000$ \\
$\mathrm{Ni}(\mathrm{II})$ & $12,000,000$ \\
$\mathrm{Cu}(\mathrm{II})$ & $2,400,000$ \\
$\mathrm{Zn}(\mathrm{II})$ & 710,000 \\
\hline
\end{tabular}


On the other hand, the structure of $\mathrm{Fe}(\mathrm{III})(\mathrm{OH})_{3}$ is amorphous, and it forms gel-like colloid particles, adsorbing the PB ion with weak coulombic interactions on the particle surfaces. Therefore, some PB can become disassociated, eventually being detected in the T-CN.

Table 3 shows the quantity of eluted Me(II) ions in the water treated with PB-X for $1 \mathrm{~h}$. PB$\mathrm{Co}(\mathrm{II})(\mathrm{OH})_{2}$ and $\mathrm{PB}-\mathrm{Ni}(\mathrm{II})(\mathrm{OH})_{2}$ show excellent Cs removal rate, but the quantities of Co and Ni eluted in the treated water were high ( 20 and $26 \mathrm{mg} / \mathrm{L}$, respectively), and the treated water was slightly colored. The eluted quantity of $\mathrm{Me}(\mathrm{II})$ corresponds to the solubility product $\left(\mathrm{K}_{\mathrm{sp}}\right)$ of $\mathrm{Me}(\mathrm{II})(\mathrm{OH})_{2}{ }^{15)}$, and the $\mathrm{PB}-\mathrm{X}$-treated water using $\mathrm{Cu}(\mathrm{II})(\mathrm{OH})_{2}$ showed the lowest metal elution, $0.03 \mathrm{mg} / \mathrm{L}$.

In our process, Cs is removed from solution by the synthesis of $\mathrm{PB}$ from ferrocyanide and $\mathrm{Fe}$ (III) salts in the contaminated water, followed by insolubilization of the soluble PB with $\mathrm{Me}(\mathrm{II})(\mathrm{OH})_{2}$. The mechanism can be expressed by formulas (2)-(6)

$$
\mathrm{Na}_{4} \mathrm{Fe}(\mathrm{II})(\mathrm{CN})_{6}+\mathrm{Fe}(\mathrm{III}) \mathrm{Cl}_{3} \rightarrow \mathrm{NaFe}(\mathrm{III}) \mathrm{Fe}(\mathrm{II})(\mathrm{CN})_{6}+3 \mathrm{NaCl}
$$

$\mathrm{Cs}^{+}$ions are captured by $\mathrm{PB}$ by the ion exchange reaction with $\mathrm{Na}^{+}$ions in $\mathrm{PB}$, as indicated in formula (3).

$$
\mathrm{Cs}^{+}+\mathrm{NaFe}(\mathrm{III}) \mathrm{Fe}(\mathrm{II})(\mathrm{CN})_{6} \rightarrow \mathrm{CsFe}(\mathrm{III}) \mathrm{Fe}(\mathrm{II})(\mathrm{CN})_{6}+\mathrm{Na}^{+}
$$

$\mathrm{Next}, \mathrm{Me}(\mathrm{II})(\mathrm{OH})_{2}$ is synthesized through hydrolytic degradation, as shown by formula (4).

$$
\mathrm{Me}(\mathrm{II}) \mathrm{X}_{2-\mathrm{n}}^{(\mathrm{n}+1)-}+2 \mathrm{NaOH} \rightarrow \mathrm{Me}(\mathrm{II})(\mathrm{OH})_{2}+2 \mathrm{Na}^{+}+\mathrm{X}_{2-\mathrm{n}}^{(\mathrm{n}+1)-}
$$

Here, when $\mathrm{n}=0$, the anion is univalent, and, when $\mathrm{n}=1$, the anion is bivalent.

The hydrolytic degradation of $\mathrm{Me}$ (II) salts is achieved through the $\mathrm{pH}$ adjustment of reaction liquid. When the $\mathrm{Me}(\mathrm{II})$ salt is added with a molar ratio, $\mathrm{x}$, of $\mathrm{Me}(\mathrm{II}) \mathrm{salt} / \mathrm{NaFe}(\mathrm{III}) \mathrm{Fe}(\mathrm{II})(\mathrm{CN})_{6}$, a novel complex $\mathrm{NaFe}(\mathrm{III}) \mathrm{Fe}(\mathrm{II})(\mathrm{CN})_{6} \cdot \mathrm{yMe}(\mathrm{II})(\mathrm{OH})_{2}$ is generated, as indicated in the reaction formulas (5) and (6), and it is considered that this insolubilizes the soluble PB. When the solubility of $\mathrm{Me}(\mathrm{II})(\mathrm{OH})_{2}$ is small, $\mathrm{x}=\mathrm{y}$.

$$
\begin{aligned}
& \mathrm{xMe}(\mathrm{II})(\mathrm{OH})_{2} \rightleftarrows \mathrm{yMe}(\mathrm{II})(\mathrm{OH})_{2}+\left(\mathrm{x}^{-} \mathrm{y}\right) \mathrm{Me}(\mathrm{II})^{2+}+2\left(\mathrm{x}^{-} \mathrm{y}\right) \mathrm{OH}^{-} \\
& \mathrm{M}(\mathrm{I}) \mathrm{Fe}(\mathrm{III}) \mathrm{Fe}(\mathrm{II})(\mathrm{CN})_{6}+\mathrm{yMe}(\mathrm{II})(\mathrm{OH})_{2} \rightarrow \mathrm{M}(\mathrm{I}) \mathrm{Fe}(\mathrm{III}) \mathrm{Fe}(\mathrm{II})(\mathrm{CN})_{6} \cdot \mathrm{yMe}(\mathrm{II})(\mathrm{OH})_{2} \\
& (\mathrm{~PB}-\mathrm{X})
\end{aligned}
$$

Here, $\mathrm{M}(\mathrm{I})$ is $\mathrm{Na}$ or Cs.

Table 3 lists the $y$ values of the novel complex in reaction formula (6) calculated from the $\mathrm{Me}(\mathrm{II})$ concentration in the treated water. If the $\mathrm{Me}$ (II) concentration is low, $\mathrm{y}$ is equal to $\mathrm{x}$. The value $y$ for $\mathrm{Mn}$ (II) that shows the maximum elution of $57 \mathrm{mg} / \mathrm{L}$ is $\mathrm{y}=1.4$, but $\mathrm{y}=2$ for $\mathrm{Cu}$ (II) because of the low concentration $(0.03 \mathrm{mg} / \mathrm{L})$ in the treatment water. The relationship of the $y$ value of the novel complex and the T-CN concentration of the treated water are discussed in the next section.

Table $3 \mathrm{Me}(\mathrm{II})$ concentrations in the treated water of the simulated seawater treated with PB-X, the solubility product $\left(\mathrm{K}_{\mathrm{sp}}\right)^{15)}$ of $\mathrm{Me}(\mathrm{II})(\mathrm{OH})_{2}$, and the $\mathrm{y}$ value in the active $\mathrm{PB}-\mathrm{yMe}(\mathrm{II})(\mathrm{OH})_{2}$ complex

\begin{tabular}{cccccccc}
\hline Me (II) & $\mathrm{Mn}$ & $\mathrm{Fe}$ & $\mathrm{Co}$ & $\mathrm{Ni}$ & $\mathrm{Cu}$ & $\mathrm{Zn}$ \\
\hline Concentration $(\mathrm{mg} / \mathrm{L})$ & 57 & 3.4 & 20 & 26 & 0.03 & 2.9 \\
\hline $\mathrm{K}_{\mathrm{sp}}$ at $25^{\circ} \mathrm{C}$ & $1.9 \times 10^{-13}$ & $8.0 \times 10^{-16}$ & $1.6 \times 10^{-15}$ & $2.0 \times 10^{-15}$ & $2.2 \times 10^{-20}$ & $1.2 \times 10^{-17}$ \\
\hline $\mathrm{y}$ & 1.39 & 1.96 & 1.80 & 1.74 & 2.00 & 1.97 \\
\hline
\end{tabular}


(c) $\mathrm{Me}(\mathrm{II})(\mathrm{OH})_{2} / \mathrm{PB}$ molar ratio, $\mathrm{y}$, and the $\mathrm{Cs}$ concentration and $\mathrm{T}-\mathrm{CN}$ of the treated water

In the PB-X treatment, the T-CN concentration of the treated water is decreased by the insolubilization of soluble $\mathrm{PB}$ by $\mathrm{Me}(\mathrm{II})(\mathrm{OH})_{2}$. The effect is related to the molar ratio, y, of $\mathrm{Me}(\mathrm{II})(\mathrm{OH})_{2}$ and $\mathrm{PB}$. The relationship between the Cs concentration and T-CN concentration of the treated water with respect to $y$ for the case of $\mathrm{Me}(\mathrm{II})=\mathrm{Cu}$ (II) in the $\mathrm{PB}-\mathrm{X}$ treatment is shown in Figure 2. The Cs concentration of the treated water is approximately constant with respect to $\mathrm{y}$, but the T-CN concentration decreases as y increases. From Figure 2, y $\geqq 1.2$ is necessary to satisfy the Fukushima Prefecture T-CN discharge limit $(<0.5 \mathrm{mg} / \mathrm{L})$ by insolubilization through the interaction of $\mathrm{Cu}(\mathrm{II})(\mathrm{OH})_{2}$ and soluble $\mathrm{PB}$. This is also true for other $\mathrm{Me}(\mathrm{II})$ species, such as $\mathrm{Mn}(\mathrm{II})$.

Unlike T-CN, the Cs concentration in the treated water shows almost no change with respect to $\mathrm{y}$. This implies that the T-CN eluted from the treated samples is derived from the PB that did not adsorb Cs, and the PB that adsorbed Cs is insoluble. Presumably this is attributable to the nature of the affinity of $\mathrm{Na}^{+}, \mathrm{K}^{+}, \mathrm{Ca}^{2}$, and $\mathrm{Cs}^{+}$for the $\mathrm{PB}$ ion $\left.\left([\mathrm{Fe}(\mathrm{III}) \mathrm{Fe}(\mathrm{II}) \mathrm{CN})_{6}\right]^{-}\right)$. This can be explained by the HSAB rules.

According to the HSAB rules, chemical species can be classified as acids or bases and hard and soft species. The hard acids and hard bases or soft acids and soft bases form stable interactions, but the hard acid and soft base or soft acid and hard base do not form stable interactions.

Cs is an alkali metal in period six, and the lowest unoccupied atomic orbital (LUAO) of $\mathrm{Cs}^{+}$ is the $6 \mathrm{~s}$ orbital. It is classified as an easily polarized soft acid compared to $\mathrm{Na}^{+}(3 \mathrm{~s})$ and $\mathrm{K}^{+}$and $\mathrm{Ca}^{2+}(4 \mathrm{~s})$. The order of softness of the cations is $\mathrm{Cs}^{+}$(soft) $\gg \mathrm{K}^{+}>\mathrm{Na}^{+}>\mathrm{Ca}^{2+}$ (hard).

On the other hand, the $\mathrm{PB}$ ion $\left(\left[\mathrm{Fe}(\mathrm{III}) \mathrm{Fe}(\mathrm{II})(\mathrm{CN})_{6}\right]^{-}\right)$is a soft base that will tightly bond with soft acid $\mathrm{Cs}^{+}$, but the bonding of hard acids $\mathrm{Ca}^{2+}, \mathrm{Na}^{+}, \mathrm{K}^{+}$, and $\left[\mathrm{Fe}(\mathrm{III}) \mathrm{Fe}(\mathrm{II})(\mathrm{CN})_{6}\right]^{-}$is weak. As a result, these complexes disassociate in aqueous solution. According to Klopman ${ }^{17}$, the formation of interactions between soft acid $\mathrm{Cs}^{+}$and soft base PB ion is dominated by the interaction of the LUAO and the frontier molecular orbitals of the highest unoccupied molecular orbital (HOMO) of the PB ion; presumably, the Cs-PB bond has some covalent character and may form insoluble particles.

\section{(2) Simulated fly ash extract}

$\mathrm{Mn}(\mathrm{II})$ and $\mathrm{Cu}$ (II) showed the same Cs removal rates in the PB-X treatment of simulated seawater, and a PB-X treatment was conducted on the simulated fly ash extract water containing

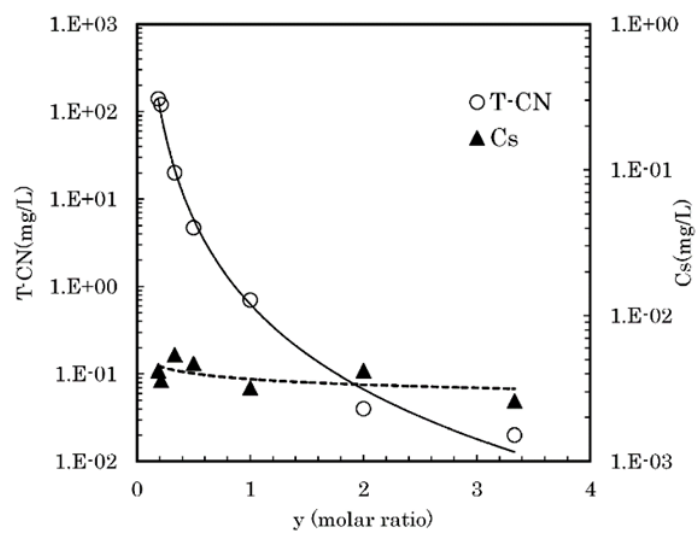

Figure 2 Effect of the $\mathrm{Cu}(\mathrm{II})(\mathrm{OH})_{2} / \mathrm{PB}$ molar ratio $(\mathrm{y})$ on the $\mathrm{T}-\mathrm{CN}$ and $\mathrm{Cs}$ concentrations in the simulated seawater treated with $\mathrm{PB}-\mathrm{Cu}(\mathrm{II})(\mathrm{OH})_{2}$ 
four levels of $\mathrm{K}^{+}$content: $0.05 \%, 0.1 \%, 0.5 \%$, and $1.0 \%$. The results acquired for the treated water and filtered residue are summarized in the next section.

(a) Influence of $\mathrm{K}^{+}$content in simulated seawater on Cs adsorption performance

The concentrations of $\mathrm{Cs}$ in the PB-X-treated water with $\mathrm{Me}(\mathrm{II})=\mathrm{Mn}$ or $\mathrm{Cu}$, T-CN concentration, and $\mathrm{K}_{\mathrm{d}}$ of the active materials under these treatment conditions are listed in Tables 4 and 5 respectively. Furthermore, the change in the Cs concentration in the treated water and $\mathrm{K}_{\mathrm{d}}$ with regard to $\mathrm{K}^{+}$content are shown in Figure 3.

The $\mathrm{K}_{\mathrm{d}}$ is $10^{4}$ to $10^{5} \mathrm{~mL} / \mathrm{g}$ for Cs in zeolite in pure water, and the inhibitory effect on Cs adsorption by $\mathrm{Na}^{+}$or $\mathrm{K}^{+}$have been reported ${ }^{6,7)}$.

Table 4 Concentrations of metal ions and T-CN in the finished water and $\mathrm{K}_{\mathrm{d}}$ for $\mathrm{Cs}$ of $\mathrm{PB} \cdot 2 \mathrm{Mn}(\mathrm{II})(\mathrm{OH})_{2}$ with respect to $\mathrm{K}^{+}$content in the simulated fly ash extract

\begin{tabular}{|c|c|c|c|c|c|c|c|c|c|}
\hline \multirow{3}{*}{$\begin{array}{l}\mathrm{K} \text { Contents in } \\
\text { the extracts }(\%)\end{array}$} & \multicolumn{9}{|c|}{ Concentration of metals and $\mathrm{T}-\mathrm{CN}$ in the finished water } \\
\hline & $\mathrm{Na}$ & $\mathrm{Mg}$ & $\mathrm{K}$ & $\mathrm{Ca}$ & $\mathrm{Mn}$ & $\mathrm{Fe}$ & Cs & $\mathrm{T}-\mathrm{CN}$ & $\mathrm{K}_{\mathrm{d}}$ \\
\hline & \multicolumn{8}{|c|}{$(\mathrm{mg} / \mathrm{L})$} & $(\mathrm{mL} / \mathrm{g})$ \\
\hline 0.05 & 4,500 & 450 & 380 & 590 & 93 & 0.13 & 0.004 & 0.08 & $2.7 \times 10^{6}$ \\
\hline 0.1 & 4,500 & 450 & 820 & 580 & 91 & 0.06 & 0.015 & 0.11 & $7.1 \times 10^{5}$ \\
\hline 0.5 & 4,400 & 440 & 4,500 & 590 & 98 & 0.03 & 0.039 & 0.05 & $2.7 \times 10^{5}$ \\
\hline 1.0 & 4,500 & 440 & 9,100 & 590 & 106 & 0.05 & 0.058 & $<0.05 \mathrm{a})$ & $1.8 \times 10^{5}$ \\
\hline
\end{tabular}

a) An inequality sign in the table indicates a value less than lower detection limit.

Table 5 Concentrations of metallic ions and T-CN in the treated water, and $\mathrm{K}_{\mathrm{d}}$ for Cs of $\mathrm{PB}-2 \mathrm{Cu}(\mathrm{II})(\mathrm{OH})_{2}$ with respect to $\mathrm{K}^{+}$content in the simulated fly ash extracts

\begin{tabular}{ccccccccccc}
\hline \multirow{2}{*}{$\begin{array}{l}\text { K Content in } \\
\text { the extracts (\%) }\end{array}$} & $\mathrm{Na}$ & $\mathrm{Mg}$ & $\mathrm{K}$ & $\mathrm{Ca}$ & $\mathrm{Cu}$ & $\mathrm{Fe}$ & $\mathrm{Cs}$ & $\mathrm{T}-\mathrm{CN}$ & $\begin{array}{c}\mathrm{K}_{\mathrm{d}} \\
(\mathrm{mL} / \mathrm{g})\end{array}$ \\
\cline { 2 - 11 } & & & \multicolumn{7}{c}{ Concentration of metals and T-CN in the finished water } \\
\hline 0.05 & 4,800 & 470 & 460 & 600 & 0.02 & 0.03 & 0.005 & $<0.05$ & $2.1 \times 10^{6}$ \\
\hline 0.1 & 4,700 & 460 & 900 & 590 & 0.01 & 0.04 & 0.007 & $<0.05$ & $1.5 \times 10^{6}$ \\
\hline 0.5 & 4,600 & 440 & 4,600 & 590 & 0.03 & 0.02 & 0.008 & $<0.05$ & $1.3 \times 10^{6}$ \\
\hline 1.0 & 4,600 & 450 & 9,300 & 590 & 0.01 & 0.04 & 0.012 & $<0.05$ & $8.6 \times 10^{5}$ \\
\hline
\end{tabular}

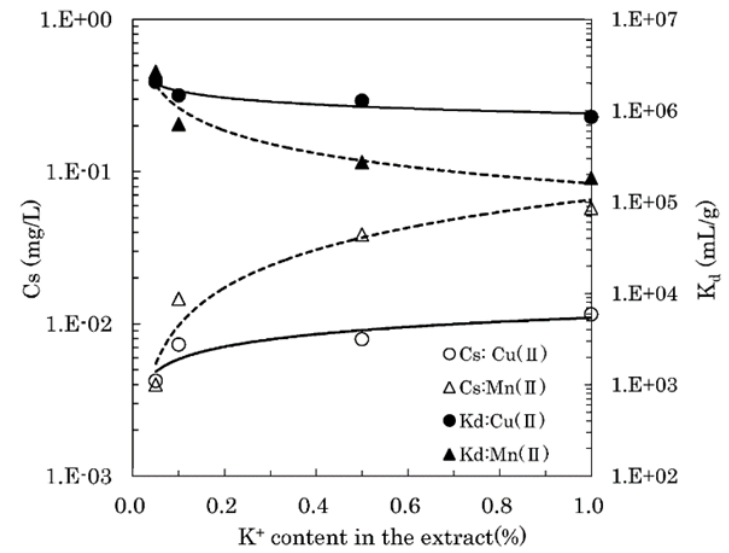

Figure 3 Effect of $\mathrm{K}^{+}$content in the simulated fly ash extracts on the concentration of $\mathrm{Cs}$ in the treated water, and the distribution coefficient $\left(\mathrm{K}_{\mathrm{d}}\right)$ of $\mathrm{Cs}$ for $\mathrm{PB} \cdot 2 \mathrm{Cu}(\mathrm{II})(\mathrm{OH})_{2}$ and $\mathrm{PB} \cdot 2 \mathrm{Mn}(\mathrm{II})(\mathrm{OH})_{2}$ 
For example, the $\mathrm{K}_{\mathrm{d}}$ of fly ash cleaning water containing $\mathrm{Na}^{+}(1,500 \mathrm{mg} / \mathrm{L}), \mathrm{K}^{+}(2,800 \mathrm{mg} / \mathrm{L})$, and $\mathrm{Ca}^{2+}(1,300 \mathrm{mg} / \mathrm{L})$ treated for $24 \mathrm{~h}$ using zeolite is $800(\mathrm{~mL} / \mathrm{g})$, and the adsorption performance is extremely inhibited by $\mathrm{Na}^{+}$and $\mathrm{K}^{+}$. The concentration of cations other than $\mathrm{K}^{+}$in the simulated fly ash extract water in this study are high $\left(\mathrm{Na}^{+}: 5,000 \mathrm{mg} / \mathrm{L}, \mathrm{Mg}^{2+}: 500 \mathrm{mg} / \mathrm{L}\right.$, and $\left.\mathrm{Ca}^{2+}: 3,000 \mathrm{mg} / \mathrm{L}\right)$, and even at a $\mathrm{K}^{+}$content of $1 \%(10,000 \mathrm{mg} / \mathrm{L})$ a high level $\mathrm{K}_{\mathrm{d}}$ of Cs, more than $10^{5}(\mathrm{~mL} / \mathrm{g})$, can be maintained through PB-X treatment as shown in Figure 3. In particular, when $\mathrm{Me}(\mathrm{II})=\mathrm{Cu}$, the inhibition of Cs adsorption by $\mathrm{K}^{+}$is lower than that with $\mathrm{Mn}^{+}$, and it showed excellent Cs adsorption performance $\left(\mathrm{K}_{\mathrm{d}}=8.6=10^{5} \mathrm{~mL} / \mathrm{g}\right.$ and a Cs removal rate of $99.88 \%$ ) for the simulated fly ash extract with a $\mathrm{K}^{+}$concentration of $1.0 \%$. Based on these results, $\mathrm{PB}-\mathrm{X}$ is an excellent material for the treatment of Cs-contaminated water containing a high concentration of salts.

(b) Elution test on PB-X treated filtered residues

Elution tests were carried out according to notification No. 13 from the Ministry of the Environment ${ }^{14}$ ) on the residues acquired by filtration of PB-X treated simulated fly ash extract. The results are shown in Table 6 and Figure 4. The weight of the filtered residues decreased to approximately $1 \mathrm{~g}$ when dried. To maintain the quantity of the analyzed specimen, the elution tests were conducted in the wet state (moisture content approximately 90\%) using distilled

Table 6 Effects of $\mathrm{K}^{+}$content in the simulated fly ash extract on $\mathrm{K}$, Cs, T-CN, and transition metal $(\mathrm{Cu}$ or $\mathrm{Mn})$ concentrations in the leachates in leaching tests

\begin{tabular}{clcccc}
\hline \multirow{2}{*}{$\mathrm{Me}(\mathrm{II})$} & $\mathrm{K}, \mathrm{Cs}, \mathrm{T}-\mathrm{Cn}$, & \multicolumn{5}{c}{$\mathrm{K}^{+}$content $(\%)$} \\
\cline { 3 - 6 } & $\mathrm{Cu}, \mathrm{Mn}$ & 0.05 & 0.1 & 0.5 & 1.0 \\
\hline \multirow{3}{*}{$\mathrm{Cu}(\mathrm{II})$} & $\mathrm{K}(\mathrm{mg} / \mathrm{L})$ & 68 & 101 & 398 & 851 \\
\cline { 2 - 6 } & $\mathrm{Cs}(\mathrm{mg} / \mathrm{L})$ & 0.001 & 0.002 & 0.001 & 0.002 \\
\cline { 2 - 6 } & $\mathrm{T}-\mathrm{CN}(\mathrm{mg} / \mathrm{L})$ & $<0.05$ & $<0.05$ & 0.05 & $<0.05$ \\
\cline { 2 - 6 } & $\mathrm{Cu}(\mathrm{mg} / \mathrm{L})$ & 0.001 & 0.005 & 0.003 & 0.006 \\
\hline \multirow{3}{*}{$\mathrm{Mn}(\mathrm{II})$} & $\mathrm{K}(\mathrm{mg} / \mathrm{L})$ & 40 & 94 & 460 & 981 \\
\cline { 2 - 6 } & $\mathrm{Cs}(\mathrm{mg} / \mathrm{L})$ & 0.001 & 0.003 & 0.006 & 0.011 \\
\cline { 2 - 6 } & $\mathrm{T}-\mathrm{CN}(\mathrm{mg} / \mathrm{L})$ & 1.0 & 0.57 & 0.52 & 0.06 \\
\cline { 2 - 6 } & $\mathrm{Mn}(\mathrm{mg} / \mathrm{L})$ & 15.8 & 17.1 & 11.6 & 11.4 \\
\hline
\end{tabular}

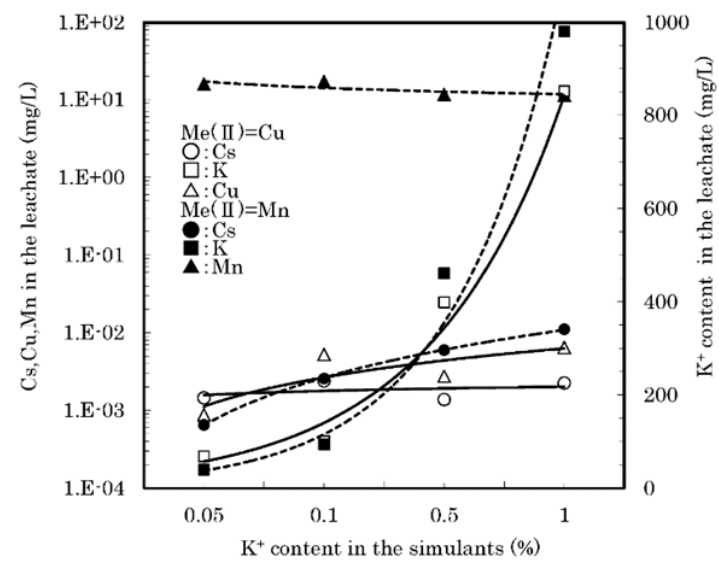

Figure 4 Relationship between $\mathrm{K}^{+}$content in the simulated fly ash extracts and the leachability of Cs, transition metals $(\mathrm{Mn}, \mathrm{Cu})$, and $\mathrm{K}^{+}$in the leachates of the filtration residues obtained after $\mathrm{PB}-\mathrm{X}(\mathrm{Me}(\mathrm{II})=$ $\mathrm{Cu}, \mathrm{Mn})$ treatment 
water (10 times as much as the filtered residues).

In the elution test of the PB-X-treated filtered residues, the elution of $\mathrm{Cs}$ and $\mathrm{CN}^{-}$was inhibited more by the presence of $\mathrm{Cu}$ (II) than $\mathrm{Mn}$ (II). Furthermore, the elution $\mathrm{Cu}$ (II) was very low, especially compared to that of Mn. The quantity of Cs eluted from the PB-X-treated filtered residues was low $(0.001-0.002 \mathrm{mg} / \mathrm{L})$ for $\mathrm{Me}(\mathrm{II})=\mathrm{Cu}$, and the presence of $\mathrm{K}^{+}$did not have an effect on the Cs elution quantity. Furthermore, the T-CN was very low, indicating stable fixation of insolubilized Cs and soluble PB through the formation of $\mathrm{PB} \cdot 2 \mathrm{Cu}(\mathrm{OH})_{2}$.

When $\mathrm{Me}(\mathrm{II})$ was $\mathrm{Mn}$, the presence of $\mathrm{K}^{+}$in the elution liquid affected the quantity of Cs eluted. The reason for the high T-CN elution is considered to be the inhibited insolubilization of soluble PB by the interaction of soluble $\mathrm{PB}$ molecules and $\mathrm{Mn}(\mathrm{II})(\mathrm{OH})_{2}$ because some portion of the $\mathrm{Mn}(\mathrm{II})$ was oxidized to $\mathrm{Mn}(\mathrm{III})$ or $\mathrm{Mn}(\mathrm{IV})$ during the 6-h shaking in the elution test. Furthermore, when the $\mathrm{d}-\pi$ interactions of the d-orbitals of $\mathrm{Mn}$ (II) and $\mathrm{Cu}(\mathrm{II})$ and the $\pi$-electrons of $\mathrm{CN}$ of $\mathrm{PB}$ are compared, the $\mathrm{d}-\pi$ interaction of $\mathrm{Cu}(\mathrm{II})-\mathrm{CN}$ is expected to be stronger than that of $\mathrm{Mn}(\mathrm{II})-\mathrm{CN}$ based on the HSAB rules ${ }^{16)}$. Thus, the superiority of using $\mathrm{Cu}$ over $\mathrm{Mn}$ in the $\mathrm{PB}-\mathrm{X}$ treatment can be explained based on the assumed stronger interaction of $\mathrm{PB}$ with $\mathrm{Cu}$ (II) $(\mathrm{OH})_{2}$ than $\mathrm{Mn}(\mathrm{II})(\mathrm{OH})_{2}$.

\section{PB-X Treatment of Radioactive-Cs-contaminated Fly Ash Extract}

In the purification test of radioactive-Cs-contaminated fly ash extract, the PB-X treatment used $\mathrm{Cu}$ (II) salts based on the results obtained using the simulated fly ash extract water.

The results are as follows:

\section{(1) Radioactive Cs-contaminated fly ash extract}

The measurement of radioactivity for Cases 1-3 of the radioactive-Cs-contaminated fly ash extract water are listed in Table 7, and the concentrations of ion species contained in the cleaning water are listed in Table 8.

The fly ash extract samples have high $\mathrm{K}^{+}$content (1.9-2.4\%), which could inhibit Cs adsorption, and the rubidium ion $\left(\mathrm{Rb}^{+}\right)$is content is about $70 \mathrm{mg} / \mathrm{L}$.

\section{(2) Analysis of treated water}

Table 9 lists the concentrations of radioactive $\mathrm{Cs}, \mathrm{T}-\mathrm{CN}, \mathrm{Fe}$, and $\mathrm{Cu}$ in the treated water acquired in Cases $1-3$ of $\mathrm{PB}-\mathrm{X}(\mathrm{Me}(\mathrm{II})=\mathrm{Cu})$ treatment of fly ash extract water.

Table 7 Radioactive Cs concentration in the fly ash extracts

\begin{tabular}{cccc}
\hline Fly ash extracts & $\begin{array}{c}{ }^{134} \mathrm{Cs} \\
(\mathrm{Bq} / \mathrm{L})\end{array}$ & $\begin{array}{c}{ }^{137} \mathrm{Cs} \\
(\mathrm{Bq} / \mathrm{L})\end{array}$ & Total Cs $(\mathrm{Bq} / \mathrm{L})$ \\
\hline Case 1 & $2,720 \pm 56$ & $6,190 \pm 64$ & 8.910 \\
\hline Case 2 & $2,540 \pm 39$ & $6,000 \pm 62$ & 8,540 \\
\hline Case 3 & $4,000 \pm 49$ & $9,000 \pm 76$ & 13,000 \\
\hline
\end{tabular}

Table 8 Ion concentrations in the fly ash extracts contaminated with radiocesium

\begin{tabular}{ccccccccccc}
\hline \multirow{2}{*}{$\begin{array}{c}\text { Fly ash } \\
\text { extract }\end{array}$} & $\mathrm{Na}$ & $\mathrm{Mg}$ & $\mathrm{K}$ & $\mathrm{Ca}$ & $\mathrm{Rb}$ & $\mathrm{Sr}$ & ${ }^{133} \mathrm{Cs}$ & $\mathrm{Cl}$ & $\mathrm{NO}_{3}$ & $\mathrm{SO}_{4}$ \\
\cline { 2 - 12 } & \multicolumn{7}{c}{$(\mathrm{mg} / \mathrm{L})$} \\
\hline Case 1 & 3,200 & 510 & 24,000 & 1,320 & 72 & 22 & 0.63 & 23,000 & 280 & 5,000 \\
\hline Case 2 & 2,570 & 830 & 22,900 & 1,580 & 68 & 31 & 0.60 & 21,000 & 280 & 8,600 \\
\hline Case 3 & 2,760 & 520 & 19,000 & 1,350 & 79 & 24 & 0.78 & 18,000 & 280 & 6,200 \\
\hline
\end{tabular}


Table 9 Concentrations of radiocesium, T-CN, Fe, $\mathrm{Cu}$, and ${ }^{133} \mathrm{Cs}$ in the filtrates obtained by PB-X treatment of the fly ash extract

\begin{tabular}{cccccccc}
\hline Filtrates & $\begin{array}{c}{ }^{134} \mathrm{Cs} \\
(\mathrm{Bq} / \mathrm{L})\end{array}$ & $\begin{array}{c}{ }^{137} \mathrm{Cs} \\
(\mathrm{Bq} / \mathrm{L})\end{array}$ & $\begin{array}{c}\text { Total Cs } \\
(\mathrm{Bq} / \mathrm{L})\end{array}$ & $\begin{array}{c}\mathrm{T}-\mathrm{CN} \\
(\mathrm{mg} / \mathrm{L})\end{array}$ & $\begin{array}{c}\mathrm{Fe} \\
(\mathrm{mg} / \mathrm{L})\end{array}$ & $\begin{array}{c}\mathrm{Cu} \\
(\mathrm{mg} / \mathrm{L})\end{array}$ & $\begin{array}{c}{ }^{133} \mathrm{Cs} \\
(\mathrm{mg} / \mathrm{L})\end{array}$ \\
\hline Case 1 & $<5$ & $<8$ & $<10$ & 0.08 & 0.14 & 7.0 & $<0.001$ \\
\hline Case 2 & $<5$ & $<7$ & $<9$ & $<0.05$ & 0.06 & 6.8 & $<0.001$ \\
\hline Case 3 & $<4$ & $<6$ & $<8$ & $<0.05$ & 0.05 & 1.8 & $<0.001$ \\
\hline
\end{tabular}

Table 10 Concentrations of radiocesium, T-CN, and heavy metals in the leachate from the leaching test of the residue obtained after $\mathrm{PB}-\mathrm{X}$ treatment of Case 1

\begin{tabular}{cccccc}
\hline $\begin{array}{c}{ }^{134} \mathrm{Cs} \\
(\mathrm{Bq} / \mathrm{L})\end{array}$ & $\begin{array}{c}{ }^{137} \mathrm{Cs} \\
(\mathrm{Bq} / \mathrm{L})\end{array}$ & $\begin{array}{c}\text { Total Cs } \\
(\mathrm{Bq} / \mathrm{L})\end{array}$ & $\begin{array}{c}\mathrm{T}-\mathrm{CN} \\
(\mathrm{mg} / \mathrm{L})\end{array}$ & $\begin{array}{c}\mathrm{Fe} \\
(\mathrm{mg} / \mathrm{L})\end{array}$ & $\begin{array}{c}\mathrm{Cu} \\
(\mathrm{mg} / \mathrm{L})\end{array}$ \\
\hline$<5$ & $<7$ & $<9$ & $<0.05$ & 0.08 & 2.8 \\
\hline
\end{tabular}

Cases 1 and 2 are the PB-X treatment using the fly ash extract water having similar ion concentrations or radioactive $\mathrm{Cs}$ concentrations. These were used to observe the reproducibility of the decontamination effect by adjusting the amount of added $\mathrm{NaFe}(\mathrm{II})(\mathrm{CN})_{6} \cdot 10 \mathrm{H}_{2} \mathrm{O}$ and $\mathrm{Cu}(\mathrm{II}) \mathrm{SO}_{4}$ required to form $3.5 \mathrm{mmol} / \mathrm{L}(1.7 \mathrm{~g} / \mathrm{L})$ of active material for Cs adsorption, i.e., $\mathrm{PB} \cdot 2 \mathrm{Cu}(\mathrm{II})(\mathrm{OH})_{2}$.

The radioactive $\mathrm{Cs}$ in the treated water was not detected in Cases 1 and 2, and the discharge limit $(<0.5 \mathrm{mg} / \mathrm{L})$ of Fukushima Prefecture code was satisfied. In Case 3, the treatment of water contaminated with radioactive $\mathrm{Cs}(13,000 \mathrm{~Bq} / \mathrm{kg})$ resulted in the removal of Cs and T-CN at an active material concentration of $4 \mathrm{mmol} / \mathrm{L}$.

The $K_{d}$ of the active material material for Cs was calculated to exceed $9.5 \times 10^{5} \mathrm{~mL} / \mathrm{g}$. Excellent Cs adsorption performance of PB-X treatment was also demonstrated for the contaminated water containing a high concentration of salts. The eluted $\mathrm{Cu}$ concentration in the treated water was higher than that of the simulated fly ash extract. All treated water in Cases 1-3 showed a high chemical oxygen demand (COD), and the amber color was easily removed using active charcoal. We believe that a lignin decomposition component was contained in the simulated fly ash extract water. Consequently, it is considered that the formation of a complex of lignin with $\mathrm{Cu}$ increased the $\mathrm{Cu}$ elution in the treated water.

\section{(3) Analysis of filtered residues}

The concentrations of radioactive $\mathrm{Cs}, \mathrm{T}-\mathrm{CN}, \mathrm{Fe}$, and $\mathrm{Cu}$ in the eluted liquid from the elution tests were determined based on notification No. 13 from the Ministry of the Environment and are listed in Table 10 for Case 1. Neither radioactive Cs nor T-CN was detected in the eluted liquid, and it was confirmed that $\mathrm{Cs}$ was firmly fixed on the $\mathrm{PB} \cdot 2 \mathrm{Cu}(\mathrm{OH})_{2}$, the active material, and, thus, the PB molecule was insoluble

\section{Summary}

A quick and highly efficient method for the removal of Cs from contaminated water was confirmed, via the synthesis of $\mathrm{PB}-\mathrm{X}$, a novel complex of $\mathrm{PB}$ and the hydroxide of a bivalent transition metal $\left(\mathrm{Me}(\mathrm{II})(\mathrm{OH})_{2}\right)$.

Furthermore, for the case where $\mathrm{Me}(\mathrm{II})=\mathrm{Cu}(\mathrm{II})$, it was demonstrated that the T-CN concentration of the treated water was below the discharge limit stipulated by Fukushima Prefecture. 
This was achieved by adjusting the $\mathrm{Cu}(\mathrm{II})(\mathrm{OH})_{2} / \mathrm{PB}$ molar ratio, $\mathrm{y}$, to above 1.2.

For the case of $\mathrm{Me}(\mathrm{II})=\mathrm{Cu}$ in $\mathrm{PB}-\mathrm{X}$, we found that the Cs removal inhibition effect was mitigated by $\mathrm{K}^{+}$. The radioactive concentration in the treated water was below the detectable level $(<10 \mathrm{~Bq} / \mathrm{L})$. This was also true for the fly ash water with a $\mathrm{K}^{+}$concentration of $2 \%$ and radioactive concentration of $13,000 \mathrm{~Bq} / \mathrm{L}$. The $\mathrm{K}_{\mathrm{d}}$ of the $\mathrm{PB} \cdot 2 \mathrm{Cu}(\mathrm{II})(\mathrm{OH})_{2}$ active material exceeded $9 \times 10^{5}(\mathrm{~mL} / \mathrm{g})$. Additionally, $\mathrm{CN}^{-}$was not detectable $(<0.05 \mathrm{mg} / \mathrm{L})$.

Furthermore, neither Cs nor T-CN were detectable in the eluted liquids prepared from the elution tests conducted on the filtered residues of PB-X treatment carried out in accordance with notification No. 13 from the Ministry of the Environment, and it was confirmed that Cs was stably trapped by $\mathrm{PB}-\mathrm{X}$, and no elution of $\mathrm{PB}$ molecules took place.

Based on the above results, the PB-X treatment is a quick and excellent adsorption process with high selectivity (better than zeolite) for Cs removal from water, also quickly reducing the T-CN of treated water to under the environmental limit, a result that powdered PB did not achieve. Consequently, the PB-X treatment is a promising Cs-removal technology for incineration fly ash cleaning water obtained after the burning of vegetation containing a high concentration of salts such as $\mathrm{Na}^{+}$and $\mathrm{K}^{+}$, seawater, as well as refuse leachate.

Currently the possibility of the application of the PB-X principle to ferrocyanide complexes of $\mathrm{Mn}(\mathrm{II}), \mathrm{Co}(\mathrm{II}), \mathrm{Ni}(\mathrm{II}), \mathrm{Fe}(\mathrm{II}), \mathrm{Cu}(\mathrm{II})$, and $\mathrm{Zn}(\mathrm{II})$, the so-called "PB analogs" is under investigation.

In addition, the PB-X was granulated and used in the study of Cs removal using the column method. Additionally, to clarify the Cs adsorption mechanism, a structural analysis of PB-X has been conducted, and the results will be reported soon.

Finally, the purification demonstration test for radioactive Cs-contaminated fly ash cleaning water in this study was conducted in Kawauchi Village, Fukushima Prefecture from October 30 until November 1,2013. The fly ash cleaning water was obtained and the measurement of radioactive Cs and T-CN was conducted by kind cooperation with Tokyo Power Technology, Ltd. We would like to take this opportunity to express our sincere appreciation to the people of Kawauchi Village and Tokyo Power Technology, Ltd. who understood, cooperated, and gave us permission for the reported experiments.

\section{References}

1) IAEA Report of United Nations Project E 11, The Use of Prussian Blue to Reduce Radiocaesium Contamination of Milk and Meat Produced on Territories Affected by the Chernobyl Accident February 1997.

2) V. Nigrovic, "Enhancement of excretion of radiocaesium in rats by ferric cyanoferrate (II)," Int. J. Radiat. Biol., 7, 307-309 (1963).

3) N. L. Torad, M. Hu, M. Imura et al., "Large Cs adsorption capability of nanostructured Prussian blue particles with high accessible surface areas,” J. Mater. Chem., 22, 18261-18267 (2012).

4) L. L. Burger, D. A. Reynolds, W. W. Schulz et al., A Summary of Available Information on Ferrocyanide Tank Wastes, October 1991, Prepared for the U.S. Department of Department of Energy. http://www.osti. gov/scitech/servlets/purl/6126105.

5) A. Omura, Y. Moritomo, $\mathrm{Cs}^{+}$Trapping in size-controlled nanospaces of hexacyanoferrates, Appl. Phys. Express, 5, 057101, (2012). http://www. tsukuba.ac.jp/public/press/120413.pdf.

6) Y. Ban, I. Yamagishi, Y. Morita, "Adsorption properties of Cs by inorganic adsorbents in sodium chloride solution," JAEA Res., 2011-037. [in Japanese]

7) NIES, Center for Material Cycles and Waste Management Research, Technical data, Fourth edition March 28th, 2014. Adequate Treatment of Radioactive Wastes from the Standpoint of Behavior of Radioactive Substances. http://www.nies.go.jp/shinsai/enpdf/hrfba3000009yxpa-att/techrepo_r4_140328. pdf. [in Japanese]

8) S. Inamori, R. Inamori, N. Murata, K. Kakazu, "The effectiveness of radioactive cesium removal 
measures using Prussian blue non-woven fabric proposed by International Research Institute for Nuclear Decommissioning," Yousui Haisui, 56, 231-237 (2014). [in Japanese]

9) Encyclopaedia Chimica, 1993.

10) Z. Liu, M. Osada, T. Sasaki et al., "Selective and controlled synthesis of A- and B-cobalt hydroxides in highly developed hexagonal platelets," J. Am. Chem. Soc., 127, 13869-13874, (2005).

11) J. Yuan, K. Laubernds, Q. Zhang, S. L. Suib, "Self-assembly of microporous manganese oxide octahedral molecular sieve hexagonal flakes into mesoporous hollow nanospheres," J. Am. Chem. Soc., 125, 4966-4967, (2003).

12) S. Ida, D. Shiga, M. Koinuma, Y. Matsumoto, "Synthesis of hexagonal nickel hydroxide nanosheets by exfoliation of layered nickel hydroxide intercalated with dodecyl sulfate ions," J. Am. Chem. Soc., 130, 14038-14039, (2008).

13) S. Takahashi, T. Iwasa, Y. Kanazawa, "Formation of organic anion-pillared layered zinc hydroxides by coprecipitation," Nippon Kagaku Kaishi, 7, 502-507, (1997). [in Japanese]

14) Ministry of Environment Minister's Secretariat Waste Management and Recycling Department, Analysis Operation Manual regarding the Industrial Waste Verification Method: Revised Version, May 2013.

15) Chemistry 112 HP, Reference Materials, Solubility Product Constants near 25 C, http://bilbo.chm.uri. edu/CHM112/tables/Ksp Table.htm.

16) R. G. Pearson, "Hard and soft acids and bases," J. Am. Chem. Soc., 85, 3533-3539, (1963).

17) G. Klopman, "Chemical reactivity and concept of charge- and frontier-controlled reactions," J. Am. Chem. Soc., 90, 223-234, (1968).

18) H. Ubara, K. Tsukiyama, M. Kamizawa et al., Method for Processing of Radioactive Cs Contaminated Water, Japanese Pat. No. 5352853. [in Japanese]

19) J. D. Bernal, D. R. Dasgupta, A. L. Mackay, "The oxide and hydroxides of iron and their structural interrelationships," Clay Miner. Bull., 4, 15-30, (1959).

20) Dainichiseika Color \& Chemicals Mfg. Co., Ltd. Product Brochure, http://www.daicolor.co.jp/products_i/pro_i pig/miloriblueqa.html. 


\title{
Estimation of Time History of I-131 \\ Concentration in Air Using NaI(Tl) Detector Pulse Height Distribution at Monitoring Posts in Fukushima Prefecture
}

\author{
Hideo HIRAYAMA ${ }^{1, *}$, Hiroshi MATSUMURA ${ }^{1}$, \\ Yoshihito NAMITO ${ }^{1}$ and Toshiya SANAMI ${ }^{1}$ \\ ${ }^{1}$ High Energy Accelerator Research Organization, 1-1 Oho, Tsukuba-shi, Ibaraki 305-0801, Japan
}

\begin{abstract}
Time histories of the I-131 concentration in air at monitoring posts in Fukushima Prefecture in March 2011 were estimated using the pulse height distribution of a $\mathrm{NaI}(\mathrm{Tl})$ detector, which was opened to the public. Several corrections to the pulse height distribution were necessary owing to high count rates. The contribution to the count rates from I-131 accumulated around the monitoring post was estimated on the basis of the time history of the peak count rate by the method proposed by the authors. The concentrations of I-131 in air were converted from the peak count rates using the calculated response of the $\mathrm{NaI}(\mathrm{Tl})$ detector with egs 5 for a model of a plume containing I-131 uniformly. The obtained time histories of the I-131 concentration in air at a fixed point in March 2011 were the first ones for Fukushima Prefecture. The results at 3 monitoring posts, Naraha Town Shoukan, Hirono Town Futatunuma and Fukushima City Momijiyama, which can be analyzed during almost all of March, show that a plume including I-131 arrived after March 15. The results at other monitoring posts near Fukushima Daiichi Nuclear Power Plant are used to characterize plume diffusion at the early period of the accident before March 15. The I-131 time-integrated concentrations in air at several monitoring posts were compared with those given in UNSCEAR 2013 ANNEX A, which were obtained using estimated time-dependent rates of release to the atmosphere. The agreement between the two results varies depending on the places compared, owing to the large uncertainties in the estimated release rate used in UNSCEAR. The results obtained in this study can be used to increase the accuracy of the time-dependent release rate estimation.
\end{abstract}

KEYWORDS: iodine 131, concentration in air, time history, Fukushima, Monte Carlo, egs5, NaI scintillator

\section{Introduction}

The time history of the I-131 concentration in air released to the environment resulting from the accident that occurred at the Fukushima Daiichi Nuclear Power Plant (hereinafter referred to as the "1F plant") is important in evaluating the initial internal exposure. In particular,

* Corresponding author, E-mail: hideo.hirayama@kek.jp

DOI : 10.15669 /fukushimainsights.Vol.4.323

(C) 2021 Atomic Energy Society of Japan. All rights reserved.

Originally published in Transactions of the Atomic Energy Society of Japan (ISSN 1347-2879), Vol. 14, No. 1, p.1-11 (2015)

in Japanese. (Japanese version accepted: October 14, 2014) 
insufficient data were obtained in Fukushima Prefecture during the early stage of the accident, a period that needs to be evaluated. An attempt needs to be made to estimate the time variation in I-131 emissions using various data such as dose rate information including the air absorbed dose rate and ambient dose equivalent rate (for which there is an abundance of measurement data) and the I-131 concentration deposited on the ground surface. These data can serve as the basis for the estimation of the concentration in air. Although the time history of dose rate is important information to determine the arrival of a plume, the analysis of the monitoring post data conducted by the Nuclear Science Research Institute (NSRI) of the Japan Atomic Energy Agency (JAEA) showed that the changes in the dose rate do not correspond to the changes in the I-131 concentration in air ${ }^{1)}$. In addition, because the density of I-131 deposited on the ground is an integral value accompanying several plume arrivals, and it is extremely difficult to obtain the time history of the I-131 emission from these data. However, if a $\mathrm{NaI}(\mathrm{Tl})$ detector was used in the dose measurement at monitoring posts, pulse height distribution data may be available. In this case, it is possible to obtain data on the radionuclides in the air from the pulse height distribution. Fukushima Prefecture publicly disclosed the numeric data of the pulse height distribution obtained at the monitoring posts in March 2011 on a website ${ }^{2}$.

Applying a following method proposed by authors for the open data of Fukushima Prefecture to estimate the concentration of I-131 in air at monitoring posts aftermath of the accident in March. The method is that estimates the contribution of radionuclides deposited on the surroundings separately from the contribution of radionuclides contained in air, from the time histories of gamma ray total absorption peak count rates ${ }^{1}$. Concerning three monitoring posts (Syoukan in Naraha Town, Futatunuma in Hirono Town, and Momijiyama in Fukushima City) at which analyzable data were obtained for the whole of March, we estimated the time history of the I-131 concentration in air at locations in Fukushima Prefecture located far away from the $1 \mathrm{~F}$ plant during this month. However, at most of the monitoring posts near the $1 \mathrm{~F}$ plant, not only were data obtained only until the middle of March but it also was impossible to perform peak analysis based on the pulse height distribution because of the pulse pile-up on and after March 15 even if data were available. Therefore, only the time history of concentration in air before March 15 was estimated.

Using the time history of the concentration in air obtained, we determined the time integration I-131 concentration in air to compare it to the estimated value described in UNSCEAR 2013 ANNEXA $^{3)}$.

\section{Values Measured at Monitoring Posts}

\section{Disclosed Pulse Height Distribution Data Obtained at Monitoring Posts}

Fukushima Prefecture disclosed the pulse height distribution data obtained for $10 \mathrm{~min}$ and $1 \mathrm{~h}$ using $\mathrm{NaI}(\mathrm{Tl})$ detectors at 13 monitoring posts as shown in Table $\mathbf{1}^{2}$. The directions and distances from the $1 \mathrm{~F}$ plant described in the table are quoted from the website ${ }^{4)}$ a . The $\mathrm{NaI}(\mathrm{Tl})$ detectors used were $\phi 2^{\prime \prime} \times 2^{\prime \prime}$ in size and placed at a height of $3 \mathrm{~m}$ above the ground surface. Among the 13 locations, Kamihatori in Futaba Town was excluded from the concentration estimation shown because only the data obtained until 11:00 p.m. on March 12 were available and the count rates were too high to perform peak analysis at and after 8:00 on that day. The data

\footnotetext{
${ }^{a}$ Because Momijiyama in Fukushima City was not included in the monitoring posts described on the website, we estimated it from the address.
} 
Table 1 Monitoring posts information made publicly available by Fukushima Prefecture

\begin{tabular}{|c|c|c|c|c|c|}
\hline $\begin{array}{c}\text { Name of city or } \\
\text { town }\end{array}$ & Name of post & $\begin{array}{l}\text { Distance from } \\
\text { Fukushima } \\
\text { Daiichi Nuclear } \\
\text { Power Plant }\end{array}$ & $\begin{array}{l}\text { Data collection } \\
\text { period during } \\
\text { March } 2011\end{array}$ & $\begin{array}{c}\text { Analyzable data } \\
\text { period }\end{array}$ & $\begin{array}{c}\text { Measurement } \\
\text { duration } \\
\text { (minutes) }\end{array}$ \\
\hline \multirow{3}{*}{ Futaba-town } & Koriyama & NNW $2.8 \mathrm{~km}$ & till 14th 24:00 & till 14th 20:00 & 10 \\
\hline & Yamada & WNW $4.1 \mathrm{~km}$ & till 31th 24:00 & till 15th 11:00 & 60 \\
\hline & Kamihatori & NW5.6 km & till 12th 24:00 & & 60 \\
\hline \multirow{4}{*}{ Okuma-town } & Mukaihata & $\mathrm{SW} 4.0 \mathrm{~km}$ & till 15th 2:00 & till 14th 20:00 & 60 \\
\hline & Oono & WSW $4.9 \mathrm{~km}$ & till 16th 16:00 & till 14th 21:00 & 60 \\
\hline & Minamidai & $\mathrm{SW} 2.4 \mathrm{~km}$ & till 14th 20:00 & till 14th 20:00 & 60 \\
\hline & Ottozawa & $\mathrm{SSW} 1.6 \mathrm{~km}$ & till 15 th $7: 00$ & till 14th 19:00 & 60 \\
\hline \multirow{2}{*}{ Naraha-town } & Shoukan & $\mathrm{SSW} 14.2 \mathrm{~km}$ & till 31th 24:00 & till 31th 24:00 & 60 \\
\hline & Shigeoka & $\mathrm{SSW} 14.2 \mathrm{~km}$ & till 14th 24:00 & till 14th 22:20 & 10 \\
\hline \multirow{2}{*}{ Tomioka-town } & Simokoriyama & $\mathrm{SSW} 11.8 \mathrm{~km}$ & till 15th 8:00 & till 14th 21:00 & 60 \\
\hline & Tomioka & SSW $9.4 \mathrm{~km}$ & till 13th 24:00 & till 13th 24:00 & 10 \\
\hline Hirono-town & Futatunuma & $\mathrm{S} 21.4 \mathrm{~km}$ & till 31th 24:00 & till 31th 24:00 & 60 \\
\hline Fukushima-city & Momijiyama & $\mathrm{NW} 61.3 \mathrm{~km}$ & till 28th $18: 00$ & till 28th $18: 00$ & 60 \\
\hline
\end{tabular}

enabling peak analysis were obtained for the whole of March at Shoukan in Naraha Town and Futatunuma in Hirono Town and until March 28 at Momijiyama in Fukushima City. However, at the other monitoring posts, no data were available on and after March 15 or the count rates were too high to perform peak analysis even if the data were available. Therefore, we used the data obtained there to study the time variation in the I-131 concentration in air in the areas around the $1 \mathrm{~F}$ plant before a drastic increase occurred at midnight on March $14^{\mathrm{b}}$.

\section{Correction of Pulse Height Distribution Data}

\section{(1) Correction of digit overflow}

Most of the pulse height distribution data are disclosed as the integration values of $1 \mathrm{~h}$. Because the count values of the respective channels are displayed in six digits, when a count value becomes a number with six or more digits, only the upper six digits are shown. As an example, Figure 1 shows the pulse height distribution obtained at 2:00-3:00 p.m. on March 13 at Minamidai in Okuma Town. It is apparent that the disclosed data of the 4-45 channels caused digit overflow. The data of the 12-18 channels were centupled $(\times 100)$ and other data causing digit overflow were decoupled $(\times 10)$ to obtain the corrected result. Taking this step enabled us to obtain a normal pulse height distribution. Based on this result, we conducted the same consideration for all the pulse height distribution data and corrected the data by centupling and decoupling the digit overflow (digit overflow correction).

\section{(2) Correction of live time}

In the case where the measurement data indicated a high dose rate, it is highly likely that there was a difference between the live time, which is the net measurement time, and real time, which is the real measurement time. In the data obtained at Koriyama in Futaba Town at 10-min intervals, both the live time and real time are shown. Figure 2 shows the relation between the total count value of the pulse height distribution (cps) and (live time) / (real time). As shown in

\footnotetext{
${ }^{\mathrm{b}}$ Although the data obtained at Tomioka in Tomioka Town were only available until March 13, no I-131 was detected during that period. Therefore, we determined that no plume had arrived by March 13 and we used this information.
} 


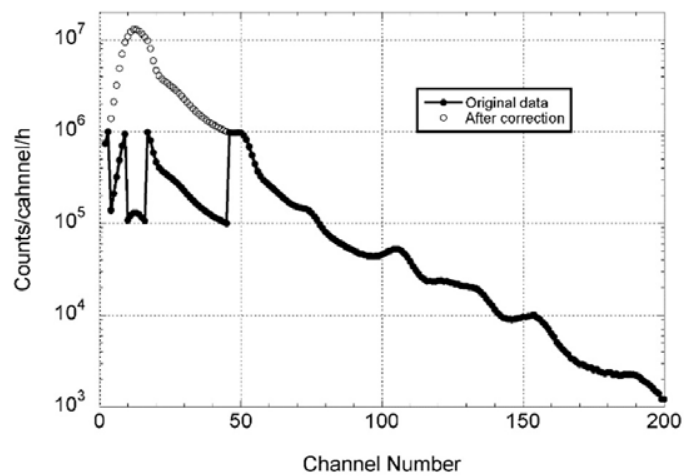

Figure 1 Pulse height distribution of $\mathrm{NaI}(\mathrm{Tl})$ detector at Okuma Town Minamidai at 14:00 15:00 March 13, 2011

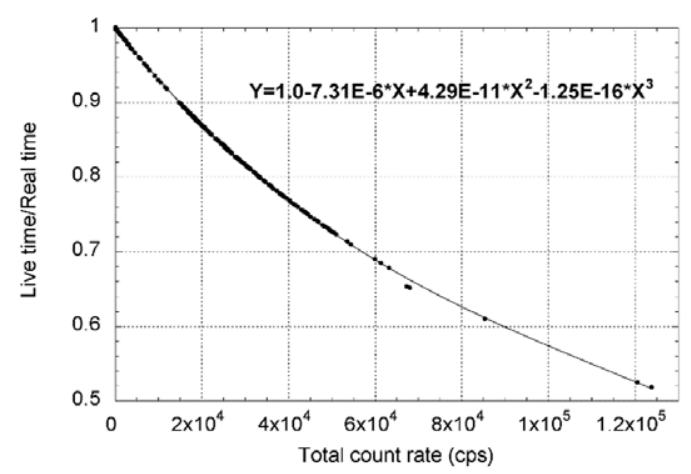

Figure 2 Relation between total count rate (cps) and (live time)/(real time) obtained from pulse-height distribution data at Futaba Town Koriyama

the figure, the relation between the two can be approximated using the following polynomial equation:

$$
\frac{\text { live time }}{\text { real time }}=1.00-7.31 \times 10^{-6} \times c+4.29 \times 10^{-11} \times c^{2}-1.25 \times 10^{-16} \times c^{3}
$$

where $c$ is the count value per second obtained by integrating a pulse height distribution. Although both live time and real time are 3,600 s in terms of hourly data, it was determined from the data obtained at 10-min intervals that the live time of 3,600 s was incorrect. As a result, all data were corrected using the value of (live time) / (real time) obtained using equation (1) (live time correction).

\section{(3) Check of correction result}

To confirm the effectiveness of "digit overflow correction" and "live time correction," we compared the total count rates of the pulse height distribution after completing both corrections using the air absorbed dose rate disclosed on Fukushima Prefecture's website. Figure 3 shows the comparison result in the case of Minamidai in Okuma Town. The low-level air absorbed dose rates were measured using $\mathrm{NaI}(\mathrm{Tl})$ detectors and the high-level air absorbed dose rates were measured using ionization chambers. At $100 \mathrm{nGy} / \mathrm{h}$ and higher, the low and high dose rate values were the same. Therefore, it would appear that the dose rate data obtained using a 
$\mathrm{NaI}(\mathrm{Tl})$ detector for the measurement of low dose rates showed normal values even in the high dose rate range. Because the corrected count rates showed the same time history as both air absorbed dose rates, we can state that we could confirm the appropriateness of the aforementioned two corrections.

\section{Peak Count Rates of I-131}

Using the corrected pulse height distribution data obtained using a $\mathrm{NaI}(\mathrm{Tl})$ detector, we determined the net count rates corresponding to the total absorption peak of I-131 with 365 $\mathrm{keV}$ as the peak count rate. The number of counts of the background components of the peak were approximated by the area of a trapezoid created by connecting the counts on both sides of the peak and then the net count rate was deduced by subtracting the background counts from the entire peak counts. The error includes the statistical error deriving from only the peak count. Figure 4 shows the time history of the peak count rates and air absorbed dose rates obtained at Koriyama in Futaba Town. As the figure shows, when the air absorbed dose rate significantly increased, the peak count rates of I-131 decreased abnormally. Figure 5 shows the pulse height distributions obtained at Koriyama in Futaba Town between 4:00 and 5:00 as well as 8:00 and 9:00 on March 12. Based on the time history of the air absorbed dose rate, the plumes arrived at approximately 4:30 and 8:10. Because there was no peak of I-131 in the pulse

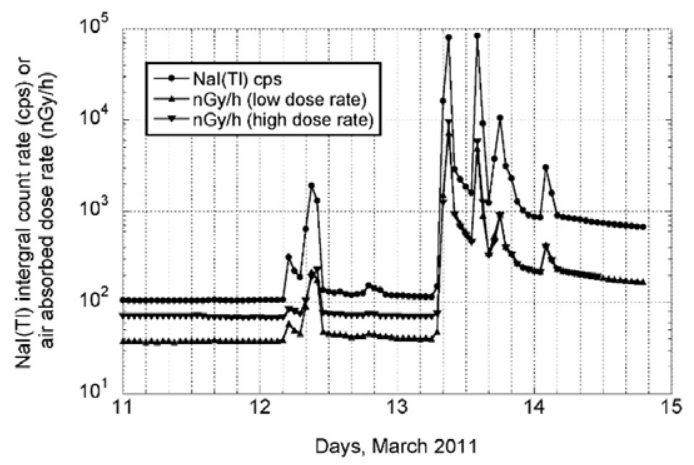

Figure 3 Comparison between corrected total count rates and air absorbed dose rates at Okuma Town Minamidai

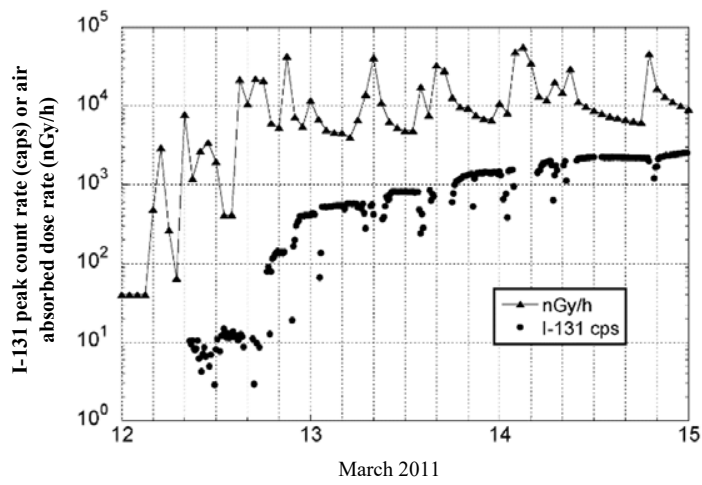

Figure 4 Comparison of time histories between I-131 peak count rates and air absorbed dose rates at Futaba Town Koriyama 


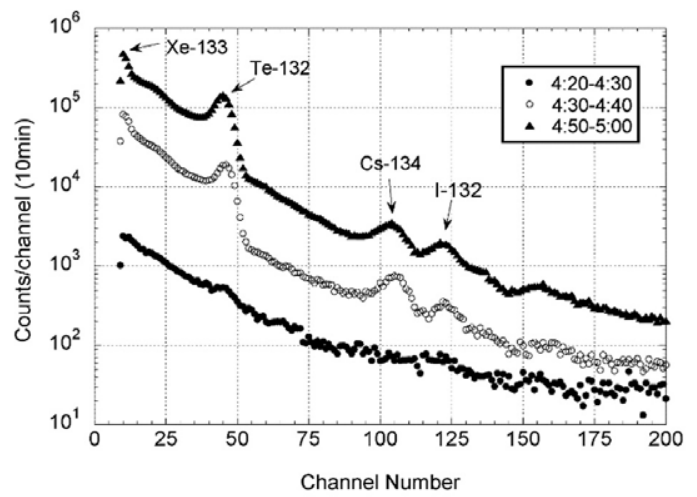

(a) 4:20-4:30, 4:30-4:40 and 4:50-5:00

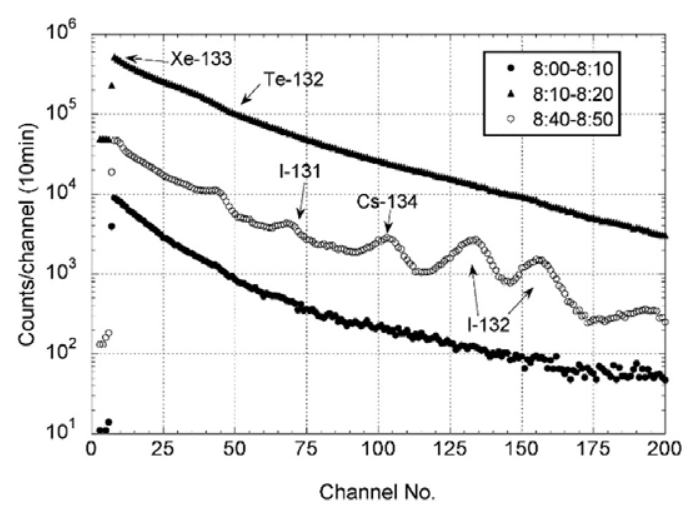

(b) $8: 00-8: 10,8: 10-8: 20$ and $8: 40-8: 50$

Figure 5 Pulse height distribution of $\mathrm{NaI}(\mathrm{Tl})$ detector at Futaba Town Koriyama in March 12

(a) 4:20-4:30, 4:30-4:40 and 4:50-5:00

(b) $8: 00-8: 10,8: 10-8: 20$ and $8: 40-8: 50$

height distributions during these periods and Xe-133 as well as Te-132 increased significantly, the dose rates increased because of these nuclides. If I-131 was contained in the plumes that arrived, a deposition proportional to the concentration in the plumes occurred to increase the peak count rates, as seen in the time series variation in the peak count rates of I-131 in Figure 4. However, after the plume at approximately 4:30 passed, the pulse height distribution returned to a state close to normal at 8:00 in which no peak of radionuclides was detected.

This indicates that the plume contained minimal I-131. The peak of I-131 became clear after $8: 40$. In this manner, it is known that the increase in the I-131 concentration in air because of the arrival of plume had a tendency to be later than that of Xe-133 and Te-132. A more significant increase in the concentrations of Xe-133 and Te-132 than that of I-131 affected the calculation of the I-131 peak area and resulted in an abnormal situation where it became smaller than the peak count rates based on I-131, which was deposited before the plumes arrived. In addition, if there were some time slots during which peak analysis could not be performed during the early stage of an increase in the concentration because of the arrival of a plume, the information obtained during that period was reflected in the I-131 peak count rate later. Given this, we interpolated the data to facilitate a smooth variation in the previous and subsequent count rates, for the time slots during which the peak count rates decreased abnormally after the dose rates increased rapidly and the time periods during which the count rates were too high 
to calculate the peak count rate. Figure 6 shows the time history of the I-131 peak count rates after data interpolation. The same data interpolation was applied to other monitoring posts to estimate the I-131 concentration in air. Figure 7 shows the time history of the I-131 peak count rates after correction observed at Syoukan in Naraha Town and Futatunuma in Hirono Town until 24:00 on March 31 as well as at Momijiyama in Fukushima City until 18:00 on March 28. This figure also shows the I-131 decay curve. Concerning the plume observed on March 16 at Shoukan in Naraha Town, the reduction rate of peak count rate after the plume passed was slightly less than decay of I-131. During the pulse height analysis at the maximum peak count rate, it is possible that the contribution of other nuclides was large and the I-131 peak count rates were underestimated.

It was not only demonstrated in the results of the measurements obtained at a number of expressways in Fukushima Prefecture using $\mathrm{LaBr}_{3}$ on March 15 by Matsumura, et al ${ }^{5}$. but also observed in the analysis at the monitoring posts of the NSRI described in reference 1 that the composition of nuclides contained in the plumes varied depending on the place and time of measurement. The form of the pulse height distribution was also confirmed to vary among the pulse height distributions observed at the monitoring posts used in this study. Figure 8 shows a comparison between Oono in Okuma Town on March 13 and Momijiyama in Fukushima City on March 15 as a typical example. At Oono in Okuma Town, it was found that the radionuclides

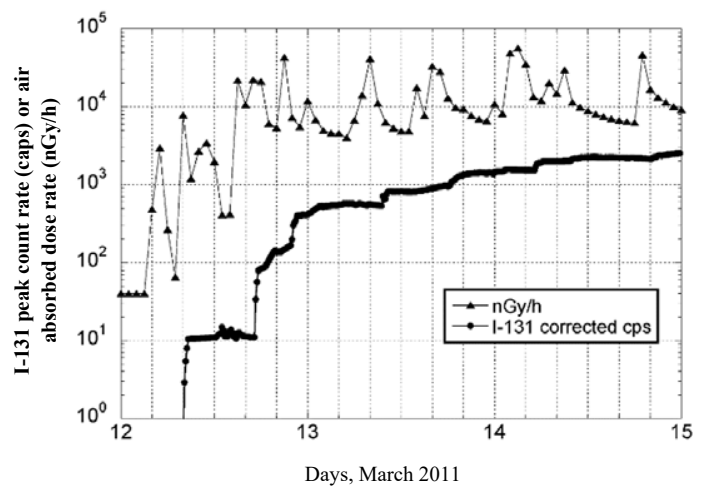

Figure 6 Comparison of time histories between corrected I-131 peak count rates and air absorbed dose rates at Futaba Town Koriyama

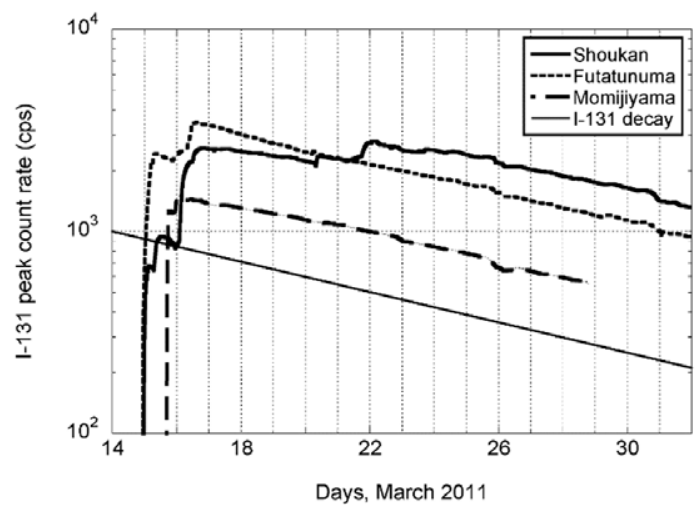

Figure 7 Time histories of corrected peak count rates at Naraha Town Shoukan, Hirono Town Futatunuma and Fukushima City Momijiyama 


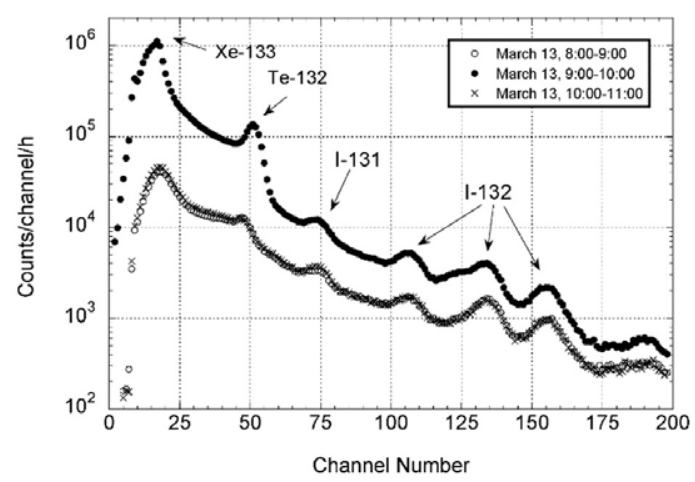

(a) Okuma-town Oono

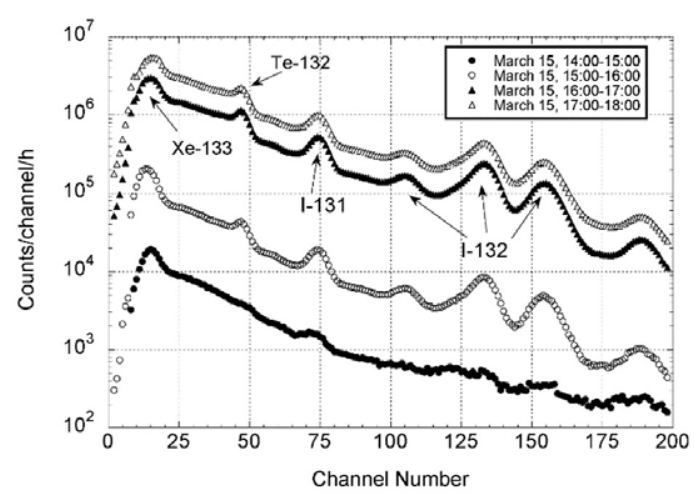

(b) Fukushima-city Momijiyama

Figure 8 Comparison of pulse height distributions of $\mathrm{NaI}(\mathrm{Tl}$ ) detector at Okuma Town Oono (March 13) and Fukushima City Momijiyama (March 15)

(a)Okuma Town Oono, (b)Fukushima City Momijiyama

in the plumes that arrived between 9:00 and 10:00 mostly consisted of Xe-133, Te-132, and I-132, which is a progeny nuclide of Te-132, and the percentage of I-131 was very low. However, at Momijiyama in Fukushima City, I-131 increased at the same rate as Xe-133 and Te-132. The variation in the nuclide composition depending on the location and time of measurement is an important matter that needs to be considered when estimating the release amounts using air absorbed dose and $1-\mathrm{cm}$ dose equivalent rates.

\section{Estimation of the Time History of the I-131 Concentration in Air}

\section{Time History of the Peak Count Rates Caused by the I-131 in the Arrived Plumes}

In the same manner as described in reference 1 for the time history of the peak count rates corresponding to $365 \mathrm{keV}$ of the respective monitoring posts, we determined the time of measurement when an increase in a count rate was observed as the plume start time and the time of the completion of measurement which was started from the moment when the count rate 
Table 2 Start and stop time of plume identified at monitoring posts in Futaba Town and Okuma Town together with average and time-integrated concentrations of I-131 in air

\begin{tabular}{|c|c|c|c|c|}
\hline Monitoring post & $\begin{array}{c}\text { Start time } \\
(\mathrm{dd} \mathrm{hh}: \mathrm{mm})\end{array}$ & $\begin{array}{c}\text { Stop time } \\
\text { (dd } \mathrm{hh}: \mathrm{mm})\end{array}$ & $\begin{array}{l}\text { Average } \\
\left(\mathrm{Bq} / \mathrm{m}^{3}\right)\end{array}$ & $\begin{array}{l}\text { Time-integrated } \\
\left(\mathrm{Bq} \mathrm{h} / \mathrm{m}^{3}\right)\end{array}$ \\
\hline \multirow{2}{*}{ Futaba-town Yamada } & $12^{\text {th }} 8: 00$ & $12^{\text {th }} 22: 00$ & 290 & 4,050 \\
\hline & $13^{\text {th }} 7: 00$ & $14^{\text {th }} 0: 00$ & 681 & 11,600 \\
\hline \multirow{4}{*}{ Futaba-town Koriyama } & $12^{\text {th }} 7: 00$ & $12^{\text {th }} 14: 00$ & 47.4 & 379 \\
\hline & $12^{\text {th }} 16: 00$ & $13^{\text {th }} 7: 00$ & 1,340 & 21,500 \\
\hline & $13^{\text {th }} 8: 00$ & $13^{\text {th }} 11: 00$ & 1,100 & 4,390 \\
\hline & $13^{\text {th }} 14: 00$ & $14^{\text {th }} 13: 00$ & 1,660 & 39,800 \\
\hline Okuma-town Mukaihata & $13^{\text {th }} 7: 00$ & $13^{\text {th }} 20: 00$ & 66.7 & 867 \\
\hline \multirow{3}{*}{ Okuma-town Oono } & $12^{\text {th }} 12: 00$ & $13^{\text {th }} 3: 00$ & 8.09 & 212 \\
\hline & $13^{\text {th }} 7: 00$ & $13^{\text {th }} 20: 00$ & 350 & 4,550 \\
\hline & $14^{\text {th }} 7: 00$ & $14^{\text {th }} 21: 00$ & 33.9 & 373 \\
\hline \multirow{2}{*}{ Okuma-town Minamidai } & $12^{\text {th }} 4: 00$ & $12^{\text {th }} 16: 00$ & 3.33 & 39.9 \\
\hline & $13^{\text {th }} 1: 00$ & $13^{\text {th }} 23: 00$ & 335 & 7,030 \\
\hline \multirow{3}{*}{ Okuma-town Ottozawa } & $12^{\text {th }} 2: 00$ & $12^{\text {th }} 15: 00$ & 9.47 & 123 \\
\hline & $13^{\text {th }} 6: 00$ & $13^{\text {th }} 15: 00$ & 906 & 8,150 \\
\hline & $14^{\text {th }} 0: 00$ & $14^{\text {th }} 7: 00$ & 136 & 949 \\
\hline
\end{tabular}

a) Average concentration in air.
b) Time-intergrated concentration in air.

Table 3 Start and stop time of plume identified at monitoring posts in Naraha Town Shoukan, Hirono Town Futatunuma and Fukushima City Momijiyama together with average and time-integrated concentrations of I-131 in air

\begin{tabular}{ccccc}
\hline Monitoring post & $\begin{array}{c}\text { Start time } \\
(\mathrm{dd} \mathrm{hh}: \mathrm{mm})\end{array}$ & $\begin{array}{c}\text { Stop time } \\
(\mathrm{dd} \mathrm{hh}: \mathrm{mm})\end{array}$ & $\begin{array}{c}\text { Average } \\
\left(\mathrm{Bq} / \mathrm{m}^{3}\right)\end{array}$ & $\begin{array}{c}\text { Time-integrated } \\
\left(\mathrm{Bq} \mathrm{h} / \mathrm{m}^{3}\right)\end{array}$ \\
\hline \multirow{2}{*}{ Naraha-town Shoukan } & $14^{\text {th }} 21: 00$ & $15^{\text {th }} 16: 00$ & 1,800 & 34,200 \\
\cline { 2 - 5 } & $15^{\text {th }} 22: 00$ & $16^{\text {th }} 20: 00$ & 1,680 & 37,100 \\
\cline { 2 - 5 } & $20^{\text {th }} 7: 00$ & $20^{\text {th }} 15: 00$ & 1,060 & 4,440 \\
\hline \multirow{2}{*}{ Hirono-town Futatunuma } & $21^{\text {th }} 15: 00$ & $22^{\text {th }} 7: 00$ & 4,080 & 65,300 \\
\cline { 2 - 5 } & $14^{\text {th }} 21: 00$ & $15^{\text {th }} 12: 00$ & 5,100 & 76,500 \\
\hline Fukushima-city Momijiyama & $15^{\text {th }} 21: 00$ & $16^{\text {th }} 20: 00$ & 2,930 & 67,400 \\
\hline
\end{tabular}

a) Average concentration in air.

b) Time-integrated concentration in air.

stopped increasing and started to attenuate according to the decay of I-131 half-life as the plume stop time. In the case of Koriyama in Futaba Town, data obtained every 10 min were available, but a large amount of data was required for the correction of peak count rates. Therefore, we used the hourly-averaged peak count rates for the analysis. Tables $\mathbf{2}$ and $\mathbf{3}$ show the estimated plume start and stop times based on this concept.

The time history of the I-131 peak count rates in the plumes were determined using the estimation method based on the time history of the peak count rates suggested in reference 1 .

\section{Peak Detection Efficiency of NaI(Tl) Detectors for $365-\mathrm{keV} \gamma$ ray of I-131 in the Plumes}

In the same manner as described in reference 1, we calculated the photon spectra of the respective angle sections which enter a detector placed at a height of $3 \mathrm{~m}$ above the ground 
surface in the air of the NTP state $\left(20^{\circ} \mathrm{C}, 1 \mathrm{~atm}\right)$, by applying the method of replacing the gamma-ray flux from a plane isotropic source with a point isotropic source and plane detector ${ }^{6}$ to egs $5^{7}$, based on the assumption that I-131 is infinitely present in a horizontal direction and from the ground surface to the plume height in a vertical direction at a uniform concentration. We calculated the peak detection efficiency using egs 5 assuming that the photons with spectra of the respective angle sections obtained enter the detector at corresponding angles. Because the lower structure of the detector and the structure of the monitoring post contribute to scattered radiation but have a minimal impact on un-scattered photons that may cause a total absorption peak, we considered only the aluminum cover of the $\mathrm{NaI}(\mathrm{Tl})$ detector and the $\mathrm{NaI}(\mathrm{Tl})$ detector in the calculation of the peak detection efficiency. For the peak detection efficiencies corresponding to $365-\mathrm{keV}$ gamma radiation of I-131, we obtained $0.0524,0.0574$, and 0.0585 cps per $\mathrm{Bq} / \mathrm{m}^{3}$ for heights of $100 \mathrm{~m}, 200 \mathrm{~m}$, and $300 \mathrm{~m}$, respectively.

Although the plume heights are within the range between $100 \mathrm{~m}$ and 1,000 m, considering that the difference resulting from plume height is small and the distance from the $1 \mathrm{~F}$ plant is short, we used the peak detection efficiency in the case of a plume height of $100 \mathrm{~m}$ in which the concentration is the highest for the same count rate as the factor for converting the peak count rate based on the I- 131 concentration in air. If this conversion factor is used, an overestimation of approximately $10 \%$ occurs when the plume height is $300 \mathrm{~m}$.

\section{Result and Discussion}

\section{I-131 Concentration in Air on or before March 15}

Using the peak count rates based on the I-131 in the plumes that arrived as well as the conversion factor obtained using the method described in Chapter III, we determined the time history of the I-131 concentration in air during the period prior to a drastic increase that occurred at midnight on March 14. Figure 9 shows the results obtained at the monitoring posts at Koriyama in Futaba Town, Yamada in Futaba Town, Mukaihata in Okuma Town, Oono in Okuma Town, Minamidai in Okuma Town and Ottozawa in Okuma Town on March 12, 13, and 14. At the monitoring posts in Naraha Town, Tomioka Town, Hirono Town and Fukushima City, an increase in the concentration in air was not observed during this period.

On March 12, at the monitoring posts at Koriyama and Yamada in Futaba Town on the north side of the $1 \mathrm{~F}$ plant, a relatively high I-131 concentration was observed although the concentration and time variation were different between them. However, at the monitoring posts in Okuma Town south of the 1F plant, I-131 was not detected or detected as a very small amount. The result corresponded to the estimation based on the weather information that the I-131 emitted on March 12 spread mainly to the north. On March 13, an increase in the concentration occurred because of the arrival of plumes at all monitoring posts although the time and the concentration in air differed. On March 14, the same level of increase as that of March 13 was observed at Koriyama in Futaba Town. Although a slight increase was also observed at Ottozawa and Oono in Okuma Town, an increase resulting from the arrival of plumes was not observed at the other monitoring posts. At midnight on March 14, the count rates recorded at many monitoring posts increased significantly and it became impossible to conduct peak analysis. The final time at which data could be analyzed shown in Table 1 corresponds to the time of the arrival of a high-concentration plume. While an increase at the monitoring posts around the 1F plant including Shoukan in Naraha Town and Futatunuma in Hirono Town began at 20:00 or 21:00 on March 14, no increase was observed by 24:00 on March 14 at Koriyama in Futaba 


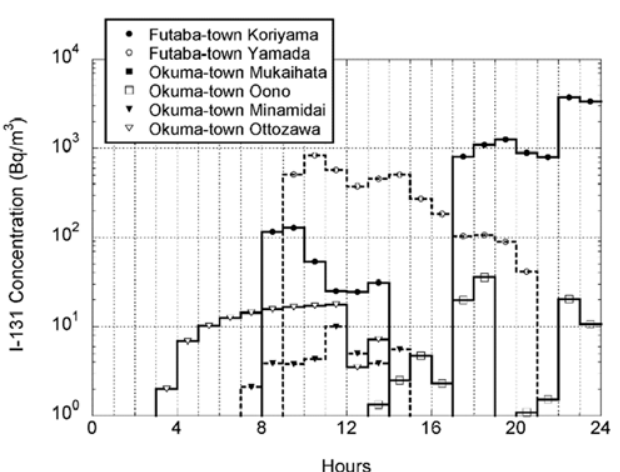

(a) March 12, 2011

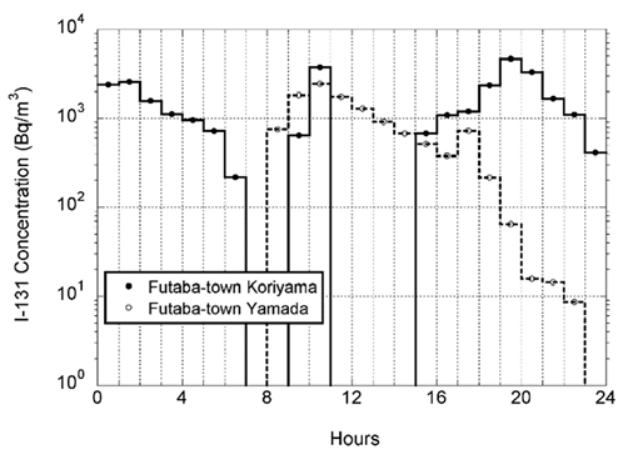

(b) Futaba-town, March 13, 2011

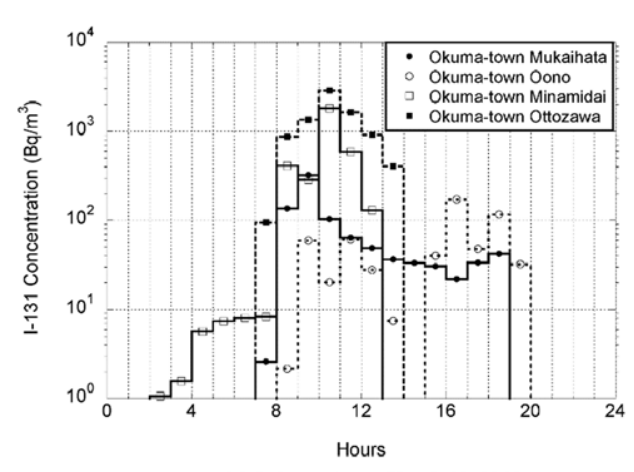

(c) Ookuma-town, March 13, 2011

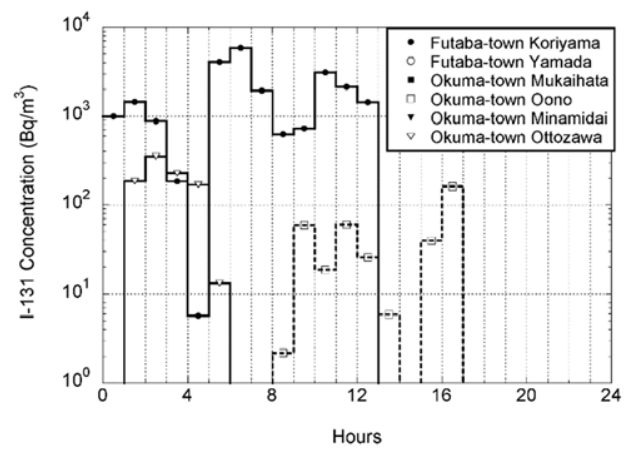

(d) March 14, 2011

Figure 9 Time histories of I-131 concentration in air at monitoring posts in Futaba Town and Okuma Town

(a) March 12, 2011, (b) Futaba Town, March 13, 2011, (c) Okuma Town, March 13, 2011, (d)March 14,2011

Town where data were available and an increase started as late as 11:00 on March 15 at Yamada in Futaba Town. These differences likely reflect weather conditions such as the direction of the wind. Although the locations and times are limited, the I-131 concentrations in air measured by sampling the air are shown on the website of the Ministry of Economy, Trade and Industry ${ }^{8}$. As the only result of measurements conducted in Futaba and Okuma towns, $12 \mathrm{~Bq} / \mathrm{m}^{3}$ was recorded between 8:37 and 8:47 at Ottozawa Chojahara in Okuma Town. The estimated value between 8:00 and 9:00 at the monitoring post at Ottozawa in Okuma Town is $16 \mathrm{~Bq} / \mathrm{m}^{3}$, nearly identical to the value recorded at Ottozawa Chojahara in Okuma Town.

When considering the influence of I-131 in the air, not only the I-131 concentration in air when a plume arrives but also the duration of the plume needs to be considered. Table 2 shows the average concentration in air as well as the time-integrated concentration in air during the estimated arrival of respective plumes. Figure 10 shows the locations of respective monitoring posts on the map ${ }^{9)}$ and the average concentration in air during the arrival of plumes to clarify the difference in the average concentration in air depending on the location of the $1 \mathrm{~F}$ plant and respective monitoring posts. The width of the bar graph indicates the duration for which the plume persisted and its area corresponds to the time-integrated concentration in air.

The I-131 emissions in the air estimated by Kobayashi, et al. ${ }^{10)}$ are also shown. The variation in the average concentration on March 12 differed markedly between Yamada and Koriyama in Futaba Town, both of which are at nearly the same distance from the 1F plant. Although an increase in the concentration corresponding to an increase in the estimated emission was 


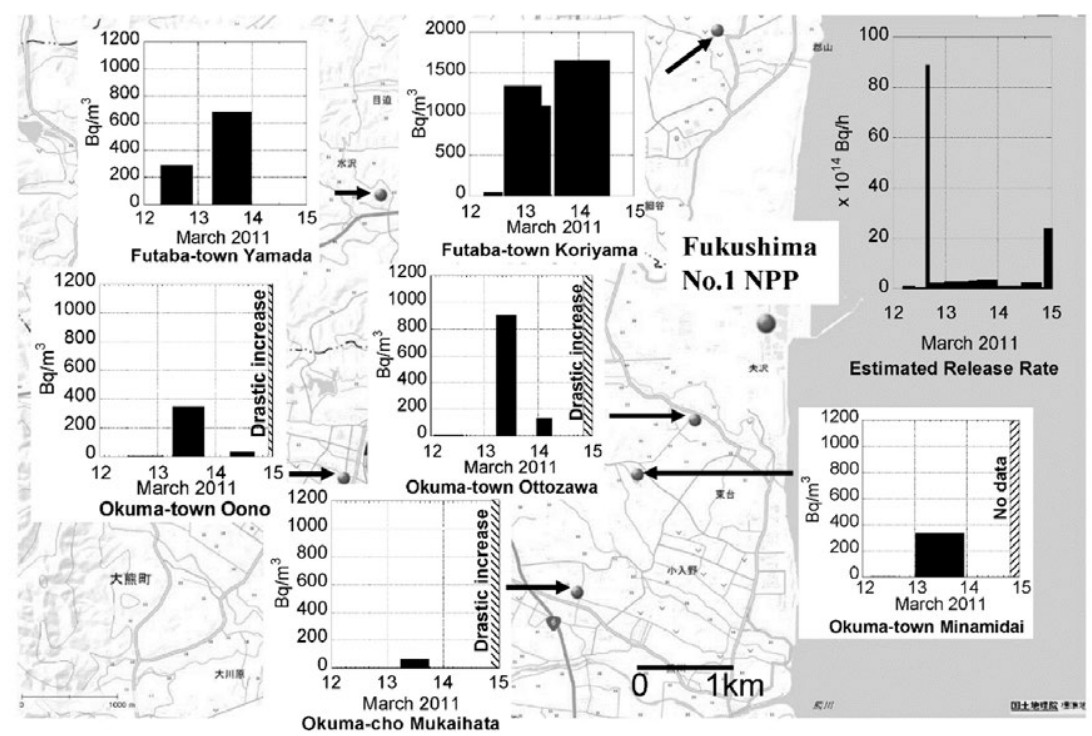

Figure 10 Average concentration of I-131 in air $\left(\mathrm{Bq} / \mathrm{m}^{3}\right)$ at monitoring posts in Futaba Town and Okuma Town together with I-131 estimated release rate $\left(10^{14} \mathrm{~Bq} / \mathrm{h}\right)^{10)}$

observed at Koriyama in Futaba Town, it was not clear at Yamada. This undoubtedly indicates that the average concentrations on March 13 differed depending on the distance and direction from the $1 \mathrm{~F}$ plant. For the monitoring posts where the analysis could not be conducted because of a drastic increase in emissions that occurred at midnight on March 14, "drastic increase" is displayed at and after the time of the occurrence of the increase and "no data" is displayed if no data are available. According to the figure, no increase occurred on March 14 at Yamada and Koriyama in Futaba Town.

\section{I-131 in the Air during March}

Figures 11-13 show the time history of the I-131 concentration in air at Shoukan in Naraha Town and Futatunuma in Hirono Town where the data obtained until 24:00 on March 31 were available and at Momijiyama in Fukushima City where data obtained until 18:00 on March 21 were available. Because of an increase in the count rates caused by the I-131 deposited in the surrounding areas as a result of the arrival of plumes, the detectable lowest detection concentration increases when the method of estimating the arrival of plumes from the time series shows a variation in peak count rates. When the error in determining a net count rate is $\sigma$ (cps), it is assumed that an increase in peak count rate can be detected in the event of a change of $3 \sigma$ or greater. Because the conversion factor of I-131 from the peak count rate to the concentration in air used in the analysis performed at the monitoring posts in Fukushima was $0.0524 \mathrm{cps}$ per $\mathrm{Bq} / \mathrm{m}^{3}$, the detection concentration limit $D_{\text {limit }}$ becomes

$$
D_{\text {limit }}\left(B q / m^{3}\right)=\frac{3 \sigma}{0.0524}
$$

Figures 11-13 also show the detection concentration limits obtained using equation (2).

Although a concentration in air comparable to the concentration measured on March 15 was observed from March 20 to 22 at Shoukan in Naraha Town, no corresponding increase 


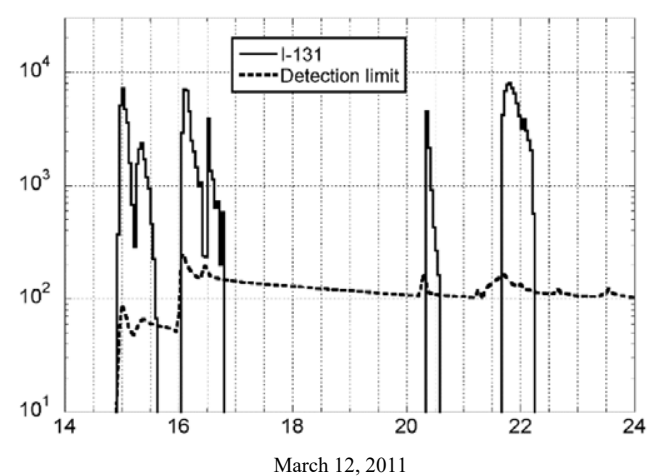

Figure 11 Time histories of I-131 concentration in air at Naraha Town Shoukan

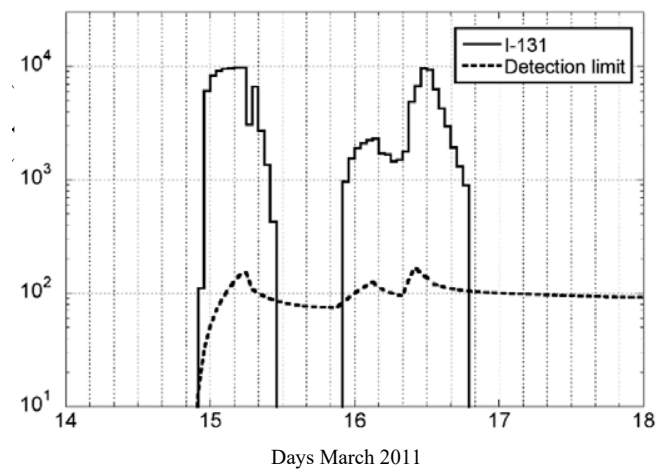

Figure 12 Time histories of I-131 concentration in air at Hirono Town Futatunuma

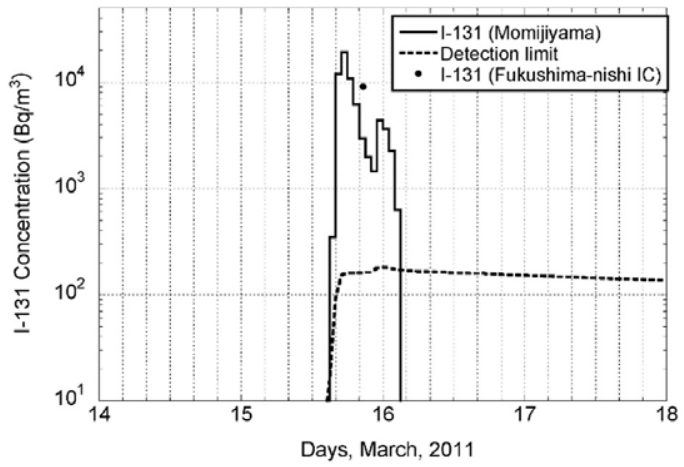

Figure 13 Time histories of I-131 concentration in air at Fukushima City Momijiyama together with estimated concentration at Fukushima-nishi IC obtained with the same method described in Ref. 11 using the $\mathrm{LaBr}_{3}$ pulse height distribution

was seen in the other two areas. In all three areas, no arrival of plumes containing I-131 at a concentration higher than the detection limit occurred on or after March 23. Figure 13 also shows the estimated I-131 concentration in air at Fukushima Nishi IC (4 km from Momijiyama in Fukushima City) determined using the pulse height distribution ${ }^{5)}$ of $\mathrm{LaBr}_{3}$ obtained with the same method as that described in reference 11. Considering that the values based on the 


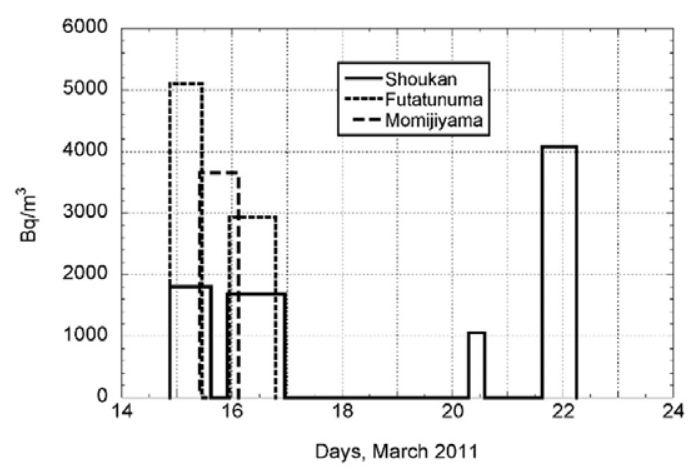

Figure 14 Comparison of average concentration of I-131 in air between Naraha Town Shoukan, Hirono Town Futatunuma and Fukushima City Momijiyama.

measurement using $\mathrm{LaBr}_{3}$ includes the impact of deposition and the concentration in air may be overestimated, it can be stated that the estimated values at Fukushima Nishi IC are the concentrations that correspond to the results obtained in this study.

Table 3 shows the average concentration in air and the time-integrated concentration in air during the arrival of plumes. Figure 14 shows a comparison of the average concentration in air among three locations. The 1F plant is $14.2 \mathrm{~km}$ from Shoukan in Naraha Town, $21.4 \mathrm{~km}$ from Futatunuma in Hirono Town, and $61.3 \mathrm{~km}$ from Momijiyama in Fukushima City. The times at which the I-131 concentration reached its maximum level because of the plumes that arrived between midnight on March 14 and midnight on March 15 are 0:00-1:00 on March 15 at Shoukan in Naraha Town, 5:00-6:00 on March 15 at Futatunuma in Hirono Town, and 17:00-18:00 on March 15 at Momijiyama in Fukushima City; the concentrations at these times were 7,220, 9,790 , and $19,100 \mathrm{~Bq} / \mathrm{m}^{3}$, respectively. Although the time-integrated concentration in air is important when considering the impact on internal exposure, as Table 3 shows, the time-integrated concentrations in air obtained by 24:00 on March 16 at Shoukan in Naraha Town and Momijiyama in Fukushima City were nearly the same, and that observed at Futatunuma in Hirono Town was approximately twice as high as those obtained at the other two locations. As described in Chapter II Section 3, because it would appear that the results obtained at Shoukan in Naraha Town between midnight on March 15 and March 16 underestimated the peak count rates around the time at which the peak count rates reached their maximum levels, the concentration in air may have been underestimated.

\section{Comparison with the Estimated Values Described in UNSCEAR ANNEX A ${ }^{3)}$}

UNSCEAR 2013 ANNEX A (hereinafter referred to as "UNSCEAR") includes Table B10 which shows the time-integrated I-131 concentrations in air at several locations. These concentrations were obtained using the estimated values of the time-dependent I-131 emissions from the $1 \mathrm{~F}$ plant as well as the atmospheric dispersion models. The time-integrated concentrations in air shown in the table were estimated using the methods employed by the U.S. National Oceanic and Atmospheric Administration - Global Data Assimilation System (NOAA-GDAS) and Institute for Radiological Protection and Nuclear Safety-European Centre for Medium Range Weather Forecasting (IRSN-ECMWF). The time-dependent emissions estimated by Terada, et al. ${ }^{12)}$ were used in NOAA-GDAS and those estimated by Saunier, et al. ${ }^{13)}$ were used in IRSN-ECMWF. They both estimated the emissions based on the measured radiation 
Table 4 Comparison of time-integrated concentration of I-131 in air between the results obtained in this study and estimated in UNSCEAR 2013 ANNEX A $^{3)}$

\begin{tabular}{|c|c|c|c|c|c|c|}
\hline \multirow{3}{*}{ Location } & \multirow{2}{*}{\multicolumn{2}{|c|}{ Time period March, 2011}} & \multirow{3}{*}{$\begin{array}{l}\text { Distance from Fukushima } \\
\text { Daiichi Nuclear Power } \\
\text { Station }\end{array}$} & \multirow{3}{*}{ This work } & \multicolumn{2}{|l|}{$\mathrm{Bq} \mathrm{h} / \mathrm{m}^{3}$} \\
\hline & & & & & UNSCEAR 20 & 3 ANNEX- ${ }^{3)}$ \\
\hline & $\begin{array}{c}\text { From } \\
\text { (dd hh: mm) }\end{array}$ & $\begin{array}{c}\text { To } \\
\text { (dd hh : mm) }\end{array}$ & & & NOAA-GDAS ${ }^{a)}$ & IRSN-ECMWF'b) \\
\hline $\begin{array}{l}\text { Futaba-town } \\
\text { Yamada }\end{array}$ & \multirow{3}{*}{$11^{\text {th }} 21: 23$} & \multirow{3}{*}{$12^{\text {th }} 21: 00$} & WNW 4.1 km & 2,440 & & \\
\hline $\begin{array}{l}\text { Futaba-town } \\
\text { Koriyama }\end{array}$ & & & NNW $2.8 \mathrm{~km}$ & 5,240 & & \\
\hline Futaba-town & & & & & 23,300 & \\
\hline $\begin{array}{l}\text { Naraha-town } \\
\text { Shoukan }\end{array}$ & \multirow{3}{*}{$12^{\text {th }} 13: 00$} & \multirow{3}{*}{$16^{\text {th }} 15: 00$} & SSW $14.2 \mathrm{~km}$ & 69,100 & & \\
\hline $\begin{array}{l}\text { Hirono-town } \\
\text { Futatunuma }\end{array}$ & & & $\mathrm{S} 21.4 \mathrm{~km}$ & 137,000 & & \\
\hline Iwaki-city & & & $\mathrm{S} 42.5 \mathrm{~km}$ & & 61,100 & 30,600 \\
\hline $\begin{array}{c}\text { Fukushima-city } \\
\text { Momijiyama }\end{array}$ & \multirow{2}{*}{$12^{\text {th }} 8: 00$} & \multirow{2}{*}{$19^{\text {th }} 8: 00$} & NW $61.3 \mathrm{~km}$ & 65,700 & & \\
\hline $\begin{array}{l}\text { Kawamata } \\
\text { elementary school }\end{array}$ & & & NW 51.7 km & & 18,900 & 20,800 \\
\hline
\end{tabular}

levels, and UNSCEAR indicates that the estimated values obtained using this method have high uncertainty even if the radioactive concentration in air is used. The time-dependent measurements of the concentration in air associated with the accident that occurred at the $1 \mathrm{~F}$ plant were limited to the those obtained after March 15 in Ibaraki Prefecture (Tokai Village and Tsukuba City) and Chiba Prefecture (Chiba City), which are located far away from the 1F plant. Before March 15 and in other areas, the radioactivity density on the ground surface and the dose rate information obtained at monitoring posts were used. Because it is difficult to obtain the time history data of the I-131 concentration in air from the radioactivity density on the soil surface and the variation in dose rate does not correspond with the variation in the I-131 concentration in air, the estimation based on these data has a higher uncertainty than the estimation based on the radioactivity concentration in air. Although the estimations had high uncertainty, to the best of our knowledge, no data were available to which they could be directly compared. Therefore, we compared the time-integrated concentrations in air, obtained at the locations, which could be associated with monitoring posts, among those shown in Table B10, with the time-integrated concentrations in air obtained in this study. Table 4 shows the results ${ }^{c}$. The period subject to integration is as shown in Table B10, and the values obtained in this study are the integrated values for the same period.

In a comparison on the north side of the 1F plant from 21:23 on March 11, which was immediately after the accident, to 21:00 on March 12, the time-integrated concentration in air, which was measured in Futaba Town and described in UNSCEAR, was approximately 10 and 4 times greater than the that measured at Yamada and Koriyama in Futaba Town, respectively. In a comparison, on the south side of the 1F plant from 3:00 on March 13 to 15:00 on March 16, the time-integrated concentration in air measured at Futatunuma in Hirono Town was twice as high as that measured at Shoukan in Naraha Town. However, as previously described, the results obtained at Shoukan in Naraha Town may have been underestimated. The estimated values obtained in Iwaki City and described in UNSCEAR were one-half (NOAA-GDAS) or one-quarter (IRSN-ECMWF) of those obtained at Futatunuma. In a comparison on the northwest side of the 1F plant from 8:00 March 12 to 8:00 March 19, the time-integrated concentration in air

\footnotetext{
${ }^{\mathrm{c}}$ Table 4 shows the values obtained by converting Bq s/ $\mathrm{m}^{3}$ values described in UNSCEAR into Bq $\mathrm{h} / \mathrm{m}^{3}$ values.
} 
measured at Momijiyama in Fukushima City, $61.3 \mathrm{~km}$ from the 1F plant, was approximately three times higher than that measured at Kawamata Elementary School in Kawamata Town, $51.7 \mathrm{~km}$ from the $1 \mathrm{~F}$ plant, as described in UNSCEAR. As previously mentioned, the results obtained in this study correspond to the results described in the UNSCEAR in terms of order, but the relation between both results differs depending on the time and location.

Although there is some uncertainty in the time-integrated concentration in air as estimated in this study associated with the method used to determine the absolute I-131 concentration in air from the time history pulse height distribution ${ }^{\mathrm{d}}$, it is based on the time history of the concentration in air estimated from the actual pulse height distribution data of the $\mathrm{NaI}(\mathrm{Tl})$ detector. However, because it is assumed, in the calculation of the conversion factor from the peak count rate to concentration in air, that the I-131 contained in the plumes distributed from the ground surface to the plume heights is of a uniform concentration, there may be a large error at the monitoring posts near the $1 \mathrm{~F}$ plant. Because the time histories of the concentration in air are not described in UNSCEAR, it is impossible to compare them. However, the estimated values described in UNSCEAR are based on time-dependent emissions determined using the radioactivity density deposited on the ground as well as the dose rate information obtained at the monitoring posts. Therefore, the estimated values obtained in this study better reflect actual conditions at least at the monitoring posts $10 \mathrm{~km}$ or farther from the $1 \mathrm{~F}$ plant. It would appear that the information on the time history of the I-131 concentration in air obtained in Fukushima Prefecture including the area around the $1 \mathrm{~F}$ plant in this study is useful in improving the precision of the estimation of time-dependent emissions using radioactivity data.

\section{Conclusions}

We estimated the I-131 concentrations in air in areas around the monitoring posts during March 2011 using the pulse height distribution data of a $\mathrm{NaI}(\mathrm{Tl})$ detector obtained at the monitoring posts and disclosed on the website of Fukushima Prefecture. Because the count rates were much higher than is typical because of the influence of high-concentration radionuclides emitted as a result of the accident that occurred at the $1 \mathrm{~F}$ plant, we performed digit overflow and (live time) / (real time) corrections of the disclosed pulse height distribution before using the data in the analysis. In addition, when a discontinuous tendency was observed in the time series variation in the peak count rates of I-131 because of the difference in the composition of radionuclides during the arrival of plumes, we interpolated the data after checking the pulse height distribution to avoid discontinuous variation. These corrections caused some uncertainty in the results obtained in this study, but it was necessary to make use of limited information.

The results obtained in this study are the first on the I-131 concentration in air continuously measured at fixed locations in Fukushima Prefecture during March 2011. In the areas near the $1 \mathrm{~F}$ plant, the results showed that while the plume mainly moved to the north on March 12, it moved in various directions on March 13, although data were limited to before the massive emission on March 15. We could confirm that no plume arrived before March 15 at Shoukan in Naraha Town, Futatunuma in Hirono Town, and Momijiyama in Fukushima City, where we could analyze the data obtained throughout nearly the whole of March. The plumes mainly arrived on March 15 and 16 and no plume containing a high-concentration of I-131 arrived at

\footnotetext{
${ }^{\mathrm{d}}$ As a result of comparing the estimated values to the air monitoring results concerning the time-integrated concentration of I-131 in air based on the data used in ref. 1 the ratio between them was within a range of from 0.2 to 4 , depending on period and location of monitoring posts.
} 
Futatunuma and Momijiyama.

In this study, we compared the time-integrated I-131 concentrations in air measured at several locations with the concentrations obtained using the estimated time-dependent emissions from the $1 \mathrm{~F}$ plant described in UNSCEAR and atmospheric dispersion models. The results corresponded to the results described in UNSCEAR in terms of order, but the relation between both results differed depending on the time and location. The major factor in this difference was the high uncertainty in the time-dependent emissions estimated using the radioactivity measurements of UNSCEAR.

From the results of this study, we found that it was possible to obtain the time history of the I-131 concentration in air as information on the conditions of I-131 diffusion resulting from the accident if the pulse height distribution data obtained at the monitoring posts were available. It would appear that analyzing not only the data that were obtained in Fukushima Prefecture and used in this study but also the data obtained at various monitoring posts during March results in more information on the time-dependent I-131 radioactivity concentration in air being available. This also contributes to an improvement in the accuracy of the estimation of time-dependent emissions as well as the estimation of the evaluation of time-dependent I-131 concentrations in air at various locations using time-dependent emission and atmospheric dispersion models.

We could not have performed this study without the disclosure of numerical pulse height distribution data obtained at the monitoring posts by Fukushima Prefecture. We would like to express our deepest gratitude to the Fukushima Nuclear Power Center for their endeavors to process an enormous quantity of data to post on the website.

\section{References}

1) H. Hirayama, M. Kawasaki, H. Matsumura, T. Ohkura, Y. Namito, T. Sanami, M. Taki, T. Oishi, M. Yoshizawa, "Estimation of I-131 concentration by using time history of pulse height distribution at monitoring post and detector response for radionuclide in plume," Trans. At. Energy Soc. Jpn., 13, 119126 (2014). [in Japanese]

2) http://www.atom-moc.pref.fukushima.jp/monitoring/monitoring201103/201103_mpdata.html, 2014.10.14 final confirmation.

3) UNSCEAR 2013 ANNEX A, Levels and Effects of Radiation Exposure due to the Nuclear Accident after the 2011 Great East-Japan Earthquake and Tsunami, United Nations, New York, 2014.

4) http://www.pref.fukushima.lg.jp/sec_file/monitoring/etc/post5-20120921.pdf, 2014.10 .14 final confirmation.

5) H. Matsumura, K. Saito, J. Ishioka, Y. Uwamino, "Diffusion of radioactive materials from Fukushima Daiichi Nuclear Power Station obtained by gamma-ray measurements on expressways," Trans. At. Energy Soc. Jpn., 10, 152-162 (2011). [in Japanese]

6) Y. Namito, H. Nakamura, A. Toyoda, K. Iijima, H. Iwase, S. Ban, H. Hirayama, "Transformation of a system consisting of plane isotropic source and unit sphere detector into a system consisting of point isotropic source and plane detector in Monte Carlo calculation," J. Nucl. Sci. Technol, 49, 167-172 (2012).

7) H. Hirayama, Y. Namito, A. F. Bielajew, S. J. Wilderman, W. R. Nelson, The EGS5 Code System, SLAC-R-730 (2005) and KEK Report 2005-8 (2005).

8) http://www.meti.go.jp/press/2011/06/20110603019/20110603019.html, 2014.10.14 final confirmation.

9) GSI Maps, Geospatial Information Authority of Japan.

10) T. Kobayashi, H. Nagai, M. Chino, H. Kawamura, "Source term estimation of atmospheric release due to the Fukushima Dai-ichi Nuclear Power Plant accident by atmospheric and oceanic dispersion simulations," J. Nucl. Sci. Technol., 50, 255-264 (2013).

11) H. Hirayama, H. Matsumura, Y. Namito, T. Sanami, "Estimation of radionuclide concentration in plume using pulse height distribution measured by $\mathrm{LaBr}_{3}$ scintillation detector and its response to radionuclides in plume calculated with egs5," Trans. At. Energy Soc. Jpn., 12, 304-310 (2012). [in Japanese]

12) H. Terada, G. Katata, M. Chino et al., "Atmospheric discharge and dispersion of radionuclides during the 
Fukushima Dai-ichi Nuclear Power Plant accident. Part II: verification of the source term and analysis of regional-scale atmospheric dispersion," J. Environ. Radioact., 112, 141-154 (2012).

13) O. Saunier, A. Mathieu, D. Didier et al., "An inverse modeling method to assess the source term of the Fukushima Nuclear Power Plant accident using gamma dose rate observations," Atmos. Chem. Phys., 13 [22], 11403-11421 (2013) 


\title{
Effect of $\mathrm{B}_{4} \mathrm{C}$ Absorber Material on Melt Progression and Chemical Forms of Iodine or Cesium under Severe Accident Conditions
}

\author{
Akihide HIDAKA ${ }^{1, *,+}$ \\ ${ }^{1}$ Nuclear Safety Research Center, Japan Atomic Energy Agency, \\ 2-4 shirane, Shirakata, Tokai-mura, Naka-gun, Ibaraki 319-1195, Japan
}

\begin{abstract}
Boron carbide $\left(\mathrm{B}_{4} \mathrm{C}\right)$ used for BWR or EPR absorbers could cause phenomena that never occur in PWR with silver-indium-cadmium absorbers during a severe accident. $\mathrm{B}_{4} \mathrm{C}$ would undergo a eutectic interaction with stainless steel and enhance core melt relocation. Boron oxidation could increase $\mathrm{H}_{2}$ generation, and the change of liberated carbon to $\mathrm{CH}_{4}$ could enhance the generation of organic iodide $\left(\mathrm{CH}_{3} \mathrm{I}\right)$. $\mathrm{HBO}_{2}$ generated during $\mathrm{B}_{4} \mathrm{C}$ oxidation could be changed to cesium borate $\left(\mathrm{CsBO}_{2}\right)$ by combining it with cesium. This may increase cesium deposition into the reactor coolant system. There could be differences in the configuration, surface area, and stainless-steel to $\mathrm{B}_{4} \mathrm{C}$ weight ratio between the $\mathrm{B}_{4} \mathrm{C}$ powder absorber and pellet absorber. The present task is to clarify the effect of these differences on melt progression, $\mathrm{B}_{4} \mathrm{C}$ oxidation, and the iodine or cesium source term. Advancement of this research field could contribute to further sophistication of prediction tools for melt progression and source terms of the Fukushima Accident, and the treatment of organic iodide formation in safety evaluation.
\end{abstract}

KEYWORDS: $B_{4} C$ powder absorber, $B_{4} C$ pellet absorber, eutectic reaction, organic iodide, cesium borate, melt progression, iodine source term, cesium source term

\section{Introduction}

Boron carbide is used as an absorber for nuclear reactors such as boiling water reactors (BWRs), European pressurized reactors (EPRs), and Russian pressurized reactors (Voda Energo Reactor/VVER). $\mathrm{B}_{4} \mathrm{C}$ could cause phenomena during a severe accident that never occur in pressurized water reactors (PWRs) in which silver-indium-cadmium (S-I-C) absorbers are widely used, and may influence the melting of the reactor core, hydrogen generation, source terms, and other processes.

The cooling function of the reactor is lost during a severe accident, and as a result, the temperature of $\mathrm{UO}_{2}$ fuel rods, absorber rods, and the surrounding reactor structures will increase because of insufficient removal of decay heat. During such a situation, if different types of metals or ceramics are in contact with each other, melting will start at a lower temperature

\footnotetext{
* Corresponding author, E-mail: hidaka.akihide@jaea.go.jp

$\dagger$ Currently, Nuclear Human Resource Development Center, Japan Atomic Energy Agency

DOI : 10.15669 /fukushimainsights.Vol.4.341

(C) 2021 Atomic Energy Society of Japan. All rights reserved.

Originally published in Transactions of the Atomic Energy Society of Japan (ISSN 1347-2879), Vol. 14, No. 1, p.51-61

(2015) in Japanese. (Japanese version accepted: October 7, 2014)
} 
than the individual melting points due to eutectic reactions. For a $\mathrm{B}_{4} \mathrm{C}$ control rod, the stainless steel cladding tube and $\mathrm{B}_{4} \mathrm{C}$ (melting point $2,620 \mathrm{~K}$ ) will liquify and flow downwards at temperatures less than the melting point of stainless steel $(1,673-1,723 \mathrm{~K})$. The molten substances will solidify when cooled in the lower part of the reactor core, but portions will travel further down in the reactor pressure vessel. Moreover, reaction heat, and $\mathrm{H}_{2}$ will be generated by the oxidation of $\mathrm{B}_{4} \mathrm{C}$ itself at around $1,500 \mathrm{~K}$.

As the temperature increases further, the fuel cladding tubes and $\mathrm{UO}_{2}$ fuel will be melted, and the molten substances will flow down around the fuel rod like candle wax (candling phenomenon) and fall down to the bottom of the reactor core or the reactor pressure vessel, as same as the case of $\mathrm{B}_{4} \mathrm{C}$ control rods. These molten materials will finally form molten debris that contains the previously melted absorber materials and reactor core structures including the $\mathrm{UO}_{2}$ fuel. The radioactive materials in the molten debris will react with dissolved oxygen and absorber materials in the debris, and their chemical form including melting point and boiling point will vary.

For the aforementioned reasons, a change in the absorber material influences the reactor core melting process, the quantity of $\mathrm{H}_{2}$ generated, and the amount, kind and timing (source terms) of the radioactive materials released into the environment.

Research into progress of core melting phenomena, especially after the TMI-2 accident ${ }^{1)}$ that occurred in the USA in 1979, has focused mainly on that of PWRs using S-I-C absorbers. Through numerous international experiments, various insights into the influence of absorber materials on the progress of reactor core melting of PWRs have been obtained ${ }^{2)}$. However, only a few experiments have been done on BWRs, partly because of the small number of reactors that use $\mathrm{B}_{4} \mathrm{C}$ absorbers. Recently, after EPRs began using the $\mathrm{B}_{4} \mathrm{C}$ absorbers, the effect of $\mathrm{B}_{4} \mathrm{C}$ on the chemical forms of iodine released from the fuel during a severe accident has been studied more extensively. In particular, studies have examined the association of $\mathrm{B}_{4} \mathrm{C}$ with the generation of gaseous iodine (organic iodine, $\mathrm{CH}_{3} \mathrm{I}$ ), which is difficult to remove with containment spray or filters, and is important for the evaluation of exposure. These topics have been investigated mainly in Europe ${ }^{3)}$. Ironically, it was a BWR that caused a reactor core meltdown in the accident at TEPCO's Fukushima Dai-ichi Nuclear Power Plant ${ }^{4}$. In the process of removing the debris from the reactor core, restoring the environment, identifying the causes and process of the reactor core meltdown, and studying the source terms in detail in the future, it is essential to understand the influence of $\mathrm{B}_{4} \mathrm{C}$ absorbers. Based on such future needs in Japan, this study was intended to summarize the results and leftover issues of existing typical experiments to examine chemical reactions involving $\mathrm{B}_{4} \mathrm{C}$ and the effect of $\mathrm{B}_{4} \mathrm{C}$ absorber materials on the reactor core melting process, and the comparison between $\mathrm{B}_{4} \mathrm{C}$ and S-I-C control rods.

\section{Chemical Reactions}

In a $\mathrm{B}_{4} \mathrm{C}$ oxidation process, similar to $\mathrm{Zr}$ oxidation, not only are large amounts of reaction heat and $\mathrm{H}_{2}$ generated, but also $\mathrm{CO}, \mathrm{CO}_{2}$, and $\mathrm{CH}_{4}$ that may influence the chemical forms of iodine. The main chemical reactions are listed along with the $\mathrm{Zr}$ oxidation reaction formula, as follows: ${ }^{5,6)}$

Oxidation of $\mathrm{B}_{4} \mathrm{C}^{5}$

$$
\mathrm{B}_{4} \mathrm{C}+7 \mathrm{H}_{2} \mathrm{O}(\mathrm{g}) \rightarrow 2 \mathrm{~B}_{2} \mathrm{O}_{3}+\mathrm{CO}(\mathrm{g})+7 \mathrm{H}_{2}(\mathrm{~g}) \quad \Delta \mathrm{H}=-738 \mathrm{~kJ} / \mathrm{mol}
$$




$$
\begin{array}{ll}
\mathrm{B}_{4} \mathrm{C}+8 \mathrm{H}_{2} \mathrm{O}(\mathrm{g}) \rightarrow 2 \mathrm{~B}_{2} \mathrm{O}_{3}+\mathrm{CO}_{2}(\mathrm{~g})+8 \mathrm{H}_{2}(\mathrm{~g}) & \Delta \mathrm{H}=-768 \mathrm{~kJ} / \mathrm{mol} \\
\mathrm{B}_{4} \mathrm{C}+6 \mathrm{H}_{2} \mathrm{O}(\mathrm{g}) \rightarrow 2 \mathrm{~B}_{2} \mathrm{O}_{3}+\mathrm{CH}_{4}(\mathrm{~g})+4 \mathrm{H}_{2}(\mathrm{~g}) & \Delta \mathrm{H}=-965 \mathrm{~kJ} / \mathrm{mol}
\end{array}
$$

Reactions related to boron oxide $\left(\mathrm{B}_{2} \mathrm{O}_{3}\right)^{5)}$

$$
\begin{array}{ll}
\mathrm{B}_{2} \mathrm{O}_{3}+\mathrm{H}_{2} \mathrm{O}(\mathrm{g}) \rightarrow 2 \mathrm{HBO}_{2} \text { (metaboric acid) } & \Delta \mathrm{H}=332 \mathrm{~kJ} / \mathrm{mol} \\
\mathrm{B}_{2} \mathrm{O}_{3}+3 \mathrm{H}_{2} \mathrm{O} \rightarrow 2 \mathrm{H}_{3} \mathrm{BO}_{3} \text { (orthoboric acid) } & \Delta \mathrm{H}=-40 \mathrm{~kJ} / \mathrm{mol} \\
\mathrm{B}_{2} \mathrm{O}_{3}(\mathrm{l}) \rightarrow \mathrm{B}_{2} \mathrm{O}_{3}(\mathrm{~g}) \quad \Delta \mathrm{H}=383 \mathrm{~kJ} / \mathrm{mol} &
\end{array}
$$

Reactions related to decomposition of $\left.\mathrm{CH}_{4}{ }^{6}\right)$

$$
\begin{aligned}
& \mathrm{CH}_{4}(\mathrm{~g})+\mathrm{H}_{2} \mathrm{O}(\mathrm{g}) \rightarrow \mathrm{CO}(\mathrm{g})+3 \mathrm{H}_{2}(\mathrm{~g}) \quad \Delta \mathrm{H}=228 \mathrm{~kJ} / \mathrm{mol} \\
& \mathrm{CH}_{4}(\mathrm{~g})+2 \mathrm{H}_{2} \mathrm{O}(\mathrm{g}) \rightarrow \mathrm{CO}_{2}(\mathrm{~g})+4 \mathrm{H}_{2}(\mathrm{~g}) \quad \Delta \mathrm{H}=197 \mathrm{~kJ} / \mathrm{mol}
\end{aligned}
$$

Oxidation of $\mathrm{Zr}$

$$
\mathrm{Zr}+2 \mathrm{H}_{2} \mathrm{O}(\text { steam }) \rightarrow \mathrm{ZrO}_{2}+2 \mathrm{H}_{2} \quad \Delta \mathrm{H}=-582 \mathrm{~kJ} / \mathrm{mol}
$$

where, the enthalpies of the reactions in formulas (1)-(9) were calculated using the formation enthalpy at $1,500 \mathrm{~K}$ in the cited literature ${ }^{7)}$.

In the process of $\mathrm{B}_{4} \mathrm{C}$ oxidation, similar to that of $\mathrm{Zr}$, a partial reaction starts at around $1,473 \mathrm{~K}$ and rapidly progresses to more than $1,543 \mathrm{~K}^{8)}$. Notably, the aforementioned chemical reaction formulas indicate that, relative to the oxidation of the same mass of $\mathrm{Zr}$ (density $6.53 \mathrm{~g}$ / $\mathrm{cm}^{3}$ ), the reaction heat from the oxidation of $\mathrm{B}_{4} \mathrm{C}$ (density $2.5 \mathrm{~g} / \mathrm{cm}^{3}$ ) is three to four times higher, and the quantity of hydrogen generated is six to seven times higher .

$\mathrm{CH}_{3} \mathrm{I}$ is believed to be generated from reactions between $\mathrm{CH}_{4}$ and $\mathrm{HI}$ or $\mathrm{I}_{2} \cdot \mathrm{CH}_{4}$ is generated by the oxidation of $\mathrm{B}_{4} \mathrm{C}$ as shown in formula (3) or generated by the reverse reactions (i.e., the reaction of $\mathrm{H}_{2}$ and $\mathrm{CO}$ or $\mathrm{CO}_{2}$ ) of formulas (7) and (8) in the low temperature nuclear reactor coolant system $^{9)}$. However, the detailed reaction processes involved are not well known ${ }^{10)}$. For instance, formulas (7) and (8), which describe the decomposition of $\mathrm{CH}_{4}$, were introduced as indicators of the small quantity of $\mathrm{CH}_{4}$ generation in a series of experiments conducted in Europe on the individual effects of $\mathrm{B}_{4} \mathrm{C}$ oxidation.

\section{Past Experimental Studies on the Influence of $\mathrm{B}_{4} \mathrm{C}$ Absorber Materials}

This section discusses the past experiments DF- $4^{11,12)}$ and XR2-1 ${ }^{13)}$, conducted by Sandia National Laboratories (SNL), USA on the control rod blades for a BWR, CORA-16 ${ }^{14}$ by Forschungszentrum Karlsruhe (FZK), Germany on the $\mathrm{B}_{4} \mathrm{C}$ pellet control rods for an EPR, and FPT- $3^{9,16-18)}$ and BECARRE ${ }^{19)}$ conducted by the Institut de Radioprotection et de Surete Nucleaire (IRSN), France.

\section{DF-4 Experiment (SNL) ${ }^{11,12)}$}

After the TMI-2 accident, the United States Nuclear Regulatory Commission (USNRC) conducted the DF-4 experiment in 1989 as the fourth in the series of damaged fuel (DF) experiments in the Severe Fuel Damage (SFD) Program enforced during the period from 1982 to 
1992 using the annular core research reactor (ACRR) of SNL. The purpose of this experiment was to acquire general information regarding the reactor core damage process in the initial stage of a severe BWR accident. The experimental core section consisted of 14 fuel rods $(0.5 \mathrm{~m}$ long), $\mathrm{B}_{4} \mathrm{C}$ (powder) control rods + simulated channel box (Figure 1), and the simulated fuel zone was heated by nuclear fission in a steam atmosphere. The measurements included the measurement of temperature by thermocouples (TC) and $\mathrm{H}_{2}$ concentration focusing on heat generation during $\mathrm{CuO}$ reduction.

One of the major outcomes was the observation of melting of stainless steel at a temperature below the melting point of stainless steel due to a eutectic reaction with $\mathrm{B}_{4} \mathrm{C}$, and the relocation of $\mathrm{B}_{4} \mathrm{C}$ to a lower position as a result of being captured in the molten stainless steel, and a chemical reaction involving $\mathrm{B}_{4} \mathrm{C}$ mainly occurred at this lower position with Cs (alkali metal), $\mathrm{Sr}$ (alkali earth metal), and other elements. No reaction with iodine was observed before the relocation.

However, because the experimental core section was made on a small scale, further verification was required in order to apply the acquired results to real reactors.

\section{XR2-1 Experiment (SNL) ${ }^{13)}$}

The USNRC conducted an XR2-1 experiment in 1996 using an apparatus installed in an experimental vessel of the Fully Instrumented Test Site (FITS) as the third in a series of Ex-Reactor (XR) experiments. When a short-term station blackout occurs while a depressurized condition exists in the reactor coolant system of a BWR, the reactor core is expected to be in a dry core condition (i.e., the coolant remains in the bottom part of the reactor pressure vessel and only a small quantity of steam passes through the reactor core). The objective of this experiment was to investigate the reactor core melting process under such a condition. Note that, in the TMI-2 accident in which the reactor coolant system was not depressurized, it was reported that the reactor core bottom was flooded, and because of the wet core conditions, the cooling water was boiling due to the decay heat, which meant that a large quantity of steam was continuously supplied to the upper part of reactor core. As a result, a metal crust was generated at lower part of the reactor core, and the molten substances were maintained on the crust.

In the experiment, as shown in Figure 2 and Figure 3, from the upper part of the testing section, which consisted of 64 fuel rods $\left(0.5 \mathrm{~m}\right.$ long) and $\mathrm{B}_{4} \mathrm{C}$ (powder) control rods, molten substances generated by induction heating of wire $\mathrm{Zr}$ and stainless steel- $\mathrm{B}_{4} \mathrm{C}$ were dropped.

The main result was that the type of large-scale metal crust found in the TMI- 2 accident was not generated under the fuel rods, and the molten substances continuously dropped to the

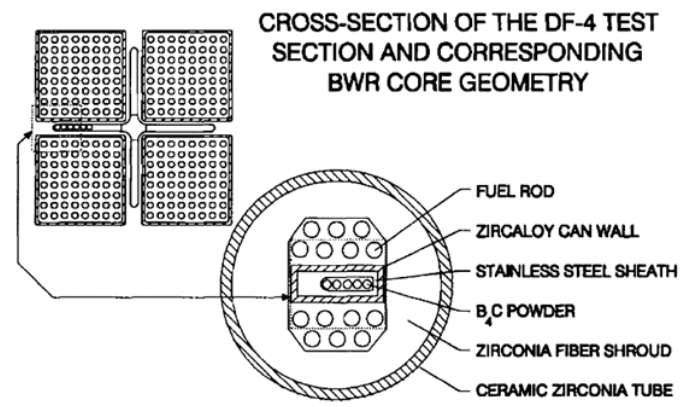

Figure 1 Cross-section of DF-4 test section and corresponding BWR core geometry ${ }^{11}$ 


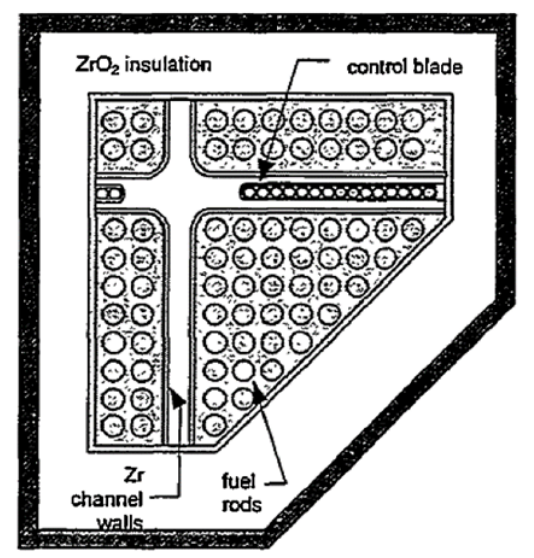

Figure 2 Cross-section of simulated reactor core of XR-2 test ${ }^{13)}$

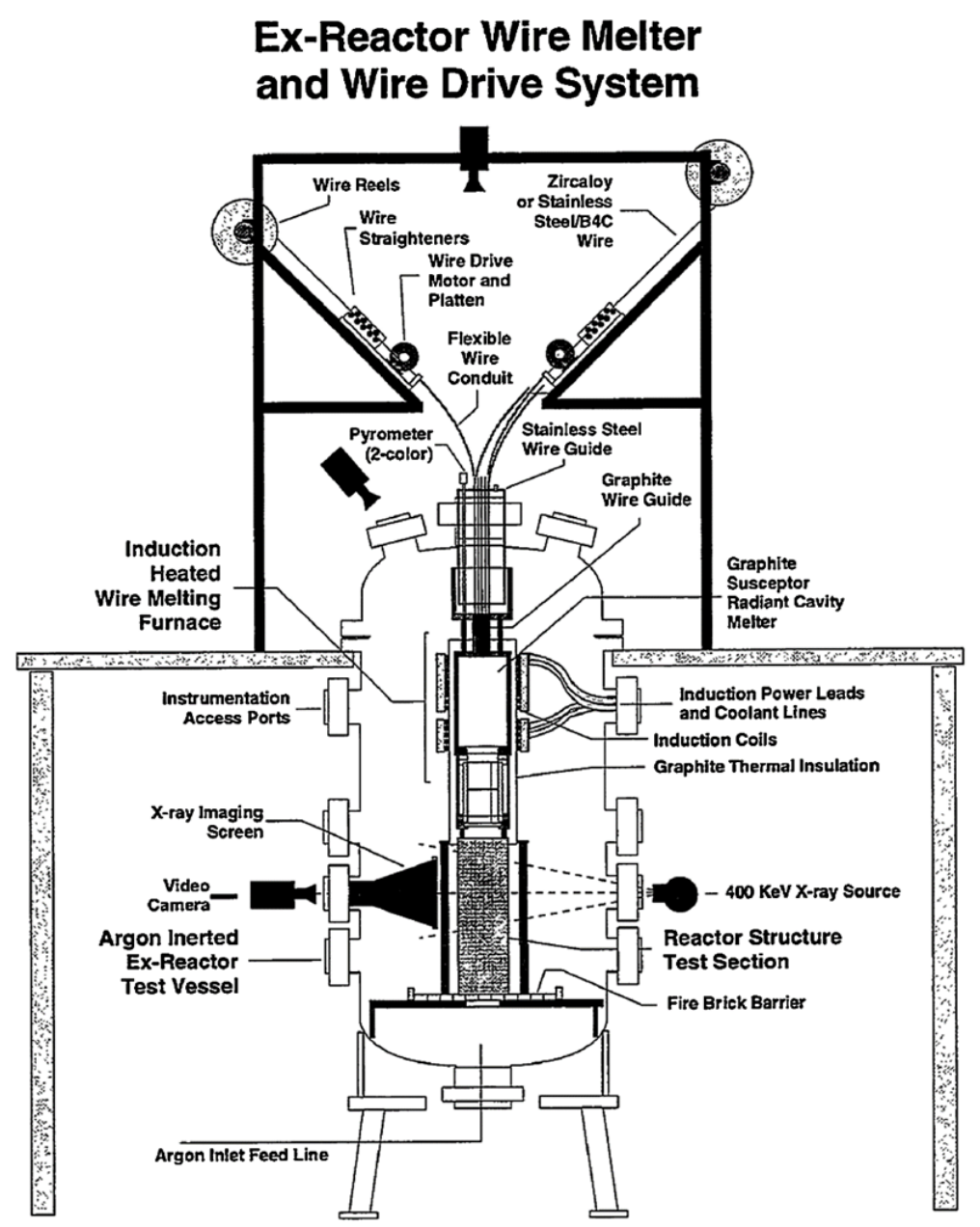

Figure 3 XR-2 experimental device installed using existing FITS (Fully Instrumented Test Site) experiment vessel $^{13)}$ 
lower plenum section through the gaps between the existing structures. However, some minor blockages were caused by the molten substances. The test apparatus had a temperature gradient of $2,000 \mathrm{~K} / \mathrm{m}$ in the vertical direction, and it was noted that the results might vary if the experimental conditions were changed.

Additionally, the experimental results were analyzed using the MERIS ${ }^{20)}$ reactor core melting process analysis code that was under development at SNL, which predicted the clogged flow path in the reactor core zone with the molten materials but could not predict molten materials dropping down into the lower plenum section. This unsolved issue was reserved as a subject for future study.

\section{CORA-16 Experiment (KfK) ${ }^{14)}$}

The Nuclear Research Center Karlsruhe (KfK, presently FZK) in Germany started the PSF Program (nuclear safety research program) in January 1992 to evaluate the effectiveness of severe accident management devices that were to be installed in the EPR. In this program, the CORA experiment (Ex-Reactor experiment), to investigate the damage and melting processes of fuel assemblies, and the BETA experiment that examined the molten core concrete interaction, were performed.

The test zone of the CORA experiment consisted of simulated fuel rods implemented with an electric heater with a $1 \mathrm{~m}$ long heating zone, simulated fuel rods (not heated), and control rods. The main achievements of the CORA experiment were the discovery of "candling" during the heating process, the occurrence of the acceleration of oxidation of the upper fuel cladding tube in the reflooding process when the immediate prior condition was under steam starvation, and the increased release of radioactive materials due to fuel smashing during reflooding.

The influence of the BWR control rods was investigated in the CORA-16 experiment. The main findings comprised the discovery of melting of $\mathrm{B}_{4} \mathrm{C}$ through a eutectic reaction with stainless steel at around $1,500 \mathrm{~K}$, the inhibition of the reaction of $\mathrm{B}_{4} \mathrm{C}$ by steam, and the generation of $\mathrm{HBO}_{2}$ due to the reaction of the molten substances with the fuel cladding tube after penetrating the channel box. However, it was understood that further verification was necessary because $\mathrm{B}_{4} \mathrm{C}$ caused melting by means of a eutectic reaction with zircalloy at over 1,920 K.

\section{QUENCH-07 and QUENCH-09 Experiments (FZK) ${ }^{5,15)}$}

FZK Germany conducted the QUENCH experiments as a follow-up to the CORA experiments to investigate the generation behavior of $\mathrm{H}_{2}$ during the reflooding of the fuel rods. The front view of the experimental device is shown in Figure 4. The simulated reactor core consists of 20 simulated fuel rods (approximately $1 \mathrm{~m}$ long) and a fuel rod or a control rod positioned in the center (Figure 5).

In the experiment, the reflooding condition was simulated by injecting water or steam from the area at the bottom of the fuel rods heated up to $2,000 \mathrm{~K}$ in advance. The simulated control rods used for the QUENCH-07 and QUENCH-09 experiments (conducted in 2001 and 2002, respectively) were $\mathrm{B}_{4} \mathrm{C}$ pellets within stainless steel-clad tubes and zircalloy- 4 guide tubes.

The experiments confirmed that molten boron, carbon, and $\mathrm{Zr}$ were generated at a lower temperature $(1,520 \mathrm{~K})$ than the melting point of $\mathrm{Zr}(2,030 \mathrm{~K})$ through a eutectic reaction between $\mathrm{B}_{4} \mathrm{C}$ and $\mathrm{Zr}$, and the melting of the reactor core was accelerated compared to the experiments conducted with S-I-C control rods (Figure 6). Furthermore, it was confirmed that when the saturated steam was injected from the bottom zone of the fuel rods, $\mathrm{H}_{2}, \mathrm{CO}, \mathrm{CO}_{2}$, and $\mathrm{CH}_{4}$ were 


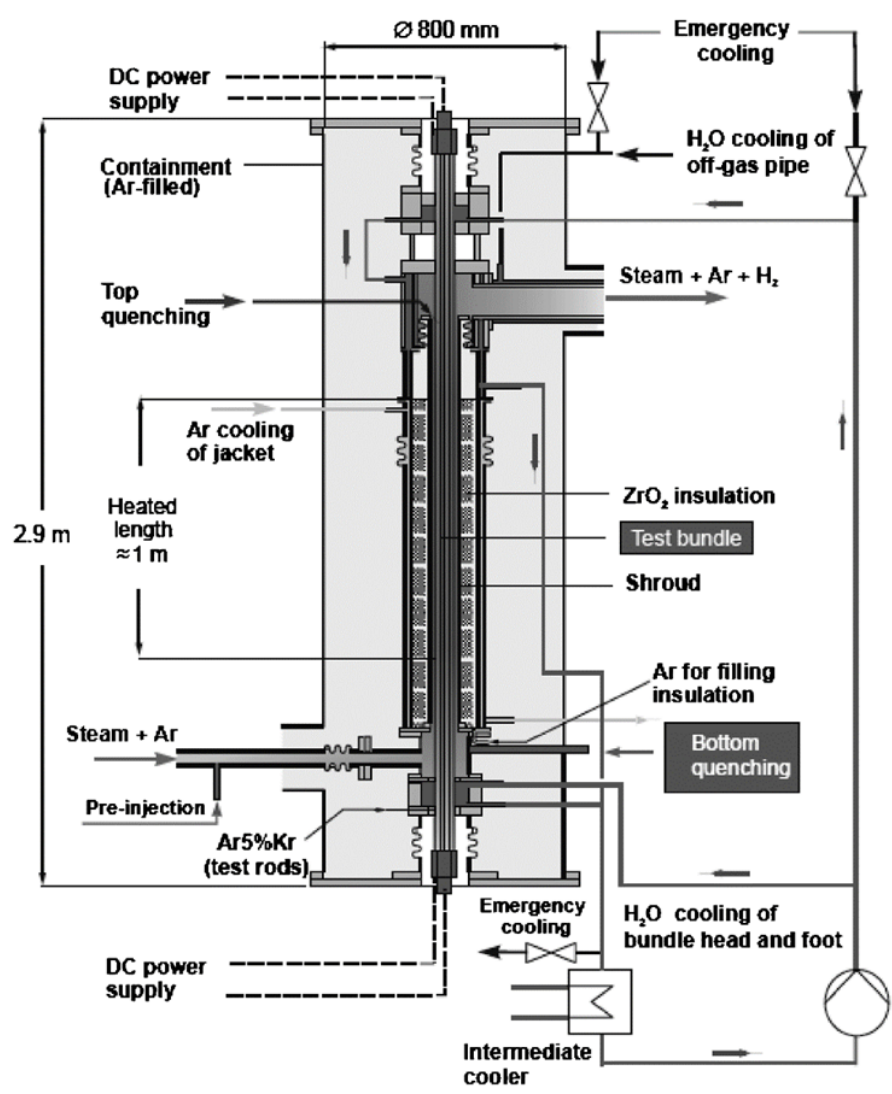

Figure 4 Front view of QUENCH experimental device ${ }^{15)}$

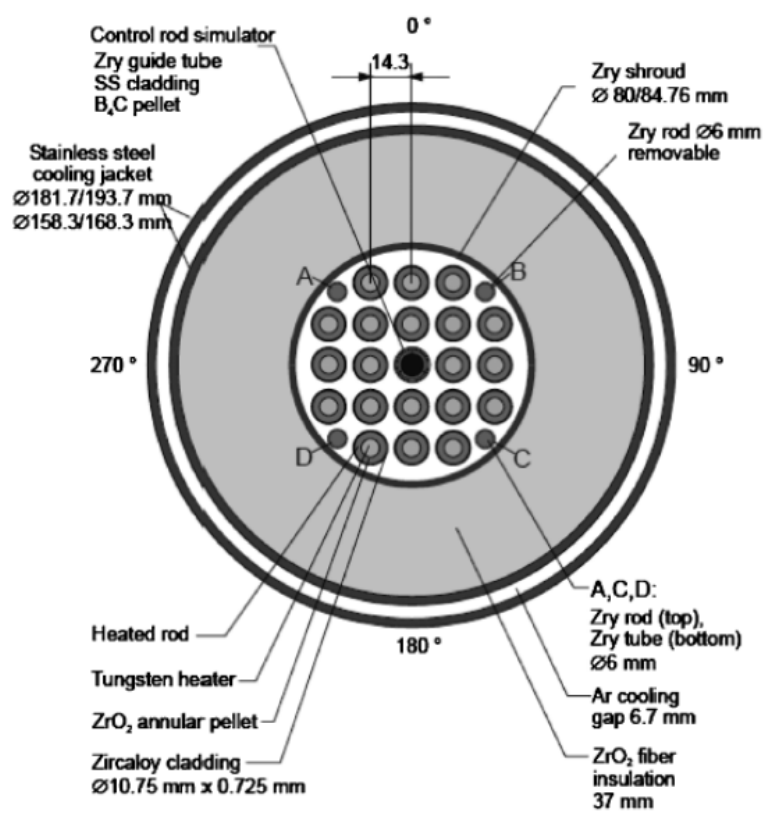

Figure 5 Cross-section of simulated reactor core for QUENCH experiment ${ }^{15}$ ) 

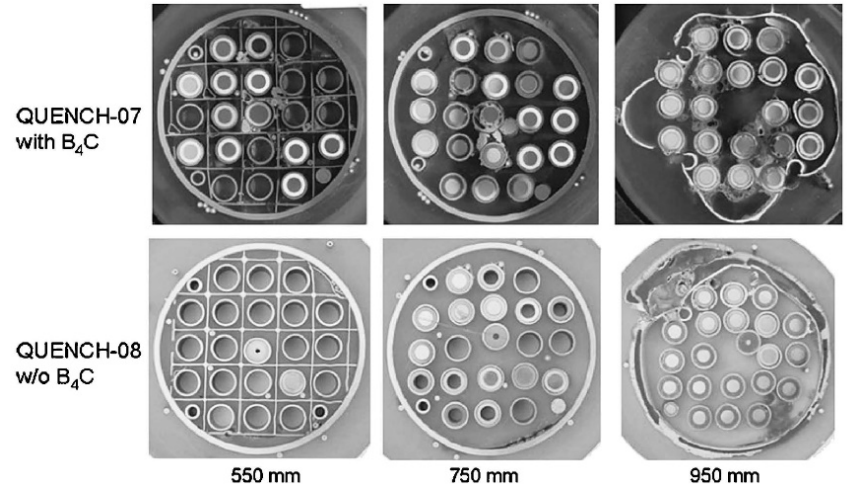

Figure 6 Comparison of cross-sections of simulated reactor core after QUENCH heating experiment between for presence and absence of $\mathrm{B}_{4} \mathrm{C}^{9)}$

generated due to the oxidation of molten substances. However, although only a small amount of $\mathrm{CH}_{4}$ was generated in this experiment, it was noted that $\mathrm{CH}_{4}$ could be generated by the reaction of $\mathrm{CO}$ or $\mathrm{CO}_{2}$ with $\mathrm{H}_{2}$ at lower temperatures in the piping of the reactor coolant system ${ }^{5)}$.

The oxidation ratio in the QUENCH-07 and QUENCH-09 experiments was 20\% and 50\%, respectively, and the contribution rate to the total quantity of $\mathrm{H}_{2}$ generation was $2.4 \%$ and $2.2 \%$, respectively. The quantity of $\mathrm{H}_{2}$ generated was $0.4 \mathrm{~kg}$ from QUENCH-09 and $0.12 \mathrm{~kg}$ from QUENCH-07; the reason for the difference was due to the steam starvation that was simulated by reducing the steam flow to the test zone from $50 \mathrm{~g} / \mathrm{s}$ to $3.3-0.4 \mathrm{~g} / \mathrm{s}$ for $11 \mathrm{~min}$ before reflooding in the QUENCH-09 experiment, while this reduction did not occur in the QUENCH-07 experiment ${ }^{5)}$. A plausible explanation for the increase in hydrogen generation is that, when the oxidized fuel rod is left in a condition where the steam supply is limited, the $\mathrm{ZrO}_{2}$ layer surrounding the cladding tube decreases and becomes $\mathrm{Zr}$ because of the reduction, which allows oxygen to be captured easily when the steam supply resumes ${ }^{5)}$.

\section{FPT-3 Experiment (IRSN) ${ }^{9,16-18)}$}

The FPT-3 experiment was conducted within the framework of the international severe accident research program PHEBUS/FP in the Cadarache Research Center at the initiative of IRSN/France and the European Community (EC). The goal was to investigate the melting of fuel, release of radioactive materials from fuel, and transportation of radioactive materials in the reactor coolant system and containment.

In the PHEBUS/FP experiment, 20 fuel rods ( $1 \mathrm{~m} \mathrm{long}$ ) and one simulated control rod were heated by nuclear fission in the PHEBUS reactor, and the released radioactive materials were captured in a steam generator or containment vessel that simulated a 900 MWe PWR at a 1/5,000 scale. The cross-section and front view of the simulated reactor core are shown in Figure 7, and the experiment matrix is shown in Table 1.

The FPT-3 experiment aimed at investigating the influence of $\mathrm{B}_{4} \mathrm{C}$ pellet control rods used in EPR and was conducted in 2004 as the fifth and final experiment in the same program. The main finding was the observation of a significant increase in the gaseous (organic) iodine content in the simulated containment vessel compared to other PHEBUS/FP experiments that used S-I-C control rods. The organic iodine $\left(\mathrm{CH}_{3} \mathrm{I}\right)$ content in the gaseous iodine in the simulated containment vessel was approximately $5 \%$ when the fuel was damaged, the maximum content was approximately $30 \%$, but was kept within $10-20 \%$ for an extended period afterwards 


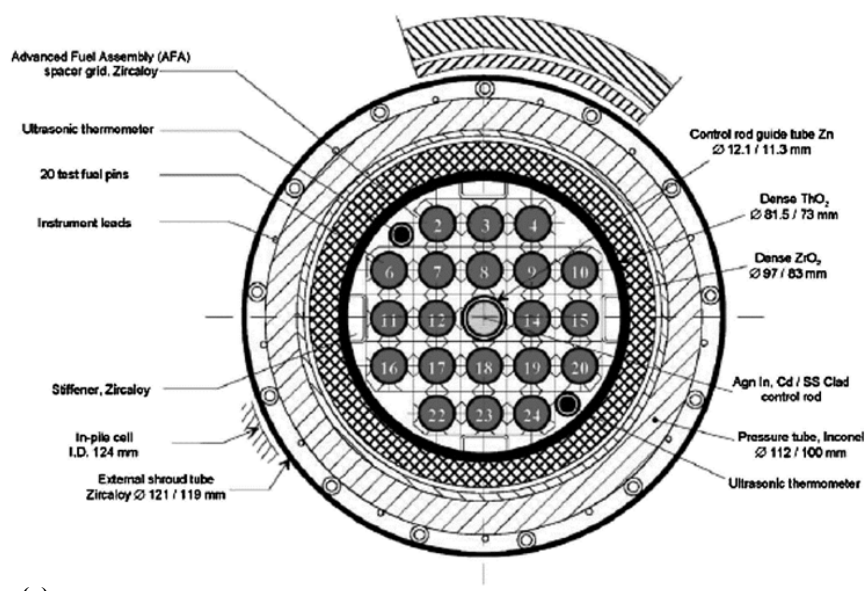

(a)

Figure 7 Cross-section and front view of simulated reactor core for PHEBUS/FP experiment ${ }^{16)}$

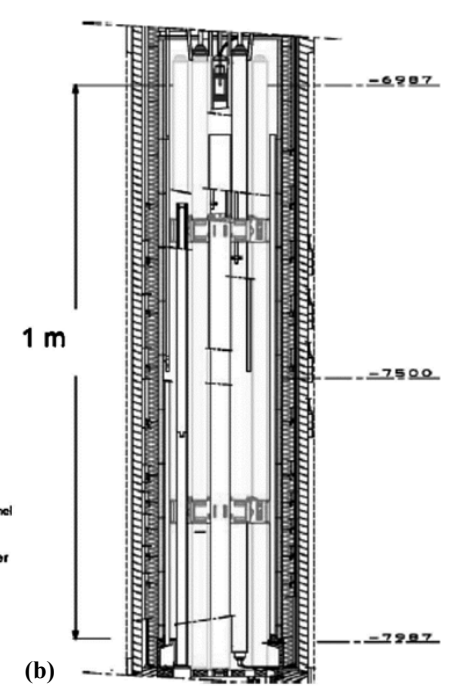

Table 1 PHEBUS/FP experiment matrix

\begin{tabular}{|c|c|c|c|c|c|}
\hline & $\begin{array}{l}\text { Fresh Fuel + Irradiated Fuel } \\
+ \text { Number of Control Rods }\end{array}$ & $\begin{array}{c}\text { Fuel } \\
\text { Burnup } \\
(\mathrm{GWd} / \mathrm{tU})\end{array}$ & Atmosphere & $\begin{array}{l}\text { Containment Sump } \\
\text { Water Condition }\end{array}$ & Date of Test \\
\hline FPT-0 & $20+0+1 S-I-C$ & 0 & Steam & pH 5, Not Boiling & $12 / 2,1993$ \\
\hline FPT-1 & $2+18+1$ S-I-C & $\sim 23$ & Steam & pH 5, Not Boiling & $7 / 26,1996$ \\
\hline FPT-2 & $2+18+1 \mathrm{~S}-\mathrm{I}-\mathrm{C}$ & $\sim 32$ & $\begin{array}{l}\text { Reductive Atmosphere } \\
+ \text { Boric Acid }\end{array}$ & pH 9, Boiling & $10 / 12,2000$ \\
\hline FPT-3 & $2+18+1 \mathrm{~B}_{4} \mathrm{C}$ & $\sim 24$ & Steam & pH 5, Boiling & $11 / 18,2004$ \\
\hline FPT-4 & $\begin{array}{l}\text { Debris bed } \\
\text { without control rod }\end{array}$ & $\sim 38$ & Steam $+\mathrm{H}_{2}$ & - & 7/22, 1999 \\
\hline
\end{tabular}

S-I-C: silver indium cadmium

(Figure 8). Based on a calculation using the SOPHAEROS ${ }^{21)}$ thermodynamic chemical equilibrium computation code, an increase in gaseous iodine was predicted. Furthermore, a clogged flow path was observed in the upstream of the simulated steam generator tube during the FPT-3 experiment, but it is highly probable that this was caused by the generation of cesium boric acid $\left(\mathrm{CsBO}_{2}\right)$ and deposition in the tube ${ }^{9)}$.

\section{BECARRE Experiment (IRSN) ${ }^{19)}$}

The BECARRE experiment was conducted during the period from 2005 to 2010 within the framework of IRSN's International Source Term Program (ISTP) with the objective of investigating the oxidation of $\mathrm{B}_{4} \mathrm{C}$ pellets or $\mathrm{B}_{4} \mathrm{C}$-stainless steel eutectic molten material, the collapse process of $\mathrm{B}_{4} \mathrm{C}$ control rods, and the challenges faced during the FPT-3 experiment (generation of $\mathrm{CH}_{4}$ due to oxidation of $\mathrm{B}_{4} \mathrm{C}$ ).

The test zone of the BECARRE experimental device is shown in Figure 9. In this experiment, the $0.6 \mathrm{~m}$ long an array of $\mathrm{B}_{4} \mathrm{C}$ pellets in a stainless steel cladding tube and zircalloy control rod guide tube were heated up to $1,953 \mathrm{~K}$.

The main findings were the increase in $\mathrm{CO}$ and $\mathrm{CO}_{2}$ generation as a result of the oxidation 
Table 2 Comparison of measurements and SOPHAEROS analyses on the percentage of gaseous iodine (to the quantity released from fuel) in simulated containment vessel in PHEBUS/FP experiment ${ }^{18)}$

\begin{tabular}{|c|c|c|}
\hline & Basic analysis & Experiment \\
\hline \multicolumn{3}{|c|}{ Total volatile iodine $(\% \text { i.b.i. })^{a)}$} \\
\hline FPT-0 & $\sim 2.6$ & $\leq 2$ \\
\hline FPT-1 & $\sim 0.052$ & $<1$ \\
\hline FPT-2-case $\mathrm{A}^{\mathrm{b})}$ & $\sim 0.03$ & $<0.1$ \\
\hline FPT-2-case $\mathrm{B}^{\mathrm{b})}$ & $\sim 9$ & \\
\hline FPT-3 & $\sim 18$ & $\sim 27$ \\
\hline
\end{tabular}

a) Released from the bundle, which reaches the containment vessel in gaseous (as $\mathrm{HI}$ ) and vapor form (as $\mathrm{SnI}_{2}, \mathrm{SnI}_{4}, \mathrm{I}_{2} \mathrm{MoO}_{2}$ or others).

${ }^{b)}$ Cases $\mathrm{A}$ and $\mathrm{B}$ correspond respectively to assumed non-limited and limited $\mathrm{Cd}$ release kinetics.

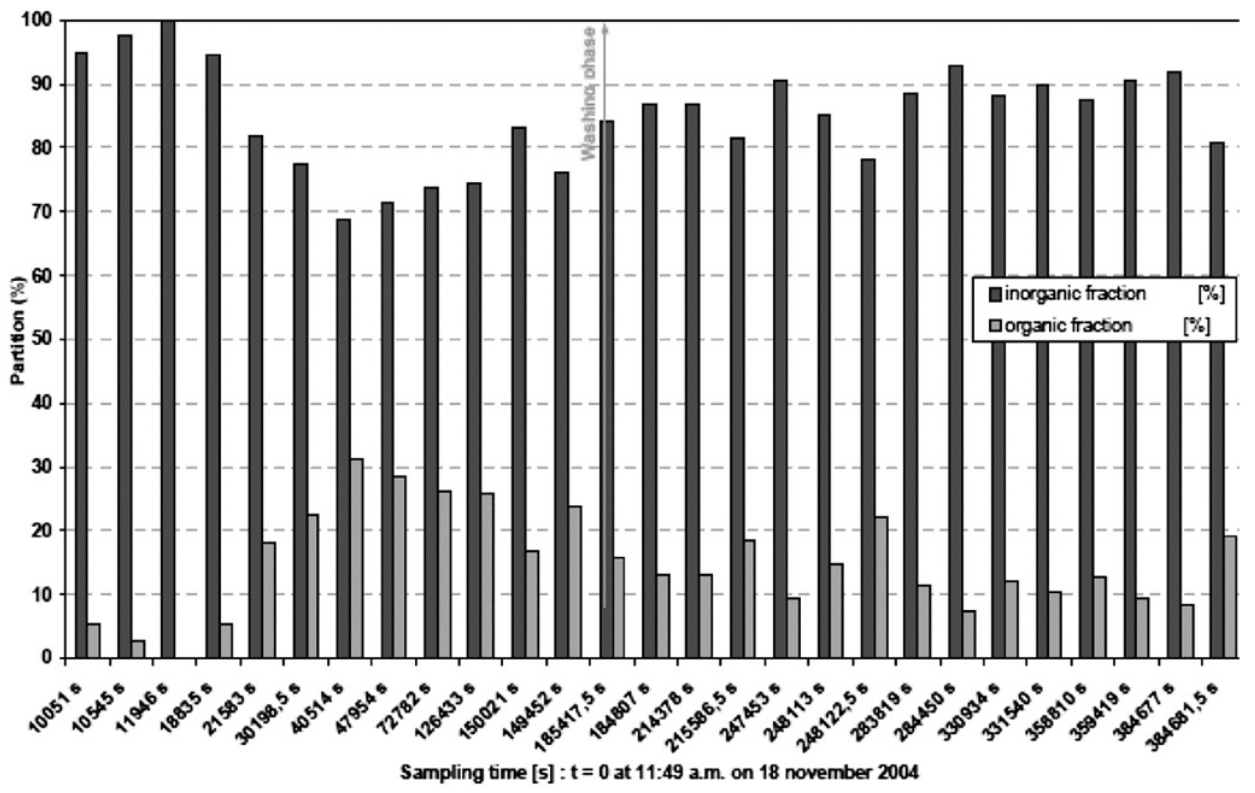

Figure 8 Time-dependent changes in percentage of inorganic iodine and organic iodine observed in simulated containment vessel of FPT-3 experiment ${ }^{17}$ )

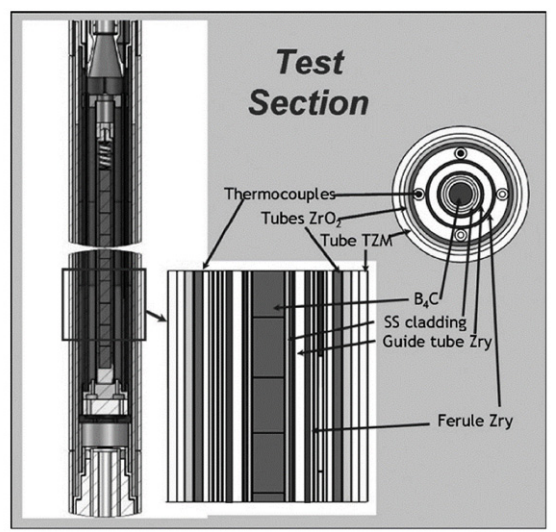

Figure 9 Test zone of BECARRE experimental device ${ }^{19)}$ 
of $\mathrm{B}_{4} \mathrm{C}$ and the decrease in $\mathrm{H}_{2}$ generation due to the consumption of oxygen by $\mathrm{B}_{4} \mathrm{C}$. However, almost no $\mathrm{CH}_{4}$ was observed. A study based on the thermochemical equilibrium theory for this outcome found that $\mathrm{CH}_{4}$ generation hardly occurs under the low pressure conditions ( 2 bar) that existed during the BECARRE experiment, but occurs readily at temperatures and pressures exceeding $1,500 \mathrm{~K}$ and 100 bar, respectively ${ }^{19}$, which supported the very low $\mathrm{CH}_{4}$ generation observed in the experiment.

Concerning the oxidation of $\mathrm{B}_{4} \mathrm{C}$, the low viscosity molten materials formed by the eutectic reaction between $\mathrm{B}_{4} \mathrm{C}$ and the stainless steel cladding flowed down between the inner surface of the stainless steel (SS) cladding tube and outer surface of the $\mathrm{B}_{4} \mathrm{C}$ pellets, and solidified at the lower part of the cladding tube where the temperature was 1,073-1,173 K. As a result, not much unoxidized $\mathrm{B}_{4} \mathrm{C}$ located above the solidification point was oxidized. In addition, the $\mathrm{Zr}$ control rod guide tube that surrounds the SS cladding obstructed contact between unoxidized $\mathrm{B}_{4} \mathrm{C}$ and steam. Consequently, hydrogen generation by $\mathrm{B}_{4} \mathrm{C}$ oxidation was limited ${ }^{19)}$. A photo of the radial cross-section after the heating experiment on the test zone of the BECARRE experimental device is shown in Figure 10.

\section{Other Individual Effects Tests}

FZK Germany conducted QUENCH-07 and QUENCH-09 experiments, and the Boron Carbide Oxidation (BOX) experiment during the period from 1998 to 2002 to investigate the oxidation behavior of $\mathrm{B}_{4} \mathrm{C}$ under high temperatures in the COLOSS (core loss during a severe accident) program ${ }^{22}$, conducted in support of the FPT-3 experiments at the initiative of IRSN as an EC project ${ }^{8,23)}$.

In the experiment, the $\mathrm{B}_{4} \mathrm{C}$ specimens (pellets fabricated by FRAMATOME/France, CODEX/Hungary, ESK/Germany, and powder by ESK/Germany) were heated in an inert atmosphere, and the generated gases $\left(\mathrm{H}_{2}, \mathrm{CO}, \mathrm{CO}_{2}\right.$, and $\left.\mathrm{CH}_{4}\right)$ were measured by supplying a steam flow after the target temperature was reached. The quantity of generation was in the order of $\mathrm{H}_{2}$ $>\mathrm{CO}_{2}>\mathrm{CO}>\mathrm{CH}_{4}$. More gas was generated from the powder than from the pellets. $\mathrm{H}_{2}$ generation increased in proportion to the temperature in the range of $800-1,400^{\circ} \mathrm{C}$, and conversely, $\mathrm{CH}_{4}$ generation reached maximum at $800^{\circ} \mathrm{C}$. According to the thermodynamic equilibrium

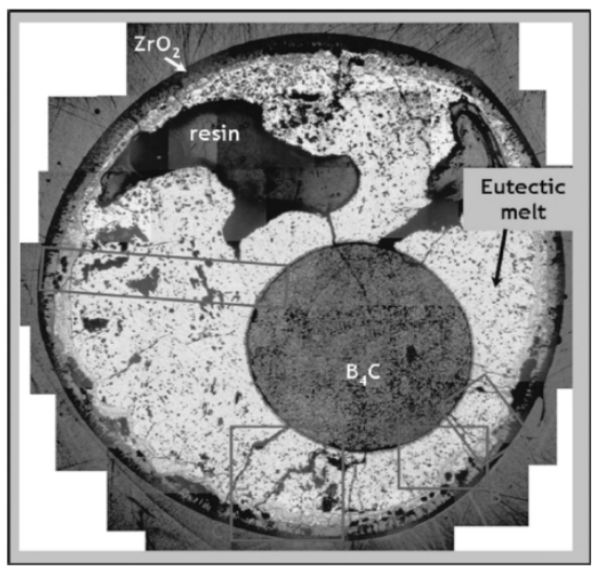

Figure 10 Photo of radial cross-section after heating experiment at $31.1 \mathrm{~cm}$ in height from bottom of BECARRE experiment testing zone (areas surrounded by square frame are locations of EPMA analysis) ${ }^{19)}$ 
calculation, $\mathrm{CH}_{4}$ generation accelerates at temperatures below $700^{\circ} \mathrm{C}$, while $\mathrm{CO}$ and $\mathrm{CO}_{2}$ generation are predominant at temperatures above this value. The transition temperature from $\mathrm{CH}_{4}$ generation predominant to $\mathrm{CO}+\mathrm{CO}_{2}$ generation predominant was dependent on the oxygen potential, and it shifted toward the high temperature side as the $\mathrm{H}_{2}$ concentration increased. Furthermore, the generation of gaseous $\mathrm{HBO}_{2}$ and $\mathrm{B}_{2} \mathrm{O}_{3}$ increased at temperatures over $1,200^{\circ} \mathrm{C}$.

Nagase et al. of the former Japan Atomic Energy Research Institute (JAERI) conducted experimental research on the eutectic reaction between $\mathrm{B}_{4} \mathrm{C}$ in the form of powder or pellets and stainless steel (SUS304) in the temperature range of 1,073-1,623 $\mathrm{K}^{24)}$. They discovered that the reaction speed of $\mathrm{B}_{4} \mathrm{C}(\mathrm{MP} 2,620 \mathrm{~K}$ ) and SUS304 (MP 1,673-1,723 K) approximately followed the Arrhenius type of reaction. The reaction speed between 1,473 $\mathrm{K}$ and 1,498 $\mathrm{K}$ was noncontiguous due to the formation of a liquid layer on the contacting surface of $\mathrm{B}_{4} \mathrm{C}$ and SUS304, and liquification was significantly accelerated near the contacting surface above $1,498 \mathrm{~K}$. In addition, the reaction speed of powder $\mathrm{B}_{4} \mathrm{C}$ was higher than that of pellet $\mathrm{B}_{4} \mathrm{C}$.

Furthermore, Minato conducted a thermodynamic chemical equilibrium calculation on the chemical form of iodine and $\mathrm{Cs}$ in the reactor cooling system under severe accident conditions and predicted $\mathrm{CsBO}_{2}$ generation ${ }^{25)}$. In the Wide Range Piping Integrity Demonstration (WIND) study, Shibazaki et al. confirmed by experiment that CsI changes to $\mathrm{CsBO}_{2}$, which has a slightly lower vapor pressure than CsI in the presence of boric acid, and the amount of deposition in the pipe increased compared to the experiment conducted in the absence of boric acid ${ }^{26}$.

\section{Experimental Research on Influence of S-I-C Control Materials}

In the case of S-I-C absorbers, there is a high possibility that, in the event of a severe accident, the Cd (MT $594 \mathrm{~K}$, BT 1,040 K) gasifies and In (MT $430 \mathrm{~K}$, BT 2,346 K) liquifies before fracture of the cladding tube of the control rod, and when the cladding tube of the control rods fractures $(1,550-1,620 \mathrm{~K})$, these elements will blow out as a liquid aerosol ${ }^{2}$. Ag (MT 1,235 K, BT $2,483 \mathrm{~K}$ ) is considered to be released a little later due to a slightly higher melting point ${ }^{2}$. Figure 11 shows a picture of the blown out S-I-C that solidified in the bottom zone of the simulated fuel bundle after a fracture of the cladding tube of the S-I-C control rod (observed in QUENCH-13 experiment). It should be noted that the particles of $\mathrm{Ag}$, In, and $\mathrm{Cd}$ that were released in the reactor as aerosols captured the radioactive material and increased deposition on the reactor cooling system piping.

Regarding the relationship with iodine, it was explained that $\mathrm{Cd}$ changed to $\mathrm{CdI}_{2}$ and

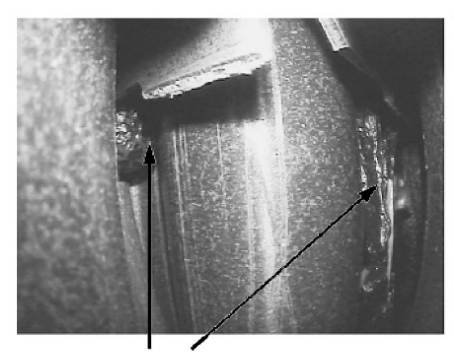

$550 \mathrm{~mm}$, AIC melt at lower edge of SG 3

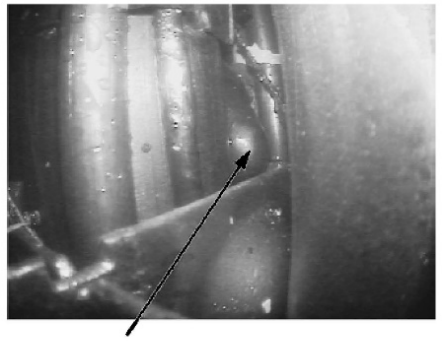

$-50 \mathrm{~mm}$, AIC melt at upper edge of SG1

Figure 11 View of blown out Ag, In, and Cd by fracture of cladding tube for S-I-C control rod solidified in the bottom of simulated fuel bundle (observed in QUENCH-13 experiment) ${ }^{2)}$ 
condensed in the heat transfer tube of the steam generator. In addition, Ag formed insoluble $\mathrm{AgI}$ and inhibited the revolatilization of iodine from the containment sump water.

Additionally, Mo that was generated through nuclear fission formed $\mathrm{Cs}_{2} \mathrm{MoO}_{4}$ first, and then increased the amount of Cs deposition in the pipe. Furthermore, the possibility of generating $\mathrm{CsOH}$, which was previously considered to be a typical chemical form of Cs, was indicated by the reaction of $\mathrm{Cs}_{2} \mathrm{MoO}_{4}$ with water ${ }^{27}$. On the other hand, in the case of the $\mathrm{B}_{4} \mathrm{C}$ control rod, it was noted that not much $\mathrm{Cs}_{2} \mathrm{MoO}_{4}$ was generated due to consumption by the generation of $\mathrm{CsBO}_{2}{ }^{18}$ ).

A comparison of the influences of S-I-C and $\mathrm{B}_{4} \mathrm{C}$ absorbers on the reactor melting process investigated in sections III and IV are listed in Table $\mathbf{3}$.

\section{Discussion}

\section{Weight Ratio of $\mathrm{B}_{4} \mathrm{C}$ and Stainless Steel}

According to the DF-4 experiment, most of the $\mathrm{B}_{4} \mathrm{C}$ relocated to the lower part of reactor core without being oxidized, and no reaction with iodine was observed before the relocation. This is considered to be due to the relocation of $\mathrm{B}_{4} \mathrm{C}$ from a high temperature zone to a low temperature zone by being captured by the molten stainless steel after the complete melting of $\mathrm{B}_{4} \mathrm{C}$ due to a eutectic reaction with stainless steel. In the reactor core of a BWR, the ratio of stainless steel to $\mathrm{B}_{4} \mathrm{C}$ is high. Therefore, it is highly probable that the generation of a large amount of organic iodine that was observed in the FPT- 3 experiment, in which the $\mathrm{B}_{4} \mathrm{C}$ control rod was simulated, did not take place.

The weight ratio of $\mathrm{B}_{4} \mathrm{C}$ to stainless steel could be an important factor in the process of collapse of pellet-type $\mathrm{B}_{4} \mathrm{C}$ or powder-type $\mathrm{B}_{4} \mathrm{C}$ control rod blades. The ratio of stainless steel (density $7.8 \mathrm{~g} / \mathrm{cm}^{3}$ ) to $\mathrm{B}_{4} \mathrm{C}\left(\right.$ density $2.5 \mathrm{~g} / \mathrm{cm}^{3}$ ) is approximately 3.5 (volumes of the two are almost equal) in the FPT-3 experiment and EPR, while that of BWR5/GE is approximately 10 . Where the percentage of stainless steel is high, based on the experience in the DF-4 experiment, most of the $\mathrm{B}_{4} \mathrm{C}$ will melt into the stainless steel (due to the eutectic reaction) and will be relocated to the low temperature zone in the lower part of the reactor keeping these conditions. Where the

Table 3 Comparison of influence of S-I-C and $\mathrm{B}_{4} \mathrm{C}$ absorbers on reactor melting process

\begin{tabular}{|c|c|c|}
\hline & PWR (S-I-C Control Rod) & BWR ( B $_{4}$ C Control Rod) \\
\hline $\begin{array}{l}\text { Failure Temperature } \\
\text { of Control Rod }\end{array}$ & $1,550-1,620 \mathrm{~K}$ & $1,520 \mathrm{~K}$ \\
\hline $\begin{array}{l}\text { Behavior of absorbers } \\
\text { immediately after } \\
\text { failure }\end{array}$ & $\begin{array}{l}\text { Cd (MT } 594 \mathrm{~K}, \mathrm{BT} 1,040 \mathrm{~K} \text { ) gasifies and In (MT } \\
430 \mathrm{~K} \text {, BT 2,346 K) liquifies inside before failure } \\
\text { of the cladding tube of control rod and blows out } \\
\text { as an aerosol when the cladded tube of the control } \\
\text { rods failures. Ag (MT } 1,235 \mathrm{~K} \text {, BT } 2,483 \mathrm{~K} \text { ) is } \\
\text { released a little later. }\end{array}$ & $\begin{array}{l}\text { The control rods, consisting of } \mathrm{B}_{4} \mathrm{C}(\mathrm{MT} 2,620 \mathrm{~K}) \\
\text { and stainless steel (MT } 1,673-1,723 \mathrm{~K}) \text {, liquify } \\
\text { at a temperature lower than the melting points } \\
\text { due to the eutectic reaction and start to collapse. } \\
\text { The weight ratio of } \mathrm{B}_{4} \mathrm{C} \text { to stainless steel varies } \\
\text { depending on whether } \mathrm{B}_{4} \mathrm{C} \text { is in powder or pellet } \\
\text { form, and } \mathrm{B}_{4} \mathrm{C} \text { oxidation is influenced by steam. }\end{array}$ \\
\hline $\begin{array}{l}\text { Influence on source } \\
\text { terms }\end{array}$ & $\begin{array}{l}\mathrm{Ag}, \mathrm{In} \text {, and } \mathrm{Cd} \text { are released into the reactor in the } \\
\text { form of aerosols, the particles grow by capturing } \\
\mathrm{FP} \text { and accelerate the deposition in the cooling } \\
\text { system piping. In relation to iodine, } \mathrm{Cd} \text { changes } \\
\text { its chemical form to } \mathrm{CdI}_{2} \text { and condenses in the } \\
\text { steam generator tubes. } \mathrm{Ag} \text { forms insoluble } \mathrm{AgI} \\
\text { and inhibits the generation of volatile iodine in } \\
\text { the containment vessel. }\end{array}$ & $\begin{array}{l}\mathrm{HBO}_{2} \text { generated by the oxidation of } \mathrm{B}_{4} \mathrm{C} \\
\text { combines with } \mathrm{Cs} \text { and forms } \mathrm{CsBO}_{2} \text {, which } \\
\text { increases the quantity of } \mathrm{Cs} \text { deposition. } \\
\text { Furthermore, } \mathrm{CH}_{4} \text { generated through oxidation } \\
\text { of } \mathrm{B}_{4} \mathrm{C} \text { or the reaction of } \mathrm{H}_{2} \text { with } \mathrm{CO} \text { or } \mathrm{CO}_{2} \\
\text { reacts with } \mathrm{HI} \text { or } \mathrm{I}_{2} \text { and forms organic iodine } \\
\left(\mathrm{CH}_{3} \mathrm{I}\right) \text {, which increases the source terms (one of } \\
\text { the causes is the absence of } \mathrm{Ag} \text { that inhibits the } \\
\text { volatilization of iodine). }\end{array}$ \\
\hline Main chemical forms & $\mathrm{AgI}, \mathrm{CdI}_{2}, \mathrm{Cs}_{2} \mathrm{MoO}_{4}, \mathrm{CsI}$ & $\mathrm{CsBO}_{2}, \mathrm{CH}_{3} \mathrm{I}, \mathrm{CsI}, \mathrm{HI}{ }^{18)}$ \\
\hline
\end{tabular}


ratio is low, as in the FPT-3 experiment, the integrity of most of the $\mathrm{B}_{4} \mathrm{C}$ is maintained in the reactor core, and it is highly probable that the $\mathrm{B}_{4} \mathrm{C}$ is oxidized by steam. Furthermore, during the oxidation process, release of iodine and Cs occurs almost at the same time, and it is necessary to consider the influence of these releases. In the BECARRE experiment, the reason why the $\mathrm{B}_{4} \mathrm{C}$ pellets were not oxidized to any great extent was because, in addition to the highest temperature of 1,953 K, which was lower than that of the FPT-3 experiment, the absence of fuel rods in the periphery also differed from the configuration of the FPT-3 experiment ${ }^{19)}$.

Thus, the findings of the experiments using powder- and pellet-type $\mathrm{B}_{4} \mathrm{C}$ are different. Therefore, great care must be taken when making predictions using different results. Furthermore, in the FPT-2 experiments with mixed boron in steam, the generation of gaseous iodine was not observed. This requires detailed study in the future.

\section{Generation Ratio of Organic Iodine}

Not all of the results of the PHEBUS/FPT-3 experiment have yet been disclosed (as of Oct. 2014). An estimation was conducted on the percentage of gaseous inorganic iodine and organic iodine in the containment vessel under severe accident conditions by using the release of almost $100 \%$ of the iodine from fuel, as in the FPT- 0 experiment, and the information contained in Table 2. Where the fractional release rate of iodine from the fuel in the FPT-3 experiment is assumed to be $100 \%$, the percentage of gaseous iodine in the containment vessel is $27 \%$ of the reactor core inventory according to Table 2 , and the percentage of organic iodine in the containment vessel is approximately $30 \%$, as shown in Figure 8. Consequently, a maximum of about $8 \%$ (i.e., $27 \% \times 0.3$ ) of organic iodine and about $19 \%$ of inorganic iodine was present in the containment vessel at the time in the FPT-3 experiment.

Table 4 shows a comparison of the "Guidelines for Safety Evaluation (hypothetical accident, loss-of-coolant accident condition)" ${ }^{28)}$ in Japan with the evaluation results of the FPT-3 experiment. This table indicates that the percentage of organic iodine in the current "Guidelines for Safety Evaluation" is slightly lower than that obtained in the FPT-3 experiment. However, it is considered too early to adopt this comparison as a basis for making any decision, especially in consideration of the arguments posed in section V-1 as well.

Recently, in the site evaluation guideline subcommittee of the former Nuclear Safety Commission, there was an argument about changing the percentage of organic iodine in the iodine released in the containment from $10 \%$ to $1 \%$, with reference to the insights from E.C. Beahm, et. al., bibliography item 10 that the percentage of organic iodine in gaseous iodine is generally less than $1 \%$ and that of the new source terms from NUREG-1465 of USNRC ${ }^{29}$ ) (Organic

Table 4 Comparison of "Guidelines for Safety Evaluation (hypothetical accident, loss-of-coolant accident condition)" and the FPT-3 experiment ${ }^{28)}$

\begin{tabular}{lc}
\hline $\begin{array}{l}\text { Guidelines for Safety Evaluation (hypothetical accident, loss-of- } \\
\text { coolant accident condition) }\end{array}$ & FPT-3 Experiment \\
\hline Percentage of iodine released in the containment vessel: $50 \%$ & Percentage of release from fuel $100 \%{ }^{\text {a) }}$ \\
\hline Inorganic iodine: $50 \%$ (fractional release rate) $\times 0.9=45 \%$ & Not disclosed \\
\hline $45 \% \times 0.5$ (deposition rate in containment vessel) $=22.5 \%$ & Deposition rate in containment vessel: Not disclosed \\
\hline Gaseous inorganic iodine & Approx. $19 \%$ \\
\hline $22.5 \% \div 100$ (gas-liquid partition coefficient) $=0.225 \%$ & Approx. $8 \%$ \\
\hline Organic iodine: $50 \%$ (fractional release rate) $\times 0.1=5 \%$ & Approx. $27 \%$ \\
\hline Total gaseous iodine: $0.225 \%+5 \%=5.23 \%$ & \\
a) $\begin{array}{l}\text { The data from the FPT- } 0 \text { experiment was adopted as a reference because the iodine release rate from the fuel in the FPT-3 experiment } \\
\text { had not been disclosed as of Oct. } 2014 .\end{array}$
\end{tabular}


iodine is $3 \%$ of inorganic iodine $)^{30}$. However, while this change may be appropriate for PWRs, further detailed studies are necessary for application to BWRs.

\section{Summary}

$\mathrm{B}_{4} \mathrm{C}$ used as absorber of control rods for reactors including BWR, EPR, and VVER, causes a phenomenon during a severe accident that is not observed in PWR, in which silver-indium-cadmium (S-I-C) absorbers are widely used, and may influence the reactor core melting process, $\mathrm{H}_{2}$ generation quantity, source terms, and other processes. The following insights have been acquired from previous experimental studies:

- During the reactor core melting process, $\mathrm{B}_{4} \mathrm{C}$ causes a eutectic reaction with stainless steel-cladding tubes, which liquefies the stainless steel at temperatures less than the melting point of stainless steel and accelerates melting of the reactor core.

- When $\mathrm{B}_{4} \mathrm{C}$ is oxidized, similar to the oxidation reaction of $\mathrm{Zr}$, reaction heat and $\mathrm{H}_{2}$ are generated. Furthermore, most of the separated $\mathrm{C}$ changes to $\mathrm{CO}$ or $\mathrm{CO}_{2}$, and some to $\mathrm{CH}_{4}$. The observed $\mathrm{CH}_{4}$ generation in the examination of individual effects was minimal, but an unignorable quantity of organic iodine $\left(\mathrm{CH}_{3} \mathrm{I}\right)$ was observed by the combination of $\mathrm{CH}_{4}$ and iodine (HI or $\mathrm{I}_{2}$ ) in the FPT-3 experiment, which was an integrated experiment. Currently, the processes that generate $\mathrm{CH}_{4}$ and $\mathrm{CH}_{3} \mathrm{I}$ when the oxidation of $\mathrm{B}_{4} \mathrm{C}$ and release of iodine occur simultaneously are not sufficiently understood.

- The metaboric acid $\left(\mathrm{HBO}_{2}\right)$ produced during the oxidation of $\mathrm{B}_{4} \mathrm{C}$ generates cesium boric acid $\left(\mathrm{CsBO}_{2}\right)$, which increases the deposition of $\mathrm{Cs}$ in the reactor coolant system.

Thus, insights have been obtained for several individual phenomena, but the real system-level behavior is not sufficiently understood. The most pressing subject we need to investigate is how the differences in shape and surface area between powder $\mathrm{B}_{4} \mathrm{C}$ control rod blades and pellet $\mathrm{B}_{4} \mathrm{C}$ control rods, and the difference in weight ratio of $\mathrm{B}_{4} \mathrm{C}$ and stainless steel, affect the actual progress of melting, $\mathrm{B}_{4} \mathrm{C}$ oxidation, chemical formation of radioactive materials, and release into the environment. It is also expected that further research in this field will provide useful knowledge and insights for reducing uncertainty in understanding how melting of the reactor core progresses and the source terms in the accident at Fukushima, and for establishing the ratio of organic iodine in the safety evaluation.

The author is grateful to Dr. Dana Auburn Powers of Sandia National Laboratories (SNL) US and Dr. Bernard Clément of the Institut de radioprotection et de sûreté nucléaire (IRSN) France who kindly gave him valuable suggestions and comments regarding the differences in the melting behavior of powder $\mathrm{B}_{4} \mathrm{C}$ control rod blades and pellet $\mathrm{B}_{4} \mathrm{C}$ control rods.

\section{References}

1) Nuclear Safety Commission, "US Nuclear Power Station Accident Special Committee - 3rd Report," May 1981.

2) R. Dubourg, H. Austregesilo, C. Bals et al., "Understanding the behavior of absorber elements in silver-indium-cadmium control rods during PWR severe accident sequences," Prog. Nucl. Energy, 52, 97-198 (2010).

3) D. Magallon, A. Mailliat, J.-M. Seiler et al., "European expert network for the reduction of uncertainties in severe accident safety issues (EURSAFE)," Nucl. Eng. Des., 235, 309-346 (2005).

4) Nuclear Emergency Response Headquarters, "Report of Japanese Government to the IAEA Ministerial Conference on Nuclear Safety-The Accident at TEPCO's Fukushima Nuclear Power Stations," June 
2011.

5) L. Sepold, G. Schanz, M. Steinbrück et al., "Results of the QUENCH-09 experiment compared to QUENCH-07 with incorporation of $\mathrm{B}_{4} \mathrm{C}$ absorber," Nucl. Technol., 154, 107-116 (2006).

6) G. Repetto, O. de Luze, N. Seiler et al., " $\mathrm{B}_{4} \mathrm{C}$ oxidation modelling in severe accident codes: Applications to PHEBUS and QUENCH experiments," Prog. Nucl. Energy, 52, 37-45 (2010).

7) L. Barin, Thermochemical Data of Pure Substances, VCH Verlagsgesellschaft, Weihelm, Germany (1989).

8) M. Steinbrück, A. Meier, U. Stegmaier et al., "Oxidation of B4C at high temperatures," 11th Int. Conf. on Nucl. Eng. (ICONE11)-36063, Tokyo (2003).

9) T. Haste, F. Payot, C. Dominguez et al., "Study of boron behavior in the primary circuit of water reactors under severe accident conditions: A comparison of Phebus FPT3 results with other recent integral and separate-effects data," Nucl. Eng. Des., 246, 147-156 (2012).

10) E. C. Beahm, C. F. Weber, T. S. Kress et al., Iodine Chemical Forms in LWR Severe Accidents, NUREG/ CR-5732 (1992).

11) R. O. Gauntt, R. D. Gasser, L. J. Ott, The DF-4 Fuel Damage Experiment in ACRR with a BWR Control Blade and Channel Box, NUREG/CR-4671 (1989).

12) R. O. Gauntt, R. D. Gasser, "Results of the DF-4 BWR control blade-channel box test," Proc. Eighteenth Water Reactor Safety Information Meeting, NUREG/CP-0114, 2, 25-40 (1990).

13) R. O. Gauntt, L. L. Humphries, Final Results of the XR2-1 BWR Metallic Melt Relocation Experiment, NUREG/CR-6527 (1997).

14) P. Hofmann, M. Markiewicz, J. Spino, "Reaction behavior of $B_{4} C$ absorber material with stainless steel and zircaloy in severe LWR accidents," Nucl. Technol., 90, 226-244 (1990).

15) A. Miassoedov, C. Homann, G. Schanz et al., "Results of the QUENCH-09 experiment on the investigation of the cool down behavior of the fuel rod simulators," 11th Int. Conf. on Nucl. Eng. (ICONE11)-36035, Tokyo (2003).

16) B. Clément, N. Girault, G. Repetto et al., "LWR severe accident simulation: synthesis of the results and interpretation of the first Phebus FP experiment FPT0," Nucl. Eng. Des., 226, 5-82 (2003).

17) F. Payot, P. March, B. Biard et al., Phebus FPT3: Main Relevant Results Likely to Impact Safety, EUROSAFE, http://www.eurosafe-forum.org/files/seminar1_01_2007.pdf.

18) N. Girault, C. Fiche, A. Bujan et al., "Towards a better understanding of iodine chemistry in RCS of nuclear reactors," Nucl. Eng. Des., 239, 1162-1170 (2009).

19) O. de Luze, "Degradation and oxidation of $\mathrm{B}_{4} \mathrm{C}$ control rod segments at high temperatures. A review and code interpretation of the BECARRE program," Nucl. Eng. Des., 259, 150-165 (2013).

20) R. C. Schmidt, MERIS: A Model for Melt Relocation in Core Structures of Nuclear Reactors during Severe Accident Conditions, NRC FIN L-2452, Sandia National Laboratories, Albuqerque, NM, Oct. 1993.

21) A. Bujan, L. Ammirabile, J. Slaby, "ASTEC V2.0 computational evaluation of source term and its chemical forms under accidental conditions during mid-loop operation," Prog. Nucl. Energy, 53, 438-448 (2011).

22) B. Adroguer, F. Bertrand, P. Chatelard et al., "Core loss during a severe accident (COLOSS)," Nucl. Eng. Des., 235, 173-198 (2005).

23) M. Steinbrück, A. Meier, U. Stegmaier et al., Experiments on the Oxidation of Boron Carbide at High Temperatures, FZKA 6979 (2004).

24) F. Nagase, H. Uetsuka, T. Otomo, "Chemical interactions between $\mathrm{B}_{4} \mathrm{C}$ and stainless steel at high temperatures,” J. Nucl. Mater., 245, 52-59 (1997).

25) K. Minato, "Thermodynamic analysis of cesium and iodine behavior in severe light water reactor accidents," J. Nucl. Mater., 185, 154-158 (1991).

26) H. Shibazaki, Y. Maruyama, T. Kudo et al., "Revaporization of a CsI aerosol in a horizontal straight pipe in a severe accident condition," Nucl. Technol., 134, 62-70 (2001).

27) M. P. Kissane, I. Drosik, "Interpretation of fission-product transport behaviour in the Phebus FPT0 and FPT1 tests," Nucl. Eng. Des., 236, 1210-1223 (2006).

28) Nuclear Safety Commission, "Regulatory Guide for Reviewing Safety Assessment of Light Water Nuclear Power Reactor Facilities," Published in Aug.30. 1990, Latest revision Mar. 29. 2001.

29) U. S. Nuclear Regulatory Commission, "Accident Source Terms for Light-Water Nuclear Power Plants," NUREG-1465 (1995).

30) Special Committee of Nuclear Safety Standards/Guidelines, Subcommittee to discuss "Regulatory Guide for Reviewing Nuclear Reactor Site Evaluation and Application Criteria," and the revisions on relevant safety examination guidelines "Interim Report of Investigation and Examination Statuses," Mar. 30. 2010. 


\title{
Article
}

\section{Survey of Tsuruga Inhabitants Concerning Radiation and Its Risks}

\author{
Yoshihiko SHINODA ${ }^{1,3, *}$ and Naoki YAMANO ${ }^{2}$ \\ ${ }^{1}$ Department of Nuclear Power and Energy Safety Engineering, Graduate School of Engineering, University of Fukui, \\ 3-9-1 Bunkyo, Fukui-shi, Fukui 910-8507, Japan \\ ${ }^{2}$ Research Institute of Nuclear Engineering, University of Fukui, 1-2-4 Kanawa-cho, Tsuruga-shi, Fukui 914-0055, Japan \\ ${ }^{3}$ Japan Atomic Energy Agency, Tsuruga Head Office, 65-20 Kizaki, Tsuruga-shi, Fukui 914-8585, Japan
}

\begin{abstract}
The Fukushima Daiichi Nuclear Accident has led to changes in the acceptance of nuclear power in many people. The authors conducted an opinion survey of 300 adult inhabitants of Tsuruga city in Fukui Prefecture, Japan. The aim of this survey is to obtain people's opinions concerning radiation and its risks. Authors classified Tsuruga inhabitants on the basis of responses to questions on the concept and knowledge of risk and the cognition of radiation by factor and cluster analyses of multivariable analysis. Using the results of these analyses, Tsuruga inhabitants have been assigned to five categories: "acceptance group," "anxiety group," and three intermediate groups.
\end{abstract}

KEYWORDS: opinion survey, Tsuruga City, ionizing radiation, health effect, risk communication, low dose, co evolution

\section{Introduction}

The accident at the Fukushima Daiichi Nuclear Power Plants in the wake of the Great East Japan Earthquake (hereinafter called the "Fukushima Accident"), on March 11, 2011, released radioactive materials into the environment and, consequently, forced local residents to evacuate the area. The released radioactive materials had an impact not only on the local residents but also, more or less, on society as a whole and aroused nationwide interest and concern.

Since 2013, the authors have undertaken practical research activities in Tsuruga City, Fukui Prefecture, concerning the health risks of low-dose radiation through an association with and the cooperation of Tsuruga City residents ${ }^{1}$. This activity aims to model a new risk communication method and establish its implementation concerning the health effects of low-dose radiation, which has become a social issue after the Fukushima Accident. As a part of this study activity, we conducted an awareness survey (hereinafter called the "Survey") of residents in Tsuruga to understand their attitudes towards radiation and the notion of the "level of risk" (this refers to the meaning of the term "risk").

* Corresponding author, E-mail: shinoda.yoshihiko@plum.plala.or.jp

DOI : 10.15669 /fukushimainsights.Vol.4.357

(C) 2021 Atomic Energy Society of Japan. All rights reserved.

Originally published in Transactions of the Atomic Energy Society of Japan (ISSN 1347-2879), Vol. 14, No. 2, p.95-112

(2015) in Japanese. (Japanese version accepted: November 27, 2014) 


\section{Survey Overview}

\section{Purpose of the Survey}

The purpose of the survey is to ascertain the awareness of risks and the level of knowledge on radiation among residents of Tsuruga City, an area where nuclear power plants are located (hereinafter called the "Power Plant Located Area"), after the Fukushima Accident. In the survey, we first try to ascertain the level of knowledge and perception of radiation and low-dose exposure among residents as well as their awareness of the associated impacts (e.g. what images are brought to mind and how they think about them). Considering the current status where such impacts are observed daily, we think that we will be able to measure the level of awareness with high sensitivity by asking questions related to everyday life. Diet is an essential part of life, and, therefore, interest in food and radiation has increased after the Fukushima Accident. Therefore, we decided to study awareness of the health effects of low-dose radiation together with the degree of awareness of food contamination and the notion of risk. We think that by obtaining responses about these issues and exploring the relationship between them will allow us to obtain substantial information.

However, it should be noted that there are variations in the interpretation of the notion of risk and it is hard to say that the notion of risk is unambiguously recognized as a common concept. This study is intended to explore how far the subjects recognize the concept of risk and how they interpret the concept and to focus on the relationship between the their awareness towards the risk concept and the radiation.

\section{Overview of Relevant Awareness Surveys}

In order to develop survey questions, we examined awareness surveys previously performed in Japan as reference materials. Table 1 shows representative awareness surveys for which results and survey forms are open to the public.

In surveys on radiation and radioactivity ${ }^{2-4)}$ performed before the Fukushima Accident, the focus was placed on understanding people's knowledge and attitudes to radiation. In 2012, after the accident, awareness surveys were conducted with residents of Fukushima City as subjects. These included one to help promote reconstruction plans in Fukushima Prefecture, including radiation reduction measures ${ }^{6}$, and one to determine residents' awareness of decontamination implementation plans ${ }^{7}$. In these surveys, questions were given under the assumption that radiation exposure, depending on the level, could potentially influence the health of residents. Also, an awareness survey concerning the Fukushima Accident ${ }^{8,9)}$ included questions on radiation and radioactivity. Countless awareness surveys, performed before and after the Fukushima Accident ${ }^{10,11)}$ regarding nuclear power in general, included questions on radiation. Awareness surveys on food safety ${ }^{12-14)}$ performed after the Fukushima Accident focus on consumer consciousness of radiation and low-dose exposure. However, in many of these surveys, the questions were restricted and, therefore, the results are superficial. In previous studies ${ }^{15-27)}$, no questions were asked in relation to public awareness of the effects of exposure to low-dose radiation or the level of public knowledge on the notion of risk. Such questions are asked in this study for the first time.

\section{Survey Structure}

We analyzed previous study examples and designed and created a questionnaire and survey method. The structure of the survey is as follows. 
Table 1 Overview of relevant awareness surveys

\begin{tabular}{|c|c|c|c|c|c|}
\hline & Survey date & Surveyed area & Main surveyed items & Items related to radioactivity & Reference \\
\hline \multirow{13}{*}{$\begin{array}{l}\text { Radiation/ } \\
\text { Radioactivity }\end{array}$} & 1963 & Nationwide & Radiation/Radioactivity & Knowledge and interest & 2) \\
\hline & 2001 & Nationwide & Radiation/Radioactivity & Perception and knowledge & 3) \\
\hline & 2001 & $\begin{array}{l}\text { Service areas of the Kansai } \\
\text { Electric Power }\end{array}$ & Radiation/Radioactivity & $\begin{array}{l}\text { Risks, perception and } \\
\text { concerns }\end{array}$ & 4) \\
\hline & 2012 & $\begin{array}{l}\text { Service areas of the Kansai } \\
\text { Electric Power }\end{array}$ & Radiation/Radioactivity & $\begin{array}{l}\text { Knowledge, education and } \\
\text { perception }\end{array}$ & 5) \\
\hline & 2012 & $\begin{array}{l}\text { Fukushima City (including } \\
\text { those who evacuated the } \\
\text { city) }\end{array}$ & $\begin{array}{l}\text { The Fukushima Accident } \\
\text { and Radiation/Radioactivity }\end{array}$ & $\begin{array}{l}\text { Concerns and response actions } \\
\text { after the Fukushima Accident }\end{array}$ & 6) \\
\hline & 2013 & Fukushima Prefecture & $\begin{array}{l}\text { Decontamination } \\
\text { implementation plans }\end{array}$ & $\begin{array}{l}\text { Radiation exposure level and } \\
\text { effects on health }\end{array}$ & 7) \\
\hline & 2013 & $\begin{array}{l}\text { Tokyo metropolitan area, } \\
\text { Kansai region and power } \\
\text { plant located area }\end{array}$ & Earthquake and tsunami & $\begin{array}{l}\text { Response actions after the } \\
\text { Fukushima Accident and } \\
\text { knowledge }\end{array}$ & 8) \\
\hline & 2011 & Nationwide & $\begin{array}{l}\text { Living conditions after } \\
\text { disaster }\end{array}$ & fears, and concerns & 9) \\
\hline & $2007-2013$ & Nationwide & $\begin{array}{l}\text { Nuclear power generation } \\
\text { (hereinafter called "nuclear } \\
\text { power") }\end{array}$ & $\begin{array}{l}\text { Necessity of nuclear power } \\
\text { use, perception }\end{array}$ & 10) \\
\hline & $2007-2014$ & Tokyo metropolitan area & Nuclear power and energy & $\begin{array}{l}\text { Concerns about and awareness } \\
\text { of environmental pollution }\end{array}$ & 11) \\
\hline & 2011 & Nationwide & Food safety & Impacts and knowledge & 12) \\
\hline & 2013-2014 & Nationwide & Food purchasing & Knowledge and impacts & 13) \\
\hline & 2011 & $\begin{array}{l}\text { Tokyo metropolitan area } \\
\text { and Kansai region }\end{array}$ & Food safety & $\begin{array}{l}\text { Concerns about radiation } \\
\text { exposure }\end{array}$ & 14) \\
\hline \multirow{14}{*}{$\begin{array}{l}\text { Including } \\
\text { power plant } \\
\text { located } \\
\text { area and } \\
\text { neighboring } \\
\text { areas }\end{array}$} & 1979 & Tsuruga City & $\begin{array}{l}\text { Knowledge and information } \\
\text { on nuclear power and } \\
\text { contribution to communities }\end{array}$ & No direct questions & $15,16)$ \\
\hline & 1980 & $\begin{array}{l}\text { Kasiwazaki City, Niigata } \\
\text { Prefecture }\end{array}$ & $\begin{array}{l}\text { Knowledge on nuclear } \\
\text { power, information and } \\
\text { contribution to communities }\end{array}$ & No direct questions & 17) \\
\hline & 1981 & $\begin{array}{l}\text { Hamaoka-cho, Shizuoka } \\
\text { Prefecture (at that time) }\end{array}$ & $\begin{array}{l}\text { Knowledge on nuclear } \\
\text { power, information and } \\
\text { contribution to communities }\end{array}$ & No direct questions & 18) \\
\hline & 1998 & $\begin{array}{l}\text { Kasiwazaki City and } \\
\text { Futaba-gun, Fukushima } \\
\text { Prefecture }\end{array}$ & $\begin{array}{l}\text { Risk awareness and nuclear } \\
\text { power }\end{array}$ & $\begin{array}{l}\text { Radioactive contamination } \\
\text { (comparison of hazardous } \\
\text { events) }\end{array}$ & 19) \\
\hline & 2000 & $\begin{array}{l}\text { Kasiwazaki-city and } \\
\text { Futaba-gun }\end{array}$ & $\begin{array}{l}\text { Community development } \\
\text { and energy policy }\end{array}$ & No direct questions & 20) \\
\hline & 2001 & $\begin{array}{l}\text { Iwaki City, Fukushima } \\
\text { Prefecture, etc. }\end{array}$ & $\begin{array}{l}\text { Community development } \\
\text { and energy policy }\end{array}$ & No direct questions & 20) \\
\hline & 2005 & $\begin{array}{l}\text { Kasiwazaki City, Niigata } \\
\text { City, etc. }\end{array}$ & $\begin{array}{l}\text { Economic advantages and } \\
\text { reasons for avoidance }\end{array}$ & $\begin{array}{l}\text { Concerns about radiation } \\
\text { exposure }\end{array}$ & 21) \\
\hline & 2006 & Fukui Prefecture & $\begin{array}{l}\text { Nuclear power, risk } \\
\text { awareness, and policy }\end{array}$ & No direct questions & 22) \\
\hline & 2007 & Ibaraki Prefecture & $\begin{array}{l}\text { Nuclear power, risk } \\
\text { awareness, and policy }\end{array}$ & No direct questions & 23) \\
\hline & 2008 & $\begin{array}{l}\text { Neighboring villages/ } \\
\text { town in Tsuruga City and } \\
\text { Mihama City }\end{array}$ & $\begin{array}{l}\text { Benefits and changes in } \\
\text { living environment }\end{array}$ & No direct questions & 24) \\
\hline & 2010 & $\begin{array}{l}\text { Tokai Village, Ibaraki } \\
\text { Prefecture and neighboring } \\
\text { municipalities }\end{array}$ & $\begin{array}{l}\text { Nuclear power, information } \\
\text { and regional community }\end{array}$ & No direct questions & 25) \\
\hline & 2011 & $\begin{array}{l}\text { Tokai Village, Ibaraki } \\
\text { Prefecture and neighboring } \\
\text { municipalities }\end{array}$ & $\begin{array}{l}\text { Nuclear power, information } \\
\text { and regional community }\end{array}$ & $\begin{array}{l}\text { Impression of explanation of } \\
\text { radiation }\end{array}$ & 25) \\
\hline & 2011 & $\begin{array}{l}\text { Mito City, Ibaraki } \\
\text { Prefecture }\end{array}$ & $\begin{array}{l}\text { Nuclear power, information } \\
\text { and regional community }\end{array}$ & $\begin{array}{l}\text { Impression of explanation of } \\
\text { radiation }\end{array}$ & 26) \\
\hline & 2012 & $\begin{array}{l}\text { Matsue City, Shimane } \\
\text { Prefecture }\end{array}$ & $\begin{array}{l}\text { Reopening of Shimane } \\
\text { Nuclear Power Plant }\end{array}$ & No direct questions & 27) \\
\hline
\end{tabular}


Respondents: $\quad$ Male and female residents 18 years and older, who live in Tsuruga City.

Number of respondents: 300 (sample size).

Sampling method: Quota sampling (sample 20 locations with a given probability in proportion to the population of Tsuruga City, and acquire responses from 300 respondents [20 locations $\times 15$ respondents] by allocating 15 respondents in proportion to the gender and age of the parent population of each location).

Survey method: $\quad$ Direct-visit and self-completion method.

Survey period: $\quad$ September 5-19, 2013.

Question items: $\quad$ (1) General matters;

(2) Awareness of radiation and radioactivity;

(3) Awareness of risks and food safety; and

(4) Awareness of effects of low-dose exposure.

In the survey, we avoided questions that directly asked about respondents' awareness of nuclear power generation. This was to distinguish our survey from a survey developed to determine whether respondents were for or against nuclear power. On this basis, we created a questionnaire consisting of only relevant question items. For question items (2), (3) and (4), we used a question style (hereinafter called the "5-point scale") where we provided opinions and statements and asked respondents what they thought about them (or whether they applied to them), giving five answer options: "I agree (It applies to me)," "I somewhat agree (It somewhat applies to me)," "Neither," "I somewhat disagree (It somewhat doesn't apply to me)," and "I disagree (It doesn't apply to me)." The questions used in this paper are listed in the Questionnaire (excerpted) in the Appendix. In this paper, questions are abbreviated as follows: "Q5-1." Questions 5, 6, 7 and 8 ask about residents' (a) awareness of radiation and radioactivity; (b) awareness of risks and food safety; (c) knowledge, experiences and attitude towards risks; and (d) awareness of low-dose exposure, respectively.

Also, in the figures and tables included in this study, answer options from "I agree (It applies to me)" to "I disagree (It doesn't apply to me)" are defined as "Positive," "Weak positive," "Neutral," "Weak negative," and "Negative" responses, respectively. Then, we scored the options at 1-point intervals, $-2,-1,0,1,2$, in the order from positive to negative answers, and analyzed (negative values indicate positive direction (answer) and positive values indicate negative direction (answer)).

\section{Survey Results}

\section{Survey Results}

\section{(1) Respondents' attributes}

Table 2 shows the gender and age demographics of the respondents. Regarding awareness surveys conducted in Power Plant Located Areas, we need to know in advance any connections between the respondents and the nuclear power industry. In this survey we asked the question, "Do you have any relatives living with you who are engaged in the nuclear power industry or radiation-related industries?" Several options for answers were provided, including "self" and "father/mother," allowing for multiple answers. Table 3 shows the results. It reveals that $44.0 \%$, nearly half, of respondents have relatives engaged in the nuclear power industry or radiation-related industries. To this same question, 7.7\% answered "self" and 20.3\%, the highest percentage, 
Table 2 Gender and age of respondents

\begin{tabular}{cccc}
\hline Age & Male & Female & Total \\
\hline $18-29$ & $8.0 \%$ & $7.3 \%$ & $15.3 \%$ \\
$30-39$ & $8.7 \%$ & $8.0 \%$ & $16.7 \%$ \\
$40-49$ & $8.0 \%$ & $7.3 \%$ & $15.3 \%$ \\
$50-59$ & $8.3 \%$ & $7.7 \%$ & $16.0 \%$ \\
$60-69$ & $8.3 \%$ & $8.3 \%$ & $16.6 \%$ \\
70 and over & $8.0 \%$ & $12.0 \%$ & $20.0 \%$ \\
\hline Total & $49.3 \%$ & $50.7 \%$ & $100.0 \%$ \\
\hline
\end{tabular}

Table 3 Respondents' relationships with those engaged in the nuclear power industry and radiation-related industries

\begin{tabular}{cc}
\hline \multicolumn{3}{c}{ Nuclear/radiation industry workers } \\
\hline No & $56.0 \%$ \\
\hline Yes & $44.0 \%$ \\
\hline Response rate for each option (multiple selection is allowed) \\
\hline Self & $7.7 \%$ \\
Father/Mother & $5.3 \%$ \\
Grandson/Granddaughter & $3.3 \%$ \\
Relative & $7.7 \%$ \\
Spouse & $1.3 \%$ \\
Children & $4.0 \%$ \\
Brother/Sister & $20.3 \%$ \\
Other & $4.0 \%$ \\
\hline Respondent rate for those who chose "self" (selection rate of "self") \\
1 & $36.0 \%(13.8 \%)$ \\
2 & $6.7 \%(20 \%)$ \\
3 & $1.0 \%(100 \%)$ \\
4 & $0.3 \%(100 \%)$ \\
\hline
\end{tabular}

answered "brother/sister." The maximum number of answers selected was four. Thirty-Six percent (36\%) of respondents only selected one answer; of that $36 \%, 13.8 \%$ chose "self."

It should be noted that the scope of the definition of "those engaged in the nuclear power industry and radiation-related industries" was interpreted by each respondent. Also, when we refer to the relationships with the people so engaged as "personal relationships" that may have impact on the awareness of respondents, we must interpret it as only a partial capturing of personal relationships of Tsuruga City residents because the personal relationships of each individual may also influence the respondents' judgment.

\section{(2) Questions concerning radiation and radioactivity}

In the Questionnaire listed in the Appendix (Q5), there are 15 questions concerning radiation and radioactivity and respondents were asked to register their level of agreement with each question using options on a 5-point scale. The Questionnaire included questions concerning respondents' purchasing behavior and adequacy of purchasing restrictions in light of their views towards radiation (such as whether radiation is evil or dangerous) and respondents' knowledge and concerns about nuclear power. However, some questions, such as Q5-2, which states, "I cannot classify radiation and radioactivity as either good or bad," frames the issue both in terms of a respondent's viewpoint and knowledge of radiation and radioactivity.

Table 4 shows the responses to all questions. It should be noted that the total of some 
Table 4 Responses to the questions concerning radiation and radioactivity

\begin{tabular}{|c|c|c|c|c|c|c|c|c|}
\hline & & Positive & $\begin{array}{c}\text { Weak } \\
\text { positive }\end{array}$ & Neutral & $\begin{array}{c}\text { Weak } \\
\text { negative }\end{array}$ & Negative & Average & Variance \\
\hline Q5-1 & $\begin{array}{l}\text { I think radiation and radioactivity } \\
\text { are evil. }\end{array}$ & $12.7 \%$ & $15.3 \%$ & $36.3 \%$ & $13.3 \%$ & $21.3 \%$ & 0.15 & 1.63 \\
\hline Q5-2 & $\begin{array}{l}\text { I cannot classify radiation and } \\
\text { radioactivity as good or bad. }\end{array}$ & $25.3 \%$ & $19.7 \%$ & $32.0 \%$ & $8.0 \%$ & $13.3 \%$ & -0.36 & 1.70 \\
\hline Q5-3 & $\begin{array}{l}\text { I think radiation and radioactivity } \\
\text { are horrible. }\end{array}$ & $36.7 \%$ & $28.0 \%$ & $21.3 \%$ & $5.0 \%$ & $8.0 \%$ & -0.80 & 1.48 \\
\hline Q5-4 & $\begin{array}{l}\text { I think radiation and radioactivity } \\
\text { can sometimes be useful for human } \\
\text { beings. }\end{array}$ & $53.0 \%$ & $28.3 \%$ & $14.0 \%$ & $1.0 \%$ & $3.0 \%$ & -1.27 & 0.92 \\
\hline Q5-5 & $\begin{array}{l}\text { I think radiation and radioactivity } \\
\text { can be horrible or not horrible, } \\
\text { depending on the level of radiation. }\end{array}$ & $54.3 \%$ & $24.7 \%$ & $14.0 \%$ & $1.7 \%$ & $4.3 \%$ & -1.23 & 1.10 \\
\hline Q5-6 & $\begin{array}{l}\text { I think that radiation and } \\
\text { radioactivity are around us. }\end{array}$ & $76.7 \%$ & $17.0 \%$ & $5.0 \%$ & $0.0 \%$ & $0.7 \%$ & -1.69 & 0.41 \\
\hline Q5-7 & $\begin{array}{l}\text { I think the effects of internal } \\
\text { exposure are greater than the effects } \\
\text { of external exposure in Fukushima } \\
\text { Prefecture. }\end{array}$ & $23.0 \%$ & $24.3 \%$ & $41.3 \%$ & $5.0 \%$ & $5.7 \%$ & -0.54 & 1.15 \\
\hline Q5-8 & $\begin{array}{l}\text { I am concerned about the radiation } \\
\text { and radioactivity around us. }\end{array}$ & $16.0 \%$ & $18.3 \%$ & $25.7 \%$ & $14.7 \%$ & $25.0 \%$ & 0.14 & 1.96 \\
\hline Q5-9 & $\begin{array}{l}\text { I am concerned about radioactive } \\
\text { elements in tap water. }\end{array}$ & $11.7 \%$ & $14.0 \%$ & $26.7 \%$ & $17.0 \%$ & $30.3 \%$ & 0.40 & 1.83 \\
\hline Q5-10 & $\begin{array}{l}\text { I am concerned about radioactive } \\
\text { elements in milk and dairy products. }\end{array}$ & $10.0 \%$ & $17.0 \%$ & $27.3 \%$ & $16.3 \%$ & $29.3 \%$ & 0.38 & 1.77 \\
\hline Q5-11 & $\begin{array}{l}\text { I think the current government } \\
\text { regulations ensure food safety, } \\
\text { including food from Fukushima. }\end{array}$ & $19.3 \%$ & $26.7 \%$ & $27.7 \%$ & $13.0 \%$ & $12.0 \%$ & -0.28 & 1.57 \\
\hline Q5-12 & $\begin{array}{l}\text { I want to avoid purchasing milk } \\
\text { and dairy products from Ibaraki and } \\
\text { Tochigi. }\end{array}$ & $11.7 \%$ & $17.7 \%$ & $32.3 \%$ & $13.7 \%$ & $24.3 \%$ & 0.21 & 1.71 \\
\hline Q5-13 & $\begin{array}{l}\text { I am concerned about radioactive } \\
\text { elements in food (agricultural, } \\
\text { animal and fishery products). }\end{array}$ & $19.7 \%$ & $24.3 \%$ & $26.0 \%$ & $11.0 \%$ & $18.3 \%$ & -0.16 & 1.85 \\
\hline Q5-14 & $\begin{array}{l}\text { I want to avoid purchasing food } \\
\text { (agricultural, animal and fishery } \\
\text { products) from Fukushima. }\end{array}$ & $17.3 \%$ & $19.0 \%$ & $27.7 \%$ & $14.0 \%$ & $22.0 \%$ & 0.04 & 1.91 \\
\hline Q5-15 & $\begin{array}{l}\text { I think the current regulation values } \\
\text { for radioactive materials in foods are } \\
\text { adequate. }\end{array}$ & $16.3 \%$ & $19.7 \%$ & $43.0 \%$ & $9.7 \%$ & $11.0 \%$ & -0.21 & 1.35 \\
\hline
\end{tabular}

responses does not reach 100\% due to invalid answers and rounding errors. In Figure 1, we described the distribution of answers for Q5-1 "Radiation is evil," Q5-3 "Radiation is horrible," Q5-8 "I am concerned about radiation around us," and Q5-15 "Regulation values for radioactive materials in foods are adequate," as representative examples of Question 5. The average values calculated after allocating scores to answer options are $0.15,-0.80,0.14$, and -0.21 , respectively. Among all respondents, more respondents provided negative answers for "Radiation is evil" and positive answers for "Radiation is horrible." Although respondents show different attitudes towards concerns about radiation, the results lean slightly toward the negative side.

Many respondents showed agreement with the statements in Q5-4, -5 and -6, which concern general knowledge on radiation. As for the questions regarding respondents' perception and concerns, many of the results do not follow normal distribution; for example, in some questions, the response rate for a negative answer is higher than that for a weak negative answer. 


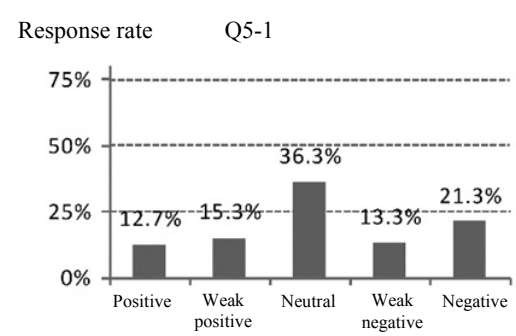

(1) Radiation is evil

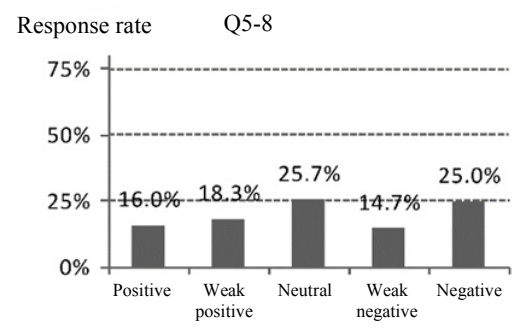

(3) I am concerned about radiation

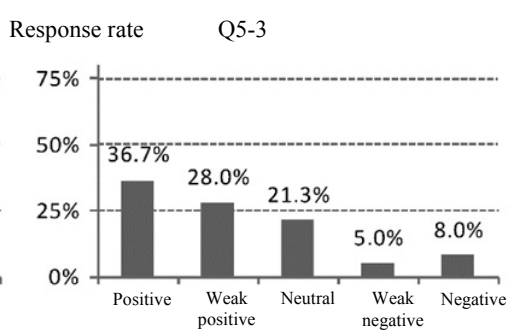

(2) Radiation is horrible

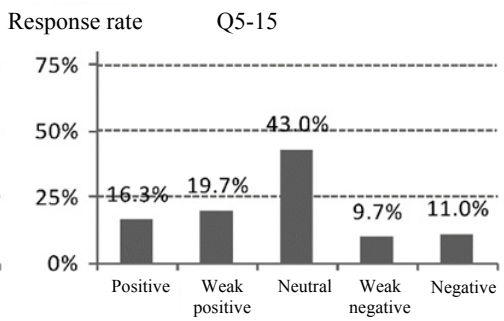

(4) Regulation values for radioactive materials in foods are adequate

"Answer options"

Positive: "I agree (It applies to me)."

5-Point Scale score

\begin{tabular}{lr}
\hline Weak positive: "I somewhat agree (It somewhat applies to me)." & -2 \\
\hline Neutral: "I neither agree nor disagree." & -1 \\
\hline Weak negative: “I somewhat disagree (It somewhat doesn't apply to me).” & 1 \\
\hline Negative: "I disagree (It doesn't apply to me)." & 2
\end{tabular}

Figure 1 Distribution of the response results of the questions concerning radiation and radioactivity (excerpted)

\section{(3) Questions concerning risks and food safety}

Q6 provides eleven questions on risks and food safety in Q6-1 to Q6-11. Q7 provides five questions on risks in Q7-1 to Q7-5.

Q6 presents typical opinions and asks respondents to register their level of agreement with them, providing multiple answer options from "I agree" to "I don't agree." Q7 measures respondents' knowledge, experience and attitudes, providing answer options from "It applies to me" to "It doesn't apply to me," and, therefore, Q7 is separate from Q6. Table $\mathbf{5}$ shows the responses to both Q6 and Q7. The results for Q6-8, concerning comparisons of the relative safety of tap water and mineral water, show that mineral water is considered safe. In Q6-11, concerning radiation materials in domestic food and imported food, more people answered that domestic food is safer. In these two questions, many respondents also had a neutral attitude and the response rates for negative answers were higher than those for weak negative answers. The results for Q7 regarding risks also showed a similar trend: the response rates for negative answers were higher than those for weak negative answers.

\section{(4) Questions concerning low-dose exposure}

Q8 provides thirteen questions on low-dose exposure, in Q8-1 to Q8-13, and asks respondents to register their level of agreement. Table 6 shows the responses. For Q8-1 "low-dose exposure is dangerous regardless of exposure dose" and Q8-13 "effects are passed on to offspring," opinions are divided. Although public trust in the government and experts is low, public trust in medical doctors is relatively high. Although we found, in Q8-9 and Q8-10, that 
Table 5 Responses to the questions concerning risks and food safety

\begin{tabular}{|c|c|c|c|c|c|c|c|c|}
\hline & & Positive & $\begin{array}{l}\text { Weak } \\
\text { Positive }\end{array}$ & Neutral & $\begin{array}{c}\text { Weak } \\
\text { Negative }\end{array}$ & Negative & Average & Variance \\
\hline Q6-1 & $\begin{array}{l}\text { I think chemical toxicity is more dangerous than } \\
\text { radioactive materials in food. }\end{array}$ & $28.0 \%$ & $25.7 \%$ & $42.3 \%$ & $1.3 \%$ & $2.3 \%$ & -0.76 & 0.91 \\
\hline Q6-2 & $\begin{array}{l}\text { We can trust the Food Safety Commission } \\
\text { regarding food safety. }\end{array}$ & $11.7 \%$ & $28.3 \%$ & $42.7 \%$ & $8.3 \%$ & $8.7 \%$ & -0.26 & 1.12 \\
\hline Q6-3 & $\begin{array}{l}\text { We can trust the Ministry of Health, Labour and } \\
\text { Welfare and Ministry of Agriculture, Forestry } \\
\text { and Fisheries of Japan regarding food safety. }\end{array}$ & $9.3 \%$ & $25.0 \%$ & $43.3 \%$ & $12.7 \%$ & $9.0 \%$ & -0.13 & 1.10 \\
\hline Q6-4 & I think there are various risks in our society & $55.3 \%$ & $32.0 \%$ & $10.3 \%$ & $0.7 \%$ & $1.0 \%$ & -1.40 & 0.62 \\
\hline Q6-5 & $\begin{array}{l}\text { I think radiation and radioactivity are just one } \\
\text { of many risks. }\end{array}$ & $43.7 \%$ & $32.7 \%$ & $16.7 \%$ & $2.7 \%$ & $4.0 \%$ & -1.09 & 1.07 \\
\hline Q6-6 & $\begin{array}{l}\text { I think some risks can have a bad influence on } \\
\text { us. }\end{array}$ & $38.0 \%$ & $32.0 \%$ & $26.0 \%$ & $2.0 \%$ & $1.3 \%$ & -1.03 & 0.85 \\
\hline Q6-7 & $\begin{array}{l}\text { I think some risks can have a good influence } \\
\text { on us. }\end{array}$ & $14.0 \%$ & $19.0 \%$ & $47.7 \%$ & $9.3 \%$ & $9.3 \%$ & -0.19 & 1.18 \\
\hline Q6-8 & I think mineral water is safer than tap water. & $15.7 \%$ & $21.3 \%$ & $43.3 \%$ & $6.0 \%$ & $13.3 \%$ & -0.20 & 1.40 \\
\hline Q6-9 & $\begin{array}{l}\text { I think the government and prefectures (local } \\
\text { government) should define criteria that clearly } \\
\text { determine what are risks. }\end{array}$ & $48.3 \%$ & $29.3 \%$ & $18.7 \%$ & $1.7 \%$ & $1.3 \%$ & -1.22 & 0.82 \\
\hline Q6-10 & $\begin{array}{l}\text { I think experts should define criteria that clearly } \\
\text { determine what are risks. }\end{array}$ & $48.3 \%$ & $28.3 \%$ & $20.0 \%$ & $1.3 \%$ & $2.0 \%$ & -1.20 & 0.88 \\
\hline Q6-11 & $\begin{array}{l}\text { I think domestic food contains less radioactive } \\
\text { materials than imported food. }\end{array}$ & $3.0 \%$ & $4.7 \%$ & $49.7 \%$ & $15.3 \%$ & $27.3 \%$ & 0.59 & 1.06 \\
\hline Q7-1 & I have thought about risks before. & $17.7 \%$ & $30.0 \%$ & $30.3 \%$ & $10.3 \%$ & $11.0 \%$ & -0.33 & 1.45 \\
\hline Q7-2 & I have been educated about risks. & $6.0 \%$ & $11.3 \%$ & $27.0 \%$ & $18.0 \%$ & $36.3 \%$ & 0.67 & 1.54 \\
\hline Q7-3 & I know how to determine risks. & $3.3 \%$ & $8.7 \%$ & $39.7 \%$ & $16.0 \%$ & $31.3 \%$ & 0.63 & 1.24 \\
\hline Q7-4 & I know the meaning of risks. & $21.7 \%$ & $29.7 \%$ & $29.0 \%$ & $7.7 \%$ & $10.3 \%$ & -0.45 & 1.46 \\
\hline Q7-5 & I determine risks by myself. & $17.3 \%$ & $30.7 \%$ & $32.3 \%$ & $6.3 \%$ & $12.0 \%$ & -0.35 & 1.43 \\
\hline
\end{tabular}

people recognize the effects of radiation on infants and toddlers, $43.3 \%$ of the respondents chose "Neither," in Q8-11, about the impact on the respondents themselves, which means they tend to be less worried about the effects of radiation on themselves.

\section{Analysis of the Survey}

\section{(1) Aggregation of the response results}

We summarized the response rates through the questions described in the above Sections III-1(2), (3) and (4). Details of the statistical methods used in this study can be found on Aoki's Website ${ }^{28)}$. From the general questions concerning radiation and risks, we can assume that respondents largely understand the risks associated with radiation. To Q5-6 "There is radiation around us," $76.7 \%$ gave positive responses and $17 \%$ gave weak positive responses, while $0.7 \%$ gave negative responses. For Q6-4 "There are various risks," the positive response rate is high, at $55.3 \%$. 
Table 6 Responses to the questions concerning low-dose exposure

\begin{tabular}{|c|c|c|c|c|c|c|c|c|}
\hline & & Positive & $\begin{array}{c}\text { Weak } \\
\text { positive }\end{array}$ & Neutral & $\begin{array}{c}\text { Weak } \\
\text { negative }\end{array}$ & Negative & Average & Variance \\
\hline Q8-1 & $\begin{array}{l}\text { I think low-dose exposure is scary regardless of } \\
\text { exposure dose. }\end{array}$ & $23.7 \%$ & $30.7 \%$ & $20.3 \%$ & $12.7 \%$ & $11.7 \%$ & -0.42 & 1.68 \\
\hline Q8-2 & $\begin{array}{l}\text { I think the effects of low-dose exposure are } \\
\text { uncertain. }\end{array}$ & $20.3 \%$ & $34.7 \%$ & $34.0 \%$ & $5.3 \%$ & $3.3 \%$ & -0.63 & 0.95 \\
\hline Q8-3 & $\begin{array}{l}\text { I think different experts say different things about } \\
\text { the effects of low-dose exposure. }\end{array}$ & $19.7 \%$ & $30.7 \%$ & $39.7 \%$ & $5.7 \%$ & $3.0 \%$ & -0.58 & 0.93 \\
\hline Q8-4 & $\begin{array}{l}\text { We cannot trust what the government says about } \\
\text { the effects of low-dose exposure. }\end{array}$ & $19.0 \%$ & $23.3 \%$ & $41.7 \%$ & $9.3 \%$ & $5.3 \%$ & -0.41 & 1.13 \\
\hline Q8-5 & $\begin{array}{l}\text { We cannot trust what experts say about effects of } \\
\text { low-dose exposure. }\end{array}$ & $11.3 \%$ & $14.7 \%$ & $51.7 \%$ & $14.3 \%$ & $6.3 \%$ & -0.10 & 0.99 \\
\hline Q8-6 & $\begin{array}{l}\text { We cannot trust what medical doctors say about } \\
\text { effects of low-dose exposure. }\end{array}$ & $8.0 \%$ & $9.3 \%$ & $53.3 \%$ & $19.3 \%$ & $8.3 \%$ & 0.11 & 0.93 \\
\hline Q8-7 & $\begin{array}{l}\text { I think one of the effects of low-dose exposure is } \\
\text { the development of cancer in the future. }\end{array}$ & $13.3 \%$ & $25.0 \%$ & $42.0 \%$ & $9.3 \%$ & $9.3 \%$ & -0.24 & 1.20 \\
\hline Q8-8 & $\begin{array}{l}\text { I think the effects of low-dose exposure not only } \\
\text { leads to the development of cancer in the future, } \\
\text { but also influences overall health. }\end{array}$ & $20.3 \%$ & $29.7 \%$ & $32.0 \%$ & $8.7 \%$ & $8.3 \%$ & -0.45 & 1.33 \\
\hline Q8-9 & $\begin{array}{l}\text { I think low-dose exposure negatively affects } \\
\text { infants. }\end{array}$ & $32.3 \%$ & $34.0 \%$ & $25.0 \%$ & $5.7 \%$ & $2.0 \%$ & -0.89 & 0.98 \\
\hline Q8-10 & $\begin{array}{l}\text { I think low-dose exposure negatively affects } \\
\text { toddlers and children. }\end{array}$ & $31.3 \%$ & $35.3 \%$ & $24.7 \%$ & $5.3 \%$ & $2.3 \%$ & -0.88 & 0.98 \\
\hline Q8-11 & $\begin{array}{l}\text { I am not concerned about any effects of low-dose } \\
\text { exposure on myself. }\end{array}$ & $13.3 \%$ & $12.7 \%$ & $43.3 \%$ & $15.7 \%$ & $14.3 \%$ & 0.05 & 1.39 \\
\hline Q8-12 & $\begin{array}{l}\text { I think the effects of low-dose exposure are } \\
\text { transmitted to others like a virus. }\end{array}$ & $2.7 \%$ & $3.0 \%$ & $28.0 \%$ & $17.7 \%$ & $47.7 \%$ & 1.05 & 1.13 \\
\hline Q8-13 & $\begin{array}{l}\text { I think the effects of low-dose exposure are } \\
\text { passed on to offspring. }\end{array}$ & $15.3 \%$ & $16.0 \%$ & $35.3 \%$ & $14.7 \%$ & $18.0 \%$ & 0.04 & 1.64 \\
\hline
\end{tabular}

To the same question, weak positive responses comprised $32 \%$. The reason people choose weak positive responses is assumed to stem from respondents' meekness and slight doubt and hesitation. For example, for Q8-10, Q8-2, and Q8-9, concerning the effects of low-dose exposure, weak positive responses were higher for these questions than for all other questions, with response rates of $35.3 \%, 34.7 \%$, and $34 \%$, respectively. As for the effects of low-dose exposure, we can say that it reflects the respondents' feeling that they cannot determine the significance of the impact even if they are aware of its existence. As for Q6-5, Q6-6 and Q6-4, concerning the notion of risk, the weak positive responses, representing $32 \%$, are difficult to analyze, but we can interpret them to mean that respondents avoided clearly indicating their responses because it is hard to understand the precise meaning of this concept.

Also, for questions where "Neither" was likely to be chosen as the response, these are considered to be questions where respondents more clearly show their hesitation (difficult to make a determination). As for Q8-6 and Q8-5, positive responses to the questions "We cannot trust what medical doctors and radiation experts say about the effects of low-dose exposure," 
were higher for both questions, at 53.3\% and 51.7\%, respectively. According to the response trends for Q8-2 and Q8-3, "Effects of low-dose exposure are uncertain and experts say different things," it would appear that respondents were hesitant to make a response about the credibility of experts and the significance of what they say.

As for the score results, the absolute values of the average values, which show the strength of positive or negative answers, in Q6-9 and Q6-10 "The government (experts) should clearly define criteria to determine what are risks," are significant at -1.22 and -1.20 , except for questions that ask respondents about their general knowledge of radiation and risks. This indicates that people have a strong desire to have risk determination criteria defined. The values of Q8-9 and Q8-10 "Low-dose exposure negatively affects infants (toddlers)" and Q5-3 "Radiation and radioactivity are horrible," are significant at $-0.88,-0.88$, and -0.80 , respectively, and respondents gave positive responses.

Evaluating values using unbiased variances, which indicate variation among responses, the value for Q5-8 is the highest at 1.96, followed by those for Q5-14, Q5-13, Q5-9, Q5-10, and Q5-12, which are related to people's concerns. This indicates that, although the level of anxiety is low overall, the variation in awareness is significant. Also, unbiased variances are large for all the questions in Q7, concerning risks. On the other hand, in the questions asking about people's general knowledge, their unbiased variances are small and that of Q5-6 "Radiation and radioactivity are around us," was the smallest at 0.41 , which means this is common knowledge. The unbiased variances of Q6-9 and Q6-10 "The government (local government) and experts should clearly define criteria to determine what are risks," are the smallest for questions that ask about the respondents' awareness, which means opinions are not so markedly split with respect to these questions.

\section{(2) Cross analysis}

First, we would like to evaluate the differences in response between males and females. As an evaluation example, we provide the cross-tabulation results and residual analysis results for Q5-14, "I want to avoid purchasing food from Fukushima," in Table 7 (1) and (2). By using cross-tabulation, we can evaluate overall relevance (independence). In addition, residual analysis allows us to test the specificity based on the difference between the statistically independent response rate and actual response rate of each cross-tabulation section. The P-value of the cross-tabulation between the response result and response difference between males and females in Q5-14, "I want to avoid purchasing food from Fukushima," is 0.0417 with $95 \%$ probability, and relevance is observed.

This means that there is a gap between males and females in their attitude as to whether food from Fukushima should be avoided. In the table, it was marked with an asterisk (*) in the upper left column.

In Table 7 (1), $14.3 \%$ of males and $7.7 \%$ of females chose negative responses. Residual analysis showed that a high percentage of males, $99 \%$ (indicated by ++ ), chose negative responses, while a low percentage of females (indicated by --), chose negative responses. Although females are more likely than males to choose positive to neutral responses and males are slightly more likely than females to choose weak negative responses, these are not statistically significant. In this example, we tend to draw the conclusion that the strength of the relevance based on cross-tabulation is significantly low, but the residual analysis revealed that there was a strong gap between males and females for negative responses. Regarding residual analysis, the meanings of symbols are provided below the table. Hereinafter, the symbols will be used for residual analysis results.

Table 7 (3) shows characteristic results from a residual analysis perspective. For Q5-1, 
Table 7 Cross-tabulation results based on the difference between males and females (excerpted)

Q5-14 I want to avoid purchasing food from Fukushima.

\begin{tabular}{|c|c|c|c|c|c|}
\hline$*$ & Positive & Weak positive & Neutral & Weak negative & Negative \\
\hline Males & $7.0 \%$ & $8.7 \%$ & $12.0 \%$ & $7.3 \%$ & $14.3 \%$ \\
\hline Females & $10.3 \%$ & $10.3 \%$ & $15.7 \%$ & $6.7 \%$ & $7.7 \%$ \\
\hline Total & $17.3 \%$ & $19.0 \%$ & $27.7 \%$ & $14.0 \%$ & $22.0 \%$ \\
\hline \multicolumn{6}{|c|}{ (1) Cross-tabulation table (Q5-14) } \\
\hline$*$ & Positive & Weak positive & Neutral & Weak negative & Negative \\
\hline Males & & & & & ++ \\
\hline Females & & & & & -- \\
\hline \multicolumn{6}{|c|}{$\begin{array}{l}\text { (2) Residual analysis results (Q5-14) } \\
\text { Q5-1 Radiation is evil. }\end{array}$} \\
\hline ** & Positive & Weak positive & Neutral & Weak negative & Negative \\
\hline Males & & - & - & & ++ \\
\hline Females & & + & + & & -- \\
\hline \multicolumn{6}{|c|}{ Q5-3 Radiation is horrible. } \\
\hline & Positive & Weak positive & Neutral & Weak negative & Negative \\
\hline \multicolumn{6}{|l|}{ Males } \\
\hline Females & & & & & -9 \\
\hline \multicolumn{6}{|c|}{ Q8-1 Low-dose exposure is scary regardless of exposure level. } \\
\hline ** & Positive & Weak positive & Neutral & Weak negative & Negative \\
\hline Males & -- & & & & ++ \\
\hline Females & ++ & & & & -- \\
\hline
\end{tabular}

(3) Characteristic of residual analysis results

\section{Upper left}

column

$* *(*)$ Relevant with $99 \%(95 \%)$ probability.

Response rate in the section is

\begin{tabular}{cl}
\hline++ & $\begin{array}{l}\text { high with } 99 \% \text { probability. } \\
\text { high with } 95 \% \text { probability. }\end{array}$ \\
\cline { 1 - 1 }+- & low with $99 \%$ probability. \\
low with $95 \%$ probability. \\
Blank: not significant but high. \\
Gray blank: not significant but low.
\end{tabular}

"Radiation is evil," the rate of negative responses was high in males and low in females. Overall, there was a difference between males and females, with $99 \%$ probability. Conversely, there was no significant difference between males and females in responses to Q5-3, "Radiation is horrible." For Q8-1 "Low-dose exposure is scary regardless of exposure level," the positive response rate was high in females and low in males, while the negative response rate was high in males and low in females. Generally, with respect to people's concerns about radiation and the impact on health of low-dose exposure, negative responses tend to be high in males and low in females. There was no significant difference between males and females regarding their knowledge of radiation itself.

Next, in Table 3, we divided and analyzed $56 \%$ of respondents who answered they had no relatives engaged in work related to nuclear power or radiation and the $44 \%$ of respondents who answered that either they, themselves, engaged in work related to nuclear power or radiation or that they had relatives who did. As indicated in Table 8 (1), a significant difference was observed for the same question as shown in Table 7 (3). However, for Q5-8 to Q8-15, regarding concerns about radiation, and Q8-7 to Q8-10, regarding future effects of low-dose exposure, there are no significant differences. This means that there is a difference in the level of anxiety 
Table 8 Results of residual analysis based on whether respondents have relatives who are engaged in radiation-related work or not (excerpted). Symbols in this table have the same meaning as those in Table 7

Q5-1 Radiation is evil.

\begin{tabular}{|c|c|c|c|c|c|}
\hline$*$ & Positive & Weak positive & Neutral & Weak negative & Negative \\
\hline No & ++ & & & & - \\
\hline Yes & -- & & & & + \\
\hline \multicolumn{6}{|c|}{ Q5-3 Radiation is horrible. } \\
\hline$* *$ & Positive & Weak positive & Neutral & Weak negative & Negative \\
\hline No & ++ & & - & & -- \\
\hline Yes & -- & & + & & ++ \\
\hline \multicolumn{6}{|c|}{ Q8-1 Low-dose exposure is scary regardless of exposure dose. } \\
\hline$* *$ & Positive & Weak positive & Neutral & Weak negative & Negative \\
\hline No & ++ & & & & - \\
\hline Yes & -- & & & & + \\
\hline \multicolumn{6}{|c|}{$\begin{array}{l}\text { (1) Residual analysis results of questions used in Table } 7 \text { (3) } \\
\text { Q6-2 We can trust the Food Safety Commission on food safety. }\end{array}$} \\
\hline$* *$ & Positive & Weak positive & Neutral & Weak negative & Negative \\
\hline No & & & -- & & \\
\hline Yes & & & ++ & & \\
\hline \multicolumn{6}{|c|}{$\begin{array}{l}\text { Q6-3 We can trust the Ministry of Health, Labour and Welfare and Ministry of Agriculture, Forestry and Fisheries of Japan } \\
\text { food safety. }\end{array}$} \\
\hline ** & Positive & Weak positive & Neutral & Weak negative & Negative \\
\hline No & & ++ & -- & & \\
\hline Yes & & -- & ++ & & \\
\hline
\end{tabular}

(2) Characteristics of residual analysis results

between those who have relatives engaged in work related to the nuclear power and radiation industries and those who do not have relatives working in these industries. However, there is no difference in the level of anxiety regarding factors for which there is uncertainty.

Characteristic differences between these two groups are seen in the responses to Q6-2 and Q6-3, concerning trust in the Food Safety Commission, the Ministry of Health, Labour and Welfare, and Ministry of Agriculture, Forestry and Fisheries of Japan, respectively, on food safety. The results are presented in Table 8 (2). Respondents with relatives who are employed in the nuclear power and radiation industries are more likely to choose neutral answers than those without such relatives. We can see that those with relatives engaged in nuclear power and radiation work tend to hesitate from expressing their opinions where the grounds for making judgments are unclear and considering that the credibility of such organizations regarding food safety is unknown.

\section{(3) Analysis of questions on risks}

In the survey, although we capitalized risks as "RISKS" in six questions in Q6 and five questions in Q7, no annotation or description is provided for risks. By capitalizing "RISKS," we thought respondents would recognize risks and respond to questions based on their perception of them. Here, we tried to evaluate the level of respondents' knowledge on the notion of risk. In the responses to Q6-4 to Q6-7 and Q7-1 to Q7-5, the degree of positive and negative attitudes indicate the level of knowledge on the notion of risk. Even if risks are not clearly stated in other questions in Q7, some are semantic questions relevant to risks. However, we do not discuss these relationships here.

We analyze the relationships between the responses to questions where risks are clearly 
Table 9 Correlation analysis of questions on RISKS

\begin{tabular}{|c|c|c|c|c|c|c|c|c|c|c|c|}
\hline & Q6-4 & Q6-5 & Q6-6 & Q6-7 & Q6-9 & Q6-10 & Q7-1 & Q7-2 & Q7-3 & Q7-4 & Q7-5 \\
\hline & $\begin{array}{l}\text { I think there } \\
\text { are various } \\
\text { RISKS in } \\
\text { our society. }\end{array}$ & $\begin{array}{l}\text { I think } \\
\text { radiation and } \\
\text { radioactivity } \\
\text { are just one } \\
\text { of many } \\
\text { RISKS. }\end{array}$ & $\begin{array}{l}\text { I think } \\
\text { some } \\
\text { RISKS can } \\
\text { have a bad } \\
\text { influence } \\
\text { on us. }\end{array}$ & $\begin{array}{l}\text { I think } \\
\text { some } \\
\text { RISKS can } \\
\text { have a good } \\
\text { influence } \\
\text { on us. }\end{array}$ & $\begin{array}{l}\text { I think the } \\
\text { government } \\
\text { and } \\
\text { prefectures } \\
\text { (local } \\
\text { government) } \\
\text { should } \\
\text { clearly define } \\
\text { criteria to } \\
\text { determine } \\
\text { what are } \\
\text { RISKS. }\end{array}$ & $\begin{array}{l}\text { I think } \\
\text { experts } \\
\text { should } \\
\text { clearly } \\
\text { define } \\
\text { criteria to } \\
\text { determine } \\
\text { RISKS. }\end{array}$ & $\begin{array}{l}\text { I have } \\
\text { thought } \\
\text { about } \\
\text { RISKS } \\
\text { before. }\end{array}$ & $\begin{array}{l}\text { I have been } \\
\text { educated } \\
\text { about } \\
\text { RISKS. }\end{array}$ & $\begin{array}{l}\text { I know } \\
\text { how to } \\
\text { determine } \\
\text { RISKS. }\end{array}$ & $\begin{array}{l}\text { I know the } \\
\text { meaning of } \\
\text { RISKS. }\end{array}$ & $\begin{array}{l}\text { I determine } \\
\text { RISKS } \\
\text { myself. }\end{array}$ \\
\hline Q6-4 & & 0.32 & 0.24 & 0.05 & 0.05 & 0.04 & 0.10 & -0.04 & -0.04 & 0.10 & 0.08 \\
\hline Q6-5 & 0.45 & & 0.19 & -0.01 & -0.05 & 0.11 & 0.02 & 0.01 & -0.02 & -0.01 & 0.07 \\
\hline Q6-6 & 0.39 & 0.34 & & 0.09 & 0.04 & 0.05 & 0.04 & -0.12 & 0.08 & 0.02 & -0.05 \\
\hline Q6-7 & 0.04 & 0.02 & 0.09 & & -0.04 & -0.01 & -0.09 & 0.03 & -0.04 & -0.01 & 0.00 \\
\hline Q6-9 & 0.22 & 0.16 & 0.20 & -0.04 & & 0.73 & -0.04 & 0.02 & -0.07 & 0.10 & 0.00 \\
\hline Q6-10 & 0.24 & 0.22 & 0.22 & -0.03 & 0.75 & & 0.02 & -0.02 & 0.01 & -0.05 & 0.01 \\
\hline Q7-1 & 0.22 & 0.14 & 0.10 & -0.10 & 0.03 & 0.04 & & 0.10 & 0.10 & 0.22 & 0.09 \\
\hline Q7-2 & 0.02 & 0.01 & -0.07 & -0.04 & -0.04 & -0.06 & 0.36 & & 0.60 & 0.17 & -0.08 \\
\hline Q7-3 & 0.06 & 0.04 & 0.01 & -0.07 & -0.06 & -0.06 & 0.38 & 0.69 & & 0.09 & 0.14 \\
\hline Q7-4 & 0.26 & 0.15 & 0.10 & -0.06 & 0.13 & 0.08 & 0.47 & 0.42 & 0.45 & & 0.49 \\
\hline Q7-5 & 0.25 & 0.18 & 0.08 & -0.05 & 0.09 & 0.07 & 0.38 & 0.28 & 0.37 & 0.63 & \\
\hline
\end{tabular}

Upper triangular portion: Partial correlation coefficient, Lower triangular portion: correlation coefficient Outline characters indicate significant values (not zero) with $99 \%$ probability.

stated and other responses. In Table 9, the lower triangular portion across the diagonal line shows the correlation coefficient calculated based on scores allocated to each response. The upper triangular portion shows a partial correlation coefficient, eliminating the impact of questions other than the two questions being focused on. Outline numbers indicate significant values (not zero) with $99 \%$ probability. When a partial correlation coefficient is a large value, we can say that there is a direct relation (causality) between the two questions

Q6-4 to Q6-7 ask about respondents' knowledge on the notion of risk in general. The correlation coefficient and partial correlation coefficient between Q6-4 and Q6-5 become large, 0.45 and 0.32 , respectively. The partial correlation coefficient between Q6-4 and Q6-6 is 0.24 , which follows after that between Q6-4 and Q6-5. This means that those who think there are various risks in society also tend to consider radiation to be one of such risks. On the other hand, in Q6-7, "Some risks can have a good influence on us," no relationship is seen with other questions and it indicates that people do not consider risks as having an acceptable trade-off between harm and convenience.

The partial correlation coefficient between Q6-9 and Q6-10 is high at 0.73, which shows that when risk determination criteria are defined, people do not mind what entity (government, local government or experts) defines them. The partial correlation coefficients between Q6-4, Q6-5, and Q6-6 are all small. No relationship is seen between Q7-1 and Q7-5, which indicates that people's demand for having risk determination criteria defined is independent of their level of knowledge on the notion of risk.

Questions Q7-1 through Q7-5 are related to each other and their correlation coefficients are all significant. Partial correlation coefficients between Q7-2 and Q7-3 are large (0.60) suggesting that having been educated (or not educated) about risks and knowing (or not knowing) how to determine RISKS are directly related. Also, the relationship between Q7-4 and Q7-5, with a partial correlation coefficient of 0.49 , suggests that knowing the meaning of risks directly relates to people's attitude about determining risks for themselves. 
It is interesting that Q7-2, "I have been educated about RISKS," and Q7-5, "I determine what RISKS are myself," are not closely related (the correlation coefficient is 0.28 ). This suggests that there is a considerable number of respondents who determine risks by themselves even if they are not educated about them. The partial correlation coefficient is nearly zero, which suggests that there are factors other than education that influence people's attitudes about their ability to determine risks for themselves.

The relationship between Q7-3, "I know how to determine RISKS," and Q7-5, "I determine what RISKS are by myself," is relatively low. The responses in Table 5 suggest that many respondents answered that they determine risks for themselves even if they do not have a method for doing so. This may be because respondents think the risks that they determine for themselves and risks that they imagine are different. Also, we must note that the order of questions has some impact on responses throughout the survey.

Table 10 shows gender differences and differences between whether respondents have or do not have relatives who are engaged in nuclear/radiation-related industries, for questions between Q7-1 and Q7-5 concerning risks. In these questions, males tend to choose positive responses compared to females. However, in Q7-3 and Q7-5, which contain the word "determine", no difference is observed between the opinion of males and females. There are no significant differences between those with and those without relatives who are engaged in nuclear/ radiation-related-fields, for both positive and negative responses. For questions Q7-1, Q7-2, and Q7-3, many respondents with relatives who are engaged in nuclear/radiation-related fields chose neutral responses, which means that they have less confidence in their response.

\section{(4) Results of multivariate analysis}

In this paper, 44 questions are discussed. When we calculate correlation coefficients between

Table 10 Impact of respondents' attributes on questions concerning RISKS (residual analysis). Symbols in this table have the same meaning as those in Table 7

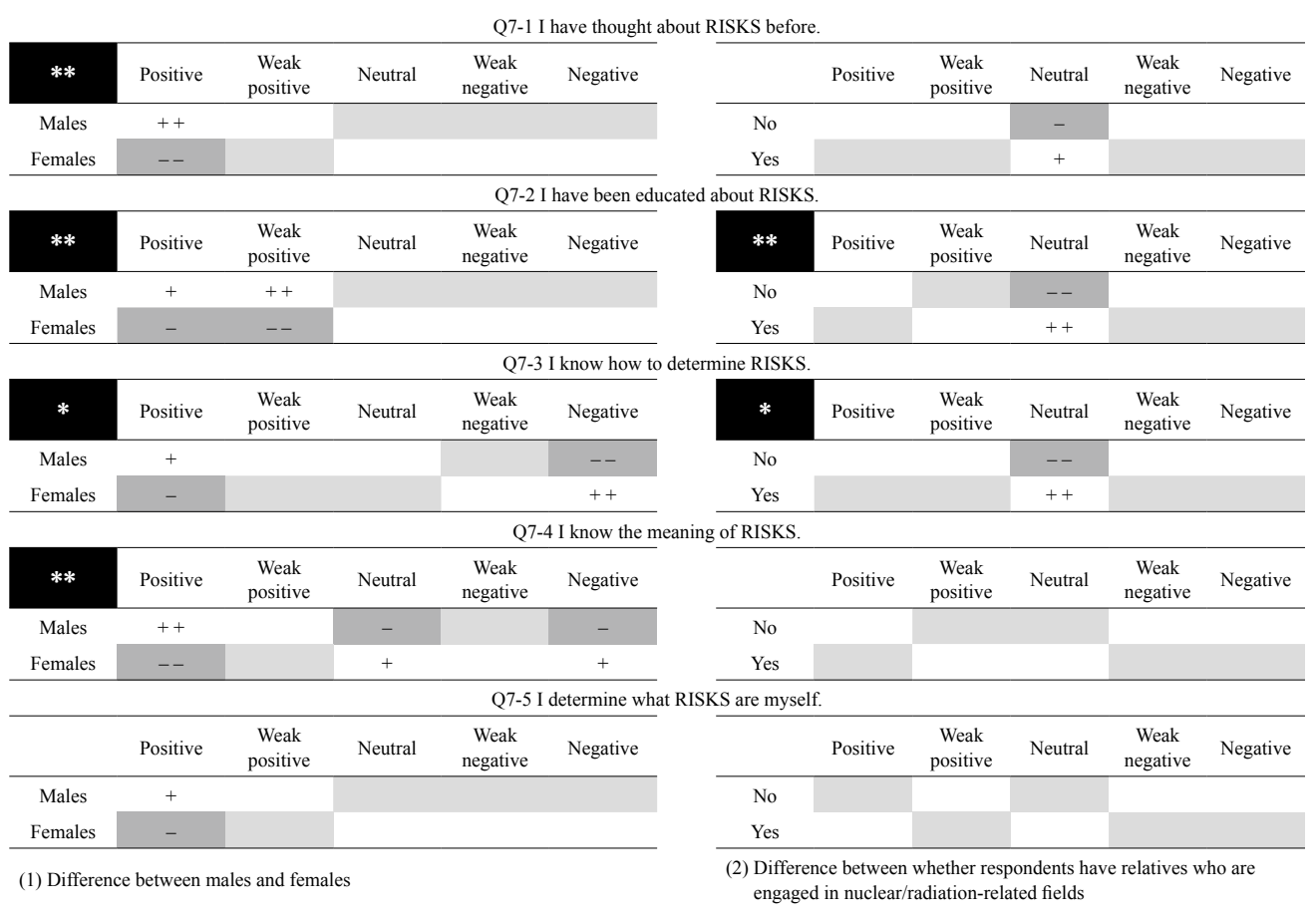


these responses, many of them are statistically significant and closely related. Response results have been analyzed individually, so far, but in this section they are analyzed using the multivariate analysis method, which uses multidimensional observed variables (*Observed variables refer to actually measured variables: Response data of the 44 questions).

(a) Adoption of factor analysis

First, we use factor analysis, which allows us to clarify latent structure. In this analysis, some observed variables may be discarded in the final analysis results because, in the course of analysis, observed variables with less commonality and those that simultaneously reflect multiple factors are eliminated and then the analysis is repeated. Also, as there are various methods used for the analysis, interpretation of results is sometimes difficult.

In this study, we used iterative principal factor analysis to extract factors considering the response distribution and Promax oblique rotation for rotation. We started with analysis of all the questions, selected questions whose absolute value of factor loading was 0.4 or more, eliminated questions that simultaneously reflect multiple factors and those with less commonality ( 0.16 and less), and then analyzed the data with an emphasis on creating a scale to show latent structure by repeating factor analysis. Table 11 shows the final results. We defined the number of factors as 5, based on the scree plot. The analysis results include $47 \%$ of all the information. The sampling adequacy ${ }^{29,30)}$ of the Kaiser-Meyer-Olkin measurement, which helps to determine the adequacy of factor analysis, is 0.8 , and we can say that it is sufficiently adequate to use factor analysis for the questions selected here. In this table, eighteen questions were excluded. These questions include those that asked about knowledge on radiation and risks.

The first factor value was large in questions from Q5-8, "I am concerned about radiation and radioactivity around us," to Q5-7, "I think there the effects of internal exposure are greater than the effects of external exposure in Fukushima Prefecture," in the list. This indicates a tendency for people to be concerned about radiation and about the effects of low-dose exposure. We call it the "Anxiety Factor." The questions include Q8-12, "I think the effects of low-dose exposure are transmitted to others like a virus," with a large Anxiety Factor value of 0.6. The second factor value was large in questions in the following order: Q7-3, Q7-2, Q7-4, Q7-1, and Q7-5. It mainly indicates that the level of knowledge on the notion of risk. We call it the "Risk Knowledge Factor." The third factor value was large in Q8-5, Q8-6, Q8-4, and Q8-3, which indicates people's distrust in the government's and experts' views regarding the effects of lowdose exposure. We call it the "Distrust Factor" and reflects distrust of sources of information on the effects of low-dose exposure. The fourth factor value was large in questions concerning food safety and indicates people's trust in food safety. We call it the "Food Safety Factor." These questions include Q8-11, "I am not concerned about any effects of low-dose exposure on myself." The fifth factor indicates people's desire to have risk determination criteria defined by the government (local government) and experts. We call it the "Demand Factor."

As we adopted the Promax method for the oblique rotation, there are correlations between the factors. Table 12 shows the correlations. Factor 1, "Anxiety Factor," has positive correlations with Factor 3, "Distrust Factor," and Factor 5, "Demand Factor," and shows negative correlations with Factor 2, "Risk Knowledge Factor," and Factor 4, "Food Safety Factor." The Risk Knowledge Factor has less of a relationship with the factors other than the Anxiety Factor. The Anxiety Factor has a negative correlation with the Food Safety Factor and a positive correlation with the Demand Factor.

Next, we provide relative features of each respondent and perform cluster analysis using factor scores, calculated for each factor, in order to get a rough estimate of the respondents' classification. For factor scores, we use the results calculated to create a scale to show latent structure. For cluster analysis, the Ward method ${ }^{31}$ (Euclidean distance) is adopted as 
Table 11 Factor analysis results

\begin{tabular}{|c|c|c|c|c|c|c|c|}
\hline & & Factor 1 & Factor 2 & Factor 3 & Factor 4 & Factor 5 & Commonality \\
\hline Q5-8 & I am concerned about radiation and radioactivity around us. & 0.80 & 0.07 & -0.09 & 0.03 & -0.04 & 0.55 \\
\hline Q5-10 & $\begin{array}{l}\text { I am concerned about radioactive elements in milk and dairy } \\
\text { products. }\end{array}$ & 0.74 & 0.09 & -0.06 & -0.01 & -0.03 & 0.50 \\
\hline Q8-1 & $\begin{array}{l}\text { I think low-dose exposure is scary regardless of exposure } \\
\text { dose. }\end{array}$ & 0.65 & -0.12 & 0.09 & 0.02 & -0.03 & 0.50 \\
\hline Q8-8 & $\begin{array}{l}\text { I think the effects of low-dose exposure have an influence on } \\
\text { overall health. (shortened) }\end{array}$ & 0.61 & -0.01 & 0.11 & -0.03 & 0.11 & 0.49 \\
\hline Q5-13 & $\begin{array}{l}\text { I am concerned about radioactive elements in food } \\
\text { (agricultural, animal and fishery products). }\end{array}$ & 0.60 & -0.04 & -0.10 & -0.16 & 0.14 & 0.48 \\
\hline Q8-13 & $\begin{array}{l}\text { I think the effects of low-dose exposure are passed on to } \\
\text { offspring. }\end{array}$ & 0.63 & 0.09 & 0.04 & -0.02 & 0.04 & 0.42 \\
\hline Q8-12 & $\begin{array}{l}\text { I think effects of low-dose exposure are transmitted to others } \\
\text { like a virus. }\end{array}$ & 0.60 & 0.06 & 0.01 & 0.14 & -0.30 & 0.32 \\
\hline Q5-3 & I think radiation and radioactivity are horrible. & 0.50 & -0.13 & -0.03 & -0.06 & 0.06 & 0.33 \\
\hline Q8-7 & $\begin{array}{l}\text { I think one of the effects of low-dose exposure is the } \\
\text { development of cancer in the future. }\end{array}$ & 0.51 & -0.07 & 0.05 & -0.06 & 0.19 & 0.42 \\
\hline Q5-7 & $\begin{array}{l}\text { I think the effects of internal exposure are greater than the } \\
\text { effects of external exposure in Fukushima Prefecture. }\end{array}$ & 0.48 & 0.04 & 0.14 & 0.19 & -0.03 & 0.23 \\
\hline Q7-3 & I know how to determine RISKS. & 0.05 & 0.78 & -0.07 & 0.02 & -0.15 & 0.60 \\
\hline Q7-2 & I have been educated about RISKS. & 0.06 & 0.71 & -0.04 & -0.02 & -0.14 & 0.49 \\
\hline Q7-4 & I know the meaning of RISKS. & 0.00 & 0.72 & -0.01 & -0.04 & 0.17 & 0.56 \\
\hline Q7-1 & I have thought about RISKS before. & -0.04 & 0.56 & 0.11 & -0.03 & 0.05 & 0.35 \\
\hline Q7-5 & I determine what RISKS are by myself. & -0.02 & 0.59 & 0.00 & -0.02 & 0.15 & 0.38 \\
\hline Q8-5 & $\begin{array}{l}\text { We cannot trust what experts say about the effects of low- } \\
\text { dose exposure. }\end{array}$ & -0.03 & -0.04 & 0.89 & -0.04 & -0.14 & 0.77 \\
\hline Q8-6 & $\begin{array}{l}\text { We cannot trust what medical doctors say about the effects of } \\
\text { low-dose exposure. }\end{array}$ & 0.00 & -0.09 & 0.69 & -0.03 & -0.09 & 0.48 \\
\hline Q8-4 & $\begin{array}{l}\text { We cannot trust what the government says about the effects } \\
\text { of low-dose exposure. }\end{array}$ & 0.07 & 0.02 & 0.66 & -0.20 & 0.08 & 0.64 \\
\hline Q8-3 & $\begin{array}{l}\text { I think different experts say different things about the effects } \\
\text { of low-dose exposure. }\end{array}$ & -0.03 & 0.08 & 0.60 & 0.11 & 0.13 & 0.38 \\
\hline Q5-11 & $\begin{array}{l}\text { I think the current government regulations ensure food } \\
\text { safety, including food from Fukushima. }\end{array}$ & -0.01 & -0.01 & -0.05 & 0.75 & -0.03 & 0.60 \\
\hline Q6-2 & We can trust the Food Safety Commission on food safety. & 0.11 & -0.04 & -0.19 & 0.64 & 0.07 & 0.45 \\
\hline Q5-15 & $\begin{array}{l}\text { I think the current regulation values for radioactive materials } \\
\text { in foods are adequate. }\end{array}$ & -0.10 & -0.02 & 0.00 & 0.64 & 0.09 & 0.47 \\
\hline Q8-11 & $\begin{array}{l}\text { I am not concerned about any effects of low-dose exposure } \\
\text { on myself. }\end{array}$ & -0.09 & 0.06 & -0.02 & 0.44 & -0.03 & 0.26 \\
\hline Q6-1 & $\begin{array}{l}\text { I think chemical toxicity is more dangerous than radioactive } \\
\text { materials in food. }\end{array}$ & -0.10 & -0.02 & 0.20 & 0.41 & 0.09 & 0.19 \\
\hline Q6-9 & $\begin{array}{l}\text { I think the government and prefectures (local government) } \\
\text { should clearly define criteria to determine RISKS. }\end{array}$ & 0.00 & -0.01 & -0.04 & 0.05 & 0.83 & 0.68 \\
\hline Q6-10 & $\begin{array}{l}\text { I think experts should clearly define criteria to determine } \\
\text { RISKS. }\end{array}$ & 0.09 & -0.01 & -0.12 & 0.02 & 0.82 & 0.68 \\
\hline \multicolumn{2}{|c|}{ Factor contribution } & 3.90 & 2.38 & 2.24 & 1.90 & 1.71 & \\
\hline \multicolumn{2}{|c|}{ Contribution rate } & 0.15 & 0.09 & 0.09 & 0.07 & 0.07 & \\
\hline \multicolumn{2}{|c|}{ Cumulative contribution } & 0.15 & 0.24 & 0.33 & 0.40 & 0.47 & \\
\hline
\end{tabular}

Table 12 Correlations between factors

\begin{tabular}{lrrrrr}
\hline & Factor 1 & Factor 2 & Factor 3 & Factor 4 & Factor 5 \\
\hline & \multicolumn{1}{c}{ Anxiety } & Risk knowledge & Distrust & Food safety & Demand \\
\hline Factor 1 & 1 & -0.21 & 0.38 & -0.44 & 0.21 \\
Factor 2 & -0.21 & 1 & 0.07 & 0.07 & -0.31 \\
Factor 3 & 0.38 & 0.07 & -0.31 & 1 & -0.20 \\
Factor 4 & -0.44 & 0.07 & 0.20 & -0.05 & 1 \\
Factor 5 & 0.21 & 0.05 & & & -0.05 \\
\hline
\end{tabular}




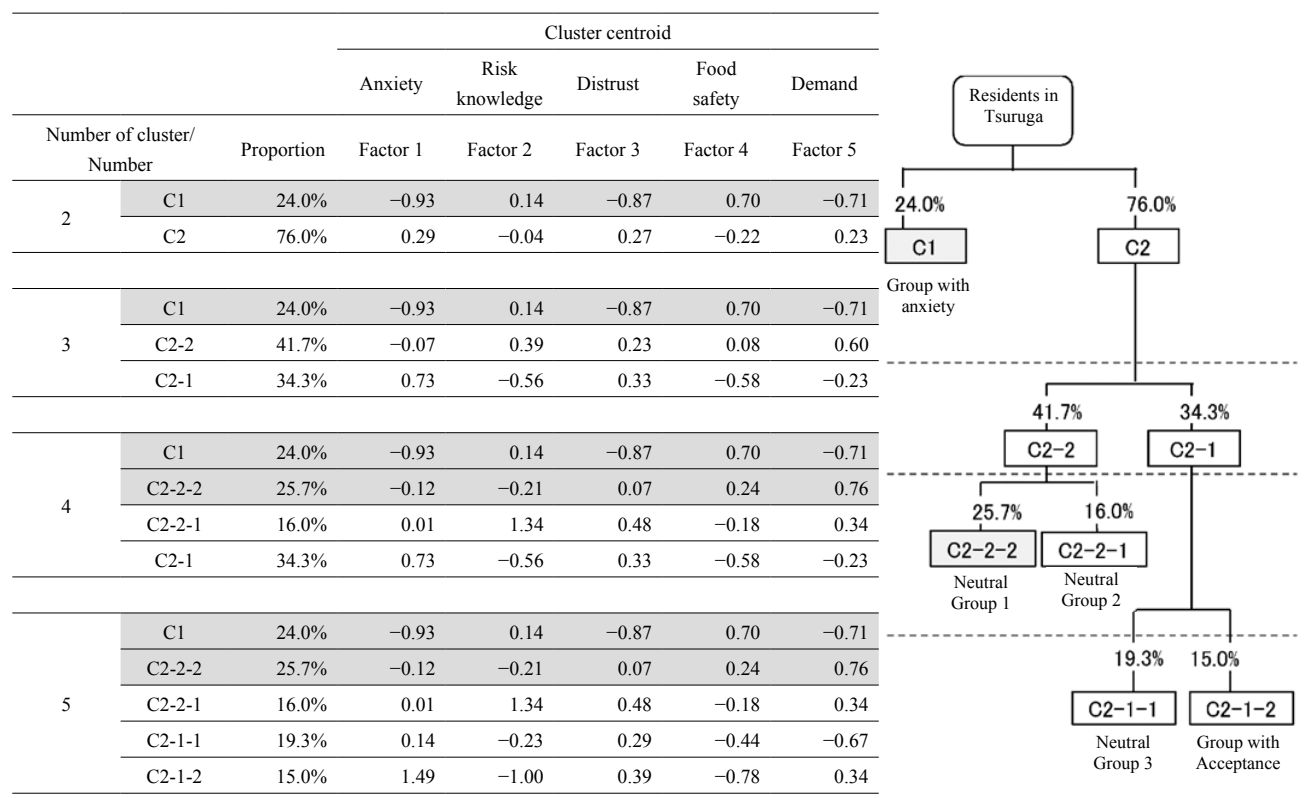

Figure 2 Results of cluster analysis based on factor scores

hierarchical clustering.

Figure 2 shows a dendrogram (tree diagram), which represents the process by which the data is summarized, and the cluster centroid of each factor per cluster classification. The cluster centroid represents the volume that indicates the features of each factor of the cluster. The positive and negative directions of values match the direction of the score allocated based on the 5-point scale (negative value indicates positive direction and positive value indicates negative direction). However, the factor score for each factor is standardized (average value: $0 /$ standard deviation: 1) in the factor and these scores represent the volumes that indicate relative features between the classified clusters.

First, we classified the respondents into two groups; Group C1 with $24 \%$ respondents and Group C2 with 76\% respondents. The cluster centroid of Factor 1, Anxiety Factor, for Group $\mathrm{C} 1$ is -0.93 , which means the respondents in this group have a high level of anxiety. Therefore, we labeled this group the "Anxiety Group." In contrast, the cluster centroid of Factor 1, Anxiety Factor, for Group C2 is 0.29 , which means that those in the group have a low level of anxiety. From the values of Factor 2, we can see that the difference in the level of knowledge on the notion of risk between the groups is small. The value of Factor 3 for Group C1 was -0.87 , which indicates that the respondents in the group distrust the views expressed about the effects of low-dose exposure. On the other hand, the value for Group C2 was 0.27 , which represents a less pronounced feeling of distrust. From the values of Factor 4, we can see that those in Group $\mathrm{C} 1$ do not have trust in food safety but those in Group C2 group do. From the values of Factor 5 , we can see that the demand for risk determination criteria to be defined is stronger among the respondents in Group C1 than it is among those in Group C2.

Next, we classified the respondents into three group; Group C2 is divided into C2-1 with $34.3 \%$ of respondents and $\mathrm{C} 2-2$ with $41.7 \%$ of respondents. From an analytical viewpoint, Group C2-1 and Group C2-2 are integrated into the C2 group. Group C1 (Anxiety) remains unchanged. Respondents in Group C2-1 tend to have less anxiety (0.73), are knowledgeable about the notion of risk $(-0.56)$, have less distrust of views on the impacts of low-dose exposure 
(0.33), have strong confidence in food safety $(-0.58)$ and demand that risk determination criteria be defined (-0.23). The features of Group C2-2 stand in contrast to those of Group C2-1 except for Factor 3, Distrust Factor and is close to those of Group C1 (Anxiety).

When we classify the respondents into four groups, Group C2-2 is divided into C2-2-1 with $16 \%$ of respondents and C2-2-2 with $25.7 \%$ of respondents. When classifying them into five groups, Group C2-1 is divided into C2-1-1 with 19.3\% and C2-1-2 with 15.0\%. Group $\mathrm{C} 1$ (Anxiety) remains unchanged. After the respondents were divided into two classifications, we can see that there is a big gap between the other groups. In cluster analysis, information to optimize the number of clusters is not given. In this study we divide respondents into five classifications, summarize the features of each group based on the classification that supports the dendrogram, and label them. In this analysis, the group showing a neutral trend was further classified into threes and we were able to provide comparative features of the five groups.

We labeled C2-2-2, C2-2-1, and C2-1-1 groups as "Neutral Group 1," "Neutral Group 2," and "Neutral Group 3," respectively. The anxiety levels of these three groups are almost the same and were at an intermediate level between Group C1 (Anxiety Group) and Group C2-1-2. As many of their other factors also show neutral features, we considered these three groups as neutral groups.

The features of Group C2-1-2 are in stark contrast to Group C1 (Anxiety Group). The respondents in C2-1-2 are different from those in other groups in having less anxiety (1.49), having knowledge on the notion of risk $(-1.00)$ and showing confidence in relation to food safety $(-0.78)$. These scores indicate those in the group accept the status quo and we labeled the group the "Acceptance Group." The features of the five classifications are clearly shown in Table 13, which indicates the order of groups on a factor basis. For example, compared to other groups, Group C1 (Anxiety Group) is ranked first in Factor 1, "Anxiety Factor," which indicates that respondents in the group have the strongest anxiety. The group is ranked fourth in Factor 2. As for the level of knowledge on the notion of risk, Group C2-2-1 (Neutral 2) is ranked fifth, the lowest.

Next, Table 14 shows features based on the differences between males and females and whether respondents have or do not have relatives who are engaged in nuclear/radiation-related fields. Slightly more females are in Group C1 (Anxiety Group) and more males are in Group C2-1-2 (Acceptance Group).

Also, more females are in C2-2-1 (Neutral 2). As for the difference based on whether respondents have or don't have relatives who are engaged in nuclear/radiation-related fields, slightly more respondents who answered "No" are in Group C1 (Anxiety Group) and more respondents who answered "Yes" are in Group C2-1-2 (Acceptance Group).

Table 13 Features of five classified groups (ranking)

\begin{tabular}{ccccccc}
\hline & Proportion & Anxiety & Risk knowledge & Distrust & Food safety & Demand \\
\cline { 2 - 7 } & Factor 1 & Factor 2 & Factor 3 & Factor 4 & Factor 5 \\
\hline $\begin{array}{c}\text { C1 } \\
\text { Anxiety Group } \\
\text { C2-2-2 } \\
\text { Neutral Group 1 } \\
\begin{array}{c}\text { C2-2-1 } \\
\text { Neutral Group 2 } \\
\text { C2-1-1 }\end{array}\end{array}$ & $24.0 \%$ & 1 & 4 & 1 & $\mathbf{5}$ & 1 \\
\hline $\begin{array}{c}\text { Neutral Group 3 } \\
\text { C2-1-2 }\end{array}$ & $16.0 \%$ & 3 & 3 & 2 & 4 & $\mathbf{5}$ \\
Acceptance Group & $15.3 \%$ & 4 & $\mathbf{5}$ & $\mathbf{5}$ & 3 & 3 \\
\hline
\end{tabular}


Table 14 Evaluation of five classified groups based on respondents' attributions. Symbols in this table comply with those in Table 7

\begin{tabular}{|c|c|c|c|c|c|}
\hline \multicolumn{6}{|c|}{ Difference among males and females } \\
\hline$* *$ & Anxiety Group & Neutral Group 1 & Neutral Group 2 & Neutral Group 3 & Acceptance Group \\
\hline Males & - & & -- & & ++ \\
\hline Females & + & & ++ & & -- \\
\hline \multicolumn{6}{|c|}{ Whether respondents have relatives who are engaged in nuclear/ radiation-related fields } \\
\hline$*$ & Anxiety Group & Neutral Group 1 & Neutral Group 2 & Neutral Group 3 & Acceptance Group \\
\hline No & + & & & & -- \\
\hline Yes & - & & & & ++ \\
\hline
\end{tabular}

(b) Application of Principal Component Analysis (PCA)

In the factor analysis, some questions were excluded from an analysis accuracy point of view and therefore it became hard to see what impact the level of knowledge on the notion of risk had. Therefore, we adopted PCA, which allows us to perform analysis using all questions. In PCA, several common components are extracted from multidimensional observed variables and are referred to as principal components. Impact levels of observed variables on the principal components are calculated. The sum of the impact level times observed variables is the principal component. The percentage of principal components that have response results information is called the contribution rate.

The contribution rates are large in the order of the first, second and third principal factors. As each principal component is uncorrelated, the evaluation is easy.

Although PCA is often used for pre-evaluation of factor analysis, the purposes of these two analyses are different. While factor analysis aims to identify potential factors that have an impact on observed variables, PCA aims to create simple (composite) variables from observed variables and allows us to develop new scales where information is aggregated, and discover the structures of observed variable groups (conscious structures).

The first principal component with a $19.5 \%$ contribution rate is an indicator that shows anxiety and safety. Fear of radiation, perception that radiation is evil, fear, concerns and distrust of viewpoints on the effects of low-dose exposure, and people's attitude of avoiding purchasing food from or around Fukushima have similar degrees of impact of and are in the same direction as anxiety. On the other hand, the attitudes that low-dose exposure is not a concern and the government (local government) and experts can be trusted have similar degrees of impact of and are in the opposite direction to anxiety. Although knowledge of radiation, which was eliminated in factor analysis, is oriented in the direction opposite to anxiety, the degree of impact is low. Similarly, as we see from the five questions in Q7 that, although the level of knowledge on risks is oriented opposite to the direction of anxiety, the degree of impact is also low. Of these questions, Q7-3 "I know how to determine RISKS" has a high impact on the sense of safety.

The second principal component with a $8.2 \%$ contribution rate has a high impact on risk awareness and has a similar degree of impact of and is in the same direction as knowledge of radiation. Anxiety about radiation has an impact in the opposite direction to the level of knowledge on the notion of risk, but the value is smaller. Concerns regarding the impacts of low-dose exposure are oriented in the same direction as the level of knowledge on the notion of risk. The third principal component with a $7.2 \%$ contribution rate connects trust and distrust and the level of knowledge on the notion of risk and distrust. The contribution rates of the fourth and the following principal components are small.

When we evaluate the structure of people's awareness based on these, "Safety-Anxiety" and "Trust-Distrust," and "Knowledge on the notion of risk" and "Knowledge on radiation" 
are strongly related in the same directions, respectively. However, the relationships between "Safety-Anxiety" - "Trust-Distrust" and "Knowledge on the notion of risk" - "Knowledge on radiation" are low. Figure 3 shows the awareness index based on the PCA results.

\section{Summary and Discussion of the Survey}

This survey was a small awareness survey with 300 respondents. Although special care is required when discussing such values as response rates, we believe the analysis and evaluation of patterns were conducted well based on the impact on health of radiation and the differences between the levels of knowledge on the notion of risk as defined in this study.

In questions that ask respondents about their knowledge of radiation and risks, Q5-6, "Radiation and radioactivity are around us," were most strongly agreed to by respondents, followed by Q6-4, Q5-4, Q5-5, Q6-5 and Q5-6. On the other hand, Q8-12 "Effects of low-dose exposure are transmitted to others like a virus" was the concept most strongly rejected by respondents. There were no significant gaps in the nuclear power-related knowledge between males and females or those with and without relatives engaged in nuclear power-related fields. This means that residents of Tsuruga, overall, have a certain level of general knowledge of radiation and its associated risks.

Regarding attitudes to radiation, Q5-3, "Radiation is horrible," which is designed to ascertain people's awareness and beliefs, was most strongly agreed to by respondents, followed by Q8-9 and Q8-10, which ask about the effects of low-dose exposure. In the awareness survey ${ }^{11}$ conducted by Kimura and others in January 2014, there was a statement question that "Radiation and radioactivity are horrible," the responses to which informed us about the awareness of residents in the Tokyo metropolitan area and members of the Atomic Energy Society of Japan around the same time. Table 15 shows the results of the comparison made in this study. The

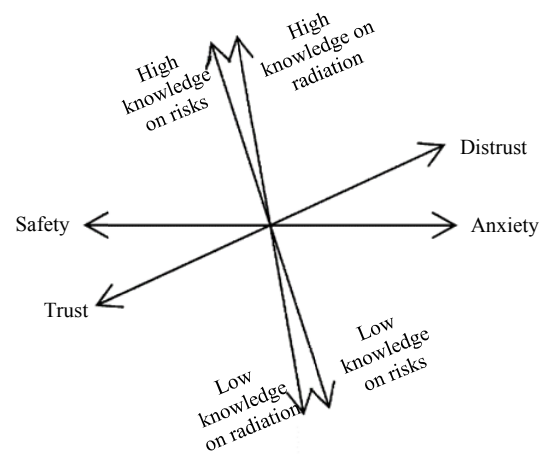

Figure 3 Awareness index based on PCA results

Table 15 Comparisons with other surveys

\begin{tabular}{lrrrrr}
\hline I think radioactivity and radiation are horrible. & \multicolumn{1}{l}{} \\
\hline & Positive & $\begin{array}{c}\text { Weak } \\
\text { positive }\end{array}$ & Neutral & $\begin{array}{c}\text { Weak } \\
\text { negative }\end{array}$ & Negative \\
\hline Residents in the Tokyo metropolitan area ${ }^{\text {a) }}$ & $63.6 \%$ & $26.6 \%$ & $8.6 \%$ & $0.8 \%$ & $0.4 \%$ \\
\hline Members of Atomic Energy Society of Japan $^{\text {a) }}$ & $8.8 \%$ & $26.2 \%$ & $25.1 \%$ & $18.3 \%$ & $21.0 \%$ \\
\hline Residents in Tsuruga city & $36.7 \%$ & $28.0 \%$ & $21.3 \%$ & $5.0 \%$ & $8.0 \%$ \\
\hline
\end{tabular}

a) Special Committee of Atomic Energy Society of Japan "Survey on energy and nuclear" Survey in FY $2013{ }^{11}$ 
differences in awareness between residents of Tsuruga and those in the Tokyo metropolitan area are assumed to be due to the distance from Fukushima and whether the area is a Power Plant Located Area. There were no marked differences in awareness between males and females, but differences were observed between those with and without relatives who are engaged in nuclear power-related fields.

As for the effects of low-dose exposure, people are concerned about this issue with respect to infants, toddlers and children, but, in Q8-7 and Q8-8, where victims are not specified, respondents show slightly less concern. In the responses to Q8-11, which showed that respondents are not concerned about such impacts on themselves, opinions were split and $43.3 \%$ of respondents answered "Neither," which shows respondents find it hard to form an opinion. Also, fewer respondents affirmed the statement in Q8-1, "Low-dose exposure is dangerous," than Q5-3, "Radiation is horrible." Differences were observed in these responses between males and females, but they were not marked between those with and without relatives who work in nuclear power-related fields.

Responses reflecting people's anxiety about radiation are widely distributed and overall such anxiety is negated. It is clear that there was a high rate of negative responses to Q5-8, Q5-9 and Q5-10, which asks respondents if they are concerned about radiation affecting their tap water, and milk, respectively. There were observable differences in these responses between males and females, but these differences were not marked between those with and without relatives working in the nuclear power field. It seems people in general are relatively unconcerned about radiation and the effects of low-dose exposure.

One of the features about the responses from residents of Tsuruga City was that, although they have a certain level of knowledge on radiation and risks and try to manage concerns about low-dose exposure, they also show anxiety at the same time. Also, overall, they feel strongly that risk determination criteria should be defined by the government and experts, but the responses were split.

We classified respondents into five groups using factor analysis and cluster analysis; Group C1 (Anxiety Group) and Group C2-1-2 (Acceptance Group) at opposite ends and three neutral groups comprising Groups C2-2-2, C2-2-1 and C2-1-1. Figure 4 shows the analysis results where the centroids of the five layered clusters organized by factor basis. The bar graph values show the relative strength of the features that each factor indicates based on the values in Figure 2.

The level of knowledge on the notion of risk of Group C1 (Anxiety Group), which has the highest level anxiety among the groups, was at moderate level and is not at a low level when compared with other groups. The anxiety and distrust shown by Group C2-2-1 (Neutral 2), which members have the lowest level of knowledge on the notion of risk, were at the intermediate level, between the other two neutral groups. The knowledge of Group C2-2-2 (Neutral 1) regarding the notion of risk was at a moderate level and the level of anxiety was higher than that in the other two neutral groups. Considering this together with the results of PCA, we can say that the level of knowledge on the notion of risk and Safety-Anxiety are not simply correlated. It was confirmed that the deficit model ${ }^{32}$, i.e., lack of knowledge on radiation and risks leads to anxiety, does not apply to residents of Tsuruga City. We found that knowing how to determine risks and the feeling of safety and security tend to be connected.

The demand that risk determination criteria be defined by the government and experts is high in Group C1 (Anxiety) and C2-1-1 (Neutral 3), and is at the same level in both groups, and is lowest in Group C-2-2 (Neutral). That in Group C2-2-1 (Neutral 2) and C2-1-2 (Acceptance) is almost the same, at the moderate level. The level of knowledge on the notion of risk is highest in Group C2-1-2 (Acceptance) and lowest in Group C2-2-1 (Neutral 2). The level of knowledge is high in Group C2-1-1 (Neutral 3), followed by C-2-2 (Neutral 1) and C1 (Anxiety) at a 


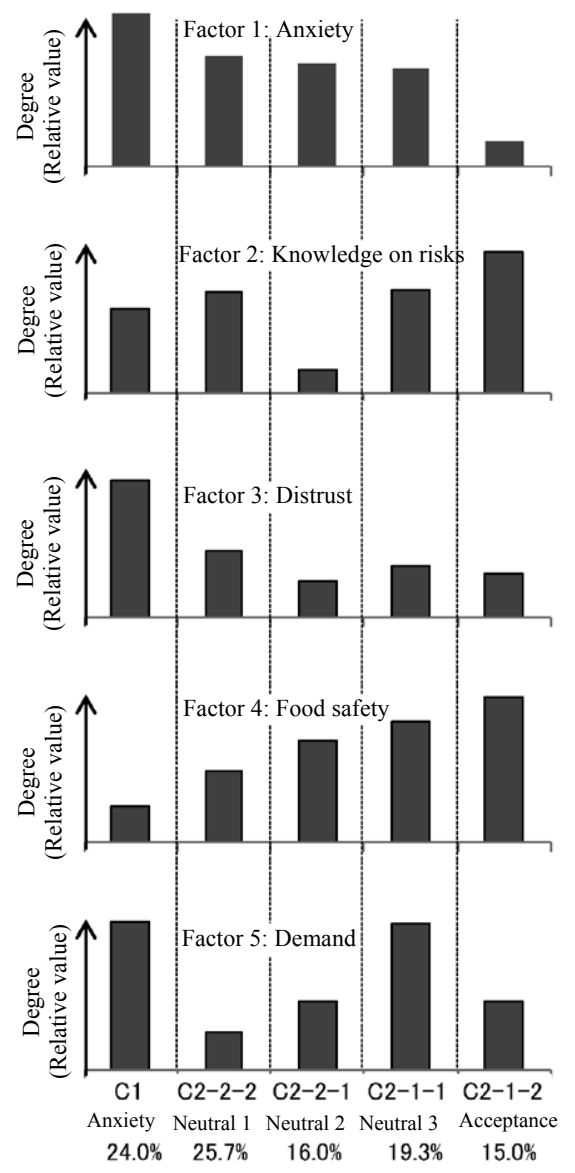

Figure 4 Features of the five classified groups

moderate level. The level of the demand to have risk determination criteria defined was about the same in Group C2-2-1 (Neutral 2), which has the highest level of knowledge on the notion of risk, as that in C2-1-2 (Acceptance), which has the lowest level of knowledge on the notion of risk. According to the correlation analysis provided in Table 9, demand for having risk determination criteria defined is independent of their level of knowledge on the notion of risk. It is explained by the features of each group.

Neutral Groups have intermediate features between Group C1 (Anxiety) and Group C2-1-2 (Acceptance) in most of the factors. For the Anxiety Factor and the Food Safety Factor, the values are high or low in the order of the groups listed in Figure 2, while, for other factors, values are irregularly distributed. Also, the table shows that knowledge on the notion of risk and distrust of experts that characterized Group C2-2-1 (Neutral 2) and the demand of Group C2-2-2 (Neutral 1) for having risk determination criteria defined exceed the features of Group C1 (Anxiety) and Group C2-1-2 (Acceptance), respectively. It is important to understand the features of the three neutral groups classified in Figure 2.

Group C2-2-2 (Neutral 1) (25.7\%) tends to choose "Neither" for questions in Tables 4 to 6 compared to other groups and this trend is observed in about half of the questions. This rate is high considering that the respondents in C2-2-1 (Neutral 2) responded "Neither" in about one third of the questions and Groups C2-1-1 (Neutral 3), C1 (Anxiety) and C2-1-2(Acceptance) in $1 / 4$ of questions. We can distinguish the features of neutral groups by looking at this 
trend of choosing "Neither." Only a few percent of all respondents responded negatively to the questions regarding their demand to have risk determination criteria defined by the government and experts, and most of them are in Group C2-1-2 (Acceptance). Responses to the questions regarding the demand to have risk determination criteria defined are concentrated in positive responses. The fact that Group C2-2-2 (Neutral 1) tended to choose "Neither" for their answers shows that their demand is weak overall. It means this group is highly likely to choose "Neither" and tends to choose "Neither" in the questions regarding demand for having risk determination criteria defined. This means they have the lowest demand for having risk determination criteria defined. We can say that Group C2-2-2 is the most typical neutral group that chooses neutral answers.

The respondents in Group C2-2-1 (Neutral 2) (16\%) think they lack knowledge regarding the notion of risk. They feel anxious about radiation to some degree, but they have the lowest level of distrust regarding information from government and experts about low-dose exposure. We thus assumed that their tendency to avoid determining whether to trust information from the government or experts led to an unwillingness to express distrust. They are considered to be a passive neutral group with little interest.

Although the characteristics of Group C2-1-2 (Neutral 3) (19.3\%) are close to those of C2-1-2 (Acceptance) as seen in Figure 2, they are very different in terms of the degree of anxiety and demand for having risk determination criteria defined. From the features of Group C2-1-2 (Acceptance), it is assumed that strong anxiety leads to strong demand. We can assume that the differences between the two groups are mostly dependent on whether they can accept the current status or not.

Both Group C1 (Anxiety) (24\%) and Group C2-1-1 (Neutral 3) (19.3\%) have high anxiety and strongly demand that risk determination criteria be defined. It shows neither group is satisfied with the current status.

It must be noted that the characteristics and rates of the groups described in the survey refer to only residents of Tsuruga City and not to people in general. Since the characteristics of residents could differ significantly depending on the area surveyed, it is desirable to compare them with similar awareness surveys conducted in other areas. We would like to analyze and evaluate the results of this study further and utilize them for research activities being undertaken in Tsuruga City in Fukui Prefecture by the authors.

\section{Conclusions}

This study performed an awareness survey and provided and discussed the analysis results related to how aware the residents of Tsuruga City are of radiation and evaluated their level knowledge on the notion of risk. In the awareness survey, we explored respondents' knowledge of and opinions about radiation and the notion of risk and tried to classify respondents based on their relevant opinions. From the classification results based on factor analysis and cluster analysis, we classified the respondents into five groups; two groups at opposite ends, the Anxiety Group (24\%) and Group of Acceptance (15\%) and three neutral groups $(25.7 \%, 16 \%$ and $19.3 \%$ respectively) and discussed their features. We think that classifying the neutral group, which tends to show a neutral attitude, into three sub-groups, based on features, provided useful information. We would like to formulate a model of a community-based risk communication method with the residents of Tsuruga City, utilizing the information acquired in this study. 
We would like to express our gratitude to Tomio Kinoshita, a fellow of International Institute for Advanced Studies, the Professor Emeritus of Kyoto University, for his useful advice and cooperation throughout the course of this study. This study was undertaken with a Grant-in-Aid for Scientific Research (25420902) from JSPS.

\section{References}

1) Naoki Yamano, Statement "How to Judge Low Dose Radiation Exposure," the eleventh page of the Mainichi Newspapers, October 29, 2013.

2) Policy and Publication Relations Office of Cabinet Office, "Public Opinion Poll on Radioactivity." http://www8.cao.go.jp/survey/s37/S37-07-37-05.html 1963. (final confirmation in May 2014).

3) Japan Atomic Energy Relations Organization, Consciousness Survey on the Word "Radiation," 2002.

4) Yuko Matsui, "The Relationship between Radiation Risks and Anxiety - From Comparison between Chest Radiography and Nuclear Power Plant -," INSS J, 10, 63-70 (2003).

5) Takeshi Murai, "Current Situation of Radiation Education and Survey on Radiation Consciousness," INSS J, 20, 28-37 (2013).

6) Fukushima City, Report on Citizen Consciousness Survey on Radiation. http://www.city.fukushima. fukushima.jp/uploaded/attachment/14143.pdf 2012. (final confirmation in May 2014).

7) Monetary Evaluation of Mitigation Measures of Nuclear Accident Research Group, Survey of Residents' Consciousness on Decontamination Implementation Plan. http://fukushima-survey.com 2013. (final confirmation in May 2014).

8) Tomoko Tsuchiya, Report on the Investigation of Citizens and Experts about Earthquakes, Tsunamis and Radiation. http://pari.u-tokyo.ac.jp/unit/ppcbpr_report2013.pdf 2013. (final confirmation in May 2014).

9) Keio University, "Special Survey on the Great East Japan Earthquake" (1st) - How did the psychology and behavior of the Japanese changed due to the earthquake?. http://www.keio.ac.jp/en/press_release/2011/kr7a430000094z75-att/120215_1.pdf 2012. (final confirmation in May 2014).

10) Japan Atomic Energy Relations Organization, Public Opinion Poll on Knowledge Dissemination and Enlightenment of Nuclear Energy Utilization. http://www.jaero.or.jp/data/01jigyou/tyousakenkyu.html 2012. (final confirmation in May 2014).

11) Special Committee of Atomic Energy Society of Japan, Questionnaire Survey on Energy and Nuclear Power (2013). http://ponpo.jp/forum/gakkai25.html 2014. (final confirmation in May 2014).

12) Consumer Affairs Agency, Consumer Consciousness Survey on Food Safety. http://www.caa.go.jp/jisin/ pdf/110715press.pdf 2011. (final confirmation in May 2014).

13) Consumer Affairs Agency, About the Results of Consumer Survey on Rumor Damage - Survey of Consciousness on Radioactive Substance etc. in Food -. http://www.caa.go.jp/safety/pdf/130311kouhyou_1. pdf 2013. (final confirmation in May 2014).

14) Environmental Marketing Theory Section, Graduate School of Global Environmental Studies, Kyoto University, Consumer Questionnaire on Radiation Exposure from Food. http://www.eeso.ges.kyoto-u. ac.jp/emm/ 2011. (final confirmation in May 2014).

15) Kazunari Minotani, "Empirical Study on Nuclear Information and Nuclear Power Consciousness" [I]: Focusing on the Statistical Survey in Tsuruga City, Fukui Prefecture, Institutional Repository of Rissho University, 1981, 29-77.

16) Kazunari Minotani, "Empirical Study on Nuclear Information and Nuclear Power Consciousness" [II]: Focusing on the Statistical Survey in Tsuruga City, Fukui Prefecture, Institutional Repository of Rissho University, 1983, 1-69.

17) Kazunari Minotani, "Empirical Study on Nuclear Information and Nuclear Power Consciousness" [I]: Focusing on the Statistical Survey in Kashiwazaki City, Niigata Prefecture, Institutional Repository of Rissho University, 1982, 61-91.

18) Kazunari Minotani, "Formation/Transformation of Nuclear Consciousness and Acceptance of Nuclear Information": Focusing on the Specimen Investigation in Hamaoka Town, Shizuoka Prefecture, Institutional Repository of Rissho University, 1985, 133-166.

19) Benibana Saru, Wataru Shōda, "A Study on Risk Cognition Attitudes of Residents near Nuclear Power Plants towards Nuclear Power," Ergonomics, 36 [4], 215-221 (2000).

20) Institute for Policy Sciences, Grasping Inhabitants Awareness of the Nuclear Location Area, Relationship with the Nuclear Location Area in the Era of Power Liberalization by Survey of Other Enterprises and Overseas Cases, etc. http://www.ifeng.or.jp/wordpress/wpcontent/uploads/2012/06/CR-2001-03. pdf 2002 (final confirmation in May 2014).

21) Takaaki Kato, Masahisa Hatta, Masashi Nishikawa, Shiro Matsumoto, "Economic and Financial Benefits 
as a Compensation for Living Near a Nuclear Power Station A Case Study of Kashiwazaki-Kariwa Nuclear Power Station,” Trans. At. Energy Soc. Jpn., 6 [4], 404-420 (2007).

22) Motoko Kosugi, Tomoko Tsuchiya, Residents' Consciousness on Nuclear Power and Energy Problems in Fukui Prefecture, Report of Central Research Institute of Electric Power Industry, 2007.

23) Motoko Kosugi, Mami Kamiya, Tomoko Tsuchiya, Residents' Consciousness on Nuclear Power and Energy Problems in Ibaraki Prefecture, Report of Central Research Institute of Electric Power Industry, 2008.

24) Megumi Ono, Yoshiumi Kawamoto, "Evaluation of Living Environment in Nuclear Location Areas," Bulletin of the Regional Environmental Research and Education Center, University of Fukui, 15, 115122 (2008).

25) Outline of the results of the Local Community and Nuclear Power Questionnaire Survey II by the Local Community and Nuclear Power research team, Regional Studies Institute, Ibaraki University, 2011.

26) Outline of the results of the Questionnaire Survey on Nuclear Accident and Everyday Life by the Local Community and Nuclear Power research team, Regional Studies Institute, Ibaraki University, 2011.

27) Masatake Kamizono, Takayasu Eguchi, Kohei Seki, "Consciousness Structure of Matsue Citizen towards the Operation of Shimane Nuclear Power Station,” San'in Research (No. 5), 2012.

28) Shigenobu Aoki, Statistics self-study note, http://aoki2.si.gunma-u.ac.jp/lecture/index.html 2011. (final confirmation in May 2014).

29) H. Kaiser, J. Rice, “Little jiffy, mark IV," Educ. Psychol. Meas., 34, 111-117 (1974).

30) H. Kaiser, "An index of factorial simplicity," Psychometrika, 39[1], 31-36 (1974).

31) J. Word, "Hierarchical grouping to optimize an objective function," J Am Stat Assoc, 58[301], 236-244 (1963).

32) J. Ziman, "Public understanding of science," Sci Technol Hum Val, 16, 99-105 (1991). 


\section{-Appendix: Survey of Tsuruga Inhabitants Concerning their Living Situation and Attitudes to Radiation (Only questions relevant to this study are excerpted. The layout of the questionnaire is provided as in the original.)-}

【Tell us your opinions concerning radiation and radioactivity.】

Q5. Do you agree or disagree with the statement in Questions 1) to 14) about radiation and radioactivity. Circle the number of the answer that best fits your opinion.

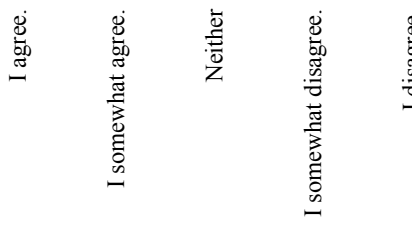

1) I think radiation and radioactivity are evil.

2) I cannot classify radiation and radioactivity as good or bad.

3) I think radiation and radioactivity are horrible.

4) I think radiation and radioactivity can sometimes be useful to human beings.

5) I think radiation and radioactivity can be horrible or not horrible, depending on the amount.

6) I think radiation and radioactivity are around us.

7) I think the effects of internal exposure are greater than the effects of external exposure in Fukushima Prefecture.

8) I am concerned about radiation and radioactivity around us.

9) I am concerned about radioactive elements in tap water.

10) I am concerned about radioactive elements in milk and dairy products.

11) I think the current government regulations ensure food safety, including food from Fukushima.

12) I want to avoid purchasing milk and dairy products from Ibaraki and Tochigi.

13) I am concerned about radioactive elements in food (agricultural, animal and fishery products).

14) I want to avoid purchasing food (agricultural, animal and fishery products) from Fukushima.

15) I think the current regulation values for radioactive materials in foods are adequate.

\begin{tabular}{|c|c|c|c|c|}
\hline & $\downarrow$ & $\downarrow$ & $\downarrow$ & $\downarrow$ \\
\hline & 2 & 3 & 4 & 5 \\
\hline 1 & 2 & 3 & 4 & 5 \\
\hline 1 & 2 & 3 & 4 & 5 \\
\hline & 1 & + & + & -1 \\
\hline 1 & 2 & 3 & 4 & 5 \\
\hline & + & + & 4 & -1 \\
\hline 1 & 2 & 3 & 4 & 5 \\
\hline 1 & 2 & 3 & 4 & 5 \\
\hline & 1 & + & + & -1 \\
\hline 1 & 2 & 3 & 4 & 5 \\
\hline 1 & 2 & 3 & 4 & 5 \\
\hline & 4 & + & + & -1 \\
\hline 1 & 2 & 3 & 4 & 5 \\
\hline 1 & 2 & 3 & 4 & 5 \\
\hline$\vdash$ & + & + & + & -1 \\
\hline 1 & 2 & 3 & 4 & 5 \\
\hline & 1 & + & + & -1 \\
\hline 1 & 2 & 3 & 4 & 5 \\
\hline 1 & 2 & 3 & 4 & 5 \\
\hline$\vdash$ & 1 & + & + & -1 \\
\hline 1 & 2 & 3 & 4 & 5 \\
\hline 1 & 2 & 3 & 4 & 5 \\
\hline ⺊ & + & 4 & & \\
\hline
\end{tabular}


【Tell us your opinions concerning RISKS and food safety.】

Q6. Do you agree or disagree with each statement of Questions 1) to 11) about RISKS and food safety. Circle the number of the answer that best fits your opinion.

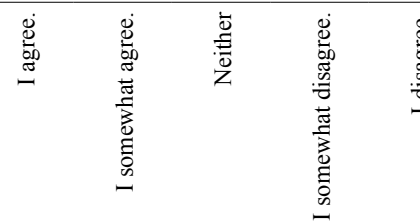

1) I think chemical toxicity is more dangerous than radioactive materials in food.

$\begin{array}{lllll}\downarrow & \downarrow & \downarrow & \downarrow & \downarrow \\ 1 & 2 & 3 & 4 & 5\end{array}$

2) We can trust the Food Safety Commission on food safety.

3) We can trust the Ministry of Health, Labour and Welfare and Ministry of Agriculture, Forestry and Fisheries of Japan on food safety.

4) I think there are various RISKS in our society.

$1+1$

$\begin{array}{lllll}1 & 2 & 3 & 4 & 5\end{array}$

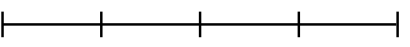

$\begin{array}{lllll}1 & 2 & 3 & 4 & 5\end{array}$

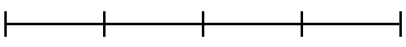

$\begin{array}{lllll}1 & 2 & 3 & 4 & 5\end{array}$

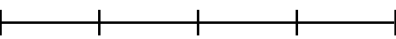

5) I think radiation and radioactivity are just one of many RISKS.

6) I think some RISKS can have a bad influence on us.

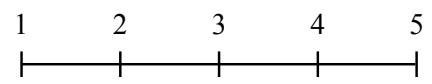

$\begin{array}{llll}2 & 3 & 4 & 5\end{array}$

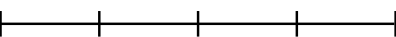

7) I think some RISKS can have a good influence on us.
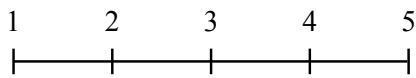

$\begin{array}{lllll}1 & 2 & 3 & 4 & 5\end{array}$

8) I think mineral water is safer than tap water.

9) I think the government and prefectures (local government) should clearly define criteria to determine RISKS.

10) I think experts should clearly define criteria to determine RISKS.

11) I think domestic food contains less radioactive materials than imported food.
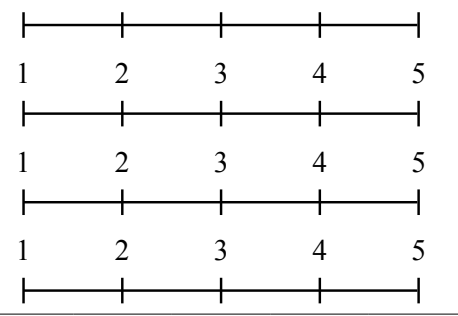

Q7. Do you agree or disagree with each statement of Questions 1) to 5) about RISKS. Circle the number of the answer that best fits your opinion.

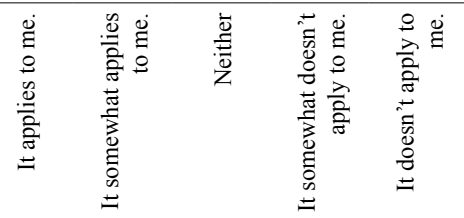

1) I have thought about RISKS before.

2) I have been educated about RISKS.

$\begin{array}{lcccc}\downarrow & \downarrow & \downarrow & \downarrow & \downarrow \\ 1 & 2 & 3 & 4 & 5 \\ 1 & \downarrow & \downarrow & + & -1\end{array}$
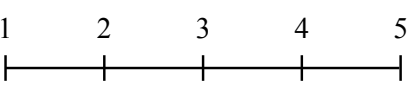

3) I know how to determine RISKS.

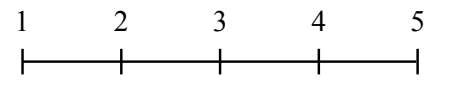

4) I know the meaning of RISKS.

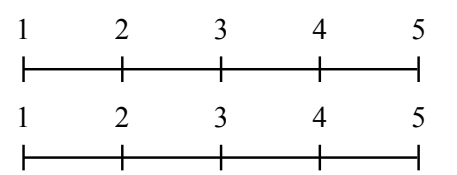

5) I determine what RISKS are by myself. 
【Tell us know your opinions concerning low-dose exposure.】

Q8. Do you agree or disagree with each statement of Questions 1) to 13) about low-dose exposure. Circle the number of the answer that best fits your opinion

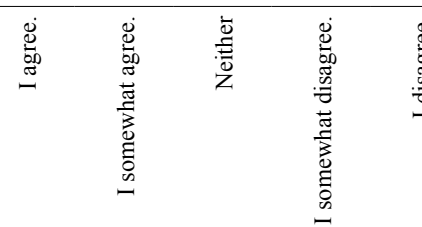

1) I think low-dose exposure is scary regardless of exposure level.

$\begin{array}{lllll}\downarrow & \downarrow & \downarrow & \downarrow & \downarrow \\ 1 & 2 & 3 & 4 & 5 \\ & \downarrow & & & 1\end{array}$

2) I think the effects of low-dose exposure are uncertain.

3) I think different experts say different things about the effects of low-dose exposure.

4) We cannot trust what the government says about the effects of low-dose exposure.

5) We cannot trust what experts say about the effects of low-dose exposure.

6) We cannot trust what doctors say about the effects of low-dose exposure.

7) I think one of the effects of low-dose exposure is the development of cancer in the future.

8) I think the effects of low-dose exposure not only leads to the development of cancer in the future, but also influences overall health.

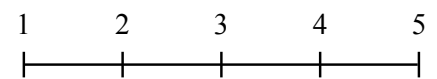

$\begin{array}{lllll}1 & 2 & 3 & 4 & 5\end{array}$

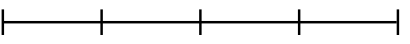

$\begin{array}{lllll}1 & 2 & 3 & 4 & 5\end{array}$

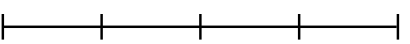

$\begin{array}{lllll}2 & 3 & 4 & 5\end{array}$

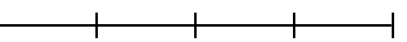

$1 \quad 2 \quad 3 \quad 4 \quad 5$

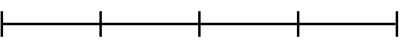

$\begin{array}{lllll}1 & 2 & 3 & 4 & 5\end{array}$
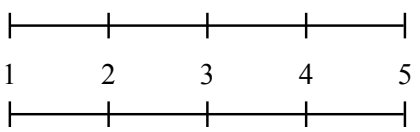

9) I think low-dose exposure negatively affects infants.

10) I think low-dose exposure negatively affects toddlers and children.

11) I am not concerned about any effects of low-dose exposure.

12) I think the effects of low-dose exposure are transmitted to others like a virus.

$\begin{array}{lllll}1 & 2 & 3 & 4 & 5\end{array}$
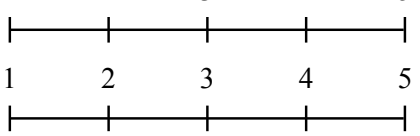

$\begin{array}{lllll}1 & 2 & 3 & 4 & 5\end{array}$
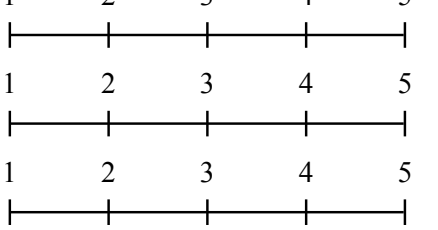

【Finally, tell us about yourself.】

F1. What is your gender? $\cdots \cdots \cdots \cdots 1$. Male 2. Female

Do you have any relatives who live with you and who are engaged in work related to nuclear power or radiation

industries? Circle the numbers of all answers that apply to you.
1. Self
3. Father/Mother
5. Grandson/Granddaughter
7. Relative
2. Spouse 4. Children
6. Brother/Sister
8. Others: 


\title{
Article
}

\section{Rapid and Simple Measurement Method of ${ }^{90} \mathrm{Sr}$ Concentration in Water by Measuring $\beta$-rays from ${ }^{90} \mathbf{Y}$}

\author{
Hideo HIRAYAMA ${ }^{1, *}$, Kenjiro KONDO ${ }^{1}$, Yasuhiro UNNO ${ }^{2}$, \\ Hiroshi MATSUMURA ${ }^{1}$, Hiroshi IWASE ${ }^{1}$, Akira YUNOKI ${ }^{2}$ \\ and Shinichi SASAKI ${ }^{1}$ \\ ${ }^{1}$ High Energy Accelerator Research Organization, 1-1 Oho, Tsukubashi, Ibaraki 305-0801, Japan \\ ${ }^{2}$ National Institute of Advanced Industrial Science and Technology, 1-1-1 Umezono, Tsukubashi, Ibaraki 305-8568, Japan
}

\begin{abstract}
A rapid and simple method to measure the concentration of ${ }^{90} \mathrm{Sr}$ in water by measuring $\beta$-rays from ${ }^{90} \mathrm{Y}$ was presented. Under the situation that ${ }^{90} \mathrm{Sr} /{ }^{90} \mathrm{Y}$, ${ }^{134} \mathrm{Cs}$ and ${ }^{137} \mathrm{Cs}$ are the main radionuclides included in the water sample, only $\beta$-rays from ${ }^{90} \mathrm{Y}$ can transmit through 1.5 -mm-thick polyethylene. From this fact, it is possible to measure $\beta$-rays from ${ }^{90} \mathrm{Y}$ using a $\beta$-ray detector, such as the GM-counter, set beneath the 1.5 -mm-thick bottom of the water bottle containing the sample with ${ }^{90} \mathrm{Sr} /{ }^{90} \mathrm{Y}$. The acrylic resin collimator having $0 \mathrm{~cm}, 1.00 \mathrm{~cm}, 1.50 \mathrm{~cm}$ or $3.00 \mathrm{~cm}$ diameter was made to detect $\beta$-rays at the fixed region of the GM-counter used. Contributions from bremsstrahlung produced by $\beta$-rays and $\gamma$-rays from radionuclides such as ${ }^{134} \mathrm{Cs}$ and ${ }^{137} \mathrm{Cs} /{ }^{137 \mathrm{~m}} \mathrm{Ba}$ are removed by subtracting the count rate measured with a $1.00 \mathrm{~cm}$ acrylic resin collimator without a hole as the background count rate. The developed method was studied using the bottle routinely used at the Fukushima Daiichi Nuclear Power Plant. It was confirmed that the developed method can be applied to measure the ${ }^{90} \mathrm{Sr}$ concentration in water to the order of several $\mathrm{Bq} / \mathrm{cm}^{3}$ if ${ }^{134} \mathrm{Cs}$ and ${ }^{137} \mathrm{Cs}$ concentrations are less than or equal to the ${ }^{90} \mathrm{Sr} /{ }^{90} \mathrm{Y}$ concentration.
\end{abstract}

KEYWORDS: Sr-90, Y-90, concentration in water, $\beta$-ray, GM-detector, range of $\beta$-ray, acrylic resin collimator

\section{Introduction}

At Fukushima Daiichi Nuclear Power Plant (hereinafter referred to as "1F"), measurement of the radioactive concentration of ${ }^{90} \mathrm{Sr}$ in numerous water samples containing ${ }^{90} \mathrm{Sr}$ in a wide range of radioactive concentrations is required. The radioactive concentrations of ${ }^{90} \mathrm{Sr}$ to be measured range widely from the effluent standard level $\left(3 \times 10^{-2} \mathrm{~Bq} / \mathrm{cm}^{3}\right)$ to several tens of $\mathrm{MBq} / \mathrm{cm}^{3}$. The types of water to be measured vary, including water retained in the nuclear reactor building, RO concentrated water from which $\mathrm{Cs}$ is eliminated using a cesium removing system (SARRY) (hereinafter referred to as "RO concentrated water"), water treated with various

* Corresponding author, E-mail: hideo.hirayama@kek.jp

DOI : $10.15669 /$ fukushimainsights. Vol.4.385

(C) 2021 Atomic Energy Society of Japan. All rights reserved.

Originally published in Transactions of the Atomic Energy Society of Japan (ISSN 1347-2879), Vol. 14, No. 3, p.141-150

(2015) in Japanese. (Japanese version accepted: April 20, 2015) 
nuclide removal equipment (ALPS), and stagnant water in the weir of the RO concentrated water tank. These water samples contain not only ${ }^{90} \mathrm{Sr}$ and ${ }^{90} \mathrm{Y}$ but also ${ }^{134} \mathrm{Cs}$ and ${ }^{137} \mathrm{Cs}$ at various radioactive concentrations. In addition, it is conceivable that the achievement of radioactive equilibrium between ${ }^{90} \mathrm{Sr}$ and ${ }^{90} \mathrm{Y}$ and the chemical dissolved state varies significantly among water samples. For water samples that are to be discharged, it is necessary to measure the radioactivity concentration of ${ }^{90} \mathrm{Sr}$ even for water samples containing ${ }^{90} \mathrm{Sr}$ at high radioactive concentrations that cannot be discharged. Tokyo Electric Power Co. measures ${ }^{90} \mathrm{Sr}$ in these water samples using a radioactive strontium analysis method requiring multistep chemical separation ${ }^{1)}$, a method of separating and concentrating ${ }^{90} \mathrm{Sr}$ through a chemical operation that allows ${ }^{90} \mathrm{Sr}$ to be measured using a $\beta$ nuclide analysis device ${ }^{2)}$, a simplified measurement method for separating and measuring ${ }^{90} \mathrm{Sr}$ with cation paper ${ }^{3)}$, and a total beta method ${ }^{4)}$.

The former two methods not only require chemical separation and advanced measuring technologies but also take a substantial amount of time to obtain measurement results, thus they are not suitable for emergency response. The cation paper method has a drawback in that it is largely dependent on the amount of dissolved ions and the dissolved state. Given this background, the total beta measurement method has been used thus far to estimate the radioactive ${ }^{90} \mathrm{Sr}$ concentration in numerous water samples generated at $1 \mathrm{~F}$.

However, as described in the introduction of "Gross beta measurement method" 4), the total beta method is intended for the measurement of environmental samples and is used to check if the radioactive concentration is below the effluent standard. Its primary purpose is to determine whether another measurement is necessary or not. From the viewpoint of measuring the concentration of Sr-90/Y-90, which has a high radioactive concentration that cannot be drained, this method is not an appropriate one. Under such circumstances, a method that enables a wide range of radioactive ${ }^{90} \mathrm{Sr}$ concentrations to be measured rapidly and easily needs to be developed.

The method proposed in this study measures water samples containing ${ }^{90} \mathrm{Sr} /{ }^{90} \mathrm{Y},{ }^{134} \mathrm{Cs}$, and ${ }^{137} \mathrm{Cs}$ as major radioactive nuclides in predetermined containers using a GM counter tube without performing any pretreatment. This is a new measurement method enables ${ }^{90} \mathrm{Sr}$ to be measured rapidly and easily at a concentration of more than several $\mathrm{Bq} / \mathrm{cm}^{3}$ by conducting the measurement twice at an interval of approximately $5 \mathrm{~h}$ even if the secular equilibrium of ${ }^{90} \mathrm{Sr}$ and ${ }^{90} \mathrm{Y}$ in the solution has not yet been confirmed. In addition to the measurement methods adopted by Tokyo Electric Power Co., the rapid analysis method ${ }^{5)}$ for discriminating radionuclides utilizing the difference in energy of $\beta$-rays using a liquid scintillation counter is also available. However, this method is intended to measure radionuclides in effluent and requires three or four days, despite its name. Because this method requires no chemical operation and can measure water samples placed in containers, it can be used to establish a system for measuring many samples in combination with a robotic operation and it is also possible to significantly shorten the time required to determine the radioactive ${ }^{90} \mathrm{Sr}$ concentration in numerous water samples generated at $1 \mathrm{~F}$.

The following section provides an outline of this method and the results of studying radioactive concentrations using known water samples containing ${ }^{90} \mathrm{Sr} /{ }^{90} \mathrm{Y},{ }^{134} \mathrm{Cs}$, or ${ }^{137} \mathrm{Cs}$. 


\section{Determining the ${ }^{90} \mathrm{Sr}$ Concentration in Water by Measuring $\beta$-rays from ${ }^{90} \mathbf{Y}$}

\section{Outline of the Method}

The following is a method to determine the radioactive ${ }^{90} \mathrm{Sr}$ concentration from the radioactive concentration of ${ }^{90} \mathrm{Y}$ obtained by measuring the number of $\beta$-rays from ${ }^{90} \mathrm{Y}$ using water samples containing ${ }^{90} \mathrm{Sr} /{ }^{90} \mathrm{Y},{ }^{134} \mathrm{Cs}$, and ${ }^{137} \mathrm{Cs}$ as the main radionuclides. It is based on the fact that $\beta$-rays from ${ }^{90} \mathrm{Sr},{ }^{134} \mathrm{Cs}$, and ${ }^{137} \mathrm{Cs}$ can be screened out by a 1.5 -mm-thick (or more) polyethylene plate and that the maximum range for $\beta$-rays from ${ }^{90} \mathrm{Y}$ is $1 \mathrm{~g} / \mathrm{cm}^{2}$.

Specifically, a water sample containing ${ }^{90} \mathrm{Sr} /{ }^{90} \mathrm{Y}$ is placed in a container with an approximately $1.5-\mathrm{mm}$-thick bottom, and the number of $\beta$-rays is measured in the range limited by a GM counter tube using a $\beta$-ray detector equipped with a 1 -cm-thick acrylic collimator. In addition, the count rate of $\beta$-rays from ${ }^{90} \mathrm{Y}$ is determined by deducting the count rate based on the bremsstrahlung X-rays generated because the $\gamma$ - and $\beta$-rays from ${ }^{134} \mathrm{Cs},{ }^{137} \mathrm{Cs} /{ }^{137 m} \mathrm{Ba}$, etc. are present in the water sample as background while blocking the $\beta$-rays from ${ }^{90} \mathrm{Y}$ with a 1 -cm-thick acrylic plate with no opening. The count rates of the $\beta$-rays from ${ }^{90} \mathrm{Y}$ are converted into radioactive concentrations using the conversion coefficient obtained from water samples with known radioactive concentrations. If it has not yet been confirmed that ${ }^{90} \mathrm{Sr}$ and ${ }^{90} \mathrm{Y}$ have achieved secular equilibrium, the measurement is conducted twice under the same conditions at an interval of approximately $5 \mathrm{~h}$ to determine the ${ }^{90} \mathrm{Sr}$ concentration from the ${ }^{90} \mathrm{Y}$ concentration obtained using the parent nuclide and daughter nuclide relation between ${ }^{90} \mathrm{Sr}$ and ${ }^{90} \mathrm{Y}$. In this case, the information on the achievement of radioactive equilibrium between ${ }^{90} \mathrm{Sr}$ and ${ }^{90} \mathrm{Y}$ during the first measurement can be obtained together from the ${ }^{90} \mathrm{Sr}$ and ${ }^{90} \mathrm{Y}$ concentrations acquired during the first measurement.

\section{2. $\beta$-Emitting Radionuclides to be Measured}

Water containing ${ }^{90} \mathrm{Sr} /{ }^{90} \mathrm{Y}$ that cannot be discharged because of high radioactive concentrations, generated at $1 \mathrm{~F}$, includes retained water such as $\mathrm{RO}$ concentrated water, revetment groundwater, and water related to leakage of reserved water, etc. The radionuclide in RO concentrated water obtained by removing cesium from stagnant water at turbine building consist mainly ${ }^{90} \mathrm{Sr} /{ }^{90} \mathrm{Y}$, except tritium, and ${ }^{134} \mathrm{Cs},{ }^{137} \mathrm{Cs},{ }^{54} \mathrm{Mn},{ }^{60} \mathrm{Co},{ }^{106} \mathrm{Ru} /{ }^{106} \mathrm{Rh}$ and ${ }^{125} \mathrm{Sb}$ at a concentration of one-thousandth or less of the ${ }^{90} \mathrm{Sr} /{ }^{90} \mathrm{Y}$ concentration ${ }^{6}$. In the case of revetment groundwater, ${ }^{90} \mathrm{Sr} /{ }^{90} \mathrm{Y}$ occur at the highest radioactive concentrations except for tritium, and there are cases in which ${ }^{134} \mathrm{Cs}$ and ${ }^{137} \mathrm{Cs}$ also occur at the same concentration in some areas. In addition, there are cases in which ${ }^{54} \mathrm{Mn},{ }^{60} \mathrm{Co},{ }^{106} \mathrm{Ru} /{ }^{106} \mathrm{Rh}$, and ${ }^{125} \mathrm{Sb}$ are also present at a concentration of one-thousandth of ${ }^{90} \mathrm{Sr} /{ }^{90} \mathrm{Y}^{7}$. Immediately after the accident, ${ }^{89} \mathrm{Sr}$ emitting $\beta$-rays with a maximum energy of $1.495 \mathrm{MeV}$ were at a level equal to ${ }^{90} \mathrm{Sr}$. However, this nuclide can be ignored because its half-life period of $50.53 \mathrm{~d}$ and 28 half-life periods have already passed. Concerning these nuclides as detected, Table 1 shows the maximum energy and emission rate of $\beta$-rays as well as the energy and emission rate of $\gamma$-rays ${ }^{8)}$. The nuclides that emit $\beta$-rays with a maximum energy of $1 \mathrm{MeV}$ or higher in addition to ${ }^{90} \mathrm{Y}$ are ${ }^{60} \mathrm{Co},{ }^{106} \mathrm{Rh}$ as a progeny nuclide of ${ }^{106} \mathrm{Ru}$, and ${ }^{137} \mathrm{Cs}$. The radioactive concentrations of ${ }^{60} \mathrm{Co}$ and ${ }^{106} \mathrm{Ru} /{ }^{106} \mathrm{Rh}$ occurring in water samples to be measured are not greater than one-thousandth of the radioactive concentrations of ${ }^{90} \mathrm{Sr} /{ }^{90} \mathrm{Y}$, thus their contributions can be ignored in the measurement of $\beta$-rays. In addition, because water samples containing ${ }^{90} \mathrm{Sr} /{ }^{90} \mathrm{Y}$ that cannot be discharged because of high radioactive concentrations are also included in the measuring objects, the contribution of $\beta$-rays from ${ }^{40} \mathrm{~K}$, 
INSIGHTS CONCERNING THE FUKUSHIMA DAIICHI NUCLEAR ACCIDENT Vol. 4

Table $1 \beta$-rays and $\gamma$-rays from main radionuclides identified in water samples at the Fukushima Daiichi Nuclear Power Station

\begin{tabular}{|c|c|c|c|c|c|c|}
\hline Nuclide & Half-life & Decay mode & $\begin{array}{l}\text { Maximum } \beta \text {-ray } \\
\text { energy }(\mathrm{MeV})\end{array}$ & Emission rate & $\begin{array}{c}\gamma \text {-ray energy } \\
(\mathrm{MeV})\end{array}$ & Emission rate \\
\hline${ }^{54} \mathrm{Mn}$ & $312.03 \mathrm{~d}$ & $\mathrm{EC}$ & & 1.0 & 0.836 & 1.0 \\
\hline \multirow{2}{*}{${ }^{60} \mathrm{Co}$} & \multirow{2}{*}{$5.2713 \mathrm{y}$} & \multirow{2}{*}{$\beta^{-}$} & 0.318 & 0.999 & 1.173 & 0.999 \\
\hline & & & 1.491 & 0.0012 & 1.333 & 1.0 \\
\hline \multirow{2}{*}{${ }^{90} \mathrm{Sr}$} & $28.79 \mathrm{y}$ & $\beta^{-}$ & 0.546 & \multicolumn{2}{|l|}{1.0} & \\
\hline & $64.00 \mathrm{~h}$ & $\beta^{-}$ & 2.28 & 1.0 & & \\
\hline \multirow[t]{2}{*}{${ }^{106} \mathrm{Ru}$} & \multirow[t]{2}{*}{$373.59 \mathrm{~d}$} & \multirow[t]{2}{*}{$\beta^{-}$} & 0.0394 & 1.0 & & \\
\hline & & & 2.407 & 0.100 & 0.512 & 0.204 \\
\hline \multirow{3}{*}{${ }^{106} \mathrm{Rh}$} & \multirow{3}{*}{$29.80 \mathrm{~s}$} & \multirow{3}{*}{$\beta^{-}$} & 3.029 & 0.081 & 0.662 & 0.099 \\
\hline & & & 3.541 & 0.786 & 1.05 & 0.016 \\
\hline & & & Others & & Others & \\
\hline \multirow{9}{*}{${ }^{125} \mathrm{Sb}$} & \multirow{9}{*}{$2.75856 \mathrm{y}$} & \multirow{9}{*}{$\beta^{-}$} & 0.0953 & 0.134 & 0.176 & 0.069 \\
\hline & & & 0.125 & 0.058 & 0.38 & 0.015 \\
\hline & & & 0.131 & 0.179 & 0.428 & 0.298 \\
\hline & & & 0.241 & 0.016 & 0.463 & 0.106 \\
\hline & & & 0.303 & 0.404 & 0.601 & 0.178 \\
\hline & & & 0.446 & 0.072 & 0.607 & 0.05 \\
\hline & & & 0.622 & 0.136 & 0.636 & 0.113 \\
\hline & & & Others & & 0.671 & 0.018 \\
\hline & & & & & Others & \\
\hline \multirow{7}{*}{${ }^{134} \mathrm{Cs}$} & \multirow{7}{*}{$2.065 \mathrm{y}$} & \multirow{7}{*}{$\beta^{-}$} & 0.0888 & 0.273 & 0.563 & 0.084 \\
\hline & & & 0.415 & 0.025 & 0.569 & 0.154 \\
\hline & & & 0.658 & 0.702 & 0.605 & 0.976 \\
\hline & & & Others & & 0.796 & 0.866 \\
\hline & & & & & 0.802 & 0.087 \\
\hline & & & & & 1.365 & 0.03 \\
\hline & & & & & Others & \\
\hline \multirow{2}{*}{${ }^{137} \mathrm{Cs}$} & \multirow{2}{*}{$30.1671 \mathrm{y}$} & \multirow{2}{*}{$\beta^{-}$} & 0.514 & 0.944 & & \\
\hline & & & 1.176 & 0.056 & & \\
\hline${ }^{137 \mathrm{~m}} \mathrm{Ba}$ & $2.532 \mathrm{~m}$ & IT & & & 0.662 & 0.851 \\
\hline
\end{tabular}

which is a form of natural radioactivity, can also be ignored.

Based on such circumstances, a method for measuring the $\beta$-rays in water samples containing ${ }^{90} \mathrm{Sr} /{ }^{90} \mathrm{Y},{ }^{134} \mathrm{Cs}$, and ${ }^{137} \mathrm{Cs}$ as the main nuclides was studied.

\section{How to Separate $\beta$-rays from ${ }^{90} \mathrm{Sr},{ }^{134} \mathrm{Cs}$, and ${ }^{137} \mathrm{Cs}$ in the Water Samples as well as $\beta$-rays from ${ }^{90} \mathrm{Y}$}

Except for the $\beta$-rays with a maximum energy of $1.176 \mathrm{MeV}$ from ${ }^{137} \mathrm{Cs}$ with an emission rate of $5.6 \%$, the maximum energy of the $\beta$-rays from ${ }^{90} \mathrm{Sr},{ }^{134} \mathrm{Cs}$, and ${ }^{137} \mathrm{Cs}$ is $0.6 \mathrm{MeV}$ or lower. The maximum range of the $\beta$-rays of $0.6 \mathrm{MeV}$ was calculated to be $0.2 \mathrm{~g} / \mathrm{cm}^{2}$ using the following equation ${ }^{9)}$ :

$$
R\left(\mathrm{~g} / \mathrm{cm}^{2}\right)=0.407 E^{1.38}
$$

where $E$ is the maximum energy of the $\beta$-rays expressed in MeV. However, the maximum range of the $\beta$-rays from ${ }^{90} \mathrm{Y}$ with a maximum energy of $2.25 \mathrm{MeV}$ is $1.05 \mathrm{~g} / \mathrm{cm}^{2}$ as determined using the following equation ${ }^{9}$ :

$$
R\left(\mathrm{~g} / \mathrm{cm}^{2}\right)=0.542 E-0.133
$$




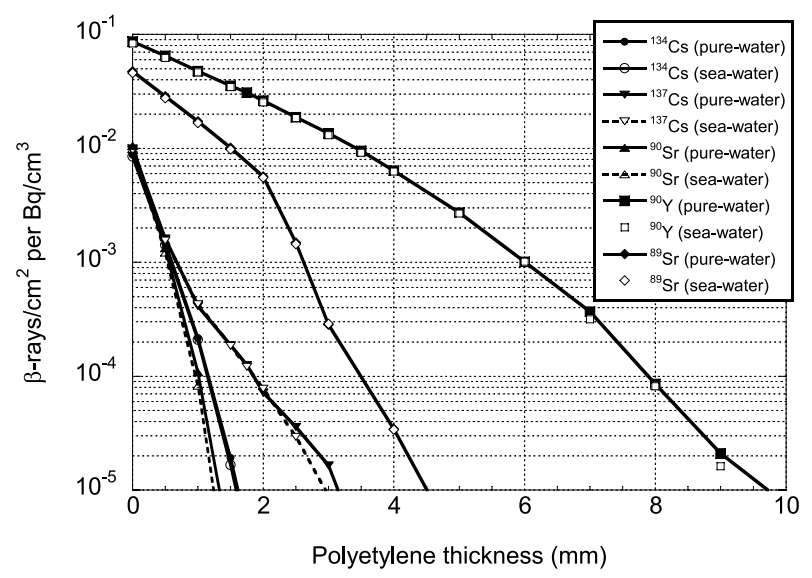

Figure 1 Calculated $\beta$-ray flux with egs5 from $1.5 \mathrm{~cm}$ pure-water or sea-water including ${ }^{90} \mathrm{Sr},{ }^{90} \mathrm{Y},{ }^{89} \mathrm{Sr},{ }^{134} \mathrm{Cs}$, or ${ }^{137} \mathrm{Cs}$ with concentration of $1 \mathrm{~Bq} / \mathrm{cm}^{3}$ as a function of polyethylene thickness inserted

These figures are different from the effective ranges because they are just relative to the maximum energy of the $\beta$-rays and the energy decreases in water if these nuclides occur in water. To more closely examine the attenuation of the actual situation, we calculated the amount of $\beta$-rays originating from a water sample that can transmit a polyethylene plate $1.5 \mathrm{~cm}$ thick containing ${ }^{90} \mathrm{Sr},{ }^{90} \mathrm{Y},{ }^{134} \mathrm{Cs}$, or ${ }^{137} \mathrm{Cs}$ at a concentration of $1 \mathrm{~Bq} / \mathrm{cm}^{3}$, using egs $5{ }^{10)}$ the electromagnetic cascade Monte Carlo code. For reference, we also calculated the $\beta$-rays from ${ }^{89} \mathrm{Sr}$ although they do not need to be considered in the case of water samples collected at $1 \mathrm{~F}$. In addition, we also performed a calculation for the case where these nuclides occur in seawater having a salt content of $3.4 \%$ and a density of $1.035 \mathrm{~g} / \mathrm{cm}^{3}$. Because the number of electrons that reached the outside of the polyethylene plate was determined in the calculation, the secondary electrons generated because of scattering with $\beta$-rays and those generated by bremsstrahlung X-rays were also included. Figure 1 shows the results. As is clear from the figure, even when ${ }^{90} \mathrm{Sr},{ }^{134} \mathrm{Cs}$, or ${ }^{137} \mathrm{Cs}$ with the same radioactive concentration as ${ }^{90} \mathrm{Y}$ is present, the number of $\beta$-rays from the respective nuclide after transmitting the $1.5-\mathrm{mm}$-thick $\left(0.136 \mathrm{~g} / \mathrm{cm}^{2}\right)$ polyethylene plate is less than one-hundredth of the number of $\beta$-rays from ${ }^{90} Y$. There was little difference found between fresh water and seawater. Therefore, assuming that the radioactive concentrations of ${ }^{134} \mathrm{Cs}$ and $137 \mathrm{Cs}$ measured are lower than the concentration of ${ }^{90} \mathrm{Y}$, the number of $\beta$-rays from ${ }^{90} \mathrm{Y}$ can be obtained by measuring the $\beta$-rays after penetrating the polyethylene plate ${ }^{\mathrm{a}}$.

\section{Elimination of the Influence of Bremsstrahlung X-Rays and $\gamma$-Rays}

$\beta$-ray measuring instruments can also sense $\gamma$-rays and X-rays, although the sensitivity

\footnotetext{
${ }^{a}$ Although different from the environment covered by this method, if ${ }^{90} \mathrm{Sr}$ and ${ }^{90} \mathrm{Y}$ are present at the same concentration, the result of the measurement conducted according to this method overestimates the concentration of ${ }^{90} \mathrm{Y}$. According to Figure 1, the number of $\beta$-rays from ${ }^{89} \mathrm{Sr}$ after penetrating a 1.5 -mm-thick polyethylene plate is approximately $30 \%$ of the number of the $\beta$-rays of ${ }^{90} \mathrm{Y}$. Therefore, the concentration of ${ }^{90} \mathrm{Y}$ which is in secular equilibrium with ${ }^{90} \mathrm{Sr}$ is overestimated by approximately $30 \%$. Even if the concentration of ${ }^{89} \mathrm{Sr}$ was the same as that of ${ }^{90} \mathrm{Sr}$ when the accident occurred, the concentration of ${ }^{89} \mathrm{Sr}$ decreases to less than $0.7 \%$ of that of ${ }^{90} \mathrm{Sr}$ over one year. Therefore, the overestimation of the concentration of ${ }^{90} \mathrm{Y}$ will be approximately $0.2 \%$ in the secular equilibrium state or approximately $2 \%$ even in an extreme case where only $10 \%$ equilibrium is achieved.
} 
varies. Because bremsstrahlung X-rays are generated in water or a container by the $\beta$-rays from ${ }^{90} \mathrm{Sr}$ and ${ }^{90} \mathrm{Y}$ in water and $\gamma$-rays are emitted when various concentrations of $\gamma$-emitting nuclides such as ${ }^{134} \mathrm{Cs}$ and ${ }^{137} \mathrm{Cs} /{ }^{137 m} \mathrm{Ba}$ occur in a water sample, it is necessary to deduct the count rates based on the $\gamma$-rays and bremsstrahlung X-rays (hereinafter referred to as "photons") as background to measure the number of $\beta$-rays.

The high-energy $\beta$-rays from ${ }^{90} \mathrm{Y}$ can also be screened out using a 1 -cm-thick acrylic plate, which is thicker than the maximum range. Although photons are slightly attenuated by an acrylic plate $1 \mathrm{~cm}$ thick, the attenuation rate is much lower than that of $\beta$-rays. Thus, the results of the measurement after penetration through the acrylic plate can be used as the background count rates based on photons.

\section{Relation between the Radioactive Concentration of ${ }^{90} \mathrm{Sr}$ and that of ${ }^{90} \mathrm{Y}$ in Water Samples}

If it has been confirmed that ${ }^{90} \mathrm{Sr}$ and ${ }^{90} \mathrm{Y}$ in a water sample have achieved secular equilibrium, the radioactive ${ }^{90} \mathrm{Sr}$ concentration can be determined from the radioactive ${ }^{90} \mathrm{Y}$ concentration obtained during the first measurement. Even if they have not yet achieved secular equilibrium, conducting the second measurement after a certain period (approximately $5 \mathrm{~h}$ ) enables the radioactive ${ }^{90} \mathrm{Sr}$ concentration to be obtained.

Because the ${ }^{90} \mathrm{Sr}$ and ${ }^{90} \mathrm{Y}$ half-life periods are $28.79 \mathrm{y}$ and $64.00 \mathrm{~h}$, respectively, their decay constants are $2.75 \times 10^{-6}\left(\mathrm{~h}^{-1}\right)$ and $1.08 \times 10^{-2}\left(\mathrm{~h}^{-1}\right)$, respectively.

Assuming the number of atoms of ${ }^{90} \mathrm{Sr}$ and ${ }^{90} \mathrm{Y}$ per $\mathrm{cm}^{3}$ at time $t=0$ is $N_{S r-90}(0)$ and $N_{Y-90}(0)$, respectively, the number of atoms of ${ }^{90} \mathrm{Y}$ per $\mathrm{cm}^{3}$ after $t$ hours $N_{Y-90}(t)$ is calculated as follows:

$$
N_{Y-90}(t)=\frac{\lambda_{S r-90}}{\lambda_{Y-90}-\lambda_{S r-90}} N_{S r-90}(0)\left[\exp \left(-\lambda_{S r-90} t\right)-\exp \left(-\lambda_{Y-90} t\right)\right]+N_{Y-90}(0) \exp \left(-\lambda_{Y-90} t\right)
$$

Because $\lambda_{Y-90} \gg \lambda_{S r-90}$, assuming $\lambda_{Y-90}-\lambda_{S r-90} \approx \lambda_{Y-90}$, the equation becomes as follows:

$$
\begin{aligned}
A_{Y-90}(t) & =\lambda_{Y-90} N_{Y-90}(t) \\
& =\lambda_{S r-90} N_{S r-90}(0)\left[\exp \left(-\lambda_{S r-90} t\right)-\exp \left(\lambda_{Y-90} t\right)\right]+\lambda_{Y-90} N_{Y-90}(0) \exp \left(-\lambda_{Y-90} t\right) \\
& =A_{S r-90}(0)\left[\exp \left(-\lambda_{S r-90} t\right)-\exp \left(-\lambda_{Y-90} t\right)\right]+A_{Y-90}(0) \exp \left(-\lambda_{Y-90} t\right)
\end{aligned}
$$

where, $A_{Y-90}(0)$ and $A_{Y-90}(t)$ are the radioactive concentrations of ${ }^{90} \mathrm{Y}$ at time $t=0$ and $t$, respectively, and $A_{S r-90}(0)$ is the radioactive ${ }^{90} \mathrm{Sr}$ concentration at time $t=0$. Based on equation (4), the radioactive concentration is calculated as follows:

$$
A_{S r-90}(0)=\frac{A_{Y-90}(t)-A_{Y-90}(0) \exp \left(-\lambda_{Y-90} t\right)}{\exp \left(-\lambda_{S r-90} t\right)-\exp \left(-\lambda_{Y-90} t\right)}
$$

If the measurement is conducted at an interval of $5 \mathrm{~h}$, it is calculated as follows:

$$
A_{S r-90}(0)=\frac{A_{Y-90}(t)-0.054 \times A_{Y-90}(0)}{0.946}
$$

Actually measuring $A_{Y-90}(0)$ and $A_{Y-90}(t)$ enables the radioactive ${ }^{90} \mathrm{Sr}$ concentration to be determined using equation (6). 


\section{Achievement of Radioactive Equilibrium between ${ }^{90} \mathrm{Sr}$ and ${ }^{90} \mathrm{Y}$ in a Solution}

The achievement of radioactive equilibrium between ${ }^{90} \mathrm{Sr}$ and ${ }^{90} \mathrm{Y}$ in a sample can possibly be used as information for determining the history of the contaminated water. In the measurement conducted according to this method, not only can the ${ }^{90} \mathrm{Sr}$ concentration in a sample be measured but also the achievement of radioactive equilibrium between ${ }^{90} \mathrm{Sr}$ and ${ }^{90} \mathrm{Y}$ at the time of the first measurement as follows:

The radioactivity ratio of ${ }^{90} \mathrm{Y}$ to ${ }^{90} \mathrm{Sr}$ at $t=0$ is determined by equation (5) as follows:

$$
f=\frac{A_{Y-90}(0)}{A_{S r-90}(0)}=\frac{A_{Y-90}(0)\left[\exp \left(-\lambda_{S r-90} t\right)-\exp \left(-\lambda_{Y-90} t\right)\right]}{A_{Y-90}(t)-A_{Y-90}(0) \exp \left(-\lambda_{Y-90} t\right)}
$$

Although $f$ can be determined as a value using equation (7) regardless of the value of $t$, it is possible that it may not be because of the influence of the statistical error of the counted value, if the measurement interval is short, when these nuclides are near radioactive equilibrium. The ratio of $A_{Y-90}(t)$ to $A_{Y-90}(0)$ is determined using equations (4) and (5) with $\mathrm{f}$ as a parameter as follows:

$$
\begin{aligned}
\frac{A_{Y-90}(t)}{A_{Y-90}(0)} & =\frac{A_{Y-90}(0) / f\left[\exp \left(-\lambda_{S r-90} t\right)-\exp \left(-\lambda_{Y-90} t\right)\right]}{A_{Y-90}(0)}+\frac{A_{Y-90}(0) \exp \left(-\lambda_{Y-90} t\right)}{A_{Y-90}(0)} \\
& =\frac{\exp \left(-\lambda_{S r-90} t\right)-\exp \left(-\lambda_{Y-90} t\right)}{f}+\exp \left(-\lambda_{Y-90} t\right)
\end{aligned}
$$

Figure 2 show the changes in $A_{Y-90}(t) / A_{Y-90}(0)$ because of $f$ when $t$ is 5,10 , and $24 \mathrm{~h}$. As shown in the figure, it is necessary to conduct the second measurement after at least $10 \mathrm{~h}$ and preferably $24 \mathrm{~h}$ to precisely determine the value of $f$ when $f$ is 0.5 or greater.

When ${ }^{90} \mathrm{Sr}$ and ${ }^{90} \mathrm{Y}$ are present in water, a bias in concentration may be caused by the formation of colloids and adsorption to suspended solids mainly because of a difference in chemical properties. In addition, immediately after the water is treated with a separation device utilizing the difference in chemical properties of both of the nuclides, there are cases in which the value of $f$ becomes greater than 1 and there are also cases in which it is less than 1. Although this does not affect the result of the radioactive ${ }^{90} \mathrm{Sr}$ concentration, if the value of $f$ is greater than 1 , attention needs to be paid to the achievement of radioactive equilibrium between ${ }^{90} \mathrm{Sr}$ and ${ }^{90} \mathrm{Y}$ in the solution.

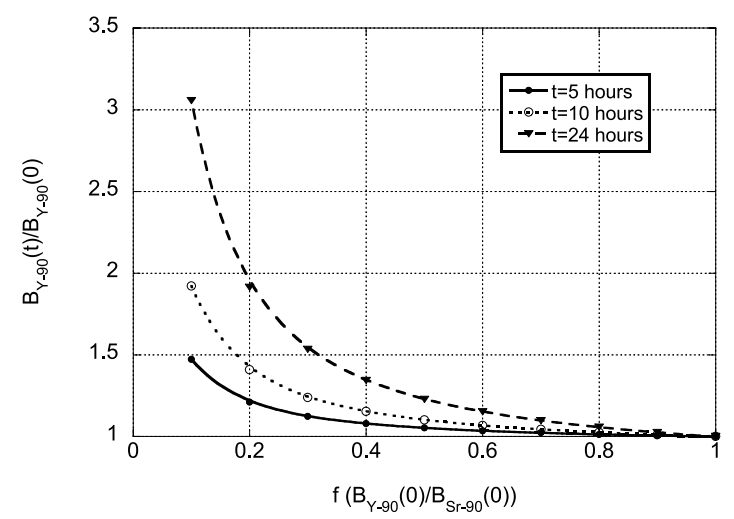

Figure 2 Increase in ${ }^{90} \mathrm{Y}$ concentration after $t$ hours as a function of the equivalent ratio between ${ }^{90} \mathrm{Y}$ and ${ }^{90} \mathrm{Sr}$ 


\section{Measurement System}

\section{1. $\beta$-Ray Detector}

Although there are various types of $\beta$-ray detectors, it is desirable if they can be handled easily because they are used for routine measurements. In the demonstration experiment described below, we used a TGS-146B ${ }^{11}$ (Hitachi-Aloka Medical) equipped with a large-area end-window organic GM tube utilized in contamination inspections as a $\beta$-ray detector, considering its sensitivity to $\beta$-rays. For precise measurement of the $\beta$-rays from ${ }^{90} \mathrm{Y}$ in a solution, the positional relation between the solution, which is a radiation source, and the detector is important. To detect the $\beta$-rays in the fixed range of the detector, we fabricated a 1.00 -cm-thick acrylic collimator and used it during the experiment. We designed a jig to make the collimator center coincide with the centers of the GM detector as well as the water sample container at all times. We used four types of collimators; those with diameters of $1.00 \mathrm{~cm}, 1.50 \mathrm{~cm}$, and $3.0 \mathrm{~cm}$ and one with no opening, for BG measurement. Figure 3 shows the collimator and jig used. The aluminum holder shown in the figure is the jig used to place the container in a predetermined position.

\section{Container Used}

Because numerous samples were measured, we adopted the container that is routinely used at $1 \mathrm{~F}$ for water samples as shown in Figure 4 (white JK wide mouth bottle manufactured by Kokugo ${ }^{12)}$, hereinafter referred to as a "T-container"). The T-container is made of polyethylene and has a bottom face the center of which is $1.5 \mathrm{~mm}$ thick and with a periphery that is $1.0 \mathrm{~mm}$ thick. The button face functions as a shield that eliminates the $\beta$-rays from the nuclides other than ${ }^{90} \mathrm{Y}$. We conducted the measurement on a $25-\mathrm{cm}^{3}$ water sample with the aforementioned concentration in the T-container. The water surface height was approximately $1.6 \mathrm{~cm}$. Because the maximum range of ${ }^{90} \mathrm{Y}$, which has the widest range, is $1 \mathrm{~cm}$ in water, it is believed that the water surface height does not influence the measurement result as long as it is $1 \mathrm{~cm}$ or higher.

During the measurement conducted to examine the influence of the uneven bottom face, we also used a U8 container, which is commonly used in radioactive sample measurement, having a bottom surface of constant thickness,.

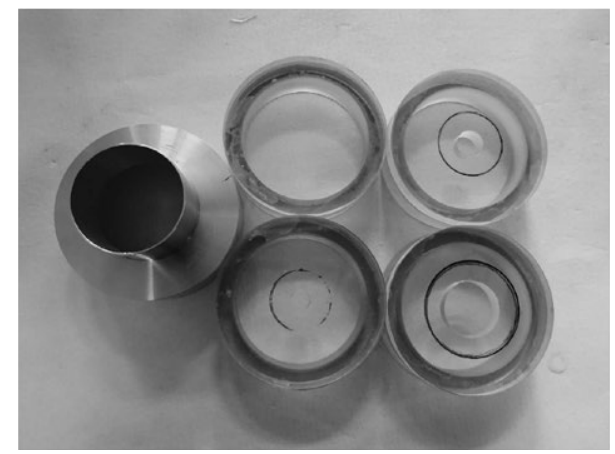

Figure 3 Picture of acrylic resin collimators and aluminum holder used for measurement 


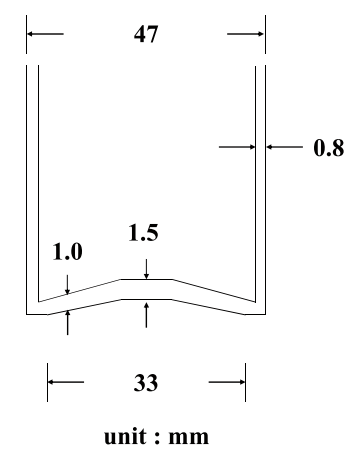

Figure 4 Schematic diagram of T-bottle used

\section{Points to Note when Preparing Samples}

The method is based on the premise that ${ }^{90} \mathrm{Y}$ generated by the decay of ${ }^{90} \mathrm{Sr}$ is uniformly distributed in a water sample. The radioactive isotopes occurring in a water sample undergoing measurement, such as ${ }^{134} \mathrm{Cs},{ }^{137} \mathrm{Cs}$, ${ }^{90} \mathrm{Sr}$, etc., are considered to be present in the carrier-free state and neutral in most cases, but there are also neutral or slightly alkaline solutions such as RO concentrated saltwater ${ }^{13)}$. It has been reported that carrier-free elements do not display their original physicochemical behavior and cause uneven solutions because of the formation of colloids and adsorption to suspended solids in some cases ${ }^{14,15}$. In particular, it is well known that carrier-free ${ }^{90} \mathrm{Y}$ causes uneven solutions because of the formation of colloids and adsorption to suspended solids when the solutions are alkaline ${ }^{14,16}$.

In RO concentrated saltwater, it has been shown that not only ${ }^{90} \mathrm{Y}$ but also other nuclides are present in the colloidal state or they are adsorbed to fine particles at a high rate ${ }^{13)}$.

To secure the uniformity of ${ }^{90} \mathrm{Y}$, which is important for this measurement method, in a water sample for measurement, it is essential to:

(1) acidify the solution to inhibit the generation of colloids and

(2) add a small amount of carrier to prevent adsorption to fine particles.

In the demonstration experiment described in the following section, we acidified the water samples containing ${ }^{90} \mathrm{Sr} /{ }^{90} \mathrm{Y},{ }^{134} \mathrm{Cs}$, or ${ }^{137} \mathrm{Cs}$ with hydrochloric acid (approximately $\mathrm{pH}=1$ ) and added the carriers of $\mathrm{Cs}, \mathrm{Sr}$, and $\mathrm{Y}$.

\section{Demonstration Experiment and Results}

\section{Radiation Source Samples and Measurement Method Used}

To validate this method, we conducted an experiment using ${ }^{90} \mathrm{Sr} /{ }^{90} \mathrm{Y}$ solutions in the radioactive equilibrium state, which were adjusted to the radioactive concentrations of 138.0, $523.9,5,244$, and $11,660 \mathrm{~Bq} / \mathrm{cm}^{3}$ as well as ${ }^{134} \mathrm{Cs}$ and ${ }^{137} \mathrm{Cs}$ solutions adjusted to the radioactive concentrations of 7,405 and $10,800 \mathrm{~Bq} / \mathrm{cm}^{3}$, respectively. These were all hydrochloric acid solutions of $0.1 \mathrm{M}$, and the carrier concentrations of the strontium chloride and yttrium chloride were adjusted to be $0.05 \mathrm{mg} / \mathrm{g}$ in the ${ }^{90} \mathrm{Sr} /{ }^{90} \mathrm{Y}$ solutions and $0.1 \mathrm{mg} / \mathrm{g}$ in the ${ }^{134} \mathrm{Cs}$ and ${ }^{137} \mathrm{Cs}$ solutions. The radioactive ${ }^{90} \mathrm{Sr} /{ }^{90} \mathrm{Y}$ concentrations in the solutions were measured according to the efficiency tracer method using a liquid scintillation counter. However, the radioactive concentrations of ${ }^{134} \mathrm{Cs}$ and ${ }^{137} \mathrm{Cs}$ were measured using the $\gamma$-ray spectrometry method with a 
high-purity germanium semiconductor detector whose counting efficiency was calibrated in advance. The expanded uncertainties of the radioactive concentrations (coverage factor of 2) were $1.4 \%$ in the case of ${ }^{90} \mathrm{Sr} /{ }^{90} \mathrm{Y}$ and $11,660 \mathrm{~Bq} / \mathrm{cm}^{3}$ and $1.5 \%$ in the case of the other nuclides.

The measurement was conducted three times for each combination of container and collimator, and the dead times were corrected using the dead time $(225 \mu \mathrm{s})$ measured in advance according to the two-source method.

\section{Variation in Results Depending on Container Type}

Because the measurement is based on the premise of the use of a T-container but not a special dedicated container as described in Section III-2, it was feared that the variation in the bottom thicknesses of T-containers used might have possibly caused a variation in measurement results. To examine the degree of variation, we conducted a measurement using 20 samples containing ${ }^{90} \mathrm{Sr} /{ }^{90} \mathrm{Y}$ at a concentration of $523.9 \mathrm{~Bq} / \mathrm{cm}^{3}$. The containers used were designated, "No. 1 Type" and "No. 2 Type", and there was a slight difference in weight between the two types. We also examined if a difference in type influenced the result. Figure 5 shows the results obtained for the collimators with diameters of $1.50 \mathrm{~cm}$ and $3.00 \mathrm{~cm}$. The lengths of the error bars in the figure represent statistical uncertainties of the count rates for $1 \mathrm{~min}$. Of course, because the variation in measurement results is affected by statistical uncertainty, the variation increases when a collimator having a smaller diameter is used. This measurement showed that

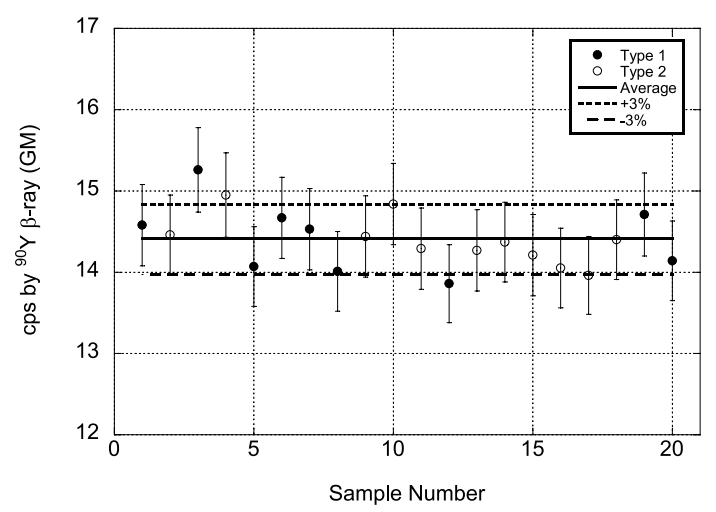

(a) $1.50 \mathrm{~cm}$ diameter collimator

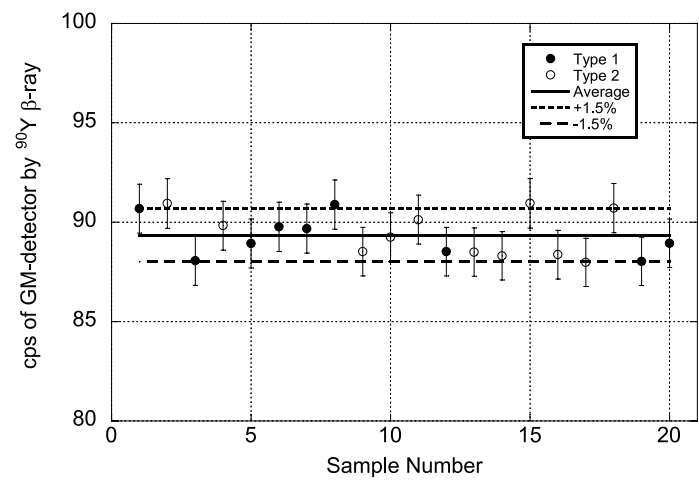

(b) $3.00 \mathrm{~cm}$ diameter collimator

Figure 5 Count rate unevenness of ${ }^{90} \mathrm{Y} \beta$-rays from water including ${ }^{90} \mathrm{Sr} /{ }^{90} \mathrm{Y}$ with concentration of $523.9 \mathrm{~Bq} / \mathrm{cm}^{3}$ due to T-bottle used (a) $1.50 \mathrm{~cm}$ diameter collimator, (b) $3.00 \mathrm{~cm}$ diameter collimator 
the variations when a 3.00-cm-diameter collimator and 1.50 -cm-diameter collimator were used were $1.5 \%$ and $3.0 \%$, respectively, which proved to be the same as the statistical uncertainty. No systematic difference was observed between the No. 1 and No. 2 types. From the aforementioned result, it was concluded that the influence of the variation among T-containers could be ignored.

\section{Influence of the Bottom Face Structure of the T-Container}

As shown in Figure 4, the T-container used in the measurement has a bottom face that is not flat but raised and thicker in the center. To examine the influence of this structure on count rates, we compared the results of the measurement conducted using a T-container containing ${ }^{90} \mathrm{Sr} /{ }^{90} \mathrm{Y}$ to a concentration of $523.9 \mathrm{~Bq} / \mathrm{cm}^{3}$ and a U8 container with a flat bottom face with the number of $\beta$-rays at the collimator exit calculated using egs5. The calculation using egs5 was made to determine the number of $\beta$-rays per second at the collimator exit when a 1.00 -cm-thick acrylic collimator is placed behind 1.5-mm-thick polyethylene plate, assuming the amounts of ${ }^{90} \mathrm{Sr}$ and ${ }^{90} \mathrm{Y}$ in the water sample are uniform. The T-container is composed of polyethylene and its bottom face is $1.5 \mathrm{~mm}$ thick at its center and $1.0 \mathrm{~mm}$ thick along its periphery.

The U8 container is composed of polystyrene and has a bottom surface with a uniform thickness of $1.5 \mathrm{~mm}$. Table 2 shows a comparison between the measured and calculated values for various collimator diameters. Although the difference from the calculation result was less than $10 \%$ for both types of containers, the measured values of the U8 container are generally in better agreement with the calculated values because the calculation was conducted assuming the bottom face of the U8 container. The count values of the U8 container were higher for the collimators with diameters of $1.00 \mathrm{~cm}$ and $1.50 \mathrm{~cm}$, apparently because of the influence of the center part of the T-container, which was slightly raised. However, the count values of the T-container were higher for the collimator with a diameter of $3.00 \mathrm{~cm}$, apparently because of the contribution of the thin polyethylene along the periphery of the bottom face of the T-container. From the aforementioned results, it is believed that the shape of the bottom face of the T-container, which is not flat, has a certain influence on the results but does not become a major factor of uncertainty as long as the conversion coefficient is determined for the container to be used.

\section{Influence of Radioactive Cesium}

To examine the influence of $\gamma$-rays when ${ }^{134} \mathrm{Cs}$ and ${ }^{137} \mathrm{Cs} /{ }^{137 m} \mathrm{Ba}$ occur in water samples, we conducted a measurement using water samples containing $7,405 \mathrm{~Bq} / \mathrm{cm}^{3}$ of ${ }^{134} \mathrm{Cs}$ or $10,800 \mathrm{~Bq} / \mathrm{cm}^{3}$ of ${ }^{137} \mathrm{Cs} /{ }^{137 m} \mathrm{Ba}$. Table 3 shows the comparison between the count rates based on the $\beta$-rays from ${ }^{90} \mathrm{Y}$ per $10 \mathrm{kBq} / \mathrm{cm}^{3}$ and the count rates of ${ }^{134} \mathrm{Cs}$ or ${ }^{137} \mathrm{Cs} /{ }^{137 m} \mathrm{Ba}$. Regardless

Table 2 Comparison between measured count rate and number of $\beta$-rays calculated with egs5

\begin{tabular}{ccccc}
\hline $\begin{array}{c}\text { Diameter of } \\
\text { collimator } \\
(\mathrm{cm})\end{array}$ & $\begin{array}{c}\text { Type of } \\
\text { bottole }\end{array}$ & $\begin{array}{c}\text { Count rate } \\
\text { (A) cps for } \\
524.6 \mathrm{~Bq} / \mathrm{cm}^{3}\end{array}$ & $\begin{array}{c}\text { egs5 calculation } \\
(\mathrm{B}) \beta \text {-rays for } \\
524.6 \mathrm{~Bq} / \mathrm{cm}^{3}\end{array}$ & $\mathrm{~A} / \mathrm{B}$ \\
\hline \multirow{2}{*}{1} & T-bottole & $4.5 \pm 0.3$ & $5.0 \pm 0.2$ & 0.91 \\
& $\mathrm{U} 8$ & $4.9 \pm 0.2$ & & 0.97 \\
\hline \multirow{5}{*}{1.5} & T-bottole & $14.4 \pm 0.9$ & $15.8 \pm 0.3$ & 0.91 \\
& $\mathrm{U} 8$ & $15.4 \pm 0.4$ & & 0.98 \\
\hline \multirow{5}{*}{3} & T-bottole & $89 \pm 2$ & \multirow{2}{*}{$90 \pm 1$} & 0.99 \\
& $\mathrm{U} 8$ & $82.8 \pm 0.6$ & & 0.92 \\
\hline
\end{tabular}


INSIGHTS CONCERNING THE FUKUSHIMA DAIICHI NUCLEAR ACCIDENT Vol. 4

Table 3 Comparison of count rate between ${ }^{134} \mathrm{Cs},{ }^{137} \mathrm{Cs}$ and ${ }^{90} \mathrm{Sr} /{ }^{90} \mathrm{Y}$

\begin{tabular}{cccccc}
\hline \multirow{3}{*}{ Colimator diameter } & \multicolumn{5}{c}{ cps for $10 \mathrm{kBq} / \mathrm{cm}^{3}$} \\
\cline { 2 - 6 } & ${ }^{90} \mathrm{Y}(\mathrm{A})$ & ${ }^{134} \mathrm{Cs}(\mathrm{B})$ & ${ }^{137} \mathrm{Cs} /{ }^{137 \mathrm{~m}} \mathrm{Ba}(\mathrm{C})$ & $(\mathrm{B}) /(\mathrm{A})$ & $(\mathrm{C}) /(\mathrm{A})$ \\
\hline $1.00 \mathrm{~cm}$ & $86.8 \pm 1.1$ & $-4.92 \pm 2.29$ & $-4.80 \pm 0.57$ & - & - \\
$1.50 \mathrm{~cm}$ & $276 \pm 0.3$ & $7.22 \pm 1.76$ & $2.25 \pm 1.20$ & $2.6 \%$ & $0.8 \%$ \\
$3.00 \mathrm{~cm}$ & $1,824 \pm 13$ & $61.6 \pm 3.1$ & $38.2 \pm 2.6$ & $3.4 \%$ & $2.1 \%$ \\
\hline
\end{tabular}

of which collimator was used, the count rates of ${ }^{134} \mathrm{Cs}$ and ${ }^{137} \mathrm{Cs} /{ }^{137 \mathrm{~m}} \mathrm{Ba}$ were less than $4 \%$ of the count rates of the $\beta$-rays from ${ }^{90} \mathrm{Y}^{\mathrm{b}}$. For the case in which a collimator with a diameter of $3 \mathrm{~cm}$ was used, we calculated the attenuation of the $\gamma$-rays from ${ }^{134} \mathrm{Cs}$ and ${ }^{137} \mathrm{Cs}$ caused by a $1-\mathrm{cm}$ thick acrylic plate using egs5. The ratio of the energy of $\gamma$-rays with and without the $1-\mathrm{cm}$ thick acrylic plate behind the 1.5 -mm-thick polyethylene plate was 0.96 for both ${ }^{134} \mathrm{Cs}$ and ${ }^{137} \mathrm{Cs} /{ }^{137 m} \mathrm{Ba}$. However, the ratio of the count rate when a collimator with a $3-\mathrm{cm}$ diameter and 1 -cm-thick acrylic plate with no opening were used was 0.85 for ${ }^{134} \mathrm{Cs}$ and 0.78 for ${ }^{137} \mathrm{Cs} /{ }^{137 \mathrm{~m}} \mathrm{Ba}$, larger than the attenuation of the penetrating energy calculated. As is clear from Figure 1, this was mainly due to the influence of $\beta$-rays originating from the difference in the sensitivity of the GM detector to $\beta$-rays and $\gamma$-rays because a small number of penetrating $\beta$-rays was present behind the 1.5-mm-thick polyethylene plate. This can also be confirmed as the attenuation of ${ }^{137} \mathrm{Cs} /{ }^{137 m} \mathrm{Ba}$ is greater. Therefore, the attenuation of $\gamma$-rays caused by the 1 -cm-thick acrylic plate is approximately $5 \%$ of the value calculated using egs 5 .

Although the influence of $\gamma$-rays increases when the concentration of ${ }^{134} \mathrm{Cs}$ or ${ }^{137} \mathrm{Cs}$ is higher than that of ${ }^{90} \mathrm{Sr} /{ }^{90} \mathrm{Y}$, overestimation can be avoided using the following method in this case. However, in the case where the concentration is 100 times or higher, the correction using this method cannot be applied because the contribution of the $\beta$-rays from ${ }^{134} \mathrm{Cs}$ and ${ }^{137} \mathrm{Cs}$ cannot be ignored.

(1) Measure the ${ }^{134} \mathrm{Cs}$ and ${ }^{137} \mathrm{Cs}$ concentrations in an aqueous solution using a Ge detector. (This concentration measurement is performed in many cases.).

(2) Determine the count rate of the $\gamma$-rays from ${ }^{134} \mathrm{Cs}$ and ${ }^{137} \mathrm{Cs} /{ }^{137 m} \mathrm{Ba}$ using the concentrations of respective nuclides and the coefficients for converting from concentration to count rate. (According to Table 3, ${ }^{134} \mathrm{Cs}: 0.0062,{ }^{137} \mathrm{Cs} /{ }^{137 m} \mathrm{Ba}: 0.0038 \mathrm{cps}$ per $\mathrm{Bq} / \mathrm{cm}^{3}$, using a 3.00 -cm-diameter collimator)

(3) Determine the count rate of the $\beta$-rays originating from ${ }^{90} \mathrm{Y}$ by deducting the count value previously determined from the count value obtained by deducting the measurement rate of the collimator with no opening from the count rate of the collimator.

\section{Confirmation of Linearity with Respect to Count Rate and ${ }^{90} Y$ Concentration as well as Determination of the Coefficient for Converting from the Count Rate of $\beta$-Rays from ${ }^{90} \mathrm{Y}$ to Concentration}

To examine the relation between the radioactive concentration of ${ }^{90} \mathrm{Y}$ obtained according to this method and the count rate of the GM detector, we conducted a measurement using samples containing ${ }^{90} \mathrm{Sr} /{ }^{90} \mathrm{Y}$ at a concentration of $138.0,523.9,5,244$, and $11,660 \mathrm{~Bq} / \mathrm{cm}^{3}$.

Figure 6 shows the results. Superior linearity was observed for all collimator diameters. The count rates per $\mathrm{Bq} / \mathrm{cm}^{3}$ for the collimators with a diameter of $1.0 \mathrm{~cm}, 1.50 \mathrm{~cm}$, and $3.00 \mathrm{~cm}$

\footnotetext{
${ }^{\mathrm{b}}$ The reason why the value is negative in the case of the collimator with a diameter of $1.00 \mathrm{~cm}$ is because the count value obtained when a 1.00 -cm-thick acrylic plate with no opening was used was deducted from the count value obtained when the collimator was used, and it is considered that both are practically the same.
} 
were $0.00871,0.0277$, and $0.181 \mathrm{cps}$, respectively.

From the measurement results, coefficients for converting from count rate to concentration using the least squares method were obtained as follows:

A collimator with a diameter of $1.00 \mathrm{~cm}: 115 \pm 0.4 \mathrm{~Bq} / \mathrm{cm}^{3}$ per cps

A collimator with a diameter of $1.50 \mathrm{~cm}: 36.2 \pm 0.1 \mathrm{~Bq} / \mathrm{cm}^{3}$ per cps

A collimator with a diameter of $3.00 \mathrm{~cm}: 5.52 \pm 0.04 \mathrm{~Bq} / \mathrm{cm}^{3}$ per $\mathrm{cps}$

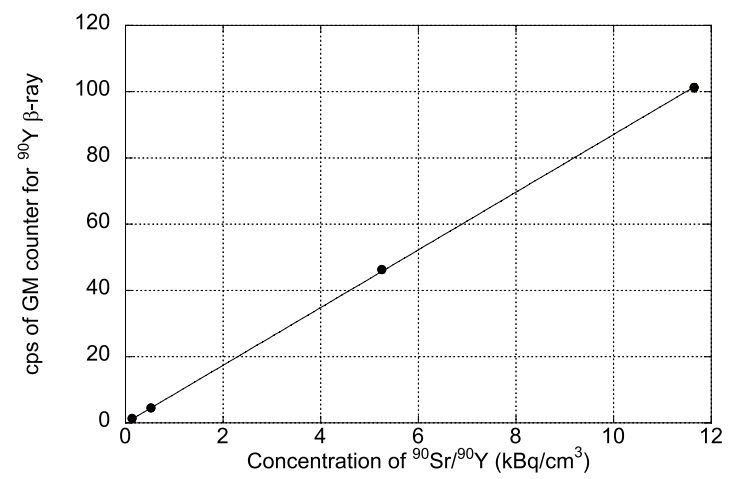

(a) $1.00 \mathrm{~cm}$ diameter collimator

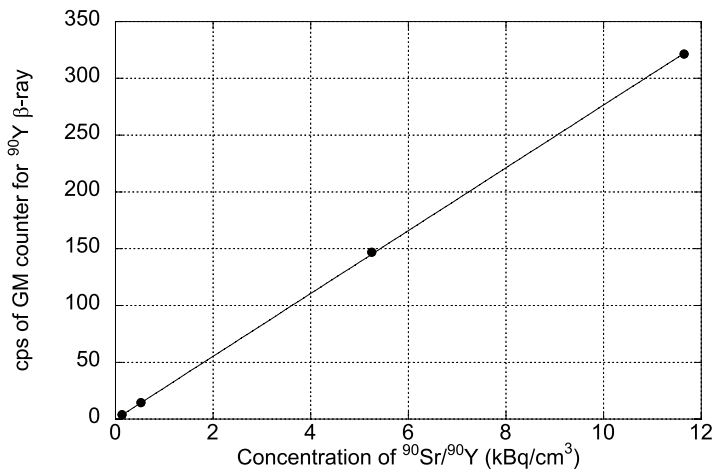

(b) $1.50 \mathrm{~cm}$ diameter collimator

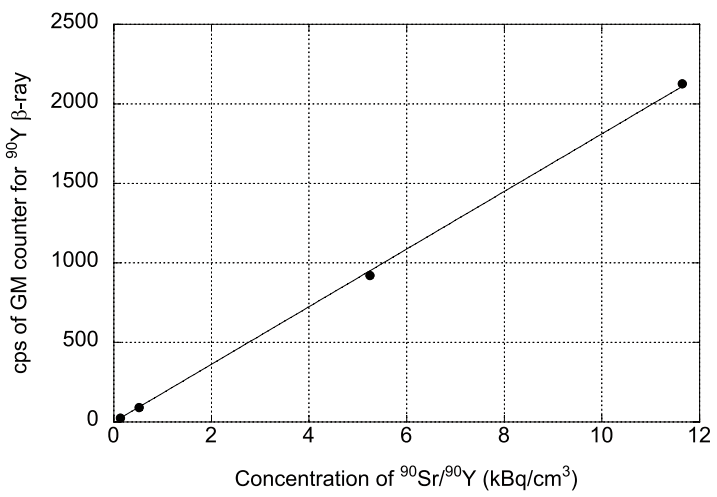

(c) $3.00 \mathrm{~cm}$ diameter collimator

Figure 6 Relation between ${ }^{90} \mathrm{Sr} /{ }^{90} \mathrm{Y}$ concentration and count rate of ${ }^{90} \mathrm{Y} \beta$-rays

(a) $1.00 \mathrm{~cm}$ diameter collimator, (b) $1.50 \mathrm{~cm}$ diameter collimator, (c) $3.00 \mathrm{~cm}$ diameter collimator 


\section{Detection Limit of This Method}

The detection limit is expressed as follows:

$$
n=(k / 2)\left(k / t+\sqrt{(k / t)^{2}+4 n_{b}\left(1 / t+1 / t_{b}\right)}\right)
$$

Where $n$ is the count rate of the detection limit (cps), $k$ is the confidence level, $t$ is the sample measurement time, $t_{b}$ is the background measurement time, and $n_{b}$ is the background count rate (cps). The measuring environment background level of the location in which the demonstration experiment was conducted when no sample was placed was $1.3 \mathrm{cps}$. The background level of the measurement site at $1 \mathrm{~F}$ may possibly be several times higher. In this method, the count rates of the photons through a collimator with no opening needs to be included in the background count rate. Because the background count rate of the bremsstrahlung X-rays based on the $\beta$-rays from ${ }^{90} \mathrm{Sr} /{ }^{90} \mathrm{Y}$ occurring in a water sample is approximately 0.0007 (cps per $\mathrm{Bq} / \mathrm{cm}^{3}$ ), the background of the measuring environment becomes dominant when the concentration of ${ }^{90} \mathrm{Sr} /{ }^{90} \mathrm{Y}$ is $1,800 \mathrm{~Bq} / \mathrm{cm}^{3}$ or less. In addition, because the background count rates based on $\gamma$-rays for ${ }^{134} \mathrm{Cs}$ and ${ }^{137} \mathrm{Cs} /{ }^{137 m} \mathrm{Ba}$ are 0.036 and $0.014\left(\mathrm{cps}\right.$ per $\mathrm{Bq} / \mathrm{cm}^{3}$ ), respectively, the background of the measuring environment dominates when the concentration of ${ }^{134} \mathrm{Cs}$ is $35 \mathrm{~Bq} / \mathrm{cm}^{3}$ or less and that of ${ }^{137} \mathrm{Cs} /{ }^{137 m} \mathrm{Ba}$ is $91 \mathrm{~Bq} / \mathrm{cm}^{3}$ or less. Assuming that $k$ is 3 and the water sample and background measurement time is $10 \mathrm{~min}$ when the background of the measuring environment is dominant, the detection limit count rate is $0.21 \mathrm{cps}$ when the background level of the measuring environment is $1.3 \mathrm{cps}$ and it is $0.31 \mathrm{cps}$ when the background level is $3 \mathrm{cps}$. Based on the conversion coefficient of the 3-cm-diameter collimator with the highest sensitivity of 5.52 $\left(\mathrm{Bq} / \mathrm{cm}^{3}\right.$ per $\left.\mathrm{cps}\right)$, the respective detection limit radioactive concentrations correspond to 1.1 and $1.7\left(\mathrm{~Bq} / \mathrm{cm}^{3}\right)$. Of course, although the detection limit is dependent on the ${ }^{134} \mathrm{Cs}$ and ${ }^{137} \mathrm{Cs}$ concentrations in the water sample as well as the background level of the measuring environment, it can be stated that the detection limit based on this method is only several $\mathrm{Bq} / \mathrm{cm}^{3}$ if the contribution of the $\gamma$-rays from ${ }^{134} \mathrm{Cs}$ and ${ }^{137} \mathrm{Cs}$ is less than the background of the measuring environment.

\section{Conclusions}

Under the condition where the main nuclides occurring in a water sample are ${ }^{90} \mathrm{Sr} /{ }^{90} \mathrm{Y},{ }^{134} \mathrm{Cs}$, and ${ }^{137} \mathrm{Cs}$, we proposed a new method that allows a wide range of radioactive ${ }^{90} \mathrm{Sr}$ concentrations to measured quickly and easily without the need for any special operations by conducting a measurement using a water sample containing ${ }^{90} \mathrm{Sr} /{ }^{90} \mathrm{Y}$ placed in a container. Based on the results of a demonstration experiment conducted using water samples, the radioactive concentrations of which were precisely evaluated, and the T-containers used routinely at $1 \mathrm{~F}$, we drew the following conclusions:

(1) The variation among $\mathrm{T}$-containers used at $1 \mathrm{~F}$ is within the range of statistical uncertainty and does not affect the result.

(2) The bottom face structure of the T-container, which is not flat, caused a slight difference from the case in which the U8 container with a flat bottom face was used. However, because the influence is small, the uncertainty of the result is not affected as long as the "conversion coefficient" is determined in advance for the container to be used.

(3) The contribution of bremsstrahlung X-rays generated by $\beta$-rays in the water samples and containers can be eliminated by deducting the count rate based on the collimator with no 
opening as the background.

(4) The influence of the $\gamma$-rays from ${ }^{134} \mathrm{Cs}$ or ${ }^{137} \mathrm{Cs} /{ }^{137 m} \mathrm{Ba}$ occurring in the water samples is $4 \%$ or less of the count value based on the $\beta$-rays from ${ }^{90} \mathrm{Y}$ having the same radioactive concentration. If the radioactive concentration of ${ }^{134} \mathrm{Cs}$ or ${ }^{137} \mathrm{Cs}$ is 100 times or less than that of ${ }^{90} \mathrm{Sr} /{ }^{90} \mathrm{Y}$, the overestimation of ${ }^{90} \mathrm{Y}$ can be avoided by deducting the count rate of $\gamma$-rays based on the radioactive concentration of ${ }^{134} \mathrm{Cs}$ or ${ }^{137} \mathrm{Cs}$ from the count value of the $\beta$-rays from ${ }^{90} \mathrm{Y}$.

(5) The relation between the count rate of the GM detector and the radioactive ${ }^{90} \mathrm{Y}$ concentration achieves superior linearity in a wide range of radioactive ${ }^{90} \mathrm{Y}$ concentrations regardless of the diameter of the collimator.

(6) Even if it has not yet been confirmed that ${ }^{90} \mathrm{Sr}$ and ${ }^{90} \mathrm{Y}$ in a water sample have achieved secular equilibrium, conducting the measurement twice at an interval of approximately $5 \mathrm{~h}$ enables the radioactive ${ }^{90} \mathrm{Sr}$ concentration to be obtained.

(7) If the contribution of the $\gamma$-rays from ${ }^{134} \mathrm{Cs}$ and ${ }^{137} \mathrm{Cs} /{ }^{137 m} \mathrm{Ba}$ is less than the background level of the measuring environment when the collimator with a diameter of $3.00 \mathrm{~cm}$ with the highest sensitivity is used, the minimum detectable radioactive concentration is only several $\mathrm{Bq} / \mathrm{cm}^{3}$, although it depends on the background level of the measuring environment.

From these results, it would appear that this method could be used to measure the radioactive ${ }^{90} \mathrm{Sr}$ concentration occurring in many water samples over a wide range of concentrations, which need to be measured at $1 \mathrm{~F}$, but the following needs to be noted when applying this method in the field.

(1) This method is based on the premise that ${ }^{90} \mathrm{Y}$ generated by the decay of ${ }^{90} \mathrm{Sr}$ is uniformly distributed in a water sample. Therefore, it is required to "acidify the solution to inhibit the generation of colloids and to add a small number of carriers to prevent adsorption to fine particles."

(2) It is necessary to determine the dead time of the GM detector to be used in advance according to the two-source method as well as to correct the dead time in the measurement results.

(3) Because the sensitivity may vary among GM detectors even if they are made by the same manufacturer, the conversion coefficient of the GM detector needs to be determined using a sample with a known radioactive concentration in the same manner as the demonstration experiment or determined in a similar manner with the TGS-146B used in this demonstration experiment.

(4) If the radioactive concentration of ${ }^{90} \mathrm{Sr} /{ }^{90} \mathrm{Y}$ in a solution is very high, it is not appropriate to use the GM detector for the measurement of the $\beta$-rays from ${ }^{90} \mathrm{Y}$ because the correction of dead time becomes significant. In such a case, a collimator with a smaller diameter ${ }^{\mathrm{c}}$ and a measuring instrument that can measure $\beta$-rays without being influenced by the dead time (such as an ionization chamber type survey meter that can be easily handled) needs to be used. It is necessary to determine the conversion coefficient using a sample with a known concentration in the same manner as in this study.

(5) To obtain precise information regarding the achievement of radioactive equilibrium between ${ }^{90} \mathrm{Sr}$ and ${ }^{90} \mathrm{Y}$ in a water sample, it is necessary to conduct measurements for at least $10 \mathrm{~h}$, preferably at an interval of 1 day.

(6) This method can be used under the condition where ${ }^{90} \mathrm{Sr} /{ }^{90} \mathrm{Y},{ }^{134} \mathrm{Cs}$ and ${ }^{137} \mathrm{Cs}$ occur as the main nuclides, such as in the water samples currently obtained at $1 \mathrm{~F}$. If a water sample containing nuclear fuel materials is obtained in the course of future decommissioning work, it will be necessary to examine the relative ratio of the nuclides occurring to determine whether

\footnotetext{
${ }^{\mathrm{c}}$ In the case of a $1 \mathrm{~cm}$ collimator with the smallest diameter used in this study, the upper limit of measurement is $10^{5} \mathrm{~Bq} / \mathrm{cm}^{3}$ if setting an upper limit of $1,000 \mathrm{cps}$. In the calculation of egs 5 , it is possible to measure up to $2 \times 10^{6} \mathrm{~Bq} / \mathrm{cm}^{3}$ in the case of a collimator with a diameter of $0.4 \mathrm{~cm}$.
} 
this method can be applied or not.

For $1 \mathrm{~F}$, it is very important to control ${ }^{90} \mathrm{Sr}$, and it is necessary to quickly and precisely measure the radioactive ${ }^{90} \mathrm{Sr}$ concentration in water samples. Although the water samples with a radioactive concentration of several $\mathrm{Bq} / \mathrm{cm}^{3}$ or higher can be the measuring objects, because no chemical operation is required and the measurement can be conducted with water samples placed in containers, it is possible to establish a system for many samples in combination with robotic operation. It is also expected that applying this method will significantly shorten the time needed to obtain a result and improve precision as well.

\section{References}

1) Ministry of Education, Culture, Sports, Science and Technology, Series of Radioactivity Measurement Method 2; Method of Radioactive Strontium Analysis [Housyano sokutei-hou shiriizu 2; Housyasei sutorontiumu bunseki-hou] (2003). [in Japanese]

2) E. Tanaka, T. Hiramoto, T. Maruyama, "Beta-spectroscopy of low level samples by a coincidence type scintillation spectrometer," J. Nucl. Sci. Technol., 1, 305-310 (1964).

3) Tokyo Electric Power Company, "Leakage of Contaminated Water at H4 Tank Area," Handout for the 5th Working Group on Managing Contaminated Water, Commission on Supervision and Evaluation of the Specified Nuclear Facility, 2013 Aug 30 (in Japanese), [Internet], http://warp.da.ndl.go.jp/info:ndljp/ pid/10953979/www.nsr.go.jp/data /000051227.pdf, cited 2018 September 19.

4) Ministry of Education, Culture, Sports, Science and Technology, Series of Radioactivity Measurement Method 1; Method of Gross Beta Measurements [Housyano sokutei-hou shiriizu 1; Zenbeta sokutei-hou] (1977). [in Japanese]

5) M. Nakano, Y. Hiyama, H. Watanabe, S. Sumiya, "Rapid analysis method of ${ }^{89} \mathrm{Sr}$ and ${ }^{90} \mathrm{Sr}$ in effluent with a liquid scintillation counter," RADIOISOTOPES, 59, 313-328 (2010). [in Japanese]

6) Tokyo Electric Power Company, "Mobile Type Strontium Removal System for Concentrated Water in RO," Handout for the 3rd Secretariat Meeting on Decommissioning and Countermeasure of Contaminated Water, 2014 February 27. [in Japanese] [Internet], http://www.meti.go.jp/earthquake/nuclear/ pdf/140227/140227_02s.pdf, cited 2015 February 27.

7) Tokyo Electric Power Company, "Results of Detailed Analysis of Radionuclide Concentrations Inside the First Port of Fukushima Daiichi Nuclear Power Station, Around the Ferry Port and the Bulkhead," 2015 January 30. [in Japanese] [Internet], http://www.tepco.co.jp/nu/fukushima-np/f1/smp/2015/images/2tb-east_15013001-j.pdf (cited 2018 September 19).

8) Japan Radioisotope Association, Radioisotope Pocket Data Book 11th Edition, Maruzen, Tokyo, ISBN978-4-89073-211-1 C3049 (2011). [in Japanese]

9) T. Nakamura, Radiation Physics and Technology for Accelerator Safety [Housyasen Buturi to Kasokuki Anzen no Kougaku], Chijin Syokan, Tokyo, ISBN4-8052-0685-3 C3042 (2001). [in Japanese]

10) H. Hirayama, Y. Namito, A. F. Bielajew, S. J. Wilderman, W. R. Nelson, The EGS5 Code System, SLAC-R-730(2005) and KEK Report 2005-8 (2005).

11) Hitachi Aloka Medical, Ltd, "GM Survey meter TGS-146B," [Internet], http://www.hitachi-aloka.co.jp/ products/data/radiation-002-TGS-146, cited 2015, February 17.

12) KOKUGO Co., Ltd., "Research and Experimental Devices, Bottles," [Internet], http://corporate.kokugo. co.jp/en/products\#chapter1, cited 2018 September 19.

13) Tokyo Electric Power Company etc., "Development Demonstration Project of High Performance Advanced Liquid Processing System: Lab Test, System Test and Evaluation Plan and Its Progress," Handout for the 2nd Task Force on High Performance Advanced Liquid Processing System, 2014 February 28. [in Japanese] [Internet], http://www.meti.go.jp/earthquake/nuclear/pdf/140228/140228_02d.pdf, cited 2018 September 19.

14) G. K. Kurbatov, M. H. Kurbatov, "Isolation of radioactive yttorium and some of its properties in minute concentrations," J. Phys. Chem., 46, 441-457 (1942).

15) Japan Radioisotope Association, Lectures and Practices on Radiations and Isotopes [Housyasen/Aisotopu Kougi to Jissyuu], Maruzen, Tokyo, ISBN 9784621037454 (1997). [in Japanese]

16) G. K. Schweitzer, B. R. Stein, W. M. Jackson, "Studies in low concentration chemistry. III. The radiocolloidal properties of yttorium-90,” J. Am. Chem. Soc., 75, 793-795 (1953). 


\title{
Article
}

\section{Simple Measurement Method with High Detection Efficiency to Evaluate Sr-90 Concentration in Water by Measuring $\beta$ rays from Y-90 Using GM-Detector}

\author{
Kenjiro KONDO ${ }^{1, *}$, Hideo HIRAYAMA ${ }^{1}$, Masafumi TAIRA ${ }^{1}$, \\ Hiroshi MATSUMURA ${ }^{1}$, Hiroshi IWASE ${ }^{1}$ and Shinichi SASAKI ${ }^{1}$ \\ ${ }^{1}$ High Energy Accelerator Research Organization, 1-1 Oho, Tsukuba-shi, Ibaraki 305-0801, Japan
}

\begin{abstract}
Strontium-90/Y-90 are major radionuclides observed in the water samples tested recently at the site of the Fukushima Daiichi Nuclear Power Plant of Tokyo Electric Power Company. A simple method of evaluating Sr-90 concentration in these water samples by measuring $\beta$ rays from Y-90 with a GM-detector setup was developed. By applying the precipitation method, Sr90 and Y-90 were separated and quantitatively collected with a filter. $\beta$ rays from Y-90 in the filter were measured two times at appropriate intervals by inserting a polyethylene plate of $2 \mathrm{~mm}$ thickness as a $\beta$-ray absorber. The contribution of $\gamma$ rays from Cs-134 and Cs- 137 to the Y-90 count rates was quantitatively evaluated using a 10 -mm-thick acrylic resin plate. From the parent-daughter relationship between Sr-90 and Y-90, the Sr-90 concentration was evaluated using the conversion coefficient of $\mathrm{Y}-90$ count rate $(\mathrm{cps})$ to $\mathrm{Sr}-90$ concentration $\left(\mathrm{Bq} / \mathrm{cm}^{3}\right)$. It was verified that $\mathrm{Sr}-90$ concentration of below $0.01 \mathrm{~Bq} / \mathrm{cm}^{3}$ in water samples can be correctly measured by this simple method.
\end{abstract}

KEYWORDS: Sr-90/Y-90, Sr-90 concentration, Y-90 $\beta$ ray, GM-detector, coprecipitation

\section{Introduction}

At the Fukushima Daiichi Nuclear Power Plant of the Tokyo Electric Power Company (hereinafter referred to as " $1 \mathrm{~F}$ power station"), various kinds of radioactive-contaminated water were generated due to the accident. Accurate determination of the radioactive concentration in the contaminated water is one of the most important tasks in the ongoing process towards recovery from the accident.

The major radionuclides contained in the contaminated water from the $1 \mathrm{~F}$ power station are Sr-90 and Y-90. In addition, Cs-134 and Cs-137 are present in low concentrations ${ }^{1)}$, which can be measured relatively easily due to the emission of $\gamma$-rays. However, $\beta$-ray-emitting nuclides such as $\mathrm{Sr}-90$ generally require complicated chemical separation and advanced measurement techniques ${ }^{2-4)}$ as well as a long time for analysis.

* Corresponding author, E-mail: kenjiro.kondo@kek.jp

DOI : 10.15669 /fukushimainsights.Vol.4.401

(C) 2021 Atomic Energy Society of Japan. All rights reserved.

Originally published in Transactions of the Atomic Energy Society of Japan (ISSN 1347-2879), Vol. 14, No. 3, p.151-160

(2015) in Japanese. (Japanese version accepted: April 9, 2015) 
Among the nuclides present in contaminated water, Sr-90 is one of the most important in terms of radiation safety management as the effluent standard prescribed under the law is as low as $0.03 \mathrm{~Bq} / \mathrm{cm}^{3}$. The provisional effluent standard to be observed for Sr-90 when discharging the stagnant water from the weir, such as a tank for storing contaminated water, at the $1 \mathrm{~F}$ power station is $0.01 \mathrm{~Bq} / \mathrm{cm}^{3}$. Therefore, a method that facilitates this concentration level to be measured easily, quickly and accurately must be developed.

In this study, low concentrations of Sr-90 were determined easily by measuring the $\beta$-rays from Y-90, concentrated and captured in a filter, using the carbonate precipitation and iron coprecipitation methods ${ }^{7,8)}$ (hereinafter referred to as "filter method").

The determination of the concentration of Sr-90 by the filter method involves the precipitation and concentration of Sr-90 or Y-90 in a filter through simplified chemical separation and subsequent measurement with a GM instrument used in the radiation management field, without requiring advanced radiochemical separation of Sr-90 or Y-90. This method was found to contribute to the efficiency of management of contaminated water at the $1 \mathrm{~F}$ power station.

\section{Water Samples to be Examined and Outline of Filter Method}

\section{Water Samples to be Examined}

Table 1 shows the major radionuclides present in RO-concentrated salt water (concentrated salt water with RO (Reverse Osmosis) membrane) stored in the $\mathrm{H} 1 \operatorname{tank}^{9)}$ (This is a facility (equipment) at the $1 \mathrm{~F}$ power station in which high concentrations of $\mathrm{Sr}-90 / \mathrm{Y}-90$ are present), H4-No.5 tank ${ }^{1)}$, and underground water (No. 1-4 observation wells) ${ }^{10)}$ as well as their concentrations, as an example of the radionuclides and their concentrations to be measured in the water sample from the 1F power station. Table 2 shows the nuclear characteristics of such nuclides ${ }^{11)}$. The proposed method of measurement is intended for water samples with relatively low concentrations of radioactive materials such as high-concentration contaminated water leaked from a tank and diluted with a large volume of rainwater or groundwater polluted with high-concentration contaminated water that has seeped underground. It is clear from Table 1 that the major nuclides are Sr-90 and Y-90 whereas the concentration of other nuclides such as Cs-134 and Cs-137 is lower than one hundredth of the concentration of Sr-90.

Table 1 Principal radioisotopes and their concentrations in the water sampled at the site of Fukushima Daiichi Nuclear Power Plant of Tokyo Electric Power Company

\begin{tabular}{|c|c|c|c|}
\hline Nuclide & $\begin{array}{c}\text { RO-concentrat- } \\
\text { ed salt-water } \\
\text { H1 tank }\end{array}$ & $\begin{array}{l}\text { RO-concentrat- } \\
\text { ed solt-water } \\
\text { H4-No.5 tank }{ }^{1)}\end{array}$ & $\begin{array}{l}\text { Underground } \\
\text { water (No.1-4 } \\
\text { observation } \\
\text { point) }{ }^{10)}\end{array}$ \\
\hline & \multicolumn{3}{|c|}{$\mathrm{Bq} / \mathrm{cm}^{3}$} \\
\hline $\mathrm{Mn}-54$ & & $1.9 \mathrm{E}+00$ & \\
\hline $\mathrm{Co}-60$ & & $1.2 \mathrm{E}+00$ & \\
\hline $\mathrm{Sr}-90$ & $7.6 \mathrm{E}+04$ & & \\
\hline $\mathrm{Cs}^{-134}$ & $4.7 \mathrm{E}+01$ & $4.6 \mathrm{E}+01$ & $1.0 \mathrm{E}-03$ \\
\hline $\mathrm{Cs}^{-137}$ & $6.6 \mathrm{E}+01$ & $1.0 \mathrm{E}+02$ & $1.1 \mathrm{E}-02$ \\
\hline Total $\beta$ & $3.9 \mathrm{E}+05$ & $8.0 \mathrm{E}+04$ & $2.4 \mathrm{E}-01$ \\
\hline
\end{tabular}


Table 2 Principal radioisotopes in the water sample and their nuclear characteristics

\begin{tabular}{|c|c|c|c|c|c|c|}
\hline Nuclide & Half-life & Decay mode & $\begin{array}{c}\text { Maximum } \beta \text { ray } \\
\text { Energy }(\mathrm{MeV})\end{array}$ & Eemission rate & $\begin{array}{c}\text { Main } \gamma \text { ray energy } \\
(\mathrm{MeV})\end{array}$ & Emission rate \\
\hline${ }^{54} \mathrm{Mn}$ & $312.03 \mathrm{~d}$ & $\mathrm{EC}$ & & 1.0 & 0.836 & 1.0 \\
\hline \multirow{2}{*}{${ }^{60} \mathrm{Co}$} & \multirow{2}{*}{$5.2713 \mathrm{y}$} & \multirow{2}{*}{$\beta^{-}$} & 0.318 & 0.999 & 1.173 & 0.999 \\
\hline & & & 1.491 & 0.0012 & 1.333 & 1.0 \\
\hline \multirow{2}{*}{$\begin{array}{l}{ }^{90} \mathrm{Sr} \\
{ }^{90} \mathrm{Y}\end{array}$} & $28.79 \mathrm{y}$ & $\beta^{-}$ & 0.546 & 1.0 & & \\
\hline & $64.00 \mathrm{~h}$ & $\beta^{-}$ & 2.28 & 1.0 & & \\
\hline \multirow{7}{*}{${ }^{134} \mathrm{Cs}$} & \multirow{7}{*}{$2.065 \mathrm{y}$} & \multirow{7}{*}{$\beta^{-}$} & 0.0888 & 0.273 & 0.563 & 0.084 \\
\hline & & & 0.415 & 0.025 & 0.569 & 0.154 \\
\hline & & & 0.658 & 0.702 & 0.605 & 0.976 \\
\hline & & & \multirow[t]{4}{*}{ Others } & & 0.796 & 0.866 \\
\hline & & & & & 0.802 & 0.087 \\
\hline & & & & & 1.365 & 0.03 \\
\hline & & & & & Others & \\
\hline \multirow{2}{*}{${ }^{137} \mathrm{Cs}$} & \multirow{2}{*}{$30.1671 \mathrm{y}$} & \multirow{2}{*}{$\beta^{-}$} & 0.514 & 0.944 & & \\
\hline & & & 1.176 & 0.056 & & \\
\hline${ }^{137 \mathrm{~m}} \mathrm{Ba}$ & $2.532 \mathrm{~m}$ & IT & & & 0.662 & 0.851 \\
\hline
\end{tabular}

\section{Outline of Filter Method}

In this method, Sr-90 and Y-90 are separated and concentrated in a filter using the carbonate precipitation and iron coprecipitation methods, and the filter is then used as a measurement sample. A polyethylene plate is inserted between the filter and GM counter tube as a $\beta$-ray absorber, and only those $\beta$-rays emitted with high energy are measured to determine the concentration of Y-90. If Sr-90 and Y-90 are confirmed to have achieved a state of secular equilibrium, the concentration of Y-90 obtained in a measurement is considered to be that of Sr-90 as well; otherwise the measurement is conducted twice after a certain interval to evaluate the concentration of Sr-90 based on the sequential decay relation between Sr-90 and Y-90.

\section{(1) Selective measurement of $\beta$-rays from Y-90 using a $\beta$-ray absorber}

Figure 1 shows the number of electrons which pass through the polyethylene plate $(1 \mathrm{~mm}$ is equivalent to $0.090 \mathrm{~g} / \mathrm{cm}^{2}$ ) and enter the GM counter, which is calculated using the electromagnetic cascade Monte Carlo calculation code egs $5^{12}$, when Sr-90, Y-90, Cs-134, Cs-137, and Co-60 are present on the filter surface with an areal density of $1 \mathrm{~Bq} / \mathrm{cm}^{2}$. The $\beta$-ray spectra of the respective nuclides are used in the calculation, and the secondary electrons generated due to the scattering of $\beta$-rays, as well as those generated by bremsstrahlung radiation, are included in the result. The system of calculation is based on the layout of the measuring instruments used in this method. As shown in the figure, the insertion of a polyethylene plate of appropriate thickness enables the removal of most of the $\beta$-rays from Co-60, Sr-90, Cs-134, and Cs- 137 to selectively measure only the $\beta$-rays from Y-90.

Although based on the premise that these nuclides are present in the same concentration, the contribution of other nuclides to the $\beta$-rays from Y-90 is $8.7 \%$ when the thickness of the polyethylene plate is $1 \mathrm{~mm}$ and $0.9 \%$ when it is $2 \mathrm{~mm}$. The contribution of the $\beta$-rays can be ignored when the thickness of the polyethylene plate is $10 \mathrm{~mm}$. With a 10 -mm-thick acrylic plate of higher density than polyethylene (Density: $1.20 \mathrm{~g} / \mathrm{cm}^{2}$ ), the contribution of the $\gamma$-rays from Cs-134 and Cs-137 can be estimated as described in a later section.

In the measurement of the $\beta$-rays emitted by Y-90, Sr-89, which is an isotope of $\mathrm{Sr}$ (Halflife: 50.5 days, maximum $\beta$-ray energy: $1.49 \mathrm{MeV}$ ), is considered to be significant because of its high $\beta$-ray energy, however, as more than three years have elapsed since the accident, its 


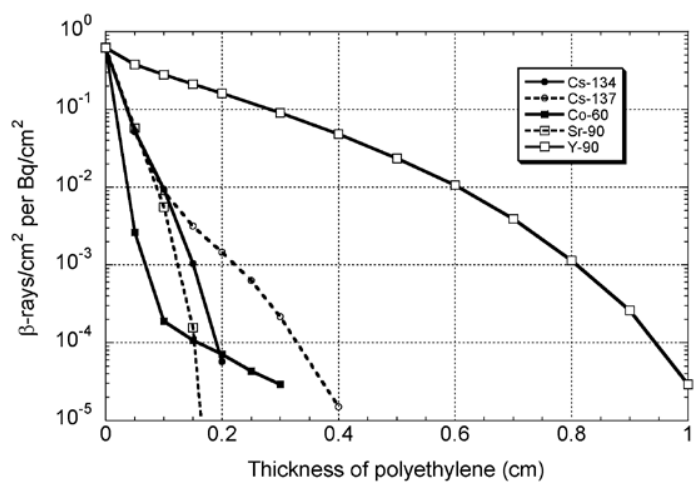

Figure 1 Relationship between thickness of polyethylene absorbers and transmission probability of $\beta$-rays from Sr-90, Y-90, Cs-134, Cs-137 and Co-60 calculated with egs5 Monte Carlo code

contribution can be ignored owing to its decay. Assuming that the contribution of Rh-106 and Ce-144, which also emit high-energy $\beta$-rays, may be ignored, a method that measures $\beta$-rays from Y-90 was used.

\section{(2) Evaluation of the radioactive concentration of $\mathrm{Sr}-90$}

This method involves the measurement of $\beta$-rays from Y-90 by concentrating and collecting the Sr-90 and Y-90 present in the water sample in a filter, regardless of the achievement of radiative equilibrium between these substances. The radioactive concentration of $\mathrm{Sr}-90$ can be determined from that of Y-90, which is measured using the polyethylene plate (with a thickness of $1 \mathrm{~mm}$ or $2 \mathrm{~mm}$ ) as a $\beta$-ray absorber. If Sr-90 and Y-90 have already achieved a state of secular equilibrium, the concentration of Sr-90 can be obtained by means of a single measurement, otherwise two measurements must be made at an interval of approximately five hours.

Assuming that the number of atoms of Sr-90 and Y-90 per $\mathrm{cm}^{3}$ when time $t$ is zero are $N_{S r-90}(0)$ and $N_{Y-90}(0)$, respectively, the number of atoms of $N_{Y-90}(t)$ after $t$ hours is determined as follows:

$$
N_{Y-90}(t)=\frac{\lambda_{S r-90}}{\lambda_{Y-90}-\lambda_{S r-90}} \times N_{S r-90}(0)\left[\exp \left(-\lambda_{S r-90} t\right)-\exp \left(-\lambda_{Y-90} t\right)\right]+N_{Y-90}(0) \exp \left(-\lambda_{Y-90} t\right)
$$

where, $\lambda_{S r-90}$ and $\lambda_{Y-90}$ are the decay constants of Sr-90 and Y-90, respectively. As $\lambda_{Y-90}-\lambda_{S r-90} \approx$ $\lambda_{Y-90}$ because $\lambda_{Y-90} \gg \lambda_{S r-90}$,

$$
\begin{aligned}
A_{Y-90}(t) & =\lambda_{Y-90} N_{Y-90}(t) \\
& =\lambda_{S r-90} N_{S r-90}(0)\left[\exp \left(-\lambda_{S r-90} t\right)-\exp \left(\lambda_{Y-90} t\right)\right]+\lambda_{Y-90} N_{Y-90}(0) \exp \left(-\lambda_{Y-90} t\right) \\
& =A_{S r-90}(0)\left[\exp \left(-\lambda_{S r-90} t\right)-\exp \left(-\lambda_{Y-90} t\right)\right]+A_{Y-90}(0) \exp \left(-\lambda_{Y-90} t\right)
\end{aligned}
$$

where, $A_{Y-90}(0)$ and $A_{Y-90}(t)$ are the radioactive concentrations of Y-90 $\left(\mathrm{Bq} / \mathrm{cm}^{3}\right)$ when time $t$ is zero and $t$, respectively, and $A_{S r-90}(0)$ is the radioactive concentration of S-90 $\left(\mathrm{Bq} / \mathrm{cm}^{3}\right)$ when time $t$ is zero. The radioactive concentration of Sr-90 can be determined by using Eq. (3) given below:

$$
A_{S r-90}(0)=\frac{A_{Y-90}(t)-A_{Y-90}(0) \exp \left(-\lambda_{Y-90} t\right)}{\exp \left(-\lambda_{S r-90} t\right)-\exp \left(-\lambda_{Y-90} t\right)}
$$

$A_{Y-90}(t)$ and $A_{Y-90}(0)$ can be obtained from the correlation (conversion coefficient) between 
the count rate of Y-90 obtained experimentally with the GM counter tube and the radioactive concentration of $\mathrm{Y}-90\left(\mathrm{~Bq} / \mathrm{cm}^{3}\right)$.

\section{Points to be Considered in the Experiment}

In order to ensure the validity of the application of the filter method, the following points must be given due consideration:

a) The collection efficiency at the time of concentrating and separating the Sr-90 and Y-90 from the water samples into the filter using the carbonate precipitation and iron coprecipitation methods.

b) The correlation between the count rate of Y-90 measured with a GM detector and radioactive concentration of Y-90 and Sr-90 using a standard Sr-90 solution (conversion coefficient).

c) The influence of seawater content, due to a possible incorporation of seawater into some water samples collected at the $1 \mathrm{~F}$ power station, on $\mathrm{Y}-90$ measurements.

d) Using the polyethylene plate as a $\beta$-ray absorber enables the measurement of the $\beta$ rays of Y-90 only, however, a GM detector has the sensitivity to detect $\gamma$-rays and bremsstrahlung radiation. Therefore, the influence of the $\gamma$-rays from the nuclides such as Cs- 134 and Cs-137, which are present in the water samples, as well as the bremsstrahlung radiation from $\beta$-rays on the measurement of Y-90, must be considered.

The experiment was conducted in view of the above-mentioned points and the results are described in the next section.

\section{Experiment and Results}

\section{Concentration, Separation and Collection Efficiencies with a Filter}

\section{(1) Separation and collection of $\mathrm{Sr}$ and $\mathrm{Y}$}

Considering that Sr-90 and Y-90, which are close to the carrier-free state, are present in the water samples collected from $1 \mathrm{~F}$ power station, $\mathrm{Sr}$ and $\mathrm{Y}$ were added as carriers in advance to study the separation conditions and the collection efficiencies of $\mathrm{Sr}$ and $\mathrm{Y}$ were determined using the carbonate precipitation and iron coprecipitation methods.

In this method, $\mathrm{Sr}$ and $\mathrm{Y}$ are separated as strontium carbonate and yttrium hydroxide, respectively. $\mathrm{Fe}^{3+}$ ions are added to facilitate the processes of sedimentation and separation due to the coprecipitation effect. As the water samples that are collected from the $1 \mathrm{~F}$ power station also contain Cs-134 and Cs-137 in the carrier-free state, Cs with a similar concentration as $\mathrm{Sr}$ is added as a carrier for the separation operation when conducting the process with actual water samples.

\section{(2) Preparation of water samples used in the experiment}

The water samples used in the experiment contained pure water, seawater, and well water collected from the High Energy Accelerator Research Organization (This was prepared by sterilizing the ground water to render it potable and is hereinafter referred to as "well water"). The well water was used as simulated groundwater obtained from the $1 \mathrm{~F}$ power station, and the water samples containing seawater were used to study the influence of the incorporation of seawater on the measurements because it was possible that seawater was mixed with some water samples collected from the $1 \mathrm{~F}$ power station. The chemicals used for calibration in the sedimentation, separation, and inductively coupled plasma (ICP) emission spectrometric 
analyses are given below:

Standard $\mathrm{Sr}^{2+}$ and $\mathrm{Y}^{3+}$ atomic absorption solutions (Kishida Chemical Co., Ltd.) containing $1,000 \mathrm{ppm}$ of $\mathrm{Sr}^{2+}$ or $\mathrm{Y}^{3+}, \mathrm{Fe}^{3+}$ prepared by mixing special grade ferric chloride (Junsei Chemical Co., Ltd.) with $0.1 \mathrm{~N}$ hydrochloric acid to $10 \mathrm{mg} / \mathrm{mL}$, and $\mathrm{Ca}^{2+}$ prepared by dissolving special grade $\mathrm{CaCO}_{3}$ (Junsei Chemical Co., Ltd.) with concentrated hydrochloric acid as $\mathrm{Ca}^{2+}$ to $50 \mathrm{mg} / \mathrm{mL}$. Granular $\mathrm{Na}_{2} \mathrm{CO}_{3}$ of special grade (Wako Pure Chemical Corporation) was used in the original form and artificial seawater was prepared using Daigo's Artificial Seawater SP (for one liter, Nihon Pharmaceutical Co., Ltd.).

\section{(3) Separation and collection operation procedures}

A solution of $100 \mathrm{~mL}$ of the water sample containing pure water, seawater, and well water was prepared by adjusting the $\mathrm{Fe}^{3+}$ concentration to $50 \mathrm{ppm}, \mathrm{Sr}^{2+}$ and $\mathrm{Y}^{3+}$ concentrations to $10 \mathrm{ppm}$ using standard solutions, and $\mathrm{Ca}^{2+}$ concentration to $250 \mathrm{ppm}$ from the prepared $\mathrm{Ca}$ solution. This solution was agitated and then heated to approximately $50^{\circ} \mathrm{C}$. After one gram of $\mathrm{Na}_{2} \mathrm{CO}_{3}$ was added, the solution was agitated vigorously with a magnetic stirrer for approximately seven minutes and then agitated gently for approximately three minutes to promote the generation of a deposit and formation of floc. The solution was subsequently left to rest for approximately three minutes for the deposit to settle at the bottom in the form of floc and then filtrated. A filtering device with a 47-mm filter holder made entirely of glass (Nihon Millipore) was used. In order to ensure the uniformity of deposition, the supernatant was first filtered and when the volume was reduced to almost half, the rest of the solution was poured into a funnel. Normally, the filtration time is approximately $30 \mathrm{~s}$. The membrane filter used was a mixed cellulose ester filter (Millipore Advantec) with a pore size of $0.65 \mu \mathrm{m}$ and diameter of $47 \mathrm{~mm}$. The filtration was carried out while reducing the pressure with an aspirator and the suction was terminated when water stopped dripping from the filter. The concentrations of $\mathrm{Sr}$ and $\mathrm{Y}$ present in the undiluted solution before addition of $\mathrm{Na}_{2} \mathrm{CO}_{3}$ and the filtered solution were measured by the ICP emission spectrometric analysis method to determine the collection efficiencies of $\mathrm{Sr}$ and $\mathrm{Y}$.

\section{(4) Collection efficiencies of $\mathrm{Sr}$ and $\mathrm{Y}$}

Table 3 shows the collection efficiencies of $\mathrm{Sr}$ and $\mathrm{Y}$ from the water samples containing pure water, seawater, and well water, as determined by the method proposed in this study. As a result of adding an excessive amount of $\mathrm{Ca}$ and allowing sufficient time for the reaction to take place

Table 3 Collection efficiencies of $\mathrm{Sr}$ and $\mathrm{Y}$

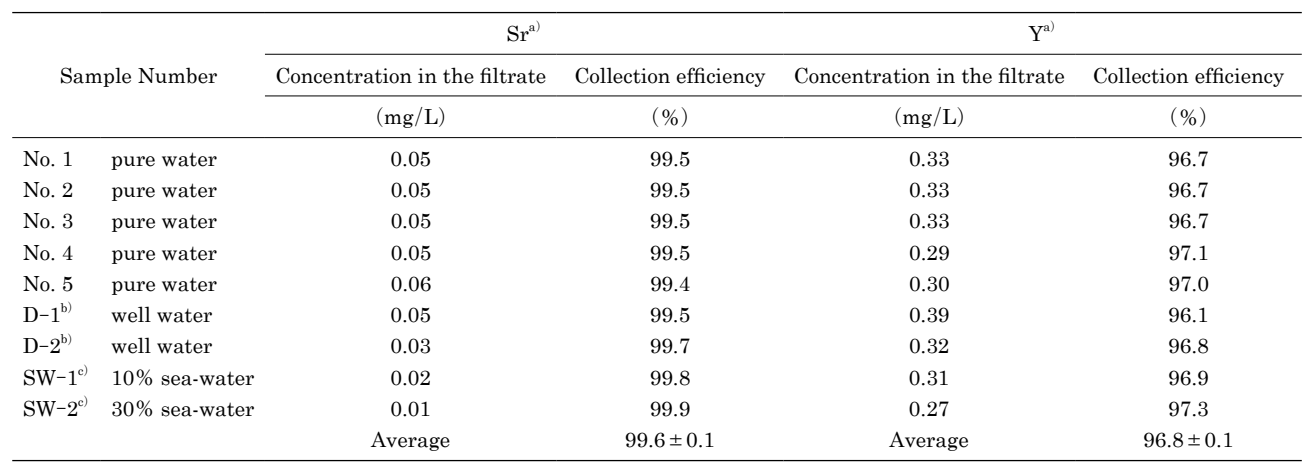

Concentration of $\mathrm{Sr}$ and/or $\mathrm{Y}$ in the start solution: $10.0 \mathrm{mg} / \mathrm{L}$

Well water: $\mathrm{Mg}^{2+}: 7 \mathrm{ppm}, \mathrm{Ca}^{2+}: 19 \mathrm{ppm}, \mathrm{Na}^{+}: 31 \mathrm{ppm}, \mathrm{K}^{+}: 4 \mathrm{ppm}, \mathrm{Cl}^{-}: 49 \mathrm{ppm}, \mathrm{SO}_{4}{ }^{2-}: 2-25 \mathrm{ppm}, \mathrm{Br}^{-}: 1 \mathrm{ppm} . \mathrm{pH}: 7.6$

Water containing seawater 
and maturation of the deposit, the collection efficiencies of $\mathrm{Sr}$ and $\mathrm{Y}$ were found to be $99.6 \%$ and $96.8 \%$, respectively. It was demonstrated that the $\mathrm{Sr}$ and $\mathrm{Y}$ in the water samples containing pure water, seawater, and well water were concentrated and captured in the filter at high collection efficiency and with high reproducibility. This result shows that the Sr-90 and Y-90 present in the stagnant water in the weir of the contaminated water tank and water samples containing underground water and seawater can be deposited and separated with high collection efficiency.

\section{Correlation between the Count rates of Y-90 and Radioactive Concentrations of Sr-90 and Y-90 (Conversion Coefficient)}

\section{(1) Concentration/Separation and radioactivity measurement of Sr-90 and Y-90 using a standard Sr-90 solution}

The relation between the count rates obtained with a GM detector and the radioactive concentrations of Sr-90 and Y-90 (conversion coefficient) was studied, where the measurement sample was the filter obtained by conducting the deposition and separation processes with the pure water-based sample containing Sr-90 of known concentration.

(a) Adjustment and concentration/collection of water samples containing Sr-90

The solutions were prepared with a concentration of $0.31 \mathrm{kBq} / \mathrm{mL}(50 \mathrm{~mL}$ measuring flask) and $3.1 \mathrm{~Bq} / \mathrm{mL}$ (100 mL measuring flask), with a standard Sr-90 solution (Eckert \& Ziegle) $\mathrm{SrCl}_{2} 0.1 \mathrm{M} \mathrm{HCl}, 15.5 \pm 0.4 \mathrm{kBq} / 5 \mathrm{~mL}$, and the specified amount from these solutions was added to adjust the concentration of Sr-90.

After adding the carriers to $100 \mathrm{~mL}$ of pure water containing Sr-90 of known concentration as described above, the deposition and separation of Sr-90 and Y-90 was carried out following the same procedure. At the $1 \mathrm{~F}$ power station, a simplified method of measurement ${ }^{13)}$ is adopted with a $500 \mathrm{~mL}$ sample water for measuring low-concentration $\mathrm{Sr}-90$, when it is imperative to determine whether the stagnant water in the weir of the RO-concentrated salt water storage tank can be discharged or not. In this study, lowering the measurable concentration by using a $400 \mathrm{~mL}$ water sample was also studied. In this case, the amount of chemicals added, except for the standard Sr-90 solution, should be four times as large as those added to $100 \mathrm{~mL}$ water samples, and the operations such as separation must be carried out according to the same procedures as the ones used for the $100 \mathrm{~mL}$ water samples. Assuming that Sr-90 and Y-90 in the standard solution achieved secular equilibrium, the analysis was conducted as described below:

(b) Measuring filter and radioactive measurement

The filter which was used in the collection process was sealed with a transparent laminate coating film. In addition, it was sealed in a polyethylene bag. Furthermore, it was placed between two plastic plates with $50 \mathrm{~mm}$ opening $(0.5 \mathrm{~mm}$ thick) for fixation. This opening was as large as the window of the GM counter tube. The size of the deposit was $37 \mathrm{~mm}$ in diameter. The center of the filter was aligned with the center of the GM counter tube and after securing it to the measuring stand the GM counter tube (Hitachi Aloka Medical, Ltd., GM survey meter TGS-146B ${ }^{14)}$ ) was firmly attached to conduct the measurement. In addition, after attaching the polyethylene plates ( $1 \mathrm{~mm}$ and $2 \mathrm{~mm}$ thick) as well as a $10-\mathrm{mm}$-thick acrylic plate $\left(1.20 \mathrm{~g} / \mathrm{cm}^{2}\right)$ close to the filter, the GM counter tube was firmly attached on top of them to conduct the measurements. The weight per unit area with the laminate coating film and polyethylene bag was approximately $0.0001 \mathrm{~g} / \mathrm{cm}^{2}$. The radioactive measurement was carried out in a box shielded with 5 -cm-thick lead. The background with no measurement sample was $0.45 \pm 0.1 \mathrm{cps}$. For the low-concentration sample, a three-minute measurement was conducted three times and for other samples, a one-minute measurement was conducted three times to determine the average 
value. The correction for the measurements was made based on a dead time of $250 \mu \mathrm{sec}$.

\section{(2) Conversion coefficient}

The results of the measurements with water samples of $100 \mathrm{~mL}$ and $400 \mathrm{~mL}$, classified into three types according to the low to high radioactive concentration range of $\mathrm{Sr}-90$ are shown in Figures 2 and 3. In the measurements using 1-mm and 2-mm $\beta$-ray absorbers, the count rates are based on the $\beta$-rays from Y-90 and the corresponding concentration is the radioactive concentration of Y-90, which is the same as the radioactive concentration of Sr-90 as it has achieved a state of secular equilibrium.

The measurements of the $\beta$-rays from Sr-90 and Y-90 were conducted directly with the filter (with no polyethylene plate) and these correspond to the radioactive concentration of Sr-90 and Y-90. For the $100 \mathrm{~mL}$ water sample, the measurement was conducted with and without the polyethylene plates $1 \mathrm{~mm}$ and $2 \mathrm{~mm}$ thick (the polyethylene plate $1 \mathrm{~mm}$ thick was $0.090 \mathrm{~g} / \mathrm{cm}^{2}$ ), and superior linearity was observed in the count rates and concentrations of Sr-90 and Y-90 at a concentration of approximately $0.03 \mathrm{~Bq} / \mathrm{cm}^{3}$ (effluent standard prescribed by law) or higher. In addition, in the case of measurements with the $400 \mathrm{~mL}$ water sample conducted in order to study the measurability of lower concentrations, superior linearity was observed in the count rates of radioactivity collected in the filter as well as the radioactive concentrations of Sr-90 and Y-90 in a wide range of concentrations at approximately $0.005 \mathrm{~Bq} / \mathrm{cm}^{3}$ or higher, as shown in Figure 3. At the 400-mL level, the sensitivity for detecting the radioactive concentrations that were much lower than the provisional effluent standard currently being used $\left(0.01 \mathrm{~Bq} / \mathrm{cm}^{3}\right)$ was confirmed, showing that the proposed method can be applied to low concentrations at around this level.

The deposits were collected in a $37 \mathrm{~mm}$ filter in diameter. The influence of self-absorption due to the amount of deposits was found to be small because high-energy $\beta$-rays from Y-90 were measured. The error bars in the figure correspond to the standard deviation $(1 \sigma)$ when the background is deducted. Table 4 shows the conversion coefficients and residual sums of squares $\left(\chi^{2}\right)$ determined by the least squares method based on the relation between the count rates (cps) and the radioactive concentrations of Sr-90 and Y-90 (Bq/ $\left.\mathrm{cm}^{3}\right)$ in the low concentration, medium concentration, and entire concentration ranges, according to the radioactive concentrations of Sr-90 used. The conversion coefficients differ depending on the thickness of the polyethylene plate and amount of water sample. As shown in Table 4, the conversion coefficients show a superior concordance in the concentration ranges of $0.5 \mathrm{~Bq} / \mathrm{cm}^{3}$ or lower as well as $6 \mathrm{~Bq} / \mathrm{cm}^{3}$ or lower in the case of $100 \mathrm{~mL}$ water samples and in the concentration ranges of $0.3 \mathrm{~Bq} / \mathrm{cm}^{3}$ or lower as well as $1 \mathrm{~Bq} / \mathrm{cm}^{3}$ or lower in the case of $400 \mathrm{~mL}$ water samples. On the other hand, they are slightly smaller in the concentration range of $0.05 \mathrm{~Bq} / \mathrm{cm}^{3}$ or lower in the case of $100 \mathrm{~mL}$ water samples and in the concentration range of $0.03 \mathrm{~Bq} / \mathrm{cm}^{3}$ or lower in the case of $400 \mathrm{~mL}$ water samples. In actual measurement, the concentration of Y-90 (or Sr-90) is evaluated using the conversion coefficient obtained for the entire concentration range with many measuring points. However, in the low concentration range, there is an overestimation of approximately $15 \%$ and $10 \%$ for the $100 \mathrm{~mL}$ and $400 \mathrm{~mL}$ water samples, respectively, when a $2 \mathrm{~mm}$-thick polyethylene plate is used. It is preferable that a polyethylene plate with a thickness of $2 \mathrm{~mm}$ is used in the measurement from the perspective that the concentration of substances such as radioactive $\mathrm{Cs}$, which are present in the deposits collected in the filter, changes significantly and only the $\beta$-rays from Y-90 are measured, even though the properties of the water sample are unknown. 


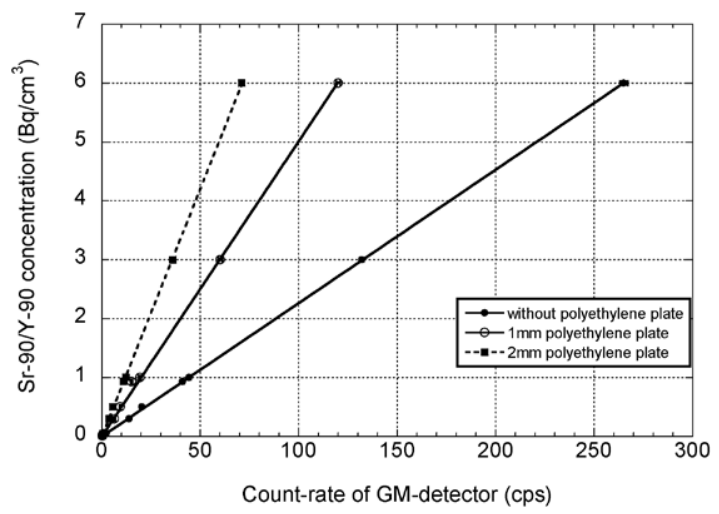

(a) the entire region of concentration: $\leq 6 \mathrm{~Bq} / \mathrm{cm}^{3}$

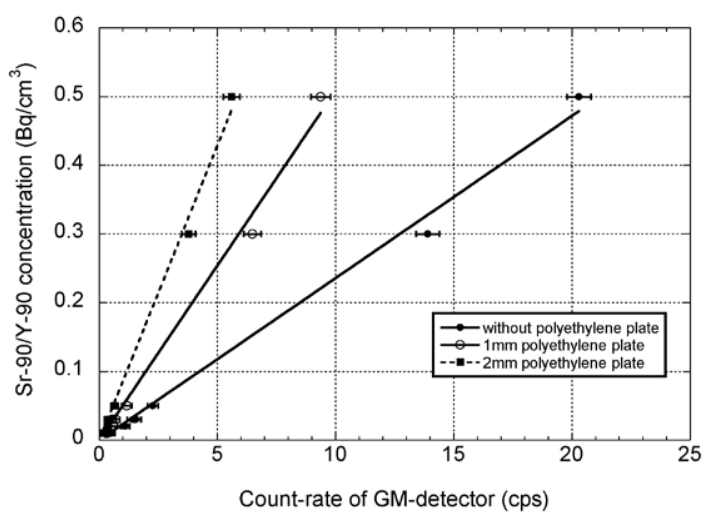

(b) the part of low concentration: $\leq 0.5 \mathrm{~Bq} / \mathrm{cm}^{3}$

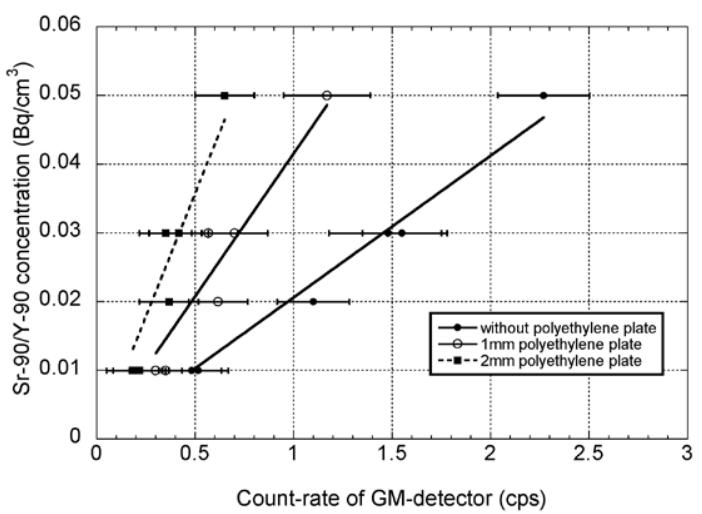

(c) the part of low concentration: $\leq 0.05 \mathrm{~Bq} / \mathrm{cm}^{3}$

Figure 2 Relationship between concentrations of $\mathrm{Sr}-90 / \mathrm{Y}-90\left(\mathrm{~Bq} / \mathrm{cm}^{3}\right)$ and count rates of GM-detector (water volume: $100 \mathrm{~mL}$, absorber: polyethylene plate)

(a) the entire region with a concentration: $\leq 6 \mathrm{~Bq} / \mathrm{cm}^{3}$

(b) the part with a low concentration: $\leq 0.5 \mathrm{~Bq} / \mathrm{cm}^{3}$

(c) the part with a low concentration: $\leq 0.05 \mathrm{~Bq} / \mathrm{cm}^{3}$ 


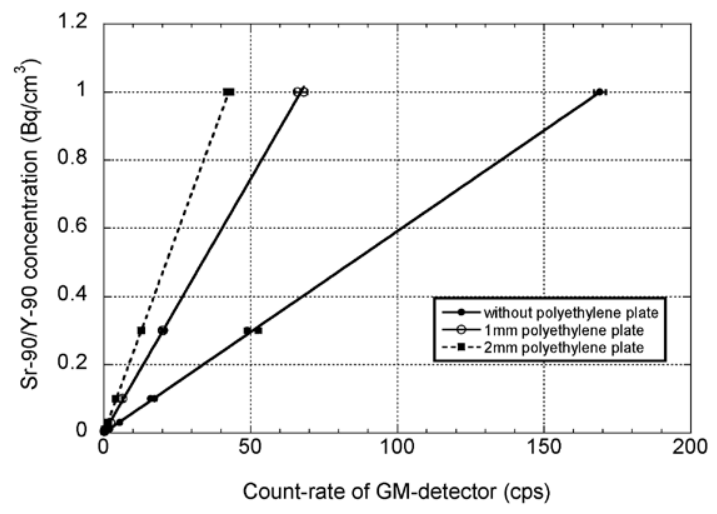

(a) the entire region of concentration: $\leq 1 \mathrm{~Bq} / \mathrm{cm}^{3}$

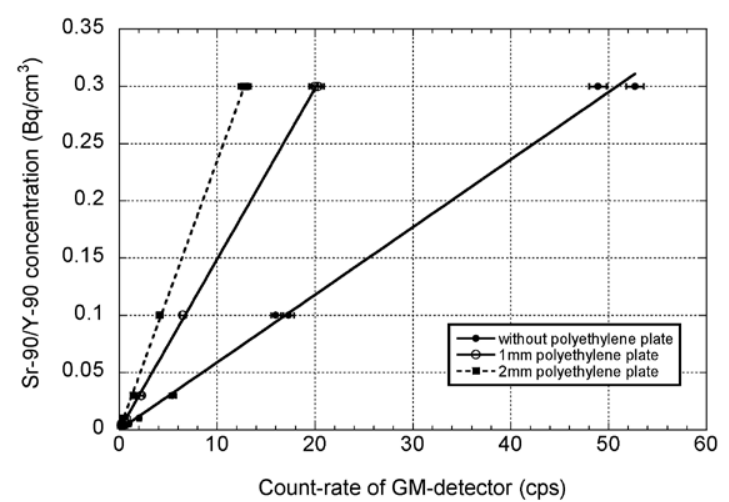

(b) the part of low concentration: $\leq 0.3 \mathrm{~Bq} / \mathrm{cm}^{3}$

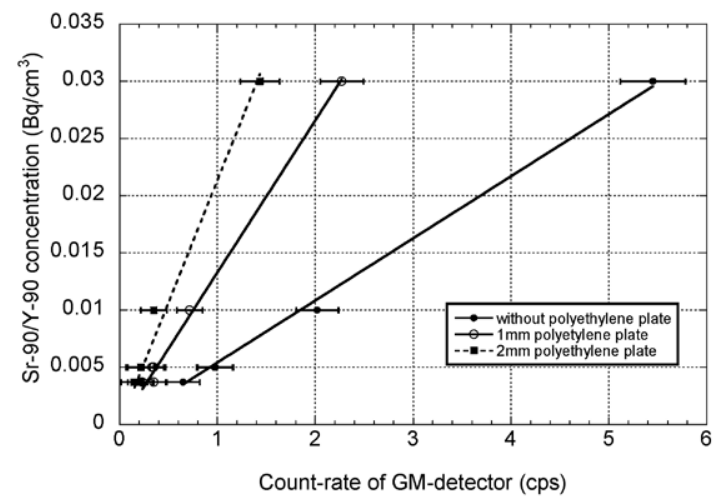

(c) the part of low concentration: $\leq 0.03 \mathrm{~Bq} / \mathrm{cm}^{3}$

Figure 3 Relation between concentrations of Sr-90/Y-90 (Bq/ $\mathrm{cm}^{3}$ ) and count rates of GM-detector (water volume: $400 \mathrm{~mL}$, absorber: polyethylene plate)
(a) the entire region with a concentration: $\leq 1 \mathrm{~Bq} / \mathrm{cm}^{3}$
(b) the part with a low concentration: $\leq 0.3 \mathrm{~Bq} / \mathrm{cm}^{3}$
(c) the part with a low concentration: $\leq 0.03 \mathrm{~Bq} / \mathrm{cm}^{3}$

\section{Influence of Seawater Concentration on the Measurements of Y-90}

As in the case for pure water, high collection efficiency was achieved in the case of well water, as shown in Table 3 (The component analysis result is shown in the Note of Table 3 ). 
Table 4 Conversion co-efficiencies of $\mathrm{cps}$ to $\mathrm{Bq} / \mathrm{cm}^{3}$

\begin{tabular}{|c|c|c|c|c|c|c|c|}
\hline \multirow{2}{*}{ Sample volume } & \multirow{2}{*}{$\begin{array}{c}\text { Polyethylene } \\
\text { plate thickness } \\
\mathrm{mm}\end{array}$} & \multicolumn{6}{|c|}{ Conversion co-efficient } \\
\hline & & $\left(\mathrm{Bq} / \mathrm{cm}^{3}\right) / \mathrm{cps}$ & $\chi^{2}$ & $\left(\mathrm{~Bq} / \mathrm{cm}^{3}\right) / \mathrm{cps}$ & $\chi^{2}$ & $\left(\mathrm{~Bq} / \mathrm{cm}^{3}\right) / \mathrm{cps}$ & $\chi^{2}$ \\
\hline \multirow{4}{*}{$100 \mathrm{~mL}$ sample } & & \multicolumn{2}{|c|}{ Concentration $\leq 6 \mathrm{~Bq} / \mathrm{cm}^{3}$} & \multicolumn{2}{|c|}{ Concentration $\leq 0.5 \mathrm{~Bq} / \mathrm{cm}^{3}$} & \multicolumn{2}{|c|}{ Concentration $\leq 0.05 \mathrm{~Bq} / \mathrm{cm}^{3}$} \\
\hline & 0 & 0.0227 & 0.00201 & 0.0236 & 0.00135 & 0.0206 & $2.19 \mathrm{E}-05$ \\
\hline & 1 & 0.0502 & 0.0329 & 0.0509 & 0.00177 & 0.0416 & 0.000103 \\
\hline & 2 & 0.0842 & 0.00540 & 0.0859 & 0.00128 & 0.0716 & 0.000116 \\
\hline \multirow{4}{*}{$400 \mathrm{~mL}$ sample } & & \multicolumn{2}{|c|}{ Concentration $\leq 1 \mathrm{~Bq} / \mathrm{cm}^{3}$} & \multicolumn{2}{|c|}{ Concentration $\leq 0.3 \mathrm{~Bq} / \mathrm{cm}^{3}$} & \multicolumn{2}{|c|}{ Concentration $\leq 0.03 \mathrm{~Bq} / \mathrm{cm}^{3}$} \\
\hline & 0 & 0.00592 & 0.000297 & 0.00590 & 0.000297 & 0.00543 & $1.34 \mathrm{E}-06$ \\
\hline & 1 & 0.0149 & 0.000588 & 0.0149 & $4.98 \mathrm{E}-05$ & 0.0133 & $1.97 \mathrm{E}-06$ \\
\hline & 2 & 0.0235 & 0.000521 & 0.0235 & $5.44 \mathrm{E}-05$ & 0.0214 & $8.13 E-06$ \\
\hline
\end{tabular}

Therefore, this would indicate that this method can be applied to most of the underground water obtained from the $1 \mathrm{~F}$ power station. Moreover, although seawater was considered to be incorporated in some water samples to be measured, high collection efficiency was also achieved for seawater concentrations of $10 \%$ and $30 \%$ as shown in Table 3, which signifies that this measurement method can be applied to seawater. However, deposits accumulate in the water samples containing seawater, and the influence of self-absorption due to such deposits must be taken into account. As a result, the influence of concentration of seawater on the measurements of Y-90 in this filter method was studied.

Using artificial seawater, $100 \mathrm{~mL}$ water samples were prepared with concentrations of 1, 2, 3, $7,10,15,20$, and $30 \%$. Carriers were added to these water samples, as in the aforementioned experiment, to prepare solutions with a Sr-90 concentration of $1.0 \mathrm{~Bq} / \mathrm{cm}^{3}$. The filter was examined after conducting the deposition and separation processes with these solutions, according to the aforementioned method. The deposits in the samples containing seawater were bulky and wet, as compared to the deposits in the $100 \mathrm{~mL}$ water sample with a seawater concentration of $0 \%$.

Table 5 shows the results of the measurement as well as the ratios of the count rates to that when the seawater concentration was $0 \%$ with a $2-\mathrm{mm}$-thick polyethylene plate. In addition to the effect of the seawater content, an increase in the amount of deposits, due to substances such as $\mathrm{Mg}$ present in the seawater, was considered to be significant. At seawater concentrations from $7 \%$ to $30 \%$, the ratio of the count rate to that when the seawater concentration was $0 \%$ was almost constant (nearly 0.86 ). If the seawater concentration is unknown, the concentration that is determined, using the count rate and conversion coefficient of Y-90 shown in Table 4 (for the total concentration range), is underestimated. If it is apparent that water sample contains seawater, the concentration of Y-90 can be estimated by using the value obtained on multiplying the count rate of Y-90 by 1.16 . However, a slight overestimation would occur at seawater concentrations of $7 \%$ or lower.

The deposits in seawater samples were susceptible to moisture, and the weight of the seawater with a concentration of $10 \%$, measured after it is dried for 24 hours in a desiccator, was $0.0042 \mathrm{~g} / \mathrm{cm}^{2}$. The actual water content of the deposits at the time of measurement was unknown, and a correlation between the amount of deposits and self-absorption was not observed.

In the actual separation process, if a water sample with a seawater concentration of $20 \%$ or higher is used, it is too difficult to conduct a quantitative measurement because the attachment of bulky deposits to the wall cannot be avoided when removing the filter and it is difficult to conduct a reproducible measurement if a water sample contains a high concentration of seawater. Therefore, in the case of such water samples, it is necessary to check the saline concentration and dilute the water sample before conducting the measurements. 
Table 5 Effect of seawater concentration on Y-90 count rates

\begin{tabular}{ccccc}
\hline \multirow{2}{*}{$\begin{array}{c}\text { Sample } \\
\text { No }\end{array}$} & $\begin{array}{c}\text { Concentra- } \\
\text { tion of Sr-90 }\end{array}$ & $\begin{array}{c}\text { Cocentra- } \\
\text { tion of sea } \\
\text { water }\end{array}$ & $\begin{array}{c}\text { Count-rate } \\
\text { with } 2 \mathrm{~mm} \\
\text { polyethylene }\end{array}$ & $\begin{array}{c}\text { Ratio to } \\
\text { A }\end{array}$ \\
\cline { 2 - 5 } & $\mathrm{Bq} / \mathrm{cm}^{3}$ & $\%$ & $\mathrm{cps}$ & $\mathrm{B} / \mathrm{A}$ \\
\hline No. S-0 & 1 & 0 & $12 \pm 0.5(\mathrm{~A})$ & - \\
No. S-1 & 1 & 1 & $11.6 \pm 0.5(\mathrm{~B})$ & 0.96 \\
No. S-2 & 1 & 2 & $11.3 \pm 0.5(\mathrm{~B})$ & 0.93 \\
No. S-3 & 1 & 3 & $11.3 \pm 0.5(\mathrm{~B})$ & 0.94 \\
No. S-4 & 1 & 7 & $10.3 \pm 0.5(\mathrm{~B})$ & 0.86 \\
No. S-5 & 1 & 7 & $10.3 \pm 0.5(\mathrm{~B})$ & 0.85 \\
No. S-6 & 1 & 10 & $10.3 \pm 0.5(\mathrm{~B})$ & 0.86 \\
No. S-7 & 1 & 15 & $10.3 \pm 0.5(\mathrm{~B})$ & 0.86 \\
No. S-8 & 1 & 20 & $10.7 \pm 0.5(\mathrm{~B})$ & 0.88 \\
No. S-9 & 1 & 30 & $10.1 \pm 0.4(\mathrm{~B})$ & 0.84 \\
\hline
\end{tabular}

\section{Influence of $\gamma$-Ray and Bremsstrahlung Radiation on the Measurements of $\gamma$-Rays from Y-90}

The GM counter tube used in the measurement is sensitive not only to $\beta$-rays but also to $\gamma$-rays and bremsstrahlung radiation (hereinafter referred to as "photon"). In the measurement of $\beta$-rays from Y-90, the influence of bremsstrahlung radiation generated by $\gamma$ - and $\beta$-rays from the nuclides contained in the water samples must be considered.

According to Figure 1, the contribution of $\beta$-rays can be ignored when an acrylic plate of thickness $10 \mathrm{~mm}\left(1.20 \mathrm{~g} / \mathrm{cm}^{2}\right)$ is used. Photons are attenuated by the $10-\mathrm{mm}$-thick acrylic plate, but the rate of attenuation caused by the acrylic plate is much lower than that caused by $\beta$-rays. This indicates that the results of measurement after passing through the 10-mm-thick acrylic plate can be used as the background measurement due to these photons. The influence of the background due to photons was studied using a standard Cs-137 planar source. In addition, according to the deposition method used in the experiment, it is considered that the rate of collection of the Cs-134 and Cs-137 in the filter is very low because most of them enter the filtrate due to their chemical properties even if they are contained in a water sample. In order to verify this, the collection efficiency was measured using a standard Cs-137 solution.

\section{(1) Evaluation of background due to Cs-137 photons}

Depending on the condition of Cs-134 and Cs-137 dissolved in the water samples from the $1 \mathrm{~F}$ power station, it is possible that some of them are collected in the filter.

Therefore, the influence of the photons from the Cs-134 and Cs-137 collected in the filter on the evaluation of the Sr-90 concentration was investigated.

In the experiment, the photons from Cs-137 were evaluated using a standard Cs-137 planar source (JRIA), sealed with plastic ( $1 \mathrm{~mm}$ thick on one side) with a diameter of $37 \mathrm{~mm}$ and radioactivity of $992 \mathrm{~Bq}$. The planar source with a diameter of $37 \mathrm{~mm}$ was as large as the deposits collected in the filter.

The 10 -mm-thick acrylic plate removes the $\beta$-rays from Cs-137 so that only the photons from Cs-137 are measured. The measurement obtained using the above standard source when the 10 -mm-thick acrylic plate was used was $1.0 \times 10^{-3} \mathrm{cps} / \mathrm{Bq}$. In the case of a 2 -mm-thick polyethylene plate, the $\beta$ rays from Cs- 137 can be ignored and only the photons from Cs-137 are measured. The measurement obtained under this condition using a standard source was $1.43 \times 10^{-3} \mathrm{cps} / \mathrm{Bq}$.

Therefore, based on the count rate $\left(C_{a c}\right)$ with a 10 -mm-thick acrylic plate, the contribution of photons for a 2-mm-thick polyethylene plate $\left(C s_{B G}\right)$ can be determined from the following 
equation using a correction coefficient of 1.43 :

$$
C s_{B G}(c p s)=1.43 \times C_{a c}
$$

In the actual measurements, the value which is obtained after deducting $C s_{B G}$ from the count rate for a 2-mm-thick polyethylene plate is used as the count rate based on the $\beta$-rays from Y-90, and the concentration of Y-90 is calculated using the conversion coefficient of Y-90.

Based on the conversion coefficient of Y-90 when a 2-mm-thick polyethylene plate is used in a $100 \mathrm{~mL}$ water sample, the count rate of $\mathrm{Y}-90$ per $\mathrm{Bq} / \mathrm{cm}^{3}$ is $11.8 \mathrm{cps}$, and this corresponds to the case where $100 \mathrm{~Bq}$ of Y-90 exists on the filter (with a diameter of $37 \mathrm{~mm}$ ) at a concentration of $0.11 \mathrm{cps}$ per Bq. On the other hand, as described above, the count rate per Bq of Cs-137 collected in the filter is $1.43 \times 10^{-3} \mathrm{cps}$, which indicates a significant difference between both the values. In addition, as described in (2) below, the filter's efficiency in collecting radioactive $\mathrm{Cs}$ is extremely low when radioactive Cs exists in the ionic state. Therefore, this suggests that the influence of the photons from Cs-134 and Cs-137 is very small.

On the basis of the above discussion, it can be inferred that the contribution of the photons from nuclides such as Cs-134 and Cs-137 to the measurements of Y-90 is approximately 1/100 even if Cs-134 and Cs-137 exist on the filter in the same radioactive concentration as Sr-90.

\section{(2) Efficiency of collecting Cs-137 in the filter}

A solution of $\mathrm{CsCl}$ was added to the aforementioned pure water-based solution used in the experiment of separating $\mathrm{Sr}$ and $\mathrm{Y}$ with a filter, to adjust the concentration of $\mathrm{Cs}^{+}$used as a carrier to $10 \mathrm{ppm}$. The same separation process was carried out after adding the standard Cs-137 solution with a concentration of $1.11 \times 10^{4} \mathrm{~Bq}$ to this solution. The deposits collected in the filter paper were dissolved in hydrochloric acid, and the solution thus prepared along with the undiluted solution before adding $\mathrm{Na}_{2} \mathrm{CO}_{3}$ were put into $20-\mathrm{mL}$ vials and measured with a Ge semiconductor detector to quantitate the Cs-137. It was found that Cs-137 collected in the filter accounted for $0.17 \%$ of the total deposits. It is considered that K-40, a naturally derived substance which may be present in the actual measurement samples, is also eliminated in the course of the deposition and separation processes due to its chemical properties.

On the other hand, it was reported ${ }^{15)}$ as an example that most of the Cs-134 and Cs-137 exist in the RO-concentrated salt water from the $1 \mathrm{~F}$ power station while adsorbing to colloids and suspended solids. In addition, it is considered that the properties of the water samples to be examined vary widely. In many cases, it is established that most of the Cs-134 and Cs-137 are collected in the filtrate and eliminated by the separation process carried out according to this method. However, the collection of these nuclides in the filter must be verified by using actual samples.

\section{Detection Limit of this Method}

The detection limit of concentration of Y-90 and Sr-90 in the measurement of $\beta$-rays from Y-90 was studied using the filter method.

The detection limit is expressed by the following equation ${ }^{16)}$ :

$$
n=(k / 2)\left(k / t+\sqrt{(k / t)^{2}+4 n_{b}\left(1 / t+1 / t_{b}\right)}\right)
$$

where, $n$ is the count rate of the detection limit (cps), $k$ is the confidence level, $t$ is the sample measurement time, $t_{b}$ is the background measurement time, and $n_{b}$ is the background count rate 
(cps).

In this experiment with standard Sr-90 samples, the background of the measurement environment where shielding with lead was applied (under the condition where there was a 10-mmthick acrylic plate and no measurement sample) was $0.45 \mathrm{cps}$. The detection limit of Y-90 was evaluated using this value. With the filter sample and background measurement time of 10 min and $k=3$, the detection limit $(n)$ was $0.13 \mathrm{cps}$. When the conversion coefficients for $100 \mathrm{~mL}$ and $400 \mathrm{~mL}$ water samples with a 2-mm-thick polyethylene plate were used, the detectable radioactive concentration limits of $\mathrm{Sr}-90$ were calculated from radioactive equilibrium as $0.012 \mathrm{~Bq} / \mathrm{cm}^{3}$ and $0.0032 \mathrm{~Bq} / \mathrm{cm}^{3}$, respectively (the radioactive concentration of $\mathrm{Y}-90$ is the same).

However, in the actual sample measurement, a 10-mm-thick acrylic plate was introduced as there was a mixing of radioactive Cs and the background, including the contribution of photons, denoted by $n_{b}$.

As described previously, most of the radioactive $\mathrm{Cs}$ is eliminated in the deposition-filtration process, and the contribution to the background is as small as $1.43 \mathrm{E}-3 \mathrm{cps}$ per Bq. Therefore, it is considered that the background specific to the measurement environment is dominant in the actual sample measurements. Based on the assumption that the background of the measurement environment at the $1 \mathrm{~F}$ power station is approximately twice $(1.0 \mathrm{cps})$ as large as that of this experiment, the detection limit was evaluated using this value as $n_{b}$. If the sample and background are both measured for $10 \mathrm{~min}$ and $k=3$, the detectable count rate limit $n$ is $0.19 \mathrm{cps}$. In this case, when the conversion coefficients for $100 \mathrm{~mL}$ and $400 \mathrm{~mL}$ water samples with 2-mm-thick polyethylene plate are also used, the detectable radioactive concentration limits of Y-90 were calculated as $0.017 \mathrm{~Bq} / \mathrm{cm}^{3}$ and $0.0045 \mathrm{~Bq} / \mathrm{cm}^{3}$, respectively. Therefore, the detection limit depends primarily on the measurement time and background of the measurement environment. As the measurement of the above level of radioactive concentration of Y-90 is achieved, it indicates that it is feasible to measure the same level of radioactive concentration of Sr-90.

\section{Conclusions}

A filter method was developed to facilitate the measurement of a relatively low level of Sr90 concentration in various types of water samples generated at the current $1 \mathrm{~F}$ power station.

1. Sr-90 and Y-90 are concentrated and collected in a filter at high collection efficiency using the carbonate precipitation and iron coprecipitation methods. A 2-mm-thick polyethylene plate ( $\beta$-ray absorber) was used as a filter to enable the selective measurement of high-energy $\beta$-rays from Y-90 with a GM counter tube.

2. Most of the radioactive Cs existing in the water samples in the ionic state was eliminated by the deposition and separation processes that were carried out according to this method. If mixed nuclides such as Cs-134 and Cs-137 existed in the filter, the contribution of the photons from such nuclides was evaluated by inserting a $10-\mathrm{mm}$-thick acrylic plate. However, even if these nuclides had the same concentration as $\mathrm{Sr}-90$, their contribution to the count rates of Y-90 was as small as 1/100.

3. In the filter method, the concentration of Sr-90 was evaluated in a single measurement as long as Sr-90 and Y-90 achieved a state of secular equilibrium. Otherwise, the radioactive concentration of Sr-90 could be evaluated with the equation derived from the sequential decay of Sr-90 and Y-90 by conducting the measurements twice at a certain interval and then determining the radioactive concentrations of Y-90 both times using the conversion 
coefficient from the obtained count rate of the $\beta$-rays from Y-90. The time taken to conduct the separation and collection processes and the measurement is $30 \mathrm{~min}$ or less, hence, it is possible to obtain results within 6 hours even if it is necessary to conduct the measurement twice. It is possible to measure concentrations that are much lower than the tentative effluent standard related to $\mathrm{Sr}-90\left(0.01 \mathrm{~Bq} / \mathrm{cm}^{3}\right)$ currently used at the $1 \mathrm{~F}$ power station in a shorter time than that taken using conventional measurement methods.

4. This is a method for measuring the radioactive concentration of Sr-90 easily and quickly without any advanced processes of chemical separation and professional measurement techniques and is considered to make a useful contribution to effluent management at the $1 \mathrm{~F}$ power station which has a large number of water samples to be examined.

When applying this method to a field site, the following points need to be noted:

1. The most important objective of the filter method is to measure high-energy $\beta$-rays from Y-90 precisely and selectively. If any nuclide other than Y-90 which emits high-energy $\beta$-rays is present (potentially, such as Rh-106 which is a daughter nuclide of Ru-106 or Pr-144 which is a daughter nuclide of Ce-144), the contribution of such a nuclide must be evaluated.

2. If nuclides which interfere with the measurement of $\beta$-rays from Y-90, such as Cs-134 and Cs-137, are present in a high concentration, it is possible to concentrate and collect these nuclides in the filter. Before applying this method, it is necessary to check the amounts of the nuclides that are concentrated and collected in the filter using an actual sample obtained from the $1 \mathrm{~F}$ power station.

3. If a water sample contains seawater at a concentration of $20 \%$ or higher, a large amount of deposit may be attached to the area around the funnel, causing difficulty in handling. The samples with high seawater concentrations need to be studied separately.

\section{References}

1) Tokyo Electric Power Company, "Leakage of Contaminated Water at H4 Tank Area," Handout for the 3rd Working Group on Managing Contaminated Water, Commission on Supervision and Evaluation of the Specified Nuclear Facility, Document No.1, 2013 Aug 21. [in Japanese]. http://www.nsr.go.jp/ data/000051216.pdf, cited 2015.Feb 26.

2) Ministry of Education, Culture, Sports, Science and Technology, Series of Radioactivity Measurement Method 2; Method of Radioactive Strontium Analysis (2003). [in Japanese]

3) Tokyo Electric Power Company, "Recent Leakage of Contaminated Water from Storage Tank and Weir, Causes and Remedies for This Matter," Handout for the 10th Working Group on Managing Contaminated Water, Commission on Supervision and Evaluation of the Specified Nuclear Facility, Document No. 2 , 2014 Jan 24. [in Japanese]. http://www.nsr.go.jp/data/000051256.pdf, cited 2015.Feb 26.

4) N. Vajda, C. Kim, "Determination of radiostrontium isotopes: A review of analytical methodology," Appl. Radiat. Isot., 68, 2306 (2010).

5) "The Law for the Regulations of Nuclear Source Material, Nuclear Fuel Material and Reactors," ed. Study Group on Legislation and Regulations on Nuclear Safety, Taisei Publishing Company, Tokyo, pp. 544 (2009). [in Japanese]

6) Tokyo Electric Power Company, "Provisional Effluent Standards for Contaminated Water," Handout for the 8th Working Group on Managing Contaminated Water, Commission on Supervision and Evaluation of the Specified Nuclear Facility, Additional Material, 2013 Oct 15. [in Japanese]. http://www.nsr.go.jp/ data/000051248.pdf (cited 2015 Feb 26)

7) Handbook of Analytical Chemistry, ed. Japan Soc. For Analytical Chemistry, Maruzen, Tokyo, pp. 132, 139 (1991). [in Japanese]

8) Japan Radioisotope Association. Radiation and Isotopes, pp. 360 (1997). [in Japanese]

9) Tokyo Electric Power Company, "Present Status of the Study in Working Group on Managing Contaminated Water," Handout for the 15th Working Group on Managing Contaminated Water, Commission on Supervision and Evaluation of the Specified Nuclear Facility, Document 3-2, 2013 Oct 30. [in Japanese]. http://www.nsr.go.jp/data/000051009.pdf, cited 2015 Feb 26. 
10) Tokyo Electric Power Company, "Present Status of the Study in Working Group on Managing Contaminated Water," Handout for the 7th Working Group on Managing Contaminated Water, Commission on Supervision and Evaluation of the Specified Nuclear Facility, Document No. 2, 2013 Sep 30. [in Japanese], http://www.nsr.go.jp/data/000051240.pdf, cited 2015 Feb 26

11) Japan Radioisotope Association, Radioisotope Pocketbook 11th edition, Maruzen, Tokyo, ISBN978-489073-211-1C3049. (2011). [in Japanese]

12) H. Hirayama, Y. Namito, A. F. Bielajew, S. J. Wilderman, W. R. Nelson, The EGS5 Code System, SLAC-R-730 (2005) and KEK Report 2005-8 (2005).

13) Tokyo Electric Power Company, "Leakage of Contaminated Water at H4 Tank Area," Handout for the 5th Working Group on Managing Contaminated Water, Commission on Supervision and Evaluation of the Specified Nuclear Facility, Document No. 2, 2013 Aug 30. [in Japanese]. [Internet], http://www.nsr. go.jp/data/000051227.pdf (cited 2015 Feb 26).

14) Hitachi Aloka Medical, Ltd, "GM Survey meter TGS-146B," [Internet], http://www.hitachi-aloka.co.jp/ products/data/radiation-002-TGS-146 (cited 2015, February 26).

15) Ministry of Economy, Trade and Industry, "Main Subjects concerning the Advanced Liquid Processing System and Purposes of Laboratory and Verification Experiments," Handout for the 1st Task Force on the Advanced Liquid Processing System, Document No 3, 2013 Nov 29. [in Japanese]. http://www.meti. go.jp/earthquake/nuclear/pdf/131129/131129_03e.pdf ( 2015.2.26 final confirmation).

16) K. Minami, "On Two Formulas Regarding Count Rates for Detection Limit." Jpn. J. Health Phys. (Hoken Butsuri), 17,79 (1982). [in Japanese] 


\title{
Article
}

\section{Multi-Physics Particle Method for the Simulation of Severe Accidents in Nuclear Power Plants}

\author{
Kenta INAGAKI ${ }^{1, *}$ \\ ${ }^{1}$ Central Research Institute of Electric Power Industry, 2-11-1 Iwado Kita, Komae-shi, Tokyo 201-8511, Japan
}

\begin{abstract}
The development of analysis methods for severe accidents in nuclear reactors is a key issue for nuclear safety. It is difficult to estimate the behaviors of several phenomena in reactor accidents, such as the melting and relocation of structural materials, the spreading of corium on the ground, and the molten core concrete interaction (MCCI), because they involve large changes of the geometry. In the present study, a new method was developed to simulate these phenomena by using a moving particle semi-implicit (MPS) method with models for surface tension, rigid bodies, melting and freezing, heat conduction, interfacial heat transfer, and heat radiation. As benchmarks, the melting of a metal cylinder on a hot plate and the freezing of a molten metallic drop in a coolant are simulated. The characteristic behaviors in each experiment agreed well with the simulation results, which indicates that the developed method is applicable for simulation to evaluate the behavior of corium in severe reactor accidents.
\end{abstract}

KEYWORDS: severe accident simulation, MPS method, heat conduction, heat transfer, melting, freezing, rigid, fluid, surface tension

\section{Introduction}

A detailed study of the progress and constituent steps of the reactor accidents that occurred in the Fukushima Daiichi Nuclear Power Plant Units 1-3 of Tokyo Electric Power Co., Inc. and TMI-2 is essential from the viewpoint of ascertaining the status inside the reactor after the accident and determining the measures for prevention of severe accidents in the future ${ }^{1)}$. For an investigation of such severe accidents (SA), an approach for predicting reactor behavior using computer simulation based on the physical phenomena is indispensable. Computational methods offer advantages such as the ability to simulate severe conditions where testing is impossible because of safety concerns, and the ability to evaluate the parameters that are difficult to measure in a test.

The melting and relocation of substances and their solidification are dominant features of various phenomena that occur under severe accident conditions. These include the melting of fuel rods and structural materials (corium), the solidification of corium in the coolant leading to the formation of debris, the spreading behavior of corium on the floor of the reactor, and the molten core concrete interaction (MCCI). Such melting and spreading behavior of corium

\footnotetext{
* Corresponding author, E-mail: inagaki@criepi.denken.or.jp

DOI : 10.15669 /fukushimainsights. Vol.4.417

(C) 2021 Atomic Energy Society of Japan. All rights reserved.

Originally published in Transactions of the Atomic Energy Society of Japan (ISSN 1347-2879), Vol. 14, No. 4, p.249-260

(2015) in Japanese. (Japanese version accepted: July 10, 2015)
} 
strongly influences the progress of phenomena in a reactor accident. On the other hand, physics-based simulation of such phenomena using mesh methods such as the finite element method (FEM) is difficult to apply because any significant change in the positions and shapes of molten substances during the progress of the accident results in heavy computational loads due to the need for remeshing near the surface. For the analysis of a system having such a free liquid surface and significant deformation, the volume of fluid (VOF) method ${ }^{2)}$ or the particle method ${ }^{3)}$ is suitable. However, various phenomena such as heat transfer via radiation, liquid phase flow, solid phase movement, and the interaction of liquid and solid phases have to be taken into consideration. To date, an analysis that considers all of these factors has not been developed. The evaluation of the influence of various conditions and physical property values, such as the temperature and atmosphere inside the reactor, and chemical composition on the behavior of molten substances is possible if an analytical method for these phenomena can be developed. Moreover, such a method makes it possible to predict the important factors affecting individual phenomena. Furthermore, when a simulation experiment is conducted on the individual phenomena that occur as a result of a severe accident, the actual test conditions can be efficiently determined by separately assessing the influence of each condition through a series of analyses with varying parameters. Furthermore, by the modeling of phenomena based on numerical experimental results, a more realistic and effective model can be recommended for the SA general analysis code represented by the Modular Accident Analysis Program (MAAP) ${ }^{4}$ ) or Methods of Estimation of Leakages and Consequences of Releases (MELCOR) ${ }^{5)}$, etc.

Hence, in this study, a simulation code is developed implementing the physical models necessary for analysis of the behavior of molten substances in the reactor. The simulation is aimed at facilitating analysis of the early phase of a reactor accident accompanied by the melting and solidification of substances.

In this approach, the moving particle semi-implicit (MPS) method ${ }^{6}$, a kind of particle method, is adopted as a basis for analysis. Previous research by Koshizuka ${ }^{7)}$ and $\mathrm{Li}^{8)}$, has also applied the MPS method to the analysis of corium in reactor accidents. However, these analyses do not include re-solidification of molten substances by cooling, nor the movement of solid materials. Therefore, they are not suitable for the analysis of a system that is accompanied by the relocation of solid materials. The method by Guo et al ${ }^{9}$.) takes into consideration the movement of the solid materials before melting, but re-solidification of multiple solid bodies and molten droplets are not taken into account. Hence, in the present method, using the MPS method for fluid analysis forms a basis which allows various existing models for solid bodies, surface tension, heat conduction, and melting-solidification to be integrated. Thus, the kinetic behaviors of liquid and solid phases generated through melting-solidification and the interaction between them can be analyzed. Furthermore, this makes it possible to analyze the mobile behavior of corium, with recurring partial melting and solidification. A model for the radiation heat transfer and interfacial heat transfer between solid bodies in the MPS method is newly developed and implemented. In order to verify the feasibility and confirm the precision of the radiation heat transfer model and the interfacial heat transfer model, analysis was conducted on a system with a theoretical solution. Furthermore, simulated calculations for experiments corresponding to the spreading of corium on the floor and corium solidification, i.e., debris generation in the coolant, were analyzed. The feasibility of this code is confirmed by comparison with experimental results, and a discussion is presented on the applicability of the present method to the simulation of individual phenomena in the case of a severe accident. 


\section{MPS Method}

\section{Fluid Analysis Algorithm}

The particle method is a Lagrange method, in which the continuous body is expressed as an aggregate of particles, and the dominant equation is discretized to calculate the forces that work between these particles and the calculated force move the particles. In the MPS method, space derivatives such as the gradient, diffusion, and Laplacian that appear in the dominant equations are discretized using the models in Eqs. (1)-(3) ${ }^{6}$.

$$
\begin{aligned}
& \nabla \phi_{i}=\frac{d}{n^{0}} \sum_{i \neq j}\left[\frac{\phi_{j}-\phi_{i}}{r_{i j}^{2}}\left(\boldsymbol{r}_{j}-\boldsymbol{r}_{i}\right) w\left(r_{i j}\right)\right] \\
& \nabla \cdot \boldsymbol{u}_{i}=\frac{2 d}{n^{0}} \sum_{i \neq j}\left[\frac{\left(\boldsymbol{u}_{j}-\boldsymbol{u}_{i}\right) \cdot\left(\boldsymbol{r}_{j}-\boldsymbol{r}_{i}\right)}{r_{i j}^{2}} w\left(r_{i j}\right)\right] \\
& \nabla^{2} \phi_{i}=\frac{2 d}{\lambda n^{0}} \sum_{i \neq j}\left[\left(\phi_{j}-\phi_{i}\right) w\left(r_{i j}\right)\right]
\end{aligned}
$$

where, $\phi$ and $u$ are physical quantities depicting the scalar and vector, respectively, $i$ and $j$ are the indexes for particles, $d$ is the number of dimensions, $r$ is the position vector and $r_{i j}$ stands for the distance between particles $i$ and $j$. $w$ denotes a weight function that indicates the intensity of interaction between particles. In this study, a newly developed weight function model defined in Eq. (4) is used.

$$
w\left(r_{i j}\right)=\left\{\begin{array}{cc}
\left(\frac{r_{e}}{r_{i j}}-1.0\right) \cdot\left(\frac{r_{0}}{r_{i j}}\right)^{\frac{3}{2}} & r_{i j}<r_{e} \\
0 & r_{i j} \geq r_{e}
\end{array}\right.
$$

where $r_{0}$ is the distance between the two closest particles in the initial scenario where the particles are lined up in a lattice configuration, and $r_{e}$ denotes the influence radius. A larger influence radius will increase the computational load, while a smaller value will produce instability in the computation, such that the optimal value has to be determined while giving consideration to balancing those tendencies. An appropriate influence radius is considered to be $2-4$ times as large as $r_{0}{ }^{6}$ e.g., $r_{e}=2.1 r_{0}$ is frequently used. On the other hand, in a method that deals with the surface tension ${ }^{10)}$, a value 3.2 times as much as $r_{0}$ is recommended. In this study, $r_{e}=3.2 r_{0}$ was adopted because the surface tension is handled. $n^{0}$, appearing in Eqs. (1)-(3) represents the representative particle number density calculated at the inner particle position of the initial configuration, and the particle number density of particle $i$ is defined as the sum of weight functions between all particles $j$ around the particle $i$. Furthermore, $\lambda$ in Eq. (3) denotes a factor for matching of the increase in statistical dispersion and the analytical solution ${ }^{3)}$, and is expressed by the following equation:

$$
\lambda=\frac{\sum_{i \neq j} r_{i j}^{2} w\left(r_{i j}\right)}{\sum_{i \neq j} w\left(r_{i j}\right)}
$$

Tamai et al ${ }^{11)}$. came up with a formulation using the higher order terms that appear in the Taylor expansion of multivariable functions in place of Eqs. (1)-(3). Thereby, they developed a higher order precision differentiation model that can develop the space derivative with an arbitrary precision, taking into account the discretization of the space derivative of the MPS method. However, a concern is that the computation will diverge when the Particle Number Density (PND) condition (described later in this paper) is applied to the pressure source term. 
Therefore, in this study, the conventional discretization model of MPS was adopted.

The dominant equation in the analysis of non-compressive fluid is the Navier-Stokes equation:

$$
\frac{\partial v}{\partial t}=-\frac{1}{\rho} \nabla P+\frac{\mu}{\rho} \nabla^{2} \boldsymbol{v}+\boldsymbol{g}+\boldsymbol{f}
$$

The right-hand side terms denote the forces generated due to the pressure gradient, viscosity, and gravity, and surface tension, respectively. $\rho$ and $\mu$ are the density and viscosity coefficient, respectively. At the first step, only the terms of viscosity, external force, and surface tension are computed by discretizing Eq. (6) into Eq. (7) to obtain the temporal velocity $v^{*}$ and position $r^{*}$.

$$
\begin{aligned}
\boldsymbol{v}_{i}^{*} & =\boldsymbol{v}_{i}+\Delta \mathrm{t}\left[\frac{\mu}{\rho} \frac{2 d}{\lambda n^{0}} \sum_{i \neq j}\left(\boldsymbol{v}_{j}-\boldsymbol{v}_{i}\right) w_{i j}+\boldsymbol{g}+\boldsymbol{f}\right] \\
\boldsymbol{r}_{i}^{*} & =\boldsymbol{r}_{i}+\Delta \mathrm{t} \boldsymbol{v}_{i}^{*}
\end{aligned}
$$

For computation of the viscosity term, the Laplacian model shown in Eq. (3) is used. Additionally, the computation of the surface tension term $f$ that appears in Eq. (7) is explained in the next section.

Next, the pressure at each particle position is computed by using the Poisson equation i.e., Eq. (9), with the particle number density $n^{*}$ and velocity $v^{*}$ computed at the particle position after computation with Eq. (8).

$$
\nabla^{2} P=\alpha \frac{\rho}{\Delta t^{2}} \frac{n^{0}-n^{*}}{n^{0}}+(1-\alpha) \nabla \cdot v^{*}
$$

The first term on the right-hand side of Eq. (9) is the PND (particle number density) condition while the second term stands for the DIV (divergence free) condition. Both of these two are derived from the incompressibility assumption of the system, and only one of the two was used in the conventional MPS method. However, there is a disadvantage in that the variation of numbers in each source term are generally large, and errors tend to accumulate. In the method adopted for this study, Eq. (9) was constructed with reference to the method proposed by Tanaka et al ${ }^{12}$., facilitating stable analysis while avoiding the accumulation of errors under an incompressibility condition by properly adjusting the parameter $\alpha$ between 0 and 1 . In this research, as a result of analyses conducted by varying the value $\alpha$ in each analysis system as explained in sections IV and V, the tendency was confirmed that a larger value of $\alpha$ makes the computation more unstable. On the other hand, if $\alpha$ is set to a value that is too small, the non-compressive condition will collapse (the spatial volume occupied by the particles gradually decreases). Thus, the upper limit for stable computation was defined as $\alpha=0.1$.

After computing the pressure, the movement of particles resulting from the pressure gradient was computed using the following equation, derived from the improved gradient model in Eq. (1):

$$
\nabla P=\frac{d}{n^{0}} \sum_{i \neq j}\left[\frac{P_{j}+P_{i}}{\left(r_{i j}^{*}\right)^{2}}\left(\boldsymbol{r}_{j}^{*}-\boldsymbol{r}_{i}^{*}\right) \cdot w\left(r_{i j}^{*}\right)\right]
$$

The details of deriving Eq. (10) are explained in the report by Oochi et al ${ }^{13)}$. This pressure gradient model is understood to compute the force applied on the particle $i$ from the pressure at the center between particles $i$ and $j$. This makes the computation stable because the resulting force from the pressure gradient is always exhibited as the repulsion force between particles. 


\section{Details of Additional Models}

In this study, the simulation of a sophisticated system including various physical phenomena was made possible by implementing the models of heat conduction, interfacial heat transfer, radiation heat transfer, surface tension, melting and solidification, and rigid body dynamics in the conventional fluid analysis method mentioned in the previous section. An overview of the algorithm of the present method is shown in Figure 1. The items surrounded with a solid frame are the models added to this research, and their details are explained as follows:

\section{(1) Heat conduction}

To conduct a thermal field analysis, the enthalpy per unit volume and temperature $T$ were given for each particle as the variables, similar to the method described by Koshizuka et al ${ }^{6}$. The heat transfer was computed according to the thermal energy preservation law in Eq. (11):

$$
\frac{\partial H}{\partial t}=K \nabla^{2} T+\widetilde{Q}
$$

where, $K$ is the heat conductivity, and $Q$ is the heat source. Furthermore, the enthalpy was set to zero at absolute zero point. Using the Laplacian model of Eq. (3), Eq. (11) is discretized to form Eq. (12).

$$
\frac{\partial H_{i}}{\partial t}=\frac{2 d}{\lambda n_{i}} K \sum_{i \neq j}\left[\left(T_{j}-T_{i}\right) w\left(r_{i j}\right)\right]+\widetilde{Q}
$$

The relation between temperature $T$ and enthalpy $H$ is approximated with Eq. (13):

$$
T_{i}=\left\{\begin{array}{cc}
H_{i} /\left(\rho c_{p}\right) & \left(H_{i}<\rho c_{p} T_{m}\right) \\
T_{m} & \left(\rho c_{p} T_{m} \leq H_{i} \leq \rho c_{p} T_{m}+\rho L\right) \\
\left(H_{i}-\rho L\right) /\left(\rho c_{p}\right) & \left(\rho c_{p} T_{m}+\rho L<H_{i}\right)
\end{array}\right.
$$

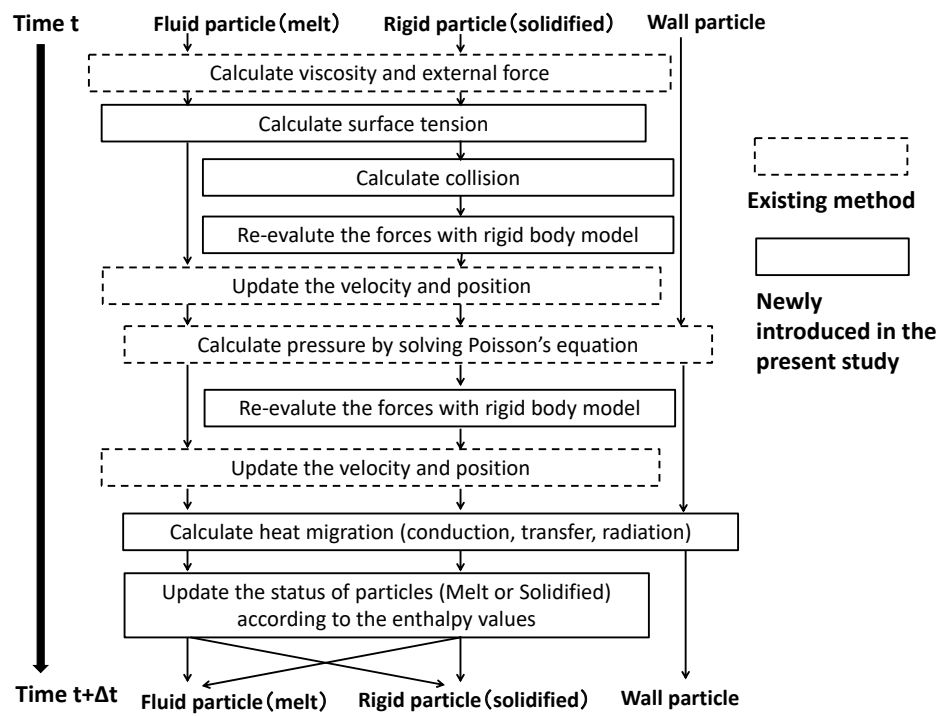

Figure 1 Schematic overview of the algorithm of the proposed method 
where, $T_{m}$ is the melting point, $L$ is the latent heat, and $c_{p}$ is the specific heat. The specific heat was assumed to have no temperature dependence. Furthermore, an identical value was used for the density and specific heat, regardless of the particle conditions (molten or solidified).

\section{(2) Interfacial heat conductance model}

When different solids are in contact, the contact thermal resistance occurs on the interface. The value of contact thermal resistance is influenced to a large extent by the contacting pressure, surface roughness, and heat conductivity of materials. In the present method, the interfacial heat conduction was treated by defining the heat transfer coefficient $h$ between different solids. In the interfacial heat transfer computation, the combination of particles in contact is not detected in particular, but when a particle $j$ belonging to a rigid particle group is detected within the influence radius of particle $i$ belonging to another rigid particle group, the heat transfer $H_{i \rightarrow j}$ between particles $i$ and $j$ is evaluated by the following equation:

$$
H_{i \rightarrow j}=\frac{h}{r_{0}}\left(T_{i}-T_{j}\right) \frac{w_{i j}}{W_{\text {sum }}} d t
$$

where, $W_{\text {sum }}$ is the total sum of the weights function of the entire combination of particles in region A and particles in region B, shown in Figure 2, and is expressed with the following equation:

$$
W_{\text {sum }}=\sum_{i \in A, j \in B} w_{i j}
$$

Using this expression, the heat quantity that moves from A to B through the interface in the center is equal to the theoretical value under the condition shown in Figure 2, where particles are configured in a lattice. To be exact, Eq. (14) is equal to the theoretical value only when the particles are configured in a lattice, but from the viewpoint of reducing the computational load, it was decided to utilize only the value computed with the lattice configuration of particles in the primary stage of computation as $W_{\text {sum }}$.

When either or both of the different phases are melted on the interface, the contact-condition dependent thermal resistance is assumed to be zero, i.e., the heat exchange between all particles that are present in the influence radius is evaluated using Eq. (12) when the particle $i$ is in a molten state. However, the heat conductivity $K$ in this state was set to an average of two particles. On the other hand, when a particle $i$ was solidified and another particle $j$ belonging to

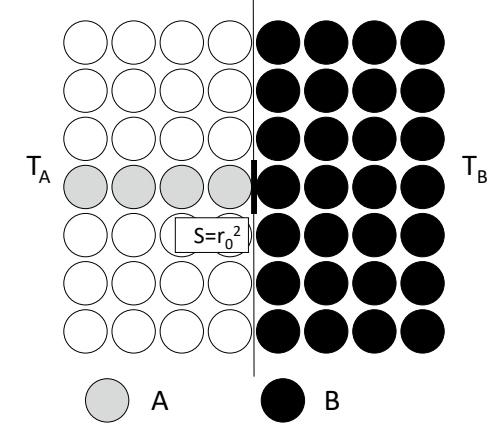

Figure 2 Hypothetical particle geometry on the interface between different phases for the estimation of several parameters 
a different rigid body particle group from particle $i$ was present within its influence radius, Eq. (14) was used for evaluation of the amount of heat exchange between $i$ and $j$.

For the particles located on the surface of the fluid or solid body, heat exchange with the atmosphere is considered. In the present method, no particle was set in the area corresponding to the atmosphere, and thus the method of Eq. (14) was not applicable. Hence, the heat transfer to the atmosphere was considered by varying the heat quantity using Eq. (16) for the surface particles.

$$
\frac{d H_{i}}{d t}=h_{a t m}\left(T_{a t m}-T_{i}\right) / r_{0}
$$

where, $h_{\text {atm }}$ denotes the heat transfer coefficient to the atmosphere, and $T_{\text {atm }}$ denotes the atmospheric temperature.

In order to find a surface particle, a method was adopted that regards the particle for which $n_{i}$ $<\beta n_{0}(\beta \fallingdotseq 0.97)$ holds true as a surface particle, making use of the fact that the value of particle number density decreases near the surface in the conventional particle method. However, the issue was that a local small value of the particle number density on the inside of a continuous body was determined as the surface. Hence, the following method was developed in consideration of the positional information of adjacent particles to avoid erroneous surface detection:

$$
\boldsymbol{a}_{i}=\sum_{i \neq j}\left(\boldsymbol{r}_{j}-\boldsymbol{r}_{i}\right) / r_{i j}
$$

First, the vector $\boldsymbol{a}_{i}$ in Eq. (17) was evaluated. This is the total sum of unit vectors in the direction of the relative positional vector of particle $i$ and adjacent particle $j$. This vector has the property of having a larger absolute value if the location of the adjacent particle is concentrated in a single direction. Using this property, the particle that satisfies one of following three conditions is determined as a surface particle:

(1) $n_{i}<0.875 \cdot n_{0} \cap\left|\boldsymbol{a}_{i}\right|>0.2$

(2) $n_{i}<1.0 \cdot n_{0} \cap\left|\boldsymbol{a}_{i}\right|>0.4$

(3) $n_{i}<0.3 \cdot n_{0}$

The coefficients that appear on the right-hand side are the values adjusted for properly determining surface particles in various shapes.

\section{(3) Radiation model}

For computing the heat transfer by radiation, a surface-to-surface model that assumes the diffusion of gray body thermal radiation was used, where the heat transfer by radiation is computed on the basis of the shape coefficient between each element for all combinations of surface elements in the system. If the surface particles defined by the method in the previous section are used as the surface elements of the surface-to-surface model, the computational load is heavy. In this method, the surface elements were defined differently from the particles used for MPS calculation and evaluation, and radiation heat transfer is calculated between newly defined surface elements, as follows. First, the analysis space in which particles are present was divided by lattice cube geometry. This lattice was termed the surface element extraction lattice. When multiple surface particles were present in a surface element extraction lattice, they were defined as a single surface element (Figure 3). By defining the width of the surface element extraction lattice as a larger size, the load for computation of radiation was decreased. Based on the balance consideration, in this study, the size of the surface element extraction lattice was 


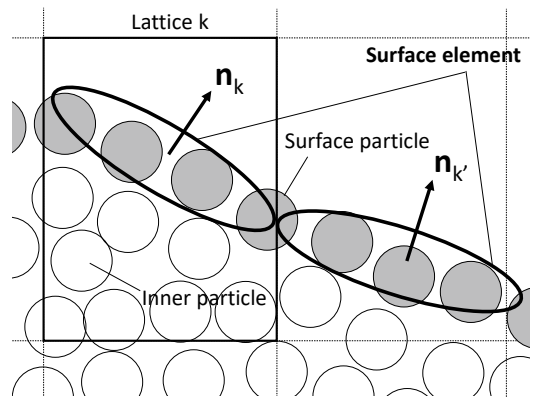

Figure 3 Definition of the surface elements

defined to be of the same dimension as the influence radius, i.e., 3.2 $r_{0}$.

Defining the size of the surface element extraction lattice as $3.2 r_{0}$ was considered to be reasonable because the surface shape was comparatively smooth in the systems analyzed in this study treated in sections IV and V.

The surface area of surface element $S_{k}$ defined in a surface element extraction lattice $k$ is expressed by the following Equation:

$$
S_{k}=r_{0}^{2} \cdot N
$$

where, $N$ is the number of surface particles present in the lattice $k$. Furthermore, the values of position vector $\boldsymbol{x}_{k}$ and normal vector $\boldsymbol{n}_{k}$ of the surface element are defined as the arithmetic average of position and normal vectors of surface particles present in the lattice $k$, respectively. Next, regarding the combination $k$ and $l$ of all surface elements, shape coefficient $F_{k, l}$ was calculated using the following equation:

$$
F_{k, l}=\frac{\cos \theta_{k} \cos \theta_{l}}{\pi r_{k l}^{2}} S_{l}
$$

where, $r_{k l}$ is the distance between surface element $k$ and $l$, and $\theta$ is the angle formed by a line connecting the surface elements and the normal vector. Both of them are computed using the position and the normal vector acquired as described above. For the surface area and the shape coefficient evaluated with Eq. (19) and Eq. (20), several validating evaluations were conducted on some simple primitive shapes. The evaluations confirmed convergence, where smaller particle diameters led each value closer to its theoretical solution.

The quantity of heat released $q_{\text {out }}$ by thermal radiation and incident heat quantity $q_{\text {in }}$ are expressed with the following equations:

$$
\begin{aligned}
& q_{\text {out }, k}=\varepsilon_{k} \sigma T_{k}^{4}+\left(1-\varepsilon_{k}\right) q_{i n, k} \\
& q_{i n, k}=\sum_{l} F_{k, l} q_{\text {out }, l}
\end{aligned}
$$

where, $\varepsilon$ and $\sigma$ are the emissivity and the Stefan-Boltzmann constant, respectively. In each time step, Eq. (21) was computed using $q_{\text {in }}=0$ as the initial value, and the acquired $q_{\text {out }}$ was substituted in Eq. (22). Next, the acquired $q_{i n}$ was substituted in Eq. (21) and such repetition was conducted until convergence was observed. The converged values of $q_{\text {in }}$ and $q_{\text {out }}$ were adopted as the quantity of heat transferred by thermalradiation. The increase or decrease of heat quantity calculated for each surface element was uniformly distributed for surface particles that 
belonged to the surface element.

\section{(4) Surface tension model}

A method using the inter-particle potential (Eq. (23)) developed by Kondo et al ${ }^{10)}$. was adopted for computation of surface tension:

$$
P(r)=C \cdot \frac{1}{3}\left(r-\frac{3}{2} r_{0}+\frac{1}{2} r_{e}\right)\left(r-r_{e}\right)^{2}
$$

The influence of surface tension was considered by applying the inter-particle potential force shown in Eq. (24) between particles $i$ and $j$. The right-hand side of Eq. (24) is a primary spatial differential of Eq. (23).

$$
\rho_{i} r_{0}^{3}\left|\frac{d v_{i}}{d t}\right|=C\left(r_{i j}-r_{0}\right)\left(r_{i j}-r_{e}\right)
$$

The coefficient $C$ in Eqs. (23) and (24) can be derived from Eq. (25) because the energy required for forming two surfaces of area $r_{0}^{2}$ by removing particle group A from particle group B in Figure 2 is equal to $2 \sigma r_{0}^{2}$, thus Eq. (25) is obtained, and then $\mathrm{C}$ was derived.

$$
2 \sigma r_{0}^{2}=\sum_{i \in A, j \in B} P\left(r_{i j}\right)
$$

The potential coefficient $C^{\prime}$ acting between the fluid particle and the wall particle was defined by the following equation:

$$
\frac{C^{\prime}}{C}=\frac{1}{2}(1+\cos \theta)
$$

where, $\theta$ is the contact angle between the fluid and wall. Furthermore, the surface tension coefficient $\sigma_{12}$ that acts between particles in different liquid phases having a surface tension coefficient $\sigma_{1}$ and $\sigma_{2}$, respectively, was defined by the following equation assuming Antonov's rule $^{14)}$ :

$$
\sigma_{12}=\left|\sigma_{1}-\sigma_{2}\right|
$$

\section{(5) Melting and solidification model}

Based on the temperature of particles evaluated using Eq. (13), whether a particle is in a molten or solidified state was determined by the following procedure. First, the particles with temperatures higher than the melting point were regarded to form a molten state, and an ordinary fluid computation process was applied for these. Next, the particles with temperatures lower than their melting points were regarded to form a solidified state, i.e., regarded as rigid body particles. The rigid body model, which is discussed later, was applied to compute the behavior of these particles. If the temperature was equal to the melting point, it was assumed that the particle is partially solidified, and the other part was in a molten state. In the methodology by $\mathrm{Guo}^{9)}$ et al., this state is treated as a fluid body, and the influence of partial solidification is simulated by increasing the viscosity. On the other hand, all the particles at temperatures below their melting point are treated as rigid body particles in this method.

The particles determined as solidified are classified according to the procedure described above into each rigid body particle group. For a cluster forming a solid body, a list is prepared of the particles that belong to it, and the ID of each of these particles is stored.

If solidified particle $i$ at time $t$ already was in a solidified state at time $t-\Delta t$, belonging to 
a rigid particle group, then it is assumed to belong to the same particle group at time $t$ as well. Furthermore, if particle $i$ was in a molten state at time $t-\Delta t$ and is in a solidified state at time $t$, it is regarded as belonging to the same solid particle group of another rigid particle $j$ in the neighborhood, which satisfies Eq. (28), indicating that particles $i$ and $j$ are close enough to each other. If such particle $j$ does not exist, a new solid particle group is defined and particle $i$ is assigned to it. Furthermore, when a particle that was in a solidified state at $t-\Delta t$ and enters a molten state at time $t$, then this particle is deleted from the solid particle group list.

$$
r_{i j} \leq \sqrt{2} r_{0}
$$

Eq. (28) is used for determining whether particles $i$ and $j$ are in contact. It is more natural to define the right-hand side as $r_{0}$, but for some cases the distance between adjacent particles is larger than $r_{0}$ due to the oscillation caused by the pressure gradient between the surrounding particles. For this reason, when the contact determination distance is defined as $r_{0}$, a tendency towards scattered solidified zones was observed. To avoid this, the standard of distance for determining the contact condition of particles was defined as $\sqrt{2} r_{0}$ which is larger than $r_{0}$.

\section{(6) Rigid body model}

The movement of solid particle group $\Pi$ is computed using the following method ${ }^{15)}$ that adopts the quaternion. First, the coordination of the gravity point of a rigid body $\Pi, r_{g, \Pi}$ is expressed as

$$
r_{g, \Pi}=\sum_{i \in \Pi} r_{i} / N_{\Pi}
$$

where, $N_{\Pi}$ is the number of particles that belong to rigid body particle group $\Pi$. Then, the mass of rigid body $M$ and inertia tensor $I$ are acquired with the following equations:

$$
\begin{aligned}
M_{\Pi} & =N_{\Pi} \cdot \rho_{i} r_{0}^{3} \\
\boldsymbol{I}_{\Pi} & =\rho_{i} r_{0}^{3} \sum_{i \in \Pi}\left[\begin{array}{lll}
r_{i g, y}^{2}+r_{i g, z}^{2} & -r_{i g, x} r_{i g, y} & -r_{i g, x} r_{i g, z} \\
-r_{i g, y} r_{i g, z} & r_{i g, x}^{2}+r_{i g, z}^{2} & -r_{i g, y} r_{i g, z} \\
-r_{i g, z} r_{i g, x} & -r_{i g, z} r_{i g, y} & r_{i g, x}^{2}+r_{i g, y}^{2}
\end{array}\right]
\end{aligned}
$$

The $r_{i g}=\left(r_{i g, x}, r_{i g, y}, r_{i g, z}\right)$ in Eq. (31) is the relative position vector of particle $i$ from the reference point of the gravity center $r_{g, I}$. In order to proceed with the rotation in three-dimensional coordinates, a four-dimensional number, named quaternion $Q$, is used. The quaternion at time $t$ is described with the following equation:

$$
\boldsymbol{Q}=\left(s, Q_{x}, Q_{y}, Q_{z}\right)=(1,0,0,0)
$$

The momentum $P_{\Pi}$ and angular momentum $L_{\Pi}$ of rigid body $\Pi$ are given according to the following equation:

$$
\begin{aligned}
\boldsymbol{P}_{\mathrm{II}} & =\sum_{i \in \Pi} \rho_{i} r_{0}^{3} \boldsymbol{v}_{i} \\
\boldsymbol{L}_{\Pi \mathrm{I}} & =\sum_{i \in \Pi} \rho_{i} r_{0}^{3}\left(\boldsymbol{r}_{i g} \times \boldsymbol{v}_{i}\right)
\end{aligned}
$$

Here, the actions by the viscosity term, external force term and surface tension terms are computed using Eq. (7) for all particles that belong to the rigid body $\Pi$, and similarly with other fluid particles. However, the positions acquired from Eq. (8) are not overwritten. Since the 
force acting on particle $i$ can be expressed as

$$
\boldsymbol{F}_{i}=\rho_{i} r_{0}^{3}\left(\boldsymbol{v}_{i}^{*}-\boldsymbol{v}_{i}\right) / d t
$$

the force and torque acting on the rigid body $\Pi$ are obtained in terms of Eqs. (36) and (37) respectively:

$$
\begin{aligned}
& \frac{d \boldsymbol{P}_{\Pi}}{d t}=\sum_{i \in \Pi} \boldsymbol{F}_{i} \\
& \frac{d \boldsymbol{L}_{\Pi}}{d t}=\sum_{i \in \Pi} \boldsymbol{r}_{i g} \times \boldsymbol{F}_{i}
\end{aligned}
$$

Applying the relation of angular momentum and angular velocity $(L=I \omega)$, the angular velocity of the rigid body at time $\mathrm{dt}$ after an action of torque is acquired in the following equation:

$$
\boldsymbol{\omega}=\boldsymbol{I}_{\mathrm{II}}^{-1}\left[\boldsymbol{L}_{\mathrm{II}}+d \boldsymbol{L}_{\mathrm{II}}\right]
$$

where, $\boldsymbol{I}^{-1}$ is an inverse matrix of inertia tensor $\boldsymbol{I}$. The quaternion $\boldsymbol{Q}^{\prime}$ after $d t$ is expressed as,

$$
Q^{\prime}=d Q \times Q
$$

where, $\boldsymbol{d} \boldsymbol{Q}$ denotes the magnitude of rotation that occurs during $d t$, expressed as $\boldsymbol{d} \boldsymbol{Q}=(\cos (\theta / 2)$, $\operatorname{asin}(\theta / 2)$ ), using the rotation axis $a=\omega /|\omega|$ and rotation angle $\theta=|\omega| d t$. The relationship between the rotation matrix $\boldsymbol{R}$ and quaternion is expressed by Eq. (39):

$$
\boldsymbol{R}=\left[\begin{array}{ccc}
1-2 Q_{y}^{2}-2 Q_{z}^{2} & 2 Q_{x} Q_{y}-2 s Q_{z} & 2 Q_{x} Q_{z}+2 s Q_{y} \\
2 Q_{x} Q_{y}+2 s Q_{z} & 1-2 Q_{x}^{2}-2 Q_{z}^{2} & 2 Q_{y} Q_{z}-2 s Q_{x} \\
2 Q_{x} Q_{z}-2 s Q_{y} & 2 Q_{y} Q_{z}+2 s Q_{x} & 1-2 Q_{x}^{2}-2 Q_{y}^{2}
\end{array}\right]
$$

Hence, the position of particle $i$ that belongs to rigid body $\Pi$ after $d t$ is acquired by Eq. (41):

$$
r_{i}^{*}=r_{g, \Pi}+\frac{1}{M_{\Pi}} d \boldsymbol{P}_{\text {II }} d t+\boldsymbol{R} \cdot r_{i g}
$$

Such computation has to be conducted again when computing the pressure gradient term following the computation of pressure.

When there is a collision between two rigid bodies or between a rigid body and the wall, it is treated by permitting a micro-overlap between particles, similar to the discrete element method $(\mathrm{DEM})^{16)}$, and generating a reaction force $f_{c}$ defined by Eq. (42).

$$
\boldsymbol{f}_{c}=-K_{c}\left(\frac{2 m_{i} m_{j}}{m_{i}+m_{j}} \frac{\left(r_{c}-r_{i j}\right)}{d t^{2}}\right) \frac{\left(r_{j}-r_{i}\right)}{r_{i j}}
$$

where, $m$ is the mass of the particle, and $r_{c}$ is the collision distance and equals to $r_{0}$. Furthermore, $K_{c}$ is a factor that determines the magnitude of the reaction force, i.e., the repulsion coefficient, and the value $K_{c}=1.0$ was used for the computation of collision between rigid bodies.

\section{Measures for Stabilizing Computation}

Generally, when the particle number density becomes locally high, the pressure increases, which results in instability in the computation. To prevent this, in the present method, the instability was avoided by specifying a reaction force between fluid particles that have approached each other beyond a certain distance. For computation of reaction force, Eq. (42) was used, 
which is identical to the equation describing the collision between rigid bodies. The collision distance of $r_{c}=0.65 r_{0}$ and the repulsion coefficient of $K_{c}=0.1$ were adopted.

Another instability risk is the zero-pressure of the surface particles, which results in the distance between surface particles becoming too close. Therefore, $r_{c}=0.75 r_{0}$ was adopted only for the case of a collision between surface particles in order to avoid an excessively close distance between surface particles. The collision distance and repulsion coefficient used in these conditions are the ones estimated as most appropriate from the viewpoint of the stability of computation.

\section{Computation for Validation}

\section{Validation Analyses of Heat Conduction and Interfacial Heat Transfer}

As a benchmark analysis of the heat conduction and interfacial heat transfer models introduced in this study, a constant heat conduction analysis on a thick cylinder was conducted using the system shown in Figure $4^{17}$. The temperature distribution in the radial direction was calculated, where the cylindrical area (Area 0$)$ and the cylinder external area $\left(\Omega_{2}\right)$ are regarded as the fixed temperature boundary of $T_{0}=333 \mathrm{~K}$ and $T_{2}=293 \mathrm{~K}$, respectively. The coefficient of heat transfer for boundary $\Omega_{1}$ between Area 0 and Area 1 and the boundary $\Omega_{2}$ between Area 1 and Area 2 were defined as $h_{1}=4,800 \mathrm{~W} / \mathrm{m} / \mathrm{m} / \mathrm{K}$ and $h_{2}=1,500 \mathrm{~W} / \mathrm{m} / \mathrm{m} / \mathrm{K}$, respectively. The heat conductivity in Area 1 was defined as $\lambda_{1}$, and the heat quantity $q_{1}$ that passes through the area in a static state is calculated by the following equation:

$$
q_{1}=\frac{2 \pi\left(T_{0}-T_{2}\right)}{\frac{1}{h_{1} r_{1}}+\frac{1}{\lambda_{1}} \ln \left(\frac{r_{2}}{r_{1}}\right)+\frac{1}{h_{2} r_{2}}}
$$

Hence, the inner wall temperature $T_{\text {in }}$ and outer wall temperature $T_{\text {out }}$ are expressed as follows:

$$
\begin{aligned}
& T_{\text {in }}=T_{0}-\frac{q}{2 \pi r_{1} h_{1}} \\
& T_{\text {out }}=T_{2}+\frac{q}{2 \pi r_{2} h_{2}}
\end{aligned}
$$

Using these equations, the theoretical solution for the inside of the cylinder is:

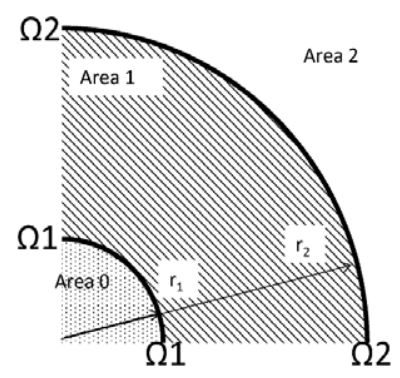

Figure 4 Configuration of the simulation of cylinders for verification of the interfacial heat transfer model 


$$
T=T_{\text {in }}-\frac{\left(T_{\text {in }}-T_{\text {out }}\right) \ln \left(\frac{r}{r_{1}}\right)}{\ln }
$$

A two dimensional analysis was made with 2907 particles where $r_{0}=0.005 \mathrm{~m}$ for configuring the inner and outer cylinders. No particle is placed outside a cylinder. The analysis result of the static state is shown in Figure 5. Furthermore, the comparison of a theoretical solution using Eq. (46) and the computed result is shown in Figure 6. The horizontal axis denotes the distance in the radial direction in Area 1 and the vertical axis denotes the temperature. In a system where the heat transfer coefficient and heat conductivity are dominant, an analysis result that agrees with the theoretical solution was acquired. A good agreement at $r=r_{1}$, where the temperature is strongly influenced by the interfacial heat transfer coefficient $h_{1}$, indicated that the interfacial heat transfer model was reasonable. Similarly, the computation of temperature at $r=r_{2}$ also showed good agreement with the theoretical solution. Consequently, the reasonability of the model of heat transfer to the atmosphere expressed by Eq. (16) was confirmed.

\section{Validation Analysis of Radiation Model}

As a benchmark analysis of the radiation model, computation of thermal migration by radiation between the inner sphere fixed in a space having a radius of $R_{1}=0.003 \mathrm{~m}$ and an outer shell having an inner radius of $R_{2}=0.01 \mathrm{~m}$ was conducted, as shown in Figure 7. The inner surface of the outer shell was defined as the fixed temperature boundary of $T_{2}=873.15 \mathrm{~K}$, and the initial temperature of inner sphere $T_{1,0}$ was defined as $1673.5 \mathrm{~K}$. Finally, the change in temperature of the inner sphere due to heat migration by radiation was computed.

The heat conductivity of the inner sphere was assumed to be sufficiently high, and the

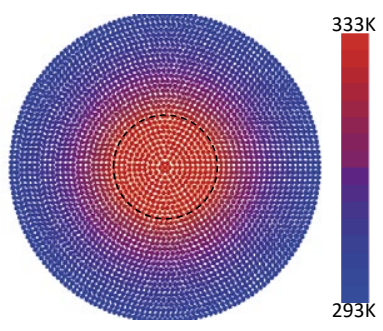

Figure 5 Temperature distribution in the simulation of cylinders in terminal static state

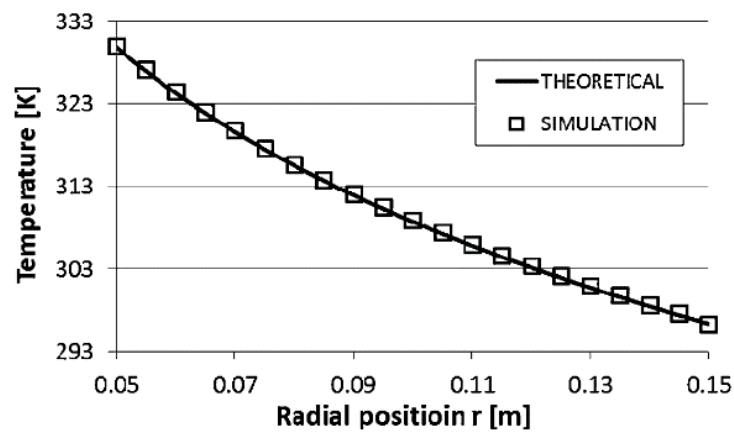

Figure 6 Comparison of theoretical and simulaiton results for temperature simulation in cylinders 
temperature distribution in the inner sphere was assumed as always uniform. The relationship between time $t$ and the inner sphere temperature $T_{1, t}$ is expressed by the following equation ${ }^{18)}$ :

$$
t=\frac{\rho V C_{p}\left[1 / \varepsilon_{1}+\left(A_{2} / A_{1}\right)\left(1 / \varepsilon_{2}-1\right)\right]}{A_{2} \sigma} \times\left[\frac{1}{4 T_{2}^{3}} \ln \left|\frac{\left(T_{1, t}+T_{2}\right) /\left(T_{1, t}-T_{2}\right)}{\left(T_{1,0}+T_{2}\right) /\left(T_{1,0}-T_{2}\right)}\right|+\frac{1}{2 T_{2}^{3}}\left(\tan ^{-1} \frac{T_{1, t}}{T_{2}}-\tan ^{-1} \frac{T_{1,0}}{T_{2}}\right)\right]
$$

where, $A_{1}$ and $A_{2}$ denote the surface area of the inner sphere and inner surface area of outer shell, respectively; $\rho, V$, and $C_{p}$ are the density, volume, and specific heat, respectively, of the inner sphere. The system analyzed with the present method is shown in Figure 8. The green particles denote the surface of the inner sphere and the red particles denote the inner surface of the outer shell. The particle diameter was $0.00035 \mathrm{~m}$, the total number of particles was 49,525, and $\mathrm{dt}$ was defined as $0.01 \mathrm{~s}$. The time progression of the inner sphere temperature acquired

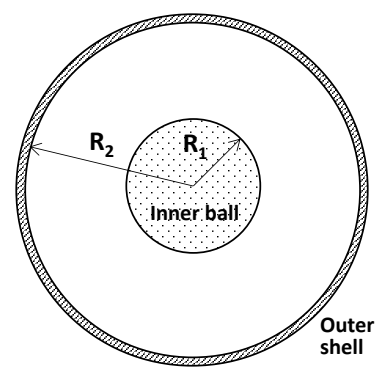

Figure 7 Simulation configuration of a sphere within an outer shell for verification of the heat radiation model

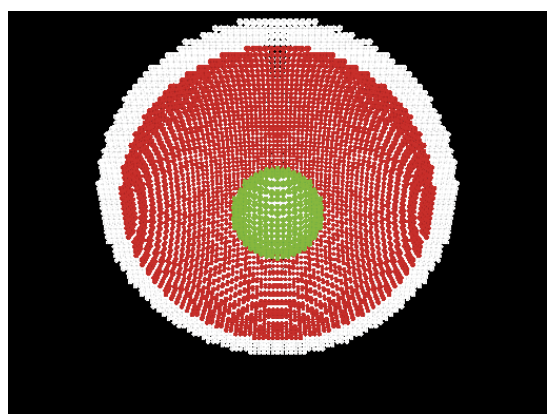

Figure 8 Configuration of the particles for the simulation of a sphere within an outer shell.

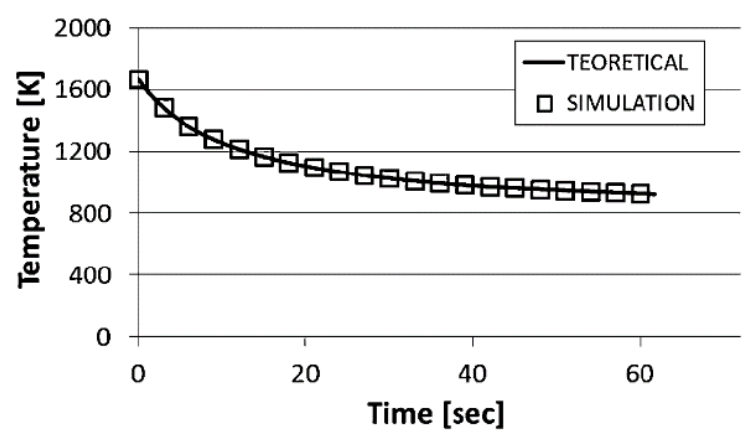

Figure 9 Comparison of theoretical and simulation results of the temperature behavior of the sphere 
by the analysis is shown in Figure 9 along with the theoretical solution using Eq. (47). The theoretical solution was consistent with the analysis results, confirming the reasonability of the radiation model developed in this study.

\section{Analysis of Cylinder Melting Test}

\section{Model and Conditions}

For the analysis of spreading of molten materials on the floor in a nuclear reactor accident, it is essential to conduct a study of a system where surface tension can be taken into consideration to a reasonable extent, since it has a significant influence on fluid behavior. Hence, as a benchmark for the present method, the analysis of a cylinder melting test described by Furuya et al ${ }^{19)}$. was conducted. In the test, a tin cylinder was placed on a $0.0025 \mathrm{~m}$ thick alumina plate in an air atmosphere held at a constant temperature, and the melting migration process was observed from three directions. The initial temperature of the alumina plate and air atmosphere was $655 \mathrm{~K}$. The tin cylinder was $0.005 \mathrm{~m}$ high, and its diameter was $0.02 \mathrm{~m}$. The physical properties of the materials used for the analysis are shown in Table 1. The heat transfer from the surface of the materials to the air atmosphere was defined as $25 \mathrm{~W} / \mathrm{m}^{2} / \mathrm{K}$, which is a typical value if air with natural convection ${ }^{20)}$ is assumed. According to Yuan et al ${ }^{21)}$., the contact angle between molten tin and alumina is approximately $160^{\circ}$, but this is a value in an environment where the oxygen partial pressure is $8.56 \times 10^{-6} \mathrm{MPa}$, and it has been reported that the contacting angle has the tendency to decrease with an increase in the oxygen partial pressure. Because this analysis was conducted in an air atmosphere, the contacting angle was assumed to have decreased and was determined as $150^{\circ}$. Furthermore, when determining the interfacial heat transfer coefficient between the tin cylinder and the alumina plate before melting, the analyses were conducted with a modified interfacial heat transfer coefficient and adjusted to acquire the same melting start time between test and analysis, as the point at which melting of the tin cylinder was set to begin was originally $1 \mathrm{~s}$ after the start of the cylinder melting test. The analyses were repeated while increasing the interfacial heat transfer coefficient by 1,000 and adopted a heat transfer of $32,000 \mathrm{~W} / \mathrm{m}^{2} / \mathrm{K}$, as the closest melting start time to the test, $1.1 \mathrm{~s}$, was acquired by the analysis using this value. In the analysis performed in this study, only the above-mentioned interfacial heat transfer coefficient was used as an adjusting parameter, while other physical properties were adopted from the literature.

In a cylinder melting test analysis using this method, there was a situation where only the particles on one perimeter of the cylinder bottom were melted immediately after melting of the cylinder started. When the pressure became zero because molten particles were determined as surface particles, a phenomenon where they were pulled into the inside of a rigid body because an attraction force due to surface tension potential was applied from the surrounding rigid body particles was observed. To avoid this, the collision distance was defined as $r_{c}=0.85 r_{0}$ only for the collision computation of fluid particles and rigid body particles. The coefficient of restitution was defined as $K_{c}=0.1$ similarly to the collision between fluid particles. All other numerical analysis parameters were adopted from those described in section II.

The parameters relevant to the particle method analysis are shown in Table 2.

The tin cylinder and alumina plate models were formed into a three-dimensional particle model. The size of the tin cylinder was designed to correspond to the actual shape and size of the physical cylinder. On the other hand, the alumina plate used for the test was a square shape of $0.1 \mathrm{~m} \times 0.1 \mathrm{~m}$, while from the viewpoint of decreasing the computational load, a disc shape 
Table 1 Physical parameters for the simulation of the melting of a metallic cylinder on a hot plate

\begin{tabular}{|c|c|c|}
\hline Physical parameters & Tin & Alumina \\
\hline Initial temp. $[\mathrm{K}]$ & 300 & 655 \\
\hline Melting temp. $[\mathrm{K}]$ & 505 & - \\
\hline Density $\left[\mathrm{kg} / \mathrm{m}^{3}\right]$ & 7,000 & 4,000 \\
\hline Heat conductivity (fluid) $[\mathrm{W} / \mathrm{m} / \mathrm{K}]$ & 30.0 & - \\
\hline Heat conductivity (solid) $[\mathrm{W} / \mathrm{m} / \mathrm{K}]$ & 66.6 & 30.0 \\
\hline Heat capacity $[\mathrm{J} / \mathrm{kg} / \mathrm{K}]$ & 228.4 & 780.0 \\
\hline Latent heat $[\mathrm{J} / \mathrm{kg}]$ & 59,600 & - \\
\hline Surface tension $[\mathrm{N} / \mathrm{m}]$ & 0.5 & - \\
\hline $\begin{array}{l}\text { Contact angle of the tin on the } \\
\text { alumina [degrees] }\end{array}$ & \multicolumn{2}{|c|}{150} \\
\hline Kinematic viscosity $\left[\mathrm{m}^{2} / \mathrm{sec}\right]$ & $1.3 \times 10^{-7}$ & - \\
\hline $\begin{array}{l}\text { Heat transfer coefficient (air) } \\
{\left[\mathrm{W} / \mathrm{m}^{2} / \mathrm{K}\right]}\end{array}$ & 25 & 25 \\
\hline $\begin{array}{l}\text { Heat transfer coefficient between solid } \\
\text { tin and alumina }\left[\mathrm{W} / \mathrm{m}^{2} / \mathrm{K}\right]\end{array}$ & \multicolumn{2}{|c|}{32,000} \\
\hline
\end{tabular}

Table 2 Particle parameters for the simulation of the melting of a metallic cylinder on a hot plate

\begin{tabular}{ccc}
\hline & Tin cylinder & Alumina plate \\
\hline Number of particles & 6,776 & 15,764 \\
Thickness $[\mathrm{m}]$ & 0.005 & 0.0025 \\
Diameter $[\mathrm{m}]$ & 0.02 & 0.04 \\
Particle size $\mathrm{r}_{0}[\mathrm{~m}]$ & \multicolumn{2}{c}{0.000625} \\
dt $[\mathrm{sec}]$ & \multicolumn{2}{c}{$2.0 \times 10^{-4}$} \\
\hline
\end{tabular}

of diameter $0.045 \mathrm{~m}$ was adopted for analysis. The total number of particles was 22,540 .

\section{Analysis Result}

A comparison of the results acquired by the analysis and testing on cylinder melting are shown in Figure 10 and the results are depicted in Table 3. In the experiment, the melting initiated at $1 \mathrm{~s}$ after start, and a change in the height was confirmed at $10 \mathrm{~s}$. The melting of the cylinder was complete after $18 \mathrm{~s}^{19}$. In the simulation, on the other hand, melting started at 1.1 s. Following this, the change in height and melting of the entire cylinder was observed at 9.5 $\mathrm{s}$ and $18.1 \mathrm{~s}$, respectively. Hence, this proved that the test results were simulated with good precision. Viewing the cylinder section in the simulation result, the melting of the cylinder was confirmed to have started from the periphery of the surface, contacting with the alumina plate and spreading to the upper side and in the cylinder center direction. Furthermore, the visible change in height was confirmed to have occurred at $9.5 \mathrm{~s}$, caused by the pushing out of the molten zone of the lower part to the outside due to compression by the remaining solid zone in the upper part after all particles in the bottom surface of cylinder had melted. The molten zone of the cylinder maintained the oval shape due to surface tension.

The interfacial heat transfer from the alumina plate to the tin cylinder was dominant immediately after test start. After this, the heat transfer to the tin cylinder was restricted by the heat conduction from the periphery of the alumina plate to the center zone, as the temperatures of the tin cylinder and the alumina plate in the zone immediately below the cylinder were almost equal after $9.5 \mathrm{~s}$ when a change in height of the cylinder was observed. In this analysis, the air flow around the cylinder was not computed, and a simplified model was used instead 


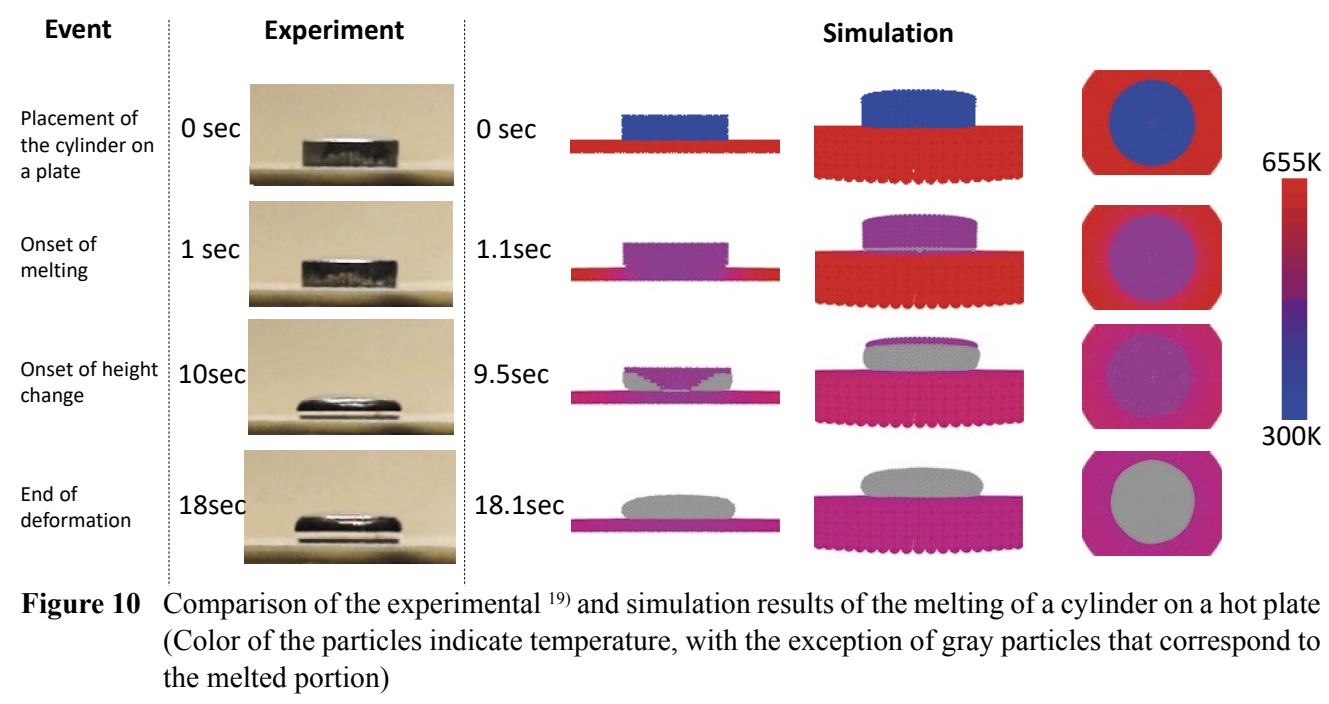

Table 3 Chronology of key phenomena in the experiment and the simulation

\begin{tabular}{ccc}
\hline & Experiment & Simulation \\
\hline Onset of melting & 1 & 1.1 \\
Onset of height change & 10 & 9.5 \\
End of deformation & 18 & 18.1 \\
\hline
\end{tabular}

that calculated the heat exchange between the cylinder surface and air with a constant heat transfer coefficient. The amount of heat exchange between the cylinder surface and air was sufficiently smaller than that from the alumina plate, and the influence was assumed to be miniscule. Regarding the comparison of shape, the experimental result was reproduced well by the simulation. The visual contact angle of less than $150^{\circ}$ was explained to be due the influence of weight of the molten tin, etc.

It was confirmed that the melting could be analyzed with a reasonable degree of precision when the influence of surface tension or the phase transition is dominant. Given that this system is the behavior of fluid aggregation by surface tension on the floor, this is a phenomenon resembling the spreading of corium on the floor as part of the elementary process of a reactor accident. As the corium spreads across the floor, reduced friction with floor due to the formation of a gaseous phase at the interface between the corium and concrete floor may occur. This method may be applicable to the analysis of such corium spreading behavior by introducing a model that accurately simulates the corium-concrete reaction.

On the other hand, a part of the melting phenomena that may occur in a reactor accident is the eutectic reaction such as Zircalloy $/ \mathrm{UO}_{2}, \mathrm{Ni} / \mathrm{Zr}$, etc. To handle this, it is necessary to consider the formation of a liquid phase that spreads with the speed controlled by the reaction between different types of substances. However, because the formation of a liquid phase area and spreading occurs on a smaller scale than the typical length of flux, it is not realistic to directly model the eutectic reaction for analysis from the viewpoint of computation load. As a solution to this issue, a method that includes the modeling of the liquid phase quantity as a function defined by time, temperature and the particle configuration may be adopted. By introducing a proper eutectic reaction model, this code will be also be applicable to the melting 
phenomena of fuel and cladded tubes.

\section{Solidification Analysis of Debris in Coolant Material}

\section{Analysis Models and Analysis Conditions}

As a benchmark for a system that includes different types of liquid phases, analysis was conducted on a testing system that simulated the solidification process of a molten substance dropped in the coolant ${ }^{22}$. For the test, a molten droplet of aluminum was dropped in a sodium pool in a stainless steel cylindrical container (I.D. $0.108 \mathrm{~m}$ and $0.3 \mathrm{~m}$ deep), and the shape of collected solidified aluminum was observed. The weight of the aluminum liquid droplet was about $5 \mathrm{~g}$, and it was dropped from a height of $0.18 \mathrm{~m}$ from the surface of the sodium pool. The physical parameters of the analysis are shown in Table $4^{22}$. Three different temperatures $960 \mathrm{~K}, 1,100 \mathrm{~K}$, and $1,200 \mathrm{~K}$ were adopted as the initial temperature of the aluminum droplet. This analysis focused on a range of conditions where boiling of sodium does not occur, and the initial temperature of the aluminum droplet was set within a range where the sodium temperature does not exceed the boiling temperature.

The parameters relevant in the particle method analysis are shown in Table 5. This analysis was conducted in two dimensions and the particle diameter was $0.001 \mathrm{~m}$. The total number of particles, including the wall particles, was 35,756. An aluminum droplet of diameter $0.02 \mathrm{~m}$ was set immediately above the sodium liquid surface and an initial downward speed of $2.0 \mathrm{~m} / \mathrm{s}$ was specified in the analysis. The parameters that are not listed in Table 5 are all adopted from the values explained in section II, and no adjustment of the parameters specific to debris solidification analysis was made.

Table 4 Physical parameters for the simulation of a molten metallic droplet being dropped into a coolant pool

\begin{tabular}{ccc}
\hline Physical parameters & $\mathrm{Al}$ & $\mathrm{Na}$ \\
\hline Initial temp. [K] & $960,1,100$, or 1,200 & 540 \\
Melting temp. [K] & 933 & - \\
Density $\left[\mathrm{kg} / \mathrm{m}^{3}\right]$ & 2,357 & 885 \\
Heat conductivity (fluid) $[\mathrm{W} / \mathrm{m} / \mathrm{K}]$ & 97.4 & 78.0 \\
Heat conductivity (solid) $[\mathrm{W} / \mathrm{m} / \mathrm{K}]$ & 238 & - \\
Heat capacity [J/kg/K] & 1,080 & 1,310 \\
Latent heat $[\mathrm{J} / \mathrm{kg}]$ & 395,000 & 115,000 \\
Surface tension $[\mathrm{N} / \mathrm{m}]$ & 0.878 & 0.179 \\
Kinematic viscosity $\left[\mathrm{m}^{2} / \mathrm{sec}\right]$ & $4.3 \times 10^{-6}$ & $4.3 \times 10^{-6}$ \\
Heat transfer coefficient $(\mathrm{air})\left[\mathrm{W} / \mathrm{m}^{2} / \mathrm{K}\right]$ & 25 & 25 \\
\hline
\end{tabular}

Table 5 Particle parameters for the simulation of a molten metallic droplet being dropped into a coolant pool

\begin{tabular}{ccc}
\hline & Al droplet & Na pool \\
\hline Number of particles & 305 & 32,591 \\
Particle size $\mathrm{r}_{0}[\mathrm{~m}]$ & \multicolumn{2}{c}{0.001} \\
dt $[\mathrm{sec}]$ & \multicolumn{2}{c}{$2.0 \times 10^{-5}$} \\
\hline
\end{tabular}




\section{Analysis Results and Discussion}

The three analysis results with different initial aluminum droplet temperatures are shown in Figure 11. In the simulation results on the right side of chart, the red particles depict aluminum in a molten state, while the black particles depict aluminum in a solidified state, and the gray particles depict the sodium. The results obtained with all initial temperatures exhibited significant deformation of the aluminum droplet that plunged into the sodium pool, due to compression by the inertia and the repulsion from the sodium pool. For the case where the initial temperature of the droplet was $960 \mathrm{~K}$, the entire bottom part of the aluminum droplet solidified due to aluminum-sodium heat transfer.

Resultantly, a cup-like crust of the aluminum drop was formed. After that, the upper part was cooled by contact with sodium and finally the whole drop solidified into a cup-like shape. On the other hand, in the case where the initial temperature of the droplet was $1,100 \mathrm{~K}$, a crust covering the entire bottom area as observed at the initial temperature of $960 \mathrm{~K}$ was not formed. The aluminum droplet separated into several masses and fragmented due to the tensile viscous forces, etc., accompanying the sodium flow. Also in the case where the initial temperature of the droplet was $1,200 \mathrm{~K}$, the tensile forces caused similar behaviors. In this case, separation into even smaller fragments occurred. For comparison, the solidified aluminum fragments obtained in the experiment are shown on the left side of Figure 11. In the experiment, when the temperature of the aluminum droplet was comparatively low $(1,131 \mathrm{~K})$, a solidified substance having a cup-like shape was formed, and when the temperature was high $(1,272 \mathrm{~K})$, branch-like and fine particle solids were formed. These results closely correspond to the appearance of the shapes acquired in the simulation. Furthermore, the sizes of solidified substances were in rough agreement between experiment and the simulation. Thus, the analysis results by the present method reproduced the phenomenon well. On the other hand, the cup-like solidified substances were generated at $960 \mathrm{~K}$, while the branch-like and fine particles were generated at initial droplet temperatures of 1,100 and $1,200 \mathrm{~K}$ in the simulation. These temperatures were comparatively

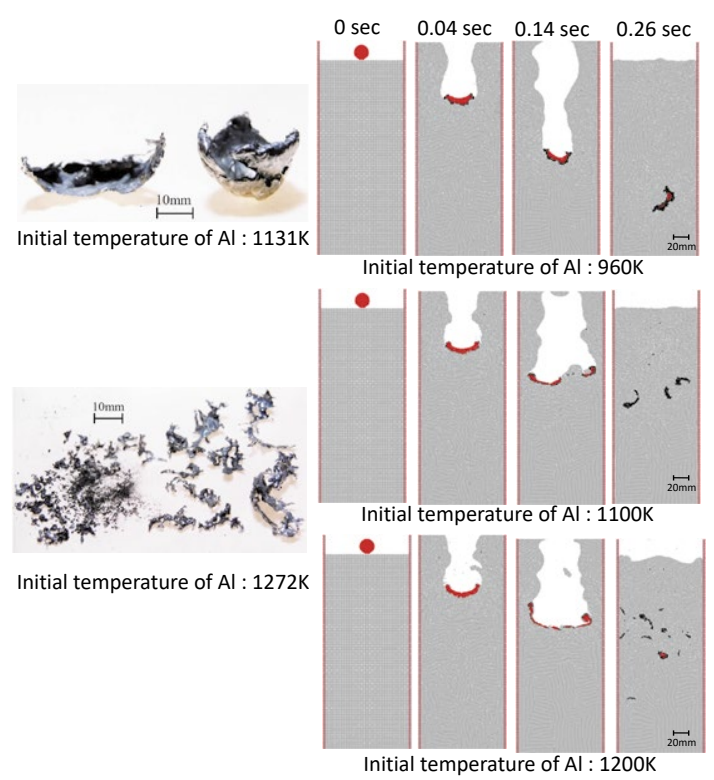

Figure 11 Appearance of the frozen Al droplet obtained in the experiment ${ }^{22)}$ (left) and the result of the simulation (right). Black: Al (Solidified), Red: Al (melt), Gray: Na (melted) 

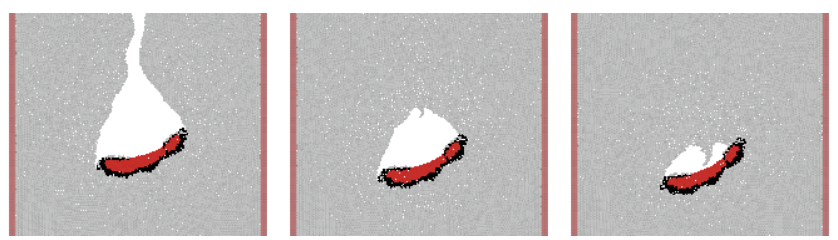

Figure 12 Appearance of the sodium jet leaping down from the sodium pool surface

lower than those in the experiment. This can be explained, as the initial temperature acquired in the experiment was measured in the upper part of the melting crucible, while the temperature decreased when collision occurred with the sodium pool, possibly due to heat exchange with the air during the fall, etc.

In the test ${ }^{22}$, a tip with a large curvature factor is formed when the sodium liquid surface rejoins after separation caused by the collision with the aluminum droplet, and a sodium jet caused by the surface tension acting at this location was observed on the liquid surface. This sodium jet is assumed to have similarly occurred in a downwards vertical direction. In this analysis, the reproduction of this downward sodium jet was confirmed as well (Figure 12).

Because the qualitative analysis of the generation of the aluminum solidified substance was successful, the analysis of solidification behavior of the metal droplet that plunged into the coolant under a range of conditions confirmed that it was not accompanied by the boiling of the coolant. Therefore, this code is expected to be similarly applicable to the analysis of the behavior of portions of corium that are generated in the reactor. Furthermore, this analysis was conducted in two dimensions in order to reduce the computational load; however, the stability and the reasonability of computation is expected to remain intact when expanded to three dimensions. Significant results could be generated by conducting three-dimensional analyses on systems that are difficult to reduce to two dimensions, such as non-symmetrical shapes.

In this analysis, the range of initial temperatures of the aluminum droplet focused on the range where the initial temperature is lower than the boiling temperature of sodium in this analysis. However, when the contact temperature of the droplet and sodium pool exceeds the boiling temperature of sodium, the evaporation of sodium and the generation of a steam film may dominate the phenomenon. Considering application to the early phase of a nuclear reactor accident, boiling of the coolant (water) in a light water reactor may influence the progress of the phenomenon. Furthermore, no particle is positioned in the air zone in this analysis, and only the heat transfer between the surface of a substance and the atmosphere is considered as an influence of the atmosphere. In an actual situation, the influence of air sucking on the sodium flux is considered to exist. As a future subject for analysis of such conditions, the evaporation of liquid and the motion of gas have to be taken into consideration.

\section{Conclusions}

The models of heat transfer, interfacial heat transfer, heat radiation, melting/solidification, rigid body, and surface tension were implemented in the MPS method code. The newly developed heat radiation model and interfacial heat transfer model in this study have been analyzed in a system with a theoretical solution, and good agreement between analysis results and theoretical values was confirmed.

As a benchmark analysis of the present method, the cylinder melting test and the debris 
solidification test in the coolant were simulated, and good reproduction of the phenomena was realized in each case. The analysis of phenomena such as the spreading of corium on the floor is possible by the present method, provided properly modeled phenomena that are assumed to occur in corium-concrete are implemented for simulation of the early phase of a reactor accident. Furthermore, with respect to debris solidification in the coolant, a more meticulous analysis is possible by implementing a model that takes into account the influence of the generation of gas phase, etc.

We would like to take this opportunity to express our sincerest gratitude to Mr. Takanari Ogata, Mr. Satoshi Nishimura, and Mr. Masahiro Furuya of the Central Research Institute of Electric Power Industry for valuable advice and comments on this study.

\section{References}

1) Division of Nuclear Fuel Molten Fuel Sub-Working Group, "Status and issues on nuclear fuel research in the field of LWR severe accident," J. Nucl. Sci. Technol., 56[9], (2014). [in Japanese]

2) C. Hirt, B. D. Nichols, "Volume of fluid method for the dynamics of free boundaries," J. Computat. Phys., 39, 201-225 (1981).

3) S. Koshizuka, Y. Oka, "Moving-particle semi-implicit method for fragmentation of incompressible fluid," J. Nucl. Sci. Eng., 123, 421-434 (1996).

4) Fauske and Associates, LLC., MAAP5-Modular Accident Analysis Program User's Mannual, 2, Part $1-3(2008)$.

5) R. O. Gauntt, R. K. Cole, M. Erickson et al., MELCOR Computer Code Manuals, Primer and Users' Guide, Version 1.8.5, NUREG/CR-6119, 1, Rev. 2 (2000).

6) S. Koshizuka, Ryuushi-hou Simulation, Maruzen (2005).

7) S. Koshizuka, J. Liu, N. Shirakawa et al., "R\&D of the next generation safety analysis methods for fast reactors with new computational science and technology (1)-(6)," Proc. 16th Int. Conf. on Nucl. Eng. (ICONE16), Orland, May 11-15 (2008).

8) X. Li, Y. Oka, "Numerical simulation of the SURC-2 and SURC-4 MCCI experiments by MPS method," Ann. Nucl. Energy, 73, 46-52 (2014).

9) L. Guo, Y. Kawano, S. Zhang, T. Suzuki, K. Morita, K.Fukuda, "Numerical simulation of theological behavior in melting metal using finite volume particle method," J. Nucl. Sci. Technol., 47[11], 1011-1022 (2010).

10) M. Kondo, S. Koshizuka, M. Takimoto, "Surface tension model using inter-particle potential force in moving particle semi-implicit method," Trans. JSCES, Paper No. 20070021, (2007). [in Japanese]

11) T. Tamai, K. Shibata, S. Koshizuka, "Development of the higher-order MPS method using the taylor expansion," Trans. JSCES, Paper No. 20130003, (2013). [in Japanese]

12) M. Tanaka, T. Masunaga, "Stabilization and smoothing of pressure on MPS method by quasi-compressibility,” Trans. JSCES, Paper No. 20080025, (2008). [in Japanese]

13) M. Oochi, S. Koshizuka, M. Sakai, "Explicit MPS algorithm for free surface flow analysis," Trans. JSCES, Paper No. 20100013, (2010). [in Japanese]

14) A. Robledo, C. Varea, "Wetting transition for the contact line and antonov's rule for the line tension," Phys. Rev. A, 45[4], 2423-2428 (1992).

15) M. Tanaka, M. Sakai, S. Koshizuka, "Particle-based rigid body simulation and coupling with fluid simulation,” Trans. JSCES, Paper No. 20070007, (2007). [in Japanese]

16) P. A. Cundall, O. D. L. Strack, "A discrete numerical model for granular assemblies," Geotechnique, 29, 47-65 (1979).

17) K. Washidu, Yuugenyouso-hou Handbook, Baifu-kan, Tokyo, ISBN 978-4563031800 (1983). [in Japanese]

18) H. T. Kim, B. W. Rhee, J. H. Park, "Benchmark calculations of a radiation heat transfer for a CANDU fuel channel analysis using the CFD code," J. Nucl. Sci. Eng., 43[11], 1422-1430 (2006).

19) M. Furuya, "Development of numerical method for fuel rod melting ((1) Experimental observation)," Proc. of Konso-ryu Symposium, Nagano, Aug. 9-11 (2013). [in Japanese]

20) Y. Katsuto, Den-netsu Gairon, Youken-do, (1964). [in Japanese]

21) Z. Yuan, K. Mukai, K. Takagi, M. Ohtaka, "Dependence of surface tension of molten tin on temperature and oxygen partial pressure," J. Jpn. Inst. Metals, 65[1], 21-28 (2001). [in Japanese] 
22) S. Nishimura, I. Kinoshita, N. Ueda et al., "Study on transformation and fragmentation behavior of molten metal in sodium pool," CRIEPI Rep., L03 (2005). [in Japanese] 


\title{
Article
}

\section{Development of a Filtered Containment Venting System for Nuclear Power Plants}

\author{
Shinichi KAWAMURA ${ }^{1, *}$, Takeo KIMURA ${ }^{1}$, Shuichi OMORI ${ }^{2}$ \\ and Tadashi NARABAYASHI ${ }^{3}$ \\ ${ }^{1}$ Nuclear Asset Management Department, Tokyo Electric Power Company \\ 1-1-3 Uchisaiwai-cho, Chiyoda-ku, Tokyo 100-8560, Japan \\ ${ }^{2}$ Research and Development Center, Tokyo Electric Power Company, \\ 4-1 Egasaki-cho, Tsurumi-ku, Yokohama 230-8510, Japan \\ ${ }^{3}$ Division of Energy and Environmental Systems, Graduate School of Engineering, \\ Hokkaido University, Kita-13, Nishi-8, Kita-ku, Sapporo 060-8628, Japan
}

\begin{abstract}
A filtered containment venting system was developed for nuclear power plants. In the Fukushima Dai-ichi Nuclear Accident, widespread land contamination was caused by cesium-137. This system was developed to filter aerosol particles and reduce the amount of radioactive particle release, while protecting the primary containment vessel from over pressure by venting gas from the vessel. Performance tests were conducted under various vent gas flow rate conditions to ascertain decontamination factors for aerosol particles with various diameters. It was observed through the tests that aerodynamic diameter was a good index for characterizing various aerosol particles for the filtered containment venting system. Test results showed that the decontamination factors were well over 1,000 for aerosol particles with aerodynamic diameters larger than $0.4 \mu \mathrm{m}$. For aerosol particles with aerodynamic diameters larger than $0.2 \mu \mathrm{m}$, the decontamination factors significantly increased with the diameter. This suggested that inertial deposition was the governing mechanism for filtering aerosols in this system. The decontamination factor of the water scrubber section of the filter increased with the increase in Stokes number.
\end{abstract}

KEYWORDS: filtered containment vent system, nuclear power plant, accident, primary containment vessel, cesium, aerosol, particle size, decontamination factor, performance, experiment

\section{Introduction}

In 2011, the Great East Japan Earthquake and the subsequent tsunami led to an accident at the Fukushima Daiichi Nuclear Power Plant. Although the reactor scram successfully inserted all the control rods into the reactor cores of operating plants during the tremors, cooling after the scram failed due to damage caused by the tsunami, which resulted in the meltdown of the core. In addition, a part of the reactor containment vessel was damaged and large amounts of

\footnotetext{
* Corresponding author, E-mail: shinichi.kawamura@tepco.co.jp

DOI : 10.15669 /fukushimainsights.Vol.4.439

(C) 2021 Atomic Energy Society of Japan. All rights reserved.

Originally published in Transactions of the Atomic Energy Society of Japan (ISSN 1347-2879), Vol. 15, No. 1, p.12-20

(2016) in Japanese. (Japanese version accepted: July 31, 2015)
} 
radioactive materials were released into the environment. Based on the lessons learned from this accident, enhanced measures that will prevent core damage have been sought. These enhancements include the protection of safety functions against not only earthquakes and tsunamis, but also a wider range of external events, the addition of alternative high-pressure injection systems for reactors, alternative operation systems for reactor depressurization, alternative low-pressure injection systems, alternative cooling systems as a contingency measure for potential accidents, the establishment of response operation procedures, and the implementation of training programs.

In addition, considering the possibility of a severe accident in which the reactor core is damaged despite these measures, a filtered containment venting system for nuclear power plants was developed. As a result of the accident that occurred at the Fukushima Daiichi Nuclear Power Plant, the long-term and widespread soil contamination, which was mainly due to radioactive cesium, has had a significant impact on the lives of residents and industrial activities, including agriculture.

A filtered containment venting system can protect a containment vessel against overpressure failure in the event of a severe accident and significantly reduce emissions of radioactive materials. Radioactive cesium is emitted mainly in particulate form. According to Soffer et al. ${ }^{1)}$ and Beahm et al. ${ }^{2}, 95 \%$ of the radioactive iodine emitted from a reactor primary system in the event of an accident is in particulate form such as cesium iodide, and the remaining $5 \%$ is in gaseous form.

When the $\mathrm{pH}$ of the water in the containment vessel is kept alkaline, a large portion of the gaseous iodine is elemental iodine, and organic iodine accounts for only $0.15 \%$ or less ${ }^{1,2}$. Since materials other than organic iodine can be absorbed into alkaline water, they can be absorbed into the scrubber water of the filtering device described in this article. This article describes the development of a filtering device for use in the filtered containment venting system and, in particular, the details of the particulate material removal process and outcomes, based on experimental results.

Experiments were conducted on filtering devices to verify their overall removal performance based on the ratio of aerosol concentration at the intake to that at the exit of the filter ${ }^{3,4}$, but detailed characteristics of the removal were not provided. However, to ensure high removal performance, it is important to understand and improve the removal performance achieved by scrubbing the aerosol-laden gas and trapping aerosolized liquid droplets containing radioactive materials and dispersed in the gaseous phase after scrubbing. Therefore, in developing this filtering device, we experimentally determined the removal performances of both scrubbing and entrained liquid droplet trapping, which finally resulted in a major improvement in the overall removal performance. We also sought to improve the removal performance significantly with innovations to the scrubbing process such as combining the scrubber nozzle and the air bubble subdividing device, water surface stabilization using the flow regulating plate located in the upper part of the scrubbing section, and removal of entrained material using metal filters.

In addition, since the aerosol removal characteristics are generally dependent on particle diameter and density, it is important to know how these factors influence the removal characteristics. This knowledge will inform procedures for selecting mitigation and vent operation methods in the event of an accident. Thus, in this article, the removal characteristics were examined in detail using particles of different sizes and densities. 


\section{Development of a Filtered Containment Venting System}

\section{Development Conditions}

Severe accidents in a light-water reactor may occur in various ways depending on the initiating event and the success or failure of components that incorporate safety functions. Moreover, to achieve cooling after a reactor scram, injection of water into damaged fuel and spraying of water into the containment vessel are needed, using permanent or portable alternative water injection equipment. However, if there is no mechanism to ultimately release the exchanged heat, inevitably the containment vessel pressure will reach its limit due to internally generated steam. At this point, venting from the containment vessel is required to enable depressurization and thermal transport to the atmosphere. In addition, since venting also discharges hydrogen from the containment vessel, it is expected to prevent explosions due to the leakage of hydrogen from the containment vessel into the reactor building, unlike what was experienced in the accident that occurred at the Fukushima Daiichi Nuclear Power Plant.

In a boiling water reactor (BWR), the process of treating the vent gas with the suppression chamber water inside the containment vessel was established as an accident management measure to reduce the level of radioactive materials to some extent before releasing the gas to the atmosphere. In a filtered containment venting system, a dedicated filtering device is added to remove particulate radioactive materials such as cesium hydroxide and cesium iodide at higher efficiency.

Thus, we established the following conditions for developing a filtered containment venting system:

- A filtered containment venting system should remain functional at the upper limits of the pressure and temperature ranges where it has been confirmed that overpressure and over temperature failure of the containment vessel will not occur even in the event of a severe accident. These limits were set as the maximum operating pressure and temperature. In the case of an advanced boiling water reactor (ABWR), the limits are $620 \mathrm{kPa}$ (gauge) and $200^{\circ} \mathrm{C}$, respectively.

- To ensure the depressurization of the containment vessel, considering the decay heat at two or three hours after a reactor scram, twice the steam flow equivalent to $1 \%$ of the rated reactor thermal output was set as the design flow rate (31.6 kg/s in the case of the ABWR).

- The decontamination factor (DF) for particulate radioactive materials of 1,000 or higher was set as the performance target.

In addition to the aforementioned conditions, we considered the following design issues from the perspective of ensuring sufficient reliability to enable operation even in the event of a severe accident:

- The necessary safety functions must not be lost due to external events that need to be considered in the design, including design basis earthquake motion and tsunami.

- Common-cause failures of safety functions must not occur between the filtered containment venting system and the design basis accident mitigation systems.

- The filtering device is made with highly corrosion-resistant materials and measures against spillover are taken to deal with unexpected leakage.

- Not only is the entire system filled with nitrogen gas on standby, but also, accumulated hydrogen is purged with nitrogen gas after venting to prevent hydrogen explosion.

- The necessary operations can be conducted safely and reliably both from the main control room and elsewhere on site.

- To reduce the influence of venting on all emergency actions, including the operation of the 
filtered containment venting system, necessary measures (such as shielding) are taken to control the exposure dose within acceptable limits.

- To maintain the water level in the filtering device within the appropriate range, a secure supply of water must be available to the device, and the discharge of water from the device into the suppression chamber in the containment vessel must also be possible even under emergency conditions (this function also involves returning the radioactive materials collected in the filtering device to the containment vessel after venting).

- The water level, $\mathrm{pH}$, inlet pressure, metal filter pressure difference, outlet radiation intensity, hydrogen concentration, and drain transfer flow rate of the filtering device must be able to be monitored during use, on standby, and after use.

- Tests or inspections must be possible to conduct, to check whether or not the necessary safety functions are being maintained.

Sodium thiosulfate and sodium hydroxide are added to the water in the filtering device to maintain an alkaline and reducing environment. Thus, the iodine ions generated from cesium iodide aerosol trapped in water remain in the water, and a chemical reduction that traps gaseous elemental iodine as $\mathrm{I}^{-}$ions in the water is also expected:

$$
4 \mathrm{I}_{2}+\mathrm{S}_{2} \mathrm{O}_{3}^{2-}+10 \mathrm{OH}^{-} \rightarrow 8 \mathrm{I}^{-}+2 \mathrm{SO}_{4}^{2-}+5 \mathrm{H}_{2} \mathrm{O}
$$

In addition, sodium thiosulfate serves as a surfactant and has the effect of decreasing the size of the bubbles in the water to increase the gas-liquid contact interface.

\section{Description of the Filtered Containment Venting System}

\section{(1) System configuration}

Figure 1 shows the system configuration of the filtered containment venting system. The gas containing radioactive materials is discharged from the gaseous phase of the suppression chamber or the dry well of the containment vessel, fed to the filtering device through a pipe, and then released at a height after it has been treated. The isolation valves of the containment vessel need to be opened at the time of venting. These valves can be operated from the main control room and elsewhere on site. To manage operator exposure, a mechanism that operates manually and remotely through the shield is provided for the on-site operation of each valve.

The gas release pipe downstream of the filtering device is equipped with a pressure release plate that serves as a barrier to fill the entire system with nitrogen gas on standby. Filling the

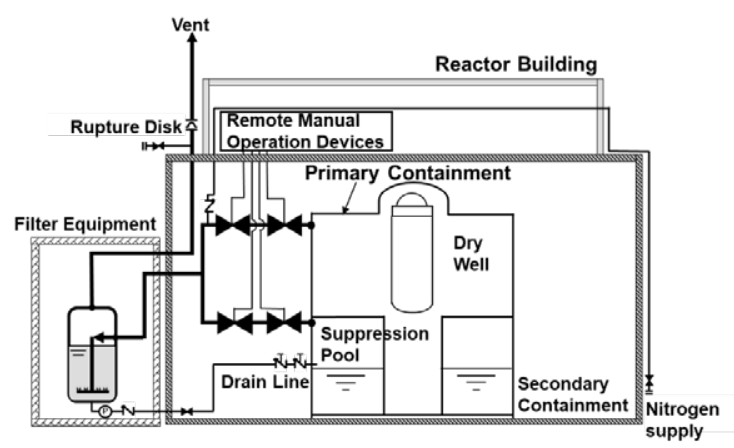

Figure 1 Basic system configuration of the filtered containment venting system 
reactor containment vessel and the inside of this system with nitrogen gas prevents hydrogen contained in the gas from burning in this system even when the steam of the vent gas condenses in the pipe at the early stage of venting. This pressure release plate is designed to operate at a pressure of $100 \mathrm{kPa}$ (gauge), which is low enough (relative to the design pressure of the containment vessel) not to interfere with the venting process.

\section{(2) Filtering device}

Figure 2 shows a schematic representation of the structure of the filtering device, which consists of the water scrubber and the metal filters. The vent gas from the containment vessel enters from the inlet nozzle in the middle to the inside and is injected into the water from 140 scrubber nozzles located in the lower portion of the device. The air bubble subdividing device installed above the nozzles enhances trapping of particulate radioactive materials by increasing the gas-liquid contact area.

The gaseous phase is in the upper portion of the device, where 128 metal filters are placed to trap radioactive fine particles that could not be removed by the water scrubber and liquid droplets. Each element has a cylindrical triple-layered structure that mainly consists of a sintered sheet of metal fibers with a diameter of $2 \mu \mathrm{m}$. This sheet has the function of removing particulates and is placed between two web layers made of metallic fibers with a diameter of $30 \mu \mathrm{m}$. The webs support the metal fiber sheet internally and externally, collect tiny liquid droplets carried by the gas that passed through the water scrubber, and return the droplets to the water scrubber through the drainage pipe in the device.

In addition, the flow regulating plate located between the water scrubber and the metal filters regulates the gas flow to the metal filter. In the event an earthquake occurs during venting, this flow regulating plate mitigates sloshing of the scrubber water.

\section{(3) Removal mechanism of the water scrubber}

In the water scrubber, the vent gas is injected into the water through nozzles. Particulate radioactive materials are trapped in the water and removed as the bubbles of the injected gas float up. The principle of the removal mechanism depends on the particle size, as described next.

First, the particles contact the gas-liquid interface as a result of inertia or gravity and become trapped in the water to be removed. This mechanism predominantly traps the larger particles, while the smaller particles, being more influenced by interaction with gas molecules, are trapped when they contact a gas-liquid interface as the result of Brownian diffusion and

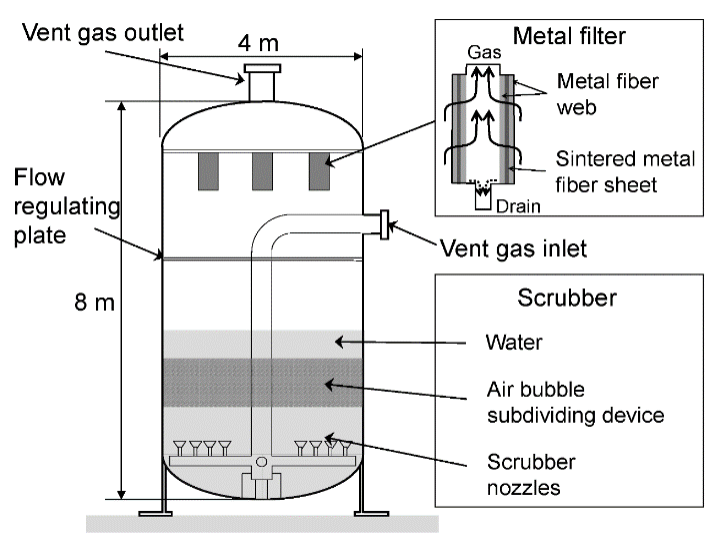

Figure 2 Schematic diagram of the filter equipment 
thermophoresis. Furthermore, when the water temperature is lower than the saturation temperature, the steam in the gas bubbles condenses and the particles become trapped as they contact the gas-liquid interface.

In addition, especially immediately after injection in the region near the scrubber nozzles, a mechanism is assumed whereby the particles are trapped in the water by their greater momentum as the lighter gas molecules in the bubble decelerate rapidly upon the injection of gas bubbles into the water.

\section{(4) Removal mechanism of the metal filter}

In the metal filter, the particulate radioactive materials are trapped and removed when the vent gas passes through the filter fibers, according to the mechanisms described next.

First, relatively large particles are blocked and trapped by the narrowness of the gaps between the metal fibers. In addition, it is assumed that the particles impact forcefully on the metal fiber surfaces and adhere to them. By these mechanisms, the larger particles are more easily trapped, while the smaller particles may be trapped, as contact with the metal filter fibers in their Brownian motion.

\section{Performance Evaluation of the Filtered Containment Venting System}

\section{Accident Scenario}

Taking the initiating event as the starting point, every accident scenario can be extracted from an event tree branched by the success or failure of the operation of a system safety function. Then, accident scenarios can be categorized into several groups. For example, in a typical accident scenario group for a BWR plant, a large break loss-of-coolant accident (LOCA), station blackout (SBO), and loss of all functions of the emergency core cooling systems (ECCS) may occur simultaneously. Assuming this scenario, we conducted experiments to confirm the performance of the filtered containment venting system using Units 6 and 7 of Kashiwazaki-Kariwa Nuclear Power Plant (ABWR plants) as an example. In terms of overpressure failure of the containment vessel, which should be preventable by the filtered containment venting system, this scenario is an extreme case in which the event progresses rapidly and the temperature and pressure of the containment vessel become high.

The event sequence of this accident scenario is as follows:

(1) A large break LOCA occurs and a large amount of coolant leaks into the containment vessel.

(2) Since an SBO and the loss of the ECCS are assumed, water cannot be injected into the reactor pressure vessel, leading to core damage.

(3) After approximately 70 minutes, an alternative low-pressure injection system powered by a gas turbine generator injects water into the reactor pressure vessel and sprays water into the containment vessel.

(4) Approximately 38 hours after the initiating event, the pressure of the containment vessel reaches the limit pressure and venting is conducted by the filtered containment venting system.

We used MAAP code for accident analysis. Figures 3 and $\mathbf{4}$ show the pressure and temperature changes in the containment vessel in this accident scenario. The charts indicate that the venting system keeps the temperature and pressure of the containment vessel at or below the limit temperature $\left(200^{\circ} \mathrm{C}\right)$ and pressure $(620 \mathrm{kPa})$, so the uncontrollable release of radioactive 


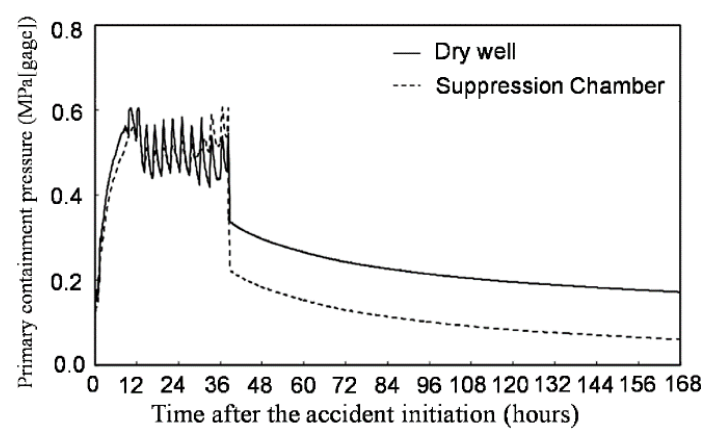

Figure 3 Primary containment pressure of an ABWR plant in a severe accident caused by a combination of LOCA, SBO, and all ECCS functional failures

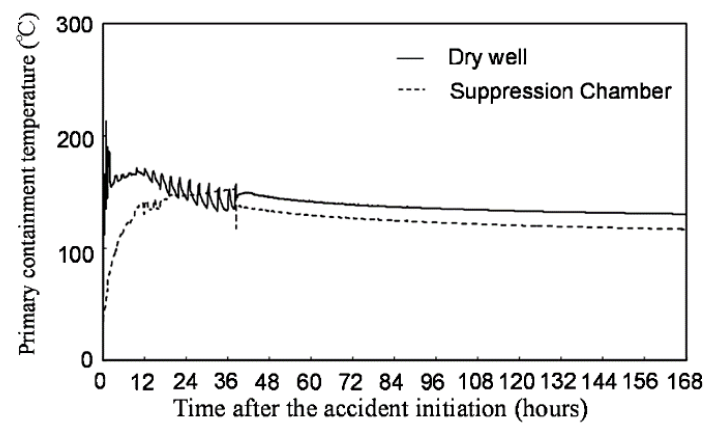

Figure 4 Primary containment temperature of an ABWR plant in a severe accident caused by a combination of LOCA, SBO, and all ECCS functional failures

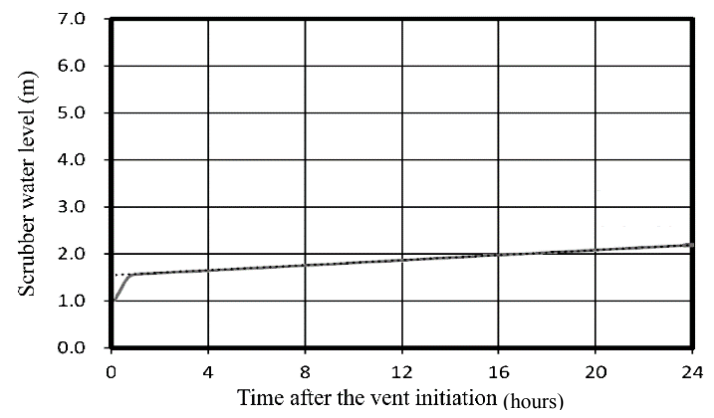

Figure 5 Predicted trend of the scrubber water level during containment venting

materials into the environment due to failure of the containment vessel can be prevented.

Continued gas venting raised the water level of the water scrubber. Figure $\mathbf{5}$ shows the predicted change of the water level. The total amount of steam condensate in the vent pipe flowing into the filtering device and that in the filtering device is larger than the amount of evaporation caused by the radioactive materials trapped in the water scrubber. Therefore, the water level continues to rise during venting. Although the installed position of the metal filters corresponds to a water level of $5 \mathrm{~m}$, it is impossible to maintain the performance of the system when the water level reaches the metal filters, and an operation to lower the water level is required. Thus, 
we incorporated a draining system into the filtering device to discharge the scrubber water into the containment vessel suppression chamber when the water level reaches $2.5 \mathrm{~m}$, considering the spout that could occur due to aeration of the vent gas, and to supply water and sodium thiosulfate and sodium hydroxide solutions to the scrubber.

\section{Performance Test Facility and Test Conditions for the Filtering Device}

\section{(1) Test facility}

Figure 6 shows the configuration of the test facility used for the performance test. By feeding air mixed with simulation particles from the aerosol generator through a test chamber designed to resemble the actual filtering device, we measured the number density and diameter of the particles at the front and back of the test chamber to calculate DF. For the measurement, we used a light-scattering type aerosol spectrometer (Welas digital $2000 \mathrm{H}$ manufactured by Palas $\mathrm{GmbH}$ ) and a scanning type mobility particle sizer (Model 3936 manufactured by TSI Inc.).

In the test chamber, we placed a scrubber nozzle and metal filter identical to those used in the actual device, and we also filled the air bubble subdividing device with the same element as that used in the actual device, up to the same height as in the actual device. The diameter of the test chamber was $0.35 \mathrm{~m}$ and its average flow passage cross sectional area was $1 / 140$ of that of the actual device, thus achieving the same operating conditions per scrubber nozzle as that of the actual device. However, since the gaseous phase between the water scrubber and the metal filter does not affect the particle removal mechanism, this section was shortened to reduce the height to $4 \mathrm{~m}$, as shown in Figure 6.

\section{(2) Test gas}

In this test, air at ordinary temperature and pressure was used as the test gas. In contrast, the gas that is vented in a severe accident mainly consists of steam at $120^{-} 170^{\circ} \mathrm{C}$. As described next, the test we conducted using air at ordinary temperature is considered conservative in

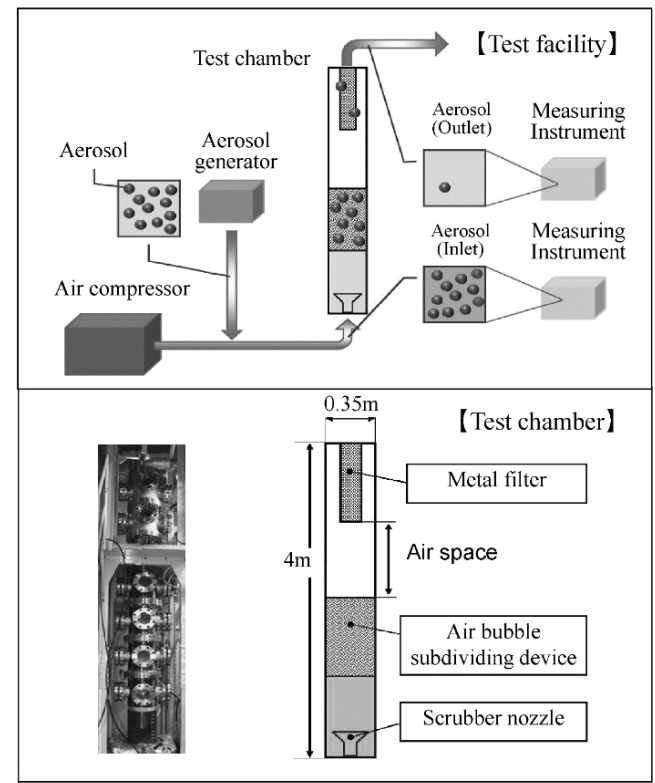

Figure 6 Performance test facility for the filter equipment 
terms of the particle trapping mechanism.

First, trapping by inertial collision produces a larger effect as the Stokes number $(\mathrm{St})^{5)}$ increases; however, there are no significant differences in St between the test gas and the vent gas when the particle diameter is $0.4 \mu \mathrm{m}$ or less. On the other hand, at larger particle diameters, St in the case of air at ordinary temperature and pressure becomes smaller than that under the steam conditions that could develop in a severe accident. Therefore, the test conducted using air at ordinary temperature and pressure is conservative in terms of the trapping effect based on inertial collision. The Stokes number is calculated by the following equations:

$$
\begin{aligned}
& \mathrm{St}=\mathrm{D}_{\mathrm{p}}^{2}\left(\rho_{\mathrm{p}}+\rho / 2\right) \mathrm{C}_{\mathrm{c}} \mathrm{u}_{0} /\left(9 \eta \mathrm{D}_{\mathrm{f}}\right) \\
& \mathrm{C}_{\mathrm{c}}=1+\mathrm{K}_{\mathrm{n}}\left(1.257+0.4 \exp \left(-1.1 / \mathrm{K}_{\mathrm{n}}\right)\right) \\
& \mathrm{K}_{\mathrm{n}}=2 \lambda_{\mathrm{g}} / \mathrm{D}_{\mathrm{p}} \\
& \lambda_{\mathrm{g}}=\eta /\left(0.499 \mathrm{P}(8 \mathrm{M} / \pi \mathrm{RT})^{1 / 2}\right)
\end{aligned}
$$

where, $\mathrm{D}_{\mathrm{p}}$ is the particle diameter, $\rho_{\mathrm{p}}$ is the particle density, $\rho$ is the gas density, $\mathrm{C}_{\mathrm{c}}$ is a slip correction coefficient, $\mathrm{u}_{0}$ is the gas flow velocity, $\eta$ is the gas viscosity, $\mathrm{D}_{\mathrm{f}}$ is a characteristic diameter in the fluid, $\mathrm{T}$ is the gas temperature, $\mathrm{P}$ is the gas molecular weight, and $\mathrm{R}$ is the gas constant.

Trapping by gravitational sedimentation is considered to produce a larger effect as the gravitational sedimentation velocity $\left(\mathrm{v}_{\mathrm{t}}\right)^{5)}$ increases. However, there is no significant difference in gravitational sedimentation velocity between the test gas and the vent gas when the particle diameter is $0.4 \mu \mathrm{m}$ or less. On the other hand, at larger particle diameters, $\mathrm{v}_{\mathrm{t}}$ in the case of air at ordinary temperature and pressure becomes smaller than that under the steam conditions that could develop in a severe accident. Therefore, the test conducted using air at ordinary temperature and pressure is conservative in terms of the trapping effect based on gravitational sedimentation. The gravitational sedimentation velocity is calculated by the following equation:

$$
\mathrm{v}_{\mathrm{t}}=\mathrm{C}_{\mathrm{c}} \mathrm{D}_{\mathrm{p}}^{2}\left(\rho_{\mathrm{p}}-\rho\right) \mathrm{g} / 18 \eta
$$

where, $\mathrm{g}$ is gravitational acceleration, and the other variables are as defined previously.

Although trapping by diffusional deposition is considered to produce a larger effect as the Brownian diffusion coefficient (D) ${ }^{5}$ increases, the Brownian diffusion coefficient in the case of air at ordinary temperature and pressure is smaller than that under the steam conditions that could develop in a severe accident. Therefore, the test conducted using air at ordinary temperature and pressure is conservative in terms of the trapping effect based on diffusional deposition. The Brownian diffusion coefficient is calculated by the following equation:

$$
\mathrm{D}=\mathrm{C}_{\mathrm{c}} \mathrm{kT} / 3 \pi \eta \mathrm{D}_{\mathrm{p}}
$$

where, $\mathrm{k}$ is the Boltzmann constant, and the other variables are as defined previously.

In addition to the aforementioned mechanisms, trapping by steam condensation and trapping by thermophoresis are also assumed under steam conditions, but the test conditions are conservative because these effects are not expected in the test using air at ordinary temperature and pressure.

\section{(3) Simulation particles}

The transition of particulate radioactive materials in the water scrubber from gas (bubbles) to water and the adhesion of particulate radioactive materials in the metal filter from gas to 
metal fiber surfaces are based on the physical movement of the particles and are not entirely dependent on their chemical properties. Therefore, for the simulation particles in our test, we used titanium oxide with a density relatively close to those of cesium hydroxide and cesium iodide, which are typical particulate radioactive materials observed during venting, and ferric oxide. The densities of $\mathrm{TiO}_{2}, \mathrm{CsOH}, \mathrm{CsI}$, and $\mathrm{Fe}_{2} \mathrm{O}_{3}$ are $4.23 \mathrm{~g} / \mathrm{cm}^{3}, 3.68 \mathrm{~g} / \mathrm{cm}^{3}, 4.51 \mathrm{~g} / \mathrm{cm}^{3}$, and $5.24 \mathrm{~g} / \mathrm{cm}^{3}$, respectively. In addition, as standard particles for the aerosol test, we used polystyrene standard particles (PSL), which are commonly employed. The density of PLS is $1.05 \mathrm{~g} / \mathrm{cm}^{3}$.

\section{(4) Test condition for scrubber water}

The water level in the test chamber was set at $1 \mathrm{~m}$ above the top edge of the scrubber nozzle, which corresponds to the water level in the actual device on standby. As shown in Figure 5, the scrubber water level rises during venting. And, as the scrubber water level increases, the vent gas goes through the water for a longer period of time, and the particles are more easily trapped. Therefore, this test condition, corresponding to the lowest water level in the actual device, is conservative.

The water in the test chamber was at ordinary temperature. In the actual device, the thermal energy of the vent gas and the decay heat of the radioactive materials raise the temperature of the scrubber water to the saturation temperature according to the pressure in the filter device. On the other hand, while the water temperature is lower than the gas temperature, as the temperature difference between the water and the gas increases, the particles are more easily trapped due to thermophoresis and the reduction of bubbles after steam condensation. However, this test condition is conservative because air at ordinary temperature and pressure was used as the test gas.

Since this test is intended to determine the particulate matter removal performance, sodium thiosulfate and sodium hydroxide, which are additives for removing gaseous inorganic iodine, were not added.

\section{(5) Gas flow rate test condition}

The gas flow rate was set to cover the conditions of use of the actual device. Figure 7 shows the change in the vent gas flow rate in the scenario that assumes an accident at an ABWR plant. The highest flow rate occurs immediately after venting at the limit pressure of the containment vessel. Subsequently, the flow rate decreases according to the amount of steam generated in the containment vessel. Therefore, we simulated three conditions in the performance test and set the test flow rate to $1 / 140$ of the corresponding volumetric flow rates in the actual device, as follows:

- Maximum flow rate condition: $33,000 \mathrm{~m}^{3} / \mathrm{h}$, which is the volumetric flow rate immediately

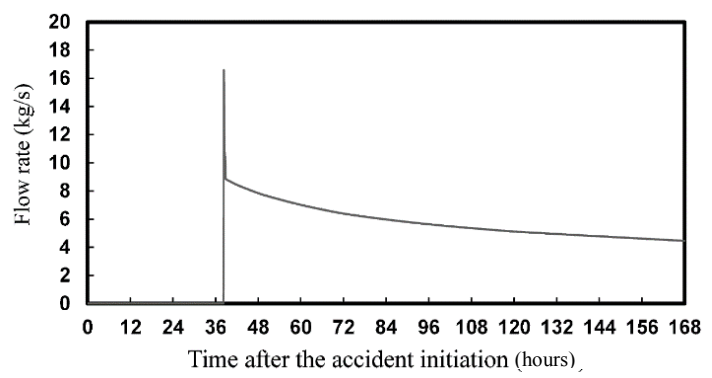

Figure 7 Predicted trend of vent gas flow rate 
after venting at twice the design pressure of the containment vessel (620 $\mathrm{kPa}$ (gage)).

- Medium flow rate condition: $27,000 \mathrm{~m}^{3} / \mathrm{h}$, which is the volumetric flow rate immediately after venting at the design pressure of the containment vessel $(310 \mathrm{kPa}$ (gage)).

- Minimum flow rate condition: $13,500 \mathrm{~m}^{3} / \mathrm{h}$, which is the volumetric flow rate set as a condition lower than the flow rate observed seven days (168 hours) after the occurrence of the accident.

\section{Results of the Removal Performance Test Conducted on the Filtering Device}

\section{(1) Particulate material removal performance}

Figures 8-10 show the results of measuring the DFs of the particulate materials.

These figures also show the distribution of particle sizes flowing into the filtered containment venting system from the containment vessel suppression chamber in the accident scenario described previously (a large break LOCA at an ABWR plant, loss of all ECCS functions, and SBO), which was obtained by analysis using MAAP code.

When the DF exceeds 10,000, particles may not be detected at the exit.

In such a case, the integrated values of the number of particles detected at the entrance of the filter during the measurement period were plotted as the reference value of DF. Although this value corresponds to the DF of the case assuming one particle is detected at the exit, no particles

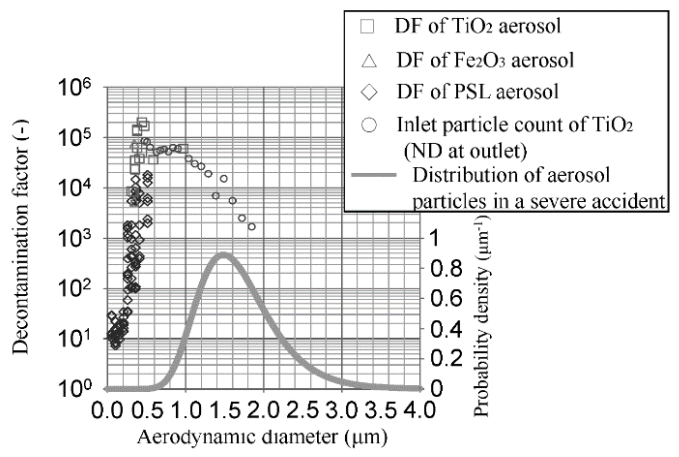

Figure 8 Measured decontamination factor at the maximum vent flow condition (a simulated venting flow rate of $33,000 \mathrm{~m}^{3} / \mathrm{h}$ at twice the maximum containment design pressure)

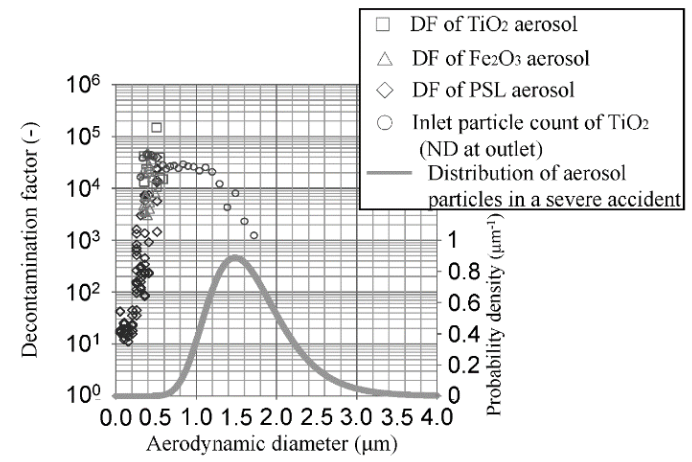

Figure 9 Measured decontamination factor at the medium vent flow condition (a simulated venting flow rate of $27,000 \mathrm{~m}^{3} / \mathrm{h}$ at the maximum containment design pressure) 


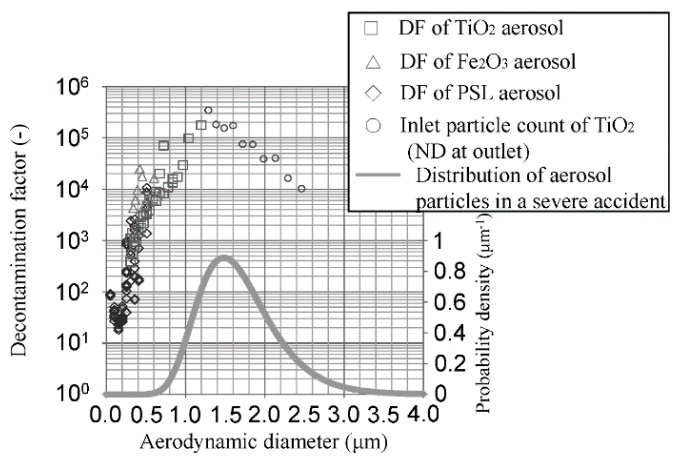

Figure 10 Measured decontamination factor at the minimum vent flow condition (a simulated venting flow rate of $13,500 \mathrm{~m}^{3} / \mathrm{h}$ )

were detected in the actual measurement. Therefore, it can be estimated that the DF was higher than this reference value. This reference DF value was used to determine whether a measured DF was equal to or higher than the target value $(1,000)$. The reference DF value decreased as the particle diameter increased because the number density of test particles decreased with the particle diameter and the integrated value of the particle number at the entrance became smaller. Therefore, it did not show a reduction tendency for DF.

In Figures 8-10, the DFs were plotted against the aerodynamic diameter of test particles with different densities on the abscissa. The aerodynamic diameter is the diameter of a hypothetical sphere with a density of $1 \mathrm{~g} / \mathrm{cm}^{3}$, which achieves the same terminal velocity as the particle to be studied in viscous fluids similar to air. The aerodynamic diameter is represented by the following equation ${ }^{6}$ :

$$
\mathrm{D}_{\mathrm{ae}}=\left(6 \rho_{\mathrm{p}} \alpha_{\mathrm{e}} / \pi \mathrm{K}_{\mathrm{R}}\right)^{1 / 2} \mathrm{D}_{\mathrm{p}}
$$

where, $D_{a e}$ is the aerodynamic diameter, $\alpha_{\mathrm{e}}$ is the volume shape factor of the particles, $K_{R}$ is the resistance shape factor of particles, and the other variables are as defined previously.

Figures 8-10 show identical DF distributions as a result of the particles organizing according to their aerodynamic diameter regardless of the test particles used. From this result, we found that organizing according to the aerodynamic diameter is effective in determining the performance of the filter.

The measured DFs increased monotonically when the aerodynamic diameter was equal to or larger than approximately $0.2 \mu \mathrm{m}$. Since the metal filter blocks particles that are larger than a certain size, and inertial collision is a dominant removal mechanism for smaller particles, we inferred that the effect of an inertial collision increased with the aerodynamic diameter. On the other hand, for the scrubber, the mechanisms whereby larger particles yield a higher removal effect include inertial collision and gravitational sedimentation, and it is possible that the effect of either one of these mechanisms increased.

Therefore, for the purpose of investigating the trapping mechanism in the scrubber, we conducted a test in which only the DFs based on the scrubber were measured, with the metal filters removed. We used $\mathrm{TiO}_{2}$ as the particles for the test. Figure 11 shows the measured DFs, which are organized according to the Stokes number. As this figure shows, the DFs increase with the Stokes number. In addition, even though the flow rates are different, the DFs shows somewhat similar tendencies when organized according to the Stokes number. Thus, we inferred that the 


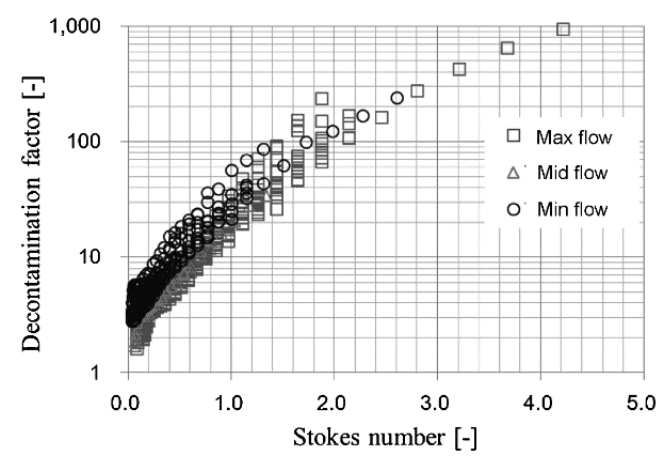

Figure 11 Relationship between measured decontamination factor of the scrubber and Stokes number ("Max flow," "Mid flow," and "Min flow" represent simulated venting flow rates of $33,000 \mathrm{~m}^{3} / \mathrm{h}, 27,000 \mathrm{~m}^{3} / \mathrm{h}$, and $13,500 \mathrm{~m}^{3} / \mathrm{h}$ respectively)

influence of inertial collision is significant in causing the increase in DF due to the scrubber.

These insights provide useful knowledge for further improving the performance of the scrubber in the future. Although lengthening the retention time in the scrubber water is required for gravitational sedimentation to have a significant influence on performance, an improvement that focuses on the Stokes number, such as increasing the ejection flow rate from the scrubber nozzle, is considered effective because inertial collision has a more significant influence.

As a particle trapping mechanism, using the scrubber under accident conditions, the effect of trapping the particles at the gas-liquid interface due to steam condensation is expected at the early stage of venting, but this effect will diminish because the scrubber water temperature increases with time. However, from our test results, we found that the required removal performance can be achieved by the trapping mechanism based on inertial collision, even if the scrubber water temperature increases as a result of continuing the venting when an accident occurs.

As mentioned earlier, the distribution of particle sizes in Figures 8-10 was calculated by MAAP code with the assumption that the vent gas was discharged from the containment vessel suppression chamber. This distribution was referred to because the DFs tend to increase when the aerodynamic diameter is equal to or greater than $0.2 \mu \mathrm{m}$ and this case gives a relatively smaller size distribution. The aerodynamic diameters in this case were distributed between $0.5 \mu \mathrm{m}$ and $4 \mu \mathrm{m}$, with the peak between $1 \mu \mathrm{m}$ and $2 \mu \mathrm{m}$. When venting from the dry well but not from the suppression chamber in the same accident scenario, the aerodynamic diameters of the particles flowing into the filtered containment venting system ranged from $0.7 \mu \mathrm{m}$ to $10 \mu \mathrm{m}$, with the peak between $3 \mu \mathrm{m}$ and $4 \mu \mathrm{m}$. The reason why the particle size distribution shifted to the larger particle diameters is that there was no scrubbing effect by the suppression chamber. In addition, when we considered an alternative accident scenario that involved injection failure at high and low pressures, we obtained an almost identical particle size distribution as when venting was conducted from the suppression chamber in the scenario of a large break LOCA at an ABWR plant, with loss of all ECCS functions, and SBO. Regarding the size distribution of the aerosol particles in the containment vessel at the time of the accident, we found several examples based on analyses or experiments in past studies ${ }^{7-9)}$ that reported distributions with median diameters of several micrometers and geometric standard deviations of several micrometers. The results of our analyses are consistent with the outcomes of these previous studies.

Considering the particle size distributions and the DFs, we found that the DFs exceeded the development target of 1,000 in the range of our test, including the range of aerodynamic diameters where no particle was detected at the exit of the filter. In addition, for aerodynamic 
diameters that exceed the range of our test, it was inferred that the DFs exceeded that obtained for the diameter at the upper limit of the test range because inertial collision increases with the aerodynamic diameter. Furthermore, for larger particle sizes, the DFs are expected to be equal to or larger than 1,000 because of the additional effect of gravitational sedimentation in the scrubber and blocking by the metal filter.

\section{(2) Liquid droplet removal performance of the metal filter}

In this test, water-insoluble solid particles were used as simulation particles. However, the main particles observed in a severe accident are cesium iodide and cesium hydroxide, which are both water soluble. The fact that the simulation particles are water-insoluble is deemed conservative for the water scrubber performance evaluation and does not affect the performance of the metal filter. Therefore, this approach is also considered to be conservative in the evaluation of the particle removal performance of the filtering device.

However, in the event of a severe accident, it is conceivable that radioactive materials dissolve in the scrubber water, and that some of them are suspended by entrainment in liquid droplets downstream of the water scrubber. The metal filter also serves as a demister that traps these liquid droplets and returns them to the water scrubber to prevent them from scattering farther downstream. We confirmed this performance in our test.

Although the basic configuration of the test device was the same as that used to measure the particulate material removal performance (as shown in Figure 6), we used clean air rather than aerosols in this test. In addition to the two particle measurement points shown in Figure 6, we

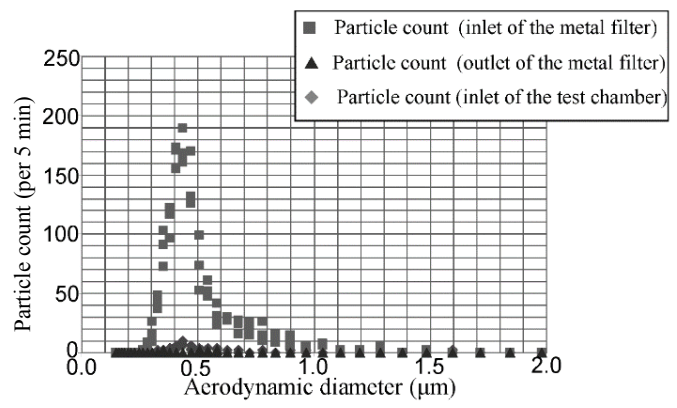

Figure 12 Comparison of the amount of entrained droplets between the inlet and the outlet of the metal filter (a simulated venting flow rate of $33,000 \mathrm{~m}^{3} / \mathrm{h}$ at twice the maximum containment design pressure)

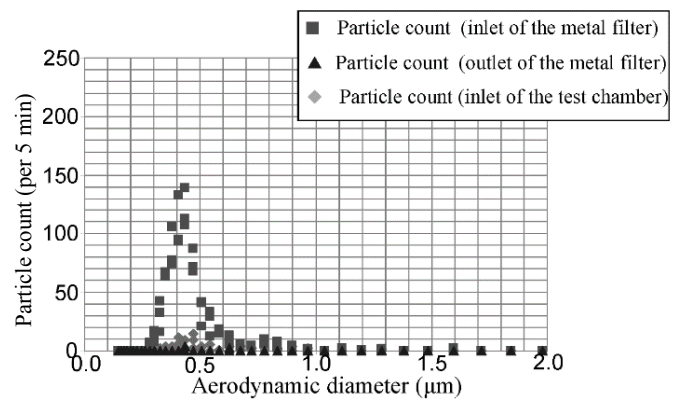

Figure 13 Comparison of the amount of entrained droplets between the inlet and the outlet of the metal filter (a simulated venting flow rate of $13,500 \mathrm{~m}^{3} / \mathrm{h}$ ) 
added a third point near the entrance of the metal filters for liquid droplet measurement. Thus, we confirmed the liquid droplet removal performance of the metal filter by comparing the numbers and sizes of liquid droplets at the entrance and exit of the metal filters.

Figures 12 and 13 show the test results under the maximum and minimum flow rate conditions. Under both conditions, we confirmed that almost no liquid droplets were detected at the exit of the metal filter, and that the filter is effective in preventing the dissolved radioactive materials from being released into the environment by entrainment.

\section{Conclusions}

Based on the lessons learned from the accident that occurred at the Fukushima Daiichi Nuclear Power Plant, enhanced protection of safety functions against external events and enhanced measures to prevent core damage have been sought. Considering a severe accident where a reactor core is damaged, we developed a filtered containment venting system. This system is deemed capable of protecting a containment vessel from overpressure failure in the event of a severe accident and of reducing emissions of particulate radioactive materials into the environment to $1 / 1,000$ or less. It was radioactive cesium that caused long-term and widespread environmental impacts from the accident that occurred at the Fukushima Daiichi Nuclear Power Plant, and cesium is emitted mainly as a particulate material. In addition, radioactive iodine is emitted mostly as particulate cesium iodide. Therefore, this system is expected to mitigate the environmental impact significantly in the event of a severe accident.

To ensure high removal performance in the filtered venting, the performance of the scrubbing section and the removal of liquid droplet entrainment containing the radioactive materials in the gaseous phase after scrubbing are important. Therefore, in developing the filtered containment venting system, we sought ways to improve the removal performance significantly by adding innovations such as combination of the scrubber nozzle with the air bubble subdividing device, water surface stabilization using the flow regulating plate located in the upper part of the scrubbing section, and removal of entrainment using metal filters.

In addition, since the aerosol removal characteristics are generally dependent on particle diameter and density, it is important to understand these characteristics in considering the procedures for selecting the mitigation and vent operation methods in the event of an accident. In this study, we confirmed the characteristics of DFs that are dependent on the particle diameter. In addition, in considering the characteristics of DFs based on particles with different densities, we confirmed that the aerodynamic diameter is an effective index. Since the characteristics of particulate materials, such as particle size distribution, vary with the stage of progress of an accident and the existence of a containment vessel spray, the insights gained from this study can contribute to plant management decisions for selecting mitigation and vent operation methods in the event of an accident and determining which method maximizes the release inhibition effect.

Furthermore, within the range of particle size distributions assumed present in a containment vessel after a severe accident, we concluded that inertial collision is a significant trapping mechanism in a filtering device, among several trapping mechanisms. In particular, for the scrubber, the DF is correlated with the particle diameters and the venting flow rates by the Stokes number. Moreover, we found that the removal performance of the scrubber was enhanced by inertial collision even when steam condensation no longer occurred due to the increase in water temperature caused by continuous venting. 


\section{References}

1) L. Soffer, S. Burson, C. Ferrell, R. Lee, J. Ridgely, Accident Source Terms for Light-water Nuclear Power Plants, U.S. Nuclear Regulatory Commission, NUREG-1465 (1995).

2) E. Beahm, C. Weber, T. Kress, G. Parker, Iodine Chemical Forms in LWR Severe Accidents, U.S. Nuclear Regulatory Commission, NUREG/CR-5732 (1992).

3) I. Wall, M. Merilo, Containment Filtration Systems Tests, Electric Power Research Institute, EPRI TR100346 (1992).

4) N. Losch, S. Buhlmann, C. Hutterer, C. Stiepani, F. Totsuka, T. Tsukahara, K. Hosomi, “AREVA's filtered containment venting system (FCVS)," Proc. 19th Power and energy technology symposium, Fukui, Japan, Jun. 26-27, 2014 (2014).

5) K. Okuyama, H. Masuda, S. Morooka, Biryuushi Kougaku, Oomu-sha, Tokyo, ISBN4-272-12900-4 (1992). [in Japanese]

6) K. Takahashi, Earozorugaku no Kiso, Morikita-syuppan, Tokyo, ISBN4-627-67251-9 (2003). [in Japanese]

7) H. Allelein, A. Auvinen, J. Ball, S. Guntay, L. Herranz, A. Hidaka, A. Jones, M. Kissane, D. Powers, G. Weber, State-of-the-art Report on Nuclear Aerosols, NEA/CSNI/R (2009) 5, Nuclear Energy Agency/ Committee on the safety of nuclear installations (2009).

8) D. Powers, S. Burson, A Simplified Model of Aerosol Removal by Containment Sprays, NUREG/CR5966, U.S. Nuclear Regulatory Commission (1993).

9) D. Powers, A Simplified Model of Decontamination by BWR Steam Suppression Pool, NUREG/CR6153, U.S. Nuclear Regulatory Commission (1997). 


\title{
Measures to Improve Robustness of Primary Containment Vessel and Operation of Filtered Containment Venting System Based on the Lessons from the Containment Leak at Fukushima Dai-ichi Nuclear Power Station Unit 2
}

\author{
Shinichi KAWAMURA ${ }^{1, *}$, Suguru OOKI ${ }^{2}$ and Tadashi NARABAYASHI ${ }^{3}$ \\ ${ }^{1}$ Nuclear Asset Management Department, Tokyo Electric Power Company, 1-1-3 Uchisaiwai-cho, Chiyoda-ku, \\ Tokyo 100-8560, Japan \\ ${ }^{2} R \& D$ Department, TEPCO Research Institute, Tokyo Electric Power Company, 4-1 Egasaki-cho, Tsurumi-ku, \\ Yokohama 230-8510, Japan \\ ${ }^{3}$ Division of Energy and Environmental Systems, Graduate School of Engineering, Hokkaido University, Kita-13, Nishi-8, Kita-ku, \\ Sapporo 060-8628, Japan
}

\begin{abstract}
Significant leakage of the primary containment vessel (PCV) occurred on March 15, 2011 at Unit 2 of the Fukushima Dai-ichi Nuclear Power Station, causing land contamination over a large area. From the radiation dose rate map of Unit 2 and the temperature trend of the PCV, degradation of the PCV top head flange gasket was identified as the cause of the leakage. The design set point of the rapture disc and operability of the valves in the hardened containment venting system were further identified as factors contributing to the leakage. Based on the lessons from the leakage, the gasket material was improved to have greater heat resistance in a steam environment, the PCV cooling system was diversified to improve reliability, and the operability and operation methods of the PCV venting were improved for greater robustness of the PCV in severe accidents.
\end{abstract}

KEYWORDS: accident, analysis, accident management, nuclear reactor, primary containment vessel, filtered containment venting system, gasket, Fukushima Dai-ichi, The Great East Japan Earthquake

\section{Introduction}

During the accident that occurred at Fukushima Dai-ichi Nuclear Power Station (hereinafter referred to as "Fukushima Dai-ichi") on March 11, 2011, a large amount of radioactive material was released into the air with serious consequences on the surrounding environment. Based on the investigation and analysis conducted thus far, which compared the results of monitoring

\footnotetext{
* Corresponding author, E-mail: shinichi.kawamura@tepco.co.jp

DOI : 10.15669/fukushimainsights.Vol.4.455

(C) 2021 Atomic Energy Society of Japan. All rights reserved.

Originally published in Transactions of the Atomic Energy Society of Japan (ISSN 1347-2879), Vol. 15, No. 2, p.53-65

(2016) in Japanese. (Japanese version accepted: November 6, 2015)
} 
the radiation outside the plant with the timing of the events that occurred in the plant, it is said that the Unit 2 reactor released the largest amount of radioactive material into the atmosphere of Units 1, 2 and 3, where core melting occurred. The Unit 2 reactor was the cause of the high level of contamination that occurred in the areas northwest of Fukushima Dai-ichi, particularly the village of Iitate ${ }^{1)}$.

In Unit 2, depressurization of the primary containment vessel (hereinafter referred to as "PCV") through venting failed. Although it is believed the leakage was a result of loss of the containment function of the PCV, the location and cause of the leakage have yet to be identified.

In addition, since PCVs are expected to maintain their containment function under conditions up to double the designed maximum working pressure and $200^{\circ} \mathrm{C}$, this is regarded as the precondition that must be taken into account by all domestic nuclear power stations when formulating severe accident measures. However, the maximum PCV pressure measured in Unit 2 was lower than this condition.

The new regulatory standards require nuclear power stations to install a PCV pressure relief system equipped with a radioactive material emissions reduction function called a filtered containment venting system (FCVS). However, for an FCVS to function effectively, a PCV needs to maintain its containment function. Otherwise, the radioactive materials have the potential to bypass the filter and be released. In addition, if the venting operation fails, as it did in Unit 2, it is possible that the containment function of the PCV may be lost before operating the vent.

Therefore, it is important to clarify the location and cause of the leaks from the PCV of Unit 2 when considering measures to operate the FCVS effectively and mitigate the consequences of an accident. In this article, we consider loss of the containment function in Unit 2 and discuss improvement measures based on our findings.

\section{Analysis of the Leakage from the PCV in Unit 2 of Fukushima Dai-ichi}

\section{Loss of the Containment Function of the PCV of Unit 2}

The actual measured pressure of the PCV of Unit 2, and the PCV pressure and temperature evaluated using MAAP code were published by the Tokyo Electric Power Company ${ }^{1)}$, as shown in Figure 1 and Figure 2.

The measured dry well pressure value of Unit 2 increased rapidly at around 22:40 on March 14 and then decreased significantly between 7:00 and 11:00 on March 15. The air dose rate near the main gate of Fukushima Dai-ichi increased rapidly from approximately $0.1 \mathrm{mSv} / \mathrm{h}$ to $10 \mathrm{mSv} / \mathrm{h}$ around the same time, but PCV venting was not conducted in Units 1 and 3 during that time. Therefore, it is believed that the PCV of Unit 2 lost its containment function around that time.

On the afternoon of March 14, the measured pressure values of the suppression chamber and dry well became significantly separated from each other and the measured pressure value of the suppression chamber decreased. Since both sections are connected via vent pipes, such separation does not occur as an actual phenomenon and is considered to be a result of a failure of the pressure indication of the suppression chamber.

The maximum pressure in the PCV measured before loss of the PCV function was approximately $0.75 \mathrm{MPa}$ [abs] and did not reach $0.95 \mathrm{MPa}$ [abs], the pressure limit for maintaining function (twice the maximum working pressure) assumed before the accident. In addition, the 


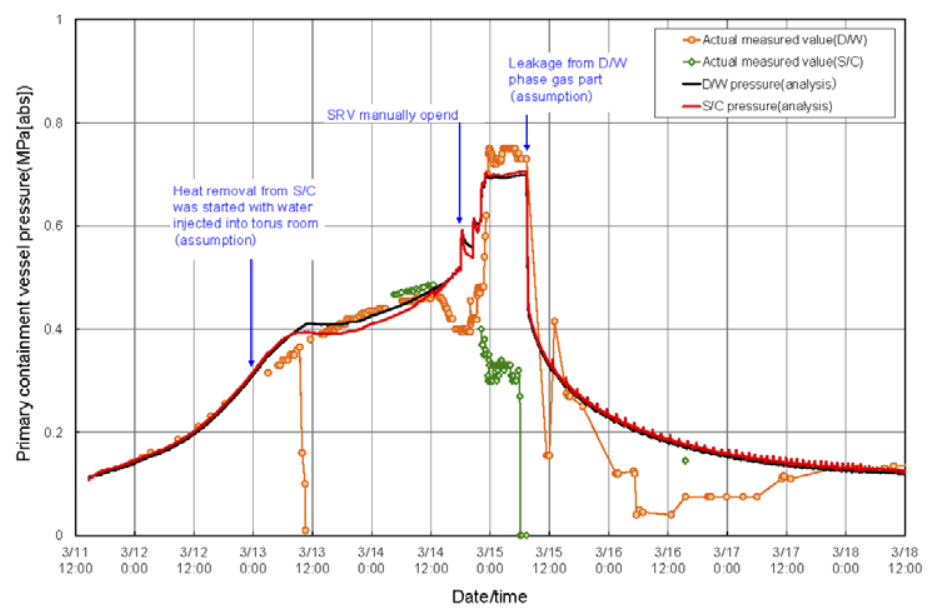

Figure 1 Pressure trend of the PCV at Fukushima Dai-ichi Unit $2^{1)}$

$\mathrm{D} / \mathrm{W}$ and S/C refer to the "dry well" and "suppression chamber" of the PCV respectively. SRV refers to the "safety relief valve."

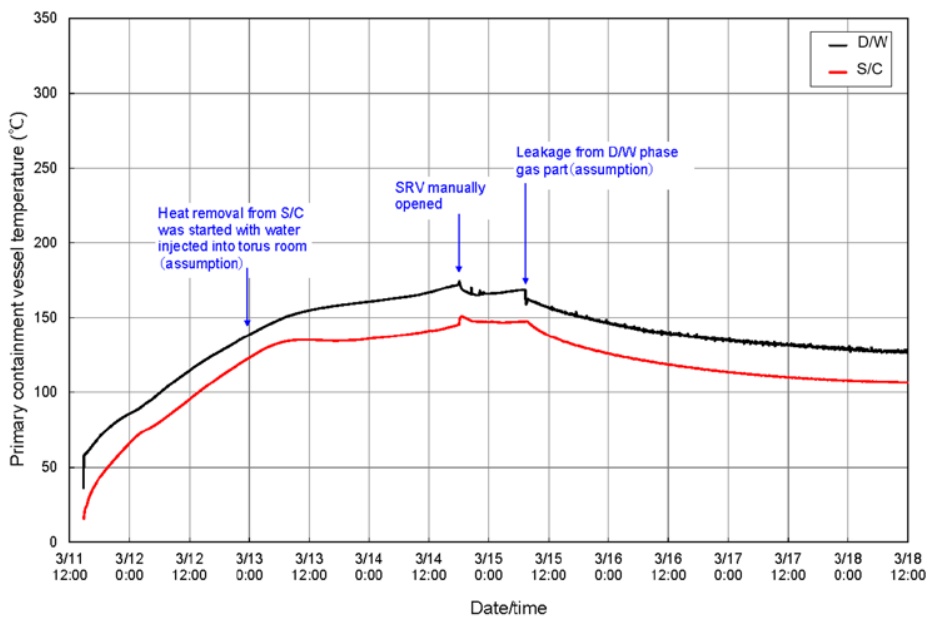

Figure 2 Temperature trend of the PCV at Fukushima Dai-ichi Unit 21) $\mathrm{D} / \mathrm{W}$ and S/C refer to the "dry well" and "suppression chamber" of the PCV respectively. SRV refers to the "safety relief valve."

maximum analytical value of the temperature in the PCV evaluated using MAAP code before loss of the containment function was approximately $175^{\circ} \mathrm{C}$ and did not reach $200^{\circ} \mathrm{C}$, the assumed temperature limit for maintaining function before the accident.

\section{Location of the Leakage that Caused Loss of the Containment Function of the PCV}

As mentioned above, loss of the containment function of the PCV of Unit 2 occurred between 7:00 and 11:00 on March 15. Figure 3 shows a photograph of Unit 2 taken on March 15. This photograph shows the steam discharged from the opening of a blow-out panel on the top 
floor of the reactor building. Figures 4-9 show the air radiation dose distributions measured in the Unit 2 reactor building after the accident. The dose rate distribution on the top floor of the reactor building (5th floor) is shown in Figure 4; the dose rate is high near the opening of the blow-out panel where the discharge of steam was observed, as shown in Figure 3. Therefore, it is estimated that a large amount of the radioactive material that leaked from the PCV due to loss of the containment function was released into the atmosphere from the opening of the blow-out panel.

In addition, according to the dose rate distribution on the 5th floor shown in Figure 4, the

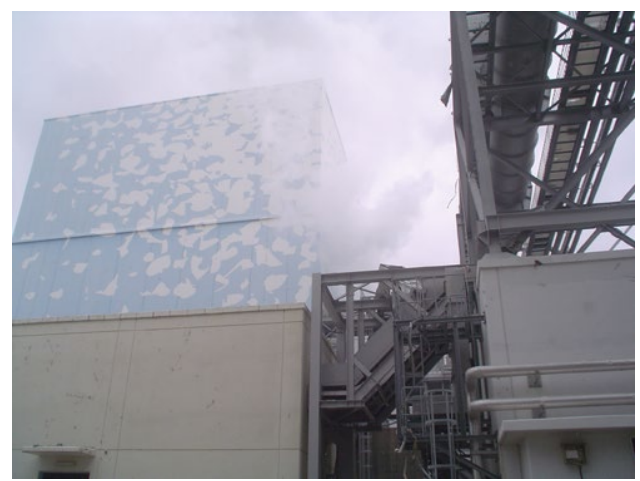

Figure 3 Steam coming out of a blow-out panel opening at the Fukushima Dai-ichi Unit 2 Reactor Building on March 15, 2011

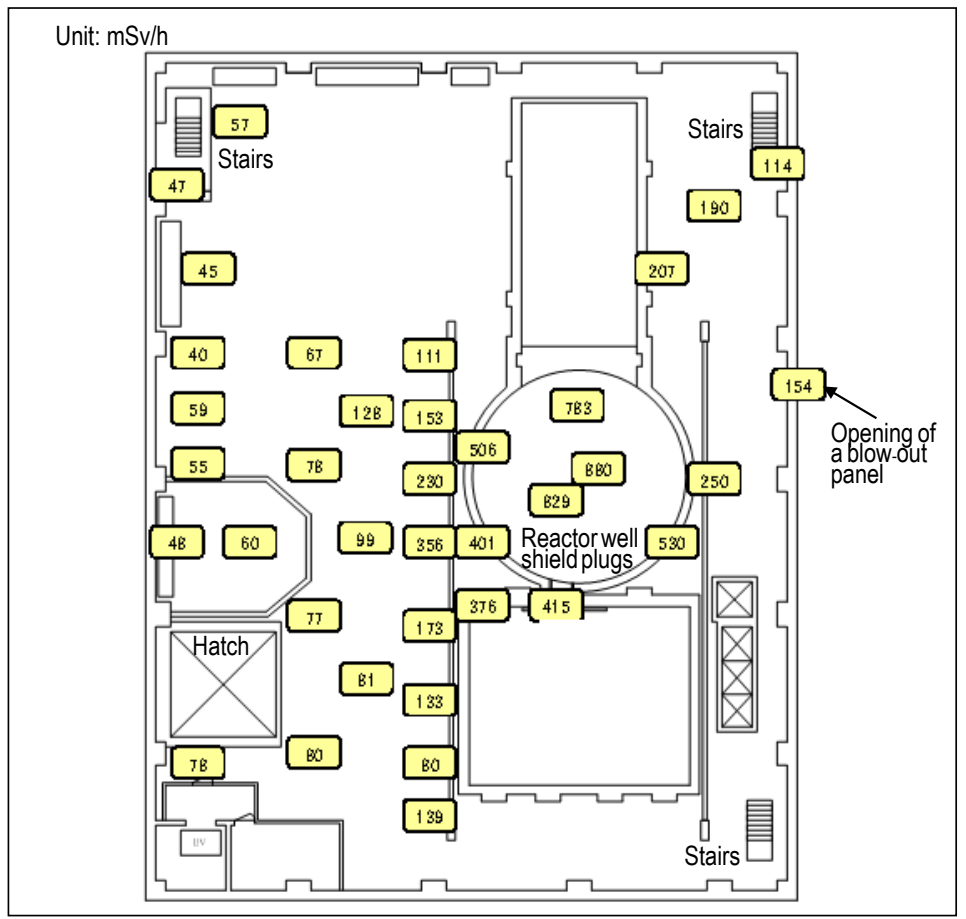

Figure 4 Spatial radiation dose rate on the 5th floor of the Fukushima Daiichi Unit 2 Reactor Building 


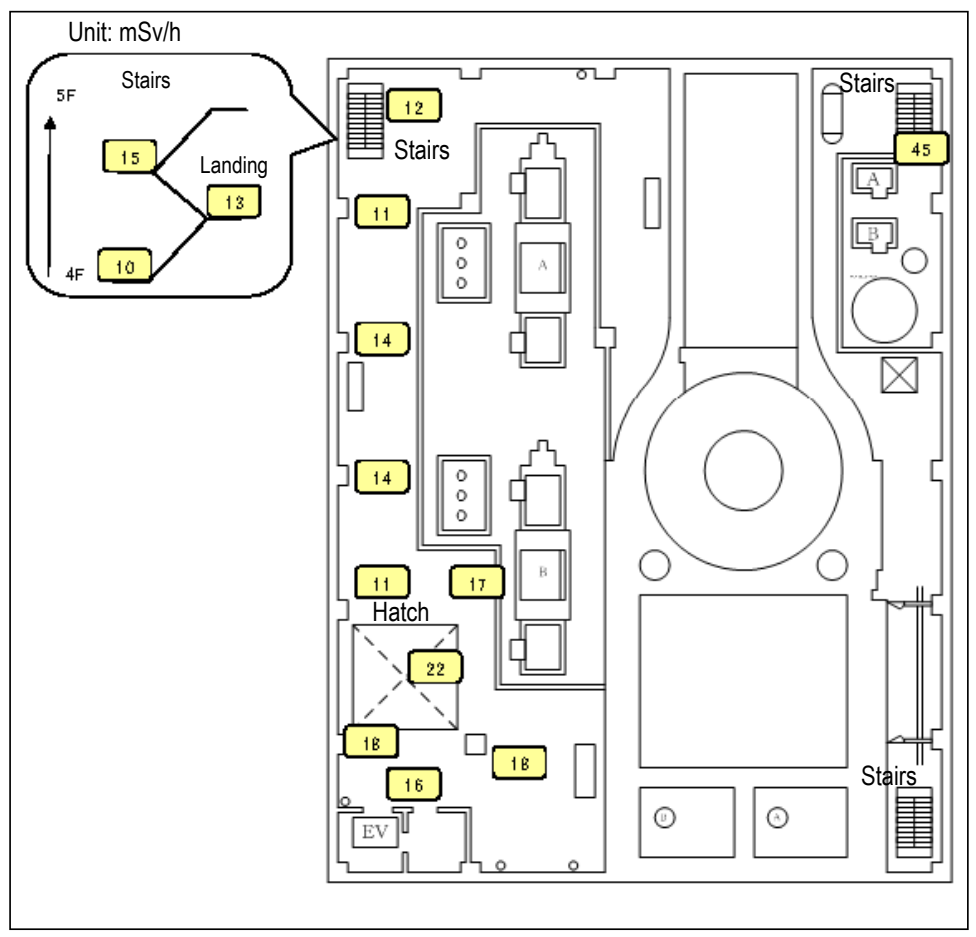

Figure 5 Spatial radiation dose rate on the 4th floor of the Fukushima Daiichi Unit 2 Reactor Building

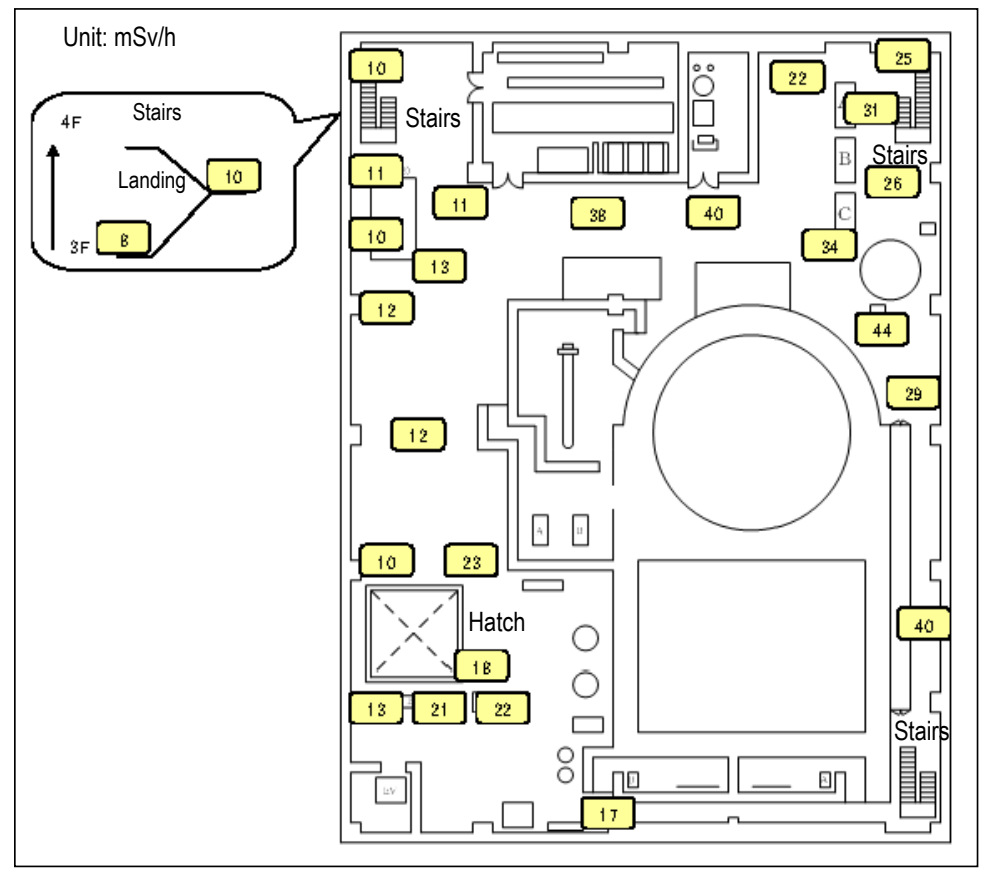

Figure 6 Spatial radiation dose rate on the 3rd floor of the Fukushima Daiichi Unit 2 Reactor Building 


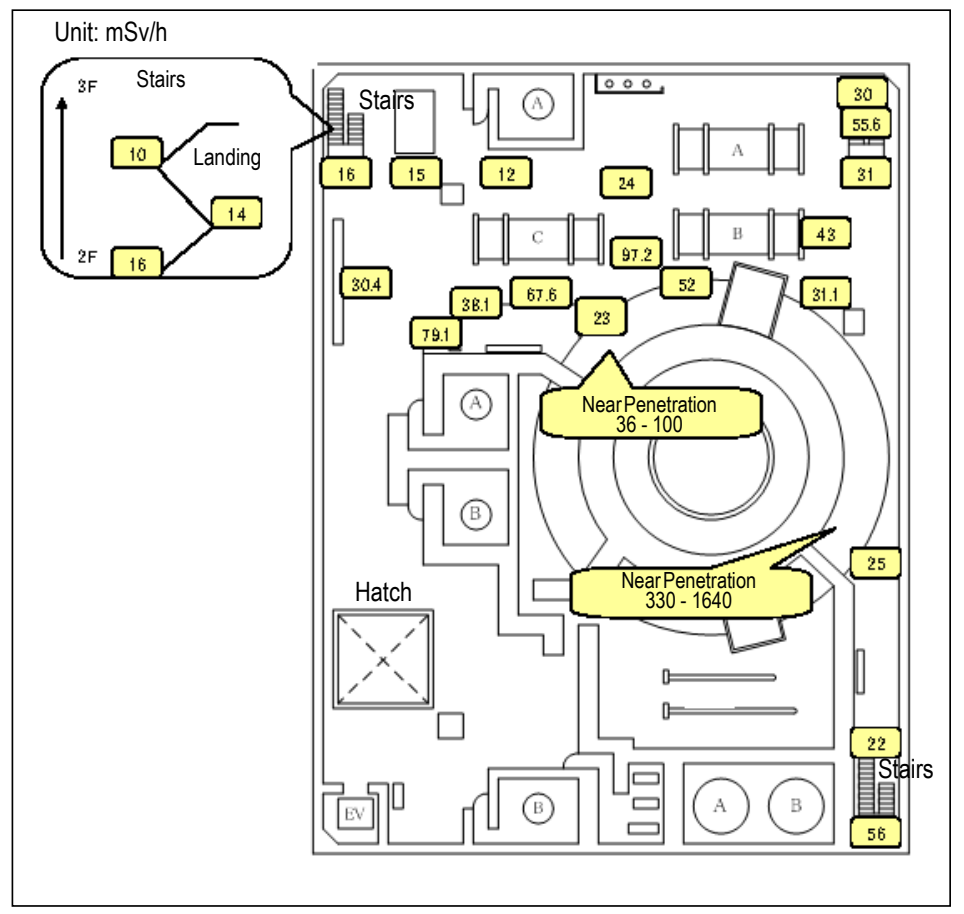

Figure 7 Spatial radiation dose rate on the 2nd floor of the Fukushima Dai-ichi Unit 2 Reactor Building

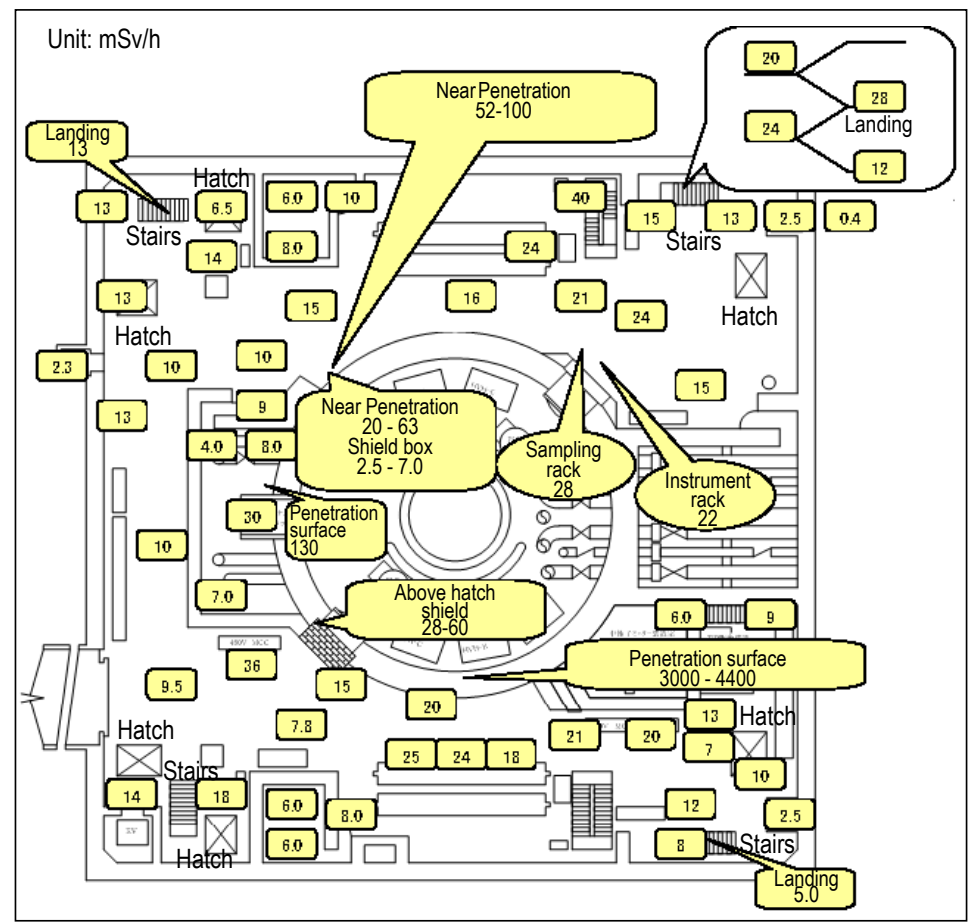

Figure 8 Spatial radiation dose rate on the 1st floor of the Fukushima Daiichi Unit 2 Reactor Building 


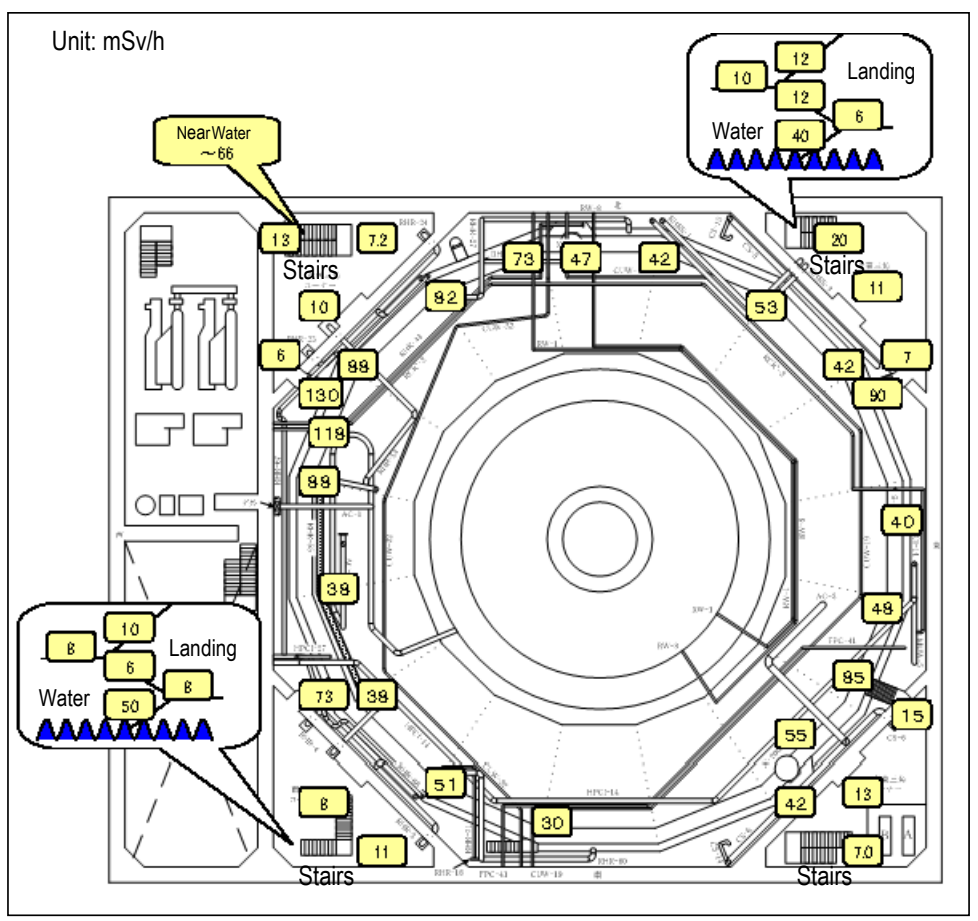

Figure 9 Spatial radiation dose rate in the middle basement of the Fukushima Dai-ichi Unit 2 reactor building

dose rates measured above the shield plug of the reactor well were higher than those measured in the surrounding area. On the other hand, the dose rates measured at the stairs connecting the 5th floor and the lower floor and near the hatch are lower than those measured above the shield plug. Furthermore, comparing the dose rates between the stairs on the 5th and 4th floors (Refer to Figure 5) and between the hatch on the 5th and 4th floor, they were higher on the 5th floor. Therefore, it seems unlikely that the leakage occurred on a lower floor and the radioactive materials reached the 5th floor through the stairs and the hatch opening and were then released into the atmosphere from the opening of the blow-out panel. Although the dose rate was not measured at the stairs shown on the lower right of the Figure, it is highly unlikely that the leakage occurred on a lower floor and the radioactive materials reached the 5th floor through the stairs because the stairs on the 4th and 3rd floors (Refer to Figure 6) passed between the shield wall of the spent fuel pool and the outer wall of the reactor building, creating no leakage path from the PCV. Additionally, the dose rate measured on the 2nd floor (Refer to Figure 7) was lower than that of the 5th floor. These investigation results suggest the possibility that a large amount of the radioactive material leaked from the PCV head flange directly beneath the shield plug of the reactor well.

Furthermore, Figures 8 and 9 show the dose rate distributions on the 1st floor and the middle basement, but the dose rates measured at the stairs and hatches were lower than those measured on the 5th floor, so it seems unlikely that they became advection paths for radioactive materials. Although high dose rates were measured near the PCV penetrations on these floors, the dose rates measured in the surrounding rooms and corridors were low. This is believed to be because of the influence of the radiation that transmitted from inside the PCV pressure boundary; however, significant leakage did not occur in these places. 
From the above, the leakage of radioactive materials into the atmosphere due to loss of the containment function of the PCV is considered to have occurred primarily from the top head of the PCV.

The water containing radioactive materials was retained in the triangular corner areas of the four corners of the reactor building shown in Figure 9. If a leakage occurred in the PCV or a system connected to the PCV such as the reactor core isolation cooling (RCIC) system in these areas, the containment function would have been lost, the PCV would depressurize, and the radioactive materials injected at high pressure would have spread high-concentration contamination to these triangular corner areas and stairs. However, the dose rates measured at those places were low, and the dose rates measured at the stairs increased with elevation. Therefore, the cause of the contamination of the retained water was distinct from the leakage that occurred when the containment function was lost - the contamination occurred after that.

The sealant for the top head flange of the PCV was made of silicone rubber. Silicone rubber is a material that can withstand relatively high temperatures because the binding energy of siloxane polymer, which is its basic structure, is greater than the carbon binding energy. However, in a high-temperature steam environment, siloxane polymer is hydrolyzed and disconnected; it is known that the performance of siloxane polymer decreases significantly at temperatures of $150^{\circ} \mathrm{C}$ or higher ${ }^{2}$. As Figure 2 shows, the dry well temperature exceeded $100^{\circ} \mathrm{C}$ on March 12 and was $150^{\circ} \mathrm{C}$ or higher on and after March 13. Therefore, it is believed that the deterioration of the silicone rubber occurred during that time. It withstood the increase in PCV pressure from 22:00 on March 14, but eventually lost its leakage resistance due to advanced deterioration.

\section{Venting Operation Problem}

It is possible that loss of the containment function of the PCV of Unit 2 could have been avoided if the pressure had been reduced by conducting containment venting before the top head flange sealant deteriorated or the PCV pressure increased rapidly in that state. However, this could not be achieved because the working venting pressure setting was high, and it was difficult to operate the venting valves.

Although Unit 2 was equipped with a hardened containment venting system as a measure to mitigate severe accidents, the set working pressure of the rupture disk incorporated into the venting system was $0.528 \mathrm{MPa}$ [abs] and was designed to allow venting at a higher pressure. This pressure was set according to the designed maximum working pressure of the PCV and was common to domestic plants before the accident occurred.

Instructions on venting preparation were issued by the head of the site emergency response organization (site superintendent) at 17:30 on March 12 when water injection into the reactor was continuously performed using the RCIC system. The site emergency response organization completed the operation for opening the valves required for venting by 11:00 on March 13 . However, as Figure 1 shows, the PCV pressure had not yet reached the set working pressure of the rupture disk at that moment, and the venting could not be started. Although the valves were kept open, it was confirmed on the site that the air-operated valve that had been kept open closed when the adjacent Unit 3 caused an explosion at 11:01 on March 14. After that, attempts were made to open the valve using a temporary air compressor, but the attempt to relieve the pressure in the PCV did not succeed, as shown in Figure 1.

Under these conditions, the PCV pressure increased rapidly at approximately 22:40 on March 14 and exceeded the set working pressure of the rupture disk for the first time.

This is believed to be because of a large amount of steam and hydrogen generated due to the water-zirconium reaction that occurred when seawater was injected by a fire engine. In 
Unit 2, water injection into the reactor was conducted using the RCIC system, but the loss of the reactor cooling function caused by the loss of the RCIC function was confirmed by the site emergency response organization at 13:25 on March 14. Because of this, the reactor water level decreased and reached the bottom of the active fuel around 18:10. It is believed that the water-zirconium reaction progressed rapidly because the fire engines were activated at 19:54 and 19:57 to inject seawater into the reactor ${ }^{1,3}$. The amount of hydrogen generated by this reaction, evaluated using the MAAP code, was approximately $460 \mathrm{~kg}$.

It could be also confirmed from the increase in the measurements of the containment atmospheric monitoring system (CAMS) that the core damage progressed after water injection into the reactor was resumed by a fire engine. Figure 10 shows the changes in the measurements of CAMS from 12:00 on March 14 to 0:00 on March 16. After water injection until around 0:00 on March 15, the measurements increased rapidly both in the dry well and the suppression chamber. This is believed to be a result of the rapid progress of the zirconium-water reaction due to water injection that caused the generation of a large amount of hydrogen and the release of fission products due to core damage. They were transferred to the suppression chamber via the main steam safety relief valves and to the dry well via the vacuum break valves. The reason why the indicated value of the suppression chamber is smaller than that of the dry well is because the CAMS on the suppression chamber side measure the dose rate outside the PCV.

Because the CAMS was unable to make measurements between 7:00 and 11:00 on March 15, when the PCV lost its containment function, the trend during that period is unknown. However, between 6:20 and 6:25, before measurement failure, the CAMS measurement of the dry well decreased rapidly from $62.7 \mathrm{~Sv} / \mathrm{h}$ to $43.0 \mathrm{~Sv} / \mathrm{h}$; it may have captured loss of the containment function in the dry well as well as the leakage into the reactor building, which is consistent with the above estimation that the containment function was lost due to leakage from the top head of the PCV. The CAMS measurement recorded its highest value at 16:10 on March 15, possibly due to the continuous progress of core damage that resulted in the relocation of the damaged core and breakage of the reactor.

As described above, it is considered that the containment function was lost due to leakage from the top head of the PCV because venting could not be conducted before a rapid increase in the PCV pressure due to the problem of the containment venting pressure setting and the operability of the valve required for venting, and because the sealant was deteriorated.

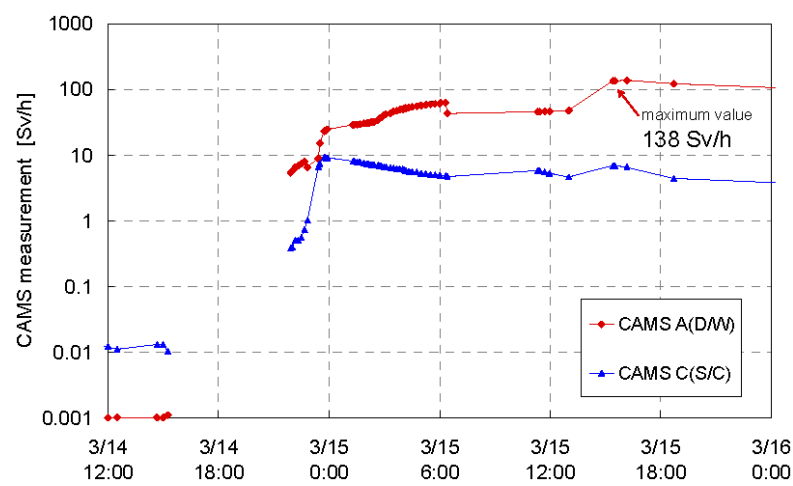

Figure 10 CAMS of the Fukushima Dai-ichi Unit 2 from March 14 to March 16, 2011

$\mathrm{D} / \mathrm{W}$ refers to the "dry well" and S/C refers to the "suppression chamber" of the PCV. 


\section{Mitigation of the Consequences after the PCV Lost its Containment Function}

Although the cause of loss of the containment function of the PCV of Unit 2 was discussed in previous sections, it is useful from the view point of resilience to consider whether the subsequent response was effective in mitigating the consequences at the time of the severe accident.

As Figure 11 shows, the CAMS measurement in Unit 2 decreased monotonically after it reached its peak at 16:10 on March 15. This indicates that continuous water injection cooled the fuel debris, which led to the suppression of the additional release of fission products.

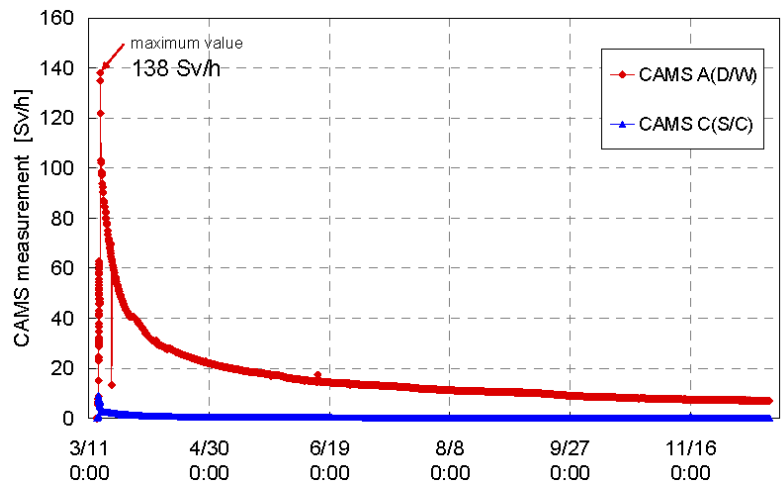

Figure 11 CAMS of the Fukushima Dai-ichi Unit 2 from March to November 2011

$\mathrm{D} / \mathrm{W}$ refers to the "dry well" and S/C refers to the "suppression chamber" of the PCV.

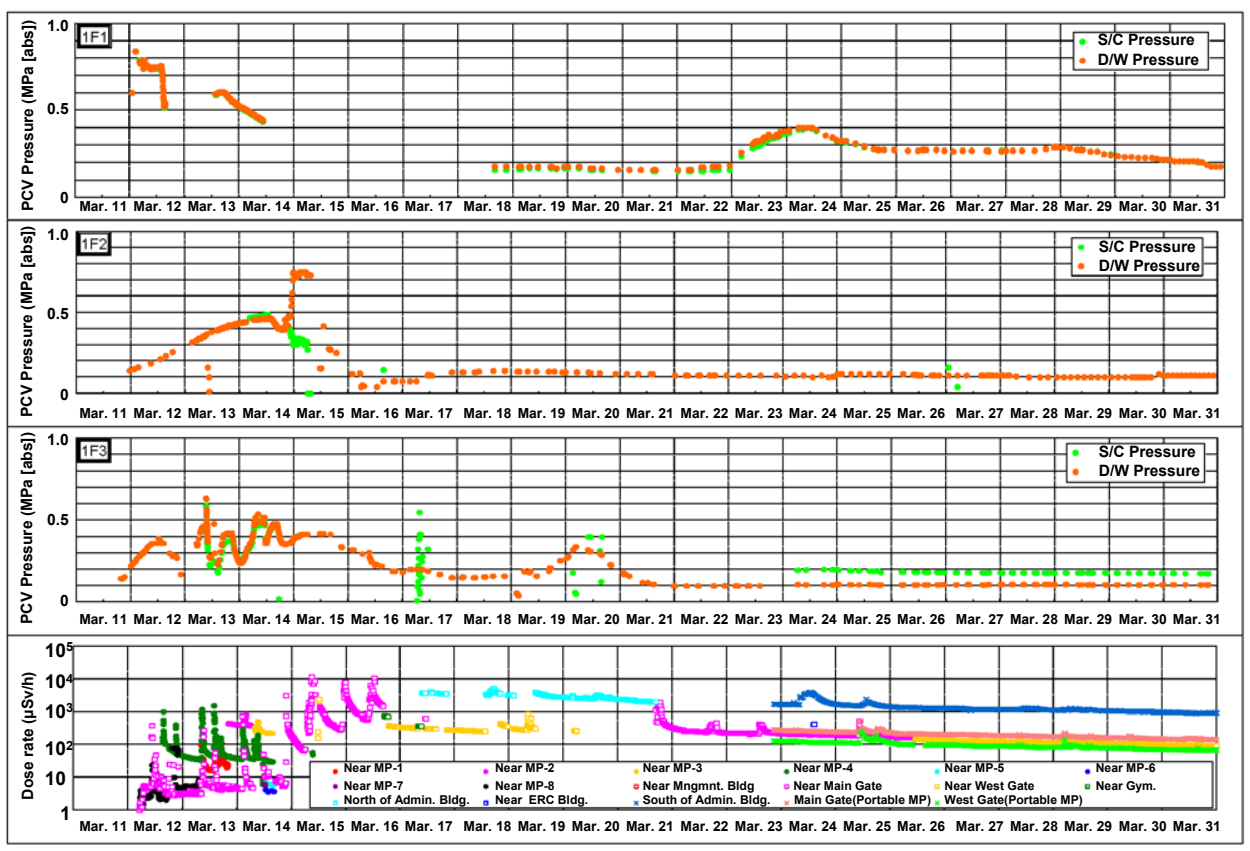

Figure 12 The PCV pressures of Units 1, 2 and $3(1 \mathrm{~F} 1,1 \mathrm{~F} 2$, and 1F3, respectively) as well as environmental radiation ${ }^{1)}$ 
The changes in the dose rate measured at the Fukushima Dai-ichi site and the changes in the PCV pressures of Units 1, 2 and 3 are as shown in Figure 12 ${ }^{1}$. It shows that continuous water injection reduced the dose rate almost monotonically on and after March 26 and the situation where a large amount of additional radioactive material could have been released into the atmosphere was avoided. In addition, the pressure in the PCV was stabilized through continuous water injection.

Therefore, continuing water injection to cool fuel debris and reduce the pressure in the PCV, even after it loses its containment function, is considered to be an effective mitigation measure.

\section{Improvement Based on the Lessons Learned from the Leakage from the PCV in Unit 2 of Fukushima Dai-ichi}

\section{Basic Policy for Improvement}

It was estimated above that the loss of the PCV's containment function in Unit 2 of Fukushima Dai-ichi occurred at the top head flange of the PCV and was caused by the deterioration of the gasket sealing material in a high-temperature steam environment and the increase in PCV pressure due to hydrogen and steam generated when water injection into the reactor was resumed. Thus, to maintain the radioactive material containment function of the PCV in a severe accident, it is important to systematically approach both temperature and pressure aspects in terms of the following:

- Mitigation of an increase in the PCV boundary temperature: Even in the situation where emergency systems have lost their function, using an alternative system, the increase in the PCV boundary temperature should be mitigated by spraying water in the PCV, injecting water immediately beneath the reactor to cool the fuel debris, and conducting water cooling of the outer surface of the top head of the PCV.

- Improvement of the heat resistance performance of the PCV boundary: Improve the sealant that constitutes part of the PCV boundary to enhance the leakage-resistance performance under a high-temperature steam environment.

- Prevention of a rapid increase in PCV pressure and an over-pressure exceeding the pressure limit: Enable the implementation of containment venting without fail before a rapid increase in pressure or an over-pressure occurs.

Of the above approaches, except for the cooling of the outer surface of the top head of the PCV by water injection, spray and water injection using an alternative system had been positioned as accident management measures before the accident at Fukushima Dai-ichi. Since the accident, further improvements, such as the addition of alternative power sources using gas turbine generators and the enhancement of the reliability in the operation of fire engines, have already been implemented.

The effectiveness of cooling a PCV using an alternative system in the event of a severe accident has been proven through an actual case. The increases in the temperature and pressure in the PCV were successfully mitigated to some extent in Units 1, 2 and 4 of the Fukushima Daini Nuclear Power Station by conducting alternative PCV spraying using make-up water condensate systems (MUWC) in the emergency situation after the Great East Japan Earthquake. In addition, enhancing the reliability of water injection in the event of a severe accident is important not only for maintaining the containment function of the PCV but also acts as a resilience measure in the event of PCV leakage, as discussed above. 
Since these measures have already been implemented, the following sections will examine methods for improving the sealant and the operation of containment venting - two important tasks to work on.

\section{Improvement of the Sealant}

\section{(1) Changing the sealing material}

For the purpose of maintaining leakage resistance performance under a high-temperature steam condition in the event of a severe accident, the material of the gasket used for the top head flange of the PCV was changed from conventional silicone rubber to improved ethylene-propylene-diene rubber (improved EPDM) ${ }^{4-6}$. The material possesses superior steam and radiation resistance and exhibited no deterioration-related behavior at the iodine concentration assumed to be present in a severe accident. Since the flange gasket of the hatches and the valve seals of the PCV were also made of silicone rubber, it was determined that improved EPDM should be utilized for these as well.

Furthermore, a backup sealing material (silicone-based one-component fire-resistant sealant) was added to the top head flange and the hatches ${ }^{4,7}$. This sealing material was applied to the flange surface located outside the gasket mounting groove. Although the leakage resistance performance was maintained by the gasket while a pressure higher than the maximum working pressure was applied to the PCV in a severe accident, the backup sealing material is intended to enhance the long-term maintenance of leakage resistance performance after the PCV pressure has been decreased through containment venting, etc. Even if the flange remains at high temperatures for a long time after the accident, the backup sealing material can maintain its leakage resistance performance independently and is also expected to reduce oxidation degradation of the improved EPDM gasket by blocking external air.

Figure 13 presents a diagram of the conceptual layout of the sealants in the top head flange of the PCV. They are arranged in the same way in the hatch flanges.

\section{(2) Environmental qualification test for the sealants made of improved EPDM}

We conducted a test to determine if the improved EPDM sealants could maintain their performance in the environment created by a severe accident.

In this test, a sealing material exposed to $\gamma$-ray irradiation of $800 \mathrm{kGy}$ was mounted on a simulated flange, exposed to a dry air environment at $200^{\circ} \mathrm{C}$ or a steam environment at $250^{\circ} \mathrm{C}$ for 168 hours, and then pressurized up to $0.9 \mathrm{MPa}$ [gage] with helium to check if airtightness was maintained. The irradiation dose was determined considering the accumulated radiation dose for seven days after the accident caused by the combined event of loss of coolant (large-break LOCA), station blackout, and a total loss of emergency core cooling system functions - a typical severe accident sequence which leads to over-pressure and over-temperature of the PCV in a boiling water reactor (BWR) plant. In addition, the high-temperature exposure condition was set

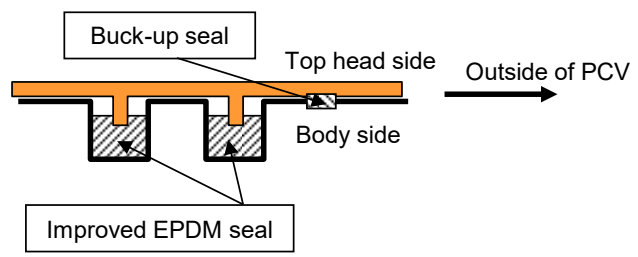

Figure 13 Seal arrangement of the PCV top head flange 
to exceed $200^{\circ} \mathrm{C}$, which was the limit temperature for the PCV leading to containment venting.

In addition to the steam environment to which the sealants are exposed in the event of an accident, they were also exposed to high temperatures generated by dry air which have the potential to lead to deterioration through an oxidation reaction.

Figure 14 shows the external appearance of the simulated flange used for the test. The seal groove of the simulated flange and the section size of the mounted sealing material were half those of the actual system. In addition, a step was provided inside the simulated flange to simulate a situation where the PCV is pressurized and the flange is opened in the event of an accident, and the sealing material was exposed to high-temperature steam while mounted on the flange. After it was exposed to a predetermined environment in the autoclave, it was pressurized by helium to check its airtightness .

In the event of an accident, the sealing material is exposed to radiation while it is mounted on the flange. In this test, post-radiation sealing material was mounted on the flange and controlled so that face-to-face dimension at the sealing material mounting groove could simulate the actual system when the flange was tightened. In this way, it is possible to determine the airtightness of the actual system because the tightening condition could be controlled without being influenced by a change in the physical properties of the sealing material due to irradiation.

In addition, in the case of domestic BWR plants where the PCV is filled with nitrogen gas, the PCV is pressurized with a gas mixture consisting mainly of nitrogen, hydrogen, and water vapor in the event of a severe accident. In this test, we used helium, which has a lower molecular weight than the average molecular weight of this mixture gas and a small molecular radius in terms of hydrogen simulation. This enabled us to easily maintain safety during the experiment. The technical standard for automobile fuel systems fueled by compressed hydrogen gas (Notice Defining the Details of the Safety Standards for Road Transport Vehicles (Attachment 100) ${ }^{8)}$ requires that the helium leak rate should be multiplied by 1.33 to estimate the hydrogen leak rate. On the other hand, the helium leak rate is multiplied by 1.41 to correct the leak rate by raising the gas constant ratio to the 0.5 th power according to the guides stipulated by U.S. Atomic Energy Commission governing the evaluation of the leak rate of PCVs ${ }^{9}$. In this test, by using an extremely low leak rate condition with no pressure decrease during airtightness testing and no bubble generation with bubble solution as the judgment criterion, we found that adaptability to the actual system could be determined using helium gas without any problems.

Table 1 shows the results of the airtightness test. After pressurized to $0.3 \mathrm{MPa}$ [gage], the test system was retained for ten minutes. Then, it was pressurized again and retained for thirty minutes after reaching at $0.65 \mathrm{MPa}$ [gage] and at $0.9 \mathrm{MPa}$ [gage]. During each retention period, no pressure drop was observed. In addition, no bubble generation was observed in the leakage check using bubble solution and superior airtightness was confirmed. Furthermore, we also conducted a helium airtightness test in the same way with the flange test piece after opening

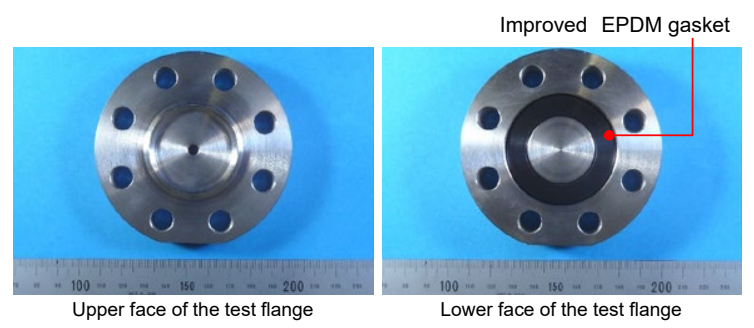

Figure 14 Test flange and improved EPDM gasket 
Table 1 Air tightness test of the improved EPDM gasket using helium after radiation exposure of $800 \mathrm{kGy}$ and thermal exposure

\begin{tabular}{ccccc}
\hline $\begin{array}{c}\text { Thermal } \\
\text { exposure }\end{array}$ & $\begin{array}{c}\text { Flange } \\
\text { opening }\end{array}$ & \multicolumn{3}{c}{ Test pressure } \\
$0.3 \mathrm{MPa}$ & $0.65 \mathrm{MPa}$ & $0.9 \mathrm{MPa}$ \\
\hline $\begin{array}{c}\text { Dry air } 200^{\circ} \mathrm{C} \\
168 \text { hours }\end{array}$ & $0.8 \mathrm{~mm}$ & $\begin{array}{c}\text { No } \\
\text { leakage }\end{array}$ & $\begin{array}{c}\text { No } \\
\text { leakage }\end{array}$ & $\begin{array}{c}\text { No } \\
\text { leakage }\end{array}$ \\
\hline $\begin{array}{c}\text { Steam } 250^{\circ} \mathrm{C} \\
168 \text { hours }\end{array}$ & $0.8 \mathrm{~mm}$ & $\begin{array}{c}\text { No } \\
\text { leakage }\end{array}$ & $\begin{array}{c}\text { No } \\
\text { leakage }\end{array}$ & $\begin{array}{c}\text { No } \\
\text { leakage }\end{array}$ \\
\hline
\end{tabular}

it by $0.8 \mathrm{~mm}$ using a jig after exposure to high temperature, considering that the flange opens when it is pressurized in the event of an accident. Since the section sizes of the simulated flange and gasket used in this test are half of those used in an actual system, an opening of $0.8 \mathrm{~mm}$ in the test system corresponded to the $1.6 \mathrm{~mm}$ opening in an actual system. In the case of a PCV in an advanced BWR (ABWR) plant, the flange of the equipment hatch has the largest opening displacement when the pressure doubles the maximum working pressure in the event of an accident. Although the displacement becomes larger on the inner gasket side than on the outer gasket side, it was approximately $1.4 \mathrm{~mm}$ on the inner gasket side, which is within the range of the opening condition set in this test. No leakage was observed in this test and it was confirmed that airtightness was maintained through the resiliency of the sealing material (which was compressed in advance and installed) even if an opening was created when the PCV was pressurized at a pressure double the maximum working pressure.

In addition, as a result of checking the external appearance of the sealing material after the test, no abnormalities such as cracks were observed on the surface.

In the case of a severe accident in an actual plant, measures are taken to decrease the temperature and pressure of the PCV by conducting containment venting or alternative recirculation cooling within a few days following the accident (less than a week). From the above test result, it is evident that the sealing material made of improved EPDM can maintain its leakage resistance performance under a severe accident environment, at least until then.

\section{(3) The environmental qualification test of the backup sealing material}

We also conducted environmental qualification test of the backup sealing material. In this test, the simulated flange to which the backup sealing material was applied was irradiated with $\gamma$-rays of $800 \mathrm{kGy}$ or higher to simulate the accumulated radiation dose seven days after an accident, and it was exposed to a steam environment of $250^{\circ} \mathrm{C}$ for 168 hours. Then, it was pressurized up to $0.9 \mathrm{MPa}$ with helium to check airtightness. Figure 15 shows the condition of the airtightness test and Table 2 shows the test results. In an actual system, the backup sealing material is used in combination with a gasket made of improved EPDM. However, in this test, only the sealing material was applied, and the gasket was not mounted to confirm the performance. The retention times for each pressure in the airtightness test were ten minutes at $0.3 \mathrm{MPa}$ and thirty minutes at $0.65 \mathrm{MPa}$ and $0.9 \mathrm{MPa}$. During each retention period, no bubble generation was observed in the leakage check using bubble solution and no pressure drop was observed. And the results demonstrated superior airtightness.

Next, in terms of maintaining the leakage resistance performance for long periods following an accident, we conducted long-term, high-temperature exposure at a temperature of $200^{\circ} \mathrm{C}$ with a combination of a gasket made of improved EPDM (irradiated with $\gamma$-rays of $800 \mathrm{kGy}$ ) and backup sealing material. After exposure, the test system was pressurized with helium and retained for ten minutes at $0.3 \mathrm{MPa}$ and thirty minutes at $0.65 \mathrm{MPa}$ and $0.9 \mathrm{MPa}$ to confirm the airtightness performance. Table 3 shows the test results. Under these conditions, no bubble 


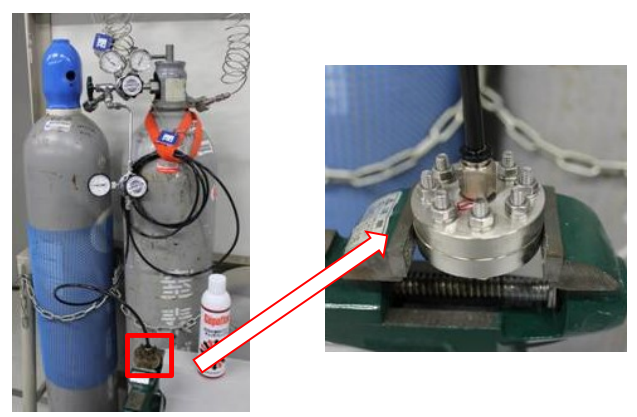

Figure 15 Air tightness test of back-up seal using helium

Table 2 Air tightness test of back-up seal using helium after radiation exposure of $819 \mathrm{kGy}$ and thermal exposure

\begin{tabular}{ccccc}
\hline $\begin{array}{c}\text { Thermal } \\
\text { exposure }\end{array}$ & $\begin{array}{c}\text { Flange } \\
\text { opening }\end{array}$ & \multicolumn{3}{c}{ Test pressure } \\
$0.3 \mathrm{MPa}$ & $0.65 \mathrm{MPa}$ & $0.9 \mathrm{MPa}$ \\
\hline $\begin{array}{c}\text { Steam } 250^{\circ} \mathrm{C} \\
168 \text { hours }\end{array}$ & $0 \mathrm{~mm}$ & $\begin{array}{c}\text { No } \\
\text { leakage }\end{array}$ & $\begin{array}{c}\text { No } \\
\text { leakage }\end{array}$ & $\begin{array}{c}\text { No } \\
\text { leakage }\end{array}$ \\
\hline
\end{tabular}

Table 3 Air tightness test of the improved EPDM gaskets and back-up seals using helium after extended periods of thermal exposure

\begin{tabular}{ccccc}
\hline $\begin{array}{c}\text { Thermal } \\
\text { exposure }\end{array}$ & $\begin{array}{c}\text { Flange } \\
\text { opening }\end{array}$ & \multicolumn{3}{c}{ Test pressure } \\
\hline $\begin{array}{c}\text { Dry air } 200^{\circ} \mathrm{C} \\
720 \text { hours }\end{array}$ & $0 \mathrm{~mm}$ & $\begin{array}{c}\text { No } \\
\text { leakage }\end{array}$ & $\begin{array}{c}\text { No } \\
\text { leakage }\end{array}$ & $\begin{array}{c}\text { No } \\
\text { leakage }\end{array}$ \\
\hline $\begin{array}{c}\text { Dry air } 200^{\circ} \mathrm{C} \\
1,080 \text { hours }\end{array}$ & $0 \mathrm{~mm}$ & $\begin{array}{c}\text { No } \\
\text { leakage }\end{array}$ & $\begin{array}{c}\text { No } \\
\text { leakage }\end{array}$ & $\begin{array}{c}\text { No } \\
\text { leakage }\end{array}$ \\
\hline $\begin{array}{c}\text { Dry air } 200^{\circ} \mathrm{C} \\
1,440 \text { hours }\end{array}$ & $0 \mathrm{~mm}$ & $\begin{array}{c}\text { No } \\
\text { leakage }\end{array}$ & $\begin{array}{c}\text { No } \\
\text { leakage }\end{array}$ & $\begin{array}{c}\text { No } \\
\text { leakage }\end{array}$ \\
\hline $\begin{array}{c}\text { Dry air } 200^{\circ} \mathrm{C} \\
1,800 \text { hours }\end{array}$ & $0 \mathrm{~mm}$ & $\begin{array}{c}\text { No } \\
\text { leakage }\end{array}$ & $\begin{array}{c}\text { No } \\
\text { leakage }\end{array}$ & $\begin{array}{c}\text { No } \\
\text { leakage }\end{array}$ \\
\hline $\begin{array}{c}\text { Dry air } 200^{\circ} \mathrm{C} \\
2,160 \text { hours }\end{array}$ & $0 \mathrm{~mm}$ & $\begin{array}{c}\text { No } \\
\text { leakage }\end{array}$ & $\begin{array}{c}\text { No } \\
\text { leakage }\end{array}$ & $\begin{array}{c}\text { No } \\
\text { leakage }\end{array}$ \\
\hline $\begin{array}{c}\text { Dry air } 200^{\circ} \mathrm{C} \\
2,520 \text { hours }\end{array}$ & $0 \mathrm{~mm}$ & $\begin{array}{c}\text { No } \\
\text { leakage }\end{array}$ & $\begin{array}{c}\text { No } \\
\text { leakage }\end{array}$ & $\begin{array}{c}\text { No } \\
\text { leakage }\end{array}$ \\
\hline
\end{tabular}

generation or pressure drop was observed in the leakage confirmation conducted using bubble solution, and superior airtightness was demonstrated. This test confirmed that the leakage resistance performance can be expected to be maintained for long periods after an accident.

\section{Improvement of the Containment Venting Operation}

The above analysis result concerning loss of the containment function of the PCV in Unit 2 suggests that it is desirable to decrease the PCV pressure by conducting venting in advance, as the PCV pressure increases rapidly when water injection into the reactor is resumed with the fuel exposed ${ }^{10}$. In addition, if the venting system is kept in service even after the pressure decrease, the influence of the generation of a large amount of non-condensable gas can be mitigated. Such operations should be considered in the future because radioactive material 
emissions due to venting can be reduced with the FCVS installed.

In the past, the containment venting conditions have been discussed in terms of the relationship with the maximum working pressure of the PCV or pressure twice the maximum working pressure and have also been reflected in the operation procedures to be followed in the event of an accident. As a result, the working pressure of the rupture disk incorporated into the venting system was set according to the maximum working pressure of the PCV. However, it is necessary to not only check whether the preset pressure is reached but to also operate containment venting flexibly to put the containment venting system into service in advance-irrespective of the pressure at the time - if a rapid increase in the PCV pressure is predicted as was the case for Unit 2 of Fukushima Dai-ichi. In addition, venting has the effect of decreasing the saturation temperature of steam through depressurization to cool the PCV and discharging hydrogen to prevent the accumulation of combustible gas. Thus, venting should be conducted in accordance with the situation. For this purpose, the rupture disk should either not be installed or should be configured to operate at low pressures if it is installed.

Furthermore, in the operation procedures to be followed in the event of a severe accident, attention must be paid to the generation of a large amount of non-condensable gas or steam when water injection into the reactor is resumed after being suspended.

In addition, in Unit 2, the pressure inside the PCV could not be relieved before loss of the containment function of the PCV because one of the valves of the containment venting system could not be kept in the open position and the operation for opening the closed valve failed. Since the valve was air-operated and the solenoid valve which controlled the air was designed to be operated remotely from the main control room, there was no on-site feature for opening and closing the valves. Therefore, in addition to remote operation from the main control room, a feature that enables the on-site, hand operation of the valves for containment venting should be provided so that venting can be conducted at any time. Figure $\mathbf{1 6}$ shows an example in which the valve operating mechanism was extended to outside the secondary containment, where the radiation level is low even in the event of an accident so that the valve can be operated

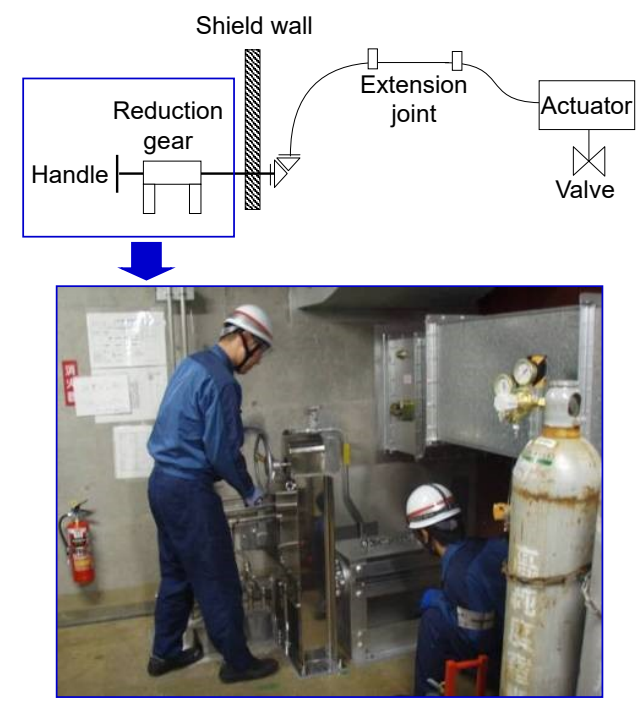

Figure 16 Example of a manual operation device for a valve in the filtered containment venting system installed outside the secondary containment boundary 
manually and kept open. This feature can be incorporated into both motor-operated valves and air-operated valves that have a ratchet mechanism in their drive mechanisms.

\section{Conclusions}

In the accident that occurred at Fukushima Dai-ichi, Unit 2 was responsible for releasing the largest amount of radioactive material into the atmosphere, which is said to be the cause of the high level of contamination found in areas northwest of Fukushima Dai-ichi, particularly the village of Iitate. In Unit 2, a significant decrease in the PCV pressure was observed on the morning of March 15 before the pressure limit was reached, at which point it was thought that containment function of the PCV was still maintained. However, it is now believed that the containment function was lost at that point in time, but details of location and cause of leakage have not been examined.

Based on the situation of the contamination in the Unit 2 reactor building and the temperature and pressure trends in the PCV at the time of the accident, we estimate that it is highly possible that this leakage occurred at the top head flange of the PCV due to the deterioration of the silicone rubber used for the flange gasket under a high-temperature steam environment.

Based on the lessons learned from this accident, it is necessary to promote the following three systematic approaches related to temperature and pressure to maintain the containment performance of the PCV in the event of a severe accident: the mitigation of an increase in the PCV boundary temperature through alternative spray and water injection; the enhancement of the heat resistance of the PCV boundary through improvement of the sealants; and the effective venting of the PCV before a rapid pressure increase or an over-pressure.

Among these approaches, this study confirmed through a test that leakage resistance performance in a high-temperature steam environment in the event of a severe accident was enhanced by using improved EPDM as the sealing material instead of conventionally used silicone rubber. In addition, we also devised a new way of applying backup sealing materials to the flange using fire-resistant sealant, which was shown to be effective in securing the reliability of leakage resistance for long periods of time after the accident. These measures are expected to enhance the heat resistance performance of the PCV boundary.

Furthermore, concerning the operation of containment venting, in addition to the traditional approach that venting is conducted depending on whether the PCV has reached a preset pressure, it was found to be beneficial to put the venting system into service in advance if there is any possibility that the PCV pressure may increase rapidly due to the generation of large amounts of non-condensable gas and steam; for example, in a case where water injection into the reactor is resumed after it was suspended. Through the operation of venting in a situation like this, the possibility of PCV failure should be reduced as much as possible. In addition, because venting is also effective in decreasing the PCV temperature and preventing an accumulation of flammable gas, it is also important to operate venting according to the situation. Such flexible operation of the containment venting should be incorporated into accident operation procedures and the improved operation mechanism should be applied to the valves in the venting system to enable such operation.

\section{References}

1) Tokyo Electric Power Company, Fukushima Nuclear Accident Analysis Report, http://www.tepco.co.jp/ en/press/corp-com/release/2012/1205638_1870.html as of November 2015, (2012). 
2) S. Takenobu, "Kakusyu gomu compound no tainetsu taisui jouki siken kekka," Valqua Rev., 21[3], 8-14 (1977). [in Japanese]

3) M. Ishikawa, Koushou Fukushima Genshiryoku Jiko, Denkikyokai Shimbunbu, Tokyo, ISBN978-4905217-34-3 C0036 (2014). [in Japanese]

4) S. Kawamura, S. Omori, T. Kimura, T. Takiguchi, T. Narabayashi, "A comprehensive approach to reducing radioactive material release in BWR containment venting," Proc. 20th Nat. Symp. on Power and Energy Systems, Sendai, Japan, Jun. 18-19, 2015 (2015). [in Japanese]

5) Y. Furuhashi, S. Ooki, Y. Ibusuki, R. Shimosakoda, A. Taniguchi, T. Kimura, N. Hiranuma, S. Murai, H. Tezuka, "Applicability evaluation of EPDM rubber sheet for butterfly valve of primary containment vessel (2) Fundamental behavior of EPDM rubber sheet under steam with iodine and its iodine durability under simulated severe accident condition," Proc. 2015 Fall Meeting of Atomic Energy Society of Japan, Shizuoka, Japan, Sep. 9-11, 2015 (2015). [in Japanese]

6) S. Ooki, Y. Ibusuki, R. Shimosakoda, A. Taniguchi, "Improvement of sealing performance at flanges and hatches in primary containment vessels," Proc. 2015 Annu. Conf. on Power and Energy Society IEE Japan, Nagoya, Japan, Aug. 25-27, 2015 (2015). [in Japanese]

7) S. Ooki, Y. Ibusuki, R. Shimosakoda, N. Hayashi, H. Abe, A. Taniguchi, "Studies on back up seal material to flanges and hatches on primary containment vessels," Proc. 2015 Annu. Conf. of Fundamentals and Materials Society IEE Japan, Kanazawa, Japan, Sep. 17-18, 2015 (2015). [in Japanese]

8) Ministry of land, infrastructure, transport and tourism, Notice Defining the Details of the Safety Standards for Road Transport Vehicles (Attachment 100), (2005). [in Japanese]

9) U. S. Atomic Energy Commission, Reactor Containment Leakage Testing and Surveillance Requirements, Safety standards, criteria, and guides for the design, location, construction, and operation of reactors, III. Technical safety guide (1966).

10) T. Narabayashi, S. Kawamura, "Development of high efficiency filtered containment venting system by using AgX," Proc. 20th Nat. Symp. on Power and Energy Systems, Sendai, Japan, Jun. 18-19, 2015(2015). [in Japanese] 


\title{
Improved Nuclear Emergency Management System Reflecting Lessons Learned from the Emergency Response at Fukushima Daini Nuclear Power Station after the Great East Japan Earthquake
}

\author{
Shinichi KAWAMURA ${ }^{1, *}$ and Tadashi NARABAYASHI ${ }^{2}$ \\ ${ }^{1}$ Nuclear Asset Management Department, Tokyo Electric Power Company, 1-1-3 Uchisaiwai-cho, Chiyoda-ku, Tokyo 100-8560, \\ Japan \\ ${ }^{2}$ Division of Energy and Environmental Systems, Graduate School of Engineering, Hokkaido University, Kita-13, Nishi-8, Kita-ku, \\ Sapporo 060-8628, Japan
}

\begin{abstract}
Three nuclear reactors at Fukushima Daini Nuclear Power Station lost all their ultimate heat sinks owing to damage from the tsunami caused by the Great East Japan Earthquake on March 11,2011 . Water was injected into the reactors by alternate measures, damaged cooling systems were restored with promptly supplied substitute materials, and all the reactors were brought to a cold shutdown state within four days. Lessons learned from this experience were identified to improve emergency management, especially in the areas of strategic response planning, logistics, and functions supporting response activities continuing over a long period. It was found that continuous planning activities reflecting information from plant parameters and response action results were important, and that relevant functions in emergency response organizations should be integrated. Logistics were handled successfully but many difficulties were experienced. Therefore, their functions should be clearly established and improved by emergency response organizations. Supporting emergency responders in the aspects of their physical and mental conditions was important for sustaining continuous response. As a platform for improvement, the concept of the Incident Command System was applied for the first time to a nuclear emergency management system, with specific improvement ideas such as a phased approach in response planning and common operation pictures.
\end{abstract}

KEYWORDS: accident, accident management, nuclear reactor, Fukushima Daini Nuclear Power Station, The Great East Japan Earthquake, emergency response, incident command, response planning, response operations, logistics

\section{Introduction}

Due to the damage from the tsunami caused by the Great East Japan Earthquake on March 11, 2011, the normal heat-removal systems of Units 1, 2 and 4, as well as all residual heat-removal

* Corresponding author, E-mail: shinichi.kawamura@tepco.co.jp

DOI : 10.15669/fukushimainsights.Vol.4.473

(C) 2021 Atomic Energy Society of Japan. All rights reserved.

Originally published in Transactions of the Atomic Energy Society of Japan (ISSN 1347-2879), Vol. 15, No. 2, p.84-96

(2016) in Japanese. (Japanese version accepted: November 14, 2015) 
(RHR) systems (there are two systems in each reactor) stopped working at the Fukushima Daini Nuclear Power Station (hereinafter referred to as "Fukushima Daini") and the nuclear reactor went into the "Loss of Ultimate Heat Sink" state. In addition, due to the loss of function of the equipment-cooling systems, the pumps of the emergency core-cooling systems (ECCS) were also disabled. While continuing water injection into the reactors from the condensate storage tanks (CST), the RHR systems whose function was lost were restored with temporary equipment, and the cold shutdown of these reactors was completed successfully by March 15 .

The facts concerning the damage and restoration of equipment in this emergency situation were published by the Tokyo Electric Power Company ${ }^{1)}$. However, this investigation report was compiled with a focus on clarifying the equipment situation, on what is called the hardware side. Therefore, human activities as part of an emergency response, on the software side, have not been organized and analyzed sufficiently.

Concerning the software side, based on what the Tokyo Electric Power Company published, the Japan Nuclear Technology Institute ${ }^{2)}$ conducted an analysis using the m-SHEL model, which is a method for analyzing a root cause to draw a lesson with the objective of preventing a mistake in the emergency response. In addition, the investigative committee of the Atomic Energy Society of Japan ${ }^{3)}$ mainly examined the accident that occurred at Tokyo Electric Power Company's Fukushima Daiichi Nuclear Power Station (hereinafter referred to as "Fukushima Daiichi"), and in the analysis, a study was conducted from the standpoint of human factors.

In an actual emergency response, especially in a situation exceeding the anticipated conditions, a response needs to be initiated with limited information, as well as the available human and material resources, in the early stages when the whole picture of the situation has not yet become clear. Therefore, while executing a response, organized activities to raise awareness regarding the situation to revise the countermeasures sequentially are very important. In addition, in order to secure and reinforce both human and material resources, unified collaboration between activities at the site and logistic support is also important. Furthermore, a system to support an ongoing response in the case of a prolonged situation is also necessary. Therefore, in addition to the past research reports and suggestions, it is important to draw lessons regarding the enhancement of organizational capacity from such perspectives.

In this paper, we analyze the case of Fukushima Daini, where the response to a situation actually exceeding the anticipated conditions succeeded, and consider how to improve the emergency management system for the purpose of improving organizational strength to respond flexibly to emergency situations exceeding the anticipated conditions.

\section{Emergency Response Taken and Lessons Learned at Fukushima Daini}

\section{Investigation Method}

The extent of the damage to significant safety equipment and major operations at Fukushima Daini were clarified in the investigation report issued by the Tokyo Electric Power Company ${ }^{1}$. However, this report failed to sufficiently clarify the organizational activities performed during the emergency. Therefore, we added the records of emergency activities gathered by the information team in the emergency response organization of Fukushima Daini, as well as the testimonies for which there was agreement among the sources regarding the objects of analysis. In this way, we attempted to clarify the whole picture of the emergency activities conducted 
at Fukushima Daini as well as to determine the effectiveness and issues regarding individual activities conducted during the emergency response.

\section{Damage to Fukushima Daini Caused by Tsunami}

Fukushima Daini is located approximately $12 \mathrm{~km}$ south of Fukushima Daiichi along the coast extending over Tomioka Town and Naraha Town of Futaba-Gun, Fukushima Prefecture. At the power station, there are four boiling water reactors (BWR5), and their rated thermal output and electric output are 3,293 MWt and 1,100 MWe per reactor, respectively. On March 11, 2011, when the Great East Japan Earthquake occurred, all four reactors were in operation at the rated outputs.

Among the seismic accelerations observed on the lowermost underground floor of each reactor building, the maximum ones were $277 \mathrm{gal}$ in the horizontal direction and 305 gal in the vertical direction. Detecting these accelerations, all reactors scrammed automatically at 14:48 and all control rods were inserted into the reactor cores. It was confirmed from the records that the scram operation and the condition of the plant systems after the scram were as originally designed, and the operations to effect a cold shutdown were conducted according to the specified procedures. Concerning the external power supply, two Tomioka lines and one Iwaido line were serviceable before the earthquake, but all lines except for one Tomioka line became unserviceable as a result of the earthquake.

After that, the tsunami arrived at the power station (the first wave was visually confirmed to have arrived at 15:22) and caused damage to the safety facilities of each reactor.

Table 1 shows the major damage. The residual heat-removal cooling (RHRC) systems and the residual heat-removal sea water (RHRS) systems for transferring the heat of nuclear reactors to the sea, which is the ultimate heat sink, as well as most of the emergency equipment-cooling water (EECW) systems became inoperable because their power panels and electric motors were underwater due to the inundation of the heat-exchanger buildings located near the water intake. In addition, inoperable cooling systems also disabled the operation of the residual

Table 1 Status of cooling systems at Fukushima Daini Nuclear Power Station after the tsunami

\begin{tabular}{|c|c|c|c|c|c|c|c|c|c|}
\hline \multicolumn{2}{|c|}{ System } & \multicolumn{2}{|r|}{ Unit 1} & \multicolumn{2}{|r|}{ Unit 2} & \multicolumn{2}{|r|}{ Unit 3} & \multicolumn{2}{|r|}{ Unit 4} \\
\hline \multirow{3}{*}{$\begin{array}{l}\mathrm{RHR}(\mathrm{A}) \\
\text { including } \\
\text { cooling } \\
\text { systems }\end{array}$} & RHR(A) & $\triangle$ & $\begin{array}{l}\text { Loss of power source } \\
\text { and cooling system }\end{array}$ & $\triangle$ & $\begin{array}{l}\text { Loss of cooling } \\
\text { system }\end{array}$ & $\triangle$ & $\begin{array}{l}\text { Loss of cooling } \\
\text { system }\end{array}$ & $\triangle$ & $\begin{array}{l}\text { Loss of cooling } \\
\text { system }\end{array}$ \\
\hline & $\begin{array}{l}\text { RHRC/RHRS } \\
\quad(\mathrm{A}, \mathrm{C})\end{array}$ & $\times$ & $\begin{array}{l}\text { Submerge of power } \\
\text { source and motor }\end{array}$ & $\triangle$ & $\begin{array}{l}\text { Loss of cooling } \\
\text { system }\end{array}$ & $\times$ & $\begin{array}{l}\text { Submerge of power } \\
\text { source and motor }\end{array}$ & $\times$ & $\begin{array}{l}\text { Submerge of power } \\
\text { source and motor }\end{array}$ \\
\hline & $\operatorname{EECW}(\mathrm{A})$ & $\times$ & $\begin{array}{l}\text { Submerge of power } \\
\text { source and motor }\end{array}$ & $\times$ & $\begin{array}{l}\text { Submerge of power } \\
\text { source and motor }\end{array}$ & $\times$ & $\begin{array}{l}\text { Submerge of power } \\
\text { source and motor }\end{array}$ & $\times$ & $\begin{array}{l}\text { Submerge of power } \\
\text { source and motor }\end{array}$ \\
\hline \multicolumn{2}{|c|}{ LPCS } & $\triangle$ & $\begin{array}{l}\text { Loss of power source } \\
\text { and cooling system }\end{array}$ & $\triangle$ & $\begin{array}{l}\text { Loss of cooling } \\
\text { system }\end{array}$ & $\triangle$ & $\begin{array}{l}\text { Loss of cooling } \\
\text { system }\end{array}$ & $\triangle$ & $\begin{array}{l}\text { Loss of cooling } \\
\text { system }\end{array}$ \\
\hline \multirow{3}{*}{$\begin{array}{l}\mathrm{RHR}(\mathrm{B}) \\
\text { including } \\
\text { cooling } \\
\text { systems }\end{array}$} & RHR(B) & $\triangle$ & $\begin{array}{l}\text { Loss of cooling } \\
\text { system }\end{array}$ & $\triangle$ & $\begin{array}{l}\text { Loss of cooling } \\
\text { system }\end{array}$ & $\bigcirc$ & Stand-by & $\triangle$ & $\begin{array}{l}\text { Loss of cooling } \\
\text { system }\end{array}$ \\
\hline & $\begin{array}{l}\text { RHRC/RHRS } \\
(\mathrm{B}, \mathrm{D})\end{array}$ & $\times$ & $\begin{array}{l}\text { Submerge of power } \\
\text { source and motor }\end{array}$ & $\times$ & $\begin{array}{l}\text { Submerge of power } \\
\text { source }\end{array}$ & 0 & Stand-by & $\times$ & $\begin{array}{l}\text { Submerge of power } \\
\text { source and motor }\end{array}$ \\
\hline & EECW (B) & $\times$ & $\begin{array}{l}\text { Submerge of power } \\
\text { source and motor }\end{array}$ & $\times$ & $\begin{array}{l}\text { Submerge of power } \\
\text { source }\end{array}$ & $\bigcirc$ & Operation & $\times$ & $\begin{array}{l}\text { Submerge of power } \\
\text { source }\end{array}$ \\
\hline \multicolumn{2}{|c|}{$\mathrm{RHR}(\mathrm{C})$} & $\triangle$ & $\begin{array}{l}\text { Loss of cooling } \\
\text { system }\end{array}$ & $\triangle$ & $\begin{array}{l}\text { Loss of cooling } \\
\text { system }\end{array}$ & 0 & Stand-by & $\triangle$ & $\begin{array}{l}\text { Loss of cooling } \\
\text { system }\end{array}$ \\
\hline \multicolumn{2}{|c|}{ RWCU } & $\triangle$ & $\begin{array}{l}\text { Loss of cooling } \\
\text { system }\end{array}$ & $\triangle$ & $\begin{array}{l}\text { Loss of cooling } \\
\text { system }\end{array}$ & $\triangle$ & $\begin{array}{l}\text { Loss of cooling } \\
\text { system }\end{array}$ & $\triangle$ & $\begin{array}{l}\text { Loss of cooling } \\
\text { system }\end{array}$ \\
\hline \multicolumn{2}{|c|}{$\begin{array}{c}\text { MUWC (alternative } \\
\text { water injection) }\end{array}$} & $\bigcirc$ & Stand-by & $\bigcirc$ & Stand-by & $\bigcirc$ & Stand-by & $\bigcirc$ & Stand-by \\
\hline \multicolumn{2}{|r|}{ RCIC } & 0 & Stand-by & 0 & Stand-by & 0 & Stand-by & 0 & Stand-by \\
\hline
\end{tabular}


heat-removal (RHR) systems and the low-pressure core spray (LPCS) systems. Furthermore, the reactor water cleanup (RWCU) systems also lost their heat-removal function. As a result, in Units 1, 2 and 4, all heat-removal facilities and ECCSs for cooling the reactor cores lost their functions. In this situation, the only facilities that could be used for injecting water into the reactor cores were the reactor core isolation cooling (RCIC) systems and the make-up water condensate (MUWC) systems, which were not designed as ECCS.

The events that occurred after the earthquake, as well as corresponding operations, restoration activities and, logistic support activities (logistics), were almost the same among Units 1, 2 and 4, and Table 2 shows these events chronologically, using Unit 1 as an example. In the following, these responses are analyzed in detail and the lessons learned are discussed.

\section{Emergency Responses Made through Operation and the Lessons Learned}

\section{(1) Assistance to the operators at the main control room}

In this emergency situation, assistance was provided to the operators at the main control room as follows. First, the work management teams, which had their offices near the main control room, rushed there to assist the operators. These teams are the organizations in charge of operation management related to maintenance work. All members of these teams are operators

Table 2 Chronology of events and response actions at Fukushima Daini Unit 1

\begin{tabular}{|c|c|c|c|c|}
\hline Date & Time & Event & Plant Operation & Restoration and logistics \\
\hline \multirow[t]{5}{*}{ March 11} & $14: 48$ & $\begin{array}{l}\text { Reactor auto-trip triggered by } \\
\text { earthquake acceleration. Two out } \\
\text { of three external power line lost } \\
\text { by earthquake damage. }\end{array}$ & $\begin{array}{l}\text { Post-scrum actions and continu- } \\
\text { ous Reactor cooling by Main Con- } \\
\text { denser. }\end{array}$ & \\
\hline & $15: 22$ & $\begin{array}{l}\text { First tsunami wave reached the } \\
\text { site. (repeated until } 17: 14 \text { ). }\end{array}$ & & \\
\hline & $15: 36$ & & $\begin{array}{l}\text { MSIV manually closed. } \\
\text { RCIC manually started. }\end{array}$ & \\
\hline & $16: 15$ & & Reactor Depressurization started. & \\
\hline & about $21: 00$ & & & $\begin{array}{l}\text { Field walkdown started for dam- } \\
\text { age identification. }\end{array}$ \\
\hline \multirow[t]{6}{*}{ March 12} & $0: 00$ & & $\begin{array}{l}\text { Low pressure alternate injection } \\
\text { to Reactor by MUWC started. }\end{array}$ & \\
\hline & about $5: 00$ & & & $\begin{array}{l}\text { Field walkdown completed. Pro- } \\
\text { curement of essential material } \\
\text { for restoration started. }\end{array}$ \\
\hline & $5: 22$ & $\begin{array}{l}\mathrm{S} / \mathrm{C} \text { temperature reached } 100^{\circ} \mathrm{C} \text {. } \\
=\text { Loss of pressure suppression } \\
\text { function of PCV. }\end{array}$ & & \\
\hline & $7: 10$ & & Alternate spray in $\mathrm{D} / \mathrm{W}$ by MUWC. & \\
\hline & $7: 37$ & & Alternate spray in $\mathrm{S} / \mathrm{C}$ by MUWC. & \\
\hline & $18: 30$ & & $\begin{array}{l}\text { Preparatory operations for PCV } \\
\text { venting completed. }\end{array}$ & \\
\hline \multirow[t]{4}{*}{ March 13} & about $7: 00$ & & & $\begin{array}{l}\text { Transportation of essential ma- } \\
\text { terial for restoration completed. }\end{array}$ \\
\hline & about $8: 30$ & & & $\begin{array}{l}\text { Cable laying and motor replace- } \\
\text { ment started for recovering RHR } \\
\text { (B). }\end{array}$ \\
\hline & $11: 32$ & & Alternate spray in $\mathrm{D} / \mathrm{W}$ by MUWC. & \\
\hline & $14: 29$ & & Alternate spray in D/W by MUWC. & \\
\hline \multirow[t]{2}{*}{ March 14} & $1: 24$ & & RHR (B) restarted. & $\begin{array}{l}\text { Restoration of RHR (B) complet- } \\
\text { ed. }\end{array}$ \\
\hline & $13: 40$ & Reactor cold shutdown achieved. & & \\
\hline
\end{tabular}

Note : MSIV : Main Steam Isolation Valve, RCIC : Reactor Core Isolation Cooling System, S/C : Suppression Chamber, D/W : Dry well, MUWC : Make-UP Water Condensate System, PCV : Primary Containment Vessel, RHR (B) : Residual Heat Removal System Div. B. 
and the leaders are qualified as operation managers (shift supervisors). With their operational skills, these teams provided effective assistance to the operators in the main control room in terms of checking the configuration of systems to be operated and reconfirming the overall status of the plant.

In the meantime, a site emergency response organization was set up in the important seismic isolated building adjacent to the administration building of the power station. Figure 1 shows the structure of the emergency response organization at that time. In this organization, the task of the plant operation team is to ascertain the operational circumstances and share them with the emergency response organization as well as to provide technical assistance to the operators under the jurisdiction of the headquarters of the site emergency response organization (Site ERC HQ). Almost simultaneously with the setup of the emergency response organization, those experienced in working as operators were dispatched to the respective main control room from this team. This enabled close and secure coordination between the emergency response organization and the main control room via the dispatched plant operation team members while allowing the operators to concentrate on gaining a clear picture of the overall plant situation and the response operations needed.

Meanwhile, at the site emergency response organization, the information on the current condition of major safety facilities and the status of water injection into the reactors were written on whiteboards so this information could be shared. Figure 2 shows some of the information written at approximately 0:00 am on March 12. On one whiteboard, important information, such as the condition of emergency power sources (high-voltage power panels (M/C), emergency generators $(\mathrm{D} / \mathrm{G})$, and direct current power sources $(\mathrm{DC}))$, the condition of the reactor water injection and heat-removal systems (such as RCIC, RHR, and MUWC), and the condition of emergency water sources (condensate storage tank (CST)) in the respective reactors $(1 \mathrm{u}, 2 \mathrm{u}, 3 \mathrm{u}$ and $4 u$ ) were summarized in an easy-to-read way. In addition to the information shown in Figure 2 , the prediction of the increase in pressure in the primary containment vessels (hereinafter called "containment vessels"), including the prediction of the time when the maximum working pressure would be reached, was also written to share this information. Although the condition of the plants and major operations was announced as needed in the site emergency response

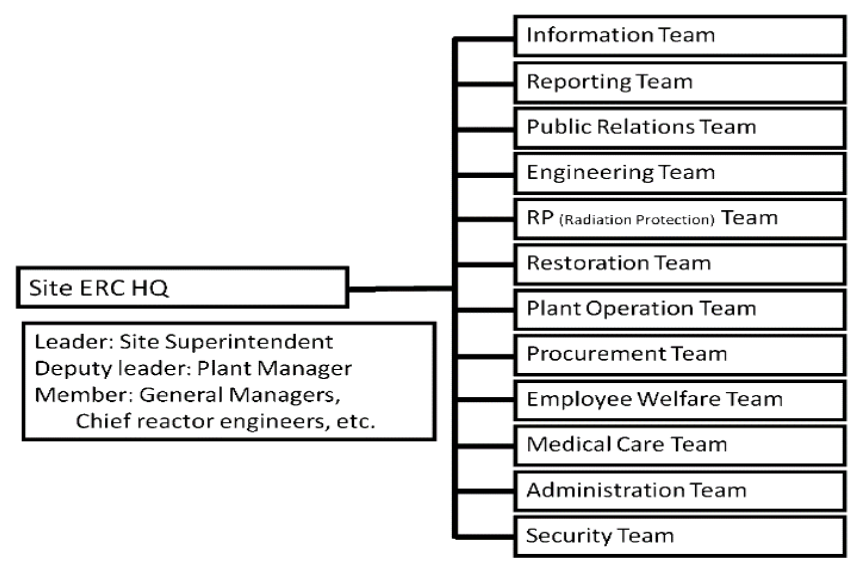

Figure 1 Organizational structure of the Fukushima Daini emergency response organization at the time of the Great East Japan Earthquake in 2011 ERC HQ means Emergency Response Center Headquarters. 


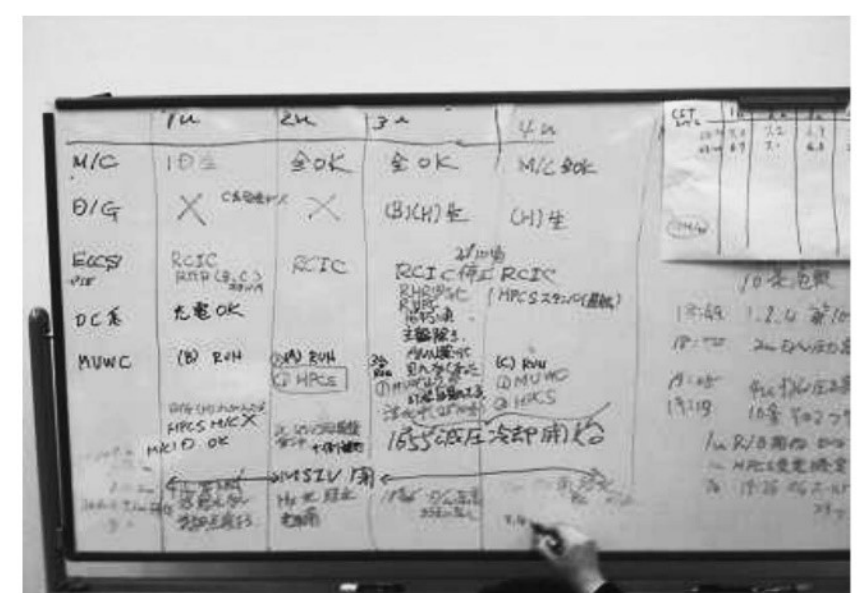

Figure 2 One of the whiteboards summarizing plant system information shared between the Fukushima Daini emergency response organization and operators in the main control rooms

Status of emergency power (M/C: metal-clad switchgear, D/G: diesel generator, DC), ECCS and injection systems such as RCIC and MUWC, injection water source (CST: condensate storage tank), availability of main condenser (status of MSIV: main steam isolation valve), and so on were summarized on this board.

organization, the condition of important facilities was kept visualized at all times. These actions like this is important for all responders and the operators at the main control room to ensure that a common perception of what is occurring is maintained when conducting activities. Besides this, the content discussed by the emergency response organization was conveyed to the main control room by the plant operation team leader. The plant operation team leader, who had been appointed due to his long experience of being responsible for operations, not only conveyed the discussion results at the site emergency response organization but also provided the necessary technical assistance to the main control room.

The main control room is designed so that emergency supervision and operations can be conducted, and the training for such activities is provided to the operators. However, in situations exceeding the anticipated conditions, like this one, constructing a structure to assist the main control room at an early stage by the personnel who have knowledge and experience in plant operation was effective in (1) allowing the operators to concentrate on operations, (2) increasing the reliability of the operators' judgement through reconfirmation, and (3) sharing information closely between the main control room and the site emergency response organization. In addition, establishing a system for sharing information and having a common understanding between the personnel of the site emergency response organization and the operators enabled them to provide appropriate assistance to the main control room. This is considered an important experience and a useful lesson for the future.

\section{(2) Water injection into reactors and containment vessel spray}

In order to secure the safety of the reactors after the scram, it is necessary to place top priority on continuing water injection into the reactors. Figure 3 shows the configuration of the facilities used to maintain continuous water injection into Units 1,2 and 4. The main steam condenser lost its heat-removal function, and the main steam isolation valves (MSIV) were closed to shut off the reactor. In addition, the RHR, RHRC, and RHRS were inoperable as mentioned above, and heat removal using these facilities could not be conducted. On the other hand, the 


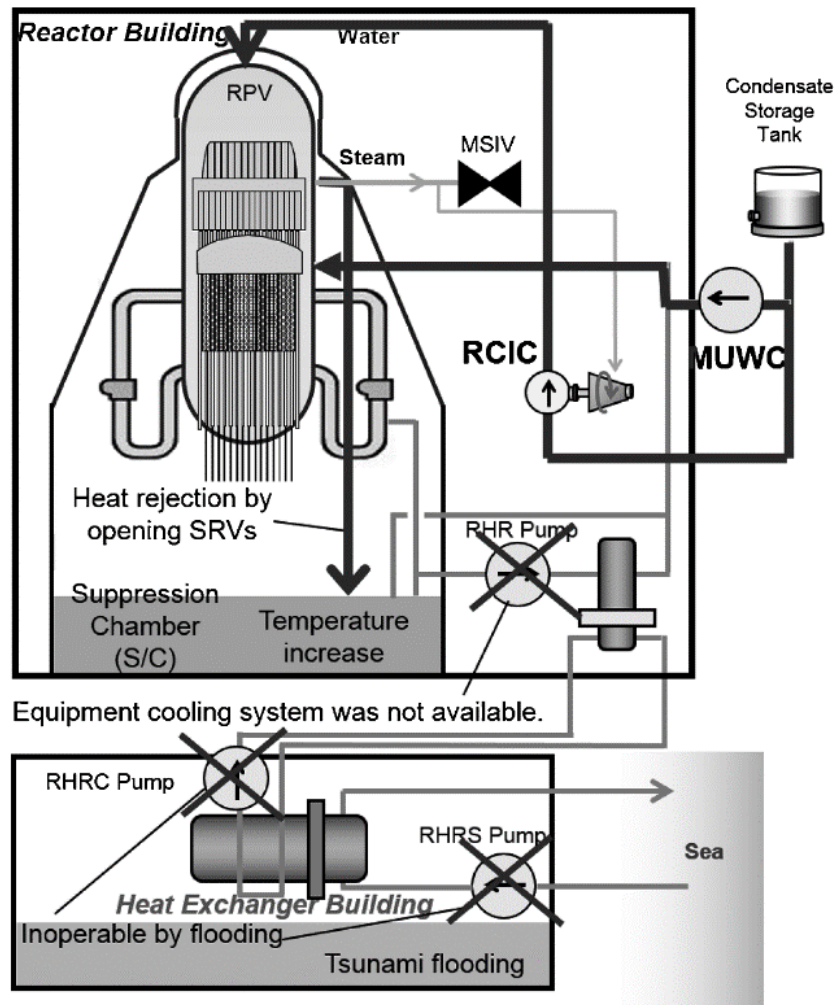

Figure 3 Emergency measures employed at Units 1, 2, and 4 to inject water into the reactor pressure vessels after losing all the safety-related injection and cooling systems including the residual heat-removal systems

steam-turbine-driven RCIC was the only system at each reactor that was operable without any equipment-cooling system among the systems that could inject water into the high-pressure reactor. Therefore, the water in the condensate storage tank was injected into the reactor using this system. After that, the reactor was depressurized using the safety relief valves (SRVs) and the water injection from the condensate storage tank was continued using the low-pressure water-injection system as an alternative to the RCIC.

For this low-pressure water injection, the MUWC that was positioned as an accident management contingency measure was used, although it was not originally designed for injecting water into the reactor. The MUWC pumps are also operable without any equipment-cooling system.

Taking Unit 1 as an example, Figure 4 shows the trend of the reactor pressure and reactor water-injection operation until the cold shutdown of the reactor. The reactor and the containment vessel were cooled with the low-pressure core injection (LPCI) function and the suppression chamber $(\mathrm{S} / \mathrm{C})$ cooling function of system B of the RHR, which was restored on March 14. The same response as that of Unit 1 was also taken for Units 2 and 4. Starting from about 15:36 on March 11, immediately after the heat-removal function of the reactor was lost, water injection through the intermittent operation of the RCIC was conducted. With this situation, the depressurization of the reactor using the SRV was started at 16:15. After that, the injection of water using the MUWC was continued from 0:00 on March 12. This shows that continuous migration from high-pressure water injection to low-pressure water injection was achieved 
with the reactor depressurization in between while maintaining the water injection function.

Figure 5 shows the trend of the reactor water level. Although the reactor water level changed significantly due to the influence of the steam discharge into the suppression chamber associated with the depressurization as well as the intermittent operation of the RCIC system, it was kept higher than the top of active fuel (TAF). In addition, in the nuclide analysis of the reactor water sampled at 8:30 on March 14, it was confirmed that the I-131 and I-132 concentrations, which would change in the case of fuel damage, were not significantly different from the analysis values obtained on February 22, before the emergency situation. This shows that the changeover from high-pressure water injection to low-pressure water injection succeeded as a result of a series of operations and fuel integrity was maintained by continuous cooling.

Meanwhile, as Figure 3 shows, the steam generated in the reactor was led to the suppression chamber in the containment vessel via the SRV. However, because there was no way to cool the water in the suppression chamber, the pressure-suppression function was lost and the pressure in the containment vessel increased after the water reached the saturation temperature.

Taking Unit 1 as an example, Figures $\mathbf{6}$ and $\mathbf{7}$ show the trends of the pressure and temperature

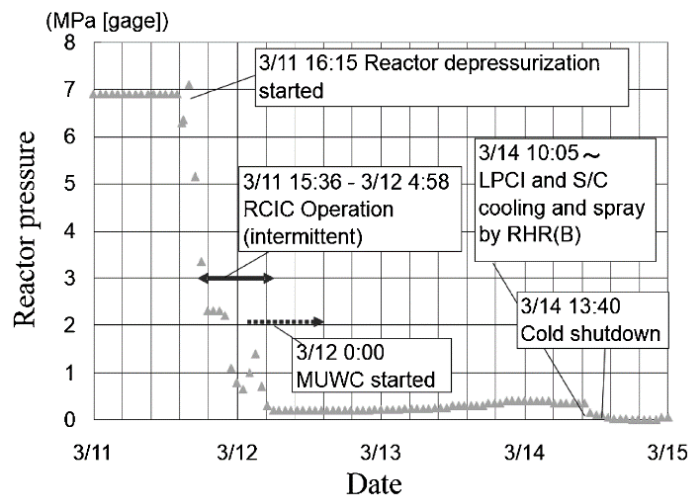

Figure 4 Reactor pressure of Unit 1

RCIC, LPCI, S/C, MUWC and RHR refer to the reactor core isolation cooling system, low-pressure core injection system, suppression chamber of primary containment vessel, make-up water condensate system, and the residual heat-removal system, respectively.

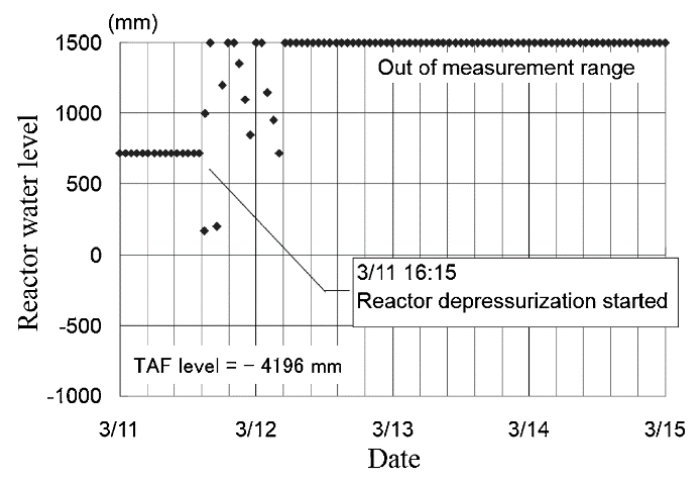

Figure 5 Reactor water level of Unit 1 TAF means Top of Active Fuel. 
in the containment vessel and major operations until the cold shutdown. First, at 6:20 on March 12 , after contacting the site emergency response organization, the operators injected low-temperature water from the MUWC into the $\mathrm{S} / \mathrm{C}$ via system $\mathrm{A}$ of the flammability control system (FCS) to mitigate the increase in the temperature. This was not what the FCS had been originally designed for, but it was a flexible response to utilize the MUWC for the FCS cooling. Next, after 7:10, the MUWC was switched from reactor water injection to containment vessel spray intermittently as an alternative measure to the original containment vessel spray using the RHR, and the increase in the containment vessel pressure was mitigated through the water spray to the dry well $(\mathrm{D} / \mathrm{W})$ and $\mathrm{S} / \mathrm{C}$.

Although these operations were effective in holding back an increase in pressure and temperature, these parameters increased again after shutdown. Therefore, in case the containment vessel exceeded the maximum working pressure, preparations for configuring the containment venting system were conducted. However, eventually, the situation was resolved before the containment vessel reached the maximum working pressure because system B of the RHR was restarted successfully at 1:24 on March 14 as a result of the remediation activity described later. Although an increase in pressure occurred on March 17, this was because water was transferred

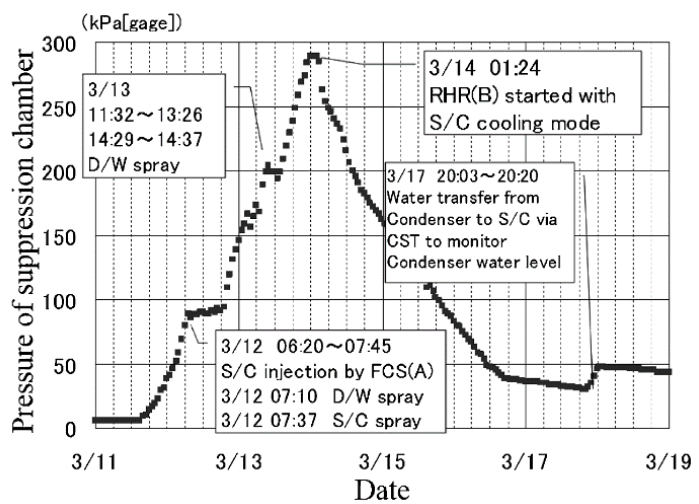

Figure 6 Pressure of Unit 1's primary containment suppression chamber $\mathrm{D} / \mathrm{W}, \mathrm{S} / \mathrm{C}$, and FCS refer to dry well, suppression chamber, and flammability control system, respectively.

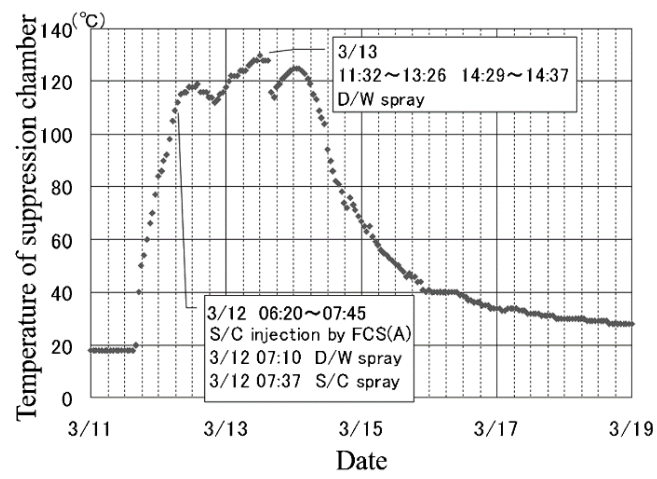

Figure 7 Temperature of Unit 1's primary containment suppression chamber $\mathrm{D} / \mathrm{W}, \mathrm{S} / \mathrm{C}$, and FCS refer to dry well, suppression chamber, and flammability control system, respectively. 
from the main steam condenser to the S/C via the condensate storage tank (CST), and not because a new event occurred on the reactor side.

The lessons learned from a series of responses include that a strategy should be developed to constantly increase the substitutability of response measures through the emergency response.

Concerning the reactor water injection, although RCIC was the only measure employed initially because the reactor was in the high-pressure stage, depressurizing the reactor increased the substitutability of water injection by a variety of means other than MUWC, which was used this time such as the utilization of a fire-extinguishing system. Meanwhile, it was possible that an unexpected situation, such as a failure of a response system, might occur over time. With this in mind, after ensuring the first response measure (water injection into the reactor using the RCIC system), action was taken continuously to progress the situation so that multiple alternative measures could be secured without becoming complacent about the first measure.

Furthermore, in order to mitigate the increase in containment vessel pressure, in addition to the alternative containment vessel spray, the number of alternative measures that could be chosen increased with time, such as by speeding up restoration of the RHR while preparing for containment venting as the next measure.

In the future, the emergency organization is required to enhance functions so that a strategy can be developed in the manner described above.

Furthermore, the function of collecting and analyzing information is essential for strategy planning as well as feeding back the execution results into strategy development. At Fukushima Daini, the strategic response as described above was possible because the functions of the instruments and control systems were secured and the site emergency response organization could ascertain the situation and predict the change in the situation based on data. In addition, it was also possible because the site emergency response organization and the main control room could share such information through establishing a means of communication and via a network and the consistency of the chain of command was maintained.

\section{(3) Summary of the lessons learned in the emergency response through operations}

The important lessons learned in operations that led to a successful response are summarized as follows:

First, in terms of implementing the emergency response effectively, it was important to provide appropriate assistance to the main control room as well as to secure the consistency of the chain of command through cooperation between the site emergency response organization and the main control room. From this perspective, the actions that were effective in the response at Fukushima Daini were as follows:

-The personnel with knowledge and experience in plant operations provided assistance to the operators in relation to technical assistance, liaison, and coordination.

-The liaison officers were dispatched from the site emergency response organization and stationed in the main control room so that the operators could concentrate on monitoring and operations, and the emergency response organization and the main control room could share information closely to secure the chain of command.

-The conditions of major safety facilities and the status of water injection into reactors were shared between the main control room and the site emergency response organization, and were also visibly posted in the emergency response organization so that all responders could maintain a common perception/understanding of events as they unfolded.

Next, as part the emergency response, the strategy of increasing the substitutability of response measures while constantly learning new information and predicting the changes was effective. With the response to a situation exceeding the anticipated conditions in mind, it is 
important to enhance the functions related to information analysis and strategy planning in the emergency response organization going forward.

In this regard, the following actions were taken at Fukushima Daini:

-The reporting and communication systems were maintained so that it was possible to understand the situation and predict changes based on the data, and it was ensured that such information could be shared between the site emergency response organization and the main control room.

-Concerning reactor water injection and containment vessel cooling, a strategy was devised whereby the substitutability of the response measures could be constantly increased based on an understanding of the situation and prediction of changes.

\section{Restoration Operations and the Lessons Learned}

\section{(1) On-site damage check}

In order to restore lost safety functions using limited human and material resources, the restoration operations should be prioritized and safely executed. For this purpose, the extent of damage at the site needs to be checked first. However, because the damaged area might have been flooded again due to tsunami waves that arrived repeatedly, dispatching the responders to the field immediately was delayed. After establishing the necessary means to secure safety, such as methods for collecting tsunami information, monitoring the situation at the site and the change in water level and issuing emergency evacuation instructions, the responders were dispatched to the damaged area around 10:00 pm. It took approximately seven hours after the tsunami hit for the responders to be dispatched to the field. In addition, in the on-site check conducted after that in the darkness, it was necessary to move carefully, avoiding scattered rubble and openings into the sea. Therefore, it took further time to reach the damaged facilities and check the situation. Including this, it took a total of seven more hours after the responders were dispatched to the field until the on-site check was completed.

Thus, as a lesson, it should be considered when designing emergency activities that ensuring the safety of responders and securing a route to the field under such an emergency situation could be difficult, and fully comprehending the damage situation can take considerable time.

In this way, the on-site check continued until around 5:00 am on March 12, and the information on damaged systems was collected at the site emergency response organization for analysis. When the components required to restore the A and B systems of the RHR of Units 1, 2 and 4 were compared, the power-supply facilities for auxiliary components were totally lost, and there was no difference between the two systems. However, as Table 1 shows, it was found that there were fewer pump motors that required replacement in the B system, and it was determined that the B system would be the subject of prioritized restoration. In addition, as part of the restoration method, it was determined by the site emergency response organization that the submerged pump motors would be replaced and temporary cables would be placed to supply electricity to the electric motors from the power panels that were not submerged or from mobile power vehicles.

\section{(2) Emergency procurement of restoration materials}

At the request of the site emergency response organization, the headquarters emergency response organization in Tokyo and Kashiwazaki Kariwa Nuclear Power Station (hereinafter referred to as "Kashiwazaki Kariwa") were in charge of the procurement and transportation of the materials required for restoration.

The major materials sent to Fukushima Daini were electric motors, power cables, mobile 
power vehicles, portable transformers, and light gas oil as the fuel for mobile power vehicles. All these materials, except for motors, could be procured relatively easily because they were highly versatile. However, for the three motors that needed replacement, it was necessary to find motors that were the same or that had similar specifications. For two out of the three motors, as suitable ones were found in the manufacturer's factory stock, three motors, including one spare motor, were shipped from Komaki Airport to Fukushima Airport by the Self-Defense Forces between 9:30 pm and 10:07 pm on March 12 and then carried by truck to arrive at Fukushima Daini at 6:33 am on March 13. For the remaining motor, because the one used at Kashiwazaki Kariwa was found to be suitable, two motors, including one spare motor, were uninstalled and then carried by truck to arrive at Fukushima Daini in the early morning of March 13.

In this way, the materials required for emergency restoration of the RHR were ready by 7:00 am on March 13. Land transportation took a long time because National Route 6 was cut off and the trucks were forced to detour due to landslides caused by the earthquake and inundation caused by the tsunami, the guide to the alternative route gave some incorrect directions resulting in a longer detour than necessary, and cell phones could not be used, making communication between the transportation team and the site emergency response organization difficult. Preparing for such situations is also an important lesson for the future.

The site emergency response organization asked the headquarters emergency response organization to arrange for the transportation of water to prevent running out of a source water for injection into the reactor, assuming a situation in which the restoration of the RHR would be delayed. However, this transportation did not come about because it was impossible to secure a way of transporting such a large amount of water. Although this problem was solved by the site emergency response organization, which decided to temporarily restore the system for taking subsoil water from the Kido river, it is necessary to fully consider which materials can be transported in an emergency, the transportation method of such materials, and the securement and storage of non-transportable materials at the power station in advance.

\section{(3) Restoration of RHR}

The full-scale restoration of the RHR was implemented on March 13, when the required materials were delivered from outside the power station. Using the road in the station, where the passage was ensured by the removal of rubble by heavy machinery the previous day, the required materials and equipment were carried into the site. In Units 1 and 4, the RHRC pump motors were replaced for cooling system B of the RHR. In addition, in Unit 1, the motor for a pump of the EECW was also replaced.

Furthermore, in parallel with these operations, the temporary restoration of the power system for operating the pumps was also conducted. Figure 8 shows the outline of the configuration of the temporary power system. In Units 1, 2 and 4, the power panels for supplying electricity to the pump motors of the RHRC, RHRS, and EECW, which were placed in the seawater heat-exchanger buildings (Hx Buildings), were totally broken by the tsunami. Therefore, the electricity was supplied to these motors from the power panels of Unit 3 and Rad-Waste Building, which were not underwater, or from the mobile power vehicles placed near the facilities via portable transformers. The temporary cables used for this restoration operation had a total length of approximately $9 \mathrm{~km}$ and were installed manually by approximately 200 people in one day on March 13.

After these operations were completed, the RHR of Unit 1 was restarted at 1:24 on March 14 to initiate heat removal from the reactor and the containment vessel. Finally, the cold shutdown of all reactors was completed by 7:15 on March 15 . 


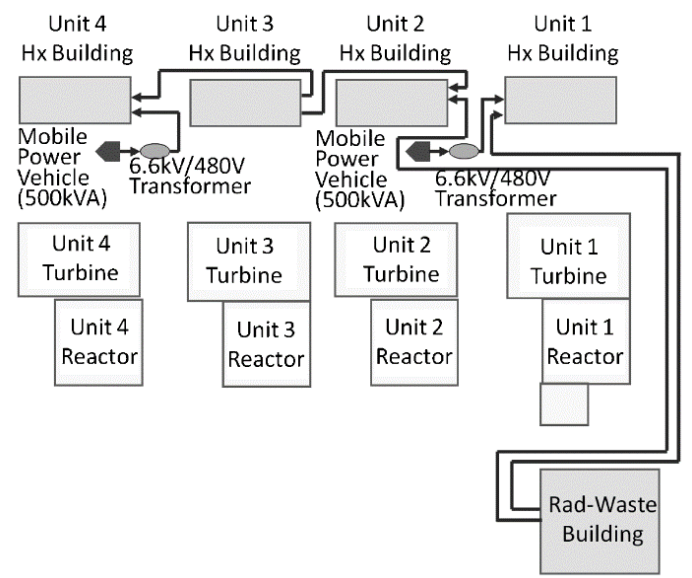

Figure 8 Layout of mobile power vehicles and temporary cables used for the recovery of cooling systems

Hx Building refers to the Heat Exchanger Building, where cooling facilities, including heat exchangers and pumps, are located.

\section{(4) Securing of stable cooling after restoration}

Although one system of the RHR was restored in each reactor and cold shutdown was achieved, there was a possibility that the means of heat removal would be lost again if the restored system failed because there was no spare heat-removal system in this situation. In addition, the possibility of deterioration in the insulation or a fire was also a concern because of the sea salt particles that had become attached to the electric facilities.

Therefore, while the restoration of standby facilities was being continuously undertaken, the vibration diagnosis, analysis of the components and cleanliness of lubricants, and diagnosis based on infrared thermography had been conducted very frequently on the restored facilities since March 14 to maintain their reliability. Among the pumps that were operable in spite of once being underwater, some experienced an increase in vibration in the vicinity of the bearings around May because of foreign materials contaminating the lubricant. Therefore, the bearings and lubricant in these pumps were replaced.

In addition, transportation of the materials for continuing the restoration work and the transportation and storage of fuel for the mobile power vehicles and service cars were needed, and some emergency response personnel were assigned to carry out these duties.

\section{(5) Summary of the lessons learned in the restoration work}

The important lessons learned in the restoration work are summarized as follows from the viewpoint of the operations and logistics supporting such operations:

The lessons learned in the restoration work are as follows:

-It is important that the condition of damaged facilities be verified in the on-site investigation and that the emergency response organization works on the restoration after clearly determining the priority of restoration operations.

-It is necessary to consider the means of securing safety in advance, assuming that on-site activities are taking place during an emergency.

-Considering that it may be impossible to dispatch responders to the damaged area at an early stage of the emergency and the on-site investigation and restoration activities may be restricted, it is important to take measures in advance to cope with the emergency even if 
on-site activities cannot be expected for a certain period of time after initial motion. In the example of Fukushima Daini, safety was temporarily secured by adopting an alternative means of water injection using a permanent facility. This measure provided sufficient time to perform restoration operations.

-It is important for the power station employees in charge of emergency response to have the skills required for emergency restoration work so that they can respond at any time, including on holidays/weekends and in the evenings.

-Facility diagnostic and repair techniques are required in order to operate the restored facilities stably for long periods after emergency restoration.

The lessons learned regarding logistics are as follows:

-Although the emergency procurement and transport could barely function as a result of flexible response, drastic improvement is required in the transportation system, as well as ascertaining the procurement and transport status and management of such information, and the means of communication between the transportation team and site emergency response organization.

-Concerning the activities conducted outside the power station, it is important to simulate the response to complex situations encountered in conjunction with natural disasters in advance.

-It is necessary to store important materials in the power station, assuming that the transportation of materials to the power station may be interrupted.

-It is necessary to prepare sufficiently for the transportation of materials in an emergency, such as organizational systems and radiation protection education for transportation personnel.

-It is necessary to establish the means to transport light gas oil and gasoline to the power station as well as to temporarily store and handle such dangerous materials in the power station in a safe manner

\section{Activities Supporting Long-Term Emergency Activities}

Although the cold shutdown of all reactors was achieved in Fukushima Daini on March 15, as described above, the activities needed to maintain a safe shutdown state continued for a long period afterward. The responders who stayed at the power station for emergency response were allowed to go home on a rotating basis starting on March 24, which was approximately two weeks after the occurrence of the event. However, even after that, many responders took turns sleeping at the power station continuously to prepare for unforeseen circumstances.

To support these long-term activities, it was necessary to cope with various issues.

First, most power station employees lived locally with their families and needed to confirm the safety of their family members and the status of their homes. Eighty-two percent of the employees lived within a twenty-kilometer radius of Fukushima Daiichi, and their families were required to evacuate. In addition, eight employees lost relatives due to the earthquake. Furthermore, forty-six percent of all employees experienced damage to their homes, ranging from total collapse to partial collapse. In order to continue emergency activities under such circumstances, it was essential to confirm the situations of the families and the condition of the homes of the responders so that necessary measures could be taken. However, it actually took ten days for all responders to contact their families; this needs to be improved further.

There were responders who developed sudden deafness or symptoms of depression due to the stress of emergency activities and required psychological support. In addition to an interview with an industrial physician and nurses, medical specialists were invited to the power 
station on April 16 and May 5 to conduct questionnaire investigations and interviews. As a result, among approximately 500 emergency responders of Fukushima Daini, more than 100 responders were diagnosed with post-traumatic stress disorder (PTSD), and periodic diagnoses were made by the medical specialists starting in May.

In addition, risk management from the aspect of hygiene was needed because many responders conducted activities and slept in the important seismically isolated building where the site emergency response organization was placed. In this form of risk management, the activities such as the procurement of food and drinking water, securing a water supply for toilets and showers through restoration of deep wells and water pipes, obtaining bedclothes, installation of laundry facilities, and management of household garbage were included.

Since almost none of the above responses had been prepared for or experienced before, they were undertaken flexibly by the teams in charge of general affairs in the emergency response organization. However, long-term emergency activities cannot be conducted without these responses, so it is necessary to take advantage of the above to prepare for the future.

\section{Reflection in the Emergency Management System}

\section{Issues in the Emergency Management System}

Although lessons were extracted from the emergency responses made at Fukushima Daini as described in the previous chapter, it is necessary to consider the way in which the entire emergency management system is implemented in order to take advantage of these lessons. This is because emergency activities are highly interconnected and management of the entire system is important in order to conduct activities effectively within a limited time and with limited human and material resources. In addition, it was revealed from the analysis described in the previous chapter that there were important functions that needed drastic enhancement, such as strategic and logistics functions, in order to cope with the situation exceeding the anticipated conditions.

The emergency organization of Fukushima Daini set up in this emergency situation was as shown in Figure 1. This organization is configured so that twelve functional teams exist in parallel under the chief of the headquarters of the site emergency response organization (Site Superintendent). It can be said that this structure enables each team to conduct activities in parallel and with the utmost promptness, according to the procedures and guides established in advance, in the case of an event within the range of the anticipated conditions. However, in situations exceeding the anticipated conditions where the procedures and guides cannot be applied without modification, it is difficult to cope with the changes in situation flexibly because the chief is required to manage a much wider range of issues alone. In Fukushima Daiichi, which had the same structure as shown in Figure 1, this problem surfaced as the situation there was far more severe than that experienced in Fukushima Daini.

As analyzed in the previous chapter, in the emergency response made at Fukushima Daini, it was important to initiate the response to the situation with limited information and limited human and material resources, enhance the situational awareness and respond to the situation while revising the response strategy. In other words, the function to develop and revise strategies (strategy-planning function) was important. However, these responses were made on an ad-hoc basis and were not assured as an emergency management system. In the structure shown in Figure 1, the functions were distributed so that information was collected by the information team, the plant behavior was predicted based on the information by the engineering team, the 
strategies related to operation were developed by the plant operation team, and the strategies for restoration were developed by the restoration team. Therefore, the functions to continuously conduct the activities such as the development of strategies and the revision of strategies through the analysis of the implementation status were not clearly determined. This needs to be improved first to realize an emergency management system which can cope with situations exceeding the anticipated conditions.

In addition, as a result of the analysis described in the previous chapter, it became clear that there were many issues to be solved concerning activities related to logistics and activities supporting long-term emergency initiatives. Because both are functions that have not yet been clearly defined, it is necessary to reflect the respective lessons related to these activities after positioning them clearly in the emergency management system.

\section{Application of the Incident Management System}

It is considered valuable to study the application of the Incident Command System ${ }^{4)}$ (ICS) developed in the United States for solving the issues concerning the emergency management system described in the previous section.

ICS is an emergency management system that was created in the US to elucidate how an emergency organization can function to enable activities in a scenario where conditions are constantly changing and under circumstances where it is not clear to what extent the situation will spread, such as wildfires and natural disasters. The effectiveness of its functions has been increased by reflecting the actual experience in disaster response, including the response to Hurricane Katrina in $2005^{5}$.

Figure 9 shows the basic configuration of the management functions of ICS. The management functions consist of the following four function modules under the Incident Command (commander) : Operations (execution team), Planning (intelligence and planning team), Logistics (logistics team), and Finance/Administration (finance and administration team). ICS is designed to cope flexibly with the changes in a situation by maintaining, reinforcing and reducing the personnel of each function module or the organizations in the module according to the situation.

The configuration of this management function is consistent with the strategy planning, logistics, and general affairs functions supporting long-term activities, which are identified as necessary based on the lessons learned at Fukushima Daini, and can be a good basis on which improvements can be considered. It is also worth considering as a management system that can handle situations exceeding the anticipated conditions because the functions are configured modularly to increase the ability to respond to changes in the situation.

However, there are no precedents in which ICS was applied to nuclear emergency

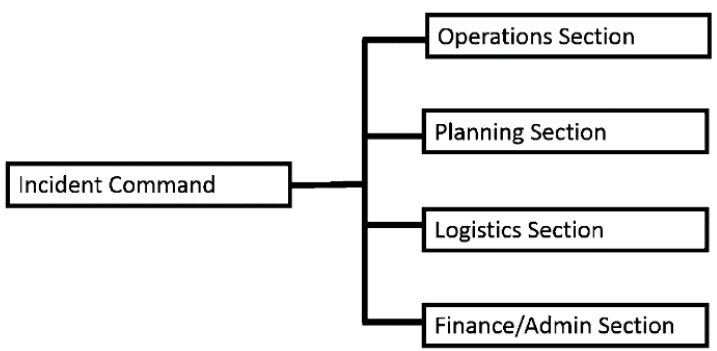

Figure 9 High-level functional structure of the Incident Command System 


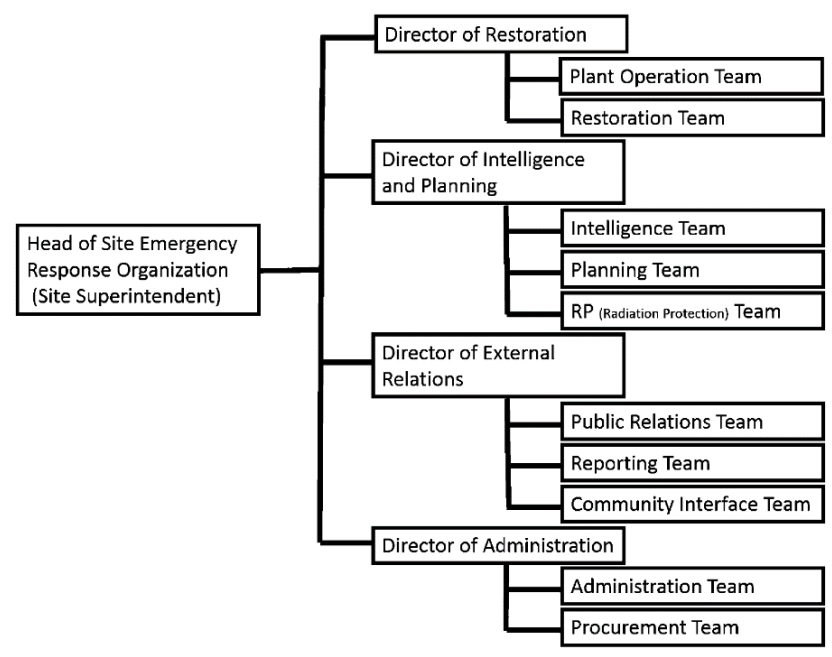

Figure 10 Functional structure of an improved site emergency response organization applying the ICS concept

management systems in the United States or Japan. Therefore, we considered the application of ICS from our own perspective, while considering the particular characteristics of nuclear emergency situations. Figure 10 shows the basic configuration of the nuclear emergency response functions to which ICS is applied. Under the head of the site emergency response organization, the persons responsible for the strategy-planning (intelligence and planning) function, execution function (such as operation and restoration), external contact (such as notifications to the central and local governments and dealing with the press), and general affairs function are positioned. This configuration basically follows ICS, but two changes were made so that it can function as a site emergency response organization.

The first change is that the external contact function was positioned as one team. This is because the activities in collaboration with the central and local governments and the activities related to the transmission of information via the press, etc., are important in nuclear emergency situations, as with a conventional nuclear emergency response organization.

The second change is that the logistics function is not positioned as a team in the site emergency response organization. This is to allow the site emergency response organization to focus on the activities conducted at the site. Instead, logistics is positioned as an important mission of the headquarters emergency response organization, which sets up the logistics bases in distant places outside the precautionary action zone (PAZ) and which is assigned the function to procure and transport necessary materials.

In the following sections, the direction of the reinforcement of respective functions are discussed reflecting the lessons learned at Fukushima Daini, based on the emergency management system to which ICS is applied.

\section{Intelligence and Planning Function (ICS's Planning Function)}

It is said that the important features of ICS's planning function are the collection, evaluation, and display of information; planning of response activities; management of human and material resources; and documentation.

In the emergency response made at Fukushima Daini, the collection, evaluation, and display 
of information had already been established as matters of primary importance. Specifically, the site emergency response organization dispatched the liaison officers to the main control room to prevent discrepant information from being collected. Then, in the site emergency response organization, the information on the condition of major safety facilities and the status of water injection into the reactors were summarized from the information conveyed from the main control room and that obtained from the safety parameter display system (SPDS), and it was written out and displayed. In this way, the operators at the main control room and the personnel of the response organization had a common and shared perception of the situation.

This is called a "common operation picture," and its information is determined by the configuration of plant facilities and emergency facilities. Therefore, the standard format of the information should be created in advance so that it can be displayed on paper and in electronic media. The SPDS is useful as a tool for sharing important plant parameters. In addition, the lessons learned at Fukushima Daini show the importance of sharing the information obtained by interpreting the parameters (e.g., the prediction of an increase in the containment vessel pressure), the information on the operability of the facilities, and the information on important restoration activities. Therefore, preparing the common operation pictures as a tool is considered to be very useful. Furthermore, standardizing especially important matters and the method of displaying them from the information shown on the whiteboards in emergency drills as the common operation picture enables continuous improvement.

Figure 11 shows an example of a common operation picture. It summarizes important information for understanding the accident conditions, such as the picture of a reactor and a containment vessel in the center, the conditions of the reactor and its control as well as the conditions of the control of the containment vessel temperature and pressure on the upper side, and the predicted time when the reactor water level reaches TAF as well as the predicted time when containment venting is required on the lower side. Such information was not only written out

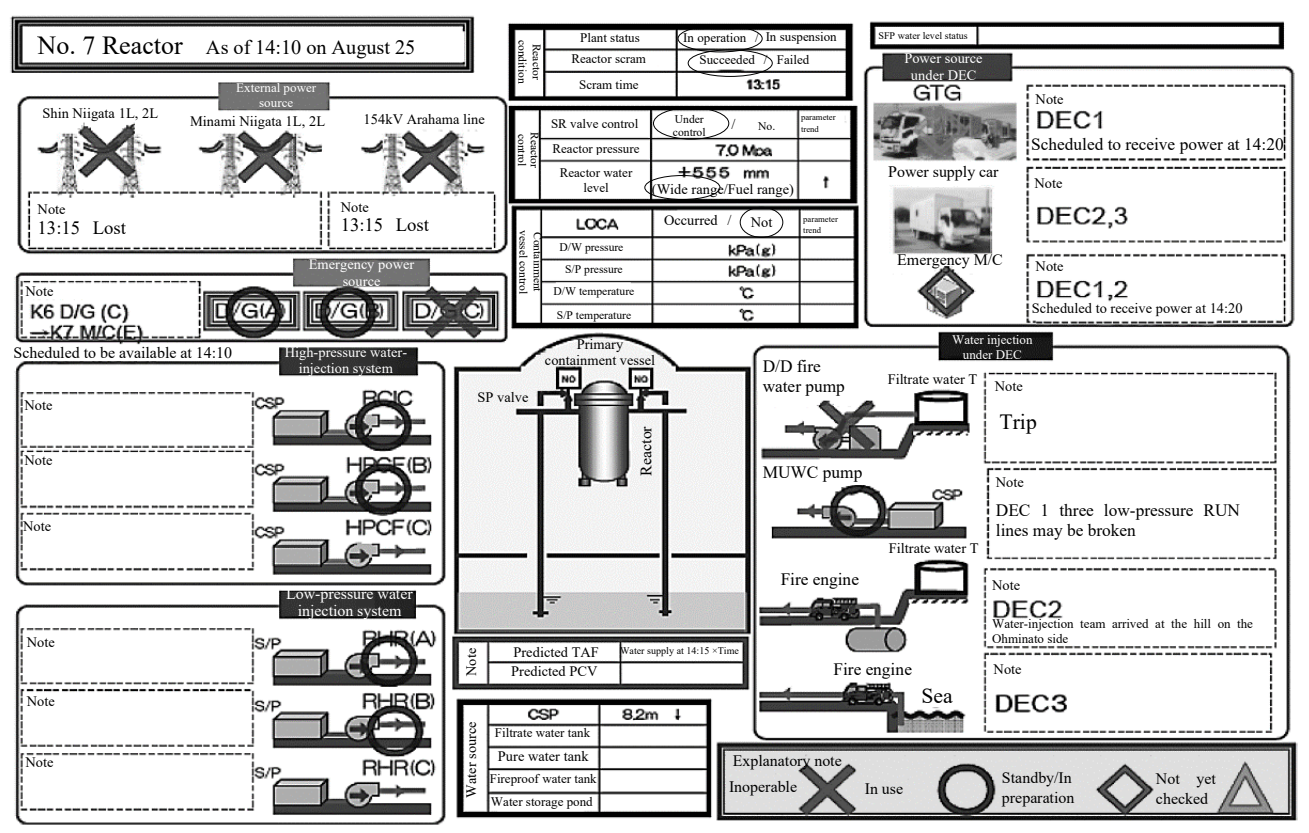

Figure 11 An example of a common operation schematic for information sharing among responders in a nuclear emergency 
on the whiteboard, but also distributed on paper so that it could be shared within the response organization of Fukushima Daini.

In the left portion of Figure 11, the information on the operability of the external power sources, emergency power sources, and high- and low-pressure water injection systems of the ECCS is summarized. The information on the operability of safety facilities was also written out, updated as needed, and shared at Fukushima Daini in a similar way as shown in Figure 2, and this also proved to be useful. In the right portion of Figure 11, the conditions that developed at major facilities related to power supply and water injection among severe accident-management facilities after the accident occurred at Fukushima Daiichi can be described. The word "DEC" in this figure represents the equipment that is usable under the design extension condition.

Furthermore, in terms of the information analysis and planning of response activities, in the emergency response made at Fukushima Daini, it was effective to constantly develop strategies that increased the substitutability of response measures while at the same time obtaining and understanding the information and predicting the changes. However, the engineering team shown in Figure 1 took charge of understanding the situations and predicting changes based on the plant parameters, the plant operation team took charge of the development of a response strategy in terms of operation, the restoration team was responsible for that strategy in relation to facility restoration, and the headquarters was responsible for making overall adjustments as needed. In order to reinforce this function further, a director should be assigned to the planning function to conduct information analysis and strategy planning in an integrated way.

In the meantime, the information team took charge of the acquisition of plant parameters and the collection of information on emergency response activities, which were utilized in information analysis as needed. It is preferable that this also be positioned and integrated as part of strategy planning.

In addition, as the lessons learned from the emergency response made at Fukushima Daini, it is important to plan strategies considering that on-site activities may initially be restricted for a certain period of time. It is preferable to prepare for the response by adopting a phased approach, where securing safety in the interim is enabled through a response using permanently installed facilities initially and then the substitutability of response measures is increased with the passage of time.

Figure 12 shows the concept of the phased approach, in which the emergency response is classified into three phases according to the time elapsed after the accident. In the first phase, mobile safety facilities cannot necessarily be expected due to constraints, such as limited human resources for on-site activities and the inability to dispatch responders to the field until their safety can be ensured. Therefore, the basic response should be conducted with permanently

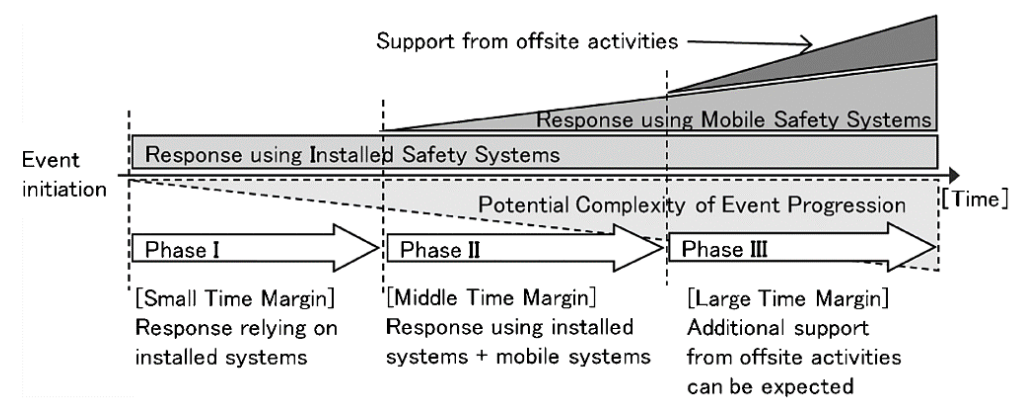

Figure 12 Concept of phased approach in emergency response 
installed facilities. In the emergency response made at Fukushima Daini, water injection into the reactors was maintained by using the RCIC and the MUWC first to secure temporary safety, which corresponds to the first phase.

In the second phase, restoration is promoted, utilizing materials such as the mobile facilities and spare items in the power station and measures for securing safety are added. In the third phase, the human and material resources from outside the power station are introduced to broaden the response as well as to ensure the continuity of safety securement. In the example of Fukushima Daini, because there were few materials and equipment available for restoration in the power station, the activities corresponding to the second phase could not be conducted sufficiently and the restoration activities corresponding to the third phase were conducted after the materials were delivered from outside the power station.

In addition, even if temporary safety is secured in the first phase, it is possible that secondary damage may spread as time passes, the safety facilities of the first phase may break down, a new problem may be found as a result of on-site investigation, etc. Therefore, it is important to continuously promote the diversification of response measures and expansion of substitutability during and after the second phase. In the example of Fukushima Daini, the response measures were added continuously in a scenario of spreading of flood damage, failure of restored safety facilities, and increase in the risk of electric fire.

The duration of each phase in an emergency situation differs depending on the situation of the accident. The strategies are developed in accordance with the phased approach concept, considering the prioritization of necessary activities. On the other hand, when designing the safety facilities or workforce configuration for emergency preparedness, it is necessary to set the estimated time of each phase as design conditions. It should be determined in consideration of important hazards, configuration of safety facilities and the geographical conditions of each power station. In the case of Fukushima Daini, it took half a day to gain access to the field and check the situation. Therefore, twelve hours after the occurrence of an accident could be a rough guide to the point at which the second phase can be expected to begin. In addition, considering the possibility that the areas outside the power station may also be in a confused state due to the influence of a significant disaster, seven days after a general disaster is considered to be a rough approximation of the amount of time that elapses before the third phase can begin.

\section{Operation and Restoration Execution Functions (Operation Functions of ICS)}

In the emergency response at Fukushima Daini, the main control room took charge of the strategy execution function from the viewpoint of operation and the restoration team took charge of this function from the viewpoint of facility restoration. In this emergency response, because the roles were divided, the operators operated undamaged facilities and the restoration personnel repaired damaged facilities, there were no special problems in their interfaces.

However, because the number of mobile safety facilities has been increasing under new regulatory standards and the number of important work-related interfaces, such as the installation of mobile facilities by the restoration personnel and the response operation utilizing such facilities by the operators, will increase, it is desirable that a director to execute functions be assigned to realize a more integrated response in the future.

In addition, as the lessons learned from the emergency response made at Fukushima Daini, the organization in charge of this execution function also needs to be prepared to ensure safety during emergency activities, such as securing working lights, communication means, and the 
removal of rubble to facilitate on-site activities. In addition, it is also necessary to enhance the skills required for emergency restoration work so that the response can be made at any time, including on holidays/weekends and in the evenings. Furthermore, it is also important to acquire component diagnosis and repair skills because restored facilities need to be operated stably for long periods after emergency restoration.

\section{Logistics Function}

In the emergency response made at Fukushima Daini, most logistics were not planned in advance, although they succeeded. Therefore, there are many lessons learned in this field, as follows:

First, most of the important restoration materials were procured through flexible response after the occurrence of the emergency situation. In order to enhance the ability to respond to situations exceeding the anticipated conditions, it is necessary to consider in advance the restoration materials required, in case the facilities important to safety are damaged, and prepare for their storage in the power station as well as the means of external procurement and transportation to the power station.

In the emergency response made at Fukushima Daini, difficulties were also experienced in the transportation of the restoration materials to the power station. It is necessary to develop a team with specialized logistics skills to enhance the response in terms of the transportation system, understanding procurement and transportation situations, the management of such information, and the means of communication between the transportation team and headquarters, etc.

In addition, especially when these activities are conducted outside the power station, the necessity of responding to complex situations due to a natural disaster needs to be assumed in advance. For example, in the emergency response made at Fukushima Daini, the transportation of restoration materials required for cold shutdown were delayed due to blockage of National Route 6 as a result of the earthquake and tsunami.

Furthermore, in order to assist restoration activities without interruption, it is necessary to develop the means of transporting light gas oil and gasoline to the power station as well as temporarily storing and safely handling these dangerous materials.

\section{General Affairs Function}

The general affairs function must also prepare for the possibility of a prolonged emergency response.

Based on the lessons learned in the emergency response at Fukushima Daini, the roles expected of the general affairs function include checking the safety of the emergency responders' families and the condition of their homes on their behalf, the construction of a mental care program, and the implementation of necessary measures in consideration of hygiene risks generated by the situation where many responders work and sleep in limited spaces. These matters were described in detail in Section 4 of the previous chapter.

\section{Conclusions}

Due to the tsunami caused by the Great East Japan Earthquake, Units 1, 2 and 4 at Fukushima 
Daini lost their heat-removal function completely, but the cold shutdown succeeded as a result of the restoration of the RHR through flexible activities. In this article, in order to reinforce the emergency management system of nuclear power stations, important success factors and issues that emerged during the emergency response were analyzed and extracted as lessons learned from Fukushima Daini, where an emergency situation exceeding the anticipated conditions was actually managed successfully.

As a result, it was found that the (1) strategy-planning function to develop strategies as well as to analyze the implementation status and revise the strategies, (2) logistics function, and (3) function to assist long-term emergency activities needed to be reinforced as the management system. Based on these findings, an emergency management system in which ICS was applied to the nuclear emergency response organization was suggested. ICS, which was developed in the United States mainly for disaster response, has achieved results in emergency activities under circumstances where it was not clear the extent to which the situation would spread. This is the first time that ICS has been applied to the management of nuclear emergencies in the US or Japan.

In addition, in applying ICS, the important points in the reinforcement of major functions were shown specifically, based on the lessons learned from the experience at Fukushima Daini. Examples of such important points are the clarification of who will be in charge of information analysis and strategy design; reinforcement of information sharing means including the utilization of a common operation picture; strategy design through a phased approach, clarification of who will be in charge of the execution of strategies such as operation of the plant, mobile facilities, and restoration activities; development of hands-on work skills among power station employees in charge of emergency response; development of a team with specialized logistics ability; improvement of material storage and procurement; and care for the personnel who are engaged in a long-term response as wells as care for their families.

It is expected that this improvement will enable a more flexible response to situations exceeding anticipated conditions. The nuclear emergency management system to which ICS was applied was introduced to Kashiwazaki Kariwa Nuclear Power Station and has been improved on a daily basis through training. The improvement under this management system has been producing good results in training sessions, which are conducted without notifying the participants of the scenario in advance and assume such a severe situation as the occurrence of multiple failures, which could lead to severe accidents occurring in several reactors. In such training programs, the site emergency response organization functioned properly to control the situations.

We intend to conduct training continuously on the management of nuclear emergencies utilizing ICS to further improve the ability to respond to the situations exceeding the anticipated conditions and to work on improving safety.

\section{References}

1) Tokyo Electric Power Company, Fukushima Nuclear Accident Investigation Report (2012).

2) Japan Nuclear Technology Institute, Tokyo Denryoku Fukushima Daini Genshiryoku Hatsudensyo Tohokuchiho Taiheiyo-oki Jishin oyobi Tsunami ni taisuru Taiojokyo no Chosa oyobi Chushutsusareru Kyokun ni Tsuite (2012). [in Japanese]

3) Atomic Energy Society of Japan, Fukushima Dai-ichi Genshiryoku Hatsudensho Jiko sono Zenbo to Asu ni Muketa Teigen, Maruzen-shuppan, Tokyo, ISBN 978-4-621-08743- 5(2014). [in Japanese]

4) Federal Emergency Management Agency, Introduction to the Incident Command System (ICS 100) (2010).

5) A. M. Howitt, H. B. Leonard, Managing Crises, CQ Press, Washington, D.C., ISBN 978-0-87289-570-6 (2009). 


\title{
Photon Spectra Measured above Operating Floor of Unit 3 Reactor at Fukushima Daiichi Nuclear Power Station
}

\author{
Yoshihiko TANIMURA ${ }^{1, *, \dagger}$, Hideo HIRAYAMA ${ }^{1,2}$, Kenjiro KONDO ${ }^{1,2}$, \\ Hiroshi NAGATA ${ }^{1}$, Kouhei IWANAGA ${ }^{1}$ and Seishirou SUZUKI ${ }^{1}$ \\ ${ }^{1}$ Secretariat of Nuclear Regulation Authority, 1-9-9 Roppongi, Minato-ku, Tokyo 106-8450, Japan \\ ${ }^{2}$ High Energy Accelerator Research Organization, 1-1 Oho, Tsukuba-shi, Ibaraki 305-0801, Japan
}

\begin{abstract}
Photon energy spectra were measured above the operating floor of unit 3 reactor at the Fukushima Daiichi Nuclear Power Station by using a CdZnTe semiconductor spectrometer. The spectrometer was installed in a lead collimator to measure the photons from the area directly below the detector. The collimator and spectrometer were lifted up by a huge crane and set above the operating floor. The photon spectra were derived by unfolding the pulse height spectra measured using the spectrometer. The response function of the spectrometer was calculated with the MCNP-4C code and was used as an input parameter of the unfolding code MAXED. It was found from the photon energy spectra that low-energy photons with energy below $0.4 \mathrm{MeV}$ were dominant above the operating floor. These spectra are fundamental data for evaluating the dose reduction effect by setting up shields on the operating floor.
\end{abstract}

KEYWORDS: photon energy spectrum, CdZnTe spectrometer, unfolding, Fukushima Daiichi Nuclear Power Station, unit 3 reactor, operating floor, ${ }^{137} \mathrm{Cs},{ }^{134} \mathrm{Cs}$

\section{Introduction}

The Unit 3 reactor at Fukushima Daiichi Nuclear Power Station was severely damaged by hydrogen explosions due to an accident attributed to the Great East Japan Earthquake that

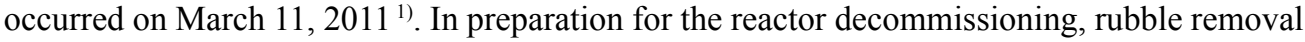
and decontamination have progressed at the operating floor on the fifth floor of the Unit 3 reactor (hereinafter referred to as "operating floor") and the removal of fuel from the spent fuel pool has been scheduled. For fuel removal, several tasks have been planned to be conducted remotely. However, some tasks need to be conducted through manned operations, such as the installation of the basement girder. Since the operating floor continues to produce a high dose rate due to the high-density contamination caused at the time of the explosion, dose reduction through decontamination measures have been implemented, such as peeling the floor surface on the assumption of surface contamination, and removing the rubble. However, sufficient

\footnotetext{
* Corresponding author, E-mail: tanimura.yoshihiko@jaea.go.jp

$\dagger$ Present, Japan Atomic Energy Agency

DOI : 10.15669 /fukushimainsights. Vol.4.495

(C) 2021 Atomic Energy Society of Japan. All rights reserved.

Originally published in Transactions of the Atomic Energy Society of Japan (ISSN 1347-2879), Vol. 15, No. 3, p.129-132

(2016) in Japanese. (Japanese version accepted: May 25, 2016)
} 
reduction has not yet been achieved. Therefore, the installation of a shield for further dose reduction is planned ${ }^{2)}$.

The Secretariat of the Nuclear Regulation Authority assumed that high-density contamination might be observed at places other than the surfaces, such as the lower surface of the shield plug located on top of the reactor, which could be a radiation source. If a place other than the surfaces is contaminated, the contribution of scattered rays with low energy will be increased by penetration through the concrete, etc. Therefore, we attempted to measure the energy spectra of the photon beams emitted upwards from the operating floor surface in order to confirm the assumption described above. The spectra thus obtained not only enable the determination of the exposure dose of workers in more detail, but also could be the basic data required to optimize the thickness and material of the shield to be installed.

\section{Measuring Method}

We used a CdZnTe semiconductor detector with a crystal size of $1 \mathrm{~cm} \times 1 \mathrm{~cm} \times 1 \mathrm{~cm}(\mathrm{GR}-1$ manufactured by Kromek) ${ }^{3)}$ for the measurement of photon beam energy spectra. By installing a CdZnTe semiconductor in a lead collimator (with a diameter of $0.8 \mathrm{~cm}$ ), we eliminated the photon beams entering from the periphery and measured only the photon beams emitted upwards from the operating floor surface. Figure 1 shows the layout of the collimator and detector.

The collimator was equipped with a tablet PC for data acquisition and mobile Wi-Fi router to measure the spectra, while monitoring the status remotely. The collimator and the detector were lifted with a 600 -t crane and moved to the measurement position on the operating floor of the Unit 3 reactor so that the sensitive volume of the CdZnTe detector was located at a height of approximately $50 \mathrm{~cm}$ above the operating floor surface. As typical samples of locations on the operating floor, 24 positions were selected, mainly on the shield plug on top of the reactor, where the dose rate is considered to be particularly high and a major radiation source, as shown in Figure 2. We conducted measurements at these 24 positions on November 19, 2015.

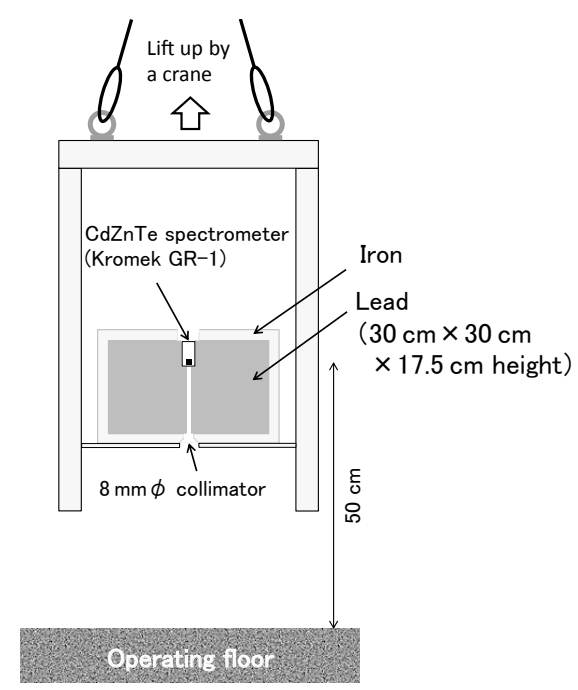

Figure 1 Schematic drawing of the lead collimator and the CdZnTe semiconductor spectrometer 

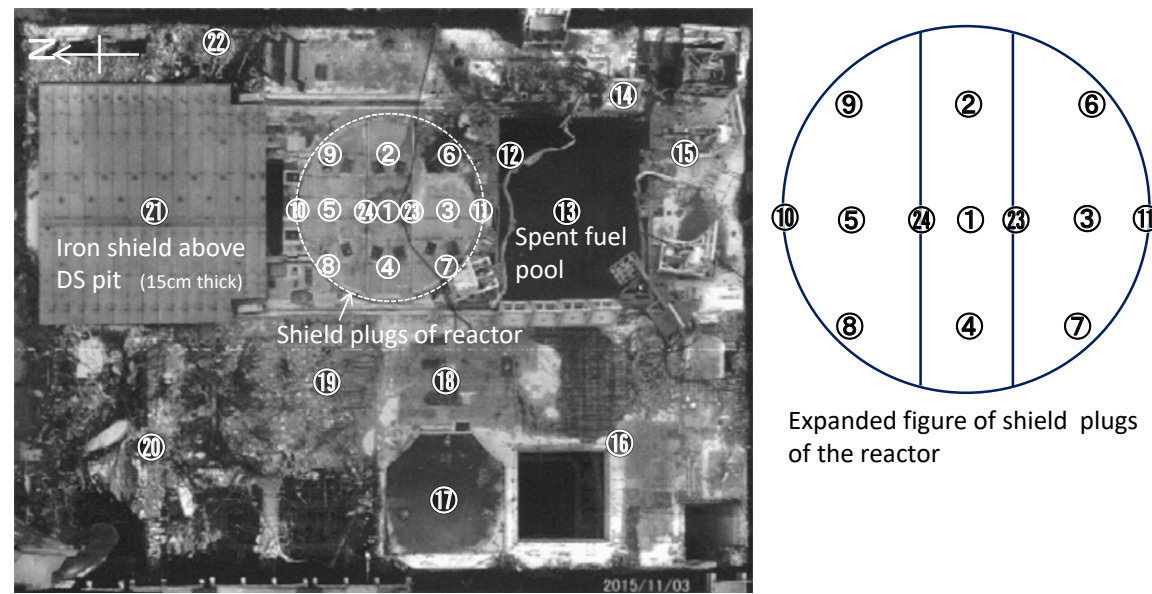

Expanded figure of shield plugs of the reactor

Figure 2 Measurement points of photon spectra above the operating floor of unit 3 reactor

\section{Measurement Results}

Figure 3 shows the pulse height distribution of the CdZnTe semiconductor detector measured at a height of $50 \mathrm{~cm}$ above the reactor shield plug, spent fuel pool and the iron shield plate on the DS pit. At the joint of the plug (Example: 24), etc.), the photoelectric peaks formed by the $0.662 \mathrm{MeV} \gamma$-rays from ${ }^{137} \mathrm{Cs}$ and the $0.605 \mathrm{MeV}$ and $0.8 \mathrm{MeV} \gamma$-rays from ${ }^{134} \mathrm{Cs}$ were observed. On the other hand, no photoelectric peak formed by $\gamma$-rays from radioactive Cs was observed on the spent fuel pool (13) and iron shield plate on the DS pit (21)), etc. In addition, at all measurement positions, no photoelectric peak formed by $\gamma$-rays from any radioactive nuclide, other than ${ }^{137} \mathrm{Cs}$ and ${ }^{134} \mathrm{Cs}$, was observed. From these results, it is estimated that the radiation dose on the operating floor is attributable to ${ }^{137} \mathrm{Cs}$ and ${ }^{134} \mathrm{Cs}$.

\section{Derivation of Photon Energy Spectra}

The photon energy spectra were derived by unfolding the pulse height distribution measured by the CdZnTe detector. The response of the detector, which was used in the unfolding procedure, was calculated as follows. First, a calculation model was created by simulating the structures of the CdZnTe semiconductor detector and collimator as closely as possible. Then, the response of the detector to the photons that passed through the collimator was calculated using the Monte Carlo code MCNP-4C ${ }^{4}$ ) and the model. Subsequently, the energy resolution of the $\mathrm{CdZnTe}$ semiconductor detector was evaluated using the pulse height distribution relative to the $\gamma$-rays from a ${ }^{137} \mathrm{Cs}$ radiation source in order to broaden the calculated response using the Gaussian distribution, which could reproduce the measured pulse height distribution ${ }^{\mathrm{a}}$. The response function of the $\mathrm{CdZnTe}$ semiconductor detector was created by changing the energy of incident photons by up to $1.5 \mathrm{MeV}$ at $10-\mathrm{keV}$ increments to calculate the pulse height distribution relative to the photon beams of a total of 150 energies.

\footnotetext{
${ }^{a}$ Compared to the photons entering from the front face of the detector (on the collimator side), the photons entering from the rear face (top) of the detector tend to cause lower resolution due to the scattering substances such as the electrical circuit. In this report, we created the response function assuming the photons entered from the front face.
} 


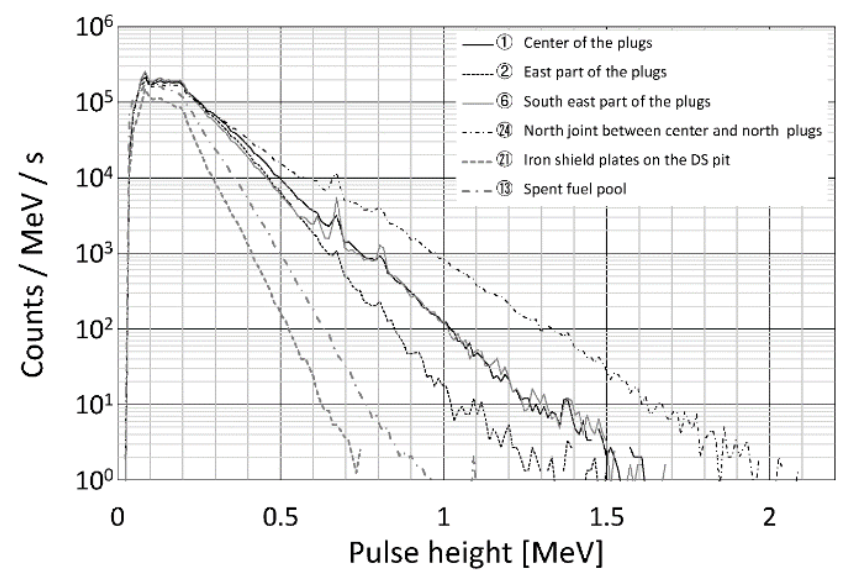

Figure 3 Pulse height spectra measured above the operating floor of unit 3 reactor

For unfolding the pulse height distribution, we used MAXED code, which was developed by PTB (Physikalisch-Technische Bundesanstalt, Germany) and included in the UMG-3.3 code package. MAXED code is based on the principle of the maximum entropy method and can estimate the photon energy spectrum, thus reproducing the measured pulse height distribution by using the above response function ${ }^{5}$. For the 23 positions excluding measurement position (11), where the influence of the pile-up could not be ignored due to the high counting rate, we derived the energy spectra of the photon beams that were emitted upwards from the operating floor surface as shown in Figure 4.

The vertical axis shows the fluence rate per unit area of the photons entering the CdZnTe semiconductor detector through the lead collimator with a diameter of $0.8 \mathrm{~cm}$.

\section{Discussion}

From the photon energy spectra shown in Figure 4, the contribution of the scattered rays with decreased energy of $0.4 \mathrm{MeV}$ or lower was large and the influence of the direct radiations from ${ }^{137} \mathrm{Cs}$ and ${ }^{134} \mathrm{Cs}$ was low at all positions. This result suggests that the contamination source is not only on the operating floor surface, but also in deeper areas, such as the lower part of the shield plug ${ }^{6}$. The dose reduction effect of the iron shield placed on the operating floor surface increases as the photon beam energy decreases. Therefore, when the thickness of the shield is determined based on the assumption of direct radiation with high energy, the dose reduction effect of the iron shield may be greater than expected in an environment where the contribution of scattered rays is large, as described above.

As shown in Figure 4, peaks were observed in the scattered rays at approximately $0.07 \mathrm{MeV}$ and $0.2 \mathrm{MeV}$, on and around the shield plug. The peak at $0.07 \mathrm{MeV}$ is caused by the characteristic X-rays of lead generated in the collimator. Table 1 shows the relation between the scattering angle based on Compton scattering and the energy of the scattered rays. As shown in Figure 1, since no shield plate for the CdZnTe semiconductor detector was placed on top of the collimator, this structure enabled photons scattering downwards in the air in the upper portion of the collimator to enter the detector. Among the scattering angles shown in Table 1, the angle of approximately $180^{\circ}$ corresponds to the position directly above the collimator, and the energy of the scattered rays is $0.18-0.2 \mathrm{MeV}$ in this case. Since this energy of the scattered 


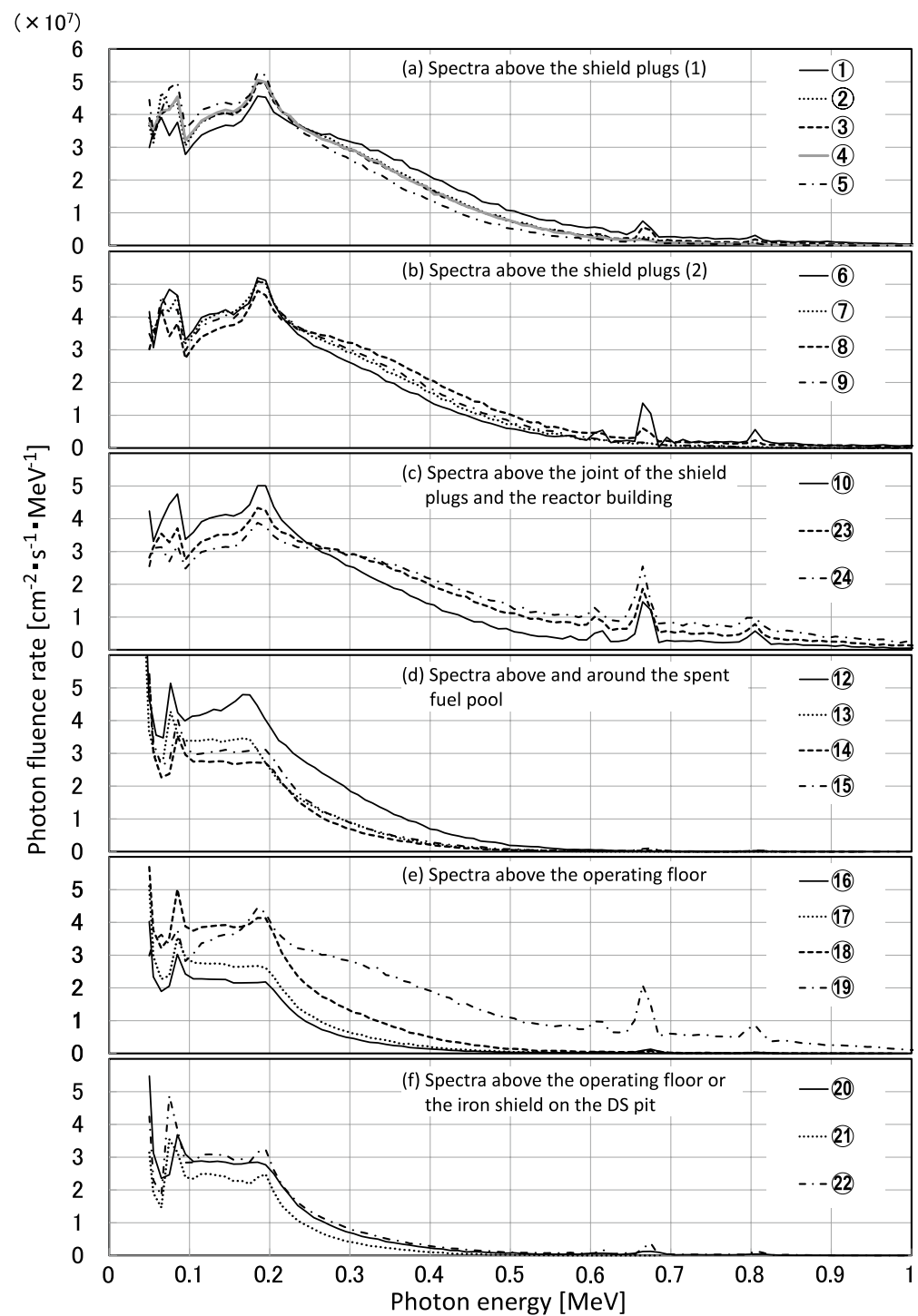

Figure 4 Photon energy spectra derived by unfolding the pulse height spectra measured above the operating floor of unit 3 reactor

Table 1 Relationship between the angle and the energy of Compton scattered photons

\begin{tabular}{cccccccc}
\hline $\begin{array}{c}\text { Scattering angle } \\
{[\text { degrees }]}\end{array}$ & 90 & 105 & 120 & 135 & 150 & 165 & 180 \\
\hline $\begin{array}{c}\text { Nuclide } \\
(\text { Energy })\end{array}$ & \multicolumn{7}{c}{ Scattered photon energy $[\mathrm{MeV}]$} \\
\hline $\begin{array}{c}{ }^{137} \mathrm{Cs} \\
(0.662 \mathrm{MeV}) \\
\begin{array}{c}{ }^{134} \mathrm{Cs} \\
(0.605 \mathrm{MeV}) \\
{ }^{134} \mathrm{Cs}\end{array} \\
(0.8 \mathrm{MeV})\end{array}$ & 0.288 & 0.252 & 0.225 & 0.206 & 0.194 & 0.187 & 0.184 \\
\hline
\end{tabular}


rays corresponds to the peak at approximately $0.2 \mathrm{MeV}$ observed in Figure 4, it would appear that the peak observed at approximately $0.2 \mathrm{MeV}$ is because of the photon beams scattered in the air above the collimator.

\section{Summary}

We measured the photon energy spectra on the operating floor of the Unit 3 reactor at Fukushima Daiichi Nuclear Power Station using a CdZnTe semiconductor detector and lead collimator. No photoelectric peak of the $\gamma$-rays from any radioactive nuclides, other than ${ }^{137} \mathrm{Cs}$ and ${ }^{134} \mathrm{Cs}$, was observed and it was estimated that radioactive Cs was the main source. In addition, from the analysis of the photon energy spectra derived from the response analysis of MCNP-4C code and the unfolding process with MAXED code, it was confirmed that the low-energy scattered component made a large contribution and the influence of direct radiation was low. The photon beam energy spectra obtained could be the basic data required for evaluating the dose reduction effect of a shield plate placed on the operating floor. In future studies, we will evaluate scattered rays in detail to estimate the location of the radiation source that contributes the most to the radiation dose based on the evaluation results.

We would like to express our deep gratitude for the advice and cooperation of the officials of the Secretariat of the Nuclear Regulation Authority, High Energy Accelerator Research Organization, Tokyo Electric Power Company, Toshiba and Kashima JV in this study.

\section{References}

1) Tokyo Electric Power Company, Fukushima Nuclear Accident Analysis Report, Tokyo Electric Power Company (20 Jun 2012). http://www.tepco.co.jp/en/press/corp-com/release/betu12_e/images/120620e0104.pdf

2) Tokyo Electric Power Company, Removal of nuclear fuel from spent fuel pool of unit 3 reactor at Fukushima Daiichi Nuclear Power Station, Handout for the 36th Commission on Supervision and Evaluation of the Specified Nuclear Facility (1 Jul 2015). [in Japanese]. http://www.nsr.go.jp/data/000112688.pdf

3) Kromek Group PLC, GR1 Spec Sheet; Revision 10, Kromek Group PLC (2015). http://www.kromek. com/index.php/products/nuclear-technology/czt/gr1-gamma-ray-spectrometer

4) J. F. Briesmeister (ed.), MCNP-A General Monte Carlo N-Particle Transport Code, Version 4C, LA13709-M, Los Alamos National Laboratory (2000).

5) M. Reginatto, P. Goldhagen, S. Neumann, "Spectrum unfolding, sensitivity analysis and propagation of uncertainties with the maximum entropy deconvolution code MAXED," Nucl. Instr Meth. A476, 242-246 (2002).

6) Secretariat of Nuclear Regulation Authority, Investigation of Radiation Sources on Operating Floor of unit 3 Reactor at Fukushima Daiichi Nuclear Power Station, Handout for the 38th Commission on Supervision and Evaluation of the Specified Nuclear Facility (18 Dec 2015). [in Japanese]. http://www. nsr.go.jp/data/000133830.pdf 


\title{
Article
}

\section{Estimation of Radionuclide Intakes by Singular Value Decomposition}

\author{
Shinji HATO ${ }^{1,2, *}$ and Sakae KINASE ${ }^{1,3}$ \\ ${ }^{1}$ Institute of Applied Beam Science, Graduate School of Science and Engineering, Ibaraki University, 2-1-1 Bunkyo, Mito-shi, \\ Ibaraki 310-8512, Japan \\ ${ }^{2}$ Visible Information Center, Inc., 440 Muramatsu, Tokai-mura, Naka-gun, Ibaraki 319-1112, Japan \\ ${ }^{3}$ Fukushima Environmental Safety Center, Japan Atomic Energy Agency, 2-4 Shirane, Shirakata, Tokai-mura, Naka-gun, \\ Ibaraki 319-1195, Japan
}

\begin{abstract}
It is important to accurately estimate the intake quantity for reliable internal exposure assessments. The intake quantity has been estimated by using the least-squares method. However, to use the least-squares method, the number of radioactivity measurements must be more than the number of intakes. To remedy this restriction, this study suggests an estimation method using singular value decomposition that is available regardless of the relation between the numbers of measurements and intakes. Moreover, this study introduces a procedure to calculate the intake quantity from the measurements with uncertainty.
\end{abstract}

KEYWORDS: internal exposure, intake estimation, radioactivity measurement, singular value decomposition

\section{Introduction}

Internal exposure occurs by the intake of radioactive materials into the human body and continues until the radioactive materials are excreted or attenuated by decay. Internal exposure can have a severe impact on the human body as the internal organs are directly exposed to the radiation. Therefore, it is necessary to consider the effects of not only strongly penetrating $\gamma$-rays, but also weakly penetrating radiation such as $\alpha$-rays emitted from the progeny nuclides produced during radioactive decay inside the human body, to evaluate their effects.

The effects of internal exposure on the human body are evaluated by the committed equivalent dose in the internal organs or the committed effective dose in the entire body accumulated over 50 years for adults and until 70 years old for children ${ }^{1)}$. These internal exposure doses are generally evaluated by multiplying the dose coefficient $[\mathrm{Sv} / \mathrm{Bq}]$, calculated using an internal exposure evaluation model devised by the International Commission on Radiological Protection (ICRP), by the intake quantity of radioactive material in the body [Bq]. Accordingly, to evaluate the internal exposure dose with a high degree of accuracy, not only the physicochemical characteristics (such as the particle size of the inhaled radioactive materials, their chemical

* Corresponding author, E-mail: hato@vic.co.jp

DOI : 10.15669/fukushimainsights.Vol.4.501

(C) 2021 Atomic Energy Society of Japan. All rights reserved.

Originally published in Transactions of the Atomic Energy Society of Japan (ISSN 1347-2879), Vol. 15, No. 3, p.146-150

(2016) in Japanese. (Japanese version accepted: March 14, 2016) 
form and the precise dose coefficient based on the exposure situation (the activity status of the examinee)) but also an evaluation of the precise intake quantity, become necessary.

The unknown intake quantity of radioactive materials due to an accident is estimated using the predicted radioactivity per unit intake and the measured radioactivity in the body or excretions after an accident. According to the ICRP publication 78 that provides a method for evaluating the internal exposure of workers ${ }^{2)}$, it is suggested that a single intake can be estimated by dividing the radioactivity measurement by the radioactivity prediction for a unit intake. Furthermore, in the IDEAS guidelines that describe the evaluation procedures for internal exposures ${ }^{3)}$, it is suggested that a single intake can be estimated from multiple measurements in which the uncertainly of measurement is considered. Generally, the intake estimation is obtained by fitting the radioactivity prediction structured with a linear combination of radioactivity prediction for the unit intake and the unknown intake quantity to the radioactivity measurement in the body or excretions. Accordingly, the estimated intake quantity is formulated as a least squares problem. The least squares method for solving this problem is only applicable when the intake frequency is lower than the number of radioactivity measurements, and there is a limit on the type of applications.

In this paper, a methodology using singular-value decomposition that allows estimation of the intake quantity using multiple intakes and multiple measurements with uncertainty is proposed. The results of the application of this estimation method to case examples in previous studies are reported.

\section{Internal Radioactivity Prediction Formula}

\section{Convolution Integration}

The internal exposure dose due to a single acute intake in the ICRP is evaluated by the multiplication of the intake quantity and dose coefficient. This dose coefficient indicates the internal exposure dose using the biokinetic model and the ICRP dose evaluation model, for the case where an acute intake of $1 \mathrm{~Bq}$ of radioactive nuclides occurred at an arbitrary age. The biokinetic model evaluates the metabolism of radioactive nuclides and predicts the radioactivity from an intake of $1 \mathrm{~Bq}$ (in the body or in excretions), i.e., the radioactivity prediction per unit intake. The internal radioactivity by chronic intake of radioactive nuclides is calculated by convolution integration on the intake rate and the radioactivity prediction per unit intake:

$$
a(T)=\int_{0}^{T} \dot{I}(t) \times q(T-t) d t
$$

where, $a(T)$ is the radioactivity prediction $[\mathrm{Bq}]$ at the age in days $T[d], \dot{I}(t)$ is the intake rate of radioactive nuclides $[\mathrm{Bq} / \mathrm{d}]$, and $q(T-t)$ is the radioactivity prediction $[\mathrm{Bq} / \mathrm{Bq}]$ per unit intake at the age of $T(T>t)$ in days when an intake occurred at the age of $t$ in days.

\section{Discretization of Integration}

By conducting convolution integration on the radioactivity prediction per unit acute intake and the intake rate of the radioactive nuclides, the radioactivity prediction of the chronic intake can be calculated as Eq. (1). Using the average intake quantity per day $\left(I\left(t_{j}\right)=\dot{I}\left(t_{j}\right) \Delta t_{j}, \Delta t_{j}=1\right)$, the integration of Eq. (1) is discretized: 


$$
a\left(T_{i}\right)=\sum_{j} q\left(T_{i}-t_{j}\right) I\left(t_{j}\right)
$$

Using Eq. (2), the prediction of radioactivity due to the chronic intake (multiple number of intakes) is evaluated based on the intake quantity expressed per the unit of one day and the prediction of radioactivity due to a single acute intake is acquired when $j=1$. Accordingly, it is considered that the intake quantity is acquired by solving Eq. (2), but it is not possible to solve as the number of unknown quantities (number of intakes) and the number of equations (number of measurements) do not necessarily match. Next, a methodology is considered for estimation of intake quantity based on the radioactivity measurements.

\section{Intake Quantity Estimation Method}

\section{Deriving the Intake Quantity Estimation Formula}

The intake quantity is estimated by fitting the radioactivity prediction of Eq. (2) to the radioactivity measurements. The fitting is conducted by the least squares method, determining the coefficient to minimize the sum of squares of residual difference of predictions and measurements. When considering the uncertainty, the maximum-likelihood method is employed, determining the coefficient in such a way that the largest value of the likelihood function is acquired. Using these two methods, the estimation of intake is possible by solving the following equation:

$$
\min \|\boldsymbol{Q}(\boldsymbol{I})-\boldsymbol{M}\|_{2}^{2}
$$

where, \|\|$_{2}$ is the two-norm. For a measurement without uncertainty, $\boldsymbol{Q}(\boldsymbol{I})=\boldsymbol{Q} \cdot \boldsymbol{I}$ is determined and expressed with the matrix $\boldsymbol{Q}$ (component: $q\left(T_{i}-t_{j}\right)$ ), intake vector $\boldsymbol{I}$ (component: $\boldsymbol{I}\left(t_{j}\right)$ ), and measurement vector $\boldsymbol{M}$ (component: $\mathrm{m}\left(T_{j}\right)$ ). On the other hand, for a measurement with uncertainty, the component of $\boldsymbol{Q}(\boldsymbol{I})$ is $\left(\ln \left(\Sigma_{j} q\left(T_{i}-t_{j}\right) I\left(t_{j}\right)\right) / \ln \left(S F_{i}\right)\right.$, and the measurement vector $\boldsymbol{M}$ is (component: $\left.\ln \left(m\left(T_{i}\right)\right) / \ln \left(S F_{i}\right)\right) . S F_{i}$ represents a scattering factor ${ }^{3)}$ that indicates the uncertainty of the radioactivity measurement and corresponds to the geometric standard deviation in the logarithmic normal distribution.

For a measurement without uncertainty, Eq. (3) is a linear equation in terms of the intake vector $\boldsymbol{I}$. Therefore, the minimum intake vector $\boldsymbol{I}$ is acquired by solving the normal equation. For a measurement with uncertainty, Eq. (3) is not linear in terms of the intake vector $I$. Therefore, the intake vector $\boldsymbol{I}$ cannot be acquired as it is. Hence, Taylor expansion of $\boldsymbol{Q}(\boldsymbol{I})$ is conducted on the trial solution $\boldsymbol{I}^{k}$ and by approximating to the first order term $\left(\boldsymbol{Q}\left(\boldsymbol{I}^{k}+\Delta \boldsymbol{I}\right) \approx \boldsymbol{Q}\left(\boldsymbol{I}^{k}\right)+J\left(\boldsymbol{I}^{k}\right)\right.$, where $\Delta \boldsymbol{I}$ and $\boldsymbol{J}\left(\boldsymbol{I}^{k}\right)$ are the Jacobian matrixes to $\left.\boldsymbol{Q}(\boldsymbol{I})\right)$. Eq. (3) then becomes:

$$
\begin{aligned}
& \min \left\|\boldsymbol{J}\left(\boldsymbol{I}^{k}\right) \boldsymbol{I}^{k+1}-\hat{\boldsymbol{M}}\left(\boldsymbol{I}^{k}\right)\right\|_{2}^{2} \\
& \boldsymbol{I}^{k+1}=\boldsymbol{I}^{k}+\Delta \boldsymbol{I} \\
& \hat{\boldsymbol{M}}\left(\boldsymbol{I}^{k}\right)=\boldsymbol{M}-\boldsymbol{Q}\left(\boldsymbol{I}^{k}\right)+\boldsymbol{J}\left(\boldsymbol{I}^{k}\right) \boldsymbol{I}^{k}
\end{aligned}
$$

Eq. (4) is a linear equation with regard to $\boldsymbol{I}^{k+1}$, therefore, the minimum $\boldsymbol{I}^{k+1}$ is derived by solving the following normal equation: 


$$
\left(\boldsymbol{J}\left(\boldsymbol{I}^{k}\right)^{T} \boldsymbol{J}\left(\boldsymbol{I}^{k}\right)\right) \boldsymbol{I}^{k+1}=\boldsymbol{J}\left(\boldsymbol{I}^{k}\right)^{T} \hat{\boldsymbol{M}}\left(\boldsymbol{I}^{k}\right)
$$

Starting from a trial solution and by applying Eq. (5) repeatedly to successively update the intake vector, the most appropriate intake vector $\boldsymbol{I}$ is acquired. However, the solution is only available when the inverse matrix $\left(\boldsymbol{J}\left(\boldsymbol{I}^{k}\right)^{T} \boldsymbol{J}\left(\boldsymbol{I}^{k}\right)\right)^{-1}$ exists. An inverse matrix exists when the number of intakes (unknown number) is equal to or lower than the number of measurements (number of equations).

Further, a method is considered to solve Eq. (5) regardless of the dimensional relationship between the number of intakes and measurements.

\section{Inverse Analysis by Singular-Value Decomposition}

A singular-value decomposition of an arbitrary matrix $\mathrm{m} \times \mathrm{n}$ called matrix $\boldsymbol{A}$ is expressed as follows:

$$
\boldsymbol{A}=\boldsymbol{U} \boldsymbol{S} \boldsymbol{V}^{T}
$$

where matrix $\boldsymbol{U}$ is an $\mathrm{m} \times \mathrm{m}$ matrix consisting of column vectors of an orthonormal basis in the m-dimensional space, matrix $\boldsymbol{V}$ is an $\mathrm{n} \times \mathrm{n}$ matrix consisting of column vectors of an orthonormal basis in the n-dimensional space, and matrix $\boldsymbol{S}$ is an $\mathrm{m} \times \mathrm{n}$ diagonal matrix with singular values ( $s_{1} \geq \ldots \geq s_{i p} \geq 0, p$ : number of positive singular values) on its diagonal line. If the Jacobian matrix $\boldsymbol{J}$ of $\mathrm{m} \times \mathrm{n}$ in Eq. (5) is replaced by $\boldsymbol{J}=\boldsymbol{U} \boldsymbol{S} \boldsymbol{V}^{T}$ and coordinated using $\boldsymbol{U} \boldsymbol{U}^{T}=$ $\boldsymbol{U}^{T} \boldsymbol{U}=\boldsymbol{E}$ and $\boldsymbol{V} \boldsymbol{V}^{T}=\boldsymbol{V}^{T} \boldsymbol{V}=\boldsymbol{E}(\boldsymbol{E}$ : Unit matrix $)$, the following equation is obtained:

$$
\boldsymbol{I}^{k+1}=\boldsymbol{V}\left(\boldsymbol{S}^{T} \boldsymbol{S}\right)^{-1} \boldsymbol{S}^{T} \boldsymbol{U}^{T} \hat{\boldsymbol{M}}
$$

Considering matrix $\boldsymbol{S}^{T} \boldsymbol{S}$ being an $\mathrm{n} \times \mathrm{n}$ diagonal matrix, Eq. (7) is expressed as the following equation:

$$
\boldsymbol{I}^{k+1}=\sum_{i=1}^{p} \frac{\left(\boldsymbol{U}_{\cdot, i}\right)^{T} \hat{\boldsymbol{M}}}{s_{i}} \boldsymbol{V}_{\cdot, i}
$$

The calculation of the singular-value decomposition is possible with an arbitrary $\mathrm{m} \times \mathrm{n}$ matrix. Therefore, using Eq. (8), the estimation of the intake quantity is possible regardless of the dimensional relationship between the number of intakes $n$ and the number of measurements $\mathrm{m}$. Furthermore, the estimation of the intake quantity is possible using Eq. (8), not only for measurements with uncertainty, but also for measurements without uncertainty.

When the number of measurements $m$ is equal to or greater than the number of intakes $\mathrm{n}(\mathrm{m} \geq \mathrm{n})$, the result of the inverse analysis using the above singular-value decomposition is equal to the result using the least squares method. This is not the case for the opposite condition $(\mathrm{m}<\mathrm{n})$. The evaluation will be in the direction from the space of lower dimensions (m-dimensional space) to that of higher dimensions (n-dimensional space) that includes a certain bias on the inverse analysis. This bias can be determined with the difference between the intake quantity acquired by an inverse analysis considering the evaluated radioactivity prediction and through a direct analysis from specific intake quantities (e.g., 2.0 Bq, etc.) as the measured values and the original specific intake quantity. 


\section{Application Examples of Intake Estimations}

\section{Measurements without Uncertainty}

As an example of intake estimation applied to measurements without uncertainty, a case of inhalation of radioactive iodine by a worker described in a report from the International Atomic Energy Agency (IAEA) ${ }^{5)}$ was evaluated. In this report, thyroid radioactivity measurements of $480 \mathrm{kBq}$ and $440 \mathrm{kBq}$ obtained respectively on Wednesday and Thursday after inhalation of ${ }^{131} \mathrm{I}$ aerosol (AMAD $5 \mu \mathrm{m}$, Type-F) by a worker are presented.

The predicted thyroid radioactivity per unit intake required for estimating the intake quantity was determined as shown in Table 1. This example involves a case where the uncertainty of measurement is not considered, and the $\boldsymbol{Q}(\boldsymbol{I})$ in the Eq. (3) was expressed as a product of matrix $\boldsymbol{Q}$ listing the predicted thyroid radioactivity per unit intake and the intake quantity $\boldsymbol{I}$. Accordingly, the Jacobian matrix $\boldsymbol{J}$ became matrix $\boldsymbol{Q}$, and the intake quantity was calculated by the singular-value decomposition of matrix $\boldsymbol{Q}$ and Eq. (8). The estimation results are presented in Table 2. The first intake was estimated as the maximum of the three intakes. On the other hand, IAEA estimated the average of the three intakes, and the total intake quantity was determined to be $4,299 \mathrm{kBq}$. This is lower than our estimated total intake of $4.425 \mathrm{kBq}$ by approximately $2.8 \%$. The intake quantity on Friday, Monday, and Tuesday was calculated by inverse analysis using a predicted value acquired by direct analysis based on the assumption that if the intake quantity was $2.00 \mathrm{~Bq}$, each of the predicted values would be $1.70 \mathrm{~Bq}, 2.05 \mathrm{~Bq}$ and $2.17 \mathrm{~Bq}$ respectively. This result explains that a bias was added through inverse analysis. The predicted thyroid radioactivity calculated from the estimated result of the intake quantity is shown in Figure 1. The predicted values correspond to the measurements.

This example involved a problem of having to estimate the three intakes from two measurements and it could not be solved using the least squares method. However, by adopting this estimation method using Eq. (8), it was possible to solve this issue regardless of the dimensional relationship between the number of intakes and measurements.

Table 1 Predicted values per unit intake for inhalation of ${ }^{131} \mathrm{I}$ by worker

\begin{tabular}{cc}
\hline Time after intake $[\mathrm{d}]$ & AMAD $5 \mu \mathrm{m}($ Type $\mathrm{F})$ Thyroid $[\mathrm{Bq} / \mathrm{Bq}]$ \\
\hline 1 & $1.222 \times 10^{-1}$ \\
2 & $1.187 \times 10^{-1}$ \\
3 & $1.084 \times 10^{-1}$ \\
4 & $9.866 \times 10^{-2}$ \\
5 & $8.976 \times 10^{-2}$ \\
6 & $8.168 \times 10^{-2}$ \\
\hline
\end{tabular}

Table 2 Estimated intakes for inhalation of ${ }^{131} \mathrm{I}$ by worker

\begin{tabular}{cccc}
\hline \multirow{2}{*}{ Time after intake } & Estimated intakes $[\mathrm{Bq}]$ & \multicolumn{2}{c}{ Thyroid } \\
\hline Friday & $1.85 \times 10^{6}$ & & \\
Monday & $1.25 \times 10^{6}$ & & \\
Tuesday & $1.33 \times 10^{6}$ & & \\
Wednesday & & $4.80 \times 10^{5}$ & $4.76 \times 10^{5}$ \\
Thursday & & $4.40 \times 10^{5}$ & $4.44 \times 10^{5}$ \\
\hline
\end{tabular}




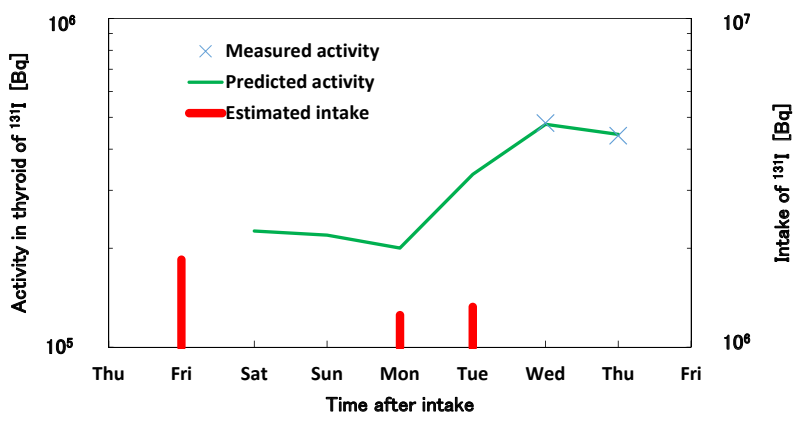

Figure 1 Predicted activity for inhalation of ${ }^{131} \mathrm{I}$ by worker

Table 3 Measured values of ${ }^{137} \mathrm{Cs}$ for worker

\begin{tabular}{cc}
\hline Time of measurement after intake $[\mathrm{d}]$ & Whole body $[\mathrm{Bq}]$ \\
\hline 1 & $8.8 \times 10^{4}$ \\
8 & $6.0 \times 10^{4}$ \\
15 & $3.0 \times 10^{4}$ \\
17 & $9.3 \times 10^{4}$ \\
30 & $8.3 \times 10^{4}$ \\
46 & $4.8 \times 10^{5}$ \\
50 & $4.6 \times 10^{5}$ \\
60 & $4.4 \times 10^{5}$ \\
\hline
\end{tabular}

\section{Measurements with Uncertainty}

A case of intake of ${ }^{137} \mathrm{Cs}$ by a worker shown in the IDEAS guidelines ${ }^{3)}$ was evaluated as an example of applying intake estimation to measurements with uncertainty. The worker (35-year-old male) was sprayed with liquid ${ }^{137} \mathrm{Cs}$ on his face when removing it from a bottle. The measured radioactivity on the entire body after exposure is presented in Table 3. The scattering factor was estimated as 1.2 for each measurement, and it was assumed that ingestion had occurred. The measurement taken on the 15th day was lower than the other measurements. It was therefore determined to be an outlier and not considered for evaluation. After the first intake, a second intake was assumed from an increase in the measurements taken between the 30th and 46th day, and the median day, the 38th, was considered as the day of intake.

The predicted radioactivity on the entire body per unit intake required for an intake estimation was evaluated, as presented in Table 4. As this example represents a measurement with uncertainly, and $\boldsymbol{Q}(\boldsymbol{I})$ from Eq. (3) was expressed using the predicted radioactivity on the entire body per unit intake, intake quantity, and logarithmic function of uncertainty. Accordingly, the intake quantity was calculated by performing singular-value decomposition from the acquired Jacobian matrix $\boldsymbol{J}$ and by the repeated application of Eq. (8). The estimated results are presented in Table 5. On the other hand, according to the IDEAS guidelines estimation, values of $93,988 \mathrm{~Bq}$ on the 0 th day and $479,100 \mathrm{~Bq}$ on the 38 th day were determined, corresponding to smaller values than ours by approximately $1 \%$. Furthermore, the intake quantity calculated with the predicted values using direct analysis based on an assumption of $2.00 \mathrm{~Bq}$ for the first and second intake quantities was $2.00 \mathrm{~Bq}$ and $2.00 \mathrm{~Bq}$, respectively, demonstrating the absence of bias in an inverse analysis. The predicted radioactivity on the entire body evaluated from the estimation result is presented in Figure 2. The predicted values match the trend of measurements. 
Table 4 Predicted values per unit intake for ingestion of ${ }^{137} \mathrm{Cs}$ by worker

\begin{tabular}{cc}
\hline Time after intake $[\mathrm{d}]$ & Whole body $[\mathrm{Bq} / \mathrm{Bq}]$ \\
\hline 1 & $9.801 \times 10^{-1}$ \\
8 & $8.596 \times 10^{-1}$ \\
12 & $8.317 \times 10^{-1}$ \\
15 & $8.146 \times 10^{-1}$ \\
17 & $8.040 \times 10^{-1}$ \\
22 & $7.785 \times 10^{-1}$ \\
30 & $7.398 \times 10^{-1}$ \\
46 & $6.682 \times 10^{-1}$ \\
50 & $6.514 \times 10^{-1}$ \\
60 & $6.112 \times 10^{-1}$ \\
\hline
\end{tabular}

Table 5 Estimated intakes for ingestion of ${ }^{137} \mathrm{Cs}$ by worker

\begin{tabular}{cccc}
\hline Time after intake [d] & Estimated intakes [Bq] & \multicolumn{2}{c}{ Whole body } \\
\hline 0 & $9.50 \times 10^{4}$ & & \\
1 & & $8.8 \times 10^{4}$ & $9.31 \times 10^{4}$ \\
8 & & $6.0 \times 10^{4}$ & $8.16 \times 10^{4}$ \\
15 & $3.0 \times 10^{4 a)}$ & $7.74 \times 10^{4}$ \\
17 & & $9.3 \times 10^{4}$ & $7.64 \times 10^{4}$ \\
30 & & $8.3 \times 10^{4}$ & $7.03 \times 10^{4}$ \\
38 & & \\
46 & $4.85 \times 10^{5}$ & $4.8 \times 10^{5}$ & \\
50 & & $4.6 \times 10^{5}$ & $4.80 \times 10^{5}$ \\
60 & & $4.4 \times 10^{5}$ & $4.65 \times 10^{5}$ \\
\hline
\end{tabular}

a) outlier

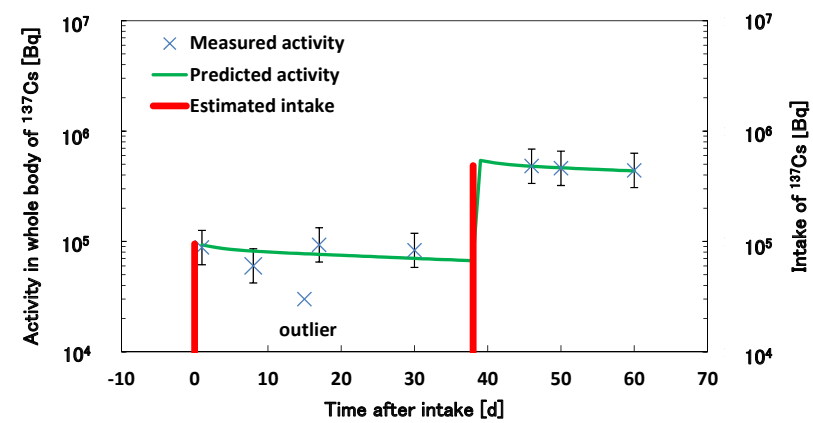

Figure 2 Predicted activity for ingestion of ${ }^{137} \mathrm{Cs}$ by worker

In this case, multiple intakes were evaluated considering the uncertainty of the measurements represented by the scattering factor. Using this estimation method, the evaluation of multiple intakes was possible for measurements with uncertainty, similarly to single intakes. 


\section{Conclusions}

To conduct a highly reliable dose evaluation of internal exposure, it was required to adequately estimate the level of intake. When an unexpected intake of radioactive materials occurs, the intake quantity is unknown and is therefore estimated by inverse calculation from the internal radioactivity or excretions, measured after intake. Estimation of the intake quantity was conducted by fitting the predicted radioactivity to the measured radioactivity values and was formulated as a least squares problem. To employ the least squares method, the number of intakes must be lower than the number of measurements. From the ICRP ${ }^{2)}$ and IDEAS ${ }^{3)}$ guidelines, only a single intake was considered, and the estimation of multiple intakes was not sufficiently conducted. Using the intake estimation method with singular-value decomposition in this research, estimation was possible regardless of the dimensional relationship between the number of intakes and the number of measurements. The effectiveness of this estimation method was demonstrated by applying it to case examples of intakes from previous studies.

Generally, for a measurement with uncertainty, the uncertainty is propagated to the results with inverse analyses. Accordingly, when the reliability of the analysis results are considered, it is essential to evaluate the propagation of uncertainty. Herein, a method permitting the evaluation regardless of dimensional differences between the number of measurements and the number of intakes was proposed. It will be necessary to consider the propagation of uncertainty to target evaluations with a higher degree of accuracy in the future.

\section{References}

1) ICRP, “1990 Recommendations of the international commission on radiological protection," ICRP Publication 60, Ann. ICRP 21 (1-3) (1991).

2) ICRP, "Individual monitoring for internal exposure of workers," ICRP Publication 78, Ann. ICRP 27 (3-4) (1997).

3) C. M. Castellani, J. W. Marsh, C. Hurtgen, E. Blanchardon, P. Berard, A. Giussani, M. A. Lopez, "IDEAS guidelines (version 2) for the estimation of committed doses from incorporation monitoring data," EURADOS Rep. 2013-01 (2013).

4) R. C. Aster, B. Borchers, C. H. Thurber, "Parameter estimation and inverse problems," Elsevier Academic Press ISBN: 0123850487 (2012).

5) IAEA, "Methods for assessing occupational radiation doses due to intakes of radionuclides," Safety Rep. Series No. 37 (2004). 


\title{
Article
}

\section{Development of an Organic Iodine Filter for Filtered Containment Venting Systems of Nuclear Power Plants}

\author{
Shinichi KAWAMURA ${ }^{1, *}$, Takeo KIMURA ${ }^{1}$, Fumitoshi WATANABE ${ }^{2}$, \\ Kazuki HIRAO ${ }^{2}$ and Tadashi NARABAYASHI ${ }^{3}$ \\ ${ }^{1}$ Nuclear Asset Management Department, Tokyo Electric Power Company Holdings, 1-1-3 Uchisaiwai-cho, Chiyoda-ku, \\ Tokyo 100-8560, Japan \\ ${ }^{2}$ R\&D Department, TEPCO Research Institute, Tokyo Electric Power Company Holdings, 4-1 Egasaki-cho, Tsurumi-ku, \\ Yokohama 230-8510, Japan \\ ${ }^{3}$ Division of Energy and Environmental Systems, Graduate School of Engineering, Hokkaido University, Kita-13, Nishi-8, Kita-ku, \\ Sapporo 060-8628, Japan
}

\begin{abstract}
A design for an organic iodine filter was developed for filtered containment venting systems of nuclear power plants. After the Fukushima Daiichi Nuclear Accident, filtered containment venting systems were developed and installed at nuclear power plants in Japan employing aerosol filters, which are typically combinations of alkaline water scrubbers and metal fiber filters. Using silver zeolite, the newly designed filter absorbs organic iodine, which cannot be captured with alkaline water scrubbers and metal fiber filters. Methyl iodide absorption performance tests were conducted with various gas velocities and compositions, which covered the operating conditions of filtered containment venting systems. The test results demonstrated that the decontamination factors of the silver zeolite were over 50 if an appropriate gas contact time was ensured, even though steam condensation and the existence of hydrogen during vent startup had negative effects on the absorption performance. It was also revealed from the tests that intermittent venting might dampen the silver zeolite and reduce its absorption performance. Flow control of the vent gas was found to be an effective countermeasure to maintain the performance even under such conditions. Filter unit tests and numerical analyses of the gas flow within the filter vessel confirmed that the gas contact time was uniform in the filter and appropriate for achieving a decontamination factor of 50 .
\end{abstract}

KEYWORDS: accident, filtered containment venting system, nuclear power plant, primary containment vessel, iodine, decontamination factor, performance, experiment

\section{Introduction}

In the accident at the Fukushima Daiichi Nuclear Power Plant, the reactor core melted down due to cooling failure resulting from the damage caused by the tsunami after the shutdown of the reactor. Part of the primary containment vessel (hereinafter referred to as "containment

* Corresponding author, E-mail: shinichi.kawamura@tepco.co.jp

DOI : 10.15669/fukushimainsights. Vol.4.509

(C) 2021 Atomic Energy Society of Japan. All rights reserved.

Originally published in Transactions of the Atomic Energy Society of Japan (ISSN 1347-2879), Vol. 15, No. 4, p.192-209

(2016) in Japanese. (Japanese version accepted: June 1, 2016) 
vessel") was damaged, and a large amount of radioactive material escaped into the environment. Based on the lessons learned from this accident, various countermeasures are being taken from the perspective of defense in depth. A part of this effort includes the development of a filtered containment venting system (hereinafter referred to as an FCVS) ${ }^{1-4)}$ to protect the containment vessel from over-pressure damage as well as to considerably reduce the emission of radioactive substances during severe accidents.

An FCVS consists of a piping system used to exhaust (vent) gas from the gas-phase of the containment vessel to a high place, and a filter system to collect radioactive substances in the gas and suppress their release into the atmosphere. The main radioactive substances released into the atmosphere from the accident at the Fukushima Daiichi Nuclear Power Plant were noble gases, cesium and iodine ${ }^{5}$. With respect to noble gases, we plan to suppress the rise in pressure and temperature in the containment vessel by spraying the vessel, or some similar activity, to delay the time when venting becomes necessary, and to attenuate the noble gases. This process can be expected to remove cesium and iodine with the filter system. Previous studies have involved the capture of cesium and inorganic iodine from the venting gas with a decontamination factor (hereinafter referred as DF) of 1,000 or more by an FCVS filter device, which is a combination of alkaline water scrubbers and metal filters ${ }^{1,3,4,6)}$. However, this device cannot effectively capture organic iodine.

Although filters that capture iodine with silver zeolite have demonstrated significant efficiency at fuel reprocessing plants, so a worthwhile capture efficiency of organic iodine might be expected, the following information suggests that such a filter would be insufficient when part of an FCVS system. Although zeolite has high hygroscopicity, iodine adsorption performance will decrease due to the absorption of moisture. However, the moisture in the FCVS, which is required for the operation of the system during a loss of AC power at the time of an accident, cannot be removed. Also, it is necessary for the FCVS to treat hydrogen-containing gas, but the hydrogen may reduce the silver and thus impair the iodine removal. Furthermore, since the pressure difference between the containment vessel and the atmosphere allows gas to flow without the use of dynamic devices such as fans, the gas flow rate changes greatly during operation. Relevant knowledge is required to deal with these issues before an organic iodine filter with silver zeolite can be developed for FCVS. There have been reports by Ishii et al. ${ }^{7}$ and Narabayashi et al. ${ }^{8)}$ on the application of silver zeolite to FCVS, but the performance characteristics in an actual device under the assumed conditions has not been presented. Kobayashi et al. ${ }^{9)}$ has reported on the adsorption performance characteristics of silver zeolite under various flow conditions, but it is necessary to understand the performance characteristics for the organic iodine concentration at a gas temperature much closer to the usage conditions of the actual device, especially during the initial venting stage when the conditions are more severe. There has been no record of development or commercialization of organic iodine filters for FCVS in Japan, including these studies.

Meanwhile, the DF requirement for inorganic iodine is 10 or greater for the seven nuclear power plants in Germany. In this case, iodine filters with molecular sieves have been installed, which also exhibit a certain organic iodine removal capability, but the performance characteristics of the filters are not clear. A filter with a DF requirement value of $10^{10)}$ was introduced to the Krško NPP in Slovenia in 2013 as a filter with a DF specification for organic iodine, but there is no published document regarding its performance characteristics.

Therefore, in this study, we consider the process of containment vessel venting subsequent to a typical severe accident at a BWR plant that involves core damage, a loss of coolant, a power station blackout, and a loss of all emergency core cooling systems due to a large break accident at an ABWR. In this scenario, we have conducted experimental studies on the performance 
characteristics of organic iodine removal using silver zeolite, and developed a filter with an organic iodine DF that exceeds 50, using silver zeolite as an absorbent. This DF target value was set at the time of development so as to be equal to or greater than the required value in Europe, as described above. The organic iodine filter developed is being introduced to the unit 6 \& 7 reactors at the Kashiwazaki Kariwa Nuclear Power Plant.

Disclosure of the performance characteristics of the absorbent is useful not only for this development but also for further performance improvement in the future. Also, it is important to conduct an investigation into the performance characteristics of the filter to determine the effectiveness of the FCVS, and this study provides a discussion from this point of view.

\section{Design of an Organic Iodine Filter for an FCVS}

\section{Overview of the Organic Iodine Filter}

Silver zeolite (Manufacturer: RASA Industries, LTD. model: AgX, particle size: 0.85 to $2.00 \mathrm{~mm}$, silver supported ratio: about $41 \mathrm{wt} \%$ ), which is used as the absorbent in the organic iodine filter, has $13 \mathrm{X}$ crystalline zeolite as its basic skeleton. In silver zeolite, the sodium ions in the skeleton of the aluminosilicate are exchanged for silver ions, and iodine is captured by chemically reacting with the silver. The silver zeolite used in this filter is shown in Figure 1.

In the crystal structure of zeolite, numerous pores, which affect adsorption performance, are formed by heating and removal of crystal water. Most of the venting gas from the containment vessel is water vapor, but when the pores come into contact with steam, water molecules are absorbed in the pores and the iodine adsorption performance declines. Thus, a design that can provide the proper temperature superheated steam at the filter inlet and verify performance based on these steam conditions is necessary.

The schematic diagram of the organic iodine filter is shown in Figure 2. The silver zeolite fills the space between the inner cylinder and the outer cylinder of a double-cylinder adsorption tower called a candle-type filter unit, and 19 candle-type filter units are installed in one filter vessel.

Figure 3 shows the basic system configuration of the filtered containment venting system with iodine filters as it is installed at the unit $6 \& 7$ reactors of the Kashiwazaki Kariwa Nuclear Power Plant. (ABWR type, electric output is $1356 \mathrm{MWe}$ each). Two organic iodine filters are installed in parallel downstream of the FCVS filter device. These filters remove particulate matter and inorganic iodine with a DF of 1,000 or greater. An FCVS is a facility used in severe

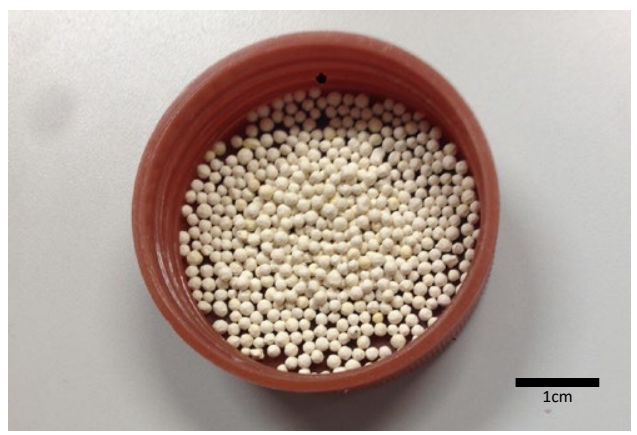

Figure 1 Silver zeolite for the organic iodine filter 


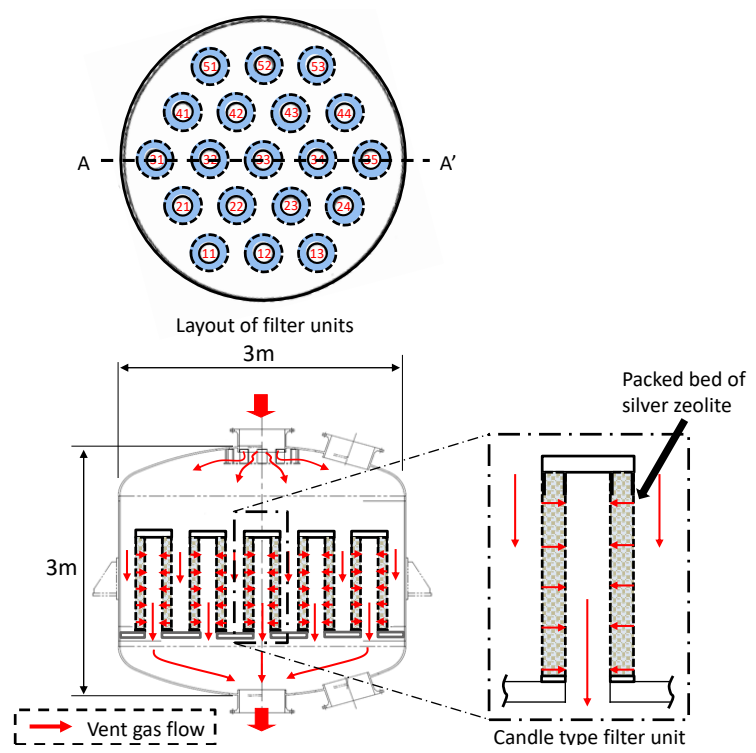

Figure 2 Schematic diagram of the organic iodine filter. Nineteen candle-type filter units, for which identification numbers are written in this figure, are installed in a filter vessel.

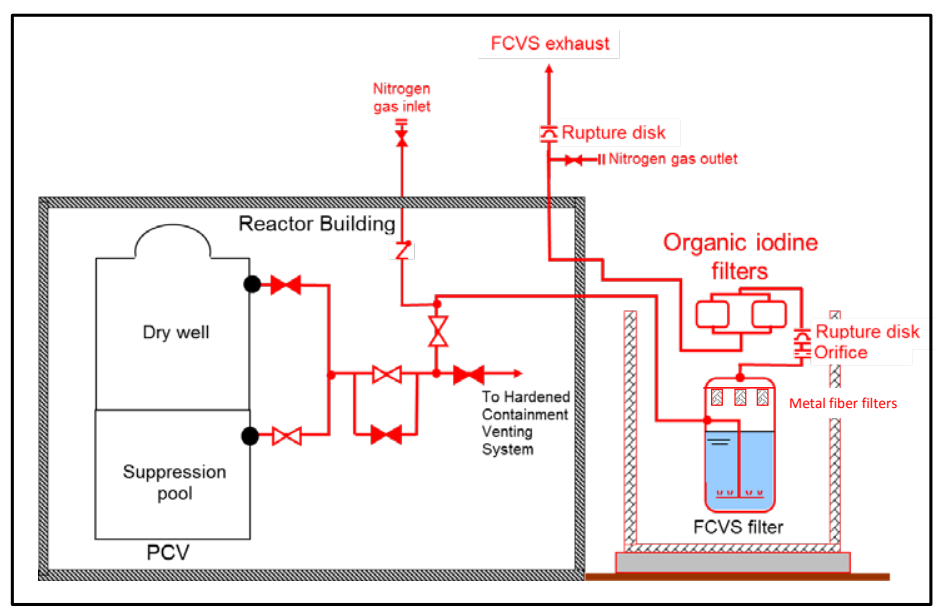

Figure 3 Basic system configuration of the filtered containment venting system with iodine filters

accidents, and it is necessary to demonstrate the required performance by manually opening the valve, as would be required in the event of loss of all AC power. Also, since a constant flow rate control cannot be achieved, the flow rate and temperature of the system change greatly during operation. And because it is necessary to prevent the performance decline of silver zeolite when it comes into contact with steam, an orifice is provided in the upstream piping of the organic iodine filter to produce an adiabatic expansion and an appropriate difference between the gas temperature and dew point, since electric heaters cannot be used. A metal filter, which acts as a demister, is installed in the FCVS, installed upstream of the organic iodine filter, and is 
designed to prevent droplets from accompanying the gas that comes from the FCVS.

A pressure release plate operating at a pressure differential of $100 \mathrm{kPa}$ is provided downstream of the organic iodine filter. With this pressure release plate as the boundary, the upstream side, including the containment vessel, will contain nitrogen gas during standby. This will prevent a high concentration of hydrogen from coming into contact with oxygen and burning in the piping, because of the steam condensed in the cold piping in the initial venting stage.

A pressure release plate operating at a differential pressure of $100 \mathrm{kPa}$ is also provided between the organic iodine filter and the FCVS filter device; because silver zeolite adsorption performance will deteriorate when it dampens, it is kept in a dry state during standby.

\section{Design Specifications of the Organic Iodine Filter}

Table 1 shows the design specifications for the organic iodine filter in the above-described system configuration.

The temperature and pressure conditions in the design of the organic iodine filter are determined from the conditions of the initial venting through the FCVS. The containment vessel is made to be able to maintain confinement at a pressure of at least twice the maximum working pressure (hereinafter $2 \mathrm{Pd}: 620 \mathrm{kPa}$ [gauge] in the case of ABWR) and $200^{\circ} \mathrm{C}^{1)}$, and venting starts up without exceeding this condition. In the route from the containment vessel to the organic iodine filter, there are pressure loss elements, such as the piping, valves, and the FCVS filter device. Therefore, even if the pressure in the containment vessel is $2 \mathrm{Pd}$, the pressure in the organic iodine filter section is lower. Therefore, the pressure loss of the FCVS system was calculated, and the maximum tolerated working pressure was set for the organic iodine filter so that it will exceed the maximum pressure that is actually generated in the organic iodine filter section. Specifically, the piping of the FCVS system can be divided into sections. The venting gas velocity in the calculation section is calculated by using the venting gas concentration calculated from the pressure and the temperature of the upstream section in sequential order, starting from the containment vessel. The local pressure losses are evaluated based on the venting gas velocity and the pressure loss coefficient (derived from the Mechanical Engineering Handbook ${ }^{11)}$, the equipment specifications, and the filter unit test results described later) of the piping (piping length / elbow / tee), valves, FCVS filter device, and organic iodine filter installed in the section under calculation, then the total pressure loss in that section is evaluated by summing all the local pressure losses. The pressure distribution of the entire FCVS system and the pressure in the organic iodine filter section are calculated by evaluating the pressure loss from the containment vessel to the exhaust outlet. Here, as a condition for the generation of the maximum pressure in the organic iodine filter, the containment vessel pressure is $2 \mathrm{Pd}$, and all

Table 1 Design specifications of the organic iodine filter for an ABWR plant

\begin{tabular}{|c|c|}
\hline & Design conditions \\
\hline Maximum design pressure ( $\mathrm{kPa}$ [gage]) & 250 \\
\hline Maximum design temperature $\left({ }^{\circ} \mathrm{C}\right)$ & 200 \\
\hline $\begin{array}{l}\text { Maximum design gas flow rate as } 100 \% \\
\text { steam condition }(\mathrm{kg} / \mathrm{s})\end{array}$ & 31.6 \\
\hline Decontamination factor & 50 \\
\hline $\begin{array}{l}\text { Maximum load of organic iodine } \\
\qquad\left(\mathrm{kg} \text { as } \mathrm{CH}_{3} \mathrm{I}\right)\end{array}$ & 1.3 \\
\hline $\begin{array}{l}\text { Maximum volume concentration of or- } \\
\text { ganic iodine (vol ppm) }\end{array}$ & 40 \\
\hline
\end{tabular}


the valves in the flow path of the venting gas are fully opened, so the pressure loss of the FCVS system can be evaluated. As a result, since the pressure in the organic iodine filter section was about $200 \mathrm{kPa}$ [gauge], the maximum working pressure in the design specification of the organic iodine filter was set to $250 \mathrm{kPa}$ [gauge] so as to exceed the above value. Also, the maximum operating temperature was $200^{\circ} \mathrm{C}$, corresponding to the condition of the containment vessel.

All of the iodine in the nuclear reactor is released to the containment vessel at the time of a severe accident. Assuming that $4 \%$ reacts with organic matter in the containment vessel to become organic iodine, it will mass about $1.3 \mathrm{~kg}$ if converted to methyl iodide, according to the Regulatory Guide $1.1951^{12)}$ of the U.S. Nuclear Regulatory Commission. According to Soffer et al. ${ }^{13)}$ and Beahm et al. ${ }^{14)}$, when maintaining the water in the containment vessel in an alkaline condition, organic iodine is suppressed to $0.15 \%$ or less of the total iodine released from the reactor primary system at the time of an accident. Since the development of a $\mathrm{pH}$ control system is in progress ${ }^{1)}$, we decided not to estimate its effect here because the organic iodine filter is expected to perform its function independently. Since the distribution of organic iodine in the containment vessel is quite uncertain, and the organic iodine concentration is conservatively estimated to be of a higher level, assuming that the full amount of the organic iodine is in the gasphase in the containment vessel suppression chamber, which is connected to the FCVS piping, the volume concentration of organic iodine is about $40 \mathrm{vol}$. ppm immediately before venting.

The flow rate and gas composition of the venting gas flowing into the organic iodine filter change after the start of venting. Considering the typical case of a severe accident involving core damage, accident analysis was conducted with MAAP code for the scenario in which a loss of coolant, a station blackout, and a loss of function of the entire emergency core cooling system occurred due to large break accident. In this analysis, the condition of the venting gas flowing into the organic iodine filter was calculated, and the results are shown in Table 2, with the results considered to be within the operating range of the organic iodine filter. The contact time is the time the gas spends passing through the silver-zeolite-packed bed, and is obtained from the following equation as the reciprocal of the space velocity SV.

$$
1 / S V=V_{A g X} / Q
$$

where, $\mathrm{V}_{\mathrm{Agx}}$ is the volume of the silver-zeolite-packed bed, and $\mathrm{Q}$ is the flow rate of the gas passing through the packed bed.

The nitrogen charged in the containment vessel at the beginning and the hydrogen accumulated after the accident account for a high proportion of the venting gas, and water vapor accounts for nearly $100 \%$ after the discharge of the venting gas. Hydrogen will reduce silver ion, which may lower the iodine adsorption performance, so it is necessary to confirm this effect by test. Also, apart from these main effects, sulfur originating in vulcanized electric cable coating material may inhibit the bonding of silver and iodine. If hydrogen sulfide is generated and reacts chemically with silver, it may inhibit the chemical reaction between iodine and silver,

Table 2 Gas conditions in the packed bed of silver zeolite for an ABWR plant

\begin{tabular}{lccc}
\hline & Vent startup & $\begin{array}{c}\text { One week after } \\
\text { continuous venting }\end{array}$ & $\begin{array}{c}\text { One month after } \\
\text { continuous venting }\end{array}$ \\
\hline Volumetric flow rate $\left(\mathrm{m}^{3} / \mathrm{s}\right)$ & 16.1 & 6.65 & 3.83 \\
Contact time $(\mathrm{s})$ & 0.222 & 0.539 & 0.934 \\
Difference between gas temperature and dew point $(\mathrm{K})$ & 82.3 & 8.6 & 7.3 \\
& Steam $10 \%$ & Steam $100 \%$ & Steam $100 \%$ \\
Gas composition other than organic iodide & Hydrogen $30 \%$ & & \\
& Nitrogen $60 \%$ & & \\
\hline
\end{tabular}


and if sulfur dioxide is generated, it may inhibit the reaction thereof by physically absorbing to zeolite. However, by keeping the scrubber water alkaline in the FCVS filter device, provided upstream of the organic iodine filter, the scrubber water will pass through these substances even if they are generated in the containment vessel. Thus it was judged unnecessary to consider their effects on the condition of the organic iodine filter.

\section{Performance Verification Test of the Organic Iodine Filter}

\section{Organic Iodine Adsorption Test}

\section{(1) Outline of the test facility}

The organic iodine removal performance of silver zeolite was confirmed by tests. Figure 4 outlines the test facility. Methyl iodide was used to represent organic iodine in the test. Since methyl iodide is highly volatile, it is thought to be the main iodine released in gaseous form at the time of an accident, and because it is small and difficult to capture, considering it as a representative gas is a conservative measure. It is also employed in the inspection of the performance of activated carbon in emergency gas treatment systems at nuclear power plants.

The gas from the methyl iodide gas generator is mixed with the steam from the boiler and introduced into a test column filled with silver zeolite. A gas injection port was provided in the pipe upstream of the test column, so that venting gas components other than water vapor, such as hydrogen and nitrogen, could be injected as needed to adjust the gas composition.

In designing a test column to be filled with silver zeolite, consideration was taken to simulate the conditions in the actual device with regard to advective diffusion, when the gas passes through the packed bed. It is expected that in the actual candle-type filter unit, the gas flows from the outside to the inside, as shown in Figure 2, because of the pressure difference inside and outside the packed bed. However, with the z-axis as the one-dimension flow direction, the equation

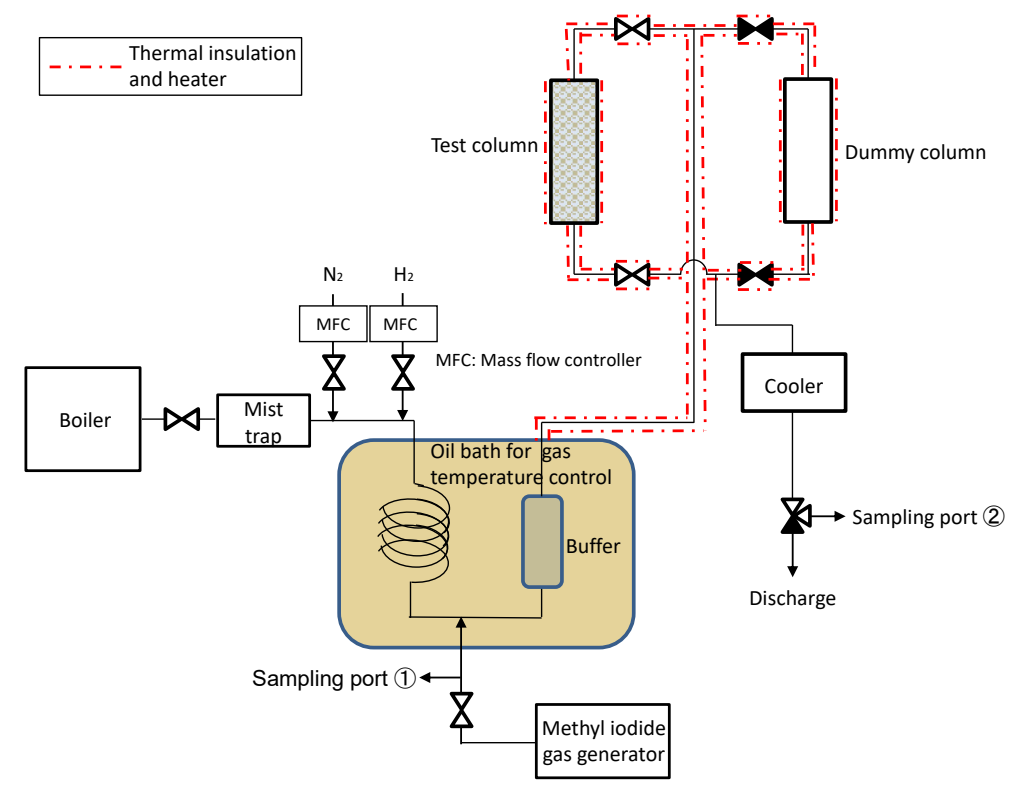

Figure 4 The test facility for methyl iodide adsorption performance of the silver zeolite 
of the advective diffusion associated with the reaction is expressed in the steady state as follows:

$$
\mathrm{u} \frac{\mathrm{dc}}{\mathrm{dz}}=D_{z} \frac{d^{2} c}{d z^{2}}-k c
$$

where, $\mathrm{c}$ is the concentration of methyl iodide, $\mathrm{u}$ is the gas velocity, $\mathrm{D}_{\mathrm{z}}$ is the mixed diffusion coefficient in the $\mathrm{z}$-direction, and $\mathrm{k}$ is the adsorption reaction speed constant of silver zeolite. Incidentally, this $\mathrm{k}$ is an overall reaction speed constant, which includes not only the chemical reaction but also factors such as the shape of silver zeolite, diffusion in silver zeolite, and the quantity of silver ions.

From this equation, we find that when filling the test column with the same silver zeolite and with the same packing as that of the actual device, basically simulating the conditions for reaction and mixing diffusion, it is necessary to adjust the distance in the flow direction, that is, the thickness of the packed bed with respect to the flow direction, as well as the gas velocity, in accordance with the actual device. Specifically, within the range that the steam production of the boiler can be adjusted, the column inner diameter is determined to ensure that the velocity in the test column can simulate the operation range of the actual device, and the packed-bed thickness (the height of the test column in Figure 4 of the test column) is set to be the same size as that of the candle-type filter unit of the actual device (the inside and outside diameter difference of the candle type filter unit of the double cylindrical structure shown in Figure 2). The influence of the gas temperature and pressure will be checked by tests.

The test column designed in this way simulates the advective diffusion in the flow direction, but the dimension in the direction orthogonal to the flow (the height of the packed bed of the candle-type filter unit in the actual device, the diameter of the test column in the test) is different, and the influence of the difference is considered as follows. First, the interior of the packed bed is filled with zeolite particles of small diameter, and it is thought that the velocity of the gas flow passing through the packed bed due to the rectification effect does not cause a large difference for most of the positions in the direction perpendicular to the flow. This will be confirmed by the filter unit test described later. However, it is thought that in such packing materials, the flow velocity increases in the vicinity of the wall in the flow paths, causing the appearance of local regions where the contact time is short and adsorption performance tends to be lower. Walls exist at the upper and lower ends of the packed portion in the candle-type filter unit of the actual device, and the inner surface of the test column is a wall, but as shown in Figure 2 and Figure 4, the wall area with respect to the packed volume is small in the actual candle-type filter unit, and the effect of the wall is considered to be smaller than that in the test column. Therefore, the adsorption performance confirmed by the test column is more conservative than the actual device. It is, therefore, considered possible to conservatively estimate the adsorption capacity in an actual device by multiplying the mass ratio of silver zeolite in both the actual device and test column by the adsorption capacity to be confirmed by the tests.

The concentration of methyl iodide supplied to the test facility was confirmed by taking a sample from sampling port 1 and analyzing it with a gas chromatograph (GC-FID: Shimadzu Corporation GC-14B, lower limit of detection concentration is 2.3 vol. ppm). On the other hand, the gas passing through the test column was diluted with nitrogen, passed through the cooling trap and was collected from sampling port 2, undergoing analysis by the GC-FID with condensed water, as samples. The outlet concentration was calculated based on this analysis value. The steam flow rate was calculated from the weight of the condensed water. 
Table 3 Test cases for methyl iodide adsorption by silver zeolite, simulating steady vent conditions

\begin{tabular}{|c|c|c|c|c|}
\hline Test number & $A-1$ & $\mathrm{~A}-2$ & $\mathrm{~A}-3$ & $\mathrm{~A}-4$ \\
\hline Contact time (s) & 0.121 & 0.119 & 0.151 & 0.134 \\
\hline Methyl iodide concentration (vol ppm) & 55 & 116 & 48 & 57 \\
\hline Difference between gas temperature and dew point & 9.1 & 10.6 & 8.5 & 12.5 \\
\hline Gas pressure ( $\mathrm{kPa}$ [gage] $)$ & 8.1 & 8.4 & 5.6 & 250.4 \\
\hline Gas composition other than methyl iodide & Steam $100 \%$ & Steam $100 \%$ & Steam $100 \%$ & Steam $100 \%$ \\
\hline Temperature of silver zeolite before testing & $\begin{array}{l}\text { Preheated to the } \\
\text { gas temperature }\end{array}$ & $\begin{array}{l}\text { Preheated to the } \\
\text { gas temperature }\end{array}$ & $\begin{array}{l}\text { Preheated to the } \\
\text { gas temperature }\end{array}$ & $\begin{array}{l}\text { Preheated to the } \\
\text { gas temperature }\end{array}$ \\
\hline $\begin{array}{l}\text { Absorbed methyl iodide before DF becomes less than } \\
50(\mathrm{mg})\end{array}$ & 1,637 & 1,644 & 2,431 & 1,985 \\
\hline Ratio of silver reacted with iodide (\%) & 6.1 & 6.1 & 9.0 & 7.4 \\
\hline $\begin{array}{l}\text { Evaluated allowable load of methyl iodide on two or- } \\
\text { ganic iodine filters for a plant (kg) }\end{array}$ & 131.0 & 131.5 & 194.5 & 158.6 \\
\hline
\end{tabular}

DF : Decontamination Factor.

\section{(2) Confirmation of basic performance}

To confirm the basic performance of organic iodine removal by silver zeolite, a test was conducted to simulate the steady venting state, excluding the initial venting. The test conditions are shown in Table 3.

With respect to the conditions shown in Table 2, in the design of the actual device, as discussed later in III-2-(2), considering the variations in the flow velocity distribution in the filter, it is necessary to ensure the performance with a contact time of $0.188 \mathrm{~s}$ or longer. The contact time in this test was set between about $0.12 \mathrm{~s}$ and $0.15 \mathrm{~s}$, a conservative shorter time condition, under which the steam flow rate was adjusted. The influence of gas flow velocity can be determined by comparing test No. A-1 with No. A-3. In test A-3, the gas flow velocity was slow, which resulted in a longer contact time. However, it was considered when setting the difference between the gas temperature and the dew point that the steam superheat temperature in the orifice decreases as the flow velocity decreases.

The concentration of methyl iodide was set to be about 50 vol. ppm, compared to 40 vol. $\mathrm{ppm}$ - the maximum value assumed for the actual device, as shown in Table 1. In test A-2, the concentration was increased to 116 vol. ppm, about twice as high. By comparing test A-1 with test A-2, it is possible to ascertain the influence of the concentration on adsorption performance.

Test A-4 used temperature and pressure settings close to the initial venting of the actual device, corresponding to a test condition assumed immediately after vent startup, in which the containment vessel pressure is $2 \mathrm{Pd}$ in the actual device. Hydrogen and nitrogen account for a high proportion of the venting gas composition at the beginning as shown in Table 2. But they were discharged promptly, and the water vapor remaining may account for $100 \%$. By comparing test A-1 with test A-4, the influence of the temperature and pressure can be determined.

Also, in the venting steady state, the silver zeolite has the same temperature as the venting gas, and it is not considered to be moist because the superheated steam is aerated. Therefore, in the test, the silver zeolite was in a dry state before the gas entered and was preheated with a heater outside of the test column so as to be at the same temperature as the venting gas; only then was the gas allowed to flow in.

Figures 5 to $\mathbf{8}$ show the changes in the inlet concentration, outlet concentration, and methyl iodide removal rate from the start of the test under each condition. The outlet concentration continues to be below the detection limit for a period after the start of the test but eventually begins to increase, and the methyl iodide removal rate also decreases.

In Table 3, the time during which DF maintains the design target of 50 or greater under each test condition, the fraction of silver, which absorbs iodine during this time, and the calculated 


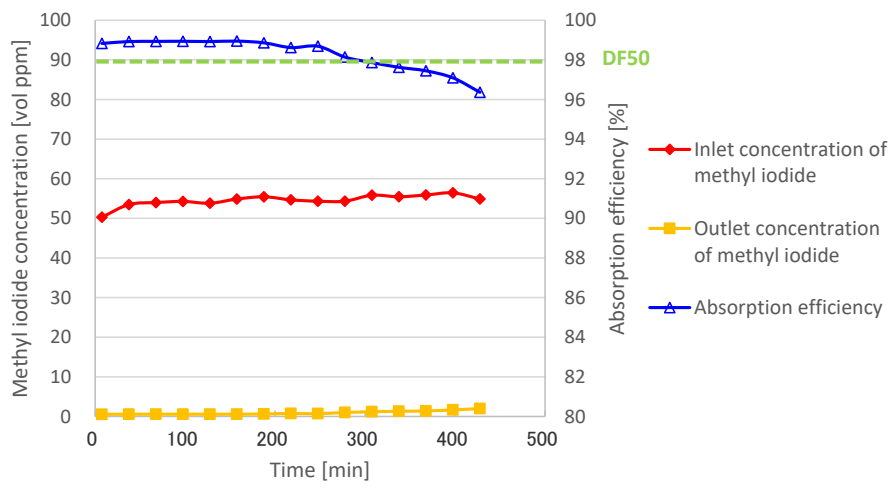

Figure 5 Results of methyl iodide adsorption performance test A-1

Test conditions are listed in Table 3 as test number A-1.

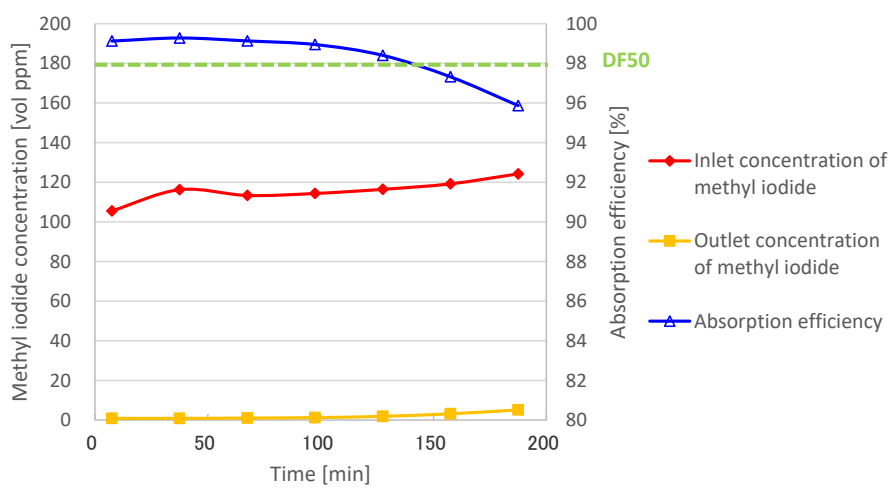

Figure 6 Results of methyl iodide adsorption performance test A-2

Test conditions are listed in Table 3 as test number A-2.

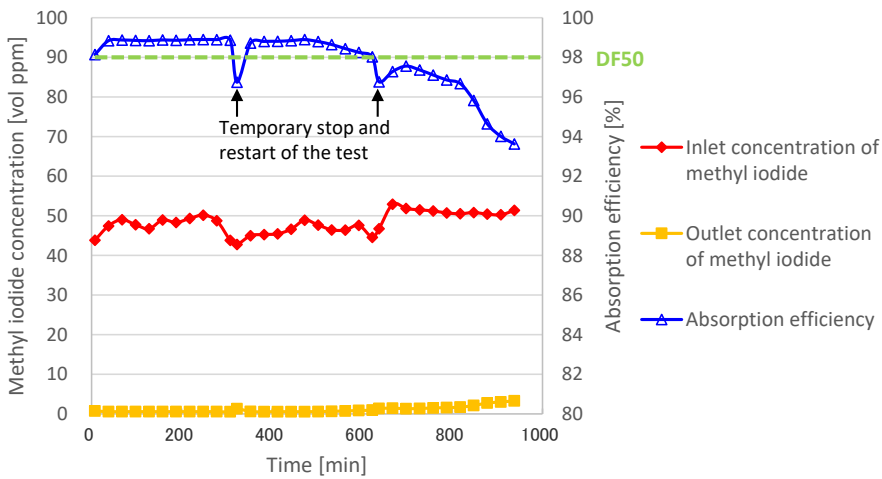

Figure 7 Results of methyl iodide adsorption performance test A-3

Test conditions are listed in Table 3 as test number A-3. Performance drops were observed when the test was suspended and resumed because residual methyl iodide was measured at the outlet. This was not considered as relevant to the performance of silver zeolite. 


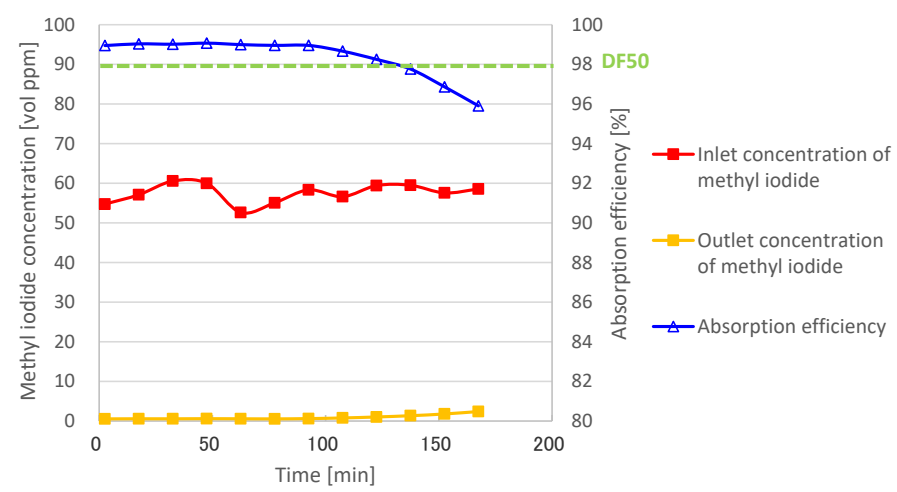

Figure 8 Results of methyl iodide absorption performance test A-4 Test conditions are listed in Table 3 as test number A-4.

result of the allowable adsorption load of the organic iodine filter are also described. The allowable adsorption load of the organic iodine filter is the total mass (as shown in Figure 3, since two filters are installed in parallel, the total value) of organic iodine (methyl iodide) absorbed by the organic iodine filters of the actual device during the period when the DF is maintained at 50 or greater, which is calculated by the following equation

$$
\mathrm{A}=\int_{0}^{T_{50}} S I\left(1-\frac{1}{D F}\right) d t \cdot A g X_{r} / A g X_{t}
$$

where $\mathrm{A}$ is the allowable adsorption load of the organic iodine filter, SI is the mass of methyl iodide supplied to the test column per unit time, $\mathrm{DF}$ is the decontamination factor, $\mathrm{AgX}_{\mathrm{t}}$ is the total mass of silver zeolite packed in the two iodine filters, $\mathrm{AgX} \mathrm{X}_{\mathrm{t}}$ is the total mass of silver zeolite packed in the test column, and $\mathrm{T}_{50}$ is the time from the start of the test until the DF becomes less than 50 .

If the allowable adsorption load of this organic iodine filter exceeds $1.3 \mathrm{~kg}$, which is the amount of organic iodine generated in the containment vessel discussed in section II-2, the total amount of organic iodine can be treated with a DF of 50 or greater. As shown in Table 3, all four test conditions satisfied this requirement, and it was confirmed that the silver zeolite has sufficient organic iodine adsorption capacity in steady state venting.

As shown in Table 3,10\% or less of the silver ions in the silver zeolite contributed to the adsorption of iodine. Methyl iodide is believed to diffuse from the surface into the interior of the silver zeolite in an accompanying reaction. Here, considering the case with high reaction speed and slow diffusion, silver ions near the surface of the zeolite particles contribute to the reaction, but silver ions deep inside the particles do not contribute to the reaction. With this mechanism, it is conceivable that silver ions contributing to the adsorption were only a portion of the total amount.

On the other hand, comparing the individual test cases with each other, the influence of the organic iodine concentration can be obtained from the comparison between Figure 5 and Figure 6. That is, in the case in Figure 6, where the concentration is about twice that of the case in Figure 5, the removal rate begins to decline at an earlier stage, and the time for which the DF can be maintained at 50 or greater is reduced by almost half. However, the fractions of silver contributing to the reaction under both test conditions are equal, and the calculated value of the allowable adsorption load of the organic iodine filter is also equivalent.

Comparison of Figures 5 and 7 shows that the removal rate begins to decline at an earlier 


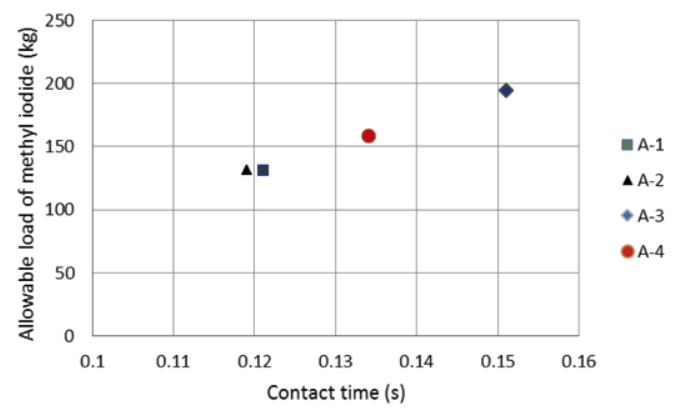

Figure 9 Relationship between contact time and allowable load of methyl iodide on two organic iodine filters for a plant

The conditions of test cases A-1 to A-4 are listed in Table 3.

stage for a shorter contact time, and the sensitivity of the contact time to the removal rate is high.

The relationship between the contact time of each test case and the allowable adsorption load obtained from equation (3) is shown in Figure 9. The amount of methyl iodide that can be absorbed while maintaining a DF of 50 or greater, including the case of changing the test temperature and pressure conditions (Test No. A-4 shown in Table 3, Figure 8), will increase with a longer contact time.

These tendencies were discussed as follows. It is considered that the flow in the packed bed is in an intermediate state of a plug flow and perfect mixing, depending on the effect of mixing diffusion. In the case of plug flow, the equation for advective diffusion in equation (2) is expressed by equation (4) since $\mathrm{D}_{\mathrm{z}}=0$, and the concentration is obtained by equation (5) by integrating equation (4)

$$
\begin{aligned}
& \mathrm{u} \frac{\mathrm{dc}}{\mathrm{dz}}=-k c \\
& \mathrm{c}=c_{0} \exp (-k z / u)
\end{aligned}
$$

where $c_{\mathrm{o}}$ is the concentration at the inlet of the packed bed. Here, when the contact time until the gas arrives at an arbitrary position $\mathrm{z}$ is set to be $\mathrm{t}$, since $\mathrm{t}=\mathrm{z} / \mathrm{u}$, the concentration, which is made non-dimensional by the inlet concentration of the packed bed, can be expressed by equation (6).

$$
c / c_{0}=\exp (-k t)
$$

The image of the non-dimensional concentration and the contact time expressed by Eq. (6) is indicated by the solid line in Figure 10. The non-dimensional concentration must be 0.02 or less to achieve a DF of 50 or greater. As shown in the figure, when the contact time is longer than the contact time limit $\mathrm{T}_{0}$, the $\mathrm{DF}$ becomes 50 or greater.

The inlet portion of the packed bed has the highest concentration, and breakthrough commences as the adsorption progresses. However, if the contact time to the outlet of the packed bed is set to be $T_{0}$, the non-dimensional concentration will immediately exceed 0.02 when breakthrough begins, and the DF will become less than 50. On the other hand, if the contact time to the outlet of the packed bed is set to be $T_{1}$, the non-dimensional outlet concentration has a margin of safety between it and the allowable value of 0.02 . As breakthrough begins, the time the gas passes through the breakthrough packed bed becomes invalid as the contact time, so the curve in this figure shifts to the right. It is thought that DF will become less than 50 when the 


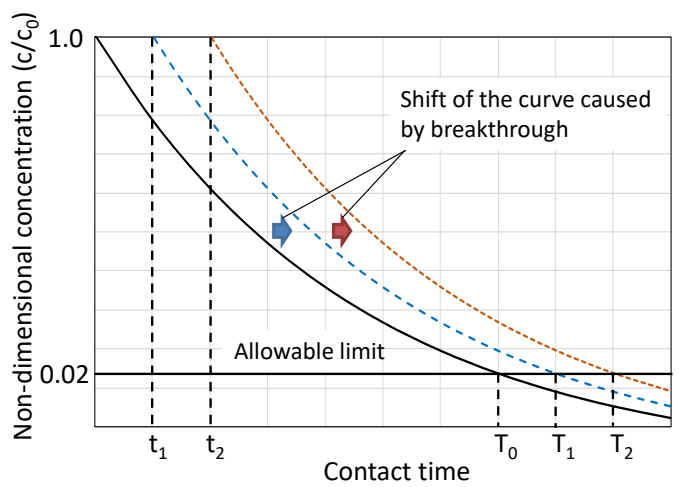

Figure 10 Typical relationship between contact time and concentration of methyl iodide in a plug flow of gas through packed silver zeolite bed. The conditions of test cases A-1 to A-4 are listed in Table 3.

breakthrough eventually progresses and reaches the state of the dashed line. In addition, when considering a mixing flow in the flow direction, instead of a plug flow, although the slope of the curve in Figure 10 becomes moderate due to the effect, the qualitative tendency is presumed to be similar.

When considering such a model, if the setting of the contact time to the outlet of the packed bed is increased from $T_{1}$ to $T_{2}$, the shiftable amount of the curve will increase, as shown in the figure, and the tolerance of invalid contact time when the gas passes through the breakthrough packed bed extends from $t_{1}$ to $t_{2}$. That is, the allowable thickness of the breakthrough packed bed increases. Accordingly, the allowable adsorption load increases by that increased thickness, but the increased amount is proportional to the length of the contact time that exceeds $\mathrm{T}_{0}$. However, in this model, it was assumed that the reaction speed constant $\mathrm{k}$ was constant downstream of the breakthrough packed bed, but in reality, silver ions, which contribute to the adsorption, are consumed by the reaction in this region, so k decreases. The slope of the curve in Figure 10 tends to moderate, especially at the position close to the breakthrough packed bed. In this case, $t_{1}$ and $t_{2}$ are smaller than the positions shown in the figure, and the proportional relationship no longer exists, but the qualitative tendency that the allowable adsorption load increases with the time exceeding $T_{o}$ is maintained. The allowable adsorption load shown in Figure 9 increases almost linearly, depending on the contact time, and demonstrates a tendency consistent with the considerations here, at least within the range of these test conditions.

On the other hand, even if the inlet concentration of methyl iodide increases, the shape of the curve does not change because the concentration is made non-dimensional by the inlet concentration, as shown in Figure 10. However, it is thought that consumption of silver ions, which can contribute to adsorption, also proceeds faster as the concentration increases. In the comparison between test A-1 and test A-2, the time for which DF can secure a value of 50 or greater in the case of A - 2, which is about double in concentration, has been almost halved, and the amount of absorbed methyl iodide is approximately equal.

In test A-3, although the conditions of temperature and pressure are different, the allowable adsorption load is in a linear relationship with the contact time indicated by the result of other test conditions, as shown in Figure 9, and the difference in contact time appears to be dominant over the adsorption load. Generally, it is predicted that the change in the temperature and in other conditions will affect reaction speed and diffusion. However, within the scope of this test condition, it is considered that these influences are not as pronounced as those of the contact time. 
Although the above discussion is based on an assumed plug flow, if we assume a different ideal flow, namely perfect mixing, the outcome is considered to be as follows. In perfect mixing, the concentration $\mathrm{c}$ in the packed bed is equal to the outlet concentration $\mathrm{c}_{\mathrm{ex}}$. Based on the material balance principle, the relationship with the inlet concentration $c_{o}$ is expressed by equation (7)

$$
\mathrm{c}=c_{e x}=c_{\mathrm{o}}-k T c_{e x}
$$

where, $\mathrm{T}$ is the average residence time of the gas in the packed bed, that is, the contact time. However, $\mathrm{t}$ in equation (6) is the contact time until position $\mathrm{z}$ is reached in the packed bed, but since it is a perfect mixing flow, $\mathrm{T}$ in equation (7) is not the contact time corresponding to the position. Based on equation (7), DF is expressed by equation (8).

$$
\mathrm{DF}=c_{0} / c_{e x}=1+k T
$$

Therefore, to reach a certain DF, the longer the contact time is, the lower the reaction speed may be. On the other hand, it is thought that the decline in the reaction speed is caused by the sequential progression of silver ions from the reactive surface of silver zeolite particles toward their interior. Therefore, if a low reaction speed is allowed, the allowable consumption of silver ions will increase accordingly, that is, the allowable adsorption load will increase.

On the other hand, when the zeolite is removed after the adsorption test, zeolite particles that have reacted with iodine and turned yellow are observed, and also zeolite particles that are still white. Therefore, it is not a perfect mixing flow in the packed bed but rather in an intermediate state of the ideal plug flow, and it is considered that the mechanism related to the adsorption capacity discussed above also coexists.

(3) Performance when the difference between gas temperature and dew-point is small

The basic performance under the conditions assumed for the actual device was confirmed by the test in III-1-(2). To confirm the design margin, a test was conducted for the case in which the difference between the gas temperature and the dew point is small, and the effect was determined.

Based on test A-4, the test condition was set with a reduced superheating degree of steam for test A-5. The conditions are described in Table 4 in comparison with the conditions of test A-4. The changes in inlet concentration, outlet concentration, and methyl iodide removal rate in test A-5 are shown in Figure 11. The period for which DF maintains the design target of 50

Table 4 Test cases for methyl iodide adsorption of silver zeolite to clarify the effects of

\begin{tabular}{|c|c|c|}
\hline Test number & A-4 (Reference) & $\mathrm{A}-5$ \\
\hline Contact time $(\mathrm{s})$ & 0.134 & 0.137 \\
\hline Methyl iodide concentration (vol ppm) & 57 & 46 \\
\hline Difference between gas temperature and dew point (K) & 12.5 & 4.6 \\
\hline Gas pressure ( $\mathrm{kPa}$ [gage]) & 250.4 & 249.9 \\
\hline Gas composition other than methyl iodide & Steam $100 \%$ & Steam $100 \%$ \\
\hline Temperature of silver zeolite before testing & $\begin{array}{l}\text { Preheated to the gas } \\
\text { temperature }\end{array}$ & $\begin{array}{l}\text { Preheated to the gas } \\
\text { temperature }\end{array}$ \\
\hline Absorbed methyl iodide before DF becomes less than 50 (mg) & 1,985 & 788 \\
\hline Ratio of silver reacted with iodide (\%) & 7.4 & 2.9 \\
\hline $\begin{array}{l}\text { Evaluated allowable load of methyl iodide on two organic iodine filters for a } \\
\text { plant }(\mathrm{kg})\end{array}$ & 158.6 & 63.0 \\
\hline
\end{tabular}
superheating level 


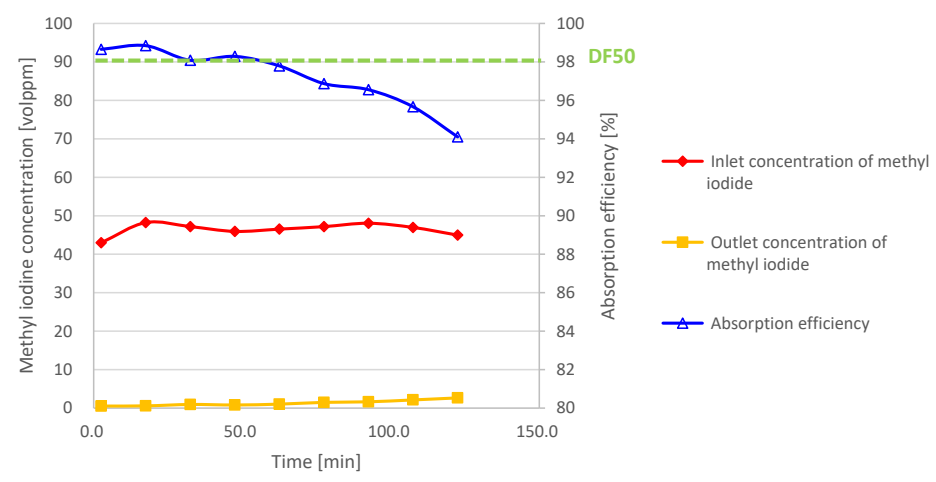

Figure 11 Results of methyl iodide adsorption performance test A-5 Test conditions are listed in Table 4 as test number A-5.

or greater in this test, the proportion of silver that absorbed iodine during that period, and the calculated result of the allowable adsorption load of the organic iodine filter are also described in Table 4. Each value is smaller than that in the test that A-4 is to be compared with, and it is understood that the decrease in the difference between gas temperature and dew-point affects the adsorption performance. However, it was confirmed that the required adsorption performance was maintained even under these conditions.

\section{(4) Confirmation of adsorption state stability}

Next, to check the adsorption state stability, we heated the absorbed silver zeolite to see whether iodine would be desorbed. In this test, the silver zeolite after the A-3 test shown in Table 3 was used as a sample. While the DF of this silver zeolite was maintained at 50 or greater, with the total amount of methyl iodide absorbed by the $50 \mathrm{~g}$ of silver zeolite packed in the test column being shown in Table 3, the total amount that remained by the end of the test was $3,678 \mathrm{mg}$. A total of $2.5 \mathrm{~g}$ of silver zeolite, which can be confirmed to turn yellow by visual check, was collected from the particles of silver zeolite removed from this test column and placed in the reaction vessel of the apparatus shown in Figure 12. If methyl iodide was uniformly absorbed by silver zeolite, $184 \mathrm{mg}$ of methyl iodide would be absorbed in this sample of $2.5 \mathrm{~g}$, but because the degree of yellow coloration varied from zeolite to zeolite, those more intensely colored were selected for the sample. Therefore, at least $184 \mathrm{mg}$ of methyl iodide, which is the average value, was thought to be absorbed in this sample.

Next, the sample temperature was raised to $100^{\circ} \mathrm{C}$ and maintained for 30 minutes while hydrogen gas flowed in. Thereafter, the temperature was raised in increments of $100^{\circ} \mathrm{C}$ and maintained for 30 minutes each time. Gas that passed through the reaction vessel was sampled from sampling port 1 with a micro syringe, and the methyl iodide concentration was measured with GC-FID. Also, gas capture was conducted by pure water in sampling port 2 and by sodium thiosulfate solution in sampling port 3, and iodine ion analysis was carried out by ion chromatography (IC: Nippon Dionex ICS-2000, lower limit of detection concentration was $0.2 \mathrm{mg} / \mathrm{L}$ ). Furthermore, the entire flow of gas was captured from sampling port 4, and the concentration of $\mathrm{I}_{2}$ was analyzed with a detection tube $(9 \mathrm{~L}$, made by GASTEC, lower limit of concentration detection: $0.2 \mathrm{vol} \mathrm{ppm}$ ).

In this test, methyl iodide was detected in the gas collected from port 1, in the process of heating the silver zeolite sample to $300-400^{\circ} \mathrm{C}$. The total amount of methyl iodide calculated 


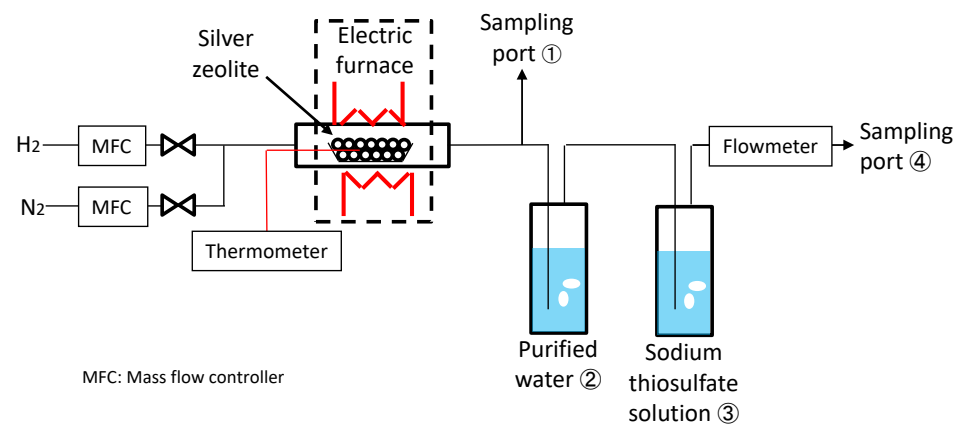

Figure 12 The test facility for iodide desorption from the silver zeolite

from that concentration was $0.04 \mathrm{mg}$. It was thought that this methyl iodide did not chemically react with silver ions and was physically absorbed by the zeolite, but the amount was as small as $0.02 \%$ of $184 \mathrm{mg}$, which is the total amount of methyl iodide absorbed by this sample. Therefore, it is considered that most of the iodine absorbed by silver zeolite chemically reacts with silver and is stably retained. Methyl iodide, which was released by heating, transfers into the gas, but in the case of the actual device, it is considered that recombination will occur when it flows through the silver-zeolite-packed bed, and the problem of re-emission will not arise in practice.

On the other hand, from the ion analysis of pure water, $\mathrm{I}^{-}$was detected when the silver zeolite temperature was $400^{\circ} \mathrm{C}$ or higher. If the $\mathrm{I}^{-}$came from methyl iodide, the total amount of $\mathrm{I}^{-}$would correspond to $10.3 \mathrm{mg}$ of methyl iodide, meaning that $5.6 \%$ of the total absorbed amount was desorbed. Furthermore, from the sodium thiosulfate solution, $\mathrm{I}^{-}$corresponding to $1.0 \mathrm{mg}$ of methyl iodide was detected. $\mathrm{I}_{2}$ was detected from the gasbag, and the total amount was $0.07 \mathrm{mg}$. The total of these is greater than the total amount estimated from the methyl iodide concentration detected at port 1 , and it is considered that silver iodide was reduced by heating in a hydrogen atmosphere, and desorbed iodine was detected.

As shown in Figure 3, there is an FCVS filter device upstream of the organic iodine filter. Fission products are collected in the scrubber. When radiolysis of water occurs, hydrogen is generated and may flow into the organic iodine filter. However, this filter device is designed in such a manner as to discharge the scrubber water into the containment vessel, in which case, it is refilled with fresh water. Therefore, operation management is necessary to suppress the generation of hydrogen by replacing scrubber water at an appropriate time, sending nitrogen gas to the organic iodine filter to lower the hydrogen concentration, and cooling down the silver zeolite during the period before water replacement.

\section{(5) Performance confirmation under initial venting conditions}

In the initial stage of venting, the iodine adsorption performance of silver zeolite may deteriorate due to the influence of hydrogen contained in the venting gas and the condensation of steam on the surface of the silver zeolite. Therefore, the test was carried out under the conditions in Table 5, and the performance under transient conditions from the start of the vent to the steady state was confirmed. Of the two test conditions, B-1 reproduces the gas composition in the containment vessel at the start of the venting, as obtained from the MAAP analysis. The vapor of this composition will flow into the FCVS system, as the scrubber water temperature is low in the FCVS filter device, which is upstream of the organic iodine filter. As the steam condenses while passing through the scrubber, a high concentration of hydrogen may flow into the organic iodine filter. Therefore, another test condition, B-2, was set, assuming that the steam 
Table 5 Test cases for methyl iodide adsorption of silver zeolite, simulating vent startup conditions

\begin{tabular}{|c|c|c|}
\hline Test number & B-1 & B-2 \\
\hline Contact time $(\mathrm{s})$ & 0.173 & 0.166 \\
\hline Methyl iodide concentration (vol ppm) & 126 & 120 \\
\hline Gas temperature $\left({ }^{\circ} \mathrm{C}\right)$ & 101 & 101 \\
\hline Gas pressure ( $\mathrm{kPa}$ [gage] $)$ & 15.9 & 10.2 \\
\hline Gas composition other than methyl iodide & $\begin{array}{c}\text { Steam } 10 \% \\
\text { Hydrogen } 30 \% \\
\text { Nitrogen } 60 \%\end{array}$ & $\begin{array}{c}\text { Hydrogen } 33 \% \\
\text { Nitrogen } 67 \%\end{array}$ \\
\hline Temperature of silver zeolite before testing & Room temperature & Room temperature \\
\hline Absorbed methyl iodide before DF becomes less than $50(\mathrm{mg})$ & 479 & 1,109 \\
\hline Ratio of silver reacted with iodide (\%) & 1.8 & 4.1 \\
\hline Evaluated allowable load of methyl iodide on two organic iodine filters for a plant $(\mathrm{kg})$ & 38.3 & 88.7 \\
\hline
\end{tabular}

DF : Decontamination Factor.

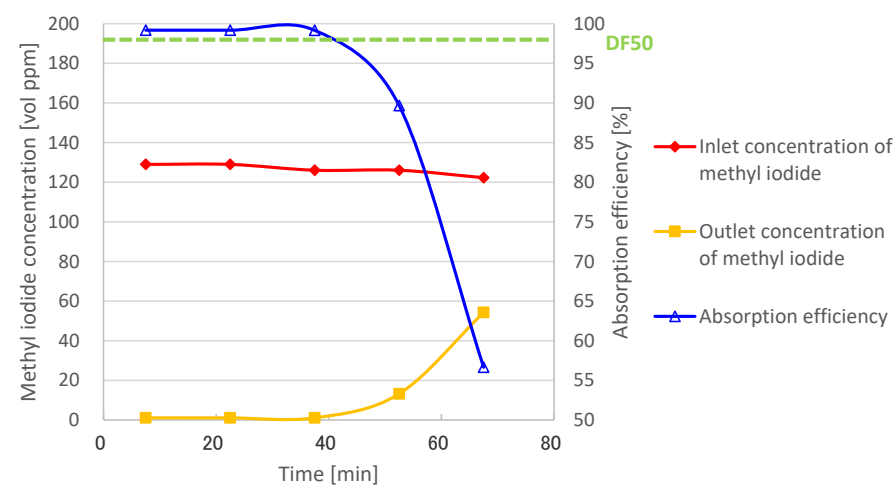

Figure 13 Results of methyl iodide adsorption performance test B-1 Test conditions are listed in Table 5 as test number B-1.

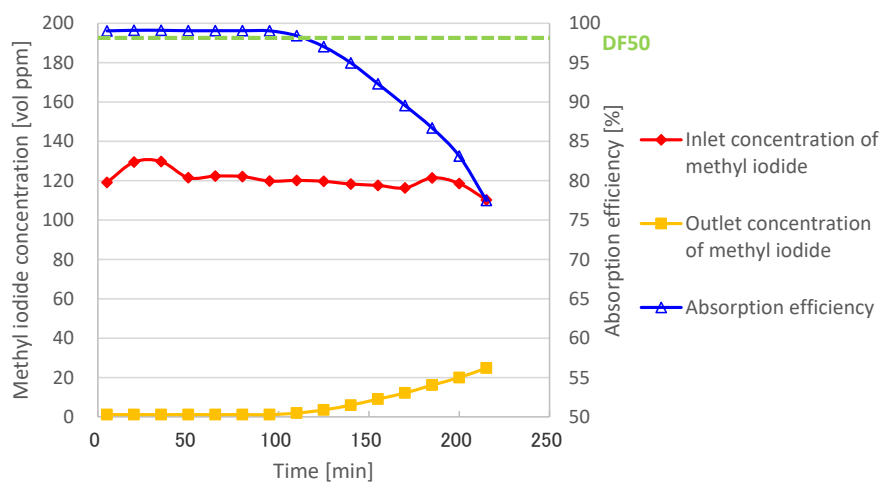

Figure 14 Results of methyl iodide adsorption performance test B-2 Test conditions are listed in Table 5 as test number B-2.

completely condensed in the scrubber at the very beginning of the venting.

Change of inlet concentration, outlet concentration and methyl iodide removal rate from the start of each test under each condition is shown in Figure 13 and Figure 14. In Table 5, the period during which DF maintains the design target of 50 or greater under each test condition, the fraction of silver, which absorbs iodine during that period, and the calculated result of the allowable adsorption load of the organic iodine filter are also described. All values are significantly lower 
than those in the steady state shown in Table 3, but a DF of 50 or greater is obtained, and the allowable adsorption load of the organic iodine filter also exceeds the organic iodine of about $1.3 \mathrm{~kg}$ actually generated in the containment vessel. It was confirmed that the organic iodine filter has sufficient effectiveness even in the transient state at the beginning of the venting.

\section{(6) Performance at restart after temporary suspension of venting}

Although it was confirmed by the previous test that the silver zeolite provides the necessary performance even at the beginning of the venting, it was found that the vapor condensation at the beginning of the venting affects the adsorption performance. However, in the first venting after the accident, as shown in Table 2, most of the gas flowing is nitrogen and hydrogen, and water vapor is about $10 \%$. The silver zeolite is dry in the initial state, and the effect of vapor condensation was considered to be limited, according to the previous test that simulated this condition.

However, a different situation may result if the venting is temporarily stopped for some reason and resumed, with vapor condensed at the time of stoppage and the silver zeolite dampened, which is different from the condition of the test described in the preceding paragraph in which venting is started when silver zeolite is dry. Therefore, a test was conducted to wet the silver zeolite and then check the performance by flowing the gas at room temperature. Steam not containing methyl iodide was flowed into the test column of the test facility in Figure 4. After the temperature and pressure reached steady state, the flow of steam was stopped. Then the heat was allowed to dissipate spontaneously to wet the silver zeolite with condensed vapor in the test column. After confirming that the test column had reached room temperature, steam containing methyl iodide was flowed into the test column, and the change in concentration was measured. In addition, because the main component of the gas is water vapor in the second and subsequent venting(s) after the accident, hydrogen and nitrogen were not mixed in this test.

The test conditions are shown in Table 6. Test C-1 used almost the same test conditions as test A-3, except that room temperature wet silver zeolite was specified as an initial condition.

The test result is shown in Figure 15. It is thought that the low adsorption performance at the beginning is due to the wet silver zeolite. After that, the adsorption performance increased with time, and about 40 minutes from the start of venting the DF exceeded 50 and demonstrated stable performance. This is thought to be due to the evaporation of the condensed water adhered to the silver zeolite by the flow of superheated steam.

Next, we checked the limit condition for maintaining the performance by decreasing the gas flow velocity in the test. As for the second and subsequent venting(s), it is not always necessary to wait until the pressure of the containment vessel reaches $2 \mathrm{Pd}$ as in the first venting, and since the decay heat of the core also decreases, it is not a necessity to apply the first venting

Table 6 Test conditions for methyl iodide adsorption of silver zeolite simulating vent restart conditions

\begin{tabular}{|c|c|c|}
\hline Test number & $\mathrm{C}-1$ & $\mathrm{C}-2$ \\
\hline Contact time (s) & 0.152 & 0.388 \\
\hline $\begin{array}{l}\text { Methyl iodide concentration } \\
\text { (vol ppm) }\end{array}$ & 56 & 46 \\
\hline $\begin{array}{l}\text { Difference between gas tem- } \\
\text { perature and dew point }(\mathrm{K})\end{array}$ & 8.4 & 8.3 \\
\hline Gas pressure (kPa [gage]) & 5.6 & 8.2 \\
\hline $\begin{array}{l}\text { Gas composition other than } \\
\text { methyl iodide }\end{array}$ & Steam $100 \%$ & Steam $100 \%$ \\
\hline $\begin{array}{l}\text { Temperature of silver zeolite } \\
\text { before testing }\end{array}$ & $\begin{array}{l}\text { Room } \\
\text { temperature }\end{array}$ & $\begin{array}{l}\text { Room } \\
\text { temperature }\end{array}$ \\
\hline
\end{tabular}




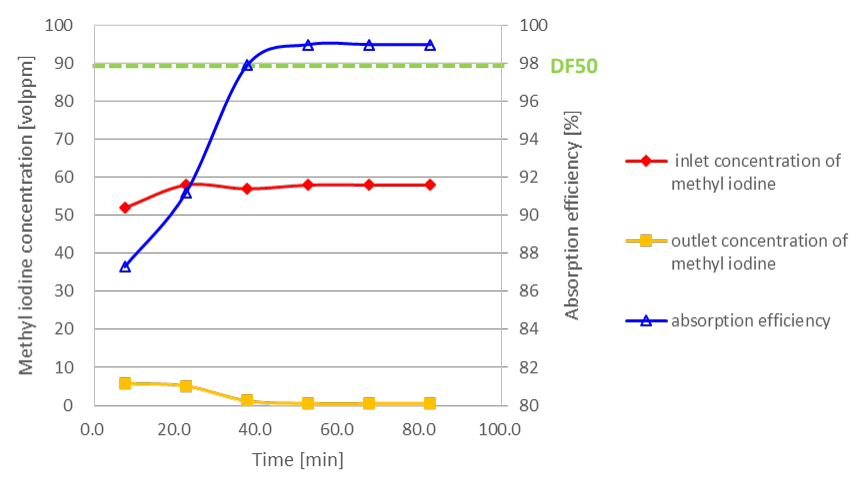

Figure 15 Results of methyl iodide adsorption performance test $\mathrm{C}-1$ Test conditions are listed in Table 6 as test number $\mathrm{C}-1$.

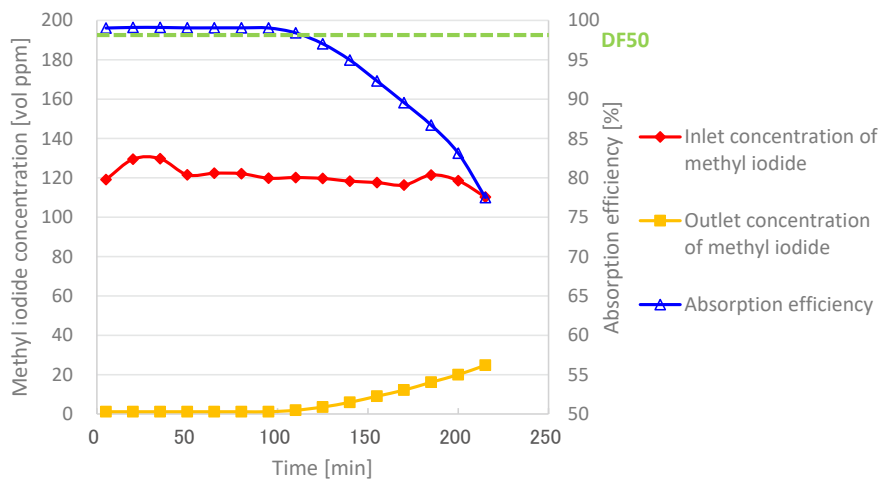

Figure 16 Results of methyl iodide adsorption performance test C-2 Test conditions are listed in Table 6 as test number $\mathrm{C}-2$.

starting condition. As a result, it was found that the target performance can almost be satisfied continuously from the start of venting, as shown in Figure 16, by reducing the gas flow rate up to the condition of test $\mathrm{C}-2$ and lengthening the contact time.

\section{(7) Discussion on operation management of the organic iodine filter}

The test results in III-1-(5) showed that the silver zeolite should be in a dry state in the initial stage of the venting. Therefore, management is necessary to keep the interior of the iodine filter dry when it is in standby. For this reason, it was decided that operation management should be conducted to isolate the front and rear of the filter, which is in a standby state with a rupture disk at a low-pressure setting. The inside of the filter is filled with nitrogen gas and kept in a dry state. In addition, the silver zeolite is removed from the inside of the iodine filter from time to time to check its condition.

Also, from the test result in III-1-(6), it was found that if venting is temporarily stopped, water vapor condenses due to heat dissipation, the silver zeolite becomes wet, and the adsorption performance is affected when venting restarts. However, for the venting of the containment vessel, since the atmosphere is expected to act as a heat sink and cool the containment vessel, venting should be continued until the containment vessel is able to resume cooling, and venting becomes unnecessary. Therefore, suspending venting and restarting it is not a good idea. However, when the venting is temporarily stopped for some reason, it is desirable to trigger an operation 
procedure, namely narrowing the opening of the adjustment valve of the vent system at the time of the venting restart, before the gas starts to flow, so that the desired performance level of the organic iodine filter is continuously secured. However, prevention of containment vessel breakage due to pressure rise takes priority, even in this case, while monitoring the tendency of the containment vessel pressure, so the opening of the valve is narrowed when the pressure decreases as a result of the venting. The findings of this study indicate that it is not necessary to fully open the valve at the time of the venting restart, so an operation based on pressure monitoring is beneficial.

Furthermore, it was found from the adsorption stability verification test of III-1-(3) that several percent of absorbed iodine may be desorbed if silver iodide is reduced by hydrogen after venting. It is important to flow the nitrogen gas to the organic iodine filter after venting, discharge the scrubber water of the upstream FCVS filter device to the containment vessel, fill in fresh water and suppress the generation of hydrogen by radiolysis of water.

\section{Filter Unit Test}

\section{(1) Outline of test facility}

It was confirmed by the previous tests that the packed bed of silver zeolite can produce a DF of 50 or greater by ensuring adequate contact time. However, since the flow velocity of the gas passing through the candle-type filter unit may not necessarily be uniform, we conducted a test using a candle-type filter unit with the same size as the actual device, and determined the distribution of the passing flow velocity. We also examined the relationship between the passing flow rate in the candle-type filter unit and the pressure loss, using the characteristics thereof to evaluate the flow rate deviation between the candle-type filter units by numerical analysis.

The outline of the test facility is shown in Figure 17. Candle-type filter units of the same size as the actual device are installed in the test chamber. Steam generated by the boiler is supplied from the upper part of the chamber like the actual organic iodine filter. It passes through the candle-type filter unit from the outside to the inside, then is discharged from the lower part of the chamber to outside the system. The amount of steam to be supplied can be set to the maximum steam flow rate per candle-type filter unit, as the actual device is designed for. An anemometer (0965-04 made by Kanomax Japan Inc) is inserted inside the candle-type filter

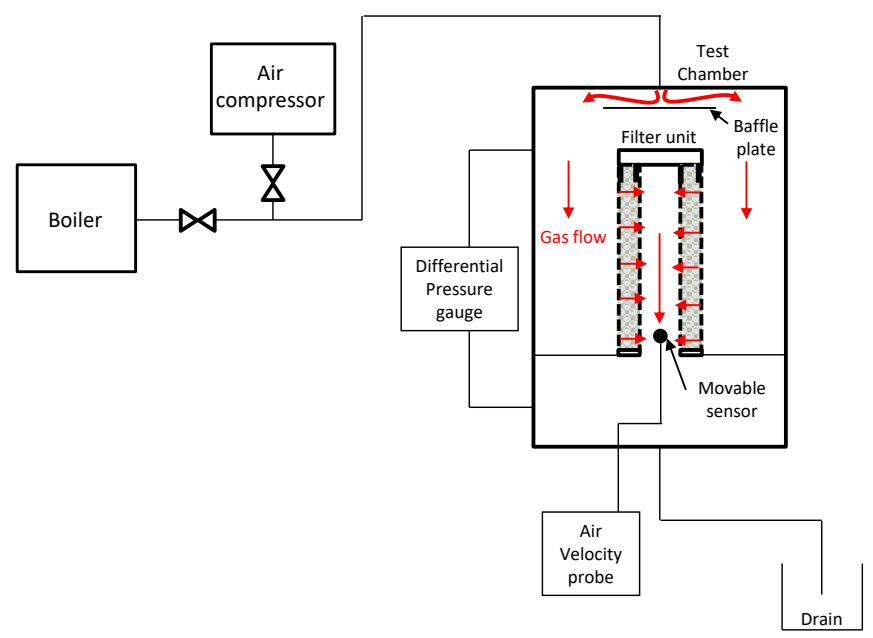

Figure 17 The filter unit test facility 
unit to measure the flow velocity by moving up and down along the central axis.

This makes it possible to track the distribution of the velocity of the steam flowing downward in the candle-type filter unit inner cylinder, after passing through the silver-zeolite-packed bed in the candle-type filter unit. When using this anemometer, we changed the steam generation rate of the boiler, confirmed that the calculated steam flow velocity value and the measured value were in a linear relationship, and judged this to be effective for the purpose of determining the relative velocity distribution within this test range.

\section{(2) Test result and evaluation}

Measurement of the gas velocity distribution when the steam flowed with the maximum steam flow rate per candle-type filter unit is shown in Figure 18. The vertical axis in this figure shows the non-dimensional flow velocity, using the flow velocity at the outlet of the candle-type filter unit as the reference. A lower position in the inner cylinder of the candle-type filter unit corresponds to a higher gas velocity, which changes almost linearly according to the height. That is, the velocity of the gas that passes through the packed bed in the inner cylinder of the candle-type filter unit increases linearly towards the outlet in the lower portion of the cylinder while being nearly uniformly added, and the flow rate of the gas that passes through the silver-zeolite-packed bed of the candle-type filter unit from the outside to the inside is almost uniform in the unit, regardless of the height.

Furthermore, since 19 candle-type filter units are installed in one filter vessel in the actual device, we evaluated the flow distribution by three-dimensional flow analysis using the general-purpose analysis code STAR-CCM+. STAR-CCM+, including its predecessor STAR-CD, is a general-purpose code that is widely used in flow analysis.

In this analysis, since the Mach number of the filter vessel inlet piping is about 0.2 at the maximum, the pressure change in the inlet piping and the filter vessel is about $5 \%$, and the concentration change is small, so the mass conservation equation and the momentum conservation equation are treated as an uncompressed steady flow. Also, the turbulence model is RANS type (Realizable k- $\varepsilon$ (two-layer type)), in which the handling of wall effects is based on the Ally+ model, which is a hybrid of a low-Reynolds-type model and a high-Reynolds-type model.

The model comprises one filter vessel and its inlet/outlet piping. From the results of the candle-type filter unit test, the correlation was obtained between the calculated value of the average velocity of the gas passing through the silver-zeolite-packed bed and the pressure loss. Giving this characteristics to the porous media by setting pressure loss coefficient per unit length, the candle-type filter unit was modeled. The analysis mesh is shown in Figure 19. We adopted a polyhedral cell, which is considered to be in good conformity with the shape and of generally

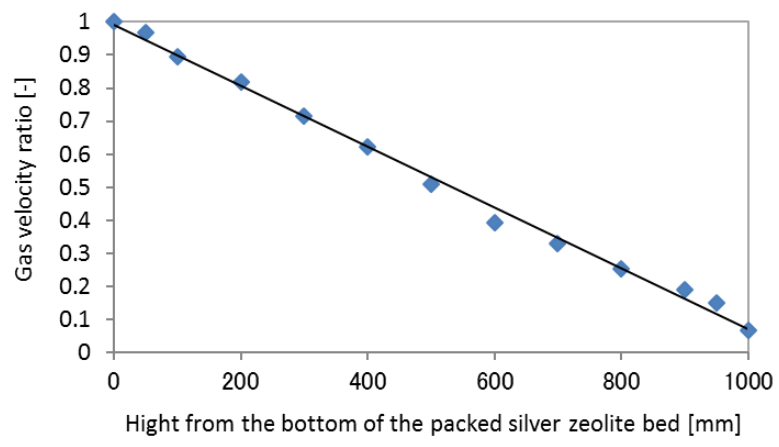

Figure 18 Gas velocity distribution along the centerline of a filter unit 
high calculation convergence and stability. Furthermore, prism cells in the form of thin plates were arranged in the vicinity of the wall surface to obtain the appropriate wall shear force. As for the boundary conditions, the velocity is given for the inlet pipe, and the pressure condition is given for the outlet pipe. As the composition of the venting gas is mostly water vapor, except during a short period of time immediately after the initiation of venting, the fluid is treated as approximately $100 \%$ water vapor and assigned the physical properties thereof.

The flow velocity distribution on the longitudinal cross section in the center of the filter vessel is shown in Figure 20, from analysis of the maximum flow rate of the actual device for one filter vessel (fluid: water vapor, volumetric flow rate: $8 \mathrm{~m}^{3} / \mathrm{s} / \mathrm{unit}$, mass flow rate: $7.9 \mathrm{~kg} / \mathrm{s} / \mathrm{unit}$ ). Gas introduced from the upper nozzle of the vessel is bent in the direction of the wall surface of the vessel by the baffle plate right under the nozzle, and turns into a complicated flow in the space above the candle-type filter unit. However, the gas flows almost uniformly into each

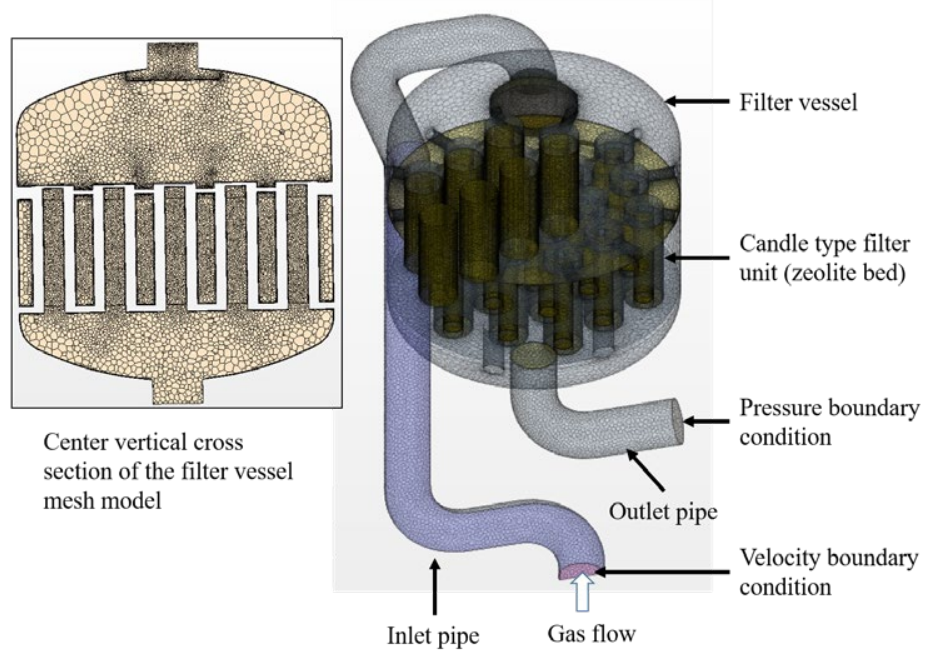

Figure 19 An analysis mesh for the organic iodine filter and its inlet and outlet pipes

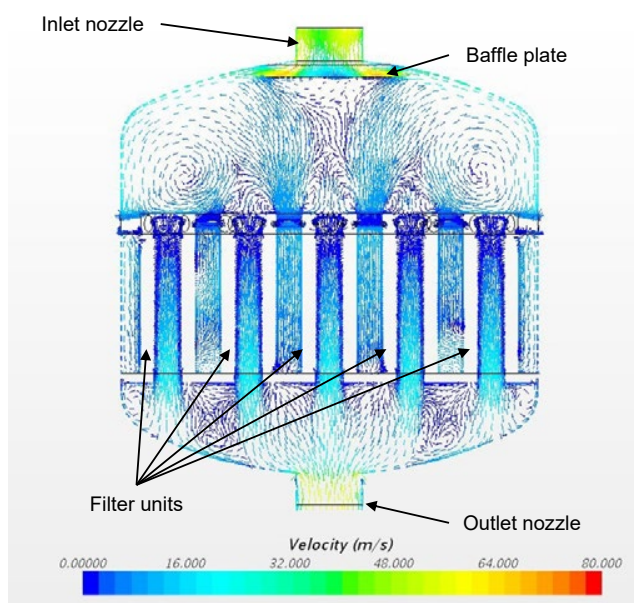

Figure 20 A numerical analysis result showing gas velocity distribution in the center cross-section of the organic iodine filter 
candle-type filter unit, and the flow velocity distribution inside the candle-type filter unit exhibits almost the same tendency among all the units. The flow rate in each candle-type filter unit and the deviation from the average value is shown in Table 7. The variation in the flow rate is within $\pm 1.0 \%$. Figure 21 shows the velocity distribution in the vertical direction of the gas passing through the packed bed in each candle-type filter unit. The variation is $\pm 11 \%$.

To provide a margin based on this result, we set the velocity variation for the gas passing through the packed bed to $15 \%$ in our design. When venting of the containment vessel is

Table 7 Numerical analysis result showing flow distribution among the filter units at the maximum venting flow conditions

\begin{tabular}{ccc}
\hline $\begin{array}{c}\text { Filter unit } \\
\text { identification number }^{\mathrm{a})}\end{array}$ & $\begin{array}{c}\text { Mass flow } \\
(\mathrm{kg} / \mathrm{s})\end{array}$ & $\begin{array}{c}\text { Deviation from the } \\
\text { average }(\%)\end{array}$ \\
\hline 11 & 0.411 & 0.4 \\
12 & 0.411 & 0.4 \\
13 & 0.412 & 0.7 \\
21 & 0.407 & -0.6 \\
22 & 0.409 & -0.2 \\
23 & 0.411 & 0.3 \\
24 & 0.409 & -0.1 \\
31 & 0.408 & -0.5 \\
32 & 0.408 & -0.5 \\
33 & 0.411 & 0.4 \\
34 & 0.409 & -0.2 \\
35 & 0.408 & -0.4 \\
41 & 0.409 & -0.2 \\
42 & 0.411 & 0.3 \\
43 & 0.410 & 0.1 \\
44 & 0.406 & -0.8 \\
51 & 0.411 & 0.4 \\
52 & 0.411 & 0.3 \\
53 & 0.411 & 0.3 \\
\hline
\end{tabular}

a) Locations and identification numbers of filter units are shown in Fig. 2.
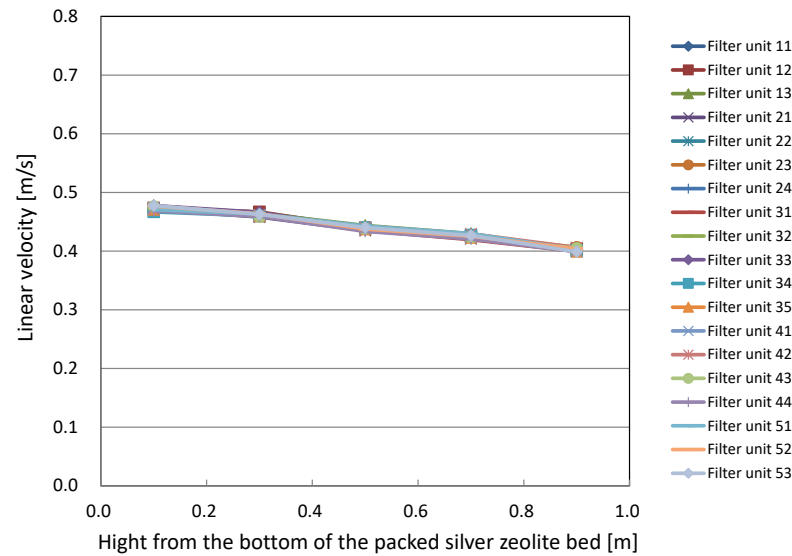

Figure 21 Numerical analysis results of the distribution of linear velocity of the gas passing through packed silver zeolite bed in candle type filter units at the maximum venting flow conditions 
performed when the containment vessel reaches twice the maximum working pressure, defined as the pressure limit in the case of an accident, the contact time of the gas in the silver zeolite packed bed in the organic iodine filter is 0.222 seconds, as shown in Table 2 . It is necessary to ensure the required adsorption performance with a contact time of 0.188 seconds because of the $15 \%$ velocity variation in our design. In the adsorption performance tests shown in Table 3 and Table 5, it has been confirmed that the DF is 50 or greater, and the allowable adsorption load is adequate, though in all cases, the target performance is achieved with a contact time less than 0.188 seconds. Therefore, it is thought that adequate performance can be secured even though there is velocity variation in the filter.

\section{Conclusions}

The development of an FCVS and introduction of the actual device are underway to protect the containment vessel from over-pressure damage as well as to considerably reduce the emission of radioactive substances at the time of severe accidents at nuclear power plants. In this study, to curb the emission of organic iodine into the environment, since it cannot be captured by a scrubber or a metal filter, we have developed a filter using silver zeolite as an absorbent, which is $13 \mathrm{X}$ crystalline zeolite as its basic skeleton with sodium ions in the aluminosilicate skeleton exchanged with the silver ions.

In the organic iodine adsorption test, we simulated the venting gas composition and vapor conditions at the time of venting of the containment vessel and measured the DF for methyl iodide. The result showed that an adsorption performance with a DF of 50 or greater is ensured by appropriately setting the contact time in the silver-zeolite-packed bed.

Also, it was found that although the allowable adsorption load declines due to condensation of steam or the presence of hydrogen at the start of venting, the DF can be kept at 50 or greater by maintaining the silver zeolite in a dry state during standby.

The findings obtained from these tests were reflected in the operation management of the venting. First, it is important to keep the inside of the filter dry during standby. It was also found that when the silver zeolite is wet after venting commences, the performance will temporarily deteriorate until it is dried again, which is reflected in the operation strategy of venting to secure a stable DF. Furthermore, it was found that when hydrogen flows into the silver-zeolite-packed bed after iodine adsorption, several percent of the absorbed iodine may be desorbed. Therefore, measures are taken in the operation management to replace the scrubber water of the FCVS filter device installed upstream at an appropriate time, and to suppress the generation of hydrogen by radiolysis of the water.

In the filter unit test, we determined the velocity distribution of the gas passing through the packed bed using a unit with the same structure and size as those of the actual device, performed flow analysis in the filter vessel, and confirmed that the contact time of the gas passing through the silver-zeolite-packed bed is longer than the contact time for which the required performance was confirmed in the organic iodine adsorption test. Based on the above, the filter developed in this study was evaluated to have a DF of 50 or greater for organic iodine. This organic iodine filter is being introduced to the unit $6 \& 7$ reactors at the Kashiwazaki Kariwa Nuclear Power Station.

We would like to express our deepest gratitude to Ms. Yoko Umeda of TEPCO Research Institute for her valuable advice in this study, and Mr. Toru Ida and Mr. Takashi Tachikawa of Kobelco Research Institute Inc. for their collaboration in the tests. 


\section{References}

1) S. Kawamura, S. Omori, T. Kimura, T. Takiguchi, T. Narabayashi, "A comprehensive approach to reducing radioactive material release in BWR containment venting," Proc. 20th Natl. Symp. on Power and Energy Systems, Sendai, Japan, Jun. 18-19, 2015 (2015). [in Japanese]

2) T. Narabayashi, S. Kawamura, "Development of high efficiency filtered containment venting system by using AgX," Proc. 20th Natl. Symp. on Power and Energy Systems, Sendai, Japan, Jun. 18-19, 2015 (2015). [in Japanese]

3) S. Kawamura, T. Kimura, "Filtered venting system no kaihatsu to Kashiwazaki Kariwa unit 6, 7 heno tekiyou," Energy Rev., 2014[11], 20-24 (2014). [in Japanese]

4) S. Kawamura, T. Kimura, S. Omori, T. Narabayashi, "Development of a filtered containment venting system for nuclear power plants," Trans. At. Energy Soc. Jpn, J14.036, Advance publication by J-Stage (2015). [in Japanese]

5) Tokyo Electric Power Company, Fukushima Nuclear Accident Analysis Report (2012).

6) T. Kanai, M. Furuya, T. Arai, Y. Nishi, K. Shirakawa, N. Tanaka, "Sophistication of filtered containment venting system operation,(5) Decontamination performance of iodine," Proc. 2015 Annu Meeting of AESJ, 20th Natl. Symp. on Power and Energy Systems, Hitachi, Japan, Mar. 20-22, 2015 (2015). in Japanese]

7) T. Ishii, N. Sato, A. Kirishima, D. Akiyama, T. Narabayashi, "Sorption of radioactive methyl iodide by silver doped zeolite for filtered venting system," Proc. 20th Natl. Symp. on Power and Energy Systems, Sendai, Japan, Jun. 18-19, 2015 (2015). [in Japanese]

8) T. Narabayashi, T. Aida, T. Kanai, T. Konno, S. Ito, "Robustization of high efficiency filtered containment venting system by using AgX," Proc. 20th Natl. Symp. on Power and Energy Systems, Sendai, Japan, Jun. 18-19, 2015 (2015). [in Japanese]

9) T. Kobayashi, J. Wang, Y. Uzuyama, K. Endo, "Application of AgX radioiodine adsorbent as severe accident countermeasures," Proc. 20th Natl. Symp. on Power and Energy Systems, Sendai, Japan, Jun. 18-19, 2015 (2015). [in Japanese]

10) OECD/NEA/CSNI, Status Report on Filtered Containment Venting, NEA/CSNI/R (2014) 7 (2014).

11) Japan Society of Mechanical Engineers, Kikaikougakubinran Kaiteidai6ban, Tokyo (1977). [ in Japanese]

12) U. S. Nuclear Regulatory Commission, Methods and Assumptions for Evaluating Radiological Consequences of Design Basis Accidents at Light-water Nuclear Power Reactors, Regulatory Guide 1.195 (2003).

13) L. Soffer, S. Burson, C. Ferrell, R. Lee, J. Ridgely, Accident Source Terms for Light-water Nuclear Power Plants, U. S. Nuclear Regulatory Commission, NUREG-1465 (1995).

14) E. Beahm, C. Weber, T. Kress, G. Parker, Iodine Chemical Forms in LWR Severe Accidents, U. S. Nuclear Regulatory Commission, NUREG/CR-5732 (1992). 